\title{
THERMODYNAMIC MODELING OF THE AWE RADIOACTIVE AQUEOUS WASTE TREATMENT PLANT EVAPORATOR
}

April 2003

C. D. Barnes, T. B. Calloway, Jr. \& R. E. Eibling

Westinghouse Savannah River Company

Savannah River Technology Center

Funded by AWE/MOD, UK

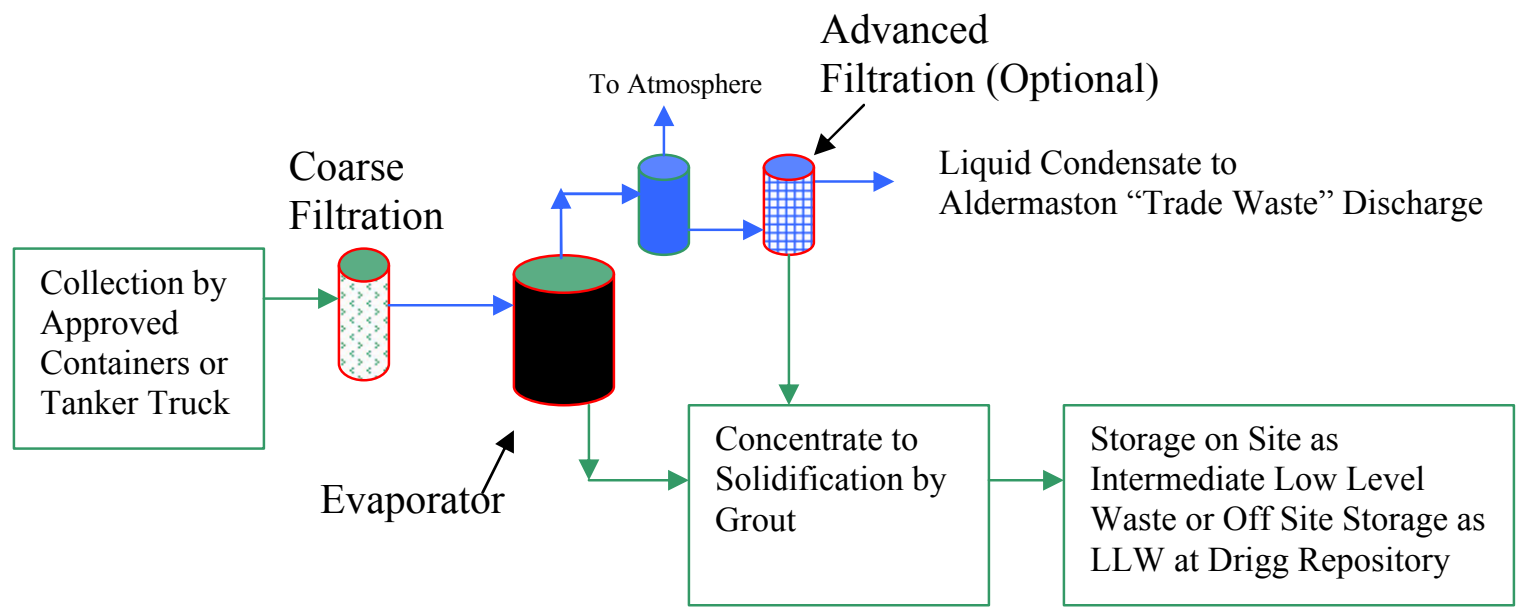

AWE-Aldermaston - Radioactive Aqueous Waste Treatment Plant -

Simplified Flowsheet

Westinghouse Savannah River Company

Savannah River Site

Aiken, SC 29808

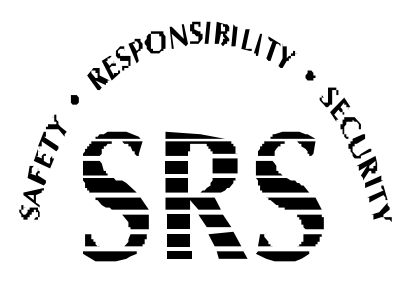

SAVANNAH RIVER SITE 
This document was prepared in conjunction with work accomplished under Contract No. DE-AC09-96SR18500 with the U. S. Department of Energy.

\section{DISCLAIMER}

This report was prepared as an account of work sponsored by an agency of the United States Government. Neither the United States Government nor any agency thereof, nor any of their employees, makes any warranty, express or implied, or assumes any legal liability or responsibility for the accuracy, completeness, or usefulness of any information, apparatus, product or process disclosed, or represents that its use would not infringe privately owned rights. Reference herein to any specific commercial product, process or service by trade name, trademark, manufacturer, or otherwise does not necessarily constitute or imply its endorsement, recommendation, or favoring by the United States Government or any agency thereof. The views and opinions of authors expressed herein do not necessarily state or reflect those of the United States Government or any agency thereof.

This report has been reproduced directly from the best available copy.

Available for sale to the public, in paper, from: U.S. Department of Commerce, National Technical Information Service, 5285 Port Royal Road, Springfield, VA 22161, phone: (800) 553-6847, fax: (703) 605-6900

email: orders@ntis.fedworld.gov

online ordering: http://www.ntis.gov/help/index.asp

Available electronically at http://www.osti.gov/bridge

Available for a processing fee to U.S. Department of Energy and its contractors, in paper, from: U.S. Department of Energy, Office of Scientific and Technical Information, P.O. Box 62, Oak Ridge, TN 37831-0062,

phone: (865)576-8401,

fax: (865)576-5728

email: $\underline{\text { reports@ adonis.osti.gov }}$ 
WSRC-TR-2002-00567

Revision 0

Table of Contents

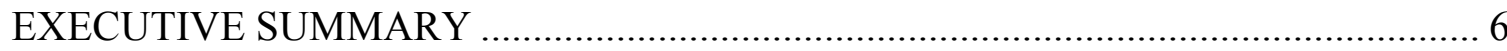

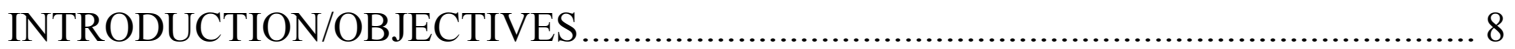

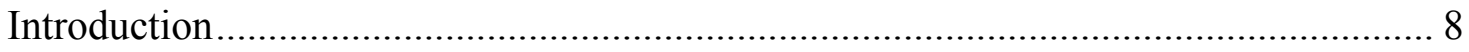

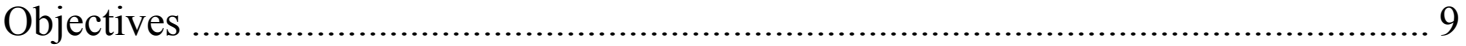

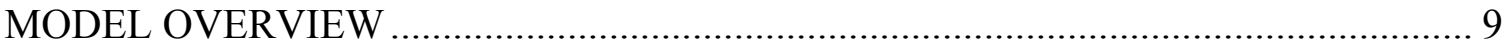

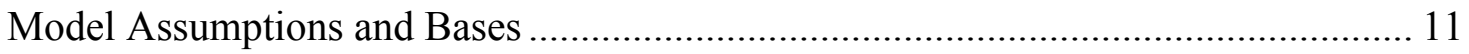

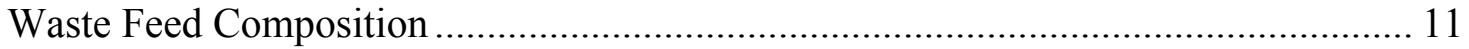

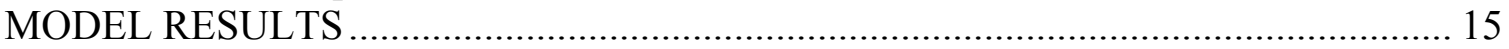

Composition of Evaporator Feed, Concentrate and Condensate ................................ 15

Concentration Factor at a Predefined Solubility Point for Insoluble Solids $=0.5 \%$... 15

Effect of Concentration on Mass Faction of Insoluble Solids formed in the Evaporator

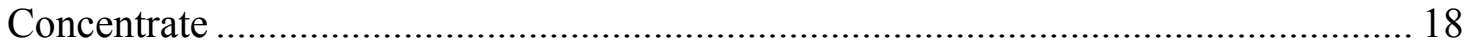

Correlation between Concentration and Density in the Evaporator Concentrate ......... 21

Feed Stock pH Comparison to Model Output............................................................ 23

Predicted Composition of Insoluble Solids in Concentrated RAWTP Waste Streams 24

Partial Validation of the OLI ESP Public Database for the $\mathrm{CaCO}_{3}$-Water and $\mathrm{CaSO}_{4}$ -

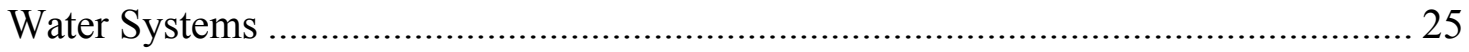

CONCLUSIONS/RECOMMENDATIONS ........................................................... 28

APPENDIX A - Predicted Composition of Insoluble Solids in the RAWTP Evaporator

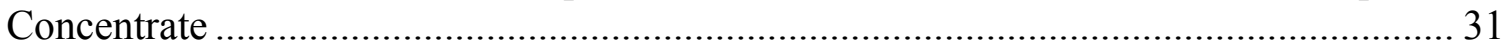

APPENDIX B - Composition of Evaporator Feed, Concentrate and Condensate for AWE

Waste - Material and Energy Balance OLI Output Files................................................. 34

AWE Low Foam Simulant - Concentrated to 30 wt. \% Total Solids ........................... 34

AWE High Foam Simulant - Concentrated to 30 wt.\% Total Solids........................... 58

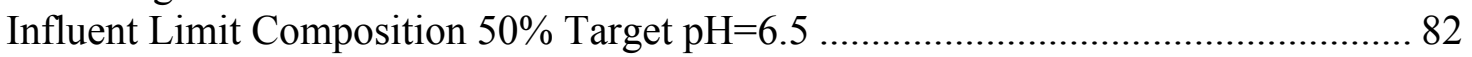

Influent Limit Composition 60\% Target $\mathrm{pH}=6.5$................................................... 113

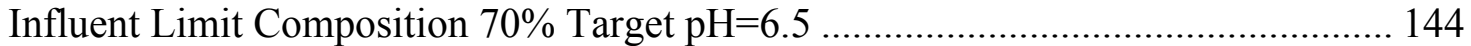

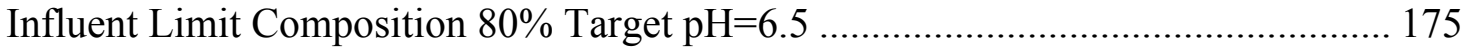

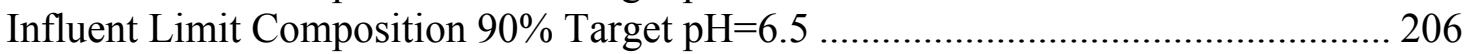

Influent Limit Composition 95\% Target $\mathrm{pH}=6.5$ ……........................................... 237

Influent Limit Composition 50\% Target $\mathrm{pH}=8.5$ ……........................................ 268

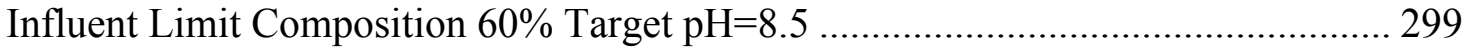

Influent Limit Composition 70\% Target $\mathrm{pH}=8.5$ …….......................................... 330

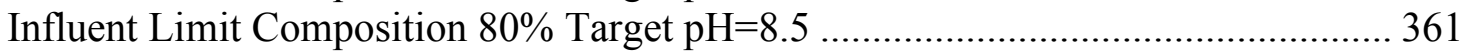

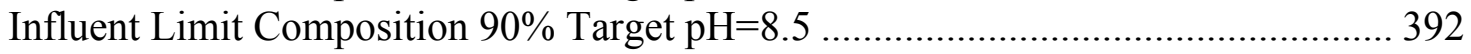

Influent Limit Composition 95\% Target $\mathrm{pH}=8.5$..................................................... 423 
WSRC-TR-2002-00567

Revision 0

\section{Table of Figure and Tables}

Table 1 - Predicted Limit Compositions of Future RAWTP Waste Streams at pH 6.5.. 12 Table 2 - Predicted Limit Compositions of Future RAWTP Waste Streams at pH 8.5.. 13

Table 3 - Cation and Anion Composition of Standard and High Foam Simulant ........... 14

Table 4 - Volume Reduction Factor at the Defined Precipitation Point (\% insoluble

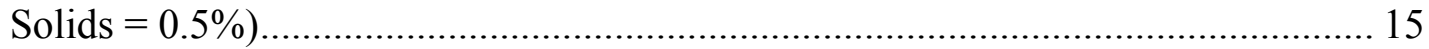

Table 5 - Predicted pH of AWE Waste Effluent and Simulants .................................... 23

Table 6 - Predicted Percentage of Carbonate and Dissolved Carbon Dioxide in the Feed

Evolving as Carbon Dioxide for the High Foam Simulant Case ............................ 25

Table 7 - Literature Solubility Values of $\mathrm{CaCO} 3$ and $\mathrm{CaSO} 4$ in water as compared to

OLI ESP Publix Database.

Table 8 - Composition of Insoluble Solids Formed in the Evaporator Concentrate for

Evaporator Feed Case $80 \%$ Waste Effluent $\mathrm{pH}=6.5$ and 8.5 as a function of

Evaporator Concentration Endpoint (0.1, 0.5, 1, 5 \% Insoluble Solids and 10, 20 and

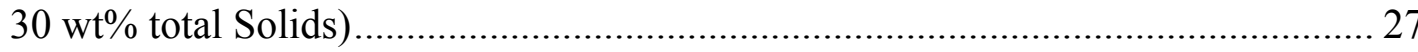

Table 9 - Insoluble Solids Formed in the Evaporator Concentrate for Feed Case 50-95\%

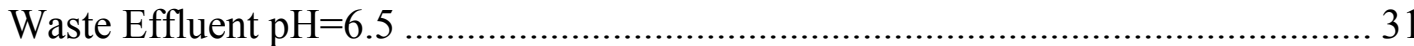

Table 10 - Insoluble Solids Formed in the Evaporator Concentrate for Feed Case 50-95\%

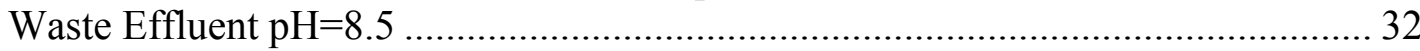

Table 11 - Composition of Insolubles Solids Formed During Evaporation of the Low and

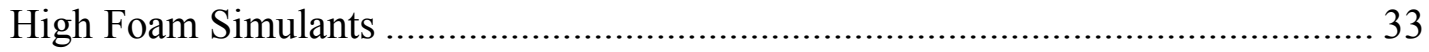

Figure 1 - Radioactive Aqueous Waste Treatment Plant - Simplified Flowsheet............ 8

Figure 2 - Schematic of AWE Evaporator Model - OLI ESP Software Blocks Names

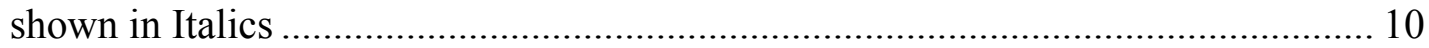

Figure 3 - Structure of Lauric Acid (Dodecanoic acid) ............................................. 14

Figure 4 - Volume Reduction Factor at the Defined Solubility Point (\% Insoluble Solids $=0.5)$ as a Function of AWE Limit Composition for Feed Case $\mathrm{pH}=6.5 \& 8.550$ $95 \%$

Figure 5 - Volume Reduction Factor as a Function of Insoluble Solids in the Evaporator Concentrate - Case RAWTP pH=6.5 for Evaporator Feed - Concentrate Target Endpoints of 0.1, 0.5, 1, 5\% Insoluble Solids and 10, 20 and 30 wt. \% Total Solids in Concentrate

Figure 6 - Volume Reduction Factor as a Function of Insoluble Solids in the Evaporator Concentrate - Case RAWTP $\mathrm{pH}=8.5$ for Evaporator Feed - Concentrate Target Endpoints of 0.1, 0.5, 1, 5\% Insoluble Solids and 10, 20 and 30 wt. \% Total Solids in Concentrate....

Figure 7 - Mass Fraction Insoluble Solids in the Evaporator Concentrate as a Function of Total Solids in the Evaporator Concentrate - Case RAWTP $\mathrm{pH}=6.5$ for Evaporator Feed Concentrate Target Endpoint of 0.1, 0.5, 1, 5\% Insoluble Solids and 10, 20 and 30 wt. \% Total Solids in Concentrate

Figure 8 - Mass Fraction Insoluble Solids in the Evaporator Concentrate as a Function of Total Solids in the Evaporator Concentrate - Case RAWTP $\mathrm{pH}=8.5$ for Evaporator Feed Concentrate Target Endpoint of 0.1, 0.5, 1, 5\% Insoluble Solids and 10, 20 and 30 wt. \% Total Solids in Concentrate 
Figure 9 - Mass Fraction Insoluble Solids in the Evaporator Concentrate as a Function of Total Solids in the Evaporator Concentrate - Case High and Low Foam Simulant Concentrated to 10,20 and $30 \mathrm{wt}$ \% total solids

Figure 10 - DOW 1520-US Antifoam Floating on Simulated Hanford Envelope C Salt

Waste.

Figure 11 - Density of the Evaporator Concentrate as a Function of Total Solids in the Evaporator Concentrate - Case RAWTP $\mathrm{pH}=6.5$ for Evaporator Feed Concentrate Target Endpoints of 0.1, 0.5, 1, 5\% Insoluble Solids and 10, 20 and 30 wt. \% Total Solids in Concentrate

Figure 12 - Density of the Evaporator Concentrate as a Function of Total Solids in the Evaporator Concentrate - Case RAWTP $\mathrm{pH}=8.5$ for Evaporator Feed Concentrate Target Endpoints of 0.1, 0.5, 1, 5\% Insoluble Solids and 10, 20 and $30 \mathrm{wt}$ \% Total Solids in Concentrate.

Figure 13 - Density of the Evaporator Concentrate as a Function of Total Solids in the Evaporator Concentrate - Case High and Low Foam Simulant Concentrated to 10, 20 and $30 \mathrm{wt}$ \% total solids 
WSRC-TR-2002-00567

Revision 0

\section{EXECUTIVE SUMMARY}

Operation of the proposed AWE Aldermaston ${ }^{1}$ Radioactive Aqueous Waste Treatment Plant evaporation system was modeled using the Environmental Simulation Program (ESP) licensed by OLI Systems, Inc. The projected RAWTP waste influents as well as two simulants (High Foam and Low Foam) were modeled to predict the composition of the feed, concentrate and condensate for projected waste influents.

Over ninety simulations were conducted and the chemical and physical properties for twelve projected waste streams and two waste simulants were calculated. Mathematical models were generated that relate the following physical parameters:

- Volume reduction factor as function of insoluble solids formed in the evaporator bottoms.

- Insoluble solids formed as a function of solids concentration in the evaporator bottoms.

- Product density as a function solids concentration in the evaporator.

The model predicted that higher volume reduction factors are obtained at the defined solubility point ( $0.5 \%$ insoluble solids in the evaporator bottoms) with acidic waste effluents (Feed Case $\mathrm{pH}=6.5)$ as compared to alkaline waste effluents $(\mathrm{pH}=8.5)$. The higher volume reduction factors are due to the higher solubility of the major analytes in acidic solutions as compared to alkaline solutions. Therefore, it is recommended that pretreatment of the RAWTP waste streams with a suitable acid (e.g. $\mathrm{HNO}_{3}$ ) and subsequent treatment of the concentrate with $\mathrm{NaOH}$ prior to grouting should be investigated as an option to evaporation of alkaline wastes.

$\mathrm{Ca}_{3}\left(\mathrm{PO}_{4}\right)_{2}$ and $\mathrm{CaSO}_{4} \cdot 2 \mathrm{H}_{2} \mathrm{O}$ are predicted to be the major insoluble species formed in the RAWTP evaporator. Generally, carbonate will evolve as $\mathrm{CO}_{2}$ in acidic to slightly basic solutions. Carbonate and dissolved $\mathrm{CO}_{2}(>99 \%)$ were predicted to evolve as carbon dioxide in the Future RAWTP waste streams. A greater percentage of the feed carbon was predicted to precipitate in the AWE High Foam Simulant as carbonate $(\approx 5 \%)$, but this is due its lower initial feed concentration; the actual carbonate concentrations and precipitated solids of the concentrated High and Low Foam simulants are very similar. Chamosite 7A $\left(\mathrm{Fe}_{2} \mathrm{Al}_{2} \mathrm{SiO}_{5}(\mathrm{OH})_{4}\right)$, is predicted to form in the RAWTP evaporator. Based upon SRS experience with evaporation, the formation of complex Fe-Al-Si minerals in the AWE evaporator could present a scaling problem on the evaporator heat exchanger

\footnotetext{
${ }^{1}$ The main center for warhead research and manufacture is located about 12 miles from the town of Reading (West of London). AWE's main site at Aldermaston has been operating since 1952, originally as part of the UK Atomic Energy Authority, more recently as government-owned, contractor-operated facility. Aldermaston employs some 5000 people and the facility covers 880 acres. AWE-Aldermaston is currently operated by an equal partnership between Lockheed Martin, BNFL Ltd.and Serco plc The AWE-Burgfield site also located in Berkshire is primarily involved with the assembly and maintenance of the British nuclear stockpile.
} 
WSRC-TR-2002-00567

Revision 0

surfaces. SRTC recommends that AWE conduct evaporator cleaning studies with the concentrated simulants obtained from pilot testing to determine suitable chemical cleaning agents and procedures for the RAWTP. Uranium was predicted to be insoluble in the RAWTP wastes. 
WSRC-TR-2002-00567

Revision 0

\section{INTRODUCTION/OBJECTIVES}

\section{$\underline{\text { Introduction }}$}

The Atomic Weapons Establishment Aldermaston site currently discharges radioactive aqueous waste through the Pangbourne pipeline (PPL) that runs underground to the Thames River. Regulatory requirements imposed by the UK Environmental Agency (EA) as well as stakeholder interests have committed AWE to cease discharges to the PPL by April 1, 2005 2,3.

AWE plans to build the Radioactive Aqueous Waste Treatment Plant (RAWTP) to treat the aqueous radioactive waste that is currently being discharged through the PPL. A general schematic of the RAWTP flowsheet is shown in Figure 1.

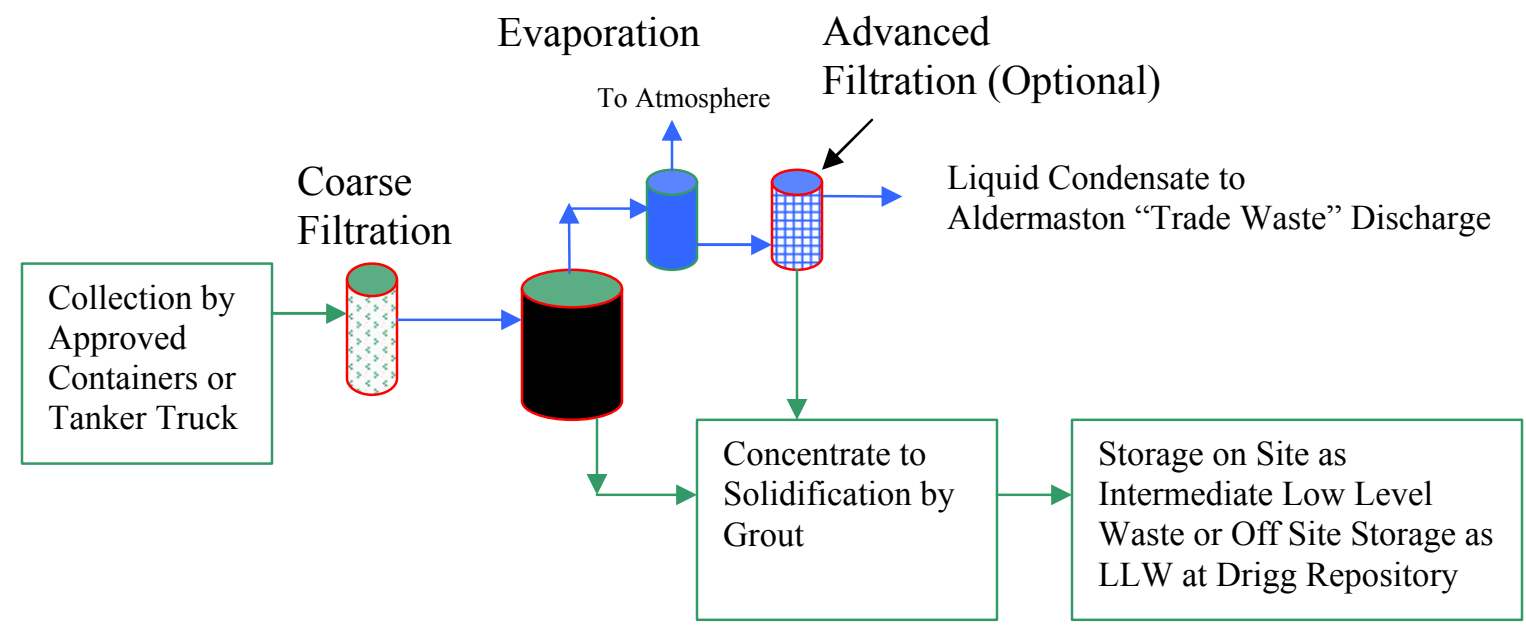

Figure 1 - Radioactive Aqueous Waste Treatment Plant - Simplified Flowsheet

\footnotetext{
${ }^{2}$ Environmental agreements signed by the UK at the Oslo and Paris Convention at Sintra Portugal in July 1998 have committed the UK to reduce radioactive and hazardous waste discharges to the marine environment. The agreement intent with respect to radioactive substances is to achieve "progressive and substantial reduction of discharges, emissions and losses" and "concentrations in the environment to near background values for naturally occurring radioactive substances and close to zero for artificial radioactive substances" ${ }^{4}$.

${ }^{3}$ Crane, A., Alternative to the Pangbourne Pipeline - Options \& Fesibility Study, AWE/DSE07/B/L/RP/EVAP/13.06.89.14/99-11, Issue 1 October 1999, Atomic Weapons Establishment, Aldermaston, Reading Berkshire RG74PR. Selected excerpts from the OSPAR Convention for the Protection of the Marine Environment of the North-East Atlantic - Ministerial Meeting of the OSPAR Commission - Sintra 22-23 July - Sintra Statement Final - Annex 45, Draft Summary Record, OSPAR 1/14/98, Annex 45. A meeting of the Oslo and Paris Commissions at Ministerial level was held in Paris on 21-22 September 1992 (MMC 1992). This meeting was attended by European Ministers responsible for the marine environment. The most important outcome of this Ministerial meeting was the adoption of a new Convention for the Protection of the Marine Environment of the North-East Atlantic (the "OSPAR Convention"), together with a Final Declaration and an Action Plan to guide the future work of the Commissions.
} 
Radioactive aqueous wastes containing low levels of uranium and plutonium from various waste generators on the Aldermaston site will be trucked or shipped by small containers to the RAWTP. The liquid waste would then be filtered and concentrated in an evaporator. The evaporator overheads are further treated by an advanced filtration (e.g. reverse osmosis) and then discharged to the Aldermaston "Trade Waste" plant. The existing "Trade Waste" plant handles mostly chemical hazardous wastes or those wastes containing only very low levels of natural or depleted uranium. The concentrated liquors from the evaporator would be immobilized in a containerized grout waste form. The immobilized waste would be stored on site as intermediate level waste or shipped to the national repository for low level waste at Drigg ${ }^{4}$.

\section{$\underline{\text { Objectives }}$}

The main objective of this work is to model the RAWTP evaporator using OLI Systems, Inc. Environmental Simulation Program (ESP) to predict the composition of the feed, concentrate and condensate for various waste feed composition vectors provided by AWE. Specific model calculations and the results of interest were agreed upon prior to starting this task and are listed below:

- Composition of Evaporator Feed, Concentrate and Condensate waste streams within the composition envelope after concentration of the evaporator feed to $30 \mathrm{wt} \%$ total solids.

- Composition of Evaporator Feed, Concentrate and Condensate for AWE High and Low Foam Simulant after concentration to $30 \mathrm{wt} \%$ total solids.

- Concentration Factor at a predefined solubility point ( $\%$ insoluble solids $=0.5 \mathrm{wt} \%$ in evaporator concentrate),

- Determine the effect of concentration on the percent of insoluble solids formed reported as plots of the percent of insoluble solids as a function of total solids in the evaporator concentrate,

- Determine the function of concentration on evaporator concentrate density reported as a function of total solids in the evaporator concentrate,

- Comparison of the Feed stock pH to the target $\mathrm{pH}$ assumed in the AWE waste feed vector.

\section{MODEL OVERVIEW}

Described in this report is a thermodynamic equilibrium model of the RAWTP evaporation process. An evaporator model constructed for the Hanford River Protection Waste Treatment Plant was modified for this task ${ }^{5}$. The model was constructed using

\footnotetext{
${ }^{4}$ Drigg is located near Sellafield on the Cumbrian Coast in Northern England.

${ }^{5}$ Barnes, C. B., Edwards, T. B. \& Choi, A. S., August 2000, Preliminary Modeling Results of Evaporatored Tc - Eluate Physical Properties, WSRC-TR-2002-00319 Rev. 0, Westinghouse Savannah River Company, Aiken SC.
} 
OLI System, Inc Environmental Simulation Program (ESP, Version 6.6). OLI uses a thermodynamic and mathematical framework for predicting the equilibrium properties of a chemical system. The software is used by DOE facilities at Hanford and Savannah River Site to model the aqueous electrolyte chemistry of waste streams generated from the Cold War. The OLI mathematical framework is based upon:

- Revised Helgeson Equation of State for predicting standard-state thermodynamic properties of all species in water;

- the Bromley-Zemaitis framework for prediction of excess thermodynamic properties of ions;

- the Pitzer and Setschenow formulation for prediction of excess thermodynamic properties of molecular species in water;

- the Enhanced SRK Equation of State for prediction of vapor and non-aqueous, liquid phase thermodynamic properties. This equation applies mostly to organics that are sparingly soluble in water.

The predictive framework is partly described in reference ${ }^{6,7}$. The OLI software contains an extensive databank of thermodynamic and physical properties containing over 3,000 inorganic and organic species. The databank supports the predictive framework ${ }^{7}$ ESP is used to simulate, design and optimize chemical processes, including complete process flow sheets. The evaporator model constructed for this effort is shown in Figure 2.

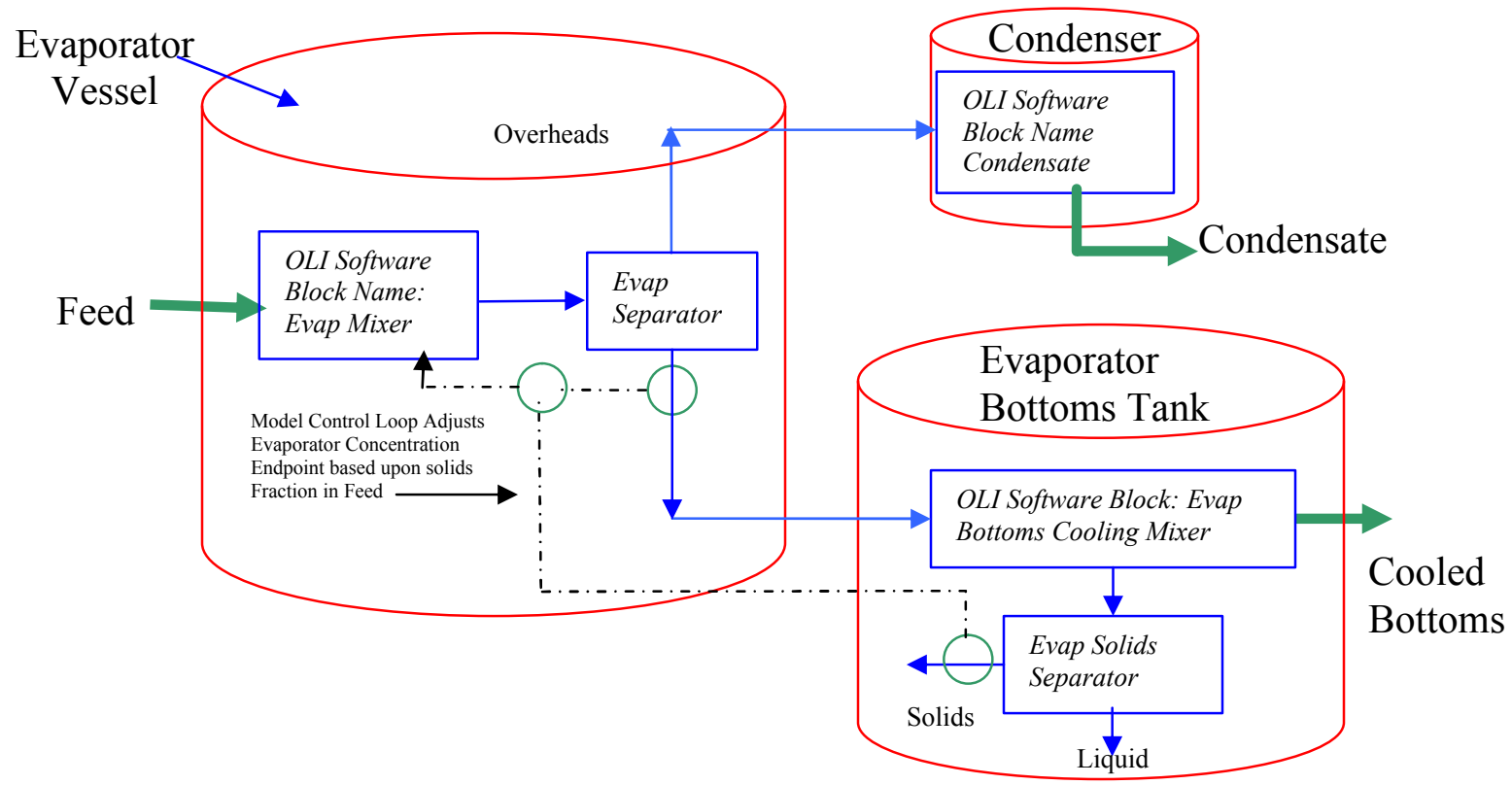

Figure 2 - Schematic of AWE Evaporator Model - OLI ESP Software Blocks Names shown in Italics

${ }^{6}$ Zemaitis, J. F., Clark, D. M., Rafal, M. and Scrivner, N. C., Handbook of Aqueous Electrolyte Thermodynamics, AICHE, Inc., New York, ISBN 0-8169-0350-6. M. Rafal is one the founders and owners of OLI Systems, Inc.

${ }^{7}$ OLI System, Inc., A Guide to Using the OLI Engine, 2002, Version 6.0 Revision 6.6, Morris Plains, NJ 07950 . 
Figure 2 shows the internal OLI/ESP software blocks that are used to simulate an evaporator, condenser and concentrated evaporator liquor bottoms tank. The model includes control loops that allow the user to concentrate the evaporator feed to a desired endpoint based upon the concentration of insoluble solids in the cooled evaporator bottoms. The model output includes the feed, condensate, cooled bottoms, evaporator contents, hot evaporator bottoms and overheads composition.

Model simulations were preformed with target evaporator endpoints of $0.1,0.5,1$, and $5 \mathrm{wt} \%$ insoluble solids in the cooled evaporator bottoms. Additional model simulations were preformed with target evaporator endpoints of 10, 20 and $30 \mathrm{wt} . \%$ total solids in the cooled evaporator bottoms.

\section{$\underline{\text { Model Assumptions and Bases }}$}

The following assumptions and conditions were used for each simulation:

- The evaporator pressure was set to a value slightly below atmospheric pressure (-5 inwc. gauge or $0.987 \mathrm{~atm}$ absolute).

- The evaporator bottoms and condensate were cooled to $25^{\circ} \mathrm{C}, 1 \mathrm{~atm}$.

- The OLI Public databank of chemical properties was used for all modeling runs.

- An evaporator feed rate of $1 \mathrm{~kg} / \mathrm{hr}$ was used for all modeling runs.

\section{$\underline{\text { Waste Feed Composition }}$}

The feed composition was provided by AWE in reference ${ }^{8}$ and summarized in Table 1 and Table 2.. The compositions are based upon an annual radioactive waste generation rate of 1500 cubic meters/yr, and presented in terms of the percentage of individual batches of waste effluent. The design basis case for the RAWTP evaporator is the $80 \%$ case shown in the tables. The compositions listed Table 1 and Table 2 differ only by the concentration of sodium and potassium which are used to charge balance ${ }^{9}$ the stream.

\footnotetext{
${ }^{8}$ Crane, A., Email to Calloway, T. B., FW: Information for Bond Calloway following Conference Call, Atomic Weapons Establishment, Aldermaston, Reading Berkshire RG74PR, 5/16/02.

${ }^{9}$ Reference [8] provided further explanation of the charge balance assumptions used by AWE. The net charge on the carbonate and phosphate anions was calculated from the dissociation constants:-
} 
WSRC-TR-2002-00567

Revision 0

This difference in concentration is due to the shift in distribution of the carbonate and phosphate species (i.e. ratio of carbonate to bicarbonate) between a $\mathrm{pH}$ of 6.5 and 8.5.

Table 1 - Predicted Limit Compositions of Future RAWTP Waste Streams at pH 6.5

\begin{tabular}{|l|r|r|r|r|r|r|}
\hline Case & $50 \%$ & $60 \%$ & $70 \%$ & $\begin{array}{r}80 \% \\
\text { Design } \\
\text { Basis }\end{array}$ & $90 \%$ & $95 \%$ \\
\hline \multicolumn{7}{|c|}{} \\
\hline $\mathrm{Al}$ & 1.7 & 2.1 & 2.4 & 2.8 & 3.2 & 6.1 \\
\hline $\mathrm{Ba}$ & 0.056 & 0.068 & 0.08 & 0.092 & 0.1 & 0.11 \\
\hline $\mathrm{Ca}$ & 29 & 32 & 35 & 40 & 50 & 58 \\
\hline $\mathrm{Cd}$ & 0.001 & 0.0019 & 0.004 & 0.0052 & 0.0066 & 0.0078 \\
\hline $\mathrm{Cl}$ & 47 & 56 & 76 & 100 & 140 & 160 \\
\hline $\mathrm{CO} 3$ & 60 & 66 & 72 & 90 & 100 & 110 \\
\hline $\mathrm{Cr}$ & 0.0028 & 0.0034 & 0.0041 & 0.0063 & 0.0096 & 0.13 \\
\hline $\mathrm{Cu}$ & 0.2 & 0.25 & 0.33 & 0.43 & 0.6 & 0.74 \\
\hline $\mathrm{Fe}$ & 3.1 & 4 & 4.8 & 5.7 & 7.3 & 8.2 \\
\hline $\mathrm{K}$ & 0.0316 & 0.0395 & 0.0521 & 0.0679 & 0.0947 & 0.1168 \\
\hline $\mathrm{Mg}$ & 6 & 6.9 & 8 & 9.2 & 13 & 14 \\
\hline $\mathrm{Na}$ & 9.9019 & 11.387 & 13.203 & 15.183 & 21.454 & 23.104 \\
\hline $\mathrm{Ni}$ & 0.055 & 0.064 & 0.076 & 0.088 & 0.12 & 0.15 \\
\hline $\mathrm{NO} 3$ & 21 & 25 & 29 & 45 & 79 & 110 \\
\hline $\mathrm{Pb}$ & 0.44 & 0.533 & 0.62 & 0.71 & 1.3 & 2.1 \\
\hline $\mathrm{PO} 4$ & 14 & 17 & 20 & 25 & 80 & 110 \\
\hline $\mathrm{SiO} 2$ & 9.9 & 11 & 12 & 14 & 15 & 16 \\
\hline $\mathrm{SO} 4$ & 47 & 56 & 71 & 92 & 120 & 160 \\
\hline $\mathrm{Sr}$ & 0.99 & 1.3 & 1.5 & 1.9 & 2.6 & 2.7 \\
\hline $\mathrm{U}$ & 0.48 & 0.58 & 0.69 & 1 & 1.9 & 2.8 \\
\hline $\mathrm{Zn}$ & 1.4 & 1.7 & 1.9 & 2.5 & 3.3 & 3.7 \\
\hline
\end{tabular}

(Carbonic acid pK $\left(25^{\circ} \mathrm{C}\right) 6.35$ and 10.33; Phosphoric acid pK $\left(25^{\circ} \mathrm{C}\right) 2.16,7.21$ and 12.32). These values were taken from the CRC Handbook, $75^{\text {th }}$ Edition. A pH of 8.5 is about halfway between the $\mathrm{pK}$ for $\mathrm{H}_{2} \mathrm{CO}_{3} / \mathrm{HCO}_{3}{ }^{-}$and the $\mathrm{pK}$ for $\mathrm{HCO}_{3}{ }^{-} / \mathrm{CO}_{3}{ }^{2-}$, so it was assumed that the small amount of $\mathrm{CO}_{3}{ }^{2-}$ present at $\mathrm{pH} 8.5$ would be balanced by the amount of $\mathrm{H}_{2} \mathrm{CO}_{3}$ present. The charge on all the other species was assumed to remain constant as the $\mathrm{pH}$ varied. It was assumed that the metals such as aluminium would be present as $\mathrm{M}^{\mathrm{n}+}$. The amounts of sodium and potassium in the varuous limit compositions were adjusted to maintain an ionic balance. 
WSRC-TR-2002-00567

Revision 0

Table 2 - Predicted Limit Compositions of Future RAWTP Waste Streams at pH 8.5

\begin{tabular}{|l|r|r|r|r|r|r|}
\hline Case & $50 \%$ & $60 \%$ & $70 \%$ & $\begin{array}{c}80 \% \\
\text { Design } \\
\text { Basis }\end{array}$ & $90 \%$ & $95 \%$ \\
\hline \multicolumn{7}{|c|}{} \\
\hline $\mathrm{Al}$ & 1.7 & 2.1 & 2.4 & 2.8 & 3.2 & 6.1 \\
\hline $\mathrm{Ba}$ & 0.056 & 0.068 & 0.08 & 0.092 & 0.1 & 0.11 \\
\hline $\mathrm{Ca}$ & 29 & 32 & 35 & 40 & 50 & 58 \\
\hline $\mathrm{Cd}$ & 0.001 & 0.0019 & 0.004 & 0.0052 & 0.0066 & 0.0078 \\
\hline $\mathrm{Cl}$ & 47 & 56 & 76 & 100 & 140 & 160 \\
\hline $\mathrm{CO} 3$ & 60 & 66 & 72 & 90 & 100 & 110 \\
\hline $\mathrm{Cr}$ & 0.0028 & 0.0034 & 0.0041 & 0.0063 & 0.0096 & 0.13 \\
\hline $\mathrm{Cu}$ & 0.2 & 0.25 & 0.33 & 0.43 & 0.6 & 0.74 \\
\hline $\mathrm{Fe}$ & 3.1 & 4 & 4.8 & 5.7 & 7.3 & 8.2 \\
\hline$K$ & 8.051 & 9.7885 & 13.719 & 20.309 & 33.243 & 42.271 \\
\hline $\mathrm{Mg}$ & 6 & 6.9 & 8 & 9.2 & 13 & 14 \\
\hline $\mathrm{Na}$ & 32.205 & 39.155 & 54.878 & 81.237 & 132.98 & 169.09 \\
\hline $\mathrm{Ni}$ & 0.055 & 0.064 & 0.076 & 0.088 & 0.12 & 0.15 \\
\hline $\mathrm{NO} 3$ & 21 & 25 & 29 & 45 & 79 & 110 \\
\hline $\mathrm{Pb}$ & 0.44 & 0.533 & 0.62 & 0.71 & 1.3 & 2.1 \\
\hline $\mathrm{PO} 4$ & 14 & 17 & 20 & 25 & 80 & 110 \\
\hline $\mathrm{SiO} 2$ & 9.9 & 11 & 12 & 14 & 15 & 16 \\
\hline $\mathrm{SO} 4$ & 47 & 56 & 71 & 92 & 120 & 160 \\
\hline $\mathrm{Sr}$ & 0.99 & 1.3 & 1.5 & 1.9 & 2.6 & 2.7 \\
\hline $\mathrm{U}$ & 0.48 & 0.58 & 0.69 & 1 & 1.9 & 2.8 \\
\hline $\mathrm{Zn}$ & 1.4 & 1.7 & 1.9 & 2.5 & 3.3 & 3.7 \\
\hline
\end{tabular}

The feed compositions presented in Table 1 and Table 2 were used for all the simulation cases. Two additional evaporator feed compositions (Standard-Low Foam Simulant and High Foam Simulant) were also modeled and are shown in Table 3. Commercial soaps and cutting oils ${ }^{10}$ present in the actual waste are added to each simulant recipe in order to observe their effect on foaming during evaporation. These simulants are made using ground water ("borehole water") containing relatively high amounts of calcium. The composition of the standard and high foam simulant was adjusted slightly for this task to maintain charge balance. The foam causing agents were simulated by adding Lauric Acid $\left(\mathrm{C}_{12} \mathrm{H}_{24} \mathrm{O}_{2}\right.$, CAS No. 143-07-7, see Figure 3$)$ in all model runs of the high and low foam

\footnotetext{
${ }^{10}$ Recipe for $1 \mathrm{~L}$ of organic mixture is: Commercial soaps added to AWE simulant include Cleenol Luxury Liquid Soap (60g), Cleenol Hair and Body Gel (40g, Cleenol) and Sprint Cream Cleaner (2.5g,S C Johnson). The cutting oil is aqueous fraction from $5 \% \mathrm{w} / \mathrm{w}$ Solcut $\mathrm{E}$ cutting fluid $(60 \mathrm{mls}$, Houghton Vaughnan). 4.5 liters of organic mixture $/ 1000 \mathrm{~kg}$ of standard simulant vs. 11 liters $/ 1000 \mathrm{~kg}$ of the high foam simulant.
} 
simulants. Lauric acid was chosen to represent the foaming agents because many common soap products contain sodium laurate. While OLI/ESP does not predict how a simulant might foam, it does capture the effects of the additional organics on the chemical equilibrium and stream compositions.

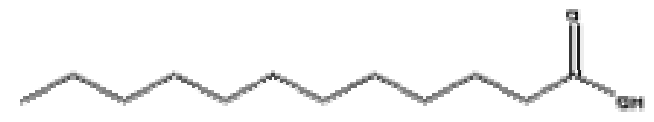

Figure 3 - Structure of Lauric Acid (Dodecanoic acid)

Table 3 - Cation and Anion Composition of Standard and High Foam Simulant

\begin{tabular}{|l|c|c|}
\hline & \multicolumn{2}{|c|}{ Concentration in mg/L } \\
\hline & $\begin{array}{c}\text { Standard } \\
\text { Simulant }\end{array}$ & $\begin{array}{c}\text { High Foam } \\
\text { Simulant }\end{array}$ \\
\hline $\mathrm{Al}$ & 3.6 & 2.4 \\
\hline $\mathrm{Ca}$ & 53.0 & 42.0 \\
\hline $\mathrm{Cl}$ & 122.0 & 65.0 \\
\hline $\mathrm{CO} 3$ & 118.0 & 82.0 \\
\hline $\mathrm{Fe}$ & 7.4 & 4.3 \\
\hline $\mathrm{K}$ & 37.0 & 12.0 \\
\hline $\mathrm{Mg}$ & 12.0 & 9.0 \\
\hline $\mathrm{Na}$ & 103.0 & 38.2 \\
\hline $\mathrm{NO} 3$ & 58.0 & 30.0 \\
\hline $\mathrm{PO} 4$ & 32.0 & 20.0 \\
\hline $\mathrm{SiO} 2$ & 18.0 & 14.0 \\
\hline $\mathrm{SO} 4$ & 118.0 & 65.0 \\
\hline $\mathrm{Zn}$ & 3.2 & 2.0 \\
\hline
\end{tabular}




\section{MODEL RESULTS}

\section{Composition of Evaporator Feed, Concentrate and Condensate}

The compositions of the evaporator feed, concentrate and condensate for each of the feed input vectors reported in Table 1, 2 and 3 (high/low foam stimulant) are shown in Appendix B. The evaporator model developed for this task was used to concentrate the feed vectors until the evaporator concentrate reached a concentration of $30 \mathrm{wt} \%$ total solids. The tables presented in Appendix B are completed material and energy balance files and also provide chemical and physical property data (e.g. density) and engineering data (e.g. enthalpy) for each stream modeled. The predicted composition of the AWE evaporator process is reported in full ionic form.

\section{Concentration Factor at a Predefined Solubility Endpoint of $5 \mathrm{wt} \%$ Insoluble Solids}

The volume reduction factor at the defined solubility point (Insoluble Solids $=0.5 \mathrm{wt} \%$ in the Evaporator Concentrate) was calculated for each of the feed compositions ( $\mathrm{pH}=6.5 \&$ 8.5: $50-95 \%$ Cases) as shown in Table 1 and Table 2. The volume reduction factors at the defined solubility endpoint are shown in Table 4 and Figure 4 . Two general trends are indicated by the data in Table 4: 1) The volume reduction factor decreases as the cation and anion concentration increases in the waste 2) Higher volume reduction factors was predicted when processing acidic wastes.

Table 4 - Volume Reduction Factor at the Defined Precipitation Endpoint (\% insoluble Solids $=0.5 \mathrm{wt} \%$ )

\begin{tabular}{|c|c|c|}
\hline $\begin{array}{c}\text { Feed Input Vector - \% } \\
\text { Waste Effluent }\end{array}$ & $\begin{array}{l}\text { Volume Reduction Factor for } \\
\text { Feed } \mathbf{p H = 6 . 5} \text { at Precipitation } \\
\text { Point (Insoluble Solids = 0.5\% }\end{array}$ & $\begin{array}{l}\text { Volume Reduction Factor for } \\
\text { Feed } \mathbf{p H = 8 . 5} \text { at Precipitation } \\
\text { Point (Insoluble Solids = 0.5\% } \\
\text { in Evaporator Concentrate) }\end{array}$ \\
\hline $\mathbf{5 0 \%}$ & 75.4 & 71.8 \\
\hline $\mathbf{6 0 \%}$ & 67.4 & 64.2 \\
\hline $\mathbf{7 0 \%}$ & 60.7 & 57.8 \\
\hline $\mathbf{8 0 \%}$ & 52.0 & 49.5 \\
\hline $\mathbf{9 0 \%}$ & 40.4 & 30.6 \\
\hline $\mathbf{9 5 \%}$ & 32.9 & 24.0 \\
\hline
\end{tabular}


WSRC-TR-2002-00567

Revision 0

Volume Reduction as a function of Waste Effluent Evaporator Feed Case

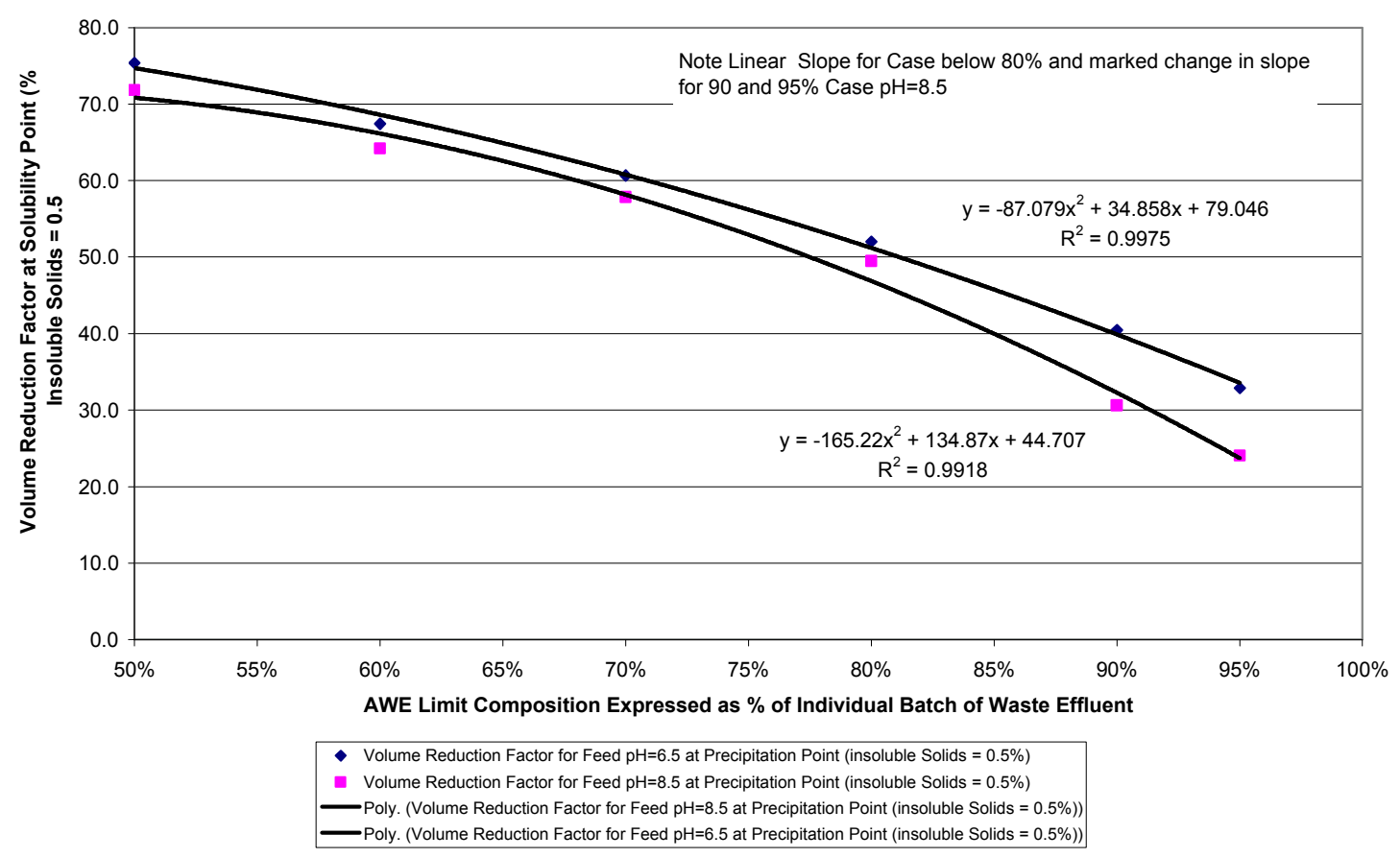

Figure 4 - Volume Reduction Factor at the Defined Solubility Point (wt\% Insoluble Solids $=0.5$ ) as a Function of AWE Limit Composition for Feed Case $\mathrm{pH}=6.5 \& 8.5$ $50-95 \%$

The higher volume reduction factors obtained with acid waste provide insight into a possible process improvement. Acid adjustment of the caustic AWE wastes might improve the overall throughput by increasing the volume reduction (i.e. increasing the solubility of the waste components (e.g. Ca) ) for a given waste stream. Additionally, acidic wastes generally have fewer tendencies to scale and foam than caustic wastes. Once the waste is concentrated and discharged from the evaporator, sodium hydroxide could be added to pretreat the concentrated waste for cementation. SRTC recommends that AWE consider evaluating acidification of the waste effluents prior to evaporation and pretreatment of the evaporator concentrate with sodium hydroxide.

The model was also used to calculate the volume reduction factor until target insoluble solids loadings of $0.1,0.5,1$ and $5 \mathrm{wt} \%$ were obtained in the evaporator concentrate. Additionally, the volume reduction factor was also calculated by allowing the model to concentrate the feed until the evaporator concentrate reached 10, 20 and $30 \mathrm{wt} \%$ total solids. The volume reduction factor as a function of insoluble solids loading for the limit compositions reported in Table 1 and Table 2 (Feed $\mathrm{pH}=6.5 \&$ 8.5: 50 - 95\%) are shown Figure 5 and Figure 6 . Correlation of the volume reduction factor as a function of insoluble solids loading is shown in each plot for the $80 \%$ limit composition case. 
WSRC-TR-2002-00567

Revision 0

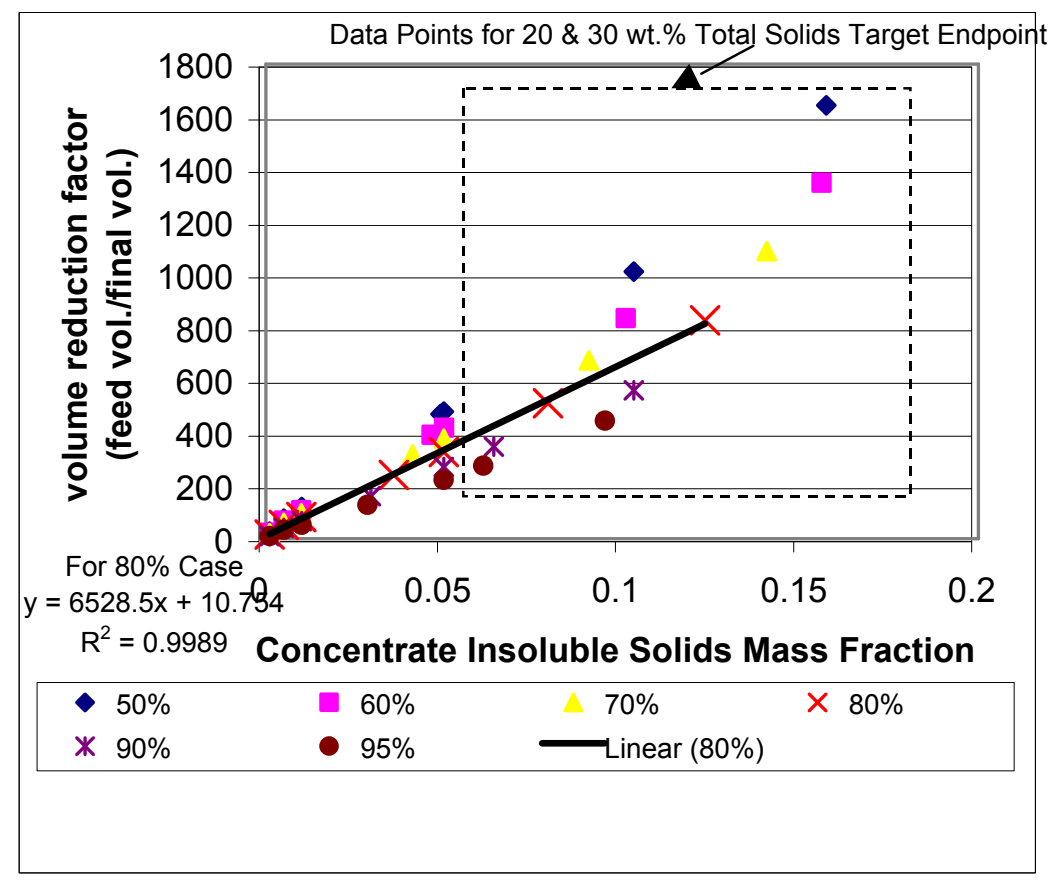

Figure 5 - Volume Reduction Factor as a Function of Insoluble Solids in the Evaporator Concentrate - Case RAWTP $\mathbf{p H}=6.5$ for Evaporator Feed Concentrate Target Endpoints of 0.1, 0.5, 1, 5wt\% Insoluble Solids and 10, 20 and 30wt\% Total Solids in Concentrate

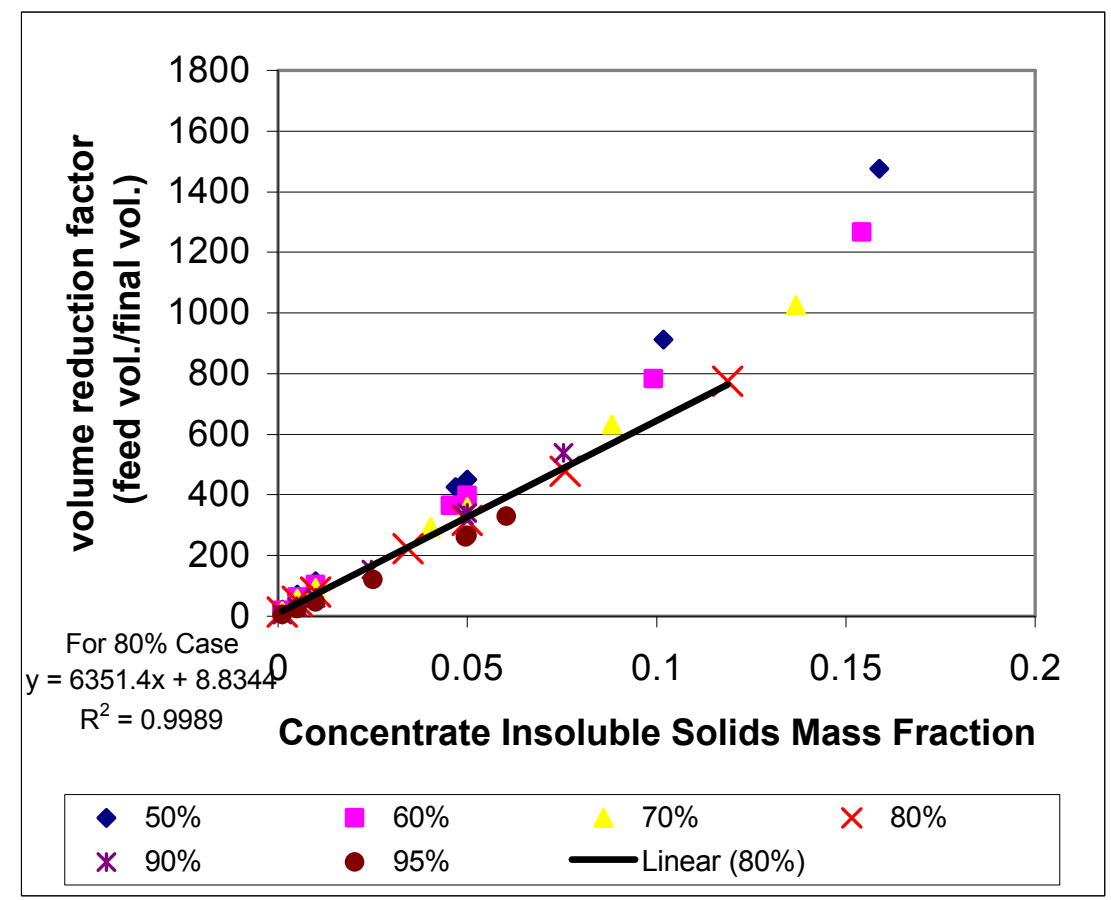

Figure 6 - Volume Reduction Factor as a Function of Insoluble Solids in the Evaporator Concentrate - Case RAWTP $\mathbf{p H}=8.5$ for Evaporator Feed Concentrate Target Endpoints of 0.1, 0.5, 1, 5wt\% Insoluble Solids and 10, 20 and $30 w t \%$ Total Solids in Concentrate 
WSRC-TR-2002-00567

Revision 0

Effect of Concentration on Mass Faction of Insoluble Solids formed in the Evaporator Concentrate

One important factor in the evaporation of wastes is the concentration and type of insoluble solids that form when the solution is concentrated. The type of insoluble species formed is discussed later in this report. The model was used to estimate the concentration of insoluble solids in the evaporator concentrate as a function of the total solids in the concentrate. The insoluble solids concentration was plotted as a function of percent total solids in the concentrate for all limit compositions outlined in Table 1 and Table 2 (Feed Case $\mathrm{pH}=6.5 \&$ 8.5: $50-95 \%$ ). The data is presented in Figure 7 and Figure 8. Correlations of insoluble solids as a function of concentrate total mass fraction are shown in each plot for the $80 \%$ limit composition case.

While there is no data at present to suggest the concentrated RAWTP evaporator concentrate will be a non-newtonian fluid as the insoluble solids loading increases, previous US experience with radioactive waste concentration suggests that insoluble solids loading above $10 \%$ will most likely produce non-newtonian flow properties ${ }^{11}$. Once a correlation between total solids and insoluble solids in the evaporator concentrate is known, measurements of the rheology (shear stress vs. shear rate, flow curves) should be developed to determine the flow properties as a function of insoluble solids and total solids loading. This type of information is very useful in the design of evaporators that are expected to handle viscous fluids. The modeling results indicate that the insoluble solids concentration will be greater than $10 \mathrm{wt} . \%$ when the total solids concentration in the evaporator reaches values in excess of $20-25 \mathrm{wt} . \%$ (For example: For the $80 \%$ case $\mathrm{pH}=6.5$, the insoluble solids concentration at 10,20 and $30 \mathrm{wt} \%$ is predicted to be $3.5,8.0$ and $12.3 \mathrm{wt} \%)$.

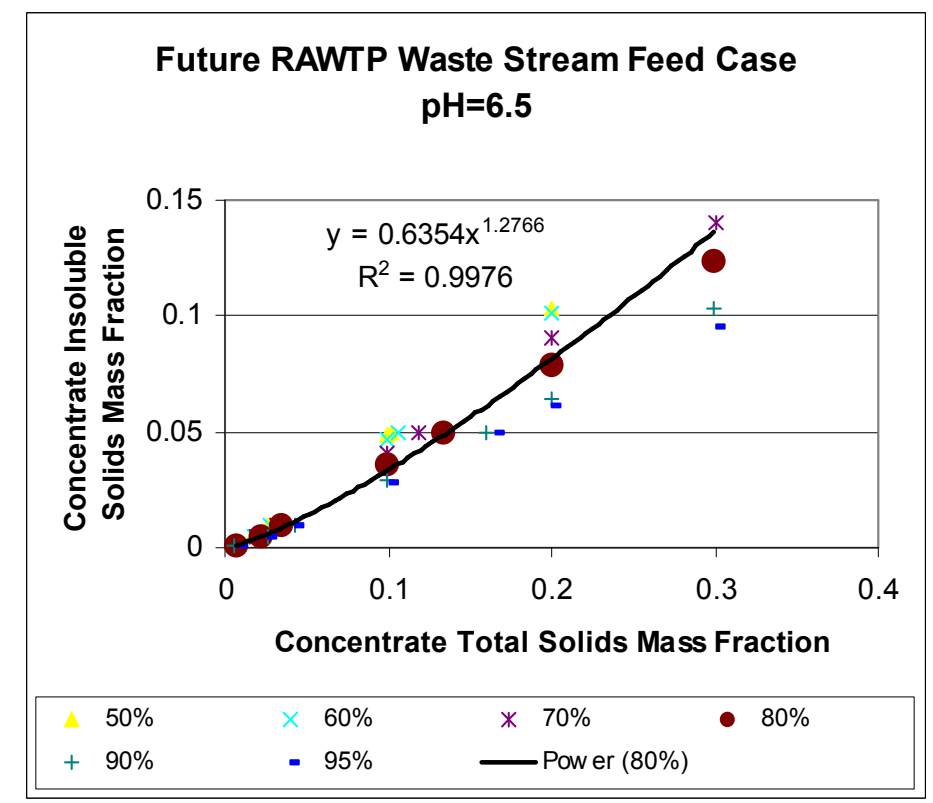

\footnotetext{
${ }^{11}$ Non-Newtonian fluids are generally more difficult to process and present more challeges to the designer of a radioactive waste treatment system.
} 
WSRC-TR-2002-00567

Revision 0

Figure 7 - Mass Fraction Insoluble Solids in the Evaporator Concentrate as a Function of Total Solids in the Evaporator Concentrate - Case RAWTP $\mathbf{p H}=6.5$ for Evaporator Feed Concentrate Target Endpoint of 0.1, 0.5, 1, 5wt\% Insoluble Solids and 10, 20 and 30wt\% Total Solids in Concentrate

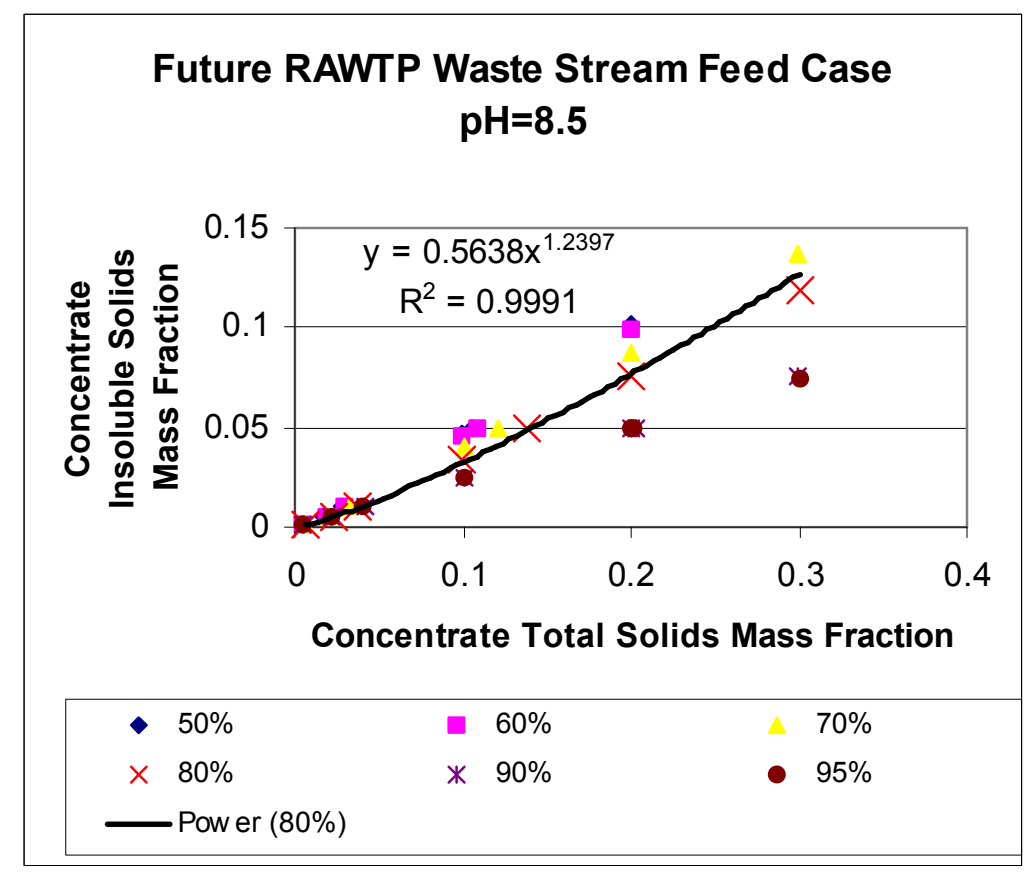

Figure 8 - Mass Fraction Insoluble Solids in the Evaporator Concentrate as a Function of Total Solids in the Evaporator Concentrate - Case RAWTP $\mathbf{p H}=8.5$ for Evaporator Feed Concentrate Target Endpoint of 0.1, 0.5, 1, 5wt\% Insoluble Solids and 10, 20 and 30wt\% Total Solids in Concentrate

The mass fractions of insoluble solids in the evaporator concentrate were also calculated as a function of the total solids for the high and low foam simulant. The results for the simulant are plotted in Figure 9. The antifoam agent expected to be used by the RAWTP was not included in the model. Gentilucci and Nash anticipated that the antifoam agent would be a significant contributor to the insoluble solids loading in the evaporator concentrate during their review of the RAWTP ${ }^{12}$. Work conducted in support of the DOE Office of River Protection has shown that most traditional silicone based antifoams are ineffective ${ }^{13}$ when the foam is caused by formation of insoluble particles and surfactants. Thus, large amounts of antifoam, well over the vendor recommended concentrations, are needed to control foaming caused by surfactants and insoluble particles. Additionally, silicone based antifoam agents can become insoluble as the electrolyte strength increases

\footnotetext{
${ }^{12}$ Gentilucci, J. A. and Nash, C. A., Independent Review of the Kvaerner "Final Concept Design Report for Radioactive Aqeous Waste Treatment Plant AWE Aldermaston, JAG Tech Services, Inc. and Westinghouse Savannah River Company, Aiken SC 29808, Novermber 2001.

${ }^{13}$ Josephs, J., Calloway, T. B. and Lambert, D. P., Foaming in the Hanford River Protection Waste Treatment Plant LAW Evaporation, WSRC-TR-2001-00561 Rev. 0, Westinghouse Savannah River Company, Aiken SC 29808, December 2001.
} 
in the evaporator (See Figure 10 for example). Therefore, the plots shown in Figure 9 should be used with caution since the antifoam agent was not modeled.

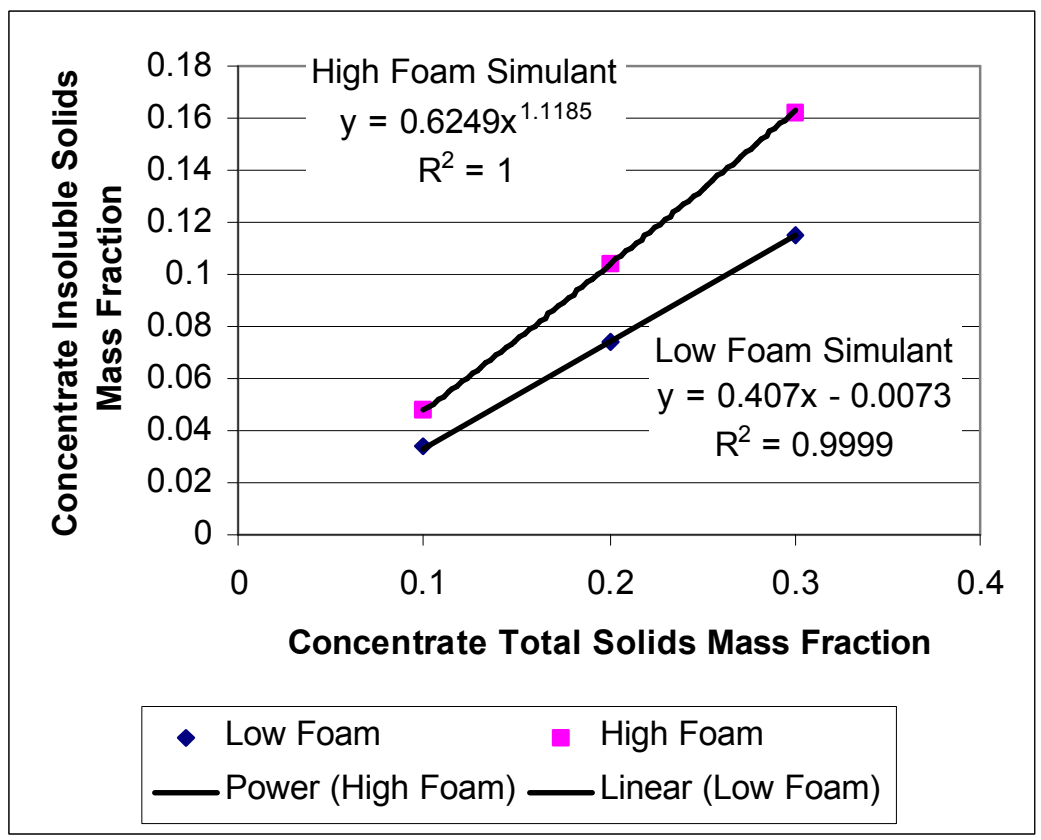

Figure 9 - Mass Fraction Insoluble Solids in the Evaporator Concentrate as a Function of Total Solids in the Evaporator Concentrate - Case High and Low Foam Simulant Concentrated to 10, 20 and $30 \mathrm{wt} \%$ total solids

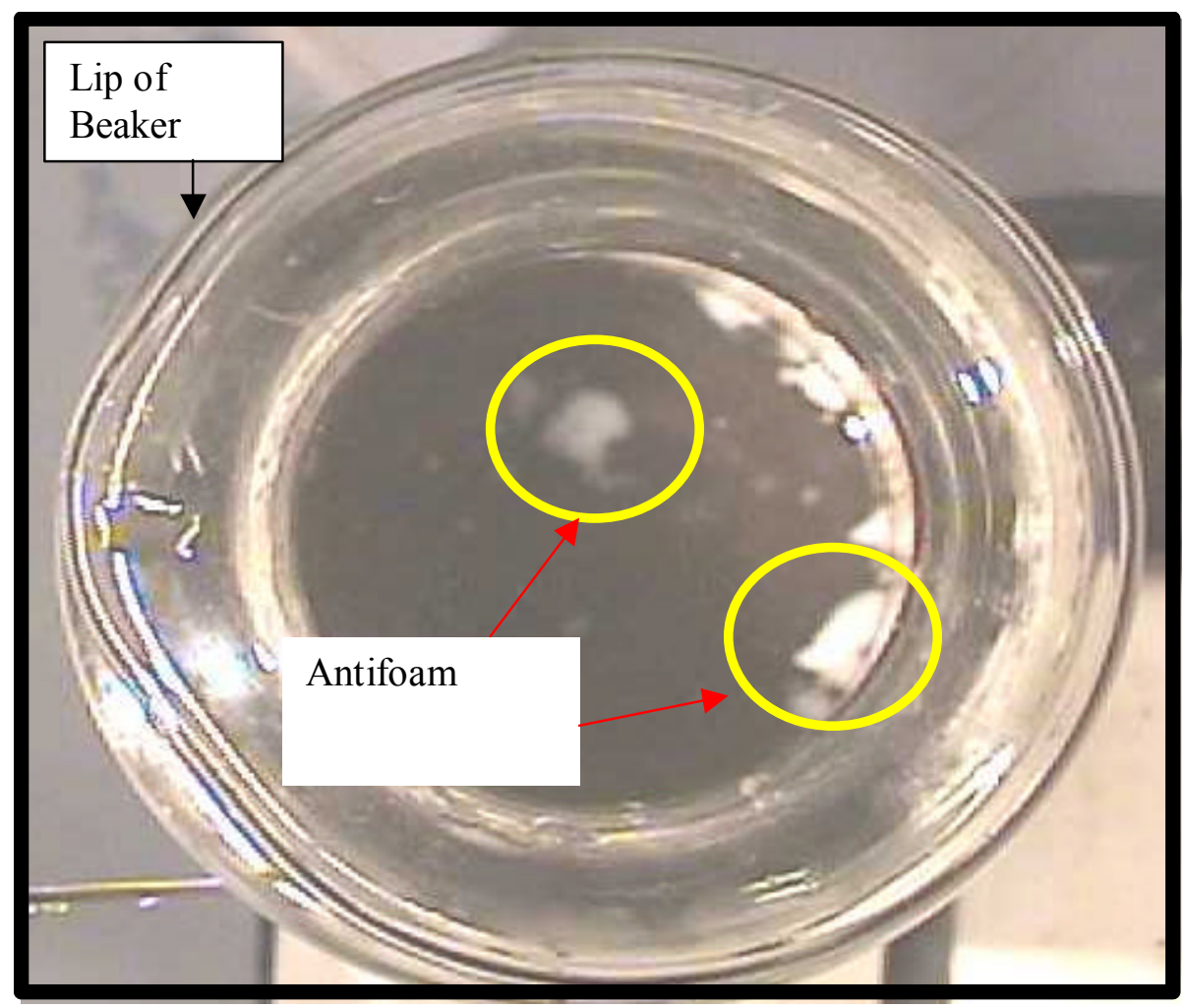


WSRC-TR-2002-00567

Revision 0

Figure 10 - DOW 1520-US Antifoam Floating on Simulated Hanford Envelope C Salt Waste

\section{Correlation between Concentration and Density in the Evaporator Concentrate}

The most frequently used evaporator process control parameter is the concentrate density. Relationships between concentration and density allow the operator to relate composition to the desired process control parameter (density). Additional relationships between temperature, density and concentration are also desirable. The percent total solids in the evaporator concentrate and the corresponding slurry density were calculated and plotted in Figure 11, Figure 12 and Figure 13 for all the feed vectors outlined in Table 1, Table 2 and Table 3 (Feed Case $\mathrm{pH}=6.5 \&$ 8.5: $50-95 \%$, High and Low Foam Simulant).

Correlations of density as a function of concentration are shown in each plot for the $80 \%$ waste effluent case. The density was calculated at $25^{\circ} \mathrm{C}$.

Concentrate density in evaporators are typically measured using pneumatic "bubbler type" differential pressure meters. Generally, density bubblers can plug and become unreliable. Coriolis type mass flow meters have the capability to measure the liquid density of the product as it exists the evaporator thus providing an alternate means of controlling the evaporator density and concentration. Some coriolis mass flow meters also measure temperature. It is recommended that AWE investigate the use coriolis mass flow meters to control the evaporator product density.

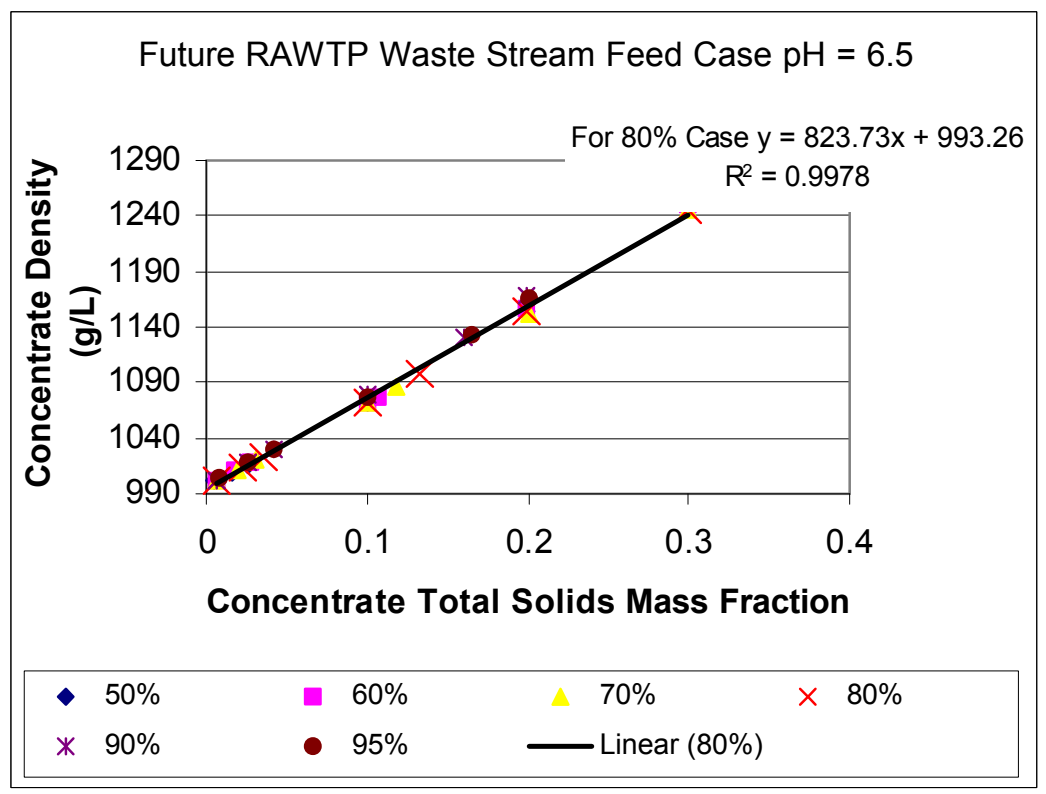

Figure 11 - Density of the Evaporator Concentrate as a Function of Total Solids in the Evaporator Concentrate - Case RAWTP $\mathrm{pH}=6.5$ for Evaporator Feed Concentrate Target Endpoints of 0.1, 0.5, 1, 5wt\% Insoluble Solids and 10, 20 and $30 w t \%$ Total Solids in Concentrate 
WSRC-TR-2002-00567

Revision 0

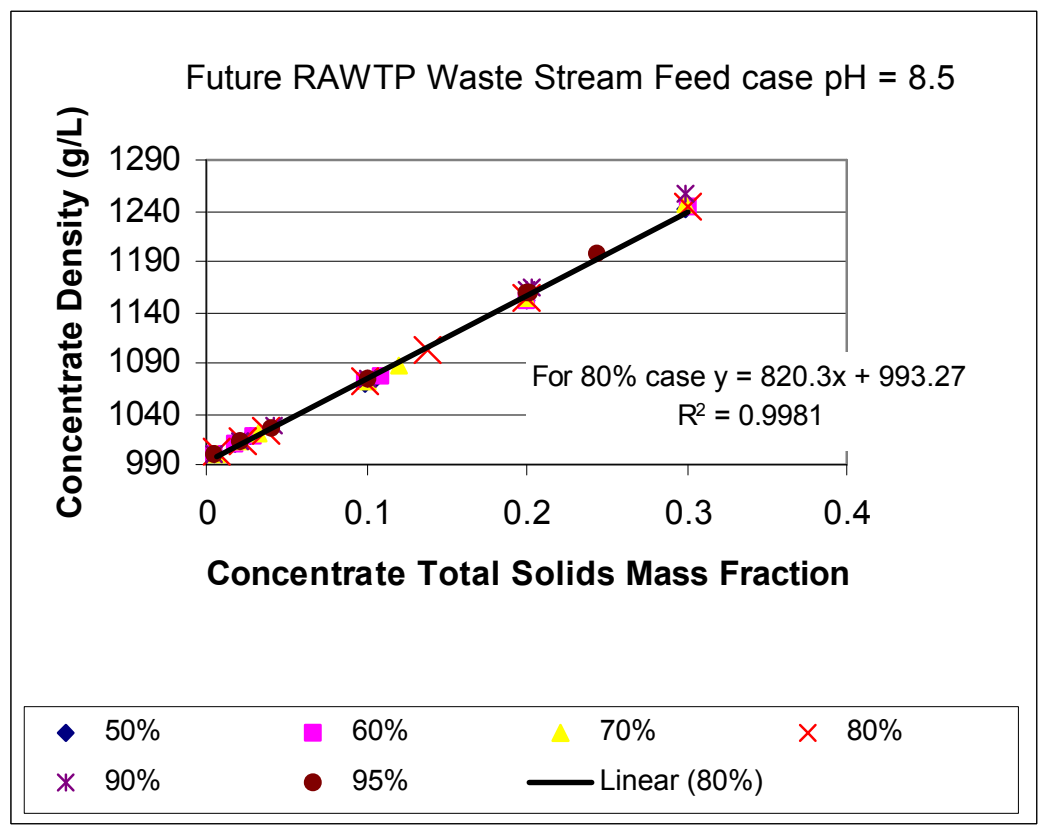

Figure 12 - Density of the Evaporator Concentrate as a Function of Total Solids in the Evaporator Concentrate - Case RAWTP $\mathbf{p H}=8.5$ for Evaporator Feed Concentrate Target Endpoints of 0.1, 0.5, 1, 5wt\% Insoluble Solids and 10, 20 and $30 w t \%$ Total Solids in Concentrate

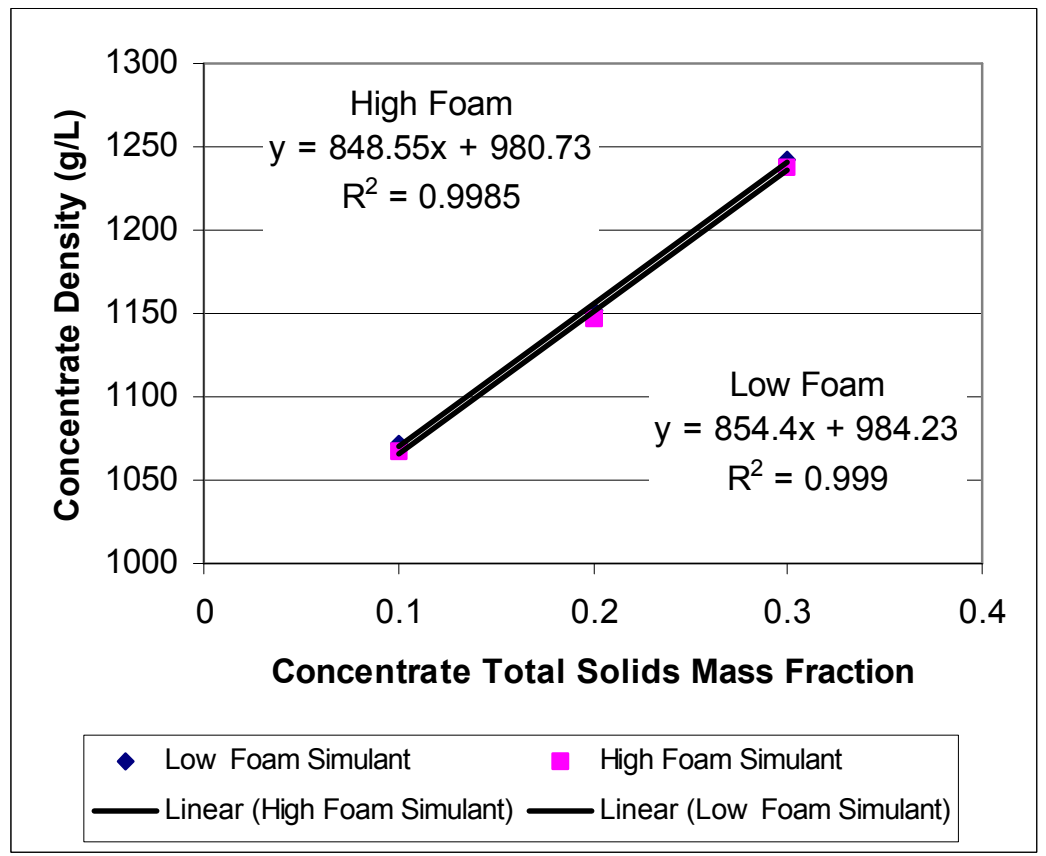

Figure 13 - Density of the Evaporator Concentrate as a Function of Total Solids in the Evaporator Concentrate - Case High and Low Foam Simulant Concentrated to 10,20 and $30 w t \%$ total solids 


\section{Feed Stock pH Comparison to Model Output}

A comparison of the OLI model calculated $\mathrm{pH}$ values for each waste feed vector and the targeted $\mathrm{pH}$ value for the waste stream $(\mathrm{pH}=6.5$ or 8.5$)$ is shown in Table 5. The model output for the predicted RAWTP waste streams at $\mathrm{pH} 6.5$ agrees fairly well with anticipated target $\mathrm{pH}$. However, for the RAWTP waste streams charge balanced to target a $\mathrm{pH}$ of 8.5 the model predicted a $\mathrm{pH}$ less than that expected by as much as $22 \%$. The predicted $\mathrm{pH}$ values for the 2 simulants are also close to the measured values $(6.5-7.5)^{14}$.

On another modeling task conducted in support of the Office of River Protection, SRTC personnel compared the OLI model $\mathrm{pH}$ predictions to high strength sodium nuclear waste simulants and found the model predictions to vary by a similar order of magnitude. SRTC personnel contacted OLI personnel concerning the validity of the OLI ESP predictions. OLI personnel informed SRTC that no attempt by OLI has been made to validate the calculated model $\mathrm{pH}$ values since the $\mathrm{pH}$ measurement techniques and data in the literature for binary and ternary aqueous solutions varies quite widely. It is possible to adjust the OLI property databases if $\mathrm{pH}$ data from simulated or actual AWE wastes become available.

SRTC recommends that AWE formulate, evaporate and measure the physical and chemical properties of simulated waste and concentrated evaporation product based upon the RAWTP limit compositions. This data and the subsequent models generated from the data could be used to construct process control models for use by the operating and engineering staff of the RAWTP. A similar effort was conducted for the Savannah River and Hanford waste evaporators.

Table 5 - Predicted pH of AWE Waste Effluent and Simulants

\begin{tabular}{|l|c|c|c|}
\hline Stream & $\begin{array}{c}\text { Feed } \\
\text { Input } \\
\text { Vector - \% } \\
\text { Waste } \\
\text { Effluent }\end{array}$ & $\begin{array}{c}\text { Predicted pH } \\
\text { for Feed } \\
\text { Vector Case } \\
\text { pH=6.5 }\end{array}$ & $\begin{array}{c}\text { Predicted pH for } \\
\text { Feed Vector Case } \\
\text { pH=8.5 }\end{array}$ \\
\cline { 1 - 2 } & $50 \%$ & 6.06 & \\
\hline Evaporator Feed & $60 \%$ & 5.90 & 7.02 \\
\hline Evaporator Feed & $70 \%$ & 5.99 & 6.97 \\
\hline Evaporator Feed & $80 \%$ & 6.00 & 6.95 \\
\hline Evaporator Feed & $90 \%$ & 5.98 & 6.92 \\
\hline Evaporator Feed & $95 \%$ & 5.64 & 6.76 \\
\hline Evaporator Feed & & & 6.60 \\
\hline Stream & & & Predicted pH for \\
\hline
\end{tabular}

\footnotetext{
${ }^{14}$ Taylor, G., RE:External: RE: Model Report Comments, email to T. B. Calloway, 2/7/2003, Atomic Weapons Establishment, Aldermaston, Reading Berkshire RG74PR.
} 
WSRC-TR-2002-00567

Revision 0

Simulant - Low

Foam

Simulant - High

Foam
7.00

7.08

\section{$\underline{\text { Predicted Composition of Insoluble Solids in Concentrated RAWTP Waste Streams }}$}

The compositions of the insoluble solids formed upon evaporation for the evaporator feed compositions shown in Table 1, Table 2 and Table 3 were calculated by the model and are shown in Appendix A. It should be noted that the model provides an estimate of the type of solids that could possibly form in the RAWTP evaporator. Experimental data and pilot tests are typically used to confirm and/or refine the model predictions.

A summary of the $80 \%$ waste effluent case (evaporator feed $\mathrm{pH}=6.5$ and 8.5 is shown in Table 8 . Several observations can be made from this table:

- $\mathrm{Ca}_{3}\left(\mathrm{PO}_{4}\right)_{2}$ and $\mathrm{CaSO}_{4} \cdot 2 \mathrm{H}_{2} \mathrm{O}$ are predicted to be the major insoluble species formed at both $\mathrm{pH}$ ranges.

- The formation of $\mathrm{Mg}(\mathrm{OH})_{2}$ in the evaporator bottoms occurs only in the alkaline evaporator feeds.

- A higher percentage of the $\mathrm{Si}$ is insoluble as $\mathrm{SiO}_{2}$ vs. Chamosite 7A $\left(\mathrm{Fe}_{2} \mathrm{Al}_{2} \mathrm{SiO}_{5}(\mathrm{OH})_{4}\right)$ in the acidic evaporator feed cases.

- A higher percentage of the $\mathrm{Si}$ is insoluble as Chamosite vs. $\mathrm{SiO}_{2}$ in the alkaline evaporator feed cases.

- $\mathrm{UO}_{2}$ was found to be insoluble in the RAWTP evaporator feed.

- Carbonate will evolve as carbon dioxide in the evaporator overheads.

These simulations show that carbonate will evolve as carbon dioxide in the evaporator overheads which explains why calcium carbonate is not predicted to precipitate in the evaporator bottoms for the $\mathrm{pH}=6.5$ and 8.5 cases. Generally, carbonate will evolve as $\mathrm{CO}_{2}$ in acidic to slightly basic solutions. The simulations for the Future RAWTP waste streams show $99+\%$ of all the carbonate evolves as carbon dioxide in the evaporator overheads. A greater percentage of the feed carbon in the AWE High Foam Simulant was predicted to precipitate as carbonate $(\approx 5 \%)$, but this is due its lower initial feed concentration; the actual carbonate concentrations and precipitated solids of the concentrated High and Low Foam simulants are very similar. Table 6 shows the percentage of carbonate and dissolved carbon dioxide predicted to evolve as $\mathrm{CO} 2$ in the evaporator overheads for the High Foam Simulant Case. 
Transport may play a role in the evolution of carbon dioxide (or precipitation of calcium carbonate) in the AWE waste. It is suggested that actual waste studies be conducted to determine the concentration of calcium carbonate in the concentrated AWE waste and the amount of carbon dioxide evolved from the waste during the evaporation process.

Evaporation of an acidic waste is likely to favor the evolution of the carbon dioxide to the evaporator overheads. Evaporation of slightly basic wastes will have reduced evolution of carbon dioxide. It is suggested that actual waste studies should be conducted to determine the concentration of calcium carbonate in the concentrated AWE waste and the amount of carbon dioxide evolved from the waste during the evaporation process. Therefore it is suggested that AWE consider acidifing the waste to improve the overall volume reduction factors.

Table 6 - Predicted Percentage of Carbonate and Dissolved Carbon Dioxide in the Feed Evolving as Carbon Dioxide for the High Foam Simulant Case

\begin{tabular}{|l|l|l|l|l|}
\hline run number & stream & $\begin{array}{l}\text { vapor moles of } \\
\text { CO2 }\end{array}$ & $\begin{array}{l}\text { \% Feed } \\
\text { CO3+CO2 going pH } \\
\text { to Overhead as } \\
\text { CO2 }\end{array}$ \\
\hline HF 10\% TS & overhead & 0.00129249 & $94.6 \%$ & $7.95 E+00$ \\
\hline HF 20\% TS & overhead & 0.00127691 & $93.4 \%$ & $7.89 E+00$ \\
\hline HF 30\% TS & overhead & 0.00126611 & $92.7 \%$ & $7.84 E+00$ \\
\hline
\end{tabular}

Based upon SRS experience with waste evaporation, the formation of complex Fe-Al-Si minerals in the AWE evaporator are likely present a scaling problem on the evaporator heat exchanger surfaces. Given the low concentration of $U$ in the AWE waste criticality is not likely to be a concern in the RAWTP evaporator. However, given the high concentration factors in the RAWTP evaporator, AWE should evaluate this data with respect to the current design and operation of the RAWTP to determine the potential for holdup of $U$ that may co-precipitate with any other scale that forms in the evaporator. Pretreatment with nitric acid prior to evaporation may reduce the quantity of complex FeAl-Si minerals formed in the evaporator. SRTC recommends that AWE conduct evaporator cleaning studies with the concentrated simulants obtained from pilot testing to determine suitable chemical cleaning agents and procedures for the RAWTP.

Partial Validation of the OLI ESP Public Database for the $\mathrm{CaCO}_{3}$-Water and $\mathrm{CaSO}_{4}=$ $\underline{\text { Water Systems }}$

The solubility of $\mathrm{CaCO} 3$ in water and $\mathrm{CaSO} 4$ in water was calculated using OLI ESP and compared with the literature solubility values ${ }^{15}$. The OLI results compare favorably with the literature values. It is important to point out that simulating waste streams are much more complicated than a single compoent system. For example the predicted solubility of calcium carbonate in a CaCO3-CaSO4-H2O system is $2.173 \mathrm{E}-04 \mathrm{~g} / 100 \mathrm{~g} \mathrm{H}_{2} \mathrm{O}$. Thus, while single component solubility values can be used to qualittatively examine trends in a

\footnotetext{
${ }^{15}$ Langes Handbook of Chemistry, Thirteenth Edition
} 
waste system, multicomponent modeling and experimentation is often needed to fully design and operate a waste treatment plant.

Table 7 - Literature Solubility Values of $\mathrm{CaCO3}$ and $\mathrm{CaSO} 4$ in water as compared to OLI ESP Publix Database.

\begin{tabular}{|l|l|l|l|}
\hline & \multicolumn{3}{|c|}{ Solubility in Water $\left(\mathbf{g} / \mathbf{1 0 0 g} \mathrm{H}_{2} \mathbf{O}\right)$} \\
\hline Compound in Water & Cold & Hot & OLI Results \\
\hline $\mathrm{CaCO}_{3}$ & $0.0014 / 0.0015$ & 0.0019 & 0.0012 \\
\hline $\mathrm{CaSO}_{4}$ & 0.209 & 0.162 & 0.206
\end{tabular}


Table 8 - Composition of Insoluble Solids Formed in the Evaporator Concentrate for Evaporator Feed Case $80 \%$ Waste Effluent pH=6.5 and 8.5 as a function of Evaporator Concentration Endpoint (0.1, 0.5, 1, $5 \mathrm{wt} \%$ Insoluble Solids and 10, 20 and $30 \mathrm{wt} \%$ total Solids)

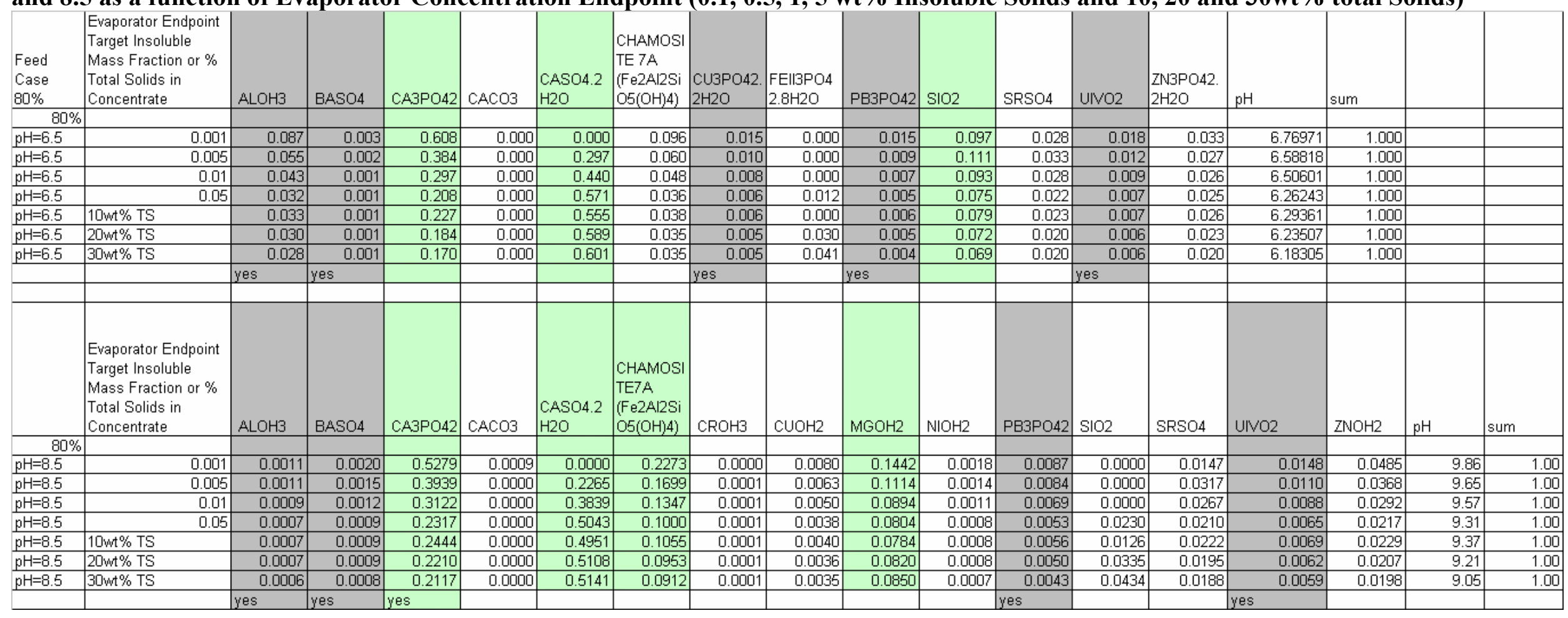


WSRC-TR-2002-00567

Revision 0

\section{CONCLUSIONS/RECOMMENDATIONS}

A model of the AWE RAWTP waste effluent evaporator was created using OLI/ESP software. Over ninety simulations were preformed using the predicted limit RAWTP waste compositions and two simulated waste stream compositions (High/Low Foam Simulant) were modeled. Physical and chemical properties for each of the waste streams were calculated. The following conclusions were drawn from the modeling of the RAWTP waste evaporation process:

- Higher volume reduction factors are predicted to be obtained with acidic waste effluents $(\mathrm{pH}=6.5)$ as compared to alkaline waste effluents $(\mathrm{pH}=8.5)$.

- $\mathrm{Ca}_{3}\left(\mathrm{PO}_{4}\right)_{2}$ and $\mathrm{CaSO}_{4} \cdot 2 \mathrm{H}_{2} \mathrm{O}$ are predicted to be the major insoluble species formed in the RAWTP evaporator.

- Over $99+\%$ of the carbonate and dissolved carbon dioxide was preicted to evolve as carbon dioxide in the evaporator overheads for the Future RAWTP waste streams.

- Approximately $93 \%$ of the carbonate and dissolved carbon dioxide (7\% predicted to precipitate in evaporator bottoms as calcium carbonate) in the high foam simulant was preicted to evolve as carbon dioxide in the evaporator overheads to due lower intial feed carbon concentration.

- It recommended that pretreatment of the RAWTP waste streams with a suitable acid (e.g. $\mathrm{HNO}_{3}$ ) and subsequent pretreatment of the concentrate with $\mathrm{NaOH}$ prior to grouting be investigated as an option to evaporation of alkaline wastes.

- Chamosite 7A $\left(\mathrm{Fe}_{2} \mathrm{Al}_{2} \mathrm{SiO}_{5}(\mathrm{OH})_{4}\right)$ is predicted to form in the RAWTP evaporator. Based upon SRS experience with evaporation of alkaline wastes contain silica and aluminum it is likely that the formation of complex Fe-Al-Si species will present a scaling problem in the RAWTP evaporator.

- A higher percentage of the $\mathrm{Si}$ is insoluble as $\mathrm{SiO}_{2}$ vs. Chamosite 7A $\left(\mathrm{Fe}_{2} \mathrm{Al}_{2} \mathrm{SiO}_{5}(\mathrm{OH})_{4}\right)$ in the acidic evaporator feed cases.

- A higher percentage of the $\mathrm{Si}$ is insoluble as Chamosite vs. $\mathrm{SiO}_{2}$ in the alkaline evaporator feed cases.

- SRTC recommends that AWE conduct evaporator cleaning studies with the concentrated simulants obtained from pilot testing to determine suitable chemical cleaning agents and procedures for the RAWTP. 
- SRTC recommends that AWE formulate, evaporate and measure the physical and chemical properties of simulated waste and concentrated evaporation product based upon the RAWTP limit compositions. This data and the models generated validated by data could be used to construct process control models for use by the operating and engineering staff of the RAWTP. A similar effort was conducted for the Savannah River and Hanford waste evaporators.

- It is recommended that AWE investigate the use of coriolis mass flow meters to control the evaporator product density. 


\section{APPROVALS:}

C.D.2,

Date: $8 / 25 / 03$

C. D. Barnes, Author, Immobilization Technology Section /SRTC

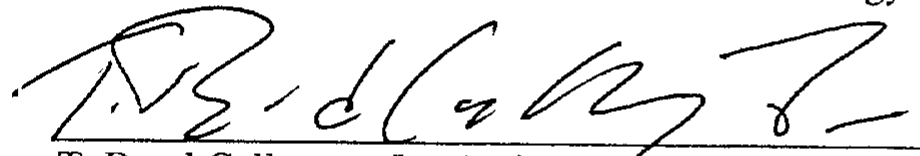

T. Bond Calloway, Jr., Author, Infrnobilization Technology Section /SRTC

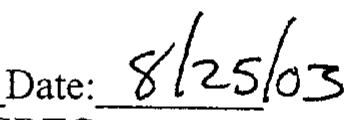

Rusell So Elobing

Date: $8-25-03$

R. E. Eibling, Author, Immobilization Technology Section /SRTC

7 rank 1 smet

Date: $8-27-03$

Frank G. Smith, Technical Reviewer, Immobilization Technology Section /SRTC

$$
\text { Q) C. Daned }
$$

Date: $8-25-03$

W. Gene Daniel, Technical Reviewer, Immobilization Technology Section /SRTC

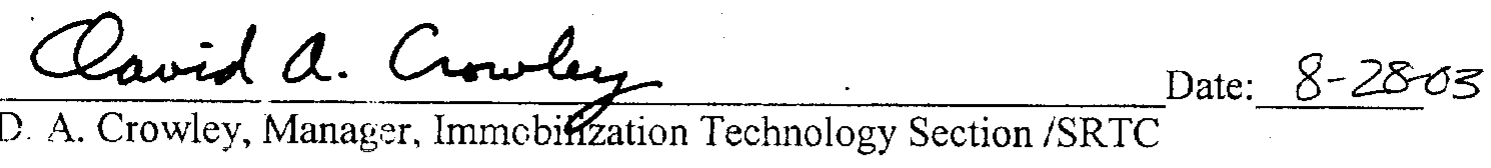




\section{APPENDIX A - Predicted Composition of Insoluble Solids in the RAWTP Evaporator Concentrate}

\section{Table 9 - Insoluble Solids Formed in the Evaporator Concentrate for Feed Case 50-95\% Waste Effluent pH=6.5}

\begin{tabular}{|c|c|c|c|c|c|c|c|c|c|c|c|c|c|c|c|c|c|c|c|c|c|}
\hline \multicolumn{2}{|c|}{ RAMTP Feed Case $\mathrm{pH}=6.5$} & \multirow[b]{2}{*}{$\begin{array}{l}\text { Evaporator } \\
\text { Concentration } \\
\text { Endpoint }\end{array}$} & \multirow[b]{2}{*}{ ALOH3 } & \multirow[b]{2}{*}{ BASO4 } & \multirow[b]{2}{*}{$\begin{array}{l}\mathrm{C} 33 \mathrm{POO} 42 . \\
2 \mathrm{H} 2 \mathrm{O}\end{array}$} & \multirow[b]{2}{*}{ PE3PO42 } & \multirow[b]{2}{*}{ UIVO2 } & \multirow[b]{2}{*}{$\mathrm{sio2}$} & \multirow[b]{2}{*}{ SRSO4 } & \multirow[b]{2}{*}{ САЗ3PO42 } & \multirow[b]{2}{*}{$\begin{array}{l}\text { CASO4. } \\
2 \mathrm{H} 2 \mathrm{O}\end{array}$} & \multirow[b]{2}{*}{$\begin{array}{l}\text { CHAMOSITE 7A } \\
\text { CHe2AIISSiO5(O) } \\
\text { (F)4) }\end{array}$} & \multirow[b]{2}{*}{$\begin{array}{l}\mathrm{FENI} 3 \mathrm{PO} 42 \\
8 \mathrm{H} 2 \mathrm{O}\end{array}$} & & & & & & & & \\
\hline stream & & & & & & & & & & & & & & $\begin{array}{l}\mathrm{ZN} 3 \mathrm{PO} 42 . \\
2 \mathrm{H} 2 \mathrm{O}\end{array}$ & С.НPPO4 & ALPO4 & $\mathrm{CROH}_{3}$ & $\mathrm{NNOH} 2$ & $\mathrm{ZNOH} 2$ & & $\mathrm{pH}$ \\
\hline formula weight & & & 78.00365 & 233.4016 & \begin{tabular}{|l|l|}
416.6114 \\
\end{tabular} & 811.5427 & 270.0257 & \begin{tabular}{|c|c|}
60.085 \\
\end{tabular} & \begin{tabular}{|l|l|}
183.68 \\
\end{tabular} & \begin{tabular}{|l|l|}
310.1767 \\
\end{tabular} & 172.17 & 341.7691 & 501.6064 & 422.1434 & \begin{tabular}{|l|}
136.057 \\
\end{tabular} & 121.95 & 103.02 & 92.68 & \begin{tabular}{|c|}
99.385 \\
\end{tabular} & Sum & \\
\hline & $50 \%$ & \begin{tabular}{|l|} 
insoluble \\
solids cor \\
total solids - \\
TSy mass \\
fraction target
\end{tabular} & & & & & & & & & & & & & & & & & & & \\
\hline $\begin{array}{l}\text { COOLED BOTTOMS } \\
\text { COOLED BOTTOMS }\end{array}$ & & \begin{tabular}{ll|}
0.001 \\
0.005
\end{tabular} & $\begin{array}{l}0.016 \\
0.009\end{array}$ & $\begin{array}{ll}0.002 \\
0.001\end{array}$ & $\begin{array}{ll}0.011 \\
0.006\end{array}$ & $\begin{array}{l}0.015 \\
0.009\end{array}$ & $\begin{array}{ll}0.014 \\
0.008\end{array}$ & $\begin{array}{ll}0.098 \\
0.099 \\
\end{array}$ & \begin{tabular}{l|l|}
0.016 \\
0.023 \\
\end{tabular} & $\begin{array}{l}0.575 \\
0.324 \\
\end{array}$ & 0.368 & $\begin{array}{ll}0.246 \\
0.141\end{array}$ & & $\begin{array}{ll}0.008 \\
0.012\end{array}$ & & & & 0.000 & 0.001 & $\frac{1.000}{1.000}$ & $\begin{array}{l}7.82 \\
7.91\end{array}$ \\
\hline COOLED BOTTOMS & & 0.01 & 0.007 & 0.001 & 0.005 & 0.007 & 0.006 & 0.085 & $\begin{array}{ll}0.023 \\
0.020 \\
\end{array}$ & $\begin{array}{l}0.253 \\
0.253 \\
\end{array}$ & 0.368 & 0.1412 & & 0.014 & & & 0.000 & \begin{tabular}{|l|}
0.0001 \\
0.001 \\
\end{tabular} & 0.001 & 1.000 & $\mid 7.90$ \\
\hline COOLED BOTTOMS & & 0.05 & 0.005 & 0.001 & 0.004 & 0.005 & 0.005 & 0.071 & 0.016 & 0.190 & 0.602 & 0.085 & & 0.014 & & & 0.000 & 0.001 & 0.001 & 1.000 & 7.82 \\
\hline COOLED BOTTOMS & & $10 \mathrm{wt} \%$ TS & 0.005 & 0.001 & 0.004 & 0.005 & 0.005 & 0.072 & 0.016 & 0.190 & 0.601 & 0.085 & & 0.014 & & & 0.000 & 0.001 & 0.001 & 1.000 & 7.83 \\
\hline COOLED BOTTOMS & & $20 \mathrm{wt} \%$ TS & 0.005 & 0.001 & 0.004 & 0.005 & 0.005 & 0.069 & 0.016 & 0.182 & 0.622 & 0.080 & & 0.010 & & & 0.000 & \begin{tabular}{|l|l|}
0.001 \\
\end{tabular} & 0.002 & 1.000 & 7.76 \\
\hline COOLED BOTTOMS & & $30 \mathrm{wt} \%$ TS & 0.005 & 0.001 & 0.004 & 0.002 & 0.005 & 0.068 & 0.016 & 0.186 & 0.631 & 0.079 & & 0.002 & & & 0.000 & 0.001 & 0.002 & 1.000 & 7.71 \\
\hline & $60 \%$ & & & & & & & & & & & & & & & & & & & & \\
\hline COOLED BOTTOMS & & 0.001 & 0.137 & 0.003 & 0.012 & 0.016 & 0.015 & 0.136 & 0.024 & 0.563 & & & & 0.020 & 0.074 & & & & & 1.000 & 5.98 \\
\hline COOLED BOTTOMS & & 0.005 & 0.081 & 0.002 & 0.007 & 0.009 & 0.009 & 0.125 & 0.029 & 0.275 & 0.304 & & & 0.019 & 0.141 & & & & & 1.000 & 5.89 \\
\hline COOLED BOTTOMS & & 0.01 & 0.063 & 0.001 & 0.006 & 0.007 & 0.007 & 0.104 & 0.024 & 0.206 & 0.437 & & & 0.020 & 0.123 & & & & & 1.000 & 5.88 \\
\hline COOLED BOTTOMS & & 0.05 & 0.047 & 0.001 & 0.004 & 0.005 & 0.005 & 0.084 & 0.019 & 0.135 & 0.563 & & 0.013 & 0.020 & 0.103 & & & & & 1.000 & 5.84 \\
\hline COOLED BOTTOMS & & $10 \mathrm{wt} \%$ TS & 0.048 & 0.001 & 0.004 & 0.005 & 0.005 & 0.085 & 0.020 & 0.139 & 0.560 & & 0.010 & 0.020 & 0.104 & & & & & 1.000 & 5.85 \\
\hline COOLED BOTTOMS & & $20 \mathrm{wt} \% \mathrm{TS}$ & 0.043 & 0.001 & 0.004 & 0.005 - 15 & 0.005 & 0.078 & 0.018 & 0.108 & 0.586 & & 0.040 & 0.017 & 0.096 & & & & & 1.000 & 5.78 \\
\hline COOLED BOTTOMS & & $30 \mathrm{wt} \%$ TS & 0.042 & 0.001 & 0.004 & 0.003 & 0.005 & 0.076 & 0.018 & 0.100 & 0.596 & & 0.054 & 0.011 & 0.091 & & & & & 1.000 & 5.71 \\
\hline & $70 \%$ & & & & & & & & & & & & & & & & & & & & \\
\hline COOLED BOTTOMS & & 0.001 & 0.107 & 0.003 & 0.014 & 0.016 & 0.016 & 0.116 & 0.026 & 0.611 & & 0.070 & & 0.021 & & & & & & 1.000 & 6.73 \\
\hline COOLED BOTTOMS & & 0.005 & 0.064 & 0.002 & 0.009 & 0.010 & 0.009 & 0.115 & 0.030 & 0.362 & 0.339 & 0.041 & & 0.020 & & & & & & 1.000 & 6.55 \\
\hline COOLED BOTTOMS & & 0.01 & 0.050 & 0.001 & 0.007 & 0.008 & 0.007 & 0.096 & 0.025 & 0.281 & 0.471 & 0.032 & & 0.021 & & & & & & 1.000 & 6.47 \\
\hline COOLED BOTTOMS & & 0.05 & 0.037 & 0.001 & 0.005 & 0.006 & 0.005 & 0.078 & 0.020 & 0.198 & 0.595 & 0.025 & 0.010 & 0.021 & & & & & & 1.000 & 6.22 \\
\hline COOLED BOTTOMS & & $10 \mathrm{wt} \%$ TS & 0.038 & 0.001 & 0.005 & 0.006 & 0.006 & 0.080 & 0.021 & 0.211 & 0.586 & 0.025 & & 0.021 & & & & & & 1.000 & 6.23 \\
\hline COOLED BOTTOMS & & $20 \mathrm{wt} \%$ TS & 0.034 & 0.001 & 0.005 & 0.005 & 0.005 & 0.073 & 0.019 & 0.169 & 0.615 & 0.023 & 0.034 & 0.019 & & & & & & 1.000 & 6.17 \\
\hline COOLED BOTTOMS & & $30 \mathrm{wt} \%$ TS & 0.033 & 0.001 & 0.004 & 0.003 & 0.005 & 0.070 & 0.019 & 0.157 & 0.625 & 0.023 & 0.045 & 0.014 & & & & & & 1.000 & 6.10 \\
\hline & $80 \%$ & & & & & & & & & & & & & & & & & & & & \\
\hline COOLED BOTTOMS & & 0.001 & 0.087 & 0.003 & 0.015 & 0.015 & 0.018 & 0.097 & 0.028 & 0.608 & & 0.096 & & 0.033 & & & & & & 1.000 & 6.77 \\
\hline COOLED BOTTOMS & & 0.005 & 0.055 & 0.002 & 0.010 & 0.009 & 0.012 & 0.111 & 0.033 & 0.384 & 0.297 & 0.060 & & 0.027 & & & & & & 1.000 & 6.59 \\
\hline COOLED BOTTOMS & & 0.01 & 0.043 & 0.001 & 0.008 & 0.007 & 0.009 & 0.093 & 0.028 & 0.297 & 0.440 & 0.048 & & 0.026 & & & & & & 1.000 & 6.51 \\
\hline COOLED BOTTOMS & & 0.05 & 0.032 & 0.001 & 0.006 & 0.005 & 0.007 & 0.075 & 0.022 & 0.208 & 0.571 & 0.036 & 0.012 & 0.025 & & & & & & 1.000 & 6.26 \\
\hline COOLED BOTTOMS & & $10 \mathrm{wt} \%$ TS & 0.033 & 0.001 & 0.006 & 0.006 & 0.007 & 0.079 & 0.023 & 0.227 & 0.555 & 0.038 & & 0.026 & & & & & & 1.000 & 6.29 \\
\hline COOLED BOTTOMS & & $20 \mathrm{wt} \%$ TS & 0.030 & 0.001 & 0.005 & 0.005 & 0.006 & 0.072 & 0.020 & 0.184 & 0.589 & 0.035 & 0.030 & 0.023 & & & & & & 1.000 & 6.24 \\
\hline COOLED BOTTOMS & & $30 \mathrm{wt} \%$ TS & 0.028 & 0.001 & 0.005 & 0.004 & 0.006 & 0.069 & 0.020 & 0.170 & 0.601 & 0.035 & 0.041 & 0.020 & & & & & & 1.000 & 6.18 \\
\hline & $90 \%$ & & & & & & & & & & & & & & & & & & & 1.000 & \\
\hline COOLED BOTTOMS & & 0.001 & 0.085 & 0.002 & 0.012 & 0.016 & 0.020 & 0.026 & 0.010 & & & & & 0.031 & 0.798 & & & & & 1.000 & 6.07 \\
\hline COOLED BOTTOMS & & 0.005 & 0.073 & 0.001 & 0.010 & 0.013 & 0.017 & 0.098 & 0.036 & & & & & 0.0014 & 0.751 & & & & & 1.000 & 5.28 \\
\hline COOLED BOTTOMS & & 0.01 & 0.056 & 0.001 & 0.008 & 0.011 & 0.014 & 0.085 & 0.031 & & 0.179 & & & & 0.611 & 0.005 & & & & 1.000 & 5.18 \\
\hline COOLED BOTTOMS & & 0.05 & 0.030 & 0.001 & 0.006 & 0.008 & 0.010 & 0.070 & 0.022 & & 0.353 & & & 0.014 & 0.463 & 0.023 & & & & 1.000 & 5.24 \\
\hline COOLED BOTTOMS & & $10 \mathrm{wt} \%$ TS & 0.034 & 0.001 & 0.007 & 0.009 & 0.011 & 0.073 & 0.025 & & 0.318 & & & 0.011 & 0.492 & 0.020 & & & & 1.000 & 5.21 \\
\hline COOLED BOTTOMS & & $20 \mathrm{wt} \%$ TS & 0.028 & 0.001 & 0.006 & 0.008 & 0.010 & 0.069 & 0.020 & & 0.369 & & 0.007 & 0.015 & 0.444 & 0.024 & & & & 1.000 & 25 \\
\hline COOLED BOTTOMS & & $30 \mathrm{wt} \%$ TS & 0.022 & 0.001 & 0.006 & 0.005 & 0.009 & 0.064 & 0.022 & & 0.410 & & 0.049 & 0.011 & 0.374 & 0.028 & & & & 1.000 & 27 \\
\hline & $95 \%$ & & & & & & & & & & & & & & & & & & & & \\
\hline COOLED BOTTOMS & & 0.001 & 0.170 & 0.002 & 0.016 & 0.028 & 0.032 & 0.049 & 0.020 & & & & & & 0.671 & 0.012 & & & & 1.000 & 5.27 \\
\hline COOLED BOTTOMS & & 0.005 & 0.003 & 0.001 & 0.010 & 0.018 & 0.020 & 0.082 & 0.029 & & 0.035 & & & & 0.628 & 0.173 & & & & 1.000 & 5.17 \\
\hline COOLED BOTTOMS & & 0.01 & & 0. & o. & 0 & 0 & o. & o. & & 0 & & & & 0.501 & 0.140 & & & & 1.000 & 5.07 \\
\hline COOLED BOTTOMS & & 0.05 & & 0. & o. & & 0 & o. & o. & & 0.395 & & & & 0.388 & 0.108 & & & & 1.000 & 4.82 \\
\hline COOLED BOTTOMS & & $10 \mathrm{wt} \%$ TS & & 0.001 & 0.007 & 0.011 & 0.013 & 0.063 & 0.021 & & 0.359 & & & & 0.410 & 0.114 & & & & 1.000 & 4.89 \\
\hline COOLED BOTTOMS & & $20 \mathrm{wt} \%$ TS & & 0.001 & 0.006 & 0.010 & 0.012 & 0.061 & 0.015 & & 0.405 & & & & 0.383 & $\begin{array}{ll}0.106 \\
\end{array}$ & & & & 1.000 & 4.82 \\
\hline COOLED BOTTOMS & & $30 \mathrm{wt} \%$ TS & & 0.001 & 0.006 & 0.008 & 0.012 & 0.059 & 0.019 & & 0.425 & & 0.006 & & 0.363 & 0.102 & & & & 1.000 & 4.90 \\
\hline
\end{tabular}




\section{Table 10 - Insoluble Solids Formed in the Evaporator Concentrate for Feed Case 50-95\% Waste Effluent $\mathbf{p H}=8.5$}

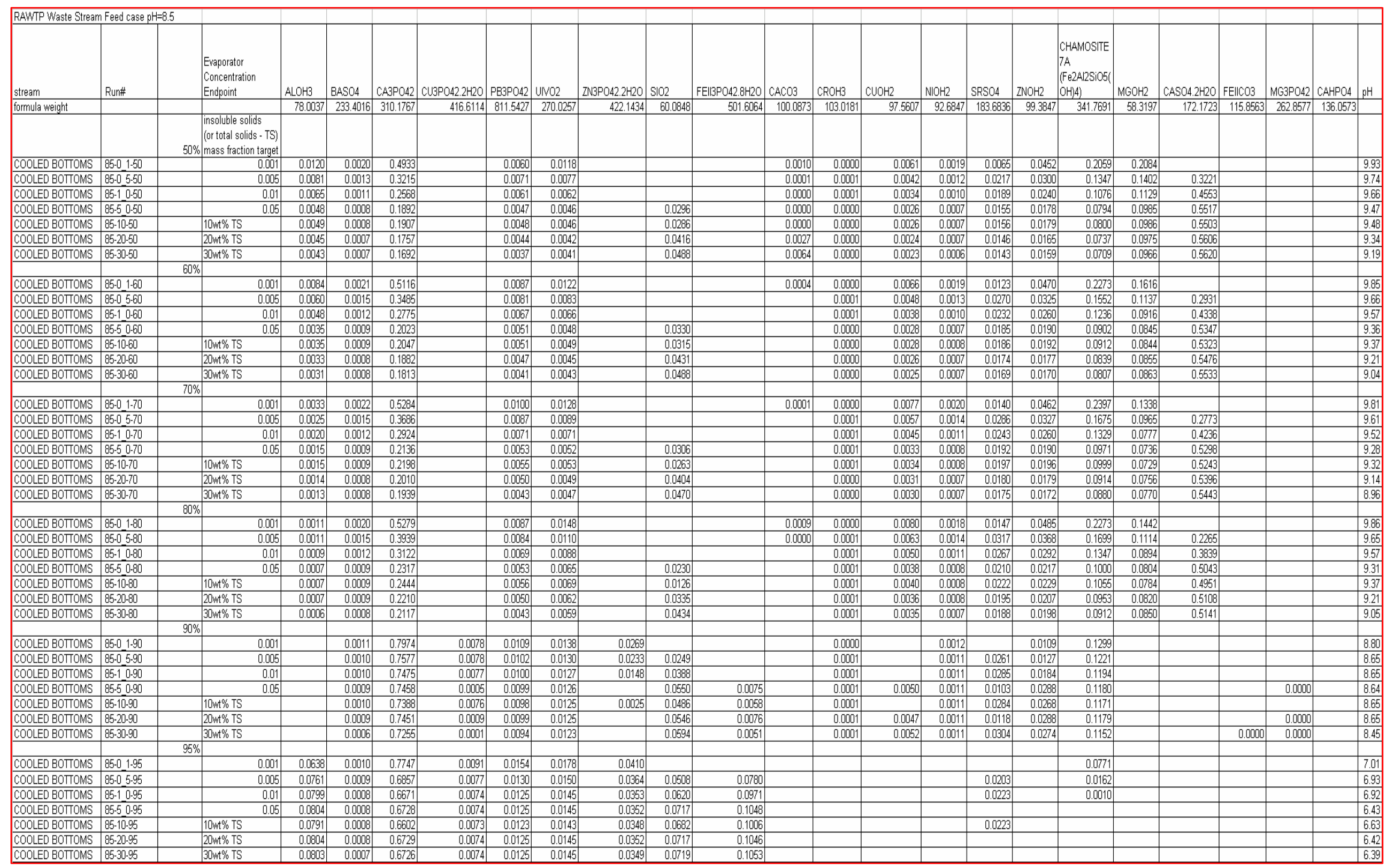


Table 11 - Composition of Insolubles Solids Formed During Evaporation of the Low and High Foam Simulants

\begin{tabular}{|c|c|c|c|c|c|c|c|c|c|c|c|c|c|}
\hline stream & Run\# & $\mathrm{ALOH} 3$ & CA.3PO42 & $\mathrm{SlO} 2$ & $\mathrm{CACO3}$ & $\mathrm{ZNOH} 2$ & CASO4.2H2O & $\begin{array}{l}\text { CHAMOSITE7A } \\
(\mathrm{Fe} 2 \mathrm{Al} 2 \mathrm{SiO5}(\mathrm{OH}) 4)\end{array}$ & $\mathrm{MGOH} 2$ & FEIICO3 & $\mathrm{ZN} 3 \mathrm{PP} 42.2 \mathrm{H} 2 \mathrm{O}$ & check & $\mathrm{pH}$ \\
\hline \multirow[t]{2}{*}{ formula weight } & & 78.0037 & 310.1767 & 60.0848 & 100.087 & 99.3847 & 172.1723 & 341.7691 & 58.3197 & 115.856 & 422.1434 & & \\
\hline & Low Foam & & & & & & & & & & & & \\
\hline COOLED BOTTOMS & $10 \mathrm{wt} \% \mathrm{TS}$ & 0.00 & 0.25 & & 0.02 & 0.02 & 0.49 & 0.11 & 0.11 & & & $1.00 \mathrm{E}+00$ & $9.53 \mathrm{E}+00$ \\
\hline COOLED BOTTOMS & $20 \mathrm{wt} \% \mathrm{TS}$ & 0.00 & 0.23 & 0.02 & 0.02 & 0.02 & 0.50 & 0.10 & 0.11 & & & $1.00 \mathrm{E}+00$ & $9.39 E+00$ \\
\hline \multirow[t]{2}{*}{ COOLED BOTTOMS } & $30 \mathrm{wt} \% \mathrm{TS}$ & 0.00 & 0.22 & 0.04 & 0.03 & 0.02 & 0.50 & 0.09 & 0.11 & & & $1.00 \mathrm{E}+00$ & $9.27 \mathrm{E}+00$ \\
\hline & High Foam & & & & & & & & & & & & \\
\hline COOLED BOTTOMS & $10 \mathrm{wt} \% \mathrm{TS}$ & 0.01 & 0.19 & 0.03 & 0.04 & 0.02 & 0.55 & 0.07 & 0.10 & & & $1.00 \mathrm{E}+00$ & $9.48 \mathrm{E}+00$ \\
\hline COOLED BOTTOMS & $20 \mathrm{wt} \% \mathrm{TS}$ & 0.00 & 0.17 & 0.04 & 0.05 & 0.02 & 0.55 & 0.07 & 0.10 & & & $1.00 \mathrm{E}+00$ & $9.33 \mathrm{E}+00$ \\
\hline COOLED BOTTOMS & $30 \mathrm{wt} \% \mathrm{TS}$ & 0.00 & 0.17 & 0.05 & 0.05 & 0.02 & 0.55 & 0.07 & 0.10 & & & $1.00 \mathrm{E}+00$ & $9.18 \mathrm{E}+00$ \\
\hline
\end{tabular}


WSRC-TR-2002-00567

Revision 0

\section{APPENDIX B - Composition of Evaporator Feed, Concentrate and Condensate for AWE Waste - Material and Energy Balance OLI Output Files}

$\underline{\text { AWE Low Foam Simulant - Concentrated to } 30 \mathrm{wt} \text {. \% Total Solids }}$

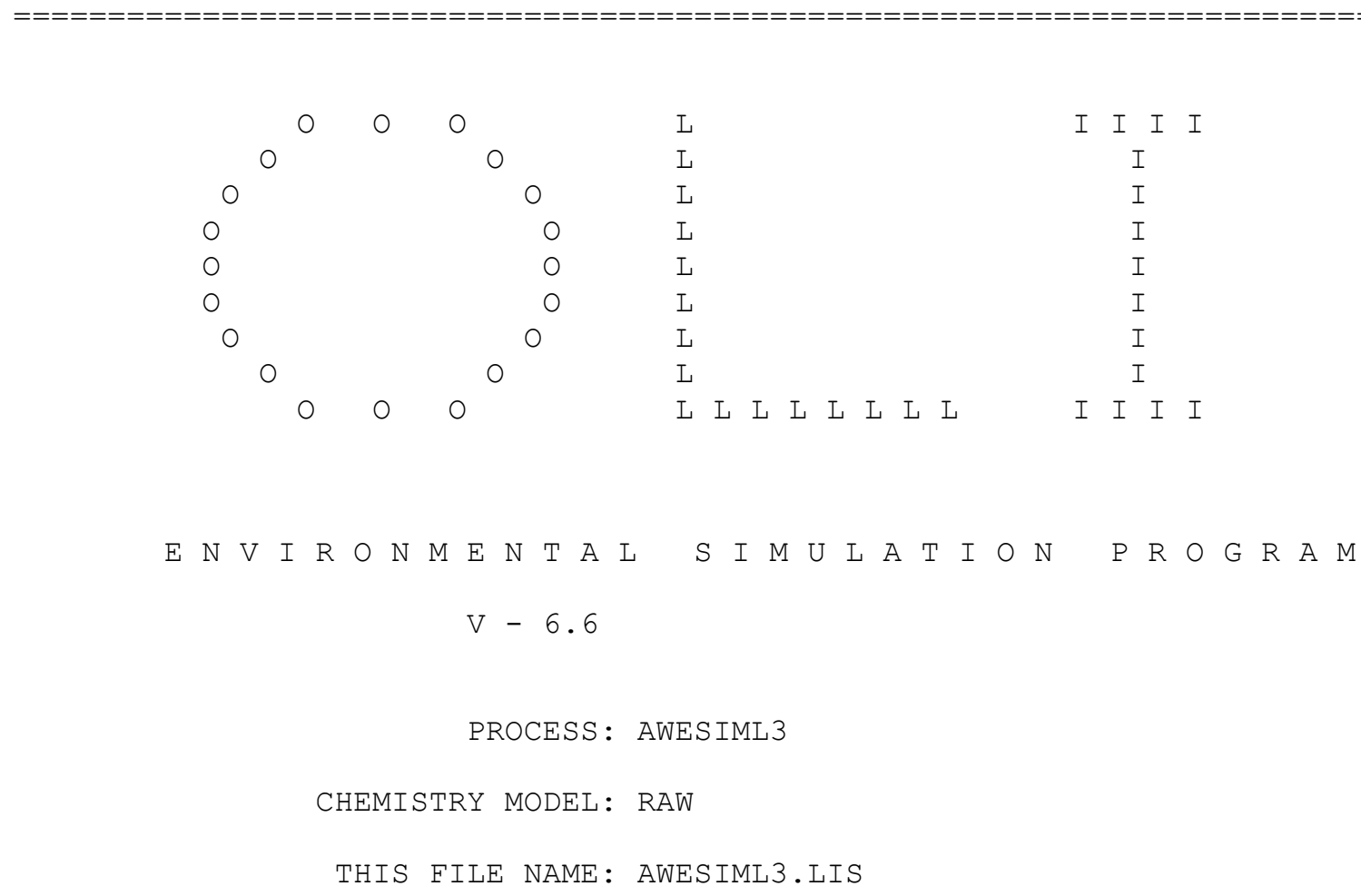


WSRC-TR-2002-00567

Revision 0

TABLE OF CONTENTS

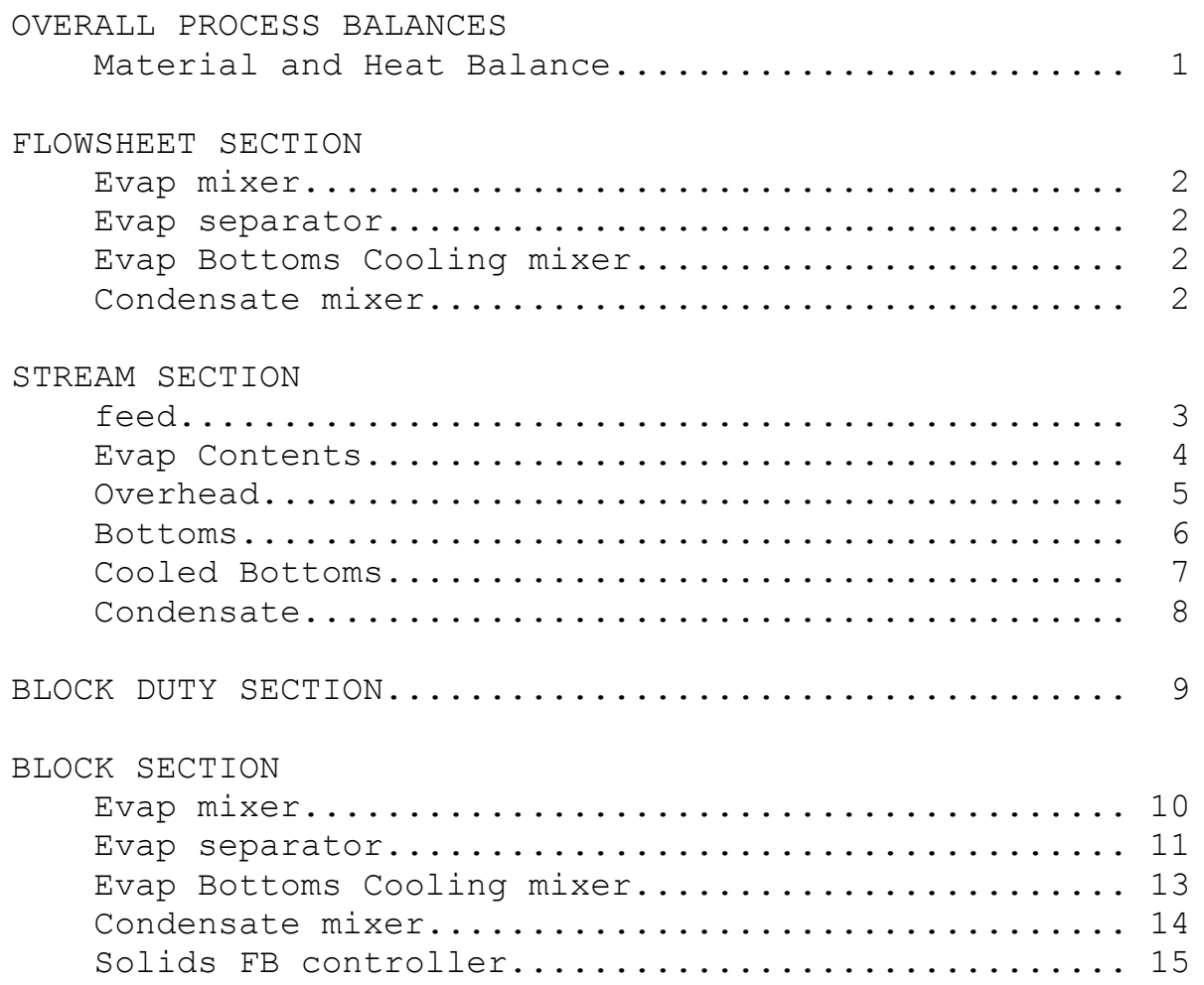


WSRC-TR-2002-00567

Revision 0

ESP V-6.6

PROCESS : AWES IML3
$11 / 21 / 2002 \quad$ PAGE 1
Overall Process Balances

\begin{tabular}{|c|c|c|}
\hline Inlet & g/hr & $\mathrm{cal} / \mathrm{hr}$ \\
\hline ----------- & ------------ & ---------1 \\
\hline FEED & $1.00000 \mathrm{D}+03$ & $-3.79081 D+06$ \\
\hline$==========$ & $=========$ & $====$ \\
\hline tal in & $1.00000 D+03$ & -3.7 \\
\hline
\end{tabular}

\begin{tabular}{lcc} 
Outlet & \multicolumn{1}{c}{ /hr } & cal/hr \\
----------- & ---------- & ---------- \\
COOLED BOTTOMS & $2.08960 \mathrm{D}+00$ & $-6.91549 \mathrm{D}+03$ \\
CONDENSATE & $9.97910 \mathrm{D}+02$ & $-3.78388 \mathrm{D}+06$ \\
$==========$ & $==========$ & $==========$ \\
Total out & $1.00000 \mathrm{D}+03$ & $-3.79079 \mathrm{D}+06$
\end{tabular}

Block Heat Duties

$$
\begin{gathered}
\text { cal/hr } \\
--------- \\
6.16760 \mathrm{D}+05 \\
-1.26083 \mathrm{D}+02 \\
-6.16621 \mathrm{D}+05 \\
========== \\
1.34336 \mathrm{D}+01
\end{gathered}
$$$$
\text { EVAP MIXER }
$$$$
\text { EVAP BOTTOMS COOLING MIXER }
$$$$
\text { CONDENSATE MIXER }
$$

$=========$

Total Duty

DIFFERENCE
REL DIEFERENCE

\begin{tabular}{|c|c|c|c|c|}
\hline Code & $\begin{array}{l}\text { Input } \\
\text { mol/hr }\end{array}$ & $\begin{array}{l}\text { Outlet } \\
\text { mol/hr }\end{array}$ & $\begin{array}{l}\text { Difference } \\
\text { mol/hr }\end{array}$ & Rel Diff \\
\hline------------ & ------------ & ------------ & ------------ & ------------1 \\
\hline $\mathrm{H}(+1)$ & $1.10941 D+02$ & $1.10941 \mathrm{D}+02$ & $1.42109 \mathrm{D}-14$ & $1.28093 \mathrm{D}-16$ \\
\hline $\mathrm{K}(+1)$ & $9.46243 \mathrm{D}-04$ & $9.46243 \mathrm{D}-04$ & $9.75782 \mathrm{D}-19$ & $1.03122 \mathrm{D}-15$ \\
\hline $\mathrm{NA}(+1)$ & $4.42632 \mathrm{D}-03$ & $4.42632 \mathrm{D}-03$ & $-8.67362 \mathrm{D}-19$ & $-1.95955 D-16$ \\
\hline $\mathrm{CA}(+2)$ & $1.30644 \mathrm{D}-03$ & $1.30644 \mathrm{D}-03$ & $0.00000 \mathrm{D}+00$ & $0.00000 \mathrm{D}+00$ \\
\hline $\mathrm{ZN}(+2)$ & $4.89521 \mathrm{D}-05$ & $4.89521 \mathrm{D}-05$ & $0.00000 \mathrm{D}+00$ & $0.00000 \mathrm{D}+00$ \\
\hline $\mathrm{FE}(+2)$ & $1.32505 \mathrm{D}-04$ & $1.32505 \mathrm{D}-04$ & $0.00000 \mathrm{D}+00$ & $0.00000 \mathrm{D}+00$ \\
\hline $\mathrm{MG}(+2)$ & $4.93583 D-04$ & $4.93583 D-04$ & $0.00000 \mathrm{D}+00$ & $0.00000 \mathrm{D}+00$ \\
\hline $\mathrm{AL}(+3)$ & $1.33425 \mathrm{D}-04$ & $1.33425 \mathrm{D}-04$ & $2.71051 D-20$ & $2.03149 D-16$ \\
\hline$O(-2)$ & $5.54852 \mathrm{D}+01$ & $5.54852 \mathrm{D}+01$ & $1.42109 \mathrm{D}-14$ & $2.56120 \mathrm{D}-16$ \\
\hline $\mathrm{CL}(-1)$ & $3.44118 D-03$ & $3.44118 D-03$ & $1.73472 \mathrm{D}-18$ & $5.04108 \mathrm{D}-16$ \\
\hline$C(+4)$ & $1.96636 \mathrm{D}-03$ & $1.96636 \mathrm{D}-03$ & $1.96501 \mathrm{D}-15$ & $9.99312 \mathrm{D}-13$ \\
\hline$P(+5)$ & $3.36943 \mathrm{D}-04$ & $3.36943 \mathrm{D}-04$ & $0.00000 \mathrm{D}+00$ & $0.00000 \mathrm{D}+00$ \\
\hline$S(+6)$ & $1.22838 D-03$ & $1.22838 D-03$ & $0.00000 \mathrm{D}+00$ & $0.00000 \mathrm{D}+00$ \\
\hline$N(+5)$ & $9.35410 \mathrm{D}-04$ & $9.35410 \mathrm{D}-04$ & $3.25261 \mathrm{D}-19$ & $3.47720 \mathrm{D}-16$ \\
\hline $\operatorname{SI}(+4)$ & $2.99577 \mathrm{D}-04$ & $2.99577 D-04$ & $5.42101 \mathrm{D}-20$ & $1.80956 \mathrm{D}-16$ \\
\hline $\operatorname{DODEC}(-1)$ & $5.57619 D-05$ & $5.57619 \mathrm{D}-05$ & $8.36358 D-15$ & $1.49987 \mathrm{D}-10$ \\
\hline
\end{tabular}

1. $36424 \mathrm{D}-12$

1. $36424 \mathrm{D}-15$

$0.00000 D+00$

$0.00000 \mathrm{D}+00$

Material Code Balances 
WSRC-TR-2002-00567

Revision 0

\begin{tabular}{|c|c|c|c|}
\hline \multicolumn{4}{|l|}{$\begin{array}{l}\text { PROCESS BLOCKS } \\
============\end{array}$} \\
\hline $\begin{array}{l}\mathrm{BLOCK} \mathrm{NAME} \\
=========================\end{array}$ & $\begin{array}{l}\text { BLOCK TYPE } \\
===========\end{array}$ & $\begin{array}{l}\text { INLET STREAM }(\mathrm{S}) \\
==============\end{array}$ & $\begin{array}{l}\text { OUTLET STREAM }(\mathrm{S}) \\
==============\end{array}$ \\
\hline Evap mixer & $\operatorname{Mix}$ & feed & Evap Contents \\
\hline Evap separator & Separate & Evap Contents & $\begin{array}{l}\text { Overhead } \\
\text { Bottoms }\end{array}$ \\
\hline Evap Bottoms Cooling mixer & Mix & Bottoms & Cooled Bottoms \\
\hline Condensate mixer & Mix & Overhead & Condensate \\
\hline
\end{tabular}


WSRC-TR-2002-00567

Revision 0

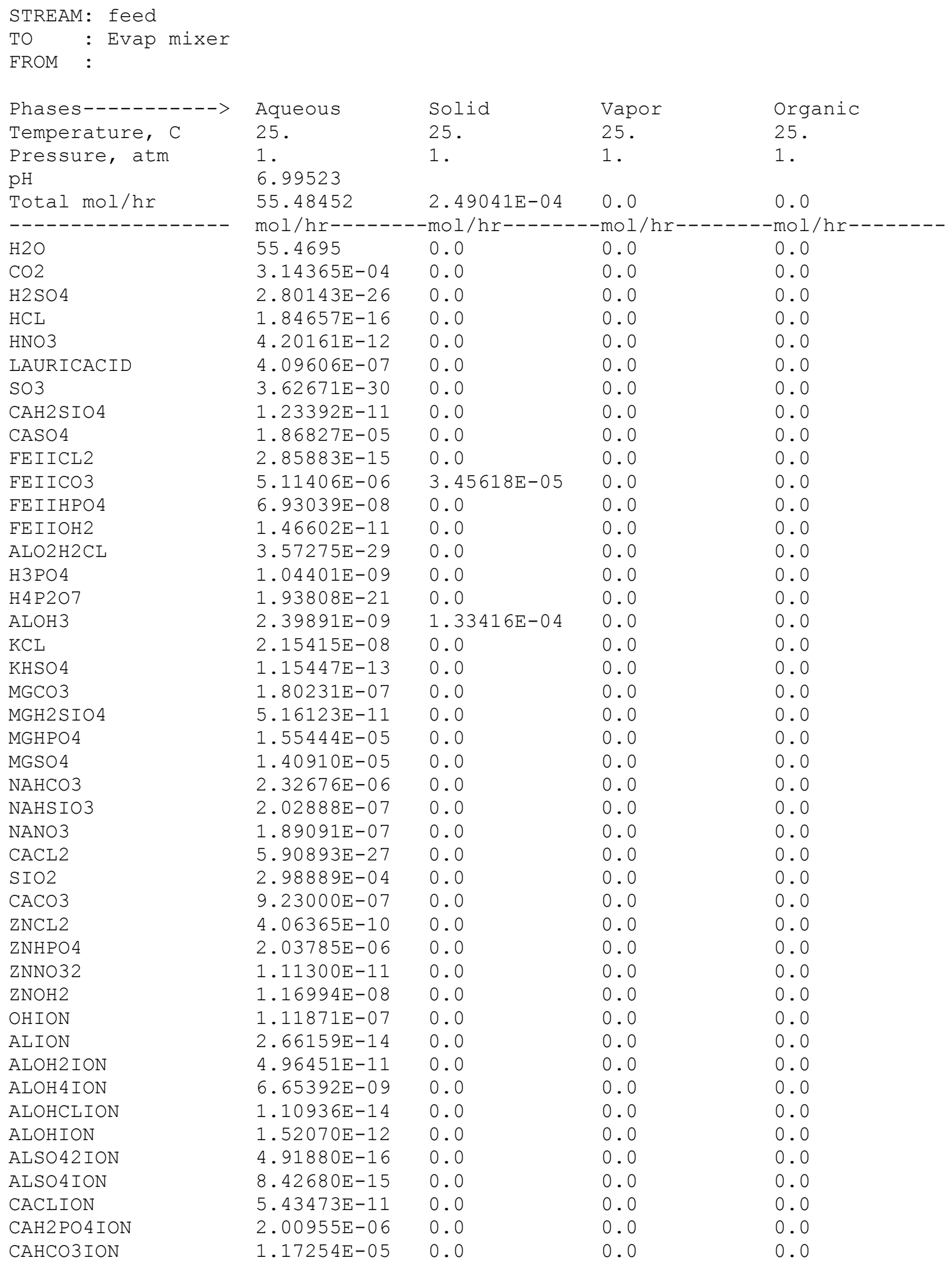




\begin{tabular}{|c|c|c|c|c|}
\hline CAHSIO3ION & $5.74946 \mathrm{E}-09$ & 0.0 & 0.0 & 0.0 \\
\hline CAION & 0.00103554 & 0.0 & 0.0 & 0.0 \\
\hline CANO3ION & $1.63421 \mathrm{E}-06$ & 0.0 & 0.0 & 0.0 \\
\hline CAOHION & $1.26675 \mathrm{E}-09$ & 0.0 & 0.0 & 0.0 \\
\hline CAPO 4 ION & $6.01092 \mathrm{E}-07$ & 0.0 & 0.0 & 0.0 \\
\hline CLION & 0.00344102 & 0.0 & 0.0 & 0.0 \\
\hline CO3ION & $1.02871 \mathrm{E}-06$ & 0.0 & 0.0 & 0.0 \\
\hline DODECION & $5.53523 E-05$ & 0.0 & 0.0 & 0.0 \\
\hline FEIICLION & $2.81091 \mathrm{E}-10$ & 0.0 & 0.0 & 0.0 \\
\hline FEIICO32ION & $1.72343 \mathrm{E}-10$ & 0.0 & 0.0 & 0.0 \\
\hline FEIIH2PO4ION & $1.59009 \mathrm{E}-08$ & 0.0 & 0.0 & 0.0 \\
\hline FEIIHCO3ION & $1.37456 \mathrm{E}-08$ & 0.0 & 0.0 & 0.0 \\
\hline FEIIION & $9.25219 \mathrm{E}-05$ & 0.0 & 0.0 & 0.0 \\
\hline FEIIOH3ION & $6.41041 \mathrm{E}-15$ & 0.0 & 0.0 & 0.0 \\
\hline FEIIOH 4 ION & $9.12118 \mathrm{E}-23$ & 0.0 & 0.0 & 0.0 \\
\hline FEIIOHION & $2.07616 \mathrm{E}-07$ & 0.0 & 0.0 & 0.0 \\
\hline H2P2O7ION & $3.83135 \mathrm{E}-11$ & 0.0 & 0.0 & 0.0 \\
\hline $\mathrm{H} 2 \mathrm{PO} 4 \mathrm{ION}$ & $8.19966 \mathrm{E}-05$ & 0.0 & 0.0 & 0.0 \\
\hline H2SIO 4 ION & $5.19387 \mathrm{E}-13$ & 0.0 & 0.0 & 0.0 \\
\hline H3P2O7ION & $6.10776 \mathrm{E}-16$ & 0.0 & 0.0 & 0.0 \\
\hline H3SIO4ION & $4.74378 E-07$ & 0.0 & 0.0 & 0.0 \\
\hline HCO3ION & 0.00157808 & 0.0 & 0.0 & 0.0 \\
\hline HION & $1.12672 \mathrm{E}-07$ & 0.0 & 0.0 & 0.0 \\
\hline HP2O 7 ION & $1.65191 \mathrm{E}-10$ & 0.0 & 0.0 & 0.0 \\
\hline HPO4 ION & $7.09697 \mathrm{E}-05$ & 0.0 & 0.0 & 0.0 \\
\hline HSO 4 ION & $7.95658 \mathrm{E}-09$ & 0.0 & 0.0 & 0.0 \\
\hline $\mathrm{KION}$ & $9.40933 \mathrm{E}-04$ & 0.0 & 0.0 & 0.0 \\
\hline KSO4 ION & $5.28886 \mathrm{E}-06$ & 0.0 & 0.0 & 0.0 \\
\hline MGH2 PO 4 ION & $1.11754 \mathrm{E}-06$ & 0.0 & 0.0 & 0.0 \\
\hline MGHCO3ION & $1.71975 \mathrm{E}-05$ & 0.0 & 0.0 & 0.0 \\
\hline MGHSIO3ION & $4.59688 \mathrm{E}-09$ & 0.0 & 0.0 & 0.0 \\
\hline MGION & $4.45070 \mathrm{E}-04$ & 0.0 & 0.0 & 0.0 \\
\hline MGOHION & $5.25563 \mathrm{E}-09$ & 0.0 & 0.0 & 0.0 \\
\hline MGP207ION & $2.71945 \mathrm{E}-09$ & 0.0 & 0.0 & 0.0 \\
\hline MGPO 4 ION & $3.70610 \mathrm{E}-07$ & 0.0 & 0.0 & 0.0 \\
\hline NACO3ION & $1.02040 \mathrm{E}-08$ & 0.0 & 0.0 & 0.0 \\
\hline NAION & 0.00439637 & 0.0 & 0.0 & 0.0 \\
\hline NASO 4 I ON & $2.72235 \mathrm{E}-05$ & 0.0 & 0.0 & 0.0 \\
\hline NO3ION & $9.33521 \mathrm{E}-04$ & 0.0 & 0.0 & 0.0 \\
\hline P207ION & $1.79354 \mathrm{E}-12$ & 0.0 & 0.0 & 0.0 \\
\hline PO4ION & $5.84268 \mathrm{E}-10$ & 0.0 & 0.0 & 0.0 \\
\hline SO4ION & 0.00116308 & 0.0 & 0.0 & 0.0 \\
\hline ZNCL3ION & $4.76171 \mathrm{E}-13$ & 0.0 & 0.0 & 0.0 \\
\hline ZNCLION & $1.31662 \mathrm{E}-07$ & 0.0 & 0.0 & 0.0 \\
\hline ZNH2PO4 ION & $7.61159 \mathrm{E}-08$ & 0.0 & 0.0 & 0.0 \\
\hline ZNHCO3ION & $8.31495 E-07$ & 0.0 & 0.0 & 0.0 \\
\hline ZNION & $3.75962 \mathrm{E}-05$ & 0.0 & 0.0 & 0.0 \\
\hline ZNNO3ION & $6.50110 \mathrm{E}-08$ & 0.0 & 0.0 & 0.0 \\
\hline ZNOH3ION & $1.27644 \mathrm{E}-12$ & 0.0 & 0.0 & 0.0 \\
\hline ZNOH 4 ION & $2.28201 \mathrm{E}-18$ & 0.0 & 0.0 & 0.0 \\
\hline ZNOHION & $3.24651 \mathrm{E}-07$ & 0.0 & 0.0 & 0.0 \\
\hline $\mathrm{CA} 3 \mathrm{PO} 42$ & 0.0 & $7.84378 \mathrm{E}-05$ & 0.0 & 0.0 \\
\hline $\mathrm{ZN} 3 \mathrm{PO} 42.2 \mathrm{H} 2 \mathrm{O}$ & 0.0 & $2.62567 \mathrm{E}-06$ & 0.0 & 0.0 \\
\hline & $=============$ & $============$ & $====$ & $====$ \\
\hline Total g/hr & 999.96 & 0.0398492 & 0.0 & 0.0 \\
\hline Volume, m3/hr & 0.00100261 & $1.29365 E-08$ & 0.0 & 0.0 \\
\hline Enthalpy, cal/hr & $-3.79068 E+06$ & -126.263 & 0.0 & 0.0 \\
\hline Density, $g / m 3$ & 997357. & $3.08037 E+06$ & & \\
\hline
\end{tabular}


WSRC-TR-2002-00567

Revision 0

$\begin{array}{lllll}\text { Vapor fraction } & 0.0 & 0.0 & 0.0 & 0.0 \\ \text { Solid fraction } & 0.0 & 1.0 .0 & 0.0 \\ \text { Organic fraction } & 0.0 & 0.0 & 0.0 & 0.0 \\ \text { Osmotic Pres, atm } & 0.400597 & & \\ \text { Redox Pot, volts } & 0.0 & 9.51583 \mathrm{E}-04 & & \\ \text { E-Con, 1/ohm-cm } & 9 & \\ \text { E-Con, cm2/ohm-mol } & 158.607 & & \\ \text { Abs Visc, cP } & 0.893063 & 1.00263 & \\ \text { Rel Visc } & 0.0114481 & & \end{array}$


WSRC-TR-2002-00567

Revision 0

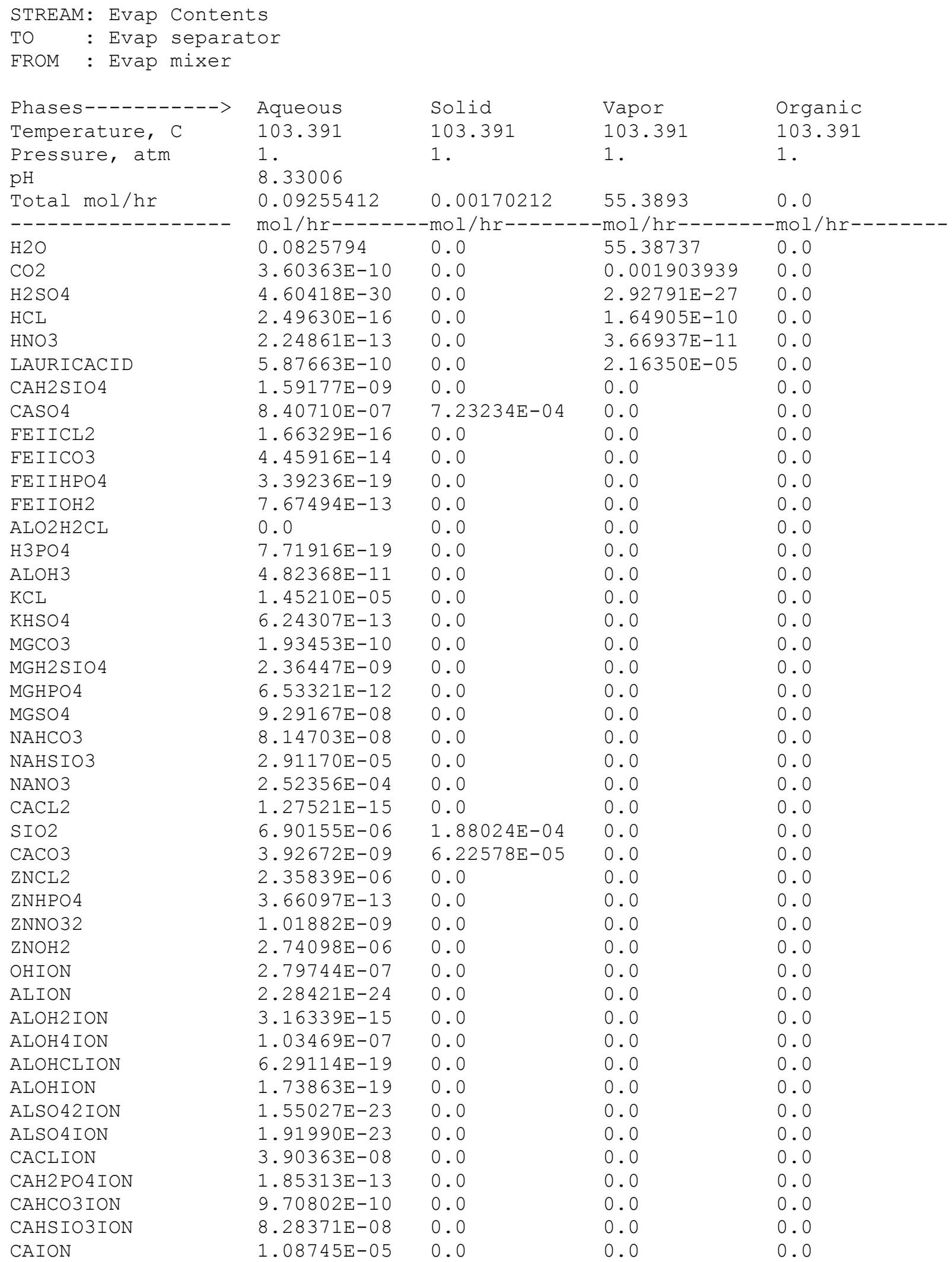




\begin{tabular}{|c|c|c|c|c|}
\hline CANO3ION & $3.66989 \mathrm{E}-06$ & 0.0 & 0.0 & 0.0 \\
\hline CAOHION & $1.66066 \mathrm{E}-08$ & 0.0 & 0.0 & 0.0 \\
\hline CAPO 4 ION & $7.48061 \mathrm{E}-11$ & 0.0 & 0.0 & 0.0 \\
\hline CLION & 0.00340754 & 0.0 & 0.0 & 0.0 \\
\hline CO3ION & $1.43269 \mathrm{E}-08$ & 0.0 & 0.0 & 0.0 \\
\hline DODECION & $3.41263 E-05$ & 0.0 & 0.0 & 0.0 \\
\hline FEIICLION & $3.94791 \mathrm{E}-14$ & 0.0 & 0.0 & 0.0 \\
\hline FEIICO32ION & $7.38409 \mathrm{E}-17$ & 0.0 & 0.0 & 0.0 \\
\hline FEIIH2PO4ION & $3.61891 \mathrm{E}-21$ & 0.0 & 0.0 & 0.0 \\
\hline FEIIHCO3ION & $1.23141 \mathrm{E}-17$ & 0.0 & 0.0 & 0.0 \\
\hline FEIIION & $5.34533 \mathrm{E}-12$ & 0.0 & 0.0 & 0.0 \\
\hline FEIIOH3ION & $1.91383 \mathrm{E}-13$ & 0.0 & 0.0 & 0.0 \\
\hline FEIIOH 4 ION & $5.80012 \mathrm{E}-17$ & 0.0 & 0.0 & 0.0 \\
\hline FEIIOHION & $5.40732 \mathrm{E}-12$ & 0.0 & 0.0 & 0.0 \\
\hline H2P2O7ION & $7.28539 \mathrm{E}-22$ & 0.0 & 0.0 & 0.0 \\
\hline H2 PO 4 ION & $1.97727 \mathrm{E}-12$ & 0.0 & 0.0 & 0.0 \\
\hline H2SIO4ION & $1.85673 \mathrm{E}-09$ & 0.0 & 0.0 & 0.0 \\
\hline H3P2O7ION & $6.91324 \mathrm{E}-29$ & 0.0 & 0.0 & 0.0 \\
\hline H3SIO 4 ION & $9.17684 \mathrm{E}-06$ & 0.0 & 0.0 & 0.0 \\
\hline HCO3ION & $5.82444 \mathrm{E}-08$ & 0.0 & 0.0 & 0.0 \\
\hline HION & $9.19279 \mathrm{E}-12$ & 0.0 & 0.0 & 0.0 \\
\hline HP2O7ION & $1.41298 \mathrm{E}-19$ & 0.0 & 0.0 & 0.0 \\
\hline HPO 4 ION & $2.34576 \mathrm{E}-10$ & 0.0 & 0.0 & 0.0 \\
\hline HSO4 ION & $8.24148 \mathrm{E}-11$ & 0.0 & 0.0 & 0.0 \\
\hline $\mathrm{KION}$ & $8.27127 \mathrm{E}-04$ & 0.0 & 0.0 & 0.0 \\
\hline KSO4 ION & $1.04595 \mathrm{E}-04$ & 0.0 & 0.0 & 0.0 \\
\hline MGH2 PO 4 ION & $6.15698 \mathrm{E}-15$ & 0.0 & 0.0 & 0.0 \\
\hline MGHCO 3 ION & $2.00704 \mathrm{E}-10$ & 0.0 & 0.0 & 0.0 \\
\hline MGHSIO3ION & $1.61067 \mathrm{E}-08$ & 0.0 & 0.0 & 0.0 \\
\hline MGION & $3.92793 E-07$ & 0.0 & 0.0 & 0.0 \\
\hline MGOHION & $1.36356 \mathrm{E}-08$ & 0.0 & 0.0 & 0.0 \\
\hline MGP207ION & $7.46318 \mathrm{E}-17$ & 0.0 & 0.0 & 0.0 \\
\hline MGPO 4 ION & $8.50099 \mathrm{E}-12$ & 0.0 & 0.0 & 0.0 \\
\hline NACO3ION & $2.26409 \mathrm{E}-09$ & 0.0 & 0.0 & 0.0 \\
\hline NAION & 0.00414476 & 0.0 & 0.0 & 0.0 \\
\hline NASO 4 I ON & $1.74217 \mathrm{E}-12$ & 0.0 & 0.0 & 0.0 \\
\hline NO3ION & $6.79343 E-04$ & 0.0 & 0.0 & 0.0 \\
\hline P207ION & $3.37841 E-19$ & 0.0 & 0.0 & 0.0 \\
\hline PO4ION & $3.89813 E-13$ & 0.0 & 0.0 & 0.0 \\
\hline SO4ION & $3.99612 \mathrm{E}-04$ & 0.0 & 0.0 & 0.0 \\
\hline ZNCL3ION & $2.78785 \mathrm{E}-06$ & 0.0 & 0.0 & 0.0 \\
\hline ZNCLION & $5.99576 \mathrm{E}-06$ & 0.0 & 0.0 & 0.0 \\
\hline ZNH2 PO 4 ION & $9.29610 \mathrm{E}-16$ & 0.0 & 0.0 & 0.0 \\
\hline ZNHCO3ION & $2.53787 \mathrm{E}-11$ & 0.0 & 0.0 & 0.0 \\
\hline ZNION & $3.87591 \mathrm{E}-07$ & 0.0 & 0.0 & 0.0 \\
\hline ZNNO3ION & $3.83502 \mathrm{E}-08$ & 0.0 & 0.0 & 0.0 \\
\hline ZNOH3ION & $1.20568 \mathrm{E}-06$ & 0.0 & 0.0 & 0.0 \\
\hline ZNOH 4 ION & $1.15294 \mathrm{E}-08$ & 0.0 & 0.0 & 0.0 \\
\hline ZNOHION & $3.34249 E-05$ & 0.0 & 0.0 & 0.0 \\
\hline ALOOH & 0.0 & $8.16392 \mathrm{E}-07$ & 0.0 & 0.0 \\
\hline $\mathrm{CA} 3 \mathrm{PO} 42$ & 0.0 & $1.68472 \mathrm{E}-04$ & 0.0 & 0.0 \\
\hline CHAMOSITE7A & 0.0 & $6.62523 E-05$ & 0.0 & 0.0 \\
\hline MGOH2 & 0.0 & $4.93064 \mathrm{E}-04$ & 0.0 & 0.0 \\
\hline Total o/hr & $\begin{array}{l}=========== \\
1.86991\end{array}$ & $\begin{array}{l}============ \\
0.219694\end{array}$ & $\begin{array}{l}============ \\
997.91\end{array}$ & $\begin{array}{l}=== \\
0.0\end{array}$ \\
\hline Volume, $\mathrm{m} 3 / \mathrm{hr}$ & $1.71908 \mathrm{E}-06$ & $6.92584 \mathrm{E}-08$ & 1.69746 & 0.0 \\
\hline Enthalpy, cal/hr & -6152.19 & -637.218 & $-3.16726 \mathrm{E}+06$ & 0.0 \\
\hline Density, $g / \mathrm{m} 3$ & $1.08773 \mathrm{E}+06$ & $3.17210 \mathrm{E}+06$ & 587.886 & \\
\hline
\end{tabular}


WSRC-TR-2002-00567

Revision 0

$\begin{array}{lll}\text { Vapor fraction } & 0.0 & 0.0 \\ \text { Solid fraction } & 0.0 & 1 . \\ \text { Organic fraction } & 0.0 & 0.0 \\ \text { Osmotic Pres, atm } & 192.486 & \\ \text { Redox Pot, volts } & 0.0 & \\ \text { E-Con, 1/ohm-cm } & 0.502653 & \\ \text { E-Con, cm2/ohm-mol } & 59.0235 & 0.424148 \\ \text { Abs Visc, cP } & 1.56016 & \\ \text { Rel Visc } & 3.66327 & \\ \text { Ionic Strength } & \end{array}$

1.

0.0

0.0

0.0

0.0 
WSRC-TR-2002-00567

Revision 0

\begin{tabular}{|c|c|c|c|c|}
\hline \\
\hline \multicolumn{5}{|c|}{$\begin{array}{l}\text { STREAM: Overhead } \\
\text { TO : Condensate mixer }\end{array}$} \\
\hline \multicolumn{5}{|c|}{ EROM : Evap separator } \\
\hline Phases-----------> & Aqueous & Solid & Vapor & Organic \\
\hline Temperature, C & 103.391 & 103.391 & 103.391 & 103.391 \\
\hline $\begin{array}{l}\text { Pressure, atm } \\
\mathrm{pH}\end{array}$ & 1. & 1 . & 1. & 1. \\
\hline $\mathrm{pH}$ & 0.0 & 0.0 & 55.3893 & 0.0 \\
\hline \multicolumn{5}{|c|}{$------------------\quad \operatorname{mol} / \mathrm{hr}--------\operatorname{mol} / \mathrm{hr}--------\mathrm{mol} / \mathrm{hr}--------\operatorname{mol} / \mathrm{hr}--------$} \\
\hline $\mathrm{H} 2 \mathrm{O}$ & 0.0 & 0.0 & 55.38737 & 0.0 \\
\hline $\mathrm{CO} 2$ & 0.0 & 0.0 & 0.001903939 & 0.0 \\
\hline $\mathrm{H} 2 \mathrm{SO} 4$ & 0.0 & 0.0 & $2.92791 E-27$ & 0.0 \\
\hline $\mathrm{HCL}$ & 0.0 & 0.0 & $1.64905 \mathrm{E}-10$ & 0.0 \\
\hline HNO3 & 0.0 & 0.0 & $3.66937 \mathrm{E}-11$ & 0.0 \\
\hline \multirow[t]{2}{*}{ LAURICACID } & 0.0 & 0.0 & $2.16350 \mathrm{E}-05$ & 0.0 \\
\hline & $===========$ & $===========$ & $===========$ & $===========$ \\
\hline Total g/hr & 0.0 & 0.0 & 997.91 & 0.0 \\
\hline Volume, m3/hr & 0.0 & 0.0 & 1.69746 & 0.0 \\
\hline $\begin{array}{l}\text { Enthalpy, cal/hr } \\
\text { Density, g/m3 }\end{array}$ & 0.0 & 0.0 & $\begin{array}{l}-3.16726 \mathrm{E}+06 \\
587.886\end{array}$ & 0.0 \\
\hline Vapor fraction & 0.0 & 0.0 & 1 . & 0.0 \\
\hline Solid fraction & 0.0 & 0.0 & 0.0 & 0.0 \\
\hline Organic fraction & 0.0 & 0.0 & 0.0 & 0.0 \\
\hline Osmotic Pres, atm & 0.0 & & & \\
\hline Redox Pot, volts & 0.0 & & & \\
\hline $\mathrm{E}-\mathrm{Con}, 1 / \mathrm{ohm}-\mathrm{cm}$ & 0.0 & & & \\
\hline E-Con, cm2/ohm-mol & 0.0 & & & \\
\hline Abs Visc, cP & 0.0 & & & \\
\hline Rel Visc & 0.0 & & & \\
\hline Ionic Strength & 0.0 & & & \\
\hline
\end{tabular}


WSRC-TR-2002-00567

Revision 0

ESP V-6.6

PROCESS : AWES IML3

$11 / 21 / 2002$

PAGE

6

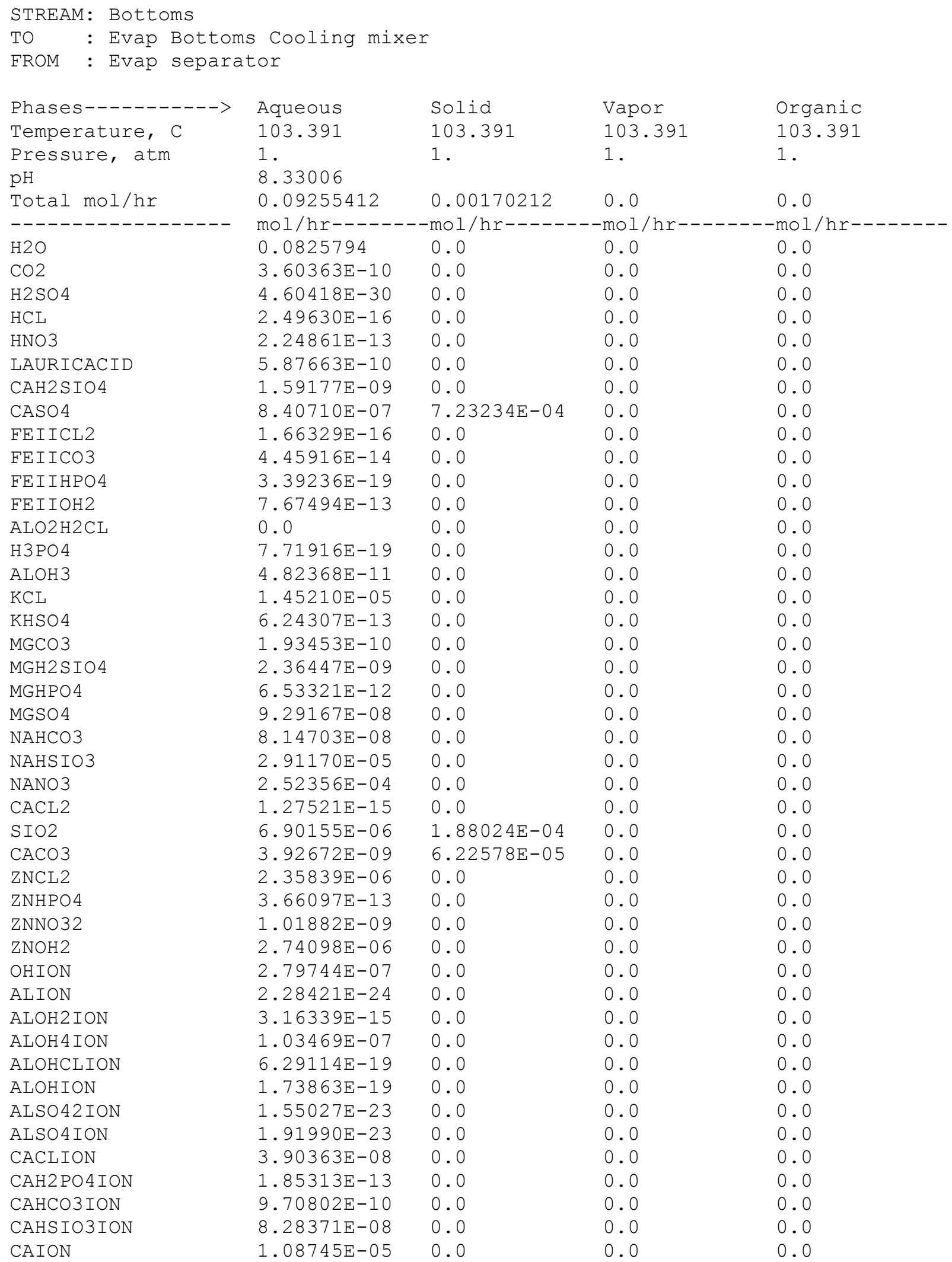




\begin{tabular}{|c|c|c|c|c|}
\hline CANO3ION & $3.66989 \mathrm{E}-06$ & 0.0 & 0.0 & 0.0 \\
\hline CAOHION & $1.66066 \mathrm{E}-08$ & 0.0 & 0.0 & 0.0 \\
\hline CAPO 4 ION & $7.48061 \mathrm{E}-11$ & 0.0 & 0.0 & 0.0 \\
\hline CLION & 0.00340754 & 0.0 & 0.0 & 0.0 \\
\hline CO3ION & $1.43269 \mathrm{E}-08$ & 0.0 & 0.0 & 0.0 \\
\hline DODECION & $3.41263 E-05$ & 0.0 & 0.0 & 0.0 \\
\hline FEIICLION & $3.94791 \mathrm{E}-14$ & 0.0 & 0.0 & 0.0 \\
\hline FEIICO32ION & $7.38409 \mathrm{E}-17$ & 0.0 & 0.0 & 0.0 \\
\hline FEIIH2PO4ION & $3.61891 \mathrm{E}-21$ & 0.0 & 0.0 & 0.0 \\
\hline FEIIHCO3ION & $1.23141 \mathrm{E}-17$ & 0.0 & 0.0 & 0.0 \\
\hline FEIIION & $5.34533 \mathrm{E}-12$ & 0.0 & 0.0 & 0.0 \\
\hline FEIIOH3ION & $1.91383 \mathrm{E}-13$ & 0.0 & 0.0 & 0.0 \\
\hline FEIIOH 4 ION & $5.80012 \mathrm{E}-17$ & 0.0 & 0.0 & 0.0 \\
\hline FEIIOHION & $5.40732 \mathrm{E}-12$ & 0.0 & 0.0 & 0.0 \\
\hline H2P2O7ION & $7.28539 \mathrm{E}-22$ & 0.0 & 0.0 & 0.0 \\
\hline H2 PO 4 ION & $1.97727 \mathrm{E}-12$ & 0.0 & 0.0 & 0.0 \\
\hline H2SIO4ION & $1.85673 \mathrm{E}-09$ & 0.0 & 0.0 & 0.0 \\
\hline H3P2O7ION & $6.91324 \mathrm{E}-29$ & 0.0 & 0.0 & 0.0 \\
\hline H3SIO 4 ION & $9.17684 \mathrm{E}-06$ & 0.0 & 0.0 & 0.0 \\
\hline HCO3ION & $5.82444 \mathrm{E}-08$ & 0.0 & 0.0 & 0.0 \\
\hline HION & $9.19279 \mathrm{E}-12$ & 0.0 & 0.0 & 0.0 \\
\hline HP2O7ION & $1.41298 \mathrm{E}-19$ & 0.0 & 0.0 & 0.0 \\
\hline HPO 4 ION & $2.34576 \mathrm{E}-10$ & 0.0 & 0.0 & 0.0 \\
\hline HSO4 ION & $8.24148 \mathrm{E}-11$ & 0.0 & 0.0 & 0.0 \\
\hline $\mathrm{KION}$ & $8.27127 \mathrm{E}-04$ & 0.0 & 0.0 & 0.0 \\
\hline KSO4 ION & $1.04595 \mathrm{E}-04$ & 0.0 & 0.0 & 0.0 \\
\hline MGH2 PO 4 ION & $6.15698 \mathrm{E}-15$ & 0.0 & 0.0 & 0.0 \\
\hline MGHCO 3 ION & $2.00704 \mathrm{E}-10$ & 0.0 & 0.0 & 0.0 \\
\hline MGHSIO3ION & $1.61067 \mathrm{E}-08$ & 0.0 & 0.0 & 0.0 \\
\hline MGION & $3.92793 E-07$ & 0.0 & 0.0 & 0.0 \\
\hline MGOHION & $1.36356 \mathrm{E}-08$ & 0.0 & 0.0 & 0.0 \\
\hline MGP207ION & $7.46318 \mathrm{E}-17$ & 0.0 & 0.0 & 0.0 \\
\hline MGPO 4 ION & $8.50099 \mathrm{E}-12$ & 0.0 & 0.0 & 0.0 \\
\hline NACO3ION & $2.26409 \mathrm{E}-09$ & 0.0 & 0.0 & 0.0 \\
\hline NAION & 0.00414476 & 0.0 & 0.0 & 0.0 \\
\hline NASO 4 I ON & $1.74217 \mathrm{E}-12$ & 0.0 & 0.0 & 0.0 \\
\hline NO3ION & $6.79343 E-04$ & 0.0 & 0.0 & 0.0 \\
\hline P207ION & $3.37841 E-19$ & 0.0 & 0.0 & 0.0 \\
\hline PO4ION & $3.89813 E-13$ & 0.0 & 0.0 & 0.0 \\
\hline SO4ION & $3.99612 \mathrm{E}-04$ & 0.0 & 0.0 & 0.0 \\
\hline ZNCL3ION & $2.78785 \mathrm{E}-06$ & 0.0 & 0.0 & 0.0 \\
\hline ZNCLION & $5.99576 \mathrm{E}-06$ & 0.0 & 0.0 & 0.0 \\
\hline ZNH2 PO 4 ION & $9.29610 \mathrm{E}-16$ & 0.0 & 0.0 & 0.0 \\
\hline ZNHCO3ION & $2.53787 \mathrm{E}-11$ & 0.0 & 0.0 & 0.0 \\
\hline ZNION & $3.87591 \mathrm{E}-07$ & 0.0 & 0.0 & 0.0 \\
\hline ZNNO3ION & $3.83502 \mathrm{E}-08$ & 0.0 & 0.0 & 0.0 \\
\hline ZNOH3ION & $1.20568 \mathrm{E}-06$ & 0.0 & 0.0 & 0.0 \\
\hline ZNOH 4 ION & $1.15294 \mathrm{E}-08$ & 0.0 & 0.0 & 0.0 \\
\hline ZNOHION & $3.34249 E-05$ & 0.0 & 0.0 & 0.0 \\
\hline ALOOH & 0.0 & $8.16392 \mathrm{E}-07$ & 0.0 & 0.0 \\
\hline $\mathrm{CA} 3 \mathrm{PO} 42$ & 0.0 & $1.68472 \mathrm{E}-04$ & 0.0 & 0.0 \\
\hline CHAMOSITE7A & 0.0 & $6.62523 E-05$ & 0.0 & 0.0 \\
\hline MGOH2 & 0.0 & $4.93064 \mathrm{E}-04$ & 0.0 & 0.0 \\
\hline Total $\mathrm{g} / \mathrm{hr}$ & $\begin{array}{l}=========== \\
1.86991\end{array}$ & $\begin{array}{l}============ \\
0.219694\end{array}$ & $\begin{array}{l}=== \\
0.0\end{array}$ & $\begin{array}{l}=== \\
0.0\end{array}$ \\
\hline Volume, $\mathrm{m} 3 / \mathrm{hr}$ & $1.71908 \mathrm{E}-06$ & $6.92584 \mathrm{E}-08$ & 0.0 & 0.0 \\
\hline Enthalpy, cal/hr & -6152.19 & -637.218 & 0.0 & 0.0 \\
\hline Density, $\mathrm{g} / \mathrm{m} 3$ & $1.08773 \mathrm{E}+06$ & $3.17210 \mathrm{E}+06$ & & \\
\hline
\end{tabular}


WSRC-TR-2002-00567

Revision 0

$\begin{array}{ll}\text { Vapor fraction } & 0.0 \\ \text { Solid fraction } & 0.0 \\ \text { Organic fraction } & 0.0 \\ \text { Osmotic Pres, atm } & 192.486 \\ \text { Redox Pot, volts } & 0.0 \\ \text { E-Con, 1/ohm-cm } & 0.502653 \\ \text { E-Con, cm2/ohm-mol } & 59.0235 \\ \text { Abs Visc, cP } & 0.424148 \\ \text { Rel Visc } & 1.56016 \\ \text { Ionic Strength } & 3.66327\end{array}$

0.0

0.0

0.0

1 .

0.0

0.0

0.0

0.0

0.0

Ionic Strength

1.56016 
WSRC-TR-2002-00567

Revision 0

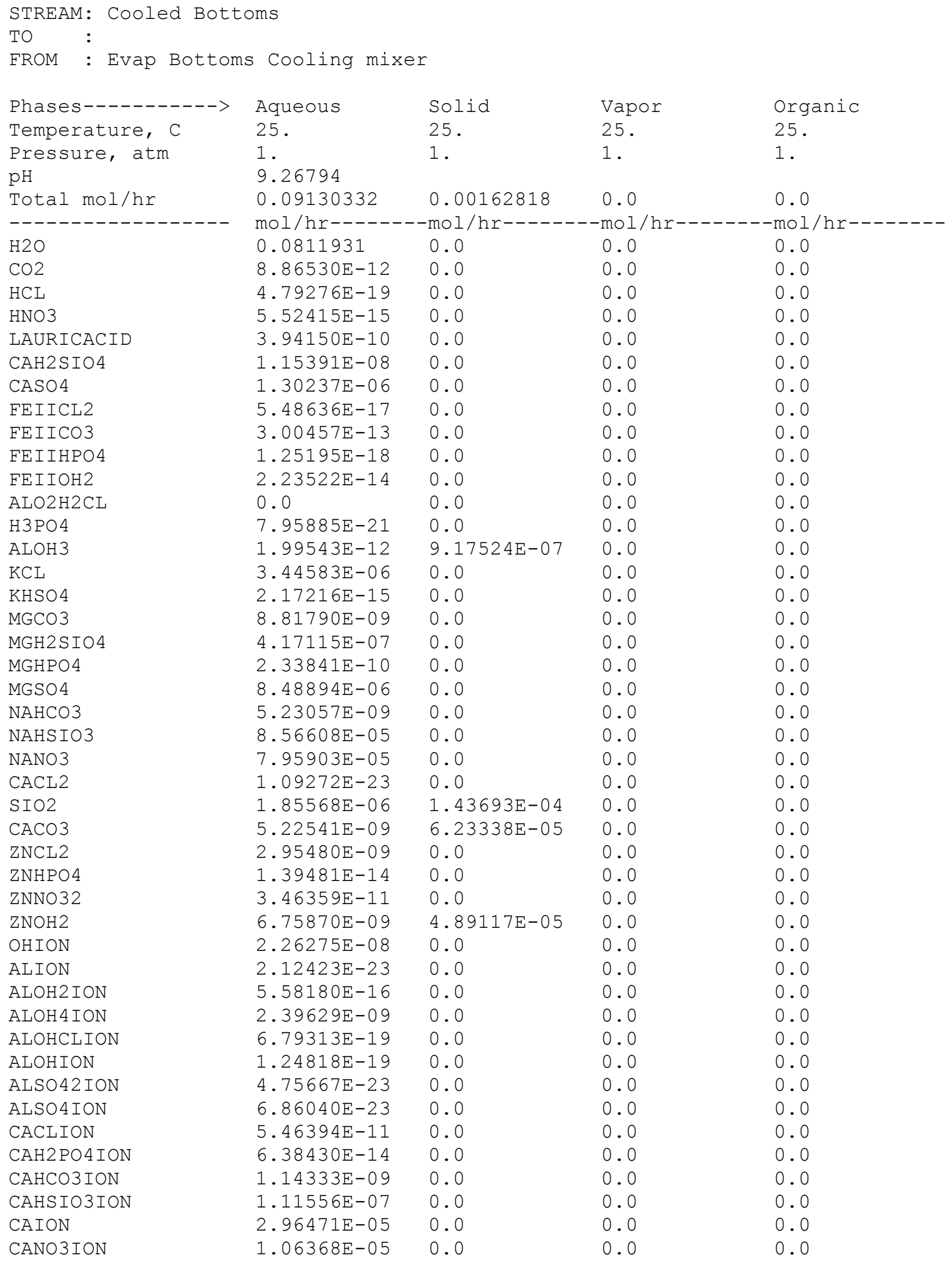




\begin{tabular}{|c|c|c|c|c|}
\hline CAOHION & $2.53167 \mathrm{E}-09$ & 0.0 & 0.0 & 0.0 \\
\hline CAPO 4 ION & $6.62076 \mathrm{E}-10$ & 0.0 & 0.0 & 0.0 \\
\hline CLION & 0.00343771 & 0.0 & 0.0 & 0.0 \\
\hline CO3ION & $1.85475 E-08$ & 0.0 & 0.0 & 0.0 \\
\hline DODECION & $3.41265 E-05$ & 0.0 & 0.0 & 0.0 \\
\hline FEIICLION & $2.24719 \mathrm{E}-14$ & 0.0 & 0.0 & 0.0 \\
\hline FEIICO32ION & $2.64096 \mathrm{E}-16$ & 0.0 & 0.0 & 0.0 \\
\hline FEIIH2PO4ION & $5.24244 \mathrm{E}-21$ & 0.0 & 0.0 & 0.0 \\
\hline FEIIHCO3ION & $1.04972 \mathrm{E}-17$ & 0.0 & 0.0 & 0.0 \\
\hline FEIIION & $1.96732 \mathrm{E}-11$ & 0.0 & 0.0 & 0.0 \\
\hline FEIIOH3ION & $5.33068 \mathrm{E}-15$ & 0.0 & 0.0 & 0.0 \\
\hline FEIIOH 4 ION & $9.47801 \mathrm{E}-20$ & 0.0 & 0.0 & 0.0 \\
\hline FEIIOHION & $4.30388 \mathrm{E}-12$ & 0.0 & 0.0 & 0.0 \\
\hline H2P2O 7 ION & $2.64443 \mathrm{E}-24$ & 0.0 & 0.0 & 0.0 \\
\hline $\mathrm{H} 2 \mathrm{PO} 4 \mathrm{ION}$ & $5.56334 \mathrm{E}-13$ & 0.0 & 0.0 & 0.0 \\
\hline H2SIO4ION & $1.82145 \mathrm{E}-09$ & 0.0 & 0.0 & 0.0 \\
\hline H3P2O7ION & 0.0 & 0.0 & 0.0 & 0.0 \\
\hline H3SIO4ION & $8.01991 \mathrm{E}-07$ & 0.0 & 0.0 & 0.0 \\
\hline HCO3ION & $2.62848 \mathrm{E}-08$ & 0.0 & 0.0 & 0.0 \\
\hline HION & $7.43792 \mathrm{E}-13$ & 0.0 & 0.0 & 0.0 \\
\hline HP2O7ION & $6.11402 \mathrm{E}-21$ & 0.0 & 0.0 & 0.0 \\
\hline HPO 4 ION & $6.63027 \mathrm{E}-10$ & 0.0 & 0.0 & 0.0 \\
\hline HSO 4 ION & $9.18255 \mathrm{E}-13$ & 0.0 & 0.0 & 0.0 \\
\hline $\mathrm{KION}$ & $8.84368 \mathrm{E}-04$ & 0.0 & 0.0 & 0.0 \\
\hline KSO4 ION & $5.84296 \mathrm{E}-05$ & 0.0 & 0.0 & 0.0 \\
\hline MGH2 PO 4 ION & $3.06826 \mathrm{E}-13$ & 0.0 & 0.0 & 0.0 \\
\hline MGHCO3ION & $1.09418 \mathrm{E}-08$ & 0.0 & 0.0 & 0.0 \\
\hline MGHSIO3ION & $7.70808 \mathrm{E}-07$ & 0.0 & 0.0 & 0.0 \\
\hline MGION & $4.31649 \mathrm{E}-05$ & 0.0 & 0.0 & 0.0 \\
\hline MGOHION & $9.07211 \mathrm{E}-08$ & 0.0 & 0.0 & 0.0 \\
\hline MGP2O7ION & $3.75513 \mathrm{E}-16$ & 0.0 & 0.0 & 0.0 \\
\hline MGPO 4 ION & $3.50013 E-09$ & 0.0 & 0.0 & 0.0 \\
\hline NACO3ION & $9.78626 \mathrm{E}-09$ & 0.0 & 0.0 & 0.0 \\
\hline NAION & 0.00412107 & 0.0 & 0.0 & 0.0 \\
\hline NASO 4 ION & $1.39982 \mathrm{E}-04$ & 0.0 & 0.0 & 0.0 \\
\hline NO3ION & $8.45181 \mathrm{E}-04$ & 0.0 & 0.0 & 0.0 \\
\hline P207ION & $7.52825 E-19$ & 0.0 & 0.0 & 0.0 \\
\hline PO4ION & $5.24084 \mathrm{E}-12$ & 0.0 & 0.0 & 0.0 \\
\hline SO4ION & $3.23197 \mathrm{E}-04$ & 0.0 & 0.0 & 0.0 \\
\hline ZNCL3ION & $4.60945 E-09$ & 0.0 & 0.0 & 0.0 \\
\hline ZNCLION & $3.98814 E-09$ & 0.0 & 0.0 & 0.0 \\
\hline $\mathrm{ZNH} 2 \mathrm{PO} 4 \mathrm{ION}$ & $9.50828 \mathrm{E}-18$ & 0.0 & 0.0 & 0.0 \\
\hline ZNHCO3ION & $3.37790 \mathrm{E}-13$ & 0.0 & 0.0 & 0.0 \\
\hline ZNION & $1.64274 \mathrm{E}-08$ & 0.0 & 0.0 & 0.0 \\
\hline ZNNO3ION & $1.66399 \mathrm{E}-09$ & 0.0 & 0.0 & 0.0 \\
\hline ZNOH3ION & $2.53580 \mathrm{E}-10$ & 0.0 & 0.0 & 0.0 \\
\hline ZNOH 4 ION & $8.90912 \mathrm{E}-13$ & 0.0 & 0.0 & 0.0 \\
\hline ZNOHION & $3.58049 \mathrm{E}-09$ & 0.0 & 0.0 & 0.0 \\
\hline $\mathrm{CA} 3 \mathrm{PO} 42$ & 0.0 & $1.68469 \mathrm{E}-04$ & 0.0 & 0.0 \\
\hline $\mathrm{CASO} 4.2 \mathrm{H} 2 \mathrm{O}$ & 0.0 & $6.96977 \mathrm{E}-04$ & 0.0 & 0.0 \\
\hline CHAMOSITE7A & 0.0 & $6.62523 \mathrm{E}-05$ & 0.0 & 0.0 \\
\hline $\mathrm{MGOH} 2$ & 0.0 & $4.40626 \mathrm{E}-04$ & 0.0 & 0.0 \\
\hline & $============$ & $============$ & $=============$ & $====$ \\
\hline Total g/hr & 1.8492 & 0.240402 & 0.0 & 0.0 \\
\hline Volume, m3/hr & $1.60244 \mathrm{E}-06$ & $7.98975 \mathrm{E}-08$ & 0.0 & 0.0 \\
\hline Enthalpy, cal/hr & -6200.08 & -715.411 & 0.0 & 0.0 \\
\hline Density, $g / \mathrm{m} 3$ & $1.15399 \mathrm{E}+06$ & $3.00888 \mathrm{E}+06$ & & \\
\hline Vapor fraction & 0.0 & 0.0 & 0.0 & 0.0 \\
\hline
\end{tabular}


WSRC-TR-2002-00567

Revision 0

\begin{tabular}{|c|c|c|c|c|}
\hline Solid fraction & 0.0 & 1 . & 0.0 & 0.0 \\
\hline Organic fraction & 0.0 & 0.0 & 0.0 & 0.0 \\
\hline Osmotic Pres, atm & 179.399 & & & \\
\hline Redox Pot, volts & 0.0 & & & \\
\hline $\mathrm{E}-\mathrm{Con}, 1 / \mathrm{ohm}-\mathrm{cm}$ & 0.198042 & & & \\
\hline E-Con, cm2/ohm-mol & 30.1681 & & & \\
\hline Abs Visc, cP & 1.31427 & & & \\
\hline Rel Visc & 1.47552 & & & \\
\hline Ionic Strength & 3.80033 & & & \\
\hline
\end{tabular}


WSRC-TR-2002-00567

Revision 0

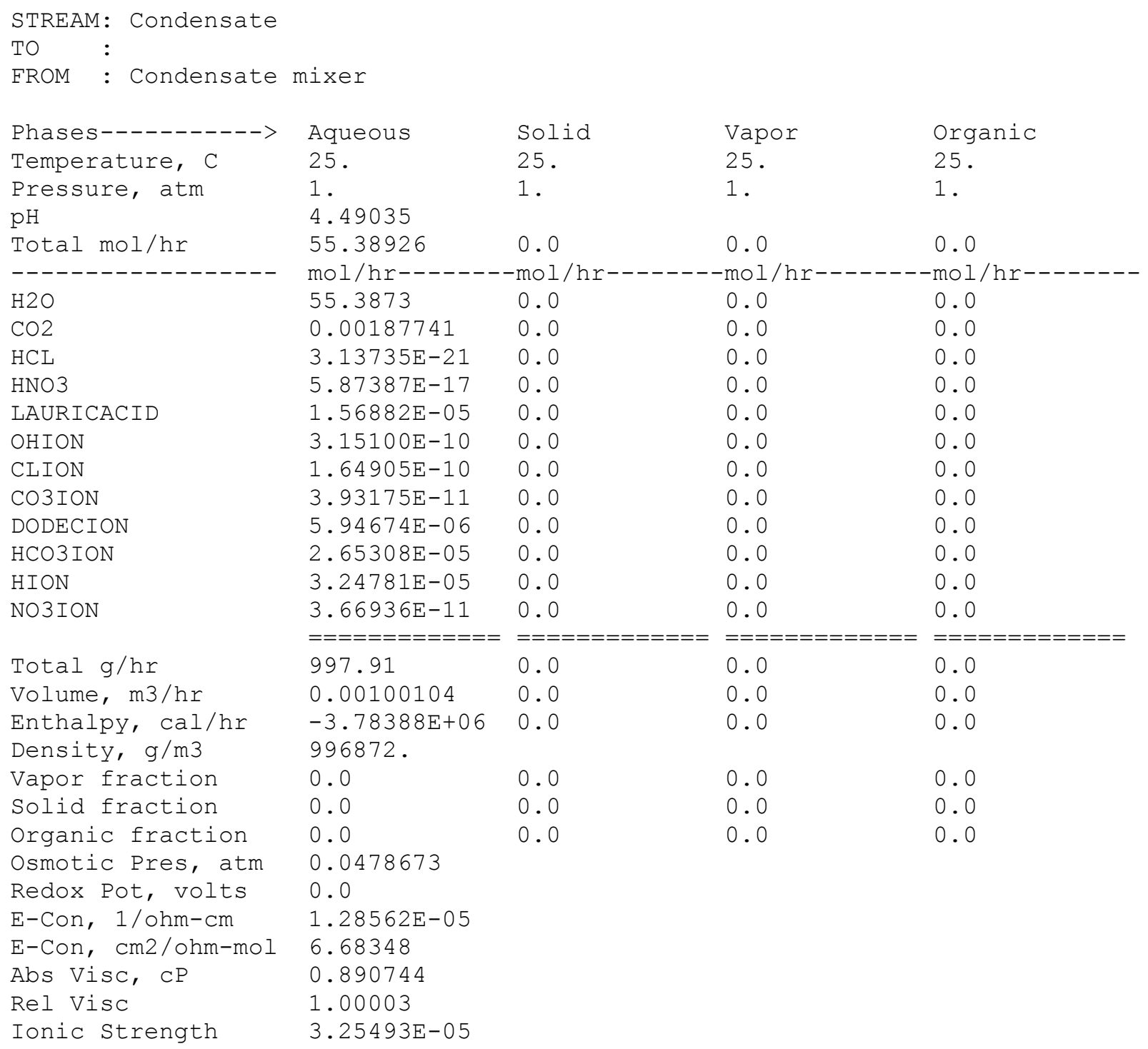


WSRC-TR-2002-00567

Revision 0

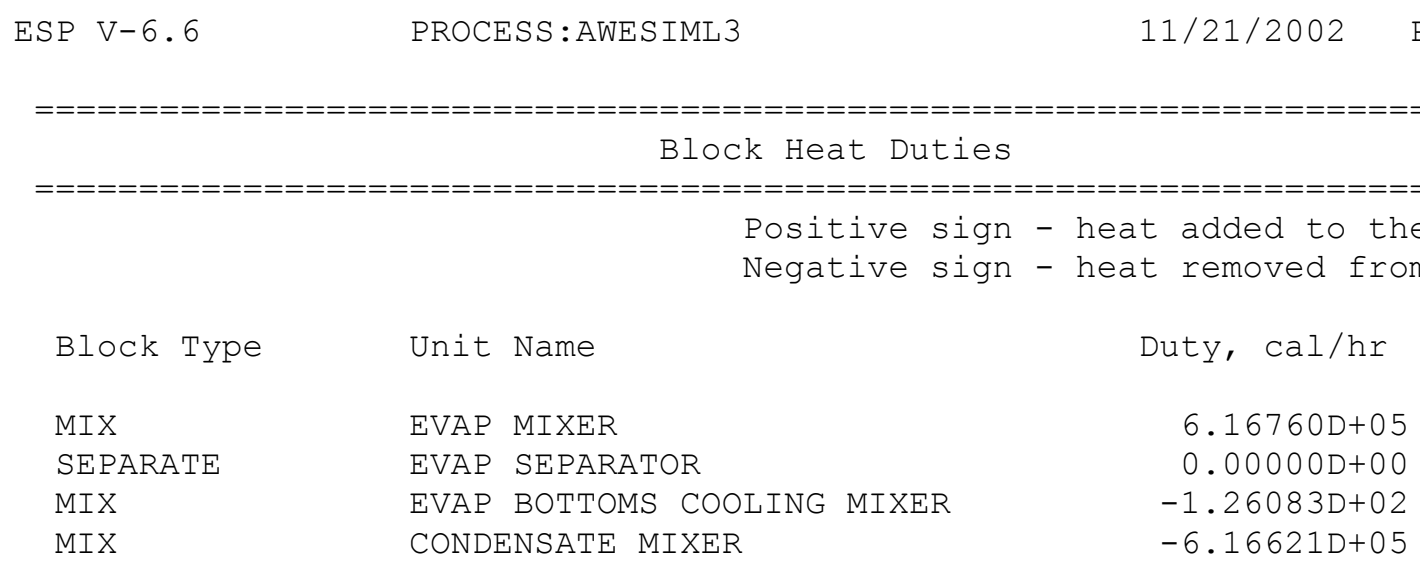


WSRC-TR-2002-00567

Revision 0

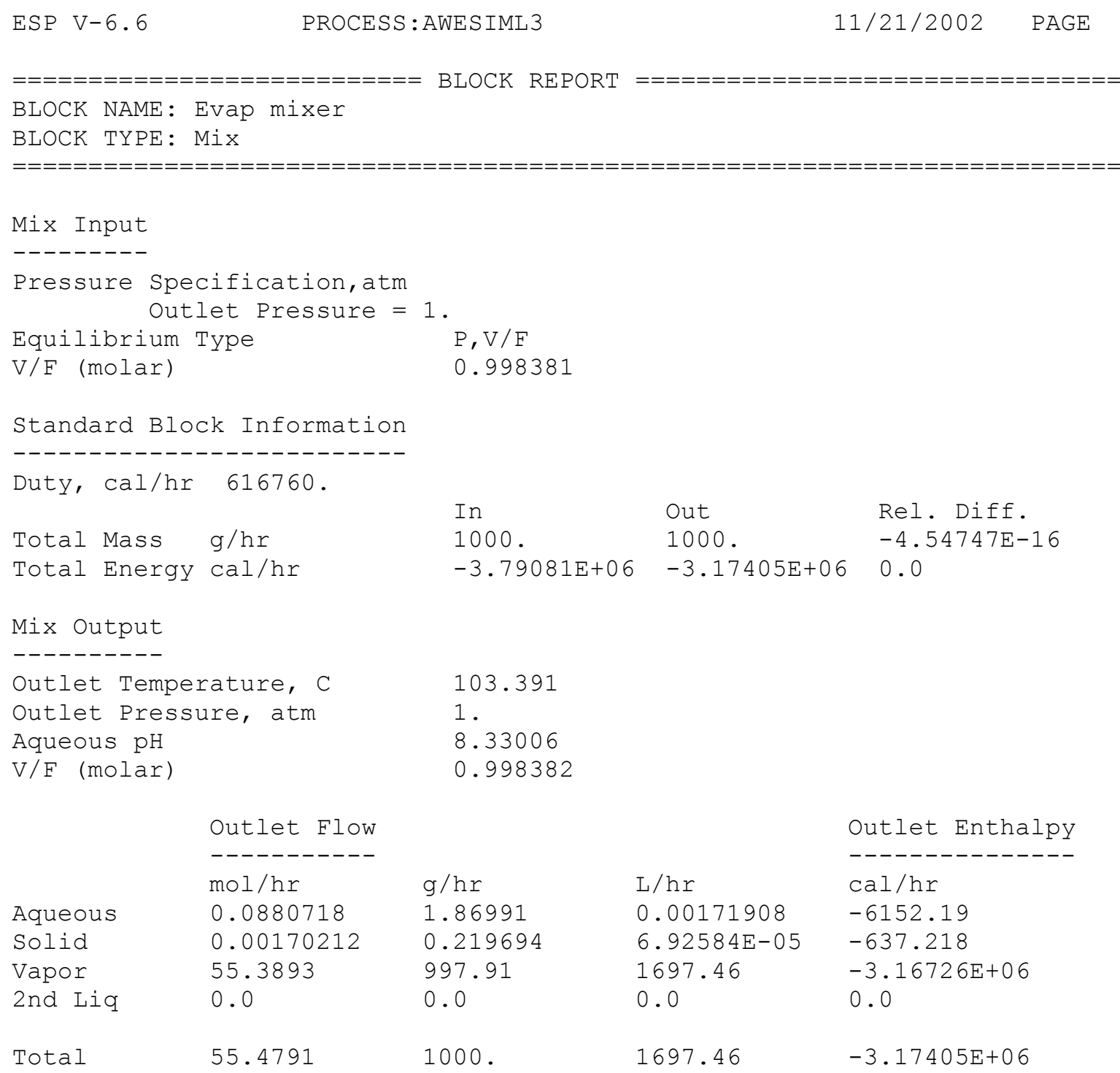


WSRC-TR-2002-00567

Revision 0

$\begin{array}{lllll}\text { ESP V-6.6 PROCESS:AWESIML3 } & 11 / 21 / 2002 & \text { PAGE } & 11\end{array}$

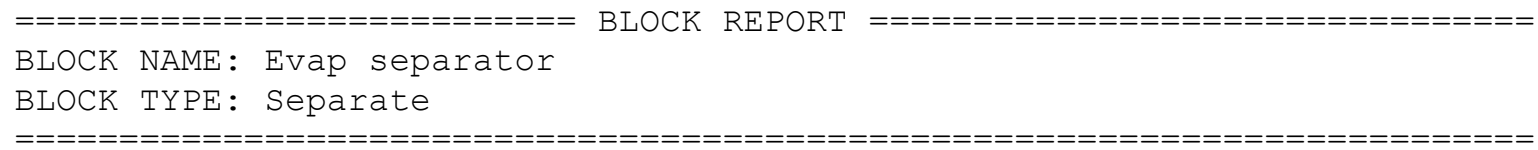

$\begin{array}{lll} & \text { Outlet Enthalpy } \\ & ----------- & \\ \text { L/hr } & \text { cal/hr } \\ 0.00171908 & -6152.19 \\ 6.92584 E-05 & -637.218 \\ 0.0 & 0.0 \\ 0.0 & 0.0 & \\ 0.00178834 & -6789.4 & \\ & 11 / 21 / 2002 \quad \text { PAGE } & 12\end{array}$


WSRC-TR-2002-00567

Revision 0

\begin{tabular}{lllll} 
Vapor Stream & \multicolumn{3}{c}{ Overhead } & \\
& Outlet Flow & & Outlet Enthalpy \\
& ------- & & \\
& mol/hr & $g / h r$ & $\mathrm{~L} / \mathrm{hr}$ & cal $/ \mathrm{hr}$ \\
Aqueous & 0.0 & 0.0 & 0.0 & 0.0 \\
Solid & 0.0 & 0.0 & 0.0 & 0.0 \\
Vapor & 55.3893 & 997.91 & 1697.46 & $-3.16726 \mathrm{E}+06$ \\
2nd Liq & 0.0 & 0.0 & 0.0 & 0.0 \\
Total & 55.3893 & 997.91 & 1697.46 & $-3.16726 \mathrm{E}+06$
\end{tabular}


WSRC-TR-2002-00567

Revision 0

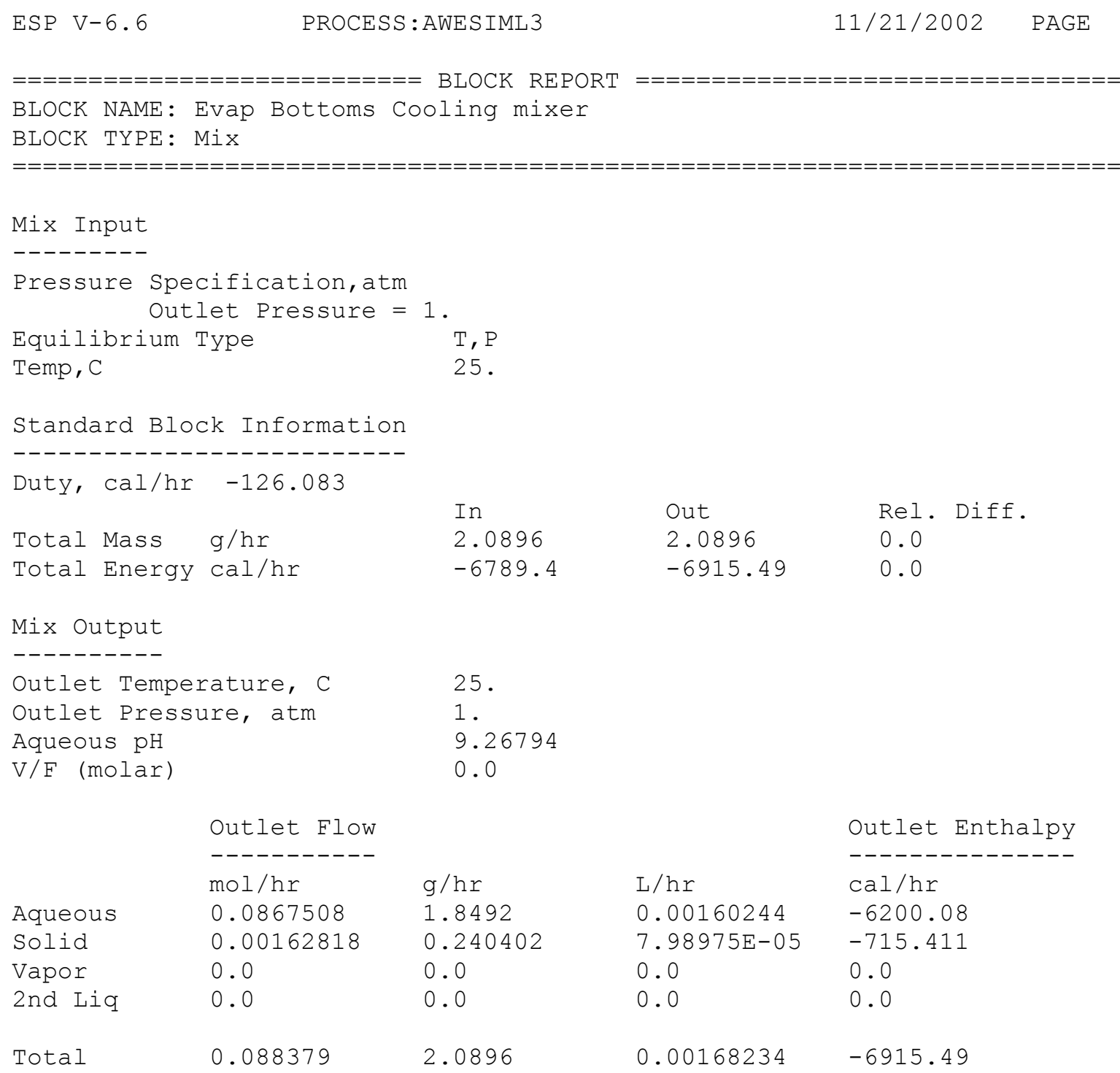


WSRC-TR-2002-00567

Revision 0

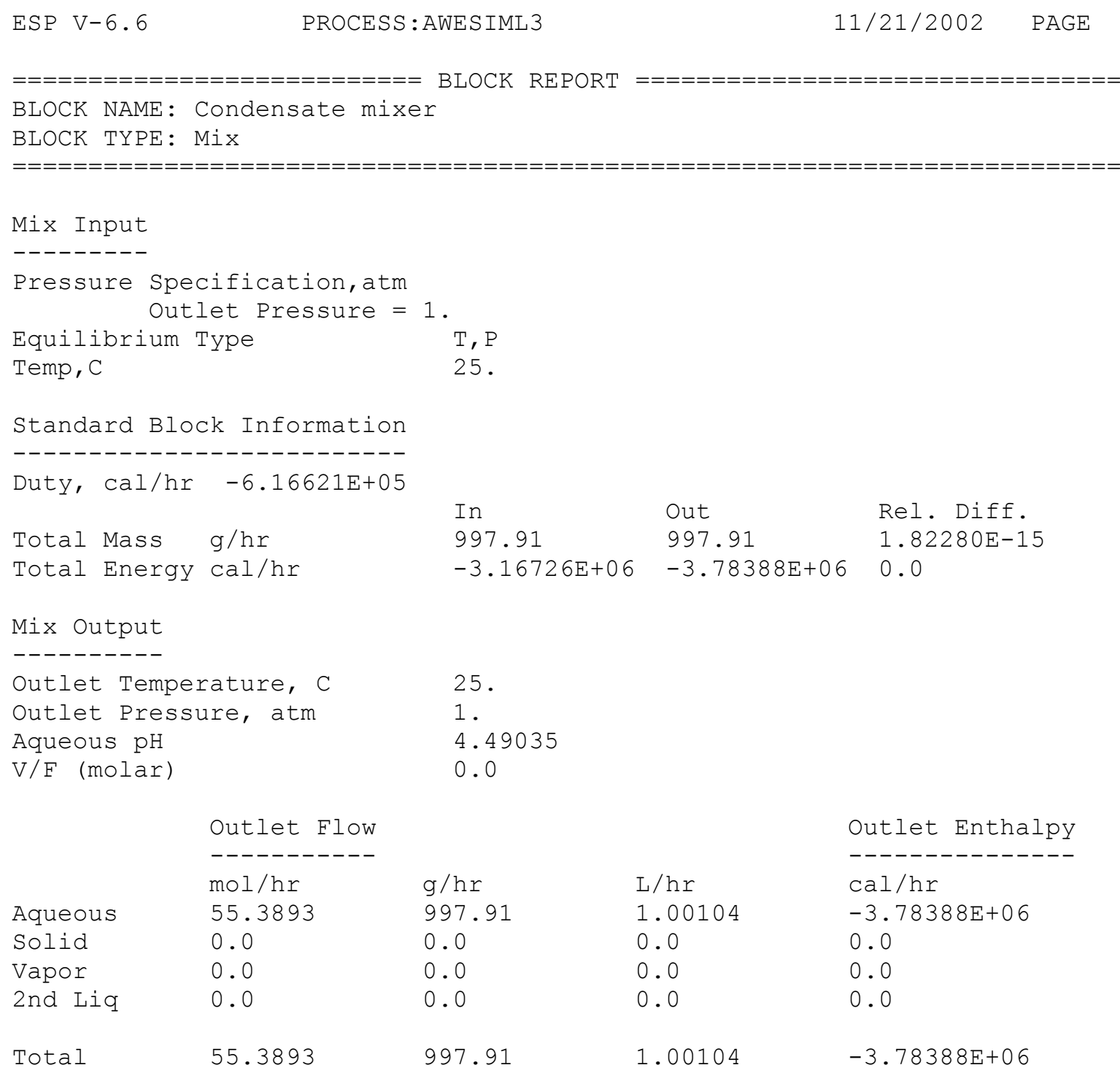


WSRC-TR-2002-00567

Revision 0

$\underline{\text { AWE High Foam Simulant - Concentrated to } 30 \mathrm{wt} . \% \text { Total Solids }}$

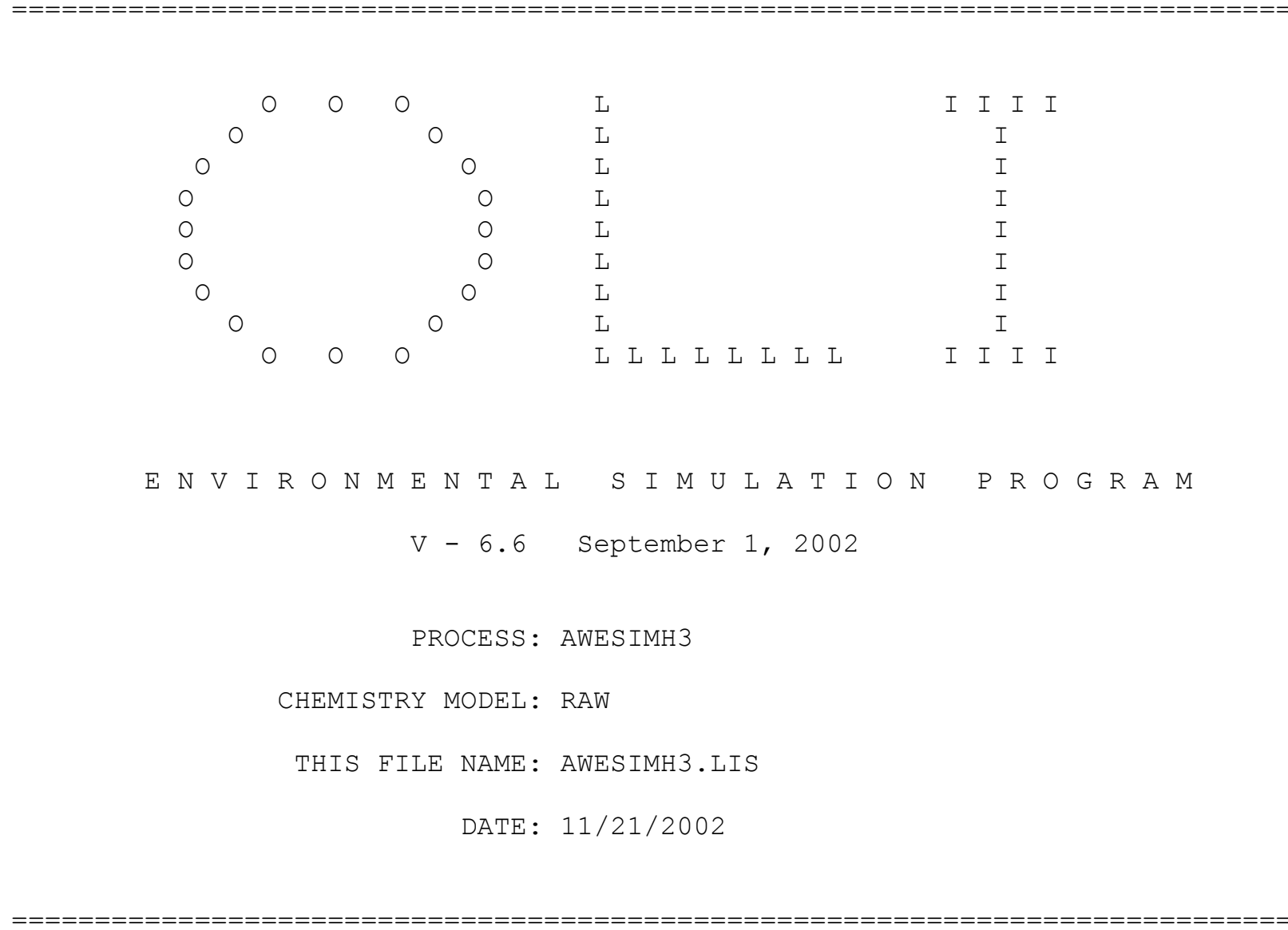


WSRC-TR-2002-00567

Revision 0

TABLE OF CONTENTS

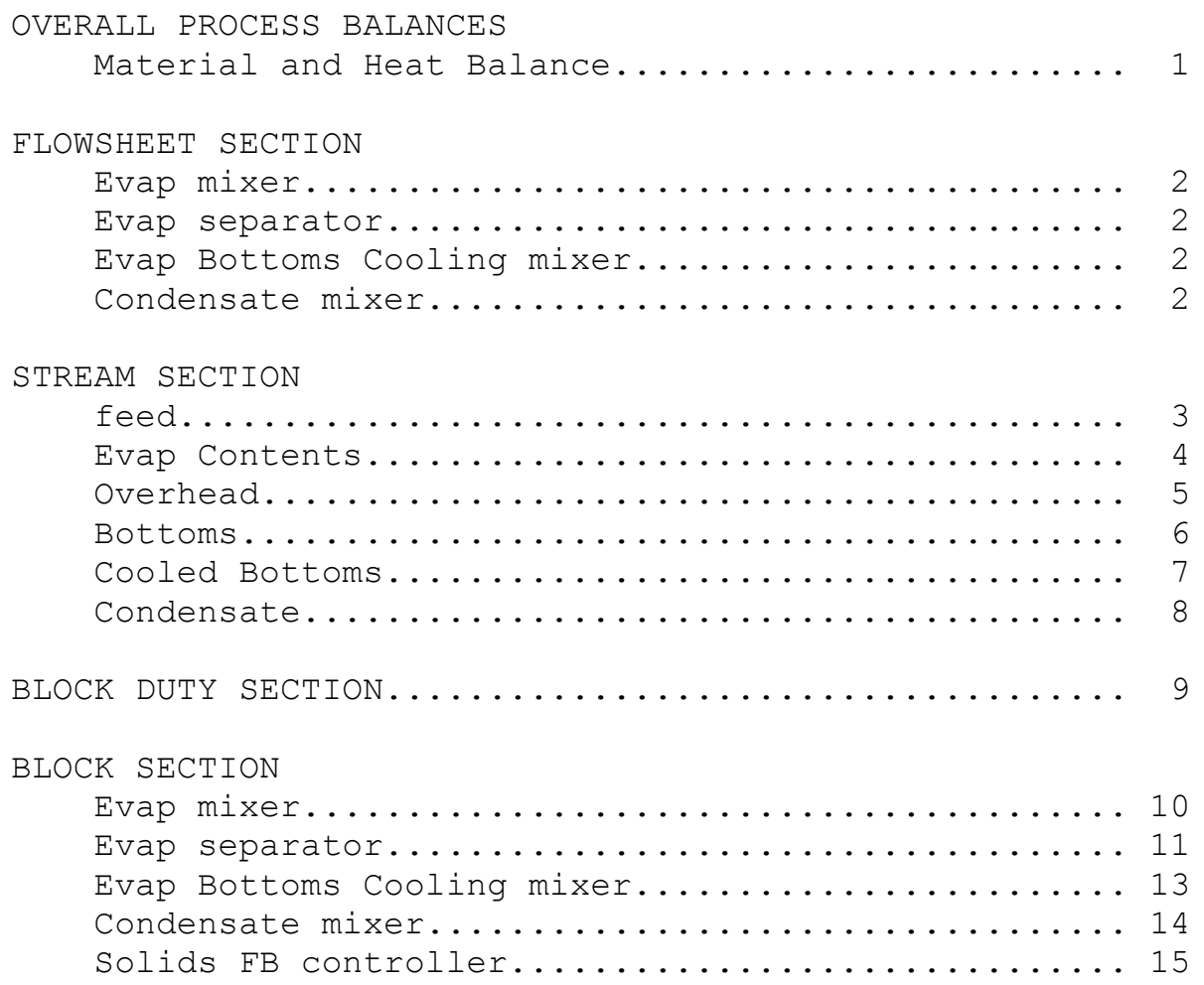


WSRC-TR-2002-00567

Revision 0

ESP V-6.6 PROCESS:AWESIMH3
$\begin{array}{lcc}\text { Overall } & \text { Process } & \text { Balances } \\ \text { Inlet } & \mathrm{g} / \mathrm{hr} & \mathrm{Cal} / \mathrm{hr} \\ ----------- & ---------- & ---------- \\ \text { FEED } & 1.00000 \mathrm{D}+03 & -3.79128 \mathrm{D}+06 \\ \text { =========== } & ========= & ========= \\ \text { Total in } & 1.00000 \mathrm{D}+03 & -3.79128 \mathrm{D}+06\end{array}$

\begin{tabular}{lrr} 
Outlet & \multicolumn{1}{c}{$g / \mathrm{hr}$} & $\mathrm{cal} / \mathrm{hr}$ \\
----------- & ---------- & ---------- \\
COOLED BOTTOMS & $1.22001 \mathrm{D}+00$ & $-4.09277 \mathrm{D}+03$ \\
CONDENSATE & $9.98780 \mathrm{D}+02$ & $-3.78717 \mathrm{D}+06$ \\
$==========$ & $==========$ & $==========$ \\
Total out & $1.00000 \mathrm{D}+03$ & $-3.79126 \mathrm{D}+06$
\end{tabular}

$11 / 21 / 2002 \quad$ PAGE 1
Block Heat Duties

EVAP MIXER

EVAP BOTTOMS COOLING MIXER CONDENSATE MIXER

$==========$

Total Duty

DIFFERENCE REL DIFEERENCE

$$
1.81899 \mathrm{D}-12-1.16415 \mathrm{D}-10
$$$$
1.81899 \mathrm{D}-15 \quad 3.07061 \mathrm{D}-17
$$

$$
\begin{gathered}
\text { cal/hr } \\
----------- \\
6.16895 \mathrm{D}+05 \\
-7.34699 \mathrm{D}+01 \\
-6.16811 \mathrm{D}+05 \\
========== \\
1.11145 \mathrm{D}+01
\end{gathered}
$$

Material Code Balances

Code
----------
$\mathrm{H}(+1)$
$\mathrm{K}(+1)$
$\mathrm{NA}(+1)$
$\mathrm{CA}(+2)$
$\mathrm{ZN}(+2)$
$\mathrm{FE}(+2)$
$\mathrm{MG}(+2)$
$\mathrm{AL}(+3)$
$\mathrm{O}(-2)$
$\mathrm{CL}(-1)$
$\mathrm{C}(+4)$
$\mathrm{P}(+5)$
$\mathrm{S}(+6)$
$\mathrm{N}(+5)$
$\mathrm{SI}(+4)$
$\mathrm{DODEC}(-1)$

Outlet $\mathrm{mol} / \mathrm{hr}$

1. $10971 \mathrm{D}+02$ 3. $06890 \mathrm{D}-04$

$1.84497 \mathrm{D}-03$

$1.16967 \mathrm{D}-03$

3. $05951 \mathrm{D}-05$

7. $69961 \mathrm{D}-05$

3. $70188 \mathrm{D}-04$

$8.89498 \mathrm{D}-05$

$5.54943 \mathrm{D}+01$

$1.83341 \mathrm{D}-03$

1. 36645D-03

$2.10590 \mathrm{D}-04$

$6.76649 \mathrm{D}-04$

4. $83833 \mathrm{D}-04$

$2.33004 \mathrm{D}-04$

1. 34809D-04
Difference Rel Diff mol/hr

$-4.26326 \mathrm{D}-14-3.84178 \mathrm{D}-16$

$-5.42101 \mathrm{D}-20-1.76644 \mathrm{D}-16$

$3.90313 \mathrm{D}-18 \quad 2.11555 \mathrm{D}-15$

$0.00000 \mathrm{D}+00 \quad 0.00000 \mathrm{D}+00$

$-1.35525 \mathrm{D}-20-4.42964 \mathrm{D}-16$

$0.00000 \mathrm{D}+00 \quad 0.00000 \mathrm{D}+00$

$-1.84314 \mathrm{D}-18-4.97895 \mathrm{D}-15$

$0.00000 \mathrm{D}+00 \quad 0.00000 \mathrm{D}+00$

$-4.26326 \mathrm{D}-14-7.68233 \mathrm{D}-16$

$-2.60209 \mathrm{D}-18-1.41926 \mathrm{D}-15$

$5.65954 \mathrm{D}-17$ 4.14177D-14

$2.71051 \mathrm{D}-20 \quad 1.28710 \mathrm{D}-16$

$-1.08420 D-19-1.60231 D-16$

$5.42101 \mathrm{D}-201.12043 \mathrm{D}-16$

5.42101D-20 2.32657D-16

$1.29842 \mathrm{D}-149.63152 \mathrm{D}-11$ 
WSRC-TR-2002-00567

Revision 0

\begin{tabular}{|c|c|c|c|}
\hline \multicolumn{4}{|l|}{$\begin{array}{l}\text { PROCESS BLOCKS } \\
===========\end{array}$} \\
\hline $\begin{array}{l}\mathrm{BLOCK} \mathrm{NAME} \\
=========================\end{array}$ & $\begin{array}{l}\text { BLOCK TYPE } \\
===========\end{array}$ & $\begin{array}{l}\text { INLET STREAM }(\mathrm{S}) \\
==============\end{array}$ & $\begin{array}{l}\text { OUTLET STREAM }(\mathrm{S}) \\
==============\end{array}$ \\
\hline Evap mixer & $\operatorname{Mix}$ & feed & Evap Contents \\
\hline Evap separator & Separate & Evap Contents & $\begin{array}{l}\text { Overhead } \\
\text { Bottoms }\end{array}$ \\
\hline Evap Bottoms Cooling mixer & Mix & Bottoms & Cooled Bottoms \\
\hline Condensate mixer & Mix & Overhead & Condensate \\
\hline
\end{tabular}


WSRC-TR-2002-00567

Revision 0 


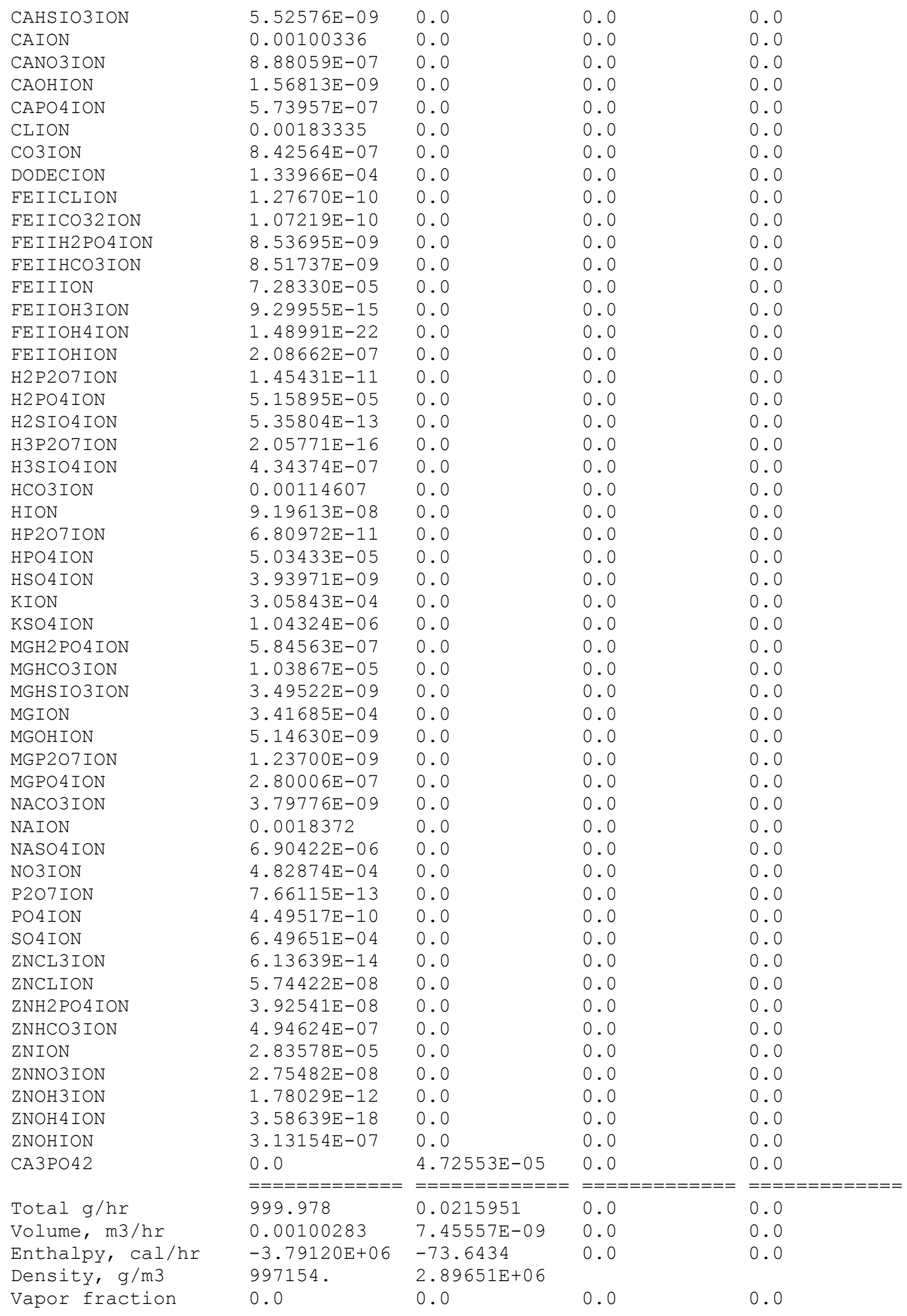


WSRC-TR-2002-00567

Revision 0

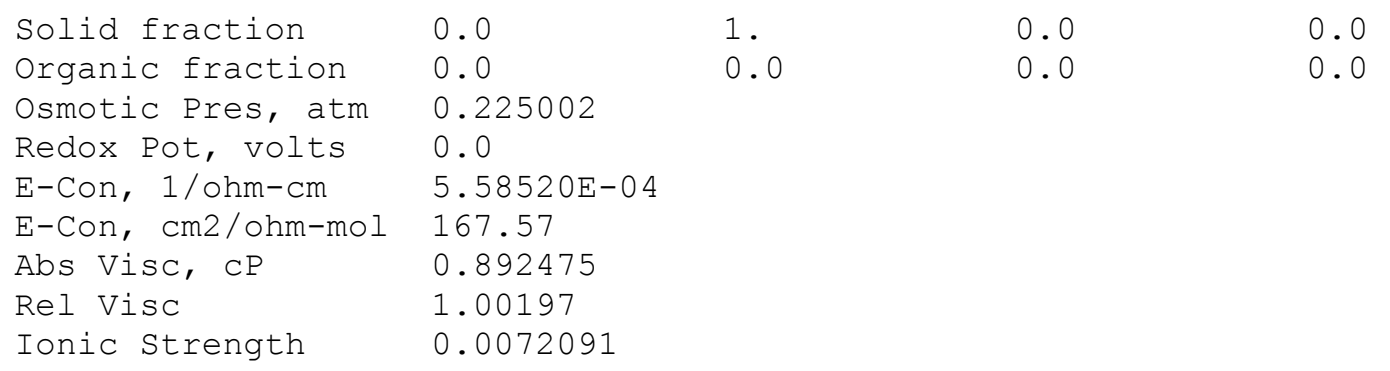


WSRC-TR-2002-00567

Revision 0

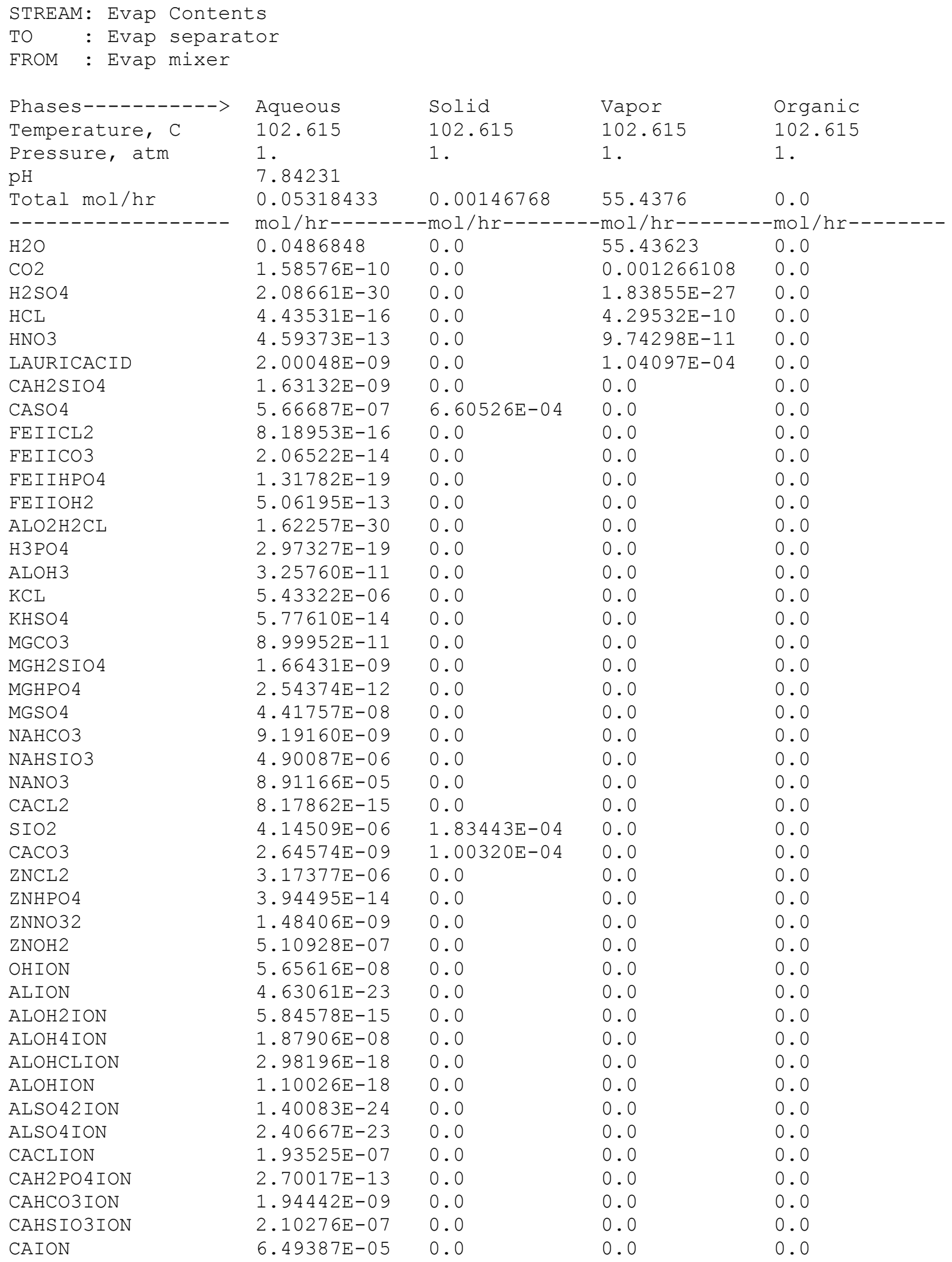




\begin{tabular}{|c|c|c|c|c|}
\hline CANO3ION & $2.69767 \mathrm{E}-05$ & 0.0 & 0.0 & 0.0 \\
\hline CAOHION & $4.39377 \mathrm{E}-08$ & 0.0 & 0.0 & 0.0 \\
\hline CAPO 4 ION & $1.09466 \mathrm{E}-11$ & 0.0 & 0.0 & 0.0 \\
\hline CLION & 0.00180449 & 0.0 & 0.0 & 0.0 \\
\hline CO3ION & $5.22192 \mathrm{E}-10$ & 0.0 & 0.0 & 0.0 \\
\hline DODECION & $3.07099 E-05$ & 0.0 & 0.0 & 0.0 \\
\hline FEIICLION & $1.87364 \mathrm{E}-13$ & 0.0 & 0.0 & 0.0 \\
\hline FEIICO32ION & $1.83172 \mathrm{E}-18$ & 0.0 & 0.0 & 0.0 \\
\hline FEIIH2PO4ION & $3.62430 \mathrm{E}-21$ & 0.0 & 0.0 & 0.0 \\
\hline FEIIHCO3ION & $1.48575 \mathrm{E}-17$ & 0.0 & 0.0 & 0.0 \\
\hline FEIIION & $2.63133 \mathrm{E}-11$ & 0.0 & 0.0 & 0.0 \\
\hline FEIIOH3ION & $3.27522 \mathrm{E}-14$ & 0.0 & 0.0 & 0.0 \\
\hline FEIIOH 4 ION & $2.91927 \mathrm{E}-18$ & 0.0 & 0.0 & 0.0 \\
\hline FEIIOHION & $9.85113 \mathrm{E}-12$ & 0.0 & 0.0 & 0.0 \\
\hline $\mathrm{H} 2 \mathrm{P} 207 \mathrm{ION}$ & $1.13932 \mathrm{E}-23$ & 0.0 & 0.0 & 0.0 \\
\hline H2 PO 4 ION & $1.86551 \mathrm{E}-13$ & 0.0 & 0.0 & 0.0 \\
\hline H2SIO4ION & $9.19757 \mathrm{E}-11$ & 0.0 & 0.0 & 0.0 \\
\hline H3P2O7ION & $4.31072 \mathrm{E}-30$ & 0.0 & 0.0 & 0.0 \\
\hline H3SIO4ION & $1.77496 \mathrm{E}-06$ & 0.0 & 0.0 & 0.0 \\
\hline HCO3ION & $7.54561 \mathrm{E}-09$ & 0.0 & 0.0 & 0.0 \\
\hline HION & $1.67080 \mathrm{E}-11$ & 0.0 & 0.0 & 0.0 \\
\hline HP207ION & $8.51041 \mathrm{E}-22$ & 0.0 & 0.0 & 0.0 \\
\hline HPO 4 ION & $6.24660 \mathrm{E}-12$ & 0.0 & 0.0 & 0.0 \\
\hline HSO 4 ION & $1.08177 \mathrm{E}-11$ & 0.0 & 0.0 & 0.0 \\
\hline KION & $2.98675 E-04$ & 0.0 & 0.0 & 0.0 \\
\hline KSO4 ION & $2.78183 \mathrm{E}-06$ & 0.0 & 0.0 & 0.0 \\
\hline MGH2 PO 4 ION & $6.25152 \mathrm{E}-15$ & 0.0 & 0.0 & 0.0 \\
\hline MGHCO3ION & $2.56294 \mathrm{E}-10$ & 0.0 & 0.0 & 0.0 \\
\hline MGHSIO3ION & $2.81759 \mathrm{E}-08$ & 0.0 & 0.0 & 0.0 \\
\hline MGION & $2.42611 \mathrm{E}-06$ & 0.0 & 0.0 & 0.0 \\
\hline MGOHION & $2.48942 E-08$ & 0.0 & 0.0 & 0.0 \\
\hline MGP2O7ION & $1.21781 \mathrm{E}-18$ & 0.0 & 0.0 & 0.0 \\
\hline MGPO 4 ION & $9.06268 \mathrm{E}-13$ & 0.0 & 0.0 & 0.0 \\
\hline NACO3ION & $7.81861 \mathrm{E}-11$ & 0.0 & 0.0 & 0.0 \\
\hline NAION & 0.00175094 & 0.0 & 0.0 & 0.0 \\
\hline NASO 4 I ON & $4.81571 \mathrm{E}-13$ & 0.0 & 0.0 & 0.0 \\
\hline NO3ION & $3.67685 \mathrm{E}-04$ & 0.0 & 0.0 & 0.0 \\
\hline P2O7ION & $4.84547 \mathrm{E}-22$ & 0.0 & 0.0 & 0.0 \\
\hline PO4ION & $3.51779 \mathrm{E}-15$ & 0.0 & 0.0 & 0.0 \\
\hline SO4ION & $1.27309 \mathrm{E}-05$ & 0.0 & 0.0 & 0.0 \\
\hline ZNCL3ION & $3.07882 \mathrm{E}-06$ & 0.0 & 0.0 & 0.0 \\
\hline ZNCLION & $7.70974 \mathrm{E}-06$ & 0.0 & 0.0 & 0.0 \\
\hline ZNH2PO 4 ION & $2.57467 \mathrm{E}-16$ & 0.0 & 0.0 & 0.0 \\
\hline ZNHCO3ION & $8.36158 \mathrm{E}-12$ & 0.0 & 0.0 & 0.0 \\
\hline ZNION & $5.52832 \mathrm{E}-07$ & 0.0 & 0.0 & 0.0 \\
\hline ZNNO3ION & $5.15105 \mathrm{E}-08$ & 0.0 & 0.0 & 0.0 \\
\hline ZNOH3 ION & $6.90395 E-08$ & 0.0 & 0.0 & 0.0 \\
\hline ZNOH 4 ION & $1.71078 \mathrm{E}-10$ & 0.0 & 0.0 & 0.0 \\
\hline ZNOHION & $1.54468 \mathrm{E}-05$ & 0.0 & 0.0 & 0.0 \\
\hline ALOOH & 0.0 & $1.19350 \mathrm{E}-05$ & 0.0 & 0.0 \\
\hline $\mathrm{CA} 3 \mathrm{PO} 42$ & 0.0 & $1.05295 \mathrm{E}-04$ & 0.0 & 0.0 \\
\hline CHAMOSITE7A & 0.0 & $3.84980 E-05$ & 0.0 & 0.0 \\
\hline MGOH2 & 0.0 & $3.67662 E-04$ & 0.0 & 0.0 \\
\hline Total g/hr & 1.04105 & 0.178963 & 998.78 & 0.0 \\
\hline Volume, m3/hr & $9.87859 \mathrm{E}-07$ & $5.86193 E-08$ & 1.69536 & 0.0 \\
\hline Enthalpy, cal/hr & -3504.77 & -514.533 & $-3.17036 \mathrm{E}+06$ & 0.0 \\
\hline Density, $g / m 3$ & $1.05384 \mathrm{E}+06$ & $3.05297 E+06$ & 589.126 & \\
\hline
\end{tabular}


WSRC-TR-2002-00567

Revision 0

$\begin{array}{lll}\text { Vapor fraction } & 0.0 & 0.0 \\ \text { Solid fraction } & 0.0 & 1 . \\ \text { Organic fraction } & 0.0 & 0.0 \\ \text { Osmotic Pres, atm } & 147.51 & \\ \text { Redox Pot, volts } & 0.0 & \\ \text { E-Con, 1/ohm-cm } & 0.437663 & \\ \text { E-Con, cm2/ohm-mol } & 52.3871 & \\ \text { Abs Visc, cP } & 0.37083 & 1.35324 \\ \text { Rel Visc } & 2.64152 & \\ \text { Ionic Strength } & \end{array}$

1.

0.0

0.0

0.0

0.0 
WSRC-TR-2002-00567

Revision 0

\begin{tabular}{|c|c|c|c|c|}
\hline \\
\hline \multicolumn{5}{|c|}{$\begin{array}{l}\text { STREAM: Overhead } \\
\text { TO : Condensate mixer }\end{array}$} \\
\hline \multicolumn{5}{|c|}{ FROM : Evap separator } \\
\hline Phases-----------> & Aqueous & Solid & Vapor & Organic \\
\hline Temperature, C & 102.615 & 102.615 & 102.615 & 102.615 \\
\hline $\begin{array}{l}\text { Pressure, atm } \\
\mathrm{pH}\end{array}$ & $\begin{array}{l}1 . \\
0.0\end{array}$ & 1 . & 1. & 1 . \\
\hline Total mol/hr & 0.0 & 0.0 & 55.4376 & 0.0 \\
\hline \multicolumn{5}{|c|}{$----------------\quad \operatorname{mol} / \mathrm{hr}--------\operatorname{mol} / \mathrm{hr}--------\operatorname{mol} / \mathrm{h} r--------\operatorname{mol} / \mathrm{hr}--------$} \\
\hline $\mathrm{H} 2 \mathrm{O}$ & 0.0 & 0.0 & 55.43623 & 0.0 \\
\hline $\mathrm{CO} 2$ & 0.0 & 0.0 & 0.001266108 & 0.0 \\
\hline $\mathrm{H} 2 \mathrm{SO} 4$ & 0.0 & 0.0 & $1.83855 E-27$ & 0.0 \\
\hline $\mathrm{HCL}$ & 0.0 & 0.0 & 4.29532E-10 & 0.0 \\
\hline HNO3 & 0.0 & 0.0 & $9.74298 E-11$ & 0.0 \\
\hline LAURICACID & $\begin{array}{l}0.0 \\
=============\end{array}$ & $\begin{array}{l}0.0 \\
=============\end{array}$ & $\begin{array}{l}\text { 1. } 04097 \mathrm{E}-04 \\
============\end{array}$ & $\begin{array}{l}0.0 \\
=============\end{array}$ \\
\hline Total g/hr & 0.0 & 0.0 & 998.78 & 0.0 \\
\hline Volume, $\mathrm{m} 3 / \mathrm{hr}$ & 0.0 & 0.0 & 1.69536 & 0.0 \\
\hline $\begin{array}{l}\text { Enthalpy, cal/hr } \\
\text { Density, } \mathrm{g} / \mathrm{m} 3\end{array}$ & 0.0 & 0.0 & $\begin{array}{l}-3.17036 \mathrm{E}+06 \\
589.126\end{array}$ & 0.0 \\
\hline Vapor fraction & 0.0 & 0.0 & 1. & 0.0 \\
\hline Solid fraction & 0.0 & 0.0 & 0.0 & 0.0 \\
\hline Organic fraction & 0.0 & 0.0 & 0.0 & 0.0 \\
\hline Osmotic Pres, atm & 0.0 & & & \\
\hline Redox Pot, volts & 0.0 & & & \\
\hline $\mathrm{E}-\mathrm{Con}, 1 / \mathrm{ohm}-\mathrm{cm}$ & 0.0 & & & \\
\hline E-Con, cm2/ohm-mol & 0.0 & & & \\
\hline Abs Visc, cP & 0.0 & & & \\
\hline Rel Visc & 0.0 & & & \\
\hline Ionic Strength & 0.0 & & & \\
\hline
\end{tabular}


WSRC-TR-2002-00567

Revision 0

ESP V-6.6

PROCESS : AWES IMH3

$11 / 21 / 2002$

PAGE

6

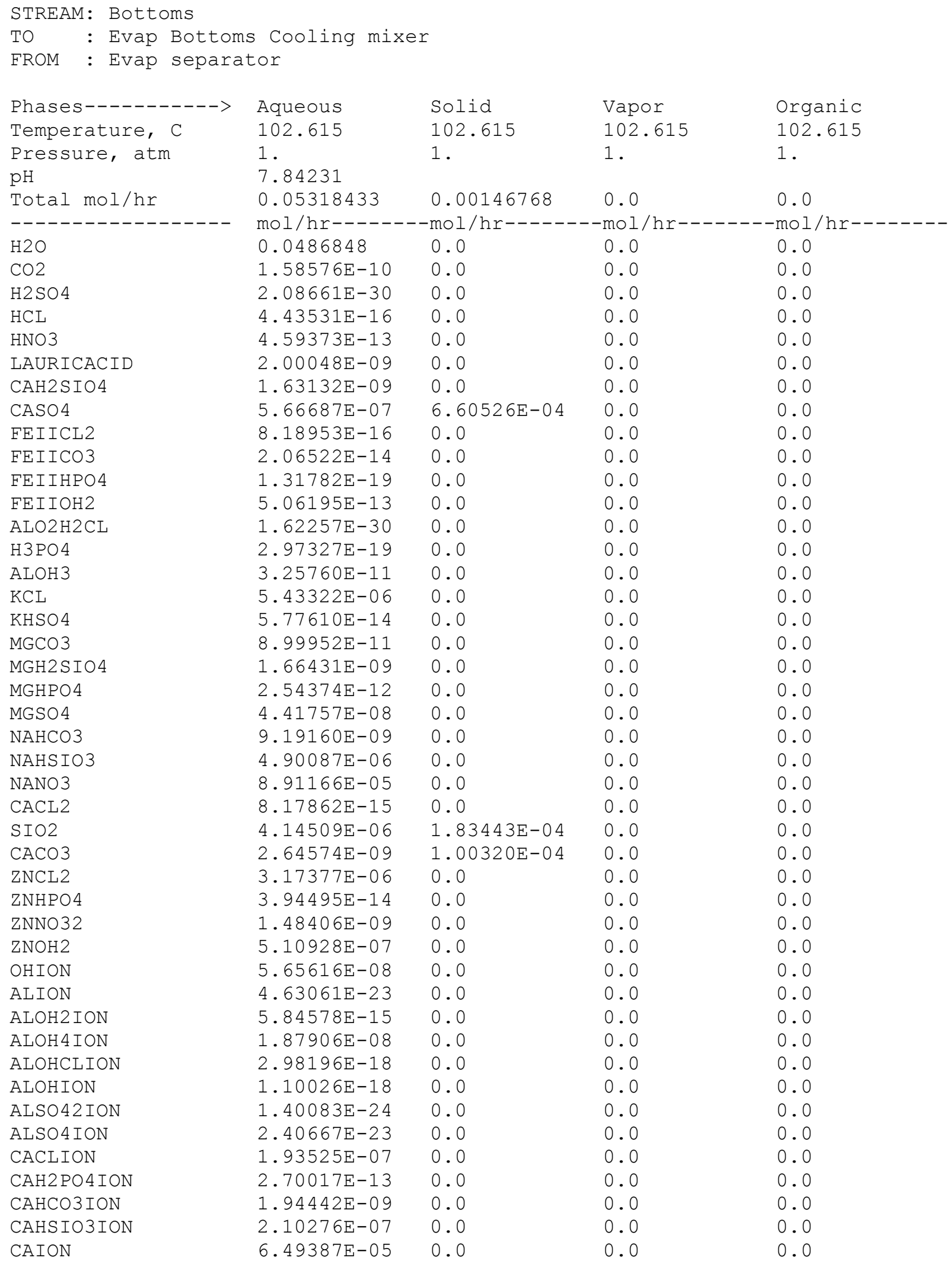




\begin{tabular}{|c|c|c|c|c|}
\hline CANO3ION & $2.69767 \mathrm{E}-05$ & 0.0 & 0.0 & 0.0 \\
\hline CAOHION & $4.39377 \mathrm{E}-08$ & 0.0 & 0.0 & 0.0 \\
\hline CAPO 4 ION & $1.09466 \mathrm{E}-11$ & 0.0 & 0.0 & 0.0 \\
\hline CLION & 0.00180449 & 0.0 & 0.0 & 0.0 \\
\hline CO3ION & $5.22192 \mathrm{E}-10$ & 0.0 & 0.0 & 0.0 \\
\hline DODECION & $3.07099 \mathrm{E}-05$ & 0.0 & 0.0 & 0.0 \\
\hline FEIICLION & $1.87364 \mathrm{E}-13$ & 0.0 & 0.0 & 0.0 \\
\hline FEIICO32ION & $1.83172 \mathrm{E}-18$ & 0.0 & 0.0 & 0.0 \\
\hline FEIIH2PO4ION & $3.62430 \mathrm{E}-21$ & 0.0 & 0.0 & 0.0 \\
\hline FEIIHCO3ION & $1.48575 \mathrm{E}-17$ & 0.0 & 0.0 & 0.0 \\
\hline FEIIION & $2.63133 \mathrm{E}-11$ & 0.0 & 0.0 & 0.0 \\
\hline FEIIOH3ION & $3.27522 \mathrm{E}-14$ & 0.0 & 0.0 & 0.0 \\
\hline FEIIOH 4 ION & $2.91927 \mathrm{E}-18$ & 0.0 & 0.0 & 0.0 \\
\hline FEIIOHION & $9.85113 \mathrm{E}-12$ & 0.0 & 0.0 & 0.0 \\
\hline H2P2O7ION & $1.13932 \mathrm{E}-23$ & 0.0 & 0.0 & 0.0 \\
\hline H2 PO 4 ION & $1.86551 \mathrm{E}-13$ & 0.0 & 0.0 & 0.0 \\
\hline H2SIO4ION & $9.19757 \mathrm{E}-11$ & 0.0 & 0.0 & 0.0 \\
\hline H3P2O7ION & $4.31072 \mathrm{E}-30$ & 0.0 & 0.0 & 0.0 \\
\hline H3SIO 4 ION & $1.77496 \mathrm{E}-06$ & 0.0 & 0.0 & 0.0 \\
\hline HCO3ION & $7.54561 \mathrm{E}-09$ & 0.0 & 0.0 & 0.0 \\
\hline $\mathrm{HION}$ & $1.67080 \mathrm{E}-11$ & 0.0 & 0.0 & 0.0 \\
\hline HP2O7ION & $8.51041 \mathrm{E}-22$ & 0.0 & 0.0 & 0.0 \\
\hline HPO 4 ION & $6.24660 \mathrm{E}-12$ & 0.0 & 0.0 & 0.0 \\
\hline HSO4 ION & $1.08177 \mathrm{E}-11$ & 0.0 & 0.0 & 0.0 \\
\hline $\mathrm{KION}$ & $2.98675 E-04$ & 0.0 & 0.0 & 0.0 \\
\hline KSO4 ION & $2.78183 E-06$ & 0.0 & 0.0 & 0.0 \\
\hline MGH2 PO 4 ION & $6.25152 E-15$ & 0.0 & 0.0 & 0.0 \\
\hline MGHCO 3 ION & $2.56294 \mathrm{E}-10$ & 0.0 & 0.0 & 0.0 \\
\hline MGHSIO3ION & $2.81759 \mathrm{E}-08$ & 0.0 & 0.0 & 0.0 \\
\hline MGION & $2.42611 \mathrm{E}-06$ & 0.0 & 0.0 & 0.0 \\
\hline MGOHION & $2.48942 \mathrm{E}-08$ & 0.0 & 0.0 & 0.0 \\
\hline MGP207ION & $1.21781 \mathrm{E}-18$ & 0.0 & 0.0 & 0.0 \\
\hline MGPO 4 ION & $9.06268 \mathrm{E}-13$ & 0.0 & 0.0 & 0.0 \\
\hline NACO3ION & $7.81861 \mathrm{E}-11$ & 0.0 & 0.0 & 0.0 \\
\hline NAION & 0.00175094 & 0.0 & 0.0 & 0.0 \\
\hline NASO 4 I ON & $4.81571 \mathrm{E}-13$ & 0.0 & 0.0 & 0.0 \\
\hline NO3ION & $3.67685 \mathrm{E}-04$ & 0.0 & 0.0 & 0.0 \\
\hline P207ION & $4.84547 \mathrm{E}-22$ & 0.0 & 0.0 & 0.0 \\
\hline PO4ION & $3.51779 \mathrm{E}-15$ & 0.0 & 0.0 & 0.0 \\
\hline SO4ION & $1.27309 \mathrm{E}-05$ & 0.0 & 0.0 & 0.0 \\
\hline ZNCL3ION & $3.07882 \mathrm{E}-06$ & 0.0 & 0.0 & 0.0 \\
\hline ZNCLION & $7.70974 \mathrm{E}-06$ & 0.0 & 0.0 & 0.0 \\
\hline ZNH2 PO 4 ION & $2.57467 \mathrm{E}-16$ & 0.0 & 0.0 & 0.0 \\
\hline ZNHCO3ION & $8.36158 \mathrm{E}-12$ & 0.0 & 0.0 & 0.0 \\
\hline ZNION & $5.52832 \mathrm{E}-07$ & 0.0 & 0.0 & 0.0 \\
\hline ZNNO3ION & $5.15105 \mathrm{E}-08$ & 0.0 & 0.0 & 0.0 \\
\hline ZNOH3ION & $6.90395 \mathrm{E}-08$ & 0.0 & 0.0 & 0.0 \\
\hline ZNOH 4 ION & $1.71078 \mathrm{E}-10$ & 0.0 & 0.0 & 0.0 \\
\hline ZNOHION & $1.54468 \mathrm{E}-05$ & 0.0 & 0.0 & 0.0 \\
\hline ALOOH & 0.0 & $1.19350 \mathrm{E}-05$ & 0.0 & 0.0 \\
\hline $\mathrm{CA} 3 \mathrm{PO} 42$ & 0.0 & $1.05295 \mathrm{E}-04$ & 0.0 & 0.0 \\
\hline CHAMOSITE7A & 0.0 & $3.84980 \mathrm{E}-05$ & 0.0 & 0.0 \\
\hline MGOH2 & 0.0 & $3.67662 \mathrm{E}-04$ & 0.0 & 0.0 \\
\hline Total $\mathrm{g} / \mathrm{hr}$ & $\begin{array}{l}============ \\
1.04105\end{array}$ & $\begin{array}{l}============ \\
0.178963\end{array}$ & $\begin{array}{l}=== \\
0.0\end{array}$ & $\begin{array}{l}=== \\
0.0\end{array}$ \\
\hline Volume, $\mathrm{m} 3 / \mathrm{hr}$ & $9.87859 \mathrm{E}-07$ & $5.86193 \mathrm{E}-08$ & 0.0 & 0.0 \\
\hline Enthalpy, cal/hr & -3504.77 & -514.533 & 0.0 & 0.0 \\
\hline Density, $g / \mathrm{m} 3$ & $1.05384 \mathrm{E}+06$ & $3.05297 E+06$ & & \\
\hline
\end{tabular}


WSRC-TR-2002-00567

Revision 0

$\begin{array}{ll}\text { Vapor fraction } & 0.0 \\ \text { Solid fraction } & 0.0 \\ \text { Organic fraction } & 0.0 \\ \text { Osmotic Pres, atm } & 147.51 \\ \text { Redox Pot, volts } & 0.0 \\ \text { E-Con, 1/ohm-cm } & 0.437663 \\ \text { E-Con, cm2/ohm-mol } & 52.3871 \\ \text { Abs Visc, cP } & 0.37083 \\ \text { Rel Visc } & 1.35324 \\ \text { Ionic Strength } & 2.64152\end{array}$

0.0

0.0

0.0

1 .

0.0

0.0

0.0

0.0

0.0

Ionic Strength

. 35324 
WSRC-TR-2002-00567

Revision 0

\begin{tabular}{|c|c|c|c|c|}
\hline Temperature, C & 25 . & 25 . & 25 & 25 \\
\hline & $\begin{array}{l}1 . \\
9.17807\end{array}$ & 1. & 1. & 1 \\
\hline Total mol/hr & $\begin{array}{l}0.05202917 \\
\mathrm{~mol} / \mathrm{hr}----\end{array}$ & $\begin{array}{l}0.0014092 \\
-\mathrm{mol} / \mathrm{hr}---.-\end{array}$ & $\begin{array}{l}0.0 \\
-\mathrm{mol} / \mathrm{hr}\end{array}$ & $\begin{array}{l}0.0 \\
-\mathrm{mol} / \mathrm{hr}-\end{array}$ \\
\hline $\begin{array}{l}\mathrm{H} 2 \mathrm{O} \\
\end{array}$ & 0.0474044 & 0.0 & 0.0 & 0.0 \\
\hline $\mathrm{CO} 2$ & $1.48981 \mathrm{E}-12$ & 0.0 & 0.0 & 0.0 \\
\hline $\mathrm{HCL}$ & $3.37411 \mathrm{E}-19$ & 0.0 & 0.0 & 0.0 \\
\hline $\mathrm{HNO} 3$ & $4.19363 \mathrm{E}-15$ & 0.0 & 0.0 & 0.0 \\
\hline LAURICACID & $5.03359 \mathrm{E}-10$ & 0.0 & 0.0 & 0.0 \\
\hline CAH2SIO4 & $2.99366 \mathrm{E}-08$ & 0.0 & 0.0 & 0.0 \\
\hline $\begin{array}{l}\mathrm{CASO} 4 \\
\text { FEIICL2 }\end{array}$ & $7.97867 \mathrm{E}-07$ & 0.0 & 0.0 & 0.0 \\
\hline FEIICL2 & $4.11805 \mathrm{E}-17$ & 0.0 & 0.0 & 0.0 \\
\hline FEIICO3 & $5.12557 \mathrm{E}-14$ & 0.0 & 0.0 & 0.0 \\
\hline FEIIHPO4 & $1.10196 \mathrm{E}-19$ & 0.0 & 0.0 & 0.0 \\
\hline FEIIOH2 & $1.52082 \mathrm{E}-14$ & 0.0 & 0.0 & 0.0 \\
\hline $\mathrm{ALO} 2 \mathrm{H} 2 \mathrm{CL}$ & 0.0 & 0.0 & 0.0 & 0.0 \\
\hline $\mathrm{H} 3 \mathrm{PO} 4$ & $7.11108 \mathrm{E}-22$ & 0.0 & 0.0 & 0.0 \\
\hline & $1.29798 \mathrm{E}-12$ & $1.19525 \mathrm{E}-05$ & 0.0 & 0.0 \\
\hline $\begin{array}{l}\mathrm{ALOH} 3 \\
\mathrm{KCL}\end{array}$ & $1.22359 \mathrm{E}-06$ & 0.0 & 0.0 & 0.0 \\
\hline $\mathrm{KHSO} 4$ & $1.78687 \mathrm{E}-16$ & 0.0 & 0.0 & 0.0 \\
\hline MGCO3 & $1.43813 \mathrm{E}-09$ & 0.0 & 0.0 & 0.0 \\
\hline MGH2SIO 4 & $2.71323 \mathrm{E}-07$ & 0.0 & 0.0 & 0.0 \\
\hline MGHPO 4 & $1.96776 \mathrm{E}-11$ & 0.0 & 0.0 & 0.0 \\
\hline MGSO 4 & $1.30392 \mathrm{E}-06$ & 0.0 & 0.0 & 0.0 \\
\hline NAHCO3 & $5.48975 \mathrm{E}-10$ & 0.0 & 0.0 & 0.0 \\
\hline NAHSIO3 & $3.47990 \mathrm{E}-05$ & 0.0 & 0.0 & 0.0 \\
\hline NANO3 & $2.68542 \mathrm{E}-05$ & 0.0 & 0.0 & 0.0 \\
\hline CACL2 & $3.12743 E-23$ & 0.0 & 0.0 & 0.0 \\
\hline $\mathrm{SIO} 2$ & $1.14051 \mathrm{E}-06$ & $1.57047 \mathrm{E}-04$ & 0.0 & 0.0 \\
\hline $\mathrm{CACO} 3$ & $3.39901 \mathrm{E}-09$ & $1.00329 \mathrm{E}-04$ & 0.0 & 0.0 \\
\hline ZNCL2 & $2.12036 \mathrm{E}-09$ & 0.0 & 0.0 & 0.0 \\
\hline ZNHPO 4 & $1.17373 \mathrm{E}-15$ & 0.0 & 0.0 & 0.0 \\
\hline ZNNO32 & $2.57764 \mathrm{E}-11$ & 0.0 & 0.0 & 0.0 \\
\hline $\mathrm{ZNOH} 2$ & $4.39637 \mathrm{E}-09$ & $3.05672 E-05$ & 0.0 & 0.0 \\
\hline OHION & $1.32885 \mathrm{E}-08$ & 0.0 & 0.0 & 0.0 \\
\hline ALION & $2.02872 \mathrm{E}-23$ & 0.0 & 0.0 & 0.0 \\
\hline ALOH 2 ION & $3.88687 \mathrm{E}-16$ & 0.0 & 0.0 & 0.0 \\
\hline $\mathrm{ALOH} 4 \mathrm{ION}$ & $1.22770 \mathrm{E}-09$ & 0.0 & 0.0 & 0.0 \\
\hline ALOHCLION & $4.80093 E-19$ & 0.0 & 0.0 & 0.0 \\
\hline ALOHION & $1.09532 \mathrm{E}-19$ & 0.0 & 0.0 & 0.0 \\
\hline ALSO 42 ION & $1.29704 \mathrm{E}-24$ & 0.0 & 0.0 & 0.0 \\
\hline ALSO 4 ION & $1.09591 \mathrm{E}-23$ & 0.0 & 0.0 & 0.0 \\
\hline CACLION & $1.41179 \mathrm{E}-10$ & 0.0 & 0.0 & 0.0 \\
\hline CAH2 PO 4 ION & $2.28391 \mathrm{E}-14$ & 0.0 & 0.0 & 0.0 \\
\hline CAHCO3ION & $7.19731 \mathrm{E}-10$ & 0.0 & 0.0 & 0.0 \\
\hline CAHSIO3ION & $2.99382 \mathrm{E}-07$ & 0.0 & 0.0 & 0.0 \\
\hline CAION & $8.65820 \mathrm{E}-05$ & 0.0 & 0.0 & 0.0 \\
\hline CANO3ION & $3.08186 \mathrm{E}-05$ & 0.0 & 0.0 & 0.0 \\
\hline
\end{tabular}




\begin{tabular}{|c|c|c|c|c|}
\hline CAOHION & $6.99502 E-09$ & 0.0 & 0.0 & 0.0 \\
\hline CAPO 4 ION & $1.46967 \mathrm{E}-10$ & 0.0 & 0.0 & 0.0 \\
\hline CLION & 0.00183217 & 0.0 & 0.0 & 0.0 \\
\hline CO3ION & $1.66401 \mathrm{E}-09$ & 0.0 & 0.0 & 0.0 \\
\hline DODECION & $3.07114 E-05$ & 0.0 & 0.0 & 0.0 \\
\hline FEIICLION & $1.69444 \mathrm{E}-14$ & 0.0 & 0.0 & 0.0 \\
\hline FEIICO32ION & $6.00560 \mathrm{E}-18$ & 0.0 & 0.0 & 0.0 \\
\hline FEIIH2PO4ION & $4.91843 E-22$ & 0.0 & 0.0 & 0.0 \\
\hline FEIIHCO3ION & $1.94694 \mathrm{E}-18$ & 0.0 & 0.0 & 0.0 \\
\hline FEIIION & $1.48815 \mathrm{E}-11$ & 0.0 & 0.0 & 0.0 \\
\hline FEIIOH3ION & $2.44124 \mathrm{E}-15$ & 0.0 & 0.0 & 0.0 \\
\hline FEIIOH 4 ION & $3.53179 \mathrm{E}-20$ & 0.0 & 0.0 & 0.0 \\
\hline FEIIOHION & $3.11863 \mathrm{E}-12$ & 0.0 & 0.0 & 0.0 \\
\hline $\mathrm{H} 2 \mathrm{P} 207 \mathrm{ION}$ & $1.46014 \mathrm{E}-26$ & 0.0 & 0.0 & 0.0 \\
\hline H2 PO 4 I ON & $2.91780 \mathrm{E}-14$ & 0.0 & 0.0 & 0.0 \\
\hline H2SIO 4 ION & $5.89477 \mathrm{E}-10$ & 0.0 & 0.0 & 0.0 \\
\hline H3SIO 4 ION & $3.99114 \mathrm{E}-07$ & 0.0 & 0.0 & 0.0 \\
\hline HCO3ION & $3.05731 \mathrm{E}-09$ & 0.0 & 0.0 & 0.0 \\
\hline HION & $5.12616 \mathrm{E}-13$ & 0.0 & 0.0 & 0.0 \\
\hline HP2O7ION & $3.57486 \mathrm{E}-23$ & 0.0 & 0.0 & 0.0 \\
\hline HPO4 ION & $2.55377 \mathrm{E}-11$ & 0.0 & 0.0 & 0.0 \\
\hline HSO 4 ION & $1.11243 \mathrm{E}-13$ & 0.0 & 0.0 & 0.0 \\
\hline KION & $3.02159 \mathrm{E}-04$ & 0.0 & 0.0 & 0.0 \\
\hline KSO4 ION & $3.50673 E-06$ & 0.0 & 0.0 & 0.0 \\
\hline MGH2 PO 4 ION & $2.75207 \mathrm{E}-14$ & 0.0 & 0.0 & 0.0 \\
\hline MGHCO3ION & $1.94057 \mathrm{E}-09$ & 0.0 & 0.0 & 0.0 \\
\hline MGHSIO3ION & $5.18658 \mathrm{E}-07$ & 0.0 & 0.0 & 0.0 \\
\hline MGION & $3.74267 \mathrm{E}-05$ & 0.0 & 0.0 & 0.0 \\
\hline MGOHION & $6.28479 E-08$ & 0.0 & 0.0 & 0.0 \\
\hline MGP207 ION & $2.17269 \mathrm{E}-18$ & 0.0 & 0.0 & 0.0 \\
\hline MGPO 4 ION & $2.04982 \mathrm{E}-10$ & 0.0 & 0.0 & 0.0 \\
\hline NACO3ION & $7.98444 \mathrm{E}-10$ & 0.0 & 0.0 & 0.0 \\
\hline NAION & 0.00177135 & 0.0 & 0.0 & 0.0 \\
\hline NASO 4 ION & $1.19658 \mathrm{E}-05$ & 0.0 & 0.0 & 0.0 \\
\hline NO3ION & $4.26158 \mathrm{E}-04$ & 0.0 & 0.0 & 0.0 \\
\hline P2O7ION & $2.58003 \mathrm{E}-21$ & 0.0 & 0.0 & 0.0 \\
\hline PO4ION & $1.90273 \mathrm{E}-13$ & 0.0 & 0.0 & 0.0 \\
\hline SO 4 ION & $2.41607 \mathrm{E}-05$ & 0.0 & 0.0 & 0.0 \\
\hline ZNCL3ION & $2.78289 \mathrm{E}-09$ & 0.0 & 0.0 & 0.0 \\
\hline ZNCLION & $2.87495 E-09$ & 0.0 & 0.0 & 0.0 \\
\hline ZNH2 PO 4 ION & $8.52843 E-19$ & 0.0 & 0.0 & 0.0 \\
\hline ZNHCO3ION & $5.87212 \mathrm{E}-14$ & 0.0 & 0.0 & 0.0 \\
\hline ZNION & $1.18092 \mathrm{E}-08$ & 0.0 & 0.0 & 0.0 \\
\hline ZNNO3ION & $1.20858 \mathrm{E}-09$ & 0.0 & 0.0 & 0.0 \\
\hline ZNOH3ION & $1.36793 \mathrm{E}-10$ & 0.0 & 0.0 & 0.0 \\
\hline ZNOH 4 ION & $3.06673 \mathrm{E}-13$ & 0.0 & 0.0 & 0.0 \\
\hline ZNOHION & $2.43128 \mathrm{E}-09$ & 0.0 & 0.0 & 0.0 \\
\hline $\mathrm{CA} 3 \mathrm{PO} 42$ & 0.0 & $1.05295 \mathrm{E}-04$ & 0.0 & 0.0 \\
\hline CASO $4.2 \mathrm{H} 2 \mathrm{O}$ & 0.0 & $6.34912 \mathrm{E}-04$ & 0.0 & 0.0 \\
\hline CHAMOSITE7A & 0.0 & $3.84979 E-05$ & 0.0 & 0.0 \\
\hline $\mathrm{MGOH} 2$ & 0.0 & $3.30600 \mathrm{E}-04$ & 0.0 & 0.0 \\
\hline Total g/hr & 1.02215 & 0.197861 & 0,0 & 0.0 \\
\hline Volume, m3/hr & $9.17119 \mathrm{E}-07$ & $6.90132 \mathrm{E}-08$ & 0.0 & 0.0 \\
\hline Enthalpy, cal/hr & -3503.97 & -588.798 & 0.0 & 0.0 \\
\hline Density, $g / \mathrm{m} 3$ & $1.11452 \mathrm{E}+06$ & $2.86701 E+06$ & & \\
\hline Vapor fraction & 0.0 & 0.0 & 0.0 & 0.0 \\
\hline Solid fraction & 0.0 & 1. & 0.0 & 0.0 \\
\hline
\end{tabular}


WSRC-TR-2002-00567

Revision 0

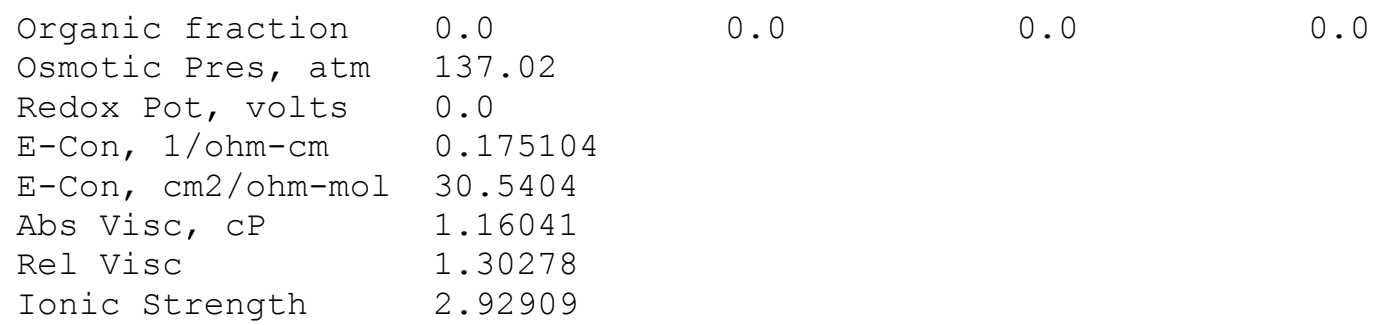


WSRC-TR-2002-00567

Revision 0

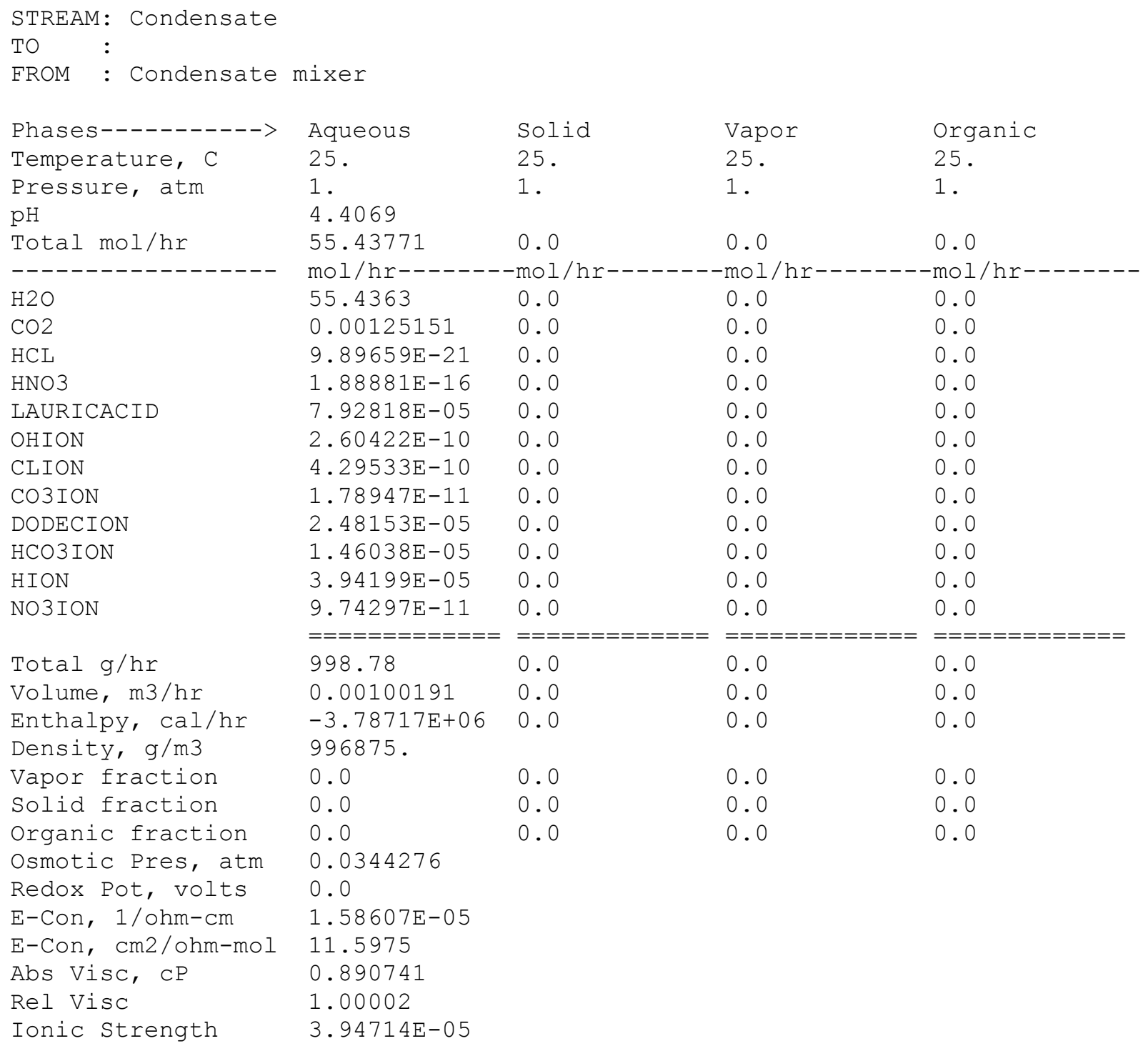


WSRC-TR-2002-00567

Revision 0

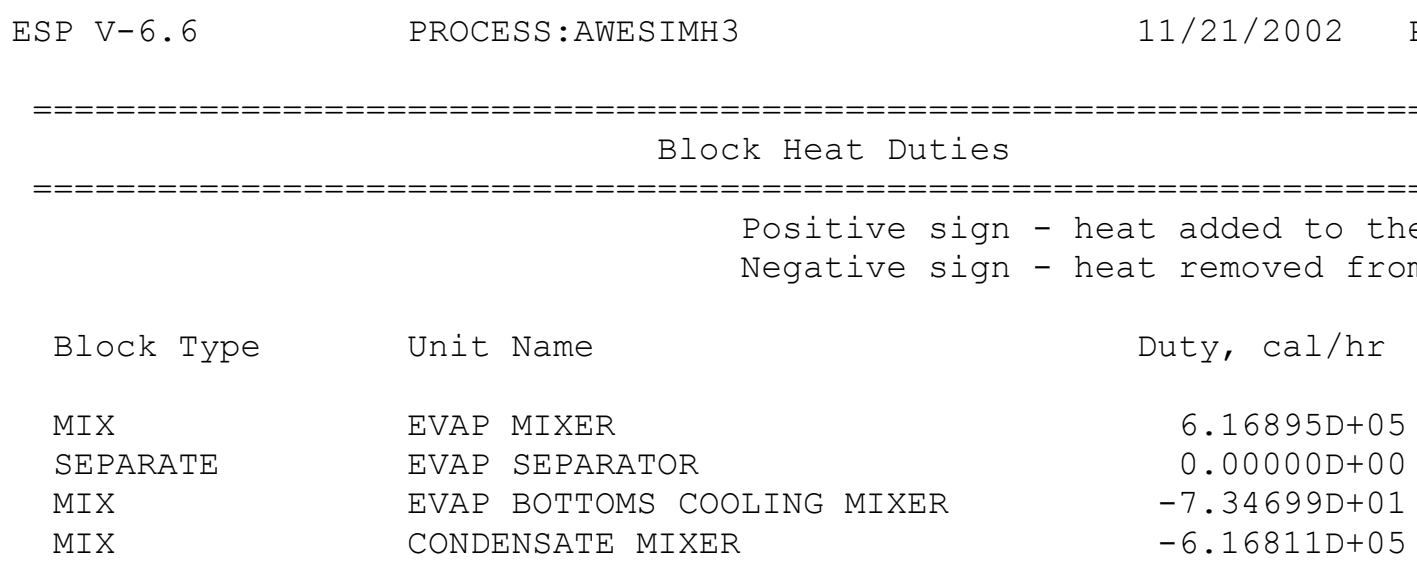


WSRC-TR-2002-00567

Revision 0

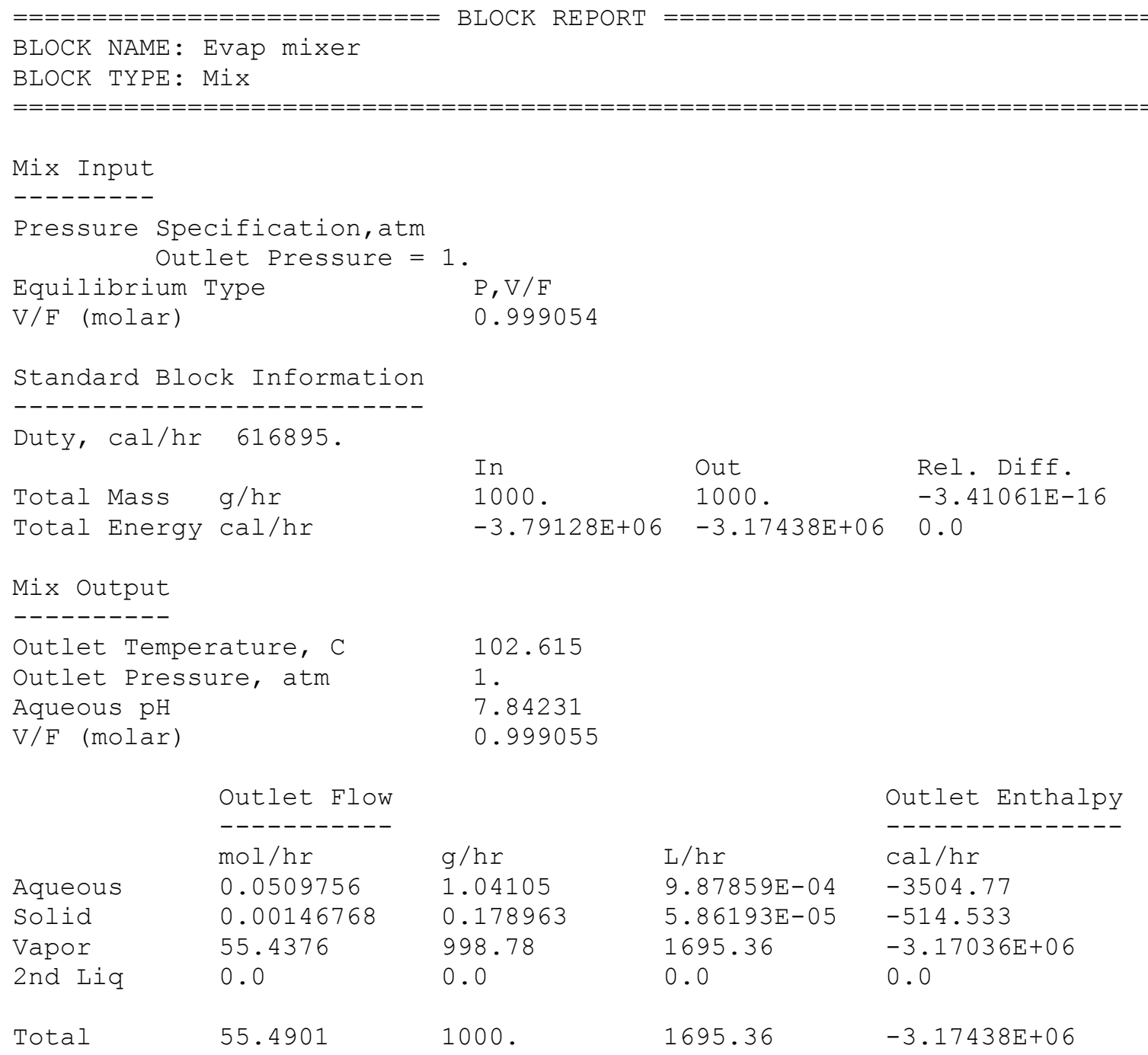


WSRC-TR-2002-00567

Revision 0

$\begin{array}{lllll}\text { ESP V-6.6 PROCESS:AWESIMH3 } & 11 / 21 / 2002 & \text { PAGE } & 11\end{array}$

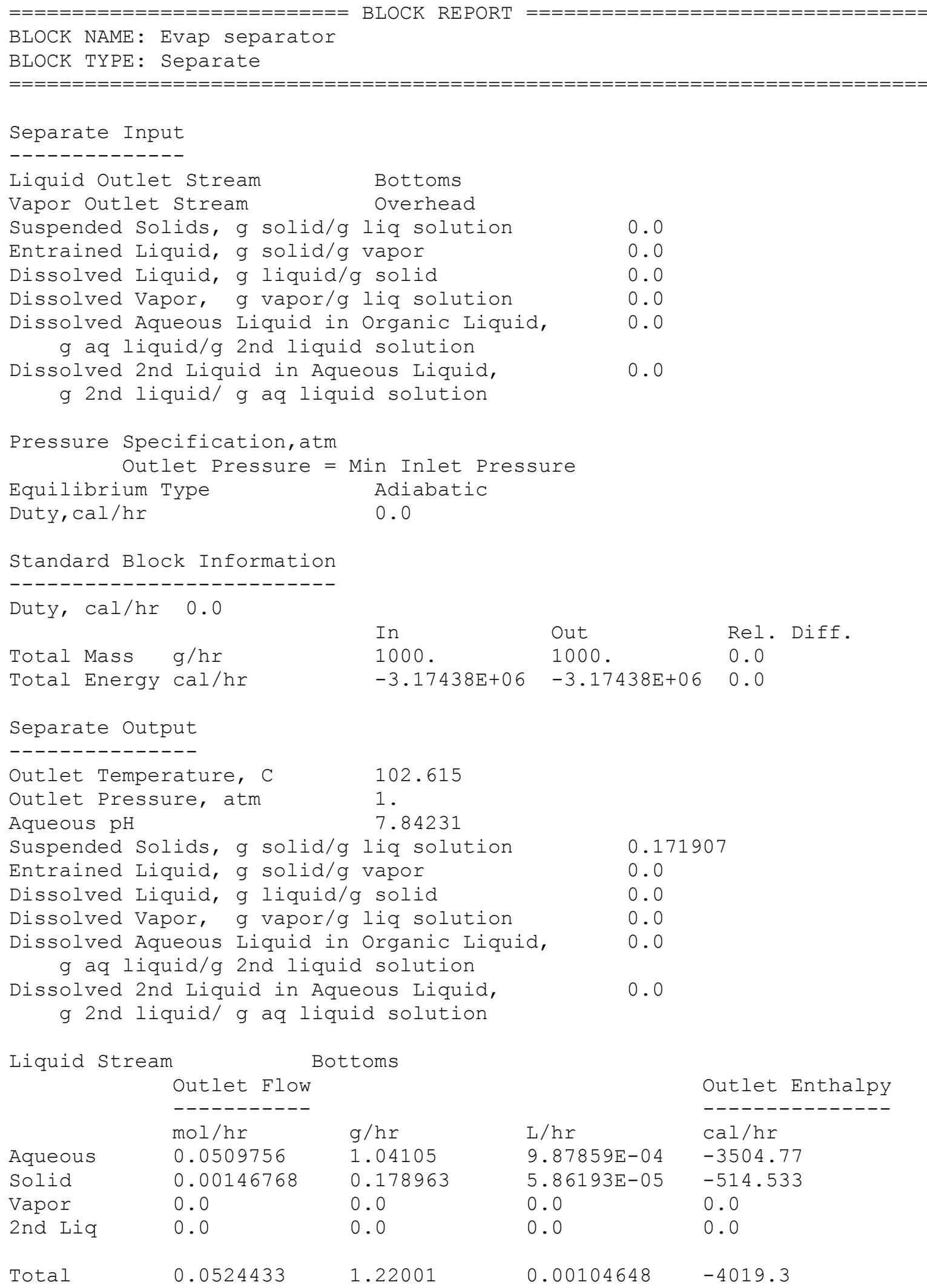


WSRC-TR-2002-00567

Revision 0

ESP V-6.6

PROCESS : AWES IMH3

Vapor stream

Outlet Flow
--------
mol/hr
0.0
0.0
55.4376
0.0

55.4376 g/hr

0.0

0.0

998.78

0.0

998.78
$11 / 21 / 2002$

PAGE

12

$\mathrm{L} / \mathrm{hr}$

0.0

0.0

1695.36

0.0

1695.36
Outlet Enthalpy

cal/hr

0.0

0.0

$-3.17036 E+06$

0.0

$-3.17036 \mathrm{E}+06$ 
WSRC-TR-2002-00567

Revision 0

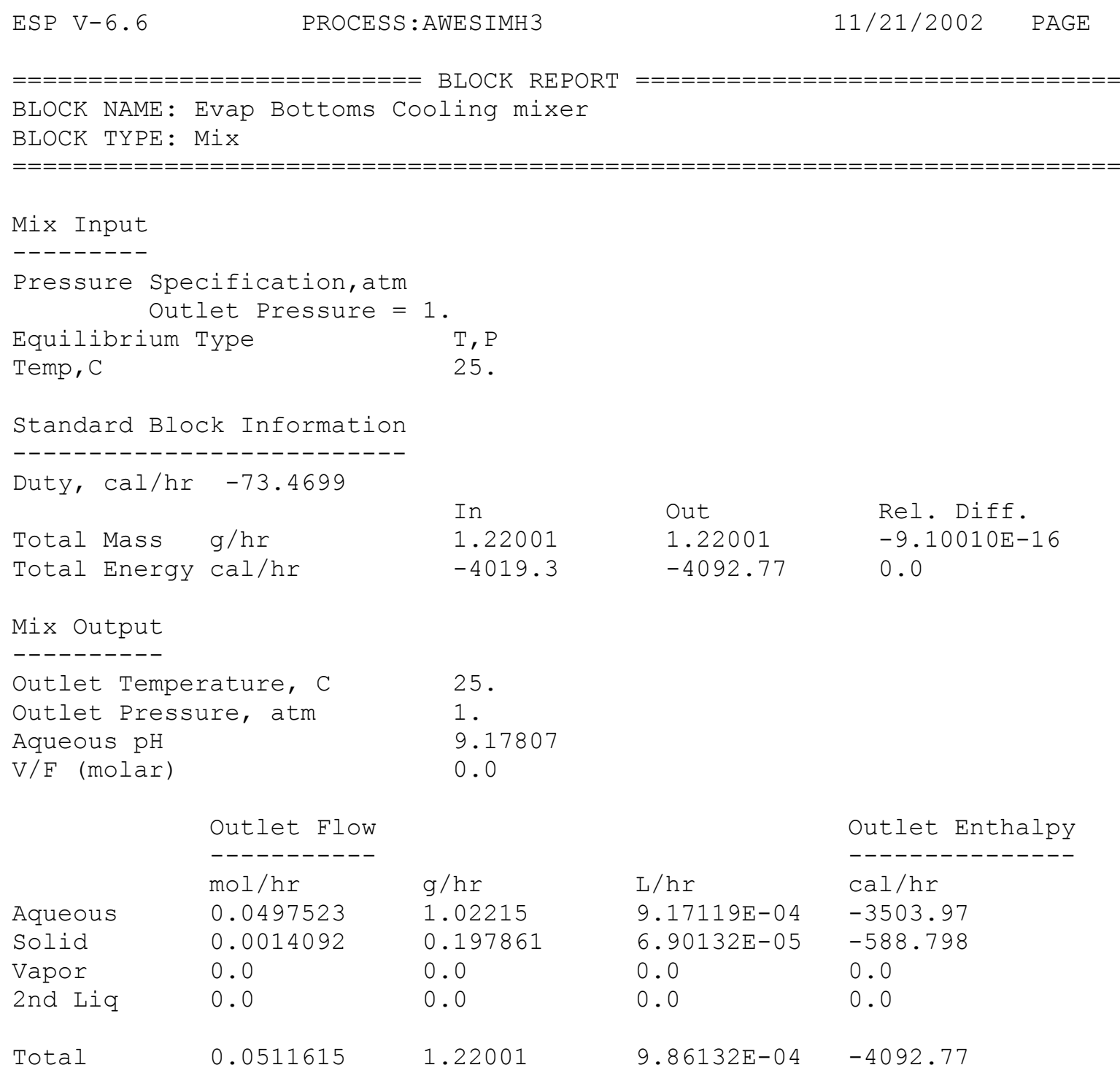


WSRC-TR-2002-00567

Revision 0

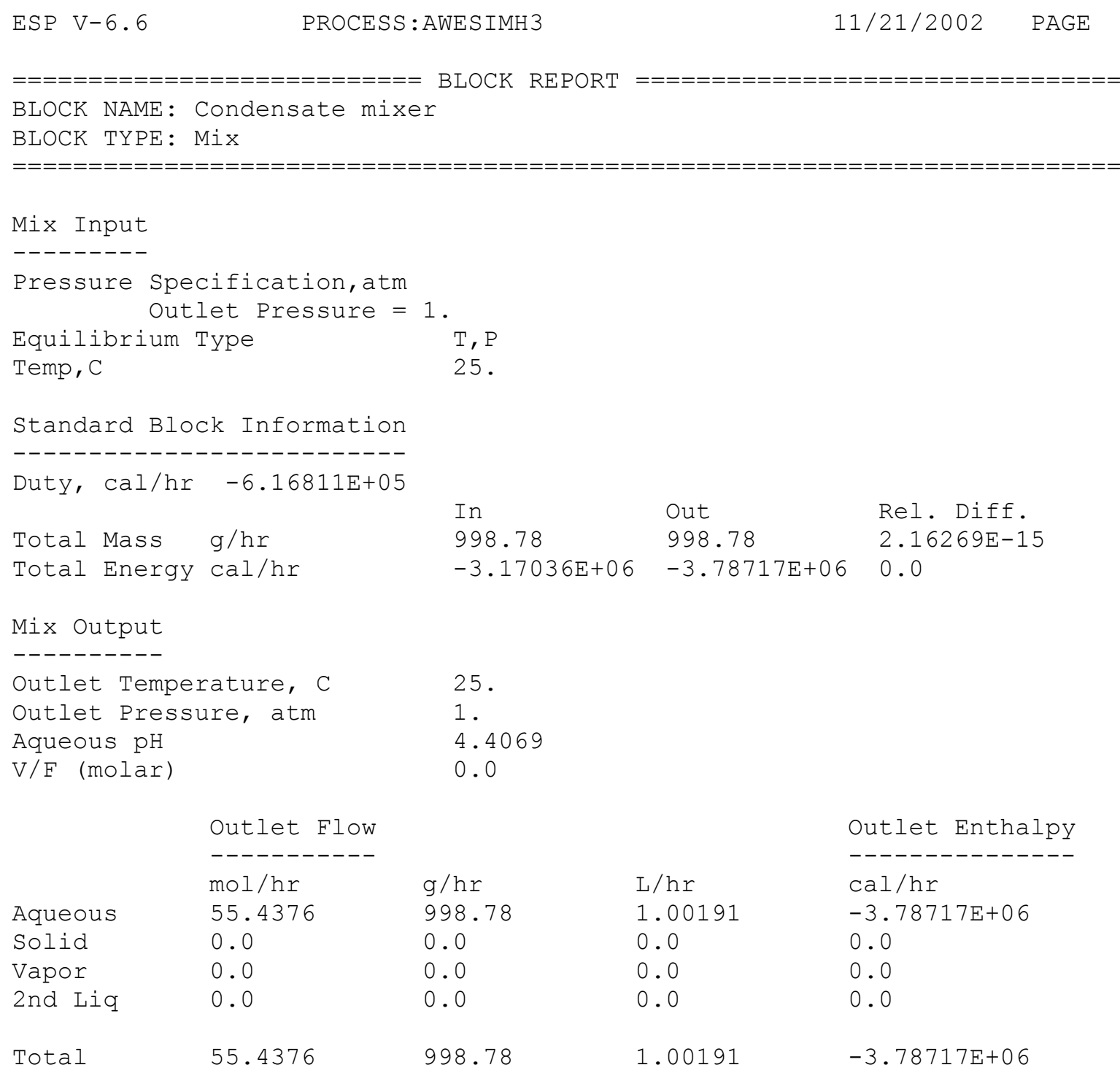


WSRC-TR-2002-00567

Revision 0

\section{Influent Limit Composition 50\% Target $\mathrm{pH}=6.5$}

AWE 6.5-50

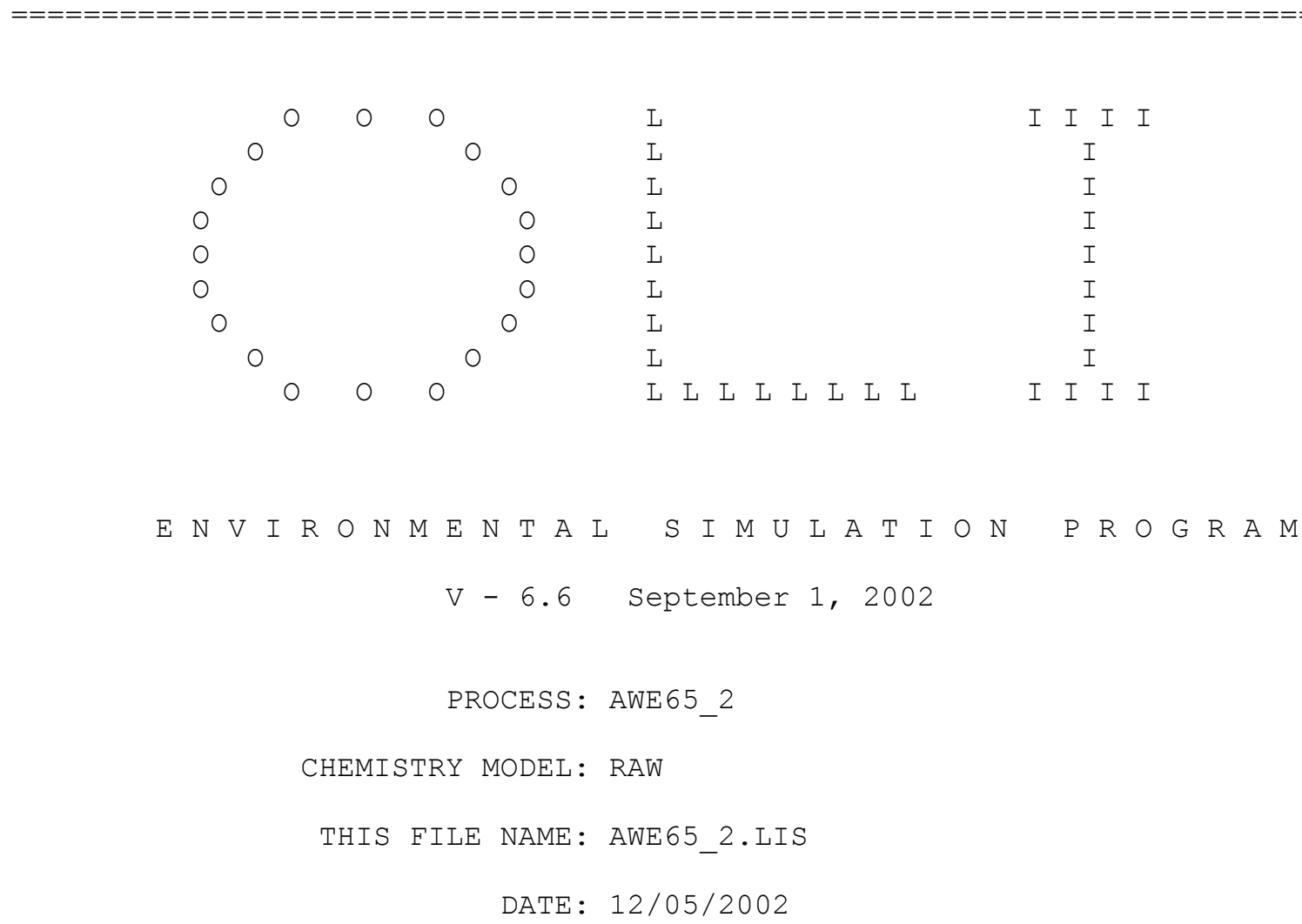


WSRC-TR-2002-00567

Revision 0

TABLE OF CONTENTS

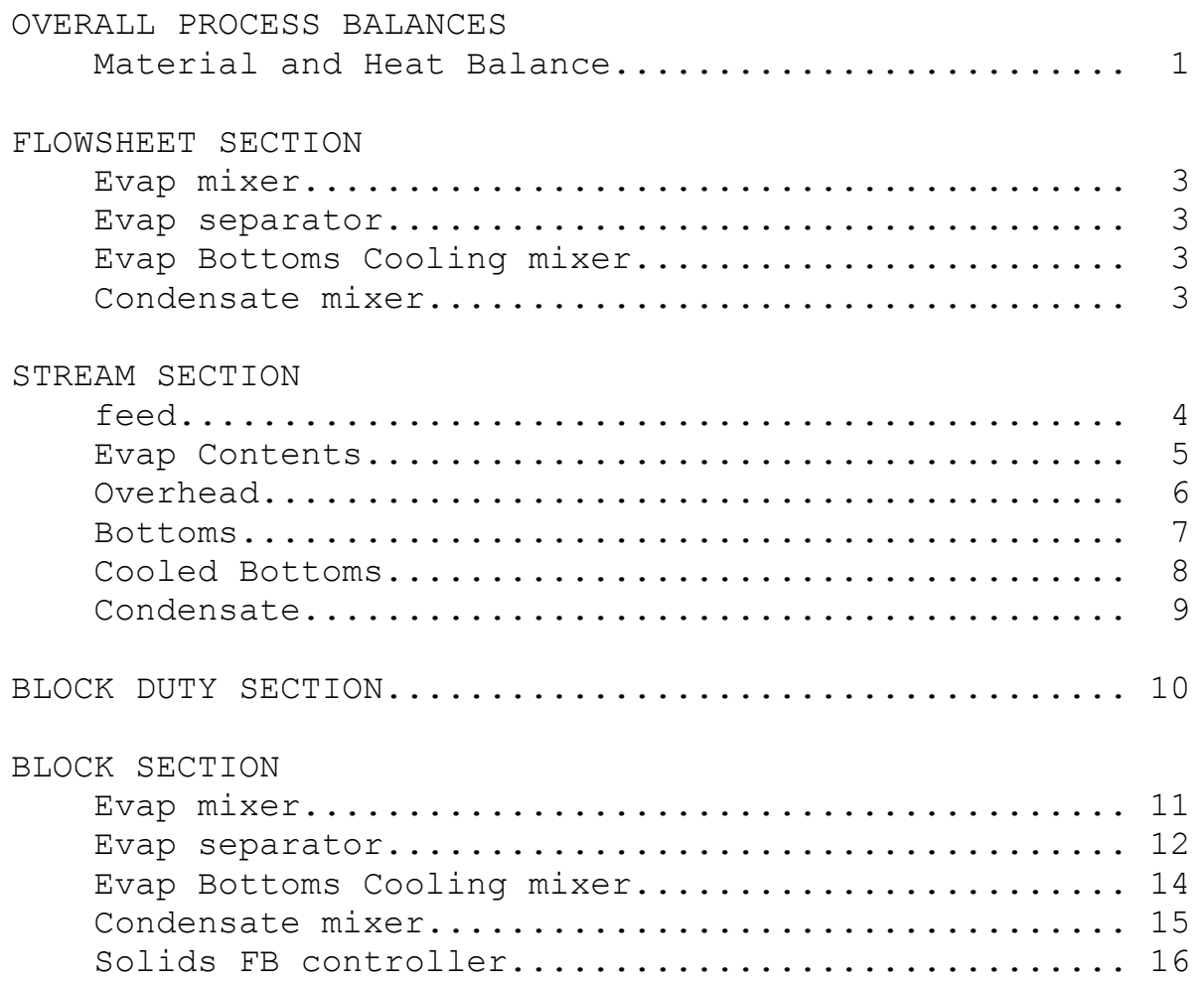


WSRC-TR-2002-00567

Revision 0

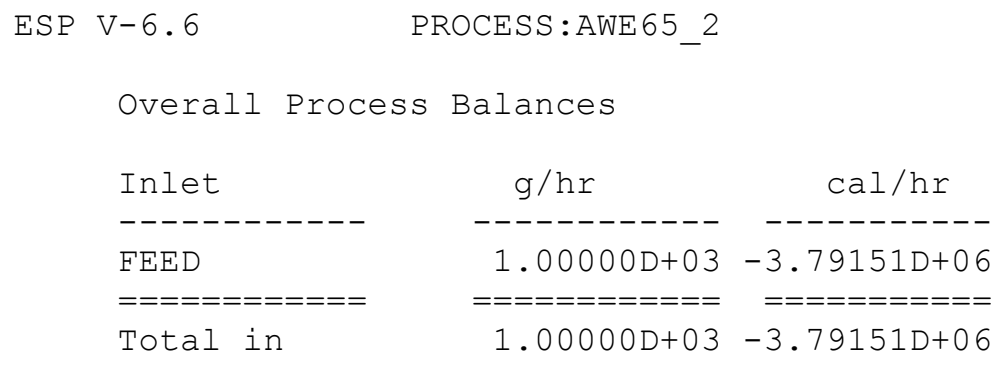

\begin{tabular}{lrr} 
Outlet & \multicolumn{1}{c}{$\mathrm{g} / \mathrm{hr}$} & $\mathrm{cal} / \mathrm{hr}$ \\
---------- & ---------- & ---------- \\
COOLED BOTTOMS & $7.62962 \mathrm{D}-01$ & $-2.54735 \mathrm{D}+03$ \\
CONDENSATE & $9.99237 \mathrm{D}+02$ & $-3.78896 \mathrm{D}+06$ \\
$==========$ & $==========$ & $=========$ \\
Total out & $1.00000 \mathrm{D}+03$ & $-3.79151 \mathrm{D}+06$
\end{tabular}

Block Heat Duties

$\mathrm{cal} / \mathrm{hr}$
--------
$6.17262 \mathrm{D}+05$
$-4.50883 \mathrm{D}+01$
$-6.17215 \mathrm{D}+05$
$==========$
$2.17588 \mathrm{D}+00$

DIFFERENCE

$0.00000 D+00-1.16415 D-10$

REL DIFFERENCE

$0.00000 \mathrm{D}+00 \quad 3.07042 \mathrm{D}-17$

Material Code Balances

\begin{tabular}{|c|c|c|c|c|}
\hline Code & $\begin{array}{l}\text { Input } \\
\text { mol/hr }\end{array}$ & $\begin{array}{l}\text { Outlet } \\
\text { mol/hr }\end{array}$ & $\begin{array}{l}\text { Difference } \\
\text { mol/hr }\end{array}$ & Rel Diff \\
\hline $\mathrm{H}(+1)$ & $1.10987 \mathrm{D}+02$ & $1.10987 \mathrm{D}+02$ & $1.42109 \mathrm{D}-14$ & $1.28040 \mathrm{D}-16$ \\
\hline$K(+1)$ & $1.37404 \mathrm{D}-04$ & $1.37404 \mathrm{D}-04$ & $-2.71051 D-20$ & $-1.97266 \mathrm{D}-16$ \\
\hline $\mathrm{NA}(+1)$ & $9.34361 \mathrm{D}-04$ & $9.34361 \mathrm{D}-04$ & $-1.19262 D-18$ & $-1.27640 \mathrm{D}-15$ \\
\hline $\mathrm{BA}(+2)$ & $4.08759 \mathrm{D}-07$ & $4.08759 \mathrm{D}-07$ & $1.05879 \mathrm{D}-22$ & $2.59026 \mathrm{D}-16$ \\
\hline$C A(+2)$ & $7.23192 \mathrm{D}-04$ & $7.23192 \mathrm{D}-04$ & $-2.16840 D-19$ & $-2.99838 D-16$ \\
\hline $\mathrm{ZN}(+2)$ & $2.14067 D-05$ & $2.14067 D-05$ & $3.38813 \mathrm{D}-21$ & $1.58274 \mathrm{D}-16$ \\
\hline $\mathrm{CU}(+2)$ & $3.14961 \mathrm{D}-06$ & $3.14961 D-06$ & $0.00000 \mathrm{D}+00$ & $0.00000 \mathrm{D}+00$ \\
\hline $\mathrm{FE}(+2)$ & $5.55556 \mathrm{D}-05$ & $5.55556 \mathrm{D}-05$ & $0.00000 D+00$ & $0.00000 \mathrm{D}+00$ \\
\hline MG (+2) & $2.46914 \mathrm{D}-04$ & $2.46914 \mathrm{D}-04$ & $0.00000 \mathrm{D}+00$ & $0.00000 \mathrm{D}+00$ \\
\hline $\mathrm{PB}(+2)$ & $2.12560 \mathrm{D}-06$ & $2.12560 \mathrm{D}-06$ & $-8.47033 D-22$ & $-3.98490 D-16$ \\
\hline $\mathrm{AL}(+3)$ & $6.29630 \mathrm{D}-05$ & $6.29630 \mathrm{D}-05$ & $0.00000 \mathrm{D}+00$ & $0.00000 \mathrm{D}+00$ \\
\hline $\mathrm{NI}(+2)$ & $9.36968 \mathrm{D}-07$ & $9.36968 \mathrm{D}-07$ & $-1.05879 D-22$ & $-1.13002 \mathrm{D}-16$ \\
\hline$O(-2)$ & $5.54997 \mathrm{D}+01$ & $5.54997 \mathrm{D}+01$ & $-1.42109 D-14$ & $-2.56053 D-16$ \\
\hline $\mathrm{CL}(-1)$ & $1.32394 \mathrm{D}-03$ & $1.32394 \mathrm{D}-03$ & $-8.67362 D-19$ & $-6.55135 D-16$ \\
\hline$C(+4)$ & $1.00000 \mathrm{D}-03$ & $1.00000 \mathrm{D}-03$ & $8.67362 \mathrm{D}-19$ & $8.67362 \mathrm{D}-16$ \\
\hline$P(+5)$ & $1.47368 \mathrm{D}-04$ & $1.47368 \mathrm{D}-04$ & $8.13152 \mathrm{D}-20$ & $5.51781 \mathrm{D}-16$ \\
\hline$S(+6)$ & $4.89583 D-04$ & $4.89583 \mathrm{D}-04$ & $-1.19262 D-18$ & $-2.43599 \mathrm{D}-15$ \\
\hline$N(+5)$ & $3.38710 \mathrm{D}-04$ & $3.38710 \mathrm{D}-04$ & $0.00000 \mathrm{D}+00$ & $0.00000 \mathrm{D}+00$ \\
\hline $\mathrm{SI}(+4)$ & $1.65000 \mathrm{D}-04$ & $1.65000 \mathrm{D}-04$ & $-2.71051 D-20$ & $-1.64273 D-16$ \\
\hline $\mathrm{SR}(+2)$ & $1.13014 \mathrm{D}-05$ & $1.13014 \mathrm{D}-05$ & $-1.69407 D-21$ & $-1.49899 D-16$ \\
\hline
\end{tabular}


WSRC-TR-2002-00567

Revision 0

$\mathrm{CD}(+2)$

$\mathrm{CR}(+3)$

$\mathrm{U}(+4)$
8. $92857 \mathrm{D}-09$

$5.38462 \mathrm{D}-08$

2. $01681 \mathrm{D}-06$
8.92857D-09

5. $38462 \mathrm{D}-08$

4.89691D-21 
WSRC-TR-2002-00567

Revision 0

ESP V-6.6

$\operatorname{DODEC}(-1)$
PROCESS:AWE 652

$3.37022 \mathrm{D}-05$
$12 / 05 / 2002$

$3.37022 \mathrm{D}-05$
1.96512D-19

$5.83082 \mathrm{D}-15$ 
WSRC-TR-2002-00567

Revision 0

\begin{tabular}{|c|c|c|c|}
\hline \multicolumn{4}{|l|}{$\begin{array}{l}\text { PROCESS BLOCKS } \\
===========\end{array}$} \\
\hline $\begin{array}{l}\mathrm{BLOCK} \mathrm{NAME} \\
=========================\end{array}$ & $\begin{array}{l}\text { BLOCK TYPE } \\
===========\end{array}$ & $\begin{array}{l}\text { INLET STREAM }(\mathrm{S}) \\
==============\end{array}$ & $\begin{array}{l}\text { OUTLET STREAM }(\mathrm{S}) \\
==============\end{array}$ \\
\hline Evap mixer & $\operatorname{Mix}$ & feed & Evap Contents \\
\hline Evap separator & Separate & Evap Contents & $\begin{array}{l}\text { Overhead } \\
\text { Bottoms }\end{array}$ \\
\hline Evap Bottoms Cooling mixer & Mix & Bottoms & Cooled Bottoms \\
\hline Condensate mixer & Mix & Overhead & Condensate \\
\hline
\end{tabular}


WSRC-TR-2002-00567

Revision 0

\begin{tabular}{|c|c|c|c|}
\hline Aqueous & Solid & Vapor & Organic \\
\hline 25 . & 25 . & 25 . & 25 . \\
\hline 1 . & 1. & 1. & 1. \\
\hline \multicolumn{4}{|l|}{6.05821} \\
\hline 55.4989 & $6.66618 \mathrm{E}-05$ & 0.0 & 0.0 \\
\hline \multicolumn{4}{|c|}{$\mathrm{mol} / \mathrm{hr}--------\mathrm{mol} / \mathrm{hr}--------\mathrm{mol} / \mathrm{hr}--------\operatorname{mol} / \mathrm{hr}--------$} \\
\hline 55.4933 & 0.0 & 0.0 & 0.0 \\
\hline $6.38321 \mathrm{E}-04$ & 0.0 & 0.0 & 0.0 \\
\hline $9.94237 \mathrm{E}-25$ & 0.0 & 0.0 & 0.0 \\
\hline $6.37324 \mathrm{E}-16$ & 0.0 & 0.0 & 0.0 \\
\hline $1.36789 \mathrm{E}-11$ & 0.0 & 0.0 & 0.0 \\
\hline $2.10382 \mathrm{E}-06$ & 0.0 & 0.0 & 0.0 \\
\hline $1.28689 E-28$ & 0.0 & 0.0 & 0.0 \\
\hline $7.23579 E-14$ & 0.0 & 0.0 & 0.0 \\
\hline $7.04016 \mathrm{E}-06$ & 0.0 & 0.0 & 0.0 \\
\hline $1.63334 \mathrm{E}-11$ & 0.0 & 0.0 & 0.0 \\
\hline $4.89720 \mathrm{E}-17$ & 0.0 & 0.0 & 0.0 \\
\hline $7.20742 \mathrm{E}-10$ & 0.0 & 0.0 & 0.0 \\
\hline $2.93720 \mathrm{E}-14$ & 0.0 & 0.0 & 0.0 \\
\hline $3.60989 E-13$ & 0.0 & 0.0 & 0.0 \\
\hline $9.57902 \mathrm{E}-09$ & 0.0 & 0.0 & 0.0 \\
\hline $4.99710 \mathrm{E}-15$ & 0.0 & 0.0 & 0.0 \\
\hline $1.08210 \mathrm{E}-09$ & 0.0 & 0.0 & 0.0 \\
\hline $3.14603 E-16$ & 0.0 & 0.0 & 0.0 \\
\hline $9.60779 E-08$ & 0.0 & 0.0 & 0.0 \\
\hline $8.92000 E-09$ & 0.0 & 0.0 & 0.0 \\
\hline $1.35847 \mathrm{E}-13$ & 0.0 & 0.0 & 0.0 \\
\hline $1.23287 \mathrm{E}-28$ & 0.0 & 0.0 & 0.0 \\
\hline $1.45258 E-08$ & 0.0 & 0.0 & 0.0 \\
\hline $3.74615 E-19$ & 0.0 & 0.0 & 0.0 \\
\hline $2.40212 \mathrm{E}-09$ & $6.29593 E-05$ & 0.0 & 0.0 \\
\hline $2.32109 E-12$ & 0.0 & 0.0 & 0.0 \\
\hline $1.29940 \mathrm{E}-09$ & 0.0 & 0.0 & 0.0 \\
\hline $7.16085 \mathrm{E}-14$ & 0.0 & 0.0 & 0.0 \\
\hline $1.98734 \mathrm{E}-10$ & 0.0 & 0.0 & 0.0 \\
\hline $3.01432 E-09$ & 0.0 & 0.0 & 0.0 \\
\hline $2.34742 E-13$ & 0.0 & 0.0 & 0.0 \\
\hline $1.78108 E-06$ & 0.0 & 0.0 & 0.0 \\
\hline $4.11835 E-06$ & 0.0 & 0.0 & 0.0 \\
\hline $1.20002 \mathrm{E}-07$ & 0.0 & 0.0 & 0.0 \\
\hline $2.84504 \mathrm{E}-09$ & 0.0 & 0.0 & 0.0 \\
\hline $1.56078 E-08$ & 0.0 & 0.0 & 0.0 \\
\hline $8.84006 \mathrm{E}-14$ & 0.0 & 0.0 & 0.0 \\
\hline $4.79754 \mathrm{E}-08$ & 0.0 & 0.0 & 0.0 \\
\hline 7. $32618 E-12$ & 0.0 & 0.0 & 0.0 \\
\hline $6.71763 E-10$ & 0.0 & 0.0 & 0.0 \\
\hline $9.25760 \mathrm{E}-14$ & 0.0 & 0.0 & 0.0 \\
\hline $6.45606 \mathrm{E}-13$ & 0.0 & 0.0 & 0.0 \\
\hline $7.46358 E-28$ & 0.0 & 0.0 & 0.0 \\
\hline $1.64968 \mathrm{E}-04$ & 0.0 & 0.0 & 0.0 \\
\hline
\end{tabular}




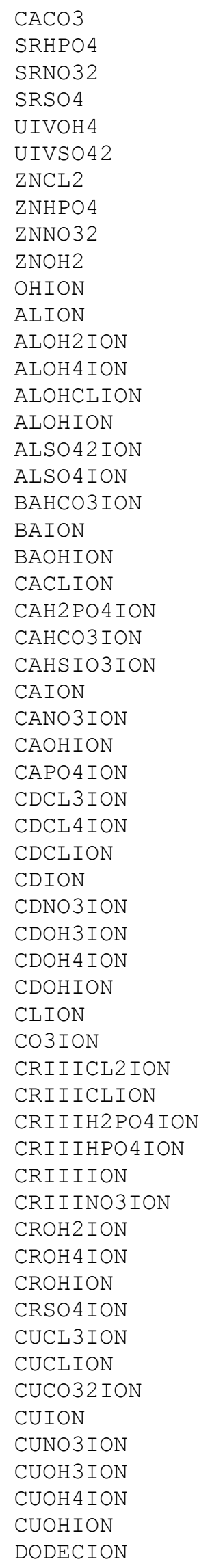

\begin{tabular}{|c|c|}
\hline $1.99032 \mathrm{E}-08$ & 0.0 \\
\hline $1.22376 \mathrm{E}-09$ & 0.0 \\
\hline $6.54426 \mathrm{E}-12$ & 0.0 \\
\hline $5.56676 \mathrm{E}-07$ & 0.0 \\
\hline $3.02313 E-10$ & 0.0 \\
\hline $1.74297 E-26$ & 0.0 \\
\hline $4.16299 E-11$ & 0.0 \\
\hline $2.44170 \mathrm{E}-07$ & 0.0 \\
\hline 1.01393E-12 & 0.0 \\
\hline 1.009 & 0.0 \\
\hline $1.24856 \mathrm{E}-08$ & 0.0 \\
\hline 1.23 & 0.0 \\
\hline 4.139 & 0.0 \\
\hline 7.41 & 0.0 \\
\hline $9 E-13$ & 0.0 \\
\hline 9.828 & 0.0 \\
\hline $6.87500 \mathrm{E}-14$ & 0.0 \\
\hline 2.4867 & 0.0 \\
\hline $1.09988 \mathrm{E}-09$ & 0.0 \\
\hline $4.07458 E-07$ & 0.0 \\
\hline $1 E-15$ & 0.0 \\
\hline$D E-11$ & 0.0 \\
\hline $9 E-06$ & 0.0 \\
\hline $2.10680 E-06$ & 0.0 \\
\hline$E-10$ & 0.0 \\
\hline $7.11076 \mathrm{E}-04$ & 0.0 \\
\hline 4.69 & 0.0 \\
\hline $1.12231 \mathrm{E}-10$ & 0.0 \\
\hline $3 E-09$ & 0.0 \\
\hline $1.03523 E-15$ & 0.0 \\
\hline $8.68388 E-19$ & 0.0 \\
\hline $6.70517 \mathrm{E}-10$ & 0.0 \\
\hline $7.51535 E-09$ & 0.0 \\
\hline $5.05302 \mathrm{E}-12$ & 0.0 \\
\hline $8.41161 E-24$ & 0.0 \\
\hline 0.0 & 0.0 \\
\hline $5.71672 \mathrm{E}-13$ & 0.0 \\
\hline 0.00132391 & 0.0 \\
\hline $2.40074 E-08$ & 0.0 \\
\hline $2.46848 E-20$ & 0.0 \\
\hline $46 E-16$ & 0.0 \\
\hline $1.31460 \mathrm{E}-13$ & 0.0 \\
\hline $54 E-08$ & 0.0 \\
\hline $86 \mathrm{E}-14$ & 0.0 \\
\hline $9 E-15$ & 0.0 \\
\hline $3 E-15$ & 0.0 \\
\hline $38 E-19$ & 0.0 \\
\hline $48 E-12$ & 0.0 \\
\hline $5 E-11$ & 0.0 \\
\hline $32 E-19$ & 0.0 \\
\hline $9 E-10$ & 0.0 \\
\hline $2 E-13$ & 0.0 \\
\hline $6 E-07$ & 0.0 \\
\hline $7 E-10$ & 0.0 \\
\hline $7 E-16$ & 0.0 \\
\hline $3 E-23$ & 0.0 \\
\hline $5 E-09$ & 0 \\
\hline $4 E-05$ & 0 . \\
\hline
\end{tabular}

0.0

0.0

0.0

0.0

0.0

0.0

0.0

0.0

0.0

0.0

0.0

0.0

0.0

0.0

0.0

0.0

0.0

0.0

0.0

0.0

0.0

0.0

0.0

0.0

0.0

0.0

0.0

0.0

0.0

0.0

0.0

0.0

0.0

0.0

0.0

0.0

0.0

0.0

0.0

0.0

0.0

0.0

0.0

0.0

0.0

0.0

0.0

0.0

0.0

0.0

0.0

0.0

0.0

0.0

0.0

0.0

0.0

0.0
0.0

0.0

0.0

0.0

0.0

0.0

0.0

0.0

0.0

0.0

0.0

0.0

0.0

0.0

0.0

0.0

0.0

0.0

0.0

0.0

0.0

0.0

0.0

0.0

0.0

0.0

0.0

0.0

0.0

0.0

0.0

0.0

0.0

0.0

0.0

0.0

0.0

0.0

0.0

0.0

0.0

0.0

0.0

0.0

0.0

0.0

0.0

0.0

0.0

0.0

0.0

0.0

0.0

0.0

0.0

0.0

0.0

0.0 


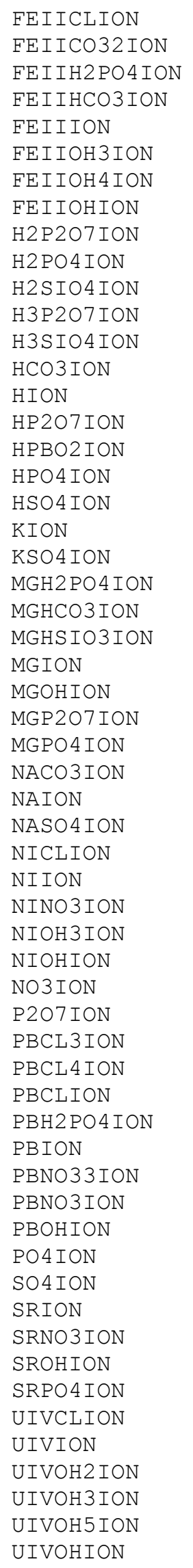

\begin{tabular}{|c|c|}
\hline $7.46945 E-11$ & 0.0 \\
\hline $7.53291 \mathrm{E}-14$ & 0.0 \\
\hline $1.70207 E-08$ & 0.0 \\
\hline $2.14931 E-09$ & 0.0 \\
\hline $5.54153 E-05$ & 0.0 \\
\hline $6.61095 E-18$ & 0.0 \\
\hline $9.68178 E-27$ & 0.0 \\
\hline $1.60186 \mathrm{E}-08$ & 0.0 \\
\hline $8.50915 E-11$ & 0.0 \\
\hline $1.26915 E-04$ & 0.0 \\
\hline $3.29439 E-15$ & 0.0 \\
\hline 1.315 & 0.0 \\
\hline $2.91873 E-08$ & 0.0 \\
\hline 3.567 & 0.0 \\
\hline 9.4117 & 0.0 \\
\hline 3.533 & 0.0 \\
\hline $8.90607 \mathrm{E}-18$ & 0.0 \\
\hline 1.133 & 0.0 \\
\hline $3.14706 \mathrm{E}-08$ & 0.0 \\
\hline 1.370 & 0.0 \\
\hline 3.6471 & 0.0 \\
\hline $1.06493 E-06$ & 0.0 \\
\hline 2.3958 & 0.0 \\
\hline $1.73845 E-10$ & 0.0 \\
\hline $2.37545 E-04$ & 0.0 \\
\hline $3.60987 \mathrm{E}-10$ & 0.0 \\
\hline 4.9563 & 0.0 \\
\hline $4.72168 E-09$ & 0.0 \\
\hline $5.85661 \mathrm{E}-11$ & 0.0 \\
\hline $9.31492 \mathrm{E}-04$ & 0.0 \\
\hline $2.73077 E-06$ & 0.0 \\
\hline $8.73738 \mathrm{E}-11$ & 0.0 \\
\hline $8.88146 E-07$ & 0.0 \\
\hline $6.44871 \mathrm{E}-10$ & 0.0 \\
\hline $1.11424 \mathrm{E}-18$ & 0.0 \\
\hline $1.13528 \mathrm{E}-10$ & 0.0 \\
\hline $3.38193 E-04$ & 0.0 \\
\hline $3.40669 \mathrm{E}-14$ & 0.0 \\
\hline $4.73877 E-15$ & 0.0 \\
\hline $4.56102 \mathrm{E}-18$ & 0.0 \\
\hline $1.76927 \mathrm{E}-09$ & 0.0 \\
\hline $2.55453 \mathrm{E}-10$ & 0.0 \\
\hline $6.54236 \mathrm{E}-08$ & 0.0 \\
\hline $5.34798 E-18$ & 0.0 \\
\hline $3.26442 \mathrm{E}-10$ & 0.0 \\
\hline $1.53261 E-09$ & 0.0 \\
\hline $40 E-12$ & 0.0 \\
\hline $4.74692 \mathrm{E}-04$ & 0.0 \\
\hline $1.07283 E-05$ & 0.0 \\
\hline $1.51741 \mathrm{E}-08$ & 0.0 \\
\hline $6.32636 \mathrm{E}-13$ & 0.0 \\
\hline $2.06402 \mathrm{E}-12$ & 0.0 \\
\hline 0.0 & 0.0 \\
\hline $2.52379 E-29$ & 0.0 \\
\hline $3.76496 \mathrm{E}-20$ & 0.0 \\
\hline 3.20 & 0 \\
\hline $1.94071 \mathrm{E}-14$ & 0.0 \\
\hline 7. $11645 E-24$ & 0 . \\
\hline
\end{tabular}

0.0

0.0

0.0

0.0

0.0

0.0

0.0

0.0

0.0

0.0

0.0

0.0

0.0

0.0

0.0

0.0

0.0

0.0

0.0

0.0

0.0

0.0

0.0

0.0

0.0

0.0

0.0

0.0

0.0

0.0

0.0

0.0

0.0

0.0

0.0

0.0

0.0

0.0

0.0

0.0

0.0

0.0

0.0

0.0

0.0

0.0

0.0

0.0

0.0

0.0

0.0

0.0

0.0

0.0

0.0

0.0

0.0

0.0
0.0

0.0

0.0

0.0

0.0

0.0

0.0

0.0

0.0

0.0

0.0

0.0

0.0

0.0

0.0

0.0

0.0

0.0

0.0

0.0

0.0

0.0

0.0

0.0

0.0

0.0

0.0

0.0

0.0

0.0

0.0

0.0

0.0

0.0

0.0

0.0

0.0

0.0

0.0

0.0

0.0

0.0

0.0

0.0

0.0

0.0

0.0

0.0

0.0

0.0

0.0

0.0

0.0

0.0

0.0

0.0

0.0

0.0 
WSRC-TR-2002-00567

Revision 0

\begin{tabular}{|c|c|c|c|c|}
\hline UIVSO4ION & $9.08925 E-27$ & 0.0 & 0.0 & 0.0 \\
\hline ZNCL3ION & $1.87119 \mathrm{E}-14$ & 0.0 & 0.0 & 0.0 \\
\hline ZNCLION & $3.25699 \mathrm{E}-08$ & 0.0 & 0.0 & 0.0 \\
\hline ZNH2 PO4 ION & $7.58481 E-08$ & 0.0 & 0.0 & 0.0 \\
\hline ZNHCO3ION & $1.20941 E-07$ & 0.0 & 0.0 & 0.0 \\
\hline ZNION & $2.08946 E-05$ & 0.0 & 0.0 & 0.0 \\
\hline ZNNO3ION & $1.51570 \mathrm{E}-08$ & 0.0 & 0.0 & 0.0 \\
\hline ZNOH3ION & $1.22600 \mathrm{E}-15$ & 0.0 & 0.0 & 0.0 \\
\hline ZNOH 4 ION & $2.25980 \mathrm{E}-22$ & 0.0 & 0.0 & 0.0 \\
\hline ZNOHION & $2.32919 E-08$ & 0.0 & 0.0 & 0.0 \\
\hline $\mathrm{CU} 3 \mathrm{PO} 42.2 \mathrm{H} 2 \mathrm{O}$ & 0.0 & $1.00082 \mathrm{E}-06$ & 0.0 & 0.0 \\
\hline $\mathrm{PB} 3 \mathrm{PO} 42$ & 0.0 & $6.85206 \mathrm{E}-07$ & 0.0 & 0.0 \\
\hline UIVO2 & $\begin{array}{l}0.0 \\
=============\end{array}$ & $\begin{array}{l}2.01650 \mathrm{E}-06 \\
============\end{array}$ & $\begin{array}{l}0.0 \\
=============\end{array}$ & $\begin{array}{l}0.0 \\
=============\end{array}$ \\
\hline Total g/hr & 999.994 & 0.00642859 & 0.0 & 0.0 \\
\hline Volume, L/hr & 1.00296 & $2.14018 E-06$ & 0.0 & 0.0 \\
\hline Enthalpy, cal/hr & $-3.79149 E+06$ & -20.2378 & 0.0 & 0.0 \\
\hline Density, g/L & 997.047 & 3003.76 & & \\
\hline Vapor fraction & 0.0 & 0.0 & 0.0 & 0.0 \\
\hline Solid fraction & 0.0 & 1 . & 0.0 & 0.0 \\
\hline Organic fraction & 0.0 & 0.0 & 0.0 & 0.0 \\
\hline Osmotic Pres, atm & 0.149686 & & & \\
\hline Redox Pot, volts & 0.0 & & & \\
\hline $\mathrm{E}-\mathrm{Con}, 1 / \mathrm{ohm}-\mathrm{cm}$ & $3.62656 \mathrm{E}-04$ & & & \\
\hline E-Con, cm2/ohm-mol & 129.216 & & & \\
\hline Abs Visc, cP & 0.891883 & & & \\
\hline Rel Visc & 1.00131 & & & \\
\hline Ionic Strength & 0.00467719 & & & \\
\hline
\end{tabular}


WSRC-TR-2002-00567

Revision 0

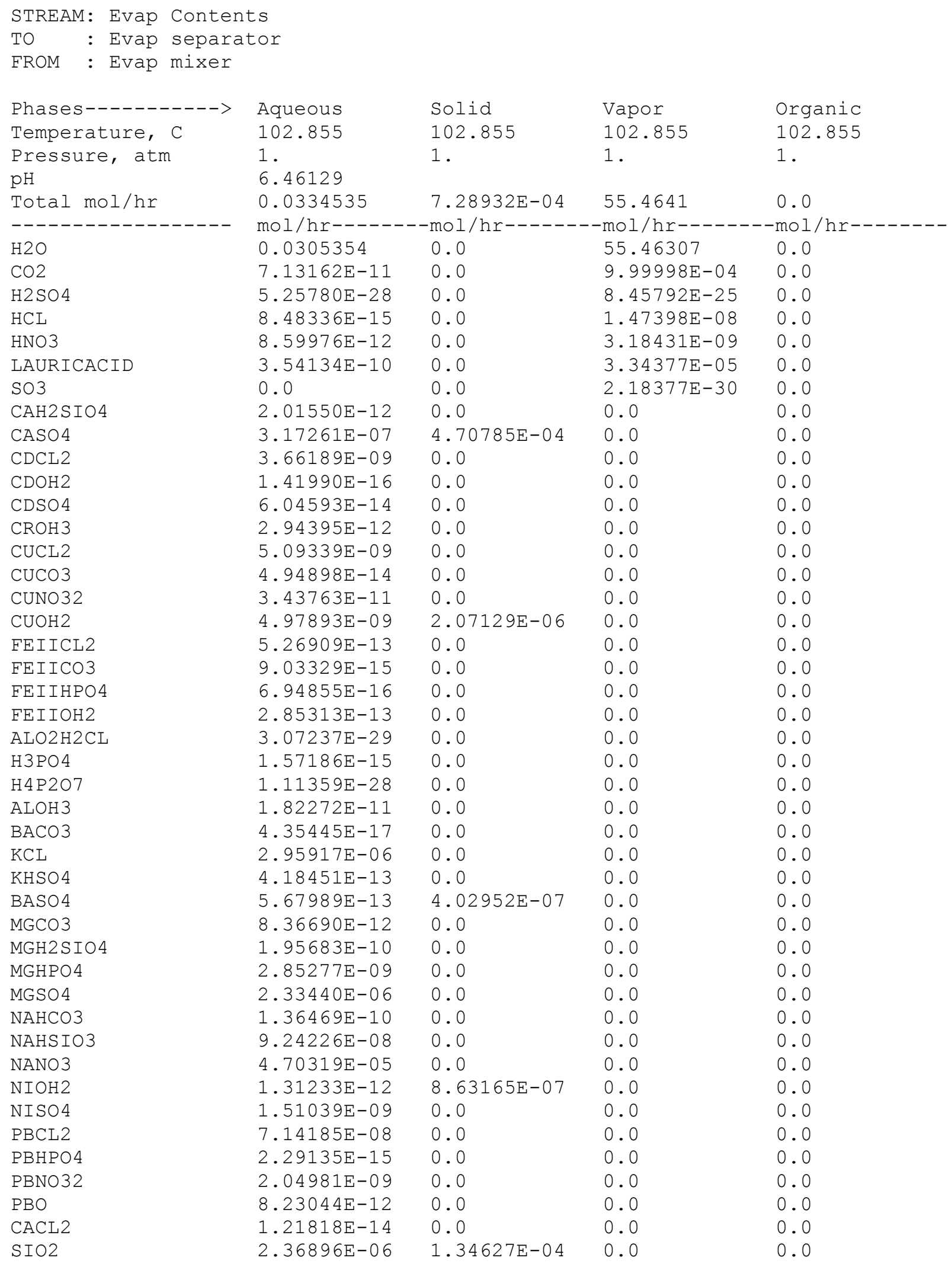




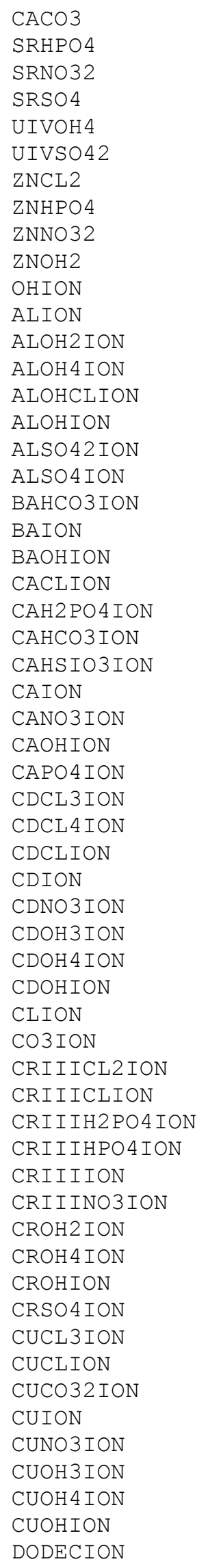

\begin{tabular}{|c|c|c|c|}
\hline $2.58890 \mathrm{E}-12$ & 0.0 & 0.0 & 0.0 \\
\hline $5.04192 \mathrm{E}-14$ & 0.0 & 0.0 & 0.0 \\
\hline $3.02682 \mathrm{E}-07$ & 0.0 & 0.0 & 0.0 \\
\hline $5.41479 \mathrm{E}-09$ & $9.31920 \mathrm{E}-06$ & 0.0 & 0.0 \\
\hline $6.88835 E-13$ & 0.0 & 0.0 & 0.0 \\
\hline 0.0 & 0.0 & 0.0 & 0.0 \\
\hline $4.38791 \mathrm{E}-06$ & 0.0 & 0.0 & 0.0 \\
\hline $4.44943 \mathrm{E}-13$ & 0.0 & 0.0 & 0.0 \\
\hline $1.83786 \mathrm{E}-09$ & 0.0 & 0.0 & 0.0 \\
\hline $6.12448 \mathrm{E}-10$ & 0.0 & 0.0 & 0.0 \\
\hline $1.61265 \mathrm{E}-09$ & 0.0 & 0.0 & 0.0 \\
\hline $4.92762 E-19$ & 0.0 & 0.0 & 0.0 \\
\hline $8.82986 \mathrm{E}-14$ & 0.0 & 0.0 & 0.0 \\
\hline $5.10748 \mathrm{E}-10$ & 0.0 & 0.0 & 0.0 \\
\hline $1.57007 \mathrm{E}-15$ & 0.0 & 0.0 & 0.0 \\
\hline $4.11036 \mathrm{E}-16$ & 0.0 & 0.0 & 0.0 \\
\hline $7.63745 \mathrm{E}-21$ & 0.0 & 0.0 & 0.0 \\
\hline $1.67556 \mathrm{E}-19$ & 0.0 & 0.0 & 0.0 \\
\hline $2.65005 \mathrm{E}-14$ & 0.0 & 0.0 & 0.0 \\
\hline $5.80637 \mathrm{E}-09$ & 0.0 & 0.0 & 0.0 \\
\hline $7.33356 \mathrm{E}-15$ & 0.0 & 0.0 & 0.0 \\
\hline $1.88046 \mathrm{E}-07$ & 0.0 & 0.0 & 0.0 \\
\hline $8.86110 \mathrm{E}-11$ & 0.0 & 0.0 & 0.0 \\
\hline $5.41842 \mathrm{E}-11$ & 0.0 & 0.0 & 0.0 \\
\hline $7.32133 \mathrm{E}-09$ & 0.0 & 0.0 & 0.0 \\
\hline $5.81133 \mathrm{E}-05$ & 0.0 & 0.0 & 0.0 \\
\hline $3.00473 E-05$ & 0.0 & 0.0 & 0.0 \\
\hline $1.48798 \mathrm{E}-09$ & 0.0 & 0.0 & 0.0 \\
\hline $6.07125 \mathrm{E}-12$ & 0.0 & 0.0 & 0.0 \\
\hline $3.87718 \mathrm{E}-09$ & 0.0 & 0.0 & 0.0 \\
\hline $1.20275 \mathrm{E}-09$ & 0.0 & 0.0 & 0.0 \\
\hline $1.82419 \mathrm{E}-10$ & 0.0 & 0.0 & 0.0 \\
\hline $3.30342 \mathrm{E}-12$ & 0.0 & 0.0 & 0.0 \\
\hline $9.64066 \mathrm{E}-13$ & 0.0 & 0.0 & 0.0 \\
\hline $3.51818 \mathrm{E}-21$ & 0.0 & 0.0 & 0.0 \\
\hline $5.01417 \mathrm{E}-26$ & 0.0 & 0.0 & 0.0 \\
\hline $1.09676 \mathrm{E}-14$ & 0.0 & 0.0 & 0.0 \\
\hline 0.00127313 & 0.0 & 0.0 & 0.0 \\
\hline $4.61301 \mathrm{E}-13$ & 0.0 & 0.0 & 0.0 \\
\hline $2.86337 \mathrm{E}-14$ & 0.0 & 0.0 & 0.0 \\
\hline $5.18398 \mathrm{E}-14$ & 0.0 & 0.0 & 0.0 \\
\hline $8.76281 \mathrm{E}-19$ & 0.0 & 0.0 & 0.0 \\
\hline $5.28457 \mathrm{E}-08$ & 0.0 & 0.0 & 0.0 \\
\hline $1.96192 \mathrm{E}-17$ & 0.0 & 0.0 & 0.0 \\
\hline $9.60792 \mathrm{E}-12$ & 0.0 & 0.0 & 0.0 \\
\hline $3.49841 \mathrm{E}-13$ & 0.0 & 0.0 & 0.0 \\
\hline $1.13772 \mathrm{E}-15$ & 0.0 & 0.0 & 0.0 \\
\hline $9.62733 \mathrm{E}-10$ & 0.0 & 0.0 & 0.0 \\
\hline $2.47639 \mathrm{E}-11$ & 0.0 & 0.0 & 0.0 \\
\hline $9.28575 \mathrm{E}-11$ & 0.0 & 0.0 & 0.0 \\
\hline $2.78203 \mathrm{E}-08$ & 0.0 & 0.0 & 0.0 \\
\hline $2.51794 \mathrm{E}-19$ & 0.0 & 0.0 & 0.0 \\
\hline $7.29312 \mathrm{E}-09$ & 0.0 & 0.0 & 0.0 \\
\hline $1.34793 \mathrm{E}-09$ & 0.0 & 0.0 & 0.0 \\
\hline $7.85487 \mathrm{E}-13$ & 0.0 & 0.0 & 0.0 \\
\hline $2.66153 \mathrm{E}-16$ & 0.0 & 0.0 & 0.0 \\
\hline $2.75749 \mathrm{E}-09$ & 0.0 & 0.0 & 0.0 \\
\hline $2.64156 \mathrm{E}-07$ & 0.0 & 0.0 & 0.0 \\
\hline
\end{tabular}




\begin{tabular}{|c|c|c|c|c|}
\hline FEIICLION & $9.65108 \mathrm{E}-11$ & 0.0 & 0.0 & 0.0 \\
\hline FEIICO32ION & $1.55296 \mathrm{E}-21$ & 0.0 & 0.0 & 0.0 \\
\hline FEIIH2PO4ION & $5.31671 \mathrm{E}-16$ & 0.0 & 0.0 & 0.0 \\
\hline FEIIHCO3ION & $1.76372 \mathrm{E}-16$ & 0.0 & 0.0 & 0.0 \\
\hline FEIIION & $1.04916 \mathrm{E}-08$ & 0.0 & 0.0 & 0.0 \\
\hline FEIIOH3ION & $6.60290 \mathrm{E}-16$ & 0.0 & 0.0 & 0.0 \\
\hline FEIIOH 4 ION & $3.65562 \mathrm{E}-21$ & 0.0 & 0.0 & 0.0 \\
\hline FEIIOHION & $1.48976 \mathrm{E}-10$ & 0.0 & 0.0 & 0.0 \\
\hline H2 P2O 7 ION & $9.80311 \mathrm{E}-19$ & 0.0 & 0.0 & 0.0 \\
\hline H2 PO 4 ION & $3.44354 \mathrm{E}-11$ & 0.0 & 0.0 & 0.0 \\
\hline H2SIO4ION & $8.56758 \mathrm{E}-14$ & 0.0 & 0.0 & 0.0 \\
\hline H3P2O 7 ION & $8.47621 \mathrm{E}-24$ & 0.0 & 0.0 & 0.0 \\
\hline H3SIO4 ION & $3.79517 \mathrm{E}-08$ & 0.0 & 0.0 & 0.0 \\
\hline HCO3ION & $1.27082 \mathrm{E}-10$ & 0.0 & 0.0 & 0.0 \\
\hline HION & $2.60573 \mathrm{E}-10$ & 0.0 & 0.0 & 0.0 \\
\hline HP207ION & $5.36617 \mathrm{E}-18$ & 0.0 & 0.0 & 0.0 \\
\hline HPBO2ION & $1.44667 \mathrm{E}-14$ & 0.0 & 0.0 & 0.0 \\
\hline HPO4 ION & $5.32651 \mathrm{E}-11$ & 0.0 & 0.0 & 0.0 \\
\hline HSO 4 ION & $1.05802 \mathrm{E}-10$ & 0.0 & 0.0 & 0.0 \\
\hline KION & $1.33467 \mathrm{E}-04$ & 0.0 & 0.0 & 0.0 \\
\hline KSO4 ION & $9.77163 \mathrm{E}-07$ & 0.0 & 0.0 & 0.0 \\
\hline MGH2 PO 4 ION & $1.94363 \mathrm{E}-10$ & 0.0 & 0.0 & 0.0 \\
\hline MGHCO3ION & $6.43762 \mathrm{E}-10$ & 0.0 & 0.0 & 0.0 \\
\hline MGHSIO3ION & $9.32615 \mathrm{E}-08$ & 0.0 & 0.0 & 0.0 \\
\hline MGION & $1.88194 \mathrm{E}-04$ & 0.0 & 0.0 & 0.0 \\
\hline MGOHION & $8.01132 \mathrm{E}-08$ & 0.0 & 0.0 & 0.0 \\
\hline MGP2O 7 ION & $2.90071 \mathrm{E}-14$ & 0.0 & 0.0 & 0.0 \\
\hline MGPO 4 ION & $3.71871 \mathrm{E}-11$ & 0.0 & 0.0 & 0.0 \\
\hline NACO3ION & $5.93776 \mathrm{E}-14$ & 0.0 & 0.0 & 0.0 \\
\hline NAION & $8.87237 \mathrm{E}-04$ & 0.0 & 0.0 & 0.0 \\
\hline NASO 4 ION & $2.30997 \mathrm{E}-14$ & 0.0 & 0.0 & 0.0 \\
\hline NICLION & $5.05030 \mathrm{E}-09$ & 0.0 & 0.0 & 0.0 \\
\hline NIION & $5.84272 \mathrm{E}-08$ & 0.0 & 0.0 & 0.0 \\
\hline NINO3ION & $8.59476 \mathrm{E}-09$ & 0.0 & 0.0 & 0.0 \\
\hline NIOH3ION & $5.10339 \mathrm{E}-16$ & 0.0 & 0.0 & 0.0 \\
\hline NIOHION & $2.19070 \mathrm{E}-10$ & 0.0 & 0.0 & 0.0 \\
\hline NO3ION & $2.59991 \mathrm{E}-04$ & 0.0 & 0.0 & 0.0 \\
\hline P207ION & $1.30450 \mathrm{E}-19$ & 0.0 & 0.0 & 0.0 \\
\hline PBCL3ION & $2.07902 \mathrm{E}-07$ & 0.0 & 0.0 & 0.0 \\
\hline PBCL 4 ION & $1.81979 \mathrm{E}-06$ & 0.0 & 0.0 & 0.0 \\
\hline PBCLION & $1.75255 \mathrm{E}-08$ & 0.0 & 0.0 & 0.0 \\
\hline PBH2 PO 4 ION & $8.55126 \mathrm{E}-16$ & 0.0 & 0.0 & 0.0 \\
\hline PBION & $4.86188 \mathrm{E}-10$ & 0.0 & 0.0 & 0.0 \\
\hline PBNO33ION & $8.74393 \mathrm{E}-11$ & 0.0 & 0.0 & 0.0 \\
\hline PBNO3ION & $4.97798 \mathrm{E}-09$ & 0.0 & 0.0 & 0.0 \\
\hline PBOHION & $1.35727 \mathrm{E}-09$ & 0.0 & 0.0 & 0.0 \\
\hline PO4ION & $1.95501 \mathrm{E}-15$ & 0.0 & 0.0 & 0.0 \\
\hline SO4ION & $5.44012 \mathrm{E}-06$ & 0.0 & 0.0 & 0.0 \\
\hline SRION & $7.21243 E-07$ & 0.0 & 0.0 & 0.0 \\
\hline SRNO3ION & $9.52826 \mathrm{E}-07$ & 0.0 & 0.0 & 0.0 \\
\hline SROHION & $1.09448 \mathrm{E}-11$ & 0.0 & 0.0 & 0.0 \\
\hline SRPO 4 ION & $1.48207 \mathrm{E}-15$ & 0.0 & 0.0 & 0.0 \\
\hline UIVOH2ION & $1.93213 \mathrm{E}-24$ & 0.0 & 0.0 & 0.0 \\
\hline UIVOH3ION & $7.56476 \mathrm{E}-20$ & 0.0 & 0.0 & 0.0 \\
\hline UIVOH5ION & $1.01271 \mathrm{E}-15$ & 0.0 & 0.0 & 0.0 \\
\hline UIVOHION & $2.89906 \mathrm{E}-28$ & 0.0 & 0.0 & 0.0 \\
\hline UIVSO 4 ION & 0.0 & 0.0 & 0.0 & 0.0 \\
\hline ZNCL3ION & $7.39732 \mathrm{E}-06$ & 0.0 & 0.0 & 0.0 \\
\hline
\end{tabular}


WSRC-TR-2002-00567

Revision 0

\begin{tabular}{|c|c|c|c|c|}
\hline ZNCLION & $8.55201 E-06$ & 0.0 & 0.0 & 0.0 \\
\hline ZNH2 PO4 ION & $8.08708 \mathrm{E}-14$ & 0.0 & 0.0 & 0.0 \\
\hline ZNHCO3ION & $2.18007 \mathrm{E}-13$ & 0.0 & 0.0 & 0.0 \\
\hline ZNION & $4.93531 E-07$ & 0.0 & 0.0 & 0.0 \\
\hline ZNNO3ION & $5.49456 \mathrm{E}-08$ & 0.0 & 0.0 & 0.0 \\
\hline ZNOH3ION & $4.34521 E-12$ & 0.0 & 0.0 & 0.0 \\
\hline ZNOH 4 ION & $5.20667 \mathrm{E}-16$ & 0.0 & 0.0 & 0.0 \\
\hline ZNOHION & $5.18556 \mathrm{E}-07$ & 0.0 & 0.0 & 0.0 \\
\hline ALOOH & 0.0 & $7.41762 \mathrm{E}-06$ & 0.0 & 0.0 \\
\hline $\mathrm{CA} 3 \mathrm{PO} 42$ & 0.0 & $5.45773 E-05$ & 0.0 & 0.0 \\
\hline CHAMOSITE7A & 0.0 & $2.77724 E-05$ & 0.0 & 0.0 \\
\hline $\mathrm{CU} 3 \mathrm{PO} 42.2 \mathrm{H} 2 \mathrm{O}$ & 0.0 & $3.42964 \mathrm{E}-07$ & 0.0 & 0.0 \\
\hline MG3PO42 & 0.0 & $1.87359 \mathrm{E}-05$ & 0.0 & 0.0 \\
\hline \multirow[t]{2}{*}{ UIVO2 } & 0.0 & $2.01681 E-06$ & 0.0 & 0.0 \\
\hline & $===========$ & $===========$ & $===========$ & $===========$ \\
\hline Total g/hr & 0.656214 & 0.106748 & 999.237 & 0.0 \\
\hline Volume, L/hr & $6.14120 \mathrm{E}-04$ & $3.13528 \mathrm{E}-05$ & 1697.27 & 0.0 \\
\hline Enthalpy, cal/hr & -2211.27 & -290.985 & $-3.17175 \mathrm{E}+06$ & 0.0 \\
\hline Density, g/L & 1068.54 & 3404.73 & 0.588731 & \\
\hline Vapor fraction & 0.0 & 0.0 & 1 . & 0.0 \\
\hline Solid fraction & 0.0 & 1 . & 0.0 & 0.0 \\
\hline Organic fraction & 0.0 & 0.0 & 0.0 & 0.0 \\
\hline Osmotic Pres, atm & 163.301 & & & \\
\hline Redox Pot, volts & 0.0 & & & \\
\hline $\mathrm{E}-\mathrm{Con}, 1 / \mathrm{ohm}-\mathrm{cm}$ & 0.432076 & & & \\
\hline E-Con, cm2/ohm-mol & 49.466 & & & \\
\hline Abs Visc, cP & 0.400031 & & & \\
\hline Rel Visc & 1.4634 & & & \\
\hline Ionic Strength & 3.29286 & & & \\
\hline
\end{tabular}


WSRC-TR-2002-00567

Revision 0

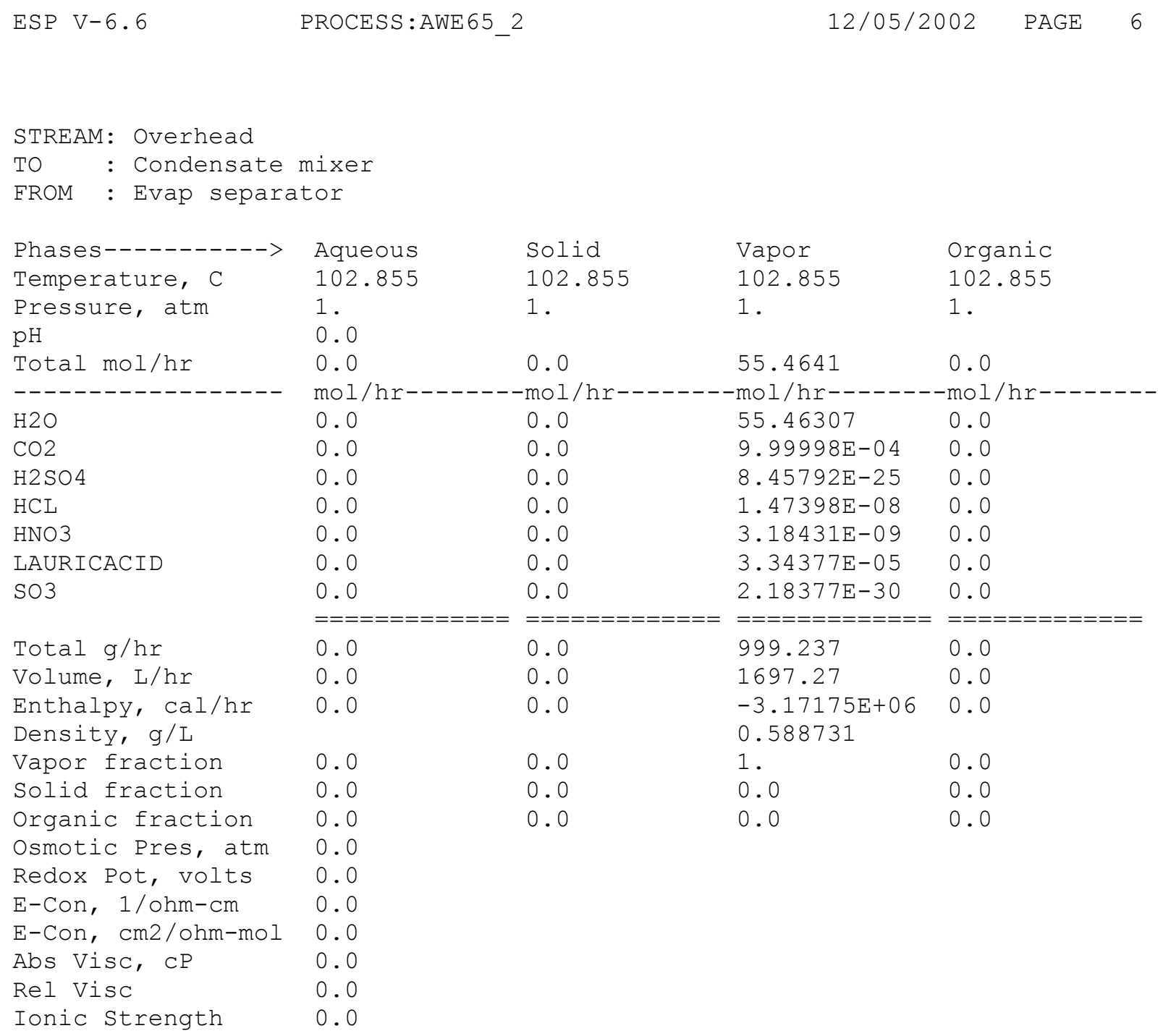


WSRC-TR-2002-00567

Revision 0

$\begin{array}{lllll}\text { ESP V-6.6 PROCESS:AWE65_2 } & \text { PAGE } 7\end{array}$

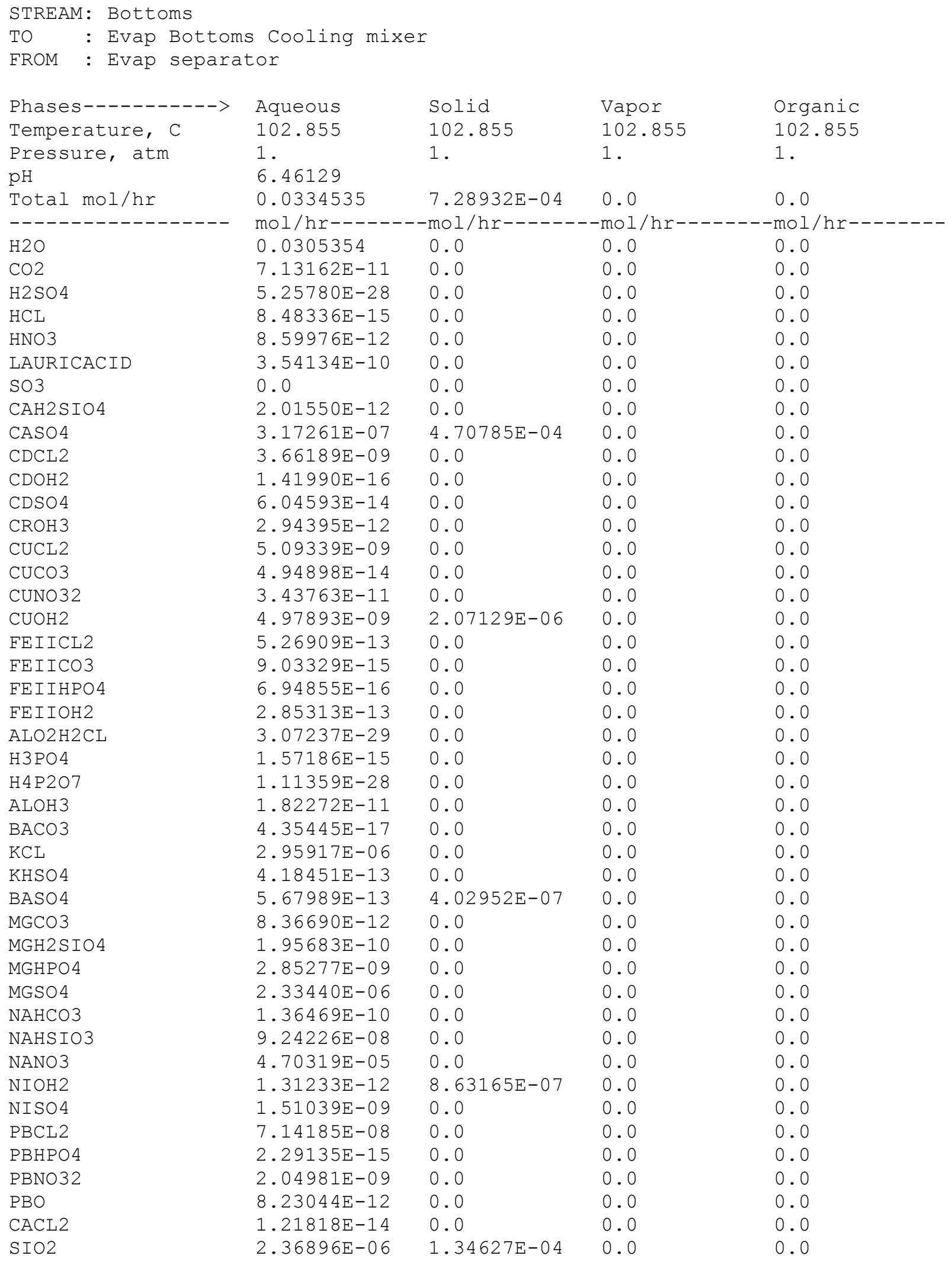




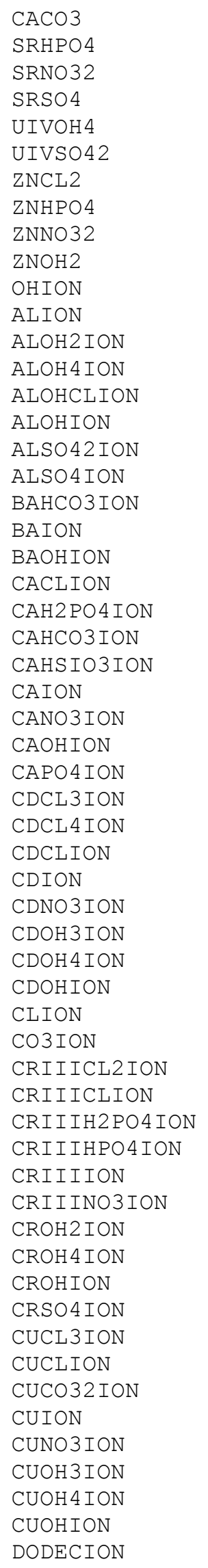

\begin{tabular}{|c|c|}
\hline $2.58890 \mathrm{E}-12$ & 0.0 \\
\hline $5.04192 \mathrm{E}-14$ & 0.0 \\
\hline $3.02682 \mathrm{E}-07$ & 0.0 \\
\hline $5.41479 E-09$ & $9.31920 \mathrm{E}-06$ \\
\hline $6.88835 \mathrm{E}-13$ & 0.0 \\
\hline 0.0 & 0.0 \\
\hline $4.38791 E-06$ & 0.0 \\
\hline $4.44943 E-13$ & 0.0 \\
\hline $1.83786 \mathrm{E}-09$ & 0.0 \\
\hline $6.12448 E-10$ & 0.0 \\
\hline $1.61265 E-09$ & 0.0 \\
\hline $4.92762 E-19$ & 0.0 \\
\hline $8.82986 \mathrm{E}-14$ & 0.0 \\
\hline $5.10748 \mathrm{E}-10$ & 0.0 \\
\hline $1.57007 \mathrm{E}-15$ & 0.0 \\
\hline $4.11036 \mathrm{E}-16$ & 0.0 \\
\hline 7. $63745 E-21$ & 0.0 \\
\hline $1.67556 \mathrm{E}-19$ & 0.0 \\
\hline $2.65005 E-14$ & 0.0 \\
\hline $5.80637 E-09$ & 0.0 \\
\hline $7.33356 \mathrm{E}-15$ & 0.0 \\
\hline $1.88046 \mathrm{E}-07$ & 0.0 \\
\hline $8.86110 E-11$ & 0.0 \\
\hline $5.41842 E-11$ & 0.0 \\
\hline 7. $32133 E-09$ & 0.0 \\
\hline $5.81133 E-05$ & 0.0 \\
\hline $3.00473 E-05$ & 0.0 \\
\hline $1.48798 E-09$ & 0.0 \\
\hline $6.07125 E-12$ & 0.0 \\
\hline $3.87718 E-09$ & 0.0 \\
\hline $1.20275 E-09$ & 0.0 \\
\hline $1.82419 \mathrm{E}-10$ & 0.0 \\
\hline $3.30342 E-12$ & 0.0 \\
\hline $9.64066 \mathrm{E}-13$ & 0.0 \\
\hline $3.51818 E-21$ & 0.0 \\
\hline $5.01417 E-26$ & 0.0 \\
\hline $1.09676 \mathrm{E}-14$ & 0.0 \\
\hline 0.00127313 & 0.0 \\
\hline 4. $61301 E-13$ & 0.0 \\
\hline $2.86337 E-14$ & 0.0 \\
\hline $5.18398 \mathrm{E}-14$ & 0.0 \\
\hline $8.76281 E-19$ & 0.0 \\
\hline $5.28457 \mathrm{E}-08$ & 0.0 \\
\hline $1.96192 \mathrm{E}-17$ & 0.0 \\
\hline $9.60792 \mathrm{E}-12$ & 0.0 \\
\hline $3.49841 E-13$ & 0.0 \\
\hline $1.13772 \mathrm{E}-15$ & 0.0 \\
\hline $9.62733 E-10$ & 0.0 \\
\hline $2.47639 \mathrm{E}-11$ & 0.0 \\
\hline $9.28575 E-11$ & 0.0 \\
\hline $2.78203 E-08$ & 0.0 \\
\hline $2.51794 \mathrm{E}-19$ & 0.0 \\
\hline $7.29312 \mathrm{E}-09$ & 0.0 \\
\hline $1.34793 E-09$ & 0.0 \\
\hline $7.85487 \mathrm{E}-13$ & 0.0 \\
\hline $2.66153 E-16$ & 0.0 \\
\hline $2.75749 E-09$ & 0.0 \\
\hline $2.64156 \mathrm{E}-07$ & 0.0 \\
\hline
\end{tabular}

0.0

0.0

0.0

0.0

0.0

0.0

0.0

0.0

0.0

0.0

0.0

0.0

0.0

0.0

0.0

0.0

0.0

0.0

0.0

0.0

0.0

0.0

0.0

0.0

0.0

0.0

0.0

0.0

0.0

0.0

0.0

0.0

0.0

0.0

0.0

0.0

0.0

0.0

0.0

0.0

0.0

0.0

0.0

0.0

0.0

0.0

0.0

0.0

0.0

0.0

0.0

0.0

0.0

0.0

0.0

0.0

0.0

0.0
0.0

0.0

0.0

0.0

0.0

0.0

0.0

0.0

0.0

0.0

0.0

0.0

0.0

0.0

0.0

0.0

0.0

0.0

0.0

0.0

0.0

0.0

0.0

0.0

0.0

0.0

0.0

0.0

0.0

0.0

0.0

0.0

0.0

0.0

0.0

0.0

0.0

0.0

0.0

0.0

0.0

0.0

0.0

0.0

0.0

0.0

0.0

0.0

0.0

0.0

0.0

0.0

0.0

0.0

0.0

0.0

0.0

0.0 


\begin{tabular}{|c|c|c|c|c|}
\hline FEIICLION & $9.65108 \mathrm{E}-11$ & 0.0 & 0.0 & 0.0 \\
\hline FEIICO32ION & $1.55296 \mathrm{E}-21$ & 0.0 & 0.0 & 0.0 \\
\hline FEIIH2PO4ION & $5.31671 \mathrm{E}-16$ & 0.0 & 0.0 & 0.0 \\
\hline FEIIHCO3ION & $1.76372 \mathrm{E}-16$ & 0.0 & 0.0 & 0.0 \\
\hline FEIIION & $1.04916 \mathrm{E}-08$ & 0.0 & 0.0 & 0.0 \\
\hline FEIIOH3ION & $6.60290 \mathrm{E}-16$ & 0.0 & 0.0 & 0.0 \\
\hline FEIIOH 4 ION & $3.65562 \mathrm{E}-21$ & 0.0 & 0.0 & 0.0 \\
\hline FEIIOHION & $1.48976 \mathrm{E}-10$ & 0.0 & 0.0 & 0.0 \\
\hline H2 P2O 7 ION & $9.80311 \mathrm{E}-19$ & 0.0 & 0.0 & 0.0 \\
\hline H2 PO 4 ION & $3.44354 \mathrm{E}-11$ & 0.0 & 0.0 & 0.0 \\
\hline H2SIO4ION & $8.56758 \mathrm{E}-14$ & 0.0 & 0.0 & 0.0 \\
\hline H3P2O 7 ION & $8.47621 \mathrm{E}-24$ & 0.0 & 0.0 & 0.0 \\
\hline H3SIO4 ION & $3.79517 \mathrm{E}-08$ & 0.0 & 0.0 & 0.0 \\
\hline HCO3ION & $1.27082 \mathrm{E}-10$ & 0.0 & 0.0 & 0.0 \\
\hline HION & $2.60573 \mathrm{E}-10$ & 0.0 & 0.0 & 0.0 \\
\hline HP207ION & $5.36617 \mathrm{E}-18$ & 0.0 & 0.0 & 0.0 \\
\hline HPBO2ION & $1.44667 \mathrm{E}-14$ & 0.0 & 0.0 & 0.0 \\
\hline HPO4 ION & $5.32651 \mathrm{E}-11$ & 0.0 & 0.0 & 0.0 \\
\hline HSO 4 ION & $1.05802 \mathrm{E}-10$ & 0.0 & 0.0 & 0.0 \\
\hline KION & $1.33467 \mathrm{E}-04$ & 0.0 & 0.0 & 0.0 \\
\hline KSO4 ION & $9.77163 \mathrm{E}-07$ & 0.0 & 0.0 & 0.0 \\
\hline MGH2 PO 4 ION & $1.94363 \mathrm{E}-10$ & 0.0 & 0.0 & 0.0 \\
\hline MGHCO3ION & $6.43762 \mathrm{E}-10$ & 0.0 & 0.0 & 0.0 \\
\hline MGHSIO3ION & $9.32615 \mathrm{E}-08$ & 0.0 & 0.0 & 0.0 \\
\hline MGION & $1.88194 \mathrm{E}-04$ & 0.0 & 0.0 & 0.0 \\
\hline MGOHION & $8.01132 \mathrm{E}-08$ & 0.0 & 0.0 & 0.0 \\
\hline MGP2O 7 ION & $2.90071 \mathrm{E}-14$ & 0.0 & 0.0 & 0.0 \\
\hline MGPO 4 ION & $3.71871 \mathrm{E}-11$ & 0.0 & 0.0 & 0.0 \\
\hline NACO3ION & $5.93776 \mathrm{E}-14$ & 0.0 & 0.0 & 0.0 \\
\hline NAION & $8.87237 \mathrm{E}-04$ & 0.0 & 0.0 & 0.0 \\
\hline NASO 4 ION & $2.30997 \mathrm{E}-14$ & 0.0 & 0.0 & 0.0 \\
\hline NICLION & $5.05030 \mathrm{E}-09$ & 0.0 & 0.0 & 0.0 \\
\hline NIION & $5.84272 \mathrm{E}-08$ & 0.0 & 0.0 & 0.0 \\
\hline NINO3ION & $8.59476 \mathrm{E}-09$ & 0.0 & 0.0 & 0.0 \\
\hline NIOH3ION & $5.10339 \mathrm{E}-16$ & 0.0 & 0.0 & 0.0 \\
\hline NIOHION & $2.19070 \mathrm{E}-10$ & 0.0 & 0.0 & 0.0 \\
\hline NO3ION & $2.59991 \mathrm{E}-04$ & 0.0 & 0.0 & 0.0 \\
\hline P207ION & $1.30450 \mathrm{E}-19$ & 0.0 & 0.0 & 0.0 \\
\hline PBCL3ION & $2.07902 \mathrm{E}-07$ & 0.0 & 0.0 & 0.0 \\
\hline PBCL 4 ION & $1.81979 \mathrm{E}-06$ & 0.0 & 0.0 & 0.0 \\
\hline PBCLION & $1.75255 \mathrm{E}-08$ & 0.0 & 0.0 & 0.0 \\
\hline PBH2 PO 4 ION & $8.55126 \mathrm{E}-16$ & 0.0 & 0.0 & 0.0 \\
\hline PBION & $4.86188 \mathrm{E}-10$ & 0.0 & 0.0 & 0.0 \\
\hline PBNO33ION & $8.74393 \mathrm{E}-11$ & 0.0 & 0.0 & 0.0 \\
\hline PBNO3ION & $4.97798 \mathrm{E}-09$ & 0.0 & 0.0 & 0.0 \\
\hline PBOHION & $1.35727 \mathrm{E}-09$ & 0.0 & 0.0 & 0.0 \\
\hline PO4ION & $1.95501 \mathrm{E}-15$ & 0.0 & 0.0 & 0.0 \\
\hline SO4ION & $5.44012 \mathrm{E}-06$ & 0.0 & 0.0 & 0.0 \\
\hline SRION & $7.21243 E-07$ & 0.0 & 0.0 & 0.0 \\
\hline SRNO3ION & $9.52826 \mathrm{E}-07$ & 0.0 & 0.0 & 0.0 \\
\hline SROHION & $1.09448 \mathrm{E}-11$ & 0.0 & 0.0 & 0.0 \\
\hline SRPO 4 ION & $1.48207 \mathrm{E}-15$ & 0.0 & 0.0 & 0.0 \\
\hline UIVOH2ION & $1.93213 \mathrm{E}-24$ & 0.0 & 0.0 & 0.0 \\
\hline UIVOH3ION & $7.56476 \mathrm{E}-20$ & 0.0 & 0.0 & 0.0 \\
\hline UIVOH5ION & $1.01271 \mathrm{E}-15$ & 0.0 & 0.0 & 0.0 \\
\hline UIVOHION & $2.89906 \mathrm{E}-28$ & 0.0 & 0.0 & 0.0 \\
\hline UIVSO 4 ION & 0.0 & 0.0 & 0.0 & 0.0 \\
\hline ZNCL3ION & $7.39732 \mathrm{E}-06$ & 0.0 & 0.0 & 0.0 \\
\hline
\end{tabular}


WSRC-TR-2002-00567

Revision 0

\begin{tabular}{|c|c|c|c|c|}
\hline ZNCLION & $8.55201 E-06$ & 0.0 & 0.0 & 0.0 \\
\hline ZNH2 PO4 ION & $8.08708 \mathrm{E}-14$ & 0.0 & 0.0 & 0.0 \\
\hline ZNHCO3ION & $2.18007 \mathrm{E}-13$ & 0.0 & 0.0 & 0.0 \\
\hline ZNION & $4.93531 E-07$ & 0.0 & 0.0 & 0.0 \\
\hline ZNNO3ION & $5.49456 \mathrm{E}-08$ & 0.0 & 0.0 & 0.0 \\
\hline ZNOH3ION & $4.34521 E-12$ & 0.0 & 0.0 & 0.0 \\
\hline ZNOH 4 ION & $5.20667 \mathrm{E}-16$ & 0.0 & 0.0 & 0.0 \\
\hline ZNOHION & $5.18556 \mathrm{E}-07$ & 0.0 & 0.0 & 0.0 \\
\hline ALOOH & 0.0 & $7.41762 \mathrm{E}-06$ & 0.0 & 0.0 \\
\hline $\mathrm{CA} 3 \mathrm{PO} 42$ & 0.0 & $5.45773 E-05$ & 0.0 & 0.0 \\
\hline CHAMOSITE7A & 0.0 & $2.77724 E-05$ & 0.0 & 0.0 \\
\hline $\mathrm{CU} 3 \mathrm{PO} 42.2 \mathrm{H} 2 \mathrm{O}$ & 0.0 & $3.42964 \mathrm{E}-07$ & 0.0 & 0.0 \\
\hline MG3PO42 & 0.0 & $1.87359 \mathrm{E}-05$ & 0.0 & 0.0 \\
\hline UIVO2 & 0.0 & $2.01681 \mathrm{E}-06$ & 0.0 & 0.0 \\
\hline & $===========$ & $===========$ & $===========$ & $===========$ \\
\hline Total g/hr & 0.656214 & 0.106748 & 0.0 & 0.0 \\
\hline Volume, L/hr & $6.14120 \mathrm{E}-04$ & $3.13528 \mathrm{E}-05$ & 0.0 & 0.0 \\
\hline Enthalpy, cal/hr & -2211.27 & -290.985 & 0.0 & 0.0 \\
\hline Density, g/L & 1068.54 & 3404.73 & & \\
\hline Vapor fraction & 0.0 & 0.0 & 0.0 & 0.0 \\
\hline Solid fraction & 0.0 & 1. & 0.0 & 0.0 \\
\hline Organic fraction & 0.0 & 0.0 & 0.0 & 0.0 \\
\hline Osmotic Pres, atm & 163.301 & & & \\
\hline Redox Pot, volts & 0.0 & & & \\
\hline $\mathrm{E}-\mathrm{Con}, 1 / \mathrm{ohm}-\mathrm{cm}$ & 0.432076 & & & \\
\hline E-Con, cm2/ohm-mol & 49.466 & & & \\
\hline Abs Visc, cP & 0.400031 & & & \\
\hline Rel Visc & 1.4634 & & & \\
\hline Ionic Strength & 3.29286 & & & \\
\hline
\end{tabular}


WSRC-TR-2002-00567

Revision 0

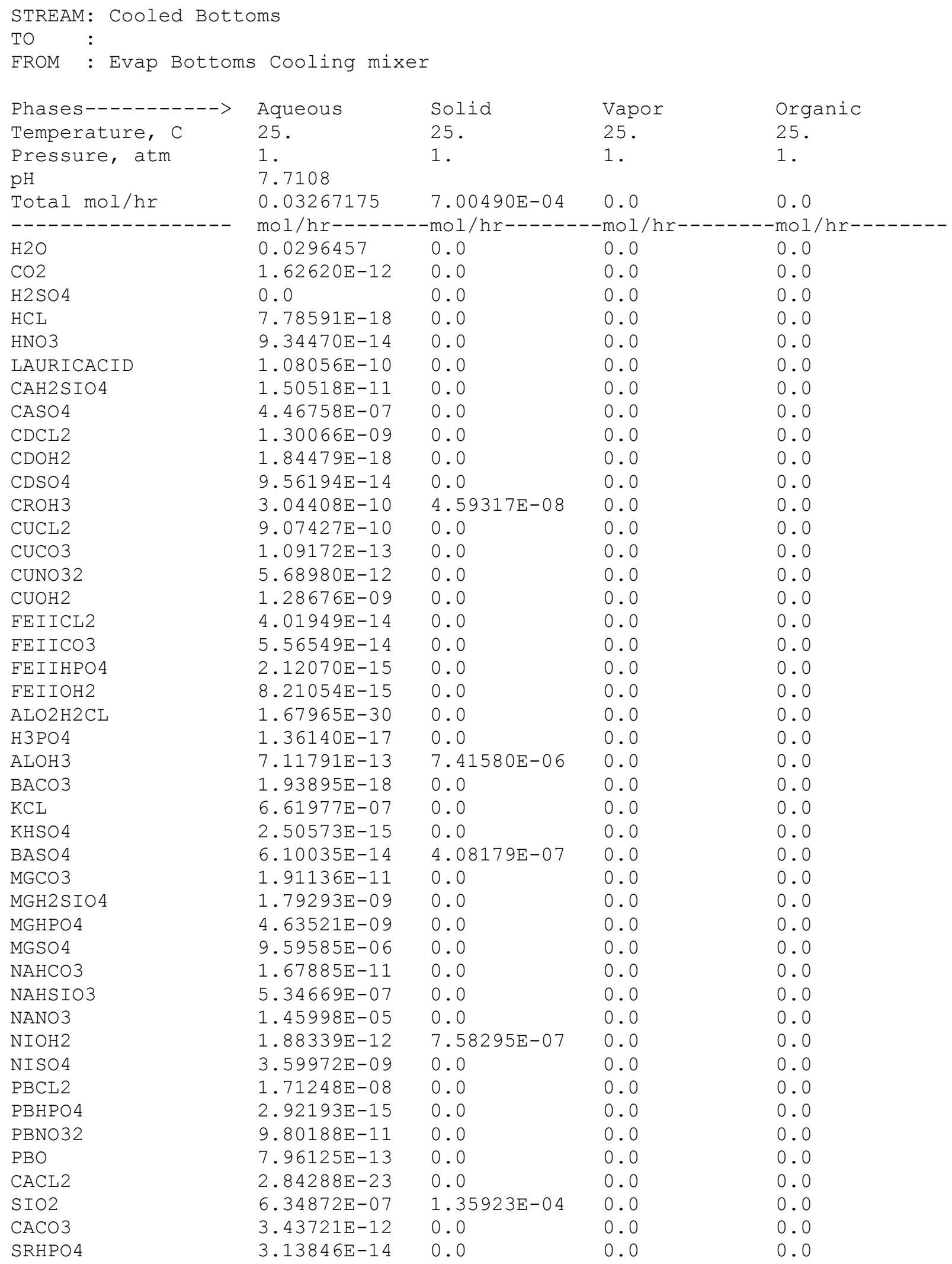




\begin{tabular}{|c|c|c|c|c|}
\hline SRNO32 & $4.08544 \mathrm{E}-08$ & 0.0 & 0.0 & 0.0 \\
\hline SRSO 4 & $1.27819 \mathrm{E}-08$ & $1.03945 E-05$ & 0.0 & 0.0 \\
\hline UIVOH 4 & $7.20293 \mathrm{E}-14$ & 0.0 & 0.0 & 0.0 \\
\hline ZNCL2 & $2.10224 \mathrm{E}-06$ & 0.0 & 0.0 & 0.0 \\
\hline ZNHPO 4 & $2.29442 E-11$ & 0.0 & 0.0 & 0.0 \\
\hline ZNNO32 & $2.31923 E-08$ & 0.0 & 0.0 & 0.0 \\
\hline ZNOH2 & $2.41090 \mathrm{E}-09$ & $2.13114 E-06$ & 0.0 & 0.0 \\
\hline OHION & $3.40030 \mathrm{E}-10$ & 0.0 & 0.0 & 0.0 \\
\hline ALION & $3.71670 \mathrm{E}-19$ & 0.0 & 0.0 & 0.0 \\
\hline ALOH2 ION & $7.14143 \mathrm{E}-15$ & 0.0 & 0.0 & 0.0 \\
\hline ALOH 4 ION & $2.77945 \mathrm{E}-11$ & 0.0 & 0.0 & 0.0 \\
\hline ALOHCLION & $3.90188 \mathrm{E}-16$ & 0.0 & 0.0 & 0.0 \\
\hline ALOHION & $5.92413 \mathrm{E}-17$ & 0.0 & 0.0 & 0.0 \\
\hline ALSO 42 ION & $3.55840 \mathrm{E}-20$ & 0.0 & 0.0 & 0.0 \\
\hline ALSO 4 ION & $2.33383 \mathrm{E}-19$ & 0.0 & 0.0 & 0.0 \\
\hline BAHCO3ION & $7.41397 \mathrm{E}-17$ & 0.0 & 0.0 & 0.0 \\
\hline BAION & $5.80000 \mathrm{E}-10$ & 0.0 & 0.0 & 0.0 \\
\hline BAOHION & $1.07484 \mathrm{E}-17$ & 0.0 & 0.0 & 0.0 \\
\hline CACLION & $2.29960 \mathrm{E}-10$ & 0.0 & 0.0 & 0.0 \\
\hline CAH2PO 4 ION & $1.41198 \mathrm{E}-11$ & 0.0 & 0.0 & 0.0 \\
\hline CAHCO3ION & $2.36882 \mathrm{E}-11$ & 0.0 & 0.0 & 0.0 \\
\hline CAHSIO3ION & $5.24702 \mathrm{E}-09$ & 0.0 & 0.0 & 0.0 \\
\hline CAION & $4.57212 \mathrm{E}-05$ & 0.0 & 0.0 & 0.0 \\
\hline CANO3ION & $2.17242 \mathrm{E}-05$ & 0.0 & 0.0 & 0.0 \\
\hline CAOHION & $1.17835 \mathrm{E}-10$ & 0.0 & 0.0 & 0.0 \\
\hline CAPO 4 ION & $1.07328 \mathrm{E}-10$ & 0.0 & 0.0 & 0.0 \\
\hline CDCL3ION & $5.53697 \mathrm{E}-10$ & 0.0 & 0.0 & 0.0 \\
\hline CDCL 4 ION & $6.99740 \mathrm{E}-09$ & 0.0 & 0.0 & 0.0 \\
\hline CDCLION & 7. $34225 \mathrm{E}-11$ & 0.0 & 0.0 & 0.0 \\
\hline CDION & $2.81217 \mathrm{E}-12$ & 0.0 & 0.0 & 0.0 \\
\hline CDNO3ION & $4.88319 E-13$ & 0.0 & 0.0 & 0.0 \\
\hline CDOH 3 ION & $4.62896 \mathrm{E}-23$ & 0.0 & 0.0 & 0.0 \\
\hline CDOH 4 ION & $1.04833 \mathrm{E}-28$ & 0.0 & 0.0 & 0.0 \\
\hline CDOHION & $1.25477 \mathrm{E}-15$ & 0.0 & 0.0 & 0.0 \\
\hline CLION & 0.00129759 & 0.0 & 0.0 & 0.0 \\
\hline CO3ION & $2.74574 \mathrm{E}-12$ & 0.0 & 0.0 & 0.0 \\
\hline CRIIICL2ION & $4.86591 \mathrm{E}-14$ & 0.0 & 0.0 & 0.0 \\
\hline CRIIICLION & $7.03122 \mathrm{E}-14$ & 0.0 & 0.0 & 0.0 \\
\hline CRIIIH2PO4ION & $8.72899 \mathrm{E}-17$ & 0.0 & 0.0 & 0.0 \\
\hline CRIIIHPO4ION & $1.97410 \mathrm{E}-10$ & 0.0 & 0.0 & 0.0 \\
\hline CRIIIION & $1.11994 \mathrm{E}-16$ & 0.0 & 0.0 & 0.0 \\
\hline CRIIINO3ION & $8.11412 \mathrm{E}-12$ & 0.0 & 0.0 & 0.0 \\
\hline CROH2ION & $4.91687 \mathrm{E}-12$ & 0.0 & 0.0 & 0.0 \\
\hline CROH 4 ION & $6.31542 \mathrm{E}-13$ & 0.0 & 0.0 & 0.0 \\
\hline CROHION & $7.31035 E-09$ & 0.0 & 0.0 & 0.0 \\
\hline CRSO 4 ION & $8.85146 \mathrm{E}-11$ & 0.0 & 0.0 & 0.0 \\
\hline CUCL3ION & $1.67398 \mathrm{E}-11$ & 0.0 & 0.0 & 0.0 \\
\hline CUCLION & $2.39615 E-09$ & 0.0 & 0.0 & 0.0 \\
\hline CUCO32ION & $1.79616 \mathrm{E}-18$ & 0.0 & 0.0 & 0.0 \\
\hline CUION & $2.00082 \mathrm{E}-09$ & 0.0 & 0.0 & 0.0 \\
\hline CUNO3ION & $4.01975 E-10$ & 0.0 & 0.0 & 0.0 \\
\hline CUOH3ION & $1.20377 \mathrm{E}-13$ & 0.0 & 0.0 & 0.0 \\
\hline CUOH 4 ION & $4.34609 \mathrm{E}-18$ & 0.0 & 0.0 & 0.0 \\
\hline CUOHION & $3.87661 \mathrm{E}-10$ & 0.0 & 0.0 & 0.0 \\
\hline DODECION & $2.64402 \mathrm{E}-07$ & 0.0 & 0.0 & 0.0 \\
\hline FEIICLION & $1.31229 \mathrm{E}-11$ & 0.0 & 0.0 & 0.0 \\
\hline FEIICO32ION & $1.98677 \mathrm{E}-20$ & 0.0 & 0.0 & 0.0 \\
\hline FEIIH2PO4ION & $3.26501 \mathrm{E}-16$ & 0.0 & 0.0 & 0.0 \\
\hline
\end{tabular}




\begin{tabular}{|c|c|c|c|c|}
\hline FEIIHCO3ION & $7.05833 \mathrm{E}-17$ & 0.0 & 0.0 & 0.0 \\
\hline EEIIION & $8.35608 \mathrm{E}-09$ & 0.0 & 0.0 & 0.0 \\
\hline FEIIOH3ION & $3.98249 \mathrm{E}-17$ & 0.0 & 0.0 & 0.0 \\
\hline FEIIOH 4 ION & $2.80141 \mathrm{E}-23$ & 0.0 & 0.0 & 0.0 \\
\hline FEIIOHION & $5.64064 \mathrm{E}-11$ & 0.0 & 0.0 & 0.0 \\
\hline H2 P2O 7 ION & $1.26416 \mathrm{E}-20$ & 0.0 & 0.0 & 0.0 \\
\hline H2 PO 4 ION & $1.59551 \mathrm{E}-11$ & 0.0 & 0.0 & 0.0 \\
\hline H2SIO4ION & $4.08128 \mathrm{E}-13$ & 0.0 & 0.0 & 0.0 \\
\hline H3P2O 7 ION & $3.59013 \mathrm{E}-27$ & 0.0 & 0.0 & 0.0 \\
\hline H3SIO4 ION & $6.96191 \mathrm{E}-09$ & 0.0 & 0.0 & 0.0 \\
\hline HCO3ION & $1.12332 \mathrm{E}-10$ & 0.0 & 0.0 & 0.0 \\
\hline HION & $9.27337 \mathrm{E}-12$ & 0.0 & 0.0 & 0.0 \\
\hline HP2O7ION & $2.13266 \mathrm{E}-18$ & 0.0 & 0.0 & 0.0 \\
\hline HPBO2ION & $1.23850 \mathrm{E}-15$ & 0.0 & 0.0 & 0.0 \\
\hline HPO 4 ION & $5.92132 \mathrm{E}-10$ & 0.0 & 0.0 & 0.0 \\
\hline HSO 4 ION & $2.18474 \mathrm{E}-12$ & 0.0 & 0.0 & 0.0 \\
\hline KION & $1.34739 \mathrm{E}-04$ & 0.0 & 0.0 & 0.0 \\
\hline KSO4 ION & $2.00278 \mathrm{E}-06$ & 0.0 & 0.0 & 0.0 \\
\hline MGH2 PO 4 ION & $2.23615 \mathrm{E}-10$ & 0.0 & 0.0 & 0.0 \\
\hline MGHCO3ION & $8.61421 \mathrm{E}-10$ & 0.0 & 0.0 & 0.0 \\
\hline MGHSIO3ION & $1.19470 \mathrm{E}-07$ & 0.0 & 0.0 & 0.0 \\
\hline MGION & $2.37175 \mathrm{E}-04$ & 0.0 & 0.0 & 0.0 \\
\hline MGOHION & $1.39145 \mathrm{E}-08$ & 0.0 & 0.0 & 0.0 \\
\hline MGP2O7ION & $2.70856 \mathrm{E}-14$ & 0.0 & 0.0 & 0.0 \\
\hline MGPO 4 ION & $1.45113 E-09$ & 0.0 & 0.0 & 0.0 \\
\hline NACO3ION & $1.04152 \mathrm{E}-12$ & 0.0 & 0.0 & 0.0 \\
\hline NAION & $9.12441 \mathrm{E}-04$ & 0.0 & 0.0 & 0.0 \\
\hline NASO 4 ION & $6.78597 \mathrm{E}-06$ & 0.0 & 0.0 & 0.0 \\
\hline NICLION & $5.41109 \mathrm{E}-09$ & 0.0 & 0.0 & 0.0 \\
\hline NIION & $1.34273 \mathrm{E}-07$ & 0.0 & 0.0 & 0.0 \\
\hline NINO3ION & $3.52460 \mathrm{E}-08$ & 0.0 & 0.0 & 0.0 \\
\hline NIOH3ION & $3.46727 \mathrm{E}-15$ & 0.0 & 0.0 & 0.0 \\
\hline NIOHION & $1.40910 \mathrm{E}-10$ & 0.0 & 0.0 & 0.0 \\
\hline NO3ION & $3.01013 E-04$ & 0.0 & 0.0 & 0.0 \\
\hline P2O7ION & $5.20041 \mathrm{E}-18$ & 0.0 & 0.0 & 0.0 \\
\hline PBCL3ION & $5.74322 \mathrm{E}-08$ & 0.0 & 0.0 & 0.0 \\
\hline PBCL 4 ION & $1.06286 \mathrm{E}-06$ & 0.0 & 0.0 & 0.0 \\
\hline PBCLION & $5.67102 \mathrm{E}-09$ & 0.0 & 0.0 & 0.0 \\
\hline PBH2 PO 4 ION & $8.96518 \mathrm{E}-17$ & 0.0 & 0.0 & 0.0 \\
\hline PBION & $2.89020 \mathrm{E}-10$ & 0.0 & 0.0 & 0.0 \\
\hline PBNO33ION & $2.55965 \mathrm{E}-11$ & 0.0 & 0.0 & 0.0 \\
\hline PBNO3ION & $9.26020 \mathrm{E}-10$ & 0.0 & 0.0 & 0.0 \\
\hline PBOHION & $9.86104 \mathrm{E}-11$ & 0.0 & 0.0 & 0.0 \\
\hline PO4ION & $2.73981 \mathrm{E}-13$ & 0.0 & 0.0 & 0.0 \\
\hline SO4ION & $1.99910 \mathrm{E}-05$ & 0.0 & 0.0 & 0.0 \\
\hline SRION & $5.77806 \mathrm{E}-07$ & 0.0 & 0.0 & 0.0 \\
\hline SRNO3ION & $2.75442 \mathrm{E}-07$ & 0.0 & 0.0 & 0.0 \\
\hline SROHION & $3.70930 \mathrm{E}-13$ & 0.0 & 0.0 & 0.0 \\
\hline SRPO 4 ION & $8.61708 \mathrm{E}-15$ & 0.0 & 0.0 & 0.0 \\
\hline UIVOH2ION & $2.75416 \mathrm{E}-26$ & 0.0 & 0.0 & 0.0 \\
\hline UIVOH3ION & $4.82499 \mathrm{E}-20$ & 0.0 & 0.0 & 0.0 \\
\hline UIVOH5ION & $6.75488 \mathrm{E}-16$ & 0.0 & 0.0 & 0.0 \\
\hline UIVOHION & 0.0 & 0.0 & 0.0 & 0.0 \\
\hline ZNCL3ION & $4.90239 \mathrm{E}-06$ & 0.0 & 0.0 & 0.0 \\
\hline ZNCLION & $2.26159 \mathrm{E}-06$ & 0.0 & 0.0 & 0.0 \\
\hline ZNH2 PO 4 ION & $5.75068 \mathrm{E}-13$ & 0.0 & 0.0 & 0.0 \\
\hline ZNHCO3ION & $2.23404 \mathrm{E}-12$ & 0.0 & 0.0 & 0.0 \\
\hline ZNION & $7.44259 \mathrm{E}-06$ & 0.0 & 0.0 & 0.0 \\
\hline
\end{tabular}


WSRC-TR-2002-00567

Revision 0

\begin{tabular}{|c|c|c|c|c|}
\hline ZNNO3ION & $9.28867 \mathrm{E}-07$ & 0.0 & 0.0 & 0.0 \\
\hline ZNOH3ION & $3.18508 \mathrm{E}-12$ & 0.0 & 0.0 & 0.0 \\
\hline ZNOH 4 ION & $2.54295 E-16$ & 0.0 & 0.0 & 0.0 \\
\hline ZNOHION & $4.61300 \mathrm{E}-08$ & 0.0 & 0.0 & 0.0 \\
\hline $\mathrm{CA} 3 \mathrm{PO} 42$ & 0.0 & $7.17841 \mathrm{E}-05$ & 0.0 & 0.0 \\
\hline $\mathrm{CASO} 4.2 \mathrm{H} 2 \mathrm{O}$ & 0.0 & $4.39942 \mathrm{E}-04$ & 0.0 & 0.0 \\
\hline CHAMOSITE7A & 0.0 & $2.77736 \mathrm{E}-05$ & 0.0 & 0.0 \\
\hline $\mathrm{CU} 3 \mathrm{PO} 42.2 \mathrm{H} 2 \mathrm{O}$ & 0.0 & $1.04740 \mathrm{E}-06$ & 0.0 & 0.0 \\
\hline РB3PO42 & 0.0 & $3.27028 E-07$ & 0.0 & 0.0 \\
\hline UIVO2 & 0.0 & $2.01681 E-06$ & 0.0 & 0.0 \\
\hline \multirow[t]{2}{*}{$\mathrm{ZN} 3 \mathrm{PO} 42.2 \mathrm{H} 2 \mathrm{O}$} & 0.0 & $5.22053 \mathrm{E}-07$ & 0.0 & 0.0 \\
\hline & $===========$ & $===========$ & $===========$ & $===========$ \\
\hline Total g/hr & 0.642955 & 0.120007 & 0.0 & 0.0 \\
\hline Volume, L/hr & $5.69674 \mathrm{E}-04$ & $4.08147 E-05$ & 0.0 & 0.0 \\
\hline Enthalpy, cal/hr & -2202.12 & -345.229 & 0.0 & 0.0 \\
\hline Density, g/L & 1128.64 & 2940.29 & & \\
\hline Vapor fraction & 0.0 & 0.0 & 0.0 & 0.0 \\
\hline Solid fraction & 0.0 & 1 . & 0.0 & 0.0 \\
\hline Organic fraction & 0.0 & 0.0 & 0.0 & 0.0 \\
\hline Osmotic Pres, atm & 153.149 & & & \\
\hline Redox Pot, volts & 0.0 & & & \\
\hline E-Con, 1/ohm-cm & 0.170265 & & & \\
\hline E-Con, cm2/ohm-mol & 31.4588 & & & \\
\hline Abs Visc, cP & 1.33985 & & & \\
\hline Rel Visc & 1.50423 & & & \\
\hline Ionic Strength & 3.68279 & & & \\
\hline
\end{tabular}


WSRC-TR-2002-00567

Revision 0

\begin{tabular}{|c|c|c|c|c|}
\hline \multicolumn{5}{|l|}{ STREAM: Condensate } \\
\hline TO : & & & & \\
\hline FROM : Condensate & mixer & & & \\
\hline Phases-----------> & Aqueous & Solid & Vapor & Organic \\
\hline Temperature, C & 25 . & 25 . & 25 . & 25 . \\
\hline $\begin{array}{l}\text { Pressure, atm } \\
\mathrm{pH}\end{array}$ & $\begin{array}{l}1 . \\
4.56912\end{array}$ & 1. & 1 . & 1 . \\
\hline \multirow{2}{*}{ Total mol/hr } & 55.46416 & 0.0 & 0.0 & 0.0 \\
\hline & $\mathrm{mol} / \mathrm{hr}------$ & $-\operatorname{mol} / \mathrm{hr}--------$ & -mol/hr------- & -mol/hr------ \\
\hline $\mathrm{H} 2 \mathrm{O}$ & 55.4631 & 0.0 & 0.0 & 0.0 \\
\hline $\mathrm{CO} 2$ & $9.83349 \mathrm{E}-04$ & 0.0 & 0.0 & 0.0 \\
\hline $\mathrm{HCL}$ & $2.34044 E-19$ & 0.0 & 0.0 & 0.0 \\
\hline HNO3 & $4.25426 \mathrm{E}-15$ & 0.0 & 0.0 & 0.0 \\
\hline LAURICACID & $2.29942 E-05$ & 0.0 & 0.0 & 0.0 \\
\hline OHION & $3.78067 \mathrm{E}-10$ & 0.0 & 0.0 & 0.0 \\
\hline CLION & $1.47398 E-08$ & 0.0 & 0.0 & 0.0 \\
\hline CO3ION & $2.95310 \mathrm{E}-11$ & 0.0 & 0.0 & 0.0 \\
\hline DODECION & $1.04435 E-05$ & 0.0 & 0.0 & 0.0 \\
\hline $\mathrm{HCO} 3 \mathrm{ION}$ & $1.66503 E-05$ & 0.0 & 0.0 & 0.0 \\
\hline HION & $2.71121 \mathrm{E}-05$ & 0.0 & 0.0 & 0.0 \\
\hline NO3ION & $\begin{array}{l}\text { 3.18430E-09 } \\
===========\end{array}$ & $\begin{array}{l}0.0 \\
============\end{array}$ & $\begin{array}{l}0.0 \\
=============\end{array}$ & $\begin{array}{l}0.0 \\
============\end{array}$ \\
\hline Total g/hr & 999.237 & 0.0 & 0.0 & 0.0 \\
\hline Volume, L/hr & 1.00238 & 0.0 & 0.0 & 0.0 \\
\hline $\begin{array}{l}\text { Enthalpy, cal/hr } \\
\text { Density, g/L }\end{array}$ & $\begin{array}{l}-3.78896 \mathrm{E}+06 \\
996.863\end{array}$ & 0.0 & 0.0 & 0.0 \\
\hline Vapor fraction & 0.0 & 0.0 & 0.0 & 0.0 \\
\hline Solid fraction & 0.0 & 0.0 & 0.0 & 0.0 \\
\hline Organic fraction & 0.0 & 0.0 & 0.0 & 0.0 \\
\hline Osmotic Pres, atm & 0.0258913 & & & \\
\hline Redox Pot, volts & 0.0 & & & \\
\hline E-Con, $1 / \mathrm{ohm}-\mathrm{cm}$ & $1.07972 \mathrm{E}-05$ & & & \\
\hline E-Con, cm2/ohm-mol & 10.4726 & & & \\
\hline Abs Visc, cP & 0.890739 & & & \\
\hline Rel Visc & 1.00002 & & & \\
\hline Ionic Strength & $2.71345 E-05$ & & & \\
\hline
\end{tabular}


WSRC-TR-2002-00567

Revision 0

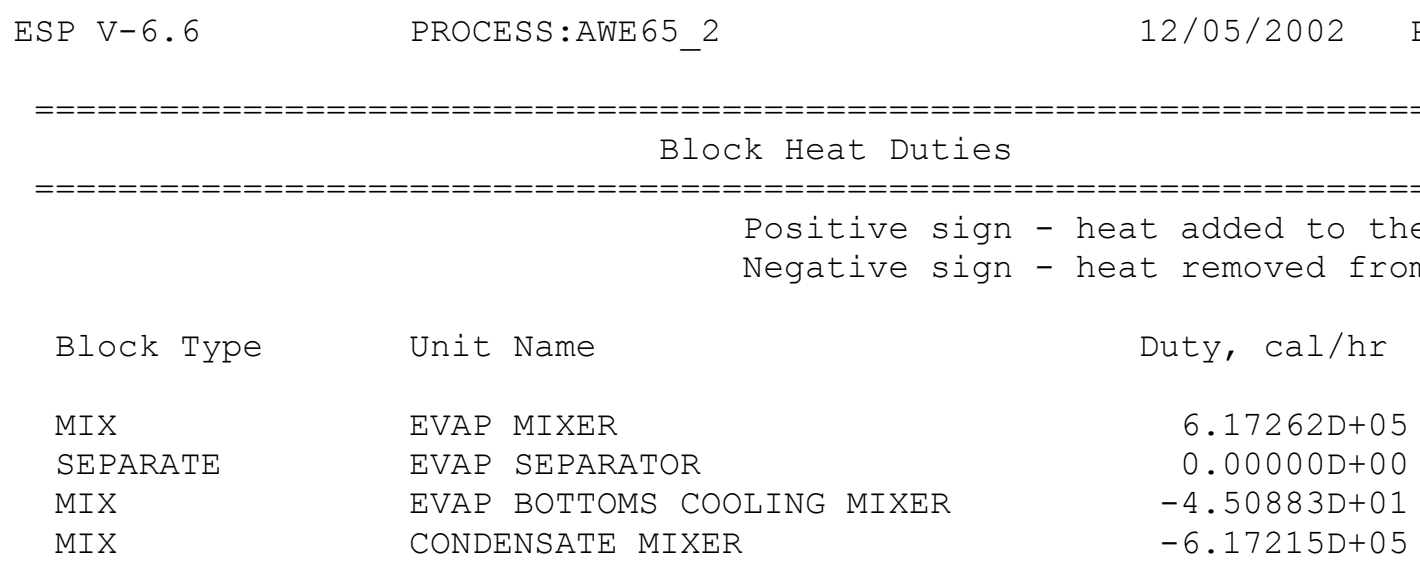


WSRC-TR-2002-00567

Revision 0

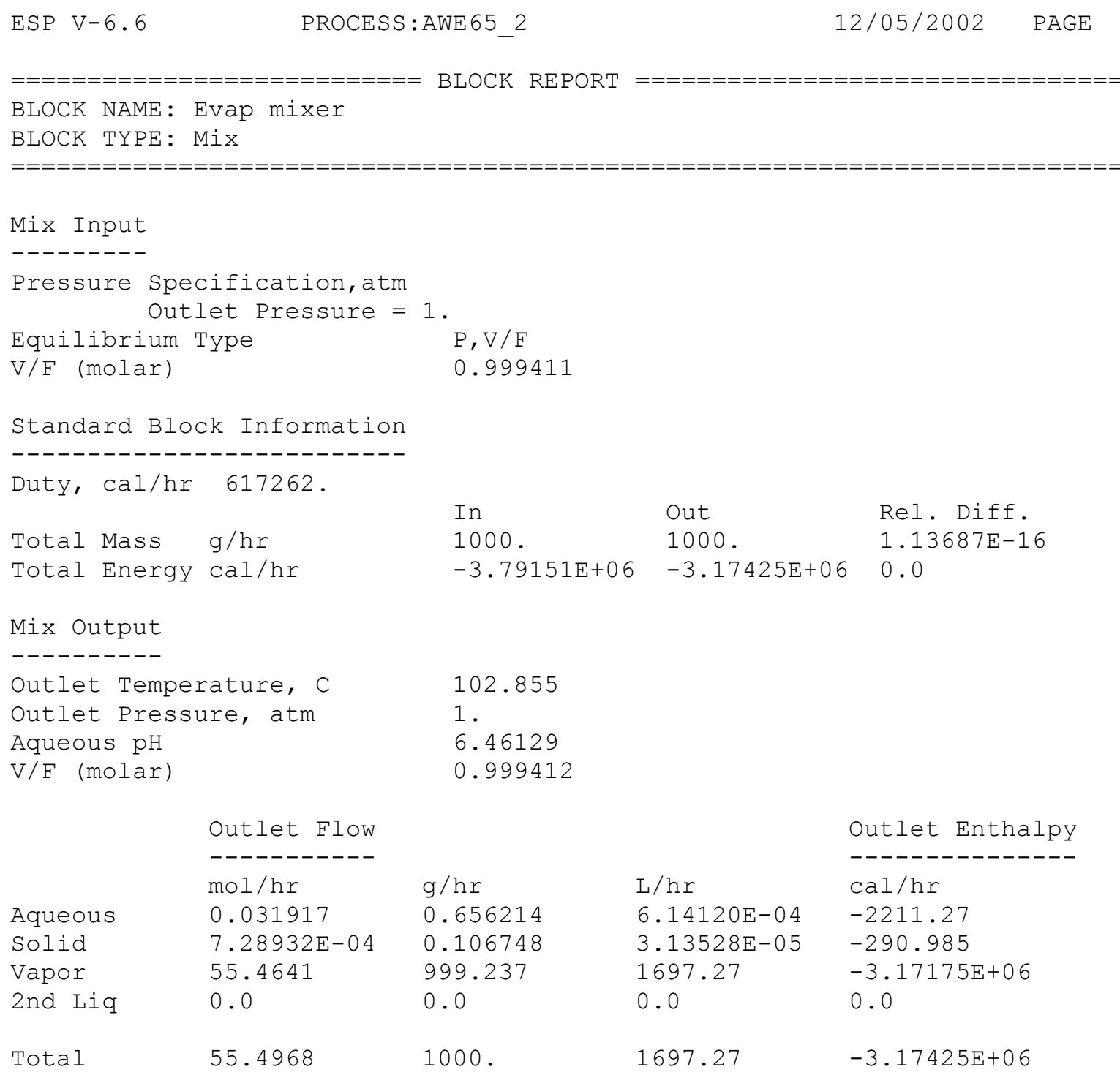


WSRC-TR-2002-00567

Revision 0

ESP V-6.6 PROCESS:AWE65_2 $\quad 12 / 05 / 2002 \quad$ PAGE 12

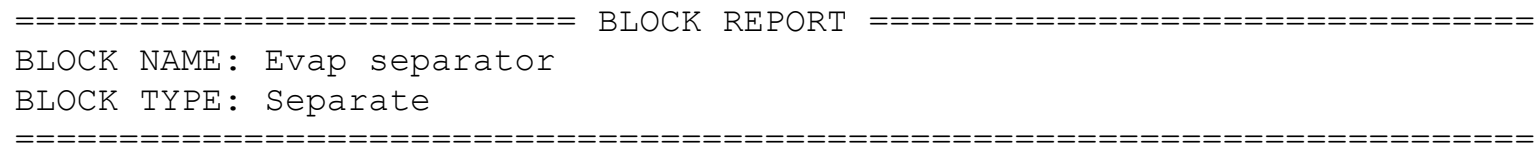

0.762962

$\mathrm{L} / \mathrm{hr}$

$0.0 \quad 0.0$

$0.0 \quad 0.0$

cal/hr

$-2211.27$

$-290.985$

$6.45473 E-04 \quad-2502.26$ 
WSRC-TR-2002-00567

Revision 0

ESP V-6.6

PROCESS:AWE 65_2

Vapor Stream

Outlet Flow
--------
mol/hr
0.0
0.0
55.4641
0.0

Total
Overhead

g/hr

0.0

0.0

999.237

0.0

999.237
$12 / 05 / 2002$

PAGE

13

$\mathrm{L} / \mathrm{hr}$

0.0

0.0

1697.27

0.0

1697.27

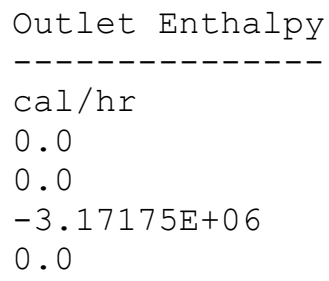

0.0

0.0

$-3.17175 E+06$

0.0

$-3.17175 E+06$ 
WSRC-TR-2002-00567

Revision 0

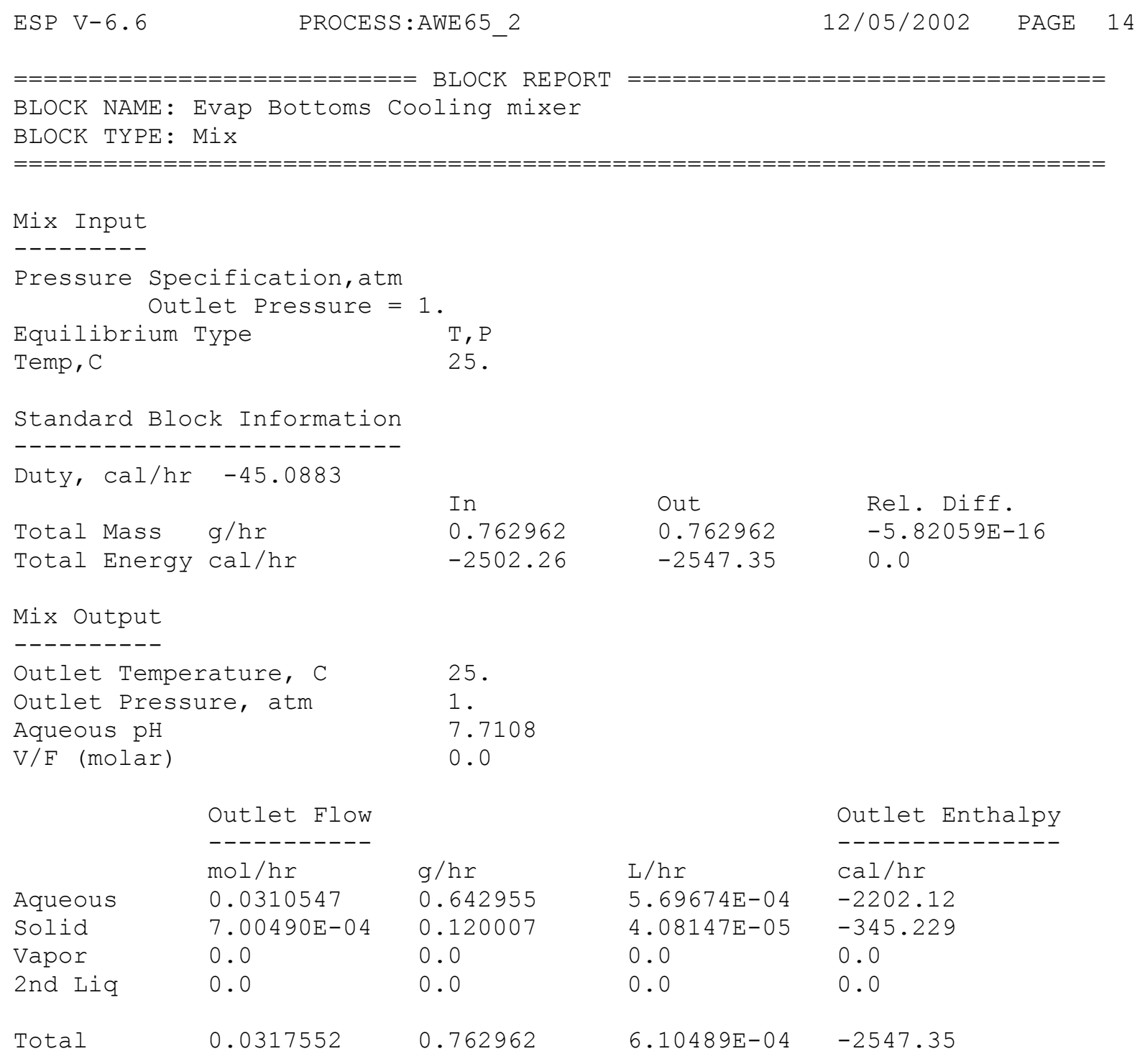


WSRC-TR-2002-00567

Revision 0

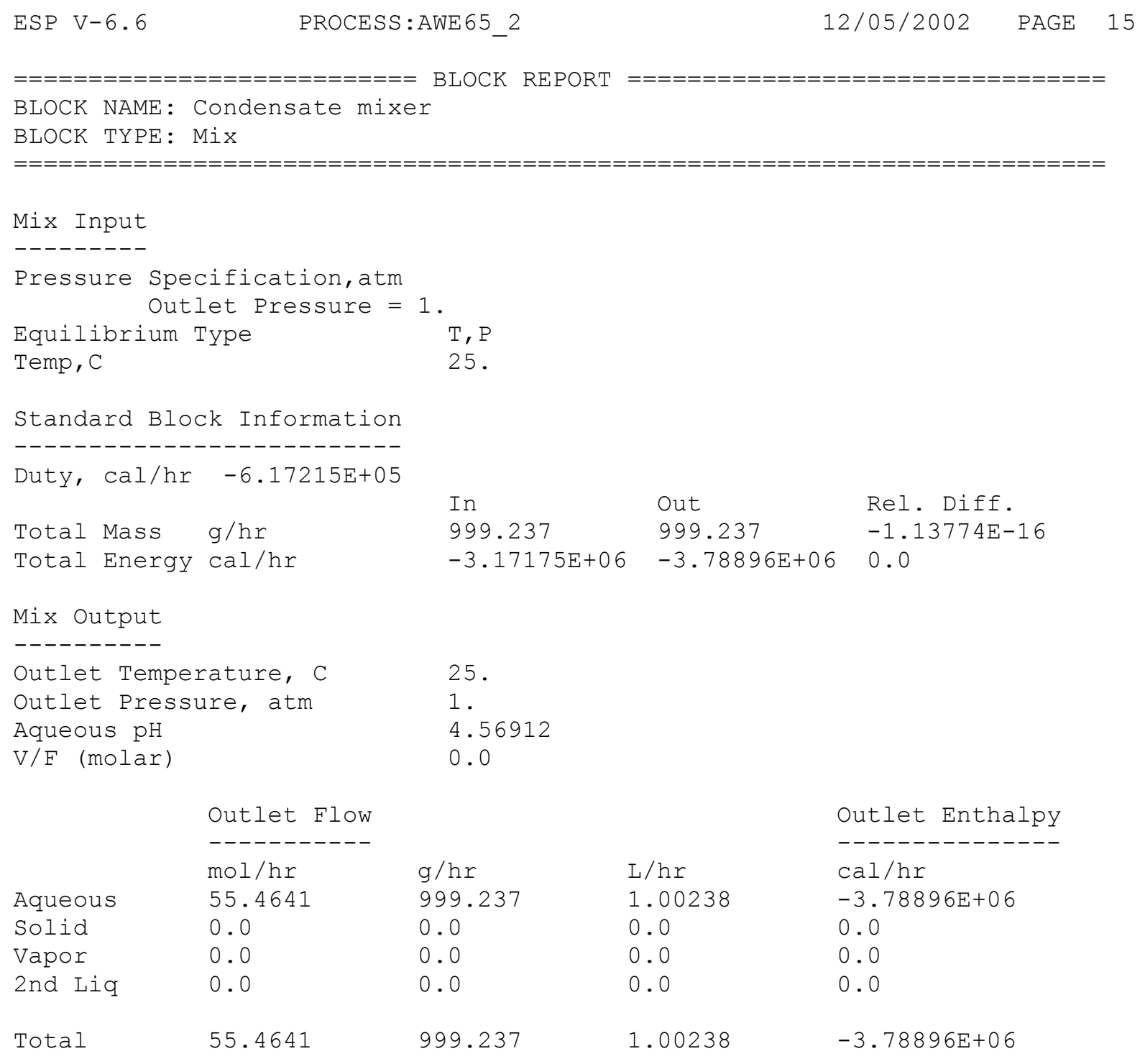


WSRC-TR-2002-00567

Revision 0

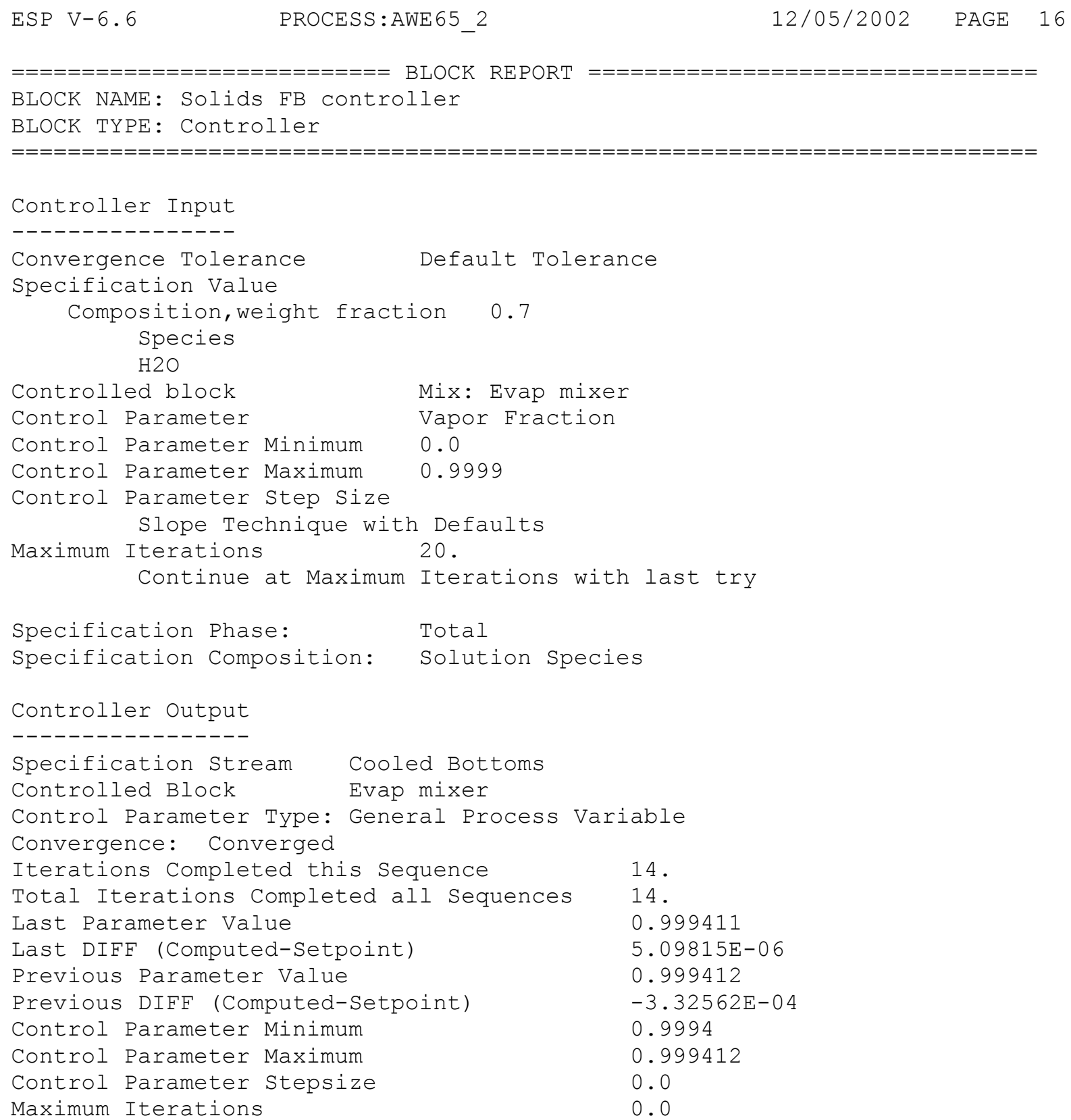


WSRC-TR-2002-00567

Revision 0

Influent Limit Composition 60\% Target $\mathrm{pH}=6.5$

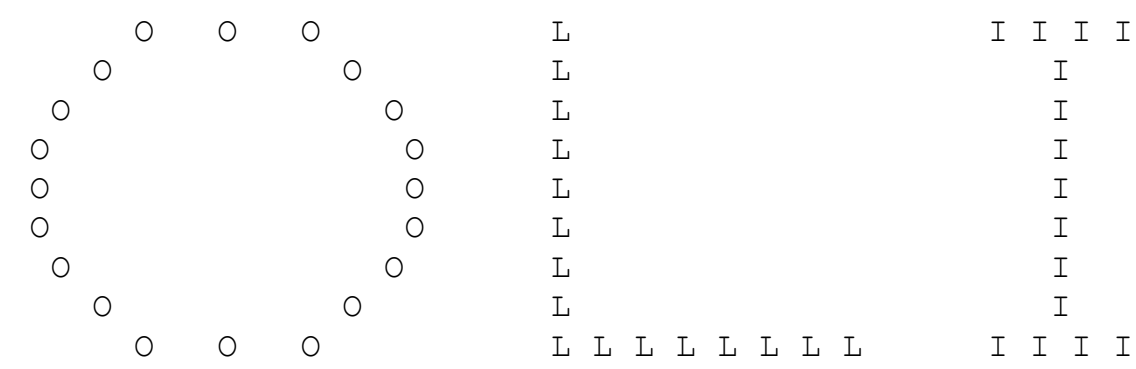

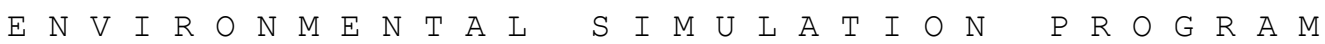

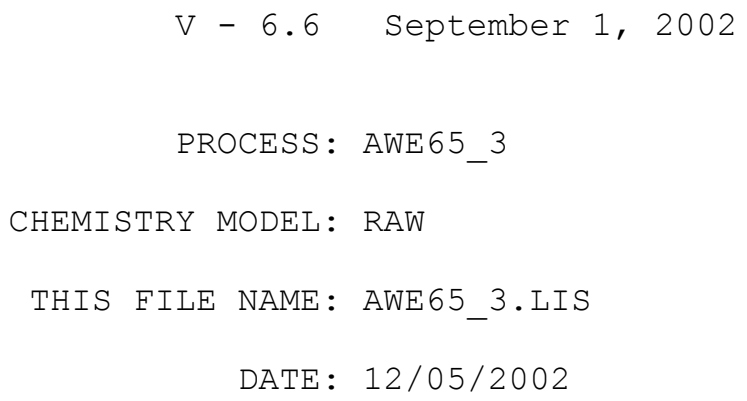




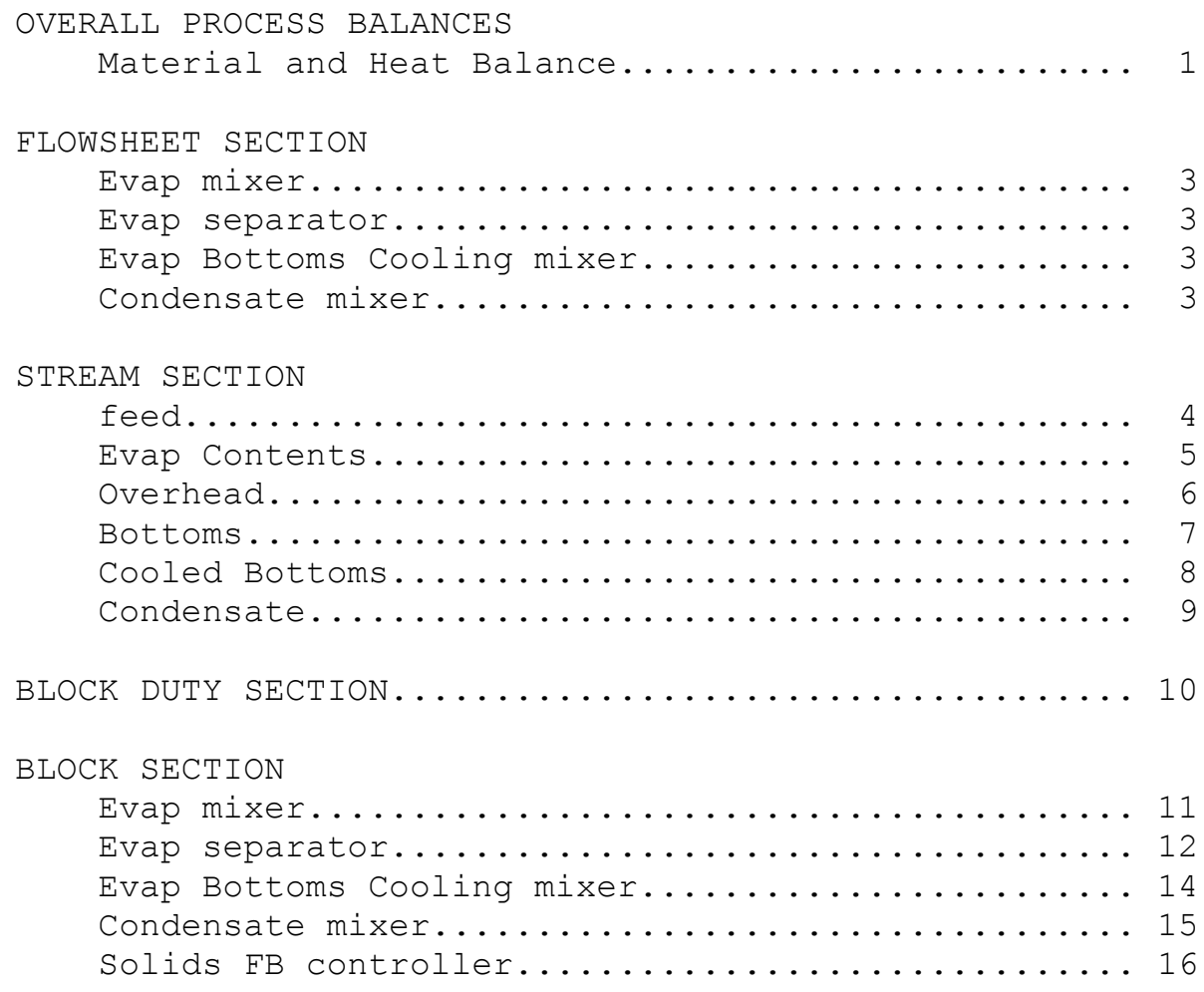


WSRC-TR-2002-00567

Revision 0

ESP V-6.6 PROCESS:AWE65_3
$\begin{array}{lcc}\text { Overall Process } & \text { Balances } \\ \text { Inlet } & \text { g/hr } & \mathrm{Cal} / \mathrm{hr} \\ ----------- & ----------- & ---------- \\ \text { FEED } & 6.28091 \mathrm{D}+01 & -2.37655 \mathrm{D}+05 \\ \text { =========== } & ========== & ========= \\ \text { Total in } & 6.28091 \mathrm{D}+01 & -2.37655 \mathrm{D}+05\end{array}$

\begin{tabular}{lrc} 
Outlet & \multicolumn{1}{c}{ g/hr } & cal/hr \\
----------- & ---------- & ---------- \\
COOLED BOTTOMS & $9.28910 \mathrm{D}-01$ & $-3.10560 \mathrm{D}+03$ \\
CONDENSATE & $6.18802 \mathrm{D}+01$ & $-2.34550 \mathrm{D}+05$ \\
$==========$ & $=========$ & $=========$ \\
Total out & $6.28091 \mathrm{D}+01$ & $-2.37655 \mathrm{D}+05$
\end{tabular}

$12 / 05 / 2002 \quad$ PAGE 1

Block Heat Duties

EVAP MIXER

EVAP BOTTOMS COOLING MIXER

CONDENSATE MIXER

$=========$

Total Duty

DIFFERENCE

REL DIFEERENCE

$7.10543 \mathrm{D}-15$

$1.13127 \mathrm{D}-1$

$$
\begin{gathered}
\mathrm{cal} / \mathrm{hr} \\
--------- \\
3.82504 \mathrm{D}+04 \\
-5.66153 \mathrm{D}+01 \\
-3.81935 \mathrm{D}+04 \\
========== \\
2.90096 \mathrm{D}-01
\end{gathered}
$$

\begin{tabular}{|c|c|c|c|c|}
\hline Code & $\begin{array}{l}\text { Input } \\
\text { mol/hr }\end{array}$ & $\begin{array}{l}\text { Outlet } \\
\text { mol/hr }\end{array}$ & $\begin{array}{l}\text { Difference } \\
\text { mol/hr }\end{array}$ & Rel Diff \\
\hline------------ & ------------ & ------------ & ------------ & ------------ \\
\hline $\mathrm{H}(+1)$ & $6.93835 D+00$ & $6.93835 \mathrm{D}+00$ & $1.77636 \mathrm{D}-15$ & $2.56020 \mathrm{D}-16$ \\
\hline$K(+1)$ & $1.73235 \mathrm{D}-04$ & $1.73235 \mathrm{D}-04$ & $8.13152 \mathrm{D}-20$ & $4.69393 \mathrm{D}-16$ \\
\hline $\mathrm{NA}(+1)$ & $1.17802 \mathrm{D}-03$ & $1.17802 \mathrm{D}-03$ & $1.30104 \mathrm{D}-18$ & $1.10443 \mathrm{D}-15$ \\
\hline $\mathrm{BA}(+2)$ & $4.96350 \mathrm{D}-07$ & $4.96350 \mathrm{D}-07$ & $-1.05879 \mathrm{D}-22$ & $-2.13315 \mathrm{D}-16$ \\
\hline $\mathrm{CA}(+2)$ & $7.98005 \mathrm{D}-04$ & $7.98005 \mathrm{D}-04$ & $-2.16840 \mathrm{D}-19$ & $-2.71728 D-16$ \\
\hline $\mathrm{ZN}(+2)$ & $2.59939 D-05$ & $2.59939 D-05$ & $3.38813 \mathrm{D}-21$ & $1.30343 \mathrm{D}-16$ \\
\hline $\mathrm{CU}(+2)$ & $3.93701 \mathrm{D}-06$ & $3.93701 \mathrm{D}-06$ & $8.47033 \mathrm{D}-22$ & $2.15146 \mathrm{D}-16$ \\
\hline $\mathrm{FE}(+2)$ & $7.16846 \mathrm{D}-05$ & $7.16846 \mathrm{D}-05$ & $0.00000 \mathrm{D}+00$ & $0.00000 \mathrm{D}+00$ \\
\hline $\mathrm{MG}(+2)$ & $2.83951 \mathrm{D}-04$ & $2.83951 \mathrm{D}-04$ & $5.42101 \mathrm{D}-20$ & $1.90914 \mathrm{D}-16$ \\
\hline $\mathrm{PB}(+2)$ & $2.57488 \mathrm{D}-06$ & $2.57488 D-06$ & $-1.69407 \mathrm{D}-21$ & $-6.57921 D-16$ \\
\hline $\mathrm{AL}(+3)$ & $7.77778 \mathrm{D}-05$ & $7.77778 \mathrm{D}-05$ & $1.35525 \mathrm{D}-20$ & $1.74247 \mathrm{D}-16$ \\
\hline $\mathrm{NI}(+2)$ & $1.09029 D-06$ & $1.09029 D-06$ & $-1.05879 D-21$ & $-9.71110 \mathrm{D}-16$ \\
\hline$O(-2)$ & $3.47629 \mathrm{D}+00$ & $3.47629 D+00$ & $0.00000 \mathrm{D}+00$ & $0.00000 \mathrm{D}+00$ \\
\hline $\operatorname{CL}(-1)$ & $1.57746 \mathrm{D}-03$ & $1.57746 \mathrm{D}-03$ & $1.08420 \mathrm{D}-18$ & $6.87307 \mathrm{D}-16$ \\
\hline$C(+4)$ & $1.10000 \mathrm{D}-03$ & $1.10000 \mathrm{D}-03$ & $-8.67362 \mathrm{D}-19$ & $-7.88511 D-16$ \\
\hline$P(+5)$ & $2.38934 \mathrm{D}-04$ & $2.38934 \mathrm{D}-04$ & $-2.71051 D-20$ & $-1.13441 D-16$ \\
\hline$S(+6)$ & $5.83333 \mathrm{D}-04$ & $5.83333 \mathrm{D}-04$ & $-1.08420 D-19$ & $-1.85863 D-16$ \\
\hline $\mathrm{N}(+5)$ & $4.03226 \mathrm{D}-04$ & $4.03226 \mathrm{D}-04$ & $3.25261 \mathrm{D}-19$ & $8.06645 \mathrm{D}-16$ \\
\hline $\operatorname{SI}(+4)$ & $1.83333 \mathrm{D}-04$ & $1.83333 \mathrm{D}-04$ & $2.71051 \mathrm{D}-20$ & $1.47846 \mathrm{D}-16$ \\
\hline $\mathrm{SR}(+2)$ & $1.48402 \mathrm{D}-05$ & $1.48402 \mathrm{D}-05$ & $1.69407 \mathrm{D}-21$ & $1.14154 \mathrm{D}-16$ \\
\hline
\end{tabular}

Material Code Balances 
WSRC-TR-2002-00567

Revision 0

\begin{abstract}
$\mathrm{CD}(+2)$
$\mathrm{CR}(+3)$

$\mathrm{U}(+4)$
\end{abstract}

$1.27340 \mathrm{D}-08$

$6.53846 \mathrm{D}-08$

2. $43698 \mathrm{D}-06$
1. $27340 \mathrm{D}-08$

$6.53846 \mathrm{D}-08$

$2.43698 \mathrm{D}-06$
$2.64698 \mathrm{D}-23$

$7.94093 \mathrm{D}-23$

$0.00000 D+00$
$2.07867 \mathrm{D}-15$

$1.21450 \mathrm{D}-15$

$0.00000 \mathrm{D}+00$ 
WSRC-TR-2002-00567

Revision 0

ESP V-6.6

$\operatorname{DODEC}(-1)$
PROCESS:AWE 653

$3.79916 \mathrm{D}-05$
$12 / 05 / 2002$

PAGE

$3.79916 \mathrm{D}-05-1.82959 \mathrm{D}-19-4.81578 \mathrm{D}-15$ 
WSRC-TR-2002-00567

Revision 0

\begin{tabular}{|c|c|c|c|}
\hline \multicolumn{4}{|l|}{$\begin{array}{l}\text { PROCESS BLOCKS } \\
===========\end{array}$} \\
\hline $\begin{array}{l}\mathrm{BLOCK} \mathrm{NAME} \\
=========================\end{array}$ & $\begin{array}{l}\text { BLOCK TYPE } \\
===========\end{array}$ & $\begin{array}{l}\text { INLET STREAM }(\mathrm{S}) \\
==============\end{array}$ & $\begin{array}{l}\text { OUTLET STREAM }(\mathrm{S}) \\
==============\end{array}$ \\
\hline Evap mixer & $\operatorname{Mix}$ & feed & Evap Contents \\
\hline Evap separator & Separate & Evap Contents & $\begin{array}{l}\text { Overhead } \\
\text { Bottoms }\end{array}$ \\
\hline Evap Bottoms Cooling mixer & Mix & Bottoms & Cooled Bottoms \\
\hline Condensate mixer & Mix & Overhead & Condensate \\
\hline
\end{tabular}


WSRC-TR-2002-00567

Revision 0

ESP V-6. 6

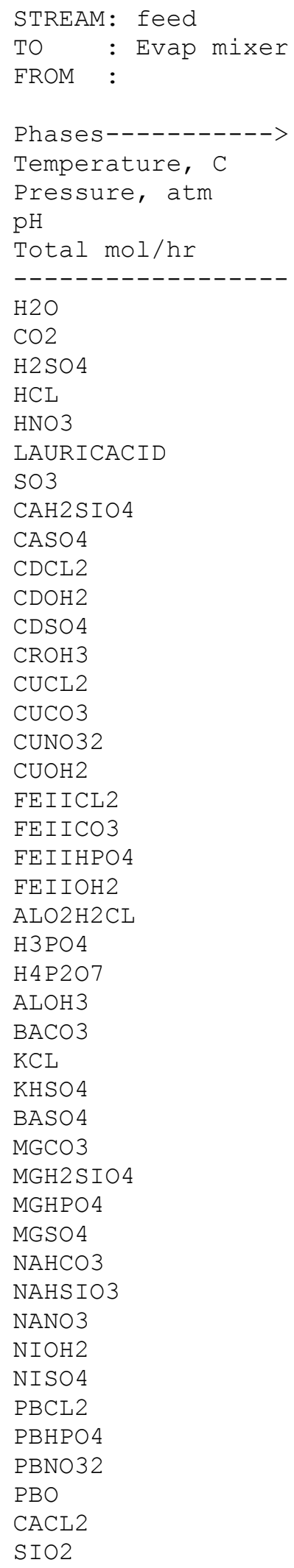

\begin{tabular}{|c|c|c|c|}
\hline Aqueous & Solid & Vapor & Organic \\
\hline 25 & 25 . & 25 . & 25 . \\
\hline 1 . & 1 . & 1 . & 1 . \\
\hline \multicolumn{4}{|l|}{5.59463} \\
\hline 3.475029 & $2.45839 \mathrm{E}-04$ & 0.0 & 0.0 \\
\hline \multicolumn{4}{|c|}{$\mathrm{mol} / \mathrm{hr}--------\mathrm{mol} / \mathrm{hr}--------\mathrm{mol} / \mathrm{hr}--------\mathrm{mol} / \mathrm{hr}--------$} \\
\hline 3.46876 & 0.0 & 0.0 & 0.0 \\
\hline $8.78408 \mathrm{E}-04$ & 0.0 & 0.0 & 0.0 \\
\hline $4.39377 \mathrm{E}-24$ & 0.0 & 0.0 & 0.0 \\
\hline $1.89132 \mathrm{E}-15$ & 0.0 & 0.0 & 0.0 \\
\hline $3.93586 \mathrm{E}-11$ & 0.0 & 0.0 & 0.0 \\
\hline $5.23869 E-06$ & 0.0 & 0.0 & 0.0 \\
\hline $5.69668 \mathrm{E}-28$ & 0.0 & 0.0 & 0.0 \\
\hline $4.52942 E-14$ & 0.0 & 0.0 & 0.0 \\
\hline $2.73422 \mathrm{E}-05$ & 0.0 & 0.0 & 0.0 \\
\hline $1.45147 \mathrm{E}-09$ & 0.0 & 0.0 & 0.0 \\
\hline $1.88749 \mathrm{E}-18$ & 0.0 & 0.0 & 0.0 \\
\hline $1.98971 \mathrm{E}-09$ & 0.0 & 0.0 & 0.0 \\
\hline $3.10237 \mathrm{E}-16$ & 0.0 & 0.0 & 0.0 \\
\hline $5.04858 E-12$ & 0.0 & 0.0 & 0.0 \\
\hline $1.29397 \mathrm{E}-09$ & 0.0 & 0.0 & 0.0 \\
\hline $6.59544 \mathrm{E}-14$ & 0.0 & 0.0 & 0.0 \\
\hline $6.56365 E-12$ & 0.0 & 0.0 & 0.0 \\
\hline $5.66444 \mathrm{E}-14$ & 0.0 & 0.0 & 0.0 \\
\hline $1.67089 \mathrm{E}-07$ & 0.0 & 0.0 & 0.0 \\
\hline $2.27118 E-08$ & 0.0 & 0.0 & 0.0 \\
\hline $1.06084 \mathrm{E}-14$ & 0.0 & 0.0 & 0.0 \\
\hline $3.66481 E-28$ & 0.0 & 0.0 & 0.0 \\
\hline $2.92216 \mathrm{E}-08$ & 0.0 & 0.0 & 0.0 \\
\hline $2.45304 \mathrm{E}-17$ & 0.0 & 0.0 & 0.0 \\
\hline $1.48707 \mathrm{E}-10$ & $7.77774 \mathrm{E}-05$ & 0.0 & 0.0 \\
\hline $4.62814 \mathrm{E}-14$ & 0.0 & 0.0 & 0.0 \\
\hline $2.19910 \mathrm{E}-08$ & 0.0 & 0.0 & 0.0 \\
\hline $1.80473 E-12$ & 0.0 & 0.0 & 0.0 \\
\hline $1.27448 \mathrm{E}-11$ & $4.87776 \mathrm{E}-07$ & 0.0 & 0.0 \\
\hline $4.22188 E-09$ & 0.0 & 0.0 & 0.0 \\
\hline $1.70435 E-13$ & 0.0 & 0.0 & 0.0 \\
\hline $3.65226 E-06$ & 0.0 & 0.0 & 0.0 \\
\hline $1.85518 \mathrm{E}-05$ & 0.0 & 0.0 & 0.0 \\
\hline $9.51033 E-07$ & 0.0 & 0.0 & 0.0 \\
\hline $1.17080 \mathrm{E}-08$ & 0.0 & 0.0 & 0.0 \\
\hline $2.61278 E-07$ & 0.0 & 0.0 & 0.0 \\
\hline $5.20166 \mathrm{E}-15$ & 0.0 & 0.0 & 0.0 \\
\hline $2.02199 E-07$ & 0.0 & 0.0 & 0.0 \\
\hline $1.02345 \mathrm{E}-10$ & 0.0 & 0.0 & 0.0 \\
\hline $1.32707 \mathrm{E}-10$ & 0.0 & 0.0 & 0.0 \\
\hline 1.22050E-12 & 0.0 & 0.0 & 0.0 \\
\hline $3.91822 E-15$ & 0.0 & 0.0 & 0.0 \\
\hline $9.33087 E-26$ & 0.0 & 0.0 & 0.0 \\
\hline $1.18247 \mathrm{E}-04$ & $6.50656 \mathrm{E}-05$ & 0.0 & 0.0 \\
\hline
\end{tabular}




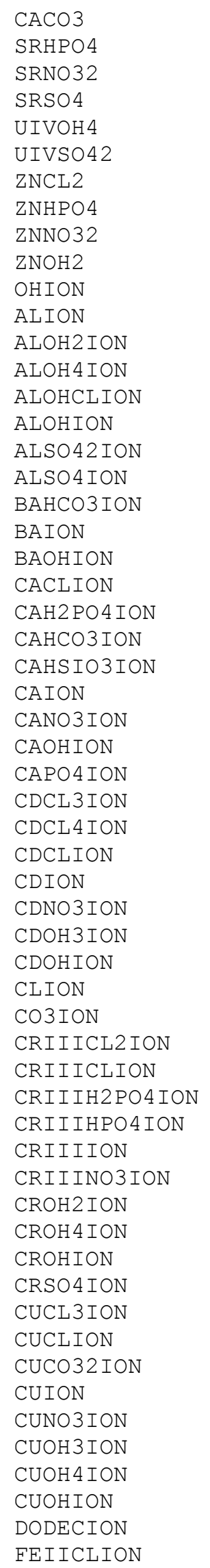

\begin{tabular}{|c|c|}
\hline $2.40341 E-08$ & 0.0 \\
\hline $2.67230 \mathrm{E}-09$ & 0.0 \\
\hline $9.53694 \mathrm{E}-10$ & 0.0 \\
\hline $2.67040 E-06$ & $2.32310 E-07$ \\
\hline $1.86522 \mathrm{E}-11$ & 0.0 \\
\hline $5.51711 E-24$ & 0.0 \\
\hline $6.38549 E-09$ & 0.0 \\
\hline $5.29631 \mathrm{E}-07$ & 0.0 \\
\hline $1.46773 \mathrm{E}-10$ & 0.0 \\
\hline $6.71404 \mathrm{E}-12$ & 0.0 \\
\hline $3.15387 \mathrm{E}-10$ & 0.0 \\
\hline $8.16271 \mathrm{E}-11$ & 0.0 \\
\hline $8.91500 \mathrm{E}-11$ & 0.0 \\
\hline 1.89985E-11 & 0.0 \\
\hline $3.33617 E-12$ & 0.0 \\
\hline $9.96534 \mathrm{E}-11$ & 0.0 \\
\hline $9.06627 \mathrm{E}-12$ & 0.0 \\
\hline $3.87419 \mathrm{E}-11$ & 0.0 \\
\hline 7. $71248 \mathrm{E}-11$ & 0.0 \\
\hline $8.48445 E-09$ & 0.0 \\
\hline $6.58659 \mathrm{E}-18$ & 0.0 \\
\hline $1.44495 \mathrm{E}-10$ & 0.0 \\
\hline $1.53563 E-05$ & 0.0 \\
\hline $8.74276 \mathrm{E}-06$ & 0.0 \\
\hline $6.18739 \mathrm{E}-10$ & 0.0 \\
\hline $6.44687 \mathrm{E}-04$ & 0.0 \\
\hline $4.16803 E-06$ & 0.0 \\
\hline $2.11420 \mathrm{E}-11$ & 0.0 \\
\hline $7.13630 \mathrm{E}-09$ & 0.0 \\
\hline $1.83572 \mathrm{E}-12$ & 0.0 \\
\hline $4.37353 E-14$ & 0.0 \\
\hline $4.34115 E-09$ & 0.0 \\
\hline $4.91774 \mathrm{E}-09$ & 0.0 \\
\hline $3.19680 \mathrm{E}-11$ & 0.0 \\
\hline $1.34727 E-25$ & 0.0 \\
\hline 7. $65899 \mathrm{E}-14$ & 0.0 \\
\hline 0.00157705 & 0.0 \\
\hline $7.87523 E-09$ & 0.0 \\
\hline $2.11958 \mathrm{E}-18$ & 0.0 \\
\hline 1.33912E-15 & 0.0 \\
\hline $7.97287 \mathrm{E}-13$ & 0.0 \\
\hline $6.53111 \mathrm{E}-08$ & 0.0 \\
\hline $5.34965 E-14$ & 0.0 \\
\hline $1.80639 \mathrm{E}-14$ & 0.0 \\
\hline $3.00152 E-16$ & 0.0 \\
\hline $1.83095 E-21$ & 0.0 \\
\hline $6.44523 E-13$ & 0.0 \\
\hline 7.20119E-11 & 0.0 \\
\hline $2.76692 \mathrm{E}-16$ & 0.0 \\
\hline $7.06319 \mathrm{E}-10$ & 0.0 \\
\hline $2.54009 \mathrm{E}-13$ & 0.0 \\
\hline $1.34441 E-08$ & 0.0 \\
\hline $1.18605 \mathrm{E}-10$ & 0.0 \\
\hline $1.81040 \mathrm{E}-18$ & 0.0 \\
\hline $1.13833 \mathrm{E}-25$ & 0.0 \\
\hline $1.18320 \mathrm{E}-10$ & 0.0 \\
\hline $3.27529 \mathrm{E}-05$ & 0.0 \\
\hline $9.79817 \mathrm{E}-10$ & 0.0 \\
\hline
\end{tabular}

0.0

0.0

0.0

0.0

0.0

0.0

0.0

0.0

0.0

0.0

0.0

0.0

0.0

0.0

0.0

0.0

0.0

0.0

0.0

0.0

0.0

0.0

0.0

0.0

0.0

0.0

0.0

0.0

0.0

0.0

0.0

0.0

0.0

0.0

0.0

0.0

0.0

0.0

0.0

0.0

0.0

0.0

0.0

0.0

0.0

0.0

0.0

0.0

0.0

0.0

0.0

0.0

0.0

0.0

0.0

0.0

0.0

0.0
0.0

0.0

0.0

0.0

0.0

0.0

0.0

0.0

0.0

0.0

0.0

0.0

0.0

0.0

0.0

0.0

0.0

0.0

0.0

0.0

0.0

0.0

0.0

0.0

0.0

0.0

0.0

0.0

0.0

0.0

0.0

0.0

0.0

0.0

0.0

0.0

0.0

0.0

0.0

0.0

0.0

0.0

0.0

0.0

0.0

0.0

0.0

0.0

0.0

0.0

0.0

0.0

0.0

0.0

0.0

0.0

0.0

0.0 


\begin{tabular}{|c|c|c|c|c|}
\hline FEIICO32ION & $7.09618 \mathrm{E}-13$ & 0.0 & 0.0 & 0.0 \\
\hline FEIIH2PO4ION & $1.52463 \mathrm{E}-07$ & 0.0 & 0.0 & 0.0 \\
\hline EEIIHCO3ION & $1.30543 \mathrm{E}-08$ & 0.0 & 0.0 & 0.0 \\
\hline FEIIION & $7.13239 \mathrm{E}-05$ & 0.0 & 0.0 & 0.0 \\
\hline FEIIOH3ION & $2.08781 \mathrm{E}-19$ & 0.0 & 0.0 & 0.0 \\
\hline FEIIOH 4 ION & $1.85379 \mathrm{E}-28$ & 0.0 & 0.0 & 0.0 \\
\hline FEIIOHION & $4.34989 \mathrm{E}-09$ & 0.0 & 0.0 & 0.0 \\
\hline H2 P2O 7 ION & $1.31601 \mathrm{E}-09$ & 0.0 & 0.0 & 0.0 \\
\hline H2 PO 4 ION & $1.03434 \mathrm{E}-04$ & 0.0 & 0.0 & 0.0 \\
\hline $\mathrm{H} 2 \mathrm{SIO} 4 \mathrm{ION}$ & $5.56977 \mathrm{E}-16$ & 0.0 & 0.0 & 0.0 \\
\hline H3P2O7 ION & $3.46322 \mathrm{E}-13$ & 0.0 & 0.0 & 0.0 \\
\hline H3SIO 4 ION & $8.39113 \mathrm{E}-09$ & 0.0 & 0.0 & 0.0 \\
\hline HCO3ION & $1.99383 \mathrm{E}-04$ & 0.0 & 0.0 & 0.0 \\
\hline HION & $2.00000 \mathrm{E}-07$ & 0.0 & 0.0 & 0.0 \\
\hline HP207ION & $4.45121 \mathrm{E}-10$ & 0.0 & 0.0 & 0.0 \\
\hline HPBO2ION & $2.23185 \mathrm{E}-20$ & 0.0 & 0.0 & 0.0 \\
\hline HPO4ION & $5.41048 \mathrm{E}-06$ & 0.0 & 0.0 & 0.0 \\
\hline HSO 4 ION & $5.59266 \mathrm{E}-08$ & 0.0 & 0.0 & 0.0 \\
\hline KION & $1.69391 \mathrm{E}-04$ & 0.0 & 0.0 & 0.0 \\
\hline KSO4 ION & $3.82168 \mathrm{E}-06$ & 0.0 & 0.0 & 0.0 \\
\hline MGH2 PO 4 ION & $7.68244 \mathrm{E}-06$ & 0.0 & 0.0 & 0.0 \\
\hline MGHCO3ION & $1.16677 \mathrm{E}-05$ & 0.0 & 0.0 & 0.0 \\
\hline MGHSIO3ION & $4.45036 \mathrm{E}-10$ & 0.0 & 0.0 & 0.0 \\
\hline MGION & $2.42387 E-04$ & 0.0 & 0.0 & 0.0 \\
\hline MGOHION & $7.89474 \mathrm{E}-11$ & 0.0 & 0.0 & 0.0 \\
\hline MGP2O7ION & $8.15919 \mathrm{E}-10$ & 0.0 & 0.0 & 0.0 \\
\hline MGPO 4 ION & $4.00246 \mathrm{E}-09$ & 0.0 & 0.0 & 0.0 \\
\hline NACO3ION & $1.92106 \mathrm{E}-10$ & 0.0 & 0.0 & 0.0 \\
\hline NAION & 0.0011483 & 0.0 & 0.0 & 0.0 \\
\hline NASO 4 ION & $2.84976 \mathrm{E}-05$ & 0.0 & 0.0 & 0.0 \\
\hline NICLION & $8.63627 \mathrm{E}-10$ & 0.0 & 0.0 & 0.0 \\
\hline NIION & $8.80975 \mathrm{E}-07$ & 0.0 & 0.0 & 0.0 \\
\hline NINO3ION & $6.22857 \mathrm{E}-09$ & 0.0 & 0.0 & 0.0 \\
\hline NIOH3ION & $2.72453 \mathrm{E}-20$ & 0.0 & 0.0 & 0.0 \\
\hline NIOHION & $2.33110 \mathrm{E}-11$ & 0.0 & 0.0 & 0.0 \\
\hline NO3ION & $3.98455 \mathrm{E}-04$ & 0.0 & 0.0 & 0.0 \\
\hline P207ION & $4.98294 \mathrm{E}-13$ & 0.0 & 0.0 & 0.0 \\
\hline PBCL3ION & 1.31253E-12 & 0.0 & 0.0 & 0.0 \\
\hline PBCL 4 ION & $3.57913 \mathrm{E}-14$ & 0.0 & 0.0 & 0.0 \\
\hline PBCLION & $1.80071 \mathrm{E}-09$ & 0.0 & 0.0 & 0.0 \\
\hline PBH2 PO 4 ION & $1.77538 \mathrm{E}-10$ & 0.0 & 0.0 & 0.0 \\
\hline PBION & $6.94184 \mathrm{E}-09$ & 0.0 & 0.0 & 0.0 \\
\hline PBNO33ION & $1.36675 \mathrm{E}-15$ & 0.0 & 0.0 & 0.0 \\
\hline PBNO3ION & $3.24657 \mathrm{E}-10$ & 0.0 & 0.0 & 0.0 \\
\hline PBOHION & $3.23735 \mathrm{E}-11$ & 0.0 & 0.0 & 0.0 \\
\hline PO4ION & $3.52231 \mathrm{E}-12$ & 0.0 & 0.0 & 0.0 \\
\hline SO4ION & $5.01469 \mathrm{E}-04$ & 0.0 & 0.0 & 0.0 \\
\hline SRION & $1.17667 \mathrm{E}-05$ & 0.0 & 0.0 & 0.0 \\
\hline SRNO3ION & $1.67144 \mathrm{E}-07$ & 0.0 & 0.0 & 0.0 \\
\hline SROHION & $1.49306 \mathrm{E}-13$ & 0.0 & 0.0 & 0.0 \\
\hline SRPO 4 ION & $1.87554 \mathrm{E}-12$ & 0.0 & 0.0 & 0.0 \\
\hline UIVCLION & $3.15521 \mathrm{E}-28$ & 0.0 & 0.0 & 0.0 \\
\hline UIVION & $1.48946 \mathrm{E}-27$ & 0.0 & 0.0 & 0.0 \\
\hline UIVOH2ION & $3.82821 \mathrm{E}-20$ & 0.0 & 0.0 & 0.0 \\
\hline UIVOH3ION & $6.87777 \mathrm{E}-16$ & 0.0 & 0.0 & 0.0 \\
\hline UIVOH5ION & $4.97591 \mathrm{E}-16$ & 0.0 & 0.0 & 0.0 \\
\hline UIVOHION & $4.90006 \mathrm{E}-23$ & 0.0 & 0.0 & 0.0 \\
\hline UIVSO 4 ION & $6.98584 \mathrm{E}-25$ & 0.0 & 0.0 & 0.0 \\
\hline
\end{tabular}


WSRC-TR-2002-00567

Revision 0

\begin{tabular}{|c|c|c|c|c|}
\hline ZNCL3ION & $5.69070 \mathrm{E}-11$ & 0.0 & 0.0 & 0.0 \\
\hline ZNCLION & $3.63972 \mathrm{E}-07$ & 0.0 & 0.0 & 0.0 \\
\hline ZNH2 PO4 ION & $5.78797 \mathrm{E}-07$ & 0.0 & 0.0 & 0.0 \\
\hline ZNHCO3ION & $6.30702 \mathrm{E}-07$ & 0.0 & 0.0 & 0.0 \\
\hline ZNION & $2.37132 E-05$ & 0.0 & 0.0 & 0.0 \\
\hline ZNNO3ION & $1.65513 \mathrm{E}-07$ & 0.0 & 0.0 & 0.0 \\
\hline ZNOH3ION & $3.36491 E-17$ & 0.0 & 0.0 & 0.0 \\
\hline ZNOH 4 ION & $3.65825 \mathrm{E}-24$ & 0.0 & 0.0 & 0.0 \\
\hline ZNOHION & $5.44966 \mathrm{E}-09$ & 0.0 & 0.0 & 0.0 \\
\hline СAHPO4 & 0.0 & $9.76768 \mathrm{E}-05$ & 0.0 & 0.0 \\
\hline $\mathrm{CU} 3 \mathrm{PO} 42.2 \mathrm{H} 2 \mathrm{O}$ & 0.0 & $1.30710 \mathrm{E}-06$ & 0.0 & 0.0 \\
\hline PB3PO4 2 & 0.0 & $8.55122 \mathrm{E}-07$ & 0.0 & 0.0 \\
\hline \multirow[t]{2}{*}{ UIVO2 } & 0.0 & $2.43696 \mathrm{E}-06$ & 0.0 & 0.0 \\
\hline & $===========$ & $===========$ & $===========$ & $===========$ \\
\hline Total g/hr & 62.7838 & 0.025319 & 0.0 & 0.0 \\
\hline Volume, L/hr & 0.0627661 & $4.45404 \mathrm{E}-06$ & 0.0 & 0.0 \\
\hline Enthalpy, cal/hr & $-2.37574 \mathrm{E}+05$ & -81.6577 & 0.0 & 0.0 \\
\hline Density, g/L & 1000.28 & 5684.52 & & \\
\hline Vapor fraction & 0.0 & 0.0 & 0.0 & 0.0 \\
\hline Solid fraction & 0.0 & 1 . & 0.0 & 0.0 \\
\hline Organic fraction & 0.0 & 0.0 & 0.0 & 0.0 \\
\hline Osmotic Pres, atm & 2.43198 & & & \\
\hline Redox Pot, volts & 0.0 & & & \\
\hline E-Con, 1/ohm-cm & 0.0050961 & & & \\
\hline E-Con, cm2/ohm-mol & 94.8793 & & & \\
\hline Abs Visc, cP & 0.90234 & & & \\
\hline Rel Visc & 1.01305 & & & \\
\hline Ionic Strength & 0.0777549 & & & \\
\hline
\end{tabular}


WSRC-TR-2002-00567

Revision 0

ESP V-6. 6

PROCESS:AWE 653
12/05/2002 PAGE 5

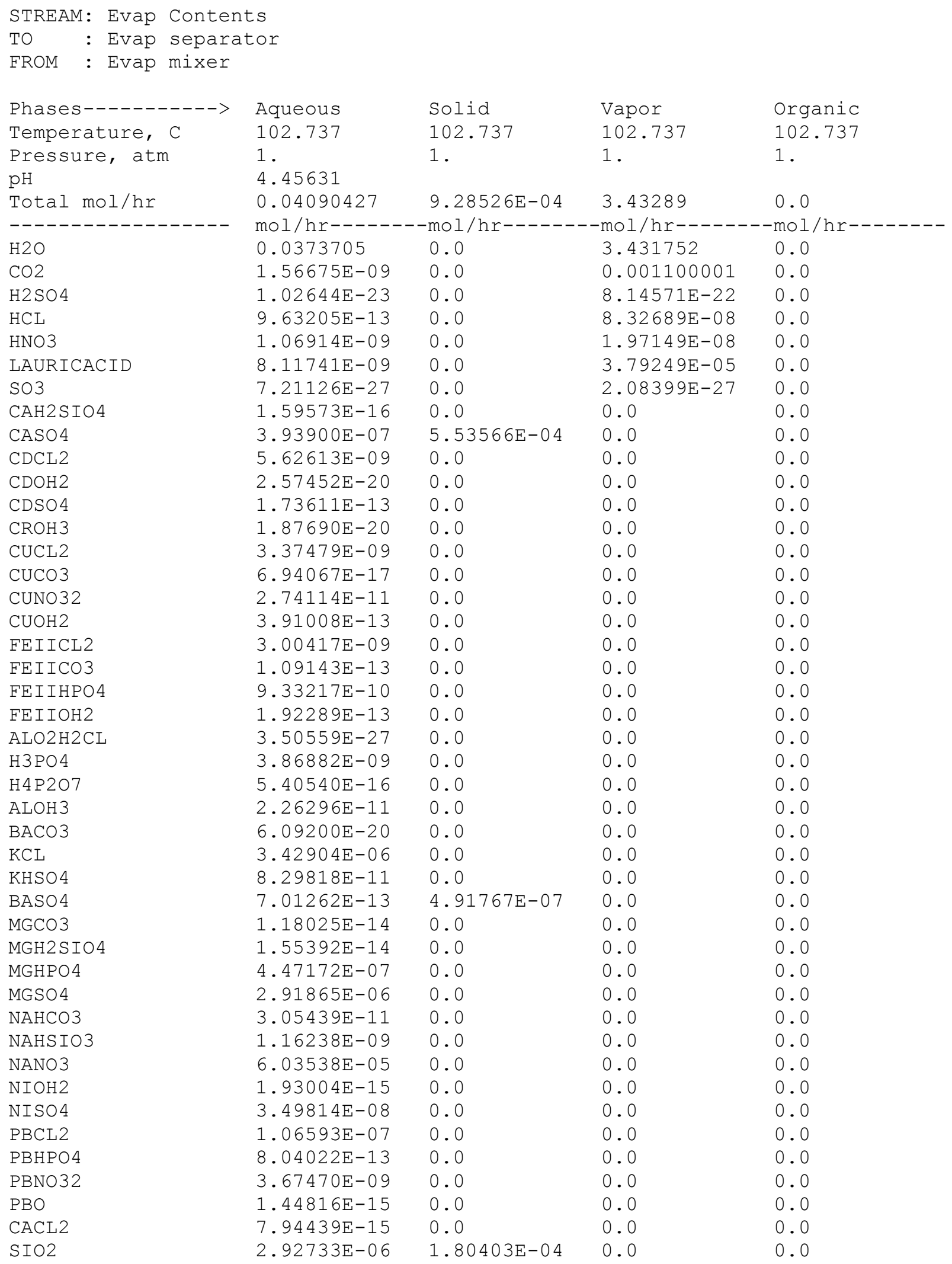




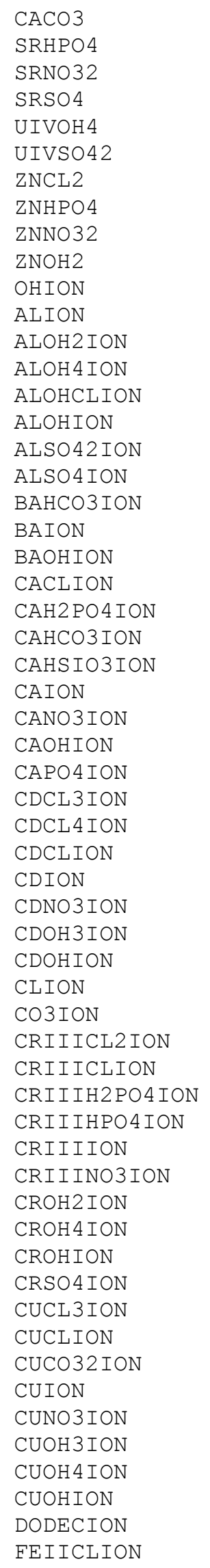

$\begin{array}{ll}3.63819 \mathrm{E}-15 & 0.0 \\ 7.87836 \mathrm{E}-12 & 0.0 \\ 2.42113 \mathrm{E}-07 & 0.0 \\ 6.70979 \mathrm{E}-09 & 1.33598 \mathrm{E}-05 \\ 8.58917 \mathrm{E}-13 & 0.0 \\ 4.83671 \mathrm{E}-24 & 0.0 \\ 5.45529 \mathrm{E}-06 & 0.0 \\ 1.30597 \mathrm{E}-10 & 0.0 \\ 2.76139 \mathrm{E}-09 & 0.0 \\ 9.04633 \mathrm{E}-14 & 0.0 \\ 1.79174 \mathrm{E}-11 & 0.0 \\ 6.25721 \mathrm{E}-13 & 0.0 \\ 1.09638 \mathrm{E}-11 & 0.0 \\ 6.15820 \mathrm{E}-12 & 0.0 \\ 1.78208 \mathrm{E}-11 & 0.0 \\ 5.18750 \mathrm{E}-12 & 0.0 \\ 2.29577 \mathrm{E}-14 & 0.0 \\ 3.26362 \mathrm{E}-13 & 0.0 \\ 3.68087 \mathrm{E}-15 & 0.0 \\ 4.58276 \mathrm{E}-09 & 0.0 \\ 5.73358 \mathrm{E}-17 & 0.0 \\ 1.38789 \mathrm{E}-07 & 0.0 \\ 1.37592 \mathrm{E}-06 & 0.0 \\ 7.57574 \mathrm{E}-12 & 0.0 \\ 5.72952 \mathrm{E}-11 & 0.0 \\ 4.64036 \mathrm{E}-05 & 0.0 \\ 2.36869 \mathrm{E}-05 & 0.0 \\ 1.16947 \mathrm{E}-11 & 0.0 \\ 9.49169 \mathrm{E}-12 & 0.0 \\ 5.31240 \mathrm{E}-09 & 0.0 \\ 1.48220 \mathrm{E}-09 & 0.0 \\ 3.05347 \mathrm{E}-10 & 0.0 \\ 5.99333 \mathrm{E}-12 & 0.0 \\ 1.75702 \mathrm{E}-12 & 0.0 \\ 6.20150 \mathrm{E}-27 & 0.0 \\ 1.99394 \mathrm{E}-16 & 0.0 \\ 0.00151648 & 0.0 \\ 1.05085 \mathrm{E}-15 & 0.0 \\ 1.52229 \mathrm{E}-16 & 0.0 \\ 3.95992 \mathrm{E}-16 & 0.0 \\ 1.09623 \mathrm{E}-16 & 0.0 \\ 6.53843 \mathrm{E}-08 & 0.0 \\ 2.14668 \mathrm{E}-19 & 0.0 \\ 6.05454 \mathrm{E}-14 & 0.0 \\ 2.22428 \mathrm{E}-19 & 0.0 \\ 7.07313 \mathrm{E}-26 & 0.0 \\ 5.78927 \mathrm{E}-14 & 0.0 \\ 2.44928 \mathrm{E}-13 & 0.0 \\ 5.49205 \mathrm{E}-11 & 0.0 \\ 2.00667 \mathrm{E}-08 & 0.0 \\ 5.96967 \mathrm{E}-25 & 0.0 \\ 5.66669 \mathrm{E}-09 & 0.0 \\ 1.05964 \mathrm{E}-09 & 0.0 \\ 5.98229 \mathrm{E}-19 & 0.0 \\ 1.97300 \mathrm{E}-24 & 0.0 \\ 2.16470 \mathrm{E}-11 & 0.0 \\ 5.85553 \mathrm{E}-08 & 0.0 \\ 5.99799 \mathrm{E}-07 & 0.0 \\ & \end{array}$

0.0

0.0

0.0

0.0

0.0

0.0

0.0

0.0

0.0

0.0

0.0

0.0

0.0

0.0

0.0

0.0

0.0

0.0

0.0

0.0

0.0

0.0

0.0

0.0

0.0

0.0

0.0

0.0

0.0

0.0

0.0

0.0

0.0

0.0

0.0

0.0

0.0

0.0

0.0

0.0

0.0

0.0

0.0

0.0

0.0

0.0

0.0

0.0

0.0

0.0

0.0

0.0

0.0

0.0

0.0

0.0

0.0

0.0

0.0 


\begin{tabular}{|c|c|c|c|c|}
\hline FEIICO32ION & $2.78794 \mathrm{E}-23$ & 0.0 & 0.0 & 0.0 \\
\hline FEIIH2PO4ION & $7.09778 \mathrm{E}-08$ & 0.0 & 0.0 & 0.0 \\
\hline FEIIHCO3ION & $2.13231 \mathrm{E}-13$ & 0.0 & 0.0 & 0.0 \\
\hline FEIIION & $7.09998 \mathrm{E}-05$ & 0.0 & 0.0 & 0.0 \\
\hline FEIIOH3ION & $4.48388 \mathrm{E}-18$ & 0.0 & 0.0 & 0.0 \\
\hline FEIIOH 4 ION & $2.07803 E-25$ & 0.0 & 0.0 & 0.0 \\
\hline FEIIOHION & $1.00702 \mathrm{E}-08$ & 0.0 & 0.0 & 0.0 \\
\hline H2P2O7ION & $4.64500 \mathrm{E}-10$ & 0.0 & 0.0 & 0.0 \\
\hline H2 PO 4 ION & $8.55701 \mathrm{E}-07$ & 0.0 & 0.0 & 0.0 \\
\hline H2SIO 4 ION & $1.17191 \mathrm{E}-17$ & 0.0 & 0.0 & 0.0 \\
\hline H3P2O 7 ION & $4.10102 \mathrm{E}-13$ & 0.0 & 0.0 & 0.0 \\
\hline H3SIO 4 ION & $5.12980 \mathrm{E}-10$ & 0.0 & 0.0 & 0.0 \\
\hline HCO3ION & $2.75406 \mathrm{E}-11$ & 0.0 & 0.0 & 0.0 \\
\hline HION & $3.24763 \mathrm{E}-08$ & 0.0 & 0.0 & 0.0 \\
\hline HP2O7ION & $2.47963 \mathrm{E}-11$ & 0.0 & 0.0 & 0.0 \\
\hline HPBO2 ION & $2.45411 \mathrm{E}-20$ & 0.0 & 0.0 & 0.0 \\
\hline HPO4ION & $1.29088 \mathrm{E}-08$ & 0.0 & 0.0 & 0.0 \\
\hline HSO 4 ION & $2.06367 \mathrm{E}-08$ & 0.0 & 0.0 & 0.0 \\
\hline KION & $1.67917 \mathrm{E}-04$ & 0.0 & 0.0 & 0.0 \\
\hline KSO4ION & $1.88841 \mathrm{E}-06$ & 0.0 & 0.0 & 0.0 \\
\hline MGH2 PO 4 ION & $3.03366 \mathrm{E}-06$ & 0.0 & 0.0 & 0.0 \\
\hline MGHCO3ION & $9.10307 \mathrm{E}-11$ & 0.0 & 0.0 & 0.0 \\
\hline MGHS IO3ION & $7.32401 \mathrm{E}-10$ & 0.0 & 0.0 & 0.0 \\
\hline MGION & $1.52423 \mathrm{E}-04$ & 0.0 & 0.0 & 0.0 \\
\hline MGOHION & $6.31977 \mathrm{E}-10$ & 0.0 & 0.0 & 0.0 \\
\hline MGP2O7ION & $8.50307 \mathrm{E}-10$ & 0.0 & 0.0 & 0.0 \\
\hline MGPO 4 ION & $6.20806 \mathrm{E}-11$ & 0.0 & 0.0 & 0.0 \\
\hline NACO3ION & $1.28421 \mathrm{E}-16$ & 0.0 & 0.0 & 0.0 \\
\hline NAION & 0.00111766 & 0.0 & 0.0 & 0.0 \\
\hline NASO 4 ION & $7.68611 \mathrm{E}-14$ & 0.0 & 0.0 & 0.0 \\
\hline NICLION & $6.82402 \mathrm{E}-08$ & 0.0 & 0.0 & 0.0 \\
\hline NIION & $8.60422 \mathrm{E}-07$ & 0.0 & 0.0 & 0.0 \\
\hline NINO3ION & $1.26614 \mathrm{E}-07$ & 0.0 & 0.0 & 0.0 \\
\hline NIOH3ION & $7.30731 \mathrm{E}-21$ & 0.0 & 0.0 & 0.0 \\
\hline NIOHION & $3.21897 \mathrm{E}-11$ & 0.0 & 0.0 & 0.0 \\
\hline NO3ION & $3.17698 \mathrm{E}-04$ & 0.0 & 0.0 & 0.0 \\
\hline P207ION & $6.16455 \mathrm{E}-15$ & 0.0 & 0.0 & 0.0 \\
\hline PBCL3ION & $2.74897 \mathrm{E}-07$ & 0.0 & 0.0 & 0.0 \\
\hline PBCL 4 ION & $2.15131 \mathrm{E}-06$ & 0.0 & 0.0 & 0.0 \\
\hline PBCLION & $2.85015 \mathrm{E}-08$ & 0.0 & 0.0 & 0.0 \\
\hline PBH2 PO 4 ION & $2.97954 \mathrm{E}-11$ & 0.0 & 0.0 & 0.0 \\
\hline PBION & $8.90113 \mathrm{E}-10$ & 0.0 & 0.0 & 0.0 \\
\hline PBNO33ION & $1.53876 \mathrm{E}-10$ & 0.0 & 0.0 & 0.0 \\
\hline PBNO3ION & $8.80386 \mathrm{E}-09$ & 0.0 & 0.0 & 0.0 \\
\hline PBOHION & $2.39698 \mathrm{E}-11$ & 0.0 & 0.0 & 0.0 \\
\hline PO4ION & $4.65813 \mathrm{E}-15$ & 0.0 & 0.0 & 0.0 \\
\hline SO4ION & $1.06528 \mathrm{E}-05$ & 0.0 & 0.0 & 0.0 \\
\hline SRION & $4.80009 \mathrm{E}-07$ & 0.0 & 0.0 & 0.0 \\
\hline SRNO3ION & $7.51546 \mathrm{E}-07$ & 0.0 & 0.0 & 0.0 \\
\hline SROHION & $8.54009 \mathrm{E}-14$ & 0.0 & 0.0 & 0.0 \\
\hline SRPO 4 ION & $2.24996 \mathrm{E}-15$ & 0.0 & 0.0 & 0.0 \\
\hline UIVCLION & $9.70065 \mathrm{E}-26$ & 0.0 & 0.0 & 0.0 \\
\hline UIVION & $2.09133 E-27$ & 0.0 & 0.0 & 0.0 \\
\hline UIVOH2ION & $2.45045 \mathrm{E}-20$ & 0.0 & 0.0 & 0.0 \\
\hline UIVOH3ION & $9.46804 \mathrm{E}-18$ & 0.0 & 0.0 & 0.0 \\
\hline UIVOH5ION & $1.23304 \mathrm{E}-17$ & 0.0 & 0.0 & 0.0 \\
\hline UIVOHION & $3.74558 \mathrm{E}-22$ & 0.0 & 0.0 & 0.0 \\
\hline UIVSO 4 ION & $8.50349 \mathrm{E}-24$ & 0.0 & 0.0 & 0.0 \\
\hline
\end{tabular}


WSRC-TR-2002-00567

Revision 0

\begin{tabular}{|c|c|c|c|c|}
\hline ZNCL3ION & $8.14736 \mathrm{E}-06$ & 0.0 & 0.0 & 0.0 \\
\hline ZNCLION & $1.15744 \mathrm{E}-05$ & 0.0 & 0.0 & 0.0 \\
\hline ZNH2 PO4 ION & $2.35833 E-09$ & 0.0 & 0.0 & 0.0 \\
\hline ZNHCO3ION & $5.71860 \mathrm{E}-14$ & 0.0 & 0.0 & 0.0 \\
\hline ZNION & $7.22646 \mathrm{E}-07$ & 0.0 & 0.0 & 0.0 \\
\hline ZNNO3ION & $8.13582 \mathrm{E}-08$ & 0.0 & 0.0 & 0.0 \\
\hline ZNOH3ION & $6.16939 \mathrm{E}-18$ & 0.0 & 0.0 & 0.0 \\
\hline ZNOH 4 ION & $7.17781 \mathrm{E}-24$ & 0.0 & 0.0 & 0.0 \\
\hline ZNOHION & $7.56697 \mathrm{E}-09$ & 0.0 & 0.0 & 0.0 \\
\hline ALPO4 & 0.0 & $3.20810 E-05$ & 0.0 & 0.0 \\
\hline ALOOH & 0.0 & $4.56967 E-05$ & 0.0 & 0.0 \\
\hline $\mathrm{CA} 3 \mathrm{PO} 42$ & 0.0 & $5.74802 \mathrm{E}-05$ & 0.0 & 0.0 \\
\hline $\mathrm{CU} 3 \mathrm{PO} 42.2 \mathrm{H} 2 \mathrm{O}$ & 0.0 & $1.30225 E-06$ & 0.0 & 0.0 \\
\hline MG3PO4 2 & 0.0 & $4.17085 \mathrm{E}-05$ & 0.0 & 0.0 \\
\hline \multirow[t]{2}{*}{ UIVO2 } & 0.0 & $2.43698 E-06$ & 0.0 & 0.0 \\
\hline & $===========$ & $===========$ & $===========$ & $===========$ \\
\hline Total g/hr & 0.803492 & 0.125418 & 61.8802 & 0.0 \\
\hline Volume, L/hr & $7.52271 \mathrm{E}-04$ & $3.90519 \mathrm{E}-05$ & 105.018 & 0.0 \\
\hline Enthalpy, cal/hr & -2697.17 & -351.817 & $-1.96356 \mathrm{E}+05$ & 0.0 \\
\hline Density, g/L & 1068.09 & 3211.57 & 0.589236 & \\
\hline Vapor fraction & 0.0 & 0.0 & 1 . & 0.0 \\
\hline Solid fraction & 0.0 & 1 . & 0.0 & 0.0 \\
\hline Organic fraction & 0.0 & 0.0 & 0.0 & 0.0 \\
\hline Osmotic Pres, atm & 156.67 & & & \\
\hline Redox Pot, volts & 0.0 & & & \\
\hline E-Con, 1/ohm-cm & 0.416283 & & & \\
\hline E-Con, cm2/ohm-mol & 51.041 & & & \\
\hline Abs Visc, cP & 0.375384 & & & \\
\hline Rel Visc & 1.37159 & & & \\
\hline Ionic Strength & 3.20213 & & & \\
\hline
\end{tabular}


WSRC-TR-2002-00567

Revision 0

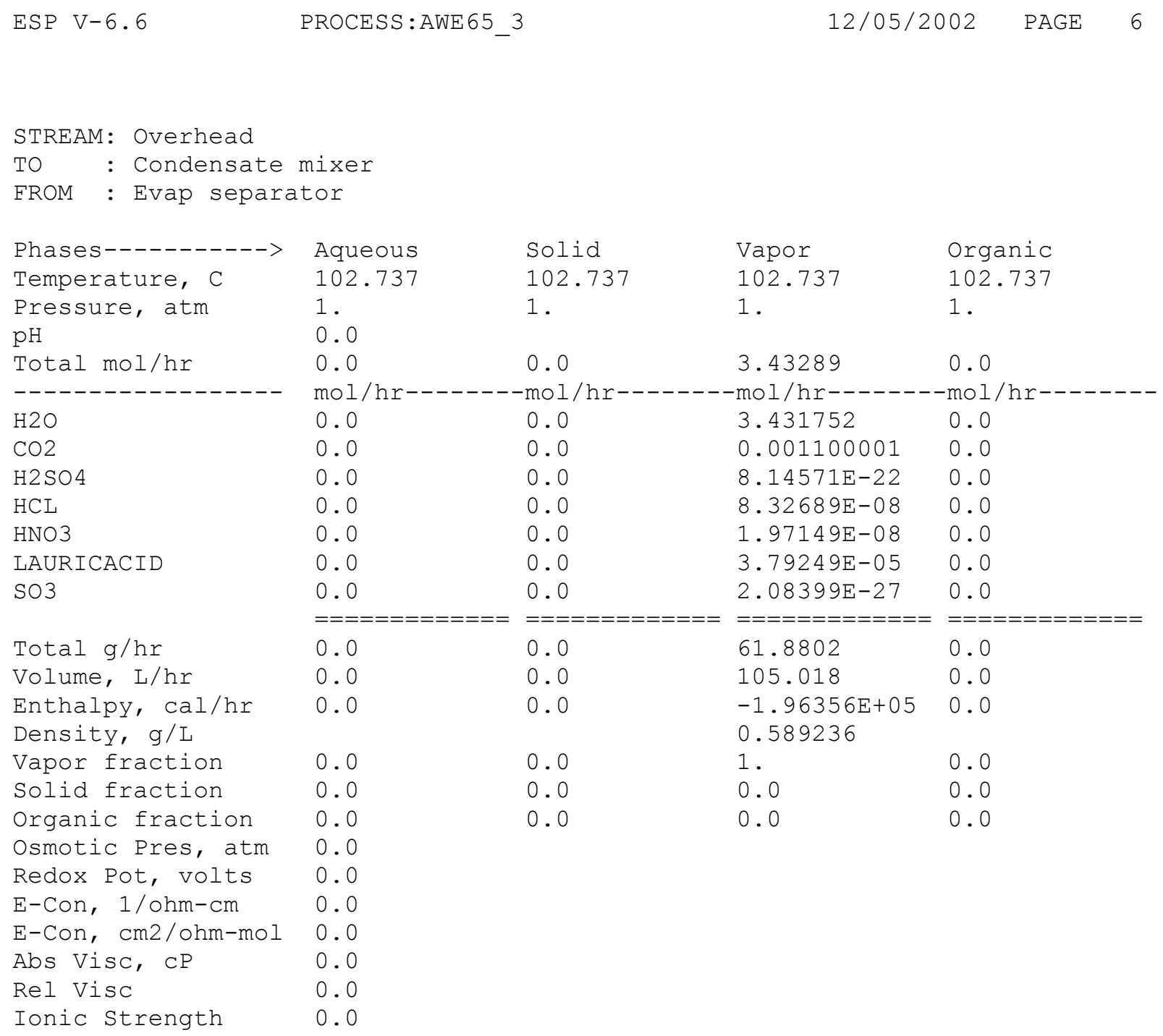


WSRC-TR-2002-00567

Revision 0

$\begin{array}{lllll}\text { ESP V-6.6 PROCESS:AWE65_3 } & \text { PAGE } 7\end{array}$

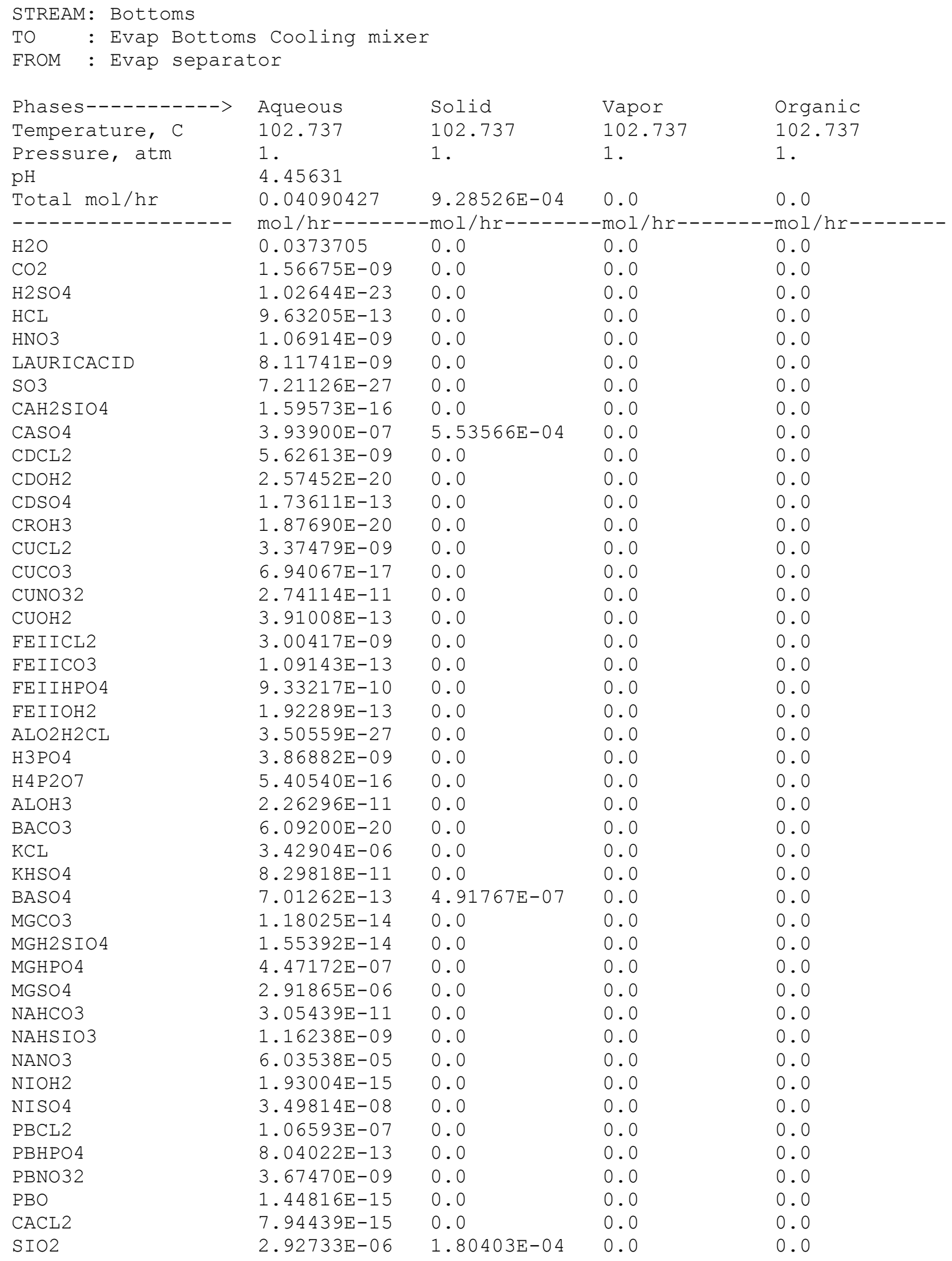




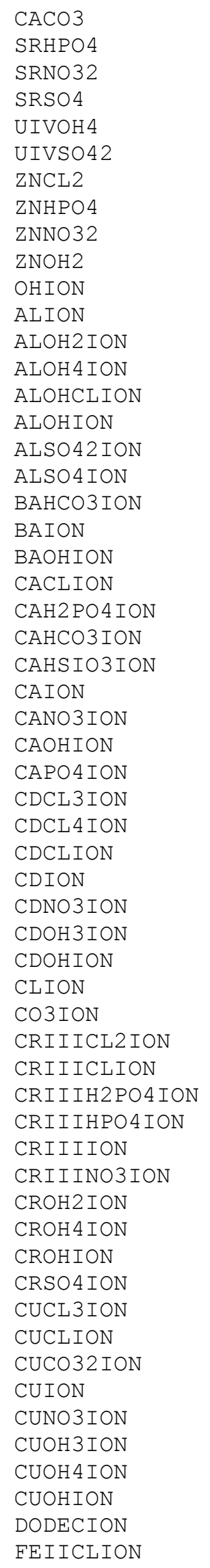

$\begin{array}{ll}3.63819 \mathrm{E}-15 & 0.0 \\ 7.87836 \mathrm{E}-12 & 0.0 \\ 2.42113 \mathrm{E}-07 & 0.0 \\ 6.70979 \mathrm{E}-09 & 1.33598 \mathrm{E}-05 \\ 8.58917 \mathrm{E}-13 & 0.0 \\ 4.83671 \mathrm{E}-24 & 0.0 \\ 5.45529 \mathrm{E}-06 & 0.0 \\ 1.30597 \mathrm{E}-10 & 0.0 \\ 2.76139 \mathrm{E}-09 & 0.0 \\ 9.04633 \mathrm{E}-14 & 0.0 \\ 1.79174 \mathrm{E}-11 & 0.0 \\ 6.25721 \mathrm{E}-13 & 0.0 \\ 1.09638 \mathrm{E}-11 & 0.0 \\ 6.15820 \mathrm{E}-12 & 0.0 \\ 1.78208 \mathrm{E}-11 & 0.0 \\ 5.18750 \mathrm{E}-12 & 0.0 \\ 2.29577 \mathrm{E}-14 & 0.0 \\ 3.26362 \mathrm{E}-13 & 0.0 \\ 3.68087 \mathrm{E}-15 & 0.0 \\ 4.58276 \mathrm{E}-09 & 0.0 \\ 5.73358 \mathrm{E}-17 & 0.0 \\ 1.38789 \mathrm{E}-07 & 0.0 \\ 1.37592 \mathrm{E}-06 & 0.0 \\ 7.57574 \mathrm{E}-12 & 0.0 \\ 5.72952 \mathrm{E}-11 & 0.0 \\ 4.64036 \mathrm{E}-05 & 0.0 \\ 2.36869 \mathrm{E}-05 & 0.0 \\ 1.16947 \mathrm{E}-11 & 0.0 \\ 9.49169 \mathrm{E}-12 & 0.0 \\ 5.31240 \mathrm{E}-09 & 0.0 \\ 1.48220 \mathrm{E}-09 & 0.0 \\ 3.05347 \mathrm{E}-10 & 0.0 \\ 5.99333 \mathrm{E}-12 & 0.0 \\ 1.75702 \mathrm{E}-12 & 0.0 \\ 6.20150 \mathrm{E}-27 & 0.0 \\ 1.99394 \mathrm{E}-16 & 0.0 \\ 0.00151648 & 0.0 \\ 1.05085 \mathrm{E}-15 & 0.0 \\ 1.52229 \mathrm{E}-16 & 0.0 \\ 3.95992 \mathrm{E}-16 & 0.0 \\ 1.09623 \mathrm{E}-16 & 0.0 \\ 6.53843 \mathrm{E}-08 & 0.0 \\ 2.14668 \mathrm{E}-19 & 0.0 \\ 6.05454 \mathrm{E}-14 & 0.0 \\ 2.22428 \mathrm{E}-19 & 0.0 \\ 7.07313 \mathrm{E}-26 & 0.0 \\ 5.78927 \mathrm{E}-14 & 0.0 \\ 2.44928 \mathrm{E}-13 & 0.0 \\ 5.49205 \mathrm{E}-11 & 0.0 \\ 2.00667 \mathrm{E}-08 & 0.0 \\ 5.96967 \mathrm{E}-25 & 0.0 \\ 5.66669 \mathrm{E}-09 & 0.0 \\ 1.05964 \mathrm{E}-09 & 0.0 \\ 5.98229 \mathrm{E}-19 & 0.0 \\ 1.97300 \mathrm{E}-24 & 0.0 \\ 2.16470 \mathrm{E}-11 & 0.0 \\ 5.85553 \mathrm{E}-08 & 0.0 \\ 5.99799 \mathrm{E}-07 & 0.0 \\ & \end{array}$

0.0

0.0

0.0

0.0

0.0

0.0

0.0

0.0

0.0

0.0

0.0

0.0

0.0

0.0

0.0

0.0

0.0

0.0

0.0

0.0

0.0

0.0

0.0

0.0

0.0

0.0

0.0

0.0

0.0

0.0

0.0

0.0

0.0

0.0

0.0

0.0

0.0

0.0

0.0

0.0

0.0

0.0

0.0

0.0

0.0

0.0

0.0

0.0

0.0

0.0

0.0

0.0

0.0

0.0

0.0

0.0

0.0

0.0

0.0 


\begin{tabular}{|c|c|c|c|c|}
\hline FEIICO32ION & $2.78794 \mathrm{E}-23$ & 0.0 & 0.0 & 0.0 \\
\hline FEIIH2PO4ION & $7.09778 \mathrm{E}-08$ & 0.0 & 0.0 & 0.0 \\
\hline EEIIHCO3ION & $2.13231 \mathrm{E}-13$ & 0.0 & 0.0 & 0.0 \\
\hline FEIIION & $7.09998 \mathrm{E}-05$ & 0.0 & 0.0 & 0.0 \\
\hline FEIIOH3ION & $4.48388 \mathrm{E}-18$ & 0.0 & 0.0 & 0.0 \\
\hline FEIIOH 4 ION & $2.07803 E-25$ & 0.0 & 0.0 & 0.0 \\
\hline FEIIOHION & $1.00702 \mathrm{E}-08$ & 0.0 & 0.0 & 0.0 \\
\hline H2 P2O 7 ION & $4.64500 \mathrm{E}-10$ & 0.0 & 0.0 & 0.0 \\
\hline H2 PO 4 ION & $8.55701 \mathrm{E}-07$ & 0.0 & 0.0 & 0.0 \\
\hline $\mathrm{H} 2 \mathrm{SIO} 4 \mathrm{ION}$ & $1.17191 \mathrm{E}-17$ & 0.0 & 0.0 & 0.0 \\
\hline H3P2O7 ION & $4.10102 \mathrm{E}-13$ & 0.0 & 0.0 & 0.0 \\
\hline H3SIO 4 ION & $5.12980 \mathrm{E}-10$ & 0.0 & 0.0 & 0.0 \\
\hline HCO3ION & $2.75406 \mathrm{E}-11$ & 0.0 & 0.0 & 0.0 \\
\hline HION & $3.24763 \mathrm{E}-08$ & 0.0 & 0.0 & 0.0 \\
\hline HP207ION & $2.47963 \mathrm{E}-11$ & 0.0 & 0.0 & 0.0 \\
\hline HPBO2ION & $2.45411 \mathrm{E}-20$ & 0.0 & 0.0 & 0.0 \\
\hline HPO4ION & $1.29088 \mathrm{E}-08$ & 0.0 & 0.0 & 0.0 \\
\hline $\mathrm{HSO} 4 \mathrm{ION}$ & $2.06367 \mathrm{E}-08$ & 0.0 & 0.0 & 0.0 \\
\hline KION & $1.67917 \mathrm{E}-04$ & 0.0 & 0.0 & 0.0 \\
\hline KSO4 ION & $1.88841 \mathrm{E}-06$ & 0.0 & 0.0 & 0.0 \\
\hline MGH2 PO 4 ION & $3.03366 \mathrm{E}-06$ & 0.0 & 0.0 & 0.0 \\
\hline MGHCO3ION & $9.10307 \mathrm{E}-11$ & 0.0 & 0.0 & 0.0 \\
\hline MGHSIO3ION & $7.32401 \mathrm{E}-10$ & 0.0 & 0.0 & 0.0 \\
\hline MGION & $1.52423 \mathrm{E}-04$ & 0.0 & 0.0 & 0.0 \\
\hline MGOHION & $6.31977 \mathrm{E}-10$ & 0.0 & 0.0 & 0.0 \\
\hline MGP2O7ION & $8.50307 \mathrm{E}-10$ & 0.0 & 0.0 & 0.0 \\
\hline MGPO 4 ION & $6.20806 \mathrm{E}-11$ & 0.0 & 0.0 & 0.0 \\
\hline NACO3ION & $1.28421 \mathrm{E}-16$ & 0.0 & 0.0 & 0.0 \\
\hline NAION & 0.00111766 & 0.0 & 0.0 & 0.0 \\
\hline NASO 4 ION & $7.68611 \mathrm{E}-14$ & 0.0 & 0.0 & 0.0 \\
\hline NICLION & $6.82402 E-08$ & 0.0 & 0.0 & 0.0 \\
\hline NIION & $8.60422 \mathrm{E}-07$ & 0.0 & 0.0 & 0.0 \\
\hline NINO3ION & $1.26614 \mathrm{E}-07$ & 0.0 & 0.0 & 0.0 \\
\hline NIOH3ION & $7.30731 \mathrm{E}-21$ & 0.0 & 0.0 & 0.0 \\
\hline NIOHION & $3.21897 \mathrm{E}-11$ & 0.0 & 0.0 & 0.0 \\
\hline NO3ION & $3.17698 \mathrm{E}-04$ & 0.0 & 0.0 & 0.0 \\
\hline P207ION & $6.16455 \mathrm{E}-15$ & 0.0 & 0.0 & 0.0 \\
\hline PBCL3ION & $2.74897 \mathrm{E}-07$ & 0.0 & 0.0 & 0.0 \\
\hline PBCL 4 ION & $2.15131 \mathrm{E}-06$ & 0.0 & 0.0 & 0.0 \\
\hline PBCLION & $2.85015 \mathrm{E}-08$ & 0.0 & 0.0 & 0.0 \\
\hline PBH2 PO 4 ION & $2.97954 \mathrm{E}-11$ & 0.0 & 0.0 & 0.0 \\
\hline PBION & $8.90113 E-10$ & 0.0 & 0.0 & 0.0 \\
\hline PBNO33ION & $1.53876 \mathrm{E}-10$ & 0.0 & 0.0 & 0.0 \\
\hline PBNO3ION & $8.80386 \mathrm{E}-09$ & 0.0 & 0.0 & 0.0 \\
\hline PBOHION & $2.39698 \mathrm{E}-11$ & 0.0 & 0.0 & 0.0 \\
\hline PO4ION & $4.65813 \mathrm{E}-15$ & 0.0 & 0.0 & 0.0 \\
\hline SO4ION & $1.06528 \mathrm{E}-05$ & 0.0 & 0.0 & 0.0 \\
\hline SRION & $4.80009 \mathrm{E}-07$ & 0.0 & 0.0 & 0.0 \\
\hline SRNO3ION & $7.51546 \mathrm{E}-07$ & 0.0 & 0.0 & 0.0 \\
\hline SROHION & $8.54009 \mathrm{E}-14$ & 0.0 & 0.0 & 0.0 \\
\hline SRPO 4 ION & $2.24996 \mathrm{E}-15$ & 0.0 & 0.0 & 0.0 \\
\hline UIVCLION & $9.70065 \mathrm{E}-26$ & 0.0 & 0.0 & 0.0 \\
\hline UIVION & $2.09133 E-27$ & 0.0 & 0.0 & 0.0 \\
\hline UIVOH2ION & $2.45045 E-20$ & 0.0 & 0.0 & 0.0 \\
\hline UIVOH3ION & $9.46804 \mathrm{E}-18$ & 0.0 & 0.0 & 0.0 \\
\hline UIVOH5ION & $1.23304 \mathrm{E}-17$ & 0.0 & 0.0 & 0.0 \\
\hline UIVOHION & $3.74558 \mathrm{E}-22$ & 0.0 & 0.0 & 0.0 \\
\hline UIVSO 4 ION & $8.50349 \mathrm{E}-24$ & 0.0 & 0.0 & 0.0 \\
\hline
\end{tabular}


WSRC-TR-2002-00567

Revision 0

\begin{tabular}{|c|c|c|c|c|}
\hline ZNCL3ION & $8.14736 \mathrm{E}-06$ & 0.0 & 0.0 & 0.0 \\
\hline ZNCLION & $1.15744 \mathrm{E}-05$ & 0.0 & 0.0 & 0.0 \\
\hline ZNH2 PO4 ION & $2.35833 E-09$ & 0.0 & 0.0 & 0.0 \\
\hline ZNHCO3ION & $5.71860 \mathrm{E}-14$ & 0.0 & 0.0 & 0.0 \\
\hline ZNION & $7.22646 \mathrm{E}-07$ & 0.0 & 0.0 & 0.0 \\
\hline ZNNO3ION & $8.13582 \mathrm{E}-08$ & 0.0 & 0.0 & 0.0 \\
\hline ZNOH3ION & $6.16939 \mathrm{E}-18$ & 0.0 & 0.0 & 0.0 \\
\hline ZNOH 4 ION & $7.17781 \mathrm{E}-24$ & 0.0 & 0.0 & 0.0 \\
\hline ZNOHION & $7.56697 \mathrm{E}-09$ & 0.0 & 0.0 & 0.0 \\
\hline ALPO4 & 0.0 & $3.20810 E-05$ & 0.0 & 0.0 \\
\hline ALOOH & 0.0 & $4.56967 E-05$ & 0.0 & 0.0 \\
\hline $\mathrm{CA} 3 \mathrm{PO} 42$ & 0.0 & $5.74802 \mathrm{E}-05$ & 0.0 & 0.0 \\
\hline $\mathrm{CU} 3 \mathrm{PO} 42.2 \mathrm{H} 2 \mathrm{O}$ & 0.0 & $1.30225 E-06$ & 0.0 & 0.0 \\
\hline MG3PO4 2 & 0.0 & $4.17085 \mathrm{E}-05$ & 0.0 & 0.0 \\
\hline \multirow[t]{2}{*}{ UIVO2 } & 0.0 & $2.43698 E-06$ & 0.0 & 0.0 \\
\hline & $===========$ & $===========$ & $===========$ & $===========$ \\
\hline Total g/hr & 0.803492 & 0.125418 & 0.0 & 0.0 \\
\hline Volume, L/hr & $7.52271 \mathrm{E}-04$ & $3.90519 \mathrm{E}-05$ & 0.0 & 0.0 \\
\hline Enthalpy, cal/hr & -2697.17 & -351.817 & 0.0 & 0.0 \\
\hline Density, g/L & 1068.09 & 3211.57 & & \\
\hline Vapor fraction & 0.0 & 0.0 & 0.0 & 0.0 \\
\hline Solid fraction & 0.0 & 1 . & 0.0 & 0.0 \\
\hline Organic fraction & 0.0 & 0.0 & 0.0 & 0.0 \\
\hline Osmotic Pres, atm & 156.67 & & & \\
\hline Redox Pot, volts & 0.0 & & & \\
\hline E-Con, 1/ohm-cm & 0.416283 & & & \\
\hline E-Con, cm2/ohm-mol & 51.041 & & & \\
\hline Abs Visc, cP & 0.375384 & & & \\
\hline Rel Visc & 1.37159 & & & \\
\hline Ionic Strength & 3.20213 & & & \\
\hline
\end{tabular}


WSRC-TR-2002-00567

Revision 0

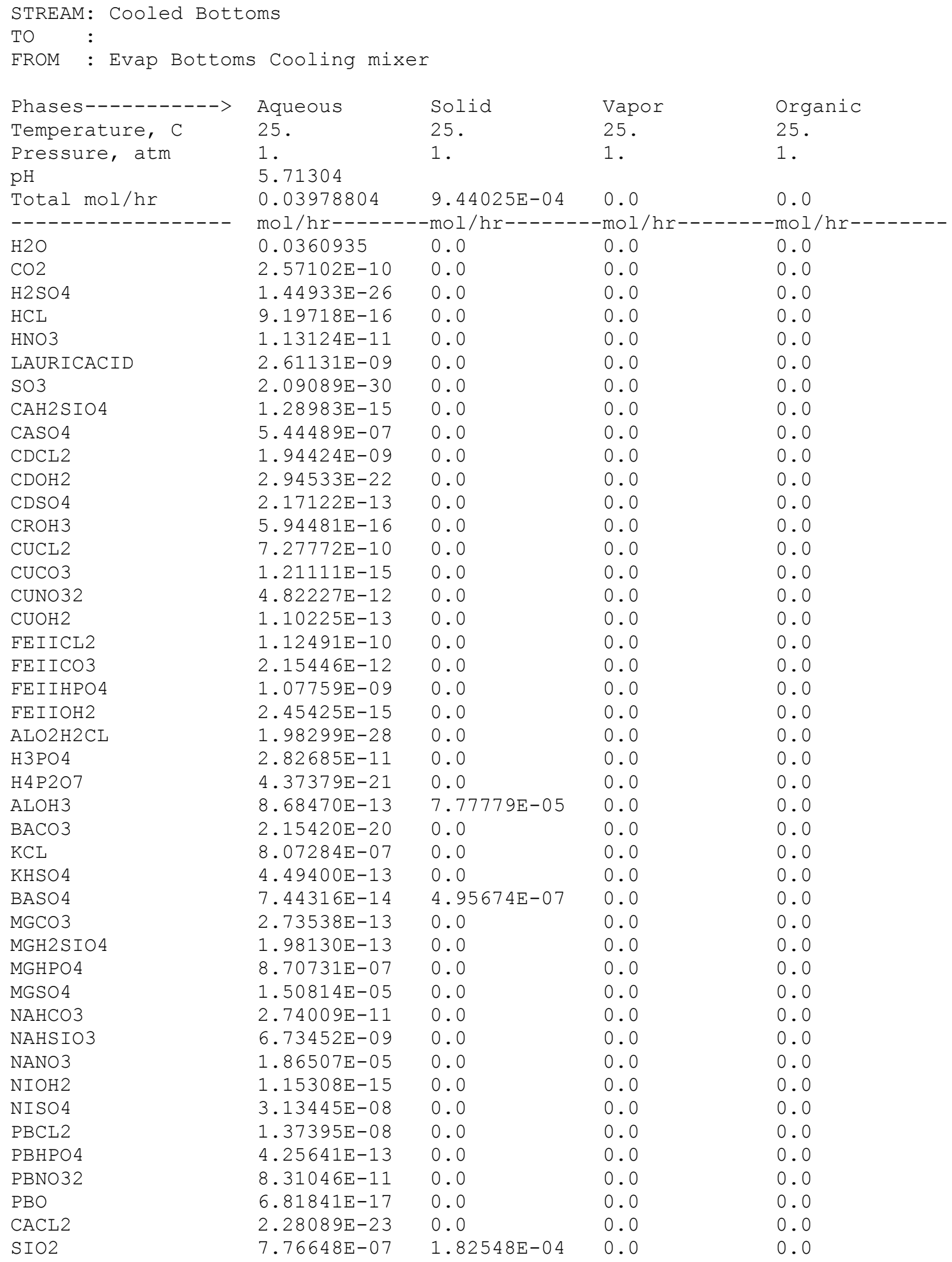




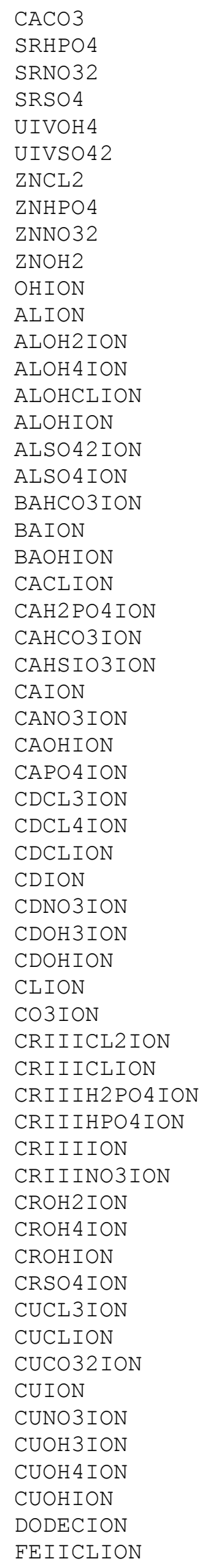

\begin{tabular}{|c|c|}
\hline $3.81452 \mathrm{E}-14$ & 0.0 \\
\hline $4.57694 \mathrm{E}-12$ & 0.0 \\
\hline $3.46769 \mathrm{E}-08$ & 0.0 \\
\hline $1.55955 \mathrm{E}-08$ & $1.39321 \mathrm{E}-05$ \\
\hline $8.79825 E-14$ & 0.0 \\
\hline $1.27264 \mathrm{E}-26$ & 0.0 \\
\hline $1.68603 \mathrm{E}-06$ & 0.0 \\
\hline $3.34107 \mathrm{E}-09$ & 0.0 \\
\hline $1.96562 \mathrm{E}-08$ & 0.0 \\
\hline $2.06520 \mathrm{E}-13$ & 0.0 \\
\hline $3.98303 E-12$ & 0.0 \\
\hline $4.57005 \mathrm{E}-13$ & 0.0 \\
\hline $8.68536 \mathrm{E}-13$ & 0.0 \\
\hline $3.38644 \mathrm{E}-13$ & 0.0 \\
\hline $4.56846 \mathrm{E}-12$ & 0.0 \\
\hline $7.23010 \mathrm{E}-13$ & 0.0 \\
\hline $8.80028 \mathrm{E}-14$ & 0.0 \\
\hline $4.01498 \mathrm{E}-13$ & 0.0 \\
\hline $8.17345 \mathrm{E}-17$ & 0.0 \\
\hline $6.76319 \mathrm{E}-10$ & 0.0 \\
\hline $9.19055 \mathrm{E}-20$ & 0.0 \\
\hline $1.81335 \mathrm{E}-10$ & 0.0 \\
\hline $2.04082 \mathrm{E}-07$ & 0.0 \\
\hline $2.66544 \mathrm{E}-11$ & 0.0 \\
\hline $4.45914 \mathrm{E}-11$ & 0.0 \\
\hline $3.94833 E-05$ & 0.0 \\
\hline $1.83685 \mathrm{E}-05$ & 0.0 \\
\hline $1.00670 \mathrm{E}-12$ & 0.0 \\
\hline $1.59594 \mathrm{E}-10$ & 0.0 \\
\hline $8.03391 \mathrm{E}-10$ & 0.0 \\
\hline $9.86787 \mathrm{E}-09$ & 0.0 \\
\hline $1.13064 \mathrm{E}-10$ & 0.0 \\
\hline $4.44687 \mathrm{E}-12$ & 0.0 \\
\hline $7.69334 \mathrm{E}-13$ & 0.0 \\
\hline $7.41373 E-29$ & 0.0 \\
\hline $1.99739 \mathrm{E}-17$ & 0.0 \\
\hline 0.00155643 & 0.0 \\
\hline $4.51588 \mathrm{E}-14$ & 0.0 \\
\hline $8.82346 \mathrm{E}-14$ & 0.0 \\
\hline $1.40799 \mathrm{E}-13$ & 0.0 \\
\hline $2.85403 E-12$ & 0.0 \\
\hline $6.49888 \mathrm{E}-08$ & 0.0 \\
\hline $2.55665 \mathrm{E}-16$ & 0.0 \\
\hline $1.55460 \mathrm{E}-11$ & 0.0 \\
\hline $9.57392 \mathrm{E}-16$ & 0.0 \\
\hline $1.23722 \mathrm{E}-20$ & 0.0 \\
\hline 1. $34400 \mathrm{E}-10$ & 0.0 \\
\hline $2.42793 \mathrm{E}-10$ & 0.0 \\
\hline $1.30317 \mathrm{E}-11$ & 0.0 \\
\hline $1.97972 \mathrm{E}-09$ & 0.0 \\
\hline $2.59999 \mathrm{E}-22$ & 0.0 \\
\hline $1.68924 \mathrm{E}-09$ & 0.0 \\
\hline $3.39643 \mathrm{E}-10$ & 0.0 \\
\hline $1.03329 \mathrm{E}-19$ & 0.0 \\
\hline $3.75100 \mathrm{E}-26$ & 0.0 \\
\hline $3.31096 \mathrm{E}-12$ & 0.0 \\
\hline $6.40614 \mathrm{E}-08$ & 0.0 \\
\hline $3.78340 \mathrm{E}-08$ & 0.0 \\
\hline
\end{tabular}

0.0

0.0

0.0

0.0

0.0

0.0

0.0

0.0

0.0

0.0

0.0

0.0

0.0

0.0

0.0

0.0

0.0

0.0

0.0

0.0

0.0

0.0

0.0

0.0

0.0

0.0

0.0

0.0

0.0

0.0

0.0

0.0

0.0

0.0

0.0

0.0

0.0

0.0

0.0

0.0

0.0

0.0

0.0

0.0

0.0

0.0

0.0

0.0

0.0

0.0

0.0

0.0

0.0

0.0

0.0

0.0

0.0

0.0

0.0 


\begin{tabular}{|c|c|c|c|c|}
\hline FEIICO32ION & $9.83131 \mathrm{E}-21$ & 0.0 & 0.0 & 0.0 \\
\hline FEIIH2PO4ION & $1.64612 \mathrm{E}-08$ & 0.0 & 0.0 & 0.0 \\
\hline EEIIHCO3ION & $2.72513 \mathrm{E}-13$ & 0.0 & 0.0 & 0.0 \\
\hline FEIIION & $2.52134 \mathrm{E}-05$ & 0.0 & 0.0 & 0.0 \\
\hline FEIIOH3ION & $1.21522 \mathrm{E}-19$ & 0.0 & 0.0 & 0.0 \\
\hline FEIIOH 4 ION & $8.26999 \mathrm{E}-28$ & 0.0 & 0.0 & 0.0 \\
\hline FEIIOHION & $1.68095 \mathrm{E}-09$ & 0.0 & 0.0 & 0.0 \\
\hline H2 P2O 7 ION & $4.55328 \mathrm{E}-12$ & 0.0 & 0.0 & 0.0 \\
\hline H2 PO 4 ION & $3.40821 \mathrm{E}-07$ & 0.0 & 0.0 & 0.0 \\
\hline $\mathrm{H} 2 \mathrm{SIO} 4 \mathrm{ION}$ & $5.17781 \mathrm{E}-17$ & 0.0 & 0.0 & 0.0 \\
\hline H3P2O7 ION & $1.28355 \mathrm{E}-16$ & 0.0 & 0.0 & 0.0 \\
\hline H3SIO 4 ION & $8.85165 \mathrm{E}-11$ & 0.0 & 0.0 & 0.0 \\
\hline HCO3ION & $1.78002 \mathrm{E}-10$ & 0.0 & 0.0 & 0.0 \\
\hline HION & $1.13910 \mathrm{E}-09$ & 0.0 & 0.0 & 0.0 \\
\hline HP207ION & $7.55724 \mathrm{E}-12$ & 0.0 & 0.0 & 0.0 \\
\hline HPBO2ION & $1.05889 \mathrm{E}-21$ & 0.0 & 0.0 & 0.0 \\
\hline HPO4ION & $1.26441 \mathrm{E}-07$ & 0.0 & 0.0 & 0.0 \\
\hline $\mathrm{HSO} 4 \mathrm{ION}$ & $3.84568 \mathrm{E}-10$ & 0.0 & 0.0 & 0.0 \\
\hline KION & $1.68830 \mathrm{E}-04$ & 0.0 & 0.0 & 0.0 \\
\hline KSO4 ION & $3.59769 \mathrm{E}-06$ & 0.0 & 0.0 & 0.0 \\
\hline MGH2 PO 4 ION & $4.16790 \mathrm{E}-06$ & 0.0 & 0.0 & 0.0 \\
\hline MGHCO3ION & $1.22942 \mathrm{E}-09$ & 0.0 & 0.0 & 0.0 \\
\hline MGHSIO3ION & $1.30930 \mathrm{E}-09$ & 0.0 & 0.0 & 0.0 \\
\hline MGION & $2.63824 \mathrm{E}-04$ & 0.0 & 0.0 & 0.0 \\
\hline MGOHION & $1.53297 \mathrm{E}-10$ & 0.0 & 0.0 & 0.0 \\
\hline MGP2O7ION & $8.71039 \mathrm{E}-10$ & 0.0 & 0.0 & 0.0 \\
\hline MGPO 4 ION & $2.80692 \mathrm{E}-09$ & 0.0 & 0.0 & 0.0 \\
\hline NACO3ION & $1.69043 \mathrm{E}-14$ & 0.0 & 0.0 & 0.0 \\
\hline NAION & 0.00114698 & 0.0 & 0.0 & 0.0 \\
\hline NASO 4 ION & $1.23821 \mathrm{E}-05$ & 0.0 & 0.0 & 0.0 \\
\hline NICLION & $3.19531 \mathrm{E}-08$ & 0.0 & 0.0 & 0.0 \\
\hline NIION & $8.14045 \mathrm{E}-07$ & 0.0 & 0.0 & 0.0 \\
\hline NINO3ION & $2.12939 \mathrm{E}-07$ & 0.0 & 0.0 & 0.0 \\
\hline NIOH3ION & $2.12794 \mathrm{E}-20$ & 0.0 & 0.0 & 0.0 \\
\hline NIOHION & $8.60165 \mathrm{E}-12$ & 0.0 & 0.0 & 0.0 \\
\hline NO3ION & $3.64846 \mathrm{E}-04$ & 0.0 & 0.0 & 0.0 \\
\hline P207ION & $1.90505 \mathrm{E}-13$ & 0.0 & 0.0 & 0.0 \\
\hline PBCL3ION & $4.45096 \mathrm{E}-08$ & 0.0 & 0.0 & 0.0 \\
\hline PBCL 4 ION & $8.05806 \mathrm{E}-07$ & 0.0 & 0.0 & 0.0 \\
\hline PBCLION & $4.69205 E-09$ & 0.0 & 0.0 & 0.0 \\
\hline PBH2 PO 4 ION & $1.29579 \mathrm{E}-12$ & 0.0 & 0.0 & 0.0 \\
\hline PBION & $2.60780 \mathrm{E}-10$ & 0.0 & 0.0 & 0.0 \\
\hline PBNO33ION & $2.16545 \mathrm{E}-11$ & 0.0 & 0.0 & 0.0 \\
\hline PBNO3ION & $7.83050 \mathrm{E}-10$ & 0.0 & 0.0 & 0.0 \\
\hline PBOHION & $8.42942 E-13$ & 0.0 & 0.0 & 0.0 \\
\hline PO4ION & $5.80138 \mathrm{E}-13$ & 0.0 & 0.0 & 0.0 \\
\hline SO4ION & $3.54647 \mathrm{E}-05$ & 0.0 & 0.0 & 0.0 \\
\hline SRION & $6.24734 \mathrm{E}-07$ & 0.0 & 0.0 & 0.0 \\
\hline SRNO3ION & $2.33098 \mathrm{E}-07$ & 0.0 & 0.0 & 0.0 \\
\hline SROHION & $3.15478 \mathrm{E}-15$ & 0.0 & 0.0 & 0.0 \\
\hline SRPO 4 ION & $1.26003 \mathrm{E}-14$ & 0.0 & 0.0 & 0.0 \\
\hline UIVCLION & $3.30738 \mathrm{E}-28$ & 0.0 & 0.0 & 0.0 \\
\hline UIVION & $1.09407 \mathrm{E}-29$ & 0.0 & 0.0 & 0.0 \\
\hline UIVOH2ION & $3.36434 \mathrm{E}-22$ & 0.0 & 0.0 & 0.0 \\
\hline UIVOH3ION & $5.87378 \mathrm{E}-18$ & 0.0 & 0.0 & 0.0 \\
\hline UIVOH5ION & $8.27698 \mathrm{E}-18$ & 0.0 & 0.0 & 0.0 \\
\hline UIVOHION & $5.77716 \mathrm{E}-25$ & 0.0 & 0.0 & 0.0 \\
\hline UIVSO 4 ION & $2.30607 \mathrm{E}-26$ & 0.0 & 0.0 & 0.0 \\
\hline
\end{tabular}


WSRC-TR-2002-00567

Revision 0

\begin{tabular}{|c|c|c|c|c|}
\hline ZNCL3ION & $3.79711 \mathrm{E}-06$ & 0.0 & 0.0 & 0.0 \\
\hline ZNCLION & $1.86856 \mathrm{E}-06$ & 0.0 & 0.0 & 0.0 \\
\hline ZNH2 PO4 ION & $8.30870 E-09$ & 0.0 & 0.0 & 0.0 \\
\hline ZNHCO3ION & $2.45923 E-12$ & 0.0 & 0.0 & 0.0 \\
\hline ZNION & $6.28873 E-06$ & 0.0 & 0.0 & 0.0 \\
\hline ZNNO3ION & $7.85165 \mathrm{E}-07$ & 0.0 & 0.0 & 0.0 \\
\hline ZNOH3ION & $2.72040 \mathrm{E}-18$ & 0.0 & 0.0 & 0.0 \\
\hline ZNOH 4 ION & $2.19835 \mathrm{E}-24$ & 0.0 & 0.0 & 0.0 \\
\hline ZNOHION & $3.91990 \mathrm{E}-10$ & 0.0 & 0.0 & 0.0 \\
\hline СAHPO4 & 0.0 & $9.69680 \mathrm{E}-05$ & 0.0 & 0.0 \\
\hline $\mathrm{CA} 3 \mathrm{PO} 42$ & 0.0 & $4.68829 \mathrm{E}-05$ & 0.0 & 0.0 \\
\hline $\mathrm{CASO} 4.2 \mathrm{H} 2 \mathrm{O}$ & 0.0 & $5.01787 \mathrm{E}-04$ & 0.0 & 0.0 \\
\hline $\mathrm{CU} 3 \mathrm{PO} 42.2 \mathrm{H} 2 \mathrm{O}$ & 0.0 & $1.31075 E-06$ & 0.0 & 0.0 \\
\hline FEII3PO $42.8 \mathrm{H} 2 \mathrm{O}$ & 0.0 & $1.54713 E-05$ & 0.0 & 0.0 \\
\hline PB3PO42 & 0.0 & $5.68327 \mathrm{E}-07$ & 0.0 & 0.0 \\
\hline UIVO2 & 0.0 & $2.43698 E-06$ & 0.0 & 0.0 \\
\hline \multirow[t]{2}{*}{$\mathrm{ZN} 3 \mathrm{PO} 42.2 \mathrm{H} 2 \mathrm{O}$} & 0.0 & $3.84553 E-06$ & 0.0 & 0.0 \\
\hline & $===========$ & $===========$ & $===========$ & $===========$ \\
\hline Total g/hr & 0.784022 & 0.144888 & 0.0 & 0.0 \\
\hline Volume, L/hr & $6.93695 \mathrm{E}-04$ & $4.92126 \mathrm{E}-05$ & 0.0 & 0.0 \\
\hline Enthalpy, cal/hr & -2683.14 & -422.457 & 0.0 & 0.0 \\
\hline Density, g/L & 1130.21 & 2944.13 & & \\
\hline Vapor fraction & 0.0 & 0.0 & 0.0 & 0.0 \\
\hline Solid fraction & 0.0 & 1 . & 0.0 & 0.0 \\
\hline Organic fraction & 0.0 & 0.0 & 0.0 & 0.0 \\
\hline Osmotic Pres, atm & 152.378 & & & \\
\hline Redox Pot, volts & 0.0 & & & \\
\hline E-Con, 1/ohm-cm & 0.169011 & & & \\
\hline E-Con, cm2/ohm-mol & 30.8339 & & & \\
\hline Abs Visc, cP & 1.3494 & & & \\
\hline Rel Visc & 1.51495 & & & \\
\hline Ionic Strength & 3.67098 & & & \\
\hline
\end{tabular}


WSRC-TR-2002-00567

Revision 0

\begin{tabular}{|c|c|c|c|c|}
\hline \multicolumn{5}{|l|}{ STREAM: Condensate } \\
\hline TO $\quad:$ & & & & \\
\hline FROM : Condensate & mixer & & & \\
\hline Phases-----------> & Aqueous & Solid & Vapor & Organic \\
\hline Temperature, C & 25 . & 25 . & 25 . & 25 . \\
\hline $\begin{array}{l}\text { Pressure, atm } \\
\mathrm{pH}\end{array}$ & $\begin{array}{l}1 . \\
3.97457\end{array}$ & 1. & 1. & 1. \\
\hline Total mol/hr & 3.432874 & $2.07641 E-05$ & 0.0 & 0.0 \\
\hline------------------ & $\mathrm{mol} / \mathrm{hr}------1$ & -mol/hr------- & -mol/hr-------- & $-\operatorname{mol} / \mathrm{hr}------1$ \\
\hline $\mathrm{H} 2 \mathrm{O}$ & 3.43175 & 0.0 & 0.0 & 0.0 \\
\hline $\mathrm{CO} 2$ & 0.00109525 & 0.0 & 0.0 & 0.0 \\
\hline $\mathrm{HCL}$ & $5.16742 \mathrm{E}-18$ & 0.0 & 0.0 & 0.0 \\
\hline HNO3 & $1.02948 \mathrm{E}-13$ & 0.0 & 0.0 & 0.0 \\
\hline LAURICACID & $1.53742 E-05$ & $2.07641 E-05$ & 0.0 & 0.0 \\
\hline OHION & $5.98394 \mathrm{E}-12$ & 0.0 & 0.0 & 0.0 \\
\hline CLION & $8.32689 E-08$ & 0.0 & 0.0 & 0.0 \\
\hline CO3ION & $2.17907 \mathrm{E}-12$ & 0.0 & 0.0 & 0.0 \\
\hline DODECION & $1.78667 \mathrm{E}-06$ & 0.0 & 0.0 & 0.0 \\
\hline HCO3ION & $4.74472 \mathrm{E}-06$ & 0.0 & 0.0 & 0.0 \\
\hline $\mathrm{HION}$ & $6.63439 E-06$ & 0.0 & 0.0 & 0.0 \\
\hline NO3ION & $\begin{array}{l}\text { 1.97148E-08 } \\
============\end{array}$ & $\begin{array}{l}0.0 \\
============\end{array}$ & $\begin{array}{l}0.0 \\
=============\end{array}$ & $\begin{array}{l}0.0 \\
=============\end{array}$ \\
\hline Total g/hr & 61.8761 & 0.00415953 & 0.0 & 0.0 \\
\hline Volume, L/hr & 0.0620562 & 0.0 & 0.0 & 0.0 \\
\hline $\begin{array}{l}\text { Enthalpy, cal/hr } \\
\text { Density, g/L }\end{array}$ & $\begin{array}{l}-2.34546 E+05 \\
997.097\end{array}$ & -3.84414 & 0.0 & 0.0 \\
\hline Vapor fraction & 0.0 & 0.0 & 0.0 & 0.0 \\
\hline Solid fraction & 0.0 & 1. & 0.0 & 0.0 \\
\hline Organic fraction & 0.0 & 0.0 & 0.0 & 0.0 \\
\hline Osmotic Pres, atm & 0.443444 & & & \\
\hline Redox Pot, volts & 0.0 & & & \\
\hline E-Con, 1/ohm-cm & $4.24985 E-05$ & & & \\
\hline E-Con, cm2/ohm-mol & 2.31743 & & & \\
\hline Abs Visc, cP & 0.890776 & & & \\
\hline Rel Visc & 1.00006 & & & \\
\hline Ionic Strength & $1.07312 \mathrm{E}-04$ & & & \\
\hline
\end{tabular}


WSRC-TR-2002-00567

Revision 0

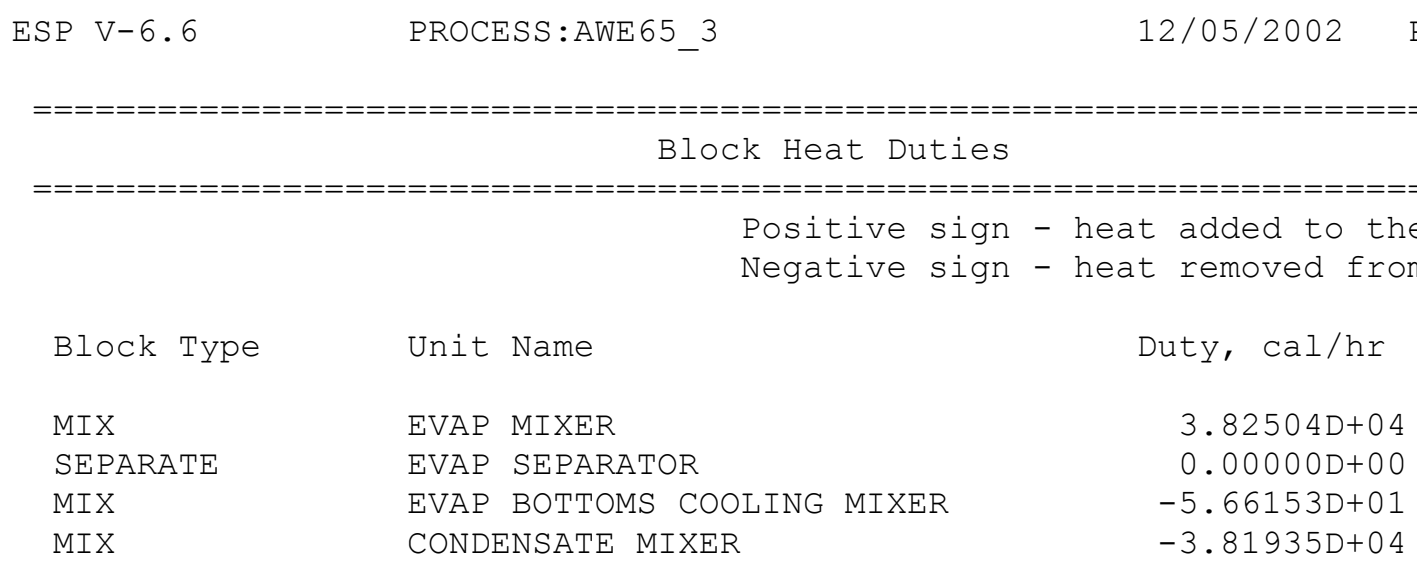


WSRC-TR-2002-00567

Revision 0

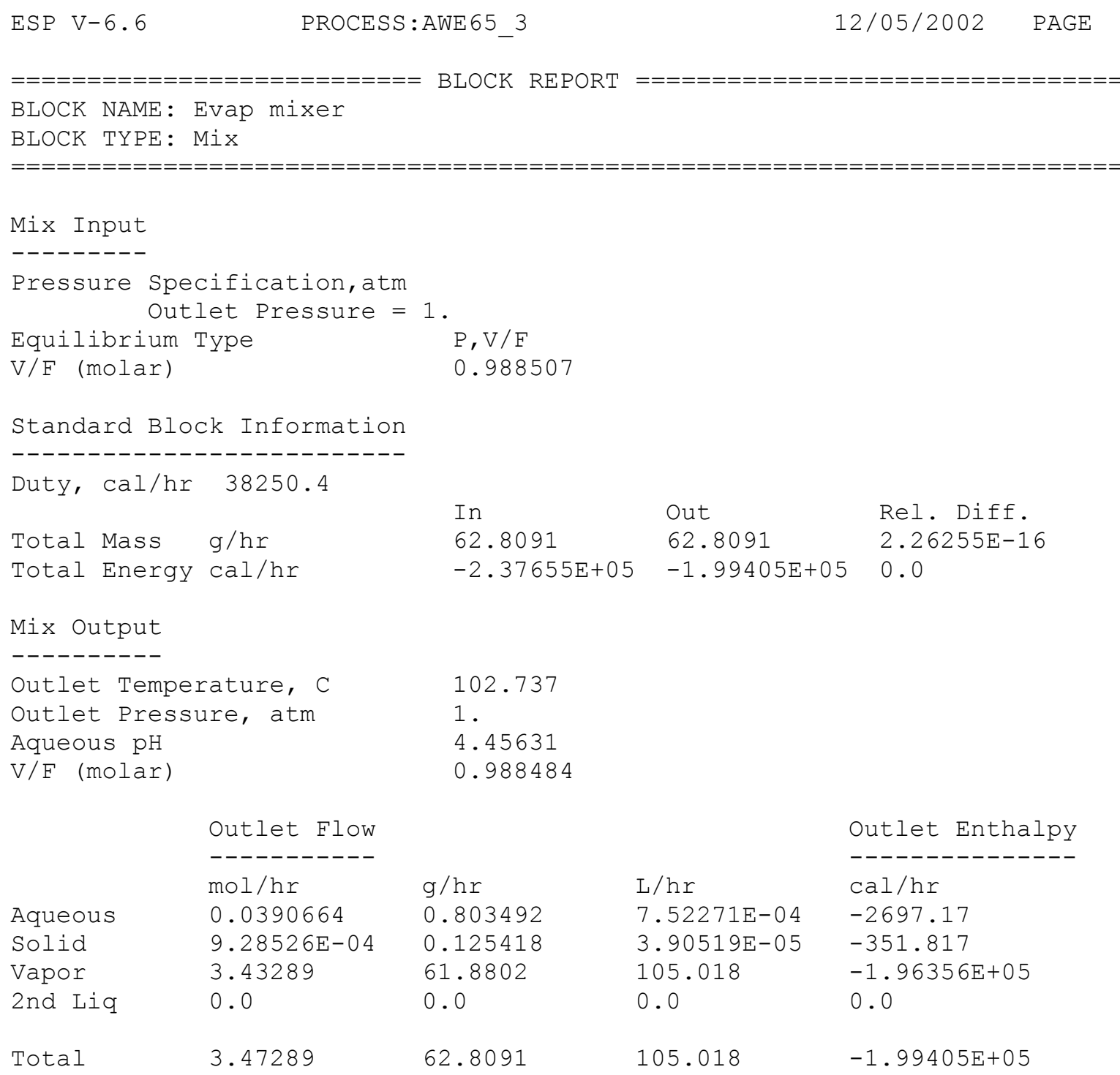


WSRC-TR-2002-00567

Revision 0

$\begin{array}{lllll}\text { ESP V-6.6 PROCESS:AWE65_3 } & 12 / 05 / 2002 & \text { PAGE } & 12\end{array}$

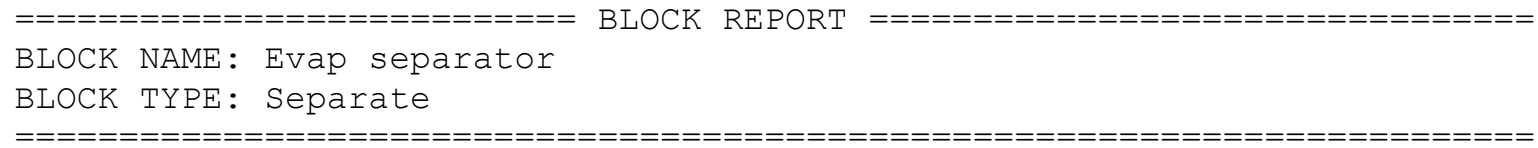


WSRC-TR-2002-00567

Revision 0

ESP V-6.6

PROCESS:AWE 65_3

Vapor Stream

Outlet F
------
mol/hr
0.0
0.0
3.43289
0.0

Total
Overhead

g/hr

0.0

0.0

61.8802

0.0

61.8802
$12 / 05 / 2002$

PAGE

13

$\mathrm{L} / \mathrm{hr}$

0.0

0.0

105.018

0.0

Outlet Enthalpy
-----------
cal/hr
0.0
0.0
$-1.96356 \mathrm{E}+05$
0.0

105.018 
WSRC-TR-2002-00567

Revision 0

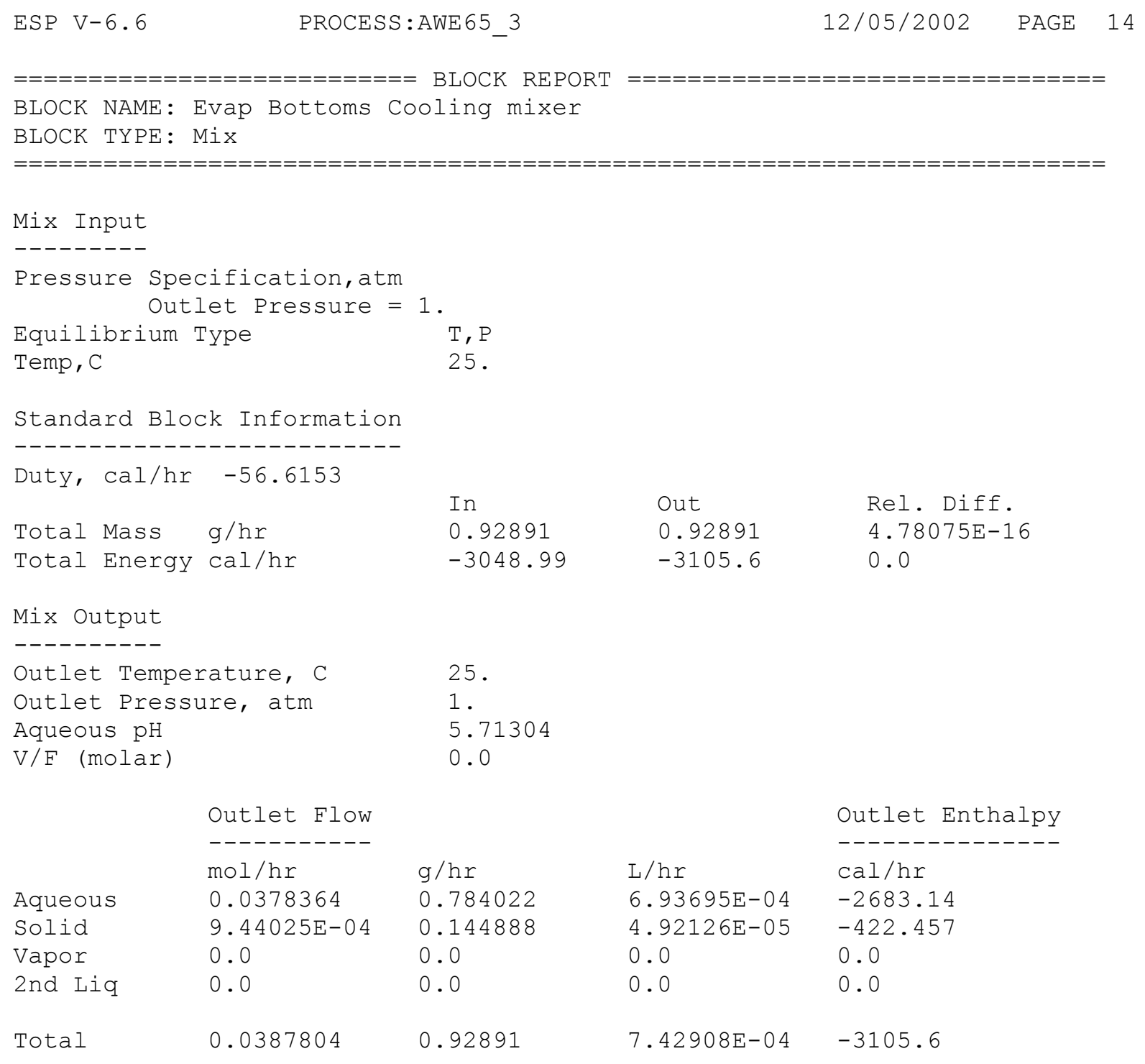


WSRC-TR-2002-00567

Revision 0

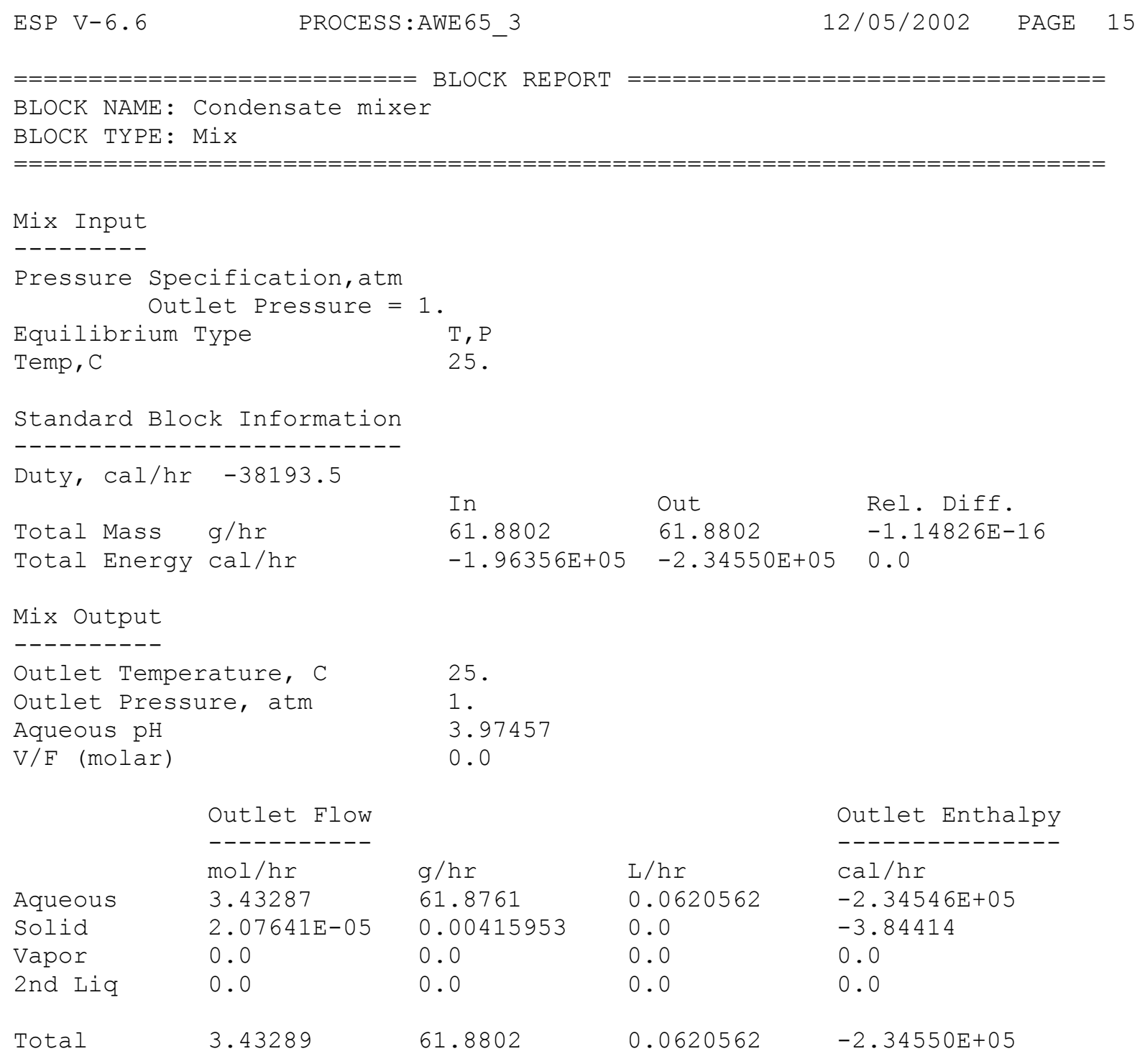


WSRC-TR-2002-00567

Revision 0

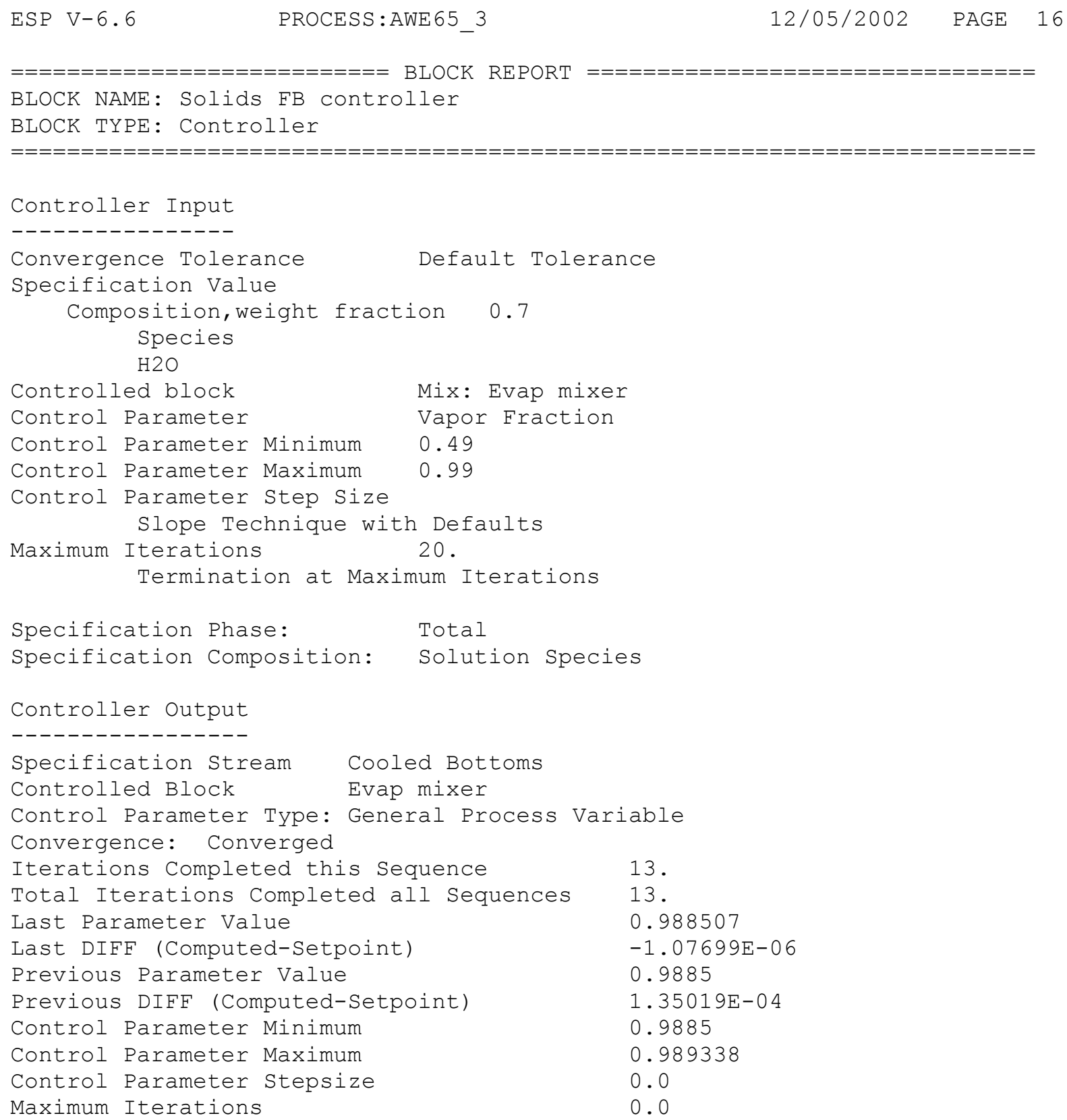


WSRC-TR-2002-00567

Revision 0

Influent Limit Composition 70\% Target $\mathrm{pH}=6.5$

$6.5-70$

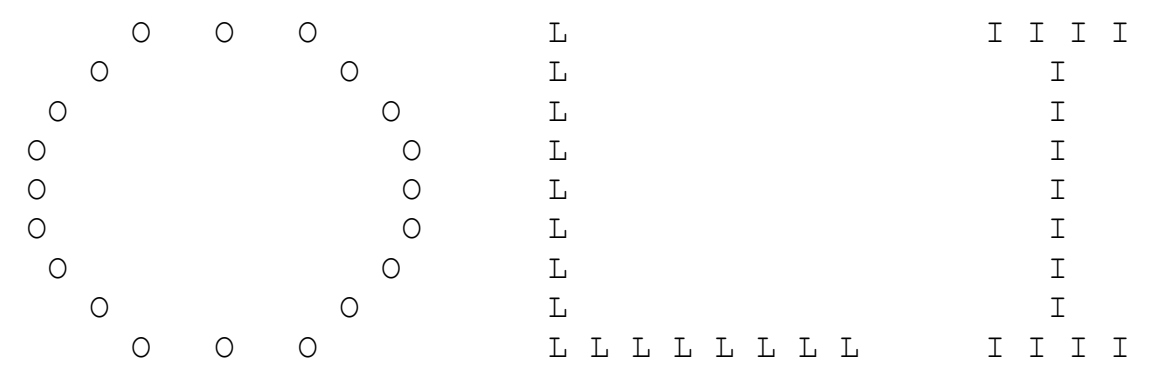

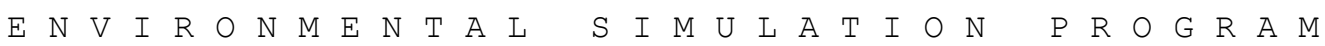

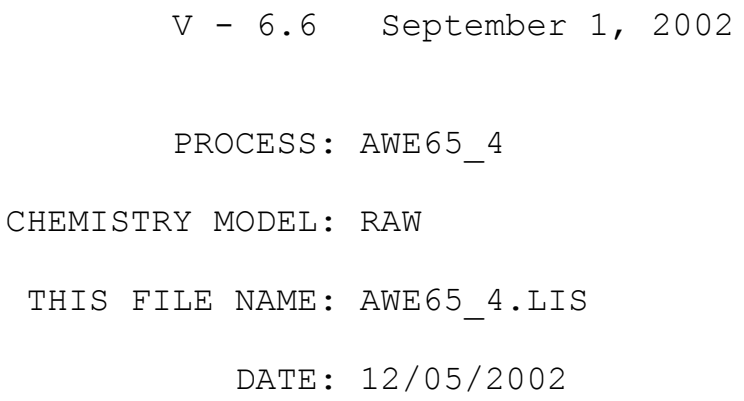




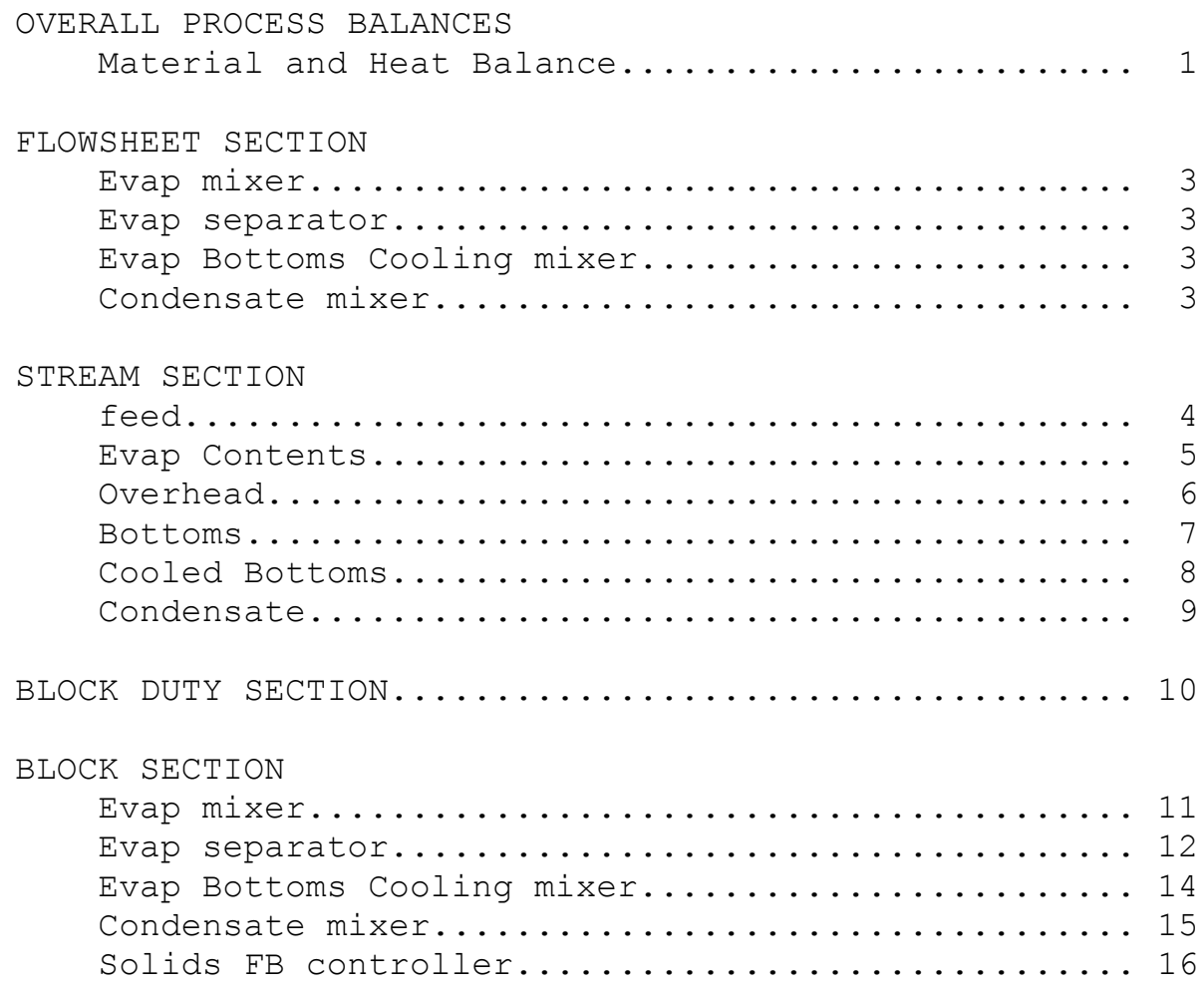


WSRC-TR-2002-00567

Revision 0

ESP V-6.6 PROCESS:AWE65_4
$\begin{array}{lcc}\text { Overall Process Balances } & \\ \text { Inlet } & \text { g/hr } & \mathrm{Cal} / \mathrm{hr} \\ ---------- & --------- & ---------- \\ \text { FEED } & 1.00000 \mathrm{D}+03 & -3.79130 \mathrm{D}+06 \\ \text { =========== } & ========= & ========= \\ \text { Total in } & 1.00000 \mathrm{D}+03 & -3.79130 \mathrm{D}+06\end{array}$

$\begin{array}{lrc}\text { Outlet } & \text { g/hr } & \text { cal/hr } \\ ----------- & ---------- & ---------- \\ \text { COOLED BOTTOMS } & 1.14440 \mathrm{D}+00 & -3.80311 \mathrm{D}+03 \\ \text { CONDENSATE } & 9.98856 \mathrm{D}+02 & -3.78750 \mathrm{D}+06 \\ ========== & ========== & ========= \\ \text { Total out } & 1.00000 \mathrm{D}+03 & -3.79130 \mathrm{D}+06\end{array}$

Block Heat Duties

EVAP MIXER

EVAP BOTTOMS COOLING MIXER CONDENSATE MIXER

$=========$

Total Duty

DIFFERENCE

REL DIFEERENCE

1.13687D-12

$1.13687 \mathrm{D}-15$
12/05/2002 PAGE 1

$$
\begin{gathered}
\text { cal/hr } \\
---------- \\
6.17140 \mathrm{D}+05 \\
-6.95779 \mathrm{D}+01 \\
-6.17069 \mathrm{D}+05 \\
========== \\
1.59220 \mathrm{D}+00
\end{gathered}
$$

\begin{tabular}{|c|c|c|c|c|}
\hline Code & $\begin{array}{l}\text { Input } \\
\text { mol/hr }\end{array}$ & $\begin{array}{l}\text { Outlet } \\
\text { mol/hr }\end{array}$ & $\begin{array}{l}\text { Difference } \\
\text { mol/hr }\end{array}$ & Rel Diff \\
\hline------------ & ------------ & ------------ & ------------ & ------------1 \\
\hline $\mathrm{H}(+1)$ & $1.10974 \mathrm{D}+02$ & $1.10974 \mathrm{D}+02$ & $7.10543 D-14$ & $6.40276 \mathrm{D}-16$ \\
\hline $\mathrm{K}(+1)$ & $2.65152 \mathrm{D}-04$ & $2.65152 \mathrm{D}-04$ & $5.42101 \mathrm{D}-20$ & $2.04449 D-16$ \\
\hline $\mathrm{NA}(+1)$ & $1.80307 D-03$ & $1.80307 \mathrm{D}-03$ & $8.67362 \mathrm{D}-19$ & $4.81048 D-16$ \\
\hline $\mathrm{BA}(+2)$ & $5.83942 \mathrm{D}-07$ & $5.83942 \mathrm{D}-07$ & $-2.32934 D-21$ & $-3.98900 \mathrm{D}-15$ \\
\hline $\mathrm{CA}(+2)$ & $8.72818 D-04$ & $8.72818 \mathrm{D}-04$ & $1.08420 \mathrm{D}-19$ & $1.24219 D-16$ \\
\hline $\mathrm{ZN}(+2)$ & $2.90520 \mathrm{D}-05$ & $2.90520 \mathrm{D}-05$ & $3.38813 \mathrm{D}-20$ & $1.16623 \mathrm{D}-15$ \\
\hline $\mathrm{CU}(+2)$ & $5.19685 \mathrm{D}-06$ & $5.19685 \mathrm{D}-06$ & $0.00000 \mathrm{D}+00$ & $0.00000 \mathrm{D}+00$ \\
\hline $\mathrm{FE}(+2)$ & $8.60215 \mathrm{D}-05$ & $8.60215 \mathrm{D}-05$ & $1.35525 \mathrm{D}-20$ & $1.57548 D-16$ \\
\hline $\mathrm{MG}(+2)$ & $3.29218 \mathrm{D}-04$ & $3.29218 \mathrm{D}-04$ & $2.16840 \mathrm{D}-19$ & $6.58653 \mathrm{D}-16$ \\
\hline $\mathrm{PB}(+2)$ & $2.99517 D-06$ & $2.99517 \mathrm{D}-06$ & $2.96462 \mathrm{D}-21$ & $9.89799 \mathrm{D}-16$ \\
\hline $\mathrm{AL}(+3)$ & $8.88889 \mathrm{D}-05$ & $8.88889 \mathrm{D}-05$ & $0.00000 \mathrm{D}+00$ & $0.00000 \mathrm{D}+00$ \\
\hline $\mathrm{NI}(+2)$ & $1.29472 \mathrm{D}-06$ & $1.29472 \mathrm{D}-06$ & $-2.11758 D-22$ & $-1.63555 D-16$ \\
\hline$O(-2)$ & $5.54954 \mathrm{D}+01$ & $5.54954 \mathrm{D}+01$ & $4.26326 \mathrm{D}-14$ & $7.68218 D-16$ \\
\hline $\mathrm{CL}(-1)$ & $2.14085 D-03$ & $2.14085 \mathrm{D}-03$ & $8.67362 \mathrm{D}-19$ & $4.05149 D-16$ \\
\hline$C(+4)$ & $1.20000 D-03$ & $1.20000 \mathrm{D}-03$ & $-8.67362 \mathrm{D}-19$ & $-7.22801 D-16$ \\
\hline$P(+5)$ & $2.10526 \mathrm{D}-04$ & $2.10526 \mathrm{D}-04$ & $0.00000 \mathrm{D}+00$ & $0.00000 \mathrm{D}+00$ \\
\hline$S(+6)$ & $7.39583 D-04$ & $7.39583 \mathrm{D}-04$ & $-1.08420 D-19$ & $-1.46596 \mathrm{D}-16$ \\
\hline $\mathrm{N}(+5)$ & $4.67742 \mathrm{D}-04$ & $4.67742 \mathrm{D}-04$ & $-1.62630 \mathrm{D}-19$ & $-3.47692 \mathrm{D}-16$ \\
\hline $\operatorname{SI}(+4)$ & $2.00000 \mathrm{D}-04$ & $2.00000 \mathrm{D}-04$ & $2.71051 \mathrm{D}-20$ & $1.35525 \mathrm{D}-16$ \\
\hline $\mathrm{SR}(+2)$ & $1.71233 \mathrm{D}-05$ & $1.71233 \mathrm{D}-05$ & $3.38813 \mathrm{D}-21$ & $1.97867 D-16$ \\
\hline
\end{tabular}

Material Code Balances

146 of 447 
WSRC-TR-2002-00567

Revision 0

$\mathrm{CD}(+2)$

$\mathrm{CR}(+3)$

$\mathrm{U}(+4)$
$3.57143 \mathrm{D}-08$

$7.88462 \mathrm{D}-08$

$2.89916 \mathrm{D}-06$
$3.57143 \mathrm{D}-08$

$7.88462 \mathrm{D}-08$

2. 89916D-06
$2.84550 \mathrm{D}-22$

$0.00000 \mathrm{D}+00 \quad 0.00000 \mathrm{D}+00$ 
WSRC-TR-2002-00567

Revision 0

ESP V-6.6

$\operatorname{DODEC}(-1)$
PROCESS:AWE $65 \quad 4$

$4.16682 \mathrm{D}-05$
$12 / 05 / 2002$

$4.16682 \mathrm{D}-05$

4. 87873D-16 
WSRC-TR-2002-00567

Revision 0

\begin{tabular}{|c|c|c|c|}
\hline \multicolumn{4}{|l|}{$\begin{array}{l}\text { PROCESS BLOCKS } \\
===========\end{array}$} \\
\hline $\begin{array}{l}\mathrm{BLOCK} \mathrm{NAME} \\
=========================\end{array}$ & $\begin{array}{l}\text { BLOCK TYPE } \\
===========\end{array}$ & $\begin{array}{l}\text { INLET STREAM }(\mathrm{S}) \\
==============\end{array}$ & $\begin{array}{l}\text { OUTLET STREAM }(\mathrm{S}) \\
==============\end{array}$ \\
\hline Evap mixer & $\operatorname{Mix}$ & feed & Evap Contents \\
\hline Evap separator & Separate & Evap Contents & $\begin{array}{l}\text { Overhead } \\
\text { Bottoms }\end{array}$ \\
\hline Evap Bottoms Cooling mixer & Mix & Bottoms & Cooled Bottoms \\
\hline Condensate mixer & Mix & Overhead & Condensate \\
\hline
\end{tabular}


WSRC-TR-2002-00567

Revision 0

ESP V-6. 6
PROCESS:AWE $65 \quad 4$
12/05/2002 PAGE 4

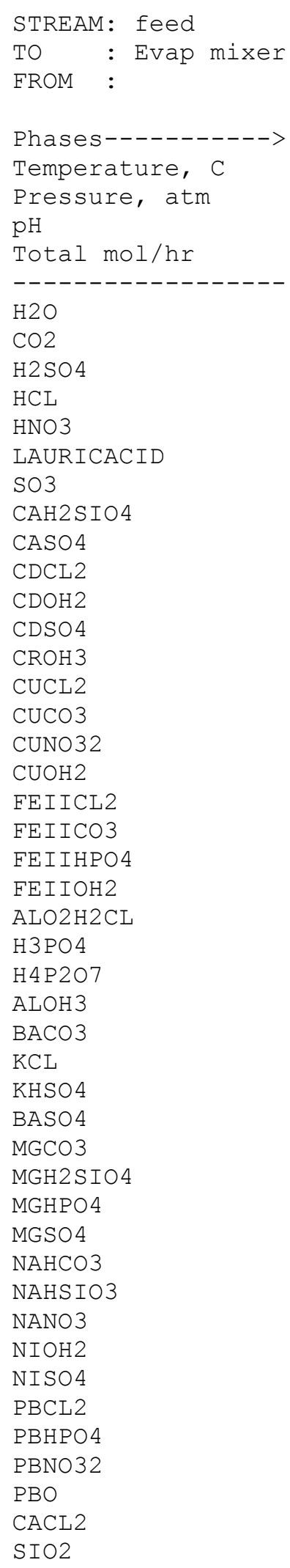

\begin{tabular}{|c|c|c|c|}
\hline Aqueous & Solid & Vapor & Organic \\
\hline 25 . & 25 . & 25 . & 25 . \\
\hline 1 . & 1 . & 1 . & 1 . \\
\hline \multicolumn{4}{|l|}{5.98541} \\
\hline 55.49506 & $9.47054 \mathrm{E}-05$ & 0.0 & 0.0 \\
\hline \multicolumn{4}{|c|}{$\mathrm{mol} / \mathrm{hr}--------\mathrm{mol} / \mathrm{hr}--------\mathrm{mol} / \mathrm{hr}--------\operatorname{mol} / \mathrm{hr}--------$} \\
\hline 55.4867 & 0.0 & 0.0 & 0.0 \\
\hline $8.06975 \mathrm{E}-04$ & 0.0 & 0.0 & 0.0 \\
\hline $1.97280 \mathrm{E}-24$ & 0.0 & 0.0 & 0.0 \\
\hline $1.20286 \mathrm{E}-15$ & 0.0 & 0.0 & 0.0 \\
\hline $2.20308 E-11$ & 0.0 & 0.0 & 0.0 \\
\hline $3.00189 \mathrm{E}-06$ & 0.0 & 0.0 & 0.0 \\
\hline $2.55365 E-28$ & 0.0 & 0.0 & 0.0 \\
\hline $7.14553 \mathrm{E}-14$ & 0.0 & 0.0 & 0.0 \\
\hline $1.13831 E-05$ & 0.0 & 0.0 & 0.0 \\
\hline 1. $47410 \mathrm{E}-10$ & 0.0 & 0.0 & 0.0 \\
\hline $1.23963 E-16$ & 0.0 & 0.0 & 0.0 \\
\hline $3.62194 E-09$ & 0.0 & 0.0 & 0.0 \\
\hline $2.14687 \mathrm{E}-14$ & 0.0 & 0.0 & 0.0 \\
\hline $9.13852 \mathrm{E}-13$ & 0.0 & 0.0 & 0.0 \\
\hline $8.60238 \mathrm{E}-09$ & 0.0 & 0.0 & 0.0 \\
\hline $9.21330 \mathrm{E}-15$ & 0.0 & 0.0 & 0.0 \\
\hline $7.68326 \mathrm{E}-10$ & 0.0 & 0.0 & 0.0 \\
\hline $1.17817 \mathrm{E}-15$ & 0.0 & 0.0 & 0.0 \\
\hline $1.27640 \mathrm{E}-07$ & 0.0 & 0.0 & 0.0 \\
\hline $1.56505 E-08$ & 0.0 & 0.0 & 0.0 \\
\hline 1. $42690 \mathrm{E}-13$ & 0.0 & 0.0 & 0.0 \\
\hline $2.32698 E-28$ & 0.0 & 0.0 & 0.0 \\
\hline $2.42516 \mathrm{E}-08$ & 0.0 & 0.0 & 0.0 \\
\hline $1.04468 E-18$ & 0.0 & 0.0 & 0.0 \\
\hline $2.40116 \mathrm{E}-09$ & $8.88852 E-05$ & 0.0 & 0.0 \\
\hline $1.53125 E-12$ & 0.0 & 0.0 & 0.0 \\
\hline $3.94455 E-09$ & 0.0 & 0.0 & 0.0 \\
\hline $2.28539 E-13$ & 0.0 & 0.0 & 0.0 \\
\hline $2.05789 \mathrm{E}-10$ & $2.66050 \mathrm{E}-07$ & 0.0 & 0.0 \\
\hline $3.42041 E-09$ & 0.0 & 0.0 & 0.0 \\
\hline $2.55355 E-13$ & 0.0 & 0.0 & 0.0 \\
\hline $2.66915 E-06$ & 0.0 & 0.0 & 0.0 \\
\hline $7.33513 E-06$ & 0.0 & 0.0 & 0.0 \\
\hline $2.44060 \mathrm{E}-07$ & 0.0 & 0.0 & 0.0 \\
\hline $5.54737 E-09$ & 0.0 & 0.0 & 0.0 \\
\hline $4.04584 E-08$ & 0.0 & 0.0 & 0.0 \\
\hline $8.13408 \mathrm{E}-14$ & 0.0 & 0.0 & 0.0 \\
\hline $8.76371 E-08$ & 0.0 & 0.0 & 0.0 \\
\hline $1.85457 \mathrm{E}-11$ & 0.0 & 0.0 & 0.0 \\
\hline $7.96709 \mathrm{E}-10$ & 0.0 & 0.0 & 0.0 \\
\hline $1.70679 \mathrm{E}-13$ & 0.0 & 0.0 & 0.0 \\
\hline $4.58409 \mathrm{E}-13$ & 0.0 & 0.0 & 0.0 \\
\hline $2.16728 \mathrm{E}-27$ & 0.0 & 0.0 & 0.0 \\
\hline $1.99964 \mathrm{E}-04$ & 0.0 & 0.0 & 0.0 \\
\hline
\end{tabular}




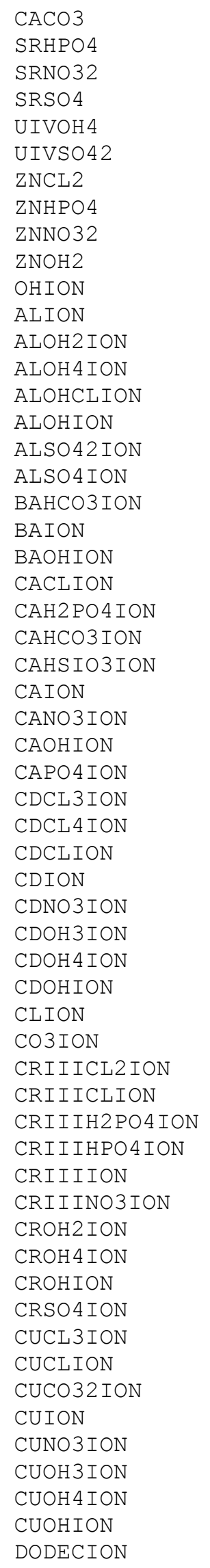

\begin{tabular}{|c|c|}
\hline $2.05025 E-08$ & 0.0 \\
\hline $.06588 \mathrm{E}-09$ & 0.0 \\
\hline $71739 \mathrm{E}-11$ & 0.0 \\
\hline $11688 \mathrm{E}-06$ & \\
\hline $59 E-10$ & 0.0 \\
\hline $5 E-26$ & 0.0 \\
\hline$E-10$ & 0.0 \\
\hline $98 E-07$ & 0.0 \\
\hline $6 E-12$ & 0.0 \\
\hline $46 \mathrm{E}-11$ & 0.0 \\
\hline $9 E-08$ & 0.0 \\
\hline $35 E-11$ & 0.0 \\
\hline $4 E-10$ & 0.0 \\
\hline $.35405 E-10$ & 0.0 \\
\hline $9 E-13$ & 0.0 \\
\hline 1. $44809 \mathrm{E}-10$ & 0.0 \\
\hline $2256 \mathrm{E}-13$ & 0.0 \\
\hline $30 E-12$ & 0.0 \\
\hline $1 E-10$ & 0.0 \\
\hline $5 E-07$ & 0.0 \\
\hline $7 E-15$ & 0.0 \\
\hline $4 E-11$ & 0.0 \\
\hline$E-06$ & 0.0 \\
\hline $2.60091 E-06$ & 0.0 \\
\hline $4 E-10$ & 0.0 \\
\hline $4 E-04$ & 0.0 \\
\hline $9 E-07$ & 0.0 \\
\hline $53 E-10$ & 0.0 \\
\hline $2 E-08$ & 0.0 \\
\hline $4 E-14$ & 0.0 \\
\hline $2 E-17$ & 0.0 \\
\hline $3 E-09$ & 0.0 \\
\hline $2 E-08$ & 0.0 \\
\hline $9 E-11$ & 0.0 \\
\hline 1.82 & 0.0 \\
\hline 0.0 & 0.0 \\
\hline $7 E-12$ & 0.0 \\
\hline 0.00 & 0.0 \\
\hline $6 E-08$ & 0.0 \\
\hline $9 E-20$ & 0.0 \\
\hline $4 E-16$ & 0.0 \\
\hline $3 E-13$ & 0.0 \\
\hline $9 E-08$ & 0.0 \\
\hline 5.4 & 0.0 \\
\hline $8 E-15$ & 0.0 \\
\hline $1 E-15$ & 0.0 \\
\hline $62 E-19$ & 0.0 \\
\hline $6 \mathrm{E}-12$ & 0.0 \\
\hline $99 E-11$ & 0.0 \\
\hline $20 E-18$ & 0.0 \\
\hline $22 E-09$ & 0.0 \\
\hline $51 E-13$ & 0.0 \\
\hline $14 \mathrm{E}-07$ & 0.0 \\
\hline $68 E-10$ & 0.0 \\
\hline $61 E-16$ & 0.0 \\
\hline $4.10087 E-23$ & 0.0 \\
\hline $45 E-09$ & 0.0 \\
\hline $563 E-05$ & 0.0 \\
\hline
\end{tabular}

0.0

0.0

0.0

0.0

0.0

0.0

0.0

0.0

0.0

0.0

0.0

0.0

0.0

0.0

0.0

0.0

0.0

0.0

0.0

0.0

0.0

0.0

0.0

0.0

0.0

0.0

0.0

0.0

0.0

0.0

0.0

0.0

0.0

0.0

0.0

0.0

0.0

0.0

0.0

0.0

0.0

0.0

0.0

0.0

0.0

0.0

0.0

0.0

0.0

0.0

0.0

0.0

0.0

0.0

0.0

0.0

0.0

0.0
0.0

0.0

0.0

0.0

0.0

0.0

0.0

0.0

0.0

0.0

0.0

0.0

0.0

0.0

0.0

0.0

0.0

0.0

0.0

0.0

0.0

0.0

0.0

0.0

0.0

0.0

0.0

0.0

0.0

0.0

0.0

0.0

0.0

0.0

0.0

0.0

0.0

0.0

0.0

0.0

0.0

0.0

0.0

0.0

0.0

0.0

0.0

0.0

0.0

0.0

0.0

0.0

0.0

0.0

0.0

0.0

0.0

0.0 


\begin{tabular}{|c|c|c|c|c|}
\hline FEIICLION & $1.77607 \mathrm{E}-10$ & 0.0 & 0.0 & 0.0 \\
\hline FEIICO32ION & $9.55931 \mathrm{E}-14$ & 0.0 & 0.0 & 0.0 \\
\hline FEIIH2PO4ION & $3.58109 \mathrm{E}-08$ & 0.0 & 0.0 & 0.0 \\
\hline FEIIHCO3ION & $3.42306 \mathrm{E}-09$ & 0.0 & 0.0 & 0.0 \\
\hline FEIIION & $8.58186 \mathrm{E}-05$ & 0.0 & 0.0 & 0.0 \\
\hline FEIIOH3ION & $5.95172 \mathrm{E}-18$ & 0.0 & 0.0 & 0.0 \\
\hline FEIIOH 4 ION & $7.68374 \mathrm{E}-27$ & 0.0 & 0.0 & 0.0 \\
\hline FEIIOHION & $2.01679 \mathrm{E}-08$ & 0.0 & 0.0 & 0.0 \\
\hline H2 P2O 7 ION & $1.79120 \mathrm{E}-10$ & 0.0 & 0.0 & 0.0 \\
\hline H2 PO 4 ION & $1.81627 \mathrm{E}-04$ & 0.0 & 0.0 & 0.0 \\
\hline H2SIO4ION & $3.01419 \mathrm{E}-15$ & 0.0 & 0.0 & 0.0 \\
\hline H3P2O 7 ION & $3.14379 \mathrm{E}-14$ & 0.0 & 0.0 & 0.0 \\
\hline H3SIO4 ION & $3.03114 \mathrm{E}-08$ & 0.0 & 0.0 & 0.0 \\
\hline HCO3ION & $3.86566 \mathrm{E}-04$ & 0.0 & 0.0 & 0.0 \\
\hline HION & $1.12730 \mathrm{E}-06$ & 0.0 & 0.0 & 0.0 \\
\hline HP207ION & $6.72131 \mathrm{E}-11$ & 0.0 & 0.0 & 0.0 \\
\hline HPBO2ION & $5.42104 \mathrm{E}-18$ & 0.0 & 0.0 & 0.0 \\
\hline HPO4 ION & $1.42888 \mathrm{E}-05$ & 0.0 & 0.0 & 0.0 \\
\hline HSO 4 ION & $5.35047 \mathrm{E}-08$ & 0.0 & 0.0 & 0.0 \\
\hline KION & $2.64150 \mathrm{E}-04$ & 0.0 & 0.0 & 0.0 \\
\hline KSO4 ION & $9.98165 \mathrm{E}-07$ & 0.0 & 0.0 & 0.0 \\
\hline MGH2 PO 4 ION & $1.91375 \mathrm{E}-06$ & 0.0 & 0.0 & 0.0 \\
\hline MGHCO3ION & $3.25864 \mathrm{E}-06$ & 0.0 & 0.0 & 0.0 \\
\hline MGHSIO3ION & $2.26790 \mathrm{E}-10$ & 0.0 & 0.0 & 0.0 \\
\hline MGION & $3.14031 \mathrm{E}-04$ & 0.0 & 0.0 & 0.0 \\
\hline MGOHION & $3.88198 \mathrm{E}-10$ & 0.0 & 0.0 & 0.0 \\
\hline MGP2O 7 ION & $9.37567 \mathrm{E}-11$ & 0.0 & 0.0 & 0.0 \\
\hline MGPO 4 ION & $6.06736 \mathrm{E}-09$ & 0.0 & 0.0 & 0.0 \\
\hline NACO3ION & $1.02117 \mathrm{E}-10$ & 0.0 & 0.0 & 0.0 \\
\hline NAION & 0.00179531 & 0.0 & 0.0 & 0.0 \\
\hline NASO 4 ION & $7.47156 \mathrm{E}-06$ & 0.0 & 0.0 & 0.0 \\
\hline NICLION & $1.81996 \mathrm{E}-10$ & 0.0 & 0.0 & 0.0 \\
\hline NIION & $1.20563 \mathrm{E}-06$ & 0.0 & 0.0 & 0.0 \\
\hline NINO3ION & $1.14663 \mathrm{E}-09$ & 0.0 & 0.0 & 0.0 \\
\hline NIOH3ION & $8.79200 \mathrm{E}-19$ & 0.0 & 0.0 & 0.0 \\
\hline NIOHION & $1.25229 \mathrm{E}-10$ & 0.0 & 0.0 & 0.0 \\
\hline NO3ION & $4.66904 \mathrm{E}-04$ & 0.0 & 0.0 & 0.0 \\
\hline P207ION & $6.02459 \mathrm{E}-14$ & 0.0 & 0.0 & 0.0 \\
\hline PBCL3ION & $1.94176 \mathrm{E}-14$ & 0.0 & 0.0 & 0.0 \\
\hline PBCL 4 ION & $3.10912 \mathrm{E}-17$ & 0.0 & 0.0 & 0.0 \\
\hline PBCLION & $2.84370 \mathrm{E}-09$ & 0.0 & 0.0 & 0.0 \\
\hline PBH2 PO 4 ION & $3.63303 \mathrm{E}-10$ & 0.0 & 0.0 & 0.0 \\
\hline PBION & $6.86049 \mathrm{E}-08$ & 0.0 & 0.0 & 0.0 \\
\hline PBNO33ION & $1.36251 \mathrm{E}-17$ & 0.0 & 0.0 & 0.0 \\
\hline PBNO3ION & $4.47883 E-10$ & 0.0 & 0.0 & 0.0 \\
\hline PBOHION & $1.30447 \mathrm{E}-09$ & 0.0 & 0.0 & 0.0 \\
\hline PO4ION & $1.02238 \mathrm{E}-11$ & 0.0 & 0.0 & 0.0 \\
\hline SO4ION & $7.10867 \mathrm{E}-04$ & 0.0 & 0.0 & 0.0 \\
\hline SRION & $1.59747 \mathrm{E}-05$ & 0.0 & 0.0 & 0.0 \\
\hline SRNO3ION & $2.96352 \mathrm{E}-08$ & 0.0 & 0.0 & 0.0 \\
\hline SROHION & $7.66723 \mathrm{E}-13$ & 0.0 & 0.0 & 0.0 \\
\hline SRPO 4 ION & $2.98812 \mathrm{E}-12$ & 0.0 & 0.0 & 0.0 \\
\hline UIVCLION & $3.28898 \mathrm{E}-30$ & 0.0 & 0.0 & 0.0 \\
\hline UIVION & $6.07933 E-29$ & 0.0 & 0.0 & 0.0 \\
\hline UIVOH2ION & $5.54752 \mathrm{E}-20$ & 0.0 & 0.0 & 0.0 \\
\hline UIVOH3ION & $3.83659 \mathrm{E}-15$ & 0.0 & 0.0 & 0.0 \\
\hline UIVOH5ION & $1.66340 \mathrm{E}-14$ & 0.0 & 0.0 & 0.0 \\
\hline UIVOHION & $1.32530 \mathrm{E}-23$ & 0.0 & 0.0 & 0.0 \\
\hline
\end{tabular}


WSRC-TR-2002-00567

Revision 0

\begin{tabular}{|c|c|c|c|c|}
\hline UIVSO4ION & $2.66369 \mathrm{E}-26$ & 0.0 & 0.0 & 0.0 \\
\hline ZNCL3ION & $9.89181 \mathrm{E}-14$ & 0.0 & 0.0 & 0.0 \\
\hline ZNCLION & $6.75359 E-08$ & 0.0 & 0.0 & 0.0 \\
\hline ZNH2PO4 ION & $1.39165 \mathrm{E}-07$ & 0.0 & 0.0 & 0.0 \\
\hline ZNHCO3ION & $1.68022 \mathrm{E}-07$ & 0.0 & 0.0 & 0.0 \\
\hline $\mathrm{ZNION}$ & $2.82510 \mathrm{E}-05$ & 0.0 & 0.0 & 0.0 \\
\hline ZNNO3ION & $2.68286 E-08$ & 0.0 & 0.0 & 0.0 \\
\hline ZNOH 3 ION & $9.62657 \mathrm{E}-16$ & 0.0 & 0.0 & 0.0 \\
\hline ZNOH 4 ION & $1.56328 \mathrm{E}-22$ & 0.0 & 0.0 & 0.0 \\
\hline ZNOHION & $2.55842 E-08$ & 0.0 & 0.0 & 0.0 \\
\hline $\mathrm{CU} 3 \mathrm{PO} 42.2 \mathrm{H} 2 \mathrm{O}$ & 0.0 & $1.68171 E-06$ & 0.0 & 0.0 \\
\hline $\mathrm{PB} 3 \mathrm{PO} 42$ & 0.0 & $9.73596 \mathrm{E}-07$ & 0.0 & 0.0 \\
\hline UIVO2 & $\begin{array}{l}0.0 \\
============\end{array}$ & $\begin{array}{l}2.89886 \mathrm{E}-06 \\
============\end{array}$ & $\begin{array}{l}0.0 \\
=============\end{array}$ & $\begin{array}{l}0.0 \\
============\end{array}$ \\
\hline Total g/hr & 999.991 & 0.00926896 & 0.0 & 0.0 \\
\hline Volume, L/hr & 1.00286 & $3.03733 E-06$ & 0.0 & 0.0 \\
\hline Enthalpy, cal/hr & $-3.79128 E+06$ & -28.8574 & 0.0 & 0.0 \\
\hline Density, g/L & 997.135 & 3051.68 & & \\
\hline Vapor fraction & 0.0 & 0.0 & 0.0 & 0.0 \\
\hline Solid fraction & 0.0 & 1 . & 0.0 & 0.0 \\
\hline Organic fraction & 0.0 & 0.0 & 0.0 & 0.0 \\
\hline Osmotic Pres, atm & 0.223532 & & & \\
\hline Redox Pot, volts & 0.0 & & & \\
\hline E-Con, 1/ohm-cm & $5.34981 E-04$ & & & \\
\hline E-Con, cm2/ohm-mol & 127.468 & & & \\
\hline Abs Visc, cP & 0.892176 & & & \\
\hline Rel Visc & 1.00164 & & & \\
\hline Ionic Strength & 0.00670117 & & & \\
\hline
\end{tabular}


WSRC-TR-2002-00567

Revision 0

\begin{tabular}{|c|c|c|c|c|}
\hline \\
\hline \multicolumn{5}{|c|}{$\begin{array}{l}\text { STREAM: Evap Contents } \\
\text { TO : Evap separator }\end{array}$} \\
\hline FROM : Evap mixer & & & & \\
\hline Phases-----------> & Aqueous & Solid & Vapor & Organic \\
\hline \multirow{2}{*}{ Temperature, C } & 103.062 & 103.062 & 103.062 & 103.062 \\
\hline & 1. & 1 . & 1. & 1 . \\
\hline $\begin{array}{l}\text { Pressure, atm } \\
\mathrm{pH}\end{array}$ & 4.65196 & & & \\
\hline Total mol/hr & 0.0506356 & 0.00108315 & 55.4426 & 0.0 \\
\hline------------------ & $\operatorname{mol} / \mathrm{hr}-----1$ & $-\operatorname{mol} / \mathrm{hr}-----$ & $-\operatorname{mol} / \mathrm{hr}----$ & -mol/hr-------- \\
\hline & 0.0458279 & 0.0 & 55.44136 & 0.0 \\
\hline $\begin{array}{ll}\mathrm{H} 2 \mathrm{O} \\
\mathrm{CO} 2\end{array}$ & $1.27929 \mathrm{E}-10$ & 0.0 & 0.001200001 & 0.0 \\
\hline & $6.05781 \mathrm{E}-24$ & 0.0 & $6.72785 E-21$ & 0.0 \\
\hline $\mathrm{HCL}$ & $7.98029 \mathrm{E}-13$ & 0.0 & $9.42925 \mathrm{E}-07$ & 0.0 \\
\hline HNO3 & $6.83897 \mathrm{E}-10$ & 0.0 & $1.81565 \mathrm{E}-07$ & 0.0 \\
\hline LAUR ICACID & $6.42487 \mathrm{E}-10$ & 0.0 & $4.16600 \mathrm{E}-05$ & 0.0 \\
\hline $\mathrm{SO} 3$ & $4.34119 \mathrm{E}-27$ & 0.0 & $1.76649 \mathrm{E}-26$ & 0.0 \\
\hline CAH2SIO4 & $3.78335 \mathrm{E}-16$ & 0.0 & 0.0 & 0.0 \\
\hline CASO 4 & $4.67951 E-07$ & $6.97740 E-04$ & 0.0 & 0.0 \\
\hline CDCL2 & $1.47042 E-08$ & 0.0 & 0.0 & 0.0 \\
\hline $\mathrm{CDOH} 2$ & $1.43310 \mathrm{E}-19$ & 0.0 & 0.0 & 0.0 \\
\hline $\mathrm{CDSO} 4$ & $4.71165 E-13$ & 0.0 & 0.0 & 0.0 \\
\hline $\mathrm{CROH} 3$ & $9.53469 \mathrm{E}-20$ & 0.0 & 0.0 & 0.0 \\
\hline CUCL2 & $3.89014 E-09$ & 0.0 & 0.0 & 0.0 \\
\hline CUCO3 & $1.12283 \mathrm{E}-17$ & 0.0 & 0.0 & 0.0 \\
\hline CUNO32 & $2.07857 \mathrm{E}-11$ & 0.0 & 0.0 & 0.0 \\
\hline CUOH2 & $9.48532 \mathrm{E}-13$ & 0.0 & 0.0 & 0.0 \\
\hline FEIICL2 & $3.11910 \mathrm{E}-09$ & 0.0 & 0.0 & 0.0 \\
\hline FEIICO3 & $1.58516 \mathrm{E}-14$ & 0.0 & 0.0 & 0.0 \\
\hline FEIIHPO4 & $6.99211 \mathrm{E}-10$ & 0.0 & 0.0 & 0.0 \\
\hline FEIIOH2 & $4.23290 \mathrm{E}-13$ & 0.0 & 0.0 & 0.0 \\
\hline ALO2H2CL & $2.86510 \mathrm{E}-27$ & 0.0 & 0.0 & 0.0 \\
\hline H3PO 4 & $1.58531 E-09$ & 0.0 & 0.0 & 0.0 \\
\hline H4 P2O 7 & $7.75501 \mathrm{E}-17$ & 0.0 & 0.0 & 0.0 \\
\hline ALOH3 & $2.68709 \mathrm{E}-11$ & 0.0 & 0.0 & 0.0 \\
\hline $\mathrm{BACO} 3$ & $9.91860 \mathrm{E}-21$ & 0.0 & 0.0 & 0.0 \\
\hline $\mathrm{KCL}$ & $5.61457 E-06$ & 0.0 & 0.0 & 0.0 \\
\hline $\mathrm{KHSO} 4$ & $9.80545 E-11$ & 0.0 & 0.0 & 0.0 \\
\hline $\mathrm{BASO} 4$ & $8.46119 \mathrm{E}-13$ & $5.79798 E-07$ & 0.0 & 0.0 \\
\hline $\mathrm{MGCO} 3$ & $1.87813 E-15$ & 0.0 & 0.0 & 0.0 \\
\hline MGH2SIO4 & $3.65383 E-14$ & 0.0 & 0.0 & 0.0 \\
\hline MGHPO4 & $3.67422 \mathrm{E}-07$ & 0.0 & 0.0 & 0.0 \\
\hline MGSO 4 & $3.40068 \mathrm{E}-06$ & 0.0 & 0.0 & 0.0 \\
\hline $\mathrm{NAHCO} 3$ & $4.82023 E-12$ & 0.0 & 0.0 & 0.0 \\
\hline NAHSIO3 & $2.72575 E-09$ & 0.0 & 0.0 & 0.0 \\
\hline NANO3 & $9.27790 \mathrm{E}-05$ & 0.0 & 0.0 & 0.0 \\
\hline $\mathrm{NIOH} 2$ & $5.82882 E-15$ & 0.0 & 0.0 & 0.0 \\
\hline NISO4 & $5.23187 \mathrm{E}-08$ & 0.0 & 0.0 & 0.0 \\
\hline PBCL2 & $1.09501 \mathrm{E}-07$ & 0.0 & 0.0 & 0.0 \\
\hline PBHPO4 & $5.99252 E-13$ & 0.0 & 0.0 & 0.0 \\
\hline PBNO32 & $2.49669 \mathrm{E}-09$ & 0.0 & 0.0 & 0.0 \\
\hline $\mathrm{PBO}$ & $3.17959 \mathrm{E}-15$ & 0.0 & 0.0 & 0.0 \\
\hline CACL2 & $9.56559 \mathrm{E}-15$ & 0.0 & 0.0 & 0.0 \\
\hline $\mathrm{SIO} 2$ & $3.56801 E-06$ & $1.85045 E-04$ & 0.0 & 0.0 \\
\hline
\end{tabular}




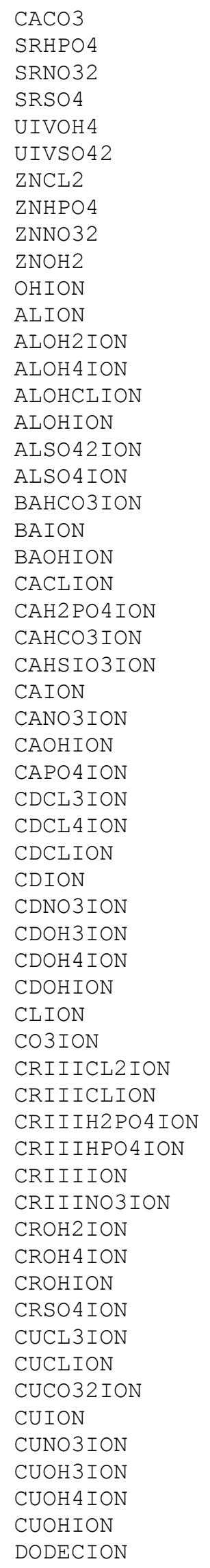

\begin{tabular}{|c|c|c|c|}
\hline $5.85043 E-16$ & 0.0 & 0.0 & 0.0 \\
\hline $6.53011 \mathrm{E}-12$ & 0.0 & 0.0 & 0.0 \\
\hline $1.81935 \mathrm{E}-07$ & 0.0 & 0.0 & 0.0 \\
\hline $8.01427 E-09$ & $1.58791 \mathrm{E}-05$ & 0.0 & 0.0 \\
\hline $1.00715 \mathrm{E}-12$ & 0.0 & 0.0 & 0.0 \\
\hline $1.40369 \mathrm{E}-24$ & 0.0 & 0.0 & 0.0 \\
\hline $6.22526 \mathrm{E}-06$ & 0.0 & 0.0 & 0.0 \\
\hline $1.06882 \mathrm{E}-10$ & 0.0 & 0.0 & 0.0 \\
\hline $2.04894 \mathrm{E}-09$ & 0.0 & 0.0 & 0.0 \\
\hline $2.15822 \mathrm{E}-13$ & 0.0 & 0.0 & 0.0 \\
\hline $3.37734 \mathrm{E}-11$ & 0.0 & 0.0 & 0.0 \\
\hline $1.46646 \mathrm{E}-13$ & 0.0 & 0.0 & 0.0 \\
\hline $8.34095 \mathrm{E}-12$ & 0.0 & 0.0 & 0.0 \\
\hline $1.19513 \mathrm{E}-11$ & 0.0 & 0.0 & 0.0 \\
\hline $9.58136 \mathrm{E}-12$ & 0.0 & 0.0 & 0.0 \\
\hline $2.25974 \mathrm{E}-12$ & 0.0 & 0.0 & 0.0 \\
\hline $1.05655 \mathrm{E}-14$ & 0.0 & 0.0 & 0.0 \\
\hline $1.25238 \mathrm{E}-13$ & 0.0 & 0.0 & 0.0 \\
\hline $3.96165 \mathrm{E}-16$ & 0.0 & 0.0 & 0.0 \\
\hline $4.14107 \mathrm{E}-09$ & 0.0 & 0.0 & 0.0 \\
\hline $9.01766 \mathrm{E}-17$ & 0.0 & 0.0 & 0.0 \\
\hline $1.73079 \mathrm{E}-07$ & 0.0 & 0.0 & 0.0 \\
\hline $7.51666 \mathrm{E}-07$ & 0.0 & 0.0 & 0.0 \\
\hline 7. $36762 \mathrm{E}-13$ & 0.0 & 0.0 & 0.0 \\
\hline $9.09607 \mathrm{E}-11$ & 0.0 & 0.0 & 0.0 \\
\hline $4.23469 \mathrm{E}-05$ & 0.0 & 0.0 & 0.0 \\
\hline $2.11073 \mathrm{E}-05$ & 0.0 & 0.0 & 0.0 \\
\hline $1.81393 \mathrm{E}-11$ & 0.0 & 0.0 & 0.0 \\
\hline $1.29262 \mathrm{E}-11$ & 0.0 & 0.0 & 0.0 \\
\hline $1.55417 \mathrm{E}-08$ & 0.0 & 0.0 & 0.0 \\
\hline $4.71238 \mathrm{E}-09$ & 0.0 & 0.0 & 0.0 \\
\hline $7.38681 \mathrm{E}-10$ & 0.0 & 0.0 & 0.0 \\
\hline $1.32499 \mathrm{E}-11$ & 0.0 & 0.0 & 0.0 \\
\hline $3.59272 \mathrm{E}-12$ & 0.0 & 0.0 & 0.0 \\
\hline $5.58545 \mathrm{E}-26$ & 0.0 & 0.0 & 0.0 \\
\hline 0.0 & 0.0 & 0.0 & 0.0 \\
\hline $7.06543 \mathrm{E}-16$ & 0.0 & 0.0 & 0.0 \\
\hline 0.00206795 & 0.0 & 0.0 & 0.0 \\
\hline $2.14167 \mathrm{E}-16$ & 0.0 & 0.0 & 0.0 \\
\hline $2.46095 \mathrm{E}-16$ & 0.0 & 0.0 & 0.0 \\
\hline $3.77128 \mathrm{E}-16$ & 0.0 & 0.0 & 0.0 \\
\hline $8.24052 \mathrm{E}-17$ & 0.0 & 0.0 & 0.0 \\
\hline $7.88455 \mathrm{E}-08$ & 0.0 & 0.0 & 0.0 \\
\hline $7.80907 E-20$ & 0.0 & 0.0 & 0.0 \\
\hline $7.83402 \mathrm{E}-14$ & 0.0 & 0.0 & 0.0 \\
\hline $7.32285 \mathrm{E}-19$ & 0.0 & 0.0 & 0.0 \\
\hline $5.76485 \mathrm{E}-25$ & 0.0 & 0.0 & 0.0 \\
\hline $1.72667 \mathrm{E}-13$ & 0.0 & 0.0 & 0.0 \\
\hline $4.12767 \mathrm{E}-13$ & 0.0 & 0.0 & 0.0 \\
\hline $7.06815 \mathrm{E}-11$ & 0.0 & 0.0 & 0.0 \\
\hline $2.14575 \mathrm{E}-08$ & 0.0 & 0.0 & 0.0 \\
\hline $1.64726 \mathrm{E}-26$ & 0.0 & 0.0 & 0.0 \\
\hline $5.69521 \mathrm{E}-09$ & 0.0 & 0.0 & 0.0 \\
\hline $9.49118 \mathrm{E}-10$ & 0.0 & 0.0 & 0.0 \\
\hline $2.33622 \mathrm{E}-18$ & 0.0 & 0.0 & 0.0 \\
\hline $1.23389 \mathrm{E}-23$ & 0.0 & 0.0 & 0.0 \\
\hline $3.38098 \mathrm{E}-11$ & 0.0 & 0.0 & 0.0 \\
\hline $7.61333 \mathrm{E}-09$ & 0.0 & 0.0 & 0.0 \\
\hline
\end{tabular}




\begin{tabular}{|c|c|c|c|c|}
\hline FEIICLION & $5.75607 \mathrm{E}-07$ & 0.0 & 0.0 & 0.0 \\
\hline FEIICO32ION & $6.86652 \mathrm{E}-25$ & 0.0 & 0.0 & 0.0 \\
\hline FEIIH2PO4ION & $3.50224 \mathrm{E}-08$ & 0.0 & 0.0 & 0.0 \\
\hline FEIIHCO3ION & $1.97544 \mathrm{E}-14$ & 0.0 & 0.0 & 0.0 \\
\hline FEIIION & $6.26309 \mathrm{E}-05$ & 0.0 & 0.0 & 0.0 \\
\hline FEIIOH3ION & $1.75691 \mathrm{E}-17$ & 0.0 & 0.0 & 0.0 \\
\hline FEIIOH 4 ION & $1.22128 \mathrm{E}-24$ & 0.0 & 0.0 & 0.0 \\
\hline FEIIOHION & $1.40936 \mathrm{E}-08$ & 0.0 & 0.0 & 0.0 \\
\hline H2 P2O 7 ION & $1.85294 \mathrm{E}-10$ & 0.0 & 0.0 & 0.0 \\
\hline H2 PO 4 ION & $6.39609 \mathrm{E}-07$ & 0.0 & 0.0 & 0.0 \\
\hline H2SIO4ION & $3.74652 \mathrm{E}-17$ & 0.0 & 0.0 & 0.0 \\
\hline H3P2O 7 ION & $9.81105 \mathrm{E}-14$ & 0.0 & 0.0 & 0.0 \\
\hline H3SIO4 ION & $9.97641 \mathrm{E}-10$ & 0.0 & 0.0 & 0.0 \\
\hline HCO3ION & $3.57887 \mathrm{E}-12$ & 0.0 & 0.0 & 0.0 \\
\hline HION & $2.35475 \mathrm{E}-08$ & 0.0 & 0.0 & 0.0 \\
\hline HP207ION & $1.18377 \mathrm{E}-11$ & 0.0 & 0.0 & 0.0 \\
\hline HPBO2ION & $8.22660 \mathrm{E}-20$ & 0.0 & 0.0 & 0.0 \\
\hline HPO4 ION & $1.53633 \mathrm{E}-08$ & 0.0 & 0.0 & 0.0 \\
\hline HSO 4 ION & $2.04036 \mathrm{E}-08$ & 0.0 & 0.0 & 0.0 \\
\hline KION & $2.55986 \mathrm{E}-04$ & 0.0 & 0.0 & 0.0 \\
\hline KSO4 ION & $3.55144 \mathrm{E}-06$ & 0.0 & 0.0 & 0.0 \\
\hline MGH2 PO 4 ION & $1.63368 \mathrm{E}-06$ & 0.0 & 0.0 & 0.0 \\
\hline MGHCO3ION & $9.16345 \mathrm{E}-12$ & 0.0 & 0.0 & 0.0 \\
\hline MGHSIO3ION & $1.15153 \mathrm{E}-09$ & 0.0 & 0.0 & 0.0 \\
\hline MGION & $1.28707 \mathrm{E}-04$ & 0.0 & 0.0 & 0.0 \\
\hline MGOHION & $9.70254 \mathrm{E}-10$ & 0.0 & 0.0 & 0.0 \\
\hline MGP2O 7 ION & $6.31406 \mathrm{E}-10$ & 0.0 & 0.0 & 0.0 \\
\hline MGPO 4 ION & $8.74582 \mathrm{E}-11$ & 0.0 & 0.0 & 0.0 \\
\hline NACO3ION & $3.07702 \mathrm{E}-17$ & 0.0 & 0.0 & 0.0 \\
\hline NAION & 0.00171029 & 0.0 & 0.0 & 0.0 \\
\hline NASO 4 ION & $1.53980 \mathrm{E}-14$ & 0.0 & 0.0 & 0.0 \\
\hline NICLION & $9.07648 \mathrm{E}-08$ & 0.0 & 0.0 & 0.0 \\
\hline NIION & $1.00975 \mathrm{E}-06$ & 0.0 & 0.0 & 0.0 \\
\hline NINO3ION & $1.41821 \mathrm{E}-07$ & 0.0 & 0.0 & 0.0 \\
\hline NIOH3ION & $3.54707 \mathrm{E}-20$ & 0.0 & 0.0 & 0.0 \\
\hline NIOHION & $6.26804 \mathrm{E}-11$ & 0.0 & 0.0 & 0.0 \\
\hline NO3ION & $3.52412 \mathrm{E}-04$ & 0.0 & 0.0 & 0.0 \\
\hline P207ION & $4.79769 \mathrm{E}-15$ & 0.0 & 0.0 & 0.0 \\
\hline PBCL3ION & $2.96895 \mathrm{E}-07$ & 0.0 & 0.0 & 0.0 \\
\hline PBCL 4 ION & $2.55122 \mathrm{E}-06$ & 0.0 & 0.0 & 0.0 \\
\hline PBCLION & $2.71145 \mathrm{E}-08$ & 0.0 & 0.0 & 0.0 \\
\hline PBH2 PO 4 ION & $1.46667 \mathrm{E}-11$ & 0.0 & 0.0 & 0.0 \\
\hline PBION & $7.00391 \mathrm{E}-10$ & 0.0 & 0.0 & 0.0 \\
\hline PBNO33ION & $9.40299 \mathrm{E}-11$ & 0.0 & 0.0 & 0.0 \\
\hline PBNO3ION & $7.09682 \mathrm{E}-09$ & 0.0 & 0.0 & 0.0 \\
\hline PBOHION & $3.35037 \mathrm{E}-11$ & 0.0 & 0.0 & 0.0 \\
\hline PO4ION & $7.24830 \mathrm{E}-15$ & 0.0 & 0.0 & 0.0 \\
\hline SO4ION & $1.78806 \mathrm{E}-05$ & 0.0 & 0.0 & 0.0 \\
\hline SRION & $3.87563 \mathrm{E}-07$ & 0.0 & 0.0 & 0.0 \\
\hline SRNO3ION & $6.66616 \mathrm{E}-07$ & 0.0 & 0.0 & 0.0 \\
\hline SROHION & $1.36533 \mathrm{E}-13$ & 0.0 & 0.0 & 0.0 \\
\hline SRPO 4 ION & $3.02899 \mathrm{E}-15$ & 0.0 & 0.0 & 0.0 \\
\hline UIVCLION & $1.43788 \mathrm{E}-26$ & 0.0 & 0.0 & 0.0 \\
\hline UIVION & $1.73756 \mathrm{E}-28$ & 0.0 & 0.0 & 0.0 \\
\hline UIVOH2ION & $1.02176 \mathrm{E}-20$ & 0.0 & 0.0 & 0.0 \\
\hline UIVOH3ION & $6.99798 \mathrm{E}-18$ & 0.0 & 0.0 & 0.0 \\
\hline UIVOH5ION & $2.31096 \mathrm{E}-17$ & 0.0 & 0.0 & 0.0 \\
\hline UIVOHION & $8.00951 \mathrm{E}-23$ & 0.0 & 0.0 & 0.0 \\
\hline
\end{tabular}


WSRC-TR-2002-00567

Revision 0

\begin{tabular}{|c|c|c|c|c|}
\hline UIVSO4ION & $2.13175 E-24$ & 0.0 & 0.0 & 0.0 \\
\hline ZNCL3 ION & $9.77488 \mathrm{E}-06$ & 0.0 & 0.0 & 0.0 \\
\hline ZNCLION & $1.22533 E-05$ & 0.0 & 0.0 & 0.0 \\
\hline ZNH2PO 4 ION & $1.27276 \mathrm{E}-09$ & 0.0 & 0.0 & 0.0 \\
\hline ZNHCO3ION & $5.98731 E-15$ & 0.0 & 0.0 & 0.0 \\
\hline ZNION & $7.11093 \mathrm{E}-07$ & 0.0 & 0.0 & 0.0 \\
\hline ZNNO3 ION & $7.18328 E-08$ & 0.0 & 0.0 & 0.0 \\
\hline ZNOH3 ION & $2.23997 \mathrm{E}-17$ & 0.0 & 0.0 & 0.0 \\
\hline ZNOH 4 ION & $4.17472 E-23$ & 0.0 & 0.0 & 0.0 \\
\hline ZNOHION & $1.21783 \mathrm{E}-08$ & 0.0 & 0.0 & 0.0 \\
\hline ALOOH & 0.0 & $6.61265 E-05$ & 0.0 & 0.0 \\
\hline $\mathrm{CA} 3 \mathrm{PO} 42$ & 0.0 & $3.67425 \mathrm{E}-05$ & 0.0 & 0.0 \\
\hline CHAMOSITE7A & 0.0 & $1.13810 \mathrm{E}-05$ & 0.0 & 0.0 \\
\hline CU3PO42.2H2O & 0.0 & $1.72157 \mathrm{E}-06$ & 0.0 & 0.0 \\
\hline MG3PO42 & 0.0 & $6.50351 E-05$ & 0.0 & 0.0 \\
\hline UIVO2 & 0.0 & $2.89915 E-06$ & 0.0 & 0.0 \\
\hline & $============$ & $============$ & $============$ & $============$ \\
\hline Total g/hr & 0.997387 & 0.147011 & 998.856 & 0.0 \\
\hline Volume, L/hr & $9.30591 E-04$ & $4.28148 E-05$ & 1697.57 & 0.0 \\
\hline Enthalpy, cal/hr & -3327.52 & -406.02 & $-3.17043 E+06$ & 0.0 \\
\hline Density, g/L & 1071.78 & 3433.64 & 0.588402 & \\
\hline Vapor fraction & 0.0 & 0.0 & 1 . & 0.0 \\
\hline Solid fraction & 0.0 & 1 . & 0.0 & 0.0 \\
\hline Organic fraction & 0.0 & 0.0 & 0.0 & 0.0 \\
\hline Osmotic Pres, atm & 175.295 & & & \\
\hline Redox Pot, volts & 0.0 & & & \\
\hline E-Con, $1 / \mathrm{ohm}-\mathrm{cm}$ & 0.457163 & & & \\
\hline E-Con, cm2/ohm-mol & 54.0155 & & & \\
\hline Abs Visc, cP & 0.388136 & & & \\
\hline Rel Visc & 1.42291 & & & \\
\hline Ionic Strength & 3.3089 & & & \\
\hline
\end{tabular}


WSRC-TR-2002-00567

Revision 0

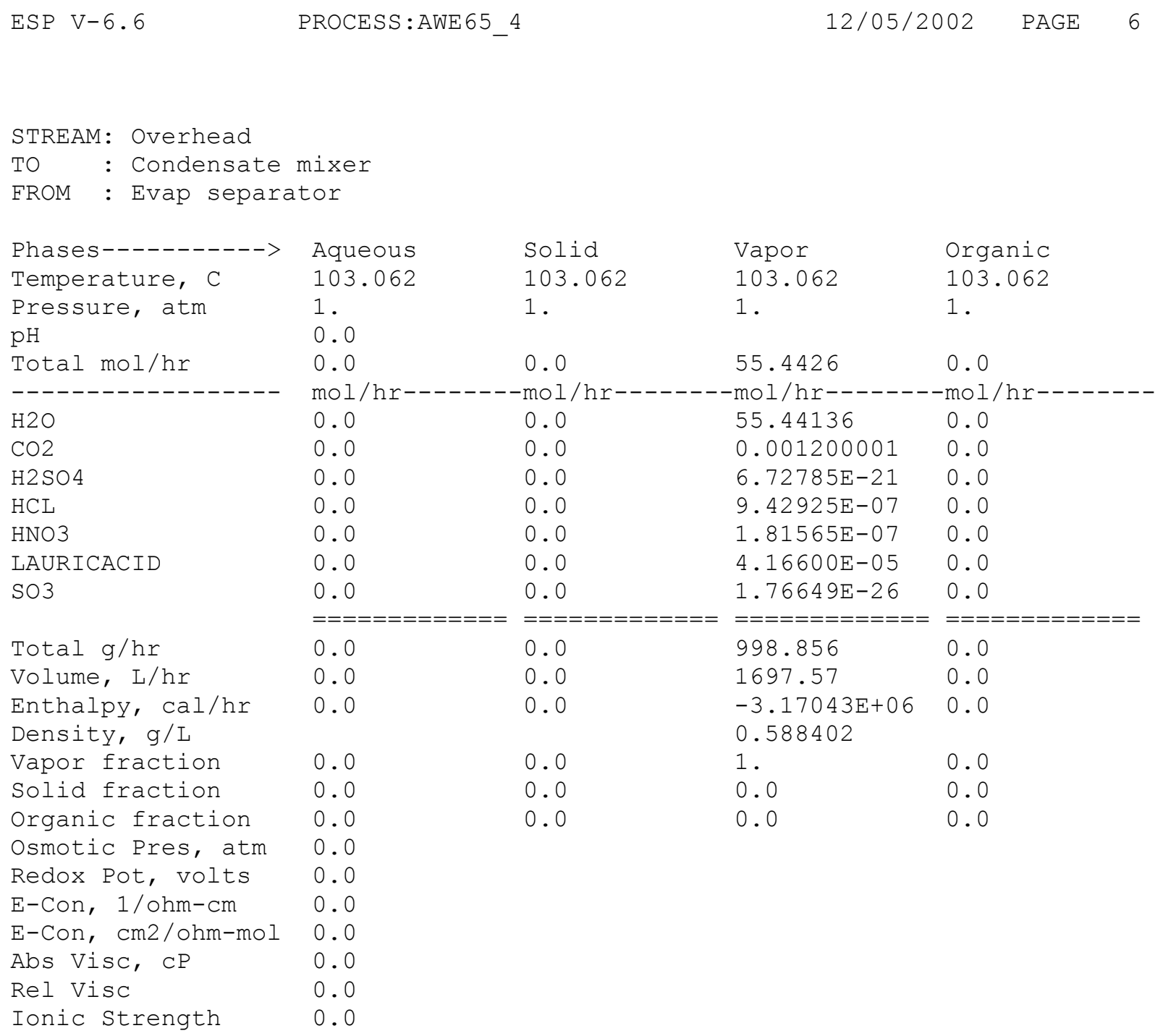


WSRC-TR-2002-00567

Revision 0

\begin{tabular}{|c|c|c|c|c|}
\hline \multicolumn{5}{|c|}{ TO : Evap Bottoms Cooling mixer } \\
\hline Phases $----------->$ & Aqueous & Solid & Vapor & Organic \\
\hline Temperature, C & 103.062 & 103.062 & 103.062 & 103.062 \\
\hline $\begin{array}{l}\text { Pressure, atm } \\
\mathrm{pH}\end{array}$ & $\begin{array}{l}1 . \\
4.65196\end{array}$ & 1. & 1 . & 1 . \\
\hline Total mol/hr & 0.0506356 & 0.00108315 & 0.0 & 0.0 \\
\hline \multicolumn{3}{|l|}{------------------} & $-\mathrm{mol} / \mathrm{hr}--$ & -mol/hr- \\
\hline $\mathrm{H} 2 \mathrm{O}$ & 0.0458279 & 0.0 & 0.0 & 0.0 \\
\hline $\mathrm{CO} 2$ & $1.27929 \mathrm{E}-10$ & 0.0 & 0.0 & 0.0 \\
\hline $\mathrm{H} 2 \mathrm{SO} 4$ & $6.05781 \mathrm{E}-24$ & 0.0 & 0.0 & 0.0 \\
\hline $\mathrm{HCL}$ & $7.98029 \mathrm{E}-13$ & 0.0 & 0.0 & 0.0 \\
\hline HNO3 & $6.83897 \mathrm{E}-10$ & 0.0 & 0.0 & 0.0 \\
\hline LAURICACID & $6.42487 E-10$ & 0.0 & 0.0 & 0.0 \\
\hline $\mathrm{SO} 3$ & $4.34119 \mathrm{E}-27$ & 0.0 & 0.0 & 0.0 \\
\hline CAH2SIO4 & $3.78335 E-16$ & 0.0 & 0.0 & 0.0 \\
\hline $\mathrm{CASO} 4$ & $4.67951 \mathrm{E}-07$ & $6.97740 E-04$ & 0.0 & 0.0 \\
\hline CDCL2 & $1.47042 E-08$ & 0.0 & 0.0 & 0.0 \\
\hline $\mathrm{CDOH} 2$ & $1.43310 \mathrm{E}-19$ & 0.0 & 0.0 & 0.0 \\
\hline $\mathrm{CDSO} 4$ & $4.71165 E-13$ & 0.0 & 0.0 & 0.0 \\
\hline $\mathrm{CROH} 3$ & $9.53469 \mathrm{E}-20$ & 0.0 & 0.0 & 0.0 \\
\hline CUCL2 & $3.89014 \mathrm{E}-09$ & 0.0 & 0.0 & 0.0 \\
\hline CUCO3 & $1.12283 \mathrm{E}-17$ & 0.0 & 0.0 & 0.0 \\
\hline CUNO32 & $2.07857 \mathrm{E}-11$ & 0.0 & 0.0 & 0.0 \\
\hline CUOH2 & $9.48532 \mathrm{E}-13$ & 0.0 & 0.0 & 0.0 \\
\hline FEIICL2 & $3.11910 \mathrm{E}-09$ & 0.0 & 0.0 & 0.0 \\
\hline FEIICO3 & $1.58516 \mathrm{E}-14$ & 0.0 & 0.0 & 0.0 \\
\hline FEIIHPO4 & $6.99211 E-10$ & 0.0 & 0.0 & 0.0 \\
\hline FEIIOH2 & $4.23290 \mathrm{E}-13$ & 0.0 & 0.0 & 0.0 \\
\hline $\mathrm{ALO} 2 \mathrm{H} 2 \mathrm{CL}$ & $2.86510 \mathrm{E}-27$ & 0.0 & 0.0 & 0.0 \\
\hline H3PO 4 & $1.58531 E-09$ & 0.0 & 0.0 & 0.0 \\
\hline H4 P 207 & $7.75501 \mathrm{E}-17$ & 0.0 & 0.0 & 0.0 \\
\hline ALOH3 & $2.68709 \mathrm{E}-11$ & 0.0 & 0.0 & 0.0 \\
\hline $\mathrm{BACO} 3$ & $9.91860 \mathrm{E}-21$ & 0.0 & 0.0 & 0.0 \\
\hline $\mathrm{KCL}$ & $5.61457 \mathrm{E}-06$ & 0.0 & 0.0 & 0.0 \\
\hline KHSO 4 & $9.80545 \mathrm{E}-11$ & 0.0 & 0.0 & 0.0 \\
\hline BASO 4 & $8.46119 E-13$ & $5.79798 E-07$ & 0.0 & 0.0 \\
\hline $\mathrm{MGCO} 3$ & $1.87813 E-15$ & 0.0 & 0.0 & 0.0 \\
\hline MGH2SIO4 & $3.65383 E-14$ & 0.0 & 0.0 & 0.0 \\
\hline MGHPO4 & $3.67422 \mathrm{E}-07$ & 0.0 & 0.0 & 0.0 \\
\hline MGSO4 & $3.40068 E-06$ & 0.0 & 0.0 & 0.0 \\
\hline NAHCO3 & $4.82023 E-12$ & 0.0 & 0.0 & 0.0 \\
\hline NAHSIO3 & $2.72575 E-09$ & 0.0 & 0.0 & 0.0 \\
\hline NANO3 & $9.27790 \mathrm{E}-05$ & 0.0 & 0.0 & 0.0 \\
\hline $\mathrm{NIOH} 2$ & $5.82882 E-15$ & 0.0 & 0.0 & 0.0 \\
\hline $\mathrm{NISO} 4$ & $5.23187 E-08$ & 0.0 & 0.0 & 0.0 \\
\hline PBCL2 & $1.09501 \mathrm{E}-07$ & 0.0 & 0.0 & 0.0 \\
\hline PBHPO4 & $5.99252 \mathrm{E}-13$ & 0.0 & 0.0 & 0.0 \\
\hline PBNO32 & $2.49669 \mathrm{E}-09$ & 0.0 & 0.0 & 0.0 \\
\hline $\mathrm{PBO}$ & $3.17959 \mathrm{E}-15$ & 0.0 & 0.0 & 0.0 \\
\hline CACL2 & $9.56559 \mathrm{E}-15$ & 0.0 & 0.0 & 0.0 \\
\hline $\mathrm{SIO} 2$ & $3.56801 E-06$ & $1.85045 E-04$ & 0.0 & 0.0 \\
\hline
\end{tabular}




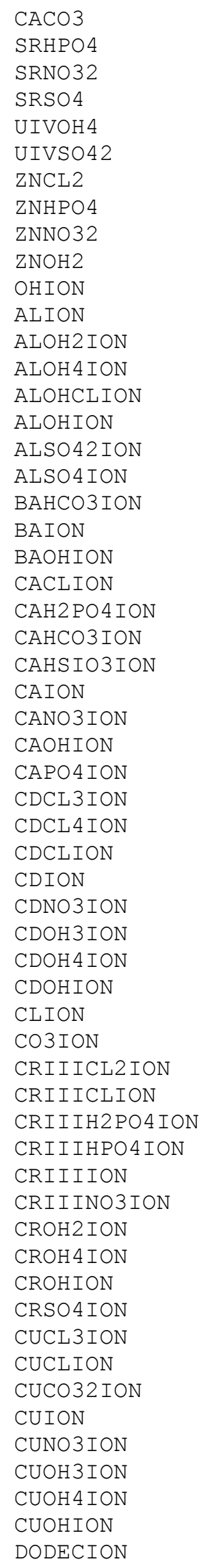

\begin{tabular}{|c|c|}
\hline $5.85043 E-16$ & 0.0 \\
\hline $6.53011 \mathrm{E}-12$ & 0.0 \\
\hline $1.81935 \mathrm{E}-07$ & 0.0 \\
\hline $8.01427 E-09$ & $1.58791 E-05$ \\
\hline $1.00715 \mathrm{E}-12$ & 0.0 \\
\hline $1.40369 \mathrm{E}-24$ & 0.0 \\
\hline $6.22526 \mathrm{E}-06$ & 0.0 \\
\hline $1.06882 \mathrm{E}-10$ & 0.0 \\
\hline $2.04894 \mathrm{E}-09$ & 0.0 \\
\hline $2.15822 \mathrm{E}-13$ & 0.0 \\
\hline $3.37734 \mathrm{E}-11$ & 0.0 \\
\hline $1.46646 \mathrm{E}-13$ & 0.0 \\
\hline $8.34095 E-12$ & 0.0 \\
\hline $1.19513 \mathrm{E}-11$ & 0.0 \\
\hline $9.58136 \mathrm{E}-12$ & 0.0 \\
\hline $2.25974 \mathrm{E}-12$ & 0.0 \\
\hline $1.05655 \mathrm{E}-14$ & 0.0 \\
\hline 1.25238E-13 & 0.0 \\
\hline $3.96165 E-16$ & 0.0 \\
\hline $4.14107 E-09$ & 0.0 \\
\hline $9.01766 \mathrm{E}-17$ & 0.0 \\
\hline $1.73079 \mathrm{E}-07$ & 0.0 \\
\hline $7.51666 \mathrm{E}-07$ & 0.0 \\
\hline $7.36762 \mathrm{E}-13$ & 0.0 \\
\hline $9.09607 \mathrm{E}-11$ & 0.0 \\
\hline $4.23469 E-05$ & 0.0 \\
\hline $2.11073 E-05$ & 0.0 \\
\hline $1.81393 \mathrm{E}-11$ & 0.0 \\
\hline $1.29262 \mathrm{E}-11$ & 0.0 \\
\hline $1.55417 \mathrm{E}-08$ & 0.0 \\
\hline $4.71238 E-09$ & 0.0 \\
\hline $7.38681 \mathrm{E}-10$ & 0.0 \\
\hline $1.32499 \mathrm{E}-11$ & 0.0 \\
\hline $3.59272 \mathrm{E}-12$ & 0.0 \\
\hline $5.58545 E-26$ & 0.0 \\
\hline 0.0 & 0.0 \\
\hline $7.06543 E-16$ & 0.0 \\
\hline 0.00206795 & 0.0 \\
\hline $2.14167 E-16$ & 0.0 \\
\hline $2.46095 E-16$ & 0.0 \\
\hline $3.77128 \mathrm{E}-16$ & 0.0 \\
\hline $8.24052 \mathrm{E}-17$ & 0.0 \\
\hline $7.88455 E-08$ & 0.0 \\
\hline $7.80907 \mathrm{E}-20$ & 0.0 \\
\hline $7.83402 \mathrm{E}-14$ & 0.0 \\
\hline 7. $32285 E-19$ & 0.0 \\
\hline $5.76485 \mathrm{E}-25$ & 0.0 \\
\hline $1.72667 \mathrm{E}-13$ & 0.0 \\
\hline $4.12767 \mathrm{E}-13$ & 0.0 \\
\hline $7.06815 \mathrm{E}-11$ & 0.0 \\
\hline $2.14575 E-08$ & 0.0 \\
\hline $1.64726 \mathrm{E}-26$ & 0.0 \\
\hline $5.69521 \mathrm{E}-09$ & 0.0 \\
\hline $9.49118 \mathrm{E}-10$ & 0.0 \\
\hline $2.33622 \mathrm{E}-18$ & 0.0 \\
\hline $1.23389 \mathrm{E}-23$ & 0.0 \\
\hline $3.38098 \mathrm{E}-11$ & 0.0 \\
\hline $7.61333 E-09$ & 0.0 \\
\hline
\end{tabular}

0.0

0.0

0.0

0.0

0.0

0.0

0.0

0.0

0.0

0.0

0.0

0.0

0.0

0.0

0.0

0.0

0.0

0.0

0.0

0.0

0.0

0.0

0.0

0.0

0.0

0.0

0.0

0.0

0.0

0.0

0.0

0.0

0.0

0.0

0.0

0.0

0.0

0.0

0.0

0.0

0.0

0.0

0.0

0.0

0.0

0.0

0.0

0.0

0.0

0.0

0.0

0.0

0.0

0.0

0.0

0.0

0.0

0.0
0.0

0.0

0.0

0.0

0.0

0.0

0.0

0.0

0.0

0.0

0.0

0.0

0.0

0.0

0.0

0.0

0.0

0.0

0.0

0.0

0.0

0.0

0.0

0.0

0.0

0.0

0.0

0.0

0.0

0.0

0.0

0.0

0.0

0.0

0.0

0.0

0.0

0.0

0.0

0.0

0.0

0.0

0.0

0.0

0.0

0.0

0.0

0.0

0.0

0.0

0.0

0.0

0.0

0.0

0.0

0.0

0.0

0.0 


\begin{tabular}{|c|c|c|c|c|}
\hline FEIICLION & $5.75607 \mathrm{E}-07$ & 0.0 & 0.0 & 0.0 \\
\hline FEIICO32ION & $6.86652 \mathrm{E}-25$ & 0.0 & 0.0 & 0.0 \\
\hline FEIIH2PO4ION & $3.50224 \mathrm{E}-08$ & 0.0 & 0.0 & 0.0 \\
\hline FEIIHCO3ION & $1.97544 \mathrm{E}-14$ & 0.0 & 0.0 & 0.0 \\
\hline FEIIION & $6.26309 \mathrm{E}-05$ & 0.0 & 0.0 & 0.0 \\
\hline FEIIOH3ION & $1.75691 \mathrm{E}-17$ & 0.0 & 0.0 & 0.0 \\
\hline FEIIOH 4 ION & $1.22128 \mathrm{E}-24$ & 0.0 & 0.0 & 0.0 \\
\hline FEIIOHION & $1.40936 \mathrm{E}-08$ & 0.0 & 0.0 & 0.0 \\
\hline H2 P2O 7 ION & $1.85294 \mathrm{E}-10$ & 0.0 & 0.0 & 0.0 \\
\hline H2 PO 4 ION & $6.39609 \mathrm{E}-07$ & 0.0 & 0.0 & 0.0 \\
\hline H2SIO4ION & $3.74652 \mathrm{E}-17$ & 0.0 & 0.0 & 0.0 \\
\hline H3P2O 7 ION & $9.81105 \mathrm{E}-14$ & 0.0 & 0.0 & 0.0 \\
\hline H3SIO4 ION & $9.97641 \mathrm{E}-10$ & 0.0 & 0.0 & 0.0 \\
\hline HCO3ION & $3.57887 \mathrm{E}-12$ & 0.0 & 0.0 & 0.0 \\
\hline HION & $2.35475 \mathrm{E}-08$ & 0.0 & 0.0 & 0.0 \\
\hline HP207ION & $1.18377 \mathrm{E}-11$ & 0.0 & 0.0 & 0.0 \\
\hline HPBO2ION & $8.22660 \mathrm{E}-20$ & 0.0 & 0.0 & 0.0 \\
\hline HPO4 ION & $1.53633 \mathrm{E}-08$ & 0.0 & 0.0 & 0.0 \\
\hline HSO 4 ION & $2.04036 \mathrm{E}-08$ & 0.0 & 0.0 & 0.0 \\
\hline KION & $2.55986 \mathrm{E}-04$ & 0.0 & 0.0 & 0.0 \\
\hline KSO4 ION & $3.55144 \mathrm{E}-06$ & 0.0 & 0.0 & 0.0 \\
\hline MGH2 PO 4 ION & $1.63368 \mathrm{E}-06$ & 0.0 & 0.0 & 0.0 \\
\hline MGHCO3ION & $9.16345 \mathrm{E}-12$ & 0.0 & 0.0 & 0.0 \\
\hline MGHSIO3ION & $1.15153 \mathrm{E}-09$ & 0.0 & 0.0 & 0.0 \\
\hline MGION & $1.28707 \mathrm{E}-04$ & 0.0 & 0.0 & 0.0 \\
\hline MGOHION & $9.70254 \mathrm{E}-10$ & 0.0 & 0.0 & 0.0 \\
\hline MGP2O 7 ION & $6.31406 \mathrm{E}-10$ & 0.0 & 0.0 & 0.0 \\
\hline MGPO 4 ION & $8.74582 \mathrm{E}-11$ & 0.0 & 0.0 & 0.0 \\
\hline NACO3ION & $3.07702 \mathrm{E}-17$ & 0.0 & 0.0 & 0.0 \\
\hline NAION & 0.00171029 & 0.0 & 0.0 & 0.0 \\
\hline NASO 4 ION & $1.53980 \mathrm{E}-14$ & 0.0 & 0.0 & 0.0 \\
\hline NICLION & $9.07648 \mathrm{E}-08$ & 0.0 & 0.0 & 0.0 \\
\hline NIION & $1.00975 \mathrm{E}-06$ & 0.0 & 0.0 & 0.0 \\
\hline NINO3ION & $1.41821 \mathrm{E}-07$ & 0.0 & 0.0 & 0.0 \\
\hline NIOH3ION & $3.54707 \mathrm{E}-20$ & 0.0 & 0.0 & 0.0 \\
\hline NIOHION & $6.26804 \mathrm{E}-11$ & 0.0 & 0.0 & 0.0 \\
\hline NO3ION & $3.52412 \mathrm{E}-04$ & 0.0 & 0.0 & 0.0 \\
\hline P207ION & $4.79769 \mathrm{E}-15$ & 0.0 & 0.0 & 0.0 \\
\hline PBCL3ION & $2.96895 \mathrm{E}-07$ & 0.0 & 0.0 & 0.0 \\
\hline PBCL 4 ION & $2.55122 \mathrm{E}-06$ & 0.0 & 0.0 & 0.0 \\
\hline PBCLION & $2.71145 \mathrm{E}-08$ & 0.0 & 0.0 & 0.0 \\
\hline PBH2 PO 4 ION & $1.46667 \mathrm{E}-11$ & 0.0 & 0.0 & 0.0 \\
\hline PBION & $7.00391 \mathrm{E}-10$ & 0.0 & 0.0 & 0.0 \\
\hline PBNO33ION & $9.40299 \mathrm{E}-11$ & 0.0 & 0.0 & 0.0 \\
\hline PBNO3ION & $7.09682 \mathrm{E}-09$ & 0.0 & 0.0 & 0.0 \\
\hline PBOHION & $3.35037 \mathrm{E}-11$ & 0.0 & 0.0 & 0.0 \\
\hline PO4ION & $7.24830 \mathrm{E}-15$ & 0.0 & 0.0 & 0.0 \\
\hline SO4ION & $1.78806 \mathrm{E}-05$ & 0.0 & 0.0 & 0.0 \\
\hline SRION & $3.87563 \mathrm{E}-07$ & 0.0 & 0.0 & 0.0 \\
\hline SRNO3ION & $6.66616 \mathrm{E}-07$ & 0.0 & 0.0 & 0.0 \\
\hline SROHION & $1.36533 \mathrm{E}-13$ & 0.0 & 0.0 & 0.0 \\
\hline SRPO 4 ION & $3.02899 \mathrm{E}-15$ & 0.0 & 0.0 & 0.0 \\
\hline UIVCLION & $1.43788 \mathrm{E}-26$ & 0.0 & 0.0 & 0.0 \\
\hline UIVION & $1.73756 \mathrm{E}-28$ & 0.0 & 0.0 & 0.0 \\
\hline UIVOH2ION & $1.02176 \mathrm{E}-20$ & 0.0 & 0.0 & 0.0 \\
\hline UIVOH3ION & $6.99798 \mathrm{E}-18$ & 0.0 & 0.0 & 0.0 \\
\hline UIVOH5ION & $2.31096 \mathrm{E}-17$ & 0.0 & 0.0 & 0.0 \\
\hline UIVOHION & $8.00951 \mathrm{E}-23$ & 0.0 & 0.0 & 0.0 \\
\hline
\end{tabular}


WSRC-TR-2002-00567

Revision 0

\begin{tabular}{|c|c|c|c|c|}
\hline UIVSO4ION & $2.13175 E-24$ & 0.0 & 0.0 & 0.0 \\
\hline ZNCL3 ION & $9.77488 \mathrm{E}-06$ & 0.0 & 0.0 & 0.0 \\
\hline ZNCLION & $1.22533 E-05$ & 0.0 & 0.0 & 0.0 \\
\hline ZNH2PO 4 ION & $1.27276 \mathrm{E}-09$ & 0.0 & 0.0 & 0.0 \\
\hline ZNHCO3ION & $5.98731 E-15$ & 0.0 & 0.0 & 0.0 \\
\hline ZNION & $7.11093 \mathrm{E}-07$ & 0.0 & 0.0 & 0.0 \\
\hline ZNNO3 ION & $7.18328 E-08$ & 0.0 & 0.0 & 0.0 \\
\hline ZNOH3 ION & $2.23997 \mathrm{E}-17$ & 0.0 & 0.0 & 0.0 \\
\hline ZNOH 4 ION & $4.17472 E-23$ & 0.0 & 0.0 & 0.0 \\
\hline ZNOHION & $1.21783 \mathrm{E}-08$ & 0.0 & 0.0 & 0.0 \\
\hline ALOOH & 0.0 & $6.61265 E-05$ & 0.0 & 0.0 \\
\hline $\mathrm{CA} 3 \mathrm{PO} 42$ & 0.0 & $3.67425 \mathrm{E}-05$ & 0.0 & 0.0 \\
\hline CHAMOSITE7A & 0.0 & $1.13810 \mathrm{E}-05$ & 0.0 & 0.0 \\
\hline CU3PO42.2H2O & 0.0 & $1.72157 \mathrm{E}-06$ & 0.0 & 0.0 \\
\hline MG3PO42 & 0.0 & $6.50351 E-05$ & 0.0 & 0.0 \\
\hline UIVO2 & 0.0 & $2.89915 E-06$ & 0.0 & 0.0 \\
\hline & $============$ & $============$ & $============$ & $============$ \\
\hline Total g/hr & 0.997387 & 0.147011 & 0.0 & 0.0 \\
\hline Volume, L/hr & $9.30591 E-04$ & $4.28148 E-05$ & 0.0 & 0.0 \\
\hline Enthalpy, cal/hr & -3327.52 & -406.02 & 0.0 & 0.0 \\
\hline Density, g/L & 1071.78 & 3433.64 & & \\
\hline Vapor fraction & 0.0 & 0.0 & 0.0 & 0.0 \\
\hline Solid fraction & 0.0 & 1 . & 0.0 & 0.0 \\
\hline Organic fraction & 0.0 & 0.0 & 0.0 & 0.0 \\
\hline Osmotic Pres, atm & 175.295 & & & \\
\hline Redox Pot, volts & 0.0 & & & \\
\hline E-Con, $1 / \mathrm{ohm}-\mathrm{cm}$ & 0.457163 & & & \\
\hline E-Con, cm2/ohm-mol & 54.0155 & & & \\
\hline Abs Visc, cP & 0.388136 & & & \\
\hline Rel Visc & 1.42291 & & & \\
\hline Ionic Strength & 3.3089 & & & \\
\hline
\end{tabular}


WSRC-TR-2002-00567

Revision 0

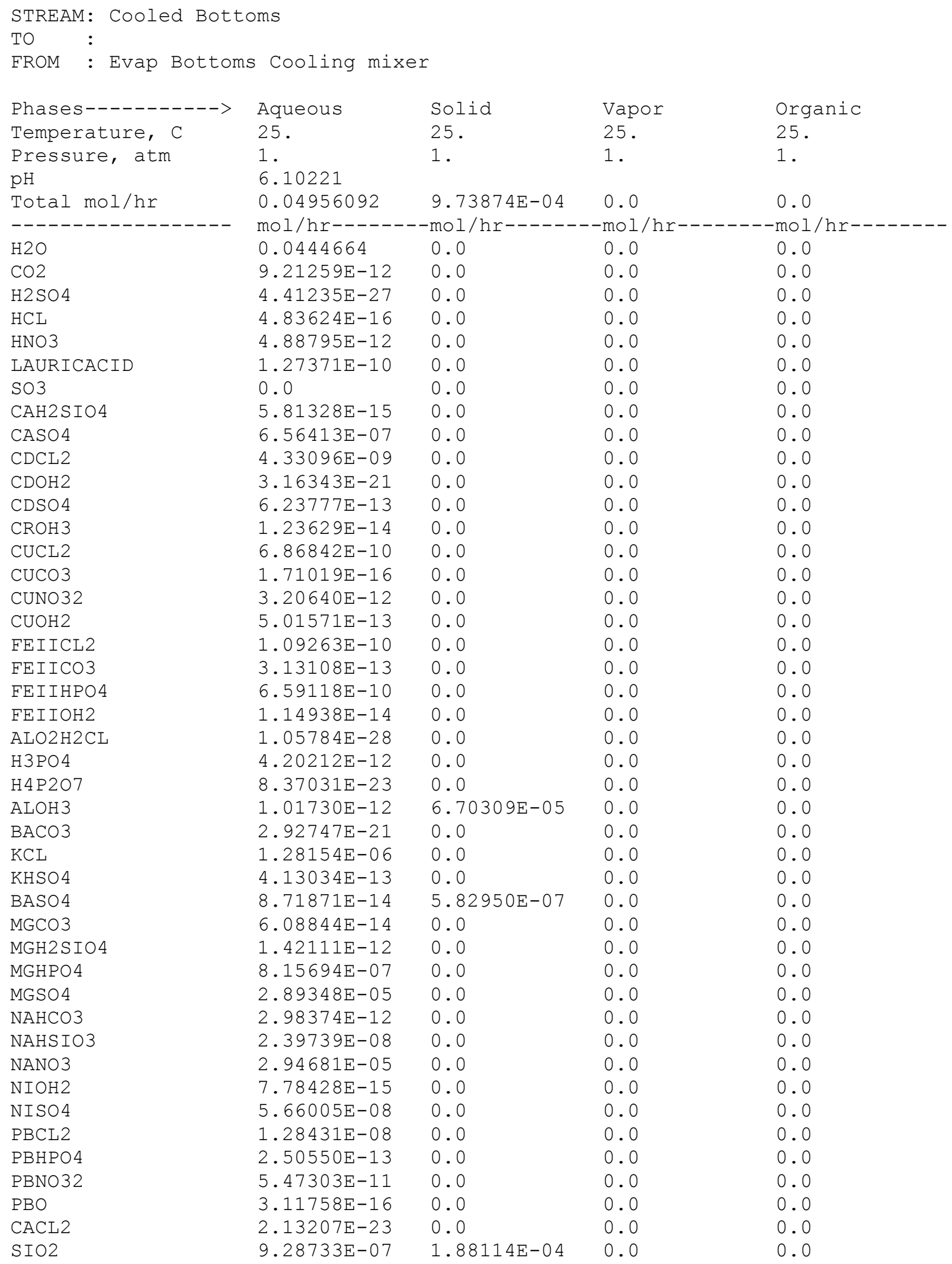




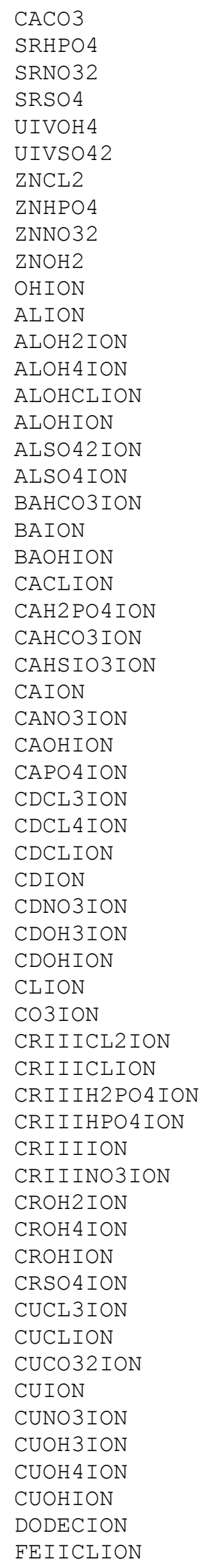

\begin{tabular}{|c|c|}
\hline $5.33504 E-15$ & 0.0 \\
\hline $2.61779 \mathrm{E}-12$ & 0.0 \\
\hline $2.21896 \mathrm{E}-08$ & 0.0 \\
\hline $1.82681 E-08$ & $1.62586 \mathrm{E}-05$ \\
\hline $1.00138 \mathrm{E}-13$ & 0.0 \\
\hline $1.03636 \mathrm{E}-27$ & 0.0 \\
\hline $1.59121 \mathrm{E}-06$ & 0.0 \\
\hline $1.98565 E-09$ & 0.0 \\
\hline $1.30697 \mathrm{E}-08$ & 0.0 \\
\hline $9.39756 \mathrm{E}-13$ & 0.0 \\
\hline $1.07930 \mathrm{E}-11$ & 0.0 \\
\hline $3.34198 E-14$ & 0.0 \\
\hline $4.34808 E-13$ & 0.0 \\
\hline $9.82520 \mathrm{E}-13$ & 0.0 \\
\hline $1.07148 \mathrm{E}-12$ & 0.0 \\
\hline $1.37992 \mathrm{E}-13$ & 0.0 \\
\hline $1.87575 \mathrm{E}-14$ & 0.0 \\
\hline $5.52756 \mathrm{E}-14$ & 0.0 \\
\hline $4.81370 \mathrm{E}-18$ & 0.0 \\
\hline $9.91162 \mathrm{E}-10$ & 0.0 \\
\hline $1.72018 \mathrm{E}-19$ & 0.0 \\
\hline $1.40432 \mathrm{E}-10$ & 0.0 \\
\hline $5.20651 E-08$ & 0.0 \\
\hline $1.65001 \mathrm{E}-12$ & 0.0 \\
\hline $8.83595 E-11$ & 0.0 \\
\hline $2.92485 E-05$ & 0.0 \\
\hline $1.39912 \mathrm{E}-05$ & 0.0 \\
\hline 1. $94063 \mathrm{E}-12$ & 0.0 \\
\hline $2.48834 \mathrm{E}-10$ & 0.0 \\
\hline $2.08986 \mathrm{E}-09$ & 0.0 \\
\hline $2.90475 E-08$ & 0.0 \\
\hline $2.35214 \mathrm{E}-10$ & 0.0 \\
\hline $8.73990 E-12$ & 0.0 \\
\hline $1.39628 \mathrm{E}-12$ & 0.0 \\
\hline $2.04180 E-27$ & 0.0 \\
\hline $9.17531 \mathrm{E}-17$ & 0.0 \\
\hline 0.00211753 & 0.0 \\
\hline $1.05138 \mathrm{E}-14$ & 0.0 \\
\hline $1.67319 \mathrm{E}-13$ & 0.0 \\
\hline $8.31272 E-14$ & 0.0 \\
\hline $1.43168 \mathrm{E}-12$ & 0.0 \\
\hline $7.76109 \mathrm{E}-08$ & 0.0 \\
\hline $4.23905 E-17$ & 0.0 \\
\hline $2.38622 E-11$ & 0.0 \\
\hline $8.51468 \mathrm{E}-15$ & 0.0 \\
\hline $6.59756 \mathrm{E}-19$ & 0.0 \\
\hline $6.21146 \mathrm{E}-10$ & 0.0 \\
\hline $5.88567 \mathrm{E}-10$ & 0.0 \\
\hline $1.43621 \mathrm{E}-11$ & 0.0 \\
\hline 1.74490E-09 & 0.0 \\
\hline 7. $26516 \mathrm{E}-24$ & 0.0 \\
\hline $1.43263 E-09$ & 0.0 \\
\hline $2.59404 \mathrm{E}-10$ & 0.0 \\
\hline $1.19580 \mathrm{E}-18$ & 0.0 \\
\hline $1.08843 E-24$ & 0.0 \\
\hline $6.44320 \mathrm{E}-12$ & 0.0 \\
\hline $8.12844 \mathrm{E}-09$ & 0.0 \\
\hline $3.43197 \mathrm{E}-08$ & 0.0 \\
\hline
\end{tabular}

0.0

0.0

0.0

0.0

0.0

0.0

0.0

0.0

0.0

0.0

0.0

0.0

0.0

0.0

0.0

0.0

0.0

0.0

0.0

0.0

0.0

0.0

0.0

0.0

0.0

0.0

0.0

0.0

0.0

0.0

0.0

0.0

0.0

0.0

0.0

0.0

0.0

0.0

0.0

0.0

0.0

0.0

0.0

0.0

0.0

0.0

0.0

0.0

0.0

0.0

0.0

0.0

0.0

0.0

0.0

0.0

0.0

0.0
0.0

0.0

0.0

0.0

0.0

0.0

0.0

0.0

0.0

0.0

0.0

0.0

0.0

0.0

0.0

0.0

0.0

0.0

0.0

0.0

0.0

0.0

0.0

0.0

0.0

0.0

0.0

0.0

0.0

0.0

0.0

0.0

0.0

0.0

0.0

0.0

0.0

0.0

0.0

0.0

0.0

0.0

0.0

0.0

0.0

0.0

0.0

0.0

0.0

0.0

0.0

0.0

0.0

0.0

0.0

0.0

0.0

0.0 


\begin{tabular}{|c|c|c|c|c|}
\hline FEIICO32ION & $2.86284 \mathrm{E}-22$ & 0.0 & 0.0 & 0.0 \\
\hline FEIIH2PO4ION & $4.36376 \mathrm{E}-09$ & 0.0 & 0.0 & 0.0 \\
\hline EEIIHCO3ION & $1.66038 \mathrm{E}-14$ & 0.0 & 0.0 & 0.0 \\
\hline FEIIION & $2.03722 \mathrm{E}-05$ & 0.0 & 0.0 & 0.0 \\
\hline FEIIOH3ION & $1.54525 \mathrm{E}-18$ & 0.0 & 0.0 & 0.0 \\
\hline FEIIOH 4 ION & $2.48680 \mathrm{E}-26$ & 0.0 & 0.0 & 0.0 \\
\hline FEIIOHION & $3.36708 \mathrm{E}-09$ & 0.0 & 0.0 & 0.0 \\
\hline H2 P2O 7 ION & $6.07062 \mathrm{E}-13$ & 0.0 & 0.0 & 0.0 \\
\hline H2 PO 4 ION & $1.46630 \mathrm{E}-07$ & 0.0 & 0.0 & 0.0 \\
\hline $\mathrm{H} 2 \mathrm{SIO} 4 \mathrm{ION}$ & $4.05681 \mathrm{E}-16$ & 0.0 & 0.0 & 0.0 \\
\hline H3P2O7 ION & $6.34856 \mathrm{E}-18$ & 0.0 & 0.0 & 0.0 \\
\hline H3SIO 4 ION & $2.59215 \mathrm{E}-10$ & 0.0 & 0.0 & 0.0 \\
\hline HCO3ION & $1.71260 \mathrm{E}-11$ & 0.0 & 0.0 & 0.0 \\
\hline HION & $5.43401 \mathrm{E}-10$ & 0.0 & 0.0 & 0.0 \\
\hline HP207ION & $1.98726 \mathrm{E}-12$ & 0.0 & 0.0 & 0.0 \\
\hline HPBO2ION & $1.17412 \mathrm{E}-20$ & 0.0 & 0.0 & 0.0 \\
\hline HPO4ION & $1.37464 \mathrm{E}-07$ & 0.0 & 0.0 & 0.0 \\
\hline $\mathrm{HSO} 4 \mathrm{ION}$ & $3.02567 \mathrm{E}-10$ & 0.0 & 0.0 & 0.0 \\
\hline KION & $2.55338 \mathrm{E}-04$ & 0.0 & 0.0 & 0.0 \\
\hline KSO4 ION & $8.53211 \mathrm{E}-06$ & 0.0 & 0.0 & 0.0 \\
\hline MGH2 PO 4 ION & $1.69219 \mathrm{E}-06$ & 0.0 & 0.0 & 0.0 \\
\hline MGHCO3ION & $1.14739 \mathrm{E}-10$ & 0.0 & 0.0 & 0.0 \\
\hline MGHSIO3ION & $4.12887 \mathrm{E}-09$ & 0.0 & 0.0 & 0.0 \\
\hline MGION & $2.97763 \mathrm{E}-04$ & 0.0 & 0.0 & 0.0 \\
\hline MGOHION & $4.70290 \mathrm{E}-10$ & 0.0 & 0.0 & 0.0 \\
\hline MGP2O7ION & $6.88864 \mathrm{E}-10$ & 0.0 & 0.0 & 0.0 \\
\hline MGPO 4 ION & $7.04726 \mathrm{E}-09$ & 0.0 & 0.0 & 0.0 \\
\hline NACO3ION & $4.51777 \mathrm{E}-15$ & 0.0 & 0.0 & 0.0 \\
\hline NAION & 0.00174777 & 0.0 & 0.0 & 0.0 \\
\hline NASO 4 ION & $2.58091 \mathrm{E}-05$ & 0.0 & 0.0 & 0.0 \\
\hline NICLION & $4.17817 \mathrm{E}-08$ & 0.0 & 0.0 & 0.0 \\
\hline NIION & $9.53400 \mathrm{E}-07$ & 0.0 & 0.0 & 0.0 \\
\hline NINO3ION & $2.42911 \mathrm{E}-07$ & 0.0 & 0.0 & 0.0 \\
\hline NIOH3ION & $3.68380 \mathrm{E}-19$ & 0.0 & 0.0 & 0.0 \\
\hline NIOHION & $2.48334 \mathrm{E}-11$ & 0.0 & 0.0 & 0.0 \\
\hline NO3ION & $4.23011 \mathrm{E}-04$ & 0.0 & 0.0 & 0.0 \\
\hline P207ION & $1.45463 \mathrm{E}-13$ & 0.0 & 0.0 & 0.0 \\
\hline PBCL3ION & $4.59495 \mathrm{E}-08$ & 0.0 & 0.0 & 0.0 \\
\hline PBCL 4 ION & $1.00273 E-06$ & 0.0 & 0.0 & 0.0 \\
\hline PBCLION & $4.09665 \mathrm{E}-09$ & 0.0 & 0.0 & 0.0 \\
\hline PBH2 PO 4 ION & $3.30580 \mathrm{E}-13$ & 0.0 & 0.0 & 0.0 \\
\hline PBION & $2.07394 \mathrm{E}-10$ & 0.0 & 0.0 & 0.0 \\
\hline PBNO33ION & $1.39785 \mathrm{E}-11$ & 0.0 & 0.0 & 0.0 \\
\hline PBNO3ION & $5.96365 \mathrm{E}-10$ & 0.0 & 0.0 & 0.0 \\
\hline PBOHION & $1.62495 \mathrm{E}-12$ & 0.0 & 0.0 & 0.0 \\
\hline PO4ION & $1.33828 \mathrm{E}-12$ & 0.0 & 0.0 & 0.0 \\
\hline SO4ION & $7.44674 \mathrm{E}-05$ & 0.0 & 0.0 & 0.0 \\
\hline SRION & $6.52698 \mathrm{E}-07$ & 0.0 & 0.0 & 0.0 \\
\hline SRNO3ION & $1.71556 \mathrm{E}-07$ & 0.0 & 0.0 & 0.0 \\
\hline SROHION & $6.06991 \mathrm{E}-15$ & 0.0 & 0.0 & 0.0 \\
\hline SRPO 4 ION & $1.87494 \mathrm{E}-14$ & 0.0 & 0.0 & 0.0 \\
\hline UIVCLION & $1.03739 \mathrm{E}-29$ & 0.0 & 0.0 & 0.0 \\
\hline UIVION & 0.0 & 0.0 & 0.0 & 0.0 \\
\hline UIVOH2ION & $6.07424 \mathrm{E}-23$ & 0.0 & 0.0 & 0.0 \\
\hline UIVOH3ION & $2.84388 \mathrm{E}-18$ & 0.0 & 0.0 & 0.0 \\
\hline UIVOH5ION & $2.41560 \mathrm{E}-17$ & 0.0 & 0.0 & 0.0 \\
\hline UIVOHION & $4.03632 E-26$ & 0.0 & 0.0 & 0.0 \\
\hline UIVSO 4 ION & $1.29292 \mathrm{E}-27$ & 0.0 & 0.0 & 0.0 \\
\hline
\end{tabular}


WSRC-TR-2002-00567

Revision 0

\begin{tabular}{|c|c|c|c|c|}
\hline ZNCL3ION & $3.95724 \mathrm{E}-06$ & 0.0 & 0.0 & 0.0 \\
\hline ZNCLION & $1.64694 \mathrm{E}-06$ & 0.0 & 0.0 & 0.0 \\
\hline ZNH2 PO4 ION & $2.14013 E-09$ & 0.0 & 0.0 & 0.0 \\
\hline ZNHCO3ION & $1.50498 \mathrm{E}-13$ & 0.0 & 0.0 & 0.0 \\
\hline ZNION & $5.32741 E-06$ & 0.0 & 0.0 & 0.0 \\
\hline ZNNO3ION & $6.03737 \mathrm{E}-07$ & 0.0 & 0.0 & 0.0 \\
\hline ZNOH3ION & $2.97727 \mathrm{E}-17$ & 0.0 & 0.0 & 0.0 \\
\hline ZNOH 4 ION & $6.42626 \mathrm{E}-23$ & 0.0 & 0.0 & 0.0 \\
\hline ZNOHION & $7.88550 \mathrm{E}-10$ & 0.0 & 0.0 & 0.0 \\
\hline $\mathrm{CA} 3 \mathrm{PO} 42$ & 0.0 & $8.15343 E-05$ & 0.0 & 0.0 \\
\hline $\mathrm{CASO} 4.2 \mathrm{H} 2 \mathrm{O}$ & 0.0 & $5.84266 \mathrm{E}-04$ & 0.0 & 0.0 \\
\hline CHAMOSITE7A & 0.0 & $1.09290 \mathrm{E}-05$ & 0.0 & 0.0 \\
\hline $\mathrm{CU} 3 \mathrm{PO} 42.2 \mathrm{H} 2 \mathrm{O}$ & 0.0 & $1.73090 \mathrm{E}-06$ & 0.0 & 0.0 \\
\hline FEII3PO $42.8 \mathrm{H} 2 \mathrm{O}$ & 0.0 & $1.45828 \mathrm{E}-05$ & 0.0 & 0.0 \\
\hline РВ3РО42 & 0.0 & $6.42890 \mathrm{E}-07$ & 0.0 & 0.0 \\
\hline UIVO2 & 0.0 & $2.89916 \mathrm{E}-06$ & 0.0 & 0.0 \\
\hline \multirow[t]{2}{*}{$\mathrm{ZN} 3 \mathrm{PO} 42.2 \mathrm{H} 2 \mathrm{O}$} & 0.0 & $5.30249 E-06$ & 0.0 & 0.0 \\
\hline & $===========$ & $===========$ & $===========$ & $===========$ \\
\hline Total g/hr & 0.983545 & 0.160852 & 0.0 & 0.0 \\
\hline Volume, L/hr & $8.61291 \mathrm{E}-04$ & $5.78477 \mathrm{E}-05$ & 0.0 & 0.0 \\
\hline Enthalpy, cal/hr & -3339.9 & -463.213 & 0.0 & 0.0 \\
\hline Density, g/L & 1141.94 & 2780.62 & & \\
\hline Vapor fraction & 0.0 & 0.0 & 0.0 & 0.0 \\
\hline Solid fraction & 0.0 & 1 . & 0.0 & 0.0 \\
\hline Organic fraction & 0.0 & 0.0 & 0.0 & 0.0 \\
\hline Osmotic Pres, atm & 173.122 & & & \\
\hline Redox Pot, volts & 0.0 & & & \\
\hline E-Con, 1/ohm-cm & 0.181849 & & & \\
\hline E-Con, cm2/ohm-mol & 30.95 & & & \\
\hline Abs Visc, $C P$ & 1.39766 & & & \\
\hline Rel Visc & 1.56914 & & & \\
\hline Ionic Strength & 3.94507 & & & \\
\hline
\end{tabular}


WSRC-TR-2002-00567

Revision 0

\begin{tabular}{|c|c|c|c|c|}
\hline \multicolumn{5}{|l|}{ STREAM: Condensate } \\
\hline TO $\quad:$ & & & & \\
\hline FROM : Condensate & mixer & & & \\
\hline Phases-----------> & Aqueous & Solid & Vapor & Organic \\
\hline Temperature, C & 25 . & 25 . & 25 . & 25 . \\
\hline $\begin{array}{l}\text { Pressure, atm } \\
\mathrm{pH}\end{array}$ & $\begin{array}{l}1 . \\
4.51483\end{array}$ & 1. & 1. & 1. \\
\hline Total mol/hr & 55.44257 & 0.0 & 0.0 & 0.0 \\
\hline------------------ & $\mathrm{mol} / \mathrm{hr}------$ & -mol/hr------- & -mol/hr-------- & $-\operatorname{mol} / \mathrm{hr}------1$ \\
\hline $\mathrm{H} 2 \mathrm{O}$ & 55.4413 & 0.0 & 0.0 & 0.0 \\
\hline $\mathrm{CO} 2$ & 0.00118233 & 0.0 & 0.0 & 0.0 \\
\hline $\mathrm{HCL}$ & $1.69591 \mathrm{E}-17$ & 0.0 & 0.0 & 0.0 \\
\hline HNO3 & $2.74765 E-13$ & 0.0 & 0.0 & 0.0 \\
\hline LAURICACID & $2.97366 \mathrm{E}-05$ & 0.0 & 0.0 & 0.0 \\
\hline OHION & $3.33640 \mathrm{E}-10$ & 0.0 & 0.0 & 0.0 \\
\hline CLION & $9.42924 E-07$ & 0.0 & 0.0 & 0.0 \\
\hline CO3ION & $2.76955 \mathrm{E}-11$ & 0.0 & 0.0 & 0.0 \\
\hline DODECION & $1.19234 \mathrm{E}-05$ & 0.0 & 0.0 & 0.0 \\
\hline HCO3 ION & $1.76739 \mathrm{E}-05$ & 0.0 & 0.0 & 0.0 \\
\hline $\mathrm{HION}$ & $3.07221 E-05$ & 0.0 & 0.0 & 0.0 \\
\hline NO3ION & $\begin{array}{l}\text { 1.81565E-07 } \\
============\end{array}$ & $\begin{array}{l}0.0 \\
============\end{array}$ & $\begin{array}{l}0.0 \\
=============\end{array}$ & $\begin{array}{l}0.0 \\
=============\end{array}$ \\
\hline Total g/hr & 998.856 & 0.0 & 0.0 & 0.0 \\
\hline Volume, L/hr & 1.002 & 0.0 & 0.0 & 0.0 \\
\hline $\begin{array}{l}\text { Enthalpy, cal/hr } \\
\text { Density, g/L }\end{array}$ & $\begin{array}{l}-3.78750 E+06 \\
996.866\end{array}$ & 0.0 & 0.0 & 0.0 \\
\hline Vapor fraction & 0.0 & 0.0 & 0.0 & 0.0 \\
\hline Solid fraction & 0.0 & 0.0 & 0.0 & 0.0 \\
\hline Organic fraction & 0.0 & 0.0 & 0.0 & 0.0 \\
\hline Osmotic Pres, atm & 0.0311278 & & & \\
\hline Redox Pot, volts & 0.0 & & & \\
\hline E-Con, 1/ohm-cm & $1.22818 \mathrm{E}-05$ & & & \\
\hline E-Con, cm2/ohm-mol & 9.90225 & & & \\
\hline Abs Visc, cP & 0.89074 & & & \\
\hline Rel Visc & 1.00002 & & & \\
\hline Ionic Strength & $3.07595 E-05$ & & & \\
\hline
\end{tabular}


WSRC-TR-2002-00567

Revision 0

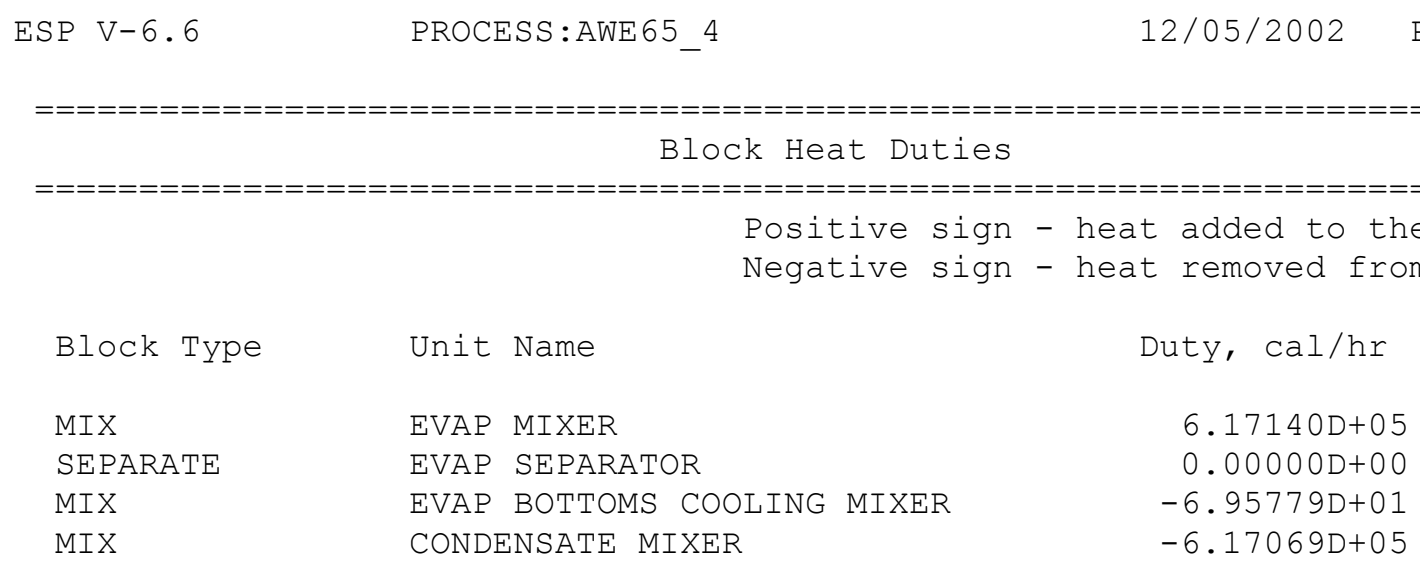


WSRC-TR-2002-00567

Revision 0

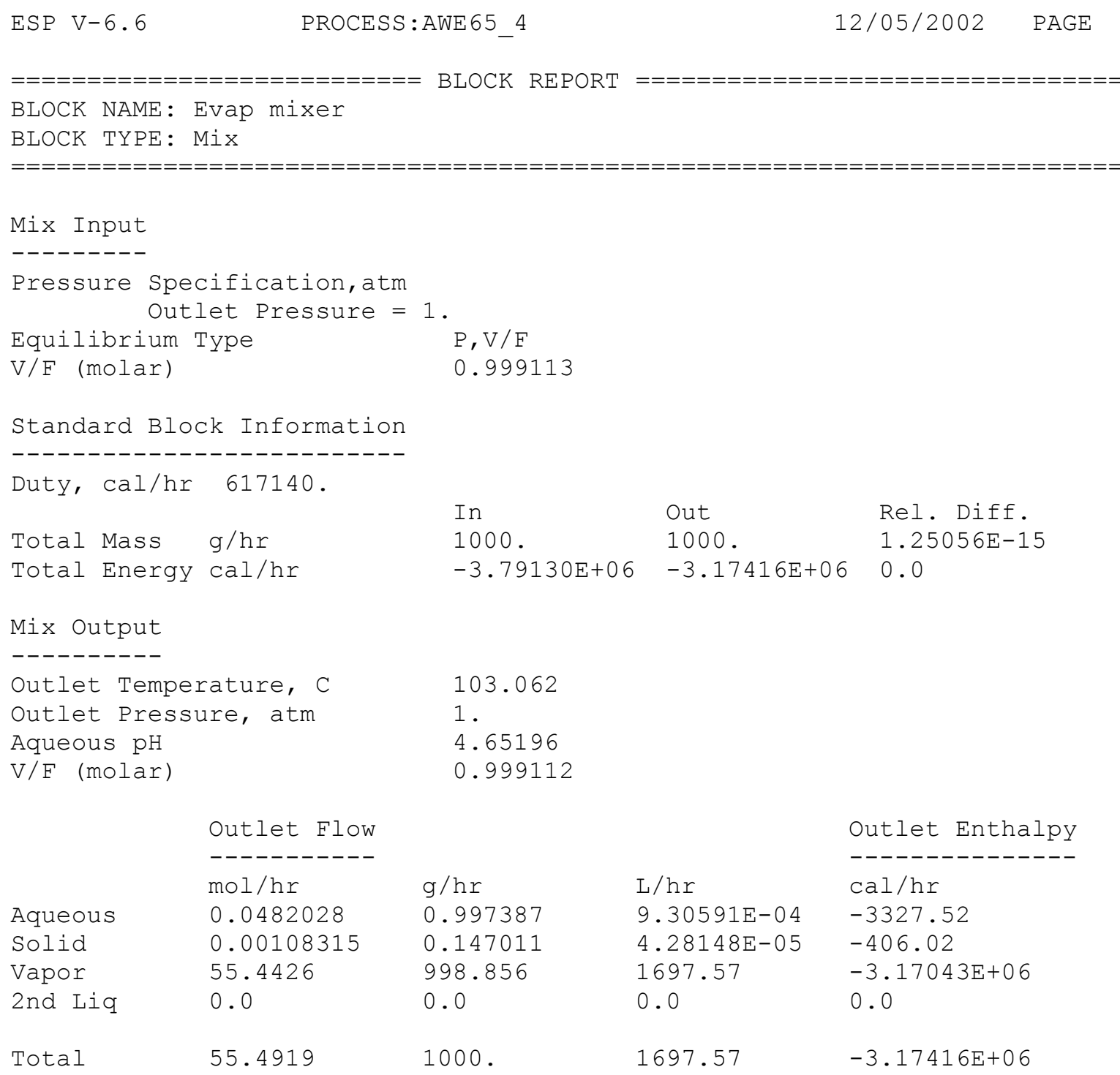


WSRC-TR-2002-00567

Revision 0

$\begin{array}{lllll}\text { ESP V-6.6 PROCESS :AWE65_4 } & \text { PAGE } & 12 / 05 / 2002\end{array}$

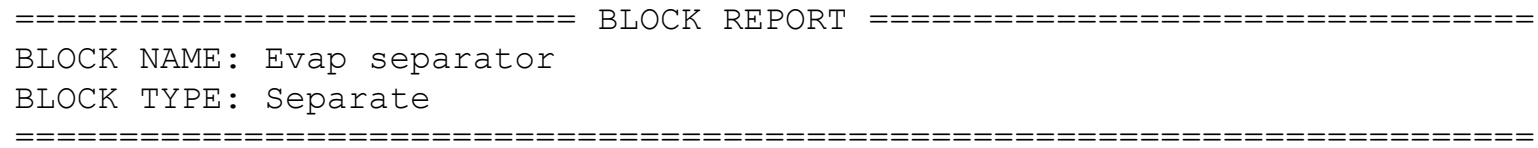

$0.049286 \quad 1.1444$

$\mathrm{L} / \mathrm{hr} \quad \mathrm{cal} / \mathrm{hr}$

4.28148E-05 -406.02

$0.0 \quad 0.0$

Total

$9.73405 E-04 \quad-3733.54$

Outlet Enthalpy 
WSRC-TR-2002-00567

Revision 0

ESP V-6.6

PROCESS:AWE 654

Vapor Stream

\begin{tabular}{ll}
\multicolumn{2}{c}{ Overhead } \\
Outlet Flow \\
-------- \\
mol/hr & $\mathrm{g} / \mathrm{hr}$ \\
0.0 & 0.0 \\
0.0 & 0.0 \\
55.4426 & 998.856 \\
0.0 & 0.0
\end{tabular}

Total

998.856
$12 / 05 / 2002$

PAGE

13

$\mathrm{L} / \mathrm{hr}$

0.0

0.0

1697.57

0.0

1697.57

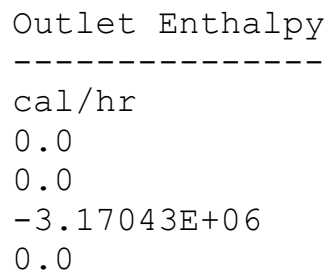

0.0

0.0

$-3.17043 E+06$

0.0

$-3.17043 E+06$ 
WSRC-TR-2002-00567

Revision 0

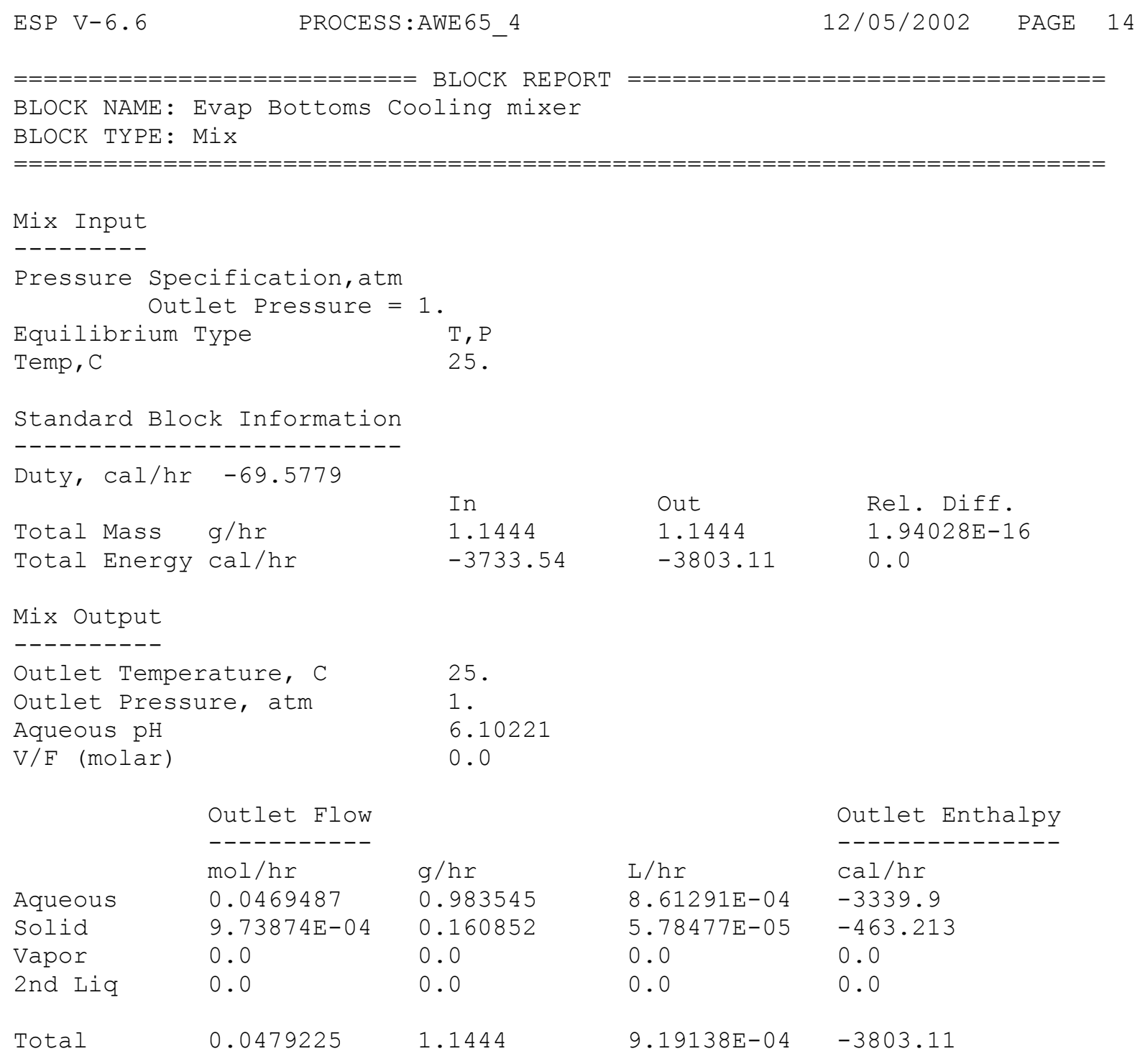


WSRC-TR-2002-00567

Revision 0

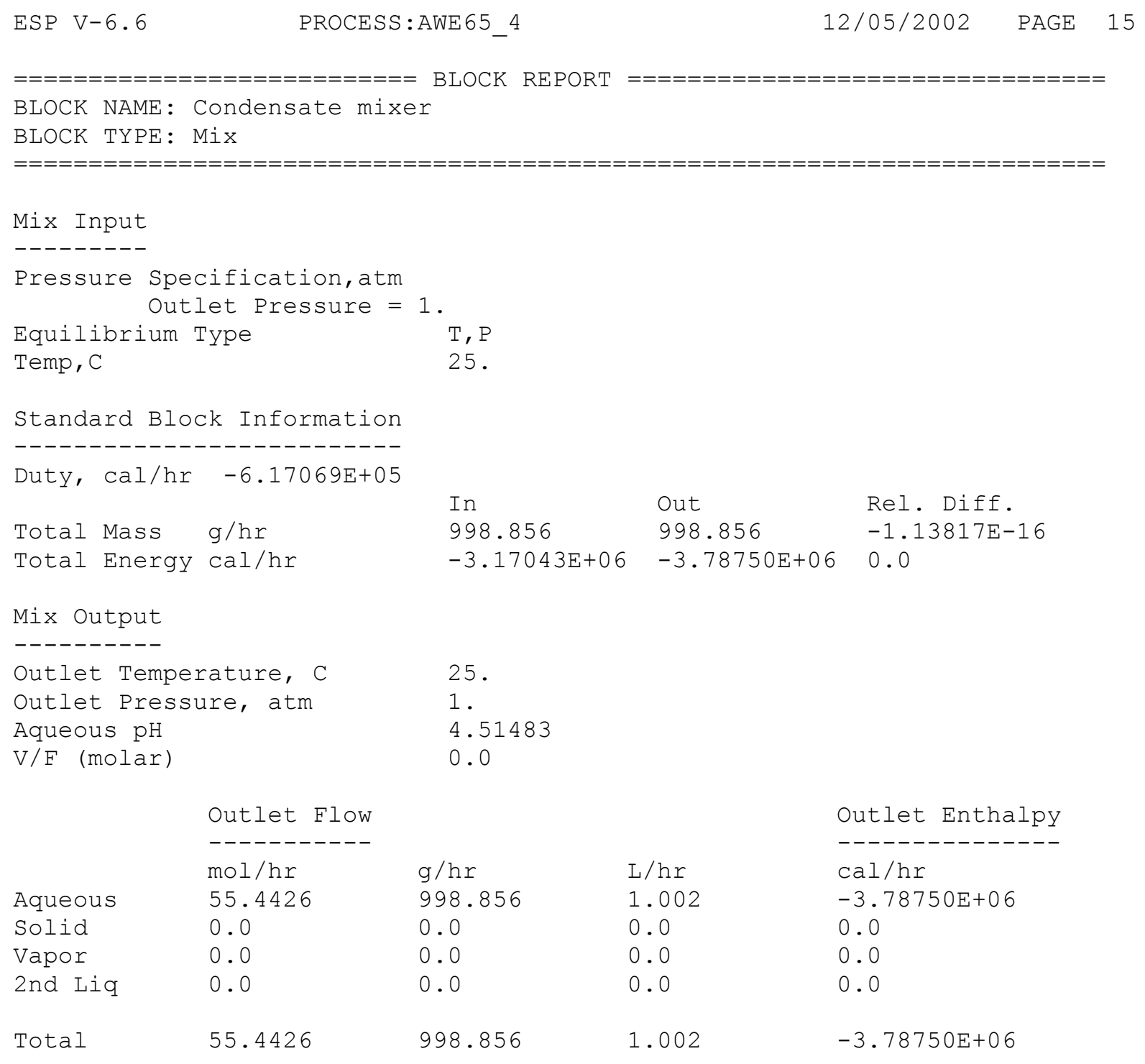


WSRC-TR-2002-00567

Revision 0

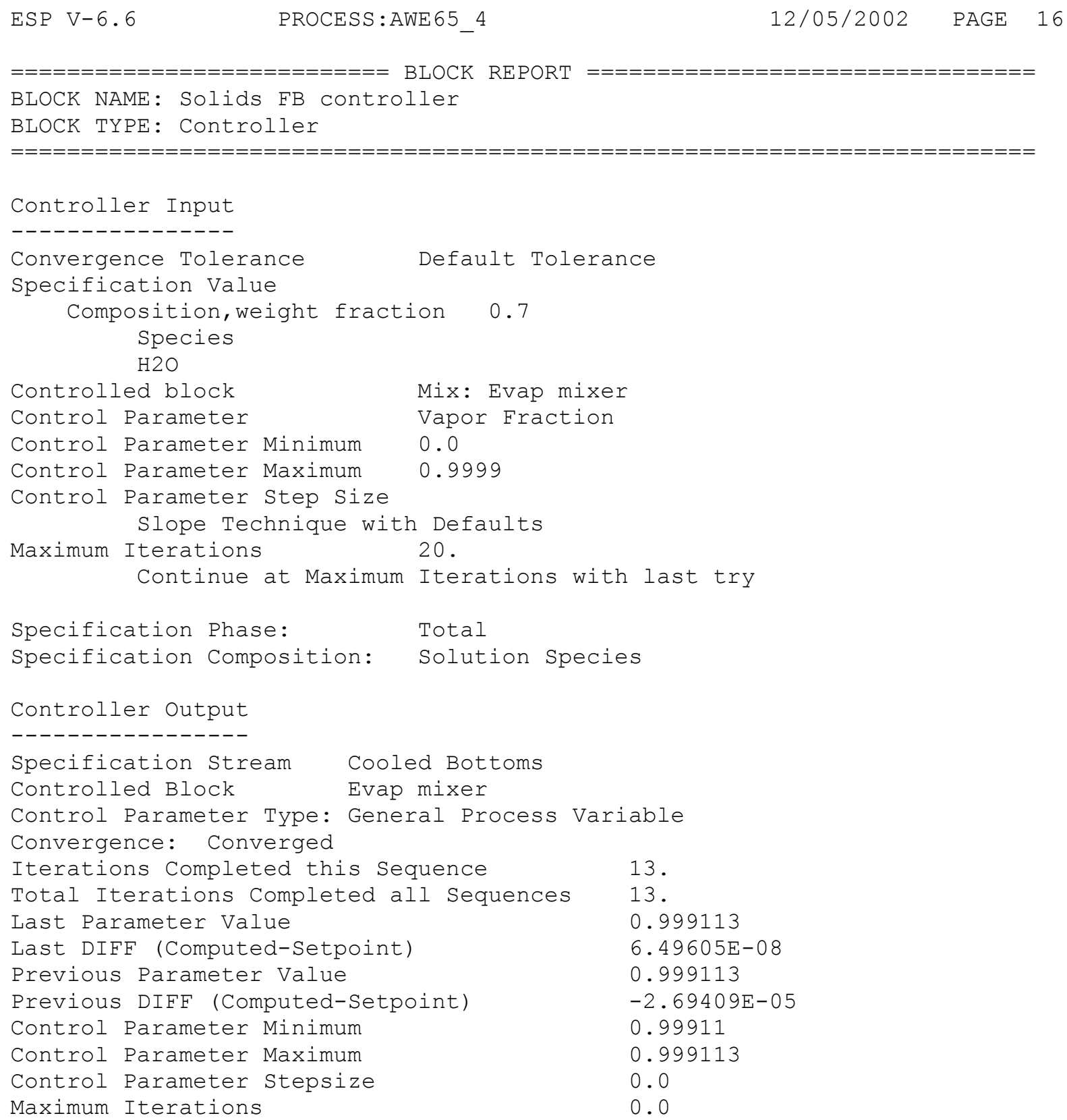


WSRC-TR-2002-00567

Revision 0

Influent Limit Composition 80\% Target $\mathrm{pH}=6.5$

Awe 6.5-80

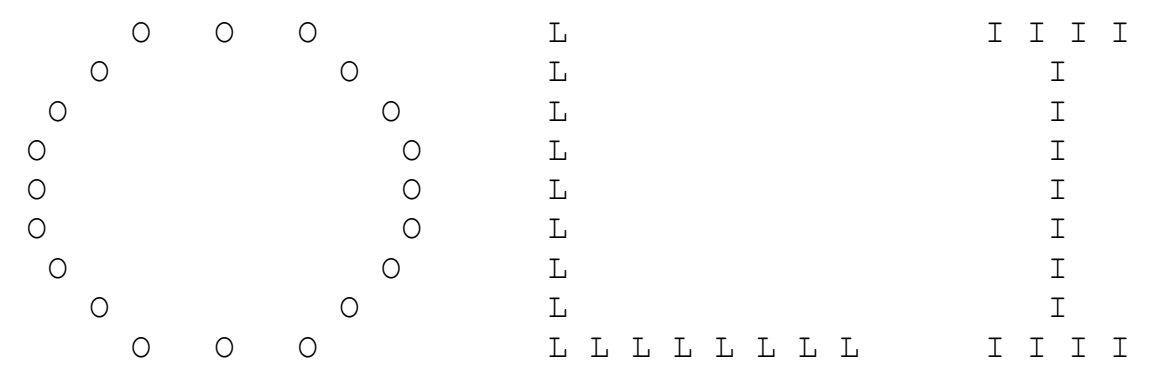

E N V I R O N M E N T A L $S$ I M U L A T I O N $P$ R O G R A M

$V-6.6 \quad$ September 1, 2002

PROCESS: AWE65_5

CHEMISTRY MODEL: RAW

THIS FILE NAME: AWE65 5.LIS

DATE : $12 / 05 / 2002$ 
WSRC-TR-2002-00567

Revision 0

TABLE OF CONTENTS

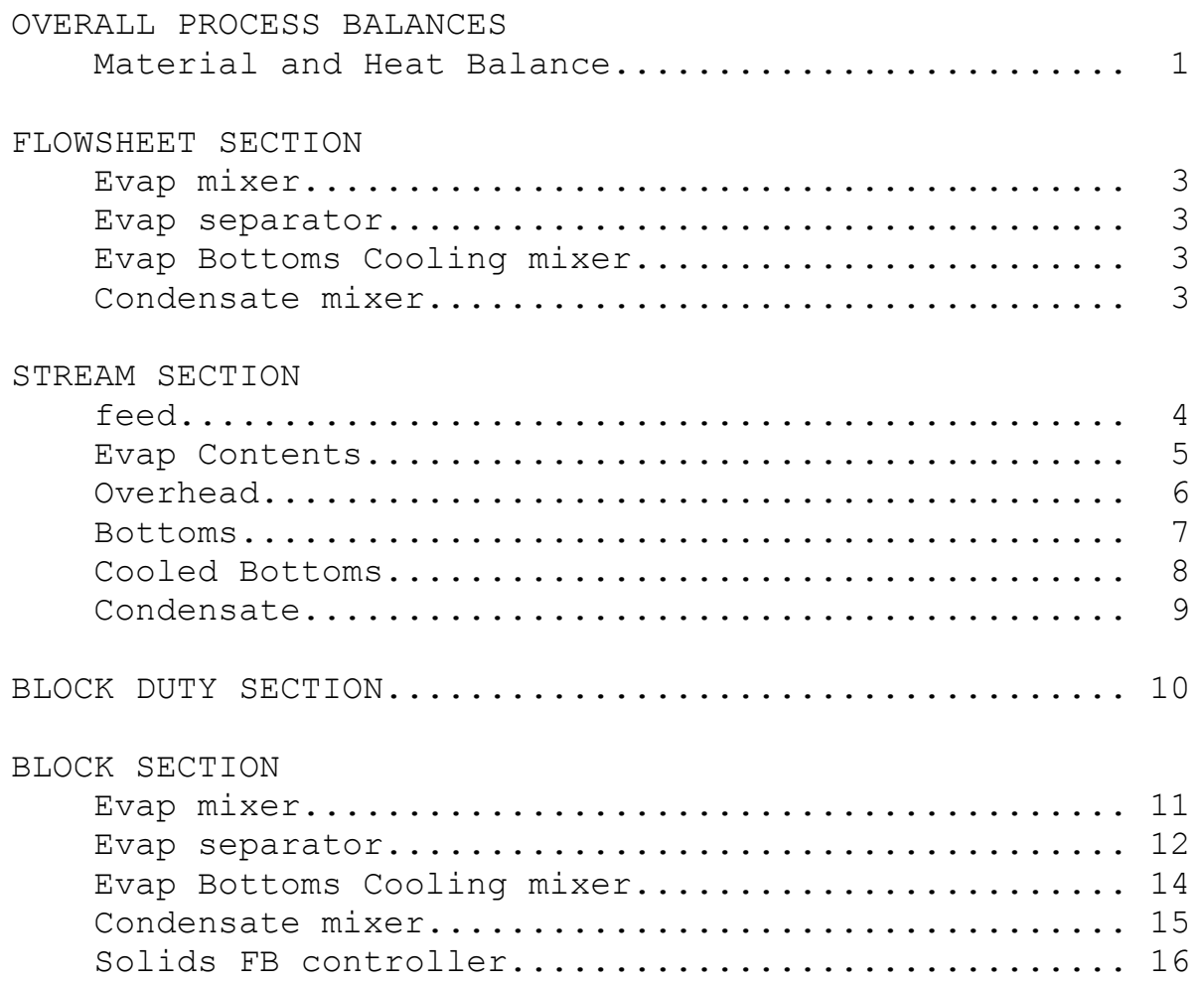


WSRC-TR-2002-00567

Revision 0

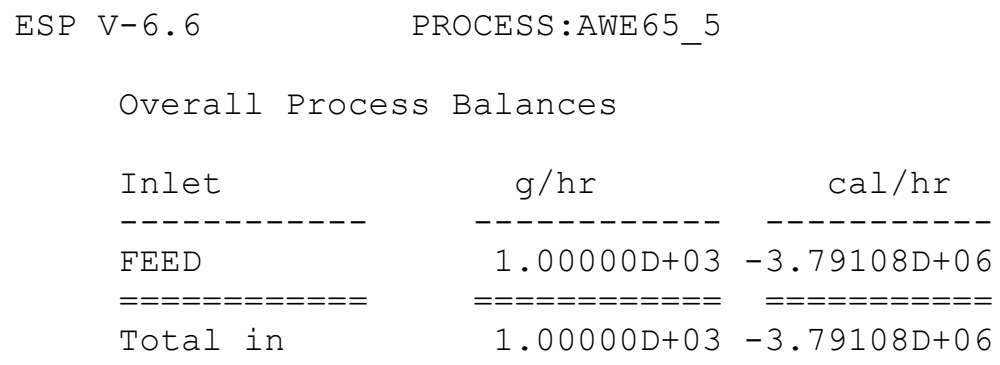

\begin{tabular}{lrr} 
Outlet & \multicolumn{1}{c}{$\mathrm{g} / \mathrm{hr}$} & $\mathrm{cal} / \mathrm{hr}$ \\
---------- & ---------- & --------- \\
COOLED BOTTOMS & $1.50849 \mathrm{D}+00$ & $-4.98377 \mathrm{D}+03$ \\
CONDENSATE & $9.98492 \mathrm{D}+02$ & $-3.78609 \mathrm{D}+06$ \\
$==========$ & $==========$ & $=========$ \\
Total out & $1.00000 \mathrm{D}+03$ & $-3.79107 \mathrm{D}+06$
\end{tabular}

Block Heat Duties

$\mathrm{cal} / \mathrm{hr}$
-----------
$6.17018 \mathrm{D}+05$
$-9.21552 \mathrm{D}+01$
$-6.16924 \mathrm{D}+05$
$==========$
$2.04442 \mathrm{D}+00$

EVAP MIXER

EVAP BOTTOMS COOLING MIXER

CONDENSATE MIXER

$===========$

Total Duty

12/05/2002 PAGE 1

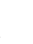

$\begin{array}{lrr}\text { DIFFERENCE } & 7.95808 \mathrm{D}-13 & -3.49246 \mathrm{D}-10 \\ \text { REL DIFFERENCE } & 7.95808 \mathrm{D}-16 & 9.21232 \mathrm{D}-17\end{array}$

Material Code Balances

\begin{tabular}{lccrr} 
Code & $\begin{array}{c}\text { Input } \\
\text { mol/hr }\end{array}$ & $\begin{array}{c}\text { Outlet } \\
\text { mol/hr }\end{array}$ & $\begin{array}{c}\text { Difference } \\
\text { mol/hr }\end{array}$ & \multicolumn{1}{c}{ Rel Diff } \\
--------- & -------- & -------- & -------- & ---------- \\
H $(+1)$ & $1.10961 \mathrm{D}+02$ & $1.10961 \mathrm{D}+02$ & $2.84217 \mathrm{D}-14$ & $2.56142 \mathrm{D}-16$ \\
$\mathrm{~K}(+1)$ & $4.12103 \mathrm{D}-04$ & $4.12103 \mathrm{D}-04$ & $1.08420 \mathrm{D}-19$ & $2.63090 \mathrm{D}-16$ \\
$\mathrm{NA}(+1)$ & $2.80236 \mathrm{D}-03$ & $2.80236 \mathrm{D}-03$ & $-4.33681 \mathrm{D}-18$ & $-1.54756 \mathrm{D}-15$ \\
$\mathrm{BA}(+2)$ & $6.71533 \mathrm{D}-07$ & $6.71533 \mathrm{D}-07$ & $-1.05879 \mathrm{D}-22$ & $-1.57668 \mathrm{D}-16$ \\
$\mathrm{CA}(+2)$ & $9.97506 \mathrm{D}-04$ & $9.97506 \mathrm{D}-04$ & $0.00000 \mathrm{D}+00$ & $0.00000 \mathrm{D}+00$ \\
$\mathrm{ZN}(+2)$ & $3.82263 \mathrm{D}-05$ & $3.82263 \mathrm{D}-05$ & $-6.77626 \mathrm{D}-21$ & $-1.77267 \mathrm{D}-16$ \\
$\mathrm{CU}(+2)$ & $6.77166 \mathrm{D}-06$ & $6.77166 \mathrm{D}-06$ & $-1.69407 \mathrm{D}-21$ & $-2.50170 \mathrm{D}-16$ \\
$\mathrm{FE}(+2)$ & $1.02151 \mathrm{D}-04$ & $1.02151 \mathrm{D}-04$ & $2.71051 \mathrm{D}-20$ & $2.65344 \mathrm{D}-16$ \\
$\mathrm{MG}(+2)$ & $3.78601 \mathrm{D}-04$ & $3.78601 \mathrm{D}-04$ & $1.62630 \mathrm{D}-19$ & $4.29556 \mathrm{D}-16$ \\
$\mathrm{~PB}(+2)$ & $3.42995 \mathrm{D}-06$ & $3.42995 \mathrm{D}-06$ & $8.47033 \mathrm{D}-22$ & $2.46952 \mathrm{D}-16$ \\
$\mathrm{AL}(+3)$ & $1.03704 \mathrm{D}-04$ & $1.03704 \mathrm{D}-04$ & $0.00000 \mathrm{D}+00$ & $0.00000 \mathrm{D}+00$ \\
$\mathrm{NI}(+2)$ & $1.49915 \mathrm{D}-06$ & $1.49915 \mathrm{D}-06$ & $8.47033 \mathrm{D}-22$ & $5.65009 \mathrm{D}-16$ \\
$\mathrm{O}(-2)$ & $5.54912 \mathrm{D}+01$ & $5.54912 \mathrm{D}+01$ & $2.13163 \mathrm{D}-14$ & $3.84138 \mathrm{D}-16$ \\
$\mathrm{CL}(-1)$ & $2.81690 \mathrm{D}-03$ & $2.81690 \mathrm{D}-03$ & $4.00721 \mathrm{D}-16$ & $1.42256 \mathrm{D}-13$ \\
$\mathrm{C}(+4)$ & $1.50000 \mathrm{D}-03$ & $1.50000 \mathrm{D}-03$ & $7.11237 \mathrm{D}-16$ & $4.74158 \mathrm{D}-13$ \\
$\mathrm{P}(+5)$ & $2.63158 \mathrm{D}-04$ & $2.63158 \mathrm{D}-04$ & $0.00000 \mathrm{D}+00$ & $0.00000 \mathrm{D}+00$ \\
$\mathrm{~S}(+6)$ & $9.58333 \mathrm{D}-04$ & $9.58333 \mathrm{D}-04$ & $1.08420 \mathrm{D}-19$ & $1.13134 \mathrm{D}-16$ \\
$\mathrm{~N}(+5)$ & $7.25807 \mathrm{D}-04$ & $7.25807 \mathrm{D}-04$ & $3.25261 \mathrm{D}-19$ & $4.48137 \mathrm{D}-16$ \\
$\mathrm{SI}(+4)$ & $2.33333 \mathrm{D}-04$ & $2.33333 \mathrm{D}-04$ & $2.71051 \mathrm{D}-20$ & $1.16165 \mathrm{D}-16$ \\
$\mathrm{SR}(+2)$ & $2.16895 \mathrm{D}-05$ & $2.16895 \mathrm{D}-05$ & $0.00000 \mathrm{D}+00$ & $0.00000 \mathrm{D}+00$
\end{tabular}


WSRC-TR-2002-00567

Revision 0

$\mathrm{CD}(+2)$

$\mathrm{CR}(+3)$

$\mathrm{U}(+4)$
$4.64286 \mathrm{D}-08$

$1.21154 \mathrm{D}-07$

4.20168D-06
4. $64286 \mathrm{D}-08$

$1.21154 \mathrm{D}-07-3.57342 \mathrm{D}-21-2.94949 \mathrm{D}-14$

4.20168D-06 0.00000D+00 0.00000D+00 
WSRC-TR-2002-00567

Revision 0

ESP V-6.6

$\operatorname{DODEC}(-1)$
PROCESS:AWE 655

$5.57619 \mathrm{D}-05$
$12 / 05 / 2002$

$5.57619 \mathrm{D}-05$

1. 45975D-15

$2.61783 D-11$ 
WSRC-TR-2002-00567

Revision 0

\begin{tabular}{|c|c|c|c|}
\hline \multicolumn{4}{|l|}{$\begin{array}{l}\text { PROCESS BLOCKS } \\
===========\end{array}$} \\
\hline $\begin{array}{l}\mathrm{BLOCK} \mathrm{NAME} \\
=========================\end{array}$ & $\begin{array}{l}\text { BLOCK TYPE } \\
===========\end{array}$ & $\begin{array}{l}\text { INLET STREAM }(\mathrm{S}) \\
==============\end{array}$ & $\begin{array}{l}\text { OUTLET STREAM }(\mathrm{S}) \\
==============\end{array}$ \\
\hline Evap mixer & $\operatorname{Mix}$ & feed & Evap Contents \\
\hline Evap separator & Separate & Evap Contents & $\begin{array}{l}\text { Overhead } \\
\text { Bottoms }\end{array}$ \\
\hline Evap Bottoms Cooling mixer & Mix & Bottoms & Cooled Bottoms \\
\hline Condensate mixer & Mix & Overhead & Condensate \\
\hline
\end{tabular}


WSRC-TR-2002-00567

Revision 0

ESP V-6. 6

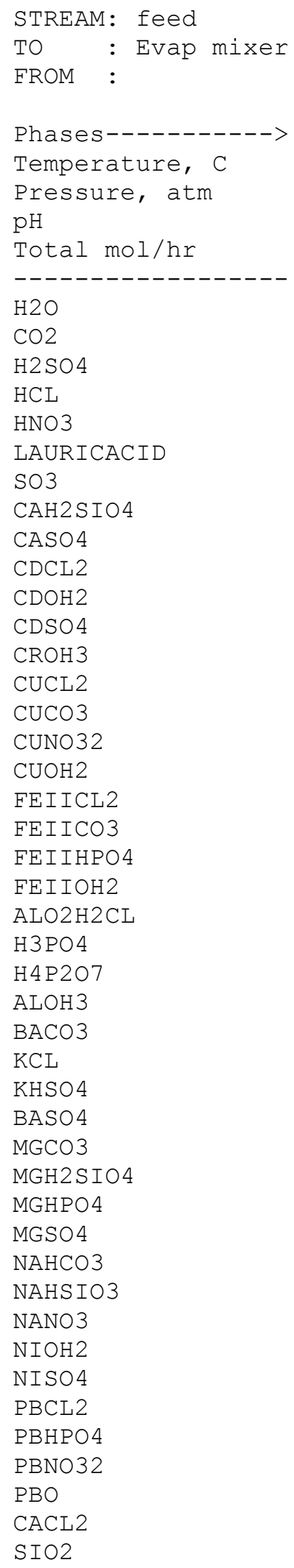

\begin{tabular}{|c|c|c|c|}
\hline Aqueous & Solid & Vapor & Organic \\
\hline 25 & 25 . & 25. & 25 \\
\hline 1 . & 1 . & 1 . & 1 . \\
\hline \multicolumn{4}{|l|}{5.9988} \\
\hline 55.49104 & $1.11635 \mathrm{E}-04$ & 0.0 & 0.0 \\
\hline \multicolumn{4}{|c|}{$\mathrm{mol} / \mathrm{hr}--------\operatorname{mol} / \mathrm{hr}--------\operatorname{mol} / \mathrm{hr}--------\operatorname{mol} / \mathrm{hr}--------$} \\
\hline 55.4798 & 0.0 & 0.0 & 0.0 \\
\hline $9.94275 \mathrm{E}-04$ & 0.0 & 0.0 & 0.0 \\
\hline $2.28637 \mathrm{E}-24$ & 0.0 & 0.0 & 0.0 \\
\hline $1.51879 \mathrm{E}-15$ & 0.0 & 0.0 & 0.0 \\
\hline $3.27818 \mathrm{E}-11$ & 0.0 & 0.0 & 0.0 \\
\hline $3.86320 E-06$ & 0.0 & 0.0 & 0.0 \\
\hline $2.95970 E-28$ & 0.0 & 0.0 & 0.0 \\
\hline $9.67582 \mathrm{E}-14$ & 0.0 & 0.0 & 0.0 \\
\hline $1.53152 \mathrm{E}-05$ & 0.0 & 0.0 & 0.0 \\
\hline $2.96900 \mathrm{E}-10$ & 0.0 & 0.0 & 0.0 \\
\hline $1.56471 \mathrm{E}-16$ & 0.0 & 0.0 & 0.0 \\
\hline $5.30100 E-09$ & 0.0 & 0.0 & 0.0 \\
\hline $2.83179 \mathrm{E}-14$ & 0.0 & 0.0 & 0.0 \\
\hline 1.29951E-12 & 0.0 & 0.0 & 0.0 \\
\hline $9.44961 \mathrm{E}-09$ & 0.0 & 0.0 & 0.0 \\
\hline $1.81988 \mathrm{E}-14$ & 0.0 & 0.0 & 0.0 \\
\hline $6.84705 \mathrm{E}-10$ & 0.0 & 0.0 & 0.0 \\
\hline $2.27604 \mathrm{E}-15$ & 0.0 & 0.0 & 0.0 \\
\hline $1.90479 \mathrm{E}-07$ & 0.0 & 0.0 & 0.0 \\
\hline $2.25097 E-08$ & 0.0 & 0.0 & 0.0 \\
\hline $1.72750 \mathrm{E}-13$ & 0.0 & 0.0 & 0.0 \\
\hline $2.93833 E-28$ & 0.0 & 0.0 & 0.0 \\
\hline $2.87967 \mathrm{E}-08$ & 0.0 & 0.0 & 0.0 \\
\hline $1.47359 \mathrm{E}-18$ & 0.0 & 0.0 & 0.0 \\
\hline $2.40024 E-09$ & $1.03700 \mathrm{E}-04$ & 0.0 & 0.0 \\
\hline $1.62720 \mathrm{E}-12$ & 0.0 & 0.0 & 0.0 \\
\hline $7.89160 \mathrm{E}-09$ & 0.0 & 0.0 & 0.0 \\
\hline $4.19670 \mathrm{E}-13$ & 0.0 & 0.0 & 0.0 \\
\hline $2.05711 \mathrm{E}-10$ & $4.00045 E-07$ & 0.0 & 0.0 \\
\hline $4.90253 E-09$ & 0.0 & 0.0 & 0.0 \\
\hline $3.46511 \mathrm{E}-13$ & 0.0 & 0.0 & 0.0 \\
\hline $3.68717 E-06$ & 0.0 & 0.0 & 0.0 \\
\hline $9.88985 E-06$ & 0.0 & 0.0 & 0.0 \\
\hline $4.76561 \mathrm{E}-07$ & 0.0 & 0.0 & 0.0 \\
\hline $1.02557 \mathrm{E}-08$ & 0.0 & 0.0 & 0.0 \\
\hline $9.54597 \mathrm{E}-08$ & 0.0 & 0.0 & 0.0 \\
\hline $9.47761 \mathrm{E}-14$ & 0.0 & 0.0 & 0.0 \\
\hline $1.18401 \mathrm{E}-07$ & 0.0 & 0.0 & 0.0 \\
\hline $2.63713 E-11$ & 0.0 & 0.0 & 0.0 \\
\hline $8.43444 \mathrm{E}-10$ & 0.0 & 0.0 & 0.0 \\
\hline $3.37126 E-13$ & 0.0 & 0.0 & 0.0 \\
\hline $4.08525 E-13$ & 0.0 & 0.0 & 0.0 \\
\hline $4.01279 E-27$ & 0.0 & 0.0 & 0.0 \\
\hline $2.33285 E-04$ & 0.0 & 0.0 & 0.0 \\
\hline
\end{tabular}




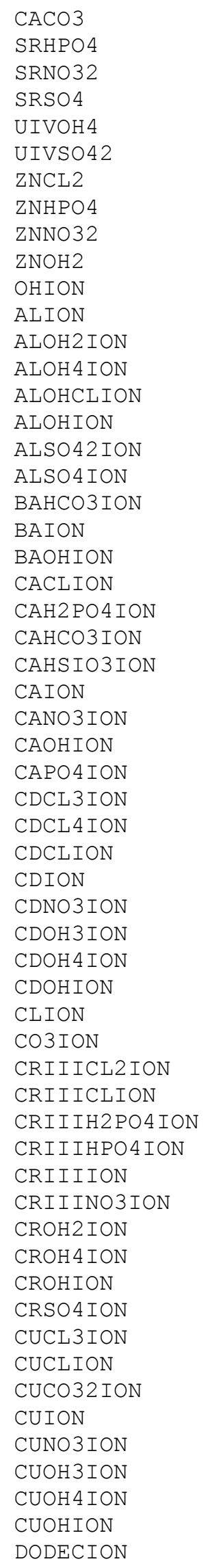

\begin{tabular}{|c|c|}
\hline $2.93244 E-08$ & 0.0 \\
\hline $3.13203 E-09$ & 0.0 \\
\hline $.85785 E-11$ & 0.0 \\
\hline $65268 E-06$ & \\
\hline$E-10$ & 0.0 \\
\hline $657 E-26$ & 0.0 \\
\hline $95 E-10$ & 0.0 \\
\hline $16 E-07$ & 0.0 \\
\hline $30 E-12$ & 0.0 \\
\hline $23190 \mathrm{E}-10$ & 0.0 \\
\hline $13 E-08$ & 0.0 \\
\hline $6 E-11$ & 0.0 \\
\hline $3 E-10$ & 0.0 \\
\hline $46 \mathrm{E}-10$ & 0.0 \\
\hline $2 E-13$ & 0.0 \\
\hline $7 E-10$ & 0.0 \\
\hline$D E-13$ & 0.0 \\
\hline $6.72500 \mathrm{E}-12$ & 0.0 \\
\hline $3 E-10$ & 0.0 \\
\hline $74 E-07$ & 0.0 \\
\hline $4 E-15$ & 0.0 \\
\hline $39 E-11$ & 0.0 \\
\hline$E-06$ & 0.0 \\
\hline $5 E-06$ & 0.0 \\
\hline $9 E-10$ & 0.0 \\
\hline $2 E-04$ & 0.0 \\
\hline $1 E-06$ & 0.0 \\
\hline $4 E-10$ & 0.0 \\
\hline $3 E-08$ & 0.0 \\
\hline $1 E-14$ & 0.0 \\
\hline $2 E-17$ & 0.0 \\
\hline $09 E-09$ & 0.0 \\
\hline $9 E-08$ & 0.0 \\
\hline $38 E-11$ & 0.0 \\
\hline $363 E-23$ & 0.0 \\
\hline 0.0 & 0.0 \\
\hline $96 E-12$ & 0.0 \\
\hline 81677 & 0.0 \\
\hline $96 E-08$ & 0.0 \\
\hline $7 E-19$ & 0.0 \\
\hline $9 E-16$ & 0.0 \\
\hline $8 E-13$ & 0.0 \\
\hline $8 E-07$ & 0.0 \\
\hline $3 E-14$ & 0.0 \\
\hline $6 E-15$ & 0.0 \\
\hline $3 E-15$ & 0.0 \\
\hline $6 E-19$ & 0.0 \\
\hline $5 E-12$ & 0.0 \\
\hline $60 \mathrm{E}-11$ & 0.0 \\
\hline $6 E-18$ & 0.0 \\
\hline $69 E-09$ & 0.0 \\
\hline $4 E-13$ & 0.0 \\
\hline $24 E-07$ & 0.0 \\
\hline $37 E-10$ & 0.0 \\
\hline $43 E-16$ & 0.0 \\
\hline $74 E-23$ & 0.0 \\
\hline $23 E-09$ & 0.0 \\
\hline $87 E-05$ & 0.0 \\
\hline
\end{tabular}

0.0

0.0

0.0

0.0

0.0

0.0

0.0

0.0

0.0

0.0

0.0

0.0

0.0

0.0

0.0

0.0

0.0

0.0

0.0

0.0

0.0

0.0

0.0

0.0

0.0

0.0

0.0

0.0

0.0

0.0

0.0

0.0

0.0

0.0

0.0

0.0

0.0

0.0

0.0

0.0

0.0

0.0

0.0

0.0

0.0

0.0

0.0

0.0

0.0

0.0

0.0

0.0

0.0

0.0

0.0

0.0

0.0

0.0
0.0

0.0

0.0

0.0

0.0

0.0

0.0

0.0

0.0

0.0

0.0

0.0

0.0

0.0

0.0

0.0

0.0

0.0

0.0

0.0

0.0

0.0

0.0

0.0

0.0

0.0

0.0

0.0

0.0

0.0

0.0

0.0

0.0

0.0

0.0

0.0

0.0

0.0

0.0

0.0

0.0

0.0

0.0

0.0

0.0

0.0

0.0

0.0

0.0

0.0

0.0

0.0

0.0

0.0

0.0

0.0

0.0

0.0 


\begin{tabular}{|c|c|c|c|c|}
\hline FEIICLION & $2.66289 \mathrm{E}-10$ & 0.0 & 0.0 & 0.0 \\
\hline FEIICO32ION & $1.95343 \mathrm{E}-13$ & 0.0 & 0.0 & 0.0 \\
\hline FEIIH2PO4ION & $5.05041 \mathrm{E}-08$ & 0.0 & 0.0 & 0.0 \\
\hline FEIIHCO3ION & $5.00781 \mathrm{E}-09$ & 0.0 & 0.0 & 0.0 \\
\hline FEIIION & $1.01858 \mathrm{E}-04$ & 0.0 & 0.0 & 0.0 \\
\hline FEIIOH3ION & $7.51207 \mathrm{E}-18$ & 0.0 & 0.0 & 0.0 \\
\hline FEIIOH 4 ION & $1.03393 \mathrm{E}-26$ & 0.0 & 0.0 & 0.0 \\
\hline FEIIOHION & $2.39324 \mathrm{E}-08$ & 0.0 & 0.0 & 0.0 \\
\hline H2 P2O 7 ION & $2.80573 \mathrm{E}-10$ & 0.0 & 0.0 & 0.0 \\
\hline H2 PO 4 ION & $2.24860 \mathrm{E}-04$ & 0.0 & 0.0 & 0.0 \\
\hline H2SIO4ION & $3.90475 E-15$ & 0.0 & 0.0 & 0.0 \\
\hline H3P2O 7 ION & $4.62135 \mathrm{E}-14$ & 0.0 & 0.0 & 0.0 \\
\hline H3SIO4 ION & $3.68495 \mathrm{E}-08$ & 0.0 & 0.0 & 0.0 \\
\hline HCO3ION & $4.96482 \mathrm{E}-04$ & 0.0 & 0.0 & 0.0 \\
\hline HION & $1.10392 \mathrm{E}-06$ & 0.0 & 0.0 & 0.0 \\
\hline HP207ION & $1.14411 \mathrm{E}-10$ & 0.0 & 0.0 & 0.0 \\
\hline HPBO2ION & $5.03651 \mathrm{E}-18$ & 0.0 & 0.0 & 0.0 \\
\hline HPO4 ION & $1.88463 \mathrm{E}-05$ & 0.0 & 0.0 & 0.0 \\
\hline HSO 4 ION & $6.46222 \mathrm{E}-08$ & 0.0 & 0.0 & 0.0 \\
\hline KION & $4.10184 \mathrm{E}-04$ & 0.0 & 0.0 & 0.0 \\
\hline KSO4 ION & $1.91159 \mathrm{E}-06$ & 0.0 & 0.0 & 0.0 \\
\hline MGH2 PO 4 ION & $2.59224 \mathrm{E}-06$ & 0.0 & 0.0 & 0.0 \\
\hline MGHCO3ION & $4.57812 \mathrm{E}-06$ & 0.0 & 0.0 & 0.0 \\
\hline MGHSIO3ION & $3.01786 \mathrm{E}-10$ & 0.0 & 0.0 & 0.0 \\
\hline MGION & $3.57839 \mathrm{E}-04$ & 0.0 & 0.0 & 0.0 \\
\hline MGOHION & $4.42445 \mathrm{E}-10$ & 0.0 & 0.0 & 0.0 \\
\hline MGP2O 7 ION & $1.71030 \mathrm{E}-10$ & 0.0 & 0.0 & 0.0 \\
\hline MGPO 4 ION & $8.74083 E-09$ & 0.0 & 0.0 & 0.0 \\
\hline NACO3ION & $2.07890 \mathrm{E}-10$ & 0.0 & 0.0 & 0.0 \\
\hline NAION & 0.00278747 & 0.0 & 0.0 & 0.0 \\
\hline NASO 4 ION & $1.43070 \mathrm{E}-05$ & 0.0 & 0.0 & 0.0 \\
\hline NICLION & $2.62616 \mathrm{E}-10$ & 0.0 & 0.0 & 0.0 \\
\hline NIION & $1.37839 \mathrm{E}-06$ & 0.0 & 0.0 & 0.0 \\
\hline NINO3ION & $1.95034 \mathrm{E}-09$ & 0.0 & 0.0 & 0.0 \\
\hline NIOH3ION & $1.06838 \mathrm{E}-18$ & 0.0 & 0.0 & 0.0 \\
\hline NIOHION & $1.43035 \mathrm{E}-10$ & 0.0 & 0.0 & 0.0 \\
\hline NO3ION & $7.24348 \mathrm{E}-04$ & 0.0 & 0.0 & 0.0 \\
\hline P207ION & $1.14049 \mathrm{E}-13$ & 0.0 & 0.0 & 0.0 \\
\hline PBCL3ION & $3.63611 \mathrm{E}-14$ & 0.0 & 0.0 & 0.0 \\
\hline PBCL 4 ION & $7.83700 \mathrm{E}-17$ & 0.0 & 0.0 & 0.0 \\
\hline PBCLION & $3.13830 \mathrm{E}-09$ & 0.0 & 0.0 & 0.0 \\
\hline PBH2 PO 4 ION & $3.77135 \mathrm{E}-10$ & 0.0 & 0.0 & 0.0 \\
\hline PBION & $6.00540 \mathrm{E}-08$ & 0.0 & 0.0 & 0.0 \\
\hline PBNO33ION & $4.17861 \mathrm{E}-17$ & 0.0 & 0.0 & 0.0 \\
\hline PBNO3ION & $5.82644 \mathrm{E}-10$ & 0.0 & 0.0 & 0.0 \\
\hline PBOHION & $1.13948 \mathrm{E}-09$ & 0.0 & 0.0 & 0.0 \\
\hline PO4ION & $1.46616 \mathrm{E}-11$ & 0.0 & 0.0 & 0.0 \\
\hline SO4ION & $9.14668 \mathrm{E}-04$ & 0.0 & 0.0 & 0.0 \\
\hline SRION & $1.99784 \mathrm{E}-05$ & 0.0 & 0.0 & 0.0 \\
\hline SRNO3ION & $5.52144 \mathrm{E}-08$ & 0.0 & 0.0 & 0.0 \\
\hline SROHION & $9.59489 \mathrm{E}-13$ & 0.0 & 0.0 & 0.0 \\
\hline SRPO 4 ION & $4.72484 \mathrm{E}-12$ & 0.0 & 0.0 & 0.0 \\
\hline UIVCLION & $4.16603 E-30$ & 0.0 & 0.0 & 0.0 \\
\hline UIVION & $6.34374 \mathrm{E}-29$ & 0.0 & 0.0 & 0.0 \\
\hline UIVOH2ION & $5.43700 \mathrm{E}-20$ & 0.0 & 0.0 & 0.0 \\
\hline UIVOH3ION & $3.75886 \mathrm{E}-15$ & 0.0 & 0.0 & 0.0 \\
\hline UIVOH5ION & $1.73392 \mathrm{E}-14$ & 0.0 & 0.0 & 0.0 \\
\hline UIVOHION & $1.32793 \mathrm{E}-23$ & 0.0 & 0.0 & 0.0 \\
\hline
\end{tabular}


WSRC-TR-2002-00567

Revision 0

\begin{tabular}{|c|c|c|c|c|}
\hline UIVSO4ION & $3.03219 E-26$ & 0.0 & 0.0 & 0.0 \\
\hline ZNCL3 ION & $2.76994 \mathrm{E}-13$ & 0.0 & 0.0 & 0.0 \\
\hline ZNCLION & $1.11455 \mathrm{E}-07$ & 0.0 & 0.0 & 0.0 \\
\hline ZNH2PO4 ION & $2.16029 \mathrm{E}-07$ & 0.0 & 0.0 & 0.0 \\
\hline ZNHCO3ION & $2.70631 E-07$ & 0.0 & 0.0 & 0.0 \\
\hline ZNION & $3.69507 \mathrm{E}-05$ & 0.0 & 0.0 & 0.0 \\
\hline ZNNO3ION & $5.21904 \mathrm{E}-08$ & 0.0 & 0.0 & 0.0 \\
\hline ZNOH3 ION & $1.33729 \mathrm{E}-15$ & 0.0 & 0.0 & 0.0 \\
\hline ZNOH 4 ION & $2.31408 E-22$ & 0.0 & 0.0 & 0.0 \\
\hline ZNOHION & $3.34288 E-08$ & 0.0 & 0.0 & 0.0 \\
\hline $\mathrm{CU} 3 \mathrm{PO} 42.2 \mathrm{H} 2 \mathrm{O}$ & 0.0 & $2.21223 E-06$ & 0.0 & 0.0 \\
\hline $\mathrm{PB} 3 \mathrm{PO} 42$ & 0.0 & $1.12126 \mathrm{E}-06$ & 0.0 & 0.0 \\
\hline \multirow[t]{2}{*}{ UIVO2 } & 0.0 & $4.20138 E-06$ & 0.0 & 0.0 \\
\hline & $===========$ & $===========$ & $===========$ & $===========$ \\
\hline Total g/hr & 999.989 & 0.0111484 & 0.0 & 0.0 \\
\hline Volume, L/hr & 1.00277 & $3.56676 \mathrm{E}-06$ & 0.0 & 0.0 \\
\hline Enthalpy, cal/hr & $-3.79104 \mathrm{E}+06$ & -34.0903 & 0.0 & 0.0 \\
\hline Density, g/L & 997.225 & 3125.64 & & \\
\hline Vapor fraction & 0.0 & 0.0 & 0.0 & 0.0 \\
\hline Solid fraction & 0.0 & 1 . & 0.0 & 0.0 \\
\hline Organic fraction & 0.0 & 0.0 & 0.0 & 0.0 \\
\hline Osmotic Pres, atm & 0.298695 & & & \\
\hline Redox Pot, volts & 0.0 & & & \\
\hline E-Con, $1 / o h m-c m$ & $7.03685 E-04$ & & & \\
\hline E-Con, cm2/ohm-mol & 122.915 & & & \\
\hline Abs Visc, cP & 0.892449 & & & \\
\hline Rel Visc & 1.00194 & & & \\
\hline Ionic Strength & 0.00862614 & & & \\
\hline
\end{tabular}


WSRC-TR-2002-00567

Revision 0

\begin{tabular}{|c|c|c|c|c|}
\hline \\
\hline \multicolumn{5}{|c|}{$\begin{array}{l}\text { STREAM: Evap Contents } \\
\text { TO : Evap separator }\end{array}$} \\
\hline FROM : Evap mixer & & & & \\
\hline Phases-----------> & Aqueous & Solid & Vapor & Organic \\
\hline & 103.256 & 103.256 & 103.256 & 103.256 \\
\hline \multirow{2}{*}{$\begin{array}{l}\text { Pressure, atm } \\
\mathrm{pH}\end{array}$} & 1. & 1 . & 1. & 1 . \\
\hline & 4.71402 & & & \\
\hline \multirow{2}{*}{ Total mol/hr } & 0.06687044 & 0.00131716 & 55.4218 & 0.0 \\
\hline & $\operatorname{mol} / \mathrm{hr}-----1$ & -mol/hr----- & $-\operatorname{mol} / \mathrm{hr}----$ & -mol/hr-------- \\
\hline $\mathrm{H} 2 \mathrm{O}$ & 0.0601048 & 0.0 & 55.42024 & 0.0 \\
\hline $\mathrm{CO} 2$ & $2.10606 \mathrm{E}-10$ & 0.0 & 0.001500001 & 0.0 \\
\hline & $1.18359 \mathrm{E}-23$ & 0.0 & $1.01684 \mathrm{E}-20$ & 0.0 \\
\hline $\mathrm{HCL}$ & $8.79749 \mathrm{E}-13$ & 0.0 & $7.92886 \mathrm{E}-07$ & 0.0 \\
\hline HNO3 & $8.29445 \mathrm{E}-10$ & 0.0 & $1.77234 \mathrm{E}-07$ & 0.0 \\
\hline LAUR ICACID & $1.11692 \mathrm{E}-09$ & 0.0 & $5.57455 E-05$ & 0.0 \\
\hline $\mathrm{SO} 3$ & $8.58407 \mathrm{E}-27$ & 0.0 & $2.71181 E-26$ & 0.0 \\
\hline CAH2SIO4 & $3.32853 E-16$ & 0.0 & 0.0 & 0.0 \\
\hline CASO 4 & $6.15209 \mathrm{E}-07$ & $8.70325 E-04$ & 0.0 & 0.0 \\
\hline CDCL2 & $1.96705 \mathrm{E}-08$ & 0.0 & 0.0 & 0.0 \\
\hline $\mathrm{CDOH} 2$ & $2.78310 \mathrm{E}-19$ & 0.0 & 0.0 & 0.0 \\
\hline $\mathrm{CDSO} 4$ & $1.34528 \mathrm{E}-12$ & 0.0 & 0.0 & 0.0 \\
\hline $\mathrm{CROH} 3$ & $9.19903 \mathrm{E}-20$ & 0.0 & 0.0 & 0.0 \\
\hline CUCL2 & $2.40718 E-09$ & 0.0 & 0.0 & 0.0 \\
\hline CUCO3 & $1.24312 \mathrm{E}-17$ & 0.0 & 0.0 & 0.0 \\
\hline CUNO32 & $1.72768 \mathrm{E}-11$ & 0.0 & 0.0 & 0.0 \\
\hline CUOH2 & $8.46048 E-13$ & 0.0 & 0.0 & 0.0 \\
\hline FEIICL2 & $2.84766 \mathrm{E}-09$ & 0.0 & 0.0 & 0.0 \\
\hline FEIICO3 & $2.58432 E-14$ & 0.0 & 0.0 & 0.0 \\
\hline FEIIHPO4 & $1.64717 \mathrm{E}-09$ & 0.0 & 0.0 & 0.0 \\
\hline FEIIOH2 & $5.59512 \mathrm{E}-13$ & 0.0 & 0.0 & 0.0 \\
\hline ALO2H2CL & $3.13307 \mathrm{E}-27$ & 0.0 & 0.0 & 0.0 \\
\hline H3 PO 4 & $3.74252 E-09$ & 0.0 & 0.0 & 0.0 \\
\hline H4 P2O 7 & $3.31730 \mathrm{E}-16$ & 0.0 & 0.0 & 0.0 \\
\hline ALOH3 & $3.53101 E-11$ & 0.0 & 0.0 & 0.0 \\
\hline $\mathrm{BACO} 3$ & $1.10215 \mathrm{E}-20$ & 0.0 & 0.0 & 0.0 \\
\hline $\mathrm{KCL}$ & $8.27813 E-06$ & 0.0 & 0.0 & 0.0 \\
\hline $\mathrm{KHSO} 4$ & $2.58215 \mathrm{E}-10$ & 0.0 & 0.0 & 0.0 \\
\hline $\mathrm{BASO} 4$ & $1.12269 \mathrm{E}-12$ & $6.68849 E-07$ & 0.0 & 0.0 \\
\hline $\mathrm{MGCO} 3$ & $2.05881 \mathrm{E}-15$ & 0.0 & 0.0 & 0.0 \\
\hline MGH2SIO4 & $3.19884 \mathrm{E}-14$ & 0.0 & 0.0 & 0.0 \\
\hline MGHPO4 & $5.82299 \mathrm{E}-07$ & 0.0 & 0.0 & 0.0 \\
\hline MGSO 4 & $4.41953 E-06$ & 0.0 & 0.0 & 0.0 \\
\hline $\mathrm{NAHCO} 3$ & $1.05426 \mathrm{E}-11$ & 0.0 & 0.0 & 0.0 \\
\hline NAHSIO3 & $4.77800 \mathrm{E}-09$ & 0.0 & 0.0 & 0.0 \\
\hline NANO3 & $1.73834 \mathrm{E}-04$ & 0.0 & 0.0 & 0.0 \\
\hline $\mathrm{NIOH} 2$ & $7.88950 \mathrm{E}-15$ & 0.0 & 0.0 & 0.0 \\
\hline NISO4 & $1.05085 \mathrm{E}-07$ & 0.0 & 0.0 & 0.0 \\
\hline PBCL2 & $1.41799 \mathrm{E}-07$ & 0.0 & 0.0 & 0.0 \\
\hline PBHPO4 & $2.00871 E-12$ & 0.0 & 0.0 & 0.0 \\
\hline PBNO32 & $4.35673 E-09$ & 0.0 & 0.0 & 0.0 \\
\hline $\mathrm{PBO}$ & $5.99092 \mathrm{E}-15$ & 0.0 & 0.0 & 0.0 \\
\hline CACL2 & $6.07322 E-15$ & 0.0 & 0.0 & 0.0 \\
\hline $\mathrm{SIO} 2$ & $4.75359 \mathrm{E}-06$ & $2.08690 \mathrm{E}-04$ & 0.0 & 0.0 \\
\hline
\end{tabular}




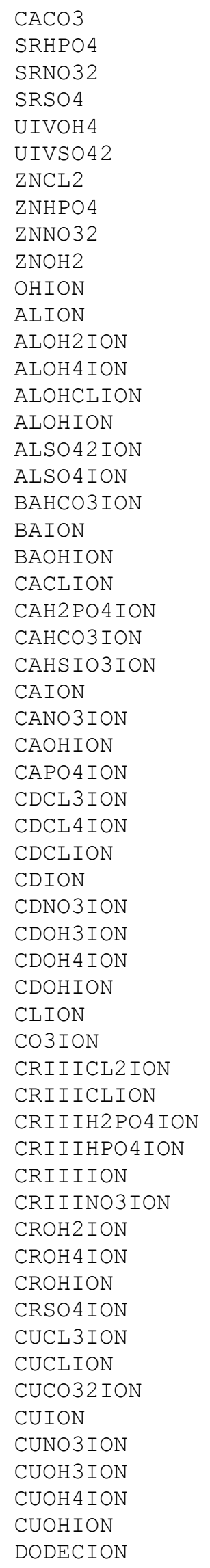

\begin{tabular}{|c|c|}
\hline $6.45329 E-16$ & 0.0 \\
\hline $1.04029 \mathrm{E}-11$ & 0.0 \\
\hline $1.50391 \mathrm{E}-07$ & 0.0 \\
\hline $1.05702 \mathrm{E}-08$ & $2.09121 E-05$ \\
\hline 1. $31334 \mathrm{E}-12$ & 0.0 \\
\hline $4.05264 \mathrm{E}-24$ & 0.0 \\
\hline $8.29540 \mathrm{E}-06$ & 0.0 \\
\hline $3.66148 E-10$ & 0.0 \\
\hline $3.64222 \mathrm{E}-09$ & 0.0 \\
\hline $4.12926 \mathrm{E}-13$ & 0.0 \\
\hline 4.99190E-11 & 0.0 \\
\hline $1.03392 \mathrm{E}-13$ & 0.0 \\
\hline $9.33354 \mathrm{E}-12$ & 0.0 \\
\hline $1.81641 \mathrm{E}-11$ & 0.0 \\
\hline $9.04588 \mathrm{E}-12$ & 0.0 \\
\hline $2.04309 E-12$ & 0.0 \\
\hline $3.45170 \mathrm{E}-14$ & 0.0 \\
\hline $2.09033 E-13$ & 0.0 \\
\hline $3.81098 E-16$ & 0.0 \\
\hline $2.68460 E-09$ & 0.0 \\
\hline $6.89548 E-17$ & 0.0 \\
\hline $1.29182 \mathrm{E}-07$ & 0.0 \\
\hline $1.03461 E-06$ & 0.0 \\
\hline $6.69060 \mathrm{E}-13$ & 0.0 \\
\hline $6.98229 E-11$ & 0.0 \\
\hline $2.77226 \mathrm{E}-05$ & 0.0 \\
\hline $1.54121 E-05$ & 0.0 \\
\hline $1.37658 \mathrm{E}-11$ & 0.0 \\
\hline $2.41417 \mathrm{E}-11$ & 0.0 \\
\hline $1.99194 \mathrm{E}-08$ & 0.0 \\
\hline $5.78739 E-09$ & 0.0 \\
\hline $1.02583 \mathrm{E}-09$ & 0.0 \\
\hline $1.83790 \mathrm{E}-11$ & 0.0 \\
\hline $5.73061 \mathrm{E}-12$ & 0.0 \\
\hline $1.24505 E-25$ & 0.0 \\
\hline 0.0 & 0.0 \\
\hline $1.16475 \mathrm{E}-15$ & 0.0 \\
\hline 0.00272497 & 0.0 \\
\hline $4.69933 E-16$ & 0.0 \\
\hline $1.43728 \mathrm{E}-16$ & 0.0 \\
\hline $2.11623 \mathrm{E}-16$ & 0.0 \\
\hline $1.07656 \mathrm{E}-16$ & 0.0 \\
\hline $1.21153 \mathrm{E}-07$ & 0.0 \\
\hline $3.60973 E-20$ & 0.0 \\
\hline $5.28570 \mathrm{E}-14$ & 0.0 \\
\hline $6.07950 \mathrm{E}-19$ & 0.0 \\
\hline $6.35217 \mathrm{E}-25$ & 0.0 \\
\hline $1.16757 \mathrm{E}-13$ & 0.0 \\
\hline $5.11347 \mathrm{E}-13$ & 0.0 \\
\hline $4.18404 \mathrm{E}-11$ & 0.0 \\
\hline $1.38017 \mathrm{E}-08$ & 0.0 \\
\hline $2.98570 E-26$ & 0.0 \\
\hline $3.66502 \mathrm{E}-09$ & 0.0 \\
\hline $7.06603 \mathrm{E}-10$ & 0.0 \\
\hline $2.37862 \mathrm{E}-18$ & 0.0 \\
\hline $1.45844 \mathrm{E}-23$ & 0.0 \\
\hline $2.58491 E-11$ & 0.0 \\
\hline $1.53321 \mathrm{E}-08$ & 0.0 \\
\hline
\end{tabular}

0.0

0.0

0.0

0.0

0.0

0.0

0.0

0.0

0.0

0.0

0.0

0.0

0.0

0.0

0.0

0.0

0.0

0.0

0.0

0.0

0.0

0.0

0.0

0.0

0.0

0.0

0.0

0.0

0.0

0.0

0.0

0.0

0.0

0.0

0.0

0.0

0.0

0.0

0.0

0.0

0.0

0.0

0.0

0.0

0.0

0.0

0.0

0.0

0.0

0.0

0.0

0.0

0.0

0.0

0.0

0.0

0.0

0.0
0.0

0.0

0.0

0.0

0.0

0.0

0.0

0.0

0.0

0.0

0.0

0.0

0.0

0.0

0.0

0.0

0.0

0.0

0.0

0.0

0.0

0.0

0.0

0.0

0.0

0.0

0.0

0.0

0.0

0.0

0.0

0.0

0.0

0.0

0.0

0.0

0.0

0.0

0.0

0.0

0.0

0.0

0.0

0.0

0.0

0.0

0.0

0.0

0.0

0.0

0.0

0.0

0.0

0.0

0.0

0.0

0.0

0.0 


\begin{tabular}{|c|c|c|c|c|}
\hline FEIICLION & $5.45085 \mathrm{E}-07$ & 0.0 & 0.0 & 0.0 \\
\hline FEIICO32ION & $1.80600 \mathrm{E}-24$ & 0.0 & 0.0 & 0.0 \\
\hline FEIIH2PO4ION & $7.12500 \mathrm{E}-08$ & 0.0 & 0.0 & 0.0 \\
\hline FEIIHCO3ION & $2.78376 \mathrm{E}-14$ & 0.0 & 0.0 & 0.0 \\
\hline FEIIION & $5.80191 \mathrm{E}-05$ & 0.0 & 0.0 & 0.0 \\
\hline FEIIOH3ION & $2.95902 \mathrm{E}-17$ & 0.0 & 0.0 & 0.0 \\
\hline FEIIOH 4 ION & $2.19888 \mathrm{E}-24$ & 0.0 & 0.0 & 0.0 \\
\hline FEIIOHION & $1.57990 \mathrm{E}-08$ & 0.0 & 0.0 & 0.0 \\
\hline H2 P2O 7 ION & $1.14896 \mathrm{E}-09$ & 0.0 & 0.0 & 0.0 \\
\hline H2 PO 4 ION & $1.96441 \mathrm{E}-06$ & 0.0 & 0.0 & 0.0 \\
\hline H2SIO4ION & $6.98870 \mathrm{E}-17$ & 0.0 & 0.0 & 0.0 \\
\hline H3P2O 7 ION & $5.04659 \mathrm{E}-13$ & 0.0 & 0.0 & 0.0 \\
\hline H3SIO4 ION & $1.55832 \mathrm{E}-09$ & 0.0 & 0.0 & 0.0 \\
\hline HCO3ION & $7.10751 \mathrm{E}-12$ & 0.0 & 0.0 & 0.0 \\
\hline HION & $2.70522 \mathrm{E}-08$ & 0.0 & 0.0 & 0.0 \\
\hline HP207ION & $6.62427 \mathrm{E}-11$ & 0.0 & 0.0 & 0.0 \\
\hline HPBO2ION & $1.68978 \mathrm{E}-19$ & 0.0 & 0.0 & 0.0 \\
\hline HPO4 ION & $5.46451 \mathrm{E}-08$ & 0.0 & 0.0 & 0.0 \\
\hline HSO 4 ION & $4.81311 \mathrm{E}-08$ & 0.0 & 0.0 & 0.0 \\
\hline KION & $3.93218 \mathrm{E}-04$ & 0.0 & 0.0 & 0.0 \\
\hline KSO4 ION & $1.06072 \mathrm{E}-05$ & 0.0 & 0.0 & 0.0 \\
\hline MGH2 PO 4 ION & $2.22957 \mathrm{E}-06$ & 0.0 & 0.0 & 0.0 \\
\hline MGHCO3ION & $8.61238 \mathrm{E}-12$ & 0.0 & 0.0 & 0.0 \\
\hline MGHSIO3ION & $8.78865 \mathrm{E}-10$ & 0.0 & 0.0 & 0.0 \\
\hline MGION & $7.86507 \mathrm{E}-05$ & 0.0 & 0.0 & 0.0 \\
\hline MGOHION & $7.31861 \mathrm{E}-10$ & 0.0 & 0.0 & 0.0 \\
\hline MGP2O 7 ION & $2.41211 \mathrm{E}-09$ & 0.0 & 0.0 & 0.0 \\
\hline MGPO 4 ION & $1.70079 \mathrm{E}-10$ & 0.0 & 0.0 & 0.0 \\
\hline NACO3ION & $7.31736 \mathrm{E}-17$ & 0.0 & 0.0 & 0.0 \\
\hline NAION & 0.00262852 & 0.0 & 0.0 & 0.0 \\
\hline NASO 4 ION & $3.79683 \mathrm{E}-14$ & 0.0 & 0.0 & 0.0 \\
\hline NICLION & $8.85479 \mathrm{E}-08$ & 0.0 & 0.0 & 0.0 \\
\hline NIION & $9.86704 \mathrm{E}-07$ & 0.0 & 0.0 & 0.0 \\
\hline NINO3ION & $1.58566 \mathrm{E}-07$ & 0.0 & 0.0 & 0.0 \\
\hline NIOH3ION & $5.49030 \mathrm{E}-20$ & 0.0 & 0.0 & 0.0 \\
\hline NIOHION & $7.27829 \mathrm{E}-11$ & 0.0 & 0.0 & 0.0 \\
\hline NO3ION & $5.35288 \mathrm{E}-04$ & 0.0 & 0.0 & 0.0 \\
\hline P2O7ION & $3.24781 \mathrm{E}-14$ & 0.0 & 0.0 & 0.0 \\
\hline PBCL3ION & $3.48883 E-07$ & 0.0 & 0.0 & 0.0 \\
\hline PBCL 4 ION & $2.88616 \mathrm{E}-06$ & 0.0 & 0.0 & 0.0 \\
\hline PBCLION & $3.64755 \mathrm{E}-08$ & 0.0 & 0.0 & 0.0 \\
\hline PBH2 PO 4 ION & $4.25292 \mathrm{E}-11$ & 0.0 & 0.0 & 0.0 \\
\hline PBION & $1.02452 \mathrm{E}-09$ & 0.0 & 0.0 & 0.0 \\
\hline PBNO33ION & $1.81187 \mathrm{E}-10$ & 0.0 & 0.0 & 0.0 \\
\hline PBNO3ION & $1.09772 \mathrm{E}-08$ & 0.0 & 0.0 & 0.0 \\
\hline PBOHION & $5.37440 \mathrm{E}-11$ & 0.0 & 0.0 & 0.0 \\
\hline PO4ION & $2.51395 \mathrm{E}-14$ & 0.0 & 0.0 & 0.0 \\
\hline SO4ION & $5.06244 \mathrm{E}-05$ & 0.0 & 0.0 & 0.0 \\
\hline SRION & $1.22731 \mathrm{E}-07$ & 0.0 & 0.0 & 0.0 \\
\hline SRNO3ION & $4.93761 \mathrm{E}-07$ & 0.0 & 0.0 & 0.0 \\
\hline SROHION & $1.05148 \mathrm{E}-13$ & 0.0 & 0.0 & 0.0 \\
\hline SRPO 4 ION & $5.55321 \mathrm{E}-15$ & 0.0 & 0.0 & 0.0 \\
\hline UIVCLION & $1.03042 \mathrm{E}-26$ & 0.0 & 0.0 & 0.0 \\
\hline UIVION & $1.02419 \mathrm{E}-28$ & 0.0 & 0.0 & 0.0 \\
\hline UIVOH2ION & $9.75263 \mathrm{E}-21$ & 0.0 & 0.0 & 0.0 \\
\hline UIVOH3ION & $7.80741 \mathrm{E}-18$ & 0.0 & 0.0 & 0.0 \\
\hline UIVOH5ION & $3.43400 \mathrm{E}-17$ & 0.0 & 0.0 & 0.0 \\
\hline UIVOHION & $6.67129 \mathrm{E}-23$ & 0.0 & 0.0 & 0.0 \\
\hline
\end{tabular}


WSRC-TR-2002-00567

Revision 0

\begin{tabular}{|c|c|c|c|c|}
\hline UIVSO4ION & $3.05256 E-24$ & 0.0 & 0.0 & 0.0 \\
\hline ZNCL3 ION & $1.18240 \mathrm{E}-05$ & 0.0 & 0.0 & 0.0 \\
\hline ZNCLION & $1.69740 \mathrm{E}-05$ & 0.0 & 0.0 & 0.0 \\
\hline ZNH2PO4 ION & $3.76830 \mathrm{E}-09$ & 0.0 & 0.0 & 0.0 \\
\hline ZNHCO3ION & $1.22763 E-14$ & 0.0 & 0.0 & 0.0 \\
\hline ZNION & $9.91599 \mathrm{E}-07$ & 0.0 & 0.0 & 0.0 \\
\hline ZNNO3 ION & $1.13381 \mathrm{E}-07$ & 0.0 & 0.0 & 0.0 \\
\hline ZNOH3 ION & $4.65286 \mathrm{E}-17$ & 0.0 & 0.0 & 0.0 \\
\hline ZNOH 4 ION & $1.00513 E-22$ & 0.0 & 0.0 & 0.0 \\
\hline ZNOHION & $2.01346 \mathrm{E}-08$ & 0.0 & 0.0 & 0.0 \\
\hline ALOOH & 0.0 & $6.39375 E-05$ & 0.0 & 0.0 \\
\hline $\mathrm{CA} 3 \mathrm{PO} 42$ & 0.0 & $2.74235 E-05$ & 0.0 & 0.0 \\
\hline CHAMOSITE7A & 0.0 & $1.98833 E-05$ & 0.0 & 0.0 \\
\hline $\mathrm{CU} 3 \mathrm{PO} 42.2 \mathrm{H} 2 \mathrm{O}$ & 0.0 & $2.25034 E-06$ & 0.0 & 0.0 \\
\hline FEII3PO42.8H2O & 0.0 & $1.24282 \mathrm{E}-06$ & 0.0 & 0.0 \\
\hline MG3PO4 2 & 0.0 & $9.75718 \mathrm{E}-05$ & 0.0 & 0.0 \\
\hline $\mathrm{NI} 3 \mathrm{PO} 42$ & 0.0 & $5.33909 \mathrm{E}-08$ & 0.0 & 0.0 \\
\hline UIVO2 & 0.0 & $4.20169 \mathrm{E}-06$ & 0.0 & 0.0 \\
\hline & $===========$ & $===========$ & $===========$ & $===========$ \\
\hline Total g/hr & 1.32597 & 0.182523 & 998.492 & 0.0 \\
\hline Volume, L/hr & 0.00123391 & $5.09465 E-05$ & 1697.83 & 0.0 \\
\hline Enthalpy, cal/hr & -4390.22 & -501.396 & $-3.16917 \mathrm{E}+06$ & 0.0 \\
\hline Density, g/L & 1074.61 & 3582.64 & 0.588099 & \\
\hline Vapor fraction & 0.0 & 0.0 & 1 . & 0.0 \\
\hline Solid fraction & 0.0 & 1 . & 0.0 & 0.0 \\
\hline Organic fraction & 0.0 & 0.0 & 0.0 & 0.0 \\
\hline Osmotic Pres, atm & 185.861 & & & \\
\hline Redox Pot, volts & 0.0 & & & \\
\hline E-Con, $1 / \mathrm{ohm}-\mathrm{cm}$ & 0.48021 & & & \\
\hline E-Con, cm2/ohm-mol & 55.9898 & & & \\
\hline Abs Visc, cP & 0.366185 & & & \\
\hline Rel Visc & 1.3451 & & & \\
\hline Ionic Strength & 3.33609 & & & \\
\hline
\end{tabular}


WSRC-TR-2002-00567

Revision 0

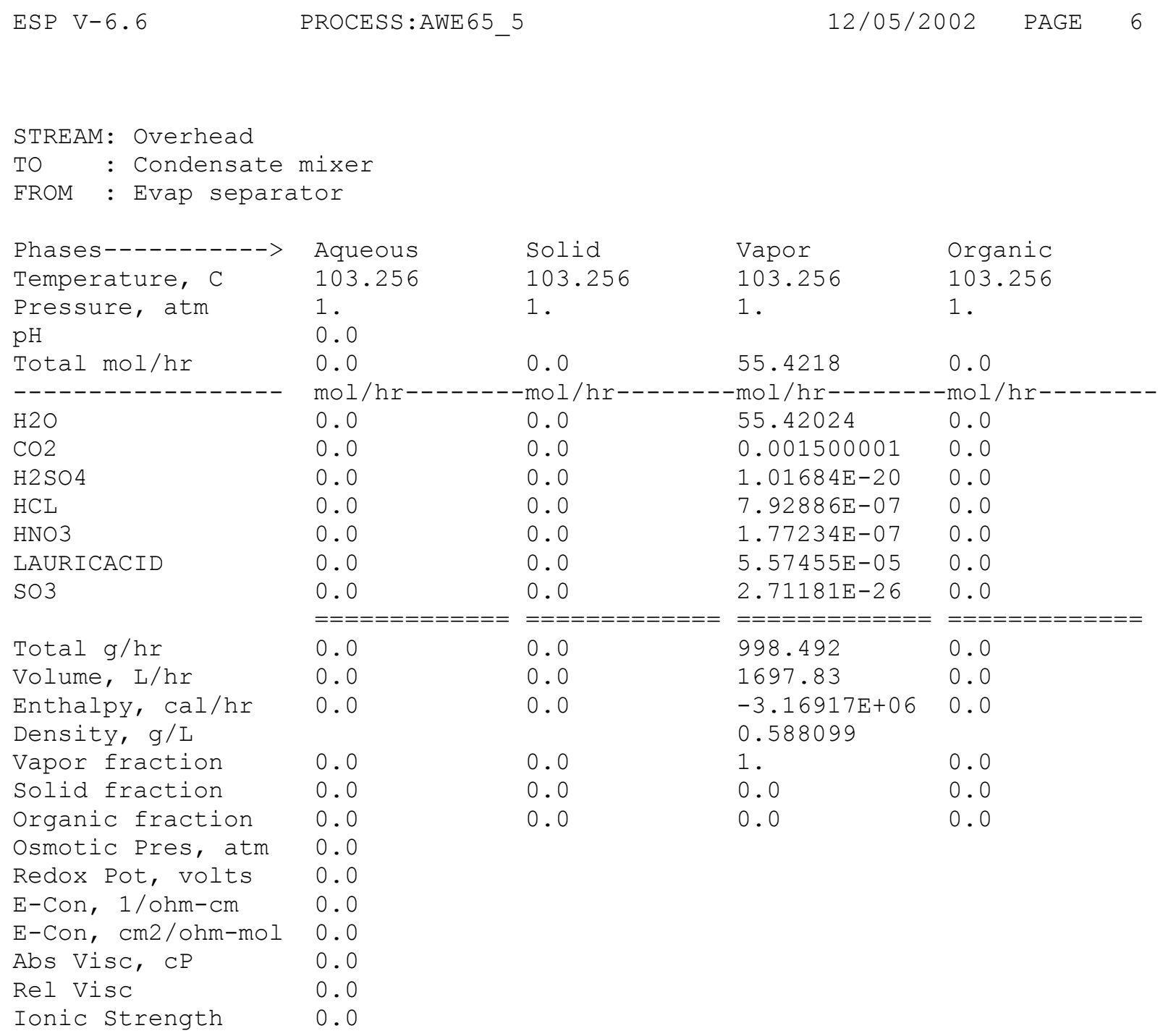


WSRC-TR-2002-00567

Revision 0

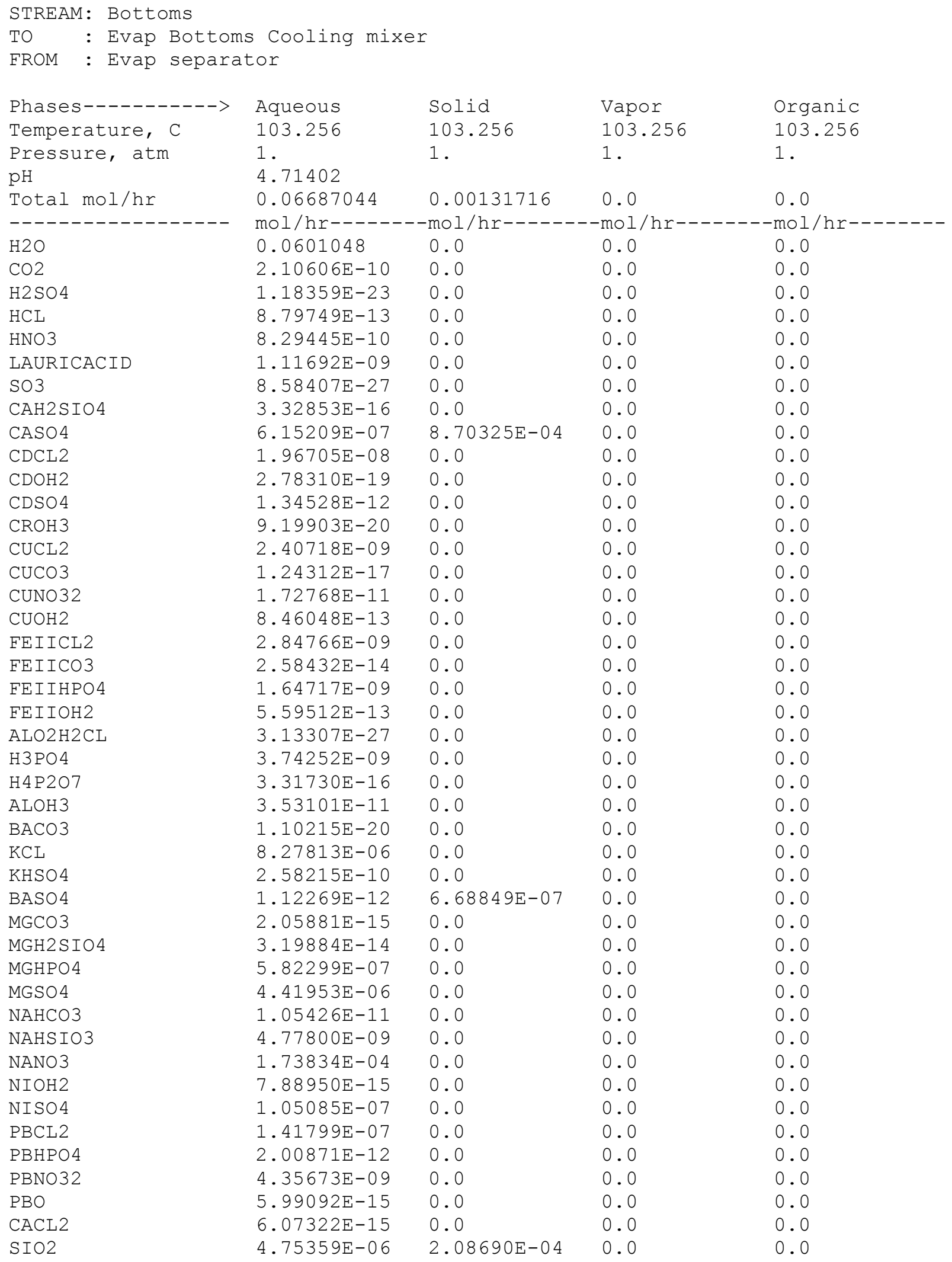




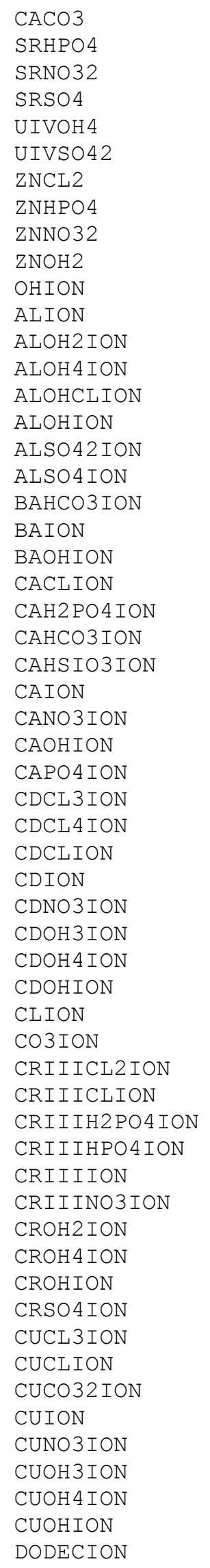

\begin{tabular}{|c|c|}
\hline $6.45329 E-16$ & 0.0 \\
\hline $1.04029 \mathrm{E}-11$ & 0.0 \\
\hline $1.50391 \mathrm{E}-07$ & 0.0 \\
\hline $1.05702 \mathrm{E}-08$ & $2.09121 E-05$ \\
\hline 1. $31334 \mathrm{E}-12$ & 0.0 \\
\hline $4.05264 \mathrm{E}-24$ & 0.0 \\
\hline $8.29540 \mathrm{E}-06$ & 0.0 \\
\hline $3.66148 E-10$ & 0.0 \\
\hline $3.64222 \mathrm{E}-09$ & 0.0 \\
\hline $4.12926 \mathrm{E}-13$ & 0.0 \\
\hline 4.99190E-11 & 0.0 \\
\hline $1.03392 \mathrm{E}-13$ & 0.0 \\
\hline $9.33354 \mathrm{E}-12$ & 0.0 \\
\hline $1.81641 \mathrm{E}-11$ & 0.0 \\
\hline $9.04588 \mathrm{E}-12$ & 0.0 \\
\hline $2.04309 E-12$ & 0.0 \\
\hline $3.45170 \mathrm{E}-14$ & 0.0 \\
\hline $2.09033 E-13$ & 0.0 \\
\hline $3.81098 E-16$ & 0.0 \\
\hline $2.68460 E-09$ & 0.0 \\
\hline $6.89548 E-17$ & 0.0 \\
\hline $1.29182 \mathrm{E}-07$ & 0.0 \\
\hline $1.03461 E-06$ & 0.0 \\
\hline $6.69060 \mathrm{E}-13$ & 0.0 \\
\hline $6.98229 E-11$ & 0.0 \\
\hline $2.77226 \mathrm{E}-05$ & 0.0 \\
\hline $1.54121 E-05$ & 0.0 \\
\hline $1.37658 \mathrm{E}-11$ & 0.0 \\
\hline $2.41417 \mathrm{E}-11$ & 0.0 \\
\hline $1.99194 \mathrm{E}-08$ & 0.0 \\
\hline $5.78739 E-09$ & 0.0 \\
\hline $1.02583 \mathrm{E}-09$ & 0.0 \\
\hline $1.83790 \mathrm{E}-11$ & 0.0 \\
\hline $5.73061 \mathrm{E}-12$ & 0.0 \\
\hline $1.24505 E-25$ & 0.0 \\
\hline 0.0 & 0.0 \\
\hline $1.16475 \mathrm{E}-15$ & 0.0 \\
\hline 0.00272497 & 0.0 \\
\hline $4.69933 E-16$ & 0.0 \\
\hline $1.43728 \mathrm{E}-16$ & 0.0 \\
\hline $2.11623 \mathrm{E}-16$ & 0.0 \\
\hline $1.07656 \mathrm{E}-16$ & 0.0 \\
\hline $1.21153 \mathrm{E}-07$ & 0.0 \\
\hline $3.60973 E-20$ & 0.0 \\
\hline $5.28570 \mathrm{E}-14$ & 0.0 \\
\hline $6.07950 \mathrm{E}-19$ & 0.0 \\
\hline $6.35217 \mathrm{E}-25$ & 0.0 \\
\hline $1.16757 \mathrm{E}-13$ & 0.0 \\
\hline $5.11347 \mathrm{E}-13$ & 0.0 \\
\hline $4.18404 \mathrm{E}-11$ & 0.0 \\
\hline $1.38017 \mathrm{E}-08$ & 0.0 \\
\hline $2.98570 E-26$ & 0.0 \\
\hline $3.66502 \mathrm{E}-09$ & 0.0 \\
\hline $7.06603 \mathrm{E}-10$ & 0.0 \\
\hline $2.37862 \mathrm{E}-18$ & 0.0 \\
\hline $1.45844 \mathrm{E}-23$ & 0.0 \\
\hline $2.58491 E-11$ & 0.0 \\
\hline $1.53321 \mathrm{E}-08$ & 0.0 \\
\hline
\end{tabular}

0.0

0.0

0.0

0.0

0.0

0.0

0.0

0.0

0.0

0.0

0.0

0.0

0.0

0.0

0.0

0.0

0.0

0.0

0.0

0.0

0.0

0.0

0.0

0.0

0.0

0.0

0.0

0.0

0.0

0.0

0.0

0.0

0.0

0.0

0.0

0.0

0.0

0.0

0.0

0.0

0.0

0.0

0.0

0.0

0.0

0.0

0.0

0.0

0.0

0.0

0.0

0.0

0.0

0.0

0.0

0.0

0.0

0.0
0.0

0.0

0.0

0.0

0.0

0.0

0.0

0.0

0.0

0.0

0.0

0.0

0.0

0.0

0.0

0.0

0.0

0.0

0.0

0.0

0.0

0.0

0.0

0.0

0.0

0.0

0.0

0.0

0.0

0.0

0.0

0.0

0.0

0.0

0.0

0.0

0.0

0.0

0.0

0.0

0.0

0.0

0.0

0.0

0.0

0.0

0.0

0.0

0.0

0.0

0.0

0.0

0.0

0.0

0.0

0.0

0.0

0.0 


\begin{tabular}{|c|c|c|c|c|}
\hline FEIICLION & $5.45085 \mathrm{E}-07$ & 0.0 & 0.0 & 0.0 \\
\hline FEIICO32ION & $1.80600 \mathrm{E}-24$ & 0.0 & 0.0 & 0.0 \\
\hline FEIIH2PO4ION & $7.12500 \mathrm{E}-08$ & 0.0 & 0.0 & 0.0 \\
\hline FEIIHCO3ION & $2.78376 \mathrm{E}-14$ & 0.0 & 0.0 & 0.0 \\
\hline FEIIION & $5.80191 \mathrm{E}-05$ & 0.0 & 0.0 & 0.0 \\
\hline FEIIOH3ION & $2.95902 \mathrm{E}-17$ & 0.0 & 0.0 & 0.0 \\
\hline FEIIOH 4 ION & $2.19888 \mathrm{E}-24$ & 0.0 & 0.0 & 0.0 \\
\hline FEIIOHION & $1.57990 \mathrm{E}-08$ & 0.0 & 0.0 & 0.0 \\
\hline H2 P2O 7 ION & $1.14896 \mathrm{E}-09$ & 0.0 & 0.0 & 0.0 \\
\hline H2 PO 4 ION & $1.96441 \mathrm{E}-06$ & 0.0 & 0.0 & 0.0 \\
\hline H2SIO4ION & $6.98870 \mathrm{E}-17$ & 0.0 & 0.0 & 0.0 \\
\hline H3P2O 7 ION & $5.04659 \mathrm{E}-13$ & 0.0 & 0.0 & 0.0 \\
\hline H3SIO4 ION & $1.55832 \mathrm{E}-09$ & 0.0 & 0.0 & 0.0 \\
\hline HCO3ION & $7.10751 \mathrm{E}-12$ & 0.0 & 0.0 & 0.0 \\
\hline HION & $2.70522 \mathrm{E}-08$ & 0.0 & 0.0 & 0.0 \\
\hline HP207ION & $6.62427 \mathrm{E}-11$ & 0.0 & 0.0 & 0.0 \\
\hline HPBO2ION & $1.68978 \mathrm{E}-19$ & 0.0 & 0.0 & 0.0 \\
\hline HPO4 ION & $5.46451 \mathrm{E}-08$ & 0.0 & 0.0 & 0.0 \\
\hline HSO 4 ION & $4.81311 \mathrm{E}-08$ & 0.0 & 0.0 & 0.0 \\
\hline KION & $3.93218 \mathrm{E}-04$ & 0.0 & 0.0 & 0.0 \\
\hline KSO4 ION & $1.06072 \mathrm{E}-05$ & 0.0 & 0.0 & 0.0 \\
\hline MGH2 PO 4 ION & $2.22957 \mathrm{E}-06$ & 0.0 & 0.0 & 0.0 \\
\hline MGHCO3ION & $8.61238 \mathrm{E}-12$ & 0.0 & 0.0 & 0.0 \\
\hline MGHSIO3ION & $8.78865 \mathrm{E}-10$ & 0.0 & 0.0 & 0.0 \\
\hline MGION & $7.86507 \mathrm{E}-05$ & 0.0 & 0.0 & 0.0 \\
\hline MGOHION & $7.31861 \mathrm{E}-10$ & 0.0 & 0.0 & 0.0 \\
\hline MGP2O 7 ION & $2.41211 \mathrm{E}-09$ & 0.0 & 0.0 & 0.0 \\
\hline MGPO 4 ION & $1.70079 \mathrm{E}-10$ & 0.0 & 0.0 & 0.0 \\
\hline NACO3ION & $7.31736 \mathrm{E}-17$ & 0.0 & 0.0 & 0.0 \\
\hline NAION & 0.00262852 & 0.0 & 0.0 & 0.0 \\
\hline NASO 4 ION & $3.79683 \mathrm{E}-14$ & 0.0 & 0.0 & 0.0 \\
\hline NICLION & $8.85479 \mathrm{E}-08$ & 0.0 & 0.0 & 0.0 \\
\hline NIION & $9.86704 \mathrm{E}-07$ & 0.0 & 0.0 & 0.0 \\
\hline NINO3ION & $1.58566 \mathrm{E}-07$ & 0.0 & 0.0 & 0.0 \\
\hline NIOH3ION & $5.49030 \mathrm{E}-20$ & 0.0 & 0.0 & 0.0 \\
\hline NIOHION & $7.27829 \mathrm{E}-11$ & 0.0 & 0.0 & 0.0 \\
\hline NO3ION & $5.35288 \mathrm{E}-04$ & 0.0 & 0.0 & 0.0 \\
\hline P2O7ION & $3.24781 \mathrm{E}-14$ & 0.0 & 0.0 & 0.0 \\
\hline PBCL3ION & $3.48883 E-07$ & 0.0 & 0.0 & 0.0 \\
\hline PBCL 4 ION & $2.88616 \mathrm{E}-06$ & 0.0 & 0.0 & 0.0 \\
\hline PBCLION & $3.64755 \mathrm{E}-08$ & 0.0 & 0.0 & 0.0 \\
\hline PBH2 PO 4 ION & $4.25292 \mathrm{E}-11$ & 0.0 & 0.0 & 0.0 \\
\hline PBION & $1.02452 \mathrm{E}-09$ & 0.0 & 0.0 & 0.0 \\
\hline PBNO33ION & $1.81187 \mathrm{E}-10$ & 0.0 & 0.0 & 0.0 \\
\hline PBNO3ION & $1.09772 \mathrm{E}-08$ & 0.0 & 0.0 & 0.0 \\
\hline PBOHION & $5.37440 \mathrm{E}-11$ & 0.0 & 0.0 & 0.0 \\
\hline PO4ION & $2.51395 \mathrm{E}-14$ & 0.0 & 0.0 & 0.0 \\
\hline SO4ION & $5.06244 \mathrm{E}-05$ & 0.0 & 0.0 & 0.0 \\
\hline SRION & $1.22731 \mathrm{E}-07$ & 0.0 & 0.0 & 0.0 \\
\hline SRNO3ION & $4.93761 \mathrm{E}-07$ & 0.0 & 0.0 & 0.0 \\
\hline SROHION & $1.05148 \mathrm{E}-13$ & 0.0 & 0.0 & 0.0 \\
\hline SRPO 4 ION & $5.55321 \mathrm{E}-15$ & 0.0 & 0.0 & 0.0 \\
\hline UIVCLION & $1.03042 \mathrm{E}-26$ & 0.0 & 0.0 & 0.0 \\
\hline UIVION & $1.02419 \mathrm{E}-28$ & 0.0 & 0.0 & 0.0 \\
\hline UIVOH2ION & $9.75263 \mathrm{E}-21$ & 0.0 & 0.0 & 0.0 \\
\hline UIVOH3ION & $7.80741 \mathrm{E}-18$ & 0.0 & 0.0 & 0.0 \\
\hline UIVOH5ION & $3.43400 \mathrm{E}-17$ & 0.0 & 0.0 & 0.0 \\
\hline UIVOHION & $6.67129 \mathrm{E}-23$ & 0.0 & 0.0 & 0.0 \\
\hline
\end{tabular}


WSRC-TR-2002-00567

Revision 0

\begin{tabular}{|c|c|c|c|c|}
\hline UIVSO4ION & $3.05256 E-24$ & 0.0 & 0.0 & 0.0 \\
\hline ZNCL3 ION & $1.18240 \mathrm{E}-05$ & 0.0 & 0.0 & 0.0 \\
\hline ZNCLION & $1.69740 \mathrm{E}-05$ & 0.0 & 0.0 & 0.0 \\
\hline ZNH2PO4 ION & $3.76830 \mathrm{E}-09$ & 0.0 & 0.0 & 0.0 \\
\hline ZNHCO3ION & $1.22763 E-14$ & 0.0 & 0.0 & 0.0 \\
\hline ZNION & $9.91599 \mathrm{E}-07$ & 0.0 & 0.0 & 0.0 \\
\hline ZNNO3 ION & $1.13381 \mathrm{E}-07$ & 0.0 & 0.0 & 0.0 \\
\hline ZNOH3 ION & $4.65286 \mathrm{E}-17$ & 0.0 & 0.0 & 0.0 \\
\hline ZNOH 4 ION & $1.00513 E-22$ & 0.0 & 0.0 & 0.0 \\
\hline ZNOHION & $2.01346 \mathrm{E}-08$ & 0.0 & 0.0 & 0.0 \\
\hline ALOOH & 0.0 & $6.39375 E-05$ & 0.0 & 0.0 \\
\hline $\mathrm{CA} 3 \mathrm{PO} 42$ & 0.0 & $2.74235 E-05$ & 0.0 & 0.0 \\
\hline CHAMOSITE7A & 0.0 & $1.98833 E-05$ & 0.0 & 0.0 \\
\hline $\mathrm{CU} 3 \mathrm{PO} 42.2 \mathrm{H} 2 \mathrm{O}$ & 0.0 & $2.25034 E-06$ & 0.0 & 0.0 \\
\hline FEII3PO42.8H2O & 0.0 & $1.24282 \mathrm{E}-06$ & 0.0 & 0.0 \\
\hline MG3PO4 2 & 0.0 & $9.75718 \mathrm{E}-05$ & 0.0 & 0.0 \\
\hline $\mathrm{NI} 3 \mathrm{PO} 42$ & 0.0 & $5.33909 \mathrm{E}-08$ & 0.0 & 0.0 \\
\hline UIVO2 & 0.0 & $4.20169 \mathrm{E}-06$ & 0.0 & 0.0 \\
\hline & $===========$ & $===========$ & $============$ & $===========$ \\
\hline Total g/hr & 1.32597 & 0.182523 & 0.0 & 0.0 \\
\hline Volume, L/hr & 0.00123391 & $5.09465 E-05$ & 0.0 & 0.0 \\
\hline Enthalpy, cal/hr & -4390.22 & -501.396 & 0.0 & 0.0 \\
\hline Density, g/L & 1074.61 & 3582.64 & & \\
\hline Vapor fraction & 0.0 & 0.0 & 0.0 & 0.0 \\
\hline Solid fraction & 0.0 & 1 . & 0.0 & 0.0 \\
\hline Organic fraction & 0.0 & 0.0 & 0.0 & 0.0 \\
\hline Osmotic Pres, atm & 185.861 & & & \\
\hline Redox Pot, volts & 0.0 & & & \\
\hline E-Con, $1 / \mathrm{ohm}-\mathrm{cm}$ & 0.48021 & & & \\
\hline E-Con, cm2/ohm-mol & 55.9898 & & & \\
\hline Abs Visc, cP & 0.366185 & & & \\
\hline Rel Visc & 1.3451 & & & \\
\hline Ionic Strength & 3.33609 & & & \\
\hline
\end{tabular}


WSRC-TR-2002-00567

Revision 0

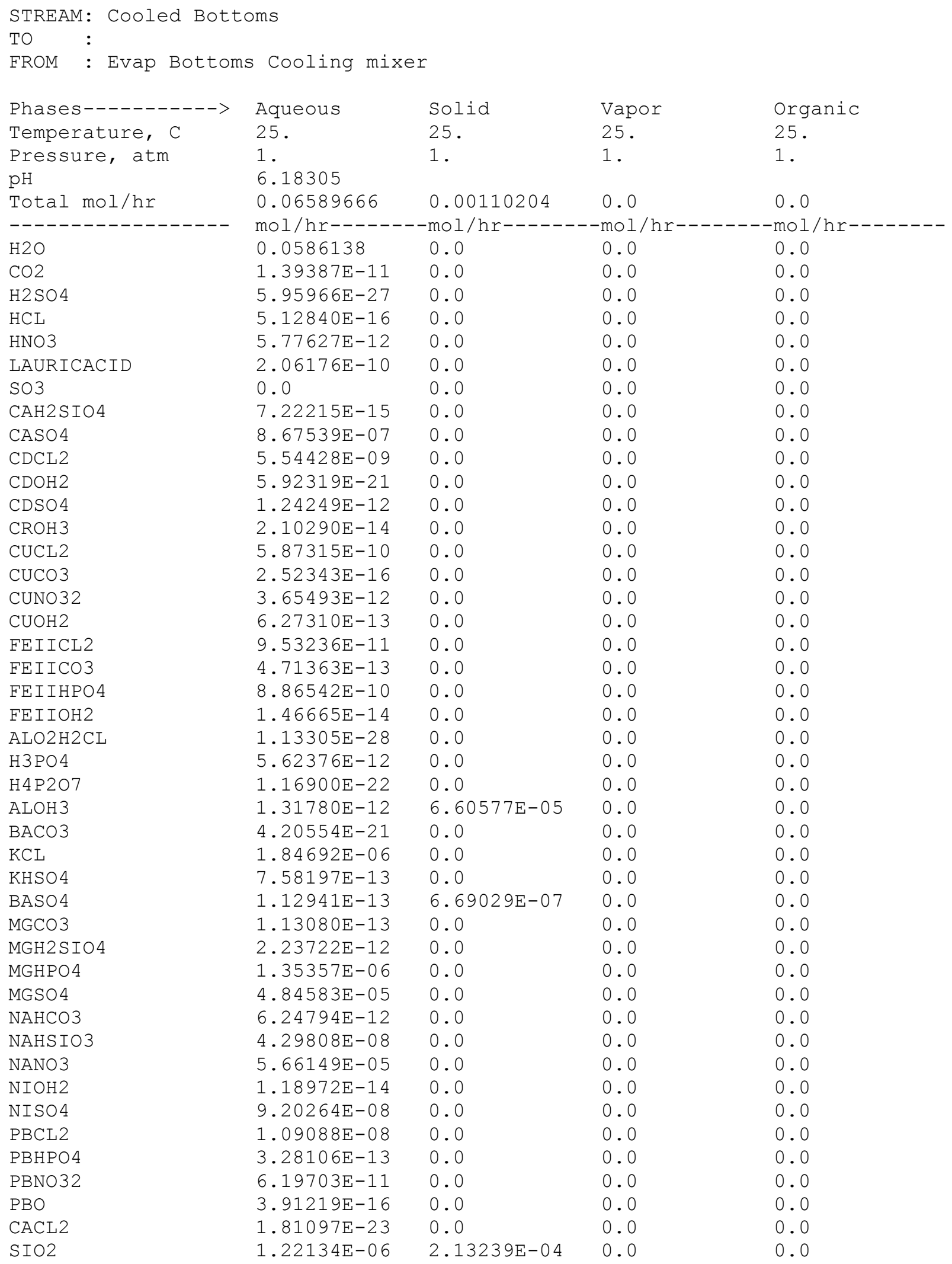




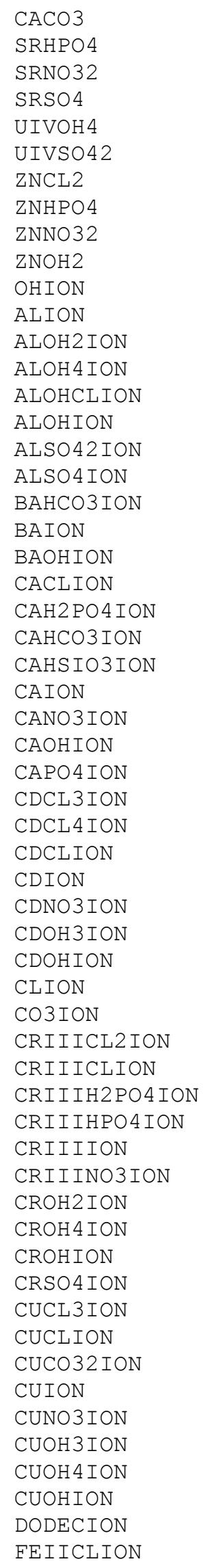

\begin{tabular}{|c|c|}
\hline $7.81954 \mathrm{E}-15$ & 0.0 \\
\hline $3.36000 \mathrm{E}-12$ & 0.0 \\
\hline $2.46259 \mathrm{E}-08$ & 0.0 \\
\hline $2.36642 E-08$ & $2.05392 E-05$ \\
\hline $1.27140 \mathrm{E}-13$ & 0.0 \\
\hline $1.48911 \mathrm{E}-27$ & 0.0 \\
\hline $1.36063 E-06$ & 0.0 \\
\hline $2.61773 E-09$ & 0.0 \\
\hline $1.48979 \mathrm{E}-08$ & 0.0 \\
\hline $1.17534 \mathrm{E}-12$ & 0.0 \\
\hline $1.55002 \mathrm{E}-11$ & 0.0 \\
\hline $2.45792 E-14$ & 0.0 \\
\hline $4.78362 E-13$ & 0.0 \\
\hline $1.50919 \mathrm{E}-12$ & 0.0 \\
\hline $9.86227 \mathrm{E}-13$ & 0.0 \\
\hline 1.22483E-13 & 0.0 \\
\hline $3.33693 E-14$ & 0.0 \\
\hline $6.54061 \mathrm{E}-14$ & 0.0 \\
\hline $5.88321 E-18$ & 0.0 \\
\hline $2.50090 E-09$ & 0.0 \\
\hline $1.77894 \mathrm{E}-19$ & 0.0 \\
\hline $1.17480 \mathrm{E}-10$ & 0.0 \\
\hline $5.80052 \mathrm{E}-08$ & 0.0 \\
\hline $2.15102 \mathrm{E}-12$ & 0.0 \\
\hline $9.43325 E-11$ & 0.0 \\
\hline $2.69377 \mathrm{E}-05$ & 0.0 \\
\hline $1.40854 \mathrm{E}-05$ & 0.0 \\
\hline $2.04979 \mathrm{E}-12$ & 0.0 \\
\hline $4.05531 E-10$ & 0.0 \\
\hline $2.70360 E-09$ & 0.0 \\
\hline $3.78520 E-08$ & 0.0 \\
\hline $3.13448 \mathrm{E}-10$ & 0.0 \\
\hline $1.19232 \mathrm{E}-11$ & 0.0 \\
\hline $2.11797 \mathrm{E}-12$ & 0.0 \\
\hline $4.67242 E-27$ & 0.0 \\
\hline $1.46064 \mathrm{E}-16$ & 0.0 \\
\hline 0.00279642 & 0.0 \\
\hline $2.40253 E-14$ & 0.0 \\
\hline $1.67213 E-13$ & 0.0 \\
\hline $4.45015 E-14$ & 0.0 \\
\hline $1.87038 \mathrm{E}-12$ & 0.0 \\
\hline $1.19578 \mathrm{E}-07$ & 0.0 \\
\hline $1.44402 \mathrm{E}-17$ & 0.0 \\
\hline $2.68686 \mathrm{E}-11$ & 0.0 \\
\hline $1.23102 \mathrm{E}-14$ & 0.0 \\
\hline $1.37155 \mathrm{E}-18$ & 0.0 \\
\hline $6.44237 \mathrm{E}-10$ & 0.0 \\
\hline $9.02751 \mathrm{E}-10$ & 0.0 \\
\hline $1.24107 \mathrm{E}-11$ & 0.0 \\
\hline $1.55320 \mathrm{E}-09$ & 0.0 \\
\hline $1.88451 \mathrm{E}-23$ & 0.0 \\
\hline $1.30502 \mathrm{E}-09$ & 0.0 \\
\hline $2.67022 \mathrm{E}-10$ & 0.0 \\
\hline $1.81489 \mathrm{E}-18$ & 0.0 \\
\hline $2.02842 E-24$ & 0.0 \\
\hline $6.84939 E-12$ & 0.0 \\
\hline 1. $62429 \mathrm{E}-08$ & 0.0 \\
\hline $3.11682 \mathrm{E}-08$ & 0.0 \\
\hline
\end{tabular}

0.0

0.0

0.0

0.0

0.0

0.0

0.0

0.0

0.0

0.0

0.0

0.0

0.0

0.0

0.0

0.0

0.0

0.0

0.0

0.0

0.0

0.0

0.0

0.0

0.0

0.0

0.0

0.0

0.0

0.0

0.0

0.0

0.0

0.0

0.0

0.0

0.0

0.0

0.0

0.0

0.0

0.0

0.0

0.0

0.0

0.0

0.0

0.0

0.0

0.0

0.0

0.0

0.0

0.0

0.0

0.0

0.0

0.0
0.0

0.0

0.0

0.0

0.0

0.0

0.0

0.0

0.0

0.0

0.0

0.0

0.0

0.0

0.0

0.0

0.0

0.0

0.0

0.0

0.0

0.0

0.0

0.0

0.0

0.0

0.0

0.0

0.0

0.0

0.0

0.0

0.0

0.0

0.0

0.0

0.0

0.0

0.0

0.0

0.0

0.0

0.0

0.0

0.0

0.0

0.0

0.0

0.0

0.0

0.0

0.0

0.0

0.0

0.0

0.0

0.0

0.0 


\begin{tabular}{|c|c|c|c|c|}
\hline FEIICO32ION & $7.64410 \mathrm{E}-22$ & 0.0 & 0.0 & 0.0 \\
\hline FEIIH2PO4ION & $4.99343 E-09$ & 0.0 & 0.0 & 0.0 \\
\hline EEIIHCO3ION & $2.13002 \mathrm{E}-14$ & 0.0 & 0.0 & 0.0 \\
\hline FEIIION & $1.87435 \mathrm{E}-05$ & 0.0 & 0.0 & 0.0 \\
\hline FEIIOH3ION & $2.56169 \mathrm{E}-18$ & 0.0 & 0.0 & 0.0 \\
\hline EEIIOH4ION & $4.74410 \mathrm{E}-26$ & 0.0 & 0.0 & 0.0 \\
\hline FEIIOHION & $3.65291 \mathrm{E}-09$ & 0.0 & 0.0 & 0.0 \\
\hline H2 P2O 7 ION & $1.36237 \mathrm{E}-12$ & 0.0 & 0.0 & 0.0 \\
\hline H2 PO 4 ION & $2.68405 \mathrm{E}-07$ & 0.0 & 0.0 & 0.0 \\
\hline $\mathrm{H} 2 \mathrm{SIO} 4 \mathrm{ION}$ & $8.21600 \mathrm{E}-16$ & 0.0 & 0.0 & 0.0 \\
\hline H3P2O7 ION & $1.09993 \mathrm{E}-17$ & 0.0 & 0.0 & 0.0 \\
\hline H3SIO 4 ION & $4.08405 \mathrm{E}-10$ & 0.0 & 0.0 & 0.0 \\
\hline HCO3ION & $3.27842 \mathrm{E}-11$ & 0.0 & 0.0 & 0.0 \\
\hline HION & $6.00687 \mathrm{E}-10$ & 0.0 & 0.0 & 0.0 \\
\hline HP207ION & $4.41398 \mathrm{E}-12$ & 0.0 & 0.0 & 0.0 \\
\hline HPBO2ION & $1.71514 \mathrm{E}-20$ & 0.0 & 0.0 & 0.0 \\
\hline HPO4ION & $3.08162 \mathrm{E}-07$ & 0.0 & 0.0 & 0.0 \\
\hline HSO 4 ION & $5.07030 \mathrm{E}-10$ & 0.0 & 0.0 & 0.0 \\
\hline KION & $3.91059 \mathrm{E}-04$ & 0.0 & 0.0 & 0.0 \\
\hline KSO4 ION & $1.91970 \mathrm{E}-05$ & 0.0 & 0.0 & 0.0 \\
\hline MGH2 PO 4 ION & $2.38894 \mathrm{E}-06$ & 0.0 & 0.0 & 0.0 \\
\hline MGHCO3ION & $1.81648 \mathrm{E}-10$ & 0.0 & 0.0 & 0.0 \\
\hline MGHSIO3ION & $5.58566 \mathrm{E}-09$ & 0.0 & 0.0 & 0.0 \\
\hline MGION & $3.26377 \mathrm{E}-04$ & 0.0 & 0.0 & 0.0 \\
\hline MGOHION & $6.29460 \mathrm{E}-10$ & 0.0 & 0.0 & 0.0 \\
\hline MGP2O7ION & $1.81206 \mathrm{E}-09$ & 0.0 & 0.0 & 0.0 \\
\hline MGPO 4 ION & $1.51367 \mathrm{E}-08$ & 0.0 & 0.0 & 0.0 \\
\hline NACO3ION & $1.11061 \mathrm{E}-14$ & 0.0 & 0.0 & 0.0 \\
\hline NAION & 0.0026881 & 0.0 & 0.0 & 0.0 \\
\hline NASO 4 ION & $5.75934 \mathrm{E}-05$ & 0.0 & 0.0 & 0.0 \\
\hline NICLION & $4.54483 E-08$ & 0.0 & 0.0 & 0.0 \\
\hline NIION & $1.06088 \mathrm{E}-06$ & 0.0 & 0.0 & 0.0 \\
\hline NINO3ION & $3.00761 \mathrm{E}-07$ & 0.0 & 0.0 & 0.0 \\
\hline NIOH3ION & $6.88200 \mathrm{E}-19$ & 0.0 & 0.0 & 0.0 \\
\hline NIOHION & $3.22598 \mathrm{E}-11$ & 0.0 & 0.0 & 0.0 \\
\hline NO3ION & $6.53765 \mathrm{E}-04$ & 0.0 & 0.0 & 0.0 \\
\hline P207ION & $4.47011 \mathrm{E}-13$ & 0.0 & 0.0 & 0.0 \\
\hline PBCL3ION & $3.75671 \mathrm{E}-08$ & 0.0 & 0.0 & 0.0 \\
\hline PBCL 4 ION & $8.72776 \mathrm{E}-07$ & 0.0 & 0.0 & 0.0 \\
\hline PBCLION & $3.62438 \mathrm{E}-09$ & 0.0 & 0.0 & 0.0 \\
\hline PBH2 PO 4 ION & $3.68296 \mathrm{E}-13$ & 0.0 & 0.0 & 0.0 \\
\hline PBION & $2.24985 \mathrm{E}-10$ & 0.0 & 0.0 & 0.0 \\
\hline PBNO33ION & $1.84673 \mathrm{E}-11$ & 0.0 & 0.0 & 0.0 \\
\hline PBNO3ION & $6.00214 \mathrm{E}-10$ & 0.0 & 0.0 & 0.0 \\
\hline PBOHION & $1.71634 \mathrm{E}-12$ & 0.0 & 0.0 & 0.0 \\
\hline PO4ION & $3.16170 \mathrm{E}-12$ & 0.0 & 0.0 & 0.0 \\
\hline SO4ION & $1.61693 \mathrm{E}-04$ & 0.0 & 0.0 & 0.0 \\
\hline SRION & $9.30505 \mathrm{E}-07$ & 0.0 & 0.0 & 0.0 \\
\hline SRNO3ION & $1.71378 \mathrm{E}-07$ & 0.0 & 0.0 & 0.0 \\
\hline SROHION & $6.34465 \mathrm{E}-15$ & 0.0 & 0.0 & 0.0 \\
\hline SRPO 4 ION & $2.97074 \mathrm{E}-14$ & 0.0 & 0.0 & 0.0 \\
\hline UIVCLION & $7.52358 \mathrm{E}-30$ & 0.0 & 0.0 & 0.0 \\
\hline UIVION & 0.0 & 0.0 & 0.0 & 0.0 \\
\hline UIVOH2ION & $5.63893 \mathrm{E}-23$ & 0.0 & 0.0 & 0.0 \\
\hline UIVOH3ION & $3.10876 \mathrm{E}-18$ & 0.0 & 0.0 & 0.0 \\
\hline UIVOH5ION & $3.74837 \mathrm{E}-17$ & 0.0 & 0.0 & 0.0 \\
\hline UIVOHION & $3.62100 \mathrm{E}-26$ & 0.0 & 0.0 & 0.0 \\
\hline UIVSO 4 ION & $1.25260 \mathrm{E}-27$ & 0.0 & 0.0 & 0.0 \\
\hline
\end{tabular}


WSRC-TR-2002-00567

Revision 0

\begin{tabular}{|c|c|c|c|c|}
\hline ZNCL3ION & $3.25672 \mathrm{E}-06$ & 0.0 & 0.0 & 0.0 \\
\hline ZNCLION & $1.46601 \mathrm{E}-06$ & 0.0 & 0.0 & 0.0 \\
\hline ZNH2 PO4 ION & $2.40029 E-09$ & 0.0 & 0.0 & 0.0 \\
\hline ZNHCO3ION & $1.88922 \mathrm{E}-13$ & 0.0 & 0.0 & 0.0 \\
\hline ZNION & $4.91505 E-06$ & 0.0 & 0.0 & 0.0 \\
\hline ZNNO3ION & $6.11711 \mathrm{E}-07$ & 0.0 & 0.0 & 0.0 \\
\hline ZNOH3ION & $4.30359 \mathrm{E}-17$ & 0.0 & 0.0 & 0.0 \\
\hline ZNOH 4 ION & $1.20560 \mathrm{E}-22$ & 0.0 & 0.0 & 0.0 \\
\hline ZNOHION & $8.36895 \mathrm{E}-10$ & 0.0 & 0.0 & 0.0 \\
\hline $\mathrm{CA} 3 \mathrm{PO} 42$ & 0.0 & $1.02120 \mathrm{E}-04$ & 0.0 & 0.0 \\
\hline $\mathrm{CASO} 4.2 \mathrm{H} 2 \mathrm{O}$ & 0.0 & $6.49195 E-04$ & 0.0 & 0.0 \\
\hline CHAMOSITE7A & 0.0 & $1.88227 E-05$ & 0.0 & 0.0 \\
\hline $\mathrm{CU} 3 \mathrm{PO} 42.2 \mathrm{H} 2 \mathrm{O}$ & 0.0 & $2.25596 \mathrm{E}-06$ & 0.0 & 0.0 \\
\hline FEII3PO $42.8 \mathrm{H} 2 \mathrm{O}$ & 0.0 & $1.52401 E-05$ & 0.0 & 0.0 \\
\hline РB3РО42 & 0.0 & $8.34719 \mathrm{E}-07$ & 0.0 & 0.0 \\
\hline UIVO2 & 0.0 & $4.20166 \mathrm{E}-06$ & 0.0 & 0.0 \\
\hline \multirow[t]{2}{*}{$\mathrm{ZN} 3 \mathrm{PO} 42.2 \mathrm{H} 2 \mathrm{O}$} & 0.0 & $8.86510 \mathrm{E}-06$ & 0.0 & 0.0 \\
\hline & $==========$ & $===========$ & $===========$ & $===========$ \\
\hline Total g/hr & 1.32258 & 0.185915 & 0.0 & 0.0 \\
\hline Volume, L/hr & 0.00114539 & $6.52136 \mathrm{E}-05$ & 0.0 & 0.0 \\
\hline Enthalpy, cal/hr & -4450.91 & -532.863 & 0.0 & 0.0 \\
\hline Density, g/L & 1154.7 & 2850.86 & & \\
\hline Vapor fraction & 0.0 & 0.0 & 0.0 & 0.0 \\
\hline Solid fraction & 0.0 & 1 . & 0.0 & 0.0 \\
\hline Organic fraction & 0.0 & 0.0 & 0.0 & 0.0 \\
\hline Osmotic Pres, atm & 187.21 & & & \\
\hline Redox Pot, volts & 0.0 & & & \\
\hline E-Con, 1/ohm-cm & 0.188655 & & & \\
\hline E-Con, cm2/ohm-mol & 30.7208 & & & \\
\hline Abs Visc, $C P$ & 1.43936 & & & \\
\hline Rel Visc & 1.61595 & & & \\
\hline Ionic Strength & 4.16529 & & & \\
\hline
\end{tabular}


WSRC-TR-2002-00567

Revision 0

\begin{tabular}{|c|c|c|c|c|}
\hline \multicolumn{5}{|l|}{ STREAM: Condensate } \\
\hline TO $\quad:$ & & & & \\
\hline FROM : Condensate & mixer & & & \\
\hline Phases-----------> & Aqueous & Solid & Vapor & Organic \\
\hline Temperature, C & 25 . & 25 . & 25 . & 25 . \\
\hline $\begin{array}{l}\text { Pressure, atm } \\
\mathrm{pH}\end{array}$ & $\begin{array}{l}1 . \\
4.45875\end{array}$ & 1. & 1. & 1. \\
\hline Total mol/hr & 55.42179 & 0.0 & 0.0 & 0.0 \\
\hline------------------ & $\mathrm{mol} / \mathrm{hr}------$ & -mol/hr------- & -mol/hr-------- & $-\operatorname{mol} / \mathrm{hr}------1$ \\
\hline $\mathrm{H} 2 \mathrm{O}$ & 55.4202 & 0.0 & 0.0 & 0.0 \\
\hline $\mathrm{CO} 2$ & 0.00148054 & 0.0 & 0.0 & 0.0 \\
\hline $\mathrm{HCL}$ & $1.62195 E-17$ & 0.0 & 0.0 & 0.0 \\
\hline HNO3 & $3.05057 \mathrm{E}-13$ & 0.0 & 0.0 & 0.0 \\
\hline LAURICACID & $4.12154 E-05$ & 0.0 & 0.0 & 0.0 \\
\hline OHION & $2.93231 \mathrm{E}-10$ & 0.0 & 0.0 & 0.0 \\
\hline CLION & $7.92885 E-07$ & 0.0 & 0.0 & 0.0 \\
\hline CO3ION & $2.68327 \mathrm{E}-11$ & 0.0 & 0.0 & 0.0 \\
\hline DODECION & $1.45300 E-05$ & 0.0 & 0.0 & 0.0 \\
\hline HCO3 ION & $1.94587 \mathrm{E}-05$ & 0.0 & 0.0 & 0.0 \\
\hline $\mathrm{HION}$ & $3.49592 \mathrm{E}-05$ & 0.0 & 0.0 & 0.0 \\
\hline NO3ION & $\begin{array}{l}\text { 1.77234E }-07 \\
============\end{array}$ & $\begin{array}{l}0.0 \\
============\end{array}$ & $\begin{array}{l}0.0 \\
=============\end{array}$ & $\begin{array}{l}0.0 \\
=============\end{array}$ \\
\hline Total g/hr & 998.492 & 0.0 & 0.0 & 0.0 \\
\hline Volume, L/hr & 1.00163 & 0.0 & 0.0 & 0.0 \\
\hline $\begin{array}{l}\text { Enthalpy, cal/hr } \\
\text { Density, g/L }\end{array}$ & $\begin{array}{l}-3.78609 E+06 \\
996.872\end{array}$ & 0.0 & 0.0 & 0.0 \\
\hline Vapor fraction & 0.0 & 0.0 & 0.0 & 0.0 \\
\hline Solid fraction & 0.0 & 0.0 & 0.0 & 0.0 \\
\hline Organic fraction & 0.0 & 0.0 & 0.0 & 0.0 \\
\hline Osmotic Pres, atm & 0.038908 & & & \\
\hline Redox Pot, volts & 0.0 & & & \\
\hline E-Con, 1/ohm-cm & $1.39848 E-05$ & & & \\
\hline E-Con, cm2/ohm-mol & 8.99814 & & & \\
\hline Abs Visc, cP & 0.890742 & & & \\
\hline Rel Visc & 1.00003 & & & \\
\hline Ionic Strength & $3.50150 E-05$ & & & \\
\hline
\end{tabular}


WSRC-TR-2002-00567

Revision 0

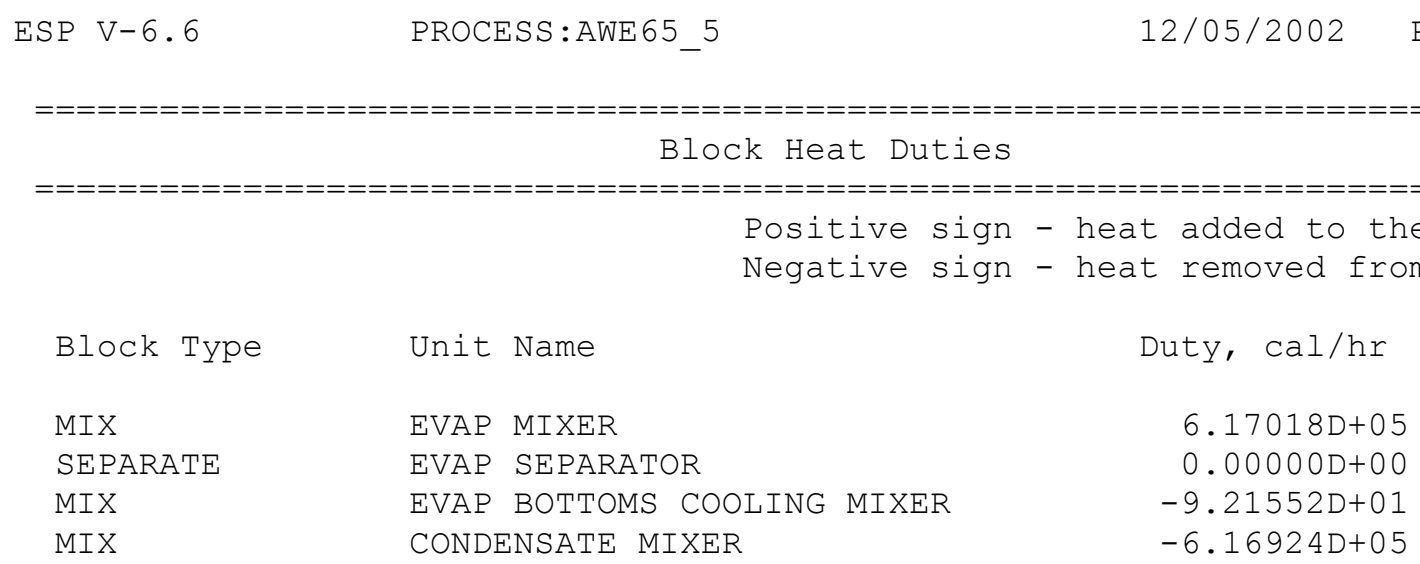


WSRC-TR-2002-00567

Revision 0

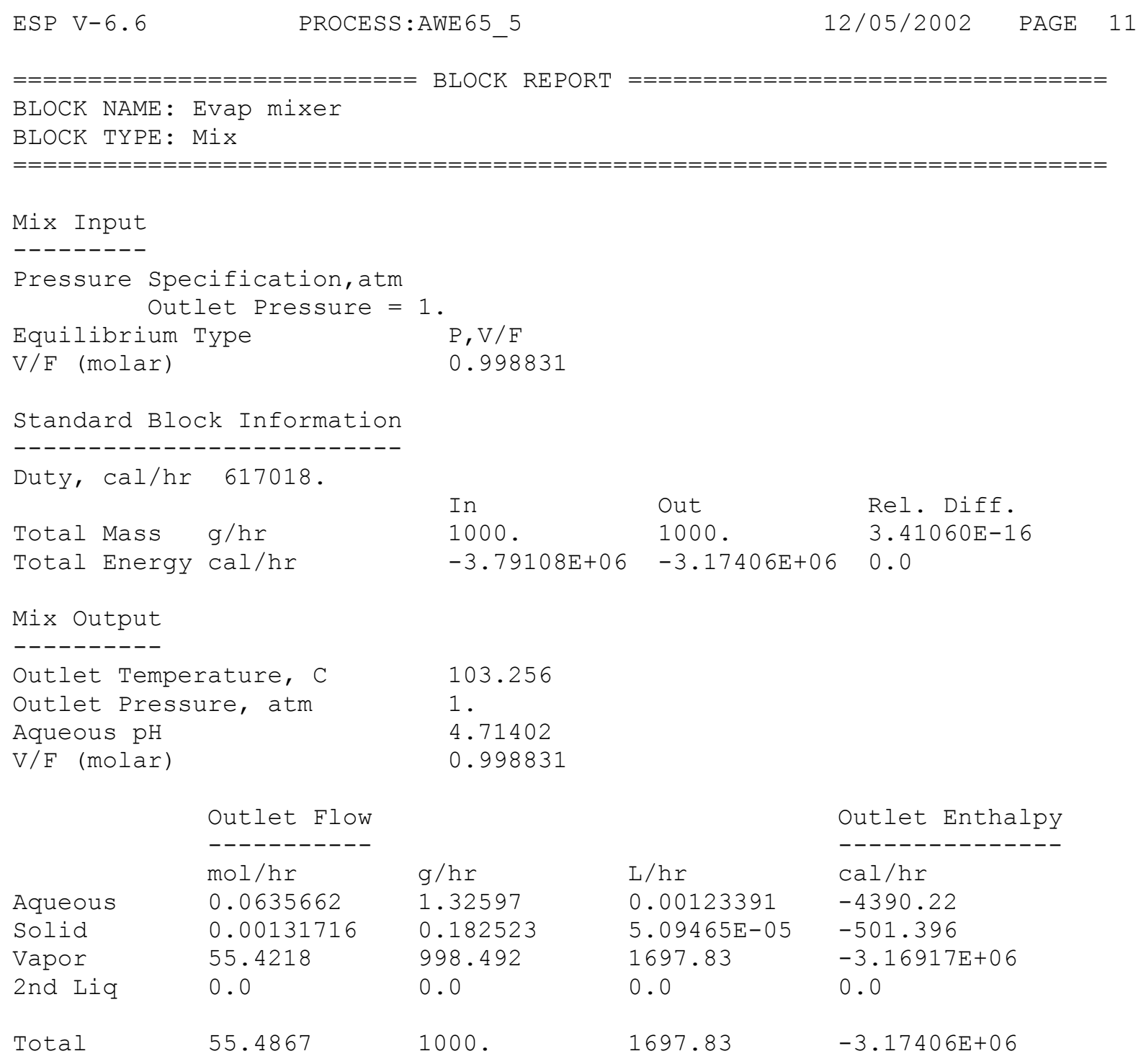


WSRC-TR-2002-00567

Revision 0

$\begin{array}{lllll}\text { ESP V-6.6 PROCESS:AWE65_5 } & 12 / 05 / 2002 & \text { PAGE } & 12\end{array}$

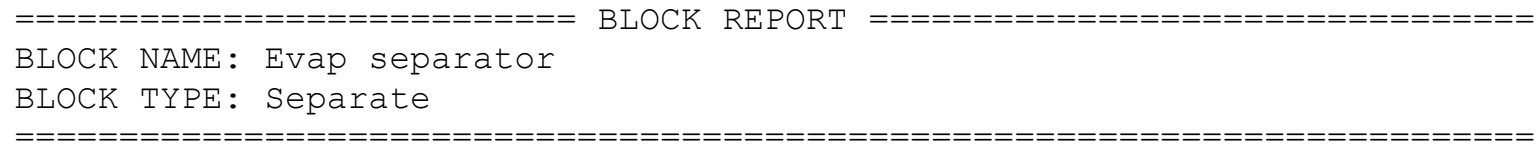

$0.0648834 \quad 1.50849$

$\mathrm{L} / \mathrm{hr} \quad \mathrm{cal} / \mathrm{hr}$

$5.09465 \mathrm{E}-05 \quad-501.396$

$0.0 \quad 0.0$

Total

$0.00128486 \quad-4891.62$

Outlet Enthalpy 
WSRC-TR-2002-00567

Revision 0

ESP V-6.6

PROCESS:AWE 65_5

Vapor Stream

\begin{tabular}{ll}
\multicolumn{2}{c}{ Overhead } \\
Outlet Flow \\
-------- \\
mol/hr & $\mathrm{g} / \mathrm{hr}$ \\
0.0 & 0.0 \\
0.0 & 0.0 \\
55.4218 & 998.492 \\
0.0 & 0.0
\end{tabular}

Total

998.492
$12 / 05 / 2002$

PAGE

13

$\mathrm{L} / \mathrm{hr}$

0.0

0.0

1697.83

0.0

1697.83

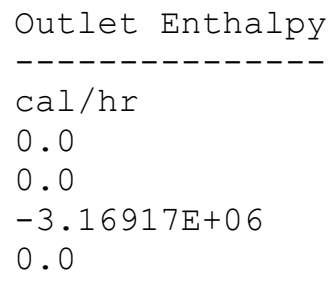

0.0

0.0

$-3.16917 E+06$

0.0

$-3.16917 E+06$ 
WSRC-TR-2002-00567

Revision 0

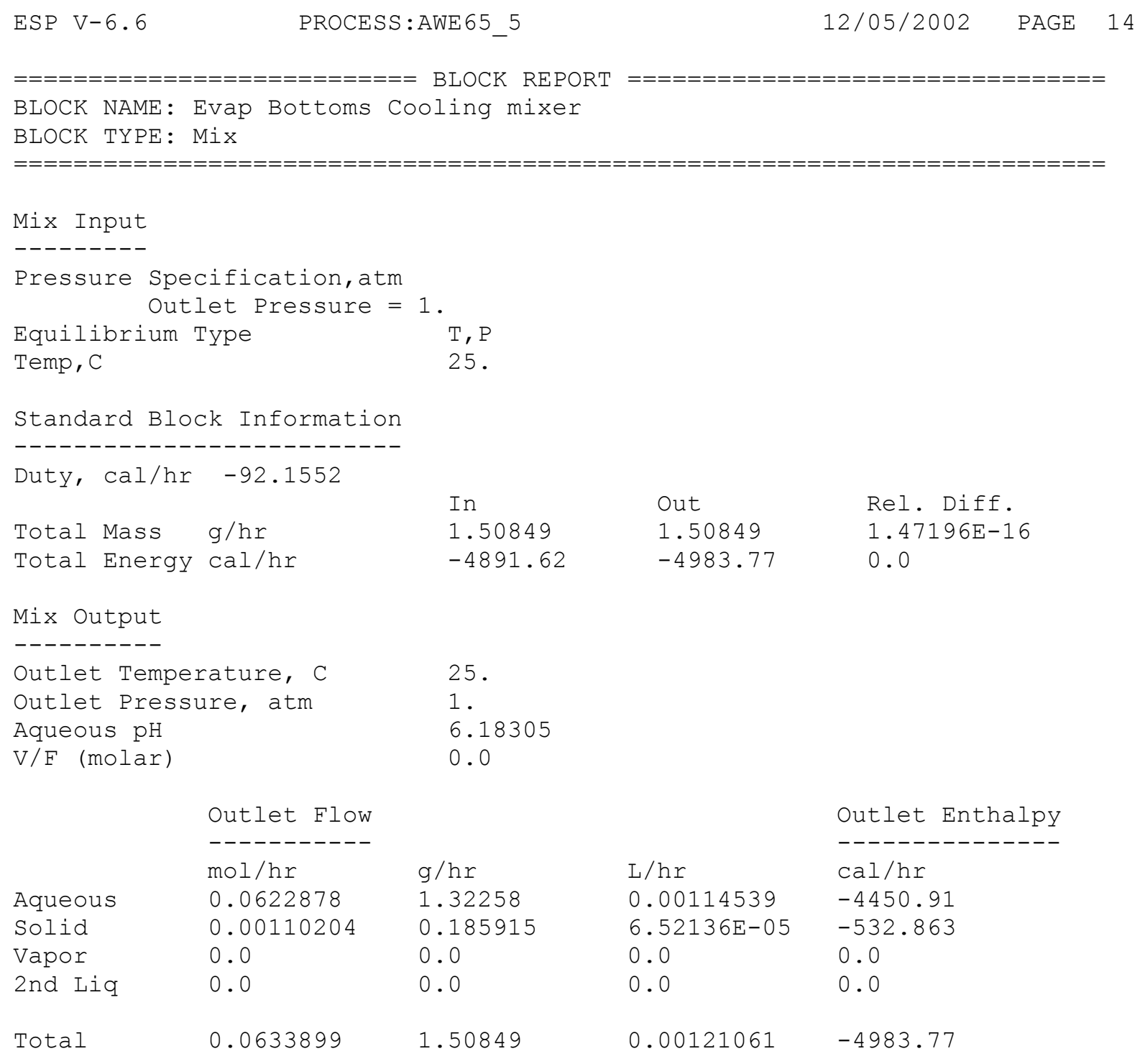


WSRC-TR-2002-00567

Revision 0

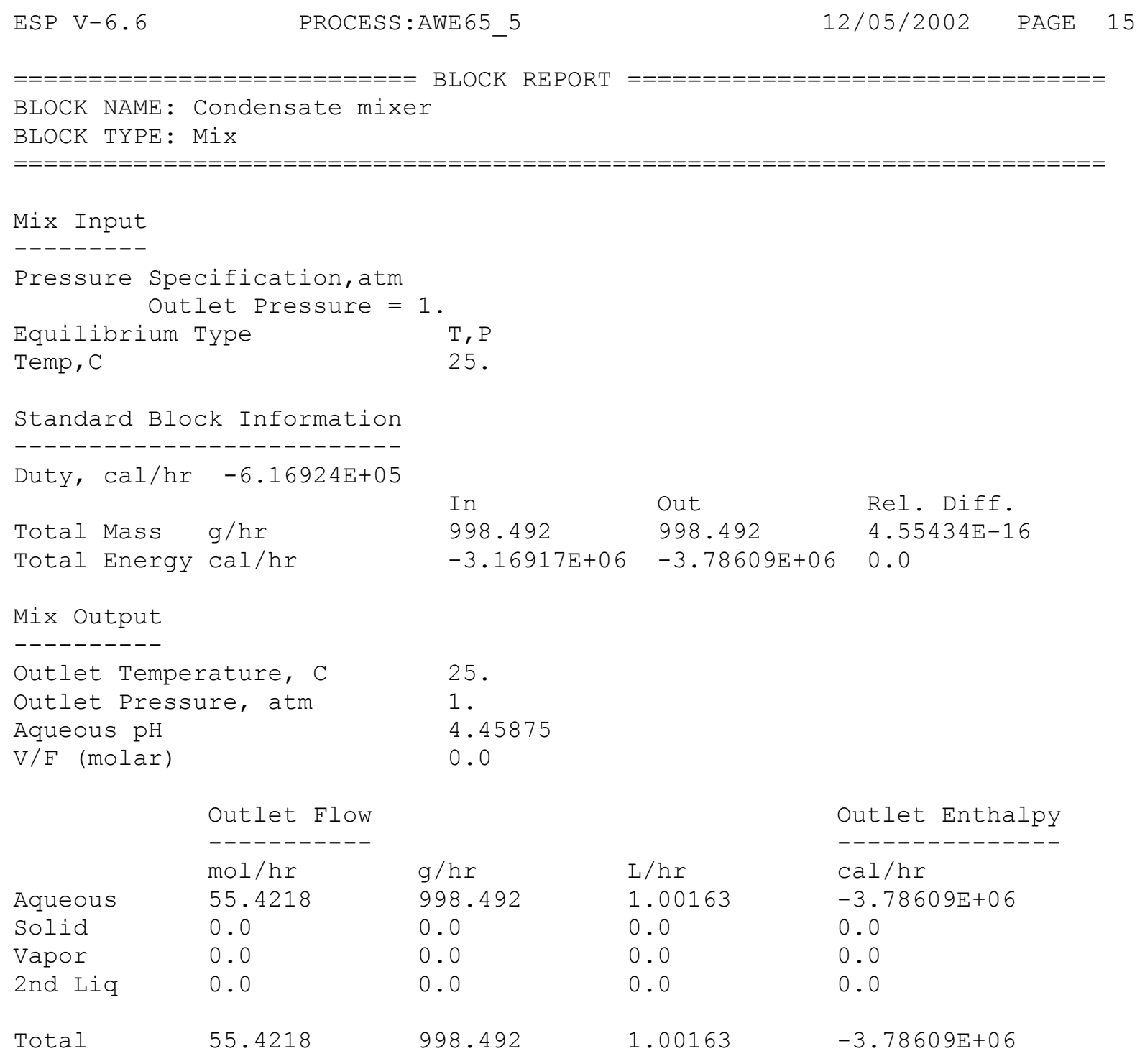


WSRC-TR-2002-00567

Revision 0

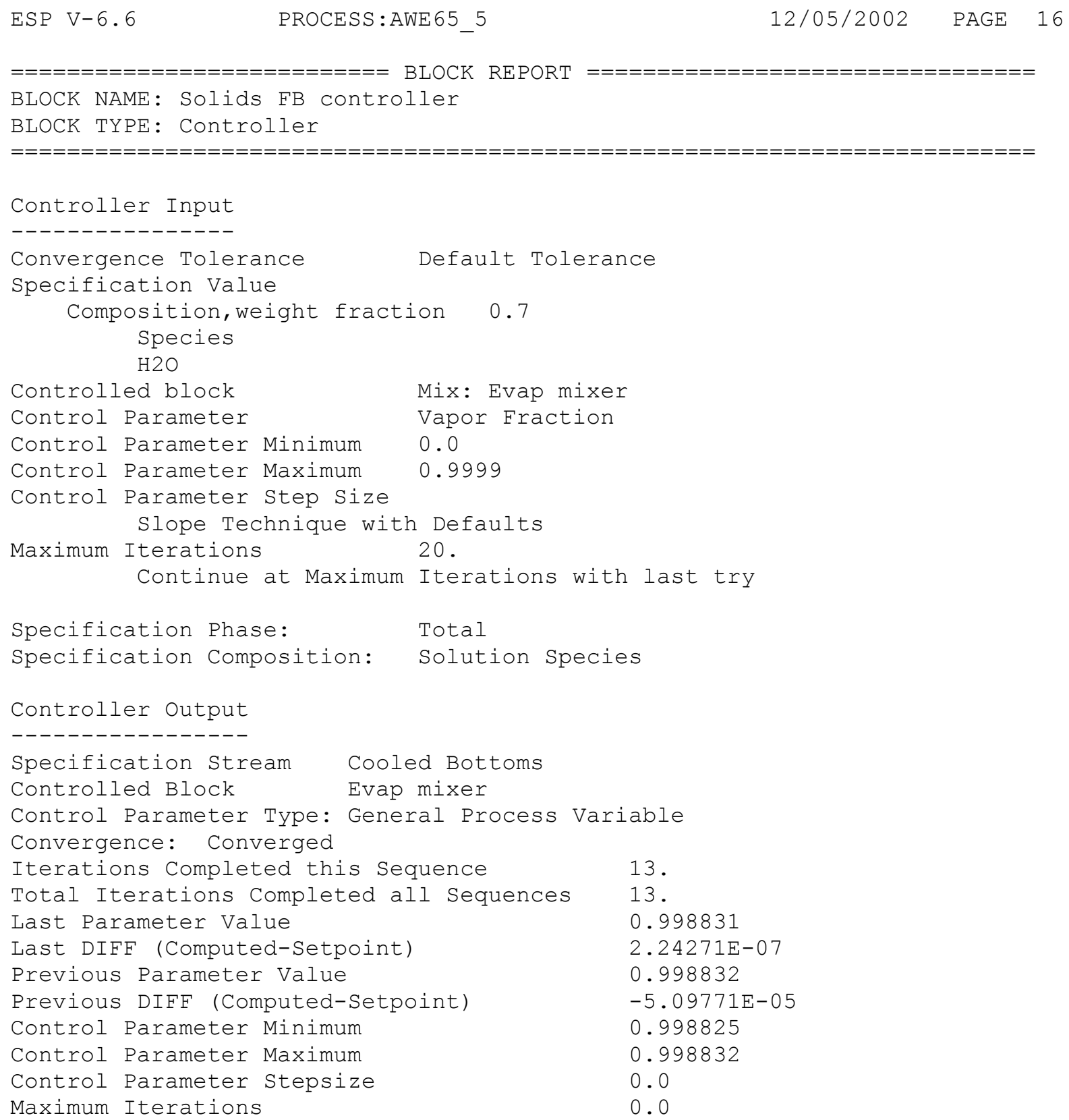


WSRC-TR-2002-00567

Revision 0

Influent Limit Composition 90\% Target $\mathrm{pH}=6.5$

6.5-90

\begin{tabular}{|c|c|c|c|c|}
\hline 0 & 0 & 0 & $\mathrm{~L}$ & $I I I I$ \\
\hline 0 & & 0 & $\mathrm{~L}$ & I \\
\hline 0 & & 0 & $\mathrm{~L}$ & I \\
\hline D & & 0 & $\mathrm{~L}$ & I \\
\hline D & & 0 & $\mathrm{~L}$ & I \\
\hline D & & 0 & $\mathrm{~L}$ & I \\
\hline 0 & & 0 & $\mathrm{~L}$ & I \\
\hline 0 & & 0 & $\mathrm{~L}$ & I \\
\hline 0 & 0 & 0 & L L L L L L L L L L & I I I \\
\hline
\end{tabular}

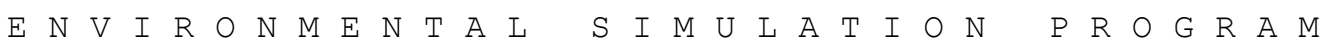

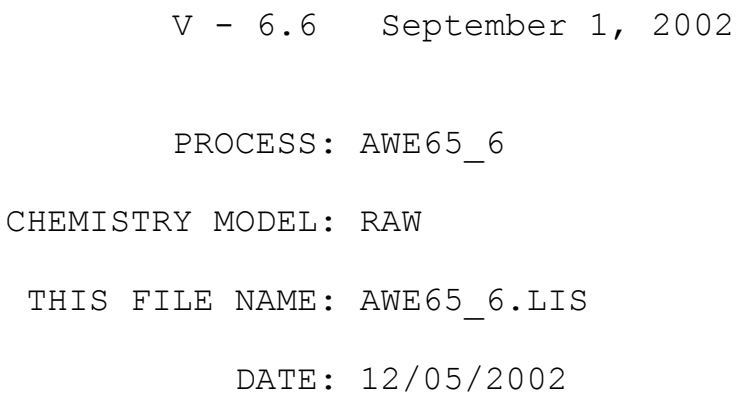


WSRC-TR-2002-00567

Revision 0

TABLE OF CONTENTS

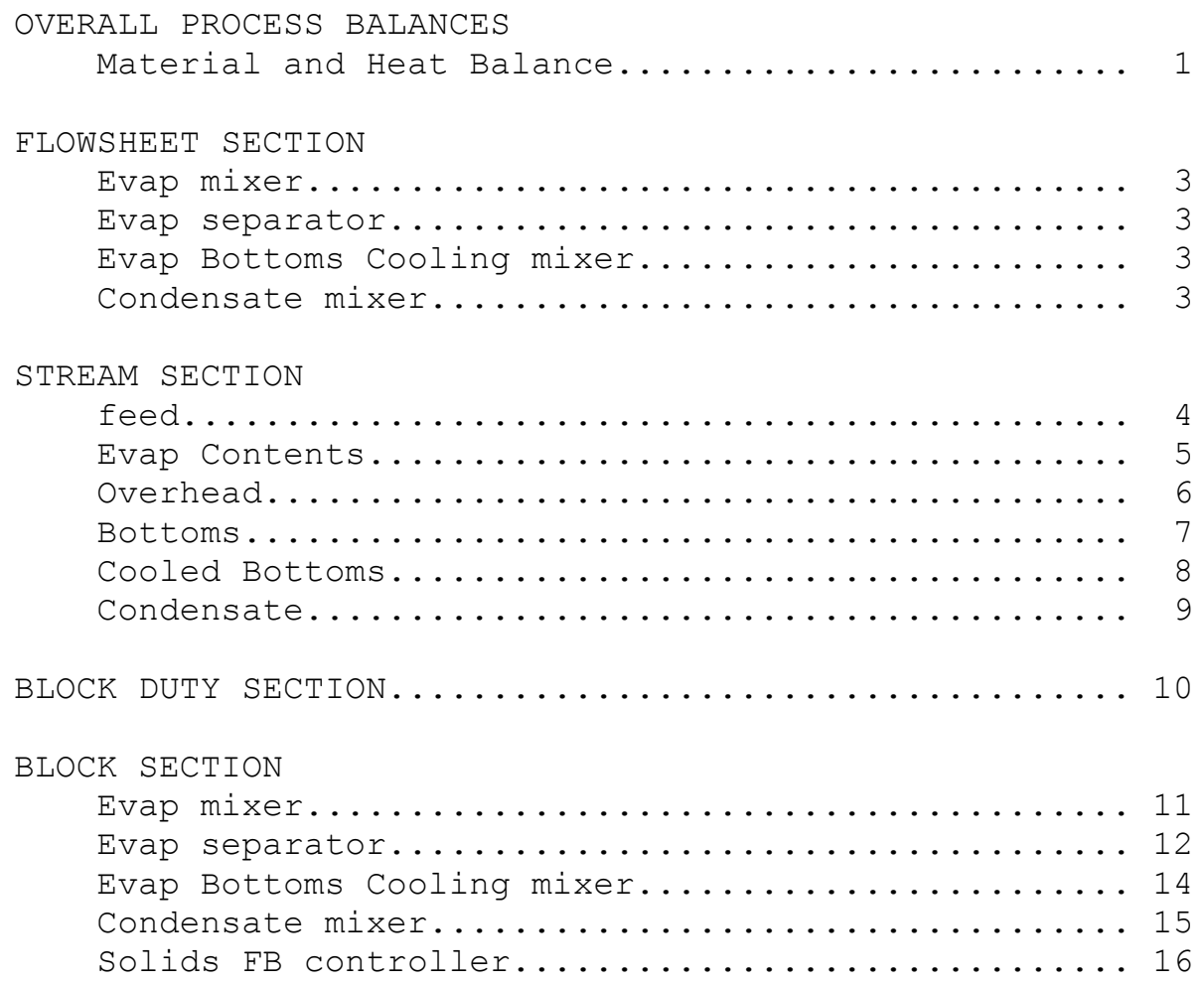


WSRC-TR-2002-00567

Revision 0

ESP V-6.6 PROCESS:AWE65_6
$\begin{array}{lcc}\text { Overall Process } & \text { Balances } \\ \text { Inlet } & \text { g/hr } & \mathrm{Cal} / \mathrm{hr} \\ ----------- & ----------- & ---------- \\ \text { FEED } & 5.00385 \mathrm{D}+02 & -1.89616 \mathrm{D}+06 \\ \text { =========== } & ========== & ======== \\ \text { Total in } & 5.00385 \mathrm{D}+02 & -1.89616 \mathrm{D}+06\end{array}$

\begin{tabular}{lrr} 
Outlet & \multicolumn{1}{c}{$g / \mathrm{hr}$} & $\mathrm{cal} / \mathrm{hr}$ \\
----------- & ---------- & ----------- \\
COOLED BOTTOMS & $2.25695 \mathrm{D}+00$ & $-7.44406 \mathrm{D}+03$ \\
CONDENSATE & $4.98128 \mathrm{D}+02$ & $-1.88871 \mathrm{D}+06$ \\
$==========$ & $==========$ & $==========$ \\
Total out & $5.00385 \mathrm{D}+02$ & $-1.89616 \mathrm{D}+06$
\end{tabular}

Block Heat Duties

EVAP MIXER

EVAP BOTTOMS COOLING MIXER

CONDENSATE MIXER

$=========$

Total Duty

DIFFERENCE

REL DIFEERENCE

$6.93490 \mathrm{D}-12$

1.38591D-14
$12 / 05 / 2002$

PAGE
1
Material Code Balances

$\begin{array}{lc}\text { Code } & \begin{array}{c}\text { Input } \\ \text { mol/hr }\end{array} \\ ---------- & --------- \\ \text { H }(+1) & 5.54695 \mathrm{D}+01 \\ \mathrm{~K}(+1) & 6.74678 \mathrm{D}-04 \\ \mathrm{NA}(+1) & 4.58790 \mathrm{D}-03 \\ \mathrm{BA}(+2) & 7.29927 \mathrm{D}-07 \\ \mathrm{CA}(+2) & 1.24688 \mathrm{D}-03 \\ \mathrm{ZN}(+2) & 5.04587 \mathrm{D}-05 \\ \mathrm{CU}(+2) & 9.44882 \mathrm{D}-06 \\ \mathrm{FE}(+2) & 1.30824 \mathrm{D}-04 \\ \mathrm{MG}(+2) & 5.34979 \mathrm{D}-04 \\ \mathrm{~PB}(+2) & 6.28019 \mathrm{D}-06 \\ \mathrm{AL}(+3) & 1.18519 \mathrm{D}-04 \\ \mathrm{NI}(+2) & 2.04429 \mathrm{D}-06 \\ \mathrm{O}(-2) & 2.77505 \mathrm{D}+01 \\ \mathrm{CL}(-1) & 3.94366 \mathrm{D}-03 \\ \mathrm{C}(+4) & 1.66667 \mathrm{D}-03 \\ \mathrm{P}(+5) & 8.42105 \mathrm{D}-04 \\ \text { S }(+6) & 1.25000 \mathrm{D}-03 \\ \mathrm{~N}(+5) & 1.27419 \mathrm{D}-03 \\ \mathrm{SI}(+4) & 2.50000 \mathrm{D}-04 \\ \text { SR }(+2) & 2.96804 \mathrm{D}-05 \\ & \end{array}$

$$
\begin{gathered}
\text { cal/hr } \\
----------- \\
3.07915 \mathrm{D}+05 \\
-1.36716 \mathrm{D}+02 \\
-3.07779 \mathrm{D}+05 \\
========== \\
-2.59294 \mathrm{D}-01
\end{gathered}
$$

1. $74623 \mathrm{D}-10$ $-9.20932 \mathrm{D}-17$ 
WSRC-TR-2002-00567

Revision 0

$\mathrm{CD}(+2)$
$\mathrm{CR}(+3)$
$\mathrm{U}(+4)$
5.89286D-08

$1.84615 \mathrm{D}-07$

7.98319D-06

$5.89286 \mathrm{D}-08$

$1.84615 \mathrm{D}-07$

7.98319D-06

$5.16161 \mathrm{D}-22$

$4.15576 \mathrm{D}-21$

$0.00000 D+00$

8. 75909D-15

$2.25103 \mathrm{D}-14$

$0.00000 \mathrm{D}+00$ 
WSRC-TR-2002-00567

Revision 0

ESP V-6.6

$\operatorname{DODEC}(-1)$
PROCESS:AWE 656

$7.96598 \mathrm{D}-05$
$12 / 05 / 2002$

$7.96598 \mathrm{D}-05$

$2.53153 \mathrm{D}-13$ 
WSRC-TR-2002-00567

Revision 0

\begin{tabular}{|c|c|c|c|}
\hline \multicolumn{4}{|l|}{$\begin{array}{l}\text { PROCESS BLOCKS } \\
===========\end{array}$} \\
\hline $\begin{array}{l}\mathrm{BLOCK} \mathrm{NAME} \\
=========================\end{array}$ & $\begin{array}{l}\text { BLOCK TYPE } \\
===========\end{array}$ & $\begin{array}{l}\text { INLET STREAM }(\mathrm{S}) \\
==============\end{array}$ & $\begin{array}{l}\text { OUTLET STREAM }(\mathrm{S}) \\
==============\end{array}$ \\
\hline Evap mixer & $\operatorname{Mix}$ & feed & Evap Contents \\
\hline Evap separator & Separate & Evap Contents & $\begin{array}{l}\text { Overhead } \\
\text { Bottoms }\end{array}$ \\
\hline Evap Bottoms Cooling mixer & Mix & Bottoms & Cooled Bottoms \\
\hline Condensate mixer & Mix & Overhead & Condensate \\
\hline
\end{tabular}


WSRC-TR-2002-00567

Revision 0

ESP V-6.6
PROCESS:AWE 656
12/05/2002 PAGE 4

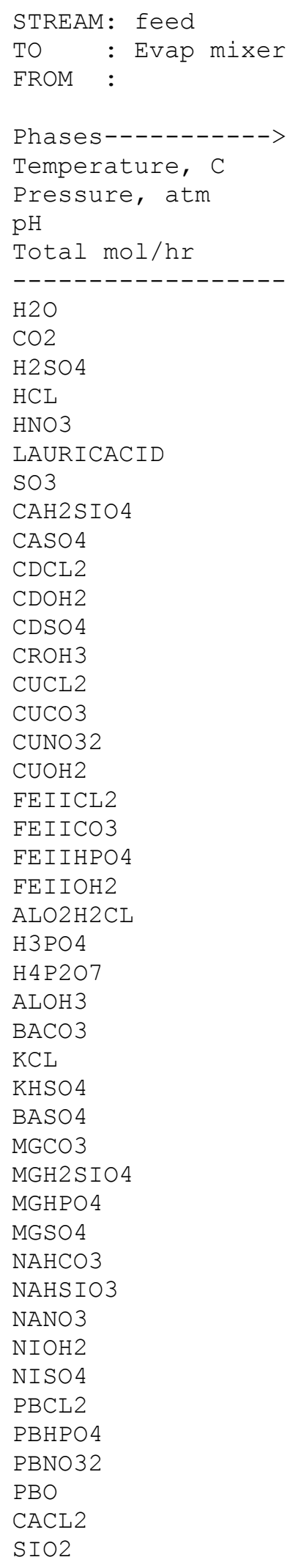

\begin{tabular}{|c|c|c|c|}
\hline Aqueous & Solid & Vapor & Organic \\
\hline 25 . & 25 . & 25 . & 25 . \\
\hline 1. & 1. & 1 . & 1 . \\
\hline \multicolumn{4}{|l|}{5.94788} \\
\hline 27.74985 & $1.32366 \mathrm{E}-04$ & 0.0 & 0.0 \\
\hline \multicolumn{4}{|c|}{$\mathrm{mol} / \mathrm{hr}--------\operatorname{mol} / \mathrm{hr}--------\operatorname{mol} / \mathrm{hr}--------\operatorname{mol} / \mathrm{hr}--------$} \\
\hline 27.7335 & 0.0 & 0.0 & 0.0 \\
\hline 0.00112051 & 0.0 & 0.0 & 0.0 \\
\hline $2.88170 \mathrm{E}-24$ & 0.0 & 0.0 & 0.0 \\
\hline $2.26781 E-15$ & 0.0 & 0.0 & 0.0 \\
\hline $6.10091 \mathrm{E}-11$ & 0.0 & 0.0 & 0.0 \\
\hline $5.82007 E-06$ & 0.0 & 0.0 & 0.0 \\
\hline $3.73181 \mathrm{E}-28$ & 0.0 & 0.0 & 0.0 \\
\hline $1.59262 \mathrm{E}-13$ & 0.0 & 0.0 & 0.0 \\
\hline $2.96963 E-05$ & 0.0 & 0.0 & 0.0 \\
\hline 1.69235E-09 & 0.0 & 0.0 & 0.0 \\
\hline $9.94750 \mathrm{E}-17$ & 0.0 & 0.0 & 0.0 \\
\hline $8.52119 E-09$ & 0.0 & 0.0 & 0.0 \\
\hline $5.48566 \mathrm{E}-15$ & 0.0 & 0.0 & 0.0 \\
\hline $1.65340 \mathrm{E}-12$ & 0.0 & 0.0 & 0.0 \\
\hline $3.03056 \mathrm{E}-09$ & 0.0 & 0.0 & 0.0 \\
\hline $3.60171 \mathrm{E}-14$ & 0.0 & 0.0 & 0.0 \\
\hline $9.71640 \mathrm{E}-11$ & 0.0 & 0.0 & 0.0 \\
\hline $1.65928 \mathrm{E}-14$ & 0.0 & 0.0 & 0.0 \\
\hline $3.50025 \mathrm{E}-07$ & 0.0 & 0.0 & 0.0 \\
\hline $1.20637 \mathrm{E}-07$ & 0.0 & 0.0 & 0.0 \\
\hline $1.40463 E-13$ & 0.0 & 0.0 & 0.0 \\
\hline $4.38913 E-28$ & 0.0 & 0.0 & 0.0 \\
\hline $9.46126 \mathrm{E}-08$ & 0.0 & 0.0 & 0.0 \\
\hline $3.18991 \mathrm{E}-17$ & 0.0 & 0.0 & 0.0 \\
\hline $1.19739 \mathrm{E}-09$ & $1.18516 \mathrm{E}-04$ & 0.0 & 0.0 \\
\hline $7.25548 \mathrm{E}-13$ & 0.0 & 0.0 & 0.0 \\
\hline $3.22694 \mathrm{E}-08$ & 0.0 & 0.0 & 0.0 \\
\hline 1. $44853 \mathrm{E}-12$ & 0.0 & 0.0 & 0.0 \\
\hline 1.02621E-10 & $6.39811 \mathrm{E}-07$ & 0.0 & 0.0 \\
\hline $9.23119 \mathrm{E}-09$ & 0.0 & 0.0 & 0.0 \\
\hline $6.19663 \mathrm{E}-13$ & 0.0 & 0.0 & 0.0 \\
\hline $2.02484 E-05$ & 0.0 & 0.0 & 0.0 \\
\hline $2.08344 \mathrm{E}-05$ & 0.0 & 0.0 & 0.0 \\
\hline $1.47379 E-06$ & 0.0 & 0.0 & 0.0 \\
\hline $3.01337 \mathrm{E}-08$ & 0.0 & 0.0 & 0.0 \\
\hline $4.89201 E-07$ & 0.0 & 0.0 & 0.0 \\
\hline $7.78821 \mathrm{E}-14$ & 0.0 & 0.0 & 0.0 \\
\hline $2.46011 E-07$ & 0.0 & 0.0 & 0.0 \\
\hline $3.35442 E-11$ & 0.0 & 0.0 & 0.0 \\
\hline $7.88699 \mathrm{E}-10$ & 0.0 & 0.0 & 0.0 \\
\hline $6.67029 E-13$ & 0.0 & 0.0 & 0.0 \\
\hline $5.79799 \mathrm{E}-14$ & 0.0 & 0.0 & 0.0 \\
\hline $2.75905 E-26$ & 0.0 & 0.0 & 0.0 \\
\hline $2.49931 E-04$ & 0.0 & 0.0 & 0.0 \\
\hline
\end{tabular}




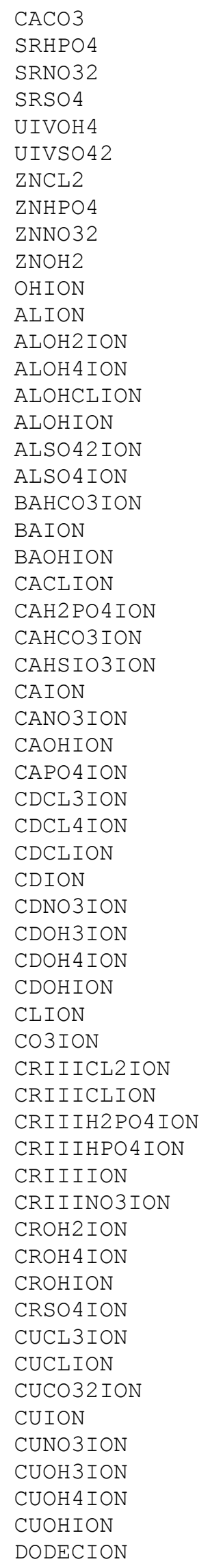

\begin{tabular}{|c|c|}
\hline $5.08222 \mathrm{E}-08$ & 0.0 \\
\hline $1.71395 E-08$ & 0.0 \\
\hline $.62488 E-10$ & 0 \\
\hline $.46941 E-06$ & 0.0 \\
\hline $.50544 \mathrm{E}-10$ & 0.0 \\
\hline $38 E-25$ & 0.0 \\
\hline $99687 E-09$ & 0.0 \\
\hline $00326 E-06$ & 0.0 \\
\hline $65347 E-11$ & 0.0 \\
\hline $51 E-11$ & 0.0 \\
\hline $95 E-09$ & 0.0 \\
\hline $10 E-11$ & 0.0 \\
\hline $88648 E-10$ & 0.0 \\
\hline $11384 \mathrm{E}-10$ & 0.0 \\
\hline $59181 \mathrm{E}-12$ & 0.0 \\
\hline $10988 \mathrm{E}-10$ & 0.0 \\
\hline $79762 E-13$ & 0.0 \\
\hline $.01253 E-11$ & 0.0 \\
\hline $82590 E-10$ & 0.0 \\
\hline $.95259 E-08$ & 0.0 \\
\hline $62622 E-16$ & 0.0 \\
\hline $.18361 \mathrm{E}-10$ & 0.0 \\
\hline $28421 E-05$ & 0.0 \\
\hline $7.51178 E-06$ & 0.0 \\
\hline $55 E-10$ & 0.0 \\
\hline 0.00117242 & 0.0 \\
\hline $4.27883 E-06$ & 0.0 \\
\hline $704 E-10$ & 0.0 \\
\hline $193 E-08$ & 0.0 \\
\hline $86 E-13$ & 0.0 \\
\hline $4 E-15$ & 0.0 \\
\hline $21 E-08$ & 0.0 \\
\hline $6 E-08$ & 0.0 \\
\hline $2 E-10$ & 0.0 \\
\hline $6 E-23$ & 0.0 \\
\hline 0.0 & 0.0 \\
\hline $8 E-12$ & 0.0 \\
\hline 0.00 & 0.0 \\
\hline $9 E-08$ & 0.0 \\
\hline $4 E-19$ & 0.0 \\
\hline $3 E-16$ & 0.0 \\
\hline $3 E-13$ & 0.0 \\
\hline $2 E-07$ & 0.0 \\
\hline $9 E-14$ & 0.0 \\
\hline$D E-15$ & 0.0 \\
\hline $67 E-15$ & 0.0 \\
\hline $3 E-20$ & 0.0 \\
\hline $0 E-12$ & 0.0 \\
\hline $8 E-11$ & 0.0 \\
\hline $9 E-17$ & 0.0 \\
\hline $9 E-10$ & 0.0 \\
\hline $0 E-13$ & 0.0 \\
\hline $66 E-08$ & 0.0 \\
\hline $5 E-10$ & 0.0 \\
\hline $7 E-17$ & 0.0 \\
\hline $2 E-24$ & 0.0 \\
\hline $78 E-10$ & 0.0 \\
\hline $8 E-05$ & 0.0 \\
\hline
\end{tabular}

0.0

0.0

0.0

0.0

0.0

0.0

0.0

0.0

0.0

0.0

0.0

0.0

0.0

0.0

0.0

0.0

0.0

0.0

0.0

0.0

0.0

0.0

0.0

0.0

0.0

0.0

0.0

0.0

0.0

0.0

0.0

0.0

0.0

0.0

0.0

0.0

0.0

0.0

0.0

0.0

0.0

0.0

0.0

0.0

0.0

0.0

0.0

0.0

0.0

0.0

0.0

0.0

0.0

0.0

0.0

0.0

0.0

0.0
0.0

0.0

0.0

0.0

0.0

0.0

0.0

0.0

0.0

0.0

0.0

0.0

0.0

0.0

0.0

0.0

0.0

0.0

0.0

0.0

0.0

0.0

0.0

0.0

0.0

0.0

0.0

0.0

0.0

0.0

0.0

0.0

0.0

0.0

0.0

0.0

0.0

0.0

0.0

0.0

0.0

0.0

0.0

0.0

0.0

0.0

0.0

0.0

0.0

0.0

0.0

0.0

0.0

0.0

0.0

0.0

0.0

0.0 


\begin{tabular}{|c|c|c|c|c|}
\hline FEIICLION & $7.73012 \mathrm{E}-10$ & 0.0 & 0.0 & 0.0 \\
\hline FEIICO32ION & $8.07377 \mathrm{E}-13$ & 0.0 & 0.0 & 0.0 \\
\hline FEIIH2PO4ION & $3.23024 \mathrm{E}-07$ & 0.0 & 0.0 & 0.0 \\
\hline FEIIHCO3ION & $1.09678 \mathrm{E}-08$ & 0.0 & 0.0 & 0.0 \\
\hline FEIIION & $1.29996 \mathrm{E}-04$ & 0.0 & 0.0 & 0.0 \\
\hline FEIIOH3ION & $5.74206 \mathrm{E}-18$ & 0.0 & 0.0 & 0.0 \\
\hline FEIIOH 4 ION & $8.39315 \mathrm{E}-27$ & 0.0 & 0.0 & 0.0 \\
\hline FEIIOHION & $2.31601 \mathrm{E}-08$ & 0.0 & 0.0 & 0.0 \\
\hline H2 P2O 7 ION & $6.01752 \mathrm{E}-09$ & 0.0 & 0.0 & 0.0 \\
\hline H2 PO 4 ION & $6.94911 \mathrm{E}-04$ & 0.0 & 0.0 & 0.0 \\
\hline H2SIO4ION & $4.14344 \mathrm{E}-15$ & 0.0 & 0.0 & 0.0 \\
\hline H3P2O 7 ION & $9.38202 \mathrm{E}-13$ & 0.0 & 0.0 & 0.0 \\
\hline H3SIO4 ION & $3.70066 \mathrm{E}-08$ & 0.0 & 0.0 & 0.0 \\
\hline HCO3ION & $5.25881 \mathrm{E}-04$ & 0.0 & 0.0 & 0.0 \\
\hline HION & $6.52978 \mathrm{E}-07$ & 0.0 & 0.0 & 0.0 \\
\hline HP207ION & $2.87004 \mathrm{E}-09$ & 0.0 & 0.0 & 0.0 \\
\hline HPBO2ION & $6.73217 \mathrm{E}-19$ & 0.0 & 0.0 & 0.0 \\
\hline HPO4 ION & $6.14529 \mathrm{E}-05$ & 0.0 & 0.0 & 0.0 \\
\hline HSO 4 ION & $7.63920 \mathrm{E}-08$ & 0.0 & 0.0 & 0.0 \\
\hline KION & $6.68417 \mathrm{E}-04$ & 0.0 & 0.0 & 0.0 \\
\hline KSO4 ION & $6.22869 \mathrm{E}-06$ & 0.0 & 0.0 & 0.0 \\
\hline MGH2 PO 4 ION & $1.69891 \mathrm{E}-05$ & 0.0 & 0.0 & 0.0 \\
\hline MGHCO3ION & $1.02651 \mathrm{E}-05$ & 0.0 & 0.0 & 0.0 \\
\hline MGHSIO3ION & $6.44628 \mathrm{E}-10$ & 0.0 & 0.0 & 0.0 \\
\hline MGION & $4.66581 \mathrm{E}-04$ & 0.0 & 0.0 & 0.0 \\
\hline MGOHION & $4.38733 E-10$ & 0.0 & 0.0 & 0.0 \\
\hline MGP2O 7 ION & $6.18522 \mathrm{E}-09$ & 0.0 & 0.0 & 0.0 \\
\hline MGPO 4 ION & $4.52793 E-08$ & 0.0 & 0.0 & 0.0 \\
\hline NACO3ION & $6.05804 \mathrm{E}-10$ & 0.0 & 0.0 & 0.0 \\
\hline NAION & 0.00453932 & 0.0 & 0.0 & 0.0 \\
\hline NASO 4 ION & $4.65888 \mathrm{E}-05$ & 0.0 & 0.0 & 0.0 \\
\hline NICLION & $7.70459 \mathrm{E}-10$ & 0.0 & 0.0 & 0.0 \\
\hline NIION & $1.79023 \mathrm{E}-06$ & 0.0 & 0.0 & 0.0 \\
\hline NINO3ION & $7.14450 \mathrm{E}-09$ & 0.0 & 0.0 & 0.0 \\
\hline NIOH3ION & $8.28794 \mathrm{E}-19$ & 0.0 & 0.0 & 0.0 \\
\hline NIOHION & $1.39987 \mathrm{E}-10$ & 0.0 & 0.0 & 0.0 \\
\hline NO3ION & 0.00126903 & 0.0 & 0.0 & 0.0 \\
\hline P2O7ION & $3.77584 \mathrm{E}-12$ & 0.0 & 0.0 & 0.0 \\
\hline PBCL3ION & $1.30433 E-13$ & 0.0 & 0.0 & 0.0 \\
\hline PBCL 4 ION & $8.89736 \mathrm{E}-16$ & 0.0 & 0.0 & 0.0 \\
\hline PBCLION & $1.58954 \mathrm{E}-09$ & 0.0 & 0.0 & 0.0 \\
\hline PBH2 PO 4 ION & $4.20872 \mathrm{E}-10$ & 0.0 & 0.0 & 0.0 \\
\hline PBION & $1.35457 \mathrm{E}-08$ & 0.0 & 0.0 & 0.0 \\
\hline PBNO33ION & $2.91475 \mathrm{E}-16$ & 0.0 & 0.0 & 0.0 \\
\hline PBNO3ION & $3.68481 \mathrm{E}-10$ & 0.0 & 0.0 & 0.0 \\
\hline PBOHION & $1.92494 \mathrm{E}-10$ & 0.0 & 0.0 & 0.0 \\
\hline PO4ION & $5.61029 \mathrm{E}-11$ & 0.0 & 0.0 & 0.0 \\
\hline SO4ION & 0.00114221 & 0.0 & 0.0 & 0.0 \\
\hline SRION & $2.59888 \mathrm{E}-05$ & 0.0 & 0.0 & 0.0 \\
\hline SRNO3ION & $2.04485 \mathrm{E}-07$ & 0.0 & 0.0 & 0.0 \\
\hline SROHION & $9.51067 \mathrm{E}-13$ & 0.0 & 0.0 & 0.0 \\
\hline SRPO 4 ION & $2.44165 \mathrm{E}-11$ & 0.0 & 0.0 & 0.0 \\
\hline UIVCLION & $1.45767 \mathrm{E}-29$ & 0.0 & 0.0 & 0.0 \\
\hline UIVION & $1.19430 \mathrm{E}-28$ & 0.0 & 0.0 & 0.0 \\
\hline UIVOH2ION & $4.26020 \mathrm{E}-20$ & 0.0 & 0.0 & 0.0 \\
\hline UIVOH3ION & $2.23102 \mathrm{E}-15$ & 0.0 & 0.0 & 0.0 \\
\hline UIVOH5ION & $8.15932 \mathrm{E}-15$ & 0.0 & 0.0 & 0.0 \\
\hline UIVOHION & $1.54259 \mathrm{E}-23$ & 0.0 & 0.0 & 0.0 \\
\hline
\end{tabular}


WSRC-TR-2002-00567

Revision 0

\begin{tabular}{|c|c|c|c|c|}
\hline UIVSO4ION & $6.09081 E-26$ & 0.0 & 0.0 & 0.0 \\
\hline ZNCL3 ION & $5.39568 \mathrm{E}-12$ & 0.0 & 0.0 & 0.0 \\
\hline ZNCLION & $3.06549 \mathrm{E}-07$ & 0.0 & 0.0 & 0.0 \\
\hline ZNH2PO4 ION & $1.30915 E-06$ & 0.0 & 0.0 & 0.0 \\
\hline ZNHCO3ION & $5.62600 \mathrm{E}-07$ & 0.0 & 0.0 & 0.0 \\
\hline ZNION & $4.50650 \mathrm{E}-05$ & 0.0 & 0.0 & 0.0 \\
\hline ZNNO3ION & $1.79237 \mathrm{E}-07$ & 0.0 & 0.0 & 0.0 \\
\hline ZNOH3 ION & $9.69977 \mathrm{E}-16$ & 0.0 & 0.0 & 0.0 \\
\hline ZNOH 4 ION & $1.77385 \mathrm{E}-22$ & 0.0 & 0.0 & 0.0 \\
\hline ZNOHION & $3.07271 E-08$ & 0.0 & 0.0 & 0.0 \\
\hline $\mathrm{CU} 3 \mathrm{PO} 42.2 \mathrm{H} 2 \mathrm{O}$ & 0.0 & $3.13913 E-06$ & 0.0 & 0.0 \\
\hline $\mathrm{PB} 3 \mathrm{PO} 42$ & 0.0 & $2.08774 \mathrm{E}-06$ & 0.0 & 0.0 \\
\hline \multirow[t]{2}{*}{ UIVO2 } & 0.0 & $7.98299 \mathrm{E}-06$ & 0.0 & 0.0 \\
\hline & $===========$ & $===========$ & $===========$ & $===========$ \\
\hline Total g/hr & 500.37 & 0.0145518 & 0.0 & 0.0 \\
\hline Volume, L/hr & 0.501393 & $4.25643 E-06$ & 0.0 & 0.0 \\
\hline Enthalpy, cal/hr & $-1.89612 \mathrm{E}+06$ & -39.7604 & 0.0 & 0.0 \\
\hline Density, g/L & 997.96 & 3418.77 & & \\
\hline Vapor fraction & 0.0 & 0.0 & 0.0 & 0.0 \\
\hline Solid fraction & 0.0 & 1 . & 0.0 & 0.0 \\
\hline Organic fraction & 0.0 & 0.0 & 0.0 & 0.0 \\
\hline Osmotic Pres, atm & 0.826227 & & & \\
\hline Redox Pot, volts & 0.0 & & & \\
\hline E-Con, $1 / o h m-c m$ & 0.00189918 & & & \\
\hline E-Con, cm2/ohm-mol & 114.997 & & & \\
\hline Abs Visc, cP & 0.894789 & & & \\
\hline Rel Visc & 1.00457 & & & \\
\hline Ionic Strength & 0.0240444 & & & \\
\hline
\end{tabular}


WSRC-TR-2002-00567

Revision 0

ESP V-6. 6

PROCESS :AWE 65 _6
12/05/2002 PAGE 5

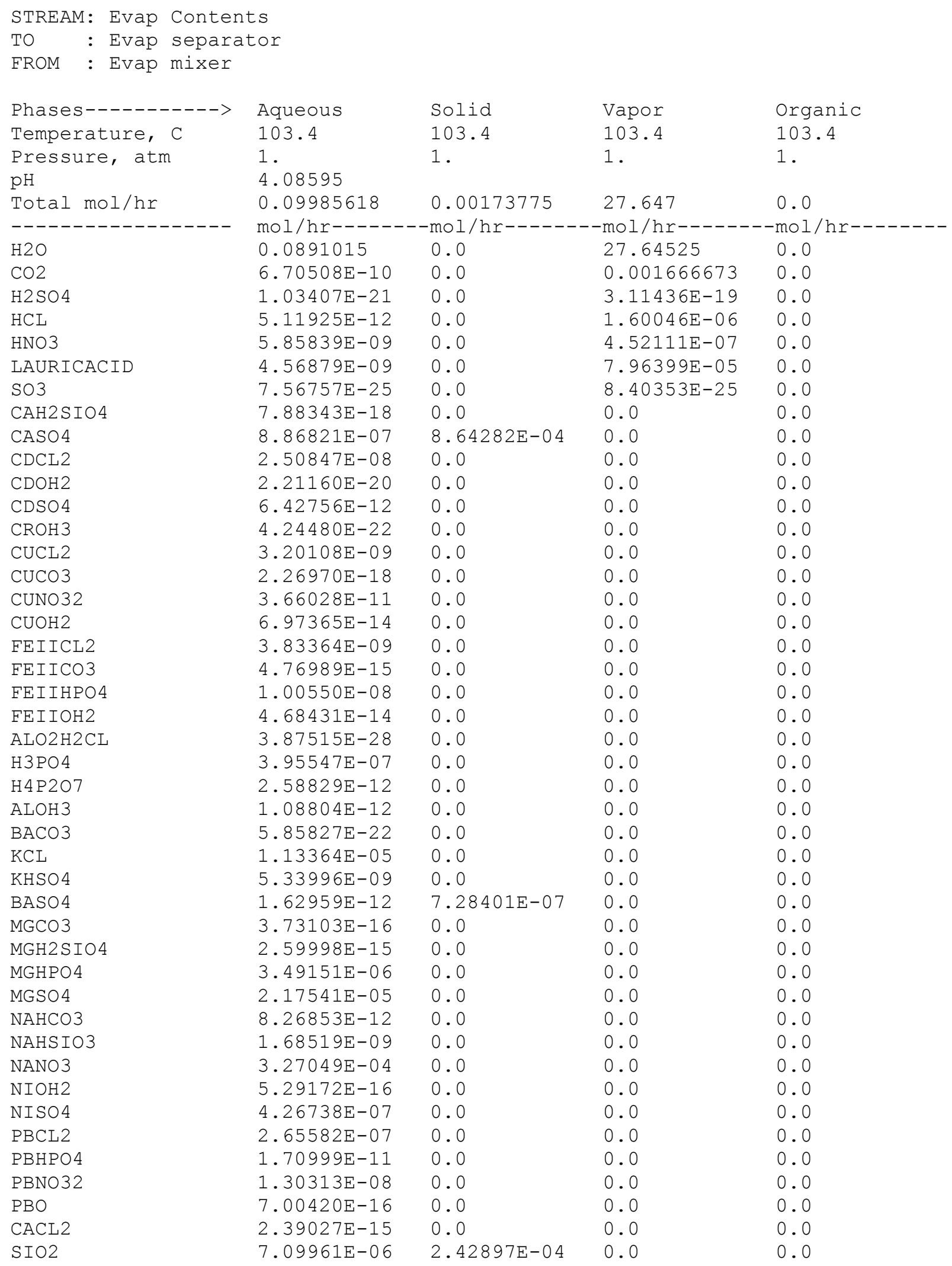




\begin{tabular}{|c|c|c|c|c|}
\hline $\mathrm{CACO} 3$ & $3.41121 E-17$ & 0.0 & 0.0 & 0.0 \\
\hline SRHPO 4 & $1.81800 \mathrm{E}-11$ & 0.0 & 0.0 & 0.0 \\
\hline SRNO32 & $9.21197 \mathrm{E}-08$ & 0.0 & 0.0 & 0.0 \\
\hline $\mathrm{SRSO} 4$ & $1.52740 \mathrm{E}-08$ & $2.93034 \mathrm{E}-05$ & 0.0 & 0.0 \\
\hline UIVOH 4 & $1.88149 \mathrm{E}-12$ & 0.0 & 0.0 & 0.0 \\
\hline UIVSO 42 & $2.13687 \mathrm{E}-20$ & 0.0 & 0.0 & 0.0 \\
\hline ZNCL2 & $1.03844 \mathrm{E}-05$ & 0.0 & 0.0 & 0.0 \\
\hline ZNHPO 4 & $2.07261 E-09$ & 0.0 & 0.0 & 0.0 \\
\hline ZNNO32 & $7.22648 \mathrm{E}-09$ & 0.0 & 0.0 & 0.0 \\
\hline ZNOH2 & $3.19463 \mathrm{E}-14$ & 0.0 & 0.0 & 0.0 \\
\hline OHION & $1.72144 \mathrm{E}-11$ & 0.0 & 0.0 & 0.0 \\
\hline ALION & $2.69032 \mathrm{E}-13$ & 0.0 & 0.0 & 0.0 \\
\hline ALOH2ION & $1.26850 \mathrm{E}-12$ & 0.0 & 0.0 & 0.0 \\
\hline ALOH 4 ION & $1.39245 \mathrm{E}-13$ & 0.0 & 0.0 & 0.0 \\
\hline ALOHCLION & $4.95935 \mathrm{E}-12$ & 0.0 & 0.0 & 0.0 \\
\hline ALOHION & $1.17526 \mathrm{E}-12$ & 0.0 & 0.0 & 0.0 \\
\hline ALSO 42 ION & $9.49590 \mathrm{E}-13$ & 0.0 & 0.0 & 0.0 \\
\hline ALSO 4 ION & $1.68883 \mathrm{E}-12$ & 0.0 & 0.0 & 0.0 \\
\hline BAHCO 3 ION & $8.99682 \mathrm{E}-17$ & 0.0 & 0.0 & 0.0 \\
\hline BAION & $1.52218 \mathrm{E}-09$ & 0.0 & 0.0 & 0.0 \\
\hline BAOHION & $7.28299 \mathrm{E}-18$ & 0.0 & 0.0 & 0.0 \\
\hline CACLION & $6.28296 \mathrm{E}-08$ & 0.0 & 0.0 & 0.0 \\
\hline CAH2 PO 4 ION & $7.98424 \mathrm{E}-06$ & 0.0 & 0.0 & 0.0 \\
\hline CAHCO3ION & $1.54236 \mathrm{E}-13$ & 0.0 & 0.0 & 0.0 \\
\hline CAHSIO3ION & $7.38842 \mathrm{E}-12$ & 0.0 & 0.0 & 0.0 \\
\hline CAION & $1.48866 \mathrm{E}-05$ & 0.0 & 0.0 & 0.0 \\
\hline CANO3ION & $8.02786 \mathrm{E}-06$ & 0.0 & 0.0 & 0.0 \\
\hline CAOHION & $1.44971 \mathrm{E}-12$ & 0.0 & 0.0 & 0.0 \\
\hline CAPO 4 ION & $1.04974 \mathrm{E}-11$ & 0.0 & 0.0 & 0.0 \\
\hline CDCL3ION & $2.50957 \mathrm{E}-08$ & 0.0 & 0.0 & 0.0 \\
\hline CDCL4 ION & $7.21990 \mathrm{E}-09$ & 0.0 & 0.0 & 0.0 \\
\hline CDCLION & $1.48420 \mathrm{E}-09$ & 0.0 & 0.0 & 0.0 \\
\hline CDION & $2.76673 \mathrm{E}-11$ & 0.0 & 0.0 & 0.0 \\
\hline CDNO3ION & $1.00774 \mathrm{E}-11$ & 0.0 & 0.0 & 0.0 \\
\hline CDOH3ION & $2.42960 \mathrm{E}-27$ & 0.0 & 0.0 & 0.0 \\
\hline CDOHION & $4.08397 \mathrm{E}-16$ & 0.0 & 0.0 & 0.0 \\
\hline CLION & 0.00381846 & 0.0 & 0.0 & 0.0 \\
\hline CO3ION & $9.22617 \mathrm{E}-17$ & 0.0 & 0.0 & 0.0 \\
\hline CRIIICL2ION & $4.78075 E-17$ & 0.0 & 0.0 & 0.0 \\
\hline CRIIICLION & $3.15242 \mathrm{E}-17$ & 0.0 & 0.0 & 0.0 \\
\hline CRIIIH2PO4ION & $7.06716 \mathrm{E}-16$ & 0.0 & 0.0 & 0.0 \\
\hline CRIIIHPO4ION & $1.84615 E-07$ & 0.0 & 0.0 & 0.0 \\
\hline CRIIIION & $3.65941 \mathrm{E}-21$ & 0.0 & 0.0 & 0.0 \\
\hline CRIIINO3ION & $2.33889 \mathrm{E}-14$ & 0.0 & 0.0 & 0.0 \\
\hline CROH2ION & $1.26271 \mathrm{E}-20$ & 0.0 & 0.0 & 0.0 \\
\hline CROH 4 ION & $7.17095 \mathrm{E}-28$ & 0.0 & 0.0 & 0.0 \\
\hline CROHION & $6.12060 \mathrm{E}-15$ & 0.0 & 0.0 & 0.0 \\
\hline CRSO 4 ION & $6.09648 \mathrm{E}-13$ & 0.0 & 0.0 & 0.0 \\
\hline CUCL3ION & $5.49053 \mathrm{E}-11$ & 0.0 & 0.0 & 0.0 \\
\hline CUCLION & $2.08433 \mathrm{E}-08$ & 0.0 & 0.0 & 0.0 \\
\hline CUCO32ION & $7.35570 \mathrm{E}-28$ & 0.0 & 0.0 & 0.0 \\
\hline CUION & $5.55397 \mathrm{E}-09$ & 0.0 & 0.0 & 0.0 \\
\hline CUNO3ION & $1.31083 \mathrm{E}-09$ & 0.0 & 0.0 & 0.0 \\
\hline CUOH3ION & $4.81485 E-20$ & 0.0 & 0.0 & 0.0 \\
\hline CUOH 4 ION & $7.34884 E-26$ & 0.0 & 0.0 & 0.0 \\
\hline CUOHION & $9.56266 \mathrm{E}-12$ & 0.0 & 0.0 & 0.0 \\
\hline DODECION & $1.55084 \mathrm{E}-08$ & 0.0 & 0.0 & 0.0 \\
\hline FEIICLION & $8.32029 E-07$ & 0.0 & 0.0 & 0 . \\
\hline
\end{tabular}




\begin{tabular}{|c|c|c|c|c|}
\hline FEIICO32ION & $4.47789 \mathrm{E}-26$ & 0.0 & 0.0 & 0.0 \\
\hline FEIIH2PO4ION & $1.91992 \mathrm{E}-06$ & 0.0 & 0.0 & 0.0 \\
\hline EEIIHCO3ION & $2.34076 \mathrm{E}-14$ & 0.0 & 0.0 & 0.0 \\
\hline FEIIION & $9.25722 \mathrm{E}-05$ & 0.0 & 0.0 & 0.0 \\
\hline FEIIOH3ION & $6.12930 \mathrm{E}-19$ & 0.0 & 0.0 & 0.0 \\
\hline EEIIOH4ION & $1.11984 \mathrm{E}-26$ & 0.0 & 0.0 & 0.0 \\
\hline FEIIOHION & $5.80707 \mathrm{E}-09$ & 0.0 & 0.0 & 0.0 \\
\hline H2 P2O 7 ION & $5.50178 \mathrm{E}-07$ & 0.0 & 0.0 & 0.0 \\
\hline H2 PO 4 ION & $5.16807 \mathrm{E}-05$ & 0.0 & 0.0 & 0.0 \\
\hline $\mathrm{H} 2 \mathrm{SIO} 4 \mathrm{ION}$ & $6.19077 \mathrm{E}-18$ & 0.0 & 0.0 & 0.0 \\
\hline H3P2O7 ION & $9.42576 \mathrm{E}-10$ & 0.0 & 0.0 & 0.0 \\
\hline H3SIO 4 ION & $5.41970 \mathrm{E}-10$ & 0.0 & 0.0 & 0.0 \\
\hline HCO3ION & $5.69292 \mathrm{E}-12$ & 0.0 & 0.0 & 0.0 \\
\hline HION & $1.79451 \mathrm{E}-07$ & 0.0 & 0.0 & 0.0 \\
\hline HP207ION & $7.43066 \mathrm{E}-09$ & 0.0 & 0.0 & 0.0 \\
\hline HPBO2ION & $4.72370 \mathrm{E}-21$ & 0.0 & 0.0 & 0.0 \\
\hline HPO4ION & $3.61131 \mathrm{E}-07$ & 0.0 & 0.0 & 0.0 \\
\hline HSO4 ION & $1.01540 \mathrm{E}-06$ & 0.0 & 0.0 & 0.0 \\
\hline KION & $6.09730 \mathrm{E}-04$ & 0.0 & 0.0 & 0.0 \\
\hline KSO4 ION & $5.36063 \mathrm{E}-05$ & 0.0 & 0.0 & 0.0 \\
\hline MGH2 PO 4 ION & $5.88870 \mathrm{E}-05$ & 0.0 & 0.0 & 0.0 \\
\hline MGHCO3ION & $6.96892 \mathrm{E}-12$ & 0.0 & 0.0 & 0.0 \\
\hline MGHSIO3ION & $3.18947 \mathrm{E}-10$ & 0.0 & 0.0 & 0.0 \\
\hline MGION & $1.25816 \mathrm{E}-04$ & 0.0 & 0.0 & 0.0 \\
\hline MGOHION & $2.64269 \mathrm{E}-10$ & 0.0 & 0.0 & 0.0 \\
\hline MGP2O7ION & $6.50020 \mathrm{E}-08$ & 0.0 & 0.0 & 0.0 \\
\hline MGPO 4 ION & $2.48473 \mathrm{E}-10$ & 0.0 & 0.0 & 0.0 \\
\hline NACO3ION & $1.36915 \mathrm{E}-17$ & 0.0 & 0.0 & 0.0 \\
\hline NAION & 0.00426085 & 0.0 & 0.0 & 0.0 \\
\hline NASO 4 ION & $6.00174 \mathrm{E}-13$ & 0.0 & 0.0 & 0.0 \\
\hline NICLION & $1.08776 \mathrm{E}-07$ & 0.0 & 0.0 & 0.0 \\
\hline NIION & $1.27239 \mathrm{E}-06$ & 0.0 & 0.0 & 0.0 \\
\hline NINO3ION & $2.36365 \mathrm{E}-07$ & 0.0 & 0.0 & 0.0 \\
\hline NIOH3ION & $9.01706 \mathrm{E}-22$ & 0.0 & 0.0 & 0.0 \\
\hline NIOHION & $2.19231 \mathrm{E}-11$ & 0.0 & 0.0 & 0.0 \\
\hline NO3ION & $9.37706 \mathrm{E}-04$ & 0.0 & 0.0 & 0.0 \\
\hline P207ION & $8.97135 \mathrm{E}-13$ & 0.0 & 0.0 & 0.0 \\
\hline PBCL3ION & $6.27270 \mathrm{E}-07$ & 0.0 & 0.0 & 0.0 \\
\hline PBCL 4 ION & $5.26273 E-06$ & 0.0 & 0.0 & 0.0 \\
\hline PBCLION & $7.75122 \mathrm{E}-08$ & 0.0 & 0.0 & 0.0 \\
\hline PBH2 PO 4 ION & $1.60020 \mathrm{E}-09$ & 0.0 & 0.0 & 0.0 \\
\hline PBION & $3.35690 \mathrm{E}-09$ & 0.0 & 0.0 & 0.0 \\
\hline PBNO33ION & $6.72975 \mathrm{E}-10$ & 0.0 & 0.0 & 0.0 \\
\hline PBNO3ION & $2.83887 \mathrm{E}-08$ & 0.0 & 0.0 & 0.0 \\
\hline PBOHION & $2.80620 \mathrm{E}-11$ & 0.0 & 0.0 & 0.0 \\
\hline PO4ION & $4.00498 \mathrm{E}-14$ & 0.0 & 0.0 & 0.0 \\
\hline SO4ION & $2.77975 \mathrm{E}-04$ & 0.0 & 0.0 & 0.0 \\
\hline SRION & $4.67426 \mathrm{E}-09$ & 0.0 & 0.0 & 0.0 \\
\hline SRNO3ION & $2.64805 \mathrm{E}-07$ & 0.0 & 0.0 & 0.0 \\
\hline SROHION & $1.10750 \mathrm{E}-14$ & 0.0 & 0.0 & 0.0 \\
\hline SRPO 4 ION & $2.38762 \mathrm{E}-15$ & 0.0 & 0.0 & 0.0 \\
\hline UIVCLION & $8.82688 \mathrm{E}-24$ & 0.0 & 0.0 & 0.0 \\
\hline UIVION & $5.75421 \mathrm{E}-26$ & 0.0 & 0.0 & 0.0 \\
\hline UIVOH2ION & $2.82580 \mathrm{E}-19$ & 0.0 & 0.0 & 0.0 \\
\hline UIVOH3ION & $4.96490 \mathrm{E}-17$ & 0.0 & 0.0 & 0.0 \\
\hline UIVOH5ION & $1.20152 \mathrm{E}-17$ & 0.0 & 0.0 & 0.0 \\
\hline UIVOHION & $1.20057 \mathrm{E}-20$ & 0.0 & 0.0 & 0.0 \\
\hline UIVSO 4 ION & $4.67507 \mathrm{E}-21$ & 0.0 & 0.0 & 0.0 \\
\hline
\end{tabular}


WSRC-TR-2002-00567

Revision 0

\begin{tabular}{|c|c|c|c|c|}
\hline ZNCL3ION & $1.42096 \mathrm{E}-05$ & 0.0 & 0.0 & 0.0 \\
\hline ZNCLION & $2.41301 E-05$ & 0.0 & 0.0 & 0.0 \\
\hline ZNH2 PO4 ION & $9.42148 E-08$ & 0.0 & 0.0 & 0.0 \\
\hline ZNHCO3ION & $9.32521 \mathrm{E}-15$ & 0.0 & 0.0 & 0.0 \\
\hline ZNION & $1.42948 E-06$ & 0.0 & 0.0 & 0.0 \\
\hline ZNNO3ION & $1.94757 \mathrm{E}-07$ & 0.0 & 0.0 & 0.0 \\
\hline ZNOH3ION & $8.61473 E-19$ & 0.0 & 0.0 & 0.0 \\
\hline ZNOH 4 ION & $4.72357 E-25$ & 0.0 & 0.0 & 0.0 \\
\hline ZNOHION & $6.84841 E-09$ & 0.0 & 0.0 & 0.0 \\
\hline СAHPO4 & 0.0 & $3.50750 \mathrm{E}-04$ & 0.0 & 0.0 \\
\hline $\mathrm{ALPO} 4$ & 0.0 & $1.18518 \mathrm{E}-04$ & 0.0 & 0.0 \\
\hline $\mathrm{CU} 3 \mathrm{PO} 42.2 \mathrm{H} 2 \mathrm{O}$ & 0.0 & $3.13926 \mathrm{E}-06$ & 0.0 & 0.0 \\
\hline FEII3PO $42.8 \mathrm{H} 20$ & 0.0 & $1.18268 E-05$ & 0.0 & 0.0 \\
\hline MG3PO4 2 & 0.0 & $1.08322 \mathrm{E}-04$ & 0.0 & 0.0 \\
\hline \multirow[t]{2}{*}{ UIVO2 } & 0.0 & $7.98317 \mathrm{E}-06$ & 0.0 & 0.0 \\
\hline & $===========$ & $===========$ & $===========$ & $===========$ \\
\hline Total g/hr & 2.01909 & 0.237857 & 498.128 & 0.0 \\
\hline Volume, L/hr & 0.00185263 & $5.57389 \mathrm{E}-05$ & 847.29 & 0.0 \\
\hline Enthalpy, cal/hr & -6633.04 & -674.306 & $-1.58093 E+06$ & 0.0 \\
\hline Density, g/L & 1089.85 & 4267.34 & 0.587907 & \\
\hline Vapor fraction & 0.0 & 0.0 & 1 . & 0.0 \\
\hline Solid fraction & 0.0 & 1 . & 0.0 & 0.0 \\
\hline Organic fraction & 0.0 & 0.0 & 0.0 & 0.0 \\
\hline Osmotic Pres, atm & 193.315 & & & \\
\hline Redox Pot, volts & 0.0 & & & \\
\hline E-Con, 1/ohm-cm & 0.483806 & & & \\
\hline E-Con, cm2/ohm-mol & 59.901 & & & \\
\hline Abs Visc, cP & 0.275692 & & & \\
\hline Rel Visc & 1.01419 & & & \\
\hline Ionic Strength & 3.71675 & & & \\
\hline
\end{tabular}


WSRC-TR-2002-00567

Revision 0

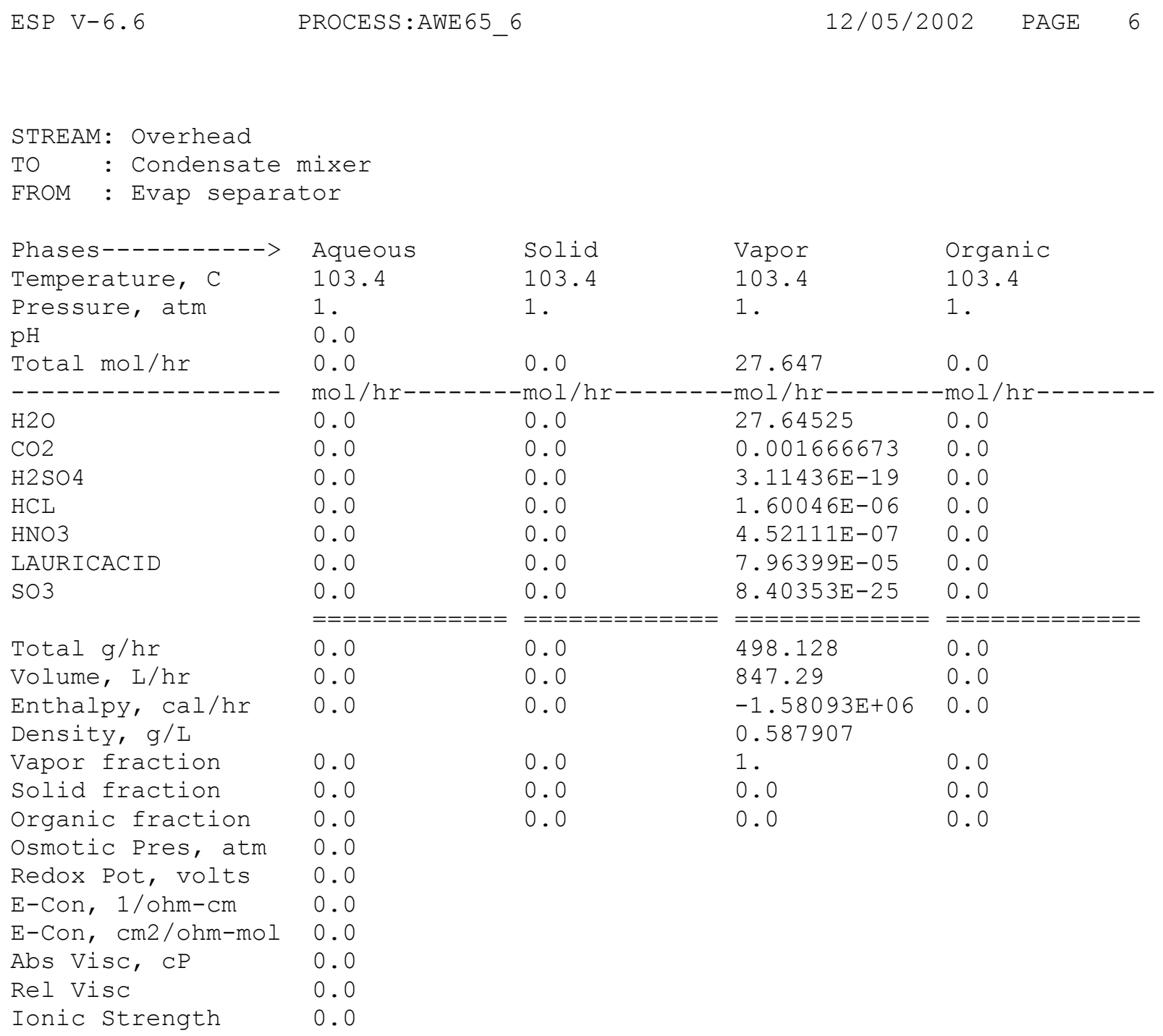


WSRC-TR-2002-00567

Revision 0

ESP V-6. 6
PROCESS:AWE 656
12/05/2002 PAGE 7

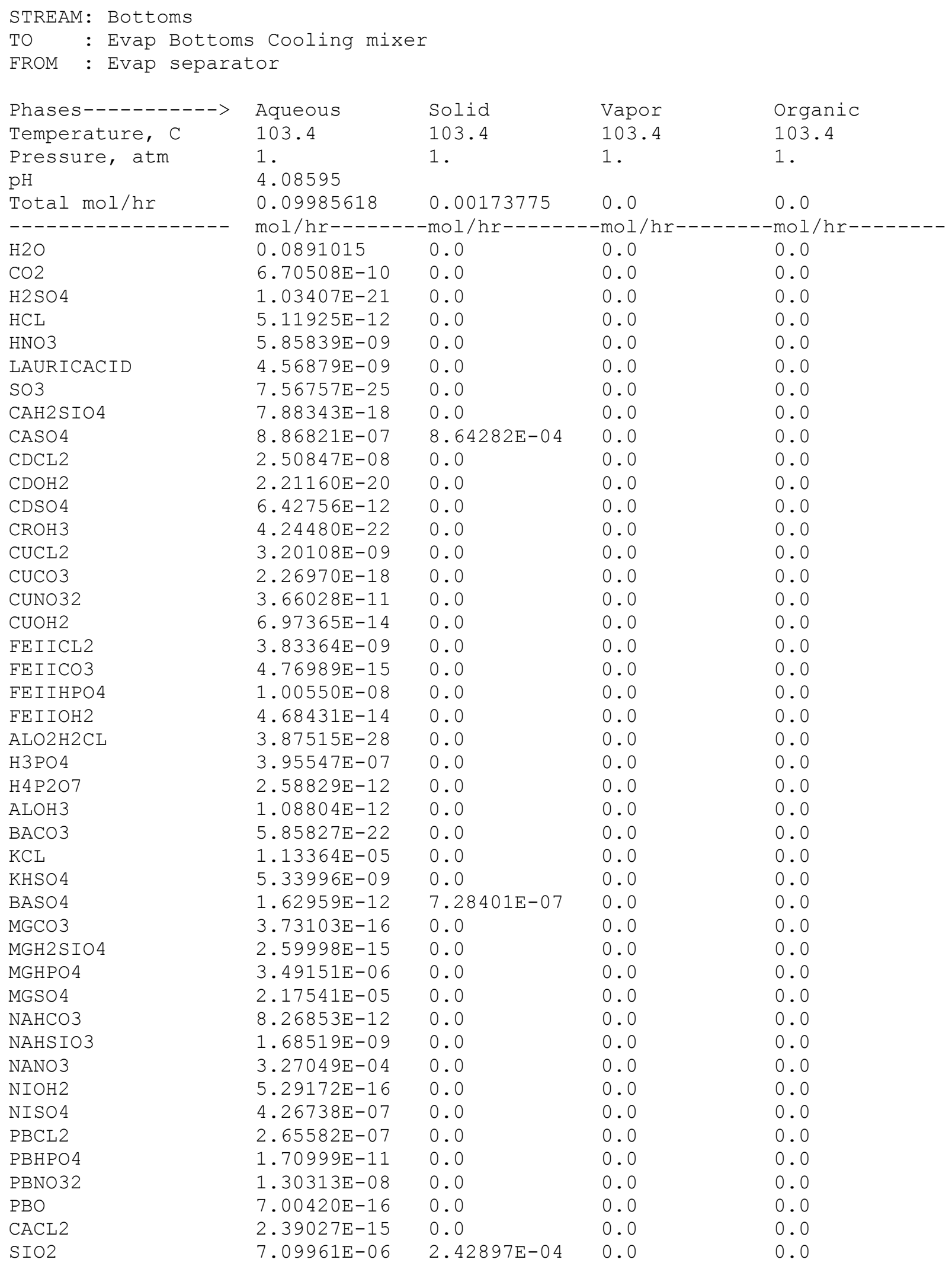




\begin{tabular}{|c|c|c|c|c|}
\hline $\mathrm{CACO} 3$ & $3.41121 E-17$ & 0.0 & 0.0 & 0.0 \\
\hline SRHPO 4 & $1.81800 \mathrm{E}-11$ & 0.0 & 0.0 & 0.0 \\
\hline SRNO32 & $9.21197 \mathrm{E}-08$ & 0.0 & 0.0 & 0.0 \\
\hline $\mathrm{SRSO} 4$ & $1.52740 \mathrm{E}-08$ & $2.93034 \mathrm{E}-05$ & 0.0 & 0.0 \\
\hline UIVOH 4 & $1.88149 \mathrm{E}-12$ & 0.0 & 0.0 & 0.0 \\
\hline UIVSO 42 & $2.13687 \mathrm{E}-20$ & 0.0 & 0.0 & 0.0 \\
\hline ZNCL2 & $1.03844 \mathrm{E}-05$ & 0.0 & 0.0 & 0.0 \\
\hline ZNHPO 4 & $2.07261 E-09$ & 0.0 & 0.0 & 0.0 \\
\hline ZNNO32 & $7.22648 \mathrm{E}-09$ & 0.0 & 0.0 & 0.0 \\
\hline ZNOH2 & $3.19463 \mathrm{E}-14$ & 0.0 & 0.0 & 0.0 \\
\hline OHION & $1.72144 \mathrm{E}-11$ & 0.0 & 0.0 & 0.0 \\
\hline ALION & $2.69032 \mathrm{E}-13$ & 0.0 & 0.0 & 0.0 \\
\hline ALOH2ION & $1.26850 \mathrm{E}-12$ & 0.0 & 0.0 & 0.0 \\
\hline ALOH 4 ION & $1.39245 \mathrm{E}-13$ & 0.0 & 0.0 & 0.0 \\
\hline ALOHCLION & $4.95935 \mathrm{E}-12$ & 0.0 & 0.0 & 0.0 \\
\hline ALOHION & $1.17526 \mathrm{E}-12$ & 0.0 & 0.0 & 0.0 \\
\hline ALSO 42 ION & $9.49590 \mathrm{E}-13$ & 0.0 & 0.0 & 0.0 \\
\hline ALSO 4 ION & $1.68883 \mathrm{E}-12$ & 0.0 & 0.0 & 0.0 \\
\hline BAHCO 3 ION & $8.99682 \mathrm{E}-17$ & 0.0 & 0.0 & 0.0 \\
\hline BAION & $1.52218 \mathrm{E}-09$ & 0.0 & 0.0 & 0.0 \\
\hline BAOHION & $7.28299 \mathrm{E}-18$ & 0.0 & 0.0 & 0.0 \\
\hline CACLION & $6.28296 \mathrm{E}-08$ & 0.0 & 0.0 & 0.0 \\
\hline CAH2 PO 4 ION & $7.98424 \mathrm{E}-06$ & 0.0 & 0.0 & 0.0 \\
\hline CAHCO3ION & $1.54236 \mathrm{E}-13$ & 0.0 & 0.0 & 0.0 \\
\hline CAHSIO3ION & $7.38842 \mathrm{E}-12$ & 0.0 & 0.0 & 0.0 \\
\hline CAION & $1.48866 \mathrm{E}-05$ & 0.0 & 0.0 & 0.0 \\
\hline CANO3ION & $8.02786 \mathrm{E}-06$ & 0.0 & 0.0 & 0.0 \\
\hline CAOHION & $1.44971 \mathrm{E}-12$ & 0.0 & 0.0 & 0.0 \\
\hline CAPO 4 ION & $1.04974 \mathrm{E}-11$ & 0.0 & 0.0 & 0.0 \\
\hline CDCL3ION & $2.50957 \mathrm{E}-08$ & 0.0 & 0.0 & 0.0 \\
\hline CDCL4 ION & $7.21990 \mathrm{E}-09$ & 0.0 & 0.0 & 0.0 \\
\hline CDCLION & $1.48420 \mathrm{E}-09$ & 0.0 & 0.0 & 0.0 \\
\hline CDION & $2.76673 \mathrm{E}-11$ & 0.0 & 0.0 & 0.0 \\
\hline CDNO3ION & $1.00774 \mathrm{E}-11$ & 0.0 & 0.0 & 0.0 \\
\hline CDOH3ION & $2.42960 \mathrm{E}-27$ & 0.0 & 0.0 & 0.0 \\
\hline CDOHION & $4.08397 \mathrm{E}-16$ & 0.0 & 0.0 & 0.0 \\
\hline CLION & 0.00381846 & 0.0 & 0.0 & 0.0 \\
\hline CO3ION & $9.22617 \mathrm{E}-17$ & 0.0 & 0.0 & 0.0 \\
\hline CRIIICL2ION & $4.78075 E-17$ & 0.0 & 0.0 & 0.0 \\
\hline CRIIICLION & $3.15242 \mathrm{E}-17$ & 0.0 & 0.0 & 0.0 \\
\hline CRIIIH2PO4ION & $7.06716 \mathrm{E}-16$ & 0.0 & 0.0 & 0.0 \\
\hline CRIIIHPO4ION & $1.84615 E-07$ & 0.0 & 0.0 & 0.0 \\
\hline CRIIIION & $3.65941 \mathrm{E}-21$ & 0.0 & 0.0 & 0.0 \\
\hline CRIIINO3ION & $2.33889 \mathrm{E}-14$ & 0.0 & 0.0 & 0.0 \\
\hline CROH2ION & $1.26271 \mathrm{E}-20$ & 0.0 & 0.0 & 0.0 \\
\hline CROH 4 ION & $7.17095 \mathrm{E}-28$ & 0.0 & 0.0 & 0.0 \\
\hline CROHION & $6.12060 \mathrm{E}-15$ & 0.0 & 0.0 & 0.0 \\
\hline CRSO 4 ION & $6.09648 \mathrm{E}-13$ & 0.0 & 0.0 & 0.0 \\
\hline CUCL3ION & $5.49053 \mathrm{E}-11$ & 0.0 & 0.0 & 0.0 \\
\hline CUCLION & $2.08433 \mathrm{E}-08$ & 0.0 & 0.0 & 0.0 \\
\hline CUCO32ION & $7.35570 \mathrm{E}-28$ & 0.0 & 0.0 & 0.0 \\
\hline CUION & $5.55397 \mathrm{E}-09$ & 0.0 & 0.0 & 0.0 \\
\hline CUNO3ION & $1.31083 \mathrm{E}-09$ & 0.0 & 0.0 & 0.0 \\
\hline CUOH3ION & $4.81485 E-20$ & 0.0 & 0.0 & 0.0 \\
\hline CUOH 4 ION & $7.34884 E-26$ & 0.0 & 0.0 & 0.0 \\
\hline CUOHION & $9.56266 \mathrm{E}-12$ & 0.0 & 0.0 & 0.0 \\
\hline DODECION & $1.55084 \mathrm{E}-08$ & 0.0 & 0.0 & 0.0 \\
\hline FEIICLION & $8.32029 E-07$ & 0.0 & 0.0 & 0 . \\
\hline
\end{tabular}




\begin{tabular}{|c|c|c|c|c|}
\hline FEIICO32ION & $4.47789 \mathrm{E}-26$ & 0.0 & 0.0 & 0.0 \\
\hline FEIIH2PO4ION & $1.91992 \mathrm{E}-06$ & 0.0 & 0.0 & 0.0 \\
\hline EEIIHCO3ION & $2.34076 \mathrm{E}-14$ & 0.0 & 0.0 & 0.0 \\
\hline FEIIION & $9.25722 \mathrm{E}-05$ & 0.0 & 0.0 & 0.0 \\
\hline FEIIOH3ION & $6.12930 \mathrm{E}-19$ & 0.0 & 0.0 & 0.0 \\
\hline EEIIOH4ION & $1.11984 \mathrm{E}-26$ & 0.0 & 0.0 & 0.0 \\
\hline FEIIOHION & $5.80707 \mathrm{E}-09$ & 0.0 & 0.0 & 0.0 \\
\hline H2 P2O 7 ION & $5.50178 \mathrm{E}-07$ & 0.0 & 0.0 & 0.0 \\
\hline H2 PO 4 ION & $5.16807 \mathrm{E}-05$ & 0.0 & 0.0 & 0.0 \\
\hline $\mathrm{H} 2 \mathrm{SIO} 4 \mathrm{ION}$ & $6.19077 \mathrm{E}-18$ & 0.0 & 0.0 & 0.0 \\
\hline H3P2O7 ION & $9.42576 \mathrm{E}-10$ & 0.0 & 0.0 & 0.0 \\
\hline H3SIO 4 ION & $5.41970 \mathrm{E}-10$ & 0.0 & 0.0 & 0.0 \\
\hline HCO3ION & $5.69292 \mathrm{E}-12$ & 0.0 & 0.0 & 0.0 \\
\hline HION & $1.79451 \mathrm{E}-07$ & 0.0 & 0.0 & 0.0 \\
\hline HP207ION & $7.43066 \mathrm{E}-09$ & 0.0 & 0.0 & 0.0 \\
\hline HPBO2ION & $4.72370 \mathrm{E}-21$ & 0.0 & 0.0 & 0.0 \\
\hline HPO4ION & $3.61131 \mathrm{E}-07$ & 0.0 & 0.0 & 0.0 \\
\hline HSO4 ION & $1.01540 \mathrm{E}-06$ & 0.0 & 0.0 & 0.0 \\
\hline KION & $6.09730 \mathrm{E}-04$ & 0.0 & 0.0 & 0.0 \\
\hline KSO4 ION & $5.36063 \mathrm{E}-05$ & 0.0 & 0.0 & 0.0 \\
\hline MGH2 PO 4 ION & $5.88870 \mathrm{E}-05$ & 0.0 & 0.0 & 0.0 \\
\hline MGHCO3ION & $6.96892 \mathrm{E}-12$ & 0.0 & 0.0 & 0.0 \\
\hline MGHSIO3ION & $3.18947 \mathrm{E}-10$ & 0.0 & 0.0 & 0.0 \\
\hline MGION & $1.25816 \mathrm{E}-04$ & 0.0 & 0.0 & 0.0 \\
\hline MGOHION & $2.64269 \mathrm{E}-10$ & 0.0 & 0.0 & 0.0 \\
\hline MGP2O7ION & $6.50020 \mathrm{E}-08$ & 0.0 & 0.0 & 0.0 \\
\hline MGPO 4 ION & $2.48473 \mathrm{E}-10$ & 0.0 & 0.0 & 0.0 \\
\hline NACO3ION & $1.36915 \mathrm{E}-17$ & 0.0 & 0.0 & 0.0 \\
\hline NAION & 0.00426085 & 0.0 & 0.0 & 0.0 \\
\hline NASO 4 ION & $6.00174 \mathrm{E}-13$ & 0.0 & 0.0 & 0.0 \\
\hline NICLION & $1.08776 \mathrm{E}-07$ & 0.0 & 0.0 & 0.0 \\
\hline NIION & $1.27239 \mathrm{E}-06$ & 0.0 & 0.0 & 0.0 \\
\hline NINO3ION & $2.36365 \mathrm{E}-07$ & 0.0 & 0.0 & 0.0 \\
\hline NIOH3ION & $9.01706 \mathrm{E}-22$ & 0.0 & 0.0 & 0.0 \\
\hline NIOHION & $2.19231 \mathrm{E}-11$ & 0.0 & 0.0 & 0.0 \\
\hline NO3ION & $9.37706 \mathrm{E}-04$ & 0.0 & 0.0 & 0.0 \\
\hline P207ION & $8.97135 \mathrm{E}-13$ & 0.0 & 0.0 & 0.0 \\
\hline PBCL3ION & $6.27270 \mathrm{E}-07$ & 0.0 & 0.0 & 0.0 \\
\hline PBCL 4 ION & $5.26273 E-06$ & 0.0 & 0.0 & 0.0 \\
\hline PBCLION & $7.75122 \mathrm{E}-08$ & 0.0 & 0.0 & 0.0 \\
\hline PBH2 PO 4 ION & $1.60020 \mathrm{E}-09$ & 0.0 & 0.0 & 0.0 \\
\hline PBION & $3.35690 \mathrm{E}-09$ & 0.0 & 0.0 & 0.0 \\
\hline PBNO33ION & $6.72975 \mathrm{E}-10$ & 0.0 & 0.0 & 0.0 \\
\hline PBNO3ION & $2.83887 \mathrm{E}-08$ & 0.0 & 0.0 & 0.0 \\
\hline PBOHION & $2.80620 \mathrm{E}-11$ & 0.0 & 0.0 & 0.0 \\
\hline PO4ION & $4.00498 \mathrm{E}-14$ & 0.0 & 0.0 & 0.0 \\
\hline SO4ION & $2.77975 \mathrm{E}-04$ & 0.0 & 0.0 & 0.0 \\
\hline SRION & $4.67426 \mathrm{E}-09$ & 0.0 & 0.0 & 0.0 \\
\hline SRNO3ION & $2.64805 \mathrm{E}-07$ & 0.0 & 0.0 & 0.0 \\
\hline SROHION & $1.10750 \mathrm{E}-14$ & 0.0 & 0.0 & 0.0 \\
\hline SRPO 4 ION & $2.38762 \mathrm{E}-15$ & 0.0 & 0.0 & 0.0 \\
\hline UIVCLION & $8.82688 \mathrm{E}-24$ & 0.0 & 0.0 & 0.0 \\
\hline UIVION & $5.75421 \mathrm{E}-26$ & 0.0 & 0.0 & 0.0 \\
\hline UIVOH2ION & $2.82580 \mathrm{E}-19$ & 0.0 & 0.0 & 0.0 \\
\hline UIVOH3ION & $4.96490 \mathrm{E}-17$ & 0.0 & 0.0 & 0.0 \\
\hline UIVOH5ION & $1.20152 \mathrm{E}-17$ & 0.0 & 0.0 & 0.0 \\
\hline UIVOHION & $1.20057 \mathrm{E}-20$ & 0.0 & 0.0 & 0.0 \\
\hline UIVSO 4 ION & $4.67507 \mathrm{E}-21$ & 0.0 & 0.0 & 0.0 \\
\hline
\end{tabular}


WSRC-TR-2002-00567

Revision 0

\begin{tabular}{|c|c|c|c|c|}
\hline ZNCL3ION & $1.42096 \mathrm{E}-05$ & 0.0 & 0.0 & 0.0 \\
\hline ZNCLION & $2.41301 E-05$ & 0.0 & 0.0 & 0.0 \\
\hline ZNH2 PO4 ION & $9.42148 E-08$ & 0.0 & 0.0 & 0.0 \\
\hline ZNHCO3ION & $9.32521 \mathrm{E}-15$ & 0.0 & 0.0 & 0.0 \\
\hline ZNION & $1.42948 E-06$ & 0.0 & 0.0 & 0.0 \\
\hline ZNNO3ION & $1.94757 \mathrm{E}-07$ & 0.0 & 0.0 & 0.0 \\
\hline ZNOH3ION & $8.61473 E-19$ & 0.0 & 0.0 & 0.0 \\
\hline ZNOH 4 ION & $4.72357 E-25$ & 0.0 & 0.0 & 0.0 \\
\hline ZNOHION & $6.84841 E-09$ & 0.0 & 0.0 & 0.0 \\
\hline СAHPO4 & 0.0 & $3.50750 \mathrm{E}-04$ & 0.0 & 0.0 \\
\hline $\mathrm{ALPO} 4$ & 0.0 & $1.18518 \mathrm{E}-04$ & 0.0 & 0.0 \\
\hline $\mathrm{CU} 3 \mathrm{PO} 42.2 \mathrm{H} 2 \mathrm{O}$ & 0.0 & $3.13926 \mathrm{E}-06$ & 0.0 & 0.0 \\
\hline FEII3PO $42.8 \mathrm{H} 20$ & 0.0 & $1.18268 E-05$ & 0.0 & 0.0 \\
\hline MG3PO4 2 & 0.0 & $1.08322 \mathrm{E}-04$ & 0.0 & 0.0 \\
\hline \multirow[t]{2}{*}{ UIVO2 } & 0.0 & $7.98317 \mathrm{E}-06$ & 0.0 & 0.0 \\
\hline & $===========$ & $===========$ & $===========$ & $===========$ \\
\hline Total g/hr & 2.01909 & 0.237857 & 0.0 & 0.0 \\
\hline Volume, L/hr & 0.00185263 & $5.57389 \mathrm{E}-05$ & 0.0 & 0.0 \\
\hline Enthalpy, cal/hr & -6633.04 & -674.306 & 0.0 & 0.0 \\
\hline Density, g/L & 1089.85 & 4267.34 & & \\
\hline Vapor fraction & 0.0 & 0.0 & 0.0 & 0.0 \\
\hline Solid fraction & 0.0 & 1 . & 0.0 & 0.0 \\
\hline Organic fraction & 0.0 & 0.0 & 0.0 & 0.0 \\
\hline Osmotic Pres, atm & 193.315 & & & \\
\hline Redox Pot, volts & 0.0 & & & \\
\hline E-Con, 1/ohm-cm & 0.483806 & & & \\
\hline E-Con, cm2/ohm-mol & 59.901 & & & \\
\hline Abs Visc, cP & 0.275692 & & & \\
\hline Rel Visc & 1.01419 & & & \\
\hline Ionic Strength & 3.71675 & & & \\
\hline
\end{tabular}


WSRC-TR-2002-00567

Revision 0

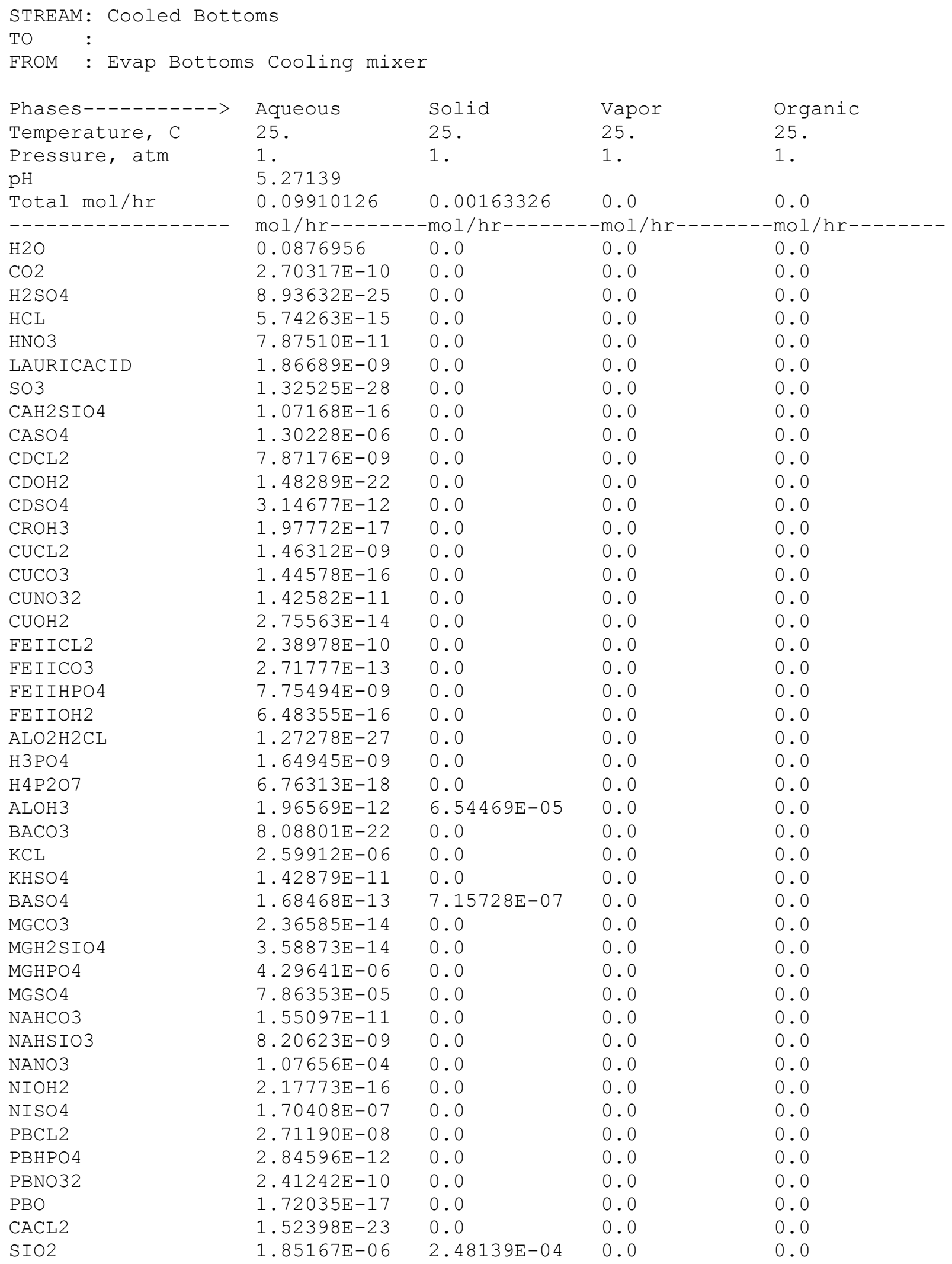




\begin{tabular}{|c|c|c|c|c|}
\hline $\mathrm{CACO} 3$ & $1.51338 \mathrm{E}-15$ & 0.0 & 0.0 & 0.0 \\
\hline SRHPO 4 & $9.80352 \mathrm{E}-12$ & 0.0 & 0.0 & 0.0 \\
\hline SRNO32 & $3.22470 \mathrm{E}-08$ & 0.0 & 0.0 & 0.0 \\
\hline $\mathrm{SRSO} 4$ & $3.52988 \mathrm{E}-08$ & $2.77081 E-05$ & 0.0 & 0.0 \\
\hline UIVOH 4 & $1.88453 \mathrm{E}-13$ & 0.0 & 0.0 & 0.0 \\
\hline UIVSO 42 & $2.25882 \mathrm{E}-23$ & 0.0 & 0.0 & 0.0 \\
\hline ZNCL2 & $3.38962 \mathrm{E}-06$ & 0.0 & 0.0 & 0.0 \\
\hline ZNHPO 4 & $2.27539 \mathrm{E}-08$ & 0.0 & 0.0 & 0.0 \\
\hline ZNNO32 & $5.81182 \mathrm{E}-08$ & 0.0 & 0.0 & 0.0 \\
\hline ZNOH2 & $5.16301 \mathrm{E}-14$ & 0.0 & 0.0 & 0.0 \\
\hline OHION & $2.63497 \mathrm{E}-12$ & 0.0 & 0.0 & 0.0 \\
\hline ALION & $2.35079 \mathrm{E}-11$ & 0.0 & 0.0 & 0.0 \\
\hline ALOH 2 ION & $5.97578 \mathrm{E}-12$ & 0.0 & 0.0 & 0.0 \\
\hline ALOH 4 ION & $2.73359 \mathrm{E}-13$ & 0.0 & 0.0 & 0.0 \\
\hline ALOHCLION & $9.16105 \mathrm{E}-11$ & 0.0 & 0.0 & 0.0 \\
\hline ALOHION & $1.29831 \mathrm{E}-11$ & 0.0 & 0.0 & 0.0 \\
\hline ALSO 42 ION & $6.26516 \mathrm{E}-11$ & 0.0 & 0.0 & 0.0 \\
\hline ALSO 4 ION & $8.15523 \mathrm{E}-11$ & 0.0 & 0.0 & 0.0 \\
\hline BAHCO3ION & $9.32665 \mathrm{E}-18$ & 0.0 & 0.0 & 0.0 \\
\hline BAION & $1.41980 \mathrm{E}-08$ & 0.0 & 0.0 & 0.0 \\
\hline BAOHION & $2.19481 \mathrm{E}-20$ & 0.0 & 0.0 & 0.0 \\
\hline CACLION & $1.23166 \mathrm{E}-10$ & 0.0 & 0.0 & 0.0 \\
\hline CAH2 PO 4 ION & $1.40270 \mathrm{E}-06$ & 0.0 & 0.0 & 0.0 \\
\hline CAHCO3ION & $3.61528 \mathrm{E}-12$ & 0.0 & 0.0 & 0.0 \\
\hline CAHSIO3ION & $1.15751 \mathrm{E}-11$ & 0.0 & 0.0 & 0.0 \\
\hline CAION & $3.12320 \mathrm{E}-05$ & 0.0 & 0.0 & 0.0 \\
\hline CANO3ION & $1.60605 \mathrm{E}-05$ & 0.0 & 0.0 & 0.0 \\
\hline CAOHION & $2.54865 E-13$ & 0.0 & 0.0 & 0.0 \\
\hline CAPO 4 ION & $1.48035 \mathrm{E}-10$ & 0.0 & 0.0 & 0.0 \\
\hline CDCL3ION & $3.56781 \mathrm{E}-09$ & 0.0 & 0.0 & 0.0 \\
\hline CDCL 4 ION & $4.69582 E-08$ & 0.0 & 0.0 & 0.0 \\
\hline CDCLION & $5.02619 \mathrm{E}-10$ & 0.0 & 0.0 & 0.0 \\
\hline CDION & $2.09822 \mathrm{E}-11$ & 0.0 & 0.0 & 0.0 \\
\hline CDNO3ION & $4.07175 \mathrm{E}-12$ & 0.0 & 0.0 & 0.0 \\
\hline CDOH 3 ION & $1.44375 E-29$ & 0.0 & 0.0 & 0.0 \\
\hline CDOHION & $3.06679 \mathrm{E}-17$ & 0.0 & 0.0 & 0.0 \\
\hline CLION & 0.00389829 & 0.0 & 0.0 & 0.0 \\
\hline CO3ION & $7.31983 \mathrm{E}-15$ & 0.0 & 0.0 & 0.0 \\
\hline CRIIICL2ION & $7.37486 \mathrm{E}-14$ & 0.0 & 0.0 & 0.0 \\
\hline CRIIICLION & $1.54006 \mathrm{E}-14$ & 0.0 & 0.0 & 0.0 \\
\hline CRIIIH2PO4ION & $2.38865 E-11$ & 0.0 & 0.0 & 0.0 \\
\hline CRIIIHPO4ION & $1.83854 \mathrm{E}-07$ & 0.0 & 0.0 & 0.0 \\
\hline CRIIIION & $6.02501 \mathrm{E}-18$ & 0.0 & 0.0 & 0.0 \\
\hline CRIIINO3ION & $1.53655 \mathrm{E}-11$ & 0.0 & 0.0 & 0.0 \\
\hline CROH2ION & $9.69462 \mathrm{E}-17$ & 0.0 & 0.0 & 0.0 \\
\hline CROH 4 ION & $1.59205 \mathrm{E}-22$ & 0.0 & 0.0 & 0.0 \\
\hline CROHION & $2.75950 \mathrm{E}-11$ & 0.0 & 0.0 & 0.0 \\
\hline CRSO 4 ION & $6.94713 \mathrm{E}-10$ & 0.0 & 0.0 & 0.0 \\
\hline CUCL3ION & $2.87370 \mathrm{E}-11$ & 0.0 & 0.0 & 0.0 \\
\hline CUCLION & $4.37003 E-09$ & 0.0 & 0.0 & 0.0 \\
\hline CUCO32ION & $2.17169 \mathrm{E}-24$ & 0.0 & 0.0 & 0.0 \\
\hline CUION & $3.94840 \mathrm{E}-09$ & 0.0 & 0.0 & 0.0 \\
\hline CUNO3ION & $9.17491 \mathrm{E}-10$ & 0.0 & 0.0 & 0.0 \\
\hline CUOH 3 ION & $9.80548 \mathrm{E}-21$ & 0.0 & 0.0 & 0.0 \\
\hline CUOH 4 ION & $1.37410 \mathrm{E}-27$ & 0.0 & 0.0 & 0.0 \\
\hline CUOHION & $2.51947 \mathrm{E}-12$ & 0.0 & 0.0 & 0.0 \\
\hline DODECION & $1.82103 \mathrm{E}-08$ & 0.0 & 0.0 & 0.0 \\
\hline FEIICLION & $8.82506 \mathrm{E}-08$ & 0.0 & 0.0 & 0. \\
\hline
\end{tabular}




\begin{tabular}{|c|c|c|c|c|}
\hline FEIICO32ION & $8.83087 \mathrm{E}-23$ & 0.0 & 0.0 & 0.0 \\
\hline FEIIH2PO4ION & $3.59738 \mathrm{E}-07$ & 0.0 & 0.0 & 0.0 \\
\hline EEIIHCO3ION & $1.04124 \mathrm{E}-13$ & 0.0 & 0.0 & 0.0 \\
\hline FEIIION & $6.24479 \mathrm{E}-05$ & 0.0 & 0.0 & 0.0 \\
\hline FEIIOH3ION & $1.44793 E-20$ & 0.0 & 0.0 & 0.0 \\
\hline EEIIOH4ION & $3.20145 \mathrm{E}-29$ & 0.0 & 0.0 & 0.0 \\
\hline FEIIOHION & $1.35309 \mathrm{E}-09$ & 0.0 & 0.0 & 0.0 \\
\hline H2 P2O 7 ION & $1.26446 \mathrm{E}-09$ & 0.0 & 0.0 & 0.0 \\
\hline H2 PO 4 ION & $1.02143 \mathrm{E}-05$ & 0.0 & 0.0 & 0.0 \\
\hline $\mathrm{H} 2 \mathrm{SIO} 4 \mathrm{ION}$ & $1.97268 \mathrm{E}-17$ & 0.0 & 0.0 & 0.0 \\
\hline H3P2O7 ION & $7.85336 \mathrm{E}-14$ & 0.0 & 0.0 & 0.0 \\
\hline H3SIO 4 ION & $7.67951 \mathrm{E}-11$ & 0.0 & 0.0 & 0.0 \\
\hline HCO3ION & $7.84616 \mathrm{E}-11$ & 0.0 & 0.0 & 0.0 \\
\hline HION & $7.88379 \mathrm{E}-09$ & 0.0 & 0.0 & 0.0 \\
\hline HP207ION & $4.59027 \mathrm{E}-10$ & 0.0 & 0.0 & 0.0 \\
\hline HPBO2ION & $9.03740 \mathrm{E}-23$ & 0.0 & 0.0 & 0.0 \\
\hline HPO4ION & $1.48016 \mathrm{E}-06$ & 0.0 & 0.0 & 0.0 \\
\hline $\mathrm{HSO} 4 \mathrm{ION}$ & $9.46041 \mathrm{E}-09$ & 0.0 & 0.0 & 0.0 \\
\hline KION & $6.27450 \mathrm{E}-04$ & 0.0 & 0.0 & 0.0 \\
\hline KSO4 ION & $4.46287 \mathrm{E}-05$ & 0.0 & 0.0 & 0.0 \\
\hline MGH2 PO 4 ION & $6.24508 \mathrm{E}-05$ & 0.0 & 0.0 & 0.0 \\
\hline MGHCO3ION & $3.22423 E-10$ & 0.0 & 0.0 & 0.0 \\
\hline MGHSIO3ION & $7.40919 \mathrm{E}-10$ & 0.0 & 0.0 & 0.0 \\
\hline MGION & $3.89572 \mathrm{E}-04$ & 0.0 & 0.0 & 0.0 \\
\hline MGOHION & $8.46065 \mathrm{E}-11$ & 0.0 & 0.0 & 0.0 \\
\hline MGP2O7ION & $1.75909 \mathrm{E}-08$ & 0.0 & 0.0 & 0.0 \\
\hline MGPO 4 ION & $6.19684 \mathrm{E}-09$ & 0.0 & 0.0 & 0.0 \\
\hline NACO3ION & $3.32561 \mathrm{E}-15$ & 0.0 & 0.0 & 0.0 \\
\hline NAION & 0.00433628 & 0.0 & 0.0 & 0.0 \\
\hline NASO 4 ION & $1.43961 \mathrm{E}-04$ & 0.0 & 0.0 & 0.0 \\
\hline NICLION & $5.32841 \mathrm{E}-08$ & 0.0 & 0.0 & 0.0 \\
\hline NIION & $1.39784 \mathrm{E}-06$ & 0.0 & 0.0 & 0.0 \\
\hline NINO3ION & $4.22757 \mathrm{E}-07$ & 0.0 & 0.0 & 0.0 \\
\hline NIOH3ION & $1.55494 \mathrm{E}-21$ & 0.0 & 0.0 & 0.0 \\
\hline NIOHION & $4.94471 \mathrm{E}-12$ & 0.0 & 0.0 & 0.0 \\
\hline NO3ION & 0.00114716 & 0.0 & 0.0 & 0.0 \\
\hline P207ION & $5.60383 \mathrm{E}-12$ & 0.0 & 0.0 & 0.0 \\
\hline PBCL3ION & $8.42728 \mathrm{E}-08$ & 0.0 & 0.0 & 0.0 \\
\hline PBCL 4 ION & $1.90395 \mathrm{E}-06$ & 0.0 & 0.0 & 0.0 \\
\hline PBCLION & $1.01711 \mathrm{E}-08$ & 0.0 & 0.0 & 0.0 \\
\hline PBH2 PO 4 ION & $2.63099 \mathrm{E}-11$ & 0.0 & 0.0 & 0.0 \\
\hline PBION & $9.89244 \mathrm{E}-10$ & 0.0 & 0.0 & 0.0 \\
\hline PBNO33ION & $8.36169 \mathrm{E}-11$ & 0.0 & 0.0 & 0.0 \\
\hline PBNO3ION & $2.02039 \mathrm{E}-09$ & 0.0 & 0.0 & 0.0 \\
\hline PBOHION & $6.29869 \mathrm{E}-13$ & 0.0 & 0.0 & 0.0 \\
\hline PO4ION & $1.75301 \mathrm{E}-12$ & 0.0 & 0.0 & 0.0 \\
\hline SO4ION & $3.97719 \mathrm{E}-04$ & 0.0 & 0.0 & 0.0 \\
\hline SRION & $1.70769 \mathrm{E}-06$ & 0.0 & 0.0 & 0.0 \\
\hline SRNO3ION & $1.96922 \mathrm{E}-07$ & 0.0 & 0.0 & 0.0 \\
\hline SROHION & $7.71831 \mathrm{E}-16$ & 0.0 & 0.0 & 0.0 \\
\hline SRPO 4 ION & $1.07305 \mathrm{E}-14$ & 0.0 & 0.0 & 0.0 \\
\hline UIVCLION & $7.08904 \mathrm{E}-26$ & 0.0 & 0.0 & 0.0 \\
\hline UIVION & $1.01234 \mathrm{E}-27$ & 0.0 & 0.0 & 0.0 \\
\hline UIVOH2ION & $6.41008 \mathrm{E}-21$ & 0.0 & 0.0 & 0.0 \\
\hline UIVOH3ION & $3.91823 \mathrm{E}-17$ & 0.0 & 0.0 & 0.0 \\
\hline UIVOH5ION & $6.85739 \mathrm{E}-18$ & 0.0 & 0.0 & 0.0 \\
\hline UIVOHION & $4.69288 \mathrm{E}-23$ & 0.0 & 0.0 & 0.0 \\
\hline UIVSO 4 ION & $1.24768 \mathrm{E}-23$ & 0.0 & 0.0 & 0.0 \\
\hline
\end{tabular}


WSRC-TR-2002-00567

Revision 0

\begin{tabular}{|c|c|c|c|c|}
\hline ZNCL3ION & $7.32237 E-06$ & 0.0 & 0.0 & 0.0 \\
\hline ZNCLION & $4.12472 \mathrm{E}-06$ & 0.0 & 0.0 & 0.0 \\
\hline ZNH2 PO4 ION & $1.71832 \mathrm{E}-07$ & 0.0 & 0.0 & 0.0 \\
\hline ZNHCO3ION & $8.92244 E-13$ & 0.0 & 0.0 & 0.0 \\
\hline ZNION & $1.49761 E-05$ & 0.0 & 0.0 & 0.0 \\
\hline ZNNO3ION & $2.06344 \mathrm{E}-06$ & 0.0 & 0.0 & 0.0 \\
\hline ZNOH3ION & $2.25749 \mathrm{E}-19$ & 0.0 & 0.0 & 0.0 \\
\hline ZNOH 4 ION & $8.20215 E-26$ & 0.0 & 0.0 & 0.0 \\
\hline ZNOHION & $2.99302 \mathrm{E}-10$ & 0.0 & 0.0 & 0.0 \\
\hline СAHPO4 & 0.0 & $6.41771 \mathrm{E}-04$ & 0.0 & 0.0 \\
\hline ALPO 4 & 0.0 & $5.30712 \mathrm{E}-05$ & 0.0 & 0.0 \\
\hline $\mathrm{CASO} 4.2 \mathrm{H} 2 \mathrm{O}$ & 0.0 & $5.55113 E-04$ & 0.0 & 0.0 \\
\hline $\mathrm{CU} 3 \mathrm{PO} 42.2 \mathrm{H} 2 \mathrm{O}$ & 0.0 & $3.14601 \mathrm{E}-06$ & 0.0 & 0.0 \\
\hline FEII3PO $42.8 \mathrm{H} 2 \mathrm{O}$ & 0.0 & $2.26397 E-05$ & 0.0 & 0.0 \\
\hline PB3PO4 2 & 0.0 & 1. $41711 \mathrm{E}-06$ & 0.0 & 0.0 \\
\hline UIVO2 & 0.0 & $7.98318 E-06$ & 0.0 & 0.0 \\
\hline \multirow[t]{2}{*}{$\mathrm{ZN} 3 \mathrm{PO} 42.2 \mathrm{H} 2 \mathrm{O}$} & 0.0 & $6.10980 \mathrm{E}-06$ & 0.0 & 0.0 \\
\hline & $===========$ & $===========$ & $===========$ & $===========$ \\
\hline Total g/hr & 2.02376 & 0.233188 & 0.0 & 0.0 \\
\hline Volume, L/hr & 0.00172725 & $5.43246 \mathrm{E}-05$ & 0.0 & 0.0 \\
\hline Enthalpy, cal/hr & -6755.25 & -688.811 & 0.0 & 0.0 \\
\hline Density, g/L & 1171.67 & 4292.49 & & \\
\hline Vapor fraction & 0.0 & 0.0 & 0.0 & 0.0 \\
\hline Solid fraction & 0.0 & 1 . & 0.0 & 0.0 \\
\hline Organic fraction & 0.0 & 0.0 & 0.0 & 0.0 \\
\hline Osmotic Pres, atm & 191.141 & & & \\
\hline Redox Pot, volts & 0.0 & & & \\
\hline E-Con, 1/ohm-cm & 0.187155 & & & \\
\hline E-Con, cm2/ohm-mol & 29.0748 & & & \\
\hline Abs Visc, cP & 1.51758 & & & \\
\hline Rel Visc & 1.70377 & & & \\
\hline Ionic Strength & 4.4033 & & & \\
\hline
\end{tabular}


WSRC-TR-2002-00567

Revision 0

\begin{tabular}{|c|c|c|c|c|}
\hline \multicolumn{5}{|l|}{ STREAM: Condensate } \\
\hline TO $\quad:$ & & & & \\
\hline FROM : Condensate & mixer & & & \\
\hline Phases-----------> & Aqueous & Solid & Vapor & Organic \\
\hline Temperature, C & 25 . & 25 . & 25 . & 25 . \\
\hline $\begin{array}{l}\text { Pressure, atm } \\
\mathrm{pH}\end{array}$ & $\begin{array}{l}1 . \\
4.23752\end{array}$ & 1. & 1. & 1. \\
\hline Total mol/hr & 27.64698 & 0.0 & 0.0 & 0.0 \\
\hline------------------ & $\mathrm{mol} / \mathrm{hr}------$ & -mol/hr------- & -mol/hr-------- & $-\operatorname{mol} / \mathrm{hr}------1$ \\
\hline $\mathrm{H} 2 \mathrm{O}$ & 27.6452 & 0.0 & 0.0 & 0.0 \\
\hline $\mathrm{CO} 2$ & 0.00165358 & 0.0 & 0.0 & 0.0 \\
\hline $\mathrm{HCL}$ & $5.43799 E-17$ & 0.0 & 0.0 & 0.0 \\
\hline HNO3 & $1.29256 \mathrm{E}-12$ & 0.0 & 0.0 & 0.0 \\
\hline LAURICACID & $6.56959 E-05$ & 0.0 & 0.0 & 0.0 \\
\hline OHION & $8.80619 \mathrm{E}-11$ & 0.0 & 0.0 & 0.0 \\
\hline CLION & $1.60046 E-06$ & 0.0 & 0.0 & 0.0 \\
\hline CO3ION & $1.09062 \mathrm{E}-11$ & 0.0 & 0.0 & 0.0 \\
\hline DODECION & $1.39438 E-05$ & 0.0 & 0.0 & 0.0 \\
\hline HCO3ION & $1.30842 E-05$ & 0.0 & 0.0 & 0.0 \\
\hline $\mathrm{HION}$ & $2.90807 \mathrm{E}-05$ & 0.0 & 0.0 & 0.0 \\
\hline NO3ION & $\begin{array}{l}\text { 4.52108E-07 } \\
============\end{array}$ & $\begin{array}{l}0.0 \\
============\end{array}$ & $\begin{array}{l}0.0 \\
=============\end{array}$ & $\begin{array}{l}0.0 \\
=============\end{array}$ \\
\hline Total g/hr & 498.128 & 0.0 & 0.0 & 0.0 \\
\hline Volume, L/hr & 0.499672 & 0.0 & 0.0 & 0.0 \\
\hline $\begin{array}{l}\text { Enthalpy, cal/hr } \\
\text { Density, g/L }\end{array}$ & $\begin{array}{l}-1.88871 E+06 \\
996.909\end{array}$ & 0.0 & 0.0 & 0.0 \\
\hline Vapor fraction & 0.0 & 0.0 & 0.0 & 0.0 \\
\hline Solid fraction & 0.0 & 0.0 & 0.0 & 0.0 \\
\hline Organic fraction & 0.0 & 0.0 & 0.0 & 0.0 \\
\hline Osmotic Pres, atm & 0.0871231 & & & \\
\hline Redox Pot, volts & 0.0 & & & \\
\hline E-Con, 1/ohm-cm & $2.34399 E-05$ & & & \\
\hline E-Con, cm2/ohm-mol & 6.699 & & & \\
\hline Abs Visc, cP & 0.890749 & & & \\
\hline Rel Visc & 1.00003 & & & \\
\hline Ionic Strength & $5.83911 E-05$ & & & \\
\hline
\end{tabular}


WSRC-TR-2002-00567

Revision 0

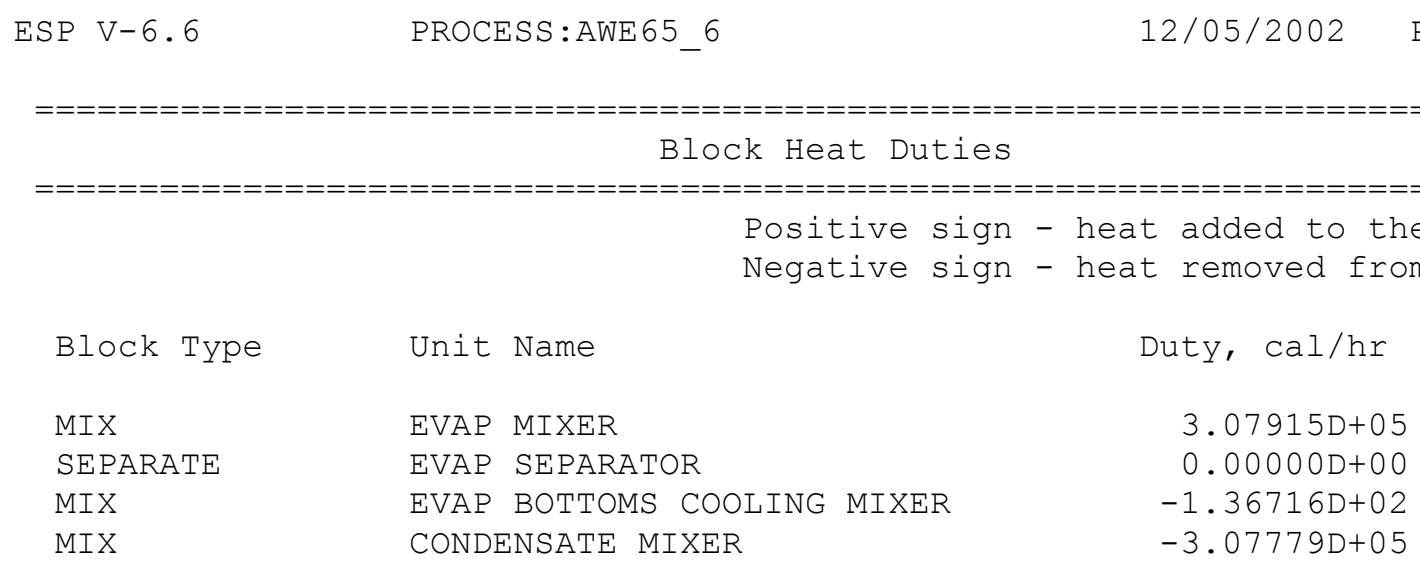


WSRC-TR-2002-00567

Revision 0

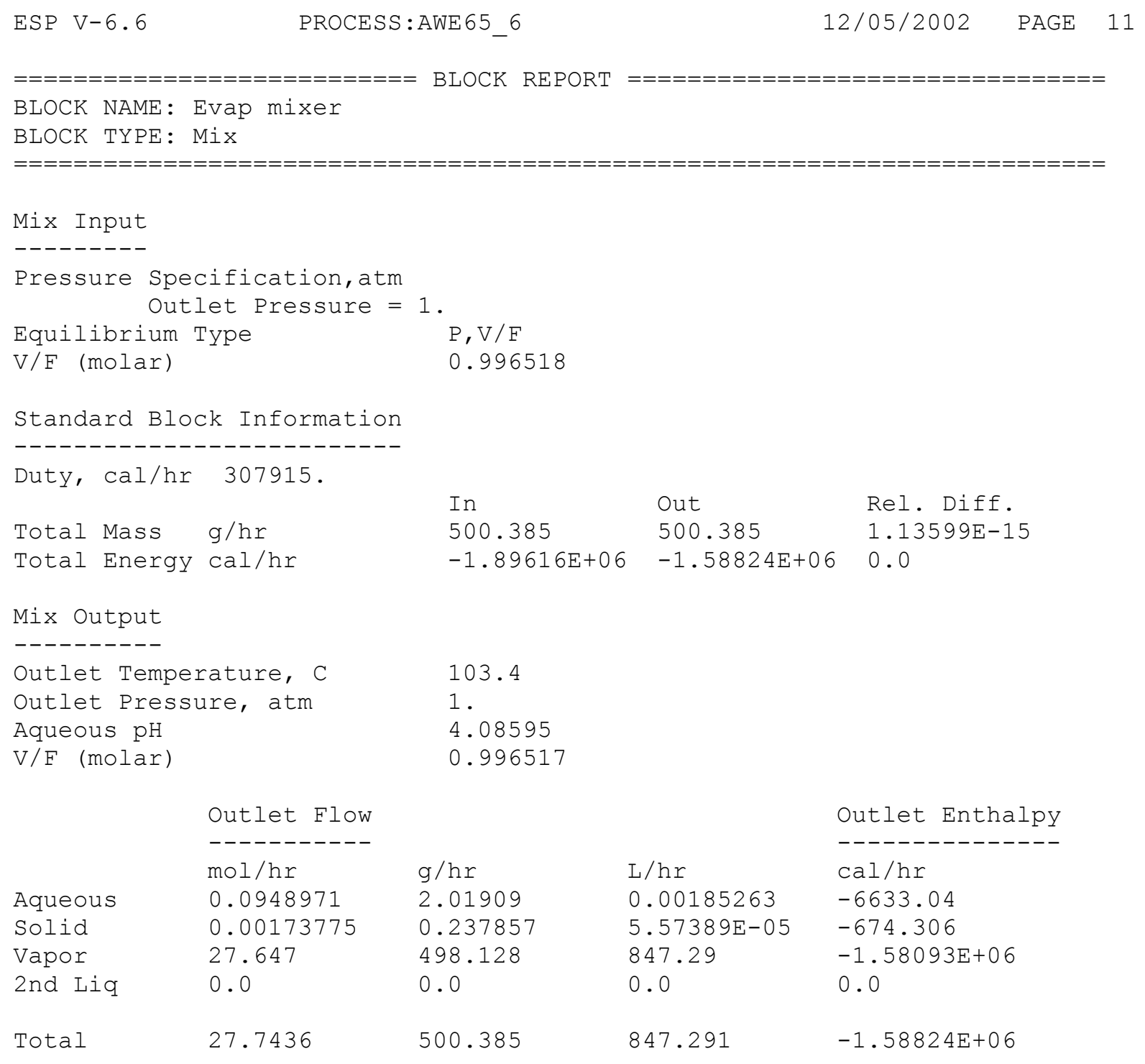


WSRC-TR-2002-00567

Revision 0

$\begin{array}{lllll}\text { ESP V-6.6 PROCESS:AWE65_6 } & \text { PAGE } & 12 / 05 / 2002\end{array}$

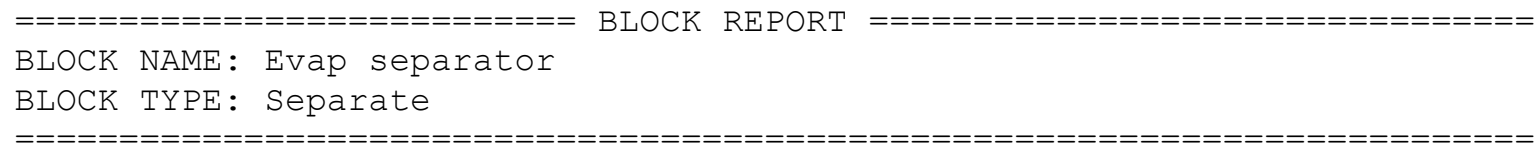

$0.0966349 \quad 2.25695$

$\mathrm{L} / \mathrm{hr} \quad \mathrm{cal} / \mathrm{hr}$

$5.57389 \mathrm{E}-05 \quad-674.306$

$0.0 \quad 0.0$

Total

$0.00190837 \quad-7307.34$

Outlet Enthalpy 
WSRC-TR-2002-00567

Revision 0

ESP V-6.6

PROCESS:AWE 656

Vapor stream

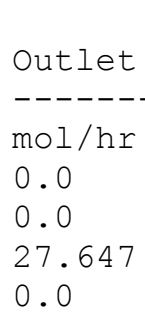

27.647
Overhead

g/hr

0.0

0.0

498.128

0.0

498.128
$12 / 05 / 2002$

PAGE

13

$\mathrm{L} / \mathrm{hr}$

0.0

0.0

847.29

0.0

Outlet Enthalpy
-----------
cal/hr
0.0
0.0
$-1.58093 \mathrm{E}+06$
0.0

847.29 
WSRC-TR-2002-00567

Revision 0

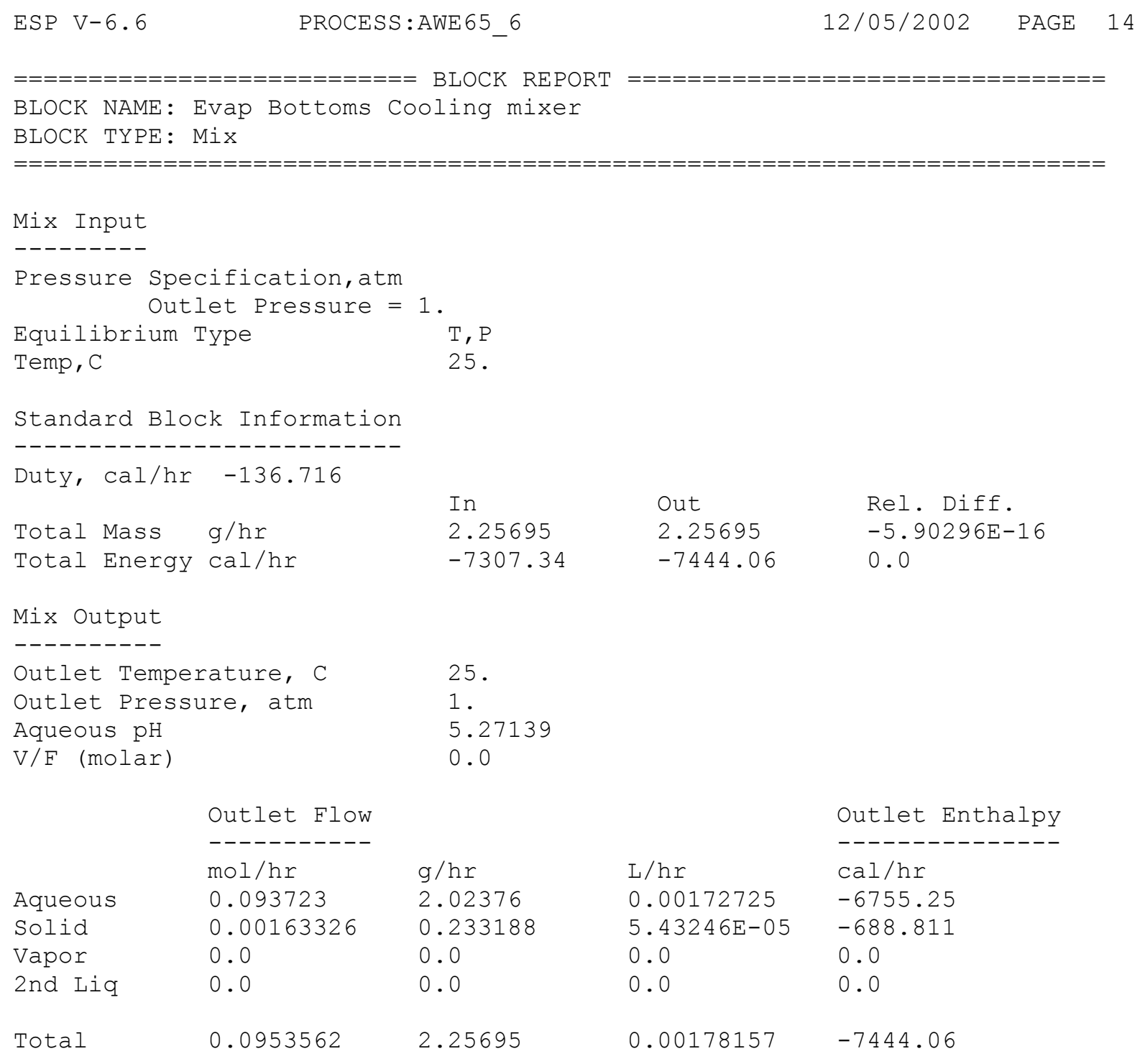


WSRC-TR-2002-00567

Revision 0

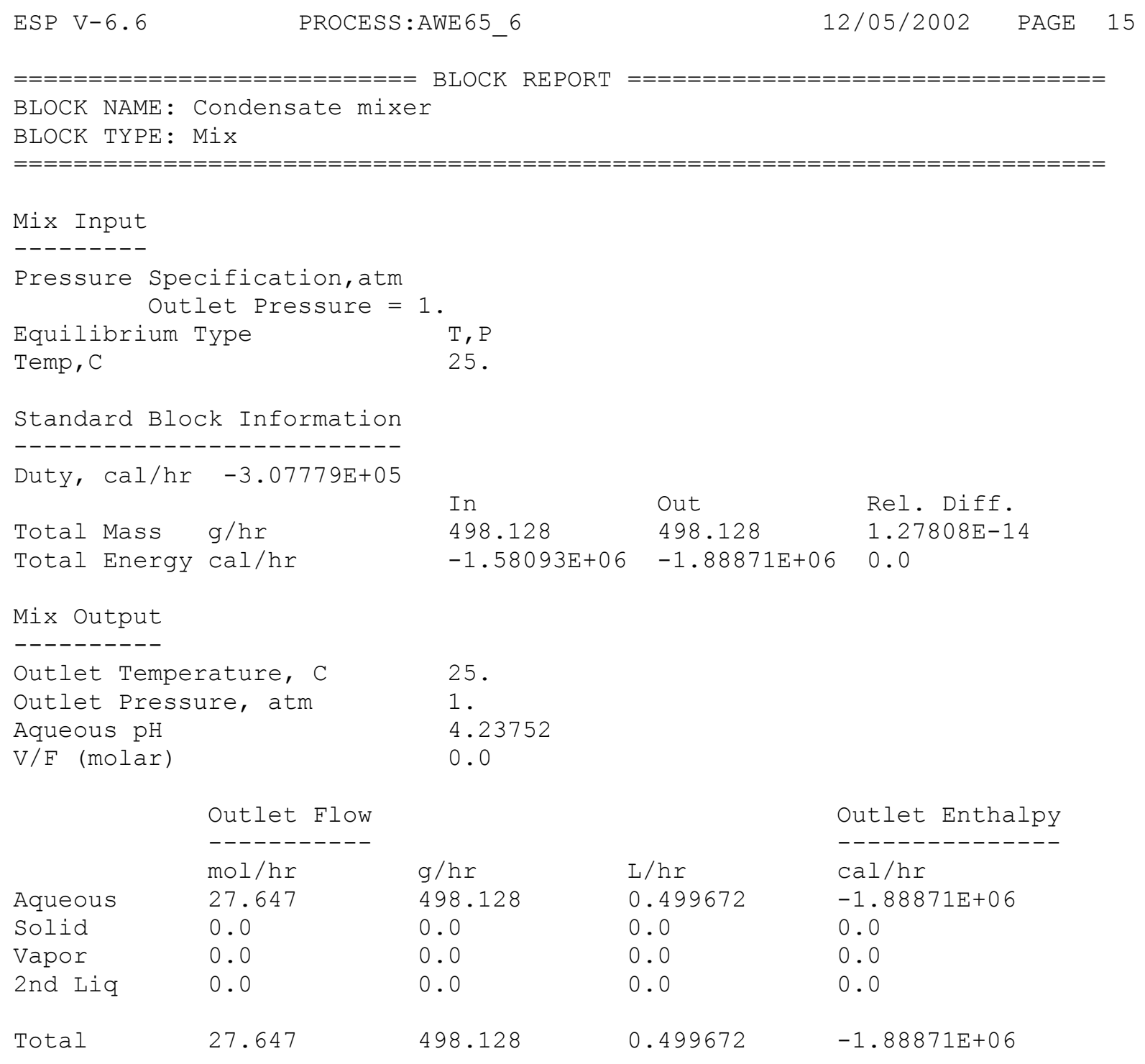


WSRC-TR-2002-00567

Revision 0

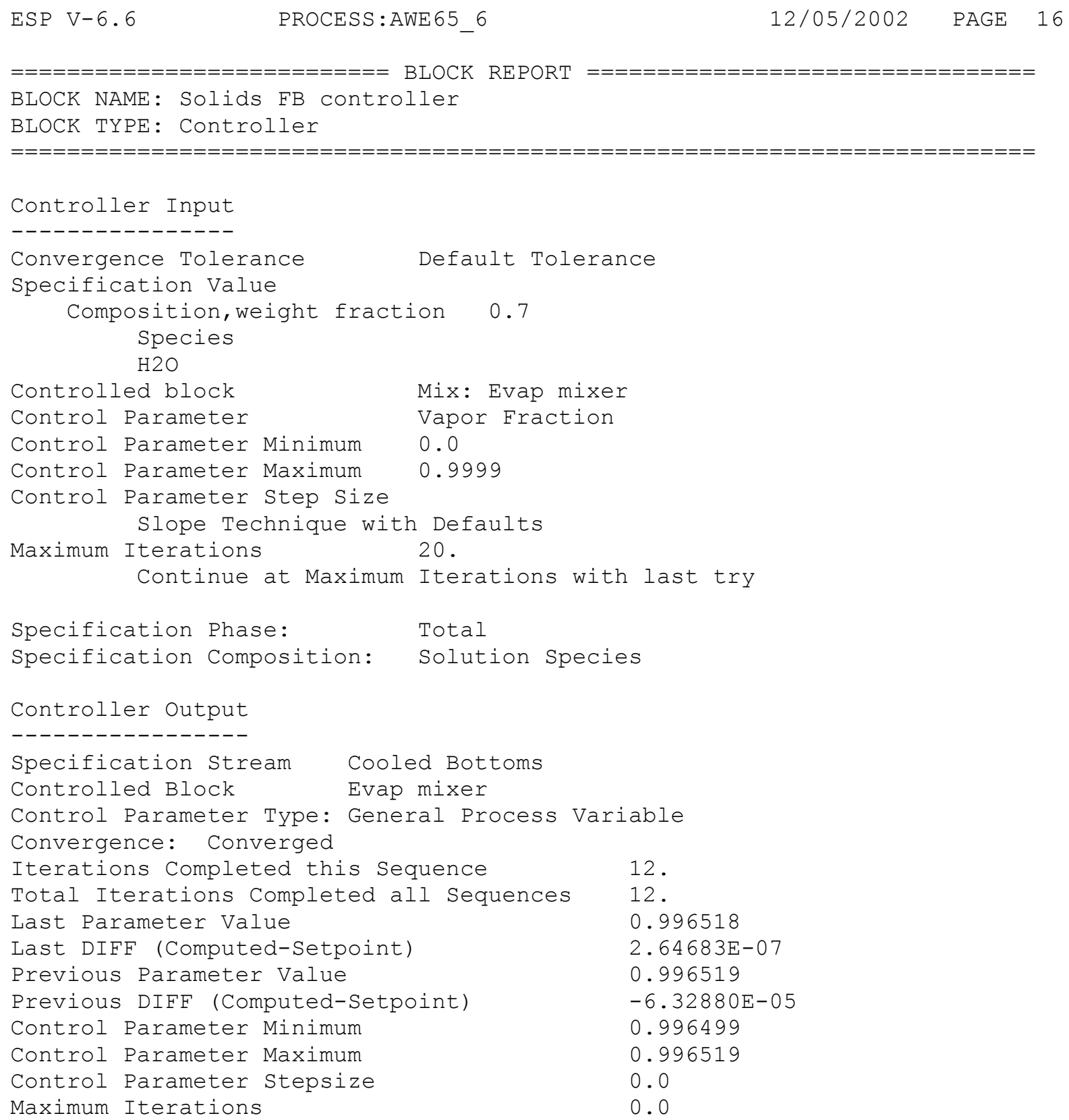


WSRC-TR-2002-00567

Revision 0

Influent Limit Composition 95\% Target $\mathrm{pH}=6.5$

AWE 6.5-95

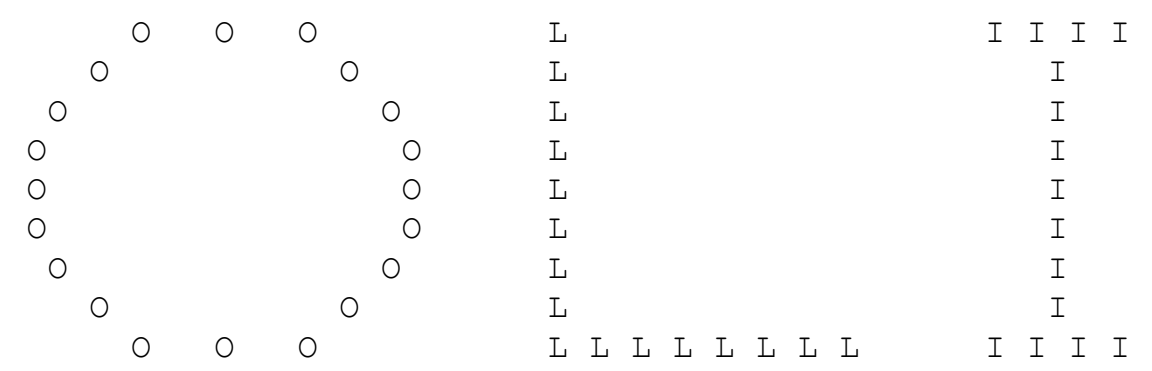

E N V I R O N M E N T A L S I M U L A T I O N P R O G R A M

$V-6.6 \quad$ September 1, 2002

PROCESS: AWE65_7

CHEMISTRY MODEL: RAW

THIS FILE NAME: AWE65 7.LIS

DATE : $12 / 05 / 2002$ 
WSRC-TR-2002-00567

Revision 0

TABLE OF CONTENTS

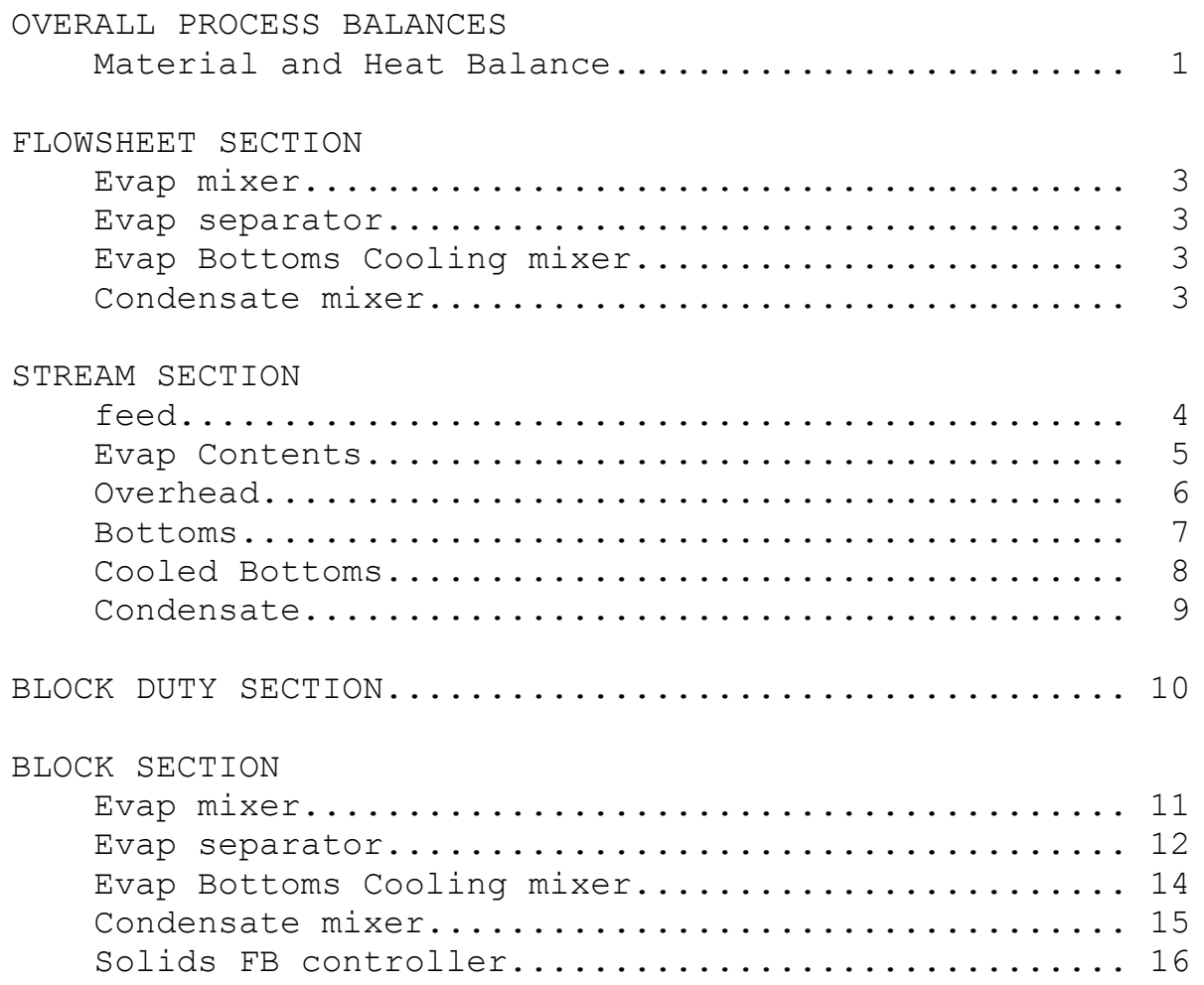


WSRC-TR-2002-00567

Revision 0

ESP V-6.6 PROCESS:AWE65_7
$\begin{array}{lcc}\text { Overall Process } & \text { Balances } \\ \text { Inlet } & \text { g/hr } & \text { Cal/hr } \\ ----------- & ---------- & ---------- \\ \text { FEED } & 5.00483 \mathrm{D}+02 & -1.89619 \mathrm{D}+06 \\ \text { ============ } & ========= & ========= \\ \text { Total in } & 5.00483 \mathrm{D}+02 & -1.89619 \mathrm{D}+06\end{array}$

\begin{tabular}{lcc} 
Outlet & \multicolumn{1}{c}{$\mathrm{g} / \mathrm{hr}$} & $\mathrm{cal} / \mathrm{hr}$ \\
---------- & ---------- & ---------- \\
COOLED BOTTOMS & $2.83132 \mathrm{D}+00$ & $-9.32613 \mathrm{D}+03$ \\
CONDENSATE & $4.97651 \mathrm{D}+02$ & $-1.88686 \mathrm{D}+06$ \\
$==========$ & $==========$ & $==========$ \\
Total out & $5.00483 \mathrm{D}+02$ & $-1.89619 \mathrm{D}+06$
\end{tabular}

12/05/2002 PAGE 1
Block Heat Duties

EVAP MIXER

EVAP BOTTOMS COOLING MIXER

CONDENSATE MIXER

Total Duty

DIFFERENCE

REL DIFEERENCE

$$
7.38964 \mathrm{D}-13 \quad 2.32831 \mathrm{D}-10
$$$$
1.47650 \mathrm{D}-15-1.22789 \mathrm{D}-16
$$

$=========$

$$
\begin{gathered}
\mathrm{cal} / \mathrm{hr} \\
---------- \\
3.07635 \mathrm{D}+05 \\
-1.67682 \mathrm{D}+02 \\
-3.07468 \mathrm{D}+05 \\
=========== \\
-7.54791 \mathrm{D}-01
\end{gathered}
$$

\begin{tabular}{|c|c|c|c|c|}
\hline Code & $\begin{array}{l}\text { Input } \\
\text { mol/hr }\end{array}$ & $\begin{array}{l}\text { Outlet } \\
\text { mol/hr }\end{array}$ & $\begin{array}{l}\text { Difference } \\
\text { mol/hr }\end{array}$ & Rel Diff \\
\hline------------ & ------------ & ------------ & ------------ & ------- \\
\hline $\mathrm{H}(+1)$ & $5.54595 \mathrm{D}+01$ & $5.54595 \mathrm{D}+01$ & $8.52651 \mathrm{D}-14$ & $1.53743 \mathrm{D}-15$ \\
\hline$K(+1)$ & $8.63895 \mathrm{D}-04$ & $8.63895 \mathrm{D}-04$ & $3.14419 \mathrm{D}-18$ & $3.63955 \mathrm{D}-15$ \\
\hline $\mathrm{NA}(+1)$ & $5.87460 \mathrm{D}-03$ & $5.87460 \mathrm{D}-03$ & $6.07153 \mathrm{D}-18$ & $1.03352 \mathrm{D}-15$ \\
\hline $\mathrm{BA}(+2)$ & $8.02920 \mathrm{D}-07$ & $8.02920 \mathrm{D}-07$ & $-1.05879 D-22$ & $-1.31868 D-16$ \\
\hline $\mathrm{CA}(+2)$ & $1.44638 D-03$ & $1.44638 D-03$ & $-2.16840 \mathrm{D}-19$ & $-1.49919 D-16$ \\
\hline $\mathrm{ZN}(+2)$ & $5.65749 \mathrm{D}-05$ & $5.65749 \mathrm{D}-05$ & $0.00000 \mathrm{D}+00$ & $0.00000 \mathrm{D}+00$ \\
\hline $\mathrm{CU}(+2)$ & $1.16535 \mathrm{D}-05$ & $1.16535 \mathrm{D}-05$ & $1.69407 \mathrm{D}-21$ & $1.45369 \mathrm{D}-16$ \\
\hline $\mathrm{FE}(+2)$ & $1.46953 \mathrm{D}-04$ & $1.46953 \mathrm{D}-04$ & $5.42101 \mathrm{D}-20$ & $3.68893 \mathrm{D}-16$ \\
\hline MG $(+2)$ & $5.76132 \mathrm{D}-04$ & $5.76132 \mathrm{D}-04$ & $-7.58942 \mathrm{D}-19$ & $-1.31731 D-15$ \\
\hline $\mathrm{PB}(+2)$ & $1.01449 \mathrm{D}-05$ & $1.01449 \mathrm{D}-05$ & $-5.08220 \mathrm{D}-21$ & $-5.00959 D-16$ \\
\hline $\mathrm{AL}(+3)$ & $2.25926 \mathrm{D}-04$ & $2.25926 \mathrm{D}-04$ & $0.00000 \mathrm{D}+00$ & $0.00000 \mathrm{D}+00$ \\
\hline $\mathrm{NI}(+2)$ & $2.55537 \mathrm{D}-06$ & $2.55537 \mathrm{D}-06$ & $-3.38813 \mathrm{D}-21$ & $-1.32589 \mathrm{D}-15$ \\
\hline$O(-2)$ & $2.77500 \mathrm{D}+01$ & $2.77500 \mathrm{D}+01$ & $3.19744 \mathrm{D}-14$ & $1.15223 \mathrm{D}-15$ \\
\hline $\mathrm{CL}(-1)$ & $4.50704 \mathrm{D}-03$ & $4.50704 \mathrm{D}-03$ & $2.60209 \mathrm{D}-18$ & $5.77338 \mathrm{D}-16$ \\
\hline$C(+4)$ & $1.83333 \mathrm{D}-03$ & $1.83333 \mathrm{D}-03$ & $3.68629 \mathrm{D}-18$ & $2.01070 \mathrm{D}-15$ \\
\hline$P(+5)$ & $1.15789 \mathrm{D}-03$ & $1.15789 \mathrm{D}-03$ & $-4.33681 D-19$ & $-3.74543 \mathrm{D}-16$ \\
\hline$S(+6)$ & $1.66667 \mathrm{D}-03$ & $1.66667 \mathrm{D}-03$ & $-4.33681 D-19$ & $-2.60209 D-16$ \\
\hline$N(+5)$ & $1.77419 \mathrm{D}-03$ & $1.77419 \mathrm{D}-03$ & $6.50521 \mathrm{D}-19$ & $3.66657 \mathrm{D}-16$ \\
\hline $\operatorname{SI}(+4)$ & $2.66667 \mathrm{D}-04$ & $2.66667 \mathrm{D}-04$ & $0.00000 \mathrm{D}+00$ & $0.00000 \mathrm{D}+00$ \\
\hline $\mathrm{SR}(+2)$ & $3.08219 \mathrm{D}-05$ & $3.08219 \mathrm{D}-05$ & $-1.35525 \mathrm{D}-20$ & $-4.39704 D-16$ \\
\hline
\end{tabular}

Material Code Balances 
WSRC-TR-2002-00567

Revision 0

$\mathrm{CD}(+2)$

$\mathrm{CR}(+3)$

$\mathrm{U}(+4)$
$6.96429 \mathrm{D}-08$

2. $50000 \mathrm{D}-06$

1.17647D-05

$6.96429 \mathrm{D}-08-8.33798 \mathrm{D}-22-1.19725 \mathrm{D}-14$

$2.50000 \mathrm{D}-06-8.04681 \mathrm{D}-21-3.21873 \mathrm{D}-15$

$1.17647 \mathrm{D}-05 \quad 0.00000 \mathrm{D}+00 \quad 0.00000 \mathrm{D}+00$ 
WSRC-TR-2002-00567

Revision 0

ESP V-6.6

$\operatorname{DODEC}(-1)$
PROCESS:AWE 657

$1.34809 D-04$

1.34809D-04

$12 / 05 / 2002$

PAGE

$4.20128 \mathrm{D}-18$

$3.11647 \mathrm{D}-14$ 
WSRC-TR-2002-00567

Revision 0

\begin{tabular}{|c|c|c|c|}
\hline \multicolumn{4}{|l|}{$\begin{array}{l}\text { PROCESS BLOCKS } \\
===========\end{array}$} \\
\hline $\begin{array}{l}\mathrm{BLOCK} \mathrm{NAME} \\
=========================\end{array}$ & $\begin{array}{l}\text { BLOCK TYPE } \\
===========\end{array}$ & $\begin{array}{l}\text { INLET STREAM }(\mathrm{S}) \\
==============\end{array}$ & $\begin{array}{l}\text { OUTLET STREAM }(\mathrm{S}) \\
==============\end{array}$ \\
\hline Evap mixer & $\operatorname{Mix}$ & feed & Evap Contents \\
\hline Evap separator & Separate & Evap Contents & $\begin{array}{l}\text { Overhead } \\
\text { Bottoms }\end{array}$ \\
\hline Evap Bottoms Cooling mixer & Mix & Bottoms & Cooled Bottoms \\
\hline Condensate mixer & Mix & Overhead & Condensate \\
\hline
\end{tabular}


WSRC-TR-2002-00567

Revision 0

ESP V-6. 6

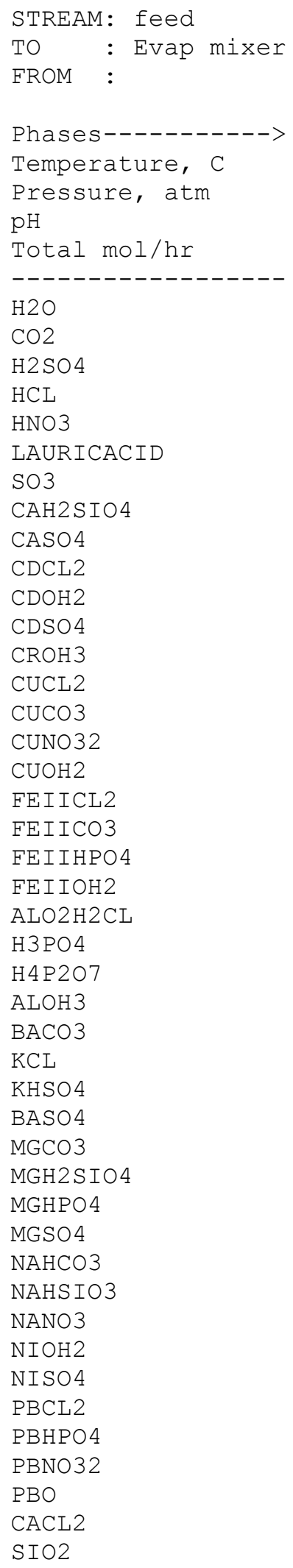

\begin{tabular}{|c|c|c|c|}
\hline Aqueous & Solid & Vapor & Organic \\
\hline 25 & 25 & 25 . & 25 . \\
\hline 1 . & 1. & 1 . & 1 . \\
\hline \multicolumn{4}{|l|}{5.60368} \\
\hline 27.74815 & $2.45642 \mathrm{E}-04$ & 0.0 & 0.0 \\
\hline \multicolumn{4}{|c|}{$\mathrm{mol} / \mathrm{hr}--------\mathrm{mol} / \mathrm{hr}--------\mathrm{mol} / \mathrm{hr}--------\mathrm{mol} / \mathrm{hr}--------$} \\
\hline 27.7281 & 0.0 & 0.0 & 0.0 \\
\hline 0.00149784 & 0.0 & 0.0 & 0.0 \\
\hline $1.76379 \mathrm{E}-23$ & 0.0 & 0.0 & 0.0 \\
\hline $5.65729 E-15$ & 0.0 & 0.0 & 0.0 \\
\hline $1.85094 \mathrm{E}-10$ & 0.0 & 0.0 & 0.0 \\
\hline $1.97534 \mathrm{E}-05$ & 0.0 & 0.0 & 0.0 \\
\hline $2.28440 \mathrm{E}-27$ & 0.0 & 0.0 & 0.0 \\
\hline $3.79299 E-14$ & 0.0 & 0.0 & 0.0 \\
\hline $4.05967 \mathrm{E}-05$ & 0.0 & 0.0 & 0.0 \\
\hline $2.29997 E-09$ & 0.0 & 0.0 & 0.0 \\
\hline $2.16848 \mathrm{E}-17$ & 0.0 & 0.0 & 0.0 \\
\hline $1.13812 \mathrm{E}-08$ & 0.0 & 0.0 & 0.0 \\
\hline $1.05212 \mathrm{E}-14$ & 0.0 & 0.0 & 0.0 \\
\hline $4.79192 \mathrm{E}-12$ & 0.0 & 0.0 & 0.0 \\
\hline $1.88499 \mathrm{E}-09$ & 0.0 & 0.0 & 0.0 \\
\hline $1.54486 \mathrm{E}-13$ & 0.0 & 0.0 & 0.0 \\
\hline $4.51695 \mathrm{E}-11$ & 0.0 & 0.0 & 0.0 \\
\hline $2.26952 \mathrm{E}-14$ & 0.0 & 0.0 & 0.0 \\
\hline $1.02746 \mathrm{E}-07$ & 0.0 & 0.0 & 0.0 \\
\hline $8.34029 E-08$ & 0.0 & 0.0 & 0.0 \\
\hline $3.08165 \mathrm{E}-14$ & 0.0 & 0.0 & 0.0 \\
\hline $1.09505 \mathrm{E}-27$ & 0.0 & 0.0 & 0.0 \\
\hline $2.97840 \mathrm{E}-07$ & 0.0 & 0.0 & 0.0 \\
\hline $3.16403 E-16$ & 0.0 & 0.0 & 0.0 \\
\hline $1.19645 \mathrm{E}-09$ & $2.25923 E-04$ & 0.0 & 0.0 \\
\hline $1.58317 \mathrm{E}-13$ & 0.0 & 0.0 & 0.0 \\
\hline $4.59431 E-08$ & 0.0 & 0.0 & 0.0 \\
\hline $5.06003 E-12$ & 0.0 & 0.0 & 0.0 \\
\hline $1.02541 \mathrm{E}-10$ & $7.25183 E-07$ & 0.0 & 0.0 \\
\hline $2.60519 E-09$ & 0.0 & 0.0 & 0.0 \\
\hline $1.39514 \mathrm{E}-13$ & 0.0 & 0.0 & 0.0 \\
\hline $1.34588 E-05$ & 0.0 & 0.0 & 0.0 \\
\hline $2.69254 \mathrm{E}-05$ & 0.0 & 0.0 & 0.0 \\
\hline $1.12537 \mathrm{E}-06$ & 0.0 & 0.0 & 0.0 \\
\hline $1.83588 E-08$ & 0.0 & 0.0 & 0.0 \\
\hline $8.48980 \mathrm{E}-07$ & 0.0 & 0.0 & 0.0 \\
\hline $1.85161 \mathrm{E}-14$ & 0.0 & 0.0 & 0.0 \\
\hline $3.58354 \mathrm{E}-07$ & 0.0 & 0.0 & 0.0 \\
\hline $9.72104 \mathrm{E}-11$ & 0.0 & 0.0 & 0.0 \\
\hline $1.15530 \mathrm{E}-09$ & 0.0 & 0.0 & 0.0 \\
\hline $2.86081 E-12$ & 0.0 & 0.0 & 0.0 \\
\hline $2.69547 E-14$ & 0.0 & 0.0 & 0.0 \\
\hline $3.83791 E-26$ & 0.0 & 0.0 & 0.0 \\
\hline $2.66629 E-04$ & 0.0 & 0.0 & 0.0 \\
\hline
\end{tabular}




\begin{tabular}{|c|c|c|c|c|}
\hline $\mathrm{CACO} 3$ & $1.51720 \mathrm{E}-08$ & 0.0 & 0.0 & 0.0 \\
\hline SRHPO 4 & $1.06902 \mathrm{E}-08$ & 0.0 & 0.0 & 0.0 \\
\hline SRNO32 & $1.02722 \mathrm{E}-09$ & 0.0 & 0.0 & 0.0 \\
\hline $\mathrm{SRSO} 4$ & $4.20736 \mathrm{E}-06$ & 0.0 & 0.0 & 0.0 \\
\hline UIVOH 4 & $1.50389 \mathrm{E}-10$ & 0.0 & 0.0 & 0.0 \\
\hline UIVSO 42 & $1.10268 \mathrm{E}-23$ & 0.0 & 0.0 & 0.0 \\
\hline ZNCL2 & $2.76132 \mathrm{E}-09$ & 0.0 & 0.0 & 0.0 \\
\hline ZNHPO 4 & $2.09918 \mathrm{E}-06$ & 0.0 & 0.0 & 0.0 \\
\hline ZNNO32 & $1.56630 \mathrm{E}-10$ & 0.0 & 0.0 & 0.0 \\
\hline ZNOH2 & $2.10506 \mathrm{E}-11$ & 0.0 & 0.0 & 0.0 \\
\hline OHION & $2.39004 \mathrm{E}-09$ & 0.0 & 0.0 & 0.0 \\
\hline ALION & $3.16038 \mathrm{E}-10$ & 0.0 & 0.0 & 0.0 \\
\hline ALOH 2 ION & $6.45509 \mathrm{E}-10$ & 0.0 & 0.0 & 0.0 \\
\hline ALOH 4 ION & $1.42738 \mathrm{E}-10$ & 0.0 & 0.0 & 0.0 \\
\hline ALOHCLION & $8.89805 \mathrm{E}-12$ & 0.0 & 0.0 & 0.0 \\
\hline ALOHION & $5.67566 \mathrm{E}-10$ & 0.0 & 0.0 & 0.0 \\
\hline ALSO 42 ION & $1.68682 \mathrm{E}-11$ & 0.0 & 0.0 & 0.0 \\
\hline ALSO 4 ION & $1.38836 \mathrm{E}-10$ & 0.0 & 0.0 & 0.0 \\
\hline BAHCO3ION & $2.35937 \mathrm{E}-10$ & 0.0 & 0.0 & 0.0 \\
\hline BAION & $7.73966 \mathrm{E}-08$ & 0.0 & 0.0 & 0.0 \\
\hline BAOHION & $9.58513 \mathrm{E}-17$ & 0.0 & 0.0 & 0.0 \\
\hline CACLION & $1.46462 \mathrm{E}-10$ & 0.0 & 0.0 & 0.0 \\
\hline CAH2 PO 4 ION & $5.17204 \mathrm{E}-05$ & 0.0 & 0.0 & 0.0 \\
\hline CAHCO3ION & $5.01896 \mathrm{E}-06$ & 0.0 & 0.0 & 0.0 \\
\hline CAHSIO3ION & $4.62499 \mathrm{E}-10$ & 0.0 & 0.0 & 0.0 \\
\hline CAION & 0.00134251 & 0.0 & 0.0 & 0.0 \\
\hline CANO3ION & $6.49272 \mathrm{E}-06$ & 0.0 & 0.0 & 0.0 \\
\hline CAOHION & $5.67770 \mathrm{E}-11$ & 0.0 & 0.0 & 0.0 \\
\hline CAPO 4 ION & $2.54255 \mathrm{E}-08$ & 0.0 & 0.0 & 0.0 \\
\hline CDCL3ION & $1.00749 \mathrm{E}-12$ & 0.0 & 0.0 & 0.0 \\
\hline CDCL 4 ION & $7.05667 \mathrm{E}-15$ & 0.0 & 0.0 & 0.0 \\
\hline CDCLION & $1.66181 \mathrm{E}-08$ & 0.0 & 0.0 & 0.0 \\
\hline CDION & $3.91504 \mathrm{E}-08$ & 0.0 & 0.0 & 0.0 \\
\hline CDNO3ION & $1.91421 \mathrm{E}-10$ & 0.0 & 0.0 & 0.0 \\
\hline CDOH 3 ION & $1.44353 \mathrm{E}-24$ & 0.0 & 0.0 & 0.0 \\
\hline CDOHION & $7.92526 \mathrm{E}-13$ & 0.0 & 0.0 & 0.0 \\
\hline CLION & 0.00450658 & 0.0 & 0.0 & 0.0 \\
\hline CO3ION & $1.01100 \mathrm{E}-08$ & 0.0 & 0.0 & 0.0 \\
\hline CRIIICL2ION & $8.84968 \mathrm{E}-18$ & 0.0 & 0.0 & 0.0 \\
\hline CRIIICLION & $1.05862 \mathrm{E}-14$ & 0.0 & 0.0 & 0.0 \\
\hline CRIIIH2PO4ION & $2.31212 \mathrm{E}-11$ & 0.0 & 0.0 & 0.0 \\
\hline CRIIIHPO4ION & $2.49887 \mathrm{E}-06$ & 0.0 & 0.0 & 0.0 \\
\hline CRIIIION & $7.78494 \mathrm{E}-13$ & 0.0 & 0.0 & 0.0 \\
\hline CRIIINO3ION & $2.42945 \mathrm{E}-13$ & 0.0 & 0.0 & 0.0 \\
\hline CROH2ION & $9.13881 \mathrm{E}-15$ & 0.0 & 0.0 & 0.0 \\
\hline CROH 4 ION & $5.79094 \mathrm{E}-20$ & 0.0 & 0.0 & 0.0 \\
\hline CROHION & $1.42971 \mathrm{E}-11$ & 0.0 & 0.0 & 0.0 \\
\hline CRSO 4 ION & $1.09027 \mathrm{E}-09$ & 0.0 & 0.0 & 0.0 \\
\hline CUCL3ION & $9.09616 \mathrm{E}-17$ & 0.0 & 0.0 & 0.0 \\
\hline CUCLION & $1.61959 \mathrm{E}-09$ & 0.0 & 0.0 & 0.0 \\
\hline CUCO32ION & $5.78504 \mathrm{E}-14$ & 0.0 & 0.0 & 0.0 \\
\hline CUION & $6.39602 \mathrm{E}-08$ & 0.0 & 0.0 & 0.0 \\
\hline CUNO3ION & $4.25079 \mathrm{E}-10$ & 0.0 & 0.0 & 0.0 \\
\hline CUOH 3 ION & $1.16224 \mathrm{E}-17$ & 0.0 & 0.0 & 0.0 \\
\hline CUOH 4 ION & $5.78570 \mathrm{E}-25$ & 0.0 & 0.0 & 0.0 \\
\hline CUOHION & $7.31630 \mathrm{E}-10$ & 0.0 & 0.0 & 0.0 \\
\hline DODECION & $1.15056 \mathrm{E}-04$ & 0.0 & 0.0 & 0.0 \\
\hline FEIICLION & $9.48384 \mathrm{E}-10$ & 0.0 & 0.0 & 0. \\
\hline
\end{tabular}




\begin{tabular}{|c|c|c|c|c|}
\hline FEIICO32ION & $6.85106 \mathrm{E}-14$ & 0.0 & 0.0 & 0.0 \\
\hline FEIIH2PO4ION & $5.00200 \mathrm{E}-07$ & 0.0 & 0.0 & 0.0 \\
\hline EEIIHCO3ION & $7.20968 \mathrm{E}-09$ & 0.0 & 0.0 & 0.0 \\
\hline FEIIION & $1.46248 \mathrm{E}-04$ & 0.0 & 0.0 & 0.0 \\
\hline FEIIOH3ION & $5.77783 \mathrm{E}-19$ & 0.0 & 0.0 & 0.0 \\
\hline FEIIOH 4 ION & $3.98331 \mathrm{E}-28$ & 0.0 & 0.0 & 0.0 \\
\hline FEIIOHION & $1.13698 \mathrm{E}-08$ & 0.0 & 0.0 & 0.0 \\
\hline H2 P2O 7 ION & $1.28836 \mathrm{E}-08$ & 0.0 & 0.0 & 0.0 \\
\hline H2 PO 4 ION & 0.00100356 & 0.0 & 0.0 & 0.0 \\
\hline $\mathrm{H} 2 \mathrm{SIO} 4 \mathrm{ION}$ & $9.53958 \mathrm{E}-16$ & 0.0 & 0.0 & 0.0 \\
\hline H3P2O7 ION & $4.26414 \mathrm{E}-12$ & 0.0 & 0.0 & 0.0 \\
\hline H3SIO 4 ION & $1.80858 \mathrm{E}-08$ & 0.0 & 0.0 & 0.0 \\
\hline HCO3ION & $3.22344 \mathrm{E}-04$ & 0.0 & 0.0 & 0.0 \\
\hline HION & $1.45839 \mathrm{E}-06$ & 0.0 & 0.0 & 0.0 \\
\hline HP207ION & $2.95633 \mathrm{E}-09$ & 0.0 & 0.0 & 0.0 \\
\hline HPBO2ION & $1.43540 \mathrm{E}-19$ & 0.0 & 0.0 & 0.0 \\
\hline HPO4ION & $4.17859 \mathrm{E}-05$ & 0.0 & 0.0 & 0.0 \\
\hline HSO4 ION & $2.14258 \mathrm{E}-07$ & 0.0 & 0.0 & 0.0 \\
\hline KION & $8.53861 \mathrm{E}-04$ & 0.0 & 0.0 & 0.0 \\
\hline KSO4 ION & $9.98780 \mathrm{E}-06$ & 0.0 & 0.0 & 0.0 \\
\hline MGH2 PO 4 ION & $2.52926 \mathrm{E}-05$ & 0.0 & 0.0 & 0.0 \\
\hline MGHCO3ION & $6.48610 \mathrm{E}-06$ & 0.0 & 0.0 & 0.0 \\
\hline MGHSIO3ION & $3.25174 \mathrm{E}-10$ & 0.0 & 0.0 & 0.0 \\
\hline MGION & $5.03949 \mathrm{E}-04$ & 0.0 & 0.0 & 0.0 \\
\hline MGOHION & $2.07074 \mathrm{E}-10$ & 0.0 & 0.0 & 0.0 \\
\hline MGP2O7ION & $2.80310 \mathrm{E}-09$ & 0.0 & 0.0 & 0.0 \\
\hline MGPO 4 ION & $1.38152 \mathrm{E}-08$ & 0.0 & 0.0 & 0.0 \\
\hline NACO3ION & $2.12187 \mathrm{E}-10$ & 0.0 & 0.0 & 0.0 \\
\hline NAION & 0.00579786 & 0.0 & 0.0 & 0.0 \\
\hline NASO 4 ION & $7.47469 \mathrm{E}-05$ & 0.0 & 0.0 & 0.0 \\
\hline NICLION & $1.02433 E-09$ & 0.0 & 0.0 & 0.0 \\
\hline NIION & $2.18436 \mathrm{E}-06$ & 0.0 & 0.0 & 0.0 \\
\hline NINO3ION & $1.15558 \mathrm{E}-08$ & 0.0 & 0.0 & 0.0 \\
\hline NIOH3ION & $9.04497 \mathrm{E}-20$ & 0.0 & 0.0 & 0.0 \\
\hline NIOHION & $7.44978 \mathrm{E}-11$ & 0.0 & 0.0 & 0.0 \\
\hline NO3ION & 0.00176629 & 0.0 & 0.0 & 0.0 \\
\hline P207ION & $1.92795 \mathrm{E}-12$ & 0.0 & 0.0 & 0.0 \\
\hline PBCL3ION & $4.32853 \mathrm{E}-13$ & 0.0 & 0.0 & 0.0 \\
\hline PBCL 4 ION & $3.47324 \mathrm{E}-15$ & 0.0 & 0.0 & 0.0 \\
\hline PBCLION & $4.13194 \mathrm{E}-09$ & 0.0 & 0.0 & 0.0 \\
\hline PBH2 PO 4 ION & $1.38084 \mathrm{E}-09$ & 0.0 & 0.0 & 0.0 \\
\hline PBION & $3.24520 \mathrm{E}-08$ & 0.0 & 0.0 & 0.0 \\
\hline PBNO33ION & $1.74308 \mathrm{E}-15$ & 0.0 & 0.0 & 0.0 \\
\hline PBNO3ION & $1.16529 \mathrm{E}-09$ & 0.0 & 0.0 & 0.0 \\
\hline PBOHION & $2.00323 E-10$ & 0.0 & 0.0 & 0.0 \\
\hline PO4ION & $1.83768 \mathrm{E}-11$ & 0.0 & 0.0 & 0.0 \\
\hline SO4ION & 0.00150889 & 0.0 & 0.0 & 0.0 \\
\hline SRION & $2.63274 \mathrm{E}-05$ & 0.0 & 0.0 & 0.0 \\
\hline SRNO3ION & $2.75445 \mathrm{E}-07$ & 0.0 & 0.0 & 0.0 \\
\hline SROHION & $4.21676 \mathrm{E}-13$ & 0.0 & 0.0 & 0.0 \\
\hline SRPO 4 ION & $6.99162 \mathrm{E}-12$ & 0.0 & 0.0 & 0.0 \\
\hline UIVCLION & $4.40388 \mathrm{E}-28$ & 0.0 & 0.0 & 0.0 \\
\hline UIVION & $3.45804 \mathrm{E}-27$ & 0.0 & 0.0 & 0.0 \\
\hline UIVOH2ION & $2.18413 \mathrm{E}-19$ & 0.0 & 0.0 & 0.0 \\
\hline UIVOH3ION & $4.98954 \mathrm{E}-15$ & 0.0 & 0.0 & 0.0 \\
\hline UIVOH5ION & $3.74159 \mathrm{E}-15$ & 0.0 & 0.0 & 0.0 \\
\hline UIVOHION & $1.86675 \mathrm{E}-22$ & 0.0 & 0.0 & 0.0 \\
\hline UIVSO 4 ION & $1.91800 \mathrm{E}-24$ & 0.0 & 0.0 & 0.0 \\
\hline
\end{tabular}


WSRC-TR-2002-00567

Revision 0

\begin{tabular}{|c|c|c|c|c|}
\hline ZNCL3ION & $8.54419 \mathrm{E}-12$ & 0.0 & 0.0 & 0.0 \\
\hline ZNCLION & $3.80237 \mathrm{E}-07$ & 0.0 & 0.0 & 0.0 \\
\hline ZNH2 PO4 ION & $2.04952 E-06$ & 0.0 & 0.0 & 0.0 \\
\hline ZNHCO3ION & $3.74037 \mathrm{E}-07$ & 0.0 & 0.0 & 0.0 \\
\hline ZNION & $5.13833 E-05$ & 0.0 & 0.0 & 0.0 \\
\hline ZNNO3ION & $2.70469 \mathrm{E}-07$ & 0.0 & 0.0 & 0.0 \\
\hline ZNOH3ION & $9.86603 E-17$ & 0.0 & 0.0 & 0.0 \\
\hline ZNOH 4 ION & $8.49963 E-24$ & 0.0 & 0.0 & 0.0 \\
\hline ZNOHION & $1.52618 \mathrm{E}-08$ & 0.0 & 0.0 & 0.0 \\
\hline $\mathrm{CU} 3 \mathrm{PO} 42.2 \mathrm{H} 2 \mathrm{O}$ & 0.0 & $3.86161 \mathrm{E}-06$ & 0.0 & 0.0 \\
\hline $\mathrm{PB} 3 \mathrm{PO} 42$ & 0.0 & $3.36810 E-06$ & 0.0 & 0.0 \\
\hline UIVO2 & 0.0 & $1.17645 \mathrm{E}-05$ & 0.0 & 0.0 \\
\hline & $============$ & $===========$ & $============$ & $===========$ \\
\hline Total g/hr & 500.457 & 0.0253109 & 0.0 & 0.0 \\
\hline Volume, L/hr & 0.501348 & $7.93309 E-06$ & 0.0 & 0.0 \\
\hline Enthalpy, cal/hr & $-1.89611 E+06$ & -73.6652 & 0.0 & 0.0 \\
\hline Density, g/L & 998.223 & 3190.55 & & \\
\hline Vapor fraction & 0.0 & 0.0 & 0.0 & 0.0 \\
\hline Solid fraction & 0.0 & 1 . & 0.0 & 0.0 \\
\hline Organic fraction & 0.0 & 0.0 & 0.0 & 0.0 \\
\hline Osmotic Pres, atm & 0.99467 & & & \\
\hline Redox Pot, volts & 0.0 & & & \\
\hline E-Con, 1/ohm-cm & 0.00228296 & & & \\
\hline E-Con, cm2/ohm-mol & 114.309 & & & \\
\hline Abs Visc, cP & 0.895376 & & & \\
\hline Rel Visc & 1.00523 & & & \\
\hline Ionic Strength & 0.029075 & & & \\
\hline
\end{tabular}


WSRC-TR-2002-00567

Revision 0

ESP V-6.6

STREAM: Evap Contents

TO : Evap separator

FROM : Evap mixer

\begin{tabular}{|c|c|c|c|c|}
\hline Phases-----------> & Aqueous & Solid & Vapor & Organic \\
\hline Temperature, C & 103.368 & 103.368 & 103.368 & 103.368 \\
\hline Pressure, atm & 1 . & 1 . & 1 . & 1 . \\
\hline $\mathrm{pH}$ & 3.92509 & & & \\
\hline Total mol/hr & 0.1250164 & 0.00201467 & 27.6197 & 0.0 \\
\hline------------------ & $\operatorname{mol} / \mathrm{hr}------$ & mol/hr------ & mol/hr------ & -mol/hr \\
\hline $\mathrm{H} 2 \mathrm{O}$ & 0.11131 & 0.0 & 27.61773 & 0.0 \\
\hline $\mathrm{CO} 2$ & $9.09648 \mathrm{E}-10$ & 0.0 & 0.001833325 & 0.0 \\
\hline $\mathrm{H} 2 \mathrm{SO} 4$ & $3.96088 \mathrm{E}-21$ & 0.0 & $9.51677 \mathrm{E}-19$ & 0.0 \\
\hline $\mathrm{HCL}$ & $8.39440 E-12$ & 0.0 & $2.09860 E-06$ & 0.0 \\
\hline HNO3 & $1.17320 \mathrm{E}-08$ & 0.0 & $7.32824 \mathrm{E}-07$ & 0.0 \\
\hline LAURICACID & $9.68366 \mathrm{E}-09$ & 0.0 & $1.34776 \mathrm{E}-04$ & 0.0 \\
\hline $\mathrm{SO} 3$ & $2.89289 \mathrm{E}-24$ & 0.0 & $2.56128 E-24$ & 0.0 \\
\hline $\mathrm{CAH} 2 \mathrm{SIO} 4$ & $3.21177 E-18$ & 0.0 & 0.0 & 0.0 \\
\hline $\mathrm{CASO} 4$ & $1.10733 E-06$ & $9.66224 \mathrm{E}-04$ & 0.0 & 0.0 \\
\hline CDCL2 & $3.11986 \mathrm{E}-08$ & 0.0 & 0.0 & 0.0 \\
\hline $\mathrm{CDOH} 2$ & $1.58946 \mathrm{E}-20$ & 0.0 & 0.0 & 0.0 \\
\hline $\mathrm{CDSO} 4$ & $1.41964 \mathrm{E}-11$ & 0.0 & 0.0 & 0.0 \\
\hline $\mathrm{CROH} 3$ & 1.29411E-21 & 0.0 & 0.0 & 0.0 \\
\hline CUCL2 & $3.27035 E-09$ & 0.0 & 0.0 & 0.0 \\
\hline CUCO3 & $1.47896 \mathrm{E}-18$ & 0.0 & 0.0 & 0.0 \\
\hline CUNO32 & $5.71729 \mathrm{E}-11$ & 0.0 & 0.0 & 0.0 \\
\hline CUOH2 & $4.12182 \mathrm{E}-14$ & 0.0 & 0.0 & 0.0 \\
\hline FEIICL2 & $3.90602 \mathrm{E}-09$ & 0.0 & 0.0 & 0.0 \\
\hline FEIICO3 & $3.10073 E-15$ & 0.0 & 0.0 & 0.0 \\
\hline FEIIHPO4 & $1.81981 E-08$ & 0.0 & 0.0 & 0.0 \\
\hline FEIIOH2 & $2.75919 \mathrm{E}-14$ & 0.0 & 0.0 & 0.0 \\
\hline $\mathrm{ALO} 2 \mathrm{H} 2 \mathrm{CL}$ & $2.07646 \mathrm{E}-28$ & 0.0 & 0.0 & 0.0 \\
\hline Н3 304 & $1.51556 \mathrm{E}-06$ & 0.0 & 0.0 & 0.0 \\
\hline H 4 P 207 & $3.03888 \mathrm{E}-11$ & 0.0 & 0.0 & 0.0 \\
\hline ALOH3 & $4.43385 E-13$ & 0.0 & 0.0 & 0.0 \\
\hline $\mathrm{BACO} 3$ & $2.62359 \mathrm{E}-22$ & 0.0 & 0.0 & 0.0 \\
\hline $\mathrm{KCL}$ & $1.23097 \mathrm{E}-05$ & 0.0 & 0.0 & 0.0 \\
\hline KHSO 4 & $1.35271 E-08$ & 0.0 & 0.0 & 0.0 \\
\hline $\mathrm{BASO} 4$ & $2.03164 \mathrm{E}-12$ & $8.01317 E-07$ & 0.0 & 0.0 \\
\hline MGCO3 & $2.43520 \mathrm{E}-16$ & 0.0 & 0.0 & 0.0 \\
\hline MGH2SIO4 & $1.54152 \mathrm{E}-15$ & 0.0 & 0.0 & 0.0 \\
\hline MGHPO4 & $6.34409 E-06$ & 0.0 & 0.0 & 0.0 \\
\hline MGSO 4 & $3.95741 E-05$ & 0.0 & 0.0 & 0.0 \\
\hline NAHCO3 & $7.69648 \mathrm{E}-12$ & 0.0 & 0.0 & 0.0 \\
\hline NAHSIO3 & $1.42408 E-09$ & 0.0 & 0.0 & 0.0 \\
\hline NANO3 & $4.48373 E-04$ & 0.0 & 0.0 & 0.0 \\
\hline $\mathrm{NIOH} 2$ & $2.78050 \mathrm{E}-16$ & 0.0 & 0.0 & 0.0 \\
\hline $\mathrm{NISO} 4$ & $6.88027 \mathrm{E}-07$ & 0.0 & 0.0 & 0.0 \\
\hline PBCL2 & $4.88626 \mathrm{E}-07$ & 0.0 & 0.0 & 0.0 \\
\hline PBHPO4 & $5.58551 \mathrm{E}-11$ & 0.0 & 0.0 & 0.0 \\
\hline PBNO32 & $3.66364 \mathrm{E}-08$ & 0.0 & 0.0 & 0.0 \\
\hline $\mathrm{PBO}$ & $7.44376 \mathrm{E}-16$ & 0.0 & 0.0 & 0.0 \\
\hline CACL2 & $1.67220 \mathrm{E}-15$ & 0.0 & 0.0 & 0.0 \\
\hline SIO2 & $9.00498 E-06$ & $2.57660 \mathrm{E}-04$ & 0.0 & 0.0 \\
\hline
\end{tabular}




\begin{tabular}{|c|c|c|c|c|}
\hline $\mathrm{CACO} 3$ & $1.52957 \mathrm{E}-17$ & 0.0 & 0.0 & 0.0 \\
\hline SRHPO 4 & $2.26977 \mathrm{E}-11$ & 0.0 & 0.0 & 0.0 \\
\hline SRNO32 & $9.90447 \mathrm{E}-08$ & 0.0 & 0.0 & 0.0 \\
\hline $\mathrm{SRSO} 4$ & $1.90615 \mathrm{E}-08$ & $3.04456 \mathrm{E}-05$ & 0.0 & 0.0 \\
\hline UIVOH 4 & $2.35248 \mathrm{E}-12$ & 0.0 & 0.0 & 0.0 \\
\hline UIVSO 42 & $2.51329 \mathrm{E}-19$ & 0.0 & 0.0 & 0.0 \\
\hline ZNCL2 & $1.10286 \mathrm{E}-05$ & 0.0 & 0.0 & 0.0 \\
\hline ZNHPO 4 & $3.91239 E-09$ & 0.0 & 0.0 & 0.0 \\
\hline ZNNO32 & $1.17474 \mathrm{E}-08$ & 0.0 & 0.0 & 0.0 \\
\hline ZNOH2 & $1.96413 \mathrm{E}-14$ & 0.0 & 0.0 & 0.0 \\
\hline OHION & $1.48263 \mathrm{E}-11$ & 0.0 & 0.0 & 0.0 \\
\hline ALION & $3.75563 \mathrm{E}-13$ & 0.0 & 0.0 & 0.0 \\
\hline ALOH2 ION & $7.62188 \mathrm{E}-13$ & 0.0 & 0.0 & 0.0 \\
\hline ALOH 4 ION & $3.97417 \mathrm{E}-14$ & 0.0 & 0.0 & 0.0 \\
\hline ALOHCLION & $3.88439 E-12$ & 0.0 & 0.0 & 0.0 \\
\hline ALOHION & $1.04993 \mathrm{E}-12$ & 0.0 & 0.0 & 0.0 \\
\hline ALSO 42 ION & $2.54508 \mathrm{E}-12$ & 0.0 & 0.0 & 0.0 \\
\hline ALSO 4 ION & $3.04857 \mathrm{E}-12$ & 0.0 & 0.0 & 0.0 \\
\hline BAHCO 3 ION & $5.88681 \mathrm{E}-17$ & 0.0 & 0.0 & 0.0 \\
\hline BAION & $1.60165 \mathrm{E}-09$ & 0.0 & 0.0 & 0.0 \\
\hline BAOHION & $4.33691 \mathrm{E}-18$ & 0.0 & 0.0 & 0.0 \\
\hline CACLION & $5.24329 \mathrm{E}-08$ & 0.0 & 0.0 & 0.0 \\
\hline CAH2 PO 4 ION & $1.44914 \mathrm{E}-05$ & 0.0 & 0.0 & 0.0 \\
\hline CAHCO3ION & $1.02705 \mathrm{E}-13$ & 0.0 & 0.0 & 0.0 \\
\hline CAHSIO3ION & $4.39217 \mathrm{E}-12$ & 0.0 & 0.0 & 0.0 \\
\hline CAION & $1.48086 \mathrm{E}-05$ & 0.0 & 0.0 & 0.0 \\
\hline CANO3ION & $7.59966 \mathrm{E}-06$ & 0.0 & 0.0 & 0.0 \\
\hline CAOHION & $8.66165 E-13$ & 0.0 & 0.0 & 0.0 \\
\hline CAPO 4 ION & $9.14515 \mathrm{E}-12$ & 0.0 & 0.0 & 0.0 \\
\hline CDCL3ION & $2.85905 E-08$ & 0.0 & 0.0 & 0.0 \\
\hline CDCL4 ION & $7.66240 \mathrm{E}-09$ & 0.0 & 0.0 & 0.0 \\
\hline CDCLION & $2.11758 \mathrm{E}-09$ & 0.0 & 0.0 & 0.0 \\
\hline CDION & $4.25223 \mathrm{E}-11$ & 0.0 & 0.0 & 0.0 \\
\hline CDNO3ION & $1.70587 \mathrm{E}-11$ & 0.0 & 0.0 & 0.0 \\
\hline CDOH3ION & $1.21701 \mathrm{E}-27$ & 0.0 & 0.0 & 0.0 \\
\hline CDOHION & $4.33904 E-16$ & 0.0 & 0.0 & 0.0 \\
\hline CLION & 0.00436098 & 0.0 & 0.0 & 0.0 \\
\hline CO3ION & $6.32999 \mathrm{E}-17$ & 0.0 & 0.0 & 0.0 \\
\hline CRIIICL2ION & $3.67597 \mathrm{E}-16$ & 0.0 & 0.0 & 0.0 \\
\hline CRIIICLION & $2.14865 E-16$ & 0.0 & 0.0 & 0.0 \\
\hline CRIIIH2PO4 ION & $1.39309 \mathrm{E}-14$ & 0.0 & 0.0 & 0.0 \\
\hline CRIIIHPO4ION & $2.49999 \mathrm{E}-06$ & 0.0 & 0.0 & 0.0 \\
\hline CRIIIION & $3.16420 \mathrm{E}-20$ & 0.0 & 0.0 & 0.0 \\
\hline CRIIINO3ION & $2.38770 \mathrm{E}-13$ & 0.0 & 0.0 & 0.0 \\
\hline CROH2ION & $5.71496 \mathrm{E}-20$ & 0.0 & 0.0 & 0.0 \\
\hline CROH 4 ION & $1.52475 \mathrm{E}-27$ & 0.0 & 0.0 & 0.0 \\
\hline CROHION & $2.48288 \mathrm{E}-14$ & 0.0 & 0.0 & 0.0 \\
\hline CRSO 4 ION & $8.05380 \mathrm{E}-12$ & 0.0 & 0.0 & 0.0 \\
\hline CUCL3ION & $5.13947 \mathrm{E}-11$ & 0.0 & 0.0 & 0.0 \\
\hline CUCLION & $2.44226 \mathrm{E}-08$ & 0.0 & 0.0 & 0.0 \\
\hline CUCO32ION & $2.61241 \mathrm{E}-28$ & 0.0 & 0.0 & 0.0 \\
\hline CUION & $6.75839 \mathrm{E}-09$ & 0.0 & 0.0 & 0.0 \\
\hline CUNO3ION & $1.84028 \mathrm{E}-09$ & 0.0 & 0.0 & 0.0 \\
\hline CUOH3ION & $1.98431 \mathrm{E}-20$ & 0.0 & 0.0 & 0.0 \\
\hline CUOH 4 ION & $2.14572 \mathrm{E}-26$ & 0.0 & 0.0 & 0.0 \\
\hline CUOHION & $8.39765 E-12$ & 0.0 & 0.0 & 0.0 \\
\hline DODECION & $2.28748 \mathrm{E}-08$ & 0.0 & 0.0 & 0.0 \\
\hline FEIICLION & $9.72624 \mathrm{E}-07$ & 0.0 & 0.0 & 0. \\
\hline
\end{tabular}




\begin{tabular}{|c|c|c|c|c|}
\hline FEIICO32ION & $1.59405 \mathrm{E}-26$ & 0.0 & 0.0 & 0.0 \\
\hline FEIIH2PO4ION & $5.05189 \mathrm{E}-06$ & 0.0 & 0.0 & 0.0 \\
\hline EEIIHCO3ION & $2.29503 \mathrm{E}-14$ & 0.0 & 0.0 & 0.0 \\
\hline FEIIION & $1.17022 \mathrm{E}-04$ & 0.0 & 0.0 & 0.0 \\
\hline FEIIOH3ION & $2.50945 E-19$ & 0.0 & 0.0 & 0.0 \\
\hline EEIIOH4ION & $3.25817 \mathrm{E}-27$ & 0.0 & 0.0 & 0.0 \\
\hline FEIIOHION & $5.03053 \mathrm{E}-09$ & 0.0 & 0.0 & 0.0 \\
\hline H2 P2O 7 ION & $3.19441 \mathrm{E}-06$ & 0.0 & 0.0 & 0.0 \\
\hline H2 PO 4 ION & $1.37043 \mathrm{E}-04$ & 0.0 & 0.0 & 0.0 \\
\hline $\mathrm{H} 2 \mathrm{SIO} 4 \mathrm{ION}$ & $3.83273 \mathrm{E}-18$ & 0.0 & 0.0 & 0.0 \\
\hline H3P2O7 ION & $7.61574 \mathrm{E}-09$ & 0.0 & 0.0 & 0.0 \\
\hline H3SIO 4 ION & $4.68407 \mathrm{E}-10$ & 0.0 & 0.0 & 0.0 \\
\hline HCO3ION & $5.64052 \mathrm{E}-12$ & 0.0 & 0.0 & 0.0 \\
\hline HION & $3.41141 \mathrm{E}-07$ & 0.0 & 0.0 & 0.0 \\
\hline HP207ION & $3.07735 \mathrm{E}-08$ & 0.0 & 0.0 & 0.0 \\
\hline HPBO2ION & $3.48379 \mathrm{E}-21$ & 0.0 & 0.0 & 0.0 \\
\hline HPO4ION & $6.86330 \mathrm{E}-07$ & 0.0 & 0.0 & 0.0 \\
\hline HSO 4 ION & $2.69338 \mathrm{E}-06$ & 0.0 & 0.0 & 0.0 \\
\hline KION & $7.56780 \mathrm{E}-04$ & 0.0 & 0.0 & 0.0 \\
\hline KSO4 ION & $9.47923 E-05$ & 0.0 & 0.0 & 0.0 \\
\hline MGH2 PO 4 ION & $1.55635 \mathrm{E}-04$ & 0.0 & 0.0 & 0.0 \\
\hline MGHCO3ION & $6.78901 \mathrm{E}-12$ & 0.0 & 0.0 & 0.0 \\
\hline MGHSIO3ION & $2.75967 \mathrm{E}-10$ & 0.0 & 0.0 & 0.0 \\
\hline MGION & $1.70664 \mathrm{E}-04$ & 0.0 & 0.0 & 0.0 \\
\hline MGOHION & $2.29827 \mathrm{E}-10$ & 0.0 & 0.0 & 0.0 \\
\hline MGP2O7ION & $1.78507 \mathrm{E}-07$ & 0.0 & 0.0 & 0.0 \\
\hline MGPO 4 ION & $3.11503 \mathrm{E}-10$ & 0.0 & 0.0 & 0.0 \\
\hline NACO3ION & $8.84772 \mathrm{E}-18$ & 0.0 & 0.0 & 0.0 \\
\hline NAION & 0.00542622 & 0.0 & 0.0 & 0.0 \\
\hline NASO 4 ION & $6.06949 \mathrm{E}-12$ & 0.0 & 0.0 & 0.0 \\
\hline NICLION & $1.13316 \mathrm{E}-07$ & 0.0 & 0.0 & 0.0 \\
\hline NIION & $1.46176 \mathrm{E}-06$ & 0.0 & 0.0 & 0.0 \\
\hline NINO3ION & $2.92246 \mathrm{E}-07$ & 0.0 & 0.0 & 0.0 \\
\hline NIOH3ION & $3.30462 E-22$ & 0.0 & 0.0 & 0.0 \\
\hline NIOHION & $1.71099 \mathrm{E}-11$ & 0.0 & 0.0 & 0.0 \\
\hline NO3ION & 0.00131627 & 0.0 & 0.0 & 0.0 \\
\hline P207ION & $2.49268 \mathrm{E}-12$ & 0.0 & 0.0 & 0.0 \\
\hline PBCL3ION & $1.05305 \mathrm{E}-06$ & 0.0 & 0.0 & 0.0 \\
\hline PBCL 4 ION & $8.31174 \mathrm{E}-06$ & 0.0 & 0.0 & 0.0 \\
\hline PBCLION & $1.63531 \mathrm{E}-07$ & 0.0 & 0.0 & 0.0 \\
\hline PBH2 PO 4 ION & $7.59706 \mathrm{E}-09$ & 0.0 & 0.0 & 0.0 \\
\hline PBION & $1.04292 \mathrm{E}-08$ & 0.0 & 0.0 & 0.0 \\
\hline PBNO33ION & $2.14491 \mathrm{E}-09$ & 0.0 & 0.0 & 0.0 \\
\hline PBNO3ION & $7.10665 \mathrm{E}-08$ & 0.0 & 0.0 & 0.0 \\
\hline PBOHION & $4.43667 \mathrm{E}-11$ & 0.0 & 0.0 & 0.0 \\
\hline PO4ION & $5.42931 \mathrm{E}-14$ & 0.0 & 0.0 & 0.0 \\
\hline SO4ION & $5.30310 \mathrm{E}-04$ & 0.0 & 0.0 & 0.0 \\
\hline SRION & $2.36081 \mathrm{E}-09$ & 0.0 & 0.0 & 0.0 \\
\hline SRNO3ION & $2.55923 \mathrm{E}-07$ & 0.0 & 0.0 & 0.0 \\
\hline SROHION & $6.53161 \mathrm{E}-15$ & 0.0 & 0.0 & 0.0 \\
\hline SRPO 4 ION & $2.07684 \mathrm{E}-15$ & 0.0 & 0.0 & 0.0 \\
\hline UIVCLION & $7.61161 \mathrm{E}-23$ & 0.0 & 0.0 & 0.0 \\
\hline UIVION & $4.33936 \mathrm{E}-25$ & 0.0 & 0.0 & 0.0 \\
\hline UIVOH2ION & $8.21567 \mathrm{E}-19$ & 0.0 & 0.0 & 0.0 \\
\hline UIVOH3ION & $9.21960 \mathrm{E}-17$ & 0.0 & 0.0 & 0.0 \\
\hline UIVOH5ION & $1.04833 \mathrm{E}-17$ & 0.0 & 0.0 & 0.0 \\
\hline UIVOHION & $6.95426 \mathrm{E}-20$ & 0.0 & 0.0 & 0.0 \\
\hline UIVSO 4 ION & $3.57697 \mathrm{E}-20$ & 0.0 & 0.0 & 0.0 \\
\hline
\end{tabular}


WSRC-TR-2002-00567

Revision 0

\begin{tabular}{|c|c|c|c|c|}
\hline ZNCL3ION & $1.37685 E-05$ & 0.0 & 0.0 & 0.0 \\
\hline ZNCLION & $2.93880 \mathrm{E}-05$ & 0.0 & 0.0 & 0.0 \\
\hline ZNH2 PO4 ION & $2.58531 E-07$ & 0.0 & 0.0 & 0.0 \\
\hline ZNHCO3ION & $9.23907 \mathrm{E}-15$ & 0.0 & 0.0 & 0.0 \\
\hline ZNION & $1.82777 \mathrm{E}-06$ & 0.0 & 0.0 & 0.0 \\
\hline ZNNO3ION & $2.81824 \mathrm{E}-07$ & 0.0 & 0.0 & 0.0 \\
\hline ZNOH3ION & $3.67602 \mathrm{E}-19$ & 0.0 & 0.0 & 0.0 \\
\hline ZNOH 4 ION & $1.44375 E-25$ & 0.0 & 0.0 & 0.0 \\
\hline ZNOHION & $6.05182 \mathrm{E}-09$ & 0.0 & 0.0 & 0.0 \\
\hline СAHPO4 & 0.0 & $4.42104 \mathrm{E}-04$ & 0.0 & 0.0 \\
\hline $\mathrm{ALPO} 4$ & 0.0 & $2.25926 \mathrm{E}-04$ & 0.0 & 0.0 \\
\hline $\mathrm{CU} 3 \mathrm{PO} 42.2 \mathrm{H} 2 \mathrm{O}$ & 0.0 & $3.87239 \mathrm{E}-06$ & 0.0 & 0.0 \\
\hline FEII3PO $42.8 \mathrm{H} 20$ & 0.0 & $7.95982 E-06$ & 0.0 & 0.0 \\
\hline MG3PO4 2 & 0.0 & $6.79118 E-05$ & 0.0 & 0.0 \\
\hline \multirow[t]{2}{*}{ UIVO2 } & 0.0 & $1.17647 \mathrm{E}-05$ & 0.0 & 0.0 \\
\hline & $===========$ & $===========$ & $===========$ & $===========$ \\
\hline Total g/hr & 2.56418 & 0.267141 & 497.651 & 0.0 \\
\hline Volume, L/hr & 0.00232949 & $6.52255 \mathrm{E}-05$ & 846.381 & 0.0 \\
\hline Enthalpy, cal/hr & -8401.44 & -757.007 & $-1.57939 \mathrm{E}+06$ & 0.0 \\
\hline Density, g/L & 1100.75 & 4095.66 & 0.587976 & \\
\hline Vapor fraction & 0.0 & 0.0 & 1 . & 0.0 \\
\hline Solid fraction & 0.0 & 1 . & 0.0 & 0.0 \\
\hline Organic fraction & 0.0 & 0.0 & 0.0 & 0.0 \\
\hline Osmotic Pres, atm & 190.63 & & & \\
\hline Redox Pot, volts & 0.0 & & & \\
\hline E-Con, 1/ohm-cm & 0.47726 & & & \\
\hline E-Con, cm2/ohm-mol & 61.0231 & & & \\
\hline Abs Visc, cP & 0.275989 & & & \\
\hline Rel Visc & 1.01495 & & & \\
\hline Ionic Strength & 3.92003 & & & \\
\hline
\end{tabular}


WSRC-TR-2002-00567

Revision 0

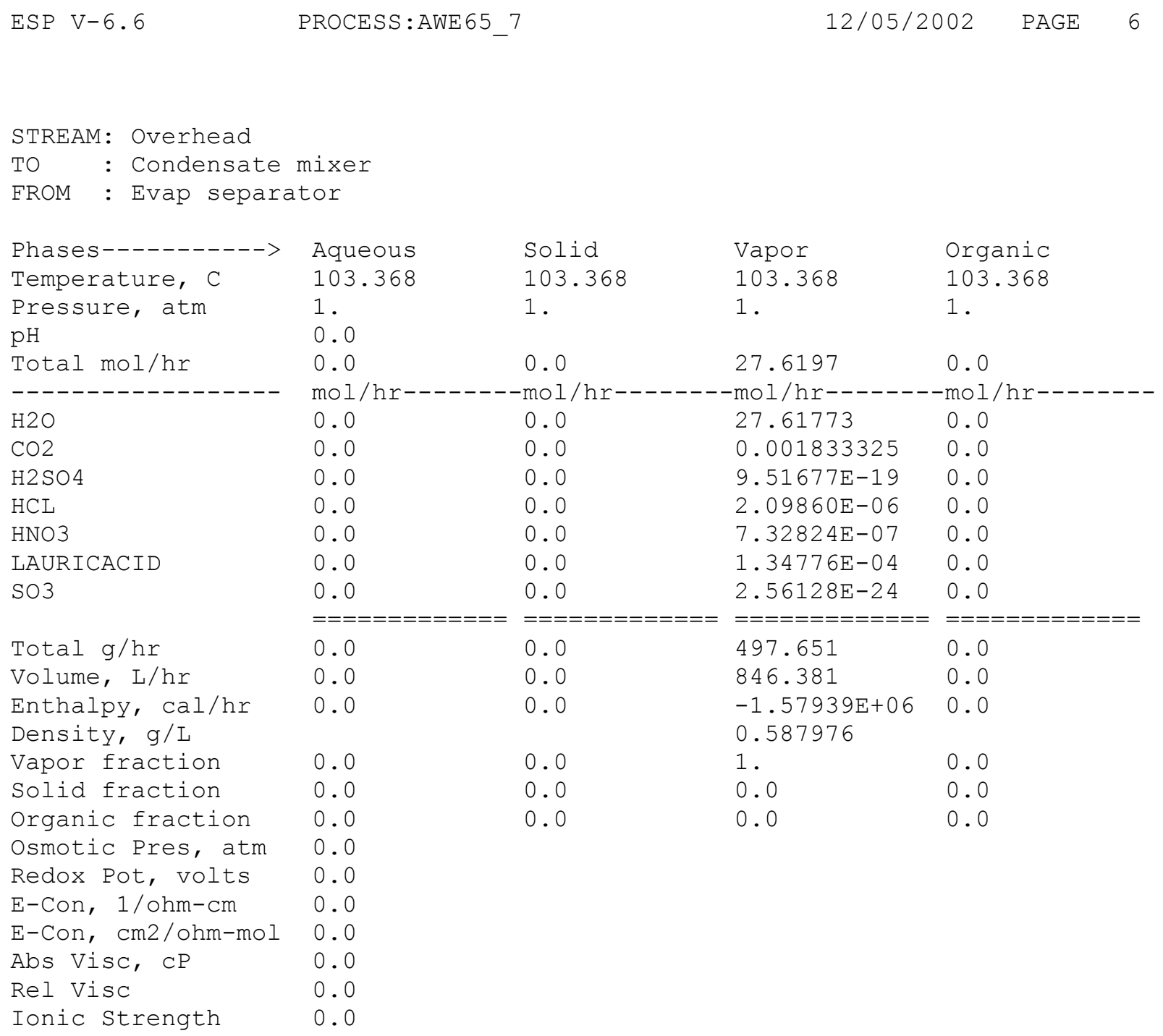


WSRC-TR-2002-00567

Revision 0

\begin{tabular}{|c|c|c|c|c|}
\hline \multicolumn{5}{|c|}{ TO : Evap Bottoms Cooling mixer } \\
\hline Phases $----------->$ & Aqueous & Solid & Vapor & Organic \\
\hline & 103.368 & 103.368 & 103.368 & 103.368 \\
\hline $\begin{array}{l}\text { Pressure, atm } \\
\mathrm{pH}\end{array}$ & $\begin{array}{l}1 . \\
3.92509\end{array}$ & 1. & 1 . & 1 . \\
\hline Total mol/hr & 0.1250164 & 0.00201467 & 0.0 & 0.0 \\
\hline \multicolumn{3}{|l|}{------------------} & $-\mathrm{mol} / \mathrm{hr}--$ & -mol/hr- \\
\hline $\mathrm{H} 2 \mathrm{O}$ & 0.11131 & 0.0 & 0.0 & 0.0 \\
\hline $\mathrm{CO} 2$ & $9.09648 \mathrm{E}-10$ & 0.0 & 0.0 & 0.0 \\
\hline $\mathrm{H} 2 \mathrm{SO} 4$ & $3.96088 \mathrm{E}-21$ & 0.0 & 0.0 & 0.0 \\
\hline $\mathrm{HCL}$ & $8.39440 \mathrm{E}-12$ & 0.0 & 0.0 & 0.0 \\
\hline HNO3 & $1.17320 \mathrm{E}-08$ & 0.0 & 0.0 & 0.0 \\
\hline LAURICACID & $9.68366 \mathrm{E}-09$ & 0.0 & 0.0 & 0.0 \\
\hline $\mathrm{SO} 3$ & $2.89289 \mathrm{E}-24$ & 0.0 & 0.0 & 0.0 \\
\hline CAH2SIO4 & $3.21177 \mathrm{E}-18$ & 0.0 & 0.0 & 0.0 \\
\hline $\mathrm{CASO} 4$ & $1.10733 \mathrm{E}-06$ & $9.66224 \mathrm{E}-04$ & 0.0 & 0.0 \\
\hline CDCL2 & $3.11986 E-08$ & 0.0 & 0.0 & 0.0 \\
\hline $\mathrm{CDOH} 2$ & $1.58946 \mathrm{E}-20$ & 0.0 & 0.0 & 0.0 \\
\hline $\mathrm{CDSO} 4$ & $1.41964 \mathrm{E}-11$ & 0.0 & 0.0 & 0.0 \\
\hline $\mathrm{CROH} 3$ & $1.29411 \mathrm{E}-21$ & 0.0 & 0.0 & 0.0 \\
\hline CUCL2 & $3.27035 E-09$ & 0.0 & 0.0 & 0.0 \\
\hline CUCO3 & $1.47896 \mathrm{E}-18$ & 0.0 & 0.0 & 0.0 \\
\hline CUNO32 & $5.71729 \mathrm{E}-11$ & 0.0 & 0.0 & 0.0 \\
\hline CUOH2 & $4.12182 \mathrm{E}-14$ & 0.0 & 0.0 & 0.0 \\
\hline FEIICL2 & $3.90602 \mathrm{E}-09$ & 0.0 & 0.0 & 0.0 \\
\hline FEIICO3 & $3.10073 E-15$ & 0.0 & 0.0 & 0.0 \\
\hline FEIIHPO4 & $1.81981 E-08$ & 0.0 & 0.0 & 0.0 \\
\hline FEIIOH2 & $2.75919 \mathrm{E}-14$ & 0.0 & 0.0 & 0.0 \\
\hline $\mathrm{ALO} 2 \mathrm{H} 2 \mathrm{CL}$ & $2.07646 \mathrm{E}-28$ & 0.0 & 0.0 & 0.0 \\
\hline H3PO 4 & $1.51556 \mathrm{E}-06$ & 0.0 & 0.0 & 0.0 \\
\hline H4 P 207 & $3.03888 \mathrm{E}-11$ & 0.0 & 0.0 & 0.0 \\
\hline ALOH3 & $4.43385 E-13$ & 0.0 & 0.0 & 0.0 \\
\hline $\mathrm{BACO} 3$ & $2.62359 \mathrm{E}-22$ & 0.0 & 0.0 & 0.0 \\
\hline $\mathrm{KCL}$ & $1.23097 \mathrm{E}-05$ & 0.0 & 0.0 & 0.0 \\
\hline KHSO 4 & $1.35271 E-08$ & 0.0 & 0.0 & 0.0 \\
\hline BASO 4 & $2.03164 E-12$ & $8.01317 E-07$ & 0.0 & 0.0 \\
\hline $\mathrm{MGCO} 3$ & $2.43520 \mathrm{E}-16$ & 0.0 & 0.0 & 0.0 \\
\hline MGH2SIO4 & $1.54152 \mathrm{E}-15$ & 0.0 & 0.0 & 0.0 \\
\hline MGHPO4 & $6.34409 E-06$ & 0.0 & 0.0 & 0.0 \\
\hline MGSO4 & $3.95741 E-05$ & 0.0 & 0.0 & 0.0 \\
\hline NAHCO3 & $7.69648 \mathrm{E}-12$ & 0.0 & 0.0 & 0.0 \\
\hline NAHSIO3 & $1.42408 E-09$ & 0.0 & 0.0 & 0.0 \\
\hline NANO3 & $4.48373 E-04$ & 0.0 & 0.0 & 0.0 \\
\hline $\mathrm{NIOH} 2$ & $2.78050 E-16$ & 0.0 & 0.0 & 0.0 \\
\hline $\mathrm{NISO} 4$ & $6.88027 E-07$ & 0.0 & 0.0 & 0.0 \\
\hline PBCL2 & $4.88626 \mathrm{E}-07$ & 0.0 & 0.0 & 0.0 \\
\hline PBHPO4 & $5.58551 \mathrm{E}-11$ & 0.0 & 0.0 & 0.0 \\
\hline PBNO32 & $3.66364 \mathrm{E}-08$ & 0.0 & 0.0 & 0.0 \\
\hline $\mathrm{PBO}$ & $7.44376 \mathrm{E}-16$ & 0.0 & 0.0 & 0.0 \\
\hline CACL2 & $1.67220 \mathrm{E}-15$ & 0.0 & 0.0 & 0.0 \\
\hline $\mathrm{SIO} 2$ & $9.00498 E-06$ & $2.57660 \mathrm{E}-04$ & 0.0 & 0.0 \\
\hline
\end{tabular}




\begin{tabular}{|c|c|c|c|c|}
\hline $\mathrm{CACO} 3$ & $1.52957 \mathrm{E}-17$ & 0.0 & 0.0 & 0.0 \\
\hline SRHPO 4 & $2.26977 \mathrm{E}-11$ & 0.0 & 0.0 & 0.0 \\
\hline SRNO32 & $9.90447 \mathrm{E}-08$ & 0.0 & 0.0 & 0.0 \\
\hline $\mathrm{SRSO} 4$ & $1.90615 \mathrm{E}-08$ & $3.04456 \mathrm{E}-05$ & 0.0 & 0.0 \\
\hline UIVOH 4 & $2.35248 \mathrm{E}-12$ & 0.0 & 0.0 & 0.0 \\
\hline UIVSO 42 & $2.51329 \mathrm{E}-19$ & 0.0 & 0.0 & 0.0 \\
\hline ZNCL2 & $1.10286 \mathrm{E}-05$ & 0.0 & 0.0 & 0.0 \\
\hline ZNHPO 4 & $3.91239 E-09$ & 0.0 & 0.0 & 0.0 \\
\hline ZNNO32 & $1.17474 \mathrm{E}-08$ & 0.0 & 0.0 & 0.0 \\
\hline ZNOH2 & $1.96413 \mathrm{E}-14$ & 0.0 & 0.0 & 0.0 \\
\hline OHION & $1.48263 \mathrm{E}-11$ & 0.0 & 0.0 & 0.0 \\
\hline ALION & $3.75563 \mathrm{E}-13$ & 0.0 & 0.0 & 0.0 \\
\hline ALOH2 ION & $7.62188 \mathrm{E}-13$ & 0.0 & 0.0 & 0.0 \\
\hline ALOH 4 ION & $3.97417 \mathrm{E}-14$ & 0.0 & 0.0 & 0.0 \\
\hline ALOHCLION & $3.88439 E-12$ & 0.0 & 0.0 & 0.0 \\
\hline ALOHION & $1.04993 \mathrm{E}-12$ & 0.0 & 0.0 & 0.0 \\
\hline ALSO 42 ION & $2.54508 \mathrm{E}-12$ & 0.0 & 0.0 & 0.0 \\
\hline ALSO 4 ION & $3.04857 \mathrm{E}-12$ & 0.0 & 0.0 & 0.0 \\
\hline BAHCO3ION & $5.88681 \mathrm{E}-17$ & 0.0 & 0.0 & 0.0 \\
\hline BAION & $1.60165 \mathrm{E}-09$ & 0.0 & 0.0 & 0.0 \\
\hline BAOHION & $4.33691 \mathrm{E}-18$ & 0.0 & 0.0 & 0.0 \\
\hline CACLION & $5.24329 \mathrm{E}-08$ & 0.0 & 0.0 & 0.0 \\
\hline CAH2 PO 4 ION & $1.44914 \mathrm{E}-05$ & 0.0 & 0.0 & 0.0 \\
\hline CAHCO3ION & $1.02705 \mathrm{E}-13$ & 0.0 & 0.0 & 0.0 \\
\hline CAHSIO3ION & $4.39217 \mathrm{E}-12$ & 0.0 & 0.0 & 0.0 \\
\hline CAION & $1.48086 \mathrm{E}-05$ & 0.0 & 0.0 & 0.0 \\
\hline CANO3ION & $7.59966 \mathrm{E}-06$ & 0.0 & 0.0 & 0.0 \\
\hline CAOHION & $8.66165 E-13$ & 0.0 & 0.0 & 0.0 \\
\hline CAPO 4 ION & $9.14515 \mathrm{E}-12$ & 0.0 & 0.0 & 0.0 \\
\hline CDCL3ION & $2.85905 \mathrm{E}-08$ & 0.0 & 0.0 & 0.0 \\
\hline CDCL 4 ION & $7.66240 \mathrm{E}-09$ & 0.0 & 0.0 & 0.0 \\
\hline CDCLION & $2.11758 \mathrm{E}-09$ & 0.0 & 0.0 & 0.0 \\
\hline CDION & $4.25223 \mathrm{E}-11$ & 0.0 & 0.0 & 0.0 \\
\hline CDNO3ION & $1.70587 \mathrm{E}-11$ & 0.0 & 0.0 & 0.0 \\
\hline CDOH 3 ION & $1.21701 \mathrm{E}-27$ & 0.0 & 0.0 & 0.0 \\
\hline CDOHION & $4.33904 E-16$ & 0.0 & 0.0 & 0.0 \\
\hline CLION & 0.00436098 & 0.0 & 0.0 & 0.0 \\
\hline CO3ION & $6.32999 \mathrm{E}-17$ & 0.0 & 0.0 & 0.0 \\
\hline CRIIICL2ION & $3.67597 \mathrm{E}-16$ & 0.0 & 0.0 & 0.0 \\
\hline CRIIICLION & $2.14865 E-16$ & 0.0 & 0.0 & 0.0 \\
\hline CRIIIH2PO4ION & $1.39309 \mathrm{E}-14$ & 0.0 & 0.0 & 0.0 \\
\hline CRIIIHPO4ION & $2.49999 \mathrm{E}-06$ & 0.0 & 0.0 & 0.0 \\
\hline CRIIIION & $3.16420 \mathrm{E}-20$ & 0.0 & 0.0 & 0.0 \\
\hline CRIIINO3ION & $2.38770 \mathrm{E}-13$ & 0.0 & 0.0 & 0.0 \\
\hline $\mathrm{CROH} 2 \mathrm{ION}$ & $5.71496 \mathrm{E}-20$ & 0.0 & 0.0 & 0.0 \\
\hline CROH 4 ION & $1.52475 \mathrm{E}-27$ & 0.0 & 0.0 & 0.0 \\
\hline CROHION & $2.48288 \mathrm{E}-14$ & 0.0 & 0.0 & 0.0 \\
\hline CRSO 4 ION & $8.05380 \mathrm{E}-12$ & 0.0 & 0.0 & 0.0 \\
\hline CUCL3ION & $5.13947 \mathrm{E}-11$ & 0.0 & 0.0 & 0.0 \\
\hline CUCLION & $2.44226 \mathrm{E}-08$ & 0.0 & 0.0 & 0.0 \\
\hline CUCO32ION & $2.61241 \mathrm{E}-28$ & 0.0 & 0.0 & 0.0 \\
\hline CUION & $6.75839 \mathrm{E}-09$ & 0.0 & 0.0 & 0.0 \\
\hline CUNO3ION & $1.84028 \mathrm{E}-09$ & 0.0 & 0.0 & 0.0 \\
\hline CUOH3ION & $1.98431 \mathrm{E}-20$ & 0.0 & 0.0 & 0.0 \\
\hline CUOH 4 ION & $2.14572 \mathrm{E}-26$ & 0.0 & 0.0 & 0.0 \\
\hline CUOHION & $8.39765 E-12$ & 0.0 & 0.0 & 0.0 \\
\hline DODECION & $2.28748 \mathrm{E}-08$ & 0.0 & 0.0 & 0.0 \\
\hline FEIICLION & $9.72624 \mathrm{E}-07$ & 0.0 & 0.0 & 0.0 \\
\hline
\end{tabular}




\begin{tabular}{|c|c|c|c|c|}
\hline FEIICO32ION & $1.59405 \mathrm{E}-26$ & 0.0 & 0.0 & 0.0 \\
\hline FEIIH2PO4ION & $5.05189 \mathrm{E}-06$ & 0.0 & 0.0 & 0.0 \\
\hline EEIIHCO3ION & $2.29503 \mathrm{E}-14$ & 0.0 & 0.0 & 0.0 \\
\hline FEIIION & $1.17022 \mathrm{E}-04$ & 0.0 & 0.0 & 0.0 \\
\hline FEIIOH3ION & $2.50945 E-19$ & 0.0 & 0.0 & 0.0 \\
\hline EEIIOH4ION & $3.25817 \mathrm{E}-27$ & 0.0 & 0.0 & 0.0 \\
\hline FEIIOHION & $5.03053 \mathrm{E}-09$ & 0.0 & 0.0 & 0.0 \\
\hline H2 P2O 7 ION & $3.19441 \mathrm{E}-06$ & 0.0 & 0.0 & 0.0 \\
\hline H2 PO 4 ION & $1.37043 \mathrm{E}-04$ & 0.0 & 0.0 & 0.0 \\
\hline $\mathrm{H} 2 \mathrm{SIO} 4 \mathrm{ION}$ & $3.83273 \mathrm{E}-18$ & 0.0 & 0.0 & 0.0 \\
\hline H3P2O7 ION & $7.61574 \mathrm{E}-09$ & 0.0 & 0.0 & 0.0 \\
\hline H3SIO 4 ION & $4.68407 \mathrm{E}-10$ & 0.0 & 0.0 & 0.0 \\
\hline HCO3ION & $5.64052 \mathrm{E}-12$ & 0.0 & 0.0 & 0.0 \\
\hline HION & $3.41141 \mathrm{E}-07$ & 0.0 & 0.0 & 0.0 \\
\hline HP207ION & $3.07735 \mathrm{E}-08$ & 0.0 & 0.0 & 0.0 \\
\hline HPBO2ION & $3.48379 \mathrm{E}-21$ & 0.0 & 0.0 & 0.0 \\
\hline HPO4ION & $6.86330 \mathrm{E}-07$ & 0.0 & 0.0 & 0.0 \\
\hline HSO 4 ION & $2.69338 \mathrm{E}-06$ & 0.0 & 0.0 & 0.0 \\
\hline KION & $7.56780 \mathrm{E}-04$ & 0.0 & 0.0 & 0.0 \\
\hline KSO4 ION & $9.47923 E-05$ & 0.0 & 0.0 & 0.0 \\
\hline MGH2 PO 4 ION & $1.55635 \mathrm{E}-04$ & 0.0 & 0.0 & 0.0 \\
\hline MGHCO3ION & $6.78901 \mathrm{E}-12$ & 0.0 & 0.0 & 0.0 \\
\hline MGHSIO3ION & $2.75967 \mathrm{E}-10$ & 0.0 & 0.0 & 0.0 \\
\hline MGION & $1.70664 \mathrm{E}-04$ & 0.0 & 0.0 & 0.0 \\
\hline MGOHION & $2.29827 \mathrm{E}-10$ & 0.0 & 0.0 & 0.0 \\
\hline MGP2O7ION & $1.78507 \mathrm{E}-07$ & 0.0 & 0.0 & 0.0 \\
\hline MGPO 4 ION & $3.11503 \mathrm{E}-10$ & 0.0 & 0.0 & 0.0 \\
\hline NACO3ION & $8.84772 \mathrm{E}-18$ & 0.0 & 0.0 & 0.0 \\
\hline NAION & 0.00542622 & 0.0 & 0.0 & 0.0 \\
\hline NASO 4 ION & $6.06949 \mathrm{E}-12$ & 0.0 & 0.0 & 0.0 \\
\hline NICLION & $1.13316 \mathrm{E}-07$ & 0.0 & 0.0 & 0.0 \\
\hline NIION & $1.46176 \mathrm{E}-06$ & 0.0 & 0.0 & 0.0 \\
\hline NINO3ION & $2.92246 \mathrm{E}-07$ & 0.0 & 0.0 & 0.0 \\
\hline NIOH3ION & $3.30462 E-22$ & 0.0 & 0.0 & 0.0 \\
\hline NIOHION & $1.71099 \mathrm{E}-11$ & 0.0 & 0.0 & 0.0 \\
\hline NO3ION & 0.00131627 & 0.0 & 0.0 & 0.0 \\
\hline P207ION & $2.49268 \mathrm{E}-12$ & 0.0 & 0.0 & 0.0 \\
\hline PBCL3ION & $1.05305 \mathrm{E}-06$ & 0.0 & 0.0 & 0.0 \\
\hline PBCL 4 ION & $8.31174 \mathrm{E}-06$ & 0.0 & 0.0 & 0.0 \\
\hline PBCLION & $1.63531 \mathrm{E}-07$ & 0.0 & 0.0 & 0.0 \\
\hline PBH2 PO 4 ION & $7.59706 \mathrm{E}-09$ & 0.0 & 0.0 & 0.0 \\
\hline PBION & $1.04292 \mathrm{E}-08$ & 0.0 & 0.0 & 0.0 \\
\hline PBNO33ION & $2.14491 \mathrm{E}-09$ & 0.0 & 0.0 & 0.0 \\
\hline PBNO3ION & $7.10665 \mathrm{E}-08$ & 0.0 & 0.0 & 0.0 \\
\hline PBOHION & $4.43667 \mathrm{E}-11$ & 0.0 & 0.0 & 0.0 \\
\hline PO4ION & $5.42931 \mathrm{E}-14$ & 0.0 & 0.0 & 0.0 \\
\hline SO4ION & $5.30310 \mathrm{E}-04$ & 0.0 & 0.0 & 0.0 \\
\hline SRION & $2.36081 \mathrm{E}-09$ & 0.0 & 0.0 & 0.0 \\
\hline SRNO3ION & $2.55923 \mathrm{E}-07$ & 0.0 & 0.0 & 0.0 \\
\hline SROHION & $6.53161 \mathrm{E}-15$ & 0.0 & 0.0 & 0.0 \\
\hline SRPO 4 ION & $2.07684 \mathrm{E}-15$ & 0.0 & 0.0 & 0.0 \\
\hline UIVCLION & $7.61161 \mathrm{E}-23$ & 0.0 & 0.0 & 0.0 \\
\hline UIVION & $4.33936 \mathrm{E}-25$ & 0.0 & 0.0 & 0.0 \\
\hline UIVOH2ION & $8.21567 \mathrm{E}-19$ & 0.0 & 0.0 & 0.0 \\
\hline UIVOH3ION & $9.21960 \mathrm{E}-17$ & 0.0 & 0.0 & 0.0 \\
\hline UIVOH5ION & $1.04833 \mathrm{E}-17$ & 0.0 & 0.0 & 0.0 \\
\hline UIVOHION & $6.95426 \mathrm{E}-20$ & 0.0 & 0.0 & 0.0 \\
\hline UIVSO 4 ION & $3.57697 \mathrm{E}-20$ & 0.0 & 0.0 & 0.0 \\
\hline
\end{tabular}


WSRC-TR-2002-00567

Revision 0

\begin{tabular}{|c|c|c|c|c|}
\hline ZNCL3ION & $1.37685 E-05$ & 0.0 & 0.0 & 0.0 \\
\hline ZNCLION & $2.93880 \mathrm{E}-05$ & 0.0 & 0.0 & 0.0 \\
\hline ZNH2 PO4 ION & $2.58531 E-07$ & 0.0 & 0.0 & 0.0 \\
\hline ZNHCO3ION & $9.23907 \mathrm{E}-15$ & 0.0 & 0.0 & 0.0 \\
\hline ZNION & $1.82777 \mathrm{E}-06$ & 0.0 & 0.0 & 0.0 \\
\hline ZNNO3ION & $2.81824 \mathrm{E}-07$ & 0.0 & 0.0 & 0.0 \\
\hline ZNOH3ION & $3.67602 \mathrm{E}-19$ & 0.0 & 0.0 & 0.0 \\
\hline ZNOH 4 ION & $1.44375 E-25$ & 0.0 & 0.0 & 0.0 \\
\hline ZNOHION & $6.05182 \mathrm{E}-09$ & 0.0 & 0.0 & 0.0 \\
\hline СAHPO4 & 0.0 & $4.42104 \mathrm{E}-04$ & 0.0 & 0.0 \\
\hline $\mathrm{ALPO} 4$ & 0.0 & $2.25926 \mathrm{E}-04$ & 0.0 & 0.0 \\
\hline $\mathrm{CU} 3 \mathrm{PO} 42.2 \mathrm{H} 2 \mathrm{O}$ & 0.0 & $3.87239 \mathrm{E}-06$ & 0.0 & 0.0 \\
\hline FEII3PO $42.8 \mathrm{H} 20$ & 0.0 & $7.95982 E-06$ & 0.0 & 0.0 \\
\hline MG3PO4 2 & 0.0 & $6.79118 E-05$ & 0.0 & 0.0 \\
\hline \multirow[t]{2}{*}{ UIVO2 } & 0.0 & $1.17647 \mathrm{E}-05$ & 0.0 & 0.0 \\
\hline & $===========$ & $===========$ & $===========$ & $===========$ \\
\hline Total g/hr & 2.56418 & 0.267141 & 0.0 & 0.0 \\
\hline Volume, L/hr & 0.00232949 & $6.52255 \mathrm{E}-05$ & 0.0 & 0.0 \\
\hline Enthalpy, cal/hr & -8401.44 & -757.007 & 0.0 & 0.0 \\
\hline Density, g/L & 1100.75 & 4095.66 & & \\
\hline Vapor fraction & 0.0 & 0.0 & 0.0 & 0.0 \\
\hline Solid fraction & 0.0 & 1 . & 0.0 & 0.0 \\
\hline Organic fraction & 0.0 & 0.0 & 0.0 & 0.0 \\
\hline Osmotic Pres, atm & 190.63 & & & \\
\hline Redox Pot, volts & 0.0 & & & \\
\hline E-Con, 1/ohm-cm & 0.47726 & & & \\
\hline E-Con, cm2/ohm-mol & 61.0231 & & & \\
\hline Abs Visc, cP & 0.275989 & & & \\
\hline Rel Visc & 1.01495 & & & \\
\hline Ionic Strength & 3.92003 & & & \\
\hline
\end{tabular}


WSRC-TR-2002-00567

Revision 0

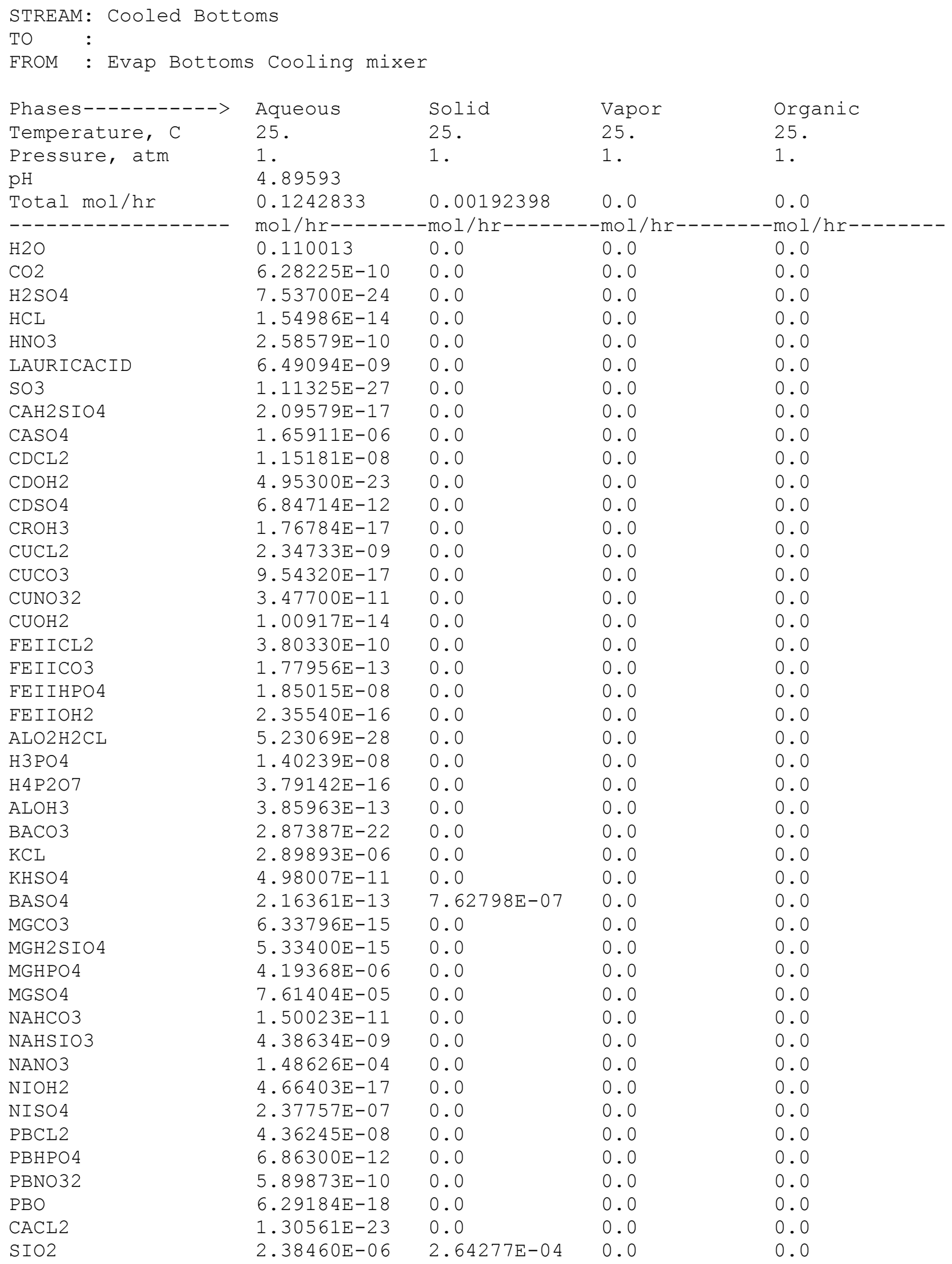




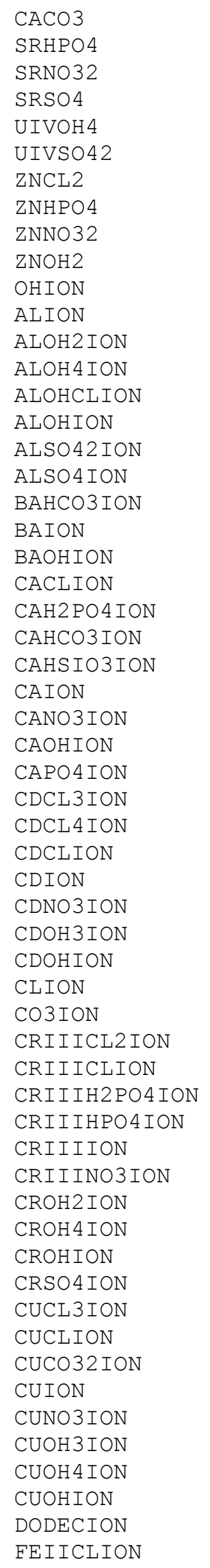

$\begin{array}{ll}5.33437 \mathrm{E}-16 & 0.0 \\ 1.26922 \mathrm{E}-11 & 0.0 \\ 4.23314 \mathrm{E}-08 & 0.0 \\ 4.53337 \mathrm{E}-08 & 2.75425 \mathrm{E}-05 \\ 2.43981 \mathrm{E}-13 & 0.0 \\ 1.24110 \mathrm{E}-21 & 0.0 \\ 5.24629 \mathrm{E}-06 & 0.0 \\ 5.27941 \mathrm{E}-08 & 0.0 \\ 1.36729 \mathrm{E}-07 & 0.0 \\ 1.82412 \mathrm{E}-14 & 0.0 \\ 1.34120 \mathrm{E}-12 & 0.0 \\ 6.94105 \mathrm{E}-11 & 0.0 \\ 2.76686 \mathrm{E}-12 & 0.0 \\ 2.21315 \mathrm{E}-14 & 0.0 \\ 8.69985 \mathrm{E}-11 & 0.0 \\ 1.49355 \mathrm{E}-11 & 0.0 \\ 2.15664 \mathrm{E}-10 & 0.0 \\ 2.40967 \mathrm{E}-10 & 0.0 \\ 7.68880 \mathrm{E}-18 & 0.0 \\ 4.01207 \mathrm{E}-08 & 0.0 \\ 1.01858 \mathrm{E}-20 & 0.0 \\ 1.21514 \mathrm{E}-10 & 0.0 \\ 4.17247 \mathrm{E}-06 & 0.0 \\ 3.06383 \mathrm{E}-12 & 0.0 \\ 5.23081 \mathrm{E}-12 & 0.0 \\ 3.80323 \mathrm{E}-05 & 0.0 \\ 1.85169 \mathrm{E}-05 & 0.0 \\ 1.17399 \mathrm{E}-13 & 0.0 \\ 7.82940 \mathrm{E}-11 & 0.0 \\ 4.51675 \mathrm{E}-09 & 0.0 \\ 5.27157 \mathrm{E}-08 & 0.0 \\ 8.38530 \mathrm{E}-10 & 0.0 \\ 3.89193 \mathrm{E}-11 & 0.0 \\ 8.01626 \mathrm{E}-12 & 0.0 \\ 1.99339 \mathrm{E}-30 & 0.0 \\ 2.41687 \mathrm{E}-17 & 0.0 \\ 0.00444476 & 0.0 \\ 3.00699 \mathrm{E}-15 & 0.0 \\ 6.67336 \mathrm{E}-13 & 0.0 \\ 2.33184 \mathrm{E}-13 & 0.0 \\ 7.64026 \mathrm{E}-10 & 0.0 \\ 2.48972 \mathrm{E}-06 & 0.0 \\ 1.94550 \mathrm{E}-16 & 0.0 \\ 1.83336 \mathrm{E}-10 & 0.0 \\ 2.04074 \mathrm{E}-16 & 0.0 \\ 5.88172 \mathrm{E}-23 & 0.0 \\ 9.68123 \mathrm{E}-11 & 0.0 \\ 9.23817 \mathrm{E}-09 & 0.0 \\ 3.98886 \mathrm{E}-11 & 0.0 \\ 7.99373 \mathrm{E}-09 & 0.0 \\ 4.52531 \mathrm{E}-25 & 0.0 \\ 7.85875 \mathrm{E}-09 & 0.0 \\ 2.00949 \mathrm{E}-09 & 0.0 \\ 1.48339 \mathrm{E}-21 & 0.0 \\ 8.81388 \mathrm{E}-29 & 0.0 \\ 2.17285 \mathrm{E}-12 & 0.0 \\ 2.60675 \mathrm{E}-08 & 0.0 \\ 1.60137 \mathrm{E}-07 & 0.0 \\ & \\ 1.07 & \end{array}$

0.0

0.0

0.0

0.0

0.0

0.0

0.0

0.0

0.0

0.0

0.0

0.0

0.0

0.0

0.0

0.0

0.0

0.0

0.0

0.0

0.0

0.0

0.0

0.0

0.0

0.0

0.0

0.0

0.0

0.0

0.0

0.0

0.0

0.0

0.0

0.0

0.0

0.0

0.0

0.0

0.0

0.0

0.0

0.0

0.0

0.0

0.0

0.0

0.0

0.0

0.0

0.0

0.0

0.0

0.0

0.0

0.0

0.0

0.0 


\begin{tabular}{|c|c|c|c|c|}
\hline FEIICO32ION & $1.80357 \mathrm{E}-23$ & 0.0 & 0.0 & 0.0 \\
\hline FEIIH2PO4ION & $1.98784 \mathrm{E}-06$ & 0.0 & 0.0 & 0.0 \\
\hline FEIIHCO3ION & $1.63360 \mathrm{E}-13$ & 0.0 & 0.0 & 0.0 \\
\hline FEIIION & $1.34910 \mathrm{E}-04$ & 0.0 & 0.0 & 0.0 \\
\hline FEIIOH3ION & $2.23557 \mathrm{E}-21$ & 0.0 & 0.0 & 0.0 \\
\hline FEIIOH 4 ION & $2.01157 \mathrm{E}-30$ & 0.0 & 0.0 & 0.0 \\
\hline FEIIOHION & $1.15784 \mathrm{E}-09$ & 0.0 & 0.0 & 0.0 \\
\hline H2P2O7ION & $1.25198 \mathrm{E}-08$ & 0.0 & 0.0 & 0.0 \\
\hline H2 PO 4 ION & $3.65593 \mathrm{E}-05$ & 0.0 & 0.0 & 0.0 \\
\hline H2SIO 4 ION & $4.57708 \mathrm{E}-18$ & 0.0 & 0.0 & 0.0 \\
\hline H3P2O 7 ION & $1.83889 \mathrm{E}-12$ & 0.0 & 0.0 & 0.0 \\
\hline H3SIO 4 ION & $4.27670 \mathrm{E}-11$ & 0.0 & 0.0 & 0.0 \\
\hline HCO3ION & $7.49496 \mathrm{E}-11$ & 0.0 & 0.0 & 0.0 \\
\hline HION & $2.52279 \mathrm{E}-08$ & 0.0 & 0.0 & 0.0 \\
\hline HP2O 7 ION & $1.83586 \mathrm{E}-09$ & 0.0 & 0.0 & 0.0 \\
\hline HPBO2 ION & $1.35596 \mathrm{E}-23$ & 0.0 & 0.0 & 0.0 \\
\hline HPO4ION & $2.22969 \mathrm{E}-06$ & 0.0 & 0.0 & 0.0 \\
\hline HSO 4 ION & $3.35763 \mathrm{E}-08$ & 0.0 & 0.0 & 0.0 \\
\hline KION & $7.97015 \mathrm{E}-04$ & 0.0 & 0.0 & 0.0 \\
\hline KSO4ION & $6.39812 \mathrm{E}-05$ & 0.0 & 0.0 & 0.0 \\
\hline MGH2 PO 4 ION & $1.41187 \mathrm{E}-04$ & 0.0 & 0.0 & 0.0 \\
\hline MGHCO3ION & $2.07001 \mathrm{E}-10$ & 0.0 & 0.0 & 0.0 \\
\hline MGHS IO3ION & $2.54475 \mathrm{E}-10$ & 0.0 & 0.0 & 0.0 \\
\hline MGION & $3.54588 \mathrm{E}-04$ & 0.0 & 0.0 & 0.0 \\
\hline MGOHION & $2.96201 \mathrm{E}-11$ & 0.0 & 0.0 & 0.0 \\
\hline MGP2O7ION & $1.96689 \mathrm{E}-08$ & 0.0 & 0.0 & 0.0 \\
\hline MGPO 4 ION & $2.59916 \mathrm{E}-09$ & 0.0 & 0.0 & 0.0 \\
\hline NACO3ION & $1.31925 \mathrm{E}-15$ & 0.0 & 0.0 & 0.0 \\
\hline NAION & 0.00549675 & 0.0 & 0.0 & 0.0 \\
\hline NASO 4 ION & $2.29223 E-04$ & 0.0 & 0.0 & 0.0 \\
\hline NICLION & $5.70001 \mathrm{E}-08$ & 0.0 & 0.0 & 0.0 \\
\hline NIION & $1.72693 \mathrm{E}-06$ & 0.0 & 0.0 & 0.0 \\
\hline NINO3ION & $5.33678 \mathrm{E}-07$ & 0.0 & 0.0 & 0.0 \\
\hline NIOH3ION & $1.37662 \mathrm{E}-22$ & 0.0 & 0.0 & 0.0 \\
\hline NIOHION & $2.49388 \mathrm{E}-12$ & 0.0 & 0.0 & 0.0 \\
\hline NO3ION & 0.00160089 & 0.0 & 0.0 & 0.0 \\
\hline P207ION & $8.85182 \mathrm{E}-12$ & 0.0 & 0.0 & 0.0 \\
\hline PBCL3ION & $1.16402 \mathrm{E}-07$ & 0.0 & 0.0 & 0.0 \\
\hline PBCL 4 ION & $2.34588 E-06$ & 0.0 & 0.0 & 0.0 \\
\hline PBCLION & $1.86546 \mathrm{E}-08$ & 0.0 & 0.0 & 0.0 \\
\hline PBH2 PO 4 ION & $1.46951 \mathrm{E}-10$ & 0.0 & 0.0 & 0.0 \\
\hline PBION & $2.61237 \mathrm{E}-09$ & 0.0 & 0.0 & 0.0 \\
\hline PBNO33ION & $2.18091 \mathrm{E}-10$ & 0.0 & 0.0 & 0.0 \\
\hline PBNO3ION & $4.37296 \mathrm{E}-09$ & 0.0 & 0.0 & 0.0 \\
\hline PBOHION & $5.44651 \mathrm{E}-13$ & 0.0 & 0.0 & 0.0 \\
\hline PO4ION & $1.07128 \mathrm{E}-12$ & 0.0 & 0.0 & 0.0 \\
\hline SO4ION & $6.01684 \mathrm{E}-04$ & 0.0 & 0.0 & 0.0 \\
\hline SRION & $2.96025 \mathrm{E}-06$ & 0.0 & 0.0 & 0.0 \\
\hline SRNO3ION & $2.31489 \mathrm{E}-07$ & 0.0 & 0.0 & 0.0 \\
\hline SROHION & $3.50809 \mathrm{E}-16$ & 0.0 & 0.0 & 0.0 \\
\hline SRPO 4 ION & $5.71801 \mathrm{E}-15$ & 0.0 & 0.0 & 0.0 \\
\hline UIVCLION & $3.55354 \mathrm{E}-24$ & 0.0 & 0.0 & 0.0 \\
\hline UIVION & $5.89233 E-26$ & 0.0 & 0.0 & 0.0 \\
\hline UIVOH2ION & $5.15857 \mathrm{E}-20$ & 0.0 & 0.0 & 0.0 \\
\hline UIVOH3ION & $1.20877 \mathrm{E}-16$ & 0.0 & 0.0 & 0.0 \\
\hline UIVOH5ION & $3.66989 \mathrm{E}-18$ & 0.0 & 0.0 & 0.0 \\
\hline UIVOHION & $1.13899 \mathrm{E}-21$ & 0.0 & 0.0 & 0.0 \\
\hline UIVSO 4 ION & $5.63131 \mathrm{E}-22$ & 0.0 & 0.0 & 0.0 \\
\hline
\end{tabular}


WSRC-TR-2002-00567

Revision 0

\begin{tabular}{|c|c|c|c|c|}
\hline ZNCL 3 ION & $9.73271 \mathrm{E}-06$ & 0.0 & 0.0 & 0.0 \\
\hline ZNCLION & $7.27891 \mathrm{E}-06$ & 0.0 & 0.0 & 0.0 \\
\hline ZNH2 PO4 ION & $9.23419 \mathrm{E}-07$ & 0.0 & 0.0 & 0.0 \\
\hline ZNHCO3ION & 1. $31823 E-12$ & 0.0 & 0.0 & 0.0 \\
\hline ZNION & $2.89067 E-05$ & 0.0 & 0.0 & 0.0 \\
\hline ZNNO3 ION & $4.29710 E-06$ & 0.0 & 0.0 & 0.0 \\
\hline ZNOH3ION & $3.26972 E-20$ & 0.0 & 0.0 & 0.0 \\
\hline ZNOH 4 ION & $5.06712 \mathrm{E}-27$ & 0.0 & 0.0 & 0.0 \\
\hline ZNOHION & $2.41132 \mathrm{E}-10$ & 0.0 & 0.0 & 0.0 \\
\hline СAHPO4 & 0.0 & $7.18655 \mathrm{E}-04$ & 0.0 & 0.0 \\
\hline $\mathrm{ALPO} 4$ & 0.0 & $2.25925 E-04$ & 0.0 & 0.0 \\
\hline $\mathrm{CASO} 4.2 \mathrm{H} 2 \mathrm{O}$ & 0.0 & $6.65346 E-04$ & 0.0 & 0.0 \\
\hline $\mathrm{CU} 3 \mathrm{PO} 42.2 \mathrm{H} 2 \mathrm{O}$ & 0.0 & $3.87775 E-06$ & 0.0 & 0.0 \\
\hline FEII3PO $42.8 \mathrm{H} 20$ & 0.0 & $3.29170 E-06$ & 0.0 & 0.0 \\
\hline $\mathrm{PB} 3 \mathrm{PO} 42$ & 0.0 & $2.53747 E-06$ & 0.0 & 0.0 \\
\hline UIVO2 & 0.0 & $1.17647 \mathrm{E}-05$ & 0.0 & 0.0 \\
\hline & $===========$ & $===========$ & $============$ & $===========$ \\
\hline Total g/hr & 2.56182 & 0.269504 & 0.0 & 0.0 \\
\hline Volume, L/hr & 0.00217405 & $6.45095 E-05$ & 0.0 & 0.0 \\
\hline Enthalpy, cal/hr & -8524.04 & -802.089 & 0.0 & 0.0 \\
\hline Density, g/L & 1178.36 & 4177.74 & & \\
\hline Vapor fraction & 0.0 & 0.0 & 0.0 & 0.0 \\
\hline Solid fraction & 0.0 & 1. & 0.0 & 0.0 \\
\hline Organic fraction & 0.0 & 0.0 & 0.0 & 0.0 \\
\hline Osmotic Pres, atm & 184.824 & & & \\
\hline Redox Pot, volts & 0.0 & & & \\
\hline $\mathrm{E}-\mathrm{Con}, 1 / \mathrm{ohm}-\mathrm{cm}$ & 0.182736 & & & \\
\hline E-Con, cm2/ohm-mol & 27.8429 & & & \\
\hline Abs Visc, cP & 1.53973 & & & \\
\hline Rel Visc & 1.72864 & & & \\
\hline Ionic Strength & 4.42278 & & & \\
\hline
\end{tabular}


WSRC-TR-2002-00567

Revision 0

\begin{tabular}{|c|c|c|c|c|}
\hline \multicolumn{5}{|l|}{ STREAM: Condensate } \\
\hline TO $\quad:$ & & & & \\
\hline FROM : Condensate & mixer & & & \\
\hline Phases-----------> & Aqueous & Solid & Vapor & Organic \\
\hline Temperature, C & 25 . & 25 . & 25 . & 25 . \\
\hline $\begin{array}{l}\text { Pressure, atm } \\
\mathrm{pH}\end{array}$ & $\begin{array}{l}1 . \\
4.15678\end{array}$ & 1. & 1. & 1. \\
\hline Total mol/hr & 27.61981 & 0.0 & 0.0 & 0.0 \\
\hline------------------ & $\mathrm{mol} / \mathrm{hr}------$ & -mol/hr------- & -mol/hr-------- & $-\operatorname{mol} / \mathrm{hr}------1$ \\
\hline $\mathrm{H} 2 \mathrm{O}$ & 27.6178 & 0.0 & 0.0 & 0.0 \\
\hline $\mathrm{CO} 2$ & 0.00182135 & 0.0 & 0.0 & 0.0 \\
\hline $\mathrm{HCL}$ & $8.58003 E-17$ & 0.0 & 0.0 & 0.0 \\
\hline HNO3 & $2.52103 E-12$ & 0.0 & 0.0 & 0.0 \\
\hline LAURICACID & $1.14568 \mathrm{E}-04$ & 0.0 & 0.0 & 0.0 \\
\hline OHION & $7.31126 \mathrm{E}-11$ & 0.0 & 0.0 & 0.0 \\
\hline CLION & $2.09861 E-06$ & 0.0 & 0.0 & 0.0 \\
\hline CO3ION & $8.31125 E-12$ & 0.0 & 0.0 & 0.0 \\
\hline DODECION & $2.02089 E-05$ & 0.0 & 0.0 & 0.0 \\
\hline HCO3ION & $1.19771 \mathrm{E}-05$ & 0.0 & 0.0 & 0.0 \\
\hline $\mathrm{HION}$ & $3.50175 E-05$ & 0.0 & 0.0 & 0.0 \\
\hline NO3ION & $\begin{array}{l}\text { 7.32823E-0 } \\
============\end{array}$ & $\begin{array}{l}0.0 \\
============\end{array}$ & $\begin{array}{l}0.0 \\
=============\end{array}$ & $\begin{array}{l}0.0 \\
=============\end{array}$ \\
\hline Total g/hr & 497.651 & 0.0 & 0.0 & 0.0 \\
\hline Volume, L/hr & 0.499184 & 0.0 & 0.0 & 0.0 \\
\hline $\begin{array}{l}\text { Enthalpy, cal/hr } \\
\text { Density, g/L }\end{array}$ & $\begin{array}{l}-1.88686 \mathrm{E}+06 \\
996.93\end{array}$ & 0.0 & 0.0 & 0.0 \\
\hline Vapor fraction & 0.0 & 0.0 & 0.0 & 0.0 \\
\hline Solid fraction & 0.0 & 0.0 & 0.0 & 0.0 \\
\hline Organic fraction & 0.0 & 0.0 & 0.0 & 0.0 \\
\hline Osmotic Pres, atm & 0.0984103 & & & \\
\hline Redox Pot, volts & 0.0 & & & \\
\hline E-Con, 1/ohm-cm & $2.83734 \mathrm{E}-05$ & & & \\
\hline E-Con, cm2/ohm-mol & 7.1862 & & & \\
\hline Abs Visc, cP & 0.89075 & & & \\
\hline Rel Visc & 1.00003 & & & \\
\hline Ionic Strength & $7.03814 \mathrm{E}-05$ & & & \\
\hline
\end{tabular}


WSRC-TR-2002-00567

Revision 0

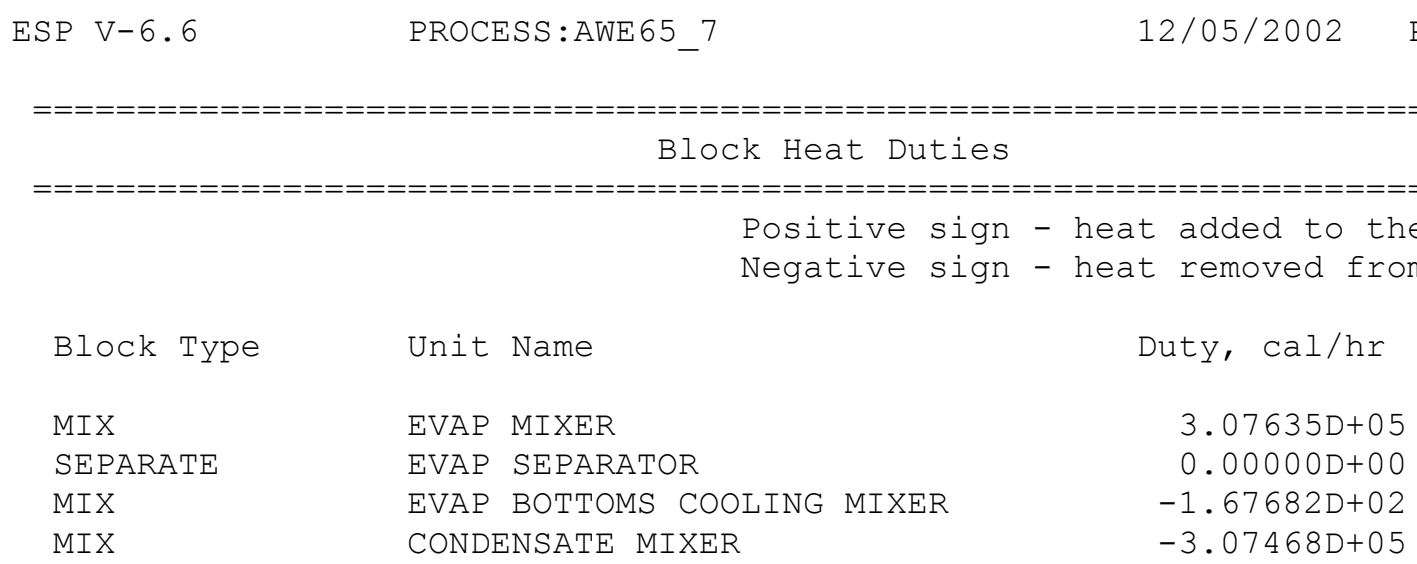


WSRC-TR-2002-00567

Revision 0

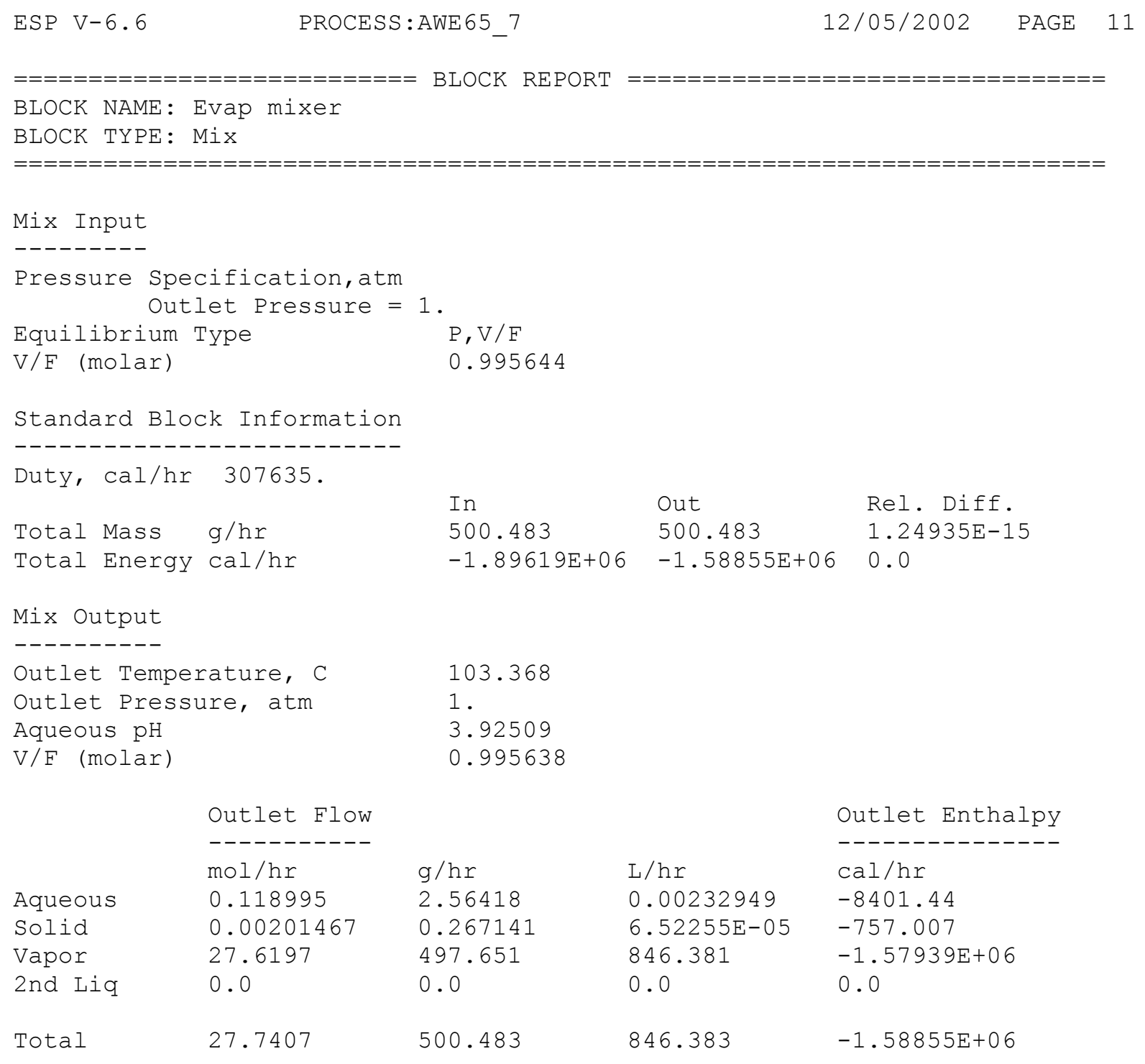


WSRC-TR-2002-00567

Revision 0

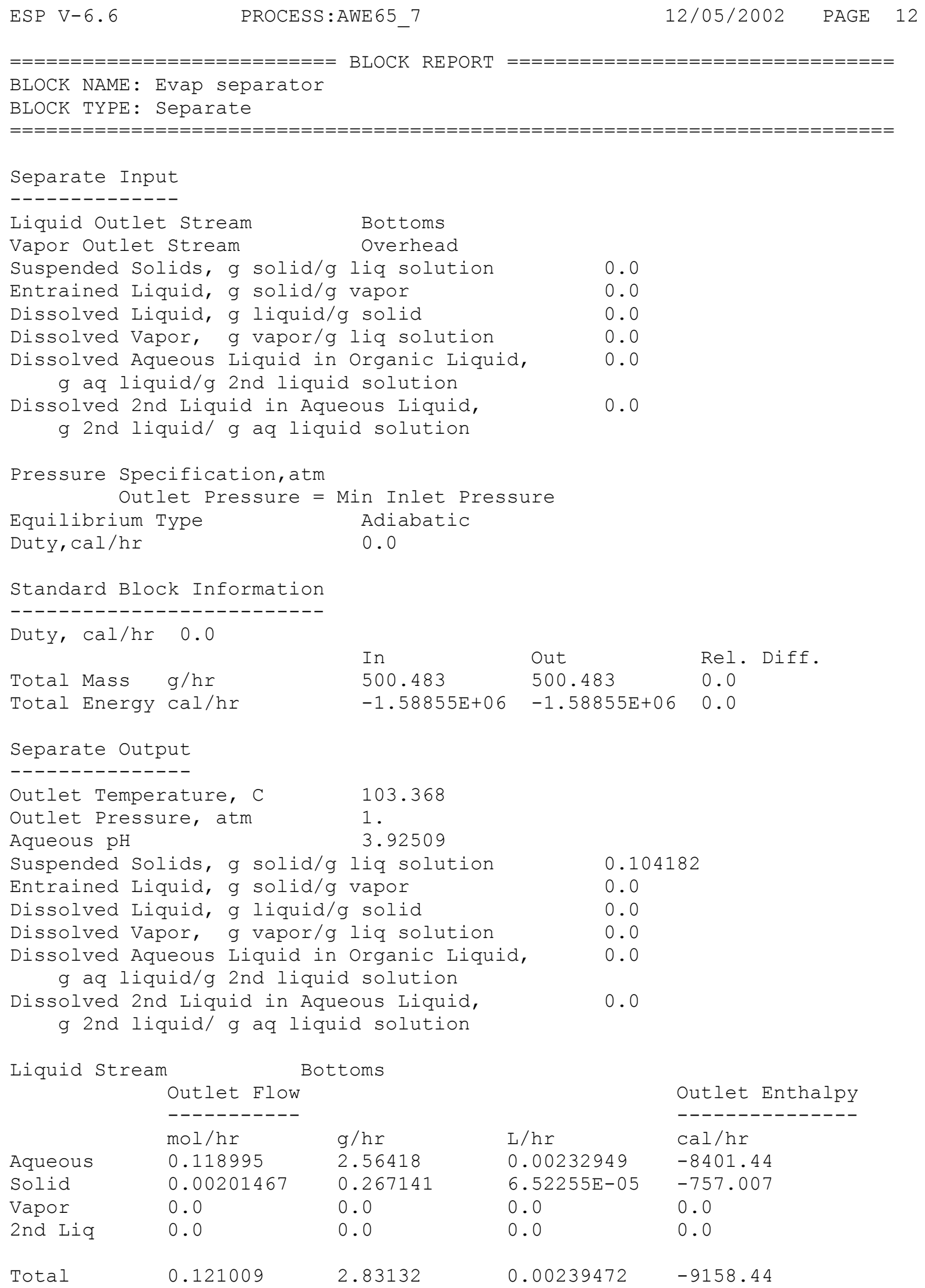


WSRC-TR-2002-00567

Revision 0

ESP V-6.6

PROCESS:AWE 657

Vapor Stream

Outlet Flow
mol/hr
0.0
0.0
27.6197
0.0

Total
Overhead

g/hr

0.0

0.0

497.651

0.0

497.651
$12 / 05 / 2002$

PAGE

13

$\mathrm{L} / \mathrm{hr}$

0.0

0.0

846.381

0.0

846.381
Outlet Enthalpy

$\mathrm{cal} / \mathrm{hr}$

0.0

0.0

$-1.57939 E+06$

0.0

$-1.57939 E+06$ 
WSRC-TR-2002-00567

Revision 0

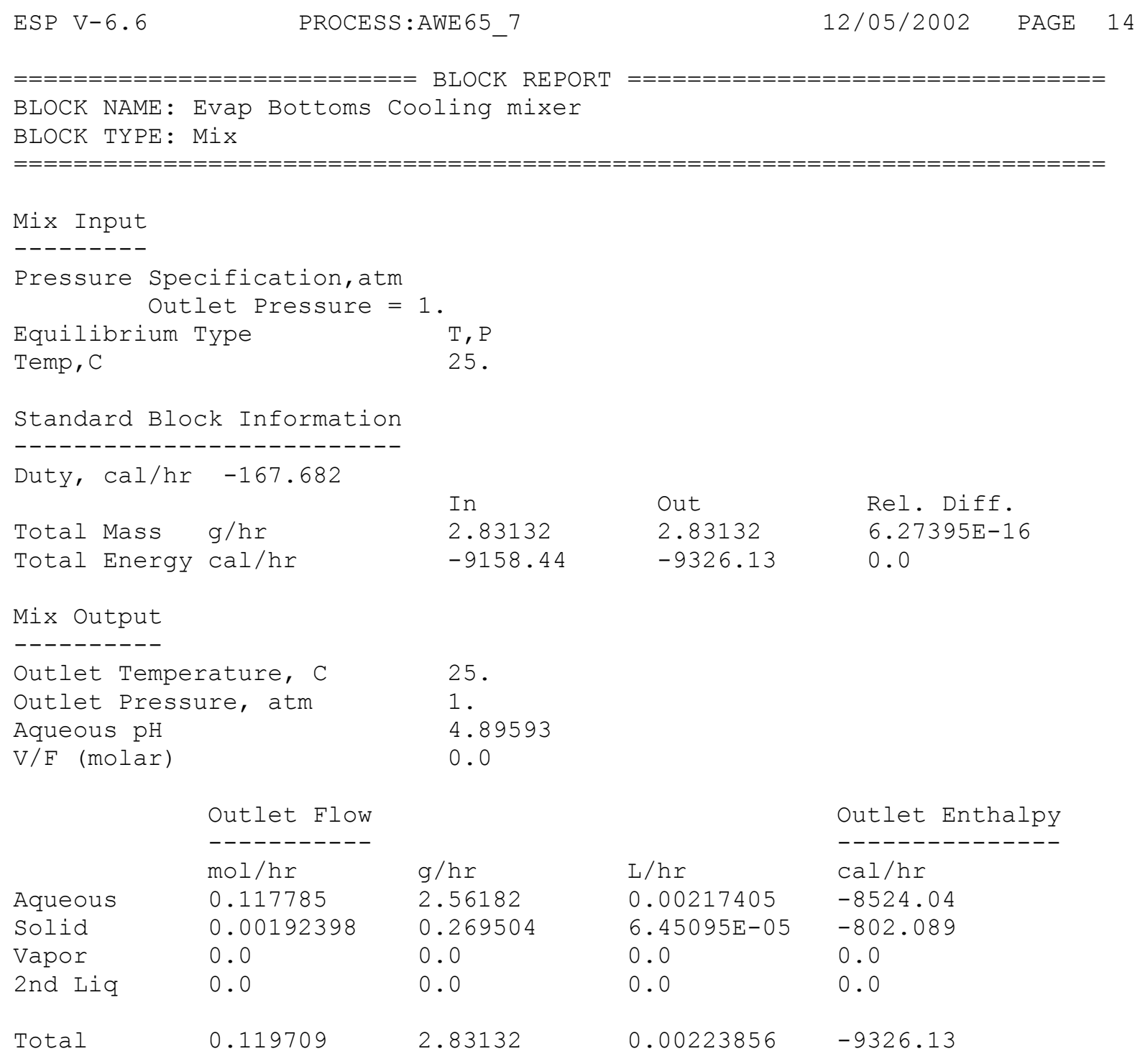


WSRC-TR-2002-00567

Revision 0

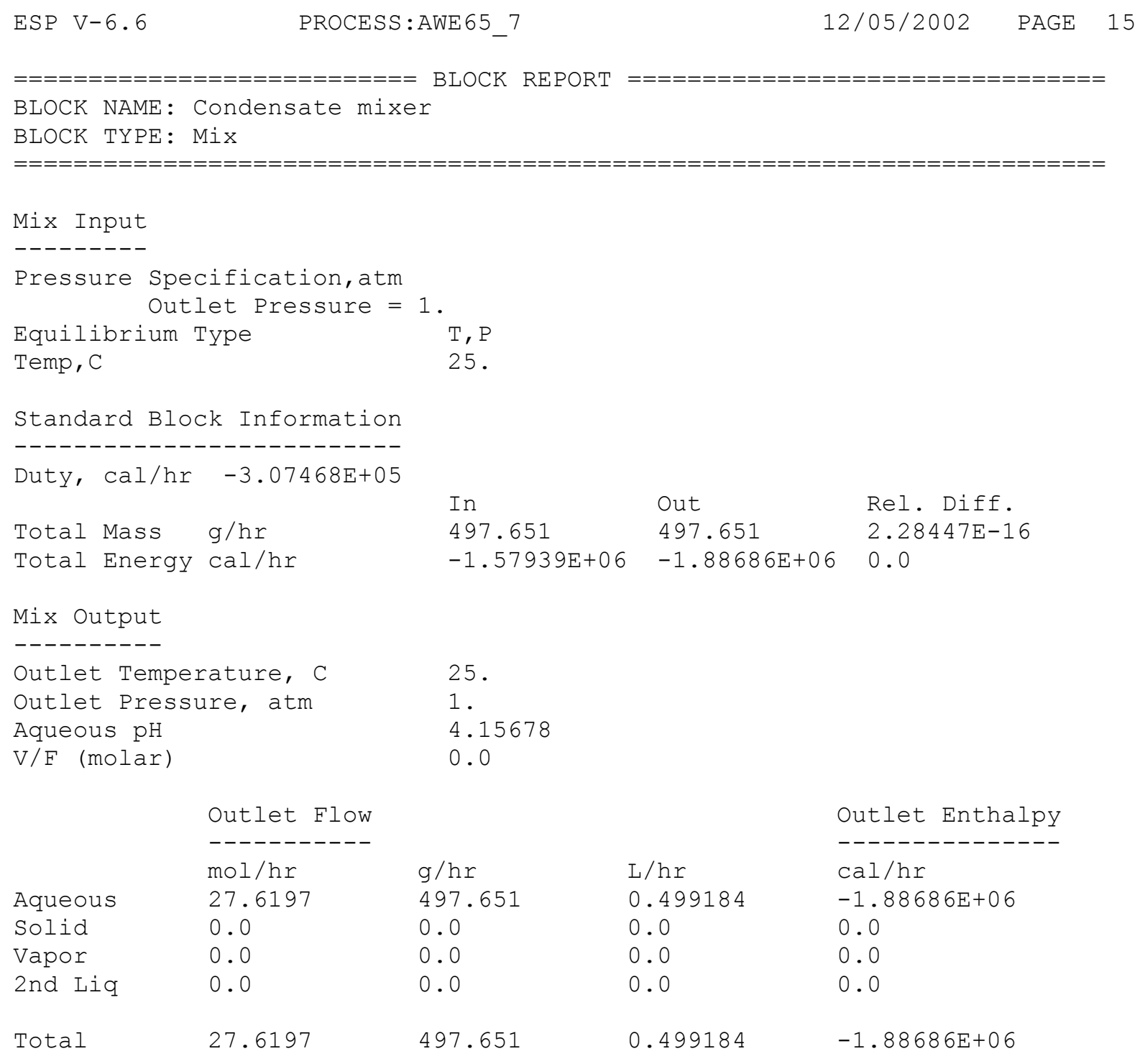


WSRC-TR-2002-00567

Revision 0

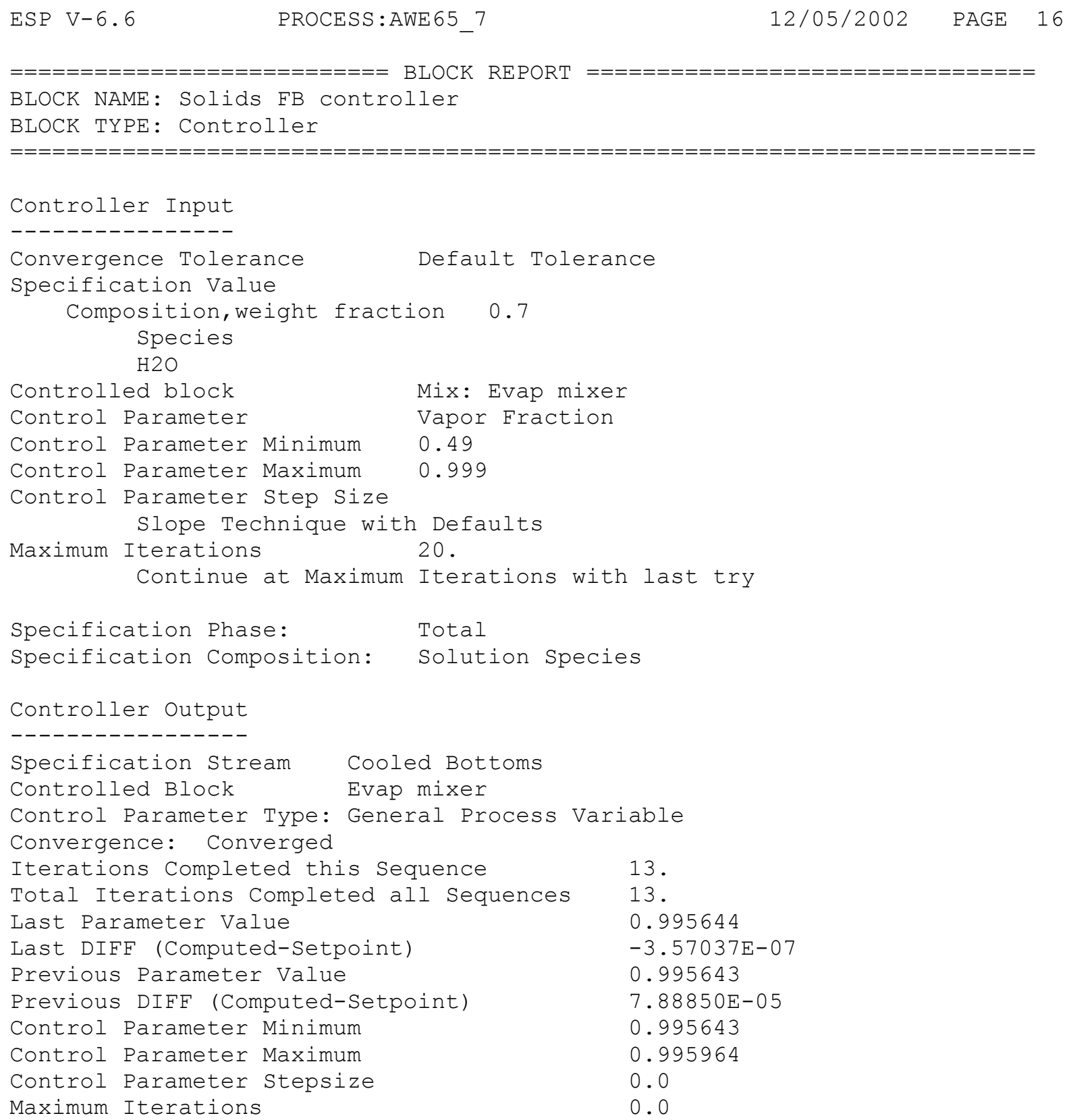


WSRC-TR-2002-00567

Revision 0

Influent Limit Composition 50\% Target $\mathrm{pH}=8.5$

8. 5-50

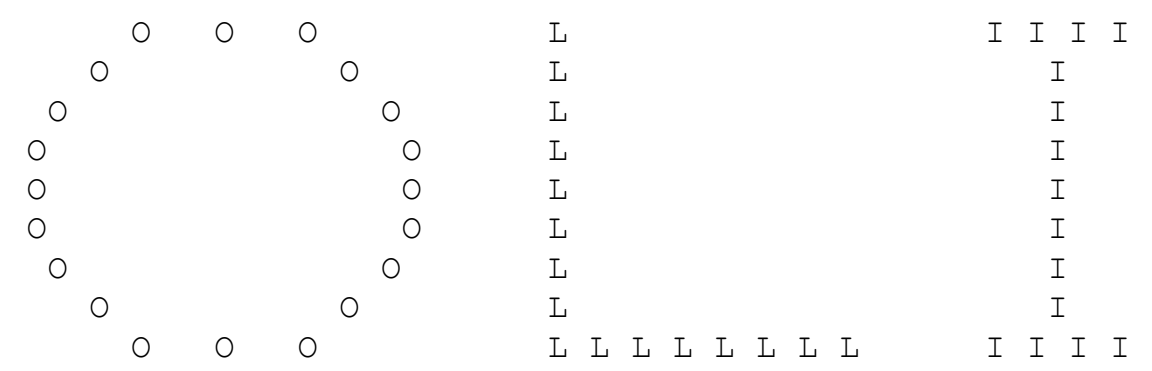

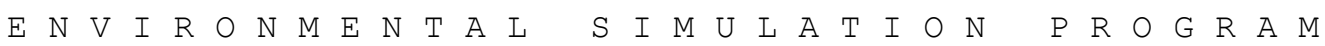

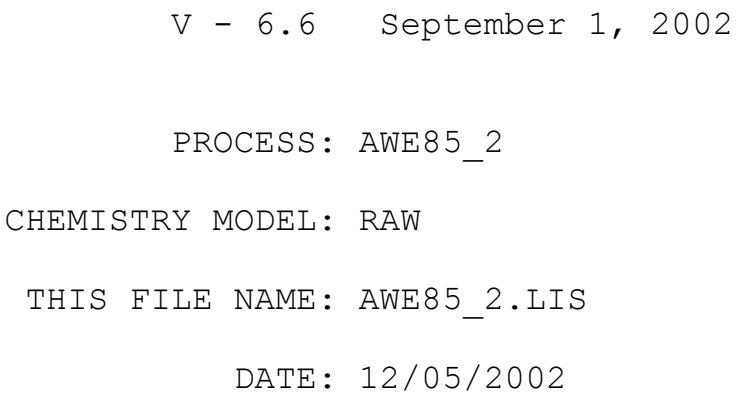


WSRC-TR-2002-00567

Revision 0

TABLE OF CONTENTS

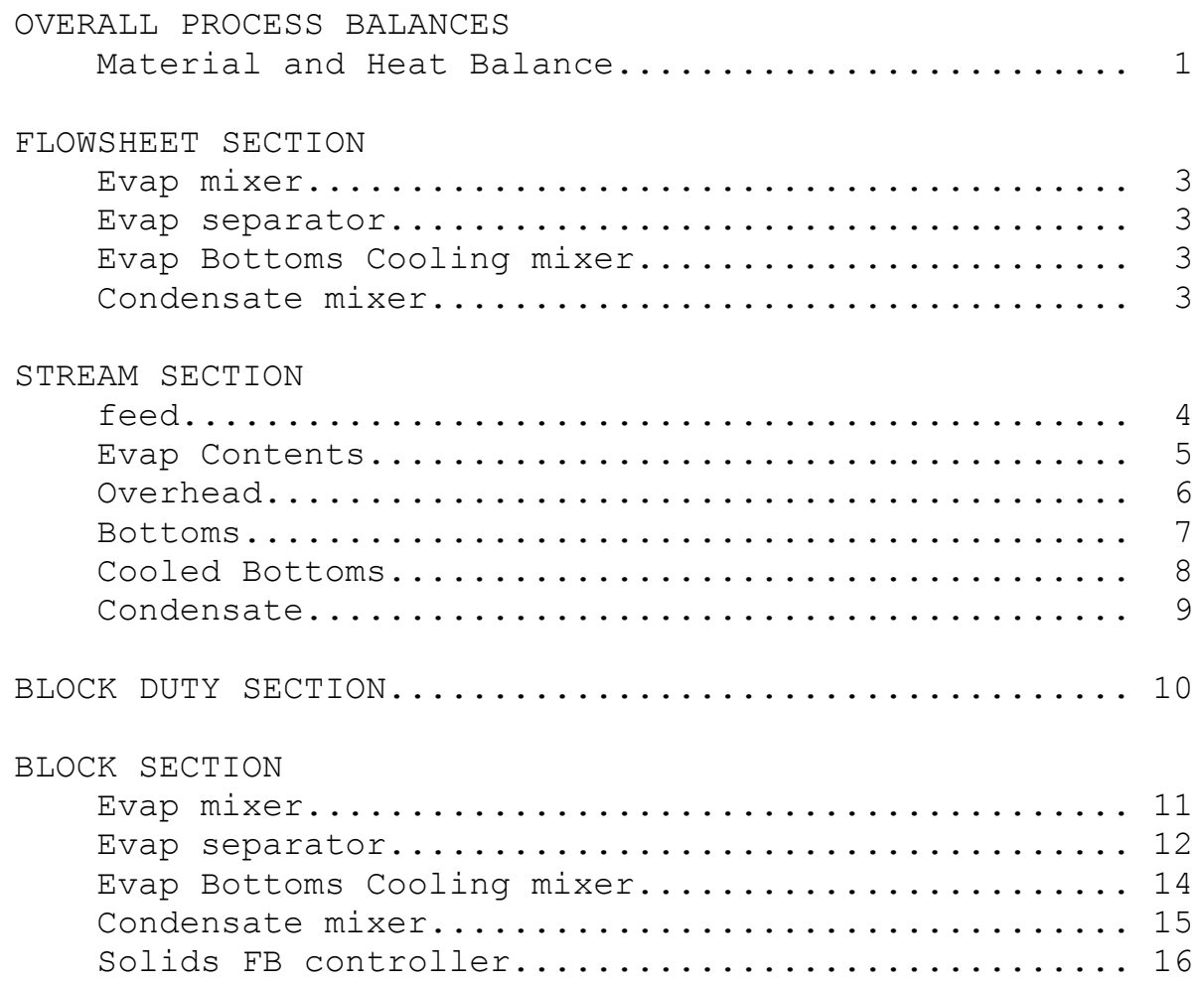


WSRC-TR-2002-00567

Revision 0

ESP V-6.6 PROCESS:AWE85_2
$\begin{array}{lcc}\text { Overall Process } & \text { Balances } \\ \text { Inlet } & \text { g/hr } & \mathrm{Cal} / \mathrm{hr} \\ ---------- & --------- & ---------- \\ \text { FEED } & 1.00000 \mathrm{D}+03 & -3.79149 \mathrm{D}+06 \\ \text { =========== } & ========= & ========= \\ \text { Total in } & 1.00000 \mathrm{D}+03 & -3.79149 \mathrm{D}+06\end{array}$

\begin{tabular}{lrr} 
Outlet & \multicolumn{1}{c}{$g / \mathrm{hr}$} & $\mathrm{cal} / \mathrm{hr}$ \\
----------- & ---------- & ----------- \\
COOLED BOTTOMS & $8.43793 \mathrm{D}-01$ & $-2.82377 \mathrm{D}+03$ \\
CONDENSATE & $9.99156 \mathrm{D}+02$ & $-3.78866 \mathrm{D}+06$ \\
$==========$ & $==========$ & $==========$ \\
Total out & $1.00000 \mathrm{D}+03$ & $-3.79149 \mathrm{D}+06$
\end{tabular}

Block Heat Duties

EVAP MIXER

EVAP BOTTOMS COOLING MIXER

CONDENSATE MIXER

$=========$

Total Duty

DIFFERENCE

REL DIFEERENCE

$5.68434 \mathrm{D}-13$

$5.68434 \mathrm{D}-16$
$12 / 05 / 2002$

PAGE

1
Material Code Balances

Code
----------
$\mathrm{H}(+1)$
$\mathrm{K}(+1)$
$\mathrm{NA}(+1)$
$\mathrm{BA}(+2)$
$\mathrm{CA}(+2)$
$\mathrm{ZN}(+2)$
$\mathrm{CU}(+2)$
$\mathrm{FE}(+2)$
$\mathrm{MG}(+2)$
$\mathrm{PB}(+2)$
$\mathrm{AL}(+3)$
$\mathrm{NI}(+2)$
$\mathrm{O}(-2)$
$\mathrm{CL}(-1)$
$\mathrm{C}(+4)$
$\mathrm{P}(+5)$
$\mathrm{S}(+6)$
$\mathrm{N}(+5)$
$\mathrm{SI}(+4)$
$\mathrm{SR}(+2)$

Input
mol/hr
---------
$1.10985 \mathrm{D}+02$
$2.06789 \mathrm{D}-04$
$1.40619 \mathrm{D}-03$
$4.08759 \mathrm{D}-07$
$7.23192 \mathrm{D}-04$
$2.14067 \mathrm{D}-05$
$3.14961 \mathrm{D}-06$
$5.55556 \mathrm{D}-05$
$2.46914 \mathrm{D}-04$
$2.12560 \mathrm{D}-06$
$6.29630 \mathrm{D}-05$
$9.36968 \mathrm{D}-07$
$5.54990 \mathrm{D}+01$
$1.32394 \mathrm{D}-03$
$1.00000 \mathrm{D}-03$
$1.47368 \mathrm{D}-04$
$4.89583 \mathrm{D}-04$
$3.38710 \mathrm{D}-04$
$1.65000 \mathrm{D}-04$
$1.13014 \mathrm{D}-05$

Outlet mol/hr

$1.10985 \mathrm{D}+02$

$2.06789 \mathrm{D}-04$

1. $40619 \mathrm{D}-03$

4.08759D-07

7.23192D-04

$2.14067 \mathrm{D}-05$

$3.14961 \mathrm{D}-06$

$5.55556 \mathrm{D}-05$

2. $46914 \mathrm{D}-04$

$2.12560 \mathrm{D}-06$

$6.29630 \mathrm{D}-05$

$9.36968 \mathrm{D}-07$

$5.54990 \mathrm{D}+01$

1. 32394D-03

$1.00000 \mathrm{D}-03$

1. $47368 \mathrm{D}-04$

4. 89583D-04

3. $38710 \mathrm{D}-04$

1. $65000 \mathrm{D}-04$

$1.13014 \mathrm{D}-05$

\begin{tabular}{rr}
$\begin{array}{r}\text { Difference } \\
\text { mol/hr }\end{array}$ & \multicolumn{1}{l}{ Rel Diff } \\
--------- & \multicolumn{1}{c}{----------} \\
$5.68434 \mathrm{D}-14$ & $5.12170 \mathrm{D}-16$ \\
$-1.08420 \mathrm{D}-19$ & $-5.24303 \mathrm{D}-16$ \\
$2.60209 \mathrm{D}-18$ & $1.85045 \mathrm{D}-15$ \\
$1.32349 \mathrm{D}-21$ & $3.23782 \mathrm{D}-15$ \\
$1.08420 \mathrm{D}-19$ & $1.49919 \mathrm{D}-16$ \\
$0.00000 \mathrm{D}+00$ & $0.00000 \mathrm{D}+00$ \\
$-4.23516 \mathrm{D}-22$ & $-1.34467 \mathrm{D}-16$ \\
$0.00000 \mathrm{D}+00$ & $0.00000 \mathrm{D}+00$ \\
$0.00000 \mathrm{D}+00$ & $0.00000 \mathrm{D}+00$ \\
$4.23516 \mathrm{D}-22$ & $1.99245 \mathrm{D}-16$ \\
$0.00000 \mathrm{D}+00$ & $0.00000 \mathrm{D}+00$ \\
$0.00000 \mathrm{D}+00$ & $0.00000 \mathrm{D}+00$ \\
$4.97380 \mathrm{D}-14$ & $8.96196 \mathrm{D}-16$ \\
$4.98733 \mathrm{D}-18$ & $3.76703 \mathrm{D}-15$ \\
$4.77049 \mathrm{D}-18$ & $4.77049 \mathrm{D}-15$ \\
$2.71051 \mathrm{D}-20$ & $1.83927 \mathrm{D}-16$ \\
$1.08420 \mathrm{D}-19$ & $2.21454 \mathrm{D}-16$ \\
$-1.08420 \mathrm{D}-19$ & $-3.20098 \mathrm{D}-16$ \\
$2.71051 \mathrm{D}-20$ & $1.64273 \mathrm{D}-16$ \\
$0.00000 \mathrm{D}+00$ & $0.00000 \mathrm{D}+00$ \\
&
\end{tabular}


WSRC-TR-2002-00567

Revision 0

$\mathrm{CD}(+2)$

$\mathrm{CR}(+3)$

$\mathrm{U}(+4)$
8. $92857 \mathrm{D}-09$

$5.38461 \mathrm{D}-08$

$2.01681 \mathrm{D}-06$
$8.92857 \mathrm{D}-09$

$5.38461 \mathrm{D}-08$

2. $01681 \mathrm{D}-06$
$-1.27386 \mathrm{D}-21-1.42672 \mathrm{D}-13$

$6.61744 \mathrm{D}-24 \quad 1.22895 \mathrm{D}-16$

$4.23516 \mathrm{D}-22 \quad 2.09994 \mathrm{D}-16$ 
WSRC-TR-2002-00567

Revision 0

ESP V- 6.6

$\operatorname{DODEC}(-1)$
PROCESS:AWE 852

$3.37022 \mathrm{D}-05$
$12 / 05 / 2002$

$3.37022 \mathrm{D}-05$
$1.52656 \mathrm{D}-16$

4. 52954D-12 
WSRC-TR-2002-00567

Revision 0

\begin{tabular}{|c|c|c|c|}
\hline \multicolumn{4}{|l|}{$\begin{array}{l}\text { PROCESS BLOCKS } \\
===========\end{array}$} \\
\hline $\begin{array}{l}\mathrm{BLOCK} \mathrm{NAME} \\
=========================\end{array}$ & $\begin{array}{l}\text { BLOCK TYPE } \\
===========\end{array}$ & $\begin{array}{l}\text { INLET STREAM }(\mathrm{S}) \\
==============\end{array}$ & $\begin{array}{l}\text { OUTLET STREAM }(\mathrm{S}) \\
==============\end{array}$ \\
\hline Evap mixer & $\operatorname{Mix}$ & feed & Evap Contents \\
\hline Evap separator & Separate & Evap Contents & $\begin{array}{l}\text { Overhead } \\
\text { Bottoms }\end{array}$ \\
\hline Evap Bottoms Cooling mixer & Mix & Bottoms & Cooled Bottoms \\
\hline Condensate mixer & Mix & Overhead & Condensate \\
\hline
\end{tabular}


WSRC-TR-2002-00567

Revision 0

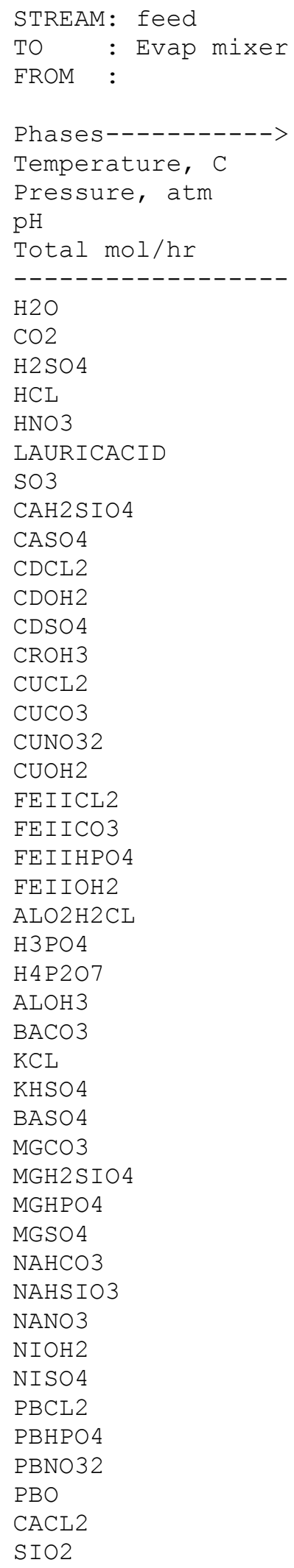

\begin{tabular}{|c|c|c|c|}
\hline Aqueous & Solid & Vapor & Organic \\
\hline 25 . & 25 . & 25 & 25 \\
\hline 1. & 1. & 1 . & 1 . \\
\hline \multicolumn{4}{|l|}{7.01951} \\
\hline 55.49823 & $6.67142 E-05$ & 0.0 & 0.0 \\
\hline \multicolumn{4}{|c|}{$\mathrm{mol} / \mathrm{hr}--------\operatorname{mol} / \mathrm{hr}--------\operatorname{mol} / \mathrm{hr}--------\operatorname{mol} / \mathrm{hr}--------$} \\
\hline 55.4921 & 0.0 & 0.0 & 0.0 \\
\hline $1.60822 \mathrm{E}-04$ & 0.0 & 0.0 & 0.0 \\
\hline $1.16750 \mathrm{E}-26$ & 0.0 & 0.0 & 0.0 \\
\hline $6.94021 \mathrm{E}-17$ & 0.0 & 0.0 & 0.0 \\
\hline $1.48942 \mathrm{E}-12$ & 0.0 & 0.0 & 0.0 \\
\hline $2.42548 E-07$ & 0.0 & 0.0 & 0.0 \\
\hline $1.51118 \mathrm{E}-30$ & 0.0 & 0.0 & 0.0 \\
\hline $5.93555 E-12$ & 0.0 & 0.0 & 0.0 \\
\hline $6.79310 E-06$ & 0.0 & 0.0 & 0.0 \\
\hline $1.60191 \mathrm{E}-11$ & 0.0 & 0.0 & 0.0 \\
\hline $4.04938 E-15$ & 0.0 & 0.0 & 0.0 \\
\hline $6.99908 \mathrm{E}-10$ & 0.0 & 0.0 & 0.0 \\
\hline $4.34229 E-12$ & 0.0 & 0.0 & 0.0 \\
\hline $2.74364 \mathrm{E}-14$ & 0.0 & 0.0 & 0.0 \\
\hline $1.54663 E-08$ & 0.0 & 0.0 & 0.0 \\
\hline $3.79737 \mathrm{E}-16$ & 0.0 & 0.0 & 0.0 \\
\hline $6.93396 E-09$ & 0.0 & 0.0 & 0.0 \\
\hline $2.96558 \mathrm{E}-16$ & 0.0 & 0.0 & 0.0 \\
\hline $1.92399 E-06$ & 0.0 & 0.0 & 0.0 \\
\hline $4.36978 E-08$ & 0.0 & 0.0 & 0.0 \\
\hline $1.07964 \mathrm{E}-11$ & 0.0 & 0.0 & 0.0 \\
\hline 1. $34256 \mathrm{E}-29$ & 0.0 & 0.0 & 0.0 \\
\hline $8.95271 E-10$ & 0.0 & 0.0 & 0.0 \\
\hline $1.42318 \mathrm{E}-21$ & 0.0 & 0.0 & 0.0 \\
\hline $2.40189 E-09$ & $6.29537 E-05$ & 0.0 & 0.0 \\
\hline $4.80173 E-11$ & 0.0 & 0.0 & 0.0 \\
\hline $1.94045 E-09$ & 0.0 & 0.0 & 0.0 \\
\hline $1.15313 E-14$ & 0.0 & 0.0 & 0.0 \\
\hline 1. $91622 \mathrm{E}-10$ & 0.0 & 0.0 & 0.0 \\
\hline $6.01490 E-08$ & 0.0 & 0.0 & 0.0 \\
\hline $1.85604 \mathrm{E}-11$ & 0.0 & 0.0 & 0.0 \\
\hline $8.69434 E-06$ & 0.0 & 0.0 & 0.0 \\
\hline $3.83028 E-06$ & 0.0 & 0.0 & 0.0 \\
\hline $4.14547 E-07$ & 0.0 & 0.0 & 0.0 \\
\hline $3.89439 E-08$ & 0.0 & 0.0 & 0.0 \\
\hline $2.33070 E-08$ & 0.0 & 0.0 & 0.0 \\
\hline 7. $28371 \mathrm{E}-12$ & 0.0 & 0.0 & 0.0 \\
\hline $4.64233 E-08$ & 0.0 & 0.0 & 0.0 \\
\hline $5.56810 \mathrm{E}-13$ & 0.0 & 0.0 & 0.0 \\
\hline $2.65334 \mathrm{E}-10$ & 0.0 & 0.0 & 0.0 \\
\hline $7.03494 E-15$ & 0.0 & 0.0 & 0.0 \\
\hline $4.13698 E-12$ & 0.0 & 0.0 & 0.0 \\
\hline $7.27332 E-28$ & 0.0 & 0.0 & 0.0 \\
\hline $1.64689 \mathrm{E}-04$ & 0.0 & 0.0 & 0.0 \\
\hline
\end{tabular}




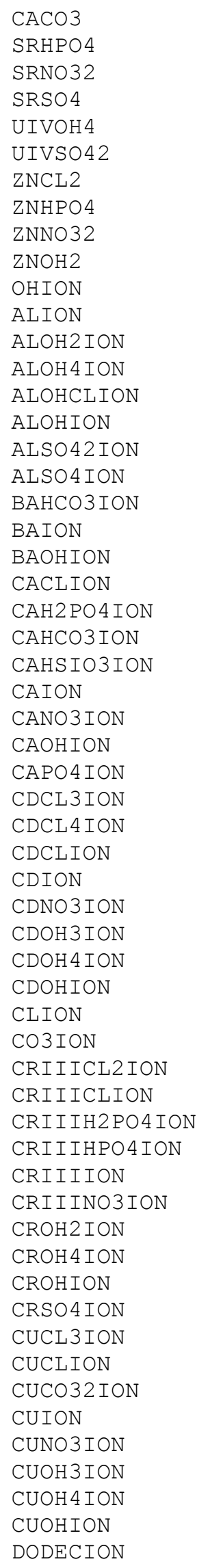

\begin{tabular}{|c|c|}
\hline $4.12042 E-07$ & 0.0 \\
\hline $6.22300 E-09$ & 0.0 \\
\hline $.40250 \mathrm{E}-12$ & 0.0 \\
\hline $39336 \mathrm{E}-07$ & \\
\hline $6 E-10$ & 0.0 \\
\hline $368 E-30$ & 0.0 \\
\hline $70 \mathrm{E}-11$ & 0.0 \\
\hline $16887 E-06$ & 0.0 \\
\hline $24 E-13$ & 0.0 \\
\hline $8 E-09$ & 0.0 \\
\hline$E-07$ & 0.0 \\
\hline $1 E-14$ & 0.0 \\
\hline $2 E-11$ & 0.0 \\
\hline $1 E-09$ & 0.0 \\
\hline$E-15$ & 0.0 \\
\hline $3 E-12$ & 0.0 \\
\hline$E-17$ & 0.0 \\
\hline $7 E-15$ & 0.0 \\
\hline$E-09$ & 0.0 \\
\hline $2 E-07$ & 0.0 \\
\hline $3 E-14$ & 0.0 \\
\hline $6 E-11$ & 0.0 \\
\hline$E-06$ & 0.0 \\
\hline $3 E-06$ & 0.0 \\
\hline $6 E-09$ & 0.0 \\
\hline $3 E-04$ & 0.0 \\
\hline $1 E-07$ & 0.0 \\
\hline $8 E-09$ & 0.0 \\
\hline $3 E-07$ & 0.0 \\
\hline $9 E-15$ & 0.0 \\
\hline $8 E-19$ & 0.0 \\
\hline $1 E-10$ & 0.0 \\
\hline $5 E-09$ & 0.0 \\
\hline $8 E-12$ & 0.0 \\
\hline $5 E-21$ & 0.0 \\
\hline $45 E-28$ & 0.0 \\
\hline $2 E-12$ & 0.0 \\
\hline 2391 & 0.0 \\
\hline $6 E-07$ & 0.0 \\
\hline $3 E-21$ & 0.0 \\
\hline $7 E-17$ & 0.0 \\
\hline $4 E-14$ & 0 . \\
\hline $5 E-08$ & 0.0 \\
\hline$E-15$ & 0.0 \\
\hline $7 E-16$ & 0.0 \\
\hline$O E-13$ & 0.0 \\
\hline $4 E-16$ & 0.0 \\
\hline $7 E-12$ & 0.0 \\
\hline $9 E-12$ & 0.0 \\
\hline$E-20$ & 0.0 \\
\hline $8 E-11$ & 0.0 \\
\hline$E-11$ & 0.0 \\
\hline $0 E-08$ & 0.0 \\
\hline $56 E-12$ & 0.0 \\
\hline $46 \mathrm{E}-14$ & 0.0 \\
\hline $62 E-20$ & 0.0 \\
\hline $77 E-09$ & 0.0 \\
\hline $97 E-05$ & 0.0 \\
\hline
\end{tabular}

0.0

0.0

0.0

0.0

0.0

0.0

0.0

0.0

0.0

0.0

0.0

0.0

0.0

0.0

0.0

0.0

0.0

0.0

0.0

0.0

0.0

0.0

0.0

0.0

0.0

0.0

0.0

0.0

0.0

0.0

0.0

0.0

0.0

0.0

0.0

0.0

0.0

0.0

0.0

0.0

0.0

0.0

0.0

0.0

0.0

0.0

0.0

0.0

0.0

0.0

0.0

0.0

0.0

0.0

0.0

0.0

0.0

0.0
0.0

0.0

0.0

0.0

0.0

0.0

0.0

0.0

0.0

0.0

0.0

0.0

0.0

0.0

0.0

0.0

0.0

0.0

0.0

0.0

0.0

0.0

0.0

0.0

0.0

0.0

0.0

0.0

0.0

0.0

0.0

0.0

0.0

0.0

0.0

0.0

0.0

0.0

0.0

0.0

0.0

0.0

0.0

0.0

0.0

0.0

0.0

0.0

0.0

0.0

0.0

0.0

0.0

0.0

0.0

0.0

0.0

0.0 


\begin{tabular}{|c|c|c|c|c|}
\hline FEIICLION & $7.09656 \mathrm{E}-11$ & 0.0 & 0.0 & 0.0 \\
\hline FEIICO32ION & $3.23108 \mathrm{E}-11$ & 0.0 & 0.0 & 0.0 \\
\hline FEIIH2PO4ION & $9.15242 \mathrm{E}-09$ & 0.0 & 0.0 & 0.0 \\
\hline FEIIHCO3ION & $4.72439 \mathrm{E}-09$ & 0.0 & 0.0 & 0.0 \\
\hline FEIIION & $5.34341 \mathrm{E}-05$ & 0.0 & 0.0 & 0.0 \\
\hline FEIIOH3ION & $4.82576 \mathrm{E}-15$ & 0.0 & 0.0 & 0.0 \\
\hline FEIIOH 4 ION & $6.54248 \mathrm{E}-23$ & 0.0 & 0.0 & 0.0 \\
\hline FEIIOHION & $1.39741 \mathrm{E}-07$ & 0.0 & 0.0 & 0.0 \\
\hline H2 P2O 7 ION & $2.74806 \mathrm{E}-11$ & 0.0 & 0.0 & 0.0 \\
\hline H2 PO 4 ION & $7.18485 \mathrm{E}-05$ & 0.0 & 0.0 & 0.0 \\
\hline H2SIO4ION & $2.79580 \mathrm{E}-13$ & 0.0 & 0.0 & 0.0 \\
\hline H3P2O 7 ION & $4.59024 \mathrm{E}-16$ & 0.0 & 0.0 & 0.0 \\
\hline H3SIO4 ION & $2.67579 \mathrm{E}-07$ & 0.0 & 0.0 & 0.0 \\
\hline HCO3ION & $8.25535 \mathrm{E}-04$ & 0.0 & 0.0 & 0.0 \\
\hline HION & $1.03270 \mathrm{E}-07$ & 0.0 & 0.0 & 0.0 \\
\hline HP207ION & $1.06353 \mathrm{E}-10$ & 0.0 & 0.0 & 0.0 \\
\hline HPBO2ION & $5.24068 \mathrm{E}-16$ & 0.0 & 0.0 & 0.0 \\
\hline HPO4 ION & $5.93972 \mathrm{E}-05$ & 0.0 & 0.0 & 0.0 \\
\hline HSO 4 ION & $3.39362 \mathrm{E}-09$ & 0.0 & 0.0 & 0.0 \\
\hline KION & $2.06248 \mathrm{E}-04$ & 0.0 & 0.0 & 0.0 \\
\hline KSO4 ION & $5.39414 \mathrm{E}-07$ & 0.0 & 0.0 & 0.0 \\
\hline MGH2 PO 4 ION & $5.70608 \mathrm{E}-07$ & 0.0 & 0.0 & 0.0 \\
\hline MGHCO3ION & $5.24665 \mathrm{E}-06$ & 0.0 & 0.0 & 0.0 \\
\hline MGHSIO3ION & $1.50880 \mathrm{E}-09$ & 0.0 & 0.0 & 0.0 \\
\hline MGION & $2.28294 \mathrm{E}-04$ & 0.0 & 0.0 & 0.0 \\
\hline MGOHION & $3.13797 \mathrm{E}-09$ & 0.0 & 0.0 & 0.0 \\
\hline MGP2O 7 ION & $1.26807 \mathrm{E}-09$ & 0.0 & 0.0 & 0.0 \\
\hline MGPO 4 ION & $2.11702 \mathrm{E}-07$ & 0.0 & 0.0 & 0.0 \\
\hline NACO3ION & $1.85791 \mathrm{E}-09$ & 0.0 & 0.0 & 0.0 \\
\hline NAION & 0.00140168 & 0.0 & 0.0 & 0.0 \\
\hline NASO 4 ION & $4.03861 \mathrm{E}-06$ & 0.0 & 0.0 & 0.0 \\
\hline NICLION & $8.60617 \mathrm{E}-11$ & 0.0 & 0.0 & 0.0 \\
\hline NIION & $8.88789 \mathrm{E}-07$ & 0.0 & 0.0 & 0.0 \\
\hline NINO3ION & $6.35138 \mathrm{E}-10$ & 0.0 & 0.0 & 0.0 \\
\hline NIOH3ION & $8.43213 E-16$ & 0.0 & 0.0 & 0.0 \\
\hline NIOHION & $1.02677 \mathrm{E}-09$ & 0.0 & 0.0 & 0.0 \\
\hline NO3ION & $3.38195 \mathrm{E}-04$ & 0.0 & 0.0 & 0.0 \\
\hline P207ION & $9.64276 \mathrm{E}-13$ & 0.0 & 0.0 & 0.0 \\
\hline PBCL3ION & $3.60198 \mathrm{E}-16$ & 0.0 & 0.0 & 0.0 \\
\hline PBCL 4 ION & $3.49513 \mathrm{E}-19$ & 0.0 & 0.0 & 0.0 \\
\hline PBCLION & $1.35530 \mathrm{E}-10$ & 0.0 & 0.0 & 0.0 \\
\hline PBH2 PO 4 ION & $1.10752 \mathrm{E}-11$ & 0.0 & 0.0 & 0.0 \\
\hline PBION & $5.09232 \mathrm{E}-09$ & 0.0 & 0.0 & 0.0 \\
\hline PBNO33ION & $4.06480 \mathrm{E}-19$ & 0.0 & 0.0 & 0.0 \\
\hline PBNO3ION & $2.50043 E-11$ & 0.0 & 0.0 & 0.0 \\
\hline PBOHION & $1.07779 \mathrm{E}-09$ & 0.0 & 0.0 & 0.0 \\
\hline PO4ION & $4.38231 \mathrm{E}-10$ & 0.0 & 0.0 & 0.0 \\
\hline SO4ION & $4.73792 \mathrm{E}-04$ & 0.0 & 0.0 & 0.0 \\
\hline SRION & $1.07407 \mathrm{E}-05$ & 0.0 & 0.0 & 0.0 \\
\hline SRNO3ION & $1.49637 \mathrm{E}-08$ & 0.0 & 0.0 & 0.0 \\
\hline SROHION & $5.72876 \mathrm{E}-12$ & 0.0 & 0.0 & 0.0 \\
\hline SRPO 4 ION & $9.64015 \mathrm{E}-11$ & 0.0 & 0.0 & 0.0 \\
\hline UIVOH2ION & $4.56739 \mathrm{E}-22$ & 0.0 & 0.0 & 0.0 \\
\hline UIVOH3ION & $3.51398 \mathrm{E}-16$ & 0.0 & 0.0 & 0.0 \\
\hline UIVOH5ION & $1.78225 \mathrm{E}-13$ & 0.0 & 0.0 & 0.0 \\
\hline UIVOHION & $9.61721 \mathrm{E}-27$ & 0.0 & 0.0 & 0.0 \\
\hline UIVSO 4 ION & $1.29621 \mathrm{E}-30$ & 0.0 & 0.0 & 0.0 \\
\hline ZNCL3ION & $1.72381 \mathrm{E}-14$ & 0.0 & 0.0 & 0.0 \\
\hline
\end{tabular}


WSRC-TR-2002-00567

Revision 0

\begin{tabular}{|c|c|c|c|c|}
\hline ZNCLION & $3.02381 E-08$ & 0.0 & 0.0 & 0.0 \\
\hline ZNH2 PO4 ION & $3.98550 \mathrm{E}-08$ & 0.0 & 0.0 & 0.0 \\
\hline ZNHCO3ION & $2.59775 \mathrm{E}-07$ & 0.0 & 0.0 & 0.0 \\
\hline ZNION & $1.96875 E-05$ & 0.0 & 0.0 & 0.0 \\
\hline ZNNO3ION & $1.40707 \mathrm{E}-08$ & 0.0 & 0.0 & 0.0 \\
\hline ZNOH3ION & $8.74325 E-13$ & 0.0 & 0.0 & 0.0 \\
\hline ZNOH 4 ION & $1.49181 \mathrm{E}-18$ & 0.0 & 0.0 & 0.0 \\
\hline ZNOHION & $1.98555 \mathrm{E}-07$ & 0.0 & 0.0 & 0.0 \\
\hline $\mathrm{CU} 3 \mathrm{PO} 42.2 \mathrm{H} 2 \mathrm{O}$ & 0.0 & $1.03769 \mathrm{E}-06$ & 0.0 & 0.0 \\
\hline РВ3РО42 & 0.0 & $7.06331 \mathrm{E}-07$ & 0.0 & 0.0 \\
\hline \multirow[t]{2}{*}{ UIVO2 } & 0.0 & $2.01650 \mathrm{E}-06$ & 0.0 & 0.0 \\
\hline & $===========$ & $===========$ & $===========$ & $===========$ \\
\hline Total g/hr & 999.994 & 0.00646066 & 0.0 & 0.0 \\
\hline Volume, L/hr & 1.00293 & $2.14241 E-06$ & 0.0 & 0.0 \\
\hline Enthalpy, cal/hr & $-3.79147 \mathrm{E}+06$ & -20.2479 & 0.0 & 0.0 \\
\hline Density, g/L & 997.073 & 3015.6 & & \\
\hline Vapor fraction & 0.0 & 0.0 & 0.0 & 0.0 \\
\hline Solid fraction & 0.0 & 1 . & 0.0 & 0.0 \\
\hline Organic fraction & 0.0 & 0.0 & 0.0 & 0.0 \\
\hline Osmotic Pres, atm & 0.166988 & & & \\
\hline Redox Pot, volts & 0.0 & & & \\
\hline E-Con, 1/ohm-cm & $4.08198 E-04$ & & & \\
\hline E-Con, cm2/ohm-mol & 139.526 & & & \\
\hline Abs Visc, cP & 0.892087 & & & \\
\hline Rel Visc & 1.00154 & & & \\
\hline Ionic Strength & 0.00522365 & & & \\
\hline
\end{tabular}


WSRC-TR-2002-00567

Revision 0

\begin{tabular}{|c|c|c|c|c|}
\hline \\
\hline \multicolumn{5}{|c|}{$\begin{array}{l}\text { STREAM: Evap Contents } \\
\text { TO : Evap separator }\end{array}$} \\
\hline FROM : Evap mixer & & & & \\
\hline Phases-----------> & Aqueous & Solid & Vapor & Organic \\
\hline & 102.754 & 102.754 & 102.754 & 102.754 \\
\hline $\begin{array}{l}\text { Pressure, atm } \\
\mathrm{pH}\end{array}$ & $\begin{array}{l}1 . \\
8.02839\end{array}$ & 1 . & 1 . & 1. \\
\hline \multirow{2}{*}{ Total mol/hr } & 0.03692398 & $9.66192 \mathrm{E}-04$ & 55.4597 & 0.0 \\
\hline & $\mathrm{mol} / \mathrm{hr}------$ & $-\operatorname{mol} / \mathrm{hr}-----$ & $-\mathrm{mol} / \mathrm{hr}----$. & $-\operatorname{mol} / \mathrm{hr}--------$ \\
\hline $\mathrm{H} 2 \mathrm{O}$ & 0.0336664 & 0.0 & 55.45868 & 0.0 \\
\hline $\mathrm{CO} 2$ & $8.45986 \mathrm{E}-11$ & 0.0 & $9.91404 \mathrm{E}-04$ & 0.0 \\
\hline & $1.10416 \mathrm{E}-30$ & 0.0 & $1.45453 E-27$ & 0.0 \\
\hline $\begin{array}{l}\mathrm{H} \angle \mathrm{SO} 4 \\
\mathrm{HCL}\end{array}$ & $2.05353 \mathrm{E}-16$ & 0.0 & $2.94330 \mathrm{E}-10$ & 0.0 \\
\hline & $2.02651 \mathrm{E}-13$ & 0.0 & $6.53194 \mathrm{E}-11$ & 0.0 \\
\hline LAURICACID & $3.31137 \mathrm{E}-10$ & 0.0 & $2.56699 \mathrm{E}-05$ & 0.0 \\
\hline & $1.40949 \mathrm{E}-09$ & 0.0 & 0.0 & 0.0 \\
\hline $\mathrm{CASO} 4$ & $3.83930 \mathrm{E}-07$ & $4.58556 \mathrm{E}-04$ & 0.0 & 0.0 \\
\hline CDCL2 & $4.69456 \mathrm{E}-09$ & 0.0 & 0.0 & 0.0 \\
\hline $\mathrm{CDOH} 2$ & $4.50076 \mathrm{E}-13$ & 0.0 & 0.0 & 0.0 \\
\hline $\mathrm{CDSO} 4$ & $3.34449 E-13$ & 0.0 & 0.0 & 0.0 \\
\hline $\mathrm{CROH} 3$ & $2.49966 \mathrm{E}-11$ & $5.38148 E-08$ & 0.0 & 0.0 \\
\hline CUCL2 & $2.47903 E-12$ & 0.0 & 0.0 & 0.0 \\
\hline CUCO3 & $5.94988 \mathrm{E}-14$ & 0.0 & 0.0 & 0.0 \\
\hline CUNO32 & $1.76863 \mathrm{E}-14$ & 0.0 & 0.0 & 0.0 \\
\hline CUOH2 & $6.01398 E-09$ & $3.14345 E-06$ & 0.0 & 0.0 \\
\hline FEIICL2 & $2.56798 \mathrm{E}-16$ & 0.0 & 0.0 & 0.0 \\
\hline FEIICO3 & $1.08858 \mathrm{E}-14$ & 0.0 & 0.0 & 0.0 \\
\hline FEIIHPO4 & $6.11399 \mathrm{E}-20$ & 0.0 & 0.0 & 0.0 \\
\hline FEIIOH2 & $3.44292 E-13$ & 0.0 & 0.0 & 0.0 \\
\hline $\mathrm{ALO} 2 \mathrm{H} 2 \mathrm{CL}$ & 0.0 & 0.0 & 0.0 & 0.0 \\
\hline H3 $\mathrm{PO} 4$ & $1.38155 \mathrm{E}-19$ & 0.0 & 0.0 & 0.0 \\
\hline ALOH3 & $2.20629 \mathrm{E}-11$ & 0.0 & 0.0 & 0.0 \\
\hline $\mathrm{BACO} 3$ & $3.00355 E-14$ & 0.0 & 0.0 & 0.0 \\
\hline $\mathrm{KCL}$ & $3.73000 \mathrm{E}-06$ & 0.0 & 0.0 & 0.0 \\
\hline $\mathrm{KHSO} 4$ & $4.55751 \mathrm{E}-14$ & 0.0 & 0.0 & 0.0 \\
\hline $\mathrm{BASO} 4$ & $6.84051 E-13$ & $4.06550 E-07$ & 0.0 & 0.0 \\
\hline $\mathrm{MGCO} 3$ & $4.73991 \mathrm{E}-11$ & 0.0 & 0.0 & 0.0 \\
\hline MGH2SIO4 & $1.11885 E-09$ & 0.0 & 0.0 & 0.0 \\
\hline MGHPO 4 & $1.17969 \mathrm{E}-12$ & 0.0 & 0.0 & 0.0 \\
\hline MGSO 4 & $2.31764 \mathrm{E}-08$ & 0.0 & 0.0 & 0.0 \\
\hline $\mathrm{NAHCO} 3$ & $8.17297 E-09$ & 0.0 & 0.0 & 0.0 \\
\hline NAHSIO3 & $5.57625 \mathrm{E}-06$ & 0.0 & 0.0 & 0.0 \\
\hline NANO3 & $7.20620 \mathrm{E}-05$ & 0.0 & 0.0 & 0.0 \\
\hline $\mathrm{NIOH} 2$ & $1.58825 \mathrm{E}-12$ & $9.36907 E-07$ & 0.0 & 0.0 \\
\hline $\mathrm{NISO} 4$ & $3.17467 \mathrm{E}-12$ & 0.0 & 0.0 & 0.0 \\
\hline PBCL2 & $1.46340 \mathrm{E}-07$ & 0.0 & 0.0 & 0.0 \\
\hline PBHPO 4 & $8.46242 E-16$ & 0.0 & 0.0 & 0.0 \\
\hline PBNO32 & $4.43245 E-09$ & 0.0 & 0.0 & 0.0 \\
\hline $\mathrm{PBO}$ & $4.16487 \mathrm{E}-08$ & 0.0 & 0.0 & 0.0 \\
\hline CACL2 & $3.36268 \mathrm{E}-15$ & 0.0 & 0.0 & 0.0 \\
\hline $\mathrm{SIO} 2$ & $2.84398 E-06$ & $1.26773 E-04$ & 0.0 & 0.0 \\
\hline $\mathrm{CACO} 3$ & $1.79262 E-09$ & $8.57765 E-06$ & 0.0 & 0.0 \\
\hline SRHPO4 & $2.54974 \mathrm{E}-15$ & 0.0 & 0.0 & 0.0 \\
\hline
\end{tabular}




\begin{tabular}{|c|c|c|c|c|}
\hline SRNO32 & $8.97745 \mathrm{E}-08$ & 0.0 & 0.0 & 0.0 \\
\hline $\mathrm{SRSO} 4$ & $6.54174 \mathrm{E}-09$ & $1.06798 \mathrm{E}-05$ & 0.0 & 0.0 \\
\hline UIVOH 4 & $8.37123 \mathrm{E}-13$ & 0.0 & 0.0 & 0.0 \\
\hline ZNCL2 & $1.78229 \mathrm{E}-06$ & 0.0 & 0.0 & 0.0 \\
\hline ZNHPO 4 & $3.26913 \mathrm{E}-14$ & 0.0 & 0.0 & 0.0 \\
\hline ZNNO32 & $7.91958 \mathrm{E}-10$ & 0.0 & 0.0 & 0.0 \\
\hline ZNOH2 & $6.18627 \mathrm{E}-07$ & 0.0 & 0.0 & 0.0 \\
\hline OHION & $5.95771 \mathrm{E}-08$ & 0.0 & 0.0 & 0.0 \\
\hline ALION & $7.85826 \mathrm{E}-24$ & 0.0 & 0.0 & 0.0 \\
\hline ALOH2 ION & $2.60627 \mathrm{E}-15$ & 0.0 & 0.0 & 0.0 \\
\hline ALOH 4 ION & $2.01890 \mathrm{E}-08$ & 0.0 & 0.0 & 0.0 \\
\hline ALOHCLION & $9.15109 \mathrm{E}-19$ & 0.0 & 0.0 & 0.0 \\
\hline ALOHION & $3.05609 \mathrm{E}-19$ & 0.0 & 0.0 & 0.0 \\
\hline ALSO 42 ION & $9.02522 \mathrm{E}-25$ & 0.0 & 0.0 & 0.0 \\
\hline ALSO 4 ION & $8.43341 \mathrm{E}-24$ & 0.0 & 0.0 & 0.0 \\
\hline BAHCO3ION & $4.37644 \mathrm{E}-13$ & 0.0 & 0.0 & 0.0 \\
\hline BAION & $2.20773 E-09$ & 0.0 & 0.0 & 0.0 \\
\hline BAOHION & $1.23723 \mathrm{E}-13$ & 0.0 & 0.0 & 0.0 \\
\hline CACLION & $8.18426 \mathrm{E}-08$ & 0.0 & 0.0 & 0.0 \\
\hline CAH2 PO 4 ION & $1.07390 \mathrm{E}-13$ & 0.0 & 0.0 & 0.0 \\
\hline CAHCO3ION & $8.47306 \mathrm{E}-10$ & 0.0 & 0.0 & 0.0 \\
\hline CAHSIO3ION & $1.22029 \mathrm{E}-07$ & 0.0 & 0.0 & 0.0 \\
\hline CAION & $2.40130 \mathrm{E}-05$ & 0.0 & 0.0 & 0.0 \\
\hline CANO3ION & $1.03754 \mathrm{E}-05$ & 0.0 & 0.0 & 0.0 \\
\hline CAOHION & $2.52168 \mathrm{E}-08$ & 0.0 & 0.0 & 0.0 \\
\hline CAPO 4 ION & $1.05563 \mathrm{E}-11$ & 0.0 & 0.0 & 0.0 \\
\hline CDCL3ION & $3.25648 \mathrm{E}-09$ & 0.0 & 0.0 & 0.0 \\
\hline CDCL 4 ION & $6.84901 \mathrm{E}-10$ & 0.0 & 0.0 & 0.0 \\
\hline CDCLION & $2.83352 \mathrm{E}-10$ & 0.0 & 0.0 & 0.0 \\
\hline CDION & $6.11628 \mathrm{E}-12$ & 0.0 & 0.0 & 0.0 \\
\hline CDNO3ION & $1.52455 \mathrm{E}-12$ & 0.0 & 0.0 & 0.0 \\
\hline CDOH3ION & $3.64377 \mathrm{E}-16$ & 0.0 & 0.0 & 0.0 \\
\hline CDOH 4 ION & $1.74748 \mathrm{E}-19$ & 0.0 & 0.0 & 0.0 \\
\hline CDOHION & $8.50008 \mathrm{E}-13$ & 0.0 & 0.0 & 0.0 \\
\hline CLION & 0.00130005 & 0.0 & 0.0 & 0.0 \\
\hline CO3ION & $6.84639 \mathrm{E}-10$ & 0.0 & 0.0 & 0.0 \\
\hline CRIIICL2ION & $2.33591 \mathrm{E}-18$ & 0.0 & 0.0 & 0.0 \\
\hline CRIIICLION & $2.46967 \mathrm{E}-17$ & 0.0 & 0.0 & 0.0 \\
\hline CRIIIH2PO4 ION & $3.19395 E-25$ & 0.0 & 0.0 & 0.0 \\
\hline CRIIIHPO4ION & $7.70390 \mathrm{E}-13$ & 0.0 & 0.0 & 0.0 \\
\hline CRIIIION & $3.42549 \mathrm{E}-20$ & 0.0 & 0.0 & 0.0 \\
\hline CRIIINO3ION & $1.01079 \mathrm{E}-15$ & 0.0 & 0.0 & 0.0 \\
\hline CROH2ION & $7.24619 \mathrm{E}-14$ & 0.0 & 0.0 & 0.0 \\
\hline CROH 4 ION & $3.16473 \mathrm{E}-13$ & 0.0 & 0.0 & 0.0 \\
\hline CROHION & $5.13133 E-12$ & 0.0 & 0.0 & 0.0 \\
\hline CRSO 4 ION & $8.63597 \mathrm{E}-15$ & 0.0 & 0.0 & 0.0 \\
\hline CUCL3ION & $2.96340 \mathrm{E}-14$ & 0.0 & 0.0 & 0.0 \\
\hline CUCLION & $1.63948 \mathrm{E}-11$ & 0.0 & 0.0 & 0.0 \\
\hline CUCO32ION & $3.24991 \mathrm{E}-16$ & 0.0 & 0.0 & 0.0 \\
\hline CUION & $5.07538 \mathrm{E}-12$ & 0.0 & 0.0 & 0.0 \\
\hline CUNO3ION & $8.04294 \mathrm{E}-13$ & 0.0 & 0.0 & 0.0 \\
\hline CUOH3ION & $3.05339 \mathrm{E}-11$ & 0.0 & 0.0 & 0.0 \\
\hline CUOH 4 ION & $3.45909 \mathrm{E}-13$ & 0.0 & 0.0 & 0.0 \\
\hline CUOHION & $8.14155 \mathrm{E}-11$ & 0.0 & 0.0 & 0.0 \\
\hline DODECION & $8.03202 \mathrm{E}-06$ & 0.0 & 0.0 & 0.0 \\
\hline FEIICLION & $5.70151 \mathrm{E}-14$ & 0.0 & 0.0 & 0.0 \\
\hline FEIICO32ION & $1.79477 \mathrm{E}-18$ & 0.0 & 0.0 & 0.0 \\
\hline FEIIH2PO4ION & $1.12139 \mathrm{E}-21$ & 0.0 & 0.0 & 0.0 \\
\hline
\end{tabular}




\begin{tabular}{|c|c|c|c|c|}
\hline FEIIHCO3ION & $5.17038 \mathrm{E}-18$ & 0.0 & 0.0 & 0.0 \\
\hline FEIIION & $7.60978 \mathrm{E}-12$ & 0.0 & 0.0 & 0.0 \\
\hline FEIIOH3ION & $3.60461 \mathrm{E}-14$ & 0.0 & 0.0 & 0.0 \\
\hline FEIIOH 4 ION & $4.85157 \mathrm{E}-18$ & 0.0 & 0.0 & 0.0 \\
\hline FEIIOHION & $4.39727 \mathrm{E}-12$ & 0.0 & 0.0 & 0.0 \\
\hline H2 P2O 7 ION & $9.13297 \mathrm{E}-24$ & 0.0 & 0.0 & 0.0 \\
\hline $\mathrm{H} 2 \mathrm{PO} 4 \mathrm{ION}$ & $1.41129 \mathrm{E}-13$ & 0.0 & 0.0 & 0.0 \\
\hline H2SIO4 ION & $1.55131 \mathrm{E}-10$ & 0.0 & 0.0 & 0.0 \\
\hline H3P2O 7 ION & $2.18083 E-30$ & 0.0 & 0.0 & 0.0 \\
\hline H3SIO 4 ION & $1.89190 \mathrm{E}-06$ & 0.0 & 0.0 & 0.0 \\
\hline HCO3ION & $6.21526 \mathrm{E}-09$ & 0.0 & 0.0 & 0.0 \\
\hline HION & $7.44004 \mathrm{E}-12$ & 0.0 & 0.0 & 0.0 \\
\hline HP2O7 ION & $9.67561 \mathrm{E}-22$ & 0.0 & 0.0 & 0.0 \\
\hline HPBO2ION & $2.17424 \mathrm{E}-09$ & 0.0 & 0.0 & 0.0 \\
\hline HPO 4 ION & $7.41704 \mathrm{E}-12$ & 0.0 & 0.0 & 0.0 \\
\hline HSO4 ION & $9.06469 \mathrm{E}-12$ & 0.0 & 0.0 & 0.0 \\
\hline KION & $1.99619 \mathrm{E}-04$ & 0.0 & 0.0 & 0.0 \\
\hline $\mathrm{KSO} 4 \mathrm{ION}$ & $3.43971 \mathrm{E}-06$ & 0.0 & 0.0 & 0.0 \\
\hline MGH2 PO 4 ION & $1.92950 \mathrm{E}-15$ & 0.0 & 0.0 & 0.0 \\
\hline MGHCO3ION & $8.86580 \mathrm{E}-11$ & 0.0 & 0.0 & 0.0 \\
\hline MGHSIO3ION & $1.27147 \mathrm{E}-08$ & 0.0 & 0.0 & 0.0 \\
\hline MGION & $6.66297 \mathrm{E}-07$ & 0.0 & 0.0 & 0.0 \\
\hline MGOHION & $1.11071 \mathrm{E}-08$ & 0.0 & 0.0 & 0.0 \\
\hline MGP2O7 ION & $9.53207 \mathrm{E}-19$ & 0.0 & 0.0 & 0.0 \\
\hline MGPO 4 ION & $6.60609 \mathrm{E}-13$ & 0.0 & 0.0 & 0.0 \\
\hline NACO3ION & $1.05927 \mathrm{E}-10$ & 0.0 & 0.0 & 0.0 \\
\hline NAION & 0.00132855 & 0.0 & 0.0 & 0.0 \\
\hline NASO 4 ION & $3.14083 \mathrm{E}-13$ & 0.0 & 0.0 & 0.0 \\
\hline NICLION & $2.98284 \mathrm{E}-12$ & 0.0 & 0.0 & 0.0 \\
\hline NIION & $4.13937 \mathrm{E}-11$ & 0.0 & 0.0 & 0.0 \\
\hline NINO3ION & $5.17392 \mathrm{E}-12$ & 0.0 & 0.0 & 0.0 \\
\hline NIOH3ION & $2.02244 \mathrm{E}-14$ & 0.0 & 0.0 & 0.0 \\
\hline NIOHION & $6.47792 \mathrm{E}-12$ & 0.0 & 0.0 & 0.0 \\
\hline NO3ION & $2.55714 \mathrm{E}-04$ & 0.0 & 0.0 & 0.0 \\
\hline P207ION & $8.94668 \mathrm{E}-22$ & 0.0 & 0.0 & 0.0 \\
\hline PBCL3ION & $2.54069 \mathrm{E}-07$ & 0.0 & 0.0 & 0.0 \\
\hline PBCL 4 ION & $1.45033 \mathrm{E}-06$ & 0.0 & 0.0 & 0.0 \\
\hline PBCLION & $4.35974 \mathrm{E}-08$ & 0.0 & 0.0 & 0.0 \\
\hline $\mathrm{PBH} 2 \mathrm{PO} 4 \mathrm{ION}$ & $7.56365 \mathrm{E}-18$ & 0.0 & 0.0 & 0.0 \\
\hline PBION & $1.57136 \mathrm{E}-09$ & 0.0 & 0.0 & 0.0 \\
\hline PBNO33ION & $1.27725 \mathrm{E}-10$ & 0.0 & 0.0 & 0.0 \\
\hline PBNO3ION & $1.25704 \mathrm{E}-08$ & 0.0 & 0.0 & 0.0 \\
\hline PBOHION & $1.68743 \mathrm{E}-07$ & 0.0 & 0.0 & 0.0 \\
\hline PO4ION & $6.14844 \mathrm{E}-15$ & 0.0 & 0.0 & 0.0 \\
\hline SO4ION & $1.60872 \mathrm{E}-05$ & 0.0 & 0.0 & 0.0 \\
\hline SRION & $1.97064 \mathrm{E}-07$ & 0.0 & 0.0 & 0.0 \\
\hline SRNO3ION & $3.27990 \mathrm{E}-07$ & 0.0 & 0.0 & 0.0 \\
\hline SROHION & $1.81995 \mathrm{E}-10$ & 0.0 & 0.0 & 0.0 \\
\hline SRPO 4 ION & $2.44782 E-15$ & 0.0 & 0.0 & 0.0 \\
\hline UIVOH2ION & $1.41254 \mathrm{E}-27$ & 0.0 & 0.0 & 0.0 \\
\hline UIVOH3ION & $2.23720 \mathrm{E}-21$ & 0.0 & 0.0 & 0.0 \\
\hline UIVOH5ION & $4.03654 \mathrm{E}-14$ & 0.0 & 0.0 & 0.0 \\
\hline ZNCL3ION & $1.79483 \mathrm{E}-06$ & 0.0 & 0.0 & 0.0 \\
\hline ZNCLION & $4.20624 \mathrm{E}-06$ & 0.0 & 0.0 & 0.0 \\
\hline ZNH2 PO 4 ION & $1.42368 \mathrm{E}-16$ & 0.0 & 0.0 & 0.0 \\
\hline ZNHCO3ION & $5.25506 \mathrm{E}-12$ & 0.0 & 0.0 & 0.0 \\
\hline ZNION & $2.88376 \mathrm{E}-07$ & 0.0 & 0.0 & 0.0 \\
\hline ZNNO3ION & $2.76236 \mathrm{E}-08$ & 0.0 & 0.0 & 0.0 \\
\hline
\end{tabular}


WSRC-TR-2002-00567

Revision 0

\begin{tabular}{|c|c|c|c|c|}
\hline ZNOH3ION & $1.28146 \mathrm{E}-07$ & 0.0 & 0.0 & 0.0 \\
\hline ZNOH $4 \mathrm{ION}$ & $4.96039 \mathrm{E}-10$ & 0.0 & 0.0 & 0.0 \\
\hline ZNOHION & $1.25593 E-05$ & 0.0 & 0.0 & 0.0 \\
\hline $\mathrm{ALOOH}$ & 0.0 & $7.38720 E-06$ & 0.0 & 0.0 \\
\hline $\mathrm{CA} 3 \mathrm{PO} 42$ & 0.0 & $7.36842 E-05$ & 0.0 & 0.0 \\
\hline CHAMOSITE7A & 0.0 & $2.77778 E-05$ & 0.0 & 0.0 \\
\hline $\mathrm{MGOH} 2$ & 0.0 & $2.46199 \mathrm{E}-04$ & 0.0 & 0.0 \\
\hline UIVO2 & 0.0 & $2.01681 E-06$ & 0.0 & 0.0 \\
\hline & $===========$ & $===========$ & $===========$ & $===========$ \\
\hline Total g/hr & 0.722739 & 0.121055 & 999.156 & 0.0 \\
\hline Volume, L/hr & $6.83304 \mathrm{E}-04$ & $3.89195 E-05$ & 1696.67 & 0.0 \\
\hline Enthalpy, cal/hr & -2430.03 & -343.728 & $-3.17154 \mathrm{E}+06$ & 0.0 \\
\hline Density, g/L & 1057.71 & 3110.38 & 0.588891 & \\
\hline Vapor fraction & 0.0 & 0.0 & 1 . & 0.0 \\
\hline Solid fraction & 0.0 & 1 . & 0.0 & 0.0 \\
\hline Organic fraction & 0.0 & 0.0 & 0.0 & 0.0 \\
\hline Osmotic Pres, atm & 156.136 & & & \\
\hline Redox Pot, volts & 0.0 & & & \\
\hline $\mathrm{E}-\mathrm{Con}, 1 / \mathrm{ohm}-\mathrm{cm}$ & 0.449358 & & & \\
\hline E-Con, cm2/ohm-mol & 51.8522 & & & \\
\hline Abs Visc, cP & 0.376299 & & & \\
\hline Rel Visc & 1.37516 & & & \\
\hline Ionic Strength & 2.71916 & & & \\
\hline
\end{tabular}


WSRC-TR-2002-00567

Revision 0

\begin{tabular}{|c|c|c|c|c|}
\hline \multicolumn{5}{|l|}{ STREAM: Overhead } \\
\hline TO : Condensate & mixer & & & \\
\hline FROM : Evap separ & tor & & & \\
\hline Phases-----------> & Aqueous & Solid & Vapor & Organic \\
\hline Temperature, C & 102.754 & 102.754 & 102.754 & 102.754 \\
\hline $\begin{array}{l}\text { Pressure, atm } \\
\mathrm{pH}\end{array}$ & $\begin{array}{l}1 . \\
0.0\end{array}$ & 1. & 1. & 1 . \\
\hline Total mol/hr & 0.0 & 0.0 & 55.4597 & 0.0 \\
\hline------------------ & $\mathrm{mol} / \mathrm{hr}-------$ & -mol/hr------- & $-\operatorname{mol} / \mathrm{hr}-------1$ & -mol/hr------ \\
\hline $\mathrm{H} 2 \mathrm{O}$ & 0.0 & 0.0 & 55.45868 & 0.0 \\
\hline $\mathrm{CO} 2$ & 0.0 & 0.0 & $9.91404 \mathrm{E}-04$ & 0.0 \\
\hline $\mathrm{H} 2 \mathrm{SO} 4$ & 0.0 & 0.0 & $1.45453 E-27$ & 0.0 \\
\hline $\mathrm{HCL}$ & 0.0 & 0.0 & $2.94330 \mathrm{E}-10$ & 0.0 \\
\hline HNO3 & 0.0 & 0.0 & $6.53194 \mathrm{E}-11$ & 0.0 \\
\hline \multirow[t]{2}{*}{ LAURICACID } & 0.0 & 0.0 & $2.56699 \mathrm{E}-05$ & 0.0 \\
\hline & $===========$ & $===========$ & $===========$ & $===========$ \\
\hline Total g/hr & 0.0 & 0.0 & 999.156 & 0.0 \\
\hline Volume, L/hr & 0.0 & 0.0 & 1696.67 & 0.0 \\
\hline $\begin{array}{l}\text { Enthalpy, cal/hr } \\
\text { Density, g/L }\end{array}$ & 0.0 & 0.0 & $\begin{array}{l}-3.17154 \mathrm{E}+06 \\
0.588891\end{array}$ & 0.0 \\
\hline Vapor fraction & 0.0 & 0.0 & 1 . & 0.0 \\
\hline Solid fraction & 0.0 & 0.0 & 0.0 & 0.0 \\
\hline Organic fraction & 0.0 & 0.0 & 0.0 & 0.0 \\
\hline Osmotic Pres, atm & 0.0 & & & \\
\hline Redox Pot, volts & 0.0 & & & \\
\hline $\mathrm{E}-\mathrm{Con}, 1 / \mathrm{ohm}-\mathrm{cm}$ & 0.0 & & & \\
\hline E-Con, cm2/ohm-mol & 0.0 & & & \\
\hline \multirow{2}{*}{$\begin{array}{l}\text { Abs Visc, cP } \\
\text { Rel Visc }\end{array}$} & 0.0 & & & \\
\hline & 0.0 & & & \\
\hline Ionic Strength & 0.0 & & & \\
\hline
\end{tabular}


WSRC-TR-2002-00567

Revision 0

$\begin{array}{lllll}\text { ESP V-6.6 PROCESS:AWE85_2 } & \text { PAGE } 7\end{array}$

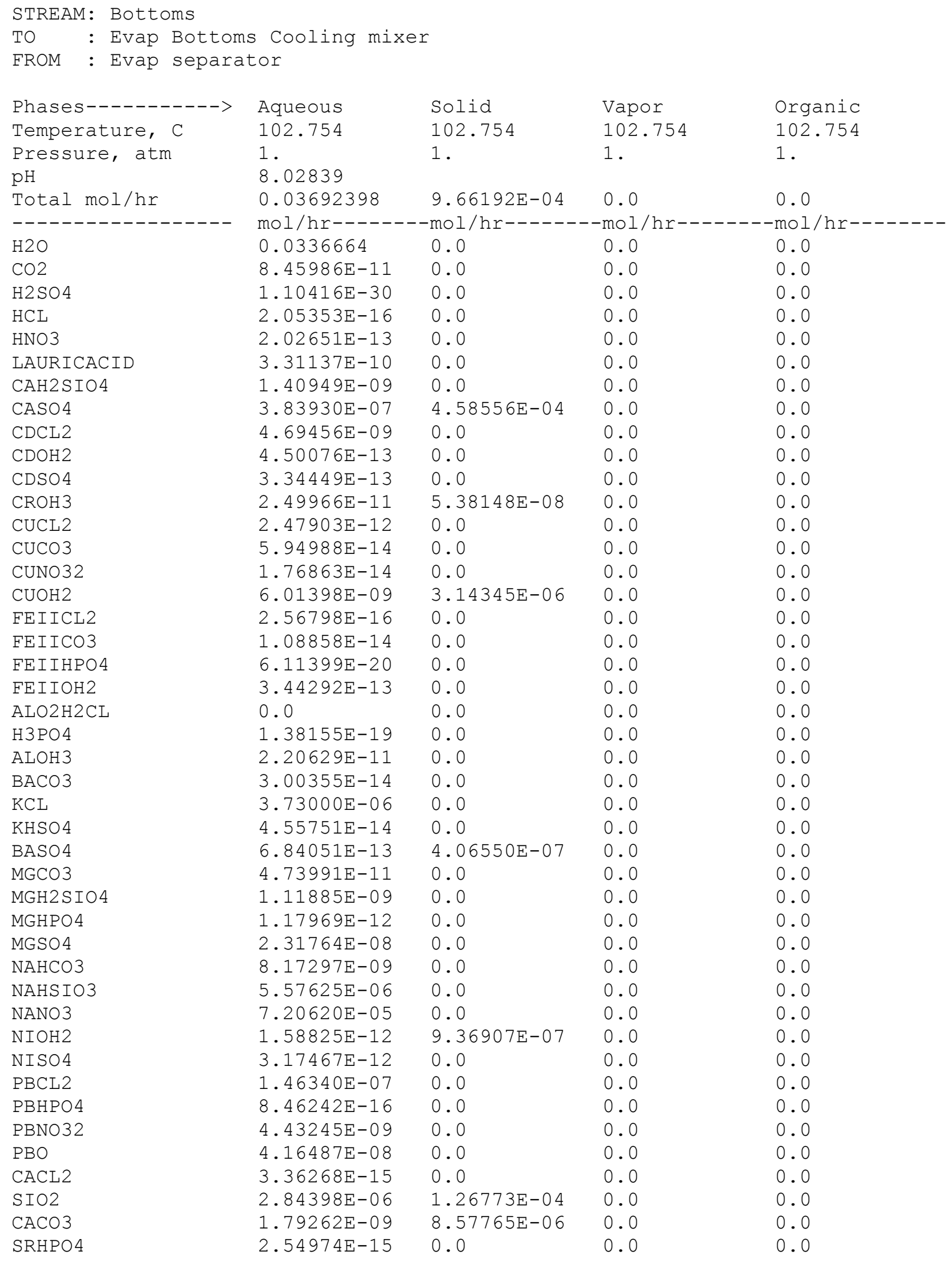




\begin{tabular}{|c|c|c|c|c|}
\hline SRNO32 & $8.97745 \mathrm{E}-08$ & 0.0 & 0.0 & 0.0 \\
\hline $\mathrm{SRSO} 4$ & $6.54174 \mathrm{E}-09$ & $1.06798 \mathrm{E}-05$ & 0.0 & 0.0 \\
\hline UIVOH 4 & $8.37123 \mathrm{E}-13$ & 0.0 & 0.0 & 0.0 \\
\hline ZNCL2 & $1.78229 \mathrm{E}-06$ & 0.0 & 0.0 & 0.0 \\
\hline ZNHPO 4 & $3.26913 \mathrm{E}-14$ & 0.0 & 0.0 & 0.0 \\
\hline ZNNO32 & $7.91958 \mathrm{E}-10$ & 0.0 & 0.0 & 0.0 \\
\hline ZNOH2 & $6.18627 \mathrm{E}-07$ & 0.0 & 0.0 & 0.0 \\
\hline OHION & $5.95771 \mathrm{E}-08$ & 0.0 & 0.0 & 0.0 \\
\hline ALION & $7.85826 \mathrm{E}-24$ & 0.0 & 0.0 & 0.0 \\
\hline ALOH2 ION & $2.60627 \mathrm{E}-15$ & 0.0 & 0.0 & 0.0 \\
\hline ALOH 4 ION & $2.01890 \mathrm{E}-08$ & 0.0 & 0.0 & 0.0 \\
\hline ALOHCLION & $9.15109 \mathrm{E}-19$ & 0.0 & 0.0 & 0.0 \\
\hline ALOHION & $3.05609 \mathrm{E}-19$ & 0.0 & 0.0 & 0.0 \\
\hline ALSO 42 ION & $9.02522 \mathrm{E}-25$ & 0.0 & 0.0 & 0.0 \\
\hline ALSO 4 ION & $8.43341 \mathrm{E}-24$ & 0.0 & 0.0 & 0.0 \\
\hline BAHCO3ION & $4.37644 \mathrm{E}-13$ & 0.0 & 0.0 & 0.0 \\
\hline BAION & $2.20773 E-09$ & 0.0 & 0.0 & 0.0 \\
\hline BAOHION & $1.23723 \mathrm{E}-13$ & 0.0 & 0.0 & 0.0 \\
\hline CACLION & $8.18426 \mathrm{E}-08$ & 0.0 & 0.0 & 0.0 \\
\hline CAH2 PO 4 ION & $1.07390 \mathrm{E}-13$ & 0.0 & 0.0 & 0.0 \\
\hline CAHCO3ION & $8.47306 \mathrm{E}-10$ & 0.0 & 0.0 & 0.0 \\
\hline CAHSIO3ION & $1.22029 \mathrm{E}-07$ & 0.0 & 0.0 & 0.0 \\
\hline CAION & $2.40130 \mathrm{E}-05$ & 0.0 & 0.0 & 0.0 \\
\hline CANO3ION & $1.03754 \mathrm{E}-05$ & 0.0 & 0.0 & 0.0 \\
\hline CAOHION & $2.52168 \mathrm{E}-08$ & 0.0 & 0.0 & 0.0 \\
\hline CAPO 4 ION & $1.05563 \mathrm{E}-11$ & 0.0 & 0.0 & 0.0 \\
\hline CDCL3ION & $3.25648 \mathrm{E}-09$ & 0.0 & 0.0 & 0.0 \\
\hline CDCL 4 ION & $6.84901 \mathrm{E}-10$ & 0.0 & 0.0 & 0.0 \\
\hline CDCLION & $2.83352 \mathrm{E}-10$ & 0.0 & 0.0 & 0.0 \\
\hline CDION & $6.11628 \mathrm{E}-12$ & 0.0 & 0.0 & 0.0 \\
\hline CDNO3ION & $1.52455 \mathrm{E}-12$ & 0.0 & 0.0 & 0.0 \\
\hline CDOH3ION & $3.64377 \mathrm{E}-16$ & 0.0 & 0.0 & 0.0 \\
\hline CDOH 4 ION & $1.74748 \mathrm{E}-19$ & 0.0 & 0.0 & 0.0 \\
\hline CDOHION & $8.50008 \mathrm{E}-13$ & 0.0 & 0.0 & 0.0 \\
\hline CLION & 0.00130005 & 0.0 & 0.0 & 0.0 \\
\hline CO3ION & $6.84639 \mathrm{E}-10$ & 0.0 & 0.0 & 0.0 \\
\hline CRIIICL2ION & $2.33591 \mathrm{E}-18$ & 0.0 & 0.0 & 0.0 \\
\hline CRIIICLION & $2.46967 \mathrm{E}-17$ & 0.0 & 0.0 & 0.0 \\
\hline CRIIIH2PO4 ION & $3.19395 E-25$ & 0.0 & 0.0 & 0.0 \\
\hline CRIIIHPO4ION & $7.70390 \mathrm{E}-13$ & 0.0 & 0.0 & 0.0 \\
\hline CRIIIION & $3.42549 \mathrm{E}-20$ & 0.0 & 0.0 & 0.0 \\
\hline CRIIINO3ION & $1.01079 \mathrm{E}-15$ & 0.0 & 0.0 & 0.0 \\
\hline CROH2ION & $7.24619 \mathrm{E}-14$ & 0.0 & 0.0 & 0.0 \\
\hline CROH 4 ION & $3.16473 \mathrm{E}-13$ & 0.0 & 0.0 & 0.0 \\
\hline CROHION & $5.13133 E-12$ & 0.0 & 0.0 & 0.0 \\
\hline CRSO 4 ION & $8.63597 \mathrm{E}-15$ & 0.0 & 0.0 & 0.0 \\
\hline CUCL3ION & $2.96340 \mathrm{E}-14$ & 0.0 & 0.0 & 0.0 \\
\hline CUCLION & $1.63948 \mathrm{E}-11$ & 0.0 & 0.0 & 0.0 \\
\hline CUCO32ION & $3.24991 \mathrm{E}-16$ & 0.0 & 0.0 & 0.0 \\
\hline CUION & $5.07538 \mathrm{E}-12$ & 0.0 & 0.0 & 0.0 \\
\hline CUNO3ION & $8.04294 \mathrm{E}-13$ & 0.0 & 0.0 & 0.0 \\
\hline CUOH3ION & $3.05339 \mathrm{E}-11$ & 0.0 & 0.0 & 0.0 \\
\hline CUOH 4 ION & $3.45909 \mathrm{E}-13$ & 0.0 & 0.0 & 0.0 \\
\hline CUOHION & $8.14155 \mathrm{E}-11$ & 0.0 & 0.0 & 0.0 \\
\hline DODECION & $8.03202 \mathrm{E}-06$ & 0.0 & 0.0 & 0.0 \\
\hline FEIICLION & $5.70151 \mathrm{E}-14$ & 0.0 & 0.0 & 0.0 \\
\hline FEIICO32ION & $1.79477 \mathrm{E}-18$ & 0.0 & 0.0 & 0.0 \\
\hline FEIIH2PO4ION & $1.12139 \mathrm{E}-21$ & 0.0 & 0.0 & 0.0 \\
\hline
\end{tabular}




\begin{tabular}{|c|c|c|c|c|}
\hline FEIIHCO3ION & $5.17038 \mathrm{E}-18$ & 0.0 & 0.0 & 0.0 \\
\hline FEIIION & $7.60978 \mathrm{E}-12$ & 0.0 & 0.0 & 0.0 \\
\hline FEIIOH3ION & $3.60461 \mathrm{E}-14$ & 0.0 & 0.0 & 0.0 \\
\hline FEIIOH 4 ION & $4.85157 \mathrm{E}-18$ & 0.0 & 0.0 & 0.0 \\
\hline FEIIOHION & $4.39727 \mathrm{E}-12$ & 0.0 & 0.0 & 0.0 \\
\hline H2 P2O 7 ION & $9.13297 \mathrm{E}-24$ & 0.0 & 0.0 & 0.0 \\
\hline $\mathrm{H} 2 \mathrm{PO} 4 \mathrm{ION}$ & $1.41129 \mathrm{E}-13$ & 0.0 & 0.0 & 0.0 \\
\hline H2SIO4 ION & $1.55131 \mathrm{E}-10$ & 0.0 & 0.0 & 0.0 \\
\hline H3P2O 7 ION & $2.18083 E-30$ & 0.0 & 0.0 & 0.0 \\
\hline H3SIO 4 ION & $1.89190 \mathrm{E}-06$ & 0.0 & 0.0 & 0.0 \\
\hline HCO3ION & $6.21526 \mathrm{E}-09$ & 0.0 & 0.0 & 0.0 \\
\hline HION & $7.44004 \mathrm{E}-12$ & 0.0 & 0.0 & 0.0 \\
\hline HP2O7 ION & $9.67561 \mathrm{E}-22$ & 0.0 & 0.0 & 0.0 \\
\hline HPBO2ION & $2.17424 \mathrm{E}-09$ & 0.0 & 0.0 & 0.0 \\
\hline HPO 4 ION & $7.41704 \mathrm{E}-12$ & 0.0 & 0.0 & 0.0 \\
\hline HSO4 ION & $9.06469 \mathrm{E}-12$ & 0.0 & 0.0 & 0.0 \\
\hline KION & $1.99619 \mathrm{E}-04$ & 0.0 & 0.0 & 0.0 \\
\hline $\mathrm{KSO} 4 \mathrm{ION}$ & $3.43971 \mathrm{E}-06$ & 0.0 & 0.0 & 0.0 \\
\hline MGH2 PO 4 ION & $1.92950 \mathrm{E}-15$ & 0.0 & 0.0 & 0.0 \\
\hline MGHCO3ION & $8.86580 \mathrm{E}-11$ & 0.0 & 0.0 & 0.0 \\
\hline MGHSIO3ION & $1.27147 \mathrm{E}-08$ & 0.0 & 0.0 & 0.0 \\
\hline MGION & $6.66297 \mathrm{E}-07$ & 0.0 & 0.0 & 0.0 \\
\hline MGOHION & $1.11071 \mathrm{E}-08$ & 0.0 & 0.0 & 0.0 \\
\hline MGP2O7 ION & $9.53207 \mathrm{E}-19$ & 0.0 & 0.0 & 0.0 \\
\hline MGPO 4 ION & $6.60609 \mathrm{E}-13$ & 0.0 & 0.0 & 0.0 \\
\hline NACO3ION & $1.05927 \mathrm{E}-10$ & 0.0 & 0.0 & 0.0 \\
\hline NAION & 0.00132855 & 0.0 & 0.0 & 0.0 \\
\hline NASO 4 ION & $3.14083 \mathrm{E}-13$ & 0.0 & 0.0 & 0.0 \\
\hline NICLION & $2.98284 \mathrm{E}-12$ & 0.0 & 0.0 & 0.0 \\
\hline NIION & $4.13937 \mathrm{E}-11$ & 0.0 & 0.0 & 0.0 \\
\hline NINO3ION & $5.17392 \mathrm{E}-12$ & 0.0 & 0.0 & 0.0 \\
\hline NIOH3ION & $2.02244 \mathrm{E}-14$ & 0.0 & 0.0 & 0.0 \\
\hline NIOHION & $6.47792 \mathrm{E}-12$ & 0.0 & 0.0 & 0.0 \\
\hline NO3ION & $2.55714 \mathrm{E}-04$ & 0.0 & 0.0 & 0.0 \\
\hline P207ION & $8.94668 \mathrm{E}-22$ & 0.0 & 0.0 & 0.0 \\
\hline PBCL3ION & $2.54069 \mathrm{E}-07$ & 0.0 & 0.0 & 0.0 \\
\hline PBCL 4 ION & $1.45033 \mathrm{E}-06$ & 0.0 & 0.0 & 0.0 \\
\hline PBCLION & $4.35974 \mathrm{E}-08$ & 0.0 & 0.0 & 0.0 \\
\hline $\mathrm{PBH} 2 \mathrm{PO} 4 \mathrm{ION}$ & $7.56365 \mathrm{E}-18$ & 0.0 & 0.0 & 0.0 \\
\hline PBION & $1.57136 \mathrm{E}-09$ & 0.0 & 0.0 & 0.0 \\
\hline PBNO33ION & $1.27725 \mathrm{E}-10$ & 0.0 & 0.0 & 0.0 \\
\hline PBNO3ION & $1.25704 \mathrm{E}-08$ & 0.0 & 0.0 & 0.0 \\
\hline PBOHION & $1.68743 \mathrm{E}-07$ & 0.0 & 0.0 & 0.0 \\
\hline PO4ION & $6.14844 \mathrm{E}-15$ & 0.0 & 0.0 & 0.0 \\
\hline SO4ION & $1.60872 \mathrm{E}-05$ & 0.0 & 0.0 & 0.0 \\
\hline SRION & $1.97064 \mathrm{E}-07$ & 0.0 & 0.0 & 0.0 \\
\hline SRNO3ION & $3.27990 \mathrm{E}-07$ & 0.0 & 0.0 & 0.0 \\
\hline SROHION & $1.81995 \mathrm{E}-10$ & 0.0 & 0.0 & 0.0 \\
\hline SRPO 4 ION & $2.44782 E-15$ & 0.0 & 0.0 & 0.0 \\
\hline UIVOH2ION & $1.41254 \mathrm{E}-27$ & 0.0 & 0.0 & 0.0 \\
\hline UIVOH3ION & $2.23720 \mathrm{E}-21$ & 0.0 & 0.0 & 0.0 \\
\hline UIVOH5ION & $4.03654 \mathrm{E}-14$ & 0.0 & 0.0 & 0.0 \\
\hline ZNCL3ION & $1.79483 \mathrm{E}-06$ & 0.0 & 0.0 & 0.0 \\
\hline ZNCLION & $4.20624 \mathrm{E}-06$ & 0.0 & 0.0 & 0.0 \\
\hline ZNH2 PO 4 ION & $1.42368 \mathrm{E}-16$ & 0.0 & 0.0 & 0.0 \\
\hline ZNHCO3ION & $5.25506 \mathrm{E}-12$ & 0.0 & 0.0 & 0.0 \\
\hline ZNION & $2.88376 \mathrm{E}-07$ & 0.0 & 0.0 & 0.0 \\
\hline ZNNO3ION & $2.76236 \mathrm{E}-08$ & 0.0 & 0.0 & 0.0 \\
\hline
\end{tabular}


WSRC-TR-2002-00567

Revision 0

\begin{tabular}{|c|c|c|c|c|}
\hline ZNOH3ION & $1.28146 \mathrm{E}-07$ & 0.0 & 0.0 & 0.0 \\
\hline ZNOH $4 \mathrm{ION}$ & $4.96039 \mathrm{E}-10$ & 0.0 & 0.0 & 0.0 \\
\hline ZNOHION & $1.25593 E-05$ & 0.0 & 0.0 & 0.0 \\
\hline $\mathrm{ALOOH}$ & 0.0 & $7.38720 E-06$ & 0.0 & 0.0 \\
\hline $\mathrm{CA} 3 \mathrm{PO} 42$ & 0.0 & $7.36842 E-05$ & 0.0 & 0.0 \\
\hline CHAMOSITE7A & 0.0 & $2.77778 E-05$ & 0.0 & 0.0 \\
\hline $\mathrm{MGOH} 2$ & 0.0 & $2.46199 \mathrm{E}-04$ & 0.0 & 0.0 \\
\hline UIVO2 & 0.0 & $2.01681 E-06$ & 0.0 & 0.0 \\
\hline & $===========$ & $===========$ & $===========$ & $===========$ \\
\hline Total g/hr & 0.722739 & 0.121055 & 0.0 & 0.0 \\
\hline Volume, L/hr & $6.83304 \mathrm{E}-04$ & $3.89195 E-05$ & 0.0 & 0.0 \\
\hline Enthalpy, cal/hr & -2430.03 & -343.728 & 0.0 & 0.0 \\
\hline Density, g/L & 1057.71 & 3110.38 & & \\
\hline Vapor fraction & 0.0 & 0.0 & 0.0 & 0.0 \\
\hline Solid fraction & 0.0 & 1 . & 0.0 & 0.0 \\
\hline Organic fraction & 0.0 & 0.0 & 0.0 & 0.0 \\
\hline Osmotic Pres, atm & 156.136 & & & \\
\hline Redox Pot, volts & 0.0 & & & \\
\hline $\mathrm{E}-\mathrm{Con}, 1 / \mathrm{ohm}-\mathrm{cm}$ & 0.449358 & & & \\
\hline E-Con, cm2/ohm-mol & 51.8522 & & & \\
\hline Abs Visc, cP & 0.376299 & & & \\
\hline Rel Visc & 1.37516 & & & \\
\hline Ionic Strength & 2.71916 & & & \\
\hline
\end{tabular}


WSRC-TR-2002-00567

Revision 0

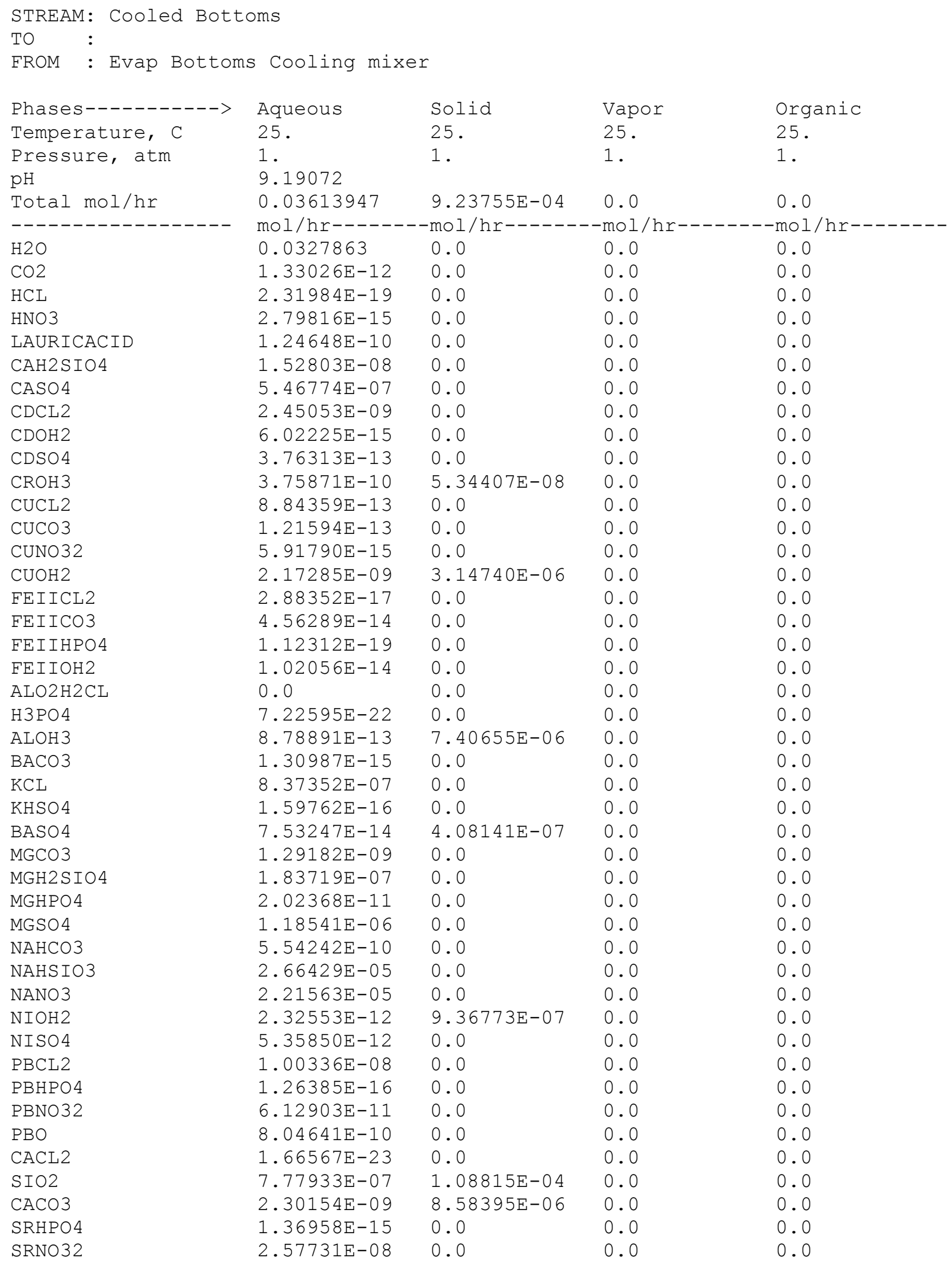




\begin{tabular}{|c|c|c|c|c|}
\hline $\mathrm{SRSO} 4$ & $1.57826 \mathrm{E}-08$ & $1.04336 \mathrm{E}-05$ & 0.0 & 0.0 \\
\hline UIVOH 4 & $8.97299 \mathrm{E}-14$ & 0.0 & 0.0 & 0.0 \\
\hline ZNCL2 & $1.49813 \mathrm{E}-09$ & 0.0 & 0.0 & 0.0 \\
\hline ZNHPO 4 & $1.20708 \mathrm{E}-15$ & 0.0 & 0.0 & 0.0 \\
\hline ZNNO32 & $1.76386 \mathrm{E}-11$ & 0.0 & 0.0 & 0.0 \\
\hline $\mathrm{ZNOH} 2$ & $2.97688 \mathrm{E}-09$ & $2.13878 E-05$ & 0.0 & 0.0 \\
\hline OHION & $9.00906 \mathrm{E}-09$ & 0.0 & 0.0 & 0.0 \\
\hline ALION & $1.18752 \mathrm{E}-23$ & 0.0 & 0.0 & 0.0 \\
\hline ALOH2 ION & $2.60133 \mathrm{E}-16$ & 0.0 & 0.0 & 0.0 \\
\hline ALOH 4 ION & $8.63300 \mathrm{E}-10$ & 0.0 & 0.0 & 0.0 \\
\hline ALOHCLION & $3.32860 \mathrm{E}-19$ & 0.0 & 0.0 & 0.0 \\
\hline ALOHION & $6.86596 \mathrm{E}-20$ & 0.0 & 0.0 & 0.0 \\
\hline ALSO 42 ION & $1.66504 \mathrm{E}-24$ & 0.0 & 0.0 & 0.0 \\
\hline ALSO 4 ION & $9.98299 \mathrm{E}-24$ & 0.0 & 0.0 & 0.0 \\
\hline BAHCO3ION & $1.44675 \mathrm{E}-15$ & 0.0 & 0.0 & 0.0 \\
\hline BAION & $6.18485 \mathrm{E}-10$ & 0.0 & 0.0 & 0.0 \\
\hline BAOHION & $3.24760 \mathrm{E}-16$ & 0.0 & 0.0 & 0.0 \\
\hline CACLION & $6.40261 \mathrm{E}-11$ & 0.0 & 0.0 & 0.0 \\
\hline CAH2 PO 4 ION & $1.76416 \mathrm{E}-14$ & 0.0 & 0.0 & 0.0 \\
\hline CAHCO3ION & $4.96869 \mathrm{E}-10$ & 0.0 & 0.0 & 0.0 \\
\hline CAHSIO3ION & $1.53175 \mathrm{E}-07$ & 0.0 & 0.0 & 0.0 \\
\hline CAION & $4.16670 \mathrm{E}-05$ & 0.0 & 0.0 & 0.0 \\
\hline CANO3ION & $1.58307 \mathrm{E}-05$ & 0.0 & 0.0 & 0.0 \\
\hline CAOHION & $3.52974 \mathrm{E}-09$ & 0.0 & 0.0 & 0.0 \\
\hline CAPO 4 ION & $1.23277 \mathrm{E}-10$ & 0.0 & 0.0 & 0.0 \\
\hline CDCL3ION & $6.62931 \mathrm{E}-10$ & 0.0 & 0.0 & 0.0 \\
\hline CDCL 4 ION & $5.63647 \mathrm{E}-09$ & 0.0 & 0.0 & 0.0 \\
\hline CDCLION & $1.68949 \mathrm{E}-10$ & 0.0 & 0.0 & 0.0 \\
\hline CDION & $8.04214 \mathrm{E}-12$ & 0.0 & 0.0 & 0.0 \\
\hline CDNO3ION & $1.14484 \mathrm{E}-12$ & 0.0 & 0.0 & 0.0 \\
\hline CDOH3ION & $3.99716 \mathrm{E}-18$ & 0.0 & 0.0 & 0.0 \\
\hline CDOH 4 ION & $2.53513 E-22$ & 0.0 & 0.0 & 0.0 \\
\hline CDOHION & $1.20873 \mathrm{E}-13$ & 0.0 & 0.0 & 0.0 \\
\hline CLION & 0.00132194 & 0.0 & 0.0 & 0.0 \\
\hline CO3ION & $1.65459 \mathrm{E}-09$ & 0.0 & 0.0 & 0.0 \\
\hline CRIIICL2ION & $9.97225 E-19$ & 0.0 & 0.0 & 0.0 \\
\hline CRIIICLION & $1.24147 \mathrm{E}-17$ & 0.0 & 0.0 & 0.0 \\
\hline CRIIIH2PO4 ION & $4.03872 \mathrm{E}-24$ & 0.0 & 0.0 & 0.0 \\
\hline CRIIIHPO4ION & $2.98671 \mathrm{E}-16$ & 0.0 & 0.0 & 0.0 \\
\hline CRIIIION & $1.03292 \mathrm{E}-19$ & 0.0 & 0.0 & 0.0 \\
\hline CRIIINO3ION & $2.22331 \mathrm{E}-16$ & 0.0 & 0.0 & 0.0 \\
\hline CROH2 ION & $1.79243 \mathrm{E}-13$ & 0.0 & 0.0 & 0.0 \\
\hline CROH 4 ION & $2.06275 \mathrm{E}-11$ & 0.0 & 0.0 & 0.0 \\
\hline CROHION & $8.75613 \mathrm{E}-12$ & 0.0 & 0.0 & 0.0 \\
\hline CRSO 4 ION & $3.78275 E-15$ & 0.0 & 0.0 & 0.0 \\
\hline CUCL3ION & $1.03673 \mathrm{E}-14$ & 0.0 & 0.0 & 0.0 \\
\hline CUCLION & $2.85207 \mathrm{E}-12$ & 0.0 & 0.0 & 0.0 \\
\hline CUCO32ION & $9.73132 E-16$ & 0.0 & 0.0 & 0.0 \\
\hline CUION & $2.90877 \mathrm{E}-12$ & 0.0 & 0.0 & 0.0 \\
\hline CUNO3ION & $4.83527 \mathrm{E}-13$ & 0.0 & 0.0 & 0.0 \\
\hline CUOH3ION & $5.30006 \mathrm{E}-12$ & 0.0 & 0.0 & 0.0 \\
\hline CUOH 4 ION & $5.41236 \mathrm{E}-15$ & 0.0 & 0.0 & 0.0 \\
\hline CUOHION & $1.93266 \mathrm{E}-11$ & 0.0 & 0.0 & 0.0 \\
\hline DODECION & $8.03223 E-06$ & 0.0 & 0.0 & 0.0 \\
\hline FEIICLION & $1.14977 \mathrm{E}-14$ & 0.0 & 0.0 & 0.0 \\
\hline FEIICO32ION & $7.72812 \mathrm{E}-18$ & 0.0 & 0.0 & 0.0 \\
\hline FEIIH2PO4ION & $4.99477 \mathrm{E}-22$ & 0.0 & 0.0 & 0.0 \\
\hline FEIIHCO3ION & $1.70543 \mathrm{E}-18$ & 0.0 & 0.0 & 0.0 \\
\hline
\end{tabular}




\begin{tabular}{|c|c|c|c|c|}
\hline FEIIION & $9.42257 \mathrm{E}-12$ & 0.0 & 0.0 & 0.0 \\
\hline FEIIOH3ION & $1.76857 \mathrm{E}-15$ & 0.0 & 0.0 & 0.0 \\
\hline FEIIOH 4 ION & $2.57951 \mathrm{E}-20$ & 0.0 & 0.0 & 0.0 \\
\hline FEIIOHION & $2.06895 \mathrm{E}-12$ & 0.0 & 0.0 & 0.0 \\
\hline H2P2O 7 ION & $2.53032 E-26$ & 0.0 & 0.0 & 0.0 \\
\hline H2 PO 4 ION & $3.26902 \mathrm{E}-14$ & 0.0 & 0.0 & 0.0 \\
\hline H2SIO 4 ION & $4.45530 \mathrm{E}-10$ & 0.0 & 0.0 & 0.0 \\
\hline H3SIO 4 ION & $2.81712 \mathrm{E}-07$ & 0.0 & 0.0 & 0.0 \\
\hline HCO3ION & $2.90276 \mathrm{E}-09$ & 0.0 & 0.0 & 0.0 \\
\hline HION & $3.38977 \mathrm{E}-13$ & 0.0 & 0.0 & 0.0 \\
\hline HP207ION & $5.85406 \mathrm{E}-23$ & 0.0 & 0.0 & 0.0 \\
\hline HPBO2 ION & $3.05670 \mathrm{E}-11$ & 0.0 & 0.0 & 0.0 \\
\hline HPO 4 ION & $3.01027 \mathrm{E}-11$ & 0.0 & 0.0 & 0.0 \\
\hline HSO 4 ION & $1.05694 \mathrm{E}-13$ & 0.0 & 0.0 & 0.0 \\
\hline KION & $2.02637 \mathrm{E}-04$ & 0.0 & 0.0 & 0.0 \\
\hline KSO4 ION & $3.31470 \mathrm{E}-06$ & 0.0 & 0.0 & 0.0 \\
\hline MGH2 PO 4 ION & $2.82005 \mathrm{E}-14$ & 0.0 & 0.0 & 0.0 \\
\hline MGHCO3ION & $1.71496 \mathrm{E}-09$ & 0.0 & 0.0 & 0.0 \\
\hline MGHSIO3ION & $3.52031 \mathrm{E}-07$ & 0.0 & 0.0 & 0.0 \\
\hline MGION & $2.32061 \mathrm{E}-05$ & 0.0 & 0.0 & 0.0 \\
\hline MGOHION & $4.20710 \mathrm{E}-08$ & 0.0 & 0.0 & 0.0 \\
\hline MGP2O7ION & $3.71584 \mathrm{E}-18$ & 0.0 & 0.0 & 0.0 \\
\hline MGPO 4 ION & $2.23481 \mathrm{E}-10$ & 0.0 & 0.0 & 0.0 \\
\hline NACO3ION & $8.27003 E-10$ & 0.0 & 0.0 & 0.0 \\
\hline NAION & 0.00134516 & 0.0 & 0.0 & 0.0 \\
\hline NASO 4 ION & $1.22281 \mathrm{E}-05$ & 0.0 & 0.0 & 0.0 \\
\hline NICLION & $4.70957 \mathrm{E}-12$ & 0.0 & 0.0 & 0.0 \\
\hline NIION & $1.46066 \mathrm{E}-10$ & 0.0 & 0.0 & 0.0 \\
\hline NINO3ION & $3.12552 \mathrm{E}-11$ & 0.0 & 0.0 & 0.0 \\
\hline NIOH3ION & $1.13268 \mathrm{E}-13$ & 0.0 & 0.0 & 0.0 \\
\hline NIOHION & $5.13684 \mathrm{E}-12$ & 0.0 & 0.0 & 0.0 \\
\hline NO3ION & $3.00468 \mathrm{E}-04$ & 0.0 & 0.0 & 0.0 \\
\hline P207ION & $4.82793 \mathrm{E}-21$ & 0.0 & 0.0 & 0.0 \\
\hline PBCL3ION & $1.97407 \mathrm{E}-08$ & 0.0 & 0.0 & 0.0 \\
\hline PBCL 4 ION & $2.60397 \mathrm{E}-07$ & 0.0 & 0.0 & 0.0 \\
\hline PBCLION & $4.06804 \mathrm{E}-09$ & 0.0 & 0.0 & 0.0 \\
\hline PBH2PO4 ION & $1.12013 \mathrm{E}-19$ & 0.0 & 0.0 & 0.0 \\
\hline PBION & $2.73966 \mathrm{E}-10$ & 0.0 & 0.0 & 0.0 \\
\hline PBNO33ION & $1.05072 \mathrm{E}-11$ & 0.0 & 0.0 & 0.0 \\
\hline PBNO3ION & $6.75140 \mathrm{E}-10$ & 0.0 & 0.0 & 0.0 \\
\hline PBOHION & $2.95949 \mathrm{E}-09$ & 0.0 & 0.0 & 0.0 \\
\hline PO4ION & $2.19382 \mathrm{E}-13$ & 0.0 & 0.0 & 0.0 \\
\hline SO4ION & $2.42876 \mathrm{E}-05$ & 0.0 & 0.0 & 0.0 \\
\hline SRION & $6.24837 \mathrm{E}-07$ & 0.0 & 0.0 & 0.0 \\
\hline SRNO3ION & $2.01350 \mathrm{E}-07$ & 0.0 & 0.0 & 0.0 \\
\hline SROHION & $1.09224 \mathrm{E}-11$ & 0.0 & 0.0 & 0.0 \\
\hline SRPO 4 ION & $9.90274 \mathrm{E}-15$ & 0.0 & 0.0 & 0.0 \\
\hline UIVOH2ION & $3.15845 E-29$ & 0.0 & 0.0 & 0.0 \\
\hline UIVOH3ION & $1.76270 \mathrm{E}-21$ & 0.0 & 0.0 & 0.0 \\
\hline UIVOH5ION & $2.22589 \mathrm{E}-14$ & 0.0 & 0.0 & 0.0 \\
\hline ZNCL3ION & $2.04823 E-09$ & 0.0 & 0.0 & 0.0 \\
\hline ZNCLION & $1.96842 \mathrm{E}-09$ & 0.0 & 0.0 & 0.0 \\
\hline ZNH2 PO 4 ION & $8.73909 \mathrm{E}-19$ & 0.0 & 0.0 & 0.0 \\
\hline ZNHCO3ION & $5.25567 \mathrm{E}-14$ & 0.0 & 0.0 & 0.0 \\
\hline ZNION & $7.89937 \mathrm{E}-09$ & 0.0 & 0.0 & 0.0 \\
\hline ZNNO3ION & $8.23693 E-10$ & 0.0 & 0.0 & 0.0 \\
\hline ZNOH3ION & $9.46295 \mathrm{E}-11$ & 0.0 & 0.0 & 0.0 \\
\hline ZNOH 4 ION & $2.26483 \mathrm{E}-13$ & 0.0 & 0.0 & 0.0 \\
\hline
\end{tabular}


WSRC-TR-2002-00567

Revision 0

\begin{tabular}{|c|c|c|c|c|}
\hline ZNOHION & $1.64828 \mathrm{E}-09$ & 0.0 & 0.0 & 0.0 \\
\hline $\mathrm{CA} 3 \mathrm{PO} 42$ & 0.0 & $7.30752 \mathrm{E}-05$ & 0.0 & 0.0 \\
\hline $\mathrm{CASO} 4.2 \mathrm{H} 2 \mathrm{O}$ & 0.0 & $4.37163 E-04$ & 0.0 & 0.0 \\
\hline CHAMOSITE7A & 0.0 & $2.77778 E-05$ & 0.0 & 0.0 \\
\hline MGOH2 & 0.0 & $2.21941 E-04$ & 0.0 & 0.0 \\
\hline РB3PO42 & 0.0 & $6.08850 \mathrm{E}-07$ & 0.0 & 0.0 \\
\hline \multirow[t]{2}{*}{ UIVO2 } & 0.0 & $2.01681 E-06$ & 0.0 & 0.0 \\
\hline & $===========$ & $===========$ & $===========$ & $===========$ \\
\hline Total g/hr & 0.709872 & 0.133922 & 0.0 & 0.0 \\
\hline Volume, L/hr & $6.33837 E-04$ & $4.59292 \mathrm{E}-05$ & 0.0 & 0.0 \\
\hline Enthalpy, cal/hr & -2431 & -392.771 & 0.0 & 0.0 \\
\hline Density, g/L & 1119.96 & 2915.83 & & \\
\hline Vapor fraction & 0.0 & 0.0 & 0.0 & 0.0 \\
\hline Solid fraction & 0.0 & 1 . & 0.0 & 0.0 \\
\hline Organic fraction & 0.0 & 0.0 & 0.0 & 0.0 \\
\hline Osmotic Pres, atm & 146.078 & & & \\
\hline Redox Pot, volts & 0.0 & & & \\
\hline E-Con, 1/ohm-cm & 0.179649 & & & \\
\hline E-Con, cm2/ohm-mol & 30.4509 & & & \\
\hline Abs Visc, cP & 1.18016 & & & \\
\hline Rel Visc & 1.32495 & & & \\
\hline Ionic Strength & 3.02289 & & & \\
\hline
\end{tabular}


WSRC-TR-2002-00567

Revision 0

\begin{tabular}{|c|c|c|c|c|}
\hline \multicolumn{5}{|l|}{ STREAM: Condensate } \\
\hline TO $\quad:$ & & & & \\
\hline FROM : Condensate & mixer & & & \\
\hline Phases-----------> & Aqueous & Solid & Vapor & Organic \\
\hline Temperature, C & 25 . & 25 . & 25 . & 25 . \\
\hline $\begin{array}{l}\text { Pressure, atm } \\
\mathrm{pH}\end{array}$ & $\begin{array}{l}1 . \\
4.5922\end{array}$ & 1. & 1. & 1. \\
\hline Total mol/hr & 55.45974 & 0.0 & 0.0 & 0.0 \\
\hline------------------ & $\mathrm{mol} / \mathrm{hr}------$ & -mol/hr------- & -mol/hr-------- & $-\operatorname{mol} / \mathrm{hr}------1$ \\
\hline $\mathrm{H} 2 \mathrm{O}$ & 55.4587 & 0.0 & 0.0 & 0.0 \\
\hline $\mathrm{CO} 2$ & $9.74015 \mathrm{E}-04$ & 0.0 & 0.0 & 0.0 \\
\hline $\mathrm{HCL}$ & $4.43231 E-21$ & 0.0 & 0.0 & 0.0 \\
\hline HNO3 & $8.27637 \mathrm{E}-17$ & 0.0 & 0.0 & 0.0 \\
\hline LAURICACID & $1.73575 E-05$ & 0.0 & 0.0 & 0.0 \\
\hline OHION & $3.98607 \mathrm{E}-10$ & 0.0 & 0.0 & 0.0 \\
\hline CLION & $2.94330 E-10$ & 0.0 & 0.0 & 0.0 \\
\hline CO3ION & $3.25101 \mathrm{E}-11$ & 0.0 & 0.0 & 0.0 \\
\hline DODECION & $8.31236 E-06$ & 0.0 & 0.0 & 0.0 \\
\hline HCO3ION & $1.73896 \mathrm{E}-05$ & 0.0 & 0.0 & 0.0 \\
\hline $\mathrm{HION}$ & $2.57028 \mathrm{E}-05$ & 0.0 & 0.0 & 0.0 \\
\hline NO3ION & $\begin{array}{l}\text { 6.53193E-11 } \\
============\end{array}$ & $\begin{array}{l}0.0 \\
============\end{array}$ & $\begin{array}{l}0.0 \\
=============\end{array}$ & $\begin{array}{l}0.0 \\
=============\end{array}$ \\
\hline Total g/hr & 999.156 & 0.0 & 0.0 & 0.0 \\
\hline Volume, L/hr & 1.0023 & 0.0 & 0.0 & 0.0 \\
\hline $\begin{array}{l}\text { Enthalpy, cal/hr } \\
\text { Density, g/L }\end{array}$ & $\begin{array}{l}-3.78866 \mathrm{E}+06 \\
996.861\end{array}$ & 0.0 & 0.0 & 0.0 \\
\hline Vapor fraction & 0.0 & 0.0 & 0.0 & 0.0 \\
\hline Solid fraction & 0.0 & 0.0 & 0.0 & 0.0 \\
\hline Organic fraction & 0.0 & 0.0 & 0.0 & 0.0 \\
\hline Osmotic Pres, atm & 0.0254588 & & & \\
\hline Redox Pot, volts & 0.0 & & & \\
\hline $\mathrm{E}-\mathrm{Con}, \quad 1 / \mathrm{ohm}-\mathrm{cm}$ & $1.02191 \mathrm{E}-05$ & & & \\
\hline E-Con, cm2/ohm-mol & 10.0707 & & & \\
\hline Abs Visc, cP & 0.890739 & & & \\
\hline Rel Visc & 1.00002 & & & \\
\hline Ionic Strength & $2.57260 E-05$ & & & \\
\hline
\end{tabular}


WSRC-TR-2002-00567

Revision 0

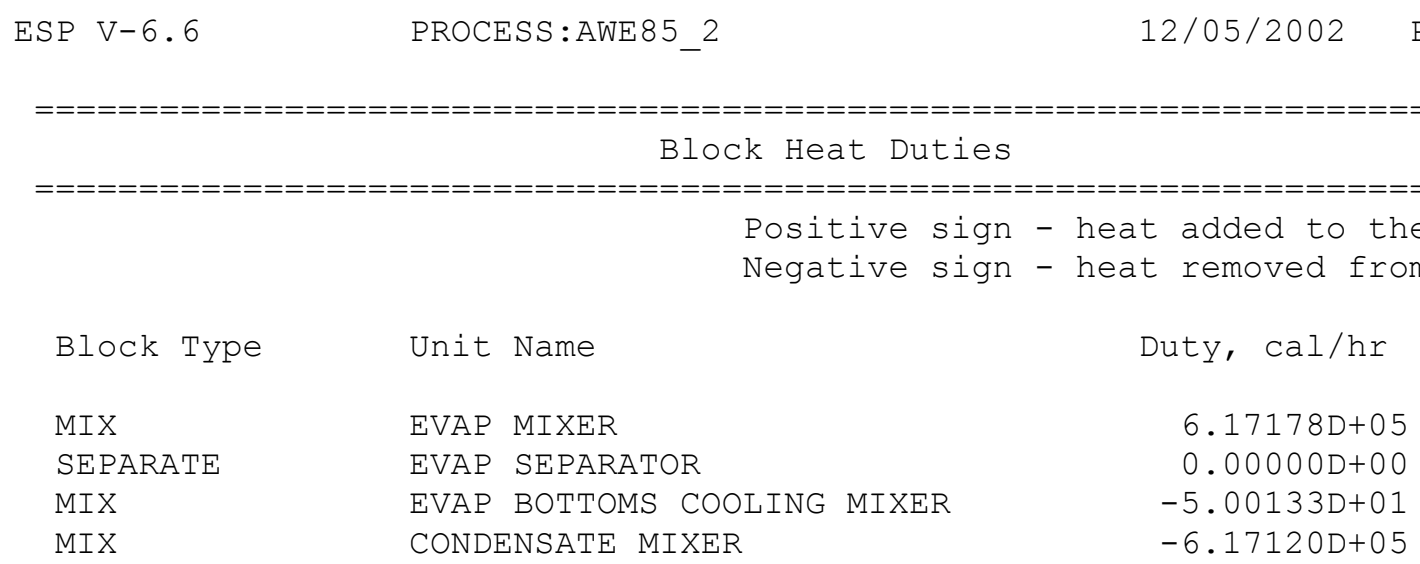


WSRC-TR-2002-00567

Revision 0

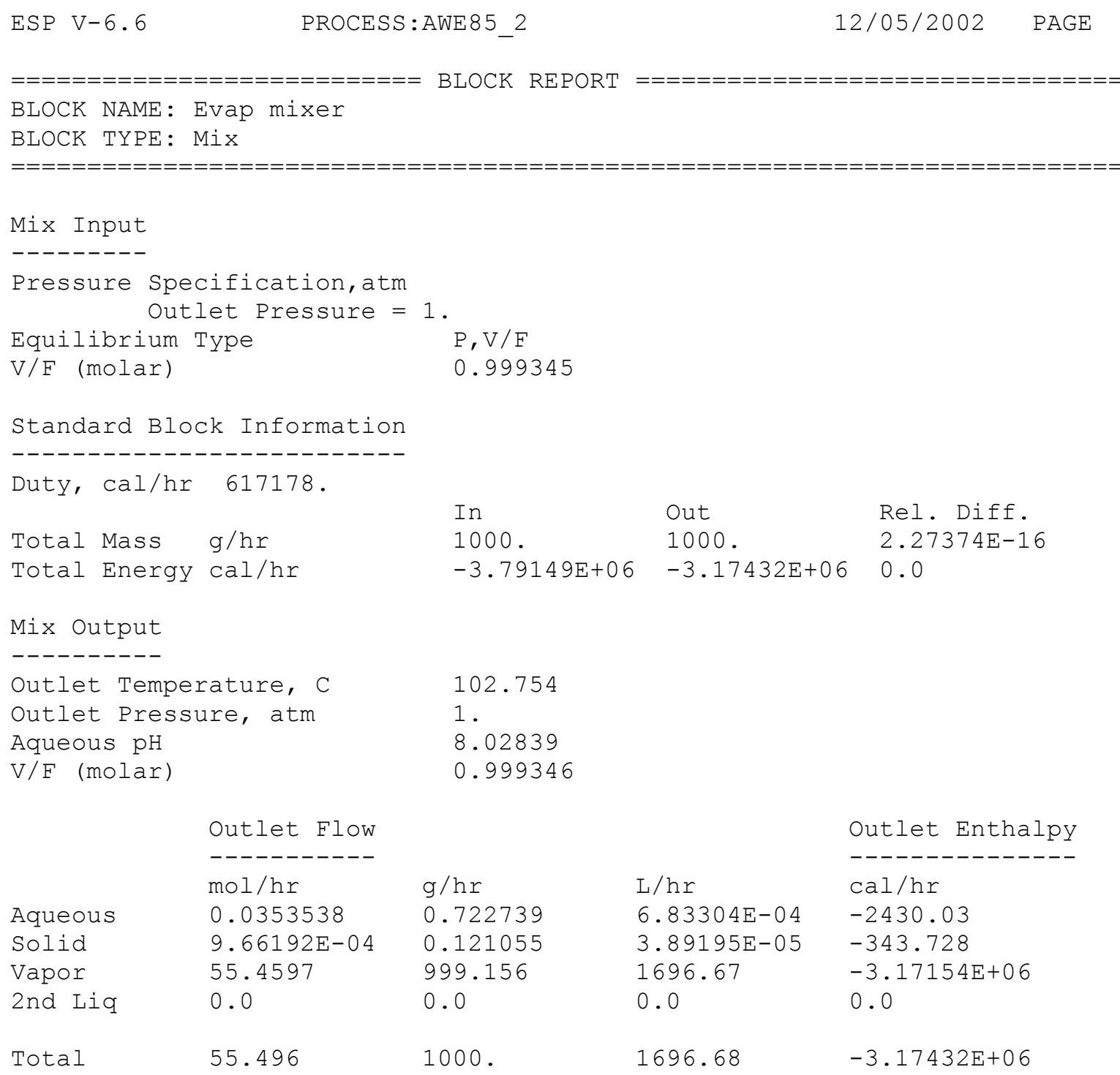


WSRC-TR-2002-00567

Revision 0

$\begin{array}{lllll}\text { ESP V-6.6 PROCESS:AWE85_2 } & 12 / 05 / 2002 & \text { PAGE } & 12\end{array}$

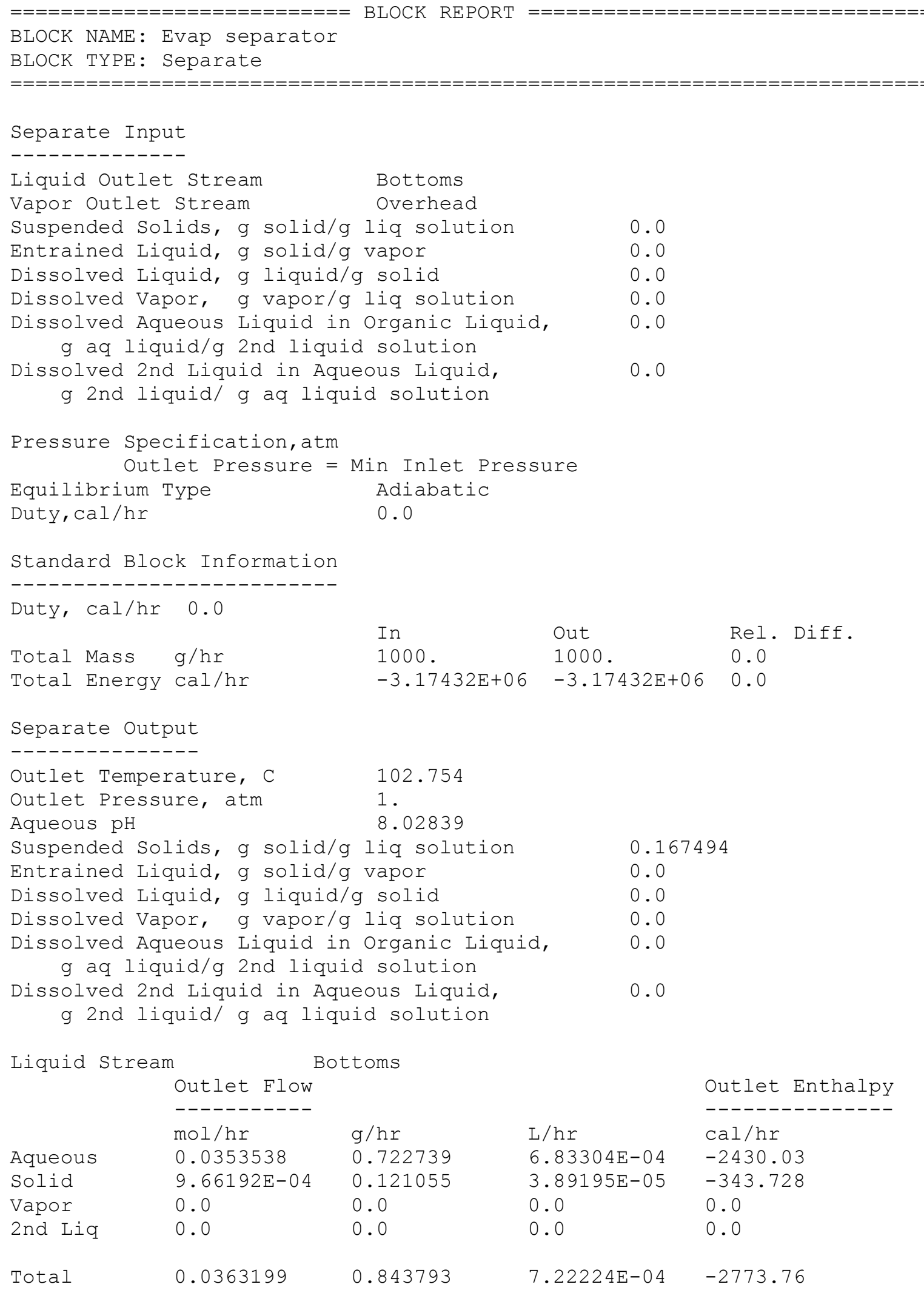


WSRC-TR-2002-00567

Revision 0

ESP V-6.6

PROCESS:AWE85 2

Vapor Stream

Outlet Flow
---------
mol/hr
0.0
0.0
55.4597
0.0

55.4597
Overhead

g/hr

0.0

0.0

999.156

0.0

999.156
$12 / 05 / 2002$

PAGE

13

$\mathrm{L} / \mathrm{hr}$

0.0

0.0

1696.67

0.0

1696.67

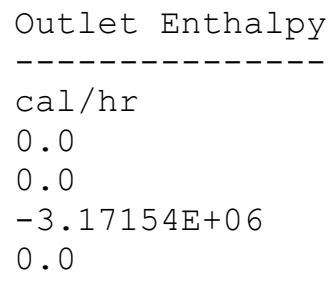

0.0

0.0

$-3.17154 E+06$

0.0

$-3.17154 E+06$ 
WSRC-TR-2002-00567

Revision 0

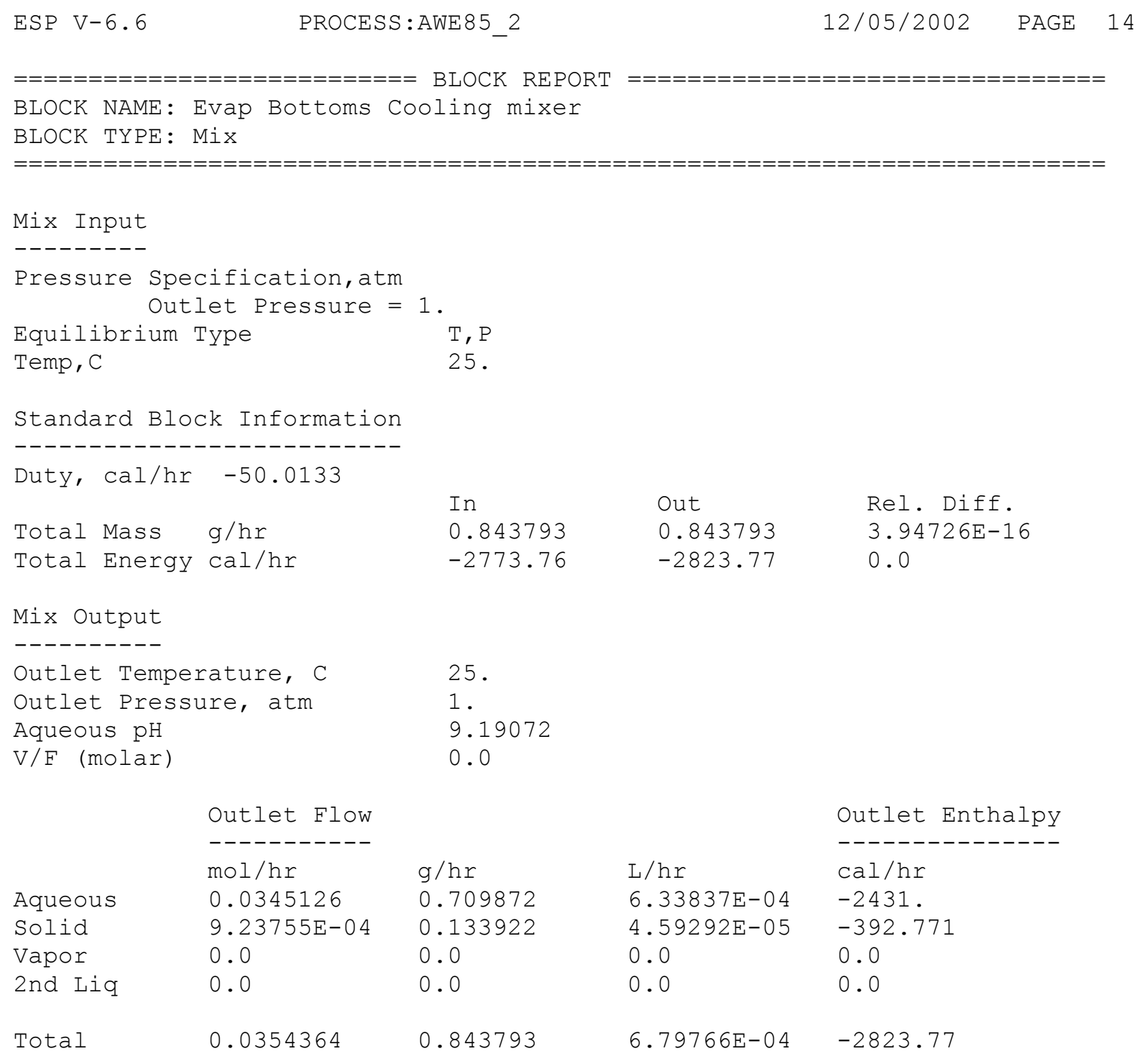


WSRC-TR-2002-00567

Revision 0

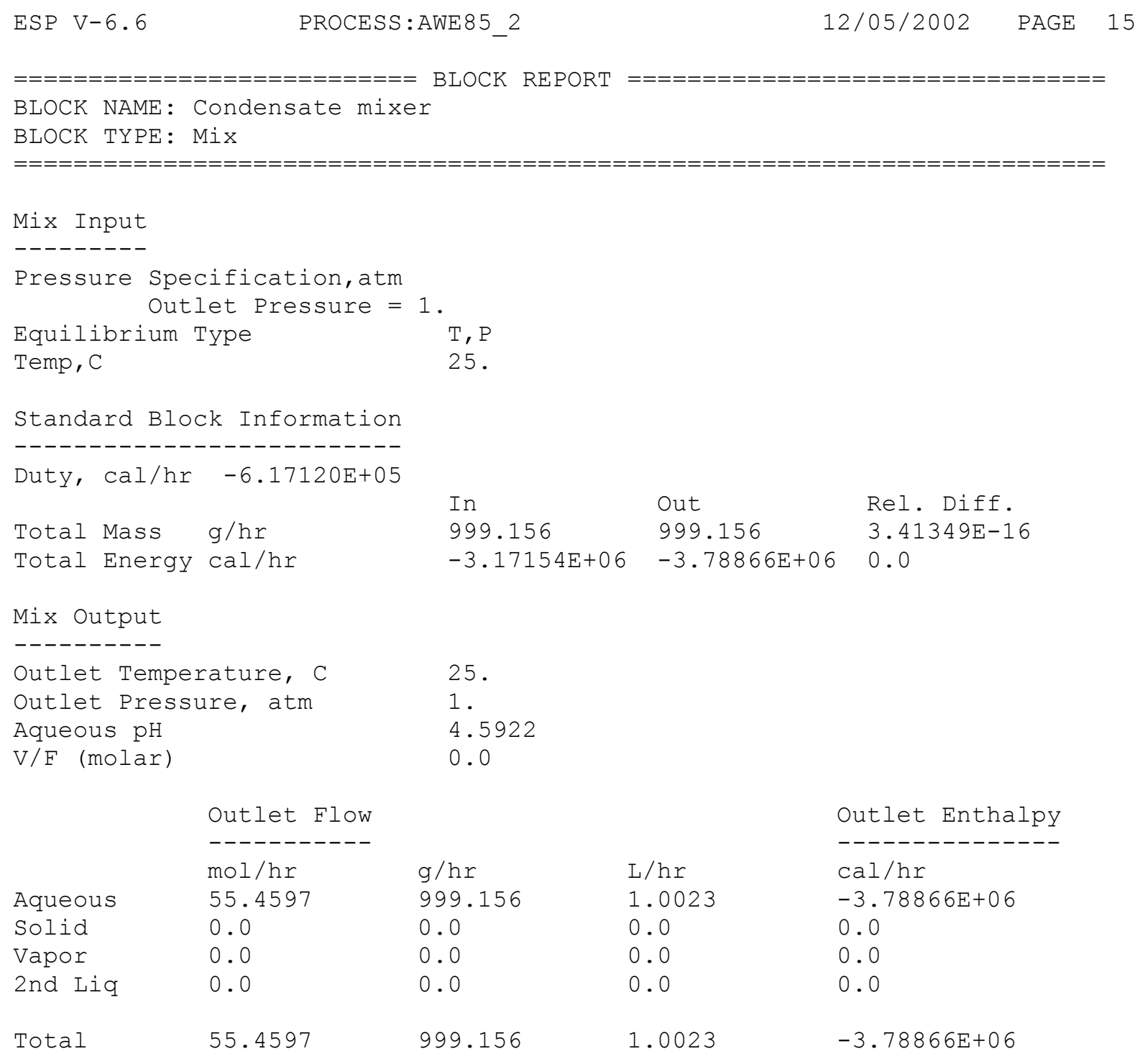


WSRC-TR-2002-00567

Revision 0

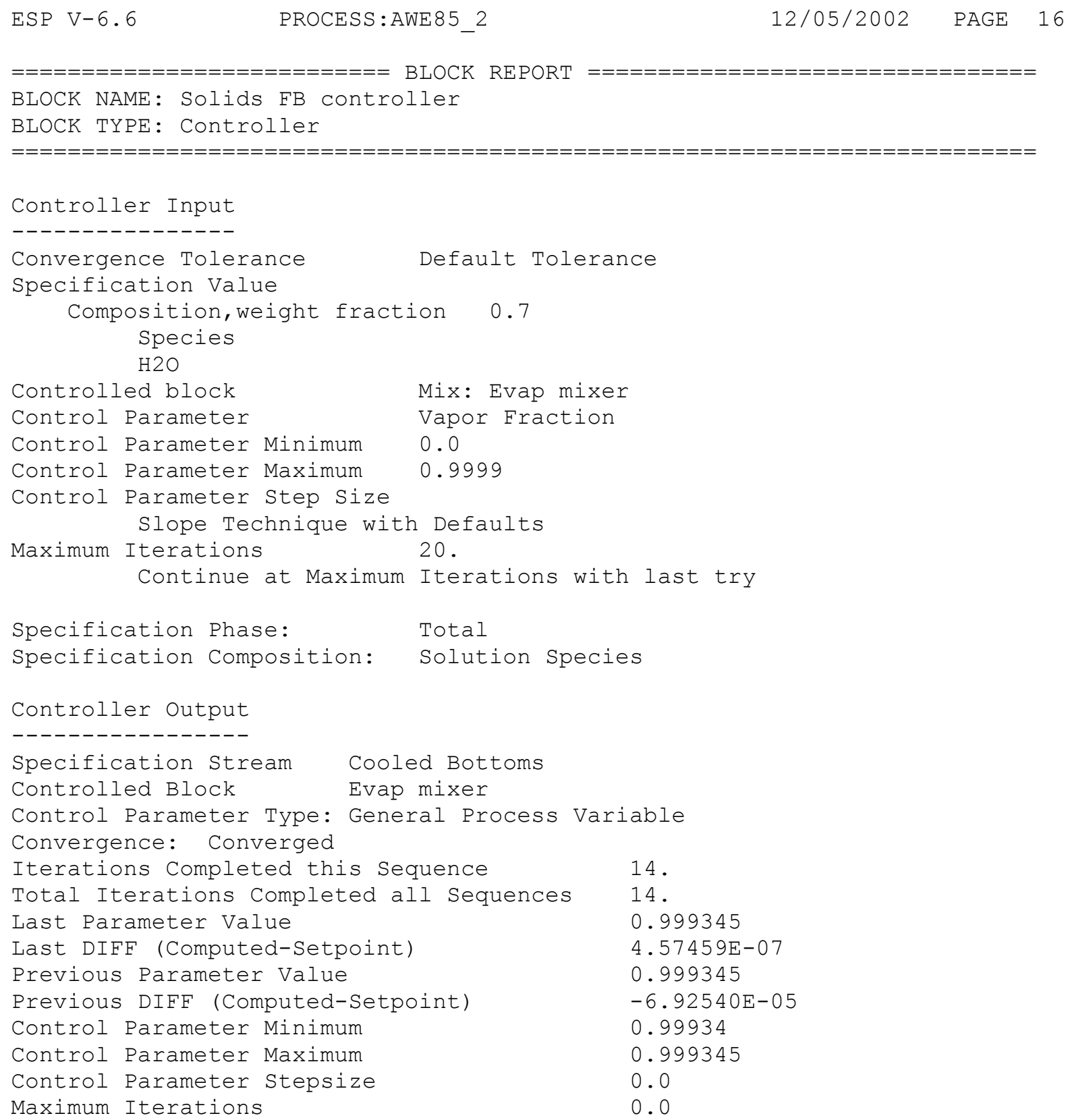


WSRC-TR-2002-00567

Revision 0

Influent Limit Composition 60\% Target $\mathrm{pH}=8.5$

$8.5-60$

\begin{tabular}{|c|c|c|c|c|}
\hline 0 & 0 & 0 & $\mathrm{~L}$ & $I I I I$ \\
\hline 0 & & 0 & $\mathrm{~L}$ & I \\
\hline 0 & & 0 & $\mathrm{~L}$ & I \\
\hline D & & 0 & $\mathrm{~L}$ & I \\
\hline D & & 0 & $\mathrm{~L}$ & I \\
\hline D & & 0 & $\mathrm{~L}$ & I \\
\hline 0 & & 0 & $\mathrm{~L}$ & I \\
\hline 0 & & 0 & $\mathrm{~L}$ & I \\
\hline 0 & 0 & 0 & L L L L L L L L L L & I I I \\
\hline
\end{tabular}

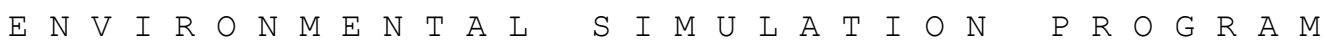

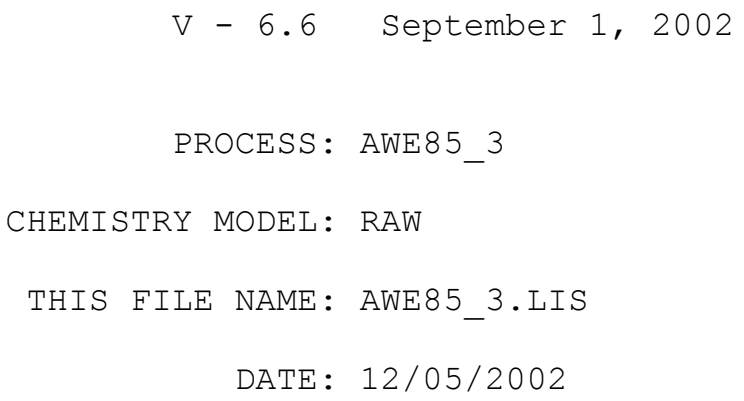




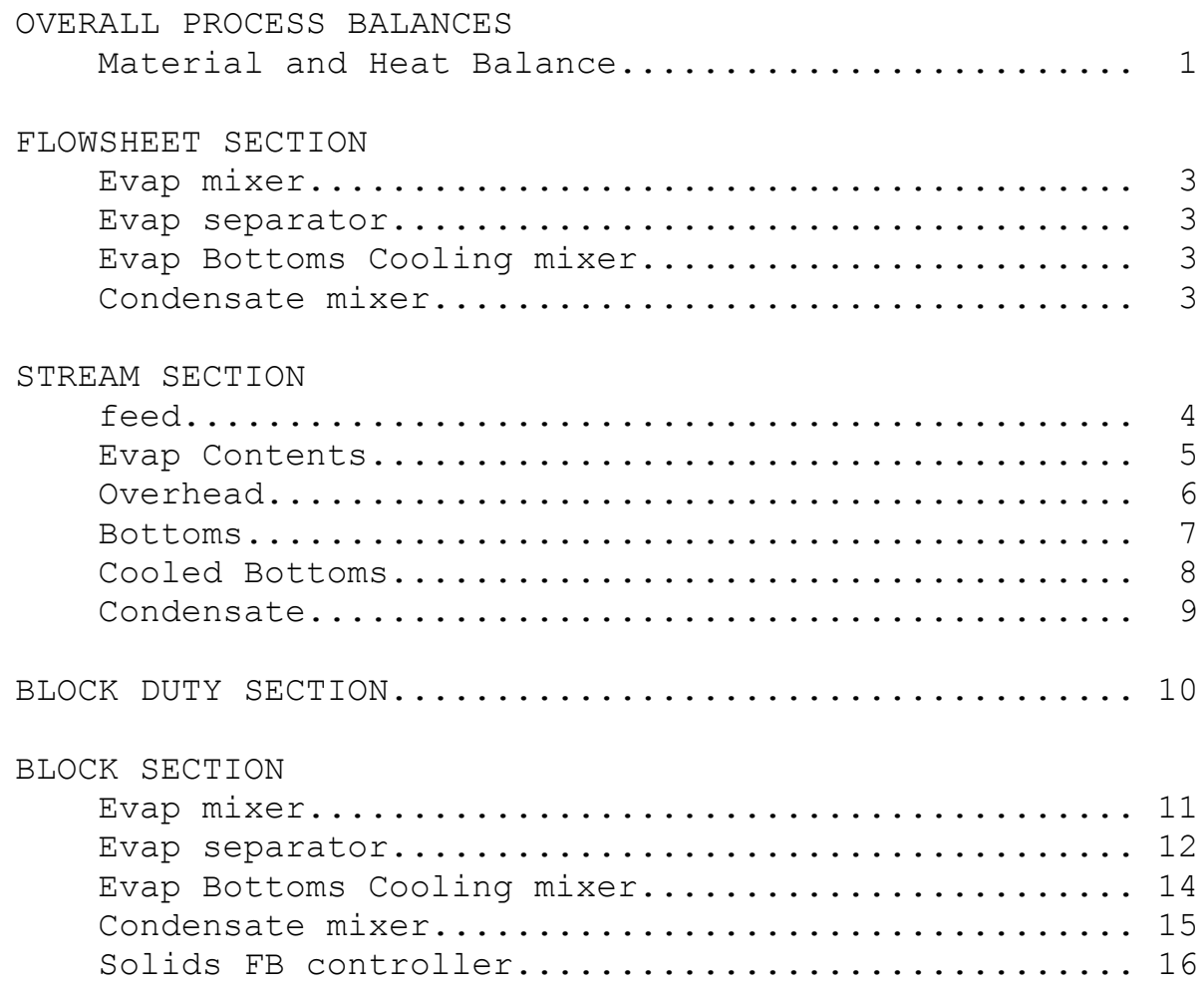


WSRC-TR-2002-00567

Revision 0

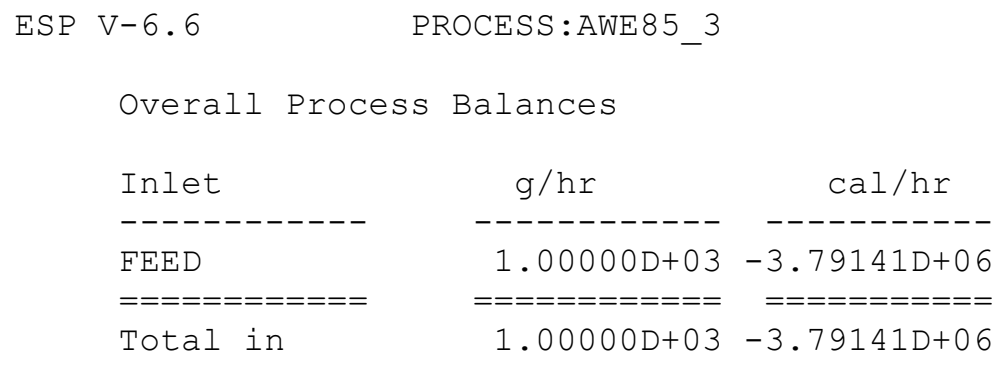

\begin{tabular}{lcc} 
Outlet & \multicolumn{1}{c}{$\mathrm{g} / \mathrm{hr}$} & $\mathrm{cal} / \mathrm{hr}$ \\
---------- & ---------- & ---------- \\
COOLED BOTTOMS & $9.84187 \mathrm{D}-01$ & $-3.28821 \mathrm{D}+03$ \\
CONDENSATE & $9.99016 \mathrm{D}+02$ & $-3.78812 \mathrm{D}+06$ \\
$==========$ & $==========$ & $=========$ \\
Total out & $1.00000 \mathrm{D}+03$ & $-3.79141 \mathrm{D}+06$
\end{tabular}

Block Heat Duties

$\mathrm{cal} / \mathrm{hr}$
--------
$6.17144 \mathrm{D}+05$

EVAP MIXER

EVAP BOTTOMS COOLING MIXER

CONDENSATE MIXER

$=======$

Total Duty

DIFFERENCE

REL DIFFERENCE
$-5.84613 D+01$

$-6.17077 \mathrm{D}+05$

$==========$

$8.45893 \mathrm{D}+00$
12/05/2002 PAGE 1

Material Code Balances

\begin{tabular}{lccrr} 
Code & $\begin{array}{c}\text { Input } \\
\text { mol/hr }\end{array}$ & $\begin{array}{c}\text { Outlet } \\
\text { mol/hr }\end{array}$ & \multicolumn{1}{c}{$\begin{array}{c}\text { Difference } \\
\text { mol/hr }\end{array}$} & \multicolumn{1}{c}{ Rel Diff } \\
-------- & ------- & -------- & -------- & --------- \\
H $(+1)$ & $1.10980 \mathrm{D}+02$ & $1.10980 \mathrm{D}+02$ & $5.68434 \mathrm{D}-14$ & $5.12194 \mathrm{D}-16$ \\
$\mathrm{~K}(+1)$ & $2.51221 \mathrm{D}-04$ & $2.51221 \mathrm{D}-04$ & $0.00000 \mathrm{D}+00$ & $0.00000 \mathrm{D}+00$ \\
$\mathrm{NA}(+1)$ & $1.70833 \mathrm{D}-03$ & $1.70833 \mathrm{D}-03$ & $-8.67362 \mathrm{D}-19$ & $-5.07724 \mathrm{D}-16$ \\
$\mathrm{BA}(+2)$ & $4.96350 \mathrm{D}-07$ & $4.96350 \mathrm{D}-07$ & $-1.48231 \mathrm{D}-21$ & $-2.98641 \mathrm{D}-15$ \\
$\mathrm{CA}(+2)$ & $7.98005 \mathrm{D}-04$ & $7.98005 \mathrm{D}-04$ & $-2.16840 \mathrm{D}-19$ & $-2.71728 \mathrm{D}-16$ \\
$\mathrm{ZN}(+2)$ & $2.59939 \mathrm{D}-05$ & $2.59939 \mathrm{D}-05$ & $0.00000 \mathrm{D}+00$ & $0.00000 \mathrm{D}+00$ \\
$\mathrm{CU}(+2)$ & $3.93701 \mathrm{D}-06$ & $3.93701 \mathrm{D}-06$ & $-8.47033 \mathrm{D}-22$ & $-2.15146 \mathrm{D}-16$ \\
$\mathrm{FE}(+2)$ & $7.16846 \mathrm{D}-05$ & $7.16846 \mathrm{D}-05$ & $0.00000 \mathrm{D}+00$ & $0.00000 \mathrm{D}+00$ \\
$\mathrm{MG}(+2)$ & $2.83951 \mathrm{D}-04$ & $2.83951 \mathrm{D}-04$ & $-5.42101 \mathrm{D}-20$ & $-1.90914 \mathrm{D}-16$ \\
$\mathrm{~PB}(+2)$ & $2.57488 \mathrm{D}-06$ & $2.57488 \mathrm{D}-06$ & $4.23516 \mathrm{D}-22$ & $1.64480 \mathrm{D}-16$ \\
$\mathrm{AL}(+3)$ & $7.77778 \mathrm{D}-05$ & $7.77778 \mathrm{D}-05$ & $0.00000 \mathrm{D}+00$ & $0.00000 \mathrm{D}+00$ \\
$\mathrm{NI}(+2)$ & $1.09029 \mathrm{D}-06$ & $1.09029 \mathrm{D}-06$ & $2.11758 \mathrm{D}-22$ & $1.94222 \mathrm{D}-16$ \\
$\mathrm{O}(-2)$ & $5.54974 \mathrm{D}+01$ & $5.54974 \mathrm{D}+01$ & $2.84217 \mathrm{D}-14$ & $5.12127 \mathrm{D}-16$ \\
$\mathrm{CL}(-1)$ & $1.57746 \mathrm{D}-03$ & $1.57746 \mathrm{D}-03$ & $-2.16840 \mathrm{D}-19$ & $-1.37461 \mathrm{D}-16$ \\
$\mathrm{C}(+4)$ & $1.10000 \mathrm{D}-03$ & $1.10000 \mathrm{D}-03$ & $6.50521 \mathrm{D}-19$ & $5.91383 \mathrm{D}-16$ \\
$\mathrm{P}(+5)$ & $1.78947 \mathrm{D}-04$ & $1.78947 \mathrm{D}-04$ & $-2.71051 \mathrm{D}-20$ & $-1.51469 \mathrm{D}-16$ \\
$\mathrm{~S}(+6)$ & $5.83333 \mathrm{D}-04$ & $5.83333 \mathrm{D}-04$ & $0.00000 \mathrm{D}+00$ & $0.00000 \mathrm{D}+00$ \\
$\mathrm{~N}(+5)$ & $4.03226 \mathrm{D}-04$ & $4.03226 \mathrm{D}-04$ & $7.04731 \mathrm{D}-19$ & $1.74773 \mathrm{D}-15$ \\
$\mathrm{SI}(+4)$ & $1.83333 \mathrm{D}-04$ & $1.83333 \mathrm{D}-04$ & $0.00000 \mathrm{D}+00$ & $0.00000 \mathrm{D}+00$ \\
$\mathrm{SR}(+2)$ & $1.48402 \mathrm{D}-05$ & $1.48402 \mathrm{D}-05$ & $-1.69407 \mathrm{D}-21$ & $-1.14154 \mathrm{D}-16$
\end{tabular}


WSRC-TR-2002-00567

Revision 0

$\mathrm{CD}(+2)$
$\mathrm{CR}(+3)$
$\mathrm{U}(+4)$
$1.69643 \mathrm{D}-08$

$6.53846 \mathrm{D}-08$

2.43697D-06

1. $69643 \mathrm{D}-08$

$6.53846 \mathrm{D}-08$

$2.43697 \mathrm{D}-06$

6. $98140 \mathrm{D}-22$

$0.00000 \mathrm{D}+00$

$0.00000 D+00$

$4.11535 D-14$

$0.00000 D+00$

$0.00000 D+00$ 
WSRC-TR-2002-00567

Revision 0

ESP V-6.6

$\operatorname{DODEC}(-1)$
PROCESS:AWE 853

$3.79916 \mathrm{D}-05$
$12 / 05 / 2002$

$3.79916 \mathrm{D}-05$
$8.80914 \mathrm{D}-20$

$2.31871 D-15$ 
WSRC-TR-2002-00567

Revision 0

\begin{tabular}{|c|c|c|c|}
\hline \multicolumn{4}{|l|}{$\begin{array}{l}\text { PROCESS BLOCKS } \\
===========\end{array}$} \\
\hline $\begin{array}{l}\mathrm{BLOCK} \mathrm{NAME} \\
=========================\end{array}$ & $\begin{array}{l}\text { BLOCK TYPE } \\
===========\end{array}$ & $\begin{array}{l}\text { INLET STREAM }(\mathrm{S}) \\
==============\end{array}$ & $\begin{array}{l}\text { OUTLET STREAM }(\mathrm{S}) \\
==============\end{array}$ \\
\hline Evap mixer & $\operatorname{Mix}$ & feed & Evap Contents \\
\hline Evap separator & Separate & Evap Contents & $\begin{array}{l}\text { Overhead } \\
\text { Bottoms }\end{array}$ \\
\hline Evap Bottoms Cooling mixer & Mix & Bottoms & Cooled Bottoms \\
\hline Condensate mixer & Mix & Overhead & Condensate \\
\hline
\end{tabular}


WSRC-TR-2002-00567

Revision 0

ESP V-6. 6

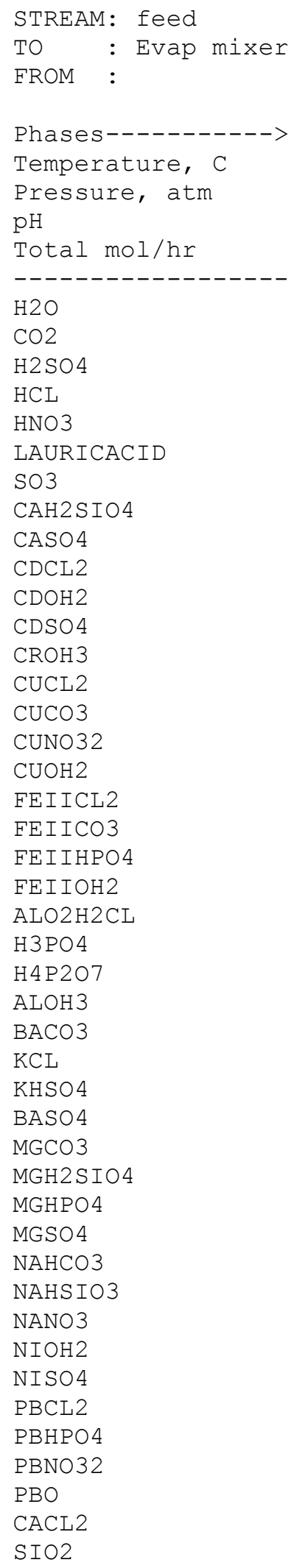

\begin{tabular}{|c|c|c|c|}
\hline Aqueous & Solid & Vapor & Organic \\
\hline 25 & 25 & 25. & 25 \\
\hline 1 . & 1 . & 1 . & 1 . \\
\hline \multicolumn{4}{|l|}{6.96924} \\
\hline 55.49656 & $8.24711 E-05$ & 0.0 & 0.0 \\
\hline \multicolumn{4}{|c|}{$\mathrm{mol} / \mathrm{hr}--------\operatorname{mol} / \mathrm{hr}--------\operatorname{mol} / \mathrm{hr}--------\operatorname{mol} / \mathrm{hr}--------$} \\
\hline 55.4894 & 0.0 & 0.0 & 0.0 \\
\hline $1.93653 \mathrm{E}-04$ & 0.0 & 0.0 & 0.0 \\
\hline $1.70876 \mathrm{E}-26$ & 0.0 & 0.0 & 0.0 \\
\hline $9.23469 \mathrm{E}-17$ & 0.0 & 0.0 & 0.0 \\
\hline $1.97945 \mathrm{E}-12$ & 0.0 & 0.0 & 0.0 \\
\hline $3.04963 E-07$ & 0.0 & 0.0 & 0.0 \\
\hline $2.21182 E-30$ & 0.0 & 0.0 & 0.0 \\
\hline $5.63823 E-12$ & 0.0 & 0.0 & 0.0 \\
\hline $8.49931 E-06$ & 0.0 & 0.0 & 0.0 \\
\hline $4.08671 \mathrm{E}-11$ & 0.0 & 0.0 & 0.0 \\
\hline $5.83272 \mathrm{E}-15$ & 0.0 & 0.0 & 0.0 \\
\hline $1.47583 E-09$ & 0.0 & 0.0 & 0.0 \\
\hline $3.31255 \mathrm{E}-12$ & 0.0 & 0.0 & 0.0 \\
\hline $3.86444 E-14$ & 0.0 & 0.0 & 0.0 \\
\hline $1.48132 \mathrm{E}-08$ & 0.0 & 0.0 & 0.0 \\
\hline $5.33608 E-16$ & 0.0 & 0.0 & 0.0 \\
\hline $5.51424 \mathrm{E}-09$ & 0.0 & 0.0 & 0.0 \\
\hline $5.27434 E-16$ & 0.0 & 0.0 & 0.0 \\
\hline $2.32682 E-06$ & 0.0 & 0.0 & 0.0 \\
\hline $6.18591 \mathrm{E}-08$ & 0.0 & 0.0 & 0.0 \\
\hline $1.08413 \mathrm{E}-11$ & 0.0 & 0.0 & 0.0 \\
\hline $1.78646 E-29$ & 0.0 & 0.0 & 0.0 \\
\hline $1.26185 \mathrm{E}-09$ & 0.0 & 0.0 & 0.0 \\
\hline $2.82778 E-21$ & 0.0 & 0.0 & 0.0 \\
\hline $2.40151 E-09$ & $7.77692 \mathrm{E}-05$ & 0.0 & 0.0 \\
\hline $4.24310 E-11$ & 0.0 & 0.0 & 0.0 \\
\hline $2.77744 \mathrm{E}-09$ & 0.0 & 0.0 & 0.0 \\
\hline $1.81550 \mathrm{E}-14$ & 0.0 & 0.0 & 0.0 \\
\hline $2.05819 \mathrm{E}-10$ & $1.08262 \mathrm{E}-07$ & 0.0 & 0.0 \\
\hline $6.42253 E-08$ & 0.0 & 0.0 & 0.0 \\
\hline $1.82888 \mathrm{E}-11$ & 0.0 & 0.0 & 0.0 \\
\hline $1.08667 \mathrm{E}-05$ & 0.0 & 0.0 & 0.0 \\
\hline $4.97121 E-06$ & 0.0 & 0.0 & 0.0 \\
\hline $5.37003 E-07$ & 0.0 & 0.0 & 0.0 \\
\hline $4.65555 E-08$ & 0.0 & 0.0 & 0.0 \\
\hline $3.33280 E-08$ & 0.0 & 0.0 & 0.0 \\
\hline $6.53357 E-12$ & 0.0 & 0.0 & 0.0 \\
\hline $6.09601 E-08$ & 0.0 & 0.0 & 0.0 \\
\hline $7.84260 \mathrm{E}-13$ & 0.0 & 0.0 & 0.0 \\
\hline $2.97462 \mathrm{E}-10$ & 0.0 & 0.0 & 0.0 \\
\hline $9.88538 E-15$ & 0.0 & 0.0 & 0.0 \\
\hline $3.28996 \mathrm{E}-12$ & 0.0 & 0.0 & 0.0 \\
\hline $1.10101 \mathrm{E}-27$ & 0.0 & 0.0 & 0.0 \\
\hline $1.83016 \mathrm{E}-04$ & 0.0 & 0.0 & 0.0 \\
\hline
\end{tabular}




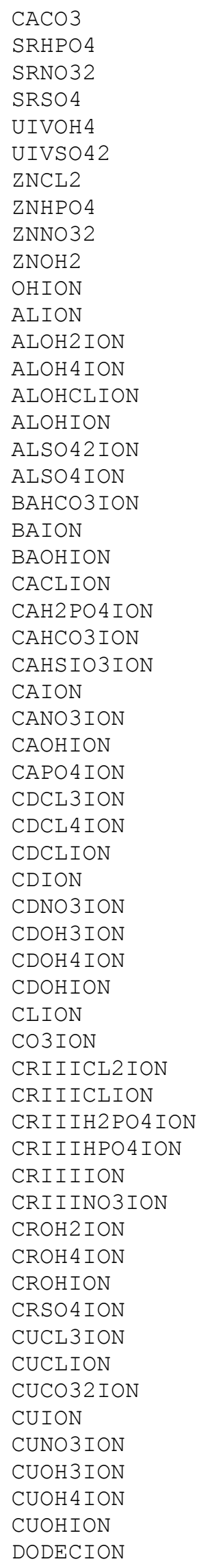

\begin{tabular}{|c|c|}
\hline $4.24133 E-07$ & 0.0 \\
\hline $8.88457 \mathrm{E}-09$ & 0.0 \\
\hline $.14573 \mathrm{E}-11$ & 0.0 \\
\hline $590 E-07$ & 0.0 \\
\hline $16 E-10$ & 0.0 \\
\hline $08 E-30$ & 0.0 \\
\hline $6 E-11$ & 0.0 \\
\hline $49 E-06$ & 0.0 \\
\hline $50 E-12$ & 0.0 \\
\hline $2 E-09$ & 0.0 \\
\hline $1 E-07$ & 0.0 \\
\hline $12 E-14$ & 0.0 \\
\hline $2 E-11$ & 0.0 \\
\hline $6.09729 \mathrm{E}-09$ & 0.0 \\
\hline $9 E-15$ & 0.0 \\
\hline $6 E-12$ & 0.0 \\
\hline $3 E-16$ & 0.0 \\
\hline $2 E-15$ & 0.0 \\
\hline$E-09$ & 0.0 \\
\hline $48 E-07$ & 0.0 \\
\hline$E-14$ & 0.0 \\
\hline $6 \mathrm{E}-11$ & 0.0 \\
\hline $8 E-06$ & 0.0 \\
\hline $6 E-06$ & 0.0 \\
\hline $7 E-09$ & 0.0 \\
\hline$E-04$ & 0.0 \\
\hline $6 E-07$ & 0.0 \\
\hline $7 E-10$ & 0.0 \\
\hline $1 E-07$ & 0.0 \\
\hline$E-15$ & 0.0 \\
\hline $9 E-18$ & 0.0 \\
\hline $5 E-09$ & 0.0 \\
\hline $8 E-08$ & 0.0 \\
\hline$E-11$ & 0.0 \\
\hline $4 E-21$ & 0.0 \\
\hline $3 E-28$ & 0.0 \\
\hline $6 \mathrm{E}-12$ & 0.0 \\
\hline 7742 & 0.0 \\
\hline $4 E-07$ & 0.0 \\
\hline $2 E-21$ & 0.0 \\
\hline $8 E-17$ & 0.0 \\
\hline $6 E-14$ & 0.0 \\
\hline $8 E-08$ & 0.0 \\
\hline$E-15$ & 0.0 \\
\hline $92 E-16$ & 0.0 \\
\hline$O E-13$ & 0.0 \\
\hline $58 E-16$ & 0.0 \\
\hline $78 E-12$ & 0.0 \\
\hline $18 \mathrm{E}-12$ & 0.0 \\
\hline $7 E-19$ & 0.0 \\
\hline $47 E-11$ & 0.0 \\
\hline $1 E-11$ & 0.0 \\
\hline $24 E-08$ & 0.0 \\
\hline $27 E-11$ & 0.0 \\
\hline $65 E-14$ & 0.0 \\
\hline $727 E-20$ & 0.0 \\
\hline $31 E-09$ & 0.0 \\
\hline $367 E-05$ & 0.0 \\
\hline
\end{tabular}

0.0

0.0

0.0

0.0

0.0

0.0

0.0

0.0

0.0

0.0

0.0

0.0

0.0

0.0

0.0

0.0

0.0

0.0

0.0

0.0

0.0

0.0

0.0

0.0

0.0

0.0

0.0

0.0

0.0

0.0

0.0

0.0

0.0

0.0

0.0

0.0

0.0

0.0

0.0

0.0

0.0

0.0

0.0

0.0

0.0

0.0

0.0

0.0

0.0

0.0

0.0

0.0

0.0

0.0

0.0

0.0

0.0

0.0
0.0

0.0

0.0

0.0

0.0

0.0

0.0

0.0

0.0

0.0

0.0

0.0

0.0

0.0

0.0

0.0

0.0

0.0

0.0

0.0

0.0

0.0

0.0

0.0

0.0

0.0

0.0

0.0

0.0

0.0

0.0

0.0

0.0

0.0

0.0

0.0

0.0

0.0

0.0

0.0

0.0

0.0

0.0

0.0

0.0

0.0

0.0

0.0

0.0

0.0

0.0

0.0

0.0

0.0

0.0

0.0

0.0

0.0 


\begin{tabular}{|c|c|c|c|c|}
\hline FEIICLION & $1.07078 \mathrm{E}-10$ & 0.0 & 0.0 & 0.0 \\
\hline FEIICO32ION & $3.81789 \mathrm{E}-11$ & 0.0 & 0.0 & 0.0 \\
\hline FEIIH2PO4ION & $1.46295 \mathrm{E}-08$ & 0.0 & 0.0 & 0.0 \\
\hline FEIIHCO3ION & $6.45090 \mathrm{E}-09$ & 0.0 & 0.0 & 0.0 \\
\hline FEIIION & $6.91162 E-05$ & 0.0 & 0.0 & 0.0 \\
\hline FEIIOH3ION & $4.33981 \mathrm{E}-15$ & 0.0 & 0.0 & 0.0 \\
\hline FEIIOH 4 ION & $5.33054 \mathrm{E}-23$ & 0.0 & 0.0 & 0.0 \\
\hline FEIIOHION & $1.58421 \mathrm{E}-07$ & 0.0 & 0.0 & 0.0 \\
\hline H2 P2O 7 ION & $4.42855 \mathrm{E}-11$ & 0.0 & 0.0 & 0.0 \\
\hline H2 PO 4 ION & $9.06949 \mathrm{E}-05$ & 0.0 & 0.0 & 0.0 \\
\hline H2SIO4ION & $2.51988 \mathrm{E}-13$ & 0.0 & 0.0 & 0.0 \\
\hline H3P2O 7 ION & $8.16713 \mathrm{E}-16$ & 0.0 & 0.0 & 0.0 \\
\hline H3SIO4 ION & $2.66269 \mathrm{E}-07$ & 0.0 & 0.0 & 0.0 \\
\hline HCO3ION & $8.90248 \mathrm{E}-04$ & 0.0 & 0.0 & 0.0 \\
\hline HION & $1.16552 \mathrm{E}-07$ & 0.0 & 0.0 & 0.0 \\
\hline HP207ION & $1.56881 \mathrm{E}-10$ & 0.0 & 0.0 & 0.0 \\
\hline HPBO2ION & $3.73294 \mathrm{E}-16$ & 0.0 & 0.0 & 0.0 \\
\hline HPO4 ION & $6.79056 \mathrm{E}-05$ & 0.0 & 0.0 & 0.0 \\
\hline HSO 4 ION & $4.44774 \mathrm{E}-09$ & 0.0 & 0.0 & 0.0 \\
\hline KION & $2.50457 \mathrm{E}-04$ & 0.0 & 0.0 & 0.0 \\
\hline KSO4 ION & $7.60756 \mathrm{E}-07$ & 0.0 & 0.0 & 0.0 \\
\hline MGH2 PO 4 ION & $8.05283 E-07$ & 0.0 & 0.0 & 0.0 \\
\hline MGHCO3ION & $6.32475 \mathrm{E}-06$ & 0.0 & 0.0 & 0.0 \\
\hline MGHSIO3ION & $1.67878 \mathrm{E}-09$ & 0.0 & 0.0 & 0.0 \\
\hline MGION & $2.60675 \mathrm{E}-04$ & 0.0 & 0.0 & 0.0 \\
\hline MGOHION & $3.14092 \mathrm{E}-09$ & 0.0 & 0.0 & 0.0 \\
\hline MGP2O 7 ION & $1.81299 \mathrm{E}-09$ & 0.0 & 0.0 & 0.0 \\
\hline MGPO 4 ION & $2.37014 \mathrm{E}-07$ & 0.0 & 0.0 & 0.0 \\
\hline NACO3ION & $2.15573 E-09$ & 0.0 & 0.0 & 0.0 \\
\hline NAION & 0.00170202 & 0.0 & 0.0 & 0.0 \\
\hline NASO 4 ION & $5.69544 \mathrm{E}-06$ & 0.0 & 0.0 & 0.0 \\
\hline NICLION & $1.16000 \mathrm{E}-10$ & 0.0 & 0.0 & 0.0 \\
\hline NIION & $1.02731 \mathrm{E}-06$ & 0.0 & 0.0 & 0.0 \\
\hline NINO3ION & $8.55139 \mathrm{E}-10$ & 0.0 & 0.0 & 0.0 \\
\hline NIOH3ION & $6.77552 \mathrm{E}-16$ & 0.0 & 0.0 & 0.0 \\
\hline NIOHION & $1.03987 \mathrm{E}-09$ & 0.0 & 0.0 & 0.0 \\
\hline NO3ION & $4.02557 \mathrm{E}-04$ & 0.0 & 0.0 & 0.0 \\
\hline P207ION & $1.31693 \mathrm{E}-12$ & 0.0 & 0.0 & 0.0 \\
\hline PBCL3ION & $6.04752 \mathrm{E}-16$ & 0.0 & 0.0 & 0.0 \\
\hline PBCL 4 ION & $7.07361 \mathrm{E}-19$ & 0.0 & 0.0 & 0.0 \\
\hline PBCLION & $1.61951 \mathrm{E}-10$ & 0.0 & 0.0 & 0.0 \\
\hline PBH2 PO 4 ION & $1.40198 \mathrm{E}-11$ & 0.0 & 0.0 & 0.0 \\
\hline PBION & $5.22063 \mathrm{E}-09$ & 0.0 & 0.0 & 0.0 \\
\hline PBNO33ION & $6.80138 \mathrm{E}-19$ & 0.0 & 0.0 & 0.0 \\
\hline PBNO3ION & $2.98458 \mathrm{E}-11$ & 0.0 & 0.0 & 0.0 \\
\hline PBOHION & $9.67677 \mathrm{E}-10$ & 0.0 & 0.0 & 0.0 \\
\hline PO4ION & $4.58669 \mathrm{E}-10$ & 0.0 & 0.0 & 0.0 \\
\hline SO4ION & $5.62432 \mathrm{E}-04$ & 0.0 & 0.0 & 0.0 \\
\hline SRION & $1.40088 \mathrm{E}-05$ & 0.0 & 0.0 & 0.0 \\
\hline SRNO3ION & $2.27466 \mathrm{E}-08$ & 0.0 & 0.0 & 0.0 \\
\hline SROHION & $6.55112 \mathrm{E}-12$ & 0.0 & 0.0 & 0.0 \\
\hline SRPO 4 ION & $1.23292 \mathrm{E}-10$ & 0.0 & 0.0 & 0.0 \\
\hline UIVOH2ION & $5.88214 \mathrm{E}-22$ & 0.0 & 0.0 & 0.0 \\
\hline UIVOH3ION & $3.96644 \mathrm{E}-16$ & 0.0 & 0.0 & 0.0 \\
\hline UIVOH5ION & $1.59621 \mathrm{E}-13$ & 0.0 & 0.0 & 0.0 \\
\hline UIVOHION & $1.42908 \mathrm{E}-26$ & 0.0 & 0.0 & 0.0 \\
\hline UIVSO 4 ION & $2.44546 \mathrm{E}-30$ & 0.0 & 0.0 & 0.0 \\
\hline ZNCL3ION & $3.41120 \mathrm{E}-14$ & 0.0 & 0.0 & 0.0 \\
\hline
\end{tabular}


WSRC-TR-2002-00567

Revision 0

\begin{tabular}{|c|c|c|c|c|}
\hline ZNCLION & $4.25876 \mathrm{E}-08$ & 0.0 & 0.0 & 0.0 \\
\hline ZNH2 PO4 ION & $5.94635 \mathrm{E}-08$ & 0.0 & 0.0 & 0.0 \\
\hline ZNHCO3ION & $3.31122 \mathrm{E}-07$ & 0.0 & 0.0 & 0.0 \\
\hline ZNION & $2.37789 \mathrm{E}-05$ & 0.0 & 0.0 & 0.0 \\
\hline ZNNO3ION & $1.97954 \mathrm{E}-08$ & 0.0 & 0.0 & 0.0 \\
\hline ZNOH3ION & $7.34006 \mathrm{E}-13$ & 0.0 & 0.0 & 0.0 \\
\hline ZNOH 4 ION & $1.13436 \mathrm{E}-18$ & 0.0 & 0.0 & 0.0 \\
\hline ZNOHION & $2.10139 \mathrm{E}-07$ & 0.0 & 0.0 & 0.0 \\
\hline $\mathrm{CU} 3 \mathrm{PO} 42.2 \mathrm{H} 2 \mathrm{O}$ & 0.0 & $1.30090 \mathrm{E}-06$ & 0.0 & 0.0 \\
\hline РВ3РО42 & 0.0 & $8.56061 \mathrm{E}-07$ & 0.0 & 0.0 \\
\hline \multirow[t]{2}{*}{ UIVO2 } & 0.0 & $2.43667 \mathrm{E}-06$ & 0.0 & 0.0 \\
\hline & $===========$ & $===========$ & $===========$ & $===========$ \\
\hline Total g/hr & 999.992 & 0.00798621 & 0.0 & 0.0 \\
\hline Volume, L/hr & 1.00289 & $2.64902 \mathrm{E}-06$ & 0.0 & 0.0 \\
\hline Enthalpy, cal/hr & $-3.79139 \mathrm{E}+06$ & -25.0603 & 0.0 & 0.0 \\
\hline Density, g/L & 997.109 & 3014.77 & & \\
\hline Vapor fraction & 0.0 & 0.0 & 0.0 & 0.0 \\
\hline Solid fraction & 0.0 & 1 . & 0.0 & 0.0 \\
\hline Organic fraction & 0.0 & 0.0 & 0.0 & 0.0 \\
\hline Osmotic Pres, atm & 0.194295 & & & \\
\hline Redox Pot, volts & 0.0 & & & \\
\hline E-Con, 1/ohm-cm & $4.73872 E-04$ & & & \\
\hline E-Con, cm2/ohm-mol & 137.534 & & & \\
\hline Abs Visc, cP & 0.89223 & & & \\
\hline Rel Visc & 1.0017 & & & \\
\hline Ionic Strength & 0.00605038 & & & \\
\hline
\end{tabular}


WSRC-TR-2002-00567

Revision 0

\begin{tabular}{|c|c|c|c|c|}
\hline \\
\hline \multicolumn{5}{|c|}{$\begin{array}{l}\text { STREAM: Evap Contents } \\
\text { TO : Evap separator }\end{array}$} \\
\hline FROM : Evap mixer & & & & \\
\hline Phases-----------> & Aqueous & Solid & Vapor & Organic \\
\hline & 102.858 & 102.858 & 102.858 & 102.858 \\
\hline $\begin{array}{l}\text { Pressure, atm } \\
\mathrm{pH}\end{array}$ & $\begin{array}{l}1 . \\
7.28941\end{array}$ & 1 . & 1 . & 1. \\
\hline \multirow{2}{*}{ Total mol/hr } & 0.04314368 & 0.00106679 & 55.4517 & 0.0 \\
\hline & $\mathrm{mol} / \mathrm{hr}------$ & $-\operatorname{mol} / \mathrm{hr}-----$ & $-\mathrm{mol} / \mathrm{hr}-----$ & $-\operatorname{mol} / \mathrm{hr}--------$ \\
\hline $\mathrm{H} 2 \mathrm{O}$ & 0.0392226 & 0.0 & 55.45056 & 0.0 \\
\hline $\mathrm{CO} 2$ & $1.06966 \mathrm{E}-10$ & 0.0 & 0.001100001 & 0.0 \\
\hline $\mathrm{H} 2 \mathrm{SO} 4$ & $8.86784 \mathrm{E}-29$ & 0.0 & $1.03674 \mathrm{E}-25$ & 0.0 \\
\hline $\mathrm{HCL}$ & $1.32974 \mathrm{E}-15$ & 0.0 & $1.67865 E-09$ & 0.0 \\
\hline HNO3 & $1.30831 \mathrm{E}-12$ & 0.0 & $3.77280 \mathrm{E}-10$ & 0.0 \\
\hline LAURICACID & $5.18994 \mathrm{E}-10$ & 0.0 & $3.56106 \mathrm{E}-05$ & 0.0 \\
\hline $\mathrm{SO} 3$ & 0.0 & 0.0 & 0.0 & 0.0 \\
\hline $\mathrm{CAH} 2 \mathrm{SIO} 4$ & $2.26277 \mathrm{E}-11$ & 0.0 & 0.0 & 0.0 \\
\hline CASO 4 & $4.36594 \mathrm{E}-07$ & $5.11611 E-04$ & 0.0 & 0.0 \\
\hline CDCL2 & $8.61753 E-09$ & 0.0 & 0.0 & 0.0 \\
\hline $\mathrm{CDOH} 2$ & $2.57653 \mathrm{E}-14$ & 0.0 & 0.0 & 0.0 \\
\hline $\mathrm{CDSO} 4$ & $1.34432 \mathrm{E}-12$ & 0.0 & 0.0 & 0.0 \\
\hline $\mathrm{CROH} 3$ & $2.83329 \mathrm{E}-11$ & $6.22196 E-08$ & 0.0 & 0.0 \\
\hline CUCL2 & $9.09203 \mathrm{E}-11$ & 0.0 & 0.0 & 0.0 \\
\hline $\mathrm{CuCO} 3$ & $7.49261 \mathrm{E}-14$ & 0.0 & 0.0 & 0.0 \\
\hline CUNO32 & $6.64122 E-13$ & 0.0 & 0.0 & 0.0 \\
\hline CUOH2 & $6.85216 E-09$ & $3.92871 E-06$ & 0.0 & 0.0 \\
\hline FEIICL2 & $9.40519 \mathrm{E}-15$ & 0.0 & 0.0 & 0.0 \\
\hline FEIICO3 & $1.36749 \mathrm{E}-14$ & 0.0 & 0.0 & 0.0 \\
\hline FEIIHPO4 & $4.10196 \mathrm{E}-17$ & 0.0 & 0.0 & 0.0 \\
\hline FEIIOH2 & $3.92671 E-13$ & 0.0 & 0.0 & 0.0 \\
\hline ALO2H2CL & $4.81508 \mathrm{E}-30$ & 0.0 & 0.0 & 0.0 \\
\hline H3PO 4 & $9.27958 \mathrm{E}-17$ & 0.0 & 0.0 & 0.0 \\
\hline H4 P2O 7 & 0.0 & 0.0 & 0.0 & 0.0 \\
\hline ALOH3 & $2.50828 E-11$ & 0.0 & 0.0 & 0.0 \\
\hline $\mathrm{BACO} 3$ & $5.37982 E-16$ & 0.0 & 0.0 & 0.0 \\
\hline $\mathrm{KCL}$ & $4.44462 E-06$ & 0.0 & 0.0 & 0.0 \\
\hline $\mathrm{KHSO} 4$ & $6.76380 \mathrm{E}-13$ & 0.0 & 0.0 & 0.0 \\
\hline $\mathrm{BASO} 4$ & $7.81771 E-13$ & $4.95158 E-07$ & 0.0 & 0.0 \\
\hline $\mathrm{MGCO} 3$ & $5.95080 \mathrm{E}-11$ & 0.0 & 0.0 & 0.0 \\
\hline MGH2SIO4 & $1.26492 \mathrm{E}-09$ & 0.0 & 0.0 & 0.0 \\
\hline MGHPO4 & $7.91226 \mathrm{E}-10$ & 0.0 & 0.0 & 0.0 \\
\hline MGSO 4 & $1.84940 \mathrm{E}-06$ & 0.0 & 0.0 & 0.0 \\
\hline $\mathrm{NAHCO} 3$ & $1.93835 E-09$ & 0.0 & 0.0 & 0.0 \\
\hline NAHSIO3 & $1.19318 \mathrm{E}-06$ & 0.0 & 0.0 & 0.0 \\
\hline NANO3 & $9.13817 \mathrm{E}-05$ & 0.0 & 0.0 & 0.0 \\
\hline $\mathrm{NIOH} 2$ & $1.80594 \mathrm{E}-12$ & $1.08823 E-06$ & 0.0 & 0.0 \\
\hline NISO4 & $2.54736 \mathrm{E}-10$ & 0.0 & 0.0 & 0.0 \\
\hline PBCL2 & $1.31329 \mathrm{E}-07$ & 0.0 & 0.0 & 0.0 \\
\hline PBHPO4 & $1.39358 \mathrm{E}-14$ & 0.0 & 0.0 & 0.0 \\
\hline PBNO32 & $4.07967 \mathrm{E}-09$ & 0.0 & 0.0 & 0.0 \\
\hline $\mathrm{PBO}$ & $1.16705 \mathrm{E}-09$ & 0.0 & 0.0 & 0.0 \\
\hline CACL2 & $1.77530 \mathrm{E}-15$ & 0.0 & 0.0 & 0.0 \\
\hline $\mathrm{SIO} 2$ & $3.28615 E-06$ & 1. $42520 \mathrm{E}-04$ & 0.0 & 0.0 \\
\hline
\end{tabular}




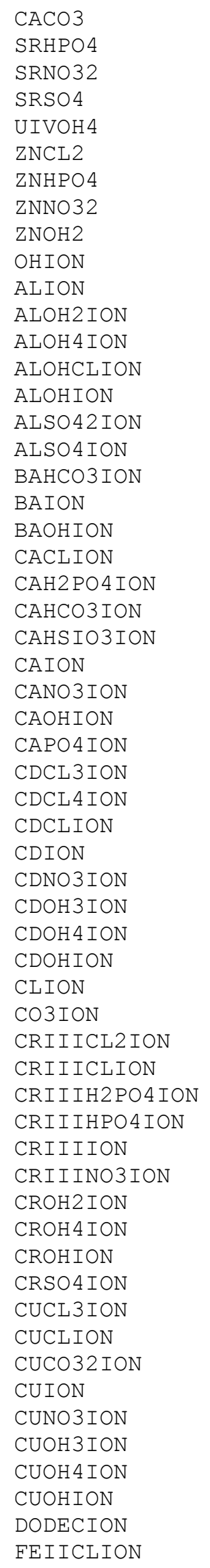

\begin{tabular}{|c|c|}
\hline $3.19806 \mathrm{E}-11$ & 0.0 \\
\hline $2.42874 \mathrm{E}-14$ & 0.0 \\
\hline $4.77103 \mathrm{E}-08$ & 0.0 \\
\hline $7.45195 \mathrm{E}-09$ & $1.45855 \mathrm{E}-05$ \\
\hline $9.47773 \mathrm{E}-13$ & 0.0 \\
\hline $4.44723 E-06$ & 0.0 \\
\hline 1. $49132 \mathrm{E}-12$ & 0.0 \\
\hline $2.01567 \mathrm{E}-09$ & 0.0 \\
\hline $4.78525 E-08$ & 0.0 \\
\hline $1.27039 \mathrm{E}-08$ & 0.0 \\
\hline $1.51543 \mathrm{E}-21$ & 0.0 \\
\hline $1.66307 \mathrm{E}-14$ & 0.0 \\
\hline $4.34362 E-09$ & 0.0 \\
\hline $3.33852 \mathrm{E}-17$ & 0.0 \\
\hline $1.06046 \mathrm{E}-17$ & 0.0 \\
\hline $9.56301 \mathrm{E}-22$ & 0.0 \\
\hline $3.79373 \mathrm{E}-21$ & 0.0 \\
\hline $4.44237 \mathrm{E}-14$ & 0.0 \\
\hline $1.18905 \mathrm{E}-09$ & 0.0 \\
\hline $1.12733 \mathrm{E}-14$ & 0.0 \\
\hline $4.42404 \mathrm{E}-08$ & 0.0 \\
\hline $5.79142 \mathrm{E}-12$ & 0.0 \\
\hline $8.40987 \mathrm{E}-11$ & 0.0 \\
\hline $1.11535 \mathrm{E}-08$ & 0.0 \\
\hline $1.27281 \mathrm{E}-05$ & 0.0 \\
\hline $5.40057 \mathrm{E}-06$ & 0.0 \\
\hline $2.29084 \mathrm{E}-09$ & 0.0 \\
\hline $1.92794 \mathrm{E}-11$ & 0.0 \\
\hline $6.38887 \mathrm{E}-09$ & 0.0 \\
\hline $1.42345 \mathrm{E}-09$ & 0.0 \\
\hline $5.19038 \mathrm{E}-10$ & 0.0 \\
\hline $1.09361 \mathrm{E}-11$ & 0.0 \\
\hline $2.82444 \mathrm{E}-12$ & 0.0 \\
\hline $3.92486 \mathrm{E}-18$ & 0.0 \\
\hline $3.52815 E-22$ & 0.0 \\
\hline $2.72848 E-13$ & 0.0 \\
\hline 0.00153247 & 0.0 \\
\hline $3.01628 \mathrm{E}-11$ & 0.0 \\
\hline $4.85063 \mathrm{E}-16$ & 0.0 \\
\hline $3.41947 \mathrm{E}-15$ & 0.0 \\
\hline $6.83285 E-21$ & 0.0 \\
\hline $2.96185 E-09$ & 0.0 \\
\hline $3.31895 \mathrm{E}-18$ & 0.0 \\
\hline $2.10757 \mathrm{E}-13$ & 0.0 \\
\hline $4.65743 E-13$ & 0.0 \\
\hline $6.73117 \mathrm{E}-14$ & 0.0 \\
\hline $1.70005 \mathrm{E}-10$ & 0.0 \\
\hline $3.87547 \mathrm{E}-12$ & 0.0 \\
\hline $1.16063 \mathrm{E}-12$ & 0.0 \\
\hline $6.00452 \mathrm{E}-10$ & 0.0 \\
\hline $1.59633 \mathrm{E}-17$ & 0.0 \\
\hline $1.80610 \mathrm{E}-10$ & 0.0 \\
\hline $2.98082 \mathrm{E}-11$ & 0.0 \\
\hline $6.54991 \mathrm{E}-12$ & 0.0 \\
\hline $1.39444 \mathrm{E}-14$ & 0.0 \\
\hline $5.24911 \mathrm{E}-10$ & 0.0 \\
\hline $2.38048 \mathrm{E}-06$ & 0.0 \\
\hline $2.08282 \mathrm{E}-12$ & 0.0 \\
\hline
\end{tabular}

0.0

0.0

0.0

0.0

0.0

0.0

0.0

0.0

0.0

0.0

0.0

0.0

0.0

0.0

0.0

0.0

0.0

0.0

0.0

0.0

0.0

0.0

0.0

0.0

0.0

0.0

0.0

0.0

0.0

0.0

0.0

0.0

0.0

0.0

0.0

0.0

0.0

0.0

0.0

0.0

0.0

0.0

0.0

0.0

0.0

0.0

0.0

0.0

0.0

0.0

0.0

0.0

0.0

0.0

0.0

0.0

0.0

0.0

0.0 


\begin{tabular}{|c|c|c|c|c|}
\hline FEIICO32ION & $8.79026 \mathrm{E}-20$ & 0.0 & 0.0 & 0.0 \\
\hline FEIIH2PO4ION & $4.25841 \mathrm{E}-18$ & 0.0 & 0.0 & 0.0 \\
\hline EEIIHCO3ION & $3.67653 \mathrm{E}-17$ & 0.0 & 0.0 & 0.0 \\
\hline FEIIION & $2.72780 \mathrm{E}-10$ & 0.0 & 0.0 & 0.0 \\
\hline FEIIOH3ION & $7.66934 \mathrm{E}-15$ & 0.0 & 0.0 & 0.0 \\
\hline EEIIOH4ION & $1.95094 \mathrm{E}-19$ & 0.0 & 0.0 & 0.0 \\
\hline FEIIOHION & $2.81092 \mathrm{E}-11$ & 0.0 & 0.0 & 0.0 \\
\hline H2 P2O 7 ION & $1.27225 \mathrm{E}-19$ & 0.0 & 0.0 & 0.0 \\
\hline H2 PO 4 ION & $1.78067 \mathrm{E}-11$ & 0.0 & 0.0 & 0.0 \\
\hline $\mathrm{H} 2 \mathrm{SIO} 4 \mathrm{ION}$ & $6.13631 \mathrm{E}-12$ & 0.0 & 0.0 & 0.0 \\
\hline H3P2O7 ION & $1.60640 \mathrm{E}-25$ & 0.0 & 0.0 & 0.0 \\
\hline H3SIO 4 ION & $3.96670 \mathrm{E}-07$ & 0.0 & 0.0 & 0.0 \\
\hline HCO3ION & $1.44851 \mathrm{E}-09$ & 0.0 & 0.0 & 0.0 \\
\hline HION & $4.81551 \mathrm{E}-11$ & 0.0 & 0.0 & 0.0 \\
\hline HP207ION & $2.51929 \mathrm{E}-18$ & 0.0 & 0.0 & 0.0 \\
\hline HPBO2ION & $1.13378 \mathrm{E}-11$ & 0.0 & 0.0 & 0.0 \\
\hline HPO4ION & $1.75428 \mathrm{E}-10$ & 0.0 & 0.0 & 0.0 \\
\hline HSO 4 ION & $1.34611 \mathrm{E}-10$ & 0.0 & 0.0 & 0.0 \\
\hline KION & $2.37198 \mathrm{E}-04$ & 0.0 & 0.0 & 0.0 \\
\hline KSO4 ION & $9.57860 \mathrm{E}-06$ & 0.0 & 0.0 & 0.0 \\
\hline MGH2 PO 4 ION & $7.31358 \mathrm{E}-12$ & 0.0 & 0.0 & 0.0 \\
\hline MGHCO3ION & $6.24930 \mathrm{E}-10$ & 0.0 & 0.0 & 0.0 \\
\hline MGHSIO3ION & $8.18028 \mathrm{E}-08$ & 0.0 & 0.0 & 0.0 \\
\hline MGION & $2.32857 \mathrm{E}-05$ & 0.0 & 0.0 & 0.0 \\
\hline MGOHION & $7.10139 \mathrm{E}-08$ & 0.0 & 0.0 & 0.0 \\
\hline MGP2O7ION & $1.33858 \mathrm{E}-14$ & 0.0 & 0.0 & 0.0 \\
\hline MGPO 4 ION & $8.11805 \mathrm{E}-11$ & 0.0 & 0.0 & 0.0 \\
\hline NACO3ION & $4.66615 \mathrm{E}-12$ & 0.0 & 0.0 & 0.0 \\
\hline NAION & 0.00161576 & 0.0 & 0.0 & 0.0 \\
\hline NASO 4 ION & $7.08753 \mathrm{E}-13$ & 0.0 & 0.0 & 0.0 \\
\hline NICLION & $1.08993 \mathrm{E}-10$ & 0.0 & 0.0 & 0.0 \\
\hline NIION & $1.46111 \mathrm{E}-09$ & 0.0 & 0.0 & 0.0 \\
\hline NINO3ION & $1.90981 \mathrm{E}-10$ & 0.0 & 0.0 & 0.0 \\
\hline NIOH3ION & $4.31767 \mathrm{E}-15$ & 0.0 & 0.0 & 0.0 \\
\hline NIOHION & $4.16995 \mathrm{E}-11$ & 0.0 & 0.0 & 0.0 \\
\hline NO3ION & $3.06082 \mathrm{E}-04$ & 0.0 & 0.0 & 0.0 \\
\hline P207ION & $4.47364 \mathrm{E}-19$ & 0.0 & 0.0 & 0.0 \\
\hline PBCL3ION & $2.40646 \mathrm{E}-07$ & 0.0 & 0.0 & 0.0 \\
\hline PBCL 4 ION & $1.47289 \mathrm{E}-06$ & 0.0 & 0.0 & 0.0 \\
\hline PBCLION & $3.90385 \mathrm{E}-08$ & 0.0 & 0.0 & 0.0 \\
\hline PBH2PO 4 ION & $7.05658 \mathrm{E}-16$ & 0.0 & 0.0 & 0.0 \\
\hline PBION & $1.50807 \mathrm{E}-09$ & 0.0 & 0.0 & 0.0 \\
\hline PBNO33ION & $1.26755 \mathrm{E}-10$ & 0.0 & 0.0 & 0.0 \\
\hline PBNO3ION & $1.13963 \mathrm{E}-08$ & 0.0 & 0.0 & 0.0 \\
\hline PBOHION & $2.66424 \mathrm{E}-08$ & 0.0 & 0.0 & 0.0 \\
\hline PO4ION & $2.74981 \mathrm{E}-14$ & 0.0 & 0.0 & 0.0 \\
\hline SO4ION & $4.47672 \mathrm{E}-05$ & 0.0 & 0.0 & 0.0 \\
\hline SRION & $2.74514 \mathrm{E}-08$ & 0.0 & 0.0 & 0.0 \\
\hline SRNO3ION & $1.71994 \mathrm{E}-07$ & 0.0 & 0.0 & 0.0 \\
\hline SROHION & $1.66468 \mathrm{E}-11$ & 0.0 & 0.0 & 0.0 \\
\hline SRPO 4 ION & $4.39386 \mathrm{E}-15$ & 0.0 & 0.0 & 0.0 \\
\hline UIVOH2ION & $4.94330 \mathrm{E}-26$ & 0.0 & 0.0 & 0.0 \\
\hline UIVOH3ION & $1.42057 \mathrm{E}-20$ & 0.0 & 0.0 & 0.0 \\
\hline UIVOH5ION & $8.56529 \mathrm{E}-15$ & 0.0 & 0.0 & 0.0 \\
\hline UIVOHION & 0.0 & 0.0 & 0.0 & 0.0 \\
\hline ZNCL3ION & $4.72385 E-06$ & 0.0 & 0.0 & 0.0 \\
\hline ZNCLION & $1.04817 \mathrm{E}-05$ & 0.0 & 0.0 & 0.0 \\
\hline ZNH2PO 4 ION & $3.67765 \mathrm{E}-14$ & 0.0 & 0.0 & 0.0 \\
\hline
\end{tabular}


WSRC-TR-2002-00567

Revision 0

\begin{tabular}{|c|c|c|c|c|}
\hline ZNHCO3ION & $2.54236 \mathrm{E}-12$ & 0.0 & 0.0 & 0.0 \\
\hline ZNION & $6.96366 \mathrm{E}-07$ & 0.0 & 0.0 & 0.0 \\
\hline ZNNO3ION & $6.93195 E-08$ & 0.0 & 0.0 & 0.0 \\
\hline ZNOH3ION & $1.84924 \mathrm{E}-09$ & 0.0 & 0.0 & 0.0 \\
\hline ZNOH $4 \mathrm{ION}$ & $1.35538 \mathrm{E}-12$ & 0.0 & 0.0 & 0.0 \\
\hline ZNOHION & $5.52373 E-06$ & 0.0 & 0.0 & 0.0 \\
\hline $\mathrm{ALOOH}$ & 0.0 & $6.08910 E-06$ & 0.0 & 0.0 \\
\hline $\mathrm{CA} 3 \mathrm{PO} 42$ & 0.0 & $8.92560 \mathrm{E}-05$ & 0.0 & 0.0 \\
\hline CHAMOSITE7A & 0.0 & $3.58420 E-05$ & 0.0 & 0.0 \\
\hline $\mathrm{MGOH} 2$ & 0.0 & $2.58659 \mathrm{E}-04$ & 0.0 & 0.0 \\
\hline $\mathrm{PB} 3 \mathrm{PO} 42$ & 0.0 & $2.15346 \mathrm{E}-07$ & 0.0 & 0.0 \\
\hline UIVO2 & 0.0 & $2.43696 \mathrm{E}-06$ & 0.0 & 0.0 \\
\hline & $============$ & $============$ & $============$ & $===========$ \\
\hline Total g/hr & 0.846469 & 0.137718 & 999.016 & 0.0 \\
\hline Volume, L/hr & $7.97549 E-04$ & $4.35179 E-05$ & 1696.91 & 0.0 \\
\hline Enthalpy, cal/hr & -2840.68 & -389.062 & $-3.17104 \mathrm{E}+06$ & 0.0 \\
\hline Density, g/L & 1061.34 & 3164.64 & 0.588726 & \\
\hline Vapor fraction & 0.0 & 0.0 & 1. & 0.0 \\
\hline Solid fraction & 0.0 & 1 . & 0.0 & 0.0 \\
\hline Organic fraction & 0.0 & 0.0 & 0.0 & 0.0 \\
\hline Osmotic Pres, atm & 162.392 & & & \\
\hline Redox Pot, volts & 0.0 & & & \\
\hline E-Con, 1/ohm-cm & 0.454821 & & & \\
\hline E-Con, cm2/ohm-mol & 52.4316 & & & \\
\hline Abs Visc, cP & 0.385228 & & & \\
\hline Rel Visc & 1.4093 & & & \\
\hline Ionic Strength & 2.87483 & & & \\
\hline
\end{tabular}


WSRC-TR-2002-00567

Revision 0

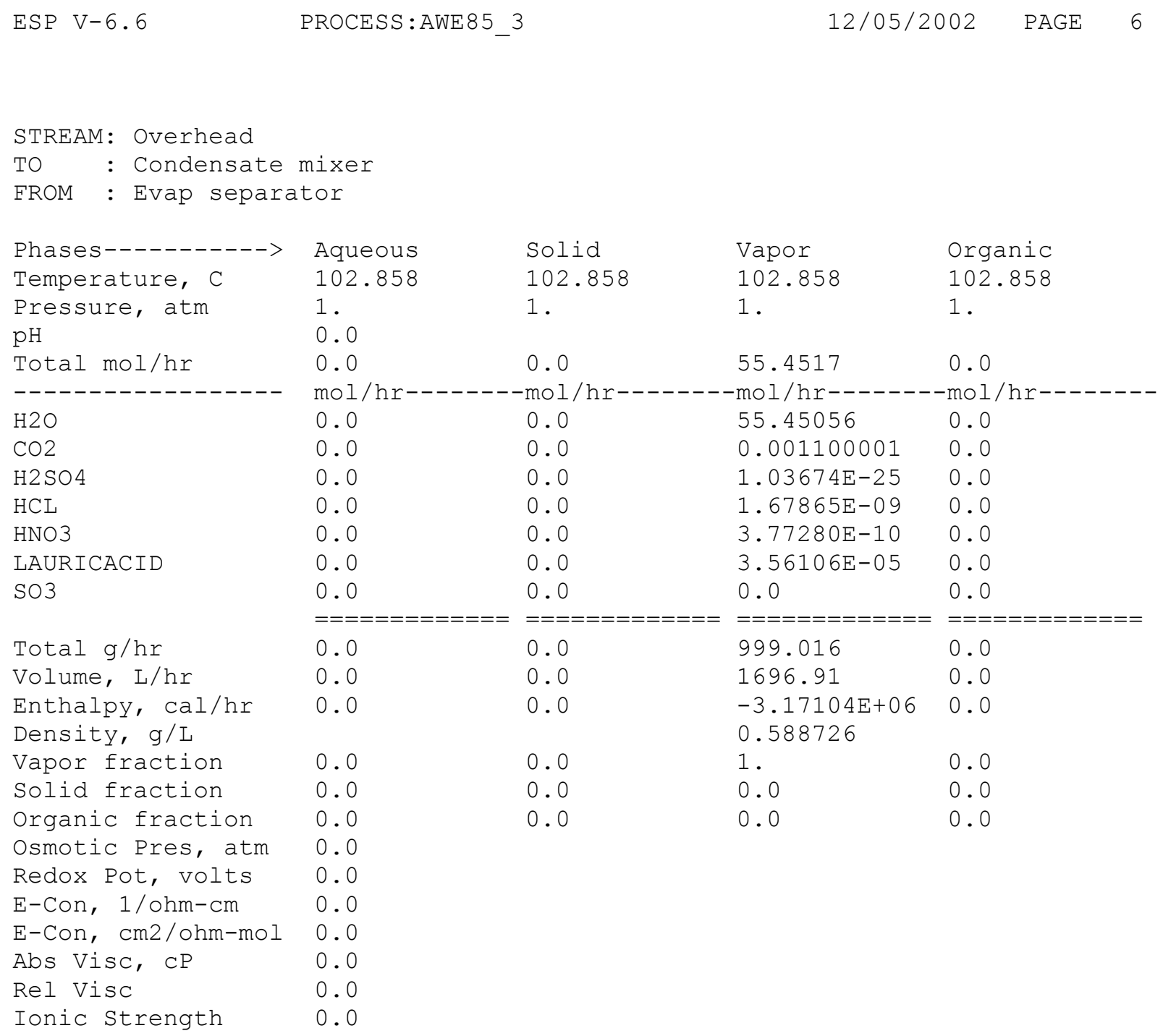


WSRC-TR-2002-00567

Revision 0

ESP V-6.6

PROCESS:AWE 85_3

12/05/2002 PAGE 7

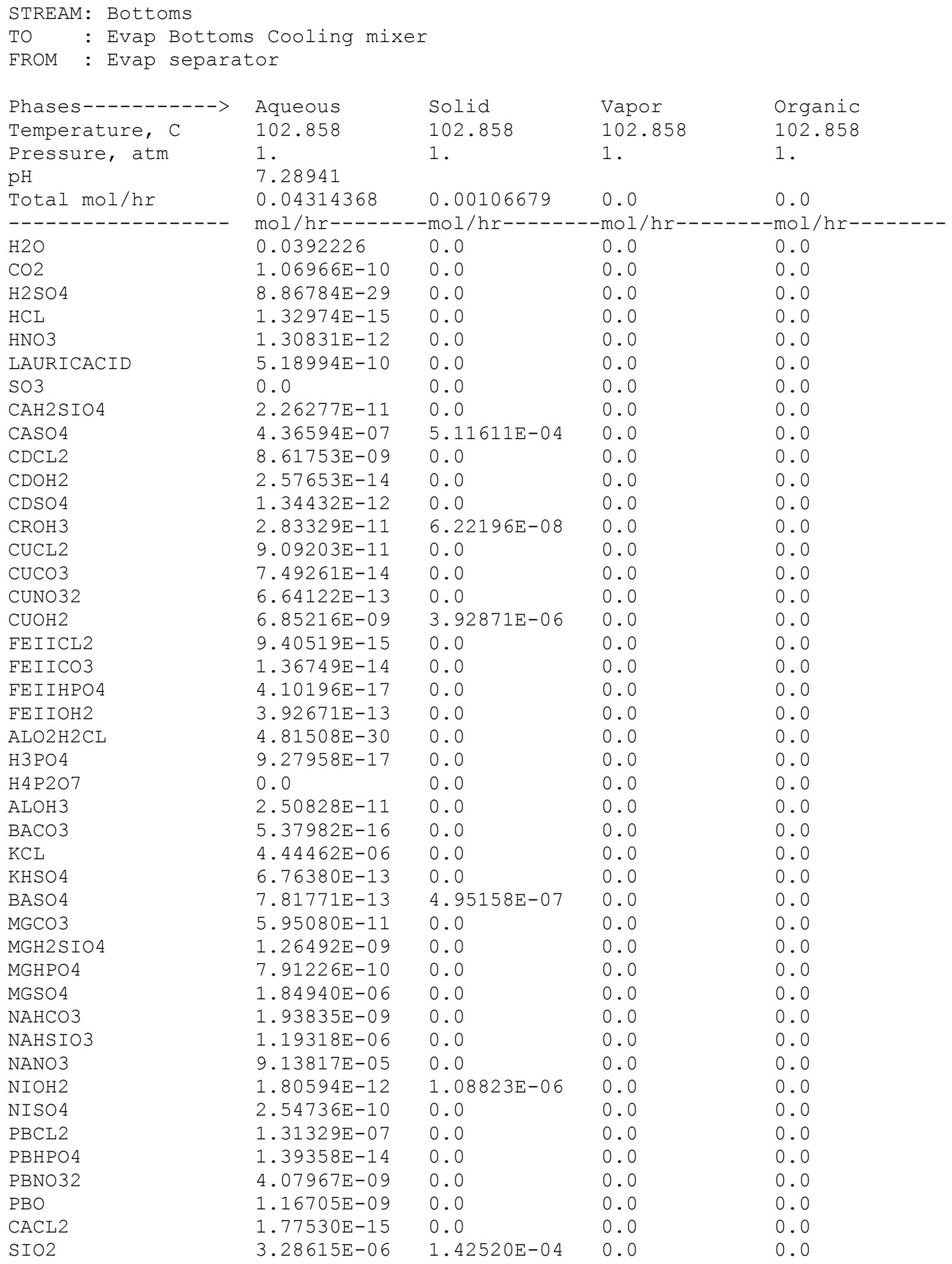




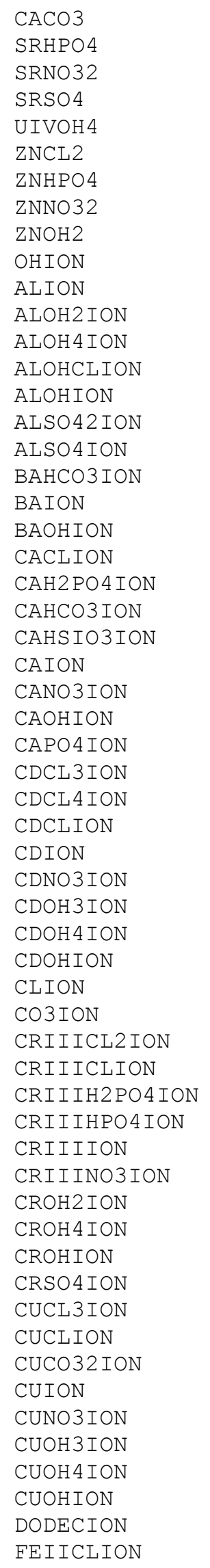

\begin{tabular}{|c|c|}
\hline $3.19806 \mathrm{E}-11$ & 0.0 \\
\hline $2.42874 \mathrm{E}-14$ & 0.0 \\
\hline $4.77103 \mathrm{E}-08$ & 0.0 \\
\hline $7.45195 \mathrm{E}-09$ & $1.45855 \mathrm{E}-05$ \\
\hline $9.47773 \mathrm{E}-13$ & 0.0 \\
\hline $4.44723 E-06$ & 0.0 \\
\hline 1. $49132 \mathrm{E}-12$ & 0.0 \\
\hline $2.01567 \mathrm{E}-09$ & 0.0 \\
\hline $4.78525 E-08$ & 0.0 \\
\hline $1.27039 \mathrm{E}-08$ & 0.0 \\
\hline $1.51543 \mathrm{E}-21$ & 0.0 \\
\hline $1.66307 \mathrm{E}-14$ & 0.0 \\
\hline $4.34362 E-09$ & 0.0 \\
\hline $3.33852 \mathrm{E}-17$ & 0.0 \\
\hline $1.06046 \mathrm{E}-17$ & 0.0 \\
\hline $9.56301 \mathrm{E}-22$ & 0.0 \\
\hline $3.79373 \mathrm{E}-21$ & 0.0 \\
\hline $4.44237 \mathrm{E}-14$ & 0.0 \\
\hline $1.18905 \mathrm{E}-09$ & 0.0 \\
\hline $1.12733 \mathrm{E}-14$ & 0.0 \\
\hline $4.42404 \mathrm{E}-08$ & 0.0 \\
\hline $5.79142 \mathrm{E}-12$ & 0.0 \\
\hline $8.40987 \mathrm{E}-11$ & 0.0 \\
\hline $1.11535 \mathrm{E}-08$ & 0.0 \\
\hline $1.27281 \mathrm{E}-05$ & 0.0 \\
\hline $5.40057 \mathrm{E}-06$ & 0.0 \\
\hline $2.29084 \mathrm{E}-09$ & 0.0 \\
\hline $1.92794 \mathrm{E}-11$ & 0.0 \\
\hline $6.38887 \mathrm{E}-09$ & 0.0 \\
\hline $1.42345 \mathrm{E}-09$ & 0.0 \\
\hline $5.19038 \mathrm{E}-10$ & 0.0 \\
\hline $1.09361 \mathrm{E}-11$ & 0.0 \\
\hline $2.82444 \mathrm{E}-12$ & 0.0 \\
\hline $3.92486 \mathrm{E}-18$ & 0.0 \\
\hline $3.52815 E-22$ & 0.0 \\
\hline $2.72848 E-13$ & 0.0 \\
\hline 0.00153247 & 0.0 \\
\hline $3.01628 \mathrm{E}-11$ & 0.0 \\
\hline $4.85063 \mathrm{E}-16$ & 0.0 \\
\hline $3.41947 \mathrm{E}-15$ & 0.0 \\
\hline $6.83285 E-21$ & 0.0 \\
\hline $2.96185 E-09$ & 0.0 \\
\hline $3.31895 \mathrm{E}-18$ & 0.0 \\
\hline $2.10757 \mathrm{E}-13$ & 0.0 \\
\hline $4.65743 E-13$ & 0.0 \\
\hline $6.73117 \mathrm{E}-14$ & 0.0 \\
\hline $1.70005 \mathrm{E}-10$ & 0.0 \\
\hline $3.87547 \mathrm{E}-12$ & 0.0 \\
\hline $1.16063 \mathrm{E}-12$ & 0.0 \\
\hline $6.00452 \mathrm{E}-10$ & 0.0 \\
\hline $1.59633 \mathrm{E}-17$ & 0.0 \\
\hline $1.80610 \mathrm{E}-10$ & 0.0 \\
\hline $2.98082 \mathrm{E}-11$ & 0.0 \\
\hline $6.54991 \mathrm{E}-12$ & 0.0 \\
\hline $1.39444 \mathrm{E}-14$ & 0.0 \\
\hline $5.24911 \mathrm{E}-10$ & 0.0 \\
\hline $2.38048 \mathrm{E}-06$ & 0.0 \\
\hline $2.08282 \mathrm{E}-12$ & 0.0 \\
\hline
\end{tabular}

0.0

0.0

0.0

0.0

0.0

0.0

0.0

0.0

0.0

0.0

0.0

0.0

0.0

0.0

0.0

0.0

0.0

0.0

0.0

0.0

0.0

0.0

0.0

0.0

0.0

0.0

0.0

0.0

0.0

0.0

0.0

0.0

0.0

0.0

0.0

0.0

0.0

0.0

0.0

0.0

0.0

0.0

0.0

0.0

0.0

0.0

0.0

0.0

0.0

0.0

0.0

0.0

0.0

0.0

0.0

0.0

0.0

0.0

0.0 


\begin{tabular}{|c|c|c|c|c|}
\hline FEIICO32ION & $8.79026 \mathrm{E}-20$ & 0.0 & 0.0 & 0.0 \\
\hline FEIIH2PO4ION & $4.25841 \mathrm{E}-18$ & 0.0 & 0.0 & 0.0 \\
\hline EEIIHCO3ION & $3.67653 \mathrm{E}-17$ & 0.0 & 0.0 & 0.0 \\
\hline FEIIION & $2.72780 \mathrm{E}-10$ & 0.0 & 0.0 & 0.0 \\
\hline FEIIOH3ION & $7.66934 \mathrm{E}-15$ & 0.0 & 0.0 & 0.0 \\
\hline EEIIOH4ION & $1.95094 \mathrm{E}-19$ & 0.0 & 0.0 & 0.0 \\
\hline FEIIOHION & $2.81092 \mathrm{E}-11$ & 0.0 & 0.0 & 0.0 \\
\hline H2 P2O 7 ION & $1.27225 \mathrm{E}-19$ & 0.0 & 0.0 & 0.0 \\
\hline H2 PO 4 ION & $1.78067 \mathrm{E}-11$ & 0.0 & 0.0 & 0.0 \\
\hline $\mathrm{H} 2 \mathrm{SIO} 4 \mathrm{ION}$ & $6.13631 \mathrm{E}-12$ & 0.0 & 0.0 & 0.0 \\
\hline H3P2O7 ION & $1.60640 \mathrm{E}-25$ & 0.0 & 0.0 & 0.0 \\
\hline H3SIO 4 ION & $3.96670 \mathrm{E}-07$ & 0.0 & 0.0 & 0.0 \\
\hline HCO3ION & $1.44851 \mathrm{E}-09$ & 0.0 & 0.0 & 0.0 \\
\hline HION & $4.81551 \mathrm{E}-11$ & 0.0 & 0.0 & 0.0 \\
\hline HP207ION & $2.51929 \mathrm{E}-18$ & 0.0 & 0.0 & 0.0 \\
\hline HPBO2ION & $1.13378 \mathrm{E}-11$ & 0.0 & 0.0 & 0.0 \\
\hline HPO4ION & $1.75428 \mathrm{E}-10$ & 0.0 & 0.0 & 0.0 \\
\hline HSO 4 ION & $1.34611 \mathrm{E}-10$ & 0.0 & 0.0 & 0.0 \\
\hline KION & $2.37198 \mathrm{E}-04$ & 0.0 & 0.0 & 0.0 \\
\hline KSO4 ION & $9.57860 \mathrm{E}-06$ & 0.0 & 0.0 & 0.0 \\
\hline MGH2 PO 4 ION & $7.31358 \mathrm{E}-12$ & 0.0 & 0.0 & 0.0 \\
\hline MGHCO3ION & $6.24930 \mathrm{E}-10$ & 0.0 & 0.0 & 0.0 \\
\hline MGHSIO3ION & $8.18028 \mathrm{E}-08$ & 0.0 & 0.0 & 0.0 \\
\hline MGION & $2.32857 \mathrm{E}-05$ & 0.0 & 0.0 & 0.0 \\
\hline MGOHION & $7.10139 \mathrm{E}-08$ & 0.0 & 0.0 & 0.0 \\
\hline MGP2O7ION & $1.33858 \mathrm{E}-14$ & 0.0 & 0.0 & 0.0 \\
\hline MGPO 4 ION & $8.11805 \mathrm{E}-11$ & 0.0 & 0.0 & 0.0 \\
\hline NACO3ION & $4.66615 \mathrm{E}-12$ & 0.0 & 0.0 & 0.0 \\
\hline NAION & 0.00161576 & 0.0 & 0.0 & 0.0 \\
\hline NASO 4 ION & $7.08753 \mathrm{E}-13$ & 0.0 & 0.0 & 0.0 \\
\hline NICLION & $1.08993 \mathrm{E}-10$ & 0.0 & 0.0 & 0.0 \\
\hline NIION & $1.46111 \mathrm{E}-09$ & 0.0 & 0.0 & 0.0 \\
\hline NINO3ION & $1.90981 \mathrm{E}-10$ & 0.0 & 0.0 & 0.0 \\
\hline NIOH3ION & $4.31767 \mathrm{E}-15$ & 0.0 & 0.0 & 0.0 \\
\hline NIOHION & $4.16995 \mathrm{E}-11$ & 0.0 & 0.0 & 0.0 \\
\hline NO3ION & $3.06082 \mathrm{E}-04$ & 0.0 & 0.0 & 0.0 \\
\hline P207ION & $4.47364 \mathrm{E}-19$ & 0.0 & 0.0 & 0.0 \\
\hline PBCL3ION & $2.40646 \mathrm{E}-07$ & 0.0 & 0.0 & 0.0 \\
\hline PBCL 4 ION & $1.47289 \mathrm{E}-06$ & 0.0 & 0.0 & 0.0 \\
\hline PBCLION & $3.90385 \mathrm{E}-08$ & 0.0 & 0.0 & 0.0 \\
\hline PBH2PO 4 ION & $7.05658 \mathrm{E}-16$ & 0.0 & 0.0 & 0.0 \\
\hline PBION & $1.50807 \mathrm{E}-09$ & 0.0 & 0.0 & 0.0 \\
\hline PBNO33ION & $1.26755 \mathrm{E}-10$ & 0.0 & 0.0 & 0.0 \\
\hline PBNO3ION & $1.13963 \mathrm{E}-08$ & 0.0 & 0.0 & 0.0 \\
\hline PBOHION & $2.66424 \mathrm{E}-08$ & 0.0 & 0.0 & 0.0 \\
\hline PO4ION & $2.74981 \mathrm{E}-14$ & 0.0 & 0.0 & 0.0 \\
\hline SO4ION & $4.47672 \mathrm{E}-05$ & 0.0 & 0.0 & 0.0 \\
\hline SRION & $2.74514 \mathrm{E}-08$ & 0.0 & 0.0 & 0.0 \\
\hline SRNO3ION & $1.71994 \mathrm{E}-07$ & 0.0 & 0.0 & 0.0 \\
\hline SROHION & $1.66468 \mathrm{E}-11$ & 0.0 & 0.0 & 0.0 \\
\hline SRPO 4 ION & $4.39386 \mathrm{E}-15$ & 0.0 & 0.0 & 0.0 \\
\hline UIVOH2ION & $4.94330 \mathrm{E}-26$ & 0.0 & 0.0 & 0.0 \\
\hline UIVOH3ION & $1.42057 \mathrm{E}-20$ & 0.0 & 0.0 & 0.0 \\
\hline UIVOH5ION & $8.56529 \mathrm{E}-15$ & 0.0 & 0.0 & 0.0 \\
\hline UIVOHION & 0.0 & 0.0 & 0.0 & 0.0 \\
\hline ZNCL3ION & $4.72385 E-06$ & 0.0 & 0.0 & 0.0 \\
\hline ZNCLION & $1.04817 \mathrm{E}-05$ & 0.0 & 0.0 & 0.0 \\
\hline ZNH2PO 4 ION & $3.67765 \mathrm{E}-14$ & 0.0 & 0.0 & 0.0 \\
\hline
\end{tabular}


WSRC-TR-2002-00567

Revision 0

\begin{tabular}{|c|c|c|c|c|}
\hline ZNHCO3ION & $2.54236 \mathrm{E}-12$ & 0.0 & 0.0 & 0.0 \\
\hline ZNION & $6.96366 \mathrm{E}-07$ & 0.0 & 0.0 & 0.0 \\
\hline ZNNO3ION & $6.93195 E-08$ & 0.0 & 0.0 & 0.0 \\
\hline ZNOH3ION & $1.84924 \mathrm{E}-09$ & 0.0 & 0.0 & 0.0 \\
\hline ZNOH $4 \mathrm{ION}$ & $1.35538 \mathrm{E}-12$ & 0.0 & 0.0 & 0.0 \\
\hline ZNOHION & $5.52373 E-06$ & 0.0 & 0.0 & 0.0 \\
\hline $\mathrm{ALOOH}$ & 0.0 & $6.08910 E-06$ & 0.0 & 0.0 \\
\hline $\mathrm{CA} 3 \mathrm{PO} 42$ & 0.0 & $8.92560 \mathrm{E}-05$ & 0.0 & 0.0 \\
\hline CHAMOSITE7A & 0.0 & $3.58420 E-05$ & 0.0 & 0.0 \\
\hline $\mathrm{MGOH} 2$ & 0.0 & $2.58659 \mathrm{E}-04$ & 0.0 & 0.0 \\
\hline $\mathrm{PB} 3 \mathrm{PO} 42$ & 0.0 & $2.15346 \mathrm{E}-07$ & 0.0 & 0.0 \\
\hline UIVO2 & 0.0 & $2.43696 \mathrm{E}-06$ & 0.0 & 0.0 \\
\hline & $============$ & $============$ & $============$ & $===========$ \\
\hline Total g/hr & 0.846469 & 0.137718 & 0.0 & 0.0 \\
\hline Volume, L/hr & $7.97549 E-04$ & $4.35179 E-05$ & 0.0 & 0.0 \\
\hline Enthalpy, cal/hr & -2840.68 & -389.062 & 0.0 & 0.0 \\
\hline Density, g/L & 1061.34 & 3164.64 & & \\
\hline Vapor fraction & 0.0 & 0.0 & 0.0 & 0.0 \\
\hline Solid fraction & 0.0 & 1 . & 0.0 & 0.0 \\
\hline Organic fraction & 0.0 & 0.0 & 0.0 & 0.0 \\
\hline Osmotic Pres, atm & 162.392 & & & \\
\hline Redox Pot, volts & 0.0 & & & \\
\hline E-Con, 1/ohm-cm & 0.454821 & & & \\
\hline E-Con, cm2/ohm-mol & 52.4316 & & & \\
\hline Abs Visc, cP & 0.385228 & & & \\
\hline Rel Visc & 1.4093 & & & \\
\hline Ionic Strength & 2.87483 & & & \\
\hline
\end{tabular}


WSRC-TR-2002-00567

Revision 0

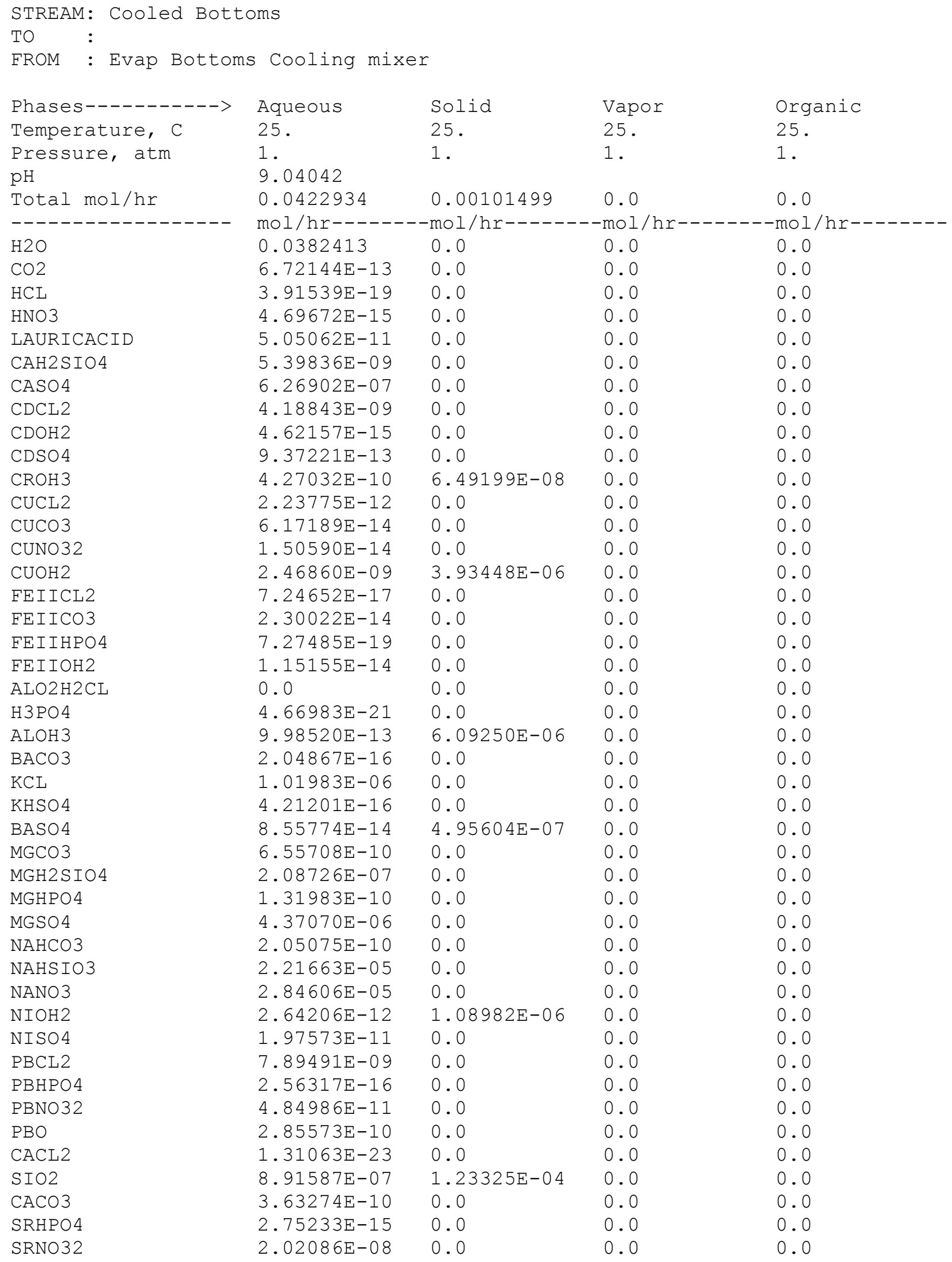




\begin{tabular}{|c|c|c|c|c|}
\hline $\mathrm{SRSO} 4$ & $1.79308 \mathrm{E}-08$ & $1.39670 \mathrm{E}-05$ & 0.0 & 0.0 \\
\hline UIVOH 4 & $1.01016 \mathrm{E}-13$ & 0.0 & 0.0 & 0.0 \\
\hline ZNCL2 & $3.79081 \mathrm{E}-09$ & 0.0 & 0.0 & 0.0 \\
\hline ZNHPO 4 & $7.87248 \mathrm{E}-15$ & 0.0 & 0.0 & 0.0 \\
\hline ZNNO32 & $4.48843 E-11$ & 0.0 & 0.0 & 0.0 \\
\hline $\mathrm{ZNOH} 2$ & $3.38208 E-09$ & $2.59522 E-05$ & 0.0 & 0.0 \\
\hline OHION & $7.32376 \mathrm{E}-09$ & 0.0 & 0.0 & 0.0 \\
\hline ALION & $4.03360 \mathrm{E}-23$ & 0.0 & 0.0 & 0.0 \\
\hline ALOH2 ION & $4.30625 E-16$ & 0.0 & 0.0 & 0.0 \\
\hline ALOH 4 ION & $7.09191 \mathrm{E}-10$ & 0.0 & 0.0 & 0.0 \\
\hline ALOHCLION & $8.28752 \mathrm{E}-19$ & 0.0 & 0.0 & 0.0 \\
\hline ALOHION & $1.60142 \mathrm{E}-19$ & 0.0 & 0.0 & 0.0 \\
\hline ALSO 42 ION & $1.44908 \mathrm{E}-23$ & 0.0 & 0.0 & 0.0 \\
\hline ALSO 4 ION & $5.40112 \mathrm{E}-23$ & 0.0 & 0.0 & 0.0 \\
\hline BAHCO3ION & $3.30762 \mathrm{E}-16$ & 0.0 & 0.0 & 0.0 \\
\hline BAION & $7.46405 \mathrm{E}-10$ & 0.0 & 0.0 & 0.0 \\
\hline BAOHION & $1.65655 \mathrm{E}-16$ & 0.0 & 0.0 & 0.0 \\
\hline CACLION & $5.17392 \mathrm{E}-11$ & 0.0 & 0.0 & 0.0 \\
\hline CAH2PO 4 ION & $5.22995 \mathrm{E}-14$ & 0.0 & 0.0 & 0.0 \\
\hline CAHCO3ION & $1.16603 \mathrm{E}-10$ & 0.0 & 0.0 & 0.0 \\
\hline CAHSIO3ION & $7.94672 \mathrm{E}-08$ & 0.0 & 0.0 & 0.0 \\
\hline CAION & $3.12183 \mathrm{E}-05$ & 0.0 & 0.0 & 0.0 \\
\hline CANO3ION & $1.22636 \mathrm{E}-05$ & 0.0 & 0.0 & 0.0 \\
\hline CAOHION & $1.81801 \mathrm{E}-09$ & 0.0 & 0.0 & 0.0 \\
\hline CAPO 4 ION & $1.86547 \mathrm{E}-10$ & 0.0 & 0.0 & 0.0 \\
\hline CDCL3ION & $1.23144 \mathrm{E}-09$ & 0.0 & 0.0 & 0.0 \\
\hline CDCL 4 ION & $1.12456 \mathrm{E}-08$ & 0.0 & 0.0 & 0.0 \\
\hline CDCLION & $2.82721 \mathrm{E}-10$ & 0.0 & 0.0 & 0.0 \\
\hline CDION & $1.31324 \mathrm{E}-11$ & 0.0 & 0.0 & 0.0 \\
\hline CDNO3ION & $1.92687 \mathrm{E}-12$ & 0.0 & 0.0 & 0.0 \\
\hline CDOH3ION & $2.23385 \mathrm{E}-18$ & 0.0 & 0.0 & 0.0 \\
\hline CDOH 4 ION & $1.01965 \mathrm{E}-22$ & 0.0 & 0.0 & 0.0 \\
\hline CDOHION & $1.35230 \mathrm{E}-13$ & 0.0 & 0.0 & 0.0 \\
\hline CLION & 0.00157532 & 0.0 & 0.0 & 0.0 \\
\hline CO3ION & $4.43457 \mathrm{E}-10$ & 0.0 & 0.0 & 0.0 \\
\hline CRIIICL2ION & $3.70542 \mathrm{E}-18$ & 0.0 & 0.0 & 0.0 \\
\hline CRIIICLION & $2.83426 \mathrm{E}-17$ & 0.0 & 0.0 & 0.0 \\
\hline CRIIIH2PO4 ION & $5.58770 \mathrm{E}-23$ & 0.0 & 0.0 & 0.0 \\
\hline CRIIIHPO4ION & $2.86041 E-15$ & 0.0 & 0.0 & 0.0 \\
\hline CRIIIION & $1.56247 \mathrm{E}-19$ & 0.0 & 0.0 & 0.0 \\
\hline CRIIINO3ION & $7.99579 \mathrm{E}-16$ & 0.0 & 0.0 & 0.0 \\
\hline CROH2ION & $2.96939 \mathrm{E}-13$ & 0.0 & 0.0 & 0.0 \\
\hline $\mathrm{CROH} 4 \mathrm{ION}$ & $1.70663 \mathrm{E}-11$ & 0.0 & 0.0 & 0.0 \\
\hline CROHION & $2.03130 \mathrm{E}-11$ & 0.0 & 0.0 & 0.0 \\
\hline CRSO 4 ION & $2.03453 \mathrm{E}-14$ & 0.0 & 0.0 & 0.0 \\
\hline CUCL3ION & $2.85105 \mathrm{E}-14$ & 0.0 & 0.0 & 0.0 \\
\hline CUCLION & $7.06569 \mathrm{E}-12$ & 0.0 & 0.0 & 0.0 \\
\hline CUCO32ION & $1.15782 \mathrm{E}-16$ & 0.0 & 0.0 & 0.0 \\
\hline CUION & $7.04789 \mathrm{E}-12$ & 0.0 & 0.0 & 0.0 \\
\hline CUNO3ION & $1.20805 \mathrm{E}-12$ & 0.0 & 0.0 & 0.0 \\
\hline CUOH3ION & $4.37950 \mathrm{E}-12$ & 0.0 & 0.0 & 0.0 \\
\hline CUOH 4 ION & $3.22615 E-15$ & 0.0 & 0.0 & 0.0 \\
\hline CUOHION & $3.20169 \mathrm{E}-11$ & 0.0 & 0.0 & 0.0 \\
\hline DODECION & $2.38095 E-06$ & 0.0 & 0.0 & 0.0 \\
\hline FEIICLION & $2.82896 \mathrm{E}-14$ & 0.0 & 0.0 & 0.0 \\
\hline FEIICO32ION & $9.18354 \mathrm{E}-19$ & 0.0 & 0.0 & 0.0 \\
\hline FEIIH2PO4ION & $4.72923 \mathrm{E}-21$ & 0.0 & 0.0 & 0.0 \\
\hline FEIIHCO3ION & $1.25046 \mathrm{E}-18$ & 0.0 & 0.0 & 0.0 \\
\hline
\end{tabular}




\begin{tabular}{|c|c|c|c|c|}
\hline FEIIION & $2.24882 \mathrm{E}-11$ & 0.0 & 0.0 & 0.0 \\
\hline FEIIOH3ION & $1.43971 \mathrm{E}-15$ & 0.0 & 0.0 & 0.0 \\
\hline FEIIOH 4 ION & $1.52546 \mathrm{E}-20$ & 0.0 & 0.0 & 0.0 \\
\hline FEIIOHION & $3.40336 \mathrm{E}-12$ & 0.0 & 0.0 & 0.0 \\
\hline H2P2O 7 ION & $4.95629 \mathrm{E}-25$ & 0.0 & 0.0 & 0.0 \\
\hline H2 PO 4 ION & $1.54608 \mathrm{E}-13$ & 0.0 & 0.0 & 0.0 \\
\hline H2SIO 4 ION & $2.65686 \mathrm{E}-10$ & 0.0 & 0.0 & 0.0 \\
\hline H3SIO 4 ION & $2.27137 \mathrm{E}-07$ & 0.0 & 0.0 & 0.0 \\
\hline HCO3ION & $1.05987 \mathrm{E}-09$ & 0.0 & 0.0 & 0.0 \\
\hline HION & $5.62641 \mathrm{E}-13$ & 0.0 & 0.0 & 0.0 \\
\hline HP207ION & $8.27993 \mathrm{E}-22$ & 0.0 & 0.0 & 0.0 \\
\hline HPBO2 ION & $7.80690 \mathrm{E}-12$ & 0.0 & 0.0 & 0.0 \\
\hline HPO 4 ION & $1.04129 \mathrm{E}-10$ & 0.0 & 0.0 & 0.0 \\
\hline HSO 4 ION & $2.76605 \mathrm{E}-13$ & 0.0 & 0.0 & 0.0 \\
\hline $\mathrm{KION}$ & $2.43816 \mathrm{E}-04$ & 0.0 & 0.0 & 0.0 \\
\hline KSO4 ION & $6.38549 \mathrm{E}-06$ & 0.0 & 0.0 & 0.0 \\
\hline MGH2 PO 4 ION & $2.68849 \mathrm{E}-13$ & 0.0 & 0.0 & 0.0 \\
\hline MGHCO3ION & $1.26584 \mathrm{E}-09$ & 0.0 & 0.0 & 0.0 \\
\hline MGHSIO3ION & $5.87318 \mathrm{E}-07$ & 0.0 & 0.0 & 0.0 \\
\hline MGION & $5.40894 \mathrm{E}-05$ & 0.0 & 0.0 & 0.0 \\
\hline MGOHION & $6.96833 \mathrm{E}-08$ & 0.0 & 0.0 & 0.0 \\
\hline MGP2O7ION & $7.32470 \mathrm{E}-17$ & 0.0 & 0.0 & 0.0 \\
\hline MGPO 4 ION & $1.03572 \mathrm{E}-09$ & 0.0 & 0.0 & 0.0 \\
\hline NACO3ION & $2.21218 \mathrm{E}-10$ & 0.0 & 0.0 & 0.0 \\
\hline NAION & 0.00163461 & 0.0 & 0.0 & 0.0 \\
\hline NASO 4 ION & $2.30933 E-05$ & 0.0 & 0.0 & 0.0 \\
\hline NICLION & $1.16675 \mathrm{E}-11$ & 0.0 & 0.0 & 0.0 \\
\hline NIION & $3.49141 \mathrm{E}-10$ & 0.0 & 0.0 & 0.0 \\
\hline NINO3ION & $7.78789 \mathrm{E}-11$ & 0.0 & 0.0 & 0.0 \\
\hline NIOH3ION & $9.37132 \mathrm{E}-14$ & 0.0 & 0.0 & 0.0 \\
\hline NIOHION & $8.50982 \mathrm{E}-12$ & 0.0 & 0.0 & 0.0 \\
\hline NO3ION & $3.62303 \mathrm{E}-04$ & 0.0 & 0.0 & 0.0 \\
\hline P207ION & $5.15491 \mathrm{E}-20$ & 0.0 & 0.0 & 0.0 \\
\hline PBCL3ION & $1.66819 \mathrm{E}-08$ & 0.0 & 0.0 & 0.0 \\
\hline PBCL 4 ION & $2.40773 \mathrm{E}-07$ & 0.0 & 0.0 & 0.0 \\
\hline PBCLION & $3.13415 \mathrm{E}-09$ & 0.0 & 0.0 & 0.0 \\
\hline PBH2PO4 ION & $3.32068 \mathrm{E}-19$ & 0.0 & 0.0 & 0.0 \\
\hline PBION & $2.18359 \mathrm{E}-10$ & 0.0 & 0.0 & 0.0 \\
\hline PBNO33ION & $9.06155 \mathrm{E}-12$ & 0.0 & 0.0 & 0.0 \\
\hline PBNO3ION & $5.23118 \mathrm{E}-10$ & 0.0 & 0.0 & 0.0 \\
\hline PBOHION & $1.52458 \mathrm{E}-09$ & 0.0 & 0.0 & 0.0 \\
\hline PO4ION & $5.53687 \mathrm{E}-13$ & 0.0 & 0.0 & 0.0 \\
\hline SO4ION & $4.66827 \mathrm{E}-05$ & 0.0 & 0.0 & 0.0 \\
\hline SRION & $6.80087 \mathrm{E}-07$ & 0.0 & 0.0 & 0.0 \\
\hline SRNO3ION & $1.54909 \mathrm{E}-07$ & 0.0 & 0.0 & 0.0 \\
\hline SROHION & $5.61184 \mathrm{E}-12$ & 0.0 & 0.0 & 0.0 \\
\hline SRPO 4 ION & $1.45597 \mathrm{E}-14$ & 0.0 & 0.0 & 0.0 \\
\hline UIVOH2ION & $7.37466 \mathrm{E}-29$ & 0.0 & 0.0 & 0.0 \\
\hline UIVOH3ION & $2.89891 \mathrm{E}-21$ & 0.0 & 0.0 & 0.0 \\
\hline UIVOH5ION & $1.82485 \mathrm{E}-14$ & 0.0 & 0.0 & 0.0 \\
\hline ZNCL3ION & $5.56616 \mathrm{E}-09$ & 0.0 & 0.0 & 0.0 \\
\hline ZNCLION & $4.87655 \mathrm{E}-09$ & 0.0 & 0.0 & 0.0 \\
\hline ZNH2 PO 4 ION & $8.33140 \mathrm{E}-18$ & 0.0 & 0.0 & 0.0 \\
\hline ZNHCO3ION & $3.89952 \mathrm{E}-14$ & 0.0 & 0.0 & 0.0 \\
\hline ZNION & $1.91637 \mathrm{E}-08$ & 0.0 & 0.0 & 0.0 \\
\hline ZNNO3ION & $2.05241 \mathrm{E}-09$ & 0.0 & 0.0 & 0.0 \\
\hline ZNOH3ION & $7.72697 \mathrm{E}-11$ & 0.0 & 0.0 & 0.0 \\
\hline ZNOH 4 ION & $1.35904 \mathrm{E}-13$ & 0.0 & 0.0 & 0.0 \\
\hline
\end{tabular}


WSRC-TR-2002-00567

Revision 0

\begin{tabular}{|c|c|c|c|c|}
\hline ZNOHION & $2.74427 E-09$ & 0.0 & 0.0 & 0.0 \\
\hline $\mathrm{CA} 3 \mathrm{PO} 42$ & 0.0 & $8.87050 \mathrm{E}-05$ & 0.0 & 0.0 \\
\hline $\mathrm{CASO} 4.2 \mathrm{H} 2 \mathrm{O}$ & 0.0 & $4.87694 \mathrm{E}-04$ & 0.0 & 0.0 \\
\hline CHAMOSITE7A & 0.0 & $3.58423 E-05$ & 0.0 & 0.0 \\
\hline MGOH2 & 0.0 & $2.24622 \mathrm{E}-04$ & 0.0 & 0.0 \\
\hline РB3PO42 & 0.0 & $7.67926 \mathrm{E}-07$ & 0.0 & 0.0 \\
\hline \multirow[t]{2}{*}{ UIVO2 } & 0.0 & $2.43697 E-06$ & 0.0 & 0.0 \\
\hline & $===========$ & $===========$ & $===========$ & $===========$ \\
\hline Total g/hr & 0.832437 & 0.15175 & 0.0 & 0.0 \\
\hline Volume, L/hr & $7.39819 E-04$ & $5.11410 \mathrm{E}-05$ & 0.0 & 0.0 \\
\hline Enthalpy, cal/hr & -2845.54 & -442.663 & 0.0 & 0.0 \\
\hline Density, g/L & 1125.19 & 2967.29 & & \\
\hline Vapor fraction & 0.0 & 0.0 & 0.0 & 0.0 \\
\hline Solid fraction & 0.0 & 1 . & 0.0 & 0.0 \\
\hline Organic fraction & 0.0 & 0.0 & 0.0 & 0.0 \\
\hline Osmotic Pres, atm & 152.855 & & & \\
\hline Redox Pot, volts & 0.0 & & & \\
\hline E-Con, 1/ohm-cm & 0.181873 & & & \\
\hline E-Con, cm2/ohm-mol & 30.4915 & & & \\
\hline Abs Visc, cP & 1.21716 & & & \\
\hline Rel Visc & 1.36649 & & & \\
\hline Ionic Strength & 3.18839 & & & \\
\hline
\end{tabular}


WSRC-TR-2002-00567

Revision 0

\begin{tabular}{|c|c|c|c|c|}
\hline \multicolumn{5}{|l|}{ STREAM: Condensate } \\
\hline TO : & & & & \\
\hline FROM : Condensate & mixer & & & \\
\hline Phases-----------> & Aqueous & Solid & Vapor & Organic \\
\hline Temperature, C & 25 . & 25 . & 25 . & 25 . \\
\hline $\begin{array}{l}\text { Pressure, atm } \\
\mathrm{pH}\end{array}$ & $\begin{array}{l}1 . \\
4.54998\end{array}$ & 1. & 1 . & 1 . \\
\hline \multirow{2}{*}{ Total mol/hr } & 55.45166 & 0.0 & 0.0 & 0.0 \\
\hline & $\mathrm{mol} / \mathrm{hr}------$ & $-\operatorname{mol} / \mathrm{hr}--------$ & -mol/hr------- & -mol/hr------ \\
\hline $\mathrm{H} 2 \mathrm{O}$ & 55.4505 & 0.0 & 0.0 & 0.0 \\
\hline $\mathrm{CO} 2$ & 0.00108245 & 0.0 & 0.0 & 0.0 \\
\hline $\mathrm{HCL}$ & $2.78519 E-20$ & 0.0 & 0.0 & 0.0 \\
\hline HNO3 & $5.26698 \mathrm{E}-16$ & 0.0 & 0.0 & 0.0 \\
\hline LAURICACID & $2.48218 E-05$ & 0.0 & 0.0 & 0.0 \\
\hline OHION & $3.61728 \mathrm{E}-10$ & 0.0 & 0.0 & 0.0 \\
\hline CLION & $1.67865 E-09$ & 0.0 & 0.0 & 0.0 \\
\hline CO3ION & $2.97799 \mathrm{E}-11$ & 0.0 & 0.0 & 0.0 \\
\hline DODECION & $1.07888 E-05$ & 0.0 & 0.0 & 0.0 \\
\hline $\mathrm{HCO} 3 \mathrm{ION}$ & $1.75403 E-05$ & 0.0 & 0.0 & 0.0 \\
\hline $\mathrm{HION}$ & $2.83315 E-05$ & 0.0 & 0.0 & 0.0 \\
\hline NO3ION & $\begin{array}{l}\text { 3. } 77280 \mathrm{E}-10 \\
===========\end{array}$ & $\begin{array}{l}0.0 \\
============\end{array}$ & $\begin{array}{l}0.0 \\
=============\end{array}$ & $\begin{array}{l}0.0 \\
============\end{array}$ \\
\hline Total g/hr & $\begin{array}{l}=========== \\
999.016\end{array}$ & $\begin{array}{l}============ \\
0.0\end{array}$ & $\begin{array}{l}============ \\
0.0\end{array}$ & $\begin{array}{l}============ \\
0.0\end{array}$ \\
\hline Volume, L/hr & 1.00216 & 0.0 & 0.0 & 0.0 \\
\hline $\begin{array}{l}\text { Enthalpy, cal/hr } \\
\text { Density, g/L }\end{array}$ & $\begin{array}{l}-3.78812 \mathrm{E}+06 \\
996.864\end{array}$ & 0.0 & 0.0 & 0.0 \\
\hline Vapor fraction & 0.0 & 0.0 & 0.0 & 0.0 \\
\hline Solid fraction & 0.0 & 0.0 & 0.0 & 0.0 \\
\hline Organic fraction & 0.0 & 0.0 & 0.0 & 0.0 \\
\hline Osmotic Pres, atm & 0.028421 & & & \\
\hline Redox Pot, volts & 0.0 & & & \\
\hline E-Con, 1/ohm-cm & $1.12896 \mathrm{E}-05$ & & & \\
\hline E-Con, cm2/ohm-mol & 9.96292 & & & \\
\hline Abs Visc, cP & 0.890739 & & & \\
\hline Rel Visc & 1.00002 & & & \\
\hline Ionic Strength & $2.83613 E-05$ & & & \\
\hline
\end{tabular}


WSRC-TR-2002-00567

Revision 0

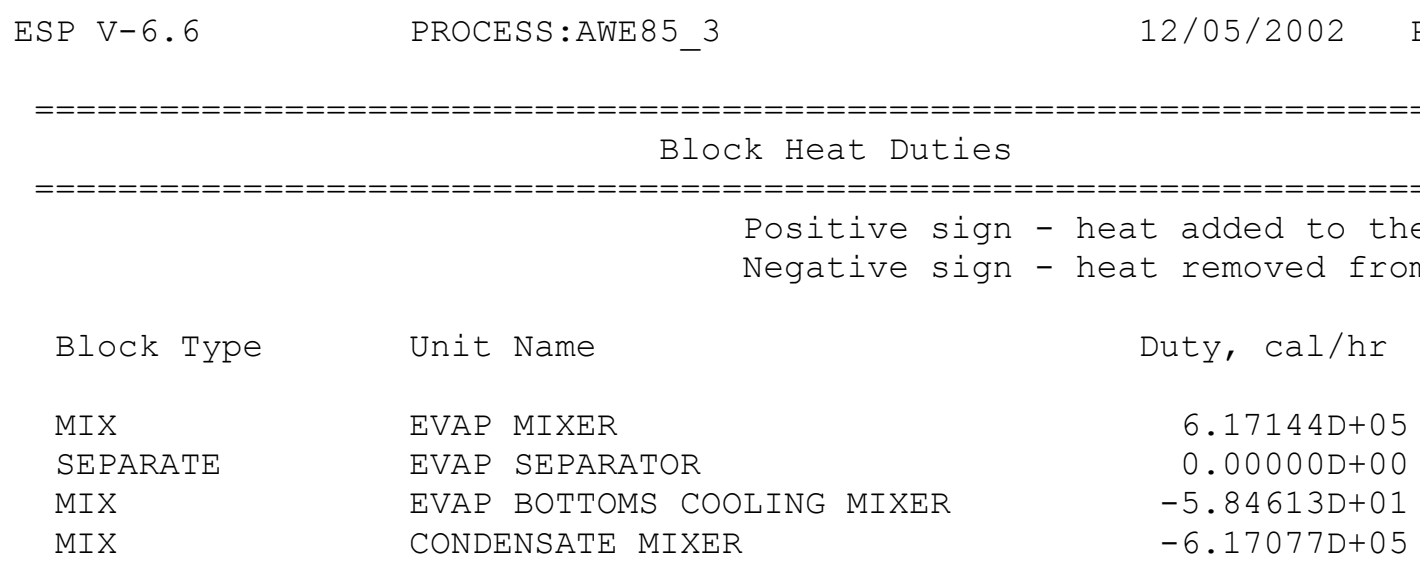


WSRC-TR-2002-00567

Revision 0

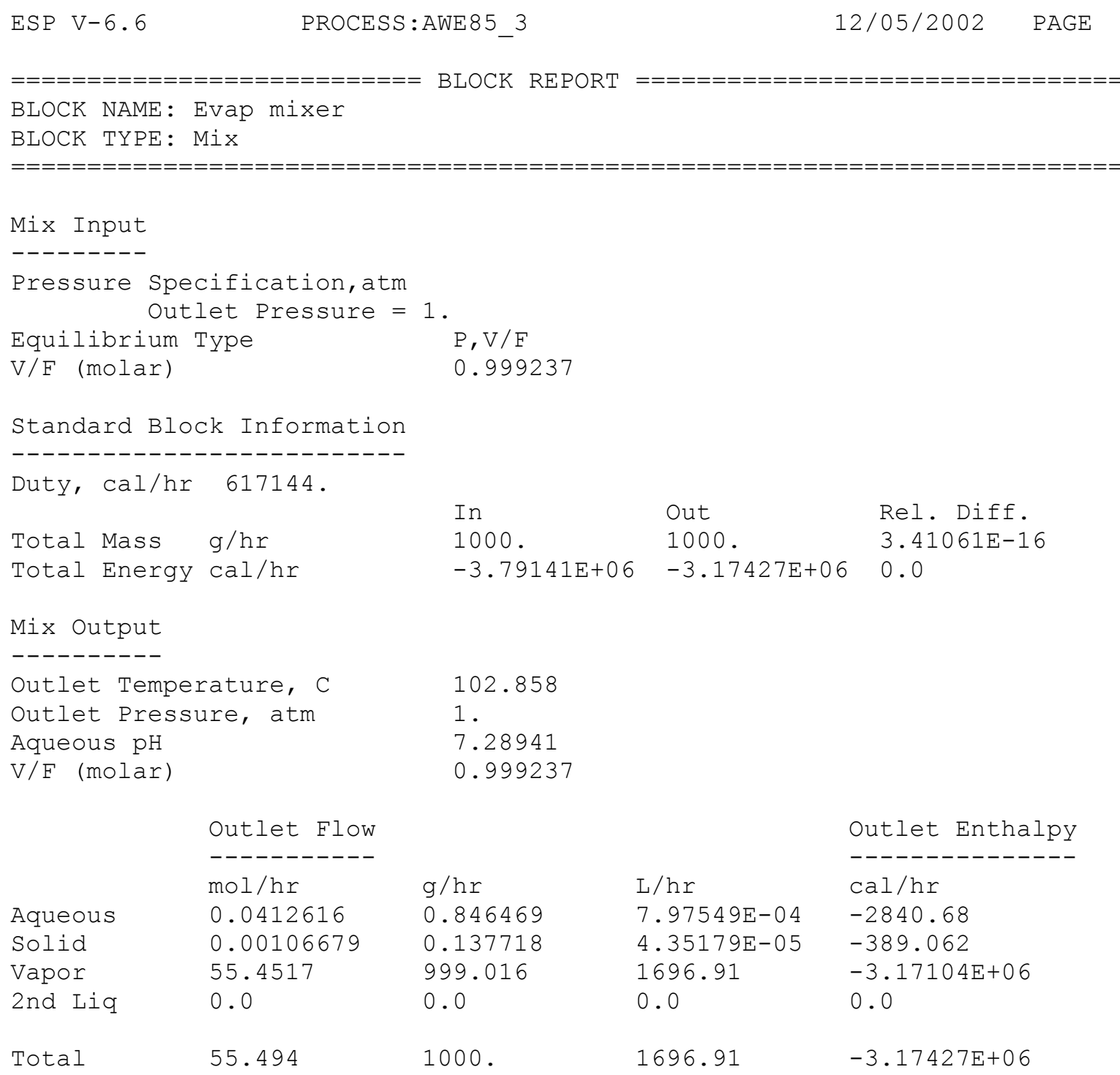


WSRC-TR-2002-00567

Revision 0

$\begin{array}{lllll}\text { ESP V-6.6 PROCESS:AWE85_3 } & 12 / 05 / 2002 & \text { PAGE } & 12\end{array}$

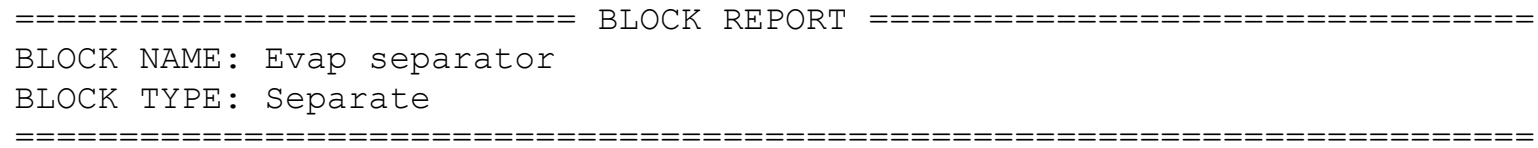

$\begin{array}{lll}\text { Total } & 0.0423284 & 0.984187\end{array}$

$\begin{array}{lll}\text { Total } & 0.0423284 & 0.984187\end{array}$

$8.41067 \mathrm{E}-04 \quad-3229.74$ 
WSRC-TR-2002-00567

Revision 0

ESP V-6.6

PROCESS:AWE 85_3

Vapor Stream

Outlet Flow
--------
mol/hr
0.0
0.0
55.4517
0.0

55.4517
Overhead

g/hr

0.0

0.0

999.016

0.0

999.016
$12 / 05 / 2002$

PAGE

13

$\mathrm{L} / \mathrm{hr}$

0.0

0.0

1696.91

0.0

1696.91

\author{
Outlet Enthalpy \\ cal/hr \\ 0.0 \\ 0.0 \\ $-3.17104 E+06$ \\ 0.0
}

$-3.17104 \mathrm{E}+06$ 
WSRC-TR-2002-00567

Revision 0

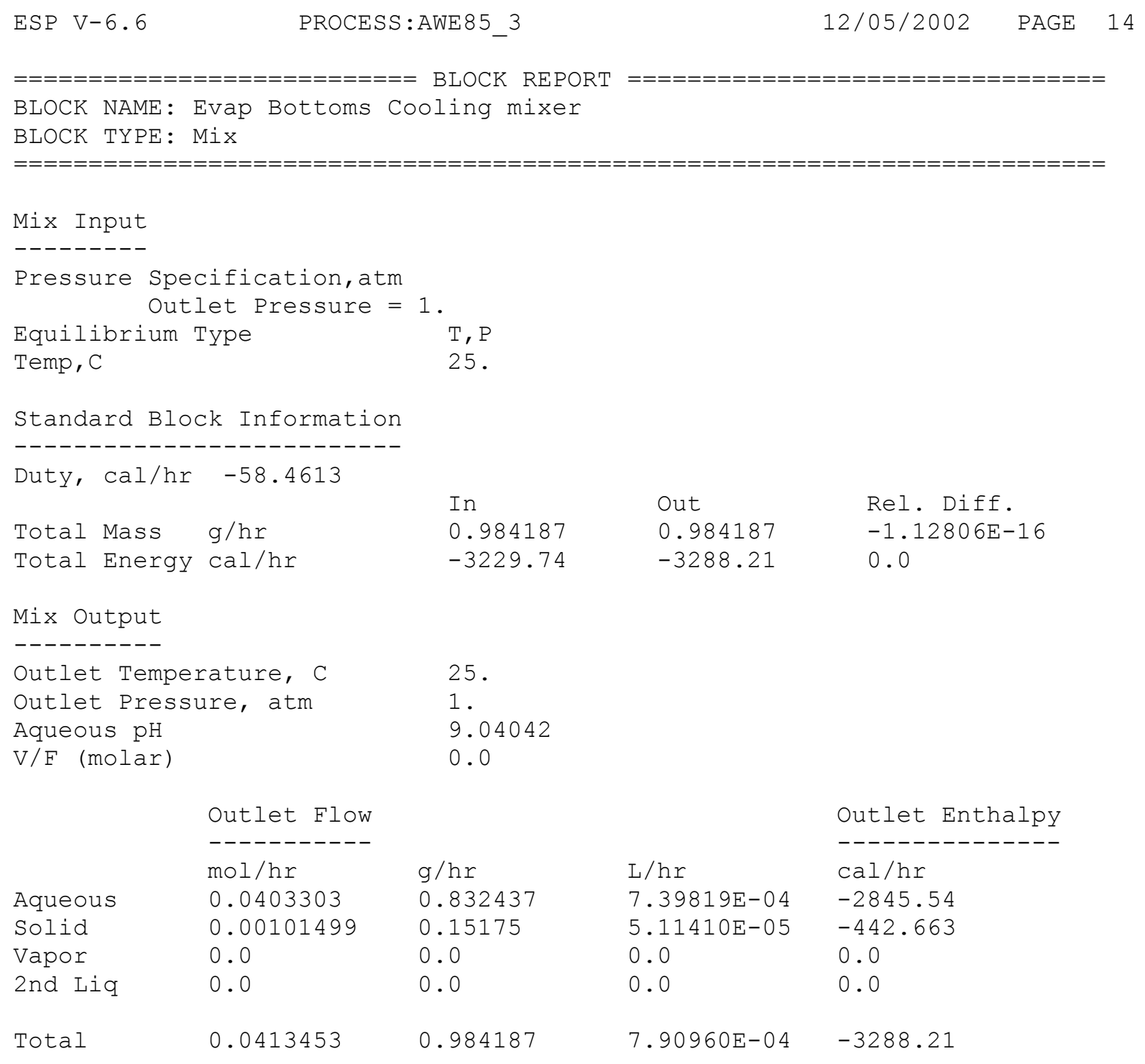


WSRC-TR-2002-00567

Revision 0

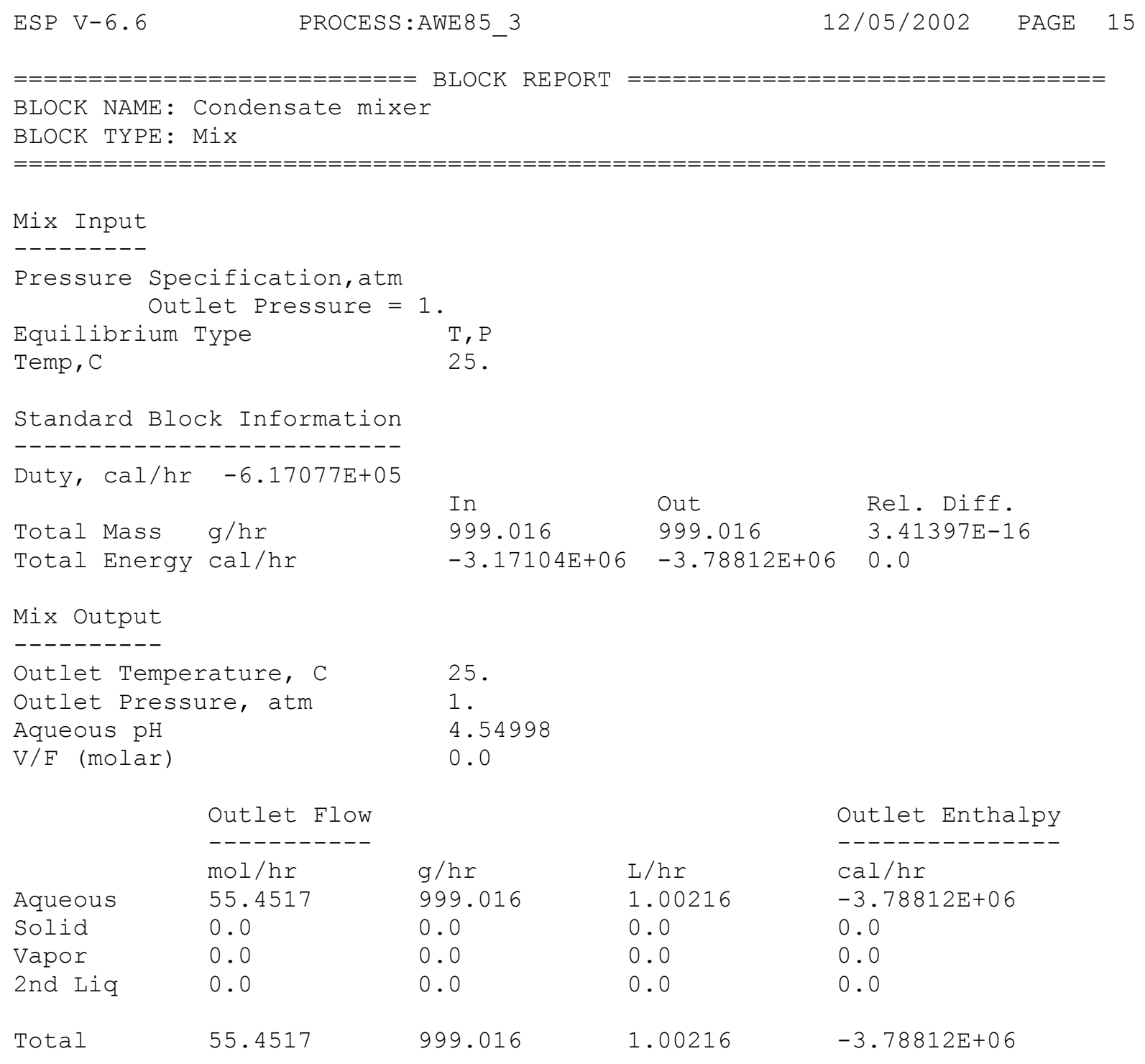


WSRC-TR-2002-00567

Revision 0

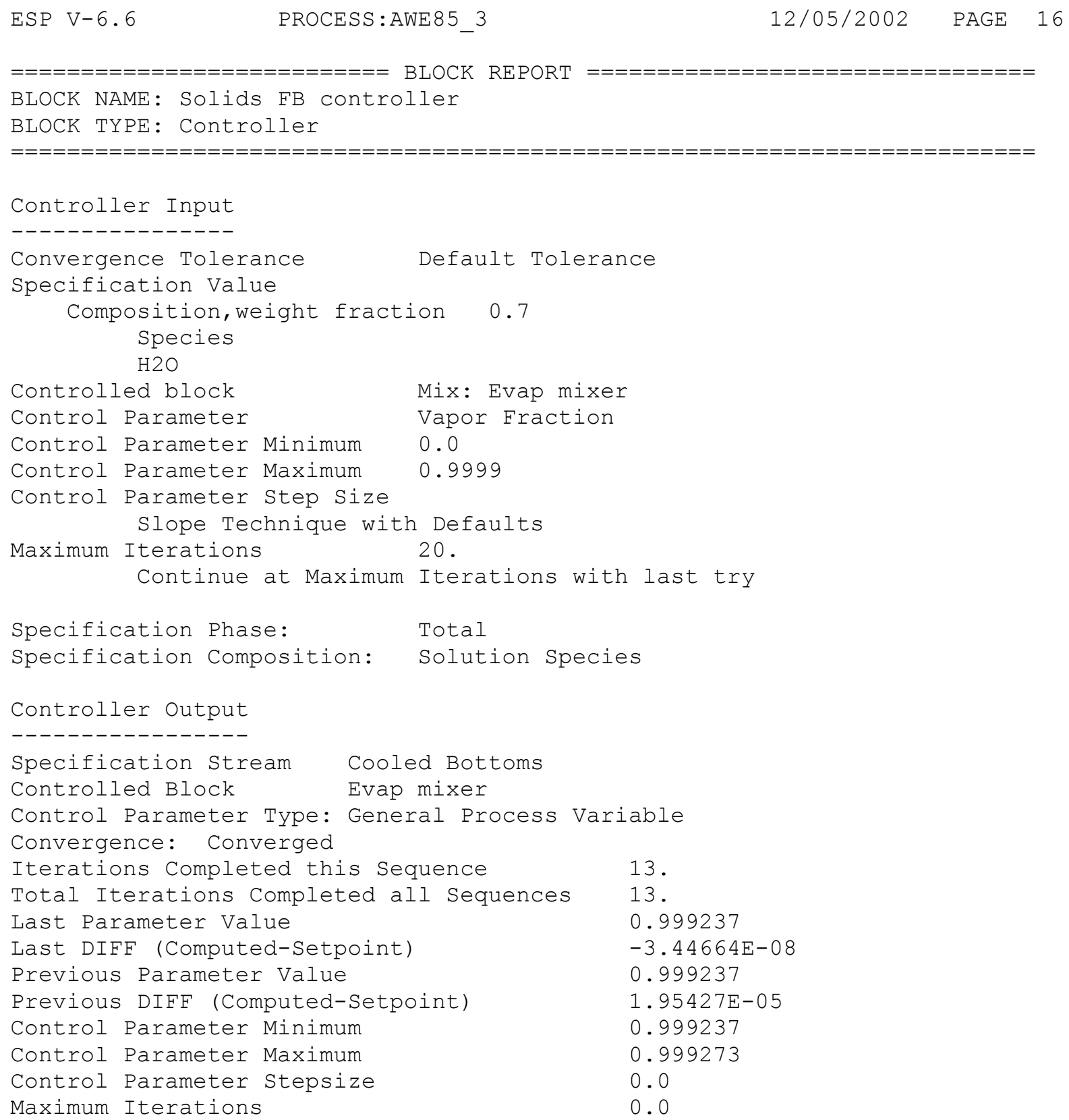


WSRC-TR-2002-00567

Revision 0

Influent Limit Composition 70\% Target $\mathrm{pH}=8.5$

$8.5-70$

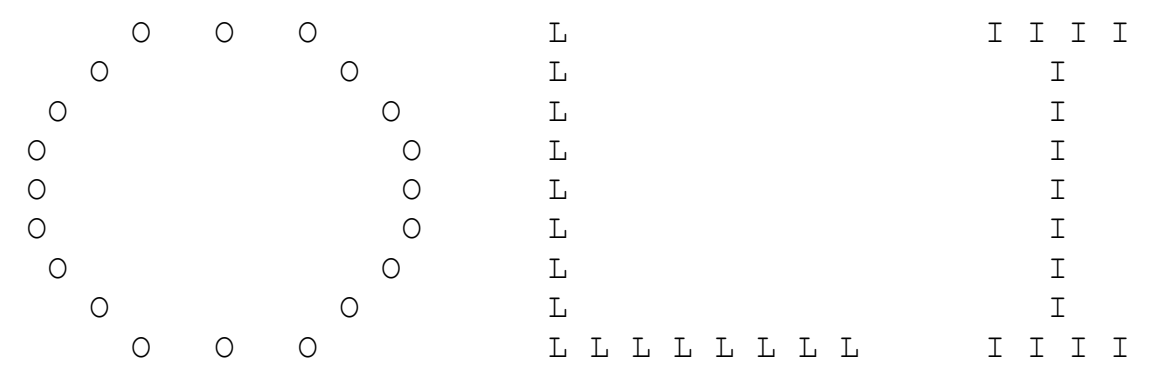

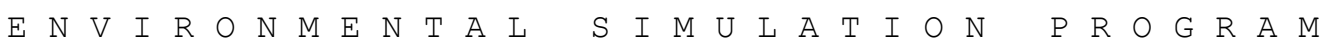

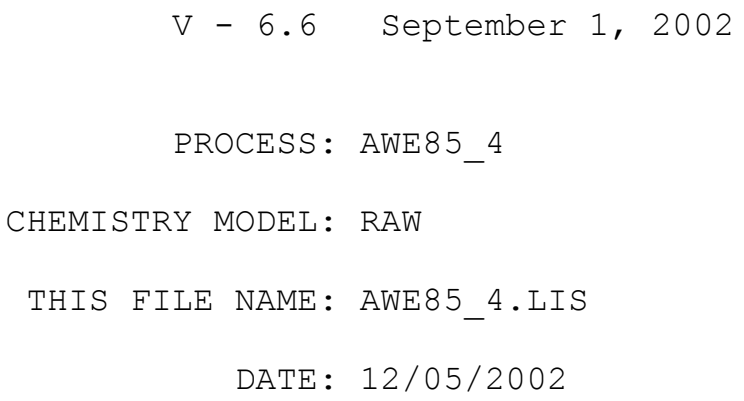




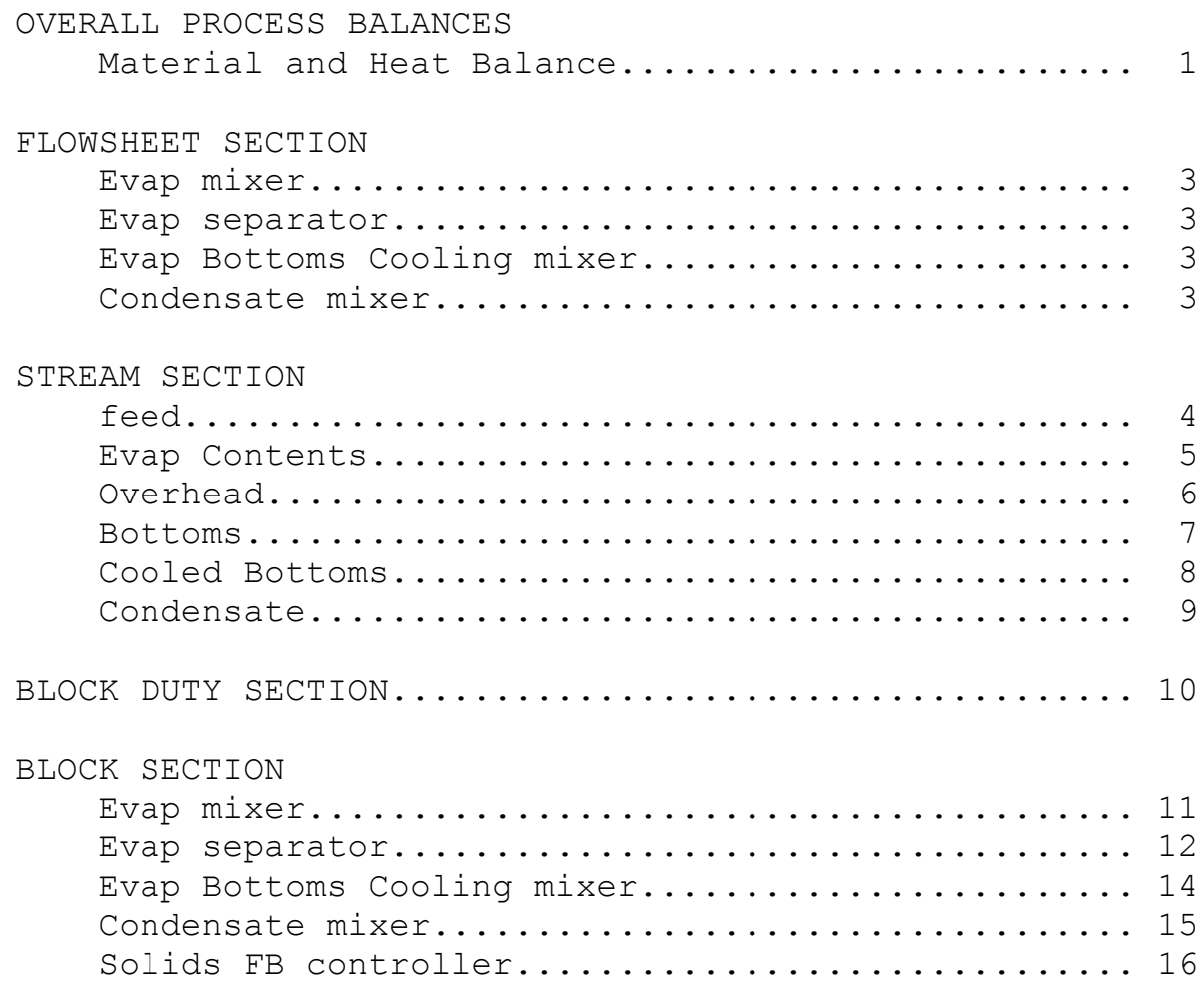


WSRC-TR-2002-00567

Revision 0

ESP V-6.6 PROCESS:AWE85_4
$\begin{array}{lcc}\text { Overall Process Balances } & \\ \text { Inlet } & \text { g/hr } & \mathrm{Cal} / \mathrm{hr} \\ ---------- & --------- & ---------- \\ \text { FEED } & 1.00000 \mathrm{D}+03 & -3.79128 \mathrm{D}+06 \\ \text { =========== } & ========= & ========= \\ \text { Total in } & 1.00000 \mathrm{D}+03 & -3.79128 \mathrm{D}+06\end{array}$

\begin{tabular}{lrr} 
Outlet & \multicolumn{1}{c}{$g / \mathrm{hr}$} & $\mathrm{cal} / \mathrm{hr}$ \\
----------- & ---------- & ----------- \\
COOLED BOTTOMS & $1.22044 \mathrm{D}+00$ & $-4.05834 \mathrm{D}+03$ \\
CONDENSATE & $9.98780 \mathrm{D}+02$ & $-3.78721 \mathrm{D}+06$ \\
$==========$ & $==========$ & $==========$ \\
Total out & $1.00000 \mathrm{D}+03$ & $-3.79127 \mathrm{D}+06$
\end{tabular}

Block Heat Duties

EVAP MIXER

EVAP BOTTOMS COOLING MIXER

CONDENSATE MIXER

$=========$

Total Duty

DIFFERENCE

REL DIFEERENCE

$2.27374 \mathrm{D}-13$

$2.27374 \mathrm{D}-16$
$12 / 05 / 2002$

PAGE
1
Material Code Balances

Code
----------
$\mathrm{H}(+1)$
$\mathrm{K}(+1)$
$\mathrm{NA}(+1)$
$\mathrm{BA}(+2)$
$\mathrm{CA}(+2)$
$\mathrm{ZN}(+2)$
$\mathrm{CU}(+2)$
$\mathrm{FE}(+2)$
$\mathrm{MG}(+2)$
$\mathrm{PB}(+2)$
$\mathrm{AL}(+3)$
$\mathrm{NI}(+2)$
$\mathrm{O}(-2)$
$\mathrm{CL}(-1)$
$\mathrm{C}(+4)$
$\mathrm{P}(+5)$
$\mathrm{S}(+6)$
$\mathrm{N}(+5)$
$\mathrm{SI}(+4)$
$\mathrm{SR}(+2)$

$$
\begin{gathered}
\text { cal/hr } \\
---------- \\
6.17162 \mathrm{D}+05 \\
-7.27961 \mathrm{D}+01 \\
-6.17081 \mathrm{D}+05 \\
========== \\
8.69082 \mathrm{D}+00
\end{gathered}
$$

$0.00000 \mathrm{D}+00$

$0.00000 \mathrm{D}+00$

Input
mol/hr
---------
$1.10972 \mathrm{D}+02$
$3.51739 \mathrm{D}-04$
$2.39187 \mathrm{D}-03$
$5.83942 \mathrm{D}-07$
$8.72818 \mathrm{D}-04$
$2.90520 \mathrm{D}-05$
$5.19685 \mathrm{D}-06$
$8.60215 \mathrm{D}-05$
$3.29218 \mathrm{D}-04$
$2.99517 \mathrm{D}-06$
$8.88889 \mathrm{D}-05$
$1.29472 \mathrm{D}-06$
$5.54945 \mathrm{D}+01$
$2.14085 \mathrm{D}-03$
$1.20000 \mathrm{D}-03$
$2.10526 \mathrm{D}-04$
$7.39583 \mathrm{D}-04$
$4.67742 \mathrm{D}-04$
$2.00000 \mathrm{D}-04$
$1.71233 \mathrm{D}-05$

Outlet mol/hr

1. $10972 \mathrm{D}+02$

3. 51739D-04

2.39187D-03

$5.83942 \mathrm{D}-07$

8. 72818D-04

2. $90520 \mathrm{D}-05$

5.19685D-06

8. $60215 \mathrm{D}-05$

3.29218D-04

2. $99517 \mathrm{D}-06$

8. 88889D-05

1. 29472D-0 6

$5.54945 \mathrm{D}+01$

2.14085D-03

1. $20000 \mathrm{D}-03$

$2.10526 \mathrm{D}-04$

7. 39583D-04

4. $67742 \mathrm{D}-04$

2.00000D-04

1. 71233D-05

\section{2 of 447}

\begin{tabular}{rr} 
Difference & \multicolumn{1}{l}{ Rel Diff } \\
mol/hr & \\
---------- & ---------- \\
$1.42109 \mathrm{D}-14$ & $1.28058 \mathrm{D}-16$ \\
$-1.62630 \mathrm{D}-18$ & $-4.62361 \mathrm{D}-15$ \\
$1.30104 \mathrm{D}-18$ & $5.43944 \mathrm{D}-16$ \\
$1.05879 \mathrm{D}-21$ & $1.81318 \mathrm{D}-15$ \\
$1.08420 \mathrm{D}-19$ & $1.24219 \mathrm{D}-16$ \\
$1.01644 \mathrm{D}-20$ & $3.49869 \mathrm{D}-16$ \\
$8.47033 \mathrm{D}-22$ & $1.62990 \mathrm{D}-16$ \\
$0.00000 \mathrm{D}+00$ & $0.00000 \mathrm{D}+00$ \\
$-5.42101 \mathrm{D}-20$ & $-1.64663 \mathrm{D}-16$ \\
$-8.47033 \mathrm{D}-22$ & $-2.82800 \mathrm{D}-16$ \\
$0.00000 \mathrm{D}+00$ & $0.00000 \mathrm{D}+00$ \\
$2.11758 \mathrm{D}-22$ & $1.63555 \mathrm{D}-16$ \\
$7.10543 \mathrm{D}-15$ & $1.28038 \mathrm{D}-16$ \\
$8.67362 \mathrm{D}-19$ & $4.05149 \mathrm{D}-16$ \\
$1.08420 \mathrm{D}-18$ & $9.03502 \mathrm{D}-16$ \\
$0.00000 \mathrm{D}+00$ & $0.00000 \mathrm{D}+00$ \\
$0.00000 \mathrm{D}+00$ & $0.00000 \mathrm{D}+00$ \\
$1.08420 \mathrm{D}-19$ & $2.31795 \mathrm{D}-16$ \\
$0.00000 \mathrm{D}+00$ & $0.00000 \mathrm{D}+00$ \\
$3.38813 \mathrm{D}-21$ & $1.97867 \mathrm{D}-16$
\end{tabular}


WSRC-TR-2002-00567

Revision 0

$\mathrm{CD}(+2)$

$\mathrm{CR}(+3)$

$\mathrm{U}(+4)$
$3.57143 \mathrm{D}-08$

$7.88462 \mathrm{D}-08$

$2.89916 \mathrm{D}-06$

$3.57143 \mathrm{D}-08$

$7.88462 \mathrm{D}-08-1.32349 \mathrm{D}-23-1.67857 \mathrm{D}-16$

$2.89916 \mathrm{D}-06 \quad 0.00000 \mathrm{D}+00 \quad 0.00000 \mathrm{D}+00$ 
WSRC-TR-2002-00567

Revision 0

$\begin{array}{crccc}\text { ESP V-6.6 } & \text { PROCESS:AWE85_4 } & 12 / 05 / 2002 & \text { PAGE } & 2 \\ \text { DODEC }(-1) & 4.16682 \mathrm{D}-05 & 4.16682 \mathrm{D}-05 & -8.80914 \mathrm{D}-20 & -2.11411 \mathrm{D}-15\end{array}$


WSRC-TR-2002-00567

Revision 0

\begin{tabular}{|c|c|c|c|}
\hline \multicolumn{4}{|l|}{$\begin{array}{l}\text { PROCESS BLOCKS } \\
===========\end{array}$} \\
\hline $\begin{array}{l}\mathrm{BLOCK} \mathrm{NAME} \\
=========================\end{array}$ & $\begin{array}{l}\text { BLOCK TYPE } \\
===========\end{array}$ & $\begin{array}{l}\text { INLET STREAM }(\mathrm{S}) \\
==============\end{array}$ & $\begin{array}{l}\text { OUTLET STREAM }(\mathrm{S}) \\
==============\end{array}$ \\
\hline Evap mixer & $\operatorname{Mix}$ & feed & Evap Contents \\
\hline Evap separator & Separate & Evap Contents & $\begin{array}{l}\text { Overhead } \\
\text { Bottoms }\end{array}$ \\
\hline Evap Bottoms Cooling mixer & Mix & Bottoms & Cooled Bottoms \\
\hline Condensate mixer & Mix & Overhead & Condensate \\
\hline
\end{tabular}


WSRC-TR-2002-00567

Revision 0

ESP V-6. 6
PROCESS:AWE85 4
12/05/2002 PAGE 4

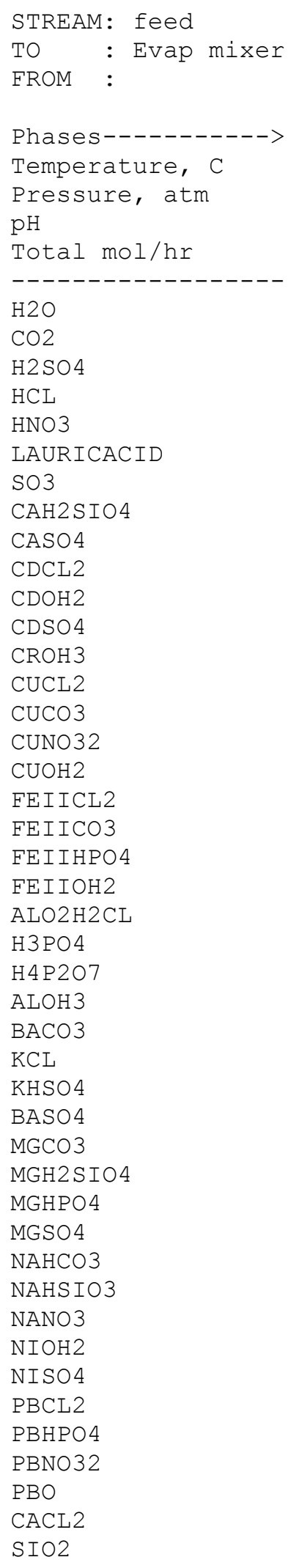

\begin{tabular}{|c|c|c|c|}
\hline Aqueous & Solid & Vapor & Organic \\
\hline 25 . & 25 . & 25 . & 25 . \\
\hline 1. & 1. & 1 . & 1 . \\
\hline \multicolumn{4}{|l|}{6.94841} \\
\hline 55.49421 & $9.47509 \mathrm{E}-05$ & 0.0 & 0.0 \\
\hline \multicolumn{4}{|c|}{$\mathrm{mol} / \mathrm{hr}--------\operatorname{mol} / \mathrm{hr}--------\mathrm{mol} / \mathrm{hr}--------\operatorname{mol} / \mathrm{hr}--------$} \\
\hline 55.4852 & 0.0 & 0.0 & 0.0 \\
\hline $2.18015 \mathrm{E}-04$ & 0.0 & 0.0 & 0.0 \\
\hline $2.29771 E-26$ & 0.0 & 0.0 & 0.0 \\
\hline $1.30470 \mathrm{E}-16$ & 0.0 & 0.0 & 0.0 \\
\hline $2.38930 \mathrm{E}-12$ & 0.0 & 0.0 & 0.0 \\
\hline $3.47879 E-07$ & 0.0 & 0.0 & 0.0 \\
\hline $2.97425 E-30$ & 0.0 & 0.0 & 0.0 \\
\hline $5.90826 \mathrm{E}-12$ & 0.0 & 0.0 & 0.0 \\
\hline $1.09798 E-05$ & 0.0 & 0.0 & 0.0 \\
\hline $1.44769 \mathrm{E}-10$ & 0.0 & 0.0 & 0.0 \\
\hline $1.03450 \mathrm{E}-14$ & 0.0 & 0.0 & 0.0 \\
\hline $3.52092 E-09$ & 0.0 & 0.0 & 0.0 \\
\hline $3.07479 \mathrm{E}-12$ & 0.0 & 0.0 & 0.0 \\
\hline $6.72897 E-14$ & 0.0 & 0.0 & 0.0 \\
\hline $1.45432 E-08$ & 0.0 & 0.0 & 0.0 \\
\hline $6.78273 E-16$ & 0.0 & 0.0 & 0.0 \\
\hline $4.80736 E-09$ & 0.0 & 0.0 & 0.0 \\
\hline $1.11350 \mathrm{E}-15$ & 0.0 & 0.0 & 0.0 \\
\hline $2.76973 E-06$ & 0.0 & 0.0 & 0.0 \\
\hline $8.02933 E-08$ & 0.0 & 0.0 & 0.0 \\
\hline $1.14595 \mathrm{E}-11$ & 0.0 & 0.0 & 0.0 \\
\hline $2.52404 E-29$ & 0.0 & 0.0 & 0.0 \\
\hline $1.54901 E-09$ & 0.0 & 0.0 & 0.0 \\
\hline $4.26255 E-21$ & 0.0 & 0.0 & 0.0 \\
\hline $2.40087 E-09$ & $8.88806 \mathrm{E}-05$ & 0.0 & 0.0 \\
\hline $3.55140 E-11$ & 0.0 & 0.0 & 0.0 \\
\hline $5.19206 \mathrm{E}-09$ & 0.0 & 0.0 & 0.0 \\
\hline $3.23011 E-14$ & 0.0 & 0.0 & 0.0 \\
\hline $2.05765 \mathrm{E}-10$ & $2.54002 \mathrm{E}-07$ & 0.0 & 0.0 \\
\hline $7.32889 E-08$ & 0.0 & 0.0 & 0.0 \\
\hline $2.02205 E-11$ & 0.0 & 0.0 & 0.0 \\
\hline $1.35217 E-05$ & 0.0 & 0.0 & 0.0 \\
\hline $6.77584 \mathrm{E}-06$ & 0.0 & 0.0 & 0.0 \\
\hline $7.99994 E-07$ & 0.0 & 0.0 & 0.0 \\
\hline $6.72004 \mathrm{E}-08$ & 0.0 & 0.0 & 0.0 \\
\hline $5.32522 E-08$ & 0.0 & 0.0 & 0.0 \\
\hline $6.75853 E-12$ & 0.0 & 0.0 & 0.0 \\
\hline $8.48219 E-08$ & 0.0 & 0.0 & 0.0 \\
\hline $1.36556 \mathrm{E}-12$ & 0.0 & 0.0 & 0.0 \\
\hline $3.18446 \mathrm{E}-10$ & 0.0 & 0.0 & 0.0 \\
\hline $1.25651 \mathrm{E}-14$ & 0.0 & 0.0 & 0.0 \\
\hline $2.86825 E-12$ & 0.0 & 0.0 & 0.0 \\
\hline $2.11194 \mathrm{E}-27$ & 0.0 & 0.0 & 0.0 \\
\hline $1.99649 E-04$ & 0.0 & 0.0 & 0.0 \\
\hline
\end{tabular}




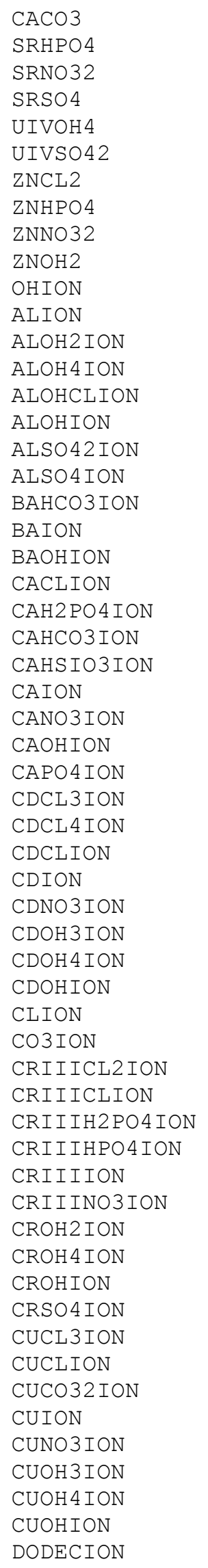

$\begin{array}{ll}4.58716 \mathrm{E}-07 & 0.0 \\ 1.09778 \mathrm{E}-08 & 0.0 \\ 1.68083 \mathrm{E}-11 & 0.0 \\ 1.08221 \mathrm{E}-06 & 0.0 \\ 3.02113 \mathrm{E}-10 & 0.0 \\ 9.31509 \mathrm{E}-30 & 0.0 \\ 1.24323 \mathrm{E}-10 & 0.0 \\ 1.85449 \mathrm{E}-06 & 0.0 \\ 2.20488 \mathrm{E}-12 & 0.0 \\ 7.18328 \mathrm{E}-09 & 0.0 \\ 9.85742 \mathrm{E}-08 & 0.0 \\ 3.08616 \mathrm{E}-14 & 0.0 \\ 5.42222 \mathrm{E}-11 & 0.0 \\ 5.85764 \mathrm{E}-09 & 0.0 \\ 8.54540 \mathrm{E}-15 & 0.0 \\ 1.74503 \mathrm{E}-12 & 0.0 \\ 2.90565 \mathrm{E}-16 & 0.0 \\ 7.53605 \mathrm{E}-15 & 0.0 \\ 2.20612 \mathrm{E}-09 & 0.0 \\ 3.27492 \mathrm{E}-07 & 0.0 \\ 1.24228 \mathrm{E}-14 & 0.0 \\ 3.02263 \mathrm{E}-11 & 0.0 \\ 2.32970 \mathrm{E}-06 & 0.0 \\ 6.36165 \mathrm{E}-06 & 0.0 \\ 3.00168 \mathrm{E}-09 & 0.0 \\ 8.51396 \mathrm{E}-04 & 0.0 \\ 7.26291 \mathrm{E}-07 & 0.0 \\ 9.91795 \mathrm{E}-10 & 0.0 \\ 5.61900 \mathrm{E}-07 & 0.0 \\ 1.48625 \mathrm{E}-14 & 0.0 \\ 2.09163 \mathrm{E}-17 & 0.0 \\ 3.80342 \mathrm{E}-09 & 0.0 \\ 2.82049 \mathrm{E}-08 & 0.0 \\ 2.44648 \mathrm{E}-11 & 0.0 \\ 1.40511 \mathrm{E}-20 & 0.0 \\ 7.95415 \mathrm{E}-28 & 0.0 \\ 1.58246 \mathrm{E}-11 & 0.0 \\ 0.00214077 & 0.0 \\ 5.30379 \mathrm{E}-07 & 0.0 \\ 1.42177 \mathrm{E}-20 & 0.0 \\ 5.11348 \mathrm{E}-17 & 0.0 \\ 2.61492 \mathrm{E}-14 & 0.0 \\ 7.88281 \mathrm{E}-08 & 0.0 \\ 1.04007 \mathrm{E}-14 & 0.0 \\ 6.82852 \mathrm{E}-16 & 0.0 \\ 1.11747 \mathrm{E}-13 & 0.0 \\ 3.45310 \mathrm{E}-16 & 0.0 \\ 6.17887 \mathrm{E}-12 & 0.0 \\ 8.62864 \mathrm{E}-12 & 0.0 \\ 2.99361 \mathrm{E}-19 & 0.0 \\ 8.26958 \mathrm{E}-11 & 0.0 \\ 1.15557 \mathrm{E}-11 & 0.0 \\ 1.02671 \mathrm{E}-08 & 0.0 \\ 1.21089 \mathrm{E}-11 & 0.0 \\ 2.52509 \mathrm{E}-14 & 0.0 \\ 2.19921 \mathrm{E}-20 & 0.0 \\ 3.25757 \mathrm{E}-09 & 0.0 \\ 4.13204 \mathrm{E}-05 & 0.0\end{array}$

\begin{tabular}{|c|c|}
\hline 0.0 & 0.0 \\
\hline 0.0 & 0.0 \\
\hline 0.0 & 0.0 \\
\hline 0.0 & 0.0 \\
\hline 0.0 & 0.0 \\
\hline 0.0 & 0.0 \\
\hline 0.0 & 0.0 \\
\hline 0.0 & 0.0 \\
\hline 0.0 & 0.0 \\
\hline 0.0 & 0.0 \\
\hline 0.0 & 0.0 \\
\hline 0.0 & 0.0 \\
\hline 0.0 & 0.0 \\
\hline 0.0 & 0.0 \\
\hline 0.0 & 0.0 \\
\hline 0.0 & 0.0 \\
\hline 0.0 & 0.0 \\
\hline 0.0 & 0.0 \\
\hline 0.0 & 0.0 \\
\hline 0.0 & 0.0 \\
\hline 0.0 & 0.0 \\
\hline 0.0 & 0.0 \\
\hline 0.0 & 0.0 \\
\hline 0.0 & 0.0 \\
\hline 0.0 & 0.0 \\
\hline 0.0 & 0.0 \\
\hline 0.0 & 0.0 \\
\hline 0.0 & 0.0 \\
\hline 0.0 & 0.0 \\
\hline 0.0 & 0.0 \\
\hline 0.0 & 0.0 \\
\hline 0.0 & 0.0 \\
\hline 0.0 & 0.0 \\
\hline 0.0 & 0.0 \\
\hline 0.0 & 0.0 \\
\hline 0.0 & 0.0 \\
\hline 0.0 & 0.0 \\
\hline 0.0 & 0.0 \\
\hline 0.0 & 0.0 \\
\hline 0.0 & 0.0 \\
\hline 0.0 & 0.0 \\
\hline 0.0 & 0.0 \\
\hline 0.0 & 0.0 \\
\hline 0.0 & 0.0 \\
\hline 0.0 & 0.0 \\
\hline 0.0 & 0.0 \\
\hline 0.0 & 0.0 \\
\hline 0.0 & 0.0 \\
\hline 0.0 & 0.0 \\
\hline 0.0 & 0.0 \\
\hline 0.0 & 0.0 \\
\hline 0.0 & 0.0 \\
\hline 0.0 & 0.0 \\
\hline 0.0 & 0.0 \\
\hline 0.0 & 0.0 \\
\hline 0.0 & 0.0 \\
\hline 0.0 & 0.0 \\
\hline 0.0 & 0.0 \\
\hline
\end{tabular}




\begin{tabular}{|c|c|c|c|c|}
\hline FEIICLION & 1.69193E-10 & 0.0 & 0.0 & 0.0 \\
\hline FEIICO32ION & $4.80262 \mathrm{E}-11$ & 0.0 & 0.0 & 0.0 \\
\hline FEIIH2PO4ION & $2.00887 \mathrm{E}-08$ & 0.0 & 0.0 & 0.0 \\
\hline FEIIHCO3ION & $8.12185 \mathrm{E}-09$ & 0.0 & 0.0 & 0.0 \\
\hline FEIIION & $8.29660 \mathrm{E}-05$ & 0.0 & 0.0 & 0.0 \\
\hline FEIIOH3ION & $4.40781 \mathrm{E}-15$ & 0.0 & 0.0 & 0.0 \\
\hline FEIIOH 4 ION & $5.28926 \mathrm{E}-23$ & 0.0 & 0.0 & 0.0 \\
\hline FEIIOHION & $1.77103 \mathrm{E}-07$ & 0.0 & 0.0 & 0.0 \\
\hline H2 P2O 7 ION & $6.26280 \mathrm{E}-11$ & 0.0 & 0.0 & 0.0 \\
\hline H2 PO 4 ION & $1.06986 \mathrm{E}-04$ & 0.0 & 0.0 & 0.0 \\
\hline H2SIO4ION & $2.57882 \mathrm{E}-13$ & 0.0 & 0.0 & 0.0 \\
\hline H3P2O 7 ION & $1.18264 \mathrm{E}-15$ & 0.0 & 0.0 & 0.0 \\
\hline H3SIO4 ION & $2.79016 \mathrm{E}-07$ & 0.0 & 0.0 & 0.0 \\
\hline HCO3ION & $9.62945 \mathrm{E}-04$ & 0.0 & 0.0 & 0.0 \\
\hline HION & $1.23206 \mathrm{E}-07$ & 0.0 & 0.0 & 0.0 \\
\hline HP207ION & $2.19892 \mathrm{E}-10$ & 0.0 & 0.0 & 0.0 \\
\hline HPBO2ION & $3.12713 \mathrm{E}-16$ & 0.0 & 0.0 & 0.0 \\
\hline HPO4 ION & $7.82197 \mathrm{E}-05$ & 0.0 & 0.0 & 0.0 \\
\hline HSO 4 ION & $5.74527 \mathrm{E}-09$ & 0.0 & 0.0 & 0.0 \\
\hline KION & $3.50433 E-04$ & 0.0 & 0.0 & 0.0 \\
\hline KSO4 ION & $1.30090 \mathrm{E}-06$ & 0.0 & 0.0 & 0.0 \\
\hline MGH2 PO 4 ION & $1.06006 \mathrm{E}-06$ & 0.0 & 0.0 & 0.0 \\
\hline MGHCO3ION & $7.63295 \mathrm{E}-06$ & 0.0 & 0.0 & 0.0 \\
\hline MGHSIO3ION & $1.96366 \mathrm{E}-09$ & 0.0 & 0.0 & 0.0 \\
\hline MGION & $2.99863 \mathrm{E}-04$ & 0.0 & 0.0 & 0.0 \\
\hline MGOHION & $3.36610 \mathrm{E}-09$ & 0.0 & 0.0 & 0.0 \\
\hline MGP2O 7 ION & $2.60073 \mathrm{E}-09$ & 0.0 & 0.0 & 0.0 \\
\hline MGPO 4 ION & $2.83445 E-07$ & 0.0 & 0.0 & 0.0 \\
\hline NACO3ION & $3.08597 \mathrm{E}-09$ & 0.0 & 0.0 & 0.0 \\
\hline NAION & 0.00238121 & 0.0 & 0.0 & 0.0 \\
\hline NASO 4 ION & $9.73707 \mathrm{E}-06$ & 0.0 & 0.0 & 0.0 \\
\hline NICLION & $1.79373 \mathrm{E}-10$ & 0.0 & 0.0 & 0.0 \\
\hline NIION & $1.20744 \mathrm{E}-06$ & 0.0 & 0.0 & 0.0 \\
\hline NINO3ION & $1.12999 \mathrm{E}-09$ & 0.0 & 0.0 & 0.0 \\
\hline NIOH3ION & $6.73633 \mathrm{E}-16$ & 0.0 & 0.0 & 0.0 \\
\hline NIOHION & $1.13774 \mathrm{E}-09$ & 0.0 & 0.0 & 0.0 \\
\hline NO3ION & $4.66907 \mathrm{E}-04$ & 0.0 & 0.0 & 0.0 \\
\hline P2O7ION & $1.86106 \mathrm{E}-12$ & 0.0 & 0.0 & 0.0 \\
\hline PBCL3ION & $1.42994 \mathrm{E}-15$ & 0.0 & 0.0 & 0.0 \\
\hline PBCL 4 ION & $2.30852 \mathrm{E}-18$ & 0.0 & 0.0 & 0.0 \\
\hline PBCLION & $2.11054 \mathrm{E}-10$ & 0.0 & 0.0 & 0.0 \\
\hline PBH2 PO 4 ION & $1.58779 \mathrm{E}-11$ & 0.0 & 0.0 & 0.0 \\
\hline PBION & $5.17468 \mathrm{E}-09$ & 0.0 & 0.0 & 0.0 \\
\hline PBNO33ION & $1.00329 \mathrm{E}-18$ & 0.0 & 0.0 & 0.0 \\
\hline PBNO3ION & $3.32377 \mathrm{E}-11$ & 0.0 & 0.0 & 0.0 \\
\hline PBOHION & $8.92262 \mathrm{E}-10$ & 0.0 & 0.0 & 0.0 \\
\hline PO4ION & $5.23834 \mathrm{E}-10$ & 0.0 & 0.0 & 0.0 \\
\hline SO4ION & $7.09359 \mathrm{E}-04$ & 0.0 & 0.0 & 0.0 \\
\hline SRION & $1.60007 \mathrm{E}-05$ & 0.0 & 0.0 & 0.0 \\
\hline SRNO3ION & $2.92379 \mathrm{E}-08$ & 0.0 & 0.0 & 0.0 \\
\hline SROHION & $6.97366 \mathrm{E}-12$ & 0.0 & 0.0 & 0.0 \\
\hline SRPO 4 ION & $1.46421 \mathrm{E}-10$ & 0.0 & 0.0 & 0.0 \\
\hline UIVOH2ION & $6.67700 \mathrm{E}-22$ & 0.0 & 0.0 & 0.0 \\
\hline UIVOH3ION & $4.19354 \mathrm{E}-16$ & 0.0 & 0.0 & 0.0 \\
\hline UIVOH5ION & $1.53363 \mathrm{E}-13$ & 0.0 & 0.0 & 0.0 \\
\hline UIVOHION & $1.76997 \mathrm{E}-26$ & 0.0 & 0.0 & 0.0 \\
\hline UIVSO 4 ION & $3.73898 \mathrm{E}-30$ & 0.0 & 0.0 & 0.0 \\
\hline ZNCL3ION & $9.04653 \mathrm{E}-14$ & 0.0 & 0.0 & 0.0 \\
\hline
\end{tabular}


WSRC-TR-2002-00567

Revision 0

\begin{tabular}{|c|c|c|c|c|}
\hline ZNCLION & $6.22483 E-08$ & 0.0 & 0.0 & 0.0 \\
\hline ZNH2 PO4 ION & $7.55331 \mathrm{E}-08$ & 0.0 & 0.0 & 0.0 \\
\hline ZNHCO3ION & $3.85725 E-07$ & 0.0 & 0.0 & 0.0 \\
\hline ZNION & $2.64246 \mathrm{E}-05$ & 0.0 & 0.0 & 0.0 \\
\hline ZNNO3ION & $2.47257 E-08$ & 0.0 & 0.0 & 0.0 \\
\hline ZNOH3ION & $6.89601 \mathrm{E}-13$ & 0.0 & 0.0 & 0.0 \\
\hline ZNOH 4 ION & $1.04082 \mathrm{E}-18$ & 0.0 & 0.0 & 0.0 \\
\hline ZNOHION & $2.17373 E-07$ & 0.0 & 0.0 & 0.0 \\
\hline $\mathrm{CU} 3 \mathrm{PO} 42.2 \mathrm{H} 2 \mathrm{O}$ & 0.0 & $1.72129 \mathrm{E}-06$ & 0.0 & 0.0 \\
\hline РВ3РО42 & 0.0 & $9.96173 E-07$ & 0.0 & 0.0 \\
\hline \multirow[t]{2}{*}{ UIVO2 } & 0.0 & $2.89886 \mathrm{E}-06$ & 0.0 & 0.0 \\
\hline & $===========$ & $===========$ & $===========$ & $===========$ \\
\hline Total g/hr & 999.991 & 0.0093006 & 0.0 & 0.0 \\
\hline Volume, L/hr & 1.00283 & $3.03914 \mathrm{E}-06$ & 0.0 & 0.0 \\
\hline Enthalpy, cal/hr & $-3.79125 \mathrm{E}+06$ & -28.8644 & 0.0 & 0.0 \\
\hline Density, g/L & 997.168 & 3060.28 & & \\
\hline Vapor fraction & 0.0 & 0.0 & 0.0 & 0.0 \\
\hline Solid fraction & 0.0 & 1 . & 0.0 & 0.0 \\
\hline Organic fraction & 0.0 & 0.0 & 0.0 & 0.0 \\
\hline Osmotic Pres, atm & 0.244569 & & & \\
\hline Redox Pot, volts & 0.0 & & & \\
\hline E-Con, 1/ohm-cm & $5.90442 E-04$ & & & \\
\hline E-Con, cm2/ohm-mol & 134.192 & & & \\
\hline Abs Visc, cP & 0.892421 & & & \\
\hline Rel Visc & 1.00191 & & & \\
\hline Ionic Strength & 0.00737689 & & & \\
\hline
\end{tabular}


WSRC-TR-2002-00567

Revision 0

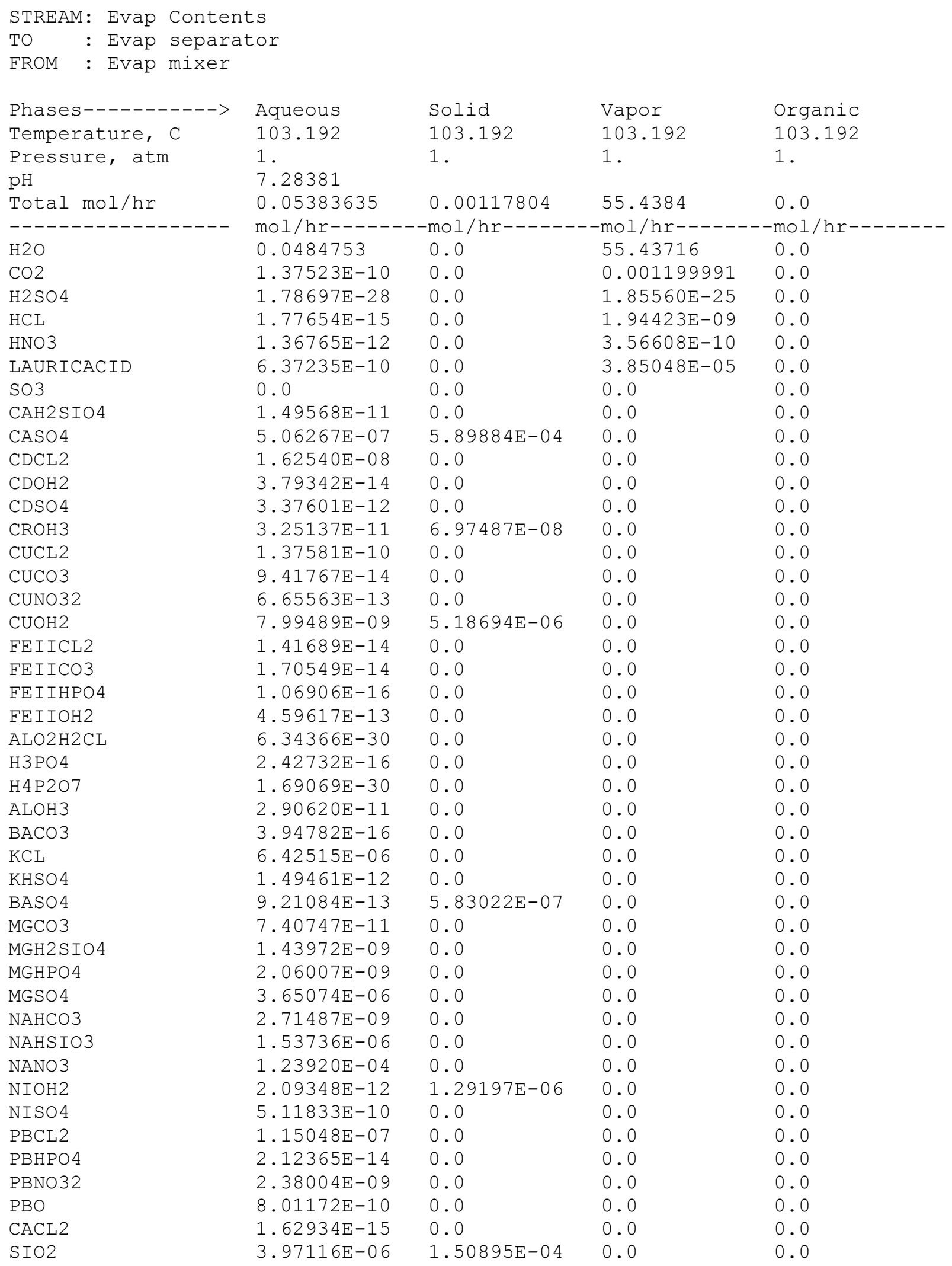




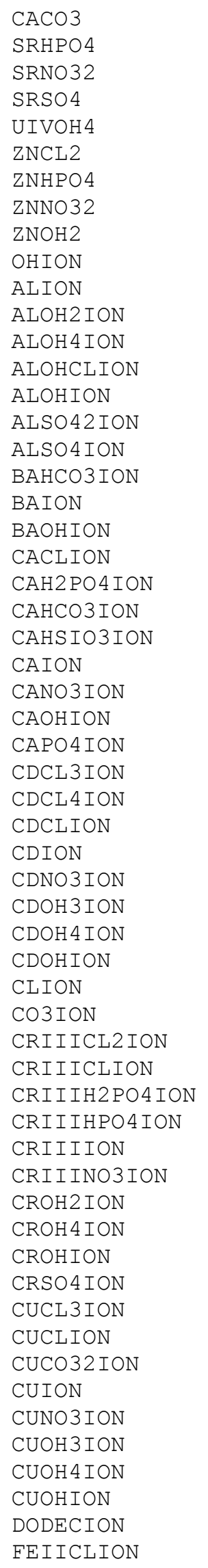

$\begin{array}{ll}2.31711 \mathrm{E}-11 & 0.0 \\ 3.67409 \mathrm{E}-14 & 0.0 \\ 2.74753 \mathrm{E}-08 & 0.0 \\ 8.68924 \mathrm{E}-09 & 1.69661 \mathrm{E}-05 \\ 1.08368 \mathrm{E}-12 & 0.0 \\ 3.94652 \mathrm{E}-06 & 0.0 \\ 2.27496 \mathrm{E}-12 & 0.0 \\ 1.17059 \mathrm{E}-09 & 0.0 \\ 3.25219 \mathrm{E}-08 & 0.0 \\ 1.52535 \mathrm{E}-08 & 0.0 \\ 1.72695 \mathrm{E}-21 & 0.0 \\ 2.05332 \mathrm{E}-14 & 0.0 \\ 5.43938 \mathrm{E}-09 & 0.0 \\ 4.81481 \mathrm{E}-17 & 0.0 \\ 1.25146 \mathrm{E}-17 & 0.0 \\ 3.47714 \mathrm{E}-21 & 0.0 \\ 8.20164 \mathrm{E}-21 & 0.0 \\ 3.58529 \mathrm{E}-14 & 0.0 \\ 9.20856 \mathrm{E}-10 & 0.0 \\ 8.22836 \mathrm{E}-15 & 0.0 \\ 4.09695 \mathrm{E}-08 & 0.0 \\ 9.60553 \mathrm{E}-12 & 0.0 \\ 6.21605 \mathrm{E}-11 & 0.0 \\ 8.21871 \mathrm{E}-09 & 0.0 \\ 9.69012 \mathrm{E}-06 & 0.0 \\ 3.67905 \mathrm{E}-06 & 0.0 \\ 1.65087 \mathrm{E}-09 & 0.0 \\ 3.12794 \mathrm{E}-11 & 0.0 \\ 1.46702 \mathrm{E}-08 & 0.0 \\ 3.84474 \mathrm{E}-09 & 0.0 \\ 9.19493 \mathrm{E}-10 & 0.0 \\ 1.77989 \mathrm{E}-11 & 0.0 \\ 4.21555 \mathrm{E}-12 & 0.0 \\ 6.15446 \mathrm{E}-18 & 0.0 \\ 5.78509 \mathrm{E}-22 & 0.0 \\ 4.25880 \mathrm{E}-13 & 0.0 \\ 0.00209468 & 0.0 \\ 4.14855 \mathrm{E}-11 & 0.0 \\ 8.04905 \mathrm{E}-16 & 0.0 \\ 1.85289 \mathrm{E}-15 & 0.0 \\ 2.09213 \mathrm{E}-20 & 0.0 \\ 8.79574 \mathrm{E}-09 & 0.0 \\ 5.11800 \mathrm{E}-19 & 0.0 \\ 2.83918 \mathrm{E}-13 & 0.0 \\ 5.81135 \mathrm{E}-13 & 0.0 \\ 8.15587 \mathrm{E}-14 & 0.0 \\ 2.60134 \mathrm{E}-10 & 0.0 \\ 8.43097 \mathrm{E}-12 & 0.0 \\ 2.13245 \mathrm{E}-12 & 0.0 \\ 8.55316 \mathrm{E}-10 & 0.0 \\ 2.40202 \mathrm{E}-17 & 0.0 \\ 2.39428 \mathrm{E}-10 & 0.0 \\ 3.54428 \mathrm{E}-11 & 0.0 \\ 8.13586 \mathrm{E}-12 & 0.0 \\ 1.82274 \mathrm{E}-14 & 0.0 \\ 6.61459 \mathrm{E}-10 & 0.0 \\ 3.16278 \mathrm{E}-06 & 0.0 \\ 2.94280 \mathrm{E}-12 & 0.0 \\ & \\ 3.0\end{array}$

0.0

0.0

0.0

0.0

0.0

0.0

0.0

0.0

0.0

0.0

0.0

0.0

0.0

0.0

0.0

0.0

0.0

0.0

0.0

0.0

0.0

0.0

0.0

0.0

0.0

0.0

0.0

0.0

0.0

0.0

0.0

0.0

0.0

0.0

0.0

0.0

0.0

0.0

0.0

0.0

0.0

0.0

0.0

0.0

0.0

0.0

0.0

0.0

0.0

0.0

0.0

0.0

0.0

0.0

0.0

0.0

0.0

0.0

0.0 


\begin{tabular}{|c|c|c|c|c|}
\hline FEIICO32ION & $1.29957 \mathrm{E}-19$ & 0.0 & 0.0 & 0.0 \\
\hline FEIIH2PO4ION & $1.21581 \mathrm{E}-17$ & 0.0 & 0.0 & 0.0 \\
\hline FEIIHCO3ION & $4.90154 \mathrm{E}-17$ & 0.0 & 0.0 & 0.0 \\
\hline FEIIION & $3.61861 \mathrm{E}-10$ & 0.0 & 0.0 & 0.0 \\
\hline FEIIOH3ION & $9.62746 \mathrm{E}-15$ & 0.0 & 0.0 & 0.0 \\
\hline FEIIOH 4 ION & $2.54081 \mathrm{E}-19$ & 0.0 & 0.0 & 0.0 \\
\hline FEIIOHION & $3.48336 \mathrm{E}-11$ & 0.0 & 0.0 & 0.0 \\
\hline H2P2O7ION & $8.46229 \mathrm{E}-19$ & 0.0 & 0.0 & 0.0 \\
\hline H2 PO 4 ION & $5.08588 \mathrm{E}-11$ & 0.0 & 0.0 & 0.0 \\
\hline H2SIO 4 ION & $7.85532 \mathrm{E}-12$ & 0.0 & 0.0 & 0.0 \\
\hline H3P2O 7 ION & $9.91442 \mathrm{E}-25$ & 0.0 & 0.0 & 0.0 \\
\hline H3SIO 4 ION & $4.74023 E-07$ & 0.0 & 0.0 & 0.0 \\
\hline HCO3ION & $1.84753 \mathrm{E}-09$ & 0.0 & 0.0 & 0.0 \\
\hline HION & $5.75886 \mathrm{E}-11$ & 0.0 & 0.0 & 0.0 \\
\hline HP2O 7 ION & $1.56469 \mathrm{E}-17$ & 0.0 & 0.0 & 0.0 \\
\hline HPBO2 ION & $7.98558 \mathrm{E}-12$ & 0.0 & 0.0 & 0.0 \\
\hline HPO4ION & $5.23140 \mathrm{E}-10$ & 0.0 & 0.0 & 0.0 \\
\hline HSO 4 ION & $2.80365 \mathrm{E}-10$ & 0.0 & 0.0 & 0.0 \\
\hline KION & $3.23039 \mathrm{E}-04$ & 0.0 & 0.0 & 0.0 \\
\hline KSO4ION & $2.22741 \mathrm{E}-05$ & 0.0 & 0.0 & 0.0 \\
\hline MGH2 PO 4 ION & $2.07577 \mathrm{E}-11$ & 0.0 & 0.0 & 0.0 \\
\hline MGHCO3ION & $8.18751 \mathrm{E}-10$ & 0.0 & 0.0 & 0.0 \\
\hline MGHS IO3ION & $1.03645 \mathrm{E}-07$ & 0.0 & 0.0 & 0.0 \\
\hline MGION & $2.68458 \mathrm{E}-05$ & 0.0 & 0.0 & 0.0 \\
\hline MGOHION & $8.79443 \mathrm{E}-08$ & 0.0 & 0.0 & 0.0 \\
\hline MGP2O7ION & $8.89815 \mathrm{E}-14$ & 0.0 & 0.0 & 0.0 \\
\hline MGPO 4 ION & $2.25681 \mathrm{E}-10$ & 0.0 & 0.0 & 0.0 \\
\hline NACO3ION & $6.66262 \mathrm{E}-12$ & 0.0 & 0.0 & 0.0 \\
\hline NAION & 0.00226641 & 0.0 & 0.0 & 0.0 \\
\hline NASO 4 ION & $2.21505 \mathrm{E}-13$ & 0.0 & 0.0 & 0.0 \\
\hline NICLION & $1.54115 \mathrm{E}-10$ & 0.0 & 0.0 & 0.0 \\
\hline NIION & $1.81544 \mathrm{E}-09$ & 0.0 & 0.0 & 0.0 \\
\hline NINO3ION & $2.26657 \mathrm{E}-10$ & 0.0 & 0.0 & 0.0 \\
\hline NIOH3ION & $5.29422 \mathrm{E}-15$ & 0.0 & 0.0 & 0.0 \\
\hline NIOHION & $5.22840 \mathrm{E}-11$ & 0.0 & 0.0 & 0.0 \\
\hline NO3ION & $3.39907 \mathrm{E}-04$ & 0.0 & 0.0 & 0.0 \\
\hline P207ION & $3.04580 \mathrm{E}-18$ & 0.0 & 0.0 & 0.0 \\
\hline PBCL3ION & $2.46053 \mathrm{E}-07$ & 0.0 & 0.0 & 0.0 \\
\hline PBCL 4 ION & $1.82272 \mathrm{E}-06$ & 0.0 & 0.0 & 0.0 \\
\hline PBCLION & $3.21393 \mathrm{E}-08$ & 0.0 & 0.0 & 0.0 \\
\hline PBH2 PO 4 ION & $1.18148 \mathrm{E}-15$ & 0.0 & 0.0 & 0.0 \\
\hline PBION & $1.18817 \mathrm{E}-09$ & 0.0 & 0.0 & 0.0 \\
\hline PBNO33ION & $7.25594 \mathrm{E}-11$ & 0.0 & 0.0 & 0.0 \\
\hline PBNO3ION & $7.92409 \mathrm{E}-09$ & 0.0 & 0.0 & 0.0 \\
\hline PBOHION & $1.95221 \mathrm{E}-08$ & 0.0 & 0.0 & 0.0 \\
\hline PO4ION & $8.04226 \mathrm{E}-14$ & 0.0 & 0.0 & 0.0 \\
\hline SO4ION & $1.05711 \mathrm{E}-04$ & 0.0 & 0.0 & 0.0 \\
\hline SRION & $3.72064 \mathrm{E}-09$ & 0.0 & 0.0 & 0.0 \\
\hline SRNO3ION & $1.17374 \mathrm{E}-07$ & 0.0 & 0.0 & 0.0 \\
\hline SROHION & $1.23354 \mathrm{E}-11$ & 0.0 & 0.0 & 0.0 \\
\hline SRPO 4 ION & $7.10798 \mathrm{E}-15$ & 0.0 & 0.0 & 0.0 \\
\hline UIVOH2ION & $5.58314 \mathrm{E}-26$ & 0.0 & 0.0 & 0.0 \\
\hline UIVOH3ION & $1.70374 \mathrm{E}-20$ & 0.0 & 0.0 & 0.0 \\
\hline UIVOH5ION & $1.03006 \mathrm{E}-14$ & 0.0 & 0.0 & 0.0 \\
\hline UIVOHION & $1.08377 \mathrm{E}-30$ & 0.0 & 0.0 & 0.0 \\
\hline ZNCL3ION & $4.88884 \mathrm{E}-06$ & 0.0 & 0.0 & 0.0 \\
\hline ZNCLION & $8.75666 \mathrm{E}-06$ & 0.0 & 0.0 & 0.0 \\
\hline ZNH2PO 4 ION & $6.15416 \mathrm{E}-14$ & 0.0 & 0.0 & 0.0 \\
\hline
\end{tabular}


WSRC-TR-2002-00567

Revision 0

\begin{tabular}{|c|c|c|c|c|}
\hline ZNHCO3ION & $2.03770 \mathrm{E}-12$ & 0.0 & 0.0 & 0.0 \\
\hline ZNION & $5.36005 \mathrm{E}-07$ & 0.0 & 0.0 & 0.0 \\
\hline ZNNO3ION & $4.81247 E-08$ & 0.0 & 0.0 & 0.0 \\
\hline ZNOH3ION & $1.29088 E-09$ & 0.0 & 0.0 & 0.0 \\
\hline ZNOH $4 \mathrm{ION}$ & $1.01438 \mathrm{E}-12$ & 0.0 & 0.0 & 0.0 \\
\hline ZNOHION & $4.21867 \mathrm{E}-06$ & 0.0 & 0.0 & 0.0 \\
\hline $\mathrm{ALOOH}$ & 0.0 & $2.86232 E-06$ & 0.0 & 0.0 \\
\hline $\mathrm{CA} 3 \mathrm{PO} 42$ & 0.0 & $8.96701 E-05$ & 0.0 & 0.0 \\
\hline CHAMOSITE7A & 0.0 & $4.30108 E-05$ & 0.0 & 0.0 \\
\hline MG3PO4 2 & 0.0 & $1.31312 \mathrm{E}-05$ & 0.0 & 0.0 \\
\hline MGOH2 & 0.0 & $2.59133 E-04$ & 0.0 & 0.0 \\
\hline PB3PO42 & 0.0 & $2.49104 \mathrm{E}-07$ & 0.0 & 0.0 \\
\hline UIVO2 & 0.0 & $2.89917 E-06$ & 0.0 & 0.0 \\
\hline $\mathrm{ZN} 3 \mathrm{PO} 42.2 \mathrm{H} 2 \mathrm{O}$ & 0.0 & $2.20740 \mathrm{E}-06$ & 0.0 & 0.0 \\
\hline & $===========$ & $===========$ & $===========$ & $===========$ \\
\hline Total g/hr & 1.06401 & 0.156425 & 998.78 & 0.0 \\
\hline Volume, L/hr & $9.93763 E-04$ & $4.75020 E-05$ & 1698.04 & 0.0 \\
\hline Enthalpy, cal/hr & -3547.01 & -438.537 & $-3.17013 E+06$ & 0.0 \\
\hline Density, g/L & 1070.69 & 3293.02 & 0.588194 & \\
\hline Vapor fraction & 0.0 & 0.0 & 1 . & 0.0 \\
\hline Solid fraction & 0.0 & 1. & 0.0 & 0.0 \\
\hline Organic fraction & 0.0 & 0.0 & 0.0 & 0.0 \\
\hline Osmotic Pres, atm & 182.086 & & & \\
\hline Redox Pot, volts & 0.0 & & & \\
\hline $\mathrm{E}-\mathrm{Con}, 1 / \mathrm{ohm}-\mathrm{cm}$ & 0.484794 & & & \\
\hline E-Con, cm2/ohm-mol & 55.4865 & & & \\
\hline Abs Visc, cP & 0.402037 & & & \\
\hline Rel Visc & 1.47583 & & & \\
\hline Ionic Strength & 3.2353 & & & \\
\hline
\end{tabular}


WSRC-TR-2002-00567

Revision 0

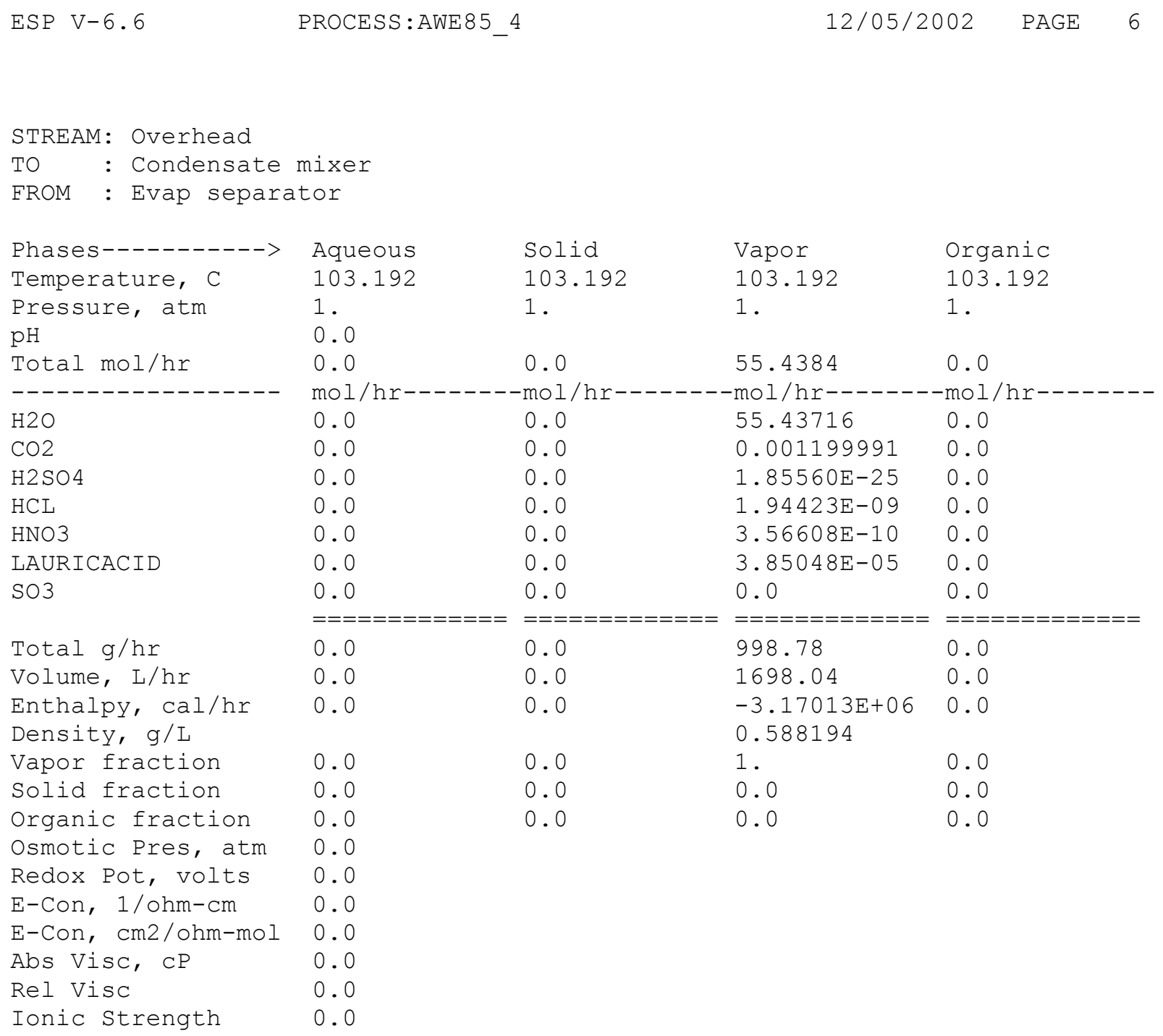


WSRC-TR-2002-00567

Revision 0

\begin{tabular}{|c|c|c|c|c|}
\hline \multicolumn{5}{|c|}{ TO : Evap Bottoms Cooling mixer } \\
\hline Phases $----------->$ & Aqueous & Solid & Vapor & Organic \\
\hline & 103.192 & 103.192 & 103.192 & 103.192 \\
\hline $\begin{array}{l}\text { Pressure, atm } \\
\mathrm{pH}\end{array}$ & $\begin{array}{l}1 . \\
7.28381\end{array}$ & 1. & 1 . & 1 . \\
\hline \multirow{2}{*}{ Total mol/hr } & 0.05383635 & 0.00117804 & 0.0 & 0.0 \\
\hline & \multicolumn{2}{|c|}{-------------------- } & $-\mathrm{mol} / \mathrm{hr}--$ & -mol/hr- \\
\hline $\mathrm{H} 2 \mathrm{O}$ & 0.0484753 & 0.0 & 0.0 & 0.0 \\
\hline $\mathrm{CO} 2$ & $1.37523 \mathrm{E}-10$ & 0.0 & 0.0 & 0.0 \\
\hline $\mathrm{H} 2 \mathrm{SO} 4$ & $1.78697 \mathrm{E}-28$ & 0.0 & 0.0 & 0.0 \\
\hline $\mathrm{HCL}$ & $1.77654 \mathrm{E}-15$ & 0.0 & 0.0 & 0.0 \\
\hline HNO3 & $1.36765 \mathrm{E}-12$ & 0.0 & 0.0 & 0.0 \\
\hline LAURICACID & $6.37235 E-10$ & 0.0 & 0.0 & 0.0 \\
\hline $\mathrm{SO} 3$ & 0.0 & 0.0 & 0.0 & 0.0 \\
\hline CAH2SIO4 & $1.49568 \mathrm{E}-11$ & 0.0 & 0.0 & 0.0 \\
\hline $\mathrm{CASO} 4$ & $5.06267 \mathrm{E}-07$ & $5.89884 \mathrm{E}-04$ & 0.0 & 0.0 \\
\hline CDCL2 & $1.62540 E-08$ & 0.0 & 0.0 & 0.0 \\
\hline $\mathrm{CDOH} 2$ & $3.79342 \mathrm{E}-14$ & 0.0 & 0.0 & 0.0 \\
\hline $\mathrm{CDSO} 4$ & $3.37601 E-12$ & 0.0 & 0.0 & 0.0 \\
\hline $\mathrm{CROH} 3$ & $3.25137 \mathrm{E}-11$ & $6.97487 E-08$ & 0.0 & 0.0 \\
\hline CUCL2 & $1.37581 \mathrm{E}-10$ & 0.0 & 0.0 & 0.0 \\
\hline CUCO3 & $9.41767 \mathrm{E}-14$ & 0.0 & 0.0 & 0.0 \\
\hline CUNO32 & $6.65563 E-13$ & 0.0 & 0.0 & 0.0 \\
\hline CUOH2 & $7.99489 E-09$ & $5.18694 \mathrm{E}-06$ & 0.0 & 0.0 \\
\hline FEIICL2 & $1.41689 \mathrm{E}-14$ & 0.0 & 0.0 & 0.0 \\
\hline FEIICO3 & $1.70549 \mathrm{E}-14$ & 0.0 & 0.0 & 0.0 \\
\hline FEIIHPO4 & $1.06906 \mathrm{E}-16$ & 0.0 & 0.0 & 0.0 \\
\hline FEIIOH2 & $4.59617 E-13$ & 0.0 & 0.0 & 0.0 \\
\hline $\mathrm{ALO} 2 \mathrm{H} 2 \mathrm{CL}$ & $6.34366 \mathrm{E}-30$ & 0.0 & 0.0 & 0.0 \\
\hline H3PO 4 & $2.42732 E-16$ & 0.0 & 0.0 & 0.0 \\
\hline H4 P 207 & $1.69069 \mathrm{E}-30$ & 0.0 & 0.0 & 0.0 \\
\hline ALOH3 & $2.90620 \mathrm{E}-11$ & 0.0 & 0.0 & 0.0 \\
\hline $\mathrm{BACO} 3$ & $3.94782 \mathrm{E}-16$ & 0.0 & 0.0 & 0.0 \\
\hline $\mathrm{KCL}$ & $6.42515 E-06$ & 0.0 & 0.0 & 0.0 \\
\hline KHSO 4 & $1.49461 \mathrm{E}-12$ & 0.0 & 0.0 & 0.0 \\
\hline BASO 4 & $9.21084 \mathrm{E}-13$ & $5.83022 E-07$ & 0.0 & 0.0 \\
\hline $\mathrm{MGCO} 3$ & $7.40747 \mathrm{E}-11$ & 0.0 & 0.0 & 0.0 \\
\hline MGH2SIO4 & $1.43972 E-09$ & 0.0 & 0.0 & 0.0 \\
\hline MGHPO4 & $2.06007 E-09$ & 0.0 & 0.0 & 0.0 \\
\hline MGSO 4 & $3.65074 E-06$ & 0.0 & 0.0 & 0.0 \\
\hline NAHCO3 & $2.71487 E-09$ & 0.0 & 0.0 & 0.0 \\
\hline NAHSIO3 & $1.53736 \mathrm{E}-06$ & 0.0 & 0.0 & 0.0 \\
\hline NANO3 & $1.23920 \mathrm{E}-04$ & 0.0 & 0.0 & 0.0 \\
\hline $\mathrm{NIOH} 2$ & $2.09348 E-12$ & $1.29197 E-06$ & 0.0 & 0.0 \\
\hline $\mathrm{NISO} 4$ & $5.11833 \mathrm{E}-10$ & 0.0 & 0.0 & 0.0 \\
\hline PBCL2 & $1.15048 \mathrm{E}-07$ & 0.0 & 0.0 & 0.0 \\
\hline PBHPO4 & $2.12365 \mathrm{E}-14$ & 0.0 & 0.0 & 0.0 \\
\hline PBNO32 & $2.38004 E-09$ & 0.0 & 0.0 & 0.0 \\
\hline $\mathrm{PBO}$ & $8.01172 \mathrm{E}-10$ & 0.0 & 0.0 & 0.0 \\
\hline CACL2 & $1.62934 \mathrm{E}-15$ & 0.0 & 0.0 & 0.0 \\
\hline $\mathrm{SIO} 2$ & $3.97116 \mathrm{E}-06$ & $1.50895 \mathrm{E}-04$ & 0.0 & 0.0 \\
\hline
\end{tabular}




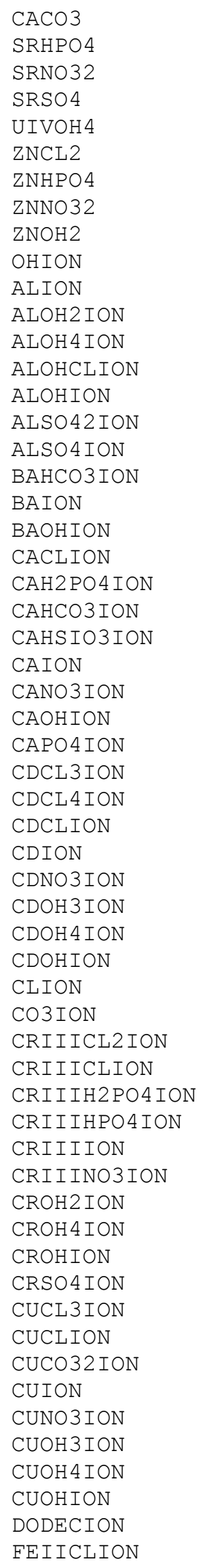

$\begin{array}{ll}2.31711 \mathrm{E}-11 & 0.0 \\ 3.67409 \mathrm{E}-14 & 0.0 \\ 2.74753 \mathrm{E}-08 & 0.0 \\ 8.68924 \mathrm{E}-09 & 1.69661 \mathrm{E}-05 \\ 1.08368 \mathrm{E}-12 & 0.0 \\ 3.94652 \mathrm{E}-06 & 0.0 \\ 2.27496 \mathrm{E}-12 & 0.0 \\ 1.17059 \mathrm{E}-09 & 0.0 \\ 3.25219 \mathrm{E}-08 & 0.0 \\ 1.52535 \mathrm{E}-08 & 0.0 \\ 1.72695 \mathrm{E}-21 & 0.0 \\ 2.05332 \mathrm{E}-14 & 0.0 \\ 5.43938 \mathrm{E}-09 & 0.0 \\ 4.81481 \mathrm{E}-17 & 0.0 \\ 1.25146 \mathrm{E}-17 & 0.0 \\ 3.47714 \mathrm{E}-21 & 0.0 \\ 8.20164 \mathrm{E}-21 & 0.0 \\ 3.58529 \mathrm{E}-14 & 0.0 \\ 9.20856 \mathrm{E}-10 & 0.0 \\ 8.22836 \mathrm{E}-15 & 0.0 \\ 4.09695 \mathrm{E}-08 & 0.0 \\ 9.60553 \mathrm{E}-12 & 0.0 \\ 6.21605 \mathrm{E}-11 & 0.0 \\ 8.21871 \mathrm{E}-09 & 0.0 \\ 9.69012 \mathrm{E}-06 & 0.0 \\ 3.67905 \mathrm{E}-06 & 0.0 \\ 1.65087 \mathrm{E}-09 & 0.0 \\ 3.12794 \mathrm{E}-11 & 0.0 \\ 1.46702 \mathrm{E}-08 & 0.0 \\ 3.84474 \mathrm{E}-09 & 0.0 \\ 9.19493 \mathrm{E}-10 & 0.0 \\ 1.77989 \mathrm{E}-11 & 0.0 \\ 4.21555 \mathrm{E}-12 & 0.0 \\ 6.15446 \mathrm{E}-18 & 0.0 \\ 5.78509 \mathrm{E}-22 & 0.0 \\ 4.25880 \mathrm{E}-13 & 0.0 \\ 0.00209468 & 0.0 \\ 4.14855 \mathrm{E}-11 & 0.0 \\ 8.04905 \mathrm{E}-16 & 0.0 \\ 1.85289 \mathrm{E}-15 & 0.0 \\ 2.09213 \mathrm{E}-20 & 0.0 \\ 8.79574 \mathrm{E}-09 & 0.0 \\ 5.11800 \mathrm{E}-19 & 0.0 \\ 2.83918 \mathrm{E}-13 & 0.0 \\ 5.81135 \mathrm{E}-13 & 0.0 \\ 8.15587 \mathrm{E}-14 & 0.0 \\ 2.60134 \mathrm{E}-10 & 0.0 \\ 8.43097 \mathrm{E}-12 & 0.0 \\ 2.13245 \mathrm{E}-12 & 0.0 \\ 8.55316 \mathrm{E}-10 & 0.0 \\ 2.40202 \mathrm{E}-17 & 0.0 \\ 2.39428 \mathrm{E}-10 & 0.0 \\ 3.54428 \mathrm{E}-11 & 0.0 \\ 8.13586 \mathrm{E}-12 & 0.0 \\ 1.82274 \mathrm{E}-14 & 0.0 \\ 6.61459 \mathrm{E}-10 & 0.0 \\ 3.16278 \mathrm{E}-06 & 0.0 \\ 2.94280 \mathrm{E}-12 & 0.0 \\ & \\ 3.0\end{array}$

0.0

0.0

0.0

0.0

0.0

0.0

0.0

0.0

0.0

0.0

0.0

0.0

0.0

0.0

0.0

0.0

0.0

0.0

0.0

0.0

0.0

0.0

0.0

0.0

0.0

0.0

0.0

0.0

0.0

0.0

0.0

0.0

0.0

0.0

0.0

0.0

0.0

0.0

0.0

0.0

0.0

0.0

0.0

0.0

0.0

0.0

0.0

0.0

0.0

0.0

0.0

0.0

0.0

0.0

0.0

0.0

0.0

0.0

0.0 


\begin{tabular}{|c|c|c|c|c|}
\hline FEIICO32ION & $1.29957 \mathrm{E}-19$ & 0.0 & 0.0 & 0.0 \\
\hline FEIIH2PO4ION & $1.21581 \mathrm{E}-17$ & 0.0 & 0.0 & 0.0 \\
\hline FEIIHCO3ION & $4.90154 \mathrm{E}-17$ & 0.0 & 0.0 & 0.0 \\
\hline FEIIION & $3.61861 \mathrm{E}-10$ & 0.0 & 0.0 & 0.0 \\
\hline FEIIOH3ION & $9.62746 \mathrm{E}-15$ & 0.0 & 0.0 & 0.0 \\
\hline FEIIOH 4 ION & $2.54081 \mathrm{E}-19$ & 0.0 & 0.0 & 0.0 \\
\hline FEIIOHION & $3.48336 \mathrm{E}-11$ & 0.0 & 0.0 & 0.0 \\
\hline H2P2O7ION & $8.46229 \mathrm{E}-19$ & 0.0 & 0.0 & 0.0 \\
\hline H2 PO 4 ION & $5.08588 \mathrm{E}-11$ & 0.0 & 0.0 & 0.0 \\
\hline H2SIO 4 ION & $7.85532 \mathrm{E}-12$ & 0.0 & 0.0 & 0.0 \\
\hline H3P2O 7 ION & $9.91442 \mathrm{E}-25$ & 0.0 & 0.0 & 0.0 \\
\hline H3SIO 4 ION & $4.74023 E-07$ & 0.0 & 0.0 & 0.0 \\
\hline HCO3ION & $1.84753 \mathrm{E}-09$ & 0.0 & 0.0 & 0.0 \\
\hline HION & $5.75886 \mathrm{E}-11$ & 0.0 & 0.0 & 0.0 \\
\hline HP2O 7 ION & $1.56469 \mathrm{E}-17$ & 0.0 & 0.0 & 0.0 \\
\hline HPBO2 ION & $7.98558 \mathrm{E}-12$ & 0.0 & 0.0 & 0.0 \\
\hline HPO4ION & $5.23140 \mathrm{E}-10$ & 0.0 & 0.0 & 0.0 \\
\hline HSO 4 ION & $2.80365 \mathrm{E}-10$ & 0.0 & 0.0 & 0.0 \\
\hline KION & $3.23039 \mathrm{E}-04$ & 0.0 & 0.0 & 0.0 \\
\hline KSO4ION & $2.22741 \mathrm{E}-05$ & 0.0 & 0.0 & 0.0 \\
\hline MGH2 PO 4 ION & $2.07577 \mathrm{E}-11$ & 0.0 & 0.0 & 0.0 \\
\hline MGHCO3ION & $8.18751 \mathrm{E}-10$ & 0.0 & 0.0 & 0.0 \\
\hline MGHS IO3ION & $1.03645 \mathrm{E}-07$ & 0.0 & 0.0 & 0.0 \\
\hline MGION & $2.68458 \mathrm{E}-05$ & 0.0 & 0.0 & 0.0 \\
\hline MGOHION & $8.79443 \mathrm{E}-08$ & 0.0 & 0.0 & 0.0 \\
\hline MGP2O7ION & $8.89815 \mathrm{E}-14$ & 0.0 & 0.0 & 0.0 \\
\hline MGPO 4 ION & $2.25681 \mathrm{E}-10$ & 0.0 & 0.0 & 0.0 \\
\hline NACO3ION & $6.66262 \mathrm{E}-12$ & 0.0 & 0.0 & 0.0 \\
\hline NAION & 0.00226641 & 0.0 & 0.0 & 0.0 \\
\hline NASO 4 ION & $2.21505 \mathrm{E}-13$ & 0.0 & 0.0 & 0.0 \\
\hline NICLION & $1.54115 \mathrm{E}-10$ & 0.0 & 0.0 & 0.0 \\
\hline NIION & $1.81544 \mathrm{E}-09$ & 0.0 & 0.0 & 0.0 \\
\hline NINO3ION & $2.26657 \mathrm{E}-10$ & 0.0 & 0.0 & 0.0 \\
\hline NIOH3ION & $5.29422 \mathrm{E}-15$ & 0.0 & 0.0 & 0.0 \\
\hline NIOHION & $5.22840 \mathrm{E}-11$ & 0.0 & 0.0 & 0.0 \\
\hline NO3ION & $3.39907 \mathrm{E}-04$ & 0.0 & 0.0 & 0.0 \\
\hline P207ION & $3.04580 \mathrm{E}-18$ & 0.0 & 0.0 & 0.0 \\
\hline PBCL3ION & $2.46053 \mathrm{E}-07$ & 0.0 & 0.0 & 0.0 \\
\hline PBCL 4 ION & $1.82272 \mathrm{E}-06$ & 0.0 & 0.0 & 0.0 \\
\hline PBCLION & $3.21393 \mathrm{E}-08$ & 0.0 & 0.0 & 0.0 \\
\hline PBH2 PO 4 ION & $1.18148 \mathrm{E}-15$ & 0.0 & 0.0 & 0.0 \\
\hline PBION & $1.18817 \mathrm{E}-09$ & 0.0 & 0.0 & 0.0 \\
\hline PBNO33ION & $7.25594 \mathrm{E}-11$ & 0.0 & 0.0 & 0.0 \\
\hline PBNO3ION & $7.92409 \mathrm{E}-09$ & 0.0 & 0.0 & 0.0 \\
\hline PBOHION & $1.95221 \mathrm{E}-08$ & 0.0 & 0.0 & 0.0 \\
\hline PO4ION & $8.04226 \mathrm{E}-14$ & 0.0 & 0.0 & 0.0 \\
\hline SO4ION & $1.05711 \mathrm{E}-04$ & 0.0 & 0.0 & 0.0 \\
\hline SRION & $3.72064 \mathrm{E}-09$ & 0.0 & 0.0 & 0.0 \\
\hline SRNO3ION & $1.17374 \mathrm{E}-07$ & 0.0 & 0.0 & 0.0 \\
\hline SROHION & $1.23354 \mathrm{E}-11$ & 0.0 & 0.0 & 0.0 \\
\hline SRPO 4 ION & $7.10798 \mathrm{E}-15$ & 0.0 & 0.0 & 0.0 \\
\hline UIVOH2ION & $5.58314 \mathrm{E}-26$ & 0.0 & 0.0 & 0.0 \\
\hline UIVOH3ION & $1.70374 \mathrm{E}-20$ & 0.0 & 0.0 & 0.0 \\
\hline UIVOH5ION & $1.03006 \mathrm{E}-14$ & 0.0 & 0.0 & 0.0 \\
\hline UIVOHION & $1.08377 \mathrm{E}-30$ & 0.0 & 0.0 & 0.0 \\
\hline ZNCL3ION & $4.88884 \mathrm{E}-06$ & 0.0 & 0.0 & 0.0 \\
\hline ZNCLION & $8.75666 \mathrm{E}-06$ & 0.0 & 0.0 & 0.0 \\
\hline ZNH2PO 4 ION & $6.15416 \mathrm{E}-14$ & 0.0 & 0.0 & 0.0 \\
\hline
\end{tabular}


WSRC-TR-2002-00567

Revision 0

\begin{tabular}{|c|c|c|c|c|}
\hline ZNHCO3ION & $2.03770 \mathrm{E}-12$ & 0.0 & 0.0 & 0.0 \\
\hline ZNION & $5.36005 \mathrm{E}-07$ & 0.0 & 0.0 & 0.0 \\
\hline ZNNO3ION & $4.81247 E-08$ & 0.0 & 0.0 & 0.0 \\
\hline ZNOH3ION & $1.29088 E-09$ & 0.0 & 0.0 & 0.0 \\
\hline ZNOH $4 \mathrm{ION}$ & $1.01438 \mathrm{E}-12$ & 0.0 & 0.0 & 0.0 \\
\hline ZNOHION & $4.21867 \mathrm{E}-06$ & 0.0 & 0.0 & 0.0 \\
\hline $\mathrm{ALOOH}$ & 0.0 & $2.86232 E-06$ & 0.0 & 0.0 \\
\hline $\mathrm{CA} 3 \mathrm{PO} 42$ & 0.0 & $8.96701 E-05$ & 0.0 & 0.0 \\
\hline CHAMOSITE7A & 0.0 & $4.30108 E-05$ & 0.0 & 0.0 \\
\hline MG3PO4 2 & 0.0 & $1.31312 \mathrm{E}-05$ & 0.0 & 0.0 \\
\hline MGOH2 & 0.0 & $2.59133 E-04$ & 0.0 & 0.0 \\
\hline PB3PO42 & 0.0 & $2.49104 \mathrm{E}-07$ & 0.0 & 0.0 \\
\hline UIVO2 & 0.0 & $2.89917 E-06$ & 0.0 & 0.0 \\
\hline $\mathrm{ZN} 3 \mathrm{PO} 42.2 \mathrm{H} 2 \mathrm{O}$ & 0.0 & $2.20740 \mathrm{E}-06$ & 0.0 & 0.0 \\
\hline & $===========$ & $===========$ & $===========$ & $===========$ \\
\hline Total g/hr & 1.06401 & 0.156425 & 0.0 & 0.0 \\
\hline Volume, L/hr & $9.93763 E-04$ & $4.75020 E-05$ & 0.0 & 0.0 \\
\hline Enthalpy, cal/hr & -3547.01 & -438.537 & 0.0 & 0.0 \\
\hline Density, g/L & 1070.69 & 3293.02 & & \\
\hline Vapor fraction & 0.0 & 0.0 & 0.0 & 0.0 \\
\hline Solid fraction & 0.0 & 1. & 0.0 & 0.0 \\
\hline Organic fraction & 0.0 & 0.0 & 0.0 & 0.0 \\
\hline Osmotic Pres, atm & 182.086 & & & \\
\hline Redox Pot, volts & 0.0 & & & \\
\hline $\mathrm{E}-\mathrm{Con}, 1 / \mathrm{ohm}-\mathrm{cm}$ & 0.484794 & & & \\
\hline E-Con, cm2/ohm-mol & 55.4865 & & & \\
\hline Abs Visc, cP & 0.402037 & & & \\
\hline Rel Visc & 1.47583 & & & \\
\hline Ionic Strength & 3.2353 & & & \\
\hline
\end{tabular}


WSRC-TR-2002-00567

Revision 0

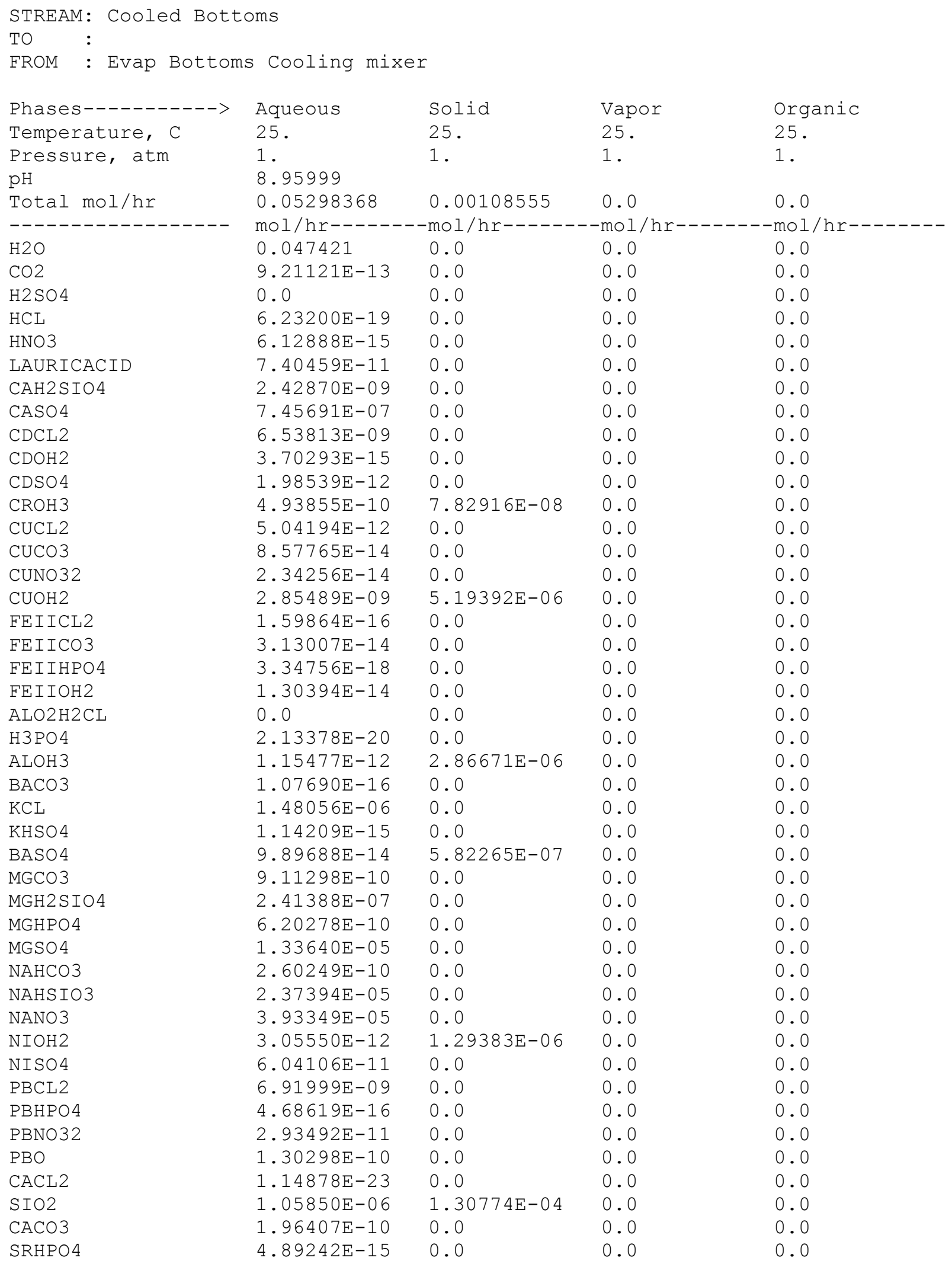




\begin{tabular}{|c|c|c|c|c|}
\hline SRNO32 & $1.18900 \mathrm{E}-08$ & 0.0 & 0.0 & 0.0 \\
\hline SRSO 4 & $2.07367 \mathrm{E}-08$ & $1.59460 \mathrm{E}-05$ & 0.0 & 0.0 \\
\hline UIVOH 4 & $1.13582 \mathrm{E}-13$ & 0.0 & 0.0 & 0.0 \\
\hline ZNCL2 & $8.54117 \mathrm{E}-09$ & 0.0 & 0.0 & 0.0 \\
\hline ZNHPO 4 & $3.69983 \mathrm{E}-14$ & 0.0 & 0.0 & 0.0 \\
\hline ZNNO32 & $6.98213 \mathrm{E}-11$ & 0.0 & 0.0 & 0.0 \\
\hline ZNOH2 & $3.91132 \mathrm{E}-09$ & $2.89682 \mathrm{E}-05$ & 0.0 & 0.0 \\
\hline OHION & $7.06169 \mathrm{E}-09$ & 0.0 & 0.0 & 0.0 \\
\hline ALION & $9.07501 \mathrm{E}-23$ & 0.0 & 0.0 & 0.0 \\
\hline ALOH2 ION & $6.48856 \mathrm{E}-16$ & 0.0 & 0.0 & 0.0 \\
\hline ALOH 4 ION & $7.16041 \mathrm{E}-10$ & 0.0 & 0.0 & 0.0 \\
\hline ALOHCLION & $1.78067 \mathrm{E}-18$ & 0.0 & 0.0 & 0.0 \\
\hline ALOHION & $2.85357 \mathrm{E}-19$ & 0.0 & 0.0 & 0.0 \\
\hline ALSO 42 ION & $1.04184 \mathrm{E}-22$ & 0.0 & 0.0 & 0.0 \\
\hline ALSO 4 ION & $2.19560 \mathrm{E}-22$ & 0.0 & 0.0 & 0.0 \\
\hline BAHCO3ION & $2.28194 \mathrm{E}-16$ & 0.0 & 0.0 & 0.0 \\
\hline BAION & $1.67775 \mathrm{E}-09$ & 0.0 & 0.0 & 0.0 \\
\hline BAOHION & $9.44075 \mathrm{E}-17$ & 0.0 & 0.0 & 0.0 \\
\hline CACLION & $4.76908 \mathrm{E}-11$ & 0.0 & 0.0 & 0.0 \\
\hline CAH2 PO 4 ION & $1.25495 \mathrm{E}-13$ & 0.0 & 0.0 & 0.0 \\
\hline CAHCO3ION & $8.39094 \mathrm{E}-11$ & 0.0 & 0.0 & 0.0 \\
\hline CAHSIO3ION & $4.75884 \mathrm{E}-08$ & 0.0 & 0.0 & 0.0 \\
\hline CAION & $2.22874 \mathrm{E}-05$ & 0.0 & 0.0 & 0.0 \\
\hline CANO3ION & $8.57126 \mathrm{E}-06$ & 0.0 & 0.0 & 0.0 \\
\hline CAOHION & $1.06705 \mathrm{E}-09$ & 0.0 & 0.0 & 0.0 \\
\hline CAPO 4 ION & $3.13772 \mathrm{E}-10$ & 0.0 & 0.0 & 0.0 \\
\hline CDCL3ION & $2.39756 \mathrm{E}-09$ & 0.0 & 0.0 & 0.0 \\
\hline CDCL 4 ION & $2.63463 \mathrm{E}-08$ & 0.0 & 0.0 & 0.0 \\
\hline CDCLION & $4.09889 \mathrm{E}-10$ & 0.0 & 0.0 & 0.0 \\
\hline CDION & $1.79166 \mathrm{E}-11$ & 0.0 & 0.0 & 0.0 \\
\hline CDNO3ION & $2.39814 \mathrm{E}-12$ & 0.0 & 0.0 & 0.0 \\
\hline CDOH 3 ION & $1.59934 \mathrm{E}-18$ & 0.0 & 0.0 & 0.0 \\
\hline CDOH 4 ION & $6.29356 \mathrm{E}-23$ & 0.0 & 0.0 & 0.0 \\
\hline CDOHION & $1.41352 \mathrm{E}-13$ & 0.0 & 0.0 & 0.0 \\
\hline CLION & 0.00213781 & 0.0 & 0.0 & 0.0 \\
\hline CO3ION & $4.81661 \mathrm{E}-10$ & 0.0 & 0.0 & 0.0 \\
\hline CRIIICL2ION & $1.11127 \mathrm{E}-17$ & 0.0 & 0.0 & 0.0 \\
\hline CRIIICLION & $2.01374 \mathrm{E}-17$ & 0.0 & 0.0 & 0.0 \\
\hline CRIIIH2PO4ION & $4.42604 \mathrm{E}-22$ & 0.0 & 0.0 & 0.0 \\
\hline CRIIIHPO4ION & $1.78935 \mathrm{E}-14$ & 0.0 & 0.0 & 0.0 \\
\hline CRIIIION & $2.92544 \mathrm{E}-20$ & 0.0 & 0.0 & 0.0 \\
\hline CRIIINO3ION & $1.90320 \mathrm{E}-15$ & 0.0 & 0.0 & 0.0 \\
\hline CROH2ION & $4.47950 \mathrm{E}-13$ & 0.0 & 0.0 & 0.0 \\
\hline CROH 4 ION & $1.76364 \mathrm{E}-11$ & 0.0 & 0.0 & 0.0 \\
\hline CROHION & $4.25994 \mathrm{E}-11$ & 0.0 & 0.0 & 0.0 \\
\hline CRSO 4 ION & $8.15133 \mathrm{E}-14$ & 0.0 & 0.0 & 0.0 \\
\hline CUCL3ION & $8.01203 \mathrm{E}-14$ & 0.0 & 0.0 & 0.0 \\
\hline CUCLION & $1.47858 \mathrm{E}-11$ & 0.0 & 0.0 & 0.0 \\
\hline CUCO32ION & $1.49321 \mathrm{E}-16$ & 0.0 & 0.0 & 0.0 \\
\hline CUION & $1.40378 \mathrm{E}-11$ & 0.0 & 0.0 & 0.0 \\
\hline CUNO3ION & $2.15383 \mathrm{E}-12$ & 0.0 & 0.0 & 0.0 \\
\hline CUOH 3ION & $4.50442 \mathrm{E}-12$ & 0.0 & 0.0 & 0.0 \\
\hline CUOH 4 ION & $2.88100 \mathrm{E}-15$ & 0.0 & 0.0 & 0.0 \\
\hline CUOHION & $4.82995 \mathrm{E}-11$ & 0.0 & 0.0 & 0.0 \\
\hline DODECION & $3.16335 \mathrm{E}-06$ & 0.0 & 0.0 & 0.0 \\
\hline FEIICLION & $5.79634 \mathrm{E}-14$ & 0.0 & 0.0 & 0.0 \\
\hline FEIICO32ION & $1.17514 \mathrm{E}-18$ & 0.0 & 0.0 & 0.0 \\
\hline FEIIH2PO4ION & $2.85615 \mathrm{E}-20$ & 0.0 & 0.0 & 0.0 \\
\hline
\end{tabular}




\begin{tabular}{|c|c|c|c|c|}
\hline FEIIHCO3ION & $2.17508 \mathrm{E}-18$ & 0.0 & 0.0 & 0.0 \\
\hline FEIIION & $4.25053 \mathrm{E}-11$ & 0.0 & 0.0 & 0.0 \\
\hline FEIIOH3ION & $1.44798 \mathrm{E}-15$ & 0.0 & 0.0 & 0.0 \\
\hline FEIIOH 4 ION & $1.33065 \mathrm{E}-20$ & 0.0 & 0.0 & 0.0 \\
\hline FEIIOHION & $5.02752 \mathrm{E}-12$ & 0.0 & 0.0 & 0.0 \\
\hline H2 P2O 7 ION & $7.33389 \mathrm{E}-24$ & 0.0 & 0.0 & 0.0 \\
\hline $\mathrm{H} 2 \mathrm{PO} 4 \mathrm{ION}$ & $6.55945 \mathrm{E}-13$ & 0.0 & 0.0 & 0.0 \\
\hline H2SIO4ION & $2.40697 \mathrm{E}-10$ & 0.0 & 0.0 & 0.0 \\
\hline H3P2O7ION & 0.0 & 0.0 & 0.0 & 0.0 \\
\hline H3SIO4ION & $2.21536 \mathrm{E}-07$ & 0.0 & 0.0 & 0.0 \\
\hline HCO3ION & $1.29974 \mathrm{E}-09$ & 0.0 & 0.0 & 0.0 \\
\hline HION & $8.29014 \mathrm{E}-13$ & 0.0 & 0.0 & 0.0 \\
\hline HP207ION & $1.00406 \mathrm{E}-20$ & 0.0 & 0.0 & 0.0 \\
\hline HPBO2ION & $3.06512 \mathrm{E}-12$ & 0.0 & 0.0 & 0.0 \\
\hline HPO 4 ION & $3.96000 \mathrm{E}-10$ & 0.0 & 0.0 & 0.0 \\
\hline HSO 4 ION & $7.10685 \mathrm{E}-13$ & 0.0 & 0.0 & 0.0 \\
\hline KION & $3.34644 \mathrm{E}-04$ & 0.0 & 0.0 & 0.0 \\
\hline $\mathrm{KSO} 4 \mathrm{ION}$ & $1.56146 \mathrm{E}-05$ & 0.0 & 0.0 & 0.0 \\
\hline MGH2 PO 4 ION & $1.65830 \mathrm{E}-12$ & 0.0 & 0.0 & 0.0 \\
\hline MGHCO3ION & $2.24904 \mathrm{E}-09$ & 0.0 & 0.0 & 0.0 \\
\hline MGHSIO3ION & $9.04093 \mathrm{E}-07$ & 0.0 & 0.0 & 0.0 \\
\hline MGION & $9.39469 \mathrm{E}-05$ & 0.0 & 0.0 & 0.0 \\
\hline MGOHION & $1.05134 \mathrm{E}-07$ & 0.0 & 0.0 & 0.0 \\
\hline MGP2O7ION & $1.09292 \mathrm{E}-15$ & 0.0 & 0.0 & 0.0 \\
\hline MGPO 4 ION & $4.31908 \mathrm{E}-09$ & 0.0 & 0.0 & 0.0 \\
\hline NACO3ION & $2.44996 \mathrm{E}-10$ & 0.0 & 0.0 & 0.0 \\
\hline NAION & 0.00227874 & 0.0 & 0.0 & 0.0 \\
\hline NASO 4 ION & $5.00583 \mathrm{E}-05$ & 0.0 & 0.0 & 0.0 \\
\hline NICLION & $2.44156 \mathrm{E}-11$ & 0.0 & 0.0 & 0.0 \\
\hline NIION & $6.51178 \mathrm{E}-10$ & 0.0 & 0.0 & 0.0 \\
\hline NINO3ION & $1.39903 \mathrm{E}-10$ & 0.0 & 0.0 & 0.0 \\
\hline NIOH3ION & $9.68434 \mathrm{E}-14$ & 0.0 & 0.0 & 0.0 \\
\hline NIOHION & $1.28376 \mathrm{E}-11$ & 0.0 & 0.0 & 0.0 \\
\hline NO3ION & $4.19703 E-04$ & 0.0 & 0.0 & 0.0 \\
\hline P2O7ION & $6.09572 \mathrm{E}-19$ & 0.0 & 0.0 & 0.0 \\
\hline PBCL3ION & $1.75620 \mathrm{E}-08$ & 0.0 & 0.0 & 0.0 \\
\hline PBCL 4 ION & $3.21733 \mathrm{E}-07$ & 0.0 & 0.0 & 0.0 \\
\hline PBCLION & $2.55140 \mathrm{E}-09$ & 0.0 & 0.0 & 0.0 \\
\hline PBH2 PO 4 ION & $7.96812 \mathrm{E}-19$ & 0.0 & 0.0 & 0.0 \\
\hline PBION & $1.76868 \mathrm{E}-10$ & 0.0 & 0.0 & 0.0 \\
\hline PBNO33ION & $5.68298 \mathrm{E}-12$ & 0.0 & 0.0 & 0.0 \\
\hline PBNO3ION & $3.65577 \mathrm{E}-10$ & 0.0 & 0.0 & 0.0 \\
\hline PBOHION & $8.94715 \mathrm{E}-10$ & 0.0 & 0.0 & 0.0 \\
\hline PO4ION & $1.79439 \mathrm{E}-12$ & 0.0 & 0.0 & 0.0 \\
\hline SO4ION & $1.15226 \mathrm{E}-04$ & 0.0 & 0.0 & 0.0 \\
\hline SRION & $1.04008 \mathrm{E}-06$ & 0.0 & 0.0 & 0.0 \\
\hline SRNO3ION & $1.04602 \mathrm{E}-07$ & 0.0 & 0.0 & 0.0 \\
\hline SROHION & $3.26311 \mathrm{E}-12$ & 0.0 & 0.0 & 0.0 \\
\hline SRPO 4 ION & $2.34506 \mathrm{E}-14$ & 0.0 & 0.0 & 0.0 \\
\hline UIVOH2ION & $1.23845 \mathrm{E}-28$ & 0.0 & 0.0 & 0.0 \\
\hline UIVOH3ION & $4.22601 \mathrm{E}-21$ & 0.0 & 0.0 & 0.0 \\
\hline UIVOH5ION & $1.83348 \mathrm{E}-14$ & 0.0 & 0.0 & 0.0 \\
\hline ZNCL3ION & $1.50630 \mathrm{E}-08$ & 0.0 & 0.0 & 0.0 \\
\hline ZNCLION & $1.02048 \mathrm{E}-08$ & 0.0 & 0.0 & 0.0 \\
\hline ZNH2 PO 4 ION & $5.13894 \mathrm{E}-17$ & 0.0 & 0.0 & 0.0 \\
\hline ZNHCO3ION & $7.11291 \mathrm{E}-14$ & 0.0 & 0.0 & 0.0 \\
\hline ZNION & $3.79511 \mathrm{E}-08$ & 0.0 & 0.0 & 0.0 \\
\hline ZNNO3ION & $3.68697 \mathrm{E}-09$ & 0.0 & 0.0 & 0.0 \\
\hline
\end{tabular}


WSRC-TR-2002-00567

Revision 0

\begin{tabular}{|c|c|c|c|c|}
\hline ZNOH3ION & $7.65316 \mathrm{E}-11$ & 0.0 & 0.0 & 0.0 \\
\hline ZNOH 4 ION & $1.23277 \mathrm{E}-13$ & 0.0 & 0.0 & 0.0 \\
\hline ZNOHION & $4.25068 E-09$ & 0.0 & 0.0 & 0.0 \\
\hline $\mathrm{CA} 3 \mathrm{PO} 42$ & 0.0 & $1.04379 E-04$ & 0.0 & 0.0 \\
\hline $\mathrm{CASO} 4.2 \mathrm{H} 2 \mathrm{O}$ & 0.0 & $5.28027 E-04$ & 0.0 & 0.0 \\
\hline CHAMOSITE7A & 0.0 & $4.30108 E-05$ & 0.0 & 0.0 \\
\hline $\mathrm{MGOH} 2$ & 0.0 & $2.20649 E-04$ & 0.0 & 0.0 \\
\hline $\mathrm{PB} 3 \mathrm{PO} 42$ & 0.0 & $8.81600 \mathrm{E}-07$ & 0.0 & 0.0 \\
\hline \multirow[t]{2}{*}{ UIVO2 } & 0.0 & $2.89916 \mathrm{E}-06$ & 0.0 & 0.0 \\
\hline & $============$ & $============$ & $===========$ & $===========$ \\
\hline Total g/hr & 1.05342 & 0.167014 & 0.0 & 0.0 \\
\hline Volume, L/hr & $9.23975 E-04$ & $5.55172 \mathrm{E}-05$ & 0.0 & 0.0 \\
\hline Enthalpy, cal/hr & -3573.27 & -485.068 & 0.0 & 0.0 \\
\hline Density, g/L & 1140.1 & 3008.32 & & \\
\hline Vapor fraction & 0.0 & 0.0 & 0.0 & 0.0 \\
\hline Solid fraction & 0.0 & 1 . & 0.0 & 0.0 \\
\hline Organic fraction & 0.0 & 0.0 & 0.0 & 0.0 \\
\hline Osmotic Pres, atm & 173.064 & & & \\
\hline Redox Pot, volts & 0.0 & & & \\
\hline E-Con, 1/ohm-cm & 0.19062 & & & \\
\hline E-Con, cm2/ohm-mol & 30.4352 & & & \\
\hline Abs Visc, cP & 1.30298 & & & \\
\hline Rel Visc & 1.46284 & & & \\
\hline Ionic Strength & 3.61777 & & & \\
\hline
\end{tabular}


WSRC-TR-2002-00567

Revision 0

\begin{tabular}{|c|c|c|c|c|}
\hline \multicolumn{5}{|l|}{ STREAM: Condensate } \\
\hline TO : & & & & \\
\hline FROM : Condensate & mixer & & & \\
\hline Phases-----------> & Aqueous & Solid & Vapor & Organic \\
\hline Temperature, C & 25 . & 25 . & 25 . & 25 . \\
\hline $\begin{array}{l}\text { Pressure, atm } \\
\mathrm{pH}\end{array}$ & $\begin{array}{l}1 . \\
4.53057\end{array}$ & 1. & 1 . & 1 . \\
\hline \multirow{2}{*}{ Total mol/hr } & 55.43837 & 0.0 & 0.0 & 0.0 \\
\hline & $\mathrm{mol} / \mathrm{hr}------1$ & $-\operatorname{mol} / \mathrm{hr}--------$ & -mol/hr------- & $-\operatorname{mol} / \mathrm{hr}------1$ \\
\hline $\mathrm{H} 2 \mathrm{O}$ & 55.4371 & 0.0 & 0.0 & 0.0 \\
\hline $\mathrm{CO} 2$ & 0.00118168 & 0.0 & 0.0 & 0.0 \\
\hline $\mathrm{HCL}$ & $3.37278 E-20$ & 0.0 & 0.0 & 0.0 \\
\hline HNO3 & $5.20516 \mathrm{E}-16$ & 0.0 & 0.0 & 0.0 \\
\hline LAURICACID & $2.71982 E-05$ & 0.0 & 0.0 & 0.0 \\
\hline OHION & $3.45885 \mathrm{E}-10$ & 0.0 & 0.0 & 0.0 \\
\hline CLION & $1.94423 E-09$ & 0.0 & 0.0 & 0.0 \\
\hline CO3ION & $2.97470 \mathrm{E}-11$ & 0.0 & 0.0 & 0.0 \\
\hline DODECION & $1.13066 \mathrm{E}-05$ & 0.0 & 0.0 & 0.0 \\
\hline $\mathrm{HCO} 3 \mathrm{ION}$ & $1.83139 \mathrm{E}-05$ & 0.0 & 0.0 & 0.0 \\
\hline HION & $2.96232 \mathrm{E}-05$ & 0.0 & 0.0 & 0.0 \\
\hline NO3ION & $\begin{array}{l}\text { 3.56607E-10 } \\
===========\end{array}$ & $\begin{array}{l}0.0 \\
============\end{array}$ & $\begin{array}{l}0.0 \\
=============\end{array}$ & $\begin{array}{l}0.0 \\
============\end{array}$ \\
\hline Total g/hr & 998.78 & 0.0 & 0.0 & 0.0 \\
\hline Volume, L/hr & 1.00192 & 0.0 & 0.0 & 0.0 \\
\hline $\begin{array}{l}\text { Enthalpy, cal/hr } \\
\text { Density, g/L }\end{array}$ & $\begin{array}{l}-3.78721 E+06 \\
996.866\end{array}$ & 0.0 & 0.0 & 0.0 \\
\hline Vapor fraction & 0.0 & 0.0 & 0.0 & 0.0 \\
\hline Solid fraction & 0.0 & 0.0 & 0.0 & 0.0 \\
\hline Organic fraction & 0.0 & 0.0 & 0.0 & 0.0 \\
\hline Osmotic Pres, atm & 0.0309725 & & & \\
\hline Redox Pot, volts & 0.0 & & & \\
\hline E-Con, $1 / \mathrm{ohm}-\mathrm{cm}$ & $1.18071 \mathrm{E}-05$ & & & \\
\hline E-Con, cm2/ohm-mol & 9.55165 & & & \\
\hline Abs Visc, cP & 0.89074 & & & \\
\hline Rel Visc & 1.00002 & & & \\
\hline Ionic Strength & $2.96615 \mathrm{E}-05$ & & & \\
\hline
\end{tabular}


WSRC-TR-2002-00567

Revision 0

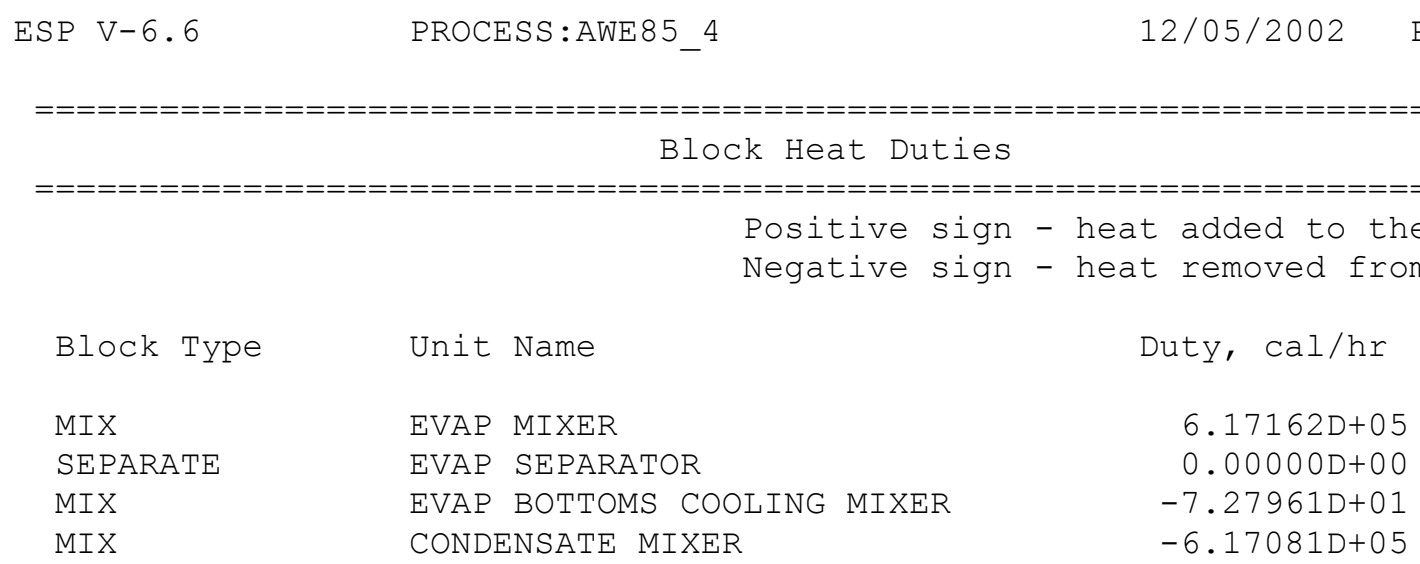


WSRC-TR-2002-00567

Revision 0

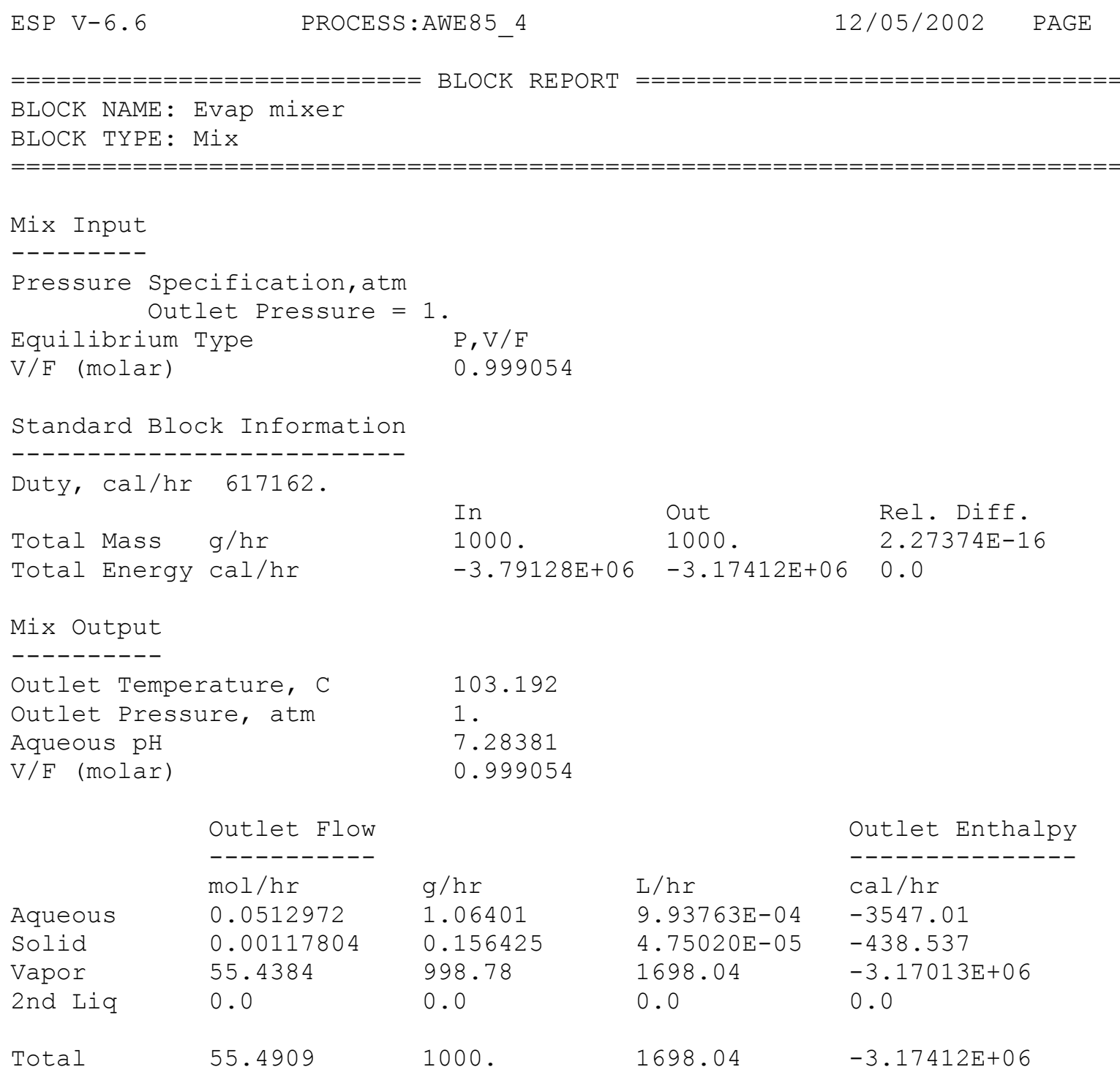


WSRC-TR-2002-00567

Revision 0

$\begin{array}{lllll}\text { ESP V-6.6 PROCESS :AWE85_4 } & \text { PAGE } & 12 / 05 / 2002\end{array}$

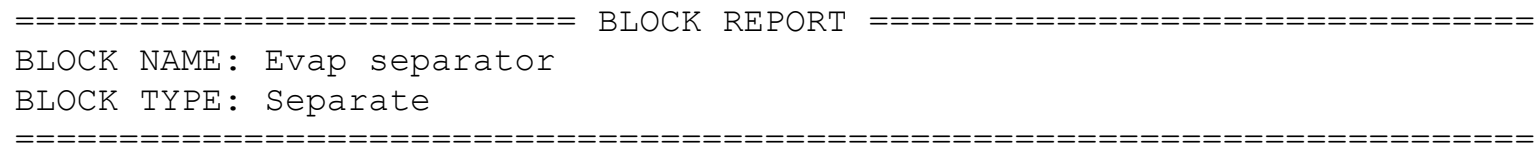


WSRC-TR-2002-00567

Revision 0

ESP V-6.6

PROCESS :AWE 85 _4

Vapor Stream

Outlet Flow
--------
mol/hr
0.0
0.0
55.4384
0.0

55.4384
Overhead

g/hr

0.0

0.0

998.78

0.0

998.78
$12 / 05 / 2002$

PAGE

13

$\mathrm{L} / \mathrm{hr}$

0.0

0.0

1698.04

0.0

Outlet Enthalpy
-----------
cal/hr
0.0
0.0
$-3.17013 \mathrm{E}+06$
0.0

1698.04 
WSRC-TR-2002-00567

Revision 0

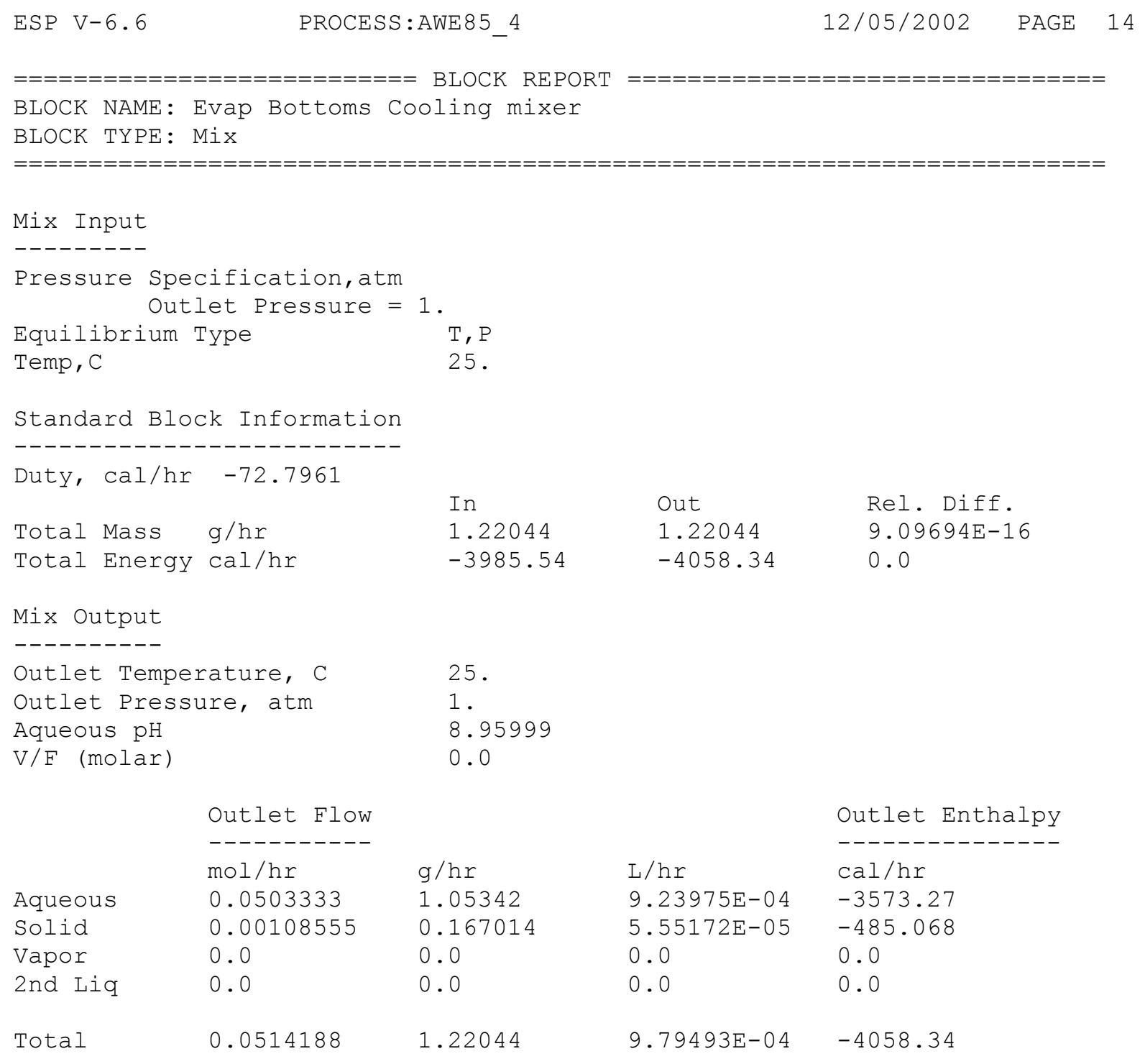


WSRC-TR-2002-00567

Revision 0

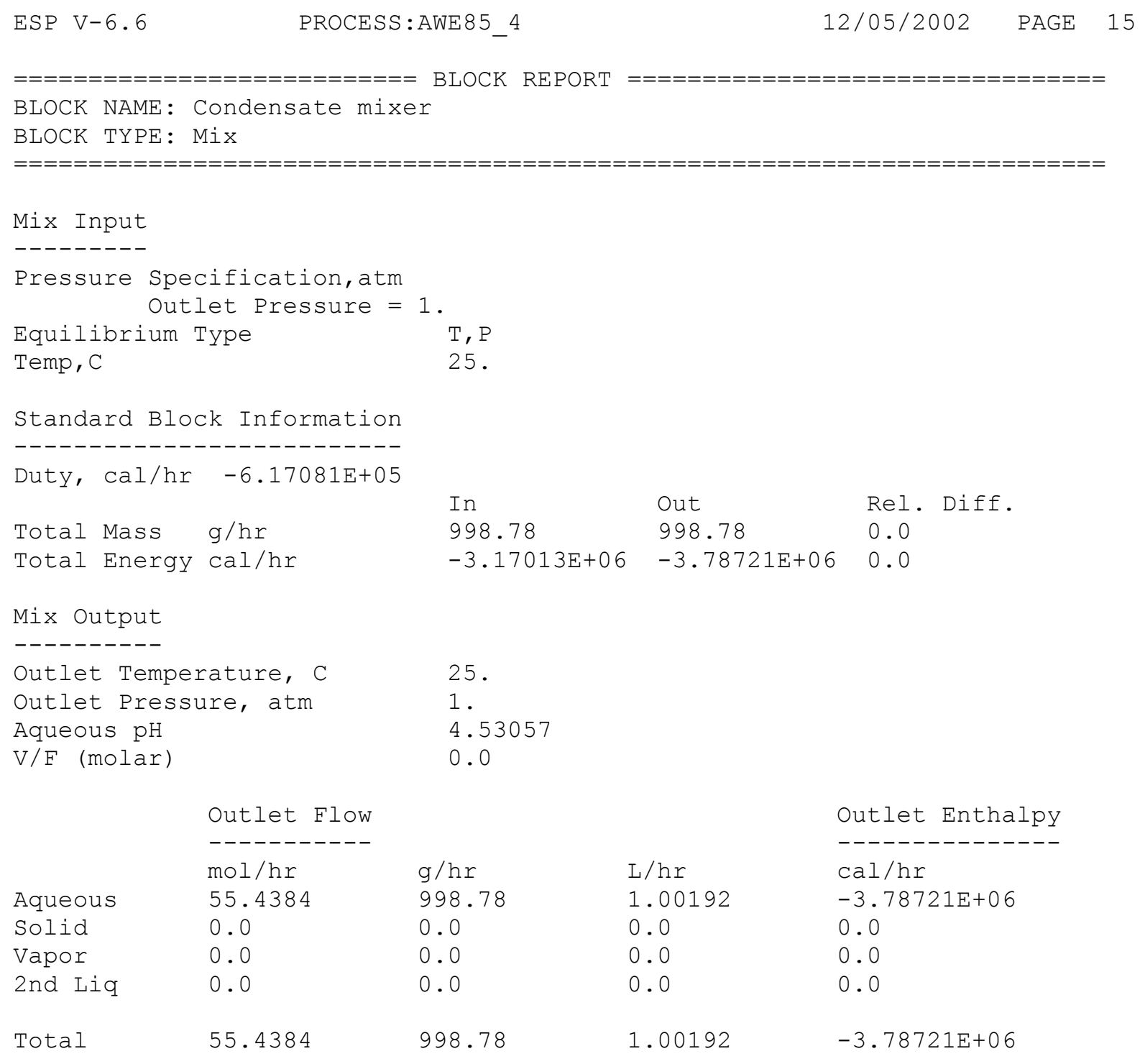


WSRC-TR-2002-00567

Revision 0

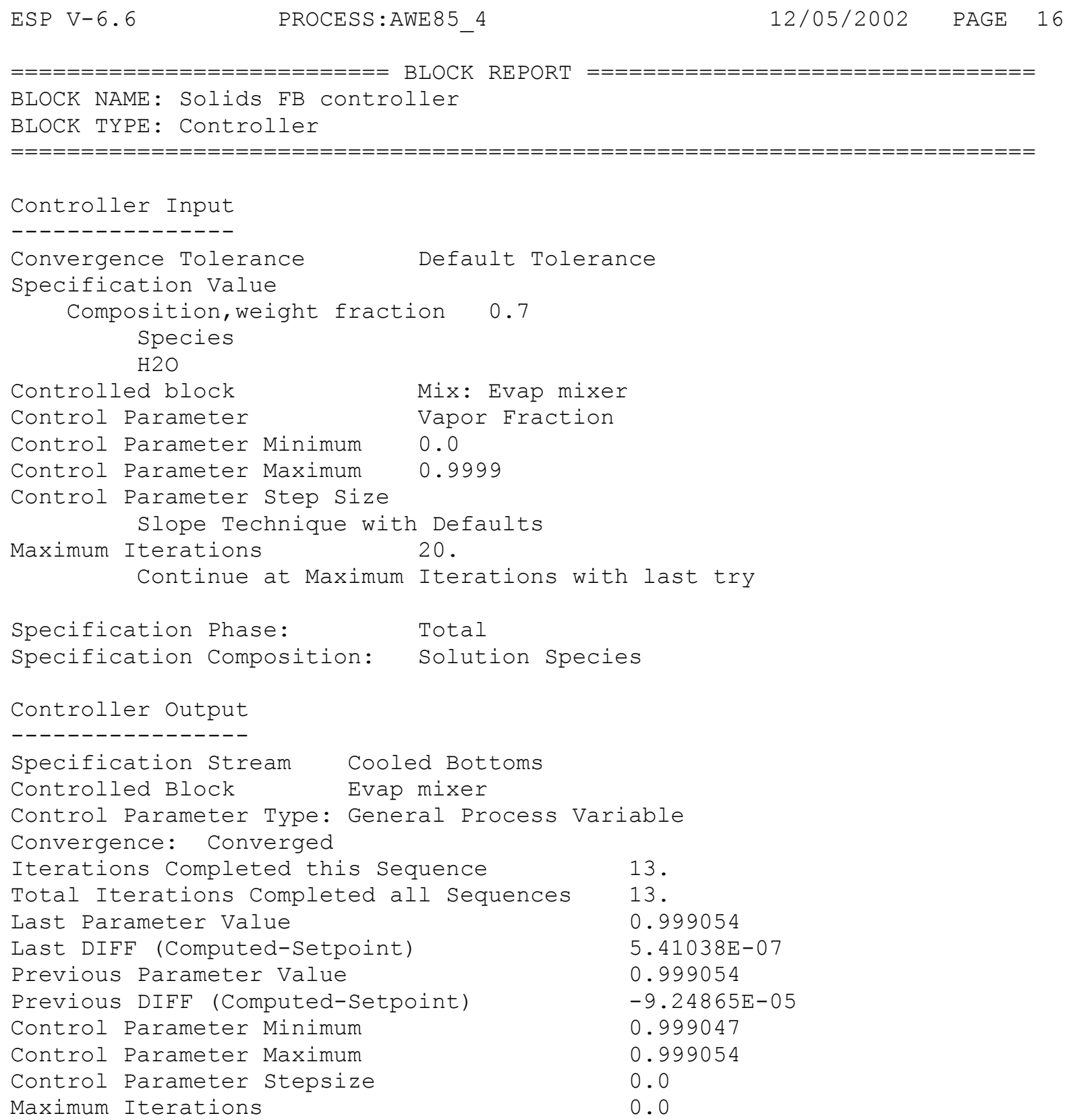


WSRC-TR-2002-00567

Revision 0

Influent Limit Composition 80\% Target $\mathrm{pH}=8.5$

$8.5-80$

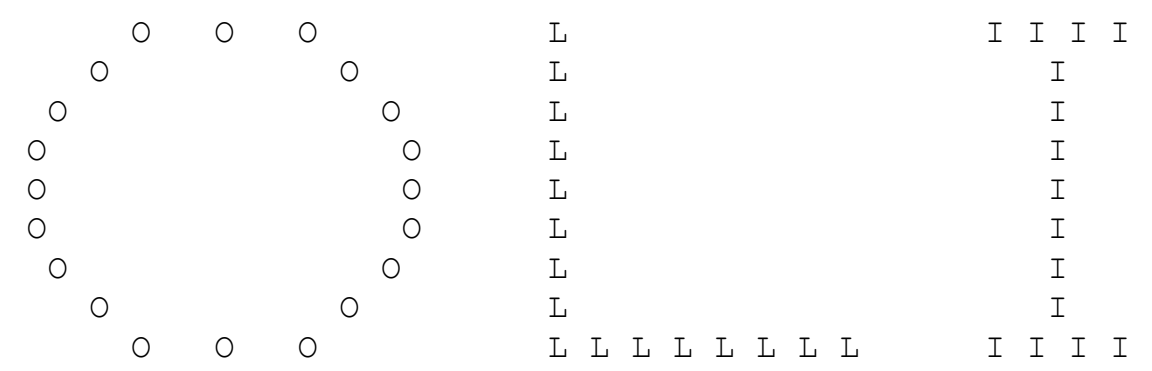

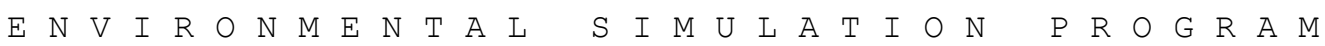

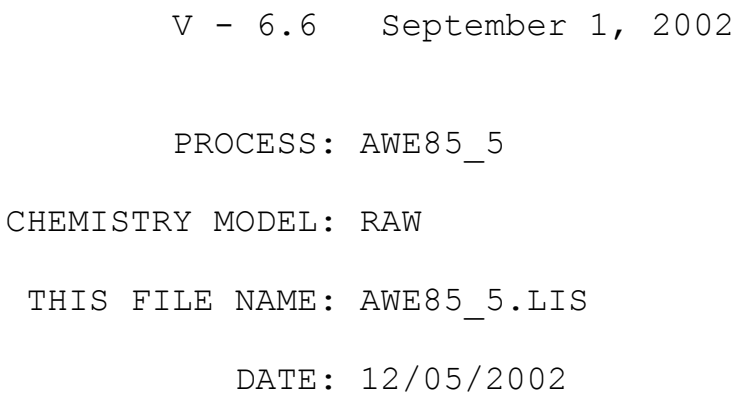


WSRC-TR-2002-00567

Revision 0

TABLE OF CONTENTS

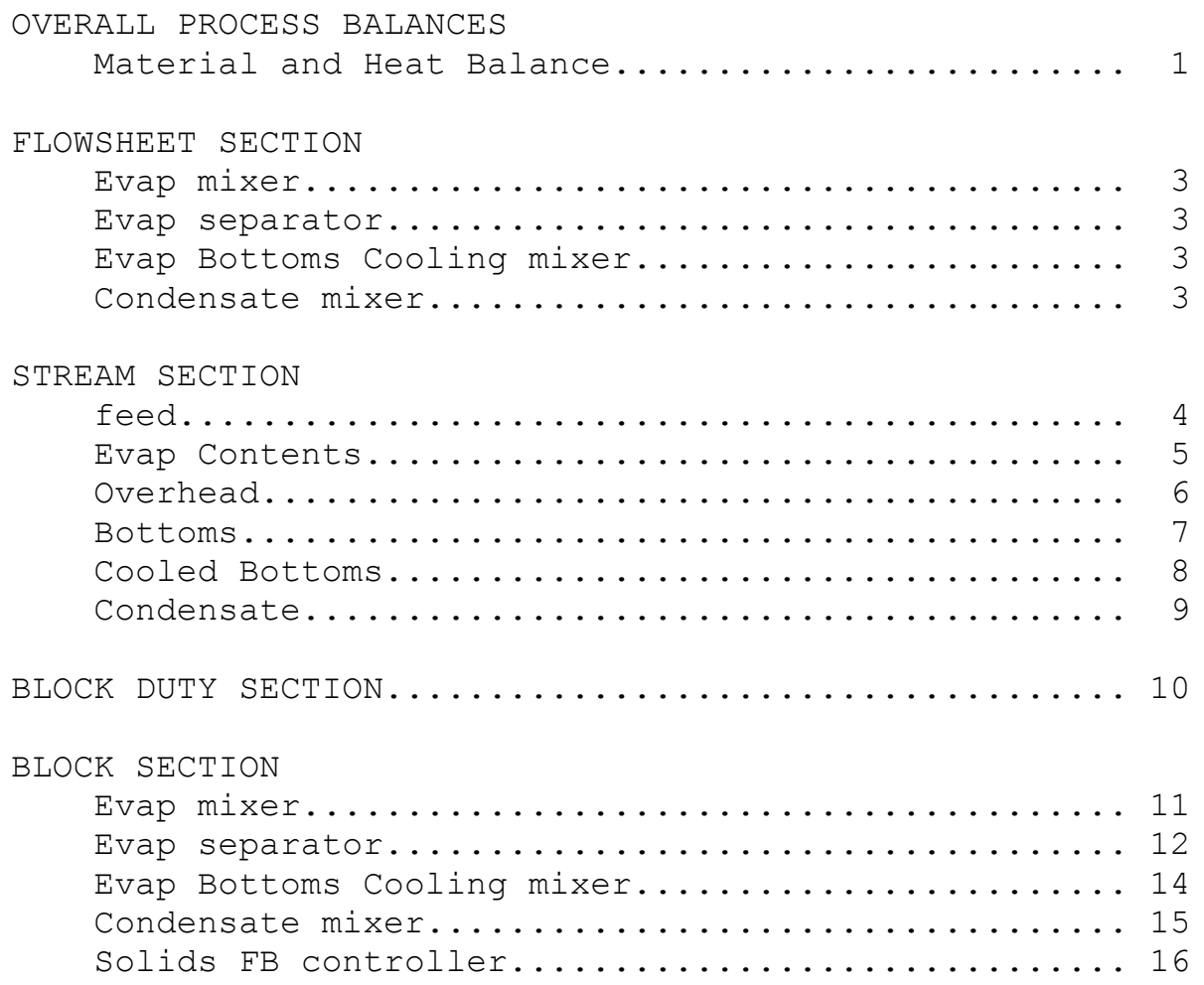


WSRC-TR-2002-00567

Revision 0

ESP V-6.6 PROCESS:AWE85_5
$\begin{array}{lcc}\text { Overall Process } & \text { Balances } \\ \text { Inlet } & \text { g/hr } & \mathrm{Cal} / \mathrm{hr} \\ ----------- & ---------- & ---------- \\ \text { FEED } & 5.00272 \mathrm{D}+02 & -1.89611 \mathrm{D}+06 \\ \text { =========== } & ========== & ======== \\ \text { Total in } & 5.00272 \mathrm{D}+02 & -1.89611 \mathrm{D}+06\end{array}$

\begin{tabular}{lrr} 
Outlet & \multicolumn{1}{c}{$g / \mathrm{hr}$} & $\mathrm{cal} / \mathrm{hr}$ \\
----------- & ---------- & ---------- \\
COOLED BOTTOMS & $1.60989 \mathrm{D}+00$ & $-5.32258 \mathrm{D}+03$ \\
CONDENSATE & $4.98663 \mathrm{D}+02$ & $-1.89077 \mathrm{D}+06$ \\
$==========$ & $==========$ & $==========$ \\
Total out & $5.00272 \mathrm{D}+02$ & $-1.89610 \mathrm{D}+06$
\end{tabular}

Block Heat Duties

EVAP MIXER

EVAP BOTTOMS COOLING MIXER

CONDENSATE MIXER

$=========$

Total Duty

DIFFERENCE

REL DIFEERENCE

$4.54747 \mathrm{D}-13$

9.08999D-16
$12 / 05 / 2002$

PAGE
1
Material Code Balances

Code
----------
$\mathrm{H}(+1)$
$\mathrm{K}(+1)$
$\mathrm{NA}(+1)$
$\mathrm{BA}(+2)$
$\mathrm{CA}(+2)$
$\mathrm{ZN}(+2)$
$\mathrm{CU}(+2)$
$\mathrm{FE}(+2)$
$\mathrm{MG}(+2)$
$\mathrm{PB}(+2)$
$\mathrm{AL}(+3)$
$\mathrm{NI}(+2)$
$\mathrm{O}(-2)$
$\mathrm{CL}(-1)$
$\mathrm{C}(+4)$
$\mathrm{P}(+5)$
$\mathrm{S}(+6)$
$\mathrm{N}(+5)$
$\mathrm{SI}(+4)$
$\mathrm{SR}(+2)$

$$
\begin{gathered}
\text { cal/hr } \\
----------- \\
3.08234 \mathrm{D}+05 \\
-9.63814 \mathrm{D}+01 \\
-3.08128 \mathrm{D}+05 \\
========== \\
9.18771 \mathrm{D}+00
\end{gathered}
$$

-----------

$0.00000 \mathrm{D}+00$

$0.00000 \mathrm{D}+00$

Input
mol/hr
--------
$5.54798 \mathrm{D}+01$
$5.20236 \mathrm{D}-04$
$3.53767 \mathrm{D}-03$
$6.71533 \mathrm{D}-07$
$9.97506 \mathrm{D}-04$
$3.82263 \mathrm{D}-05$
$6.77165 \mathrm{D}-06$
$1.02150 \mathrm{D}-04$
$3.78601 \mathrm{D}-04$
$3.42995 \mathrm{D}-06$
$1.03704 \mathrm{D}-04$
$1.49915 \mathrm{D}-06$
$2.77510 \mathrm{D}+01$
$2.81690 \mathrm{D}-03$
$1.50000 \mathrm{D}-03$
$2.63158 \mathrm{D}-04$
$9.58333 \mathrm{D}-04$
$7.25807 \mathrm{D}-04$
$2.33333 \mathrm{D}-04$
$2.16895 \mathrm{D}-05$

Outlet $\mathrm{mol} / \mathrm{hr}$

$5.54798 \mathrm{D}+01$

$5.20236 \mathrm{D}-04$

$3.53767 \mathrm{D}-03$

$6.71533 \mathrm{D}-07$

$9.97506 \mathrm{D}-04$

$3.82263 \mathrm{D}-05$

$6.77165 \mathrm{D}-06$

$1.02150 \mathrm{D}-04$

3. $78601 \mathrm{D}-04$

3. $42995 \mathrm{D}-06$

1.03704D-04

1. 49915D-06

2. $77510 \mathrm{D}+01$

2.81690D-03

1. $50000 \mathrm{D}-03$

2. $63158 \mathrm{D}-04$

9. 58333D-04

7. 25807D-04

$2.33333 \mathrm{D}-04$

2.16895D-05

363 of 447
Difference Rel Diff mol/hr

4.26326D-14

$4.33681 \mathrm{D}-19$

..33681D-19 1.22589D-16

$2.11758 \mathrm{D}-22 \quad 3.15336 \mathrm{D}-16$

$-2.16840 \mathrm{D}-19-2.17383 \mathrm{D}-16$

$0.00000 \mathrm{D}+00 \quad 0.00000 \mathrm{D}+00$

$0.00000 \mathrm{D}+00 \quad 0.00000 \mathrm{D}+00$

$2.71051 \mathrm{D}-20 \quad 2.65344 \mathrm{D}-16$

$1.08420 \mathrm{D}-192.86371 \mathrm{D}-16$

$3.38813 \mathrm{D}-219.87808 \mathrm{D}-16$

$0.00000 \mathrm{D}+00 \quad 0.00000 \mathrm{D}+00$

$-2.11758 \mathrm{D}-22-1.41252 \mathrm{D}-16$

$2.48690 \mathrm{D}-14 \quad 8.96147 \mathrm{D}-16$

$4.77049 \mathrm{D}-18 \quad 1.69352 \mathrm{D}-15$

$-1.51788 \mathrm{D}-18-1.01192 \mathrm{D}-15$

$0.00000 D+00 \quad 0.00000 D+00$

$1.08420 \mathrm{D}-19 \quad 1.13134 \mathrm{D}-16$

$-5.42101 D-19-7.46894 D-16$

$-2.71051 D-20-1.16165 \mathrm{D}-16$

$3.38813 \mathrm{D}-21 \quad 1.56211 \mathrm{D}-16$ 
WSRC-TR-2002-00567

Revision 0

$\mathrm{CD}(+2)$

$\mathrm{CR}(+3)$

$\mathrm{U}(+4)$
$4.64286 \mathrm{D}-08$

$1.21154 \mathrm{D}-07$

4.20168D-06

$4.64286 \mathrm{D}-08-4.03664 \mathrm{D}-22-8.69430 \mathrm{D}-15$

$1.21154 \mathrm{D}-07-2.64698 \mathrm{D}-23-2.18481 \mathrm{D}-16$

4.20168D-06 0.00000D+00 0.00000D+00 
WSRC-TR-2002-00567

Revision 0

ESP V-6.6

$\operatorname{DODEC}(-1)$
PROCESS:AWE 855

$5.57619 \mathrm{D}-05$
$12 / 05 / 2002$

PAGE

$5.57619 \mathrm{D}-05 \quad 1.07743 \mathrm{D}-18$

1. $93219 \mathrm{D}-14$ 
WSRC-TR-2002-00567

Revision 0

\begin{tabular}{|c|c|c|c|}
\hline \multicolumn{4}{|l|}{$\begin{array}{l}\text { PROCESS BLOCKS } \\
===========\end{array}$} \\
\hline $\begin{array}{l}\mathrm{BLOCK} \mathrm{NAME} \\
=========================\end{array}$ & $\begin{array}{l}\text { BLOCK TYPE } \\
===========\end{array}$ & $\begin{array}{l}\text { INLET STREAM }(\mathrm{S}) \\
==============\end{array}$ & $\begin{array}{l}\text { OUTLET STREAM }(\mathrm{S}) \\
==============\end{array}$ \\
\hline Evap mixer & $\operatorname{Mix}$ & feed & Evap Contents \\
\hline Evap separator & Separate & Evap Contents & $\begin{array}{l}\text { Overhead } \\
\text { Bottoms }\end{array}$ \\
\hline Evap Bottoms Cooling mixer & Mix & Bottoms & Cooled Bottoms \\
\hline Condensate mixer & Mix & Overhead & Condensate \\
\hline
\end{tabular}


WSRC-TR-2002-00567

Revision 0

ESP V-6. 6
PROCESS:AWE85 5
12/05/2002 PAGE 4

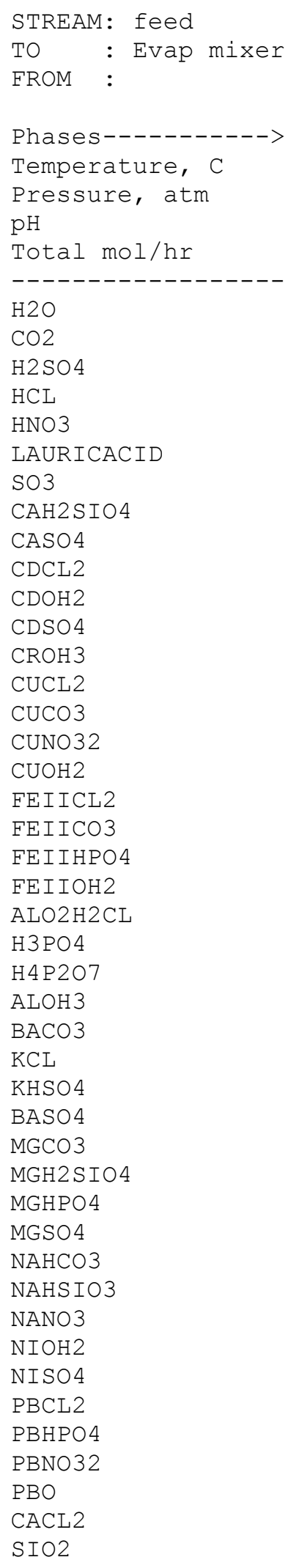

\begin{tabular}{|c|c|c|c|}
\hline Aqueous & Solid & Vapor & Organic \\
\hline 25 . & 25 . & 25 . & 25 . \\
\hline 1 . & 1 . & 1 . & 1 . \\
\hline \multicolumn{4}{|l|}{6.69293} \\
\hline 27.75084 & $1.79950 \mathrm{E}-04$ & 0.0 & 0.0 \\
\hline \multicolumn{4}{|c|}{$\mathrm{mol} / \mathrm{hr}--------\mathrm{mol} / \mathrm{hr}--------\mathrm{mol} / \mathrm{hr}--------\mathrm{mol} / \mathrm{hr}--------$} \\
\hline 27.7391 & 0.0 & 0.0 & 0.0 \\
\hline $4.06004 \mathrm{E}-04$ & 0.0 & 0.0 & 0.0 \\
\hline $7.82980 \mathrm{E}-26$ & 0.0 & 0.0 & 0.0 \\
\hline $2.96506 \mathrm{E}-16$ & 0.0 & 0.0 & 0.0 \\
\hline $6.37790 \mathrm{E}-12$ & 0.0 & 0.0 & 0.0 \\
\hline $7.95431 E-07$ & 0.0 & 0.0 & 0.0 \\
\hline $1.01381 E-29$ & 0.0 & 0.0 & 0.0 \\
\hline $3.46870 \mathrm{E}-12$ & 0.0 & 0.0 & 0.0 \\
\hline $1.88392 E-05$ & 0.0 & 0.0 & 0.0 \\
\hline $8.24872 \mathrm{E}-10$ & 0.0 & 0.0 & 0.0 \\
\hline $2.84297 E-15$ & 0.0 & 0.0 & 0.0 \\
\hline $6.60825 E-09$ & 0.0 & 0.0 & 0.0 \\
\hline $8.03814 E-13$ & 0.0 & 0.0 & 0.0 \\
\hline $3.26600 \mathrm{E}-13$ & 0.0 & 0.0 & 0.0 \\
\hline $1.27034 \mathrm{E}-08$ & 0.0 & 0.0 & 0.0 \\
\hline $4.54582 E-15$ & 0.0 & 0.0 & 0.0 \\
\hline $1.12539 \mathrm{E}-09$ & 0.0 & 0.0 & 0.0 \\
\hline $5.70810 \mathrm{E}-15$ & 0.0 & 0.0 & 0.0 \\
\hline $2.55522 \mathrm{E}-06$ & $2.05178 E-05$ & 0.0 & 0.0 \\
\hline $6.18267 E-08$ & 0.0 & 0.0 & 0.0 \\
\hline $2.83331 E-12$ & 0.0 & 0.0 & 0.0 \\
\hline $5.73773 E-29$ & 0.0 & 0.0 & 0.0 \\
\hline $2.40706 \mathrm{E}-09$ & 0.0 & 0.0 & 0.0 \\
\hline $2.06228 E-20$ & 0.0 & 0.0 & 0.0 \\
\hline $1.19860 \mathrm{E}-09$ & $1.03701 E-04$ & 0.0 & 0.0 \\
\hline $9.68675 E-12$ & 0.0 & 0.0 & 0.0 \\
\hline $1.84688 \mathrm{E}-08$ & 0.0 & 0.0 & 0.0 \\
\hline $1.72286 E-13$ & 0.0 & 0.0 & 0.0 \\
\hline $1.02726 \mathrm{E}-10$ & $5.73454 \mathrm{E}-07$ & 0.0 & 0.0 \\
\hline $7.96406 E-08$ & 0.0 & 0.0 & 0.0 \\
\hline $1.37612 \mathrm{E}-11$ & 0.0 & 0.0 & 0.0 \\
\hline $1.22640 \mathrm{E}-05$ & 0.0 & 0.0 & 0.0 \\
\hline $1.34768 E-05$ & 0.0 & 0.0 & 0.0 \\
\hline $2.33418 E-06$ & 0.0 & 0.0 & 0.0 \\
\hline $1.22831 E-07$ & 0.0 & 0.0 & 0.0 \\
\hline $2.23268 E-07$ & 0.0 & 0.0 & 0.0 \\
\hline 1. $92588 \mathrm{E}-12$ & 0.0 & 0.0 & 0.0 \\
\hline $1.65072 E-07$ & 0.0 & 0.0 & 0.0 \\
\hline $6.62673 E-12$ & 0.0 & 0.0 & 0.0 \\
\hline $2.32123 E-10$ & 0.0 & 0.0 & 0.0 \\
\hline 8. $41962 E-14$ & 0.0 & 0.0 & 0.0 \\
\hline $6.71513 E-13$ & 0.0 & 0.0 & 0.0 \\
\hline $1.10010 \mathrm{E}-26$ & 0.0 & 0.0 & 0.0 \\
\hline $2.33016 \mathrm{E}-04$ & 0.0 & 0.0 & 0.0 \\
\hline
\end{tabular}




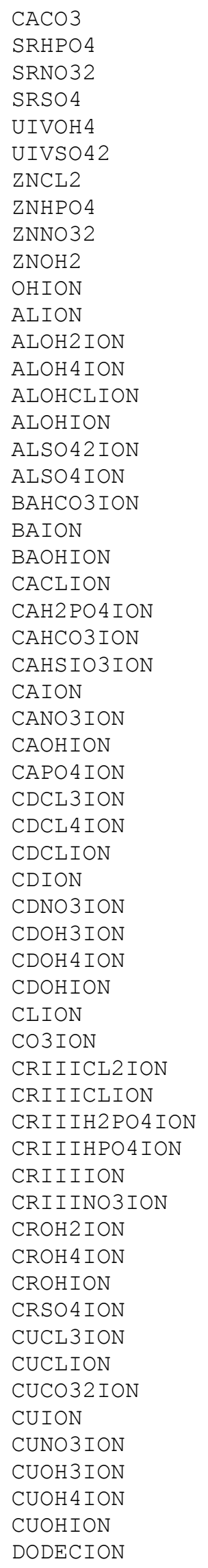

\begin{tabular}{|c|c|}
\hline $4.30015 E-07$ & 0.0 \\
\hline $1.07235 \mathrm{E}-08$ & 0.0 \\
\hline $36 \mathrm{E}-10$ & 0.0 \\
\hline $324 E-06$ & \\
\hline $43 E-10$ & 0.0 \\
\hline $16787 \mathrm{E}-28$ & 0.0 \\
\hline $34 E-10$ & 0.0 \\
\hline $.69533 E-06$ & 0.0 \\
\hline $3 E-11$ & 0.0 \\
\hline $56 E-09$ & 0.0 \\
\hline $00 E-08$ & 0.0 \\
\hline $08 E-13$ & 0.0 \\
\hline$E-11$ & 0.0 \\
\hline $1.69885 E-09$ & 0.0 \\
\hline $6 E-14$ & 0.0 \\
\hline $3.35223 E-12$ & 0.0 \\
\hline $5 E-15$ & 0.0 \\
\hline$E-14$ & 0.0 \\
\hline$E-09$ & 0.0 \\
\hline $98 E-08$ & 0.0 \\
\hline $3 E-15$ & 0.0 \\
\hline $4 E-11$ & 0.0 \\
\hline$E-06$ & 0.0 \\
\hline $5 E-05$ & 0.0 \\
\hline $8 E-09$ & 0.0 \\
\hline $9 E-04$ & 0.0 \\
\hline $5 E-06$ & 0.0 \\
\hline $3 E-10$ & 0.0 \\
\hline $\mathrm{E}-07$ & 0.0 \\
\hline $3 E-13$ & 0.0 \\
\hline $7 E-16$ & 0.0 \\
\hline $8 E-09$ & 0.0 \\
\hline $2 E-08$ & 0.0 \\
\hline $5 E-11$ & 0.0 \\
\hline$O E-21$ & 0.0 \\
\hline $7 E-29$ & 0.0 \\
\hline $7 E-12$ & 0.0 \\
\hline 1671 & 0.0 \\
\hline $1 E-07$ & 0.0 \\
\hline $7 E-19$ & 0.0 \\
\hline $3 E-16$ & 0.0 \\
\hline$L E-14$ & 0.0 \\
\hline $7 E-07$ & 0.0 \\
\hline $5 E-14$ & 0.0 \\
\hline $2 E-15$ & 0.0 \\
\hline $1 E-14$ & 0.0 \\
\hline $35 E-17$ & 0.0 \\
\hline $0 E-12$ & 0.0 \\
\hline $31 E-11$ & 0.0 \\
\hline $2 E-18$ & 0.0 \\
\hline 1. $66152 \mathrm{E}-10$ & 0.0 \\
\hline $8 E-11$ & 0.0 \\
\hline $3 E-09$ & 0.0 \\
\hline $33 E-11$ & 0.0 \\
\hline $1 E-15$ & 0.0 \\
\hline $1.90303 E-21$ & 0.0 \\
\hline $8 E-09$ & 0.0 \\
\hline $5.49665 E-05$ & 0.0 \\
\hline
\end{tabular}

0.0

0.0

0.0

0.0

0.0

0.0

0.0

0.0

0.0

0.0

0.0

0.0

0.0

0.0

0.0

0.0

0.0

0.0

0.0

0.0

0.0

0.0

0.0

0.0

0.0

0.0

0.0

0.0

0.0

0.0

0.0

0.0

0.0

0.0

0.0

0.0

0.0

0.0

0.0

0.0

0.0

0.0

0.0

0.0

0.0

0.0

0.0

0.0

0.0

0.0

0.0

0.0

0.0

0.0

0.0

0.0

0.0

0.0
0.0

0.0

0.0

0.0

0.0

0.0

0.0

0.0

0.0

0.0

0.0

0.0

0.0

0.0

0.0

0.0

0.0

0.0

0.0

0.0

0.0

0.0

0.0

0.0

0.0

0.0

0.0

0.0

0.0

0.0

0.0

0.0

0.0

0.0

0.0

0.0

0.0

0.0

0.0

0.0

0.0

0.0

0.0

0.0

0.0

0.0

0.0

0.0

0.0

0.0

0.0

0.0

0.0

0.0

0.0

0.0

0.0

0.0 


\begin{tabular}{|c|c|c|c|c|}
\hline FEIICLION & $3.59036 \mathrm{E}-10$ & 0.0 & 0.0 & 0.0 \\
\hline FEIICO32ION & $6.09771 \mathrm{E}-11$ & 0.0 & 0.0 & 0.0 \\
\hline FEIIH2PO4ION & $2.91842 \mathrm{E}-08$ & 0.0 & 0.0 & 0.0 \\
\hline FEIIHCO3ION & $1.41194 \mathrm{E}-08$ & 0.0 & 0.0 & 0.0 \\
\hline FEIIION & $7.88893 \mathrm{E}-05$ & 0.0 & 0.0 & 0.0 \\
\hline FEIIOH3ION & $6.32285 \mathrm{E}-16$ & 0.0 & 0.0 & 0.0 \\
\hline FEIIOH 4 ION & $4.83482 \mathrm{E}-24$ & 0.0 & 0.0 & 0.0 \\
\hline FEIIOHION & $8.24598 \mathrm{E}-08$ & 0.0 & 0.0 & 0.0 \\
\hline H2 P2O 7 ION & $1.11461 \mathrm{E}-10$ & 0.0 & 0.0 & 0.0 \\
\hline H2 PO 4 ION & $9.64959 \mathrm{E}-05$ & 0.0 & 0.0 & 0.0 \\
\hline H2SIO4ION & $1.10679 \mathrm{E}-13$ & 0.0 & 0.0 & 0.0 \\
\hline H3P2O 7 ION & $3.31378 \mathrm{E}-15$ & 0.0 & 0.0 & 0.0 \\
\hline H3SIO4 ION & $1.88514 \mathrm{E}-07$ & 0.0 & 0.0 & 0.0 \\
\hline HCO3ION & 0.00104005 & 0.0 & 0.0 & 0.0 \\
\hline HION & $1.15531 \mathrm{E}-07$ & 0.0 & 0.0 & 0.0 \\
\hline HP207ION & $2.69249 \mathrm{E}-10$ & 0.0 & 0.0 & 0.0 \\
\hline HPBO2ION & $4.25078 \mathrm{E}-17$ & 0.0 & 0.0 & 0.0 \\
\hline HPO4 ION & $4.47688 \mathrm{E}-05$ & 0.0 & 0.0 & 0.0 \\
\hline HSO 4 ION & $1.13390 \mathrm{E}-08$ & 0.0 & 0.0 & 0.0 \\
\hline KION & $5.16182 \mathrm{E}-04$ & 0.0 & 0.0 & 0.0 \\
\hline KSO4 ION & $4.03562 \mathrm{E}-06$ & 0.0 & 0.0 & 0.0 \\
\hline MGH2 PO 4 ION & $1.81397 \mathrm{E}-06$ & 0.0 & 0.0 & 0.0 \\
\hline MGHCO3ION & $1.56153 \mathrm{E}-05$ & 0.0 & 0.0 & 0.0 \\
\hline MGHSIO3ION & $2.52212 \mathrm{E}-09$ & 0.0 & 0.0 & 0.0 \\
\hline MGION & $3.35194 \mathrm{E}-04$ & 0.0 & 0.0 & 0.0 \\
\hline MGOHION & $1.84607 \mathrm{E}-09$ & 0.0 & 0.0 & 0.0 \\
\hline MGP2O 7 ION & $2.71716 \mathrm{E}-09$ & 0.0 & 0.0 & 0.0 \\
\hline MGPO 4 ION & $1.49454 \mathrm{E}-07$ & 0.0 & 0.0 & 0.0 \\
\hline NACO3ION & $5.22967 \mathrm{E}-09$ & 0.0 & 0.0 & 0.0 \\
\hline NAION & 0.00350481 & 0.0 & 0.0 & 0.0 \\
\hline NASO 4 ION & $3.01813 E-05$ & 0.0 & 0.0 & 0.0 \\
\hline NICLION & $4.38692 \mathrm{E}-10$ & 0.0 & 0.0 & 0.0 \\
\hline NIION & $1.32977 \mathrm{E}-06$ & 0.0 & 0.0 & 0.0 \\
\hline NINO3ION & $3.25042 E-09$ & 0.0 & 0.0 & 0.0 \\
\hline NIOH3ION & $1.11644 \mathrm{E}-16$ & 0.0 & 0.0 & 0.0 \\
\hline NIOHION & $6.10857 \mathrm{E}-10$ & 0.0 & 0.0 & 0.0 \\
\hline NO3ION & $7.23573 \mathrm{E}-04$ & 0.0 & 0.0 & 0.0 \\
\hline P207ION & $1.72366 \mathrm{E}-12$ & 0.0 & 0.0 & 0.0 \\
\hline PBCL3ION & $1.83453 \mathrm{E}-14$ & 0.0 & 0.0 & 0.0 \\
\hline PBCL 4 ION & $8.56782 \mathrm{E}-17$ & 0.0 & 0.0 & 0.0 \\
\hline PBCLION & $4.23970 \mathrm{E}-10$ & 0.0 & 0.0 & 0.0 \\
\hline PBH2 PO 4 ION & $2.18361 \mathrm{E}-11$ & 0.0 & 0.0 & 0.0 \\
\hline PBION & $4.70897 \mathrm{E}-09$ & 0.0 & 0.0 & 0.0 \\
\hline PBNO33ION & $2.09219 \mathrm{E}-17$ & 0.0 & 0.0 & 0.0 \\
\hline PBNO3ION & $7.85300 \mathrm{E}-11$ & 0.0 & 0.0 & 0.0 \\
\hline PBOHION & $3.93329 \mathrm{E}-10$ & 0.0 & 0.0 & 0.0 \\
\hline PO4ION & $2.06772 \mathrm{E}-10$ & 0.0 & 0.0 & 0.0 \\
\hline SO4ION & $8.88725 \mathrm{E}-04$ & 0.0 & 0.0 & 0.0 \\
\hline SRION & $1.92677 \mathrm{E}-05$ & 0.0 & 0.0 & 0.0 \\
\hline SRNO3ION & $9.26073 \mathrm{E}-08$ & 0.0 & 0.0 & 0.0 \\
\hline SROHION & $4.12798 \mathrm{E}-12$ & 0.0 & 0.0 & 0.0 \\
\hline SRPO 4 ION & $8.32086 \mathrm{E}-11$ & 0.0 & 0.0 & 0.0 \\
\hline UIVION & 0.0 & 0.0 & 0.0 & 0.0 \\
\hline UIVOH2ION & $1.28162 \mathrm{E}-21$ & 0.0 & 0.0 & 0.0 \\
\hline UIVOH3ION & $3.94016 \mathrm{E}-16$ & 0.0 & 0.0 & 0.0 \\
\hline UIVOH5ION & $4.45068 \mathrm{E}-14$ & 0.0 & 0.0 & 0.0 \\
\hline UIVOHION & $7.58308 \mathrm{E}-26$ & 0.0 & 0.0 & 0.0 \\
\hline UIVSO 4 ION & $4.94799 \mathrm{E}-29$ & 0.0 & 0.0 & 0.0 \\
\hline
\end{tabular}


WSRC-TR-2002-00567

Revision 0

\begin{tabular}{|c|c|c|c|c|}
\hline ZNCL3ION & $1.45559 \mathrm{E}-12$ & 0.0 & 0.0 & 0.0 \\
\hline ZNCLION & $1.56826 \mathrm{E}-07$ & 0.0 & 0.0 & 0.0 \\
\hline ZNH2 PO4 ION & $1.30277 E-07$ & 0.0 & 0.0 & 0.0 \\
\hline ZNHCO3ION & $7.97109 \mathrm{E}-07$ & 0.0 & 0.0 & 0.0 \\
\hline ZNION & $2.99922 E-05$ & 0.0 & 0.0 & 0.0 \\
\hline ZNNO3ION & $7.32658 \mathrm{E}-08$ & 0.0 & 0.0 & 0.0 \\
\hline ZNOH3ION & $1.17501 \mathrm{E}-13$ & 0.0 & 0.0 & 0.0 \\
\hline ZNOH 4 ION & $1.12660 \mathrm{E}-19$ & 0.0 & 0.0 & 0.0 \\
\hline ZNOHION & $1.20375 \mathrm{E}-07$ & 0.0 & 0.0 & 0.0 \\
\hline $\mathrm{CA} 3 \mathrm{PO} 42$ & 0.0 & $4.58136 \mathrm{E}-05$ & 0.0 & 0.0 \\
\hline $\mathrm{CU} 3 \mathrm{PO} 42.2 \mathrm{H} 2 \mathrm{O}$ & 0.0 & $2.24895 E-06$ & 0.0 & 0.0 \\
\hline РВ3РО42 & 0.0 & $1.14136 \mathrm{E}-06$ & 0.0 & 0.0 \\
\hline UIVO2 & 0.0 & $4.20151 E-06$ & 0.0 & 0.0 \\
\hline \multirow[t]{2}{*}{$\mathrm{ZN} 3 \mathrm{PO} 42.2 \mathrm{H} 2 \mathrm{O}$} & 0.0 & $1.75265 \mathrm{E}-06$ & 0.0 & 0.0 \\
\hline & $============$ & $============$ & $============$ & $============$ \\
\hline Total g/hr & 500.244 & 0.028548 & 0.0 & 0.0 \\
\hline Volume, L/hr & 0.501428 & $8.65328 E-06$ & 0.0 & 0.0 \\
\hline Enthalpy, cal/hr & $-1.89602 \mathrm{E}+06$ & -84.0643 & 0.0 & 0.0 \\
\hline Density, g/L & 997.638 & 3299.09 & & \\
\hline Vapor fraction & 0.0 & 0.0 & 0.0 & 0.0 \\
\hline Solid fraction & 0.0 & 1. & 0.0 & 0.0 \\
\hline Organic fraction & 0.0 & 0.0 & 0.0 & 0.0 \\
\hline Osmotic Pres, atm & 0.621071 & & & \\
\hline Redox Pot, volts & 0.0 & & & \\
\hline E-Con, $1 / \mathrm{ohm}-\mathrm{cm}$ & 0.00143311 & & & \\
\hline E-Con, cm2/ohm-mol & 119.881 & & & \\
\hline Abs Visc, cP & 0.894055 & & & \\
\hline Rel Visc & 1.00374 & & & \\
\hline Ionic Strength & 0.0177239 & & & \\
\hline
\end{tabular}


WSRC-TR-2002-00567

Revision 0

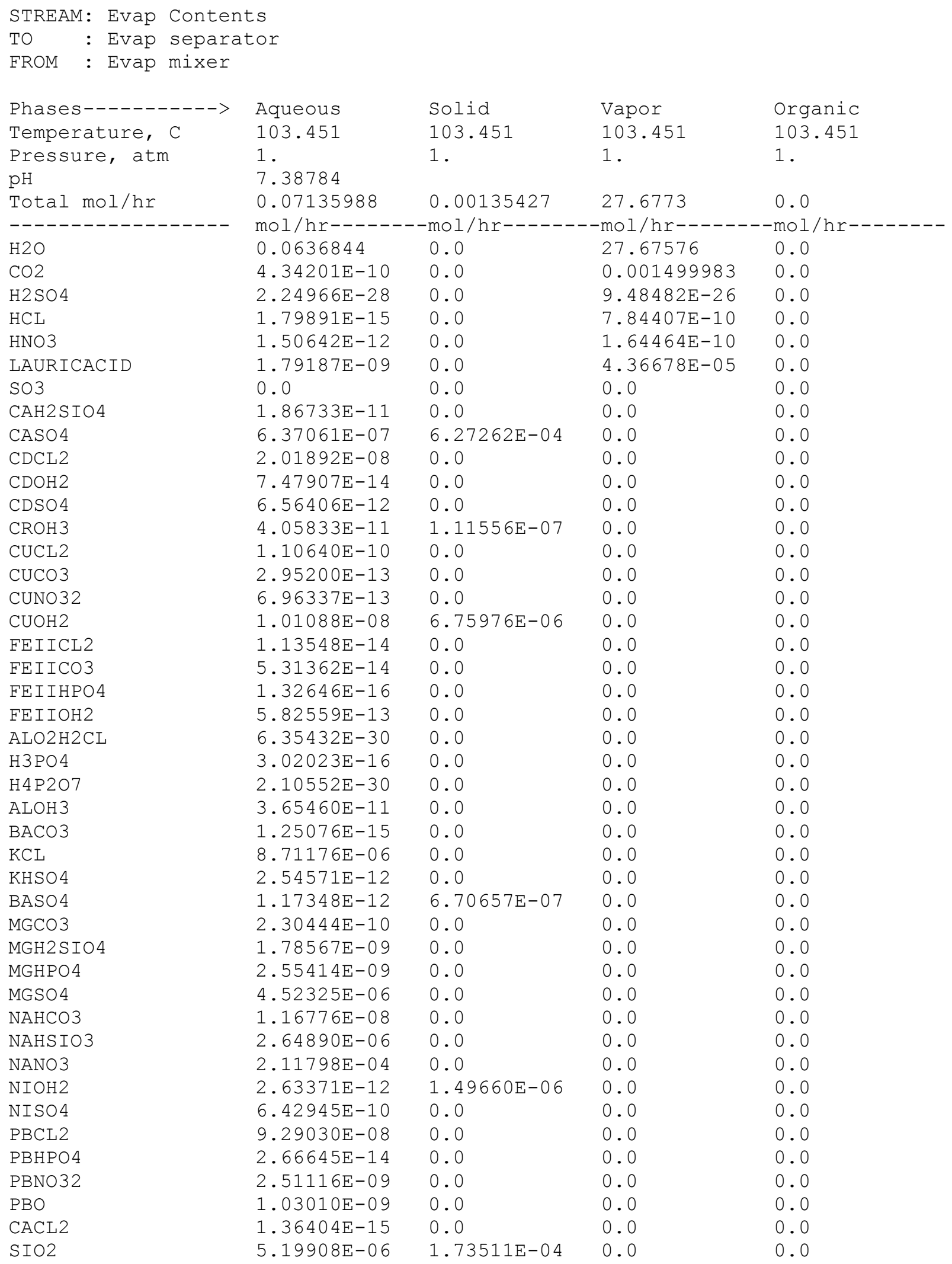




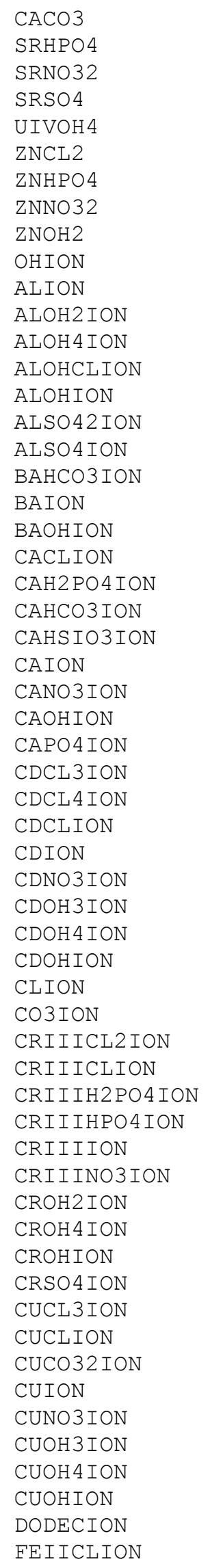

$\begin{array}{ll}7.26897 \mathrm{E}-11 & 0.0 \\ 4.58703 \mathrm{E}-14 & 0.0 \\ 2.86989 \mathrm{E}-08 & 0.0 \\ 1.09816 \mathrm{E}-08 & 2.15367 \mathrm{E}-05 \\ 1.34872 \mathrm{E}-12 & 0.0 \\ 3.20149 \mathrm{E}-06 & 0.0 \\ 2.84323 \mathrm{E}-12 & 0.0 \\ 1.22405 \mathrm{E}-09 & 0.0 \\ 4.12634 \mathrm{E}-08 & 0.0 \\ 2.50321 \mathrm{E}-08 & 0.0 \\ 1.09069 \mathrm{E}-21 & 0.0 \\ 2.11446 \mathrm{E}-14 & 0.0 \\ 9.32633 \mathrm{E}-09 & 0.0 \\ 4.01706 \mathrm{E}-17 & 0.0 \\ 9.87791 \mathrm{E}-18 & 0.0 \\ 5.76390 \mathrm{E}-21 & 0.0 \\ 8.40141 \mathrm{E}-21 & 0.0 \\ 9.49527 \mathrm{E}-14 & 0.0 \\ 8.74076 \mathrm{E}-10 & 0.0 \\ 8.64824 \mathrm{E}-15 & 0.0 \\ 3.95372 \mathrm{E}-08 & 0.0 \\ 1.00006 \mathrm{E}-11 & 0.0 \\ 1.55488 \mathrm{E}-10 & 0.0 \\ 8.66800 \mathrm{E}-09 & 0.0 \\ 9.02446 \mathrm{E}-06 & 0.0 \\ 3.45508 \mathrm{E}-06 & 0.0 \\ 1.72017 \mathrm{E}-09 & 0.0 \\ 5.26295 \mathrm{E}-11 & 0.0 \\ 1.95720 \mathrm{E}-08 & 0.0 \\ 5.42991 \mathrm{E}-09 & 0.0 \\ 1.20077 \mathrm{E}-09 & 0.0 \\ 2.31756 \mathrm{E}-11 & 0.0 \\ 6.21236 \mathrm{E}-12 & 0.0 \\ 1.62956 \mathrm{E}-17 & 0.0 \\ 2.05265 \mathrm{E}-21 & 0.0 \\ 6.85742 \mathrm{E}-13 & 0.0 \\ 0.00277442 & 0.0 \\ 2.31581 \mathrm{E}-10 & 0.0 \\ 5.39856 \mathrm{E}-16 & 0.0 \\ 4.50681 \mathrm{E}-16 & 0.0 \\ 1.79499 \mathrm{E}-20 & 0.0 \\ 9.37721 \mathrm{E}-09 & 0.0 \\ 5.62589 \mathrm{E}-20 & 0.0 \\ 2.20116 \mathrm{E}-13 & 0.0 \\ 6.08063 \mathrm{E}-13 & 0.0 \\ 1.35798 \mathrm{E}-13 & 0.0 \\ 1.70077 \mathrm{E}-10 & 0.0 \\ 8.58073 \mathrm{E}-12 & 0.0 \\ 1.83815 \mathrm{E}-12 & 0.0 \\ 7.24412 \mathrm{E}-10 & 0.0 \\ 3.35413 \mathrm{E}-16 & 0.0 \\ 1.99293 \mathrm{E}-10 & 0.0 \\ 3.40488 \mathrm{E}-11 & 0.0 \\ 1.38033 \mathrm{E}-11 & 0.0 \\ 4.16764 \mathrm{E}-14 & 0.0 \\ 6.97436 \mathrm{E}-10 & 0.0 \\ 1.20924 \mathrm{E}-05 & 0.0 \\ 2.47667 \mathrm{E}-12 & 0.0 \\ & \\ 1.0 & \end{array}$

0.0

0.0

0.0

0.0

0.0

0.0

0.0

0.0

0.0

0.0

0.0

0.0

0.0

0.0

0.0

0.0

0.0

0.0

0.0

0.0

0.0

0.0

0.0

0.0

0.0

0.0

0.0

0.0

0.0

0.0

0.0

0.0

0.0

0.0

0.0

0.0

0.0

0.0

0.0

0.0

0.0

0.0

0.0

0.0

0.0

0.0

0.0

0.0

0.0

0.0

0.0

0.0

0.0

0.0

0.0

0.0

0.0

0.0

0.0
0.0

0.0

0.0

0.0

0.0

0.0

0.0

0.0

0.0

0.0

0.0

0.0

0.0

0.0

0.0

0.0

0.0

0.0

0.0

0.0

0.0

0.0

0.0

0.0

0.0

0.0

0.0

0.0

0.0

0.0

0.0

0.0

0.0

0.0

0.0

0.0

0.0

0.0

0.0

0.0

0.0

0.0

0.0

0.0

0.0

0.0

0.0

0.0

0.0

0.0

0.0

0.0

0.0

0.0

0.0

0.0

0.0 


\begin{tabular}{|c|c|c|c|c|}
\hline FEIICO32ION & $1.78873 \mathrm{E}-18$ & 0.0 & 0.0 & 0.0 \\
\hline FEIIH2PO4ION & $1.25717 \mathrm{E}-17$ & 0.0 & 0.0 & 0.0 \\
\hline EEIIHCO3ION & $1.27982 \mathrm{E}-16$ & 0.0 & 0.0 & 0.0 \\
\hline FEIIION & $3.04354 \mathrm{E}-10$ & 0.0 & 0.0 & 0.0 \\
\hline FEIIOH3ION & $1.65772 \mathrm{E}-14$ & 0.0 & 0.0 & 0.0 \\
\hline FEIIOH 4 ION & $5.79145 \mathrm{E}-19$ & 0.0 & 0.0 & 0.0 \\
\hline FEIIOHION & $3.60199 \mathrm{E}-11$ & 0.0 & 0.0 & 0.0 \\
\hline H2 P2O 7 ION & $1.90460 \mathrm{E}-18$ & 0.0 & 0.0 & 0.0 \\
\hline H2 PO 4 ION & $8.74013 \mathrm{E}-11$ & 0.0 & 0.0 & 0.0 \\
\hline $\mathrm{H} 2 \mathrm{SIO} 4 \mathrm{ION}$ & $1.77925 \mathrm{E}-11$ & 0.0 & 0.0 & 0.0 \\
\hline H3P2O7 ION & $1.61286 \mathrm{E}-24$ & 0.0 & 0.0 & 0.0 \\
\hline H3SIO 4 ION & $7.80273 \mathrm{E}-07$ & 0.0 & 0.0 & 0.0 \\
\hline HCO3ION & $7.71356 \mathrm{E}-09$ & 0.0 & 0.0 & 0.0 \\
\hline HION & $6.04790 \mathrm{E}-11$ & 0.0 & 0.0 & 0.0 \\
\hline HP207ION & $4.30218 \mathrm{E}-17$ & 0.0 & 0.0 & 0.0 \\
\hline HPBO2ION & $1.32990 \mathrm{E}-11$ & 0.0 & 0.0 & 0.0 \\
\hline HPO4ION & $1.20700 \mathrm{E}-09$ & 0.0 & 0.0 & 0.0 \\
\hline $\mathrm{HSO} 4 \mathrm{ION}$ & $4.62419 \mathrm{E}-10$ & 0.0 & 0.0 & 0.0 \\
\hline KION & $4.61072 \mathrm{E}-04$ & 0.0 & 0.0 & 0.0 \\
\hline KSO4 ION & $5.04522 \mathrm{E}-05$ & 0.0 & 0.0 & 0.0 \\
\hline MGH2 PO 4 ION & $2.13661 \mathrm{E}-11$ & 0.0 & 0.0 & 0.0 \\
\hline MGHCO3ION & $2.09549 \mathrm{E}-09$ & 0.0 & 0.0 & 0.0 \\
\hline MGHSIO3ION & $1.08471 \mathrm{E}-07$ & 0.0 & 0.0 & 0.0 \\
\hline MGION & $2.15352 \mathrm{E}-05$ & 0.0 & 0.0 & 0.0 \\
\hline MGOHION & $9.08930 \mathrm{E}-08$ & 0.0 & 0.0 & 0.0 \\
\hline MGP2O7ION & $1.98806 \mathrm{E}-13$ & 0.0 & 0.0 & 0.0 \\
\hline MGPO 4 ION & $3.79546 \mathrm{E}-10$ & 0.0 & 0.0 & 0.0 \\
\hline NACO3ION & $3.69506 \mathrm{E}-11$ & 0.0 & 0.0 & 0.0 \\
\hline NAION & 0.00332321 & 0.0 & 0.0 & 0.0 \\
\hline NASO 4 ION & $3.92144 \mathrm{E}-13$ & 0.0 & 0.0 & 0.0 \\
\hline NICLION & $1.29786 \mathrm{E}-10$ & 0.0 & 0.0 & 0.0 \\
\hline NIION & $1.49902 \mathrm{E}-09$ & 0.0 & 0.0 & 0.0 \\
\hline NINO3ION & $2.14768 \mathrm{E}-10$ & 0.0 & 0.0 & 0.0 \\
\hline NIOH3ION & $8.89693 \mathrm{E}-15$ & 0.0 & 0.0 & 0.0 \\
\hline NIOHION & $5.49134 \mathrm{E}-11$ & 0.0 & 0.0 & 0.0 \\
\hline NO3ION & $5.10322 \mathrm{E}-04$ & 0.0 & 0.0 & 0.0 \\
\hline P207ION & $1.22128 \mathrm{E}-17$ & 0.0 & 0.0 & 0.0 \\
\hline PBCL3ION & $2.05078 \mathrm{E}-07$ & 0.0 & 0.0 & 0.0 \\
\hline PBCL 4 ION & $1.65430 \mathrm{E}-06$ & 0.0 & 0.0 & 0.0 \\
\hline PBCLION & $2.73174 \mathrm{E}-08$ & 0.0 & 0.0 & 0.0 \\
\hline PBH2PO 4 ION & $1.23910 \mathrm{E}-15$ & 0.0 & 0.0 & 0.0 \\
\hline PBION & $1.29408 \mathrm{E}-09$ & 0.0 & 0.0 & 0.0 \\
\hline PBNO33ION & $9.30935 \mathrm{E}-11$ & 0.0 & 0.0 & 0.0 \\
\hline PBNO3ION & $7.60984 \mathrm{E}-09$ & 0.0 & 0.0 & 0.0 \\
\hline PBOHION & $2.07438 \mathrm{E}-08$ & 0.0 & 0.0 & 0.0 \\
\hline PO4ION & $2.37021 \mathrm{E}-13$ & 0.0 & 0.0 & 0.0 \\
\hline SO4ION & $2.53239 \mathrm{E}-04$ & 0.0 & 0.0 & 0.0 \\
\hline SRION & $5.72314 \mathrm{E}-10$ & 0.0 & 0.0 & 0.0 \\
\hline SRNO3ION & $1.12544 \mathrm{E}-07$ & 0.0 & 0.0 & 0.0 \\
\hline SROHION & $1.30499 \mathrm{E}-11$ & 0.0 & 0.0 & 0.0 \\
\hline SRPO 4 ION & $1.19552 \mathrm{E}-14$ & 0.0 & 0.0 & 0.0 \\
\hline UIVOH2ION & $4.60321 \mathrm{E}-26$ & 0.0 & 0.0 & 0.0 \\
\hline UIVOH3ION & $1.73992 \mathrm{E}-20$ & 0.0 & 0.0 & 0.0 \\
\hline UIVOH5ION & $1.70497 \mathrm{E}-14$ & 0.0 & 0.0 & 0.0 \\
\hline UIVOHION & 0.0 & 0.0 & 0.0 & 0.0 \\
\hline ZNCL3ION & $4.09188 \mathrm{E}-06$ & 0.0 & 0.0 & 0.0 \\
\hline ZNCLION & $7.48267 \mathrm{E}-06$ & 0.0 & 0.0 & 0.0 \\
\hline ZNH2PO 4 ION & $6.41653 \mathrm{E}-14$ & 0.0 & 0.0 & 0.0 \\
\hline
\end{tabular}


WSRC-TR-2002-00567

Revision 0

\begin{tabular}{|c|c|c|c|c|}
\hline ZNHCO3ION & $5.33856 \mathrm{E}-12$ & 0.0 & 0.0 & 0.0 \\
\hline ZNION & $4.52602 \mathrm{E}-07$ & 0.0 & 0.0 & 0.0 \\
\hline ZNNO3ION & $4.59099 E-08$ & 0.0 & 0.0 & 0.0 \\
\hline ZNOH3ION & $2.12228 E-09$ & 0.0 & 0.0 & 0.0 \\
\hline ZNOH $4 \mathrm{ION}$ & $2.29426 \mathrm{E}-12$ & 0.0 & 0.0 & 0.0 \\
\hline ZNOHION & $4.49166 \mathrm{E}-06$ & 0.0 & 0.0 & 0.0 \\
\hline ALOOH & 0.0 & $1.54416 \mathrm{E}-06$ & 0.0 & 0.0 \\
\hline $\mathrm{CA} 3 \mathrm{PO} 42$ & 0.0 & $1.19026 \mathrm{E}-04$ & 0.0 & 0.0 \\
\hline CHAMOSITE7A & 0.0 & $5.10751 E-05$ & 0.0 & 0.0 \\
\hline MG3PO4 2 & 0.0 & $5.93559 \mathrm{E}-06$ & 0.0 & 0.0 \\
\hline MGOH2 & 0.0 & $3.34529 E-04$ & 0.0 & 0.0 \\
\hline PB3PO42 & 0.0 & $4.72353 E-07$ & 0.0 & 0.0 \\
\hline UIVO2 & 0.0 & $4.20168 E-06$ & 0.0 & 0.0 \\
\hline \multirow[t]{2}{*}{$\mathrm{ZN} 3 \mathrm{PO} 42.2 \mathrm{H} 2 \mathrm{O}$} & 0.0 & $6.13848 \mathrm{E}-06$ & 0.0 & 0.0 \\
\hline & $============$ & $============$ & $===========$ & $===========$ \\
\hline Total g/hr & 1.4295 & 0.18039 & 498.663 & 0.0 \\
\hline Volume, L/hr & 0.00132219 & $5.48580 \mathrm{E}-05$ & 848.335 & 0.0 \\
\hline Enthalpy, cal/hr & -4719.19 & -507.005 & $-1.58265 \mathrm{E}+06$ & 0.0 \\
\hline Density, g/L & 1081.16 & 3288.32 & 0.587813 & \\
\hline Vapor fraction & 0.0 & 0.0 & 1 . & 0.0 \\
\hline Solid fraction & 0.0 & 1. & 0.0 & 0.0 \\
\hline Organic fraction & 0.0 & 0.0 & 0.0 & 0.0 \\
\hline Osmotic Pres, atm & 196.506 & & & \\
\hline Redox Pot, volts & 0.0 & & & \\
\hline E-Con, $1 / \mathrm{ohm}-\mathrm{cm}$ & 0.501471 & & & \\
\hline E-Con, cm2/ohm-mol & 58.4688 & & & \\
\hline Abs Visc, cP & 0.421849 & & & \\
\hline Rel Visc & 1.55267 & & & \\
\hline Ionic Strength & 3.61561 & & & \\
\hline
\end{tabular}


WSRC-TR-2002-00567

Revision 0

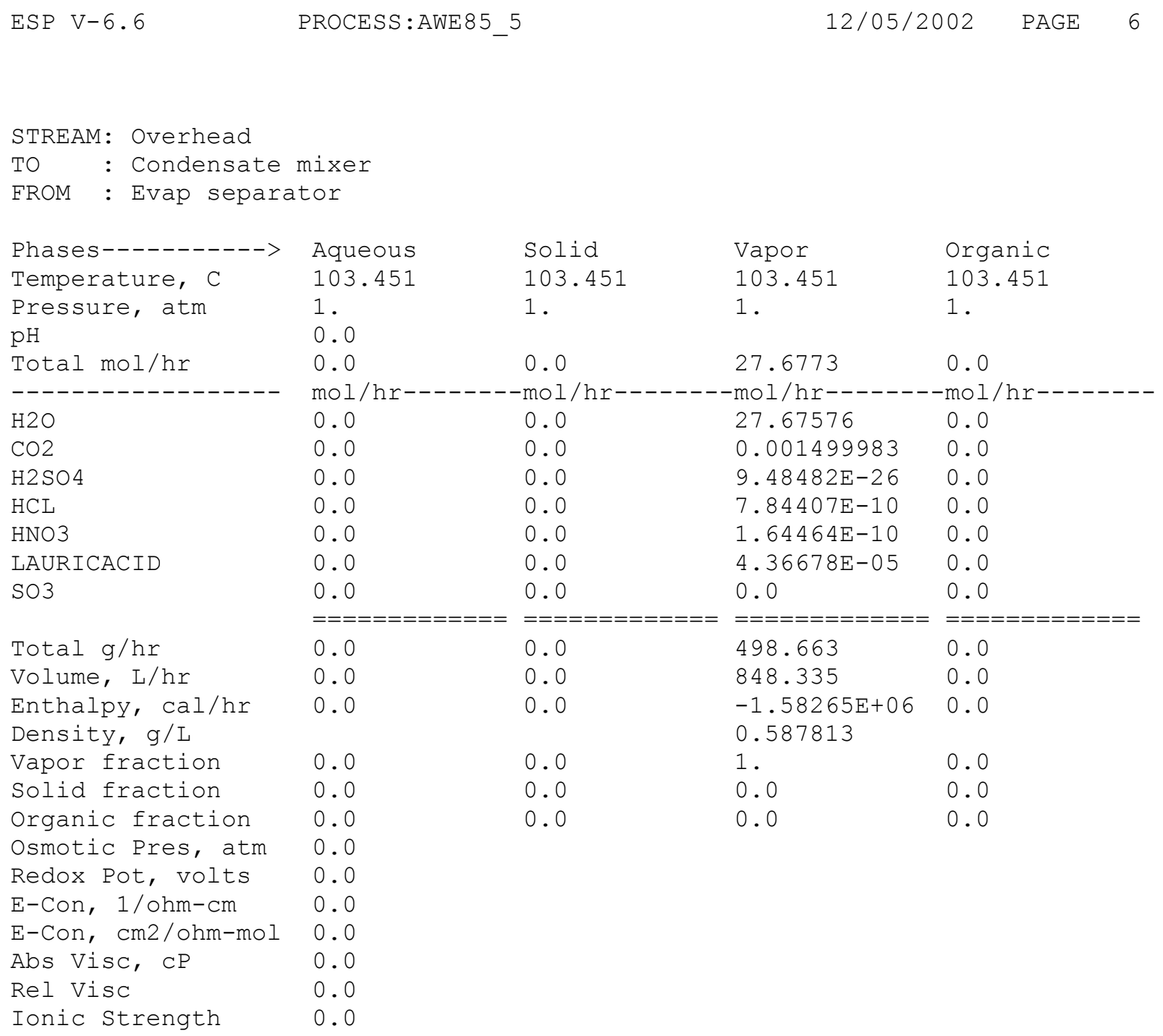


WSRC-TR-2002-00567

Revision 0

\begin{tabular}{|c|c|c|c|c|}
\hline \multicolumn{5}{|c|}{ TO : Evap Bottoms Cooling mixer } \\
\hline Phases $----------->$ & Aqueous & Solid & Vapor & Organic \\
\hline Temperature, C & 103.451 & 103.451 & 103.451 & 103.451 \\
\hline $\begin{array}{l}\text { Pressure, atm } \\
\mathrm{pH}\end{array}$ & $\begin{array}{l}1 . \\
7.38784\end{array}$ & 1. & 1 . & 1 . \\
\hline Total mol/hr & 0.07135988 & 0.00135427 & 0.0 & 0.0 \\
\hline \multicolumn{3}{|l|}{-ー-ー-ー-ー-ー-ー-ー-ー-ー } & $-\mathrm{mol} / \mathrm{hr}--$ & -mol/hr- \\
\hline $\mathrm{H} 2 \mathrm{O}$ & 0.0636844 & 0.0 & 0.0 & 0.0 \\
\hline $\mathrm{CO} 2$ & $4.34201 \mathrm{E}-10$ & 0.0 & 0.0 & 0.0 \\
\hline $\mathrm{H} 2 \mathrm{SO} 4$ & $2.24966 \mathrm{E}-28$ & 0.0 & 0.0 & 0.0 \\
\hline $\mathrm{HCL}$ & $1.79891 \mathrm{E}-15$ & 0.0 & 0.0 & 0.0 \\
\hline HNO3 & $1.50642 \mathrm{E}-12$ & 0.0 & 0.0 & 0.0 \\
\hline LAURICACID & $1.79187 E-09$ & 0.0 & 0.0 & 0.0 \\
\hline $\mathrm{SO} 3$ & 0.0 & 0.0 & 0.0 & 0.0 \\
\hline CAH2SIO4 & $1.86733 E-11$ & 0.0 & 0.0 & 0.0 \\
\hline $\mathrm{CASO} 4$ & $6.37061 \mathrm{E}-07$ & $6.27262 \mathrm{E}-04$ & 0.0 & 0.0 \\
\hline CDCL2 & $2.01892 E-08$ & 0.0 & 0.0 & 0.0 \\
\hline $\mathrm{CDOH} 2$ & $7.47907 \mathrm{E}-14$ & 0.0 & 0.0 & 0.0 \\
\hline $\mathrm{CDSO} 4$ & $6.56406 \mathrm{E}-12$ & 0.0 & 0.0 & 0.0 \\
\hline $\mathrm{CROH} 3$ & $4.05833 E-11$ & $1.11556 \mathrm{E}-07$ & 0.0 & 0.0 \\
\hline CUCL2 & $1.10640 \mathrm{E}-10$ & 0.0 & 0.0 & 0.0 \\
\hline CUCO3 & $2.95200 \mathrm{E}-13$ & 0.0 & 0.0 & 0.0 \\
\hline CUNO32 & $6.96337 E-13$ & 0.0 & 0.0 & 0.0 \\
\hline CUOH2 & $1.01088 E-08$ & $6.75976 \mathrm{E}-06$ & 0.0 & 0.0 \\
\hline FEIICL2 & $1.13548 \mathrm{E}-14$ & 0.0 & 0.0 & 0.0 \\
\hline FEIICO3 & $5.31362 \mathrm{E}-14$ & 0.0 & 0.0 & 0.0 \\
\hline FEIIHPO4 & $1.32646 \mathrm{E}-16$ & 0.0 & 0.0 & 0.0 \\
\hline FEIIOH2 & $5.82559 \mathrm{E}-13$ & 0.0 & 0.0 & 0.0 \\
\hline $\mathrm{ALO} 2 \mathrm{H} 2 \mathrm{CL}$ & $6.35432 E-30$ & 0.0 & 0.0 & 0.0 \\
\hline H3PO 4 & $3.02023 E-16$ & 0.0 & 0.0 & 0.0 \\
\hline H4 P 207 & $2.10552 \mathrm{E}-30$ & 0.0 & 0.0 & 0.0 \\
\hline ALOH3 & $3.65460 \mathrm{E}-11$ & 0.0 & 0.0 & 0.0 \\
\hline $\mathrm{BACO} 3$ & $1.25076 \mathrm{E}-15$ & 0.0 & 0.0 & 0.0 \\
\hline $\mathrm{KCL}$ & $8.71176 \mathrm{E}-06$ & 0.0 & 0.0 & 0.0 \\
\hline KHSO 4 & $2.54571 E-12$ & 0.0 & 0.0 & 0.0 \\
\hline BASO 4 & $1.17348 \mathrm{E}-12$ & $6.70657 E-07$ & 0.0 & 0.0 \\
\hline $\mathrm{MGCO} 3$ & $2.30444 \mathrm{E}-10$ & 0.0 & 0.0 & 0.0 \\
\hline MGH2SIO4 & $1.78567 E-09$ & 0.0 & 0.0 & 0.0 \\
\hline MGHPO4 & $2.55414 \mathrm{E}-09$ & 0.0 & 0.0 & 0.0 \\
\hline MGSO4 & $4.52325 E-06$ & 0.0 & 0.0 & 0.0 \\
\hline NAHCO3 & $1.16776 \mathrm{E}-08$ & 0.0 & 0.0 & 0.0 \\
\hline NAHSIO3 & $2.64890 \mathrm{E}-06$ & 0.0 & 0.0 & 0.0 \\
\hline NANO3 & $2.11798 \mathrm{E}-04$ & 0.0 & 0.0 & 0.0 \\
\hline $\mathrm{NIOH} 2$ & $2.63371 E-12$ & $1.49660 E-06$ & 0.0 & 0.0 \\
\hline $\mathrm{NISO} 4$ & $6.42945 \mathrm{E}-10$ & 0.0 & 0.0 & 0.0 \\
\hline PBCL2 & $9.29030 \mathrm{E}-08$ & 0.0 & 0.0 & 0.0 \\
\hline PBHPO4 & $2.66645 E-14$ & 0.0 & 0.0 & 0.0 \\
\hline PBNO32 & $2.51116 \mathrm{E}-09$ & 0.0 & 0.0 & 0.0 \\
\hline $\mathrm{PBO}$ & $1.03010 \mathrm{E}-09$ & 0.0 & 0.0 & 0.0 \\
\hline CACL2 & $1.36404 \mathrm{E}-15$ & 0.0 & 0.0 & 0.0 \\
\hline $\mathrm{SIO} 2$ & $5.19908 \mathrm{E}-06$ & $1.73511 E-04$ & 0.0 & 0.0 \\
\hline
\end{tabular}




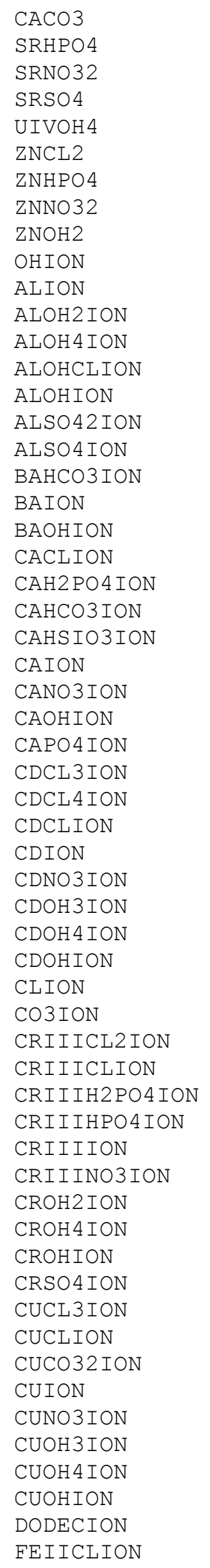

$\begin{array}{ll}7.26897 \mathrm{E}-11 & 0.0 \\ 4.58703 \mathrm{E}-14 & 0.0 \\ 2.86989 \mathrm{E}-08 & 0.0 \\ 1.09816 \mathrm{E}-08 & 2.15367 \mathrm{E}-05 \\ 1.34872 \mathrm{E}-12 & 0.0 \\ 3.20149 \mathrm{E}-06 & 0.0 \\ 2.84323 \mathrm{E}-12 & 0.0 \\ 1.22405 \mathrm{E}-09 & 0.0 \\ 4.12634 \mathrm{E}-08 & 0.0 \\ 2.50321 \mathrm{E}-08 & 0.0 \\ 1.09069 \mathrm{E}-21 & 0.0 \\ 2.11446 \mathrm{E}-14 & 0.0 \\ 9.32633 \mathrm{E}-09 & 0.0 \\ 4.01706 \mathrm{E}-17 & 0.0 \\ 9.87791 \mathrm{E}-18 & 0.0 \\ 5.76390 \mathrm{E}-21 & 0.0 \\ 8.40141 \mathrm{E}-21 & 0.0 \\ 9.49527 \mathrm{E}-14 & 0.0 \\ 8.74076 \mathrm{E}-10 & 0.0 \\ 8.64824 \mathrm{E}-15 & 0.0 \\ 3.95372 \mathrm{E}-08 & 0.0 \\ 1.00006 \mathrm{E}-11 & 0.0 \\ 1.55488 \mathrm{E}-10 & 0.0 \\ 8.66800 \mathrm{E}-09 & 0.0 \\ 9.02446 \mathrm{E}-06 & 0.0 \\ 3.45508 \mathrm{E}-06 & 0.0 \\ 1.72017 \mathrm{E}-09 & 0.0 \\ 5.26295 \mathrm{E}-11 & 0.0 \\ 1.95720 \mathrm{E}-08 & 0.0 \\ 5.42991 \mathrm{E}-09 & 0.0 \\ 1.20077 \mathrm{E}-09 & 0.0 \\ 2.31756 \mathrm{E}-11 & 0.0 \\ 6.21236 \mathrm{E}-12 & 0.0 \\ 1.62956 \mathrm{E}-17 & 0.0 \\ 2.05265 \mathrm{E}-21 & 0.0 \\ 6.85742 \mathrm{E}-13 & 0.0 \\ 0.00277442 & 0.0 \\ 2.31581 \mathrm{E}-10 & 0.0 \\ 5.39856 \mathrm{E}-16 & 0.0 \\ 4.50681 \mathrm{E}-16 & 0.0 \\ 1.79499 \mathrm{E}-20 & 0.0 \\ 9.37721 \mathrm{E}-09 & 0.0 \\ 5.62589 \mathrm{E}-20 & 0.0 \\ 2.20116 \mathrm{E}-13 & 0.0 \\ 6.08063 \mathrm{E}-13 & 0.0 \\ 1.35798 \mathrm{E}-13 & 0.0 \\ 1.70077 \mathrm{E}-10 & 0.0 \\ 8.58073 \mathrm{E}-12 & 0.0 \\ 1.83815 \mathrm{E}-12 & 0.0 \\ 7.24412 \mathrm{E}-10 & 0.0 \\ 3.35413 \mathrm{E}-16 & 0.0 \\ 1.99293 \mathrm{E}-10 & 0.0 \\ 3.40488 \mathrm{E}-11 & 0.0 \\ 1.38033 \mathrm{E}-11 & 0.0 \\ 4.16764 \mathrm{E}-14 & 0.0 \\ 6.97436 \mathrm{E}-10 & 0.0 \\ 1.20924 \mathrm{E}-05 & 0.0 \\ 2.47667 \mathrm{E}-12 & 0.0 \\ & \\ 1.0 & \end{array}$

0.0

0.0

0.0

0.0

0.0

0.0

0.0

0.0

0.0

0.0

0.0

0.0

0.0

0.0

0.0

0.0

0.0

0.0

0.0

0.0

0.0

0.0

0.0

0.0

0.0

0.0

0.0

0.0

0.0

0.0

0.0

0.0

0.0

0.0

0.0

0.0

0.0

0.0

0.0

0.0

0.0

0.0

0.0

0.0

0.0

0.0

0.0

0.0

0.0

0.0

0.0

0.0

0.0

0.0

0.0

0.0

0.0

0.0

0.0
0.0

0.0

0.0

0.0

0.0

0.0

0.0

0.0

0.0

0.0

0.0

0.0

0.0

0.0

0.0

0.0

0.0

0.0

0.0

0.0

0.0

0.0

0.0

0.0

0.0

0.0

0.0

0.0

0.0

0.0

0.0

0.0

0.0

0.0

0.0

0.0

0.0

0.0

0.0

0.0

0.0

0.0

0.0

0.0

0.0

0.0

0.0

0.0

0.0

0.0

0.0

0.0

0.0

0.0

0.0

0.0

0.0 


\begin{tabular}{|c|c|c|c|c|}
\hline FEIICO32ION & $1.78873 \mathrm{E}-18$ & 0.0 & 0.0 & 0.0 \\
\hline FEIIH2PO4ION & $1.25717 \mathrm{E}-17$ & 0.0 & 0.0 & 0.0 \\
\hline EEIIHCO3ION & $1.27982 \mathrm{E}-16$ & 0.0 & 0.0 & 0.0 \\
\hline FEIIION & $3.04354 \mathrm{E}-10$ & 0.0 & 0.0 & 0.0 \\
\hline FEIIOH3ION & $1.65772 \mathrm{E}-14$ & 0.0 & 0.0 & 0.0 \\
\hline FEIIOH 4 ION & $5.79145 \mathrm{E}-19$ & 0.0 & 0.0 & 0.0 \\
\hline FEIIOHION & $3.60199 \mathrm{E}-11$ & 0.0 & 0.0 & 0.0 \\
\hline H2 P2O 7 ION & $1.90460 \mathrm{E}-18$ & 0.0 & 0.0 & 0.0 \\
\hline H2 PO 4 ION & $8.74013 \mathrm{E}-11$ & 0.0 & 0.0 & 0.0 \\
\hline $\mathrm{H} 2 \mathrm{SIO} 4 \mathrm{ION}$ & $1.77925 \mathrm{E}-11$ & 0.0 & 0.0 & 0.0 \\
\hline H3P2O7 ION & $1.61286 \mathrm{E}-24$ & 0.0 & 0.0 & 0.0 \\
\hline H3SIO 4 ION & $7.80273 \mathrm{E}-07$ & 0.0 & 0.0 & 0.0 \\
\hline HCO3ION & $7.71356 \mathrm{E}-09$ & 0.0 & 0.0 & 0.0 \\
\hline HION & $6.04790 \mathrm{E}-11$ & 0.0 & 0.0 & 0.0 \\
\hline HP207ION & $4.30218 \mathrm{E}-17$ & 0.0 & 0.0 & 0.0 \\
\hline HPBO2ION & $1.32990 \mathrm{E}-11$ & 0.0 & 0.0 & 0.0 \\
\hline HPO4ION & $1.20700 \mathrm{E}-09$ & 0.0 & 0.0 & 0.0 \\
\hline $\mathrm{HSO} 4 \mathrm{ION}$ & $4.62419 \mathrm{E}-10$ & 0.0 & 0.0 & 0.0 \\
\hline KION & $4.61072 \mathrm{E}-04$ & 0.0 & 0.0 & 0.0 \\
\hline KSO4 ION & $5.04522 \mathrm{E}-05$ & 0.0 & 0.0 & 0.0 \\
\hline MGH2 PO 4 ION & $2.13661 \mathrm{E}-11$ & 0.0 & 0.0 & 0.0 \\
\hline MGHCO3ION & $2.09549 \mathrm{E}-09$ & 0.0 & 0.0 & 0.0 \\
\hline MGHSIO3ION & $1.08471 \mathrm{E}-07$ & 0.0 & 0.0 & 0.0 \\
\hline MGION & $2.15352 \mathrm{E}-05$ & 0.0 & 0.0 & 0.0 \\
\hline MGOHION & $9.08930 \mathrm{E}-08$ & 0.0 & 0.0 & 0.0 \\
\hline MGP2O7ION & $1.98806 \mathrm{E}-13$ & 0.0 & 0.0 & 0.0 \\
\hline MGPO 4 ION & $3.79546 \mathrm{E}-10$ & 0.0 & 0.0 & 0.0 \\
\hline NACO3ION & $3.69506 \mathrm{E}-11$ & 0.0 & 0.0 & 0.0 \\
\hline NAION & 0.00332321 & 0.0 & 0.0 & 0.0 \\
\hline NASO 4 ION & $3.92144 \mathrm{E}-13$ & 0.0 & 0.0 & 0.0 \\
\hline NICLION & $1.29786 \mathrm{E}-10$ & 0.0 & 0.0 & 0.0 \\
\hline NIION & $1.49902 \mathrm{E}-09$ & 0.0 & 0.0 & 0.0 \\
\hline NINO3ION & $2.14768 \mathrm{E}-10$ & 0.0 & 0.0 & 0.0 \\
\hline NIOH3ION & $8.89693 \mathrm{E}-15$ & 0.0 & 0.0 & 0.0 \\
\hline NIOHION & $5.49134 \mathrm{E}-11$ & 0.0 & 0.0 & 0.0 \\
\hline NO3ION & $5.10322 \mathrm{E}-04$ & 0.0 & 0.0 & 0.0 \\
\hline P207ION & $1.22128 \mathrm{E}-17$ & 0.0 & 0.0 & 0.0 \\
\hline PBCL3ION & $2.05078 \mathrm{E}-07$ & 0.0 & 0.0 & 0.0 \\
\hline PBCL 4 ION & $1.65430 \mathrm{E}-06$ & 0.0 & 0.0 & 0.0 \\
\hline PBCLION & $2.73174 \mathrm{E}-08$ & 0.0 & 0.0 & 0.0 \\
\hline PBH2PO 4 ION & $1.23910 \mathrm{E}-15$ & 0.0 & 0.0 & 0.0 \\
\hline PBION & $1.29408 \mathrm{E}-09$ & 0.0 & 0.0 & 0.0 \\
\hline PBNO33ION & $9.30935 \mathrm{E}-11$ & 0.0 & 0.0 & 0.0 \\
\hline PBNO3ION & $7.60984 \mathrm{E}-09$ & 0.0 & 0.0 & 0.0 \\
\hline PBOHION & $2.07438 \mathrm{E}-08$ & 0.0 & 0.0 & 0.0 \\
\hline PO4ION & $2.37021 \mathrm{E}-13$ & 0.0 & 0.0 & 0.0 \\
\hline SO4ION & $2.53239 \mathrm{E}-04$ & 0.0 & 0.0 & 0.0 \\
\hline SRION & $5.72314 \mathrm{E}-10$ & 0.0 & 0.0 & 0.0 \\
\hline SRNO3ION & $1.12544 \mathrm{E}-07$ & 0.0 & 0.0 & 0.0 \\
\hline SROHION & $1.30499 \mathrm{E}-11$ & 0.0 & 0.0 & 0.0 \\
\hline SRPO 4 ION & $1.19552 \mathrm{E}-14$ & 0.0 & 0.0 & 0.0 \\
\hline UIVOH2ION & $4.60321 \mathrm{E}-26$ & 0.0 & 0.0 & 0.0 \\
\hline UIVOH3ION & $1.73992 \mathrm{E}-20$ & 0.0 & 0.0 & 0.0 \\
\hline UIVOH5ION & $1.70497 \mathrm{E}-14$ & 0.0 & 0.0 & 0.0 \\
\hline UIVOHION & 0.0 & 0.0 & 0.0 & 0.0 \\
\hline ZNCL3ION & $4.09188 \mathrm{E}-06$ & 0.0 & 0.0 & 0.0 \\
\hline ZNCLION & $7.48267 \mathrm{E}-06$ & 0.0 & 0.0 & 0.0 \\
\hline ZNH2PO 4 ION & $6.41653 \mathrm{E}-14$ & 0.0 & 0.0 & 0.0 \\
\hline
\end{tabular}


WSRC-TR-2002-00567

Revision 0

\begin{tabular}{|c|c|c|c|c|}
\hline ZNHCO3ION & $5.33856 \mathrm{E}-12$ & 0.0 & 0.0 & 0.0 \\
\hline ZNION & $4.52602 \mathrm{E}-07$ & 0.0 & 0.0 & 0.0 \\
\hline ZNNO3ION & $4.59099 E-08$ & 0.0 & 0.0 & 0.0 \\
\hline ZNOH3ION & $2.12228 E-09$ & 0.0 & 0.0 & 0.0 \\
\hline ZNOH $4 \mathrm{ION}$ & $2.29426 \mathrm{E}-12$ & 0.0 & 0.0 & 0.0 \\
\hline ZNOHION & $4.49166 \mathrm{E}-06$ & 0.0 & 0.0 & 0.0 \\
\hline $\mathrm{ALOOH}$ & 0.0 & $1.54416 \mathrm{E}-06$ & 0.0 & 0.0 \\
\hline $\mathrm{CA} 3 \mathrm{PO} 42$ & 0.0 & $1.19026 \mathrm{E}-04$ & 0.0 & 0.0 \\
\hline CHAMOS ITE7A & 0.0 & $5.10751 E-05$ & 0.0 & 0.0 \\
\hline MG3PO4 2 & 0.0 & $5.93559 \mathrm{E}-06$ & 0.0 & 0.0 \\
\hline MGOH2 & 0.0 & $3.34529 E-04$ & 0.0 & 0.0 \\
\hline PB3PO42 & 0.0 & $4.72353 E-07$ & 0.0 & 0.0 \\
\hline UIVO2 & 0.0 & $4.20168 E-06$ & 0.0 & 0.0 \\
\hline $\mathrm{ZN} 3 \mathrm{PO} 42.2 \mathrm{H} 2 \mathrm{O}$ & 0.0 & $6.13848 \mathrm{E}-06$ & 0.0 & 0.0 \\
\hline & $============$ & $============$ & $===========$ & $===========$ \\
\hline Total g/hr & 1.4295 & 0.18039 & 0.0 & 0.0 \\
\hline Volume, L/hr & 0.00132219 & $5.48580 \mathrm{E}-05$ & 0.0 & 0.0 \\
\hline Enthalpy, cal/hr & -4719.19 & -507.005 & 0.0 & 0.0 \\
\hline Density, g/L & 1081.16 & 3288.32 & & \\
\hline Vapor fraction & 0.0 & 0.0 & 0.0 & 0.0 \\
\hline Solid fraction & 0.0 & 1 . & 0.0 & 0.0 \\
\hline Organic fraction & 0.0 & 0.0 & 0.0 & 0.0 \\
\hline Osmotic Pres, atm & 196.506 & & & \\
\hline Redox Pot, volts & 0.0 & & & \\
\hline E-Con, $1 / \mathrm{ohm}-\mathrm{cm}$ & 0.501471 & & & \\
\hline E-Con, cm2/ohm-mol & 58.4688 & & & \\
\hline Abs Visc, cP & 0.421849 & & & \\
\hline Rel Visc & 1.55267 & & & \\
\hline Ionic Strength & 3.61561 & & & \\
\hline
\end{tabular}


WSRC-TR-2002-00567

Revision 0

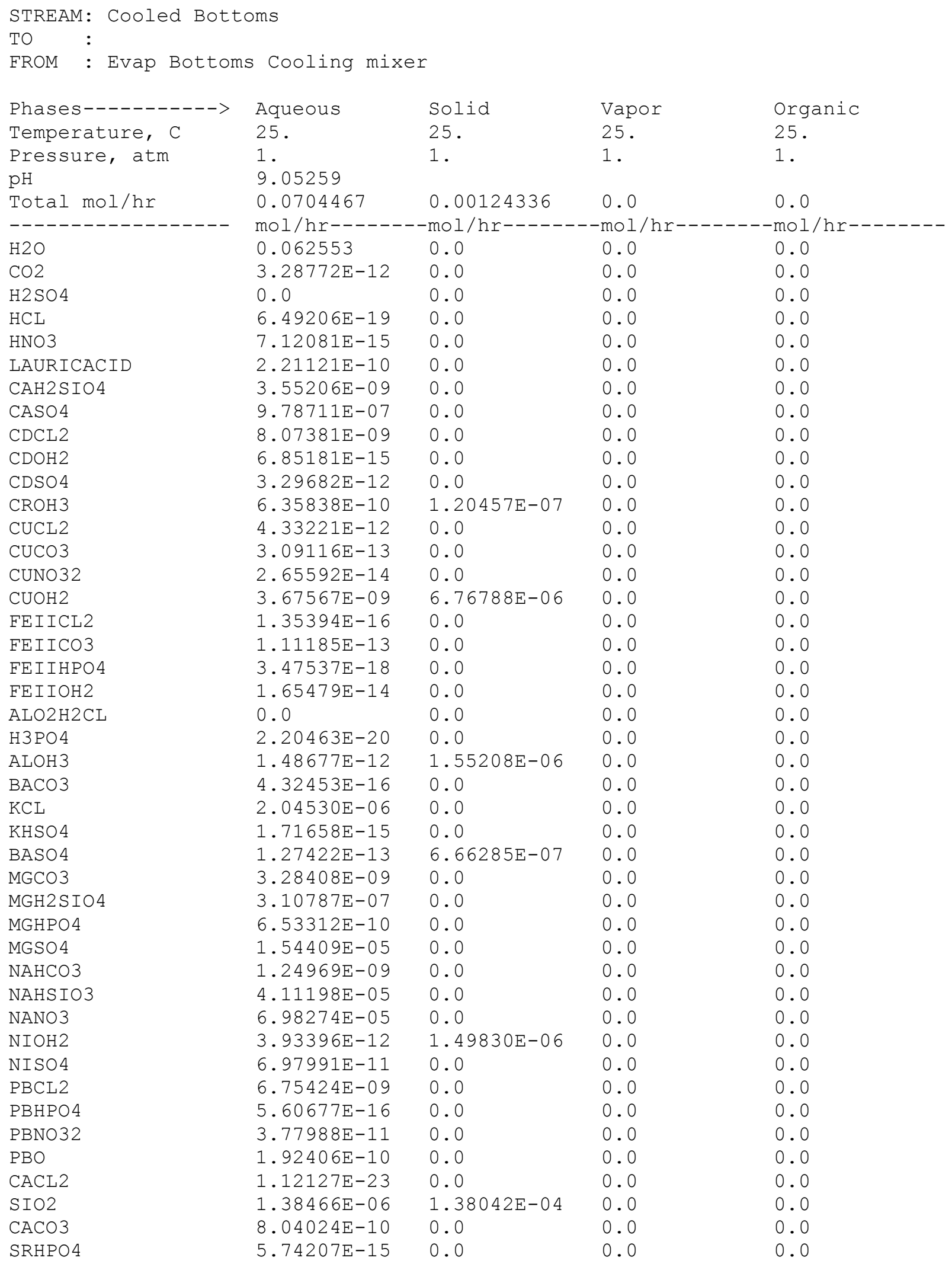




\begin{tabular}{|c|c|c|c|c|}
\hline SRNO32 & $1.50216 \mathrm{E}-08$ & 0.0 & 0.0 & 0.0 \\
\hline SRSO 4 & $2.66985 \mathrm{E}-08$ & $1.96062 \mathrm{E}-05$ & 0.0 & 0.0 \\
\hline UIVOH 4 & $1.43452 \mathrm{E}-13$ & 0.0 & 0.0 & 0.0 \\
\hline ZNCL2 & $7.33888 \mathrm{E}-09$ & 0.0 & 0.0 & 0.0 \\
\hline ZNHPO 4 & $3.89687 \mathrm{E}-14$ & 0.0 & 0.0 & 0.0 \\
\hline ZNNO32 & $7.91612 \mathrm{E}-11$ & 0.0 & 0.0 & 0.0 \\
\hline ZNOH2 & $5.03582 \mathrm{E}-09$ & $3.81487 \mathrm{E}-05$ & 0.0 & 0.0 \\
\hline OHION & $1.06480 \mathrm{E}-08$ & 0.0 & 0.0 & 0.0 \\
\hline ALION & $6.65409 \mathrm{E}-23$ & 0.0 & 0.0 & 0.0 \\
\hline ALOH2 ION & $6.99217 \mathrm{E}-16$ & 0.0 & 0.0 & 0.0 \\
\hline ALOH 4 ION & $1.14132 \mathrm{E}-09$ & 0.0 & 0.0 & 0.0 \\
\hline ALOHCLION & $1.58044 \mathrm{E}-18$ & 0.0 & 0.0 & 0.0 \\
\hline ALOHION & $2.47281 \mathrm{E}-19$ & 0.0 & 0.0 & 0.0 \\
\hline ALSO 42 ION & $1.36220 \mathrm{E}-22$ & 0.0 & 0.0 & 0.0 \\
\hline ALSO 4 ION & $2.13894 \mathrm{E}-22$ & 0.0 & 0.0 & 0.0 \\
\hline BAHCO3ION & $7.65875 \mathrm{E}-16$ & 0.0 & 0.0 & 0.0 \\
\hline BAION & $5.24773 \mathrm{E}-09$ & 0.0 & 0.0 & 0.0 \\
\hline BAOHION & $1.13365 \mathrm{E}-16$ & 0.0 & 0.0 & 0.0 \\
\hline CACLION & $5.07311 \mathrm{E}-11$ & 0.0 & 0.0 & 0.0 \\
\hline CAH2 PO 4 ION & $1.25490 \mathrm{E}-13$ & 0.0 & 0.0 & 0.0 \\
\hline CAHCO3ION & $2.97865 \mathrm{E}-10$ & 0.0 & 0.0 & 0.0 \\
\hline CAHSIO3ION & $5.87311 \mathrm{E}-08$ & 0.0 & 0.0 & 0.0 \\
\hline CAION & $2.37209 \mathrm{E}-05$ & 0.0 & 0.0 & 0.0 \\
\hline CANO3ION & $9.81903 \mathrm{E}-06$ & 0.0 & 0.0 & 0.0 \\
\hline CAOHION & $1.30771 \mathrm{E}-09$ & 0.0 & 0.0 & 0.0 \\
\hline CAPO 4 ION & $4.82510 \mathrm{E}-10$ & 0.0 & 0.0 & 0.0 \\
\hline CDCL3ION & $3.06687 \mathrm{E}-09$ & 0.0 & 0.0 & 0.0 \\
\hline CDCL 4 ION & $3.47326 \mathrm{E}-08$ & 0.0 & 0.0 & 0.0 \\
\hline CDCLION & $5.24888 \mathrm{E}-10$ & 0.0 & 0.0 & 0.0 \\
\hline CDION & $2.34515 \mathrm{E}-11$ & 0.0 & 0.0 & 0.0 \\
\hline CDNO3ION & $3.47457 \mathrm{E}-12$ & 0.0 & 0.0 & 0.0 \\
\hline CDOH 3 ION & $3.75248 \mathrm{E}-18$ & 0.0 & 0.0 & 0.0 \\
\hline CDOH 4 ION & $1.86288 \mathrm{E}-22$ & 0.0 & 0.0 & 0.0 \\
\hline CDOHION & $2.19172 \mathrm{E}-13$ & 0.0 & 0.0 & 0.0 \\
\hline CLION & 0.00281321 & 0.0 & 0.0 & 0.0 \\
\hline CO3ION & $2.79714 \mathrm{E}-09$ & 0.0 & 0.0 & 0.0 \\
\hline CRIIICL2ION & $8.05741 \mathrm{E}-18$ & 0.0 & 0.0 & 0.0 \\
\hline CRIIICLION & $7.01586 \mathrm{E}-18$ & 0.0 & 0.0 & 0.0 \\
\hline CRIIIH2PO4ION & $3.27111 \mathrm{E}-22$ & 0.0 & 0.0 & 0.0 \\
\hline CRIIIHPO4ION & $1.59035 \mathrm{E}-14$ & 0.0 & 0.0 & 0.0 \\
\hline CRIIIION & $6.34645 \mathrm{E}-21$ & 0.0 & 0.0 & 0.0 \\
\hline CRIIINO3ION & $1.54545 \mathrm{E}-15$ & 0.0 & 0.0 & 0.0 \\
\hline CROH2ION & $4.83112 \mathrm{E}-13$ & 0.0 & 0.0 & 0.0 \\
\hline CROH 4 ION & $2.87921 \mathrm{E}-11$ & 0.0 & 0.0 & 0.0 \\
\hline CROHION & $3.11504 \mathrm{E}-11$ & 0.0 & 0.0 & 0.0 \\
\hline CRSO 4 ION & $7.83022 \mathrm{E}-14$ & 0.0 & 0.0 & 0.0 \\
\hline CUCL3ION & $7.13108 \mathrm{E}-14$ & 0.0 & 0.0 & 0.0 \\
\hline CUCLION & $1.31745 \mathrm{E}-11$ & 0.0 & 0.0 & 0.0 \\
\hline CUCO32ION & $2.41259 \mathrm{E}-15$ & 0.0 & 0.0 & 0.0 \\
\hline CUION & $1.27505 \mathrm{E}-11$ & 0.0 & 0.0 & 0.0 \\
\hline CUNO3ION & $2.20233 \mathrm{E}-12$ & 0.0 & 0.0 & 0.0 \\
\hline CUOH 3ION & $7.31305 \mathrm{E}-12$ & 0.0 & 0.0 & 0.0 \\
\hline CUOH 4 ION & $5.94149 \mathrm{E}-15$ & 0.0 & 0.0 & 0.0 \\
\hline CUOHION & $5.20907 \mathrm{E}-11$ & 0.0 & 0.0 & 0.0 \\
\hline DODECION & $1.20939 \mathrm{E}-05$ & 0.0 & 0.0 & 0.0 \\
\hline FEIICLION & $5.09073 E-14$ & 0.0 & 0.0 & 0.0 \\
\hline FEIICO32ION & $1.88601 \mathrm{E}-17$ & 0.0 & 0.0 & 0.0 \\
\hline FEIIH2PO4ION & $2.47824 \mathrm{E}-20$ & 0.0 & 0.0 & 0.0 \\
\hline
\end{tabular}




\begin{tabular}{|c|c|c|c|c|}
\hline FEIIHCO3ION & $6.48261 \mathrm{E}-18$ & 0.0 & 0.0 & 0.0 \\
\hline EEIIION & $3.83770 \mathrm{E}-11$ & 0.0 & 0.0 & 0.0 \\
\hline FEIIOH3ION & $2.40431 \mathrm{E}-15$ & 0.0 & 0.0 & 0.0 \\
\hline FEIIOH 4 ION & $2.70133 \mathrm{E}-20$ & 0.0 & 0.0 & 0.0 \\
\hline FEIIOHION & $5.34641 \mathrm{E}-12$ & 0.0 & 0.0 & 0.0 \\
\hline H2 P2O 7 ION & $1.03776 \mathrm{E}-23$ & 0.0 & 0.0 & 0.0 \\
\hline H2 PO 4 ION & $9.23331 \mathrm{E}-13$ & 0.0 & 0.0 & 0.0 \\
\hline H2SIO4ION & $5.12783 \mathrm{E}-10$ & 0.0 & 0.0 & 0.0 \\
\hline H3P2O 7 ION & 0.0 & 0.0 & 0.0 & 0.0 \\
\hline H3SIO4 ION & $3.55638 \mathrm{E}-07$ & 0.0 & 0.0 & 0.0 \\
\hline HCO3ION & $5.96520 \mathrm{E}-09$ & 0.0 & 0.0 & 0.0 \\
\hline HION & $9.03027 \mathrm{E}-13$ & 0.0 & 0.0 & 0.0 \\
\hline HP2O7ION & $1.58589 \mathrm{E}-20$ & 0.0 & 0.0 & 0.0 \\
\hline HPBO2ION & $5.52188 \mathrm{E}-12$ & 0.0 & 0.0 & 0.0 \\
\hline HPO 4 ION & $7.13402 \mathrm{E}-10$ & 0.0 & 0.0 & 0.0 \\
\hline HSO 4 ION & $1.01811 \mathrm{E}-12$ & 0.0 & 0.0 & 0.0 \\
\hline KION & $4.88321 \mathrm{E}-04$ & 0.0 & 0.0 & 0.0 \\
\hline KSO4 ION & $2.98698 \mathrm{E}-05$ & 0.0 & 0.0 & 0.0 \\
\hline MGH2 PO 4 ION & $1.45978 \mathrm{E}-12$ & 0.0 & 0.0 & 0.0 \\
\hline MGHCO3ION & $6.80274 \mathrm{E}-09$ & 0.0 & 0.0 & 0.0 \\
\hline MGHSIO3ION & $9.82248 \mathrm{E}-07$ & 0.0 & 0.0 & 0.0 \\
\hline MGION & $8.28893 E-05$ & 0.0 & 0.0 & 0.0 \\
\hline MGOHION & $1.13425 \mathrm{E}-07$ & 0.0 & 0.0 & 0.0 \\
\hline MGP2O7ION & $1.52095 \mathrm{E}-15$ & 0.0 & 0.0 & 0.0 \\
\hline MGPO 4 ION & $5.98598 \mathrm{E}-09$ & 0.0 & 0.0 & 0.0 \\
\hline NACO3ION & $1.44911 \mathrm{E}-09$ & 0.0 & 0.0 & 0.0 \\
\hline NAION & 0.00333203 & 0.0 & 0.0 & 0.0 \\
\hline NASO 4 ION & $9.46937 \mathrm{E}-05$ & 0.0 & 0.0 & 0.0 \\
\hline NICLION & $2.17548 \mathrm{E}-11$ & 0.0 & 0.0 & 0.0 \\
\hline NIION & $5.92719 \mathrm{E}-10$ & 0.0 & 0.0 & 0.0 \\
\hline NINO3ION & $1.41039 \mathrm{E}-10$ & 0.0 & 0.0 & 0.0 \\
\hline NIOH3ION & $1.58101 \mathrm{E}-13$ & 0.0 & 0.0 & 0.0 \\
\hline NIOHION & $1.38453 \mathrm{E}-11$ & 0.0 & 0.0 & 0.0 \\
\hline NO3ION & $6.46007 \mathrm{E}-04$ & 0.0 & 0.0 & 0.0 \\
\hline P207ION & $1.35323 \mathrm{E}-18$ & 0.0 & 0.0 & 0.0 \\
\hline PBCL3ION & $1.70839 \mathrm{E}-08$ & 0.0 & 0.0 & 0.0 \\
\hline PBCL 4 ION & $3.38291 \mathrm{E}-07$ & 0.0 & 0.0 & 0.0 \\
\hline PBCLION & $2.58257 \mathrm{E}-09$ & 0.0 & 0.0 & 0.0 \\
\hline PBH2 PO 4 ION & $7.96781 \mathrm{E}-19$ & 0.0 & 0.0 & 0.0 \\
\hline PBION & $2.22790 \mathrm{E}-10$ & 0.0 & 0.0 & 0.0 \\
\hline PBNO33ION & $8.70895 \mathrm{E}-12$ & 0.0 & 0.0 & 0.0 \\
\hline PBNO3ION & $4.18650 \mathrm{E}-10$ & 0.0 & 0.0 & 0.0 \\
\hline PBOHION & $1.09613 \mathrm{E}-09$ & 0.0 & 0.0 & 0.0 \\
\hline PO4ION & $3.77453 \mathrm{E}-12$ & 0.0 & 0.0 & 0.0 \\
\hline SO4ION & $2.25790 \mathrm{E}-04$ & 0.0 & 0.0 & 0.0 \\
\hline SRION & $1.92270 \mathrm{E}-06$ & 0.0 & 0.0 & 0.0 \\
\hline SRNO3ION & $1.18837 \mathrm{E}-07$ & 0.0 & 0.0 & 0.0 \\
\hline SROHION & $3.94591 \mathrm{E}-12$ & 0.0 & 0.0 & 0.0 \\
\hline SRPO 4 ION & $3.52245 \mathrm{E}-14$ & 0.0 & 0.0 & 0.0 \\
\hline UIVOH2ION & $1.11603 \mathrm{E}-28$ & 0.0 & 0.0 & 0.0 \\
\hline UIVOH3ION & $4.52313 \mathrm{E}-21$ & 0.0 & 0.0 & 0.0 \\
\hline UIVOH5ION & $2.93625 \mathrm{E}-14$ & 0.0 & 0.0 & 0.0 \\
\hline ZNCL3ION & $1.28993 \mathrm{E}-08$ & 0.0 & 0.0 & 0.0 \\
\hline ZNCLION & $9.09273 E-09$ & 0.0 & 0.0 & 0.0 \\
\hline ZNH2 PO 4 ION & $4.52374 \mathrm{E}-17$ & 0.0 & 0.0 & 0.0 \\
\hline ZNHCO3ION & $2.14235 \mathrm{E}-13$ & 0.0 & 0.0 & 0.0 \\
\hline ZNION & $3.47857 \mathrm{E}-08$ & 0.0 & 0.0 & 0.0 \\
\hline ZNNO3ION & $3.71692 \mathrm{E}-09$ & 0.0 & 0.0 & 0.0 \\
\hline
\end{tabular}


WSRC-TR-2002-00567

Revision 0

\begin{tabular}{|c|c|c|c|c|}
\hline ZNOH3ION & $1.19548 \mathrm{E}-10$ & 0.0 & 0.0 & 0.0 \\
\hline ZNOH 4 ION & $2.56547 \mathrm{E}-13$ & 0.0 & 0.0 & 0.0 \\
\hline ZNOHION & $4.56650 E-09$ & 0.0 & 0.0 & 0.0 \\
\hline $\mathrm{CA} 3 \mathrm{PO} 42$ & 0.0 & $1.30554 \mathrm{E}-04$ & 0.0 & 0.0 \\
\hline $\mathrm{CASO} 4.2 \mathrm{H} 2 \mathrm{O}$ & 0.0 & $5.71260 E-04$ & 0.0 & 0.0 \\
\hline CHAMOSITE7A & 0.0 & $5.10752 \mathrm{E}-05$ & 0.0 & 0.0 \\
\hline $\mathrm{MGOH} 2$ & 0.0 & $2.78847 E-04$ & 0.0 & 0.0 \\
\hline $\mathrm{PB} 3 \mathrm{PO} 42$ & 0.0 & $1.02109 \mathrm{E}-06$ & 0.0 & 0.0 \\
\hline \multirow[t]{2}{*}{ UIVO2 } & 0.0 & $4.20168 E-06$ & 0.0 & 0.0 \\
\hline & $============$ & $============$ & $===========$ & $===========$ \\
\hline Total g/hr & 1.41859 & 0.191307 & 0.0 & 0.0 \\
\hline Volume, L/hr & 0.00123053 & $6.28103 E-05$ & 0.0 & 0.0 \\
\hline Enthalpy, cal/hr & -4766.59 & -555.986 & 0.0 & 0.0 \\
\hline Density, g/L & 1152.82 & 3045.8 & & \\
\hline Vapor fraction & 0.0 & 0.0 & 0.0 & 0.0 \\
\hline Solid fraction & 0.0 & 1 . & 0.0 & 0.0 \\
\hline Organic fraction & 0.0 & 0.0 & 0.0 & 0.0 \\
\hline Osmotic Pres, atm & 186.242 & & & \\
\hline Redox Pot, volts & 0.0 & & & \\
\hline E-Con, 1/ohm-cm & 0.195617 & & & \\
\hline E-Con, cm2/ohm-mol & 29.9256 & & & \\
\hline Abs Visc, cP & 1.35031 & & & \\
\hline Rel Visc & 1.51598 & & & \\
\hline Ionic Strength & 3.88973 & & & \\
\hline
\end{tabular}


WSRC-TR-2002-00567

Revision 0

\begin{tabular}{|c|c|c|c|c|}
\hline \multicolumn{5}{|l|}{ STREAM: Condensate } \\
\hline TO $\quad:$ & & & & \\
\hline FROM : Condensate & mixer & & & \\
\hline Phases-----------> & Aqueous & Solid & Vapor & Organic \\
\hline Temperature, C & 25 . & 25 . & 25 . & 25 . \\
\hline $\begin{array}{l}\text { Pressure, atm } \\
\mathrm{pH}\end{array}$ & $\begin{array}{l}1 . \\
4.32904\end{array}$ & 1. & 1. & 1. \\
\hline Total mol/hr & 27.67727 & 0.0 & 0.0 & 0.0 \\
\hline------------------ & $\mathrm{mol} / \mathrm{hr}------$ & -mol/hr------- & -mol/hr-------- & $-\operatorname{mol} / \mathrm{hr}------1$ \\
\hline $\mathrm{H} 2 \mathrm{O}$ & 27.6757 & 0.0 & 0.0 & 0.0 \\
\hline $\mathrm{CO} 2$ & 0.00148548 & 0.0 & 0.0 & 0.0 \\
\hline $\mathrm{HCL}$ & $2.16070 E-20$ & 0.0 & 0.0 & 0.0 \\
\hline HNO3 & $3.81185 \mathrm{E}-16$ & 0.0 & 0.0 & 0.0 \\
\hline LAURICACID & $3.46073 E-05$ & 0.0 & 0.0 & 0.0 \\
\hline OHION & $1.08744 \mathrm{E}-10$ & 0.0 & 0.0 & 0.0 \\
\hline CLION & $7.84405 E-10$ & 0.0 & 0.0 & 0.0 \\
\hline CO3ION & $1.48806 \mathrm{E}-11$ & 0.0 & 0.0 & 0.0 \\
\hline DODECION & $9.06042 \mathrm{E}-06$ & 0.0 & 0.0 & 0.0 \\
\hline HCO3ION & $1.44987 \mathrm{E}-05$ & 0.0 & 0.0 & 0.0 \\
\hline $\mathrm{HION}$ & $2.35602 \mathrm{E}-05$ & 0.0 & 0.0 & 0.0 \\
\hline NO3ION & $\begin{array}{l}\text { 1. } 64463 \mathrm{E}-10 \\
===========\end{array}$ & $\begin{array}{l}0.0 \\
============\end{array}$ & $\begin{array}{l}0.0 \\
=============\end{array}$ & $\begin{array}{l}0.0 \\
=============\end{array}$ \\
\hline Total g/hr & 498.663 & 0.0 & 0.0 & 0.0 \\
\hline Volume, L/hr & 0.500216 & 0.0 & 0.0 & 0.0 \\
\hline $\begin{array}{l}\text { Enthalpy, cal/hr } \\
\text { Density, g/L }\end{array}$ & $\begin{array}{l}-1.89077 E+06 \\
996.894\end{array}$ & 0.0 & 0.0 & 0.0 \\
\hline Vapor fraction & 0.0 & 0.0 & 0.0 & 0.0 \\
\hline Solid fraction & 0.0 & 0.0 & 0.0 & 0.0 \\
\hline Organic fraction & 0.0 & 0.0 & 0.0 & 0.0 \\
\hline Osmotic Pres, atm & 0.0766734 & & & \\
\hline Redox Pot, volts & 0.0 & & & \\
\hline E-Con, 1/ohm-cm & $1.88041 \mathrm{E}-05$ & & & \\
\hline E-Con, cm2/ohm-mol & 6.09343 & & & \\
\hline Abs Visc, cP & 0.890748 & & & \\
\hline Rel Visc & 1.00003 & & & \\
\hline Ionic Strength & $4.72543 E-05$ & & & \\
\hline
\end{tabular}


WSRC-TR-2002-00567

Revision 0

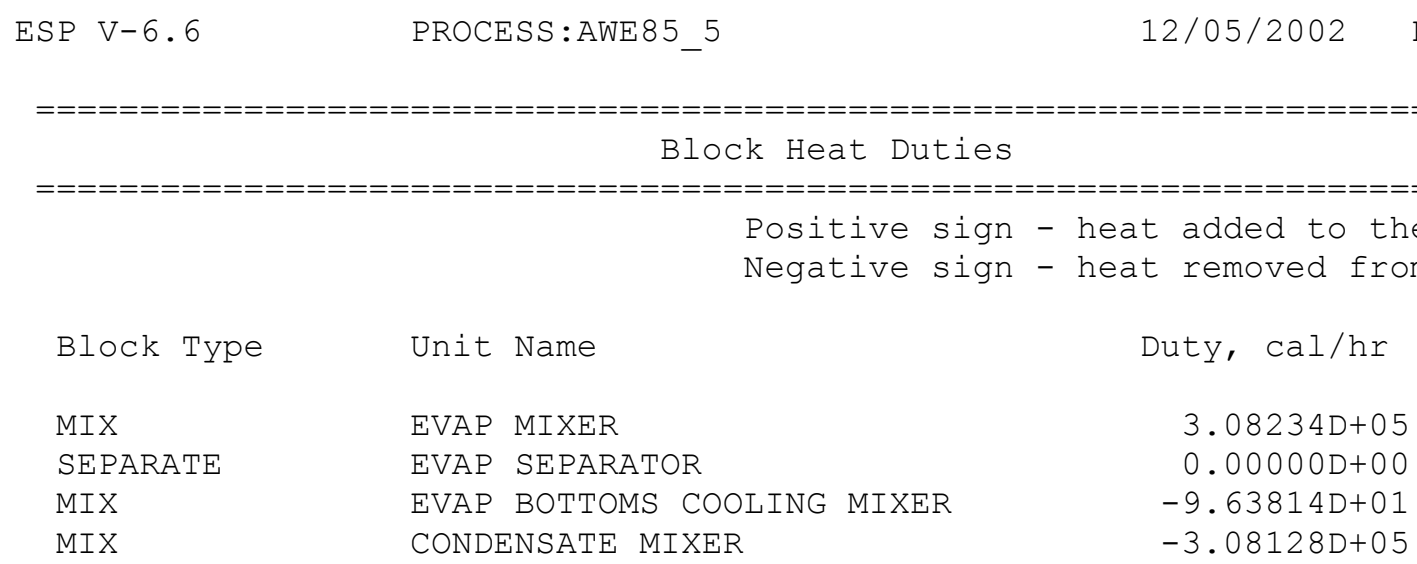


WSRC-TR-2002-00567

Revision 0

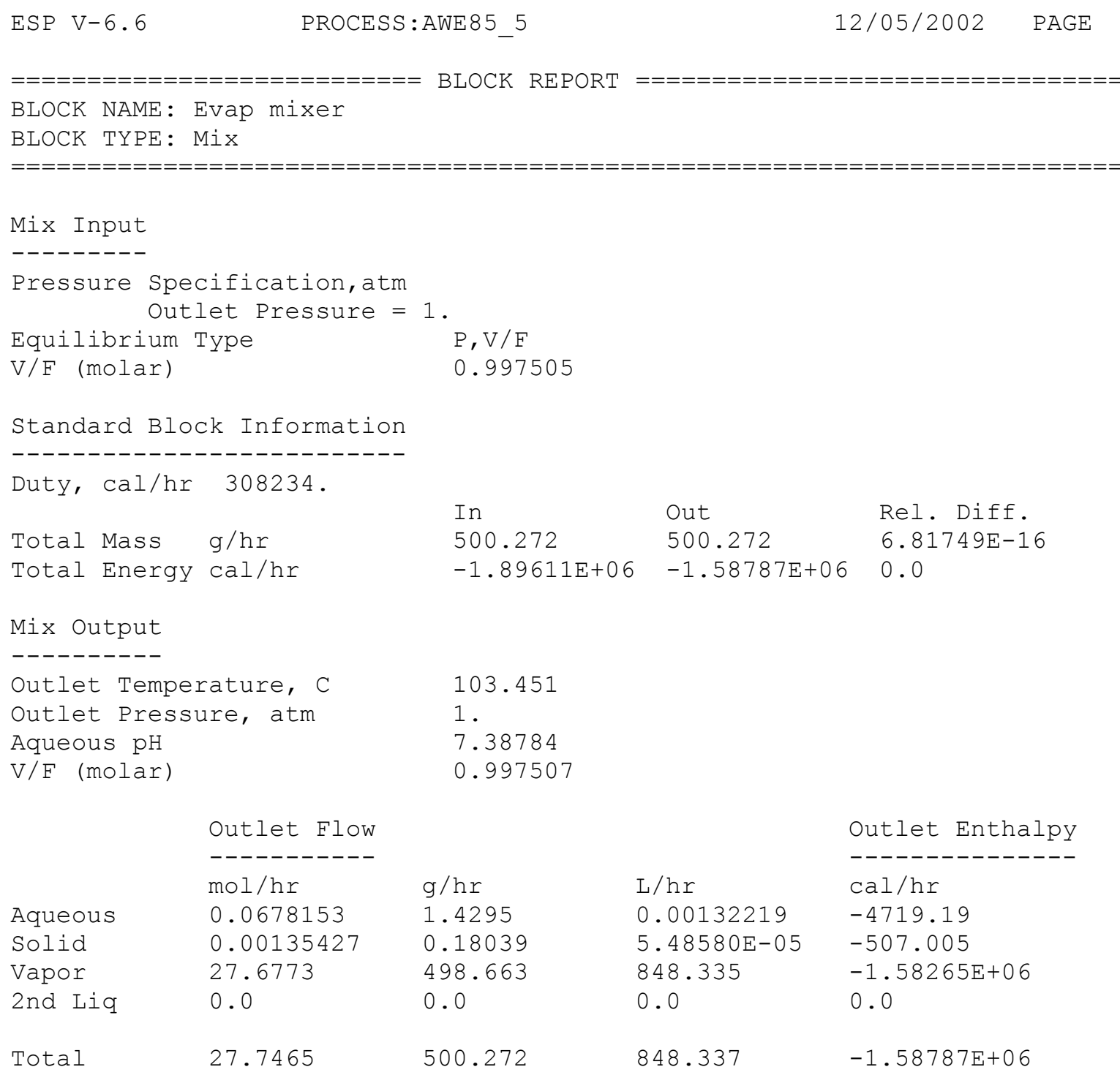


WSRC-TR-2002-00567

Revision 0

$\begin{array}{lllll}\text { ESP V-6.6 PROCESS:AWE85_5 } & 12 / 05 / 2002 & \text { PAGE } & 12\end{array}$

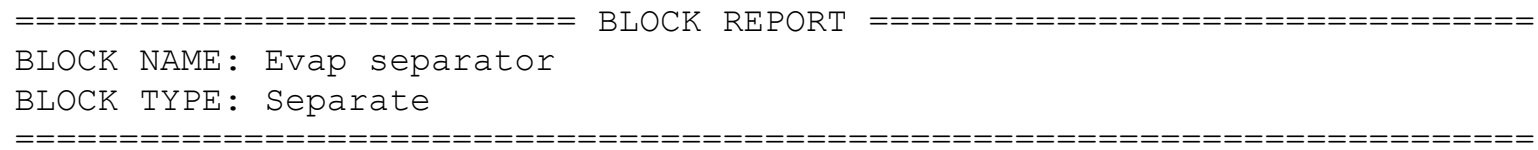

$0.0691696 \quad 1.60989$

$\mathrm{L} / \mathrm{hr} \quad \mathrm{cal} / \mathrm{hr}$

$5.48580 \mathrm{E}-05 \quad-507.005$

$0.0 \quad 0.0$

Total

$0.00137705 \quad-5226.2$

Outlet Enthalpy 
WSRC-TR-2002-00567

Revision 0

ESP V-6.6

PROCESS:AWE 85_5

Vapor Stream

Outlet Flow
--------
mol/hr
0.0
0.0
27.6773
0.0

Total
Overhead

g/hr

0.0

0.0

498.663

0.0

498.663
$12 / 05 / 2002$

PAGE

13

$\mathrm{L} / \mathrm{hr}$

0.0

0.0

848.335

0.0

Outlet Enthalpy
-----------
cal/hr
0.0
0.0
$-1.58265 \mathrm{E}+06$
0.0

848.335 
WSRC-TR-2002-00567

Revision 0

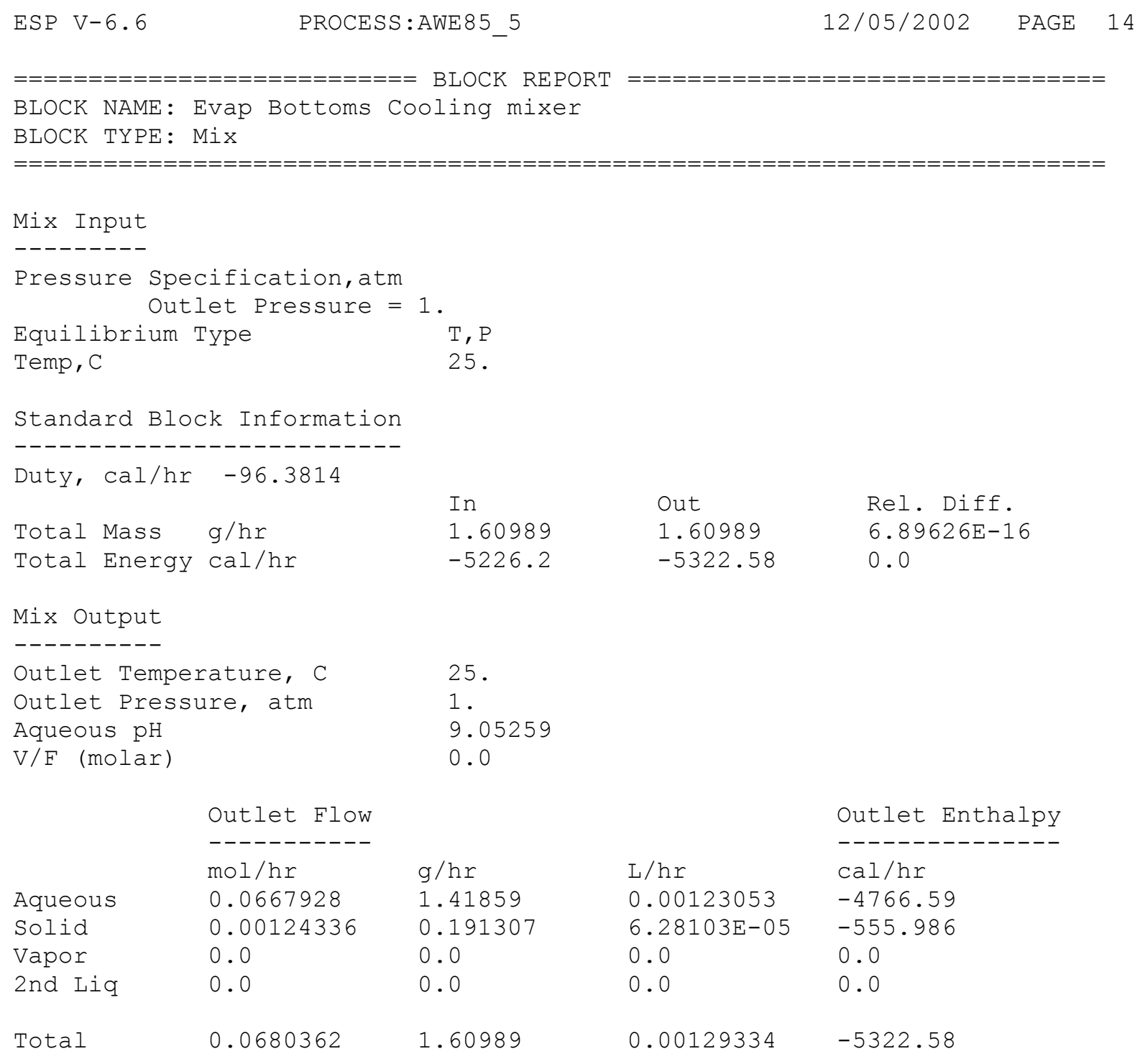


WSRC-TR-2002-00567

Revision 0

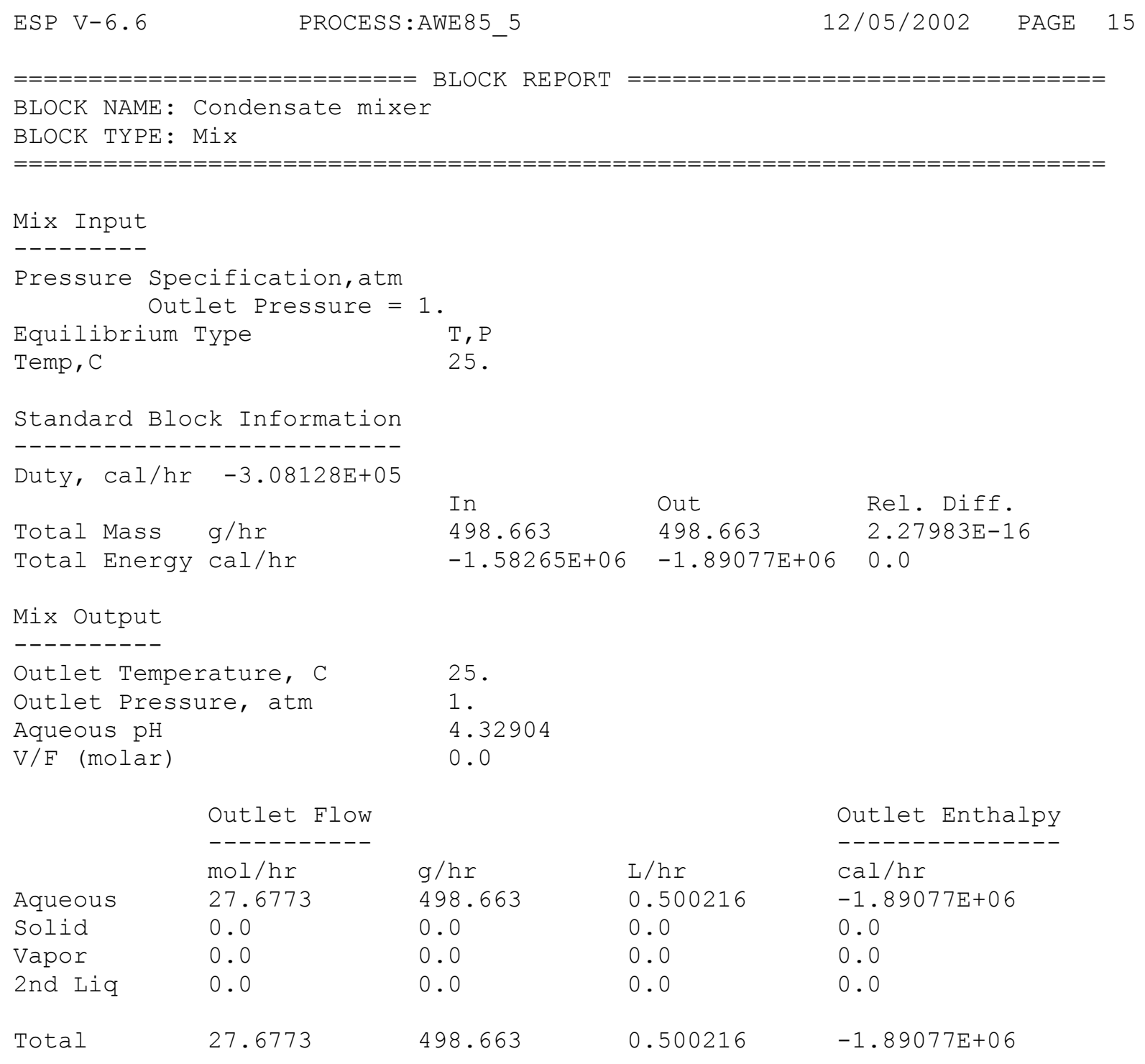


WSRC-TR-2002-00567

Revision 0

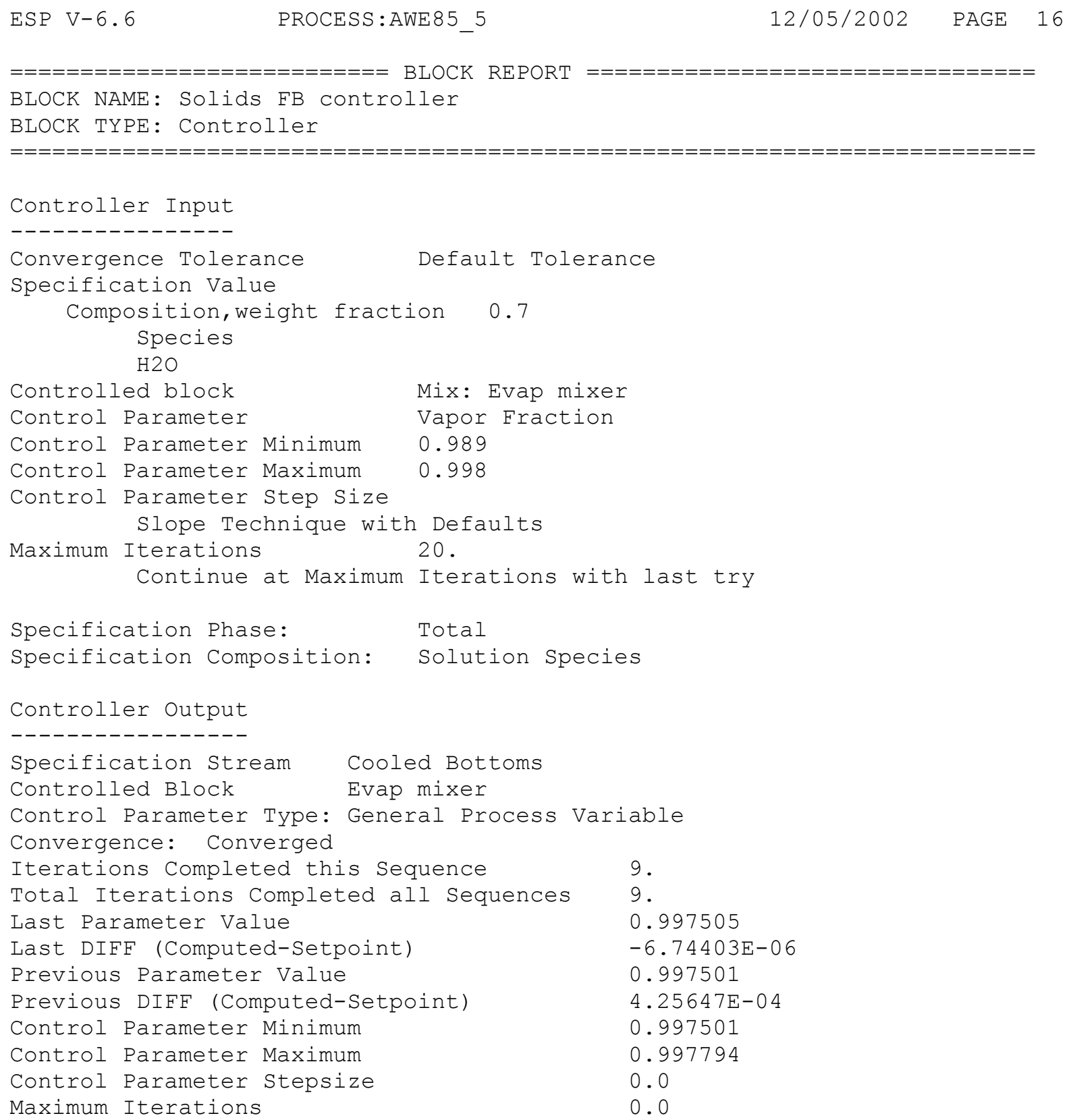


WSRC-TR-2002-00567

Revision 0

Influent Limit Composition 90\% Target $\mathrm{pH}=8.5$

8.5-90

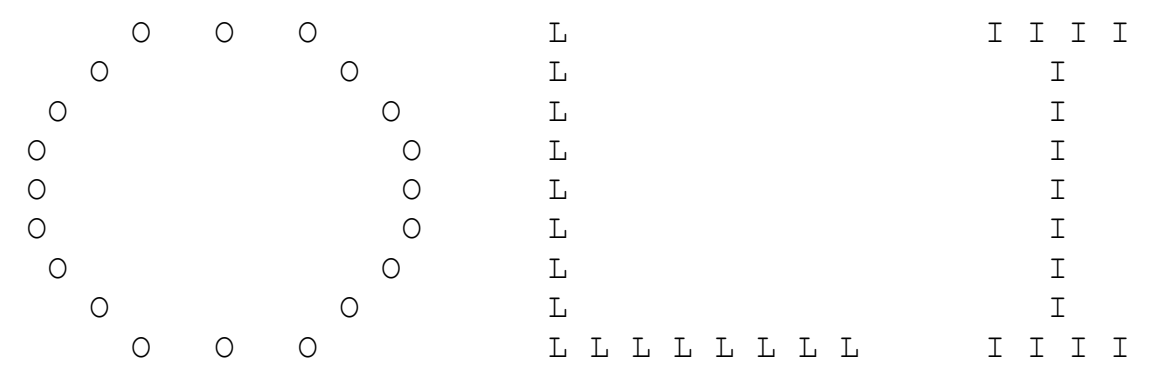

E N V I R O N M E N T A L S I M U L A T I O N P R O G R A M

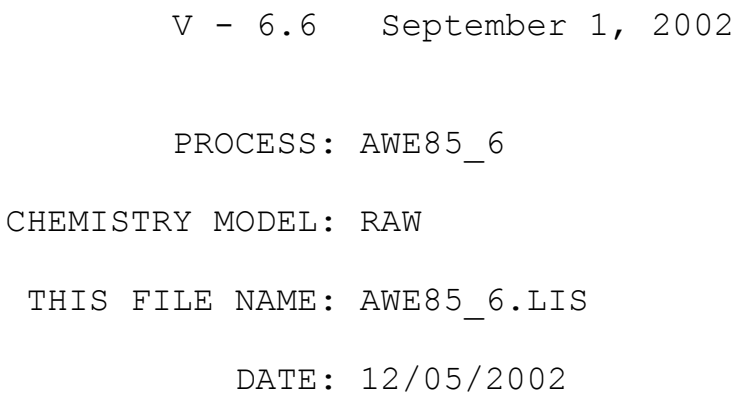




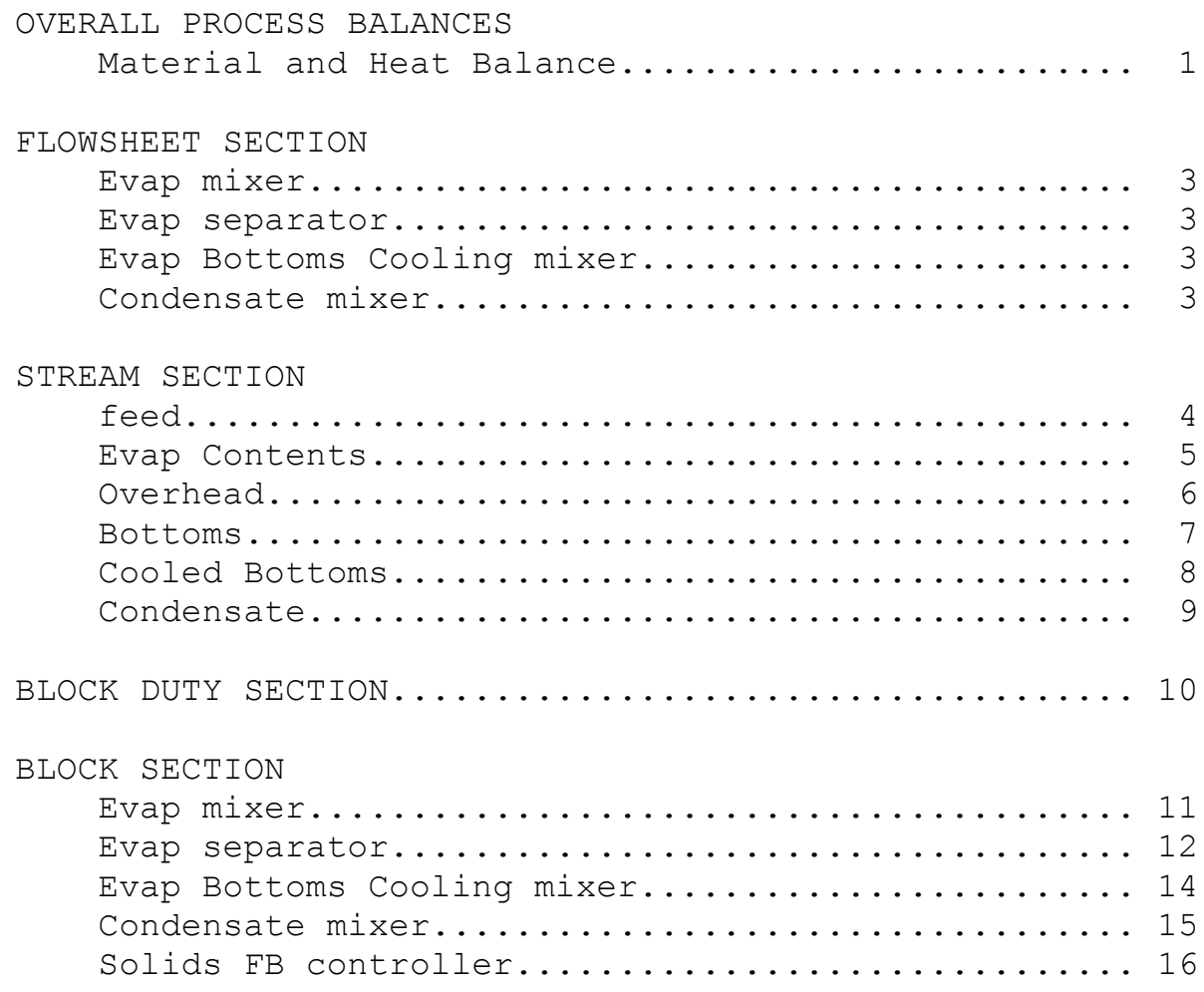


WSRC-TR-2002-00567

Revision 0

ESP V-6.6 PROCESS:AWE85_6
$\begin{array}{lcc}\text { Overall Process Balances } & \\ \text { Inlet } & \text { g/hr } & \mathrm{Cal} / \mathrm{hr} \\ ---------- & ---------- & ---------- \\ \text { FEED } & 2.50601 \mathrm{D}+02 & -9.48936 \mathrm{D}+05 \\ \text { =========== } & ========== & ========= \\ \text { Total in } & 2.50601 \mathrm{D}+02 & -9.48936 \mathrm{D}+05\end{array}$

\begin{tabular}{lrr} 
Outlet & \multicolumn{1}{c}{$g / \mathrm{hr}$} & $\mathrm{cal} / \mathrm{hr}$ \\
----------- & ---------- & ----------- \\
COOLED BOTTOMS & $2.33689 \mathrm{D}+00$ & $-7.66557 \mathrm{D}+03$ \\
CONDENSATE & $2.48264 \mathrm{D}+02$ & $-9.41267 \mathrm{D}+05$ \\
$==========$ & $==========$ & $==========$ \\
Total out & $2.50601 \mathrm{D}+02$ & $-9.48932 \mathrm{D}+05$
\end{tabular}

Block Heat Duties

cal/hr
----------
$1.53562 \mathrm{D}+05$
$-1.42231 \mathrm{D}+02$
$-1.53416 \mathrm{D}+05$
$==========$
$3.61606 \mathrm{D}+00$

DIFFERENCE

$4.37694 \mathrm{D}-12$

$0.00000 D+00$

REL DIFEERENCE

1. $74658 \mathrm{D}-14$

$0.00000 \mathrm{D}+00$

Material Code Balances

\begin{tabular}{|c|c|c|c|c|}
\hline Code & $\begin{array}{l}\text { Input } \\
\text { mol/hr }\end{array}$ & $\begin{array}{l}\text { Outlet } \\
\text { mol/hr }\end{array}$ & $\begin{array}{l}\text { Difference } \\
\text { mol/hr }\end{array}$ & Rel Diff \\
\hline------------ & ------------ & ------------ & ------------ & ------------- \\
\hline $\mathrm{H}(+1)$ & $2.77344 \mathrm{D}+01$ & $2.77344 \mathrm{D}+01$ & $2.48690 \mathrm{D}-14$ & $8.96683 \mathrm{D}-16$ \\
\hline$K(+1)$ & $8.50332 \mathrm{D}-04$ & $8.50332 \mathrm{D}-04$ & $1.08420 \mathrm{D}-18$ & $1.27503 \mathrm{D}-15$ \\
\hline $\mathrm{NA}(+1)$ & $5.78237 D-03$ & $5.78237 D-03$ & $-8.67362 D-18$ & $-1.50001 D-15$ \\
\hline $\mathrm{BA}(+2)$ & $7.29927 \mathrm{D}-07$ & $7.29927 D-07$ & $1.05879 \mathrm{D}-22$ & $1.45054 \mathrm{D}-16$ \\
\hline $\mathrm{CA}(+2)$ & $1.24688 D-03$ & $1.24688 D-03$ & $0.00000 \mathrm{D}+00$ & $0.00000 D+00$ \\
\hline $\mathrm{ZN}(+2)$ & $5.04587 \mathrm{D}-05$ & $5.04587 \mathrm{D}-05$ & $-6.77626 \mathrm{D}-21$ & $-1.34293 \mathrm{D}-16$ \\
\hline $\mathrm{CU}(+2)$ & $9.44882 \mathrm{D}-06$ & $9.44882 \mathrm{D}-06$ & $0.00000 \mathrm{D}+00$ & $0.00000 \mathrm{D}+00$ \\
\hline $\mathrm{FE}(+2)$ & $1.30824 \mathrm{D}-04$ & $1.30824 \mathrm{D}-04$ & $2.71051 \mathrm{D}-20$ & $2.07187 \mathrm{D}-16$ \\
\hline $\mathrm{MG}(+2)$ & $5.34979 D-04$ & $5.34979 D-04$ & $0.00000 \mathrm{D}+00$ & $0.00000 D+00$ \\
\hline $\mathrm{PB}(+2)$ & $6.28019 D-06$ & $6.28019 D-06$ & $2.54110 \mathrm{D}-21$ & $4.04621 \mathrm{D}-16$ \\
\hline $\mathrm{AL}(+3)$ & $1.18519 \mathrm{D}-04$ & $1.18519 \mathrm{D}-04$ & $0.00000 \mathrm{D}+00$ & $0.00000 \mathrm{D}+00$ \\
\hline $\mathrm{NI}(+2)$ & $2.04429 D-06$ & $2.04429 D-06$ & $-4.23516 \mathrm{D}-22$ & $-2.07170 \mathrm{D}-16$ \\
\hline$O(-2)$ & $1.38836 \mathrm{D}+01$ & $1.38836 \mathrm{D}+01$ & $-3.55271 D-15$ & $-2.55893 D-16$ \\
\hline $\operatorname{CL}(-1)$ & $3.94366 \mathrm{D}-03$ & $3.94366 \mathrm{D}-03$ & $-5.20417 D-18$ & $-1.31963 \mathrm{D}-15$ \\
\hline$C(+4)$ & $1.66667 \mathrm{D}-03$ & $1.66667 \mathrm{D}-03$ & $1.32273 D-17$ & $7.93636 \mathrm{D}-15$ \\
\hline$P(+5)$ & $8.42105 \mathrm{D}-04$ & $8.42105 \mathrm{D}-04$ & $0.00000 \mathrm{D}+00$ & $0.00000 \mathrm{D}+00$ \\
\hline$S(+6)$ & $1.25000 \mathrm{D}-03$ & $1.25000 \mathrm{D}-03$ & $6.50521 \mathrm{D}-19$ & $5.20417 D-16$ \\
\hline $\mathrm{N}(+5)$ & $1.27419 \mathrm{D}-03$ & $1.27419 \mathrm{D}-03$ & $2.16840 \mathrm{D}-19$ & $1.70178 D-16$ \\
\hline $\operatorname{SI}(+4)$ & $2.50000 \mathrm{D}-04$ & $2.50000 \mathrm{D}-04$ & $0.00000 \mathrm{D}+00$ & $0.00000 \mathrm{D}+00$ \\
\hline $\mathrm{SR}(+2)$ & $2.96804 \mathrm{D}-05$ & $2.96804 \mathrm{D}-05$ & $-3.38813 D-21$ & $-1.14154 \mathrm{D}-16$ \\
\hline
\end{tabular}


WSRC-TR-2002-00567

Revision 0

$\mathrm{CD}(+2)$

$\mathrm{CR}(+3)$

$\mathrm{U}(+4)$
$5.89286 \mathrm{D}-08$

$1.84615 \mathrm{D}-07$

7. $98319 \mathrm{D}-06$
$5.89286 \mathrm{D}-08-$

$1.84615 \mathrm{D}-07$

7.98319D-06
$-1.32349 \mathrm{D}-23-2.24592 \mathrm{D}-16$

$2.64698 \mathrm{D}-23 \quad 1.43378 \mathrm{D}-16$

$0.00000 \mathrm{D}+00 \quad 0.00000 \mathrm{D}+00$ 
WSRC-TR-2002-00567

Revision 0

ESP V-6.6

$\operatorname{DODEC}(-1)$
PROCESS:AWE 856

7.96599D-05
$12 / 05 / 2002$

7.96599D-05

$2.77094 \mathrm{D}-10$ 
WSRC-TR-2002-00567

Revision 0

\begin{tabular}{|c|c|c|c|}
\hline \multicolumn{4}{|l|}{$\begin{array}{l}\text { PROCESS BLOCKS } \\
============\end{array}$} \\
\hline $\begin{array}{l}\mathrm{BLOCK} \mathrm{NAME} \\
=========================\end{array}$ & $\begin{array}{l}\text { BLOCK TYPE } \\
===========\end{array}$ & $\begin{array}{l}\text { INLET STREAM }(\mathrm{S}) \\
==============\end{array}$ & $\begin{array}{l}\text { OUTLET STREAM }(\mathrm{S}) \\
==============\end{array}$ \\
\hline Evap mixer & $\operatorname{Mix}$ & feed & Evap Contents \\
\hline Evap separator & Separate & Evap Contents & $\begin{array}{l}\text { Overhead } \\
\text { Bottoms }\end{array}$ \\
\hline Evap Bottoms Cooling mixer & Mix & Bottoms & Cooled Bottoms \\
\hline Condensate mixer & Mix & Overhead & Condensate \\
\hline
\end{tabular}


WSRC-TR-2002-00567

Revision 0

ESP V-6. 6
PROCESS:AWE85 6
12/05/2002 PAGE 4

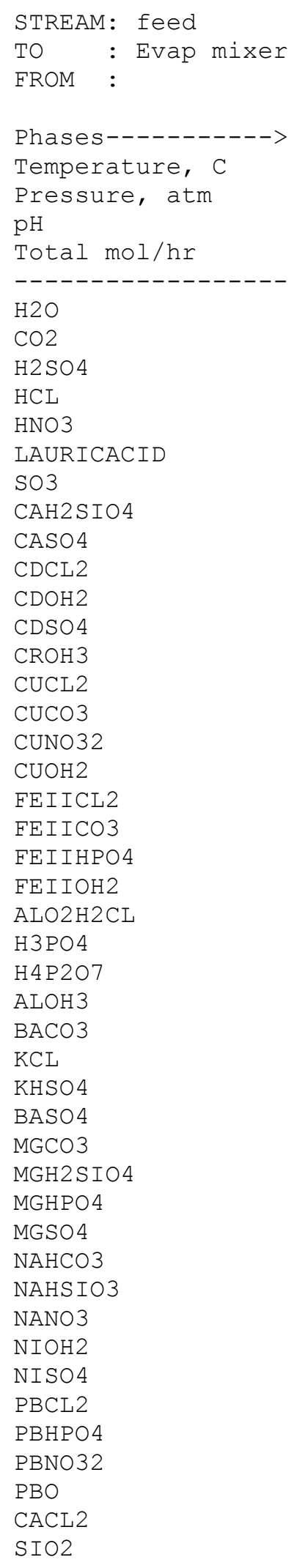

\begin{tabular}{|c|c|c|c|}
\hline Aqueous & Solid & Vapor & Organic \\
\hline 25 . & 25 . & 25 . & 25 . \\
\hline 1. & 1 . & 1 . & 1 . \\
\hline \multicolumn{4}{|l|}{6.32177} \\
\hline 13.88262 & $4.12436 \mathrm{E}-04$ & 0.0 & 0.0 \\
\hline \multicolumn{4}{|c|}{$\mathrm{mol} / \mathrm{hr}--------\operatorname{mol} / \mathrm{hr}--------\mathrm{mol} / \mathrm{hr}--------\operatorname{mol} / \mathrm{hr}---------$} \\
\hline 13.8662 & 0.0 & 0.0 & 0.0 \\
\hline $7.26281 E-04$ & 0.0 & 0.0 & 0.0 \\
\hline $4.13691 E-25$ & 0.0 & 0.0 & 0.0 \\
\hline $9.19580 \mathrm{E}-16$ & 0.0 & 0.0 & 0.0 \\
\hline $2.46275 \mathrm{E}-11$ & 0.0 & 0.0 & 0.0 \\
\hline $2.44827 E-06$ & 0.0 & 0.0 & 0.0 \\
\hline $5.36047 E-29$ & 0.0 & 0.0 & 0.0 \\
\hline $7.07109 \mathrm{E}-13$ & 0.0 & 0.0 & 0.0 \\
\hline $1.89817 E-05$ & 0.0 & 0.0 & 0.0 \\
\hline $4.12996 \mathrm{E}-09$ & 0.0 & 0.0 & 0.0 \\
\hline $3.66527 E-16$ & 0.0 & 0.0 & 0.0 \\
\hline $9.05159 \mathrm{E}-09$ & 0.0 & 0.0 & 0.0 \\
\hline $4.03820 \mathrm{E}-14$ & 0.0 & 0.0 & 0.0 \\
\hline 1. $20344 E-12$ & 0.0 & 0.0 & 0.0 \\
\hline $4.33256 \mathrm{E}-09$ & 0.0 & 0.0 & 0.0 \\
\hline $2.60427 E-14$ & 0.0 & 0.0 & 0.0 \\
\hline $1.06779 \mathrm{E}-10$ & 0.0 & 0.0 & 0.0 \\
\hline $3.07143 E-14$ & 0.0 & 0.0 & 0.0 \\
\hline $1.27261 \mathrm{E}-06$ & $5.26643 E-05$ & 0.0 & 0.0 \\
\hline $1.03020 E-07$ & 0.0 & 0.0 & 0.0 \\
\hline $3.92571 \mathrm{E}-13$ & 0.0 & 0.0 & 0.0 \\
\hline $1.78081 E-28$ & 0.0 & 0.0 & 0.0 \\
\hline $1.43957 \mathrm{E}-08$ & 0.0 & 0.0 & 0.0 \\
\hline 1. $48215 E-18$ & 0.0 & 0.0 & 0.0 \\
\hline $5.96955 \mathrm{E}-10$ & $1.18517 \mathrm{E}-04$ & 0.0 & 0.0 \\
\hline 1. $63223 E-12$ & 0.0 & 0.0 & 0.0 \\
\hline $7.41701 \mathrm{E}-08$ & 0.0 & 0.0 & 0.0 \\
\hline $1.17872 \mathrm{E}-12$ & 0.0 & 0.0 & 0.0 \\
\hline $5.11616 \mathrm{E}-11$ & $6.93444 E-07$ & 0.0 & 0.0 \\
\hline $5.42266 E-08$ & 0.0 & 0.0 & 0.0 \\
\hline $5.60329 \mathrm{E}-12$ & 0.0 & 0.0 & 0.0 \\
\hline $2.79378 E-05$ & 0.0 & 0.0 & 0.0 \\
\hline $2.71224 E-05$ & 0.0 & 0.0 & 0.0 \\
\hline $5.42249 E-06$ & 0.0 & 0.0 & 0.0 \\
\hline $1.70767 \mathrm{E}-07$ & 0.0 & 0.0 & 0.0 \\
\hline $1.12889 \mathrm{E}-06$ & 0.0 & 0.0 & 0.0 \\
\hline $3.49500 \mathrm{E}-13$ & 0.0 & 0.0 & 0.0 \\
\hline $3.18271 E-07$ & 0.0 & 0.0 & 0.0 \\
\hline $2.44058 \mathrm{E}-11$ & 0.0 & 0.0 & 0.0 \\
\hline $2.64735 \mathrm{E}-10$ & 0.0 & 0.0 & 0.0 \\
\hline $4.82115 E-13$ & 0.0 & 0.0 & 0.0 \\
\hline $6.37302 \mathrm{E}-14$ & 0.0 & 0.0 & 0.0 \\
\hline $4.05159 \mathrm{E}-26$ & 0.0 & 0.0 & 0.0 \\
\hline $2.49734 E-04$ & 0.0 & 0.0 & 0.0 \\
\hline
\end{tabular}




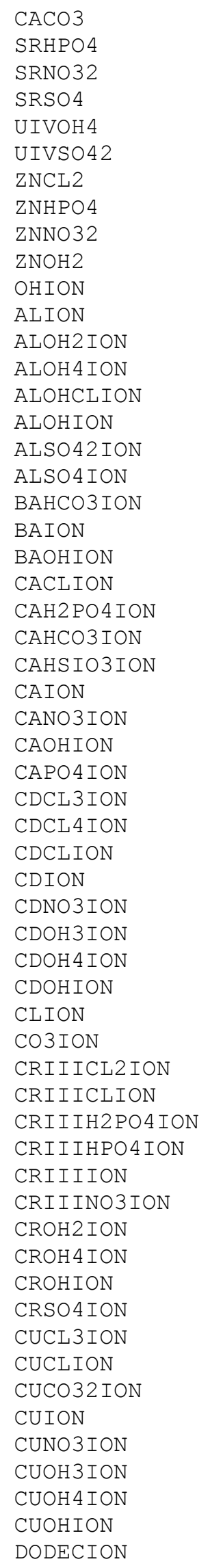

\begin{tabular}{|c|c|}
\hline $1.46587 \mathrm{E}-07$ & 0.0 \\
\hline $2.37378 E-08$ & 0.0 \\
\hline $1.67750 \mathrm{E}-09$ & 0.0 \\
\hline $4.53360 E-06$ & 0.0 \\
\hline $7.49648 \mathrm{E}-11$ & 0.0 \\
\hline $1.21692 \mathrm{E}-26$ & 0.0 \\
\hline $2.78801 E-09$ & 0.0 \\
\hline $1.93447 E-06$ & 0.0 \\
\hline $1.06153 \mathrm{E}-10$ & 0.0 \\
\hline 2.0006 & 0.0 \\
\hline $6.41241 E-09$ & 0.0 \\
\hline $7 E-12$ & 0.0 \\
\hline $6.36866 \mathrm{E}-11$ & 0.0 \\
\hline 3.84 & 0.0 \\
\hline 2.87 & 0.0 \\
\hline 1.16 & 0.0 \\
\hline $6 E-13$ & 0.0 \\
\hline $7 E-13$ & 0.0 \\
\hline $8 E-10$ & 0.0 \\
\hline $6 E-08$ & 0.0 \\
\hline 2.00 & 0.0 \\
\hline $47 E-11$ & 0.0 \\
\hline $02 E-06$ & 0.0 \\
\hline $9.59147 E-06$ & 0.0 \\
\hline $78 E-09$ & 0.0 \\
\hline $5.49487 E-04$ & 0.0 \\
\hline $3.41469 E-06$ & 0.0 \\
\hline $1.11491 \mathrm{E}-10$ & 0.0 \\
\hline $1.32786 \mathrm{E}-07$ & 0.0 \\
\hline $3.19120 \mathrm{E}-12$ & 0.0 \\
\hline $99 E-14$ & 0.0 \\
\hline $1.81243 E-08$ & 0.0 \\
\hline $5 E-08$ & 0.0 \\
\hline $1.71348 \mathrm{E}-10$ & 0.0 \\
\hline $1.32047 E-22$ & 0.0 \\
\hline $2.46651 E-30$ & 0.0 \\
\hline $2.64641 \mathrm{E}-12$ & 0.0 \\
\hline 0.00394332 & 0.0 \\
\hline $1.52054 \mathrm{E}-07$ & 0.0 \\
\hline $7.15983 E-19$ & 0.0 \\
\hline $8 E-16$ & 0.0 \\
\hline $3.61687 \mathrm{E}-13$ & 0.0 \\
\hline $574 E-07$ & 0.0 \\
\hline $2.84011 E-14$ & 0.0 \\
\hline $45 E-14$ & 0.0 \\
\hline $1 E-15$ & 0.0 \\
\hline $1.20289 \mathrm{E}-18$ & 0.0 \\
\hline $35 E-12$ & 0.0 \\
\hline 3.90 & 0.0 \\
\hline $2 E-17$ & 0.0 \\
\hline $2.47046 \mathrm{E}-10$ & 0.0 \\
\hline $19 E-12$ & 0.0 \\
\hline $32 E-09$ & 0.0 \\
\hline $5 E-11$ & 0.0 \\
\hline $12 E-16$ & 0.0 \\
\hline $2 E-23$ & 0 . \\
\hline $2 E-1 C$ & 0. \\
\hline $6 \mathrm{E}-0$ & 0 . \\
\hline
\end{tabular}

0.0

0.0

0.0

0.0

0.0

0.0

0.0

0.0

0.0

0.0

0.0

0.0

0.0

0.0

0.0

0.0

0.0

0.0

0.0

0.0

0.0

0.0

0.0

0.0

0.0

0.0

0.0

0.0

0.0

0.0

0.0

0.0

0.0

0.0

0.0

0.0

0.0

0.0

0.0

0.0

0.0

0.0

0.0

0.0

0.0

0.0

0.0

0.0

0.0

0.0

0.0

0.0

0.0

0.0

0.0

0.0

0.0

0.0
0.0

0.0

0.0

0.0

0.0

0.0

0.0

0.0

0.0

0.0

0.0

0.0

0.0

0.0

0.0

0.0

0.0

0.0

0.0

0.0

0.0

0.0

0.0

0.0

0.0

0.0

0.0

0.0

0.0

0.0

0.0

0.0

0.0

0.0

0.0

0.0

0.0

0.0

0.0

0.0

0.0

0.0

0.0

0.0

0.0

0.0

0.0

0.0

0.0

0.0

0.0

0.0

0.0

0.0

0.0

0.0

0.0

0.0 


\begin{tabular}{|c|c|c|c|c|}
\hline FEIICLION & $7.79560 \mathrm{E}-10$ & 0.0 & 0.0 & 0.0 \\
\hline FEIICO32ION & $2.56347 \mathrm{E}-11$ & 0.0 & 0.0 & 0.0 \\
\hline FEIIH2PO4ION & $1.22569 \mathrm{E}-07$ & 0.0 & 0.0 & 0.0 \\
\hline FEIIHCO3ION & $1.76700 \mathrm{E}-08$ & 0.0 & 0.0 & 0.0 \\
\hline FEIIION & $7.66149 \mathrm{E}-05$ & 0.0 & 0.0 & 0.0 \\
\hline FEIIOH3ION & $3.98161 \mathrm{E}-17$ & 0.0 & 0.0 & 0.0 \\
\hline FEIIOH 4 ION & $1.58503 \mathrm{E}-25$ & 0.0 & 0.0 & 0.0 \\
\hline FEIIOHION & $2.86488 \mathrm{E}-08$ & 0.0 & 0.0 & 0.0 \\
\hline H2 P2O 7 ION & $1.87455 \mathrm{E}-09$ & 0.0 & 0.0 & 0.0 \\
\hline H2 204 ION & $2.62704 \mathrm{E}-04$ & 0.0 & 0.0 & 0.0 \\
\hline H2SIO4ION & $2.77297 \mathrm{E}-14$ & 0.0 & 0.0 & 0.0 \\
\hline H3P2O 7 ION & $1.07634 \mathrm{E}-13$ & 0.0 & 0.0 & 0.0 \\
\hline H3SIO4ION & $9.11924 \mathrm{E}-08$ & 0.0 & 0.0 & 0.0 \\
\hline HCO3ION & $8.43696 \mathrm{E}-04$ & 0.0 & 0.0 & 0.0 \\
\hline HION & $1.43201 \mathrm{E}-07$ & 0.0 & 0.0 & 0.0 \\
\hline HP2O7ION & $2.58570 \mathrm{E}-09$ & 0.0 & 0.0 & 0.0 \\
\hline HPBO2 ION & $1.82988 \mathrm{E}-18$ & 0.0 & 0.0 & 0.0 \\
\hline HPO 4 ION & $6.29169 \mathrm{E}-05$ & 0.0 & 0.0 & 0.0 \\
\hline $\mathrm{HSO} 4 \mathrm{ION}$ & $2.70775 \mathrm{E}-08$ & 0.0 & 0.0 & 0.0 \\
\hline KION & $8.37669 \mathrm{E}-04$ & 0.0 & 0.0 & 0.0 \\
\hline KSO4 ION & $1.25893 \mathrm{E}-05$ & 0.0 & 0.0 & 0.0 \\
\hline MGH2 PO 4 ION & $1.04154 \mathrm{E}-05$ & 0.0 & 0.0 & 0.0 \\
\hline MGHCO3ION & $2.66726 \mathrm{E}-05$ & 0.0 & 0.0 & 0.0 \\
\hline MGHSIO3ION & $2.59133 \mathrm{E}-09$ & 0.0 & 0.0 & 0.0 \\
\hline MGION & $4.42601 \mathrm{E}-04$ & 0.0 & 0.0 & 0.0 \\
\hline MGOHION & $8.76849 \mathrm{E}-10$ & 0.0 & 0.0 & 0.0 \\
\hline MGP2O7ION & $1.76320 \mathrm{E}-08$ & 0.0 & 0.0 & 0.0 \\
\hline MGPO 4 ION & $1.55046 \mathrm{E}-07$ & 0.0 & 0.0 & 0.0 \\
\hline NACO3ION & $5.51493 \mathrm{E}-09$ & 0.0 & 0.0 & 0.0 \\
\hline NAION & 0.00568165 & 0.0 & 0.0 & 0.0 \\
\hline NASO 4 ION & $9.39968 \mathrm{E}-05$ & 0.0 & 0.0 & 0.0 \\
\hline NICLION & $1.24758 \mathrm{E}-09$ & 0.0 & 0.0 & 0.0 \\
\hline NIION & $1.71294 \mathrm{E}-06$ & 0.0 & 0.0 & 0.0 \\
\hline NINO3ION & $1.15514 \mathrm{E}-08$ & 0.0 & 0.0 & 0.0 \\
\hline NIOH3ION & $9.23969 \mathrm{E}-18$ & 0.0 & 0.0 & 0.0 \\
\hline NIOHION & $2.78313 \mathrm{E}-10$ & 0.0 & 0.0 & 0.0 \\
\hline NO3ION & 0.00126916 & 0.0 & 0.0 & 0.0 \\
\hline P207ION & $1.10144 \mathrm{E}-11$ & 0.0 & 0.0 & 0.0 \\
\hline PBCL3ION & $1.90983 \mathrm{E}-13$ & 0.0 & 0.0 & 0.0 \\
\hline PBCL 4 ION & $2.87853 \mathrm{E}-15$ & 0.0 & 0.0 & 0.0 \\
\hline PBCLION & $6.30075 \mathrm{E}-10$ & 0.0 & 0.0 & 0.0 \\
\hline PBH2 PO 4 ION & $6.27699 \mathrm{E}-11$ & 0.0 & 0.0 & 0.0 \\
\hline PBION & $3.20876 \mathrm{E}-09$ & 0.0 & 0.0 & 0.0 \\
\hline PBNO33ION & $4.24531 \mathrm{E}-16$ & 0.0 & 0.0 & 0.0 \\
\hline PBNO3ION & $1.45841 \mathrm{E}-10$ & 0.0 & 0.0 & 0.0 \\
\hline PBOHION & $9.36094 \mathrm{E}-11$ & 0.0 & 0.0 & 0.0 \\
\hline PO4ION & $1.67225 \mathrm{E}-10$ & 0.0 & 0.0 & 0.0 \\
\hline SO4ION & 0.00109173 & 0.0 & 0.0 & 0.0 \\
\hline SRION & $2.47871 \mathrm{E}-05$ & 0.0 & 0.0 & 0.0 \\
\hline SRNO3ION & $3.34225 \mathrm{E}-07$ & 0.0 & 0.0 & 0.0 \\
\hline SROHION & $1.91654 \mathrm{E}-12$ & 0.0 & 0.0 & 0.0 \\
\hline SRPO 4 ION & $8.40519 \mathrm{E}-11$ & 0.0 & 0.0 & 0.0 \\
\hline UIVCLION & 0.0 & 0.0 & 0.0 & 0.0 \\
\hline UIVION & $3.62921 \mathrm{E}-30$ & 0.0 & 0.0 & 0.0 \\
\hline UIVOH2ION & $4.48105 \mathrm{E}-21$ & 0.0 & 0.0 & 0.0 \\
\hline UIVOH3ION & $4.91737 \mathrm{E}-16$ & 0.0 & 0.0 & 0.0 \\
\hline UIVOH5ION & $1.00938 \mathrm{E}-14$ & 0.0 & 0.0 & 0.0 \\
\hline UIVOHION & $8.45819 \mathrm{E}-25$ & 0.0 & 0.0 & 0.0 \\
\hline
\end{tabular}


WSRC-TR-2002-00567

Revision 0

\begin{tabular}{|c|c|c|c|c|}
\hline UIVSO 4 ION & $1.88303 E-27$ & 0.0 & 0.0 & 0.0 \\
\hline ZNCL3ION & $1.51608 \mathrm{E}-11$ & 0.0 & 0.0 & 0.0 \\
\hline ZNCLION & $2.33179 E-07$ & 0.0 & 0.0 & 0.0 \\
\hline ZNH2 PO 4 ION & $3.74678 E-07$ & 0.0 & 0.0 & 0.0 \\
\hline ZNHCO3ION & $6.85473 E-07$ & 0.0 & 0.0 & 0.0 \\
\hline ZNION & $2.02850 E-05$ & 0.0 & 0.0 & 0.0 \\
\hline ZNNO3ION & $1.36132 \mathrm{E}-07$ & 0.0 & 0.0 & 0.0 \\
\hline ZNOH3ION & $5.05294 \mathrm{E}-15$ & 0.0 & 0.0 & 0.0 \\
\hline ZNOH $4 \mathrm{ION}$ & $2.50812 E-21$ & 0.0 & 0.0 & 0.0 \\
\hline ZNOHION & $2.87733 E-08$ & 0.0 & 0.0 & 0.0 \\
\hline $\mathrm{CA} 3 \mathrm{PO} 42$ & 0.0 & $2.18414 E-04$ & 0.0 & 0.0 \\
\hline $\mathrm{CU} 3 \mathrm{PO} 42.2 \mathrm{H} 2 \mathrm{O}$ & 0.0 & $3.14582 E-06$ & 0.0 & 0.0 \\
\hline PB3PO4 2 & 0.0 & $2.09192 E-06$ & 0.0 & 0.0 \\
\hline UIVO2 & 0.0 & $7.98313 E-06$ & 0.0 & 0.0 \\
\hline $\mathrm{ZN} 3 \mathrm{PO} 42.2 \mathrm{H} 2 \mathrm{O}$ & 0.0 & $8.92599 E-06$ & 0.0 & 0.0 \\
\hline & $===========$ & $===========$ & $===========$ & $===========$ \\
\hline Total g/hr & 250.509 & 0.0921869 & 0.0 & 0.0 \\
\hline Volume, L/hr & 0.250778 & $2.71286 \mathrm{E}-05$ & 0.0 & 0.0 \\
\hline Enthalpy, cal/hr & $-9.48666 \mathrm{E}+05$ & -270.041 & 0.0 & 0.0 \\
\hline Density, g/L & 998.929 & 3398.15 & & \\
\hline Vapor fraction & 0.0 & 0.0 & 0.0 & 0.0 \\
\hline Solid fraction & 0.0 & 1 . & 0.0 & 0.0 \\
\hline Organic fraction & 0.0 & 0.0 & 0.0 & 0.0 \\
\hline Osmotic Pres, atm & 1.62537 & & & \\
\hline Redox Pot, volts & 0.0 & & & \\
\hline E-Con, 1/ohm-cm & 0.00365003 & & & \\
\hline E-Con, cm2/ohm-mol & 103.795 & & & \\
\hline Abs Visc, cP & 0.897488 & & & \\
\hline Rel Visc & 1.0076 & & & \\
\hline Ionic Strength & 0.0443675 & & & \\
\hline
\end{tabular}


WSRC-TR-2002-00567

Revision 0

ESP V-6. 6

PROCESS :AWE 85 _ 6
12/05/2002 PAGE 5

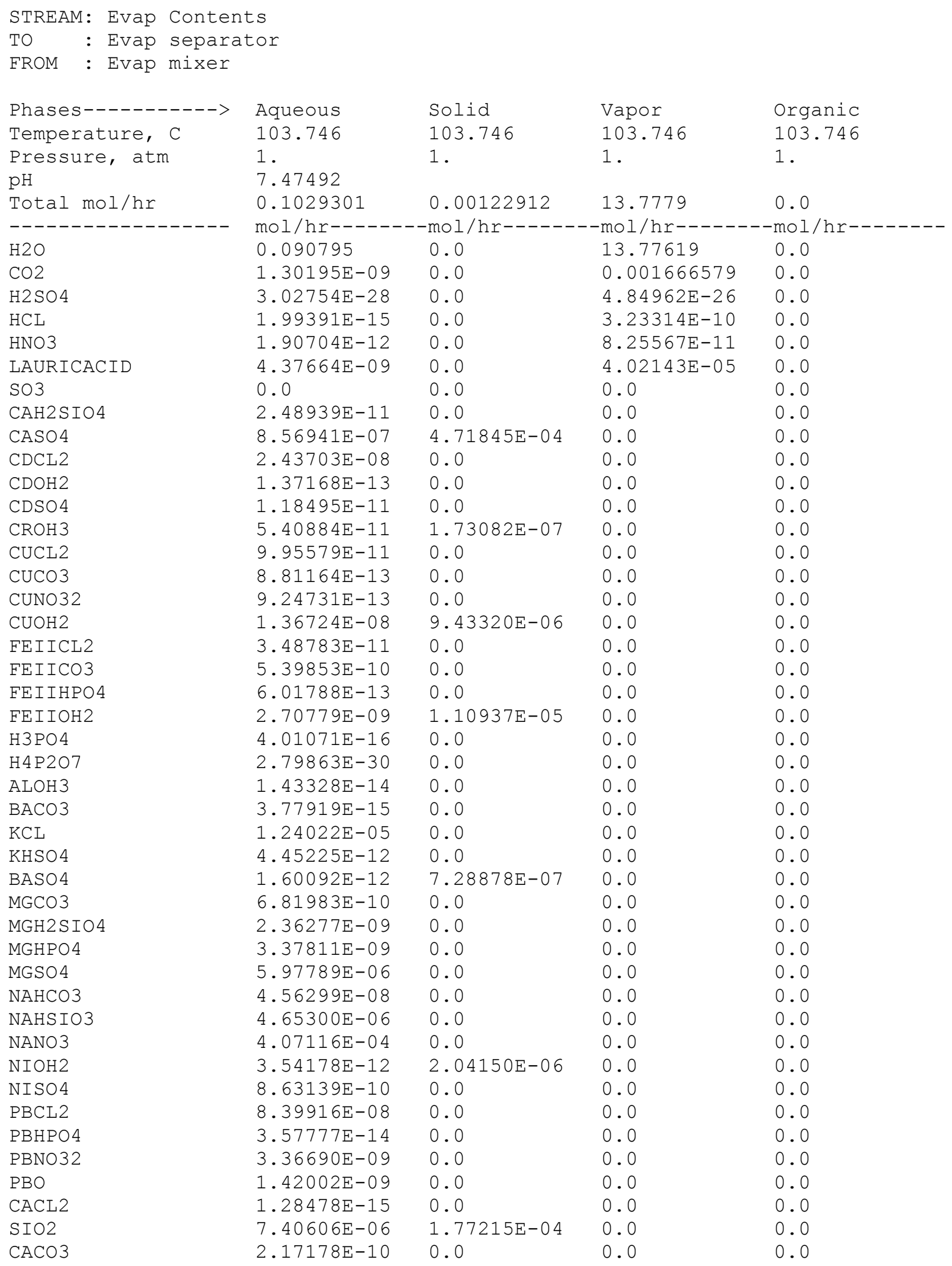




\begin{tabular}{|c|c|c|c|c|}
\hline SRHPO 4 & $6.11501 \mathrm{E}-14$ & 0.0 & 0.0 & 0.0 \\
\hline SRNO32 & $3.80408 \mathrm{E}-08$ & 0.0 & 0.0 & 0.0 \\
\hline SRSO 4 & $1.48456 \mathrm{E}-08$ & $2.94886 \mathrm{E}-05$ & 0.0 & 0.0 \\
\hline UIVOH 4 & $1.79148 \mathrm{E}-12$ & 0.0 & 0.0 & 0.0 \\
\hline ZNCL2 & $2.90958 \mathrm{E}-06$ & 0.0 & 0.0 & 0.0 \\
\hline ZNHPO 4 & $3.79499 \mathrm{E}-12$ & 0.0 & 0.0 & 0.0 \\
\hline ZNNO32 & $1.62456 \mathrm{E}-09$ & 0.0 & 0.0 & 0.0 \\
\hline ZNOH2 & $5.60310 \mathrm{E}-08$ & 0.0 & 0.0 & 0.0 \\
\hline OHION & $4.26305 \mathrm{E}-08$ & 0.0 & 0.0 & 0.0 \\
\hline ALION & $2.57599 \mathrm{E}-25$ & 0.0 & 0.0 & 0.0 \\
\hline ALOH 2 ION & $7.20260 \mathrm{E}-18$ & 0.0 & 0.0 & 0.0 \\
\hline ALOH 4 ION & $4.91951 \mathrm{E}-12$ & 0.0 & 0.0 & 0.0 \\
\hline ALOHCLION & $1.13330 \mathrm{E}-20$ & 0.0 & 0.0 & 0.0 \\
\hline ALOHION & $2.71153 \mathrm{E}-21$ & 0.0 & 0.0 & 0.0 \\
\hline ALSO 42 ION & $2.91325 E-24$ & 0.0 & 0.0 & 0.0 \\
\hline ALSO 4 ION & $2.82544 \mathrm{E}-24$ & 0.0 & 0.0 & 0.0 \\
\hline BAHCO3ION & $2.54408 \mathrm{E}-13$ & 0.0 & 0.0 & 0.0 \\
\hline BAION & $1.04645 \mathrm{E}-09$ & 0.0 & 0.0 & 0.0 \\
\hline BAOHION & $1.03338 \mathrm{E}-14$ & 0.0 & 0.0 & 0.0 \\
\hline CACLION & $4.64256 \mathrm{E}-08$ & 0.0 & 0.0 & 0.0 \\
\hline CAH2 PO 4 ION & $1.17899 \mathrm{E}-11$ & 0.0 & 0.0 & 0.0 \\
\hline CAHCO3ION & $3.90574 \mathrm{E}-10$ & 0.0 & 0.0 & 0.0 \\
\hline CAHSIO3ION & $1.03707 \mathrm{E}-08$ & 0.0 & 0.0 & 0.0 \\
\hline CAION & $1.05658 \mathrm{E}-05$ & 0.0 & 0.0 & 0.0 \\
\hline CANO3ION & $3.97499 \mathrm{E}-06$ & 0.0 & 0.0 & 0.0 \\
\hline CAOHION & $2.03656 \mathrm{E}-09$ & 0.0 & 0.0 & 0.0 \\
\hline CAPO 4 ION & $9.25742 \mathrm{E}-11$ & 0.0 & 0.0 & 0.0 \\
\hline CDCL3ION & $2.54547 \mathrm{E}-08$ & 0.0 & 0.0 & 0.0 \\
\hline CDCL 4 ION & $7.45378 \mathrm{E}-09$ & 0.0 & 0.0 & 0.0 \\
\hline CDCLION & $1.59557 \mathrm{E}-09$ & 0.0 & 0.0 & 0.0 \\
\hline CDION & $3.14176 \mathrm{E}-11$ & 0.0 & 0.0 & 0.0 \\
\hline CDNO3ION & $9.79599 \mathrm{E}-12$ & 0.0 & 0.0 & 0.0 \\
\hline CDOH 3 ION & $3.93498 \mathrm{E}-17$ & 0.0 & 0.0 & 0.0 \\
\hline CDOH 4 ION & $6.48366 \mathrm{E}-21$ & 0.0 & 0.0 & 0.0 \\
\hline CDOHION & $1.08969 \mathrm{E}-12$ & 0.0 & 0.0 & 0.0 \\
\hline CLION & 0.00389878 & 0.0 & 0.0 & 0.0 \\
\hline CO3ION & $1.17057 \mathrm{E}-09$ & 0.0 & 0.0 & 0.0 \\
\hline CRIIICL2ION & $4.29653 \mathrm{E}-16$ & 0.0 & 0.0 & 0.0 \\
\hline CRIIICLION & $8.95011 \mathrm{E}-17$ & 0.0 & 0.0 & 0.0 \\
\hline CRIIIH2PO4 ION & $1.83883 \mathrm{E}-20$ & 0.0 & 0.0 & 0.0 \\
\hline CRIIIHPO4ION & $1.13573 \mathrm{E}-08$ & 0.0 & 0.0 & 0.0 \\
\hline CRIIIION & $4.34833 E-21$ & 0.0 & 0.0 & 0.0 \\
\hline CRIIINO3ION & $2.19364 \mathrm{E}-13$ & 0.0 & 0.0 & 0.0 \\
\hline CROH2ION & $7.25861 \mathrm{E}-13$ & 0.0 & 0.0 & 0.0 \\
\hline $\mathrm{CROH} 4 \mathrm{ION}$ & $2.36483 E-13$ & 0.0 & 0.0 & 0.0 \\
\hline CROHION & $1.10910 \mathrm{E}-10$ & 0.0 & 0.0 & 0.0 \\
\hline CRSO 4 ION & $9.73065 \mathrm{E}-12$ & 0.0 & 0.0 & 0.0 \\
\hline CUCL3ION & $1.77798 \mathrm{E}-12$ & 0.0 & 0.0 & 0.0 \\
\hline CUCLION & $7.18983 \mathrm{E}-10$ & 0.0 & 0.0 & 0.0 \\
\hline CUCO32ION & $3.80102 E-15$ & 0.0 & 0.0 & 0.0 \\
\hline CUION & $1.96220 \mathrm{E}-10$ & 0.0 & 0.0 & 0.0 \\
\hline CUNO3ION & $4.04334 \mathrm{E}-11$ & 0.0 & 0.0 & 0.0 \\
\hline CUOH3ION & $2.45521 \mathrm{E}-11$ & 0.0 & 0.0 & 0.0 \\
\hline CUOH 4 ION & $9.76650 \mathrm{E}-14$ & 0.0 & 0.0 & 0.0 \\
\hline CUOHION & $8.39850 \mathrm{E}-10$ & 0.0 & 0.0 & 0.0 \\
\hline DODECION & $3.94412 E-05$ & 0.0 & 0.0 & 0.0 \\
\hline FEIICLION & $8.36379 \mathrm{E}-09$ & 0.0 & 0.0 & 0.0 \\
\hline FEIICO32ION & $6.85011 \mathrm{E}-14$ & 0.0 & 0.0 & 0.0 \\
\hline
\end{tabular}




\begin{tabular}{|c|c|c|c|c|}
\hline FEIIH2PO4ION & $5.04001 \mathrm{E}-14$ & 0.0 & 0.0 & 0.0 \\
\hline FEIIHCO3ION & $1.16685 \mathrm{E}-12$ & 0.0 & 0.0 & 0.0 \\
\hline FEIIION & $1.05556 \mathrm{E}-06$ & 0.0 & 0.0 & 0.0 \\
\hline FEIIOH3ION & $1.01884 \mathrm{E}-10$ & 0.0 & 0.0 & 0.0 \\
\hline FEIIOH4ION & $4.62955 \mathrm{E}-15$ & 0.0 & 0.0 & 0.0 \\
\hline FEIIOHION & $1.44889 \mathrm{E}-07$ & 0.0 & 0.0 & 0.0 \\
\hline H2P2O7ION & $4.34210 \mathrm{E}-18$ & 0.0 & 0.0 & 0.0 \\
\hline H2 PO 4 ION & $1.56614 \mathrm{E}-10$ & 0.0 & 0.0 & 0.0 \\
\hline H2SIO 4 ION & $4.08733 \mathrm{E}-11$ & 0.0 & 0.0 & 0.0 \\
\hline H3P2O 7 ION & $2.70101 \mathrm{E}-24$ & 0.0 & 0.0 & 0.0 \\
\hline H3SIO 4 ION & $1.32563 \mathrm{E}-06$ & 0.0 & 0.0 & 0.0 \\
\hline HCO3ION & $3.00982 \mathrm{E}-08$ & 0.0 & 0.0 & 0.0 \\
\hline HION & $7.03022 \mathrm{E}-11$ & 0.0 & 0.0 & 0.0 \\
\hline HP207ION & $1.15966 \mathrm{E}-16$ & 0.0 & 0.0 & 0.0 \\
\hline HPBO2 ION & $2.30770 \mathrm{E}-11$ & 0.0 & 0.0 & 0.0 \\
\hline HPO 4 ION & $2.83619 \mathrm{E}-09$ & 0.0 & 0.0 & 0.0 \\
\hline HSO4ION & $7.86710 \mathrm{E}-10$ & 0.0 & 0.0 & 0.0 \\
\hline KION & $7.23065 \mathrm{E}-04$ & 0.0 & 0.0 & 0.0 \\
\hline KSO4 ION & $1.14865 \mathrm{E}-04$ & 0.0 & 0.0 & 0.0 \\
\hline MGH2 PO 4 ION & $2.48639 \mathrm{E}-11$ & 0.0 & 0.0 & 0.0 \\
\hline MGHCO3ION & $5.43017 \mathrm{E}-09$ & 0.0 & 0.0 & 0.0 \\
\hline MGHSIO3ION & $1.28646 \mathrm{E}-07$ & 0.0 & 0.0 & 0.0 \\
\hline MGION & $2.08852 \mathrm{E}-05$ & 0.0 & 0.0 & 0.0 \\
\hline MGOHION & $1.06621 \mathrm{E}-07$ & 0.0 & 0.0 & 0.0 \\
\hline MGP2O7ION & $4.53546 \mathrm{E}-13$ & 0.0 & 0.0 & 0.0 \\
\hline MGPO 4 ION & $6.64250 \mathrm{E}-10$ & 0.0 & 0.0 & 0.0 \\
\hline NACO3ION & $1.80894 \mathrm{E}-10$ & 0.0 & 0.0 & 0.0 \\
\hline NAION & 0.00537055 & 0.0 & 0.0 & 0.0 \\
\hline NASO 4 ION & $1.04548 \mathrm{E}-12$ & 0.0 & 0.0 & 0.0 \\
\hline NICLION & $1.27981 \mathrm{E}-10$ & 0.0 & 0.0 & 0.0 \\
\hline NIION & $1.47881 \mathrm{E}-09$ & 0.0 & 0.0 & 0.0 \\
\hline NINO3ION & $2.50481 \mathrm{E}-10$ & 0.0 & 0.0 & 0.0 \\
\hline NIOH3ION & $1.56572 \mathrm{E}-14$ & 0.0 & 0.0 & 0.0 \\
\hline NIOHION & $6.58340 \mathrm{E}-11$ & 0.0 & 0.0 & 0.0 \\
\hline NO3ION & $8.62820 \mathrm{E}-04$ & 0.0 & 0.0 & 0.0 \\
\hline P207ION & $4.85164 \mathrm{E}-17$ & 0.0 & 0.0 & 0.0 \\
\hline PBCL3ION & $1.90182 \mathrm{E}-07$ & 0.0 & 0.0 & 0.0 \\
\hline PBCL4 ION & $1.68331 \mathrm{E}-06$ & 0.0 & 0.0 & 0.0 \\
\hline PBCLION & $2.72105 \mathrm{E}-08$ & 0.0 & 0.0 & 0.0 \\
\hline PBH2PO4 ION & $1.47298 \mathrm{E}-15$ & 0.0 & 0.0 & 0.0 \\
\hline PBION & $1.94412 E-09$ & 0.0 & 0.0 & 0.0 \\
\hline PBNO33ION & $1.61992 \mathrm{E}-10$ & 0.0 & 0.0 & 0.0 \\
\hline PBNO3ION & $9.01168 \mathrm{E}-09$ & 0.0 & 0.0 & 0.0 \\
\hline PBOHION & $2.51963 \mathrm{E}-08$ & 0.0 & 0.0 & 0.0 \\
\hline PO4ION & $6.94035 E-13$ & 0.0 & 0.0 & 0.0 \\
\hline SO4ION & $6.26220 \mathrm{E}-04$ & 0.0 & 0.0 & 0.0 \\
\hline SRION & $5.39637 \mathrm{E}-09$ & 0.0 & 0.0 & 0.0 \\
\hline SRNO3ION & $1.33340 \mathrm{E}-07$ & 0.0 & 0.0 & 0.0 \\
\hline SROHION & $1.56583 \mathrm{E}-11$ & 0.0 & 0.0 & 0.0 \\
\hline SRPO 4 ION & $2.10597 \mathrm{E}-14$ & 0.0 & 0.0 & 0.0 \\
\hline UIVOH2ION & $4.61336 \mathrm{E}-26$ & 0.0 & 0.0 & 0.0 \\
\hline UIVOH3ION & $2.01776 \mathrm{E}-20$ & 0.0 & 0.0 & 0.0 \\
\hline UIVOH5ION & $2.94892 \mathrm{E}-14$ & 0.0 & 0.0 & 0.0 \\
\hline UIVOHION & $1.01967 \mathrm{E}-30$ & 0.0 & 0.0 & 0.0 \\
\hline ZNCL3ION & $3.81445 \mathrm{E}-06$ & 0.0 & 0.0 & 0.0 \\
\hline ZNCLION & $7.50177 \mathrm{E}-06$ & 0.0 & 0.0 & 0.0 \\
\hline ZNH2 PO 4 ION & $7.57712 \mathrm{E}-14$ & 0.0 & 0.0 & 0.0 \\
\hline ZNHCO3ION & $1.41289 \mathrm{E}-11$ & 0.0 & 0.0 & 0 . \\
\hline
\end{tabular}


WSRC-TR-2002-00567

Revision 0

\begin{tabular}{|c|c|c|c|c|}
\hline ZNION & $4.53685 E-07$ & 0.0 & 0.0 & 0.0 \\
\hline ZNNO3ION & $5.39589 \mathrm{E}-08$ & 0.0 & 0.0 & 0.0 \\
\hline ZNOH3ION & $3.62949 E-09$ & 0.0 & 0.0 & 0.0 \\
\hline ZNOH $4 \mathrm{ION}$ & $5.32544 \mathrm{E}-12$ & 0.0 & 0.0 & 0.0 \\
\hline ZNOHION & $5.41976 \mathrm{E}-06$ & 0.0 & 0.0 & 0.0 \\
\hline $\mathrm{CA} 3 \mathrm{PO} 42$ & 0.0 & $2.53192 \mathrm{E}-04$ & 0.0 & 0.0 \\
\hline CHAMOSITE7A & 0.0 & $5.92592 \mathrm{E}-05$ & 0.0 & 0.0 \\
\hline MG $3 \mathrm{PO} 42$ & 0.0 & $1.56351 \mathrm{E}-04$ & 0.0 & 0.0 \\
\hline MGOH2 & 0.0 & $3.88163 E-05$ & 0.0 & 0.0 \\
\hline PB3PO42 & 0.0 & 1. $41812 \mathrm{E}-06$ & 0.0 & 0.0 \\
\hline uIVO2 & 0.0 & $7.98317 E-06$ & 0.0 & 0.0 \\
\hline $\mathrm{ZN} 3 \mathrm{PO} 42.2 \mathrm{H} 2 \mathrm{O}$ & 0.0 & $1.00814 \mathrm{E}-05$ & 0.0 & 0.0 \\
\hline & $============$ & $============$ & $============$ & $===========$ \\
\hline Total g/hr & 2.10458 & 0.232308 & 248.264 & 0.0 \\
\hline Volume, L/hr & 0.00191896 & $5.45597 \mathrm{E}-05$ & 422.644 & 0.0 \\
\hline Enthalpy, cal/hr & -6853.07 & -670.273 & $-7.87851 E+05$ & 0.0 \\
\hline Density, g/L & 1096.73 & 4257.87 & 0.587407 & \\
\hline Vapor fraction & 0.0 & 0.0 & 1 . & 0.0 \\
\hline Solid fraction & 0.0 & 1. & 0.0 & 0.0 \\
\hline Organic fraction & 0.0 & 0.0 & 0.0 & 0.0 \\
\hline Osmotic Pres, atm & 212.438 & & & \\
\hline Redox Pot, volts & 0.0 & & & \\
\hline E-Con, 1/ohm-cm & 0.517329 & & & \\
\hline E-Con, cm2/ohm-mol & 62.3255 & & & \\
\hline Abs Visc, cP & 0.453761 & & & \\
\hline Rel Visc & 1.67516 & & & \\
\hline Ionic Strength & 4.18054 & & & \\
\hline
\end{tabular}


WSRC-TR-2002-00567

Revision 0

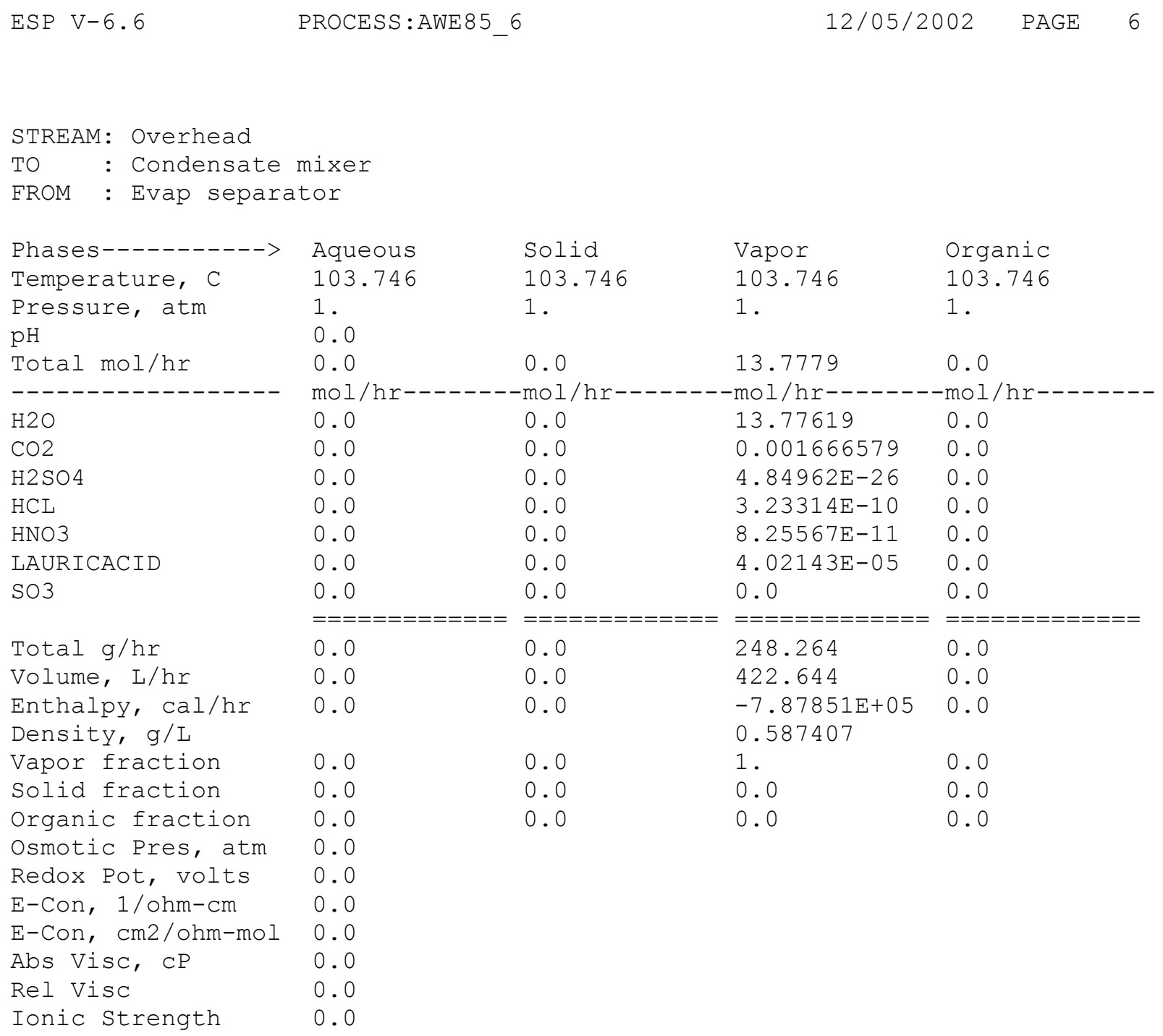


WSRC-TR-2002-00567

Revision 0

$\begin{array}{lllll}\text { ESP V-6.6 PROCESS:AWE85_6 } & \text { PAGE } 7\end{array}$

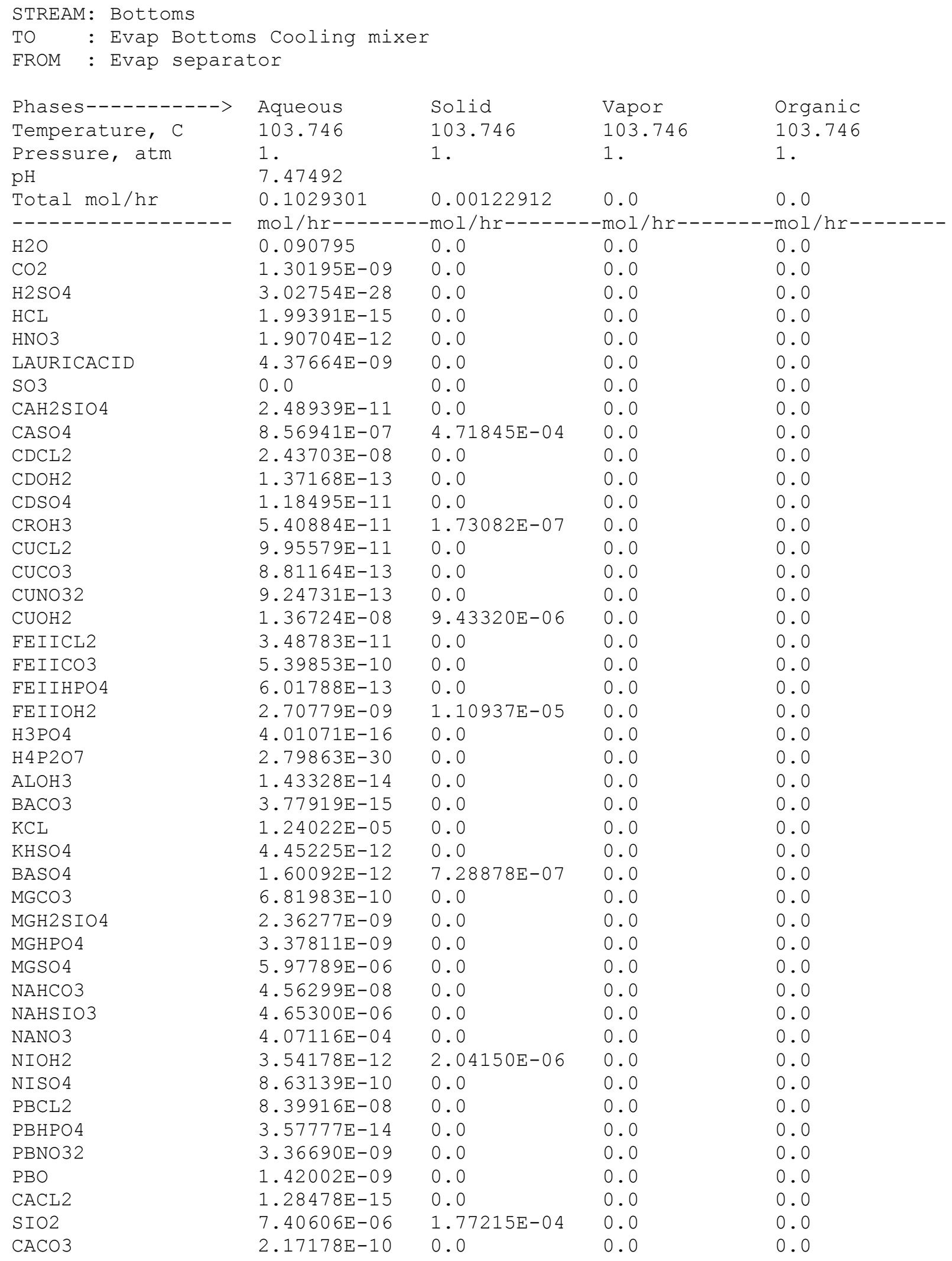




\begin{tabular}{|c|c|c|c|c|}
\hline SRHPO 4 & $6.11501 \mathrm{E}-14$ & 0.0 & 0.0 & 0.0 \\
\hline SRNO32 & $3.80408 \mathrm{E}-08$ & 0.0 & 0.0 & 0.0 \\
\hline SRSO 4 & $1.48456 \mathrm{E}-08$ & $2.94886 \mathrm{E}-05$ & 0.0 & 0.0 \\
\hline UIVOH 4 & $1.79148 \mathrm{E}-12$ & 0.0 & 0.0 & 0.0 \\
\hline ZNCL2 & $2.90958 \mathrm{E}-06$ & 0.0 & 0.0 & 0.0 \\
\hline ZNHPO 4 & $3.79499 \mathrm{E}-12$ & 0.0 & 0.0 & 0.0 \\
\hline ZNNO32 & $1.62456 \mathrm{E}-09$ & 0.0 & 0.0 & 0.0 \\
\hline ZNOH2 & $5.60310 \mathrm{E}-08$ & 0.0 & 0.0 & 0.0 \\
\hline OHION & $4.26305 \mathrm{E}-08$ & 0.0 & 0.0 & 0.0 \\
\hline ALION & $2.57599 \mathrm{E}-25$ & 0.0 & 0.0 & 0.0 \\
\hline ALOH 2 ION & $7.20260 \mathrm{E}-18$ & 0.0 & 0.0 & 0.0 \\
\hline ALOH 4 ION & $4.91951 \mathrm{E}-12$ & 0.0 & 0.0 & 0.0 \\
\hline ALOHCLION & $1.13330 \mathrm{E}-20$ & 0.0 & 0.0 & 0.0 \\
\hline ALOHION & $2.71153 \mathrm{E}-21$ & 0.0 & 0.0 & 0.0 \\
\hline ALSO 42 ION & $2.91325 E-24$ & 0.0 & 0.0 & 0.0 \\
\hline ALSO 4 ION & $2.82544 \mathrm{E}-24$ & 0.0 & 0.0 & 0.0 \\
\hline BAHCO3ION & $2.54408 \mathrm{E}-13$ & 0.0 & 0.0 & 0.0 \\
\hline BAION & $1.04645 \mathrm{E}-09$ & 0.0 & 0.0 & 0.0 \\
\hline BAOHION & $1.03338 \mathrm{E}-14$ & 0.0 & 0.0 & 0.0 \\
\hline CACLION & $4.64256 \mathrm{E}-08$ & 0.0 & 0.0 & 0.0 \\
\hline CAH2 PO 4 ION & $1.17899 \mathrm{E}-11$ & 0.0 & 0.0 & 0.0 \\
\hline CAHCO3ION & $3.90574 \mathrm{E}-10$ & 0.0 & 0.0 & 0.0 \\
\hline CAHSIO3ION & $1.03707 \mathrm{E}-08$ & 0.0 & 0.0 & 0.0 \\
\hline CAION & $1.05658 \mathrm{E}-05$ & 0.0 & 0.0 & 0.0 \\
\hline CANO3ION & $3.97499 \mathrm{E}-06$ & 0.0 & 0.0 & 0.0 \\
\hline CAOHION & $2.03656 \mathrm{E}-09$ & 0.0 & 0.0 & 0.0 \\
\hline CAPO 4 ION & $9.25742 \mathrm{E}-11$ & 0.0 & 0.0 & 0.0 \\
\hline CDCL3ION & $2.54547 \mathrm{E}-08$ & 0.0 & 0.0 & 0.0 \\
\hline CDCL 4 ION & $7.45378 \mathrm{E}-09$ & 0.0 & 0.0 & 0.0 \\
\hline CDCLION & $1.59557 \mathrm{E}-09$ & 0.0 & 0.0 & 0.0 \\
\hline CDION & $3.14176 \mathrm{E}-11$ & 0.0 & 0.0 & 0.0 \\
\hline CDNO3ION & $9.79599 \mathrm{E}-12$ & 0.0 & 0.0 & 0.0 \\
\hline CDOH 3 ION & $3.93498 \mathrm{E}-17$ & 0.0 & 0.0 & 0.0 \\
\hline CDOH 4 ION & $6.48366 \mathrm{E}-21$ & 0.0 & 0.0 & 0.0 \\
\hline CDOHION & $1.08969 \mathrm{E}-12$ & 0.0 & 0.0 & 0.0 \\
\hline CLION & 0.00389878 & 0.0 & 0.0 & 0.0 \\
\hline CO3ION & $1.17057 \mathrm{E}-09$ & 0.0 & 0.0 & 0.0 \\
\hline CRIIICL2ION & $4.29653 \mathrm{E}-16$ & 0.0 & 0.0 & 0.0 \\
\hline CRIIICLION & $8.95011 \mathrm{E}-17$ & 0.0 & 0.0 & 0.0 \\
\hline CRIIIH2PO4 ION & $1.83883 \mathrm{E}-20$ & 0.0 & 0.0 & 0.0 \\
\hline CRIIIHPO4ION & $1.13573 \mathrm{E}-08$ & 0.0 & 0.0 & 0.0 \\
\hline CRIIIION & $4.34833 E-21$ & 0.0 & 0.0 & 0.0 \\
\hline CRIIINO3ION & $2.19364 \mathrm{E}-13$ & 0.0 & 0.0 & 0.0 \\
\hline CROH2ION & $7.25861 \mathrm{E}-13$ & 0.0 & 0.0 & 0.0 \\
\hline $\mathrm{CROH} 4 \mathrm{ION}$ & $2.36483 E-13$ & 0.0 & 0.0 & 0.0 \\
\hline CROHION & $1.10910 \mathrm{E}-10$ & 0.0 & 0.0 & 0.0 \\
\hline CRSO 4 ION & $9.73065 \mathrm{E}-12$ & 0.0 & 0.0 & 0.0 \\
\hline CUCL3ION & $1.77798 \mathrm{E}-12$ & 0.0 & 0.0 & 0.0 \\
\hline CUCLION & $7.18983 \mathrm{E}-10$ & 0.0 & 0.0 & 0.0 \\
\hline CUCO32ION & $3.80102 E-15$ & 0.0 & 0.0 & 0.0 \\
\hline CUION & $1.96220 \mathrm{E}-10$ & 0.0 & 0.0 & 0.0 \\
\hline CUNO3ION & $4.04334 \mathrm{E}-11$ & 0.0 & 0.0 & 0.0 \\
\hline CUOH3ION & $2.45521 \mathrm{E}-11$ & 0.0 & 0.0 & 0.0 \\
\hline CUOH 4 ION & $9.76650 \mathrm{E}-14$ & 0.0 & 0.0 & 0.0 \\
\hline CUOHION & $8.39850 \mathrm{E}-10$ & 0.0 & 0.0 & 0.0 \\
\hline DODECION & $3.94412 E-05$ & 0.0 & 0.0 & 0.0 \\
\hline FEIICLION & $8.36379 \mathrm{E}-09$ & 0.0 & 0.0 & 0.0 \\
\hline FEIICO32ION & $6.85011 \mathrm{E}-14$ & 0.0 & 0.0 & 0.0 \\
\hline
\end{tabular}




\begin{tabular}{|c|c|c|c|c|}
\hline FEIIH2PO4ION & $5.04001 \mathrm{E}-14$ & 0.0 & 0.0 & 0.0 \\
\hline FEIIHCO3ION & $1.16685 \mathrm{E}-12$ & 0.0 & 0.0 & 0.0 \\
\hline FEIIION & $1.05556 \mathrm{E}-06$ & 0.0 & 0.0 & 0.0 \\
\hline FEIIOH3ION & $1.01884 \mathrm{E}-10$ & 0.0 & 0.0 & 0.0 \\
\hline FEIIOH4ION & $4.62955 \mathrm{E}-15$ & 0.0 & 0.0 & 0.0 \\
\hline FEIIOHION & $1.44889 \mathrm{E}-07$ & 0.0 & 0.0 & 0.0 \\
\hline H2P2O7ION & $4.34210 \mathrm{E}-18$ & 0.0 & 0.0 & 0.0 \\
\hline H2 PO 4 ION & $1.56614 \mathrm{E}-10$ & 0.0 & 0.0 & 0.0 \\
\hline H2SIO 4 ION & $4.08733 \mathrm{E}-11$ & 0.0 & 0.0 & 0.0 \\
\hline H3P2O 7 ION & $2.70101 \mathrm{E}-24$ & 0.0 & 0.0 & 0.0 \\
\hline H3SIO 4 ION & $1.32563 \mathrm{E}-06$ & 0.0 & 0.0 & 0.0 \\
\hline HCO3ION & $3.00982 \mathrm{E}-08$ & 0.0 & 0.0 & 0.0 \\
\hline HION & $7.03022 \mathrm{E}-11$ & 0.0 & 0.0 & 0.0 \\
\hline HP207ION & $1.15966 \mathrm{E}-16$ & 0.0 & 0.0 & 0.0 \\
\hline HPBO2 ION & $2.30770 \mathrm{E}-11$ & 0.0 & 0.0 & 0.0 \\
\hline HPO 4 ION & $2.83619 \mathrm{E}-09$ & 0.0 & 0.0 & 0.0 \\
\hline HSO4ION & $7.86710 \mathrm{E}-10$ & 0.0 & 0.0 & 0.0 \\
\hline KION & $7.23065 \mathrm{E}-04$ & 0.0 & 0.0 & 0.0 \\
\hline KSO4 ION & $1.14865 \mathrm{E}-04$ & 0.0 & 0.0 & 0.0 \\
\hline MGH2 PO 4 ION & $2.48639 \mathrm{E}-11$ & 0.0 & 0.0 & 0.0 \\
\hline MGHCO3ION & $5.43017 \mathrm{E}-09$ & 0.0 & 0.0 & 0.0 \\
\hline MGHSIO3ION & $1.28646 \mathrm{E}-07$ & 0.0 & 0.0 & 0.0 \\
\hline MGION & $2.08852 \mathrm{E}-05$ & 0.0 & 0.0 & 0.0 \\
\hline MGOHION & $1.06621 \mathrm{E}-07$ & 0.0 & 0.0 & 0.0 \\
\hline MGP2O7ION & $4.53546 \mathrm{E}-13$ & 0.0 & 0.0 & 0.0 \\
\hline MGPO 4 ION & $6.64250 \mathrm{E}-10$ & 0.0 & 0.0 & 0.0 \\
\hline NACO3ION & $1.80894 \mathrm{E}-10$ & 0.0 & 0.0 & 0.0 \\
\hline NAION & 0.00537055 & 0.0 & 0.0 & 0.0 \\
\hline NASO 4 ION & $1.04548 \mathrm{E}-12$ & 0.0 & 0.0 & 0.0 \\
\hline NICLION & $1.27981 \mathrm{E}-10$ & 0.0 & 0.0 & 0.0 \\
\hline NIION & $1.47881 \mathrm{E}-09$ & 0.0 & 0.0 & 0.0 \\
\hline NINO3ION & $2.50481 \mathrm{E}-10$ & 0.0 & 0.0 & 0.0 \\
\hline NIOH3ION & $1.56572 \mathrm{E}-14$ & 0.0 & 0.0 & 0.0 \\
\hline NIOHION & $6.58340 \mathrm{E}-11$ & 0.0 & 0.0 & 0.0 \\
\hline NO3ION & $8.62820 \mathrm{E}-04$ & 0.0 & 0.0 & 0.0 \\
\hline P207ION & $4.85164 \mathrm{E}-17$ & 0.0 & 0.0 & 0.0 \\
\hline PBCL3ION & $1.90182 \mathrm{E}-07$ & 0.0 & 0.0 & 0.0 \\
\hline PBCL4 ION & $1.68331 \mathrm{E}-06$ & 0.0 & 0.0 & 0.0 \\
\hline PBCLION & $2.72105 \mathrm{E}-08$ & 0.0 & 0.0 & 0.0 \\
\hline PBH2PO4 ION & $1.47298 \mathrm{E}-15$ & 0.0 & 0.0 & 0.0 \\
\hline PBION & $1.94412 E-09$ & 0.0 & 0.0 & 0.0 \\
\hline PBNO33ION & $1.61992 \mathrm{E}-10$ & 0.0 & 0.0 & 0.0 \\
\hline PBNO3ION & $9.01168 \mathrm{E}-09$ & 0.0 & 0.0 & 0.0 \\
\hline PBOHION & $2.51963 \mathrm{E}-08$ & 0.0 & 0.0 & 0.0 \\
\hline PO4ION & $6.94035 E-13$ & 0.0 & 0.0 & 0.0 \\
\hline SO4ION & $6.26220 \mathrm{E}-04$ & 0.0 & 0.0 & 0.0 \\
\hline SRION & $5.39637 \mathrm{E}-09$ & 0.0 & 0.0 & 0.0 \\
\hline SRNO3ION & $1.33340 \mathrm{E}-07$ & 0.0 & 0.0 & 0.0 \\
\hline SROHION & $1.56583 \mathrm{E}-11$ & 0.0 & 0.0 & 0.0 \\
\hline SRPO 4 ION & $2.10597 \mathrm{E}-14$ & 0.0 & 0.0 & 0.0 \\
\hline UIVOH2ION & $4.61336 \mathrm{E}-26$ & 0.0 & 0.0 & 0.0 \\
\hline UIVOH3ION & $2.01776 \mathrm{E}-20$ & 0.0 & 0.0 & 0.0 \\
\hline UIVOH5ION & $2.94892 \mathrm{E}-14$ & 0.0 & 0.0 & 0.0 \\
\hline UIVOHION & $1.01967 \mathrm{E}-30$ & 0.0 & 0.0 & 0.0 \\
\hline ZNCL3ION & $3.81445 \mathrm{E}-06$ & 0.0 & 0.0 & 0.0 \\
\hline ZNCLION & $7.50177 \mathrm{E}-06$ & 0.0 & 0.0 & 0.0 \\
\hline ZNH2 PO 4 ION & $7.57712 \mathrm{E}-14$ & 0.0 & 0.0 & 0.0 \\
\hline ZNHCO3ION & $1.41289 \mathrm{E}-11$ & 0.0 & 0.0 & 0 . \\
\hline
\end{tabular}


WSRC-TR-2002-00567

Revision 0

\begin{tabular}{|c|c|c|c|c|}
\hline ZNION & $4.53685 E-07$ & 0.0 & 0.0 & 0.0 \\
\hline ZNNO3ION & $5.39589 \mathrm{E}-08$ & 0.0 & 0.0 & 0.0 \\
\hline ZNOH3ION & $3.62949 E-09$ & 0.0 & 0.0 & 0.0 \\
\hline ZNOH $4 \mathrm{ION}$ & $5.32544 \mathrm{E}-12$ & 0.0 & 0.0 & 0.0 \\
\hline ZNOHION & $5.41976 \mathrm{E}-06$ & 0.0 & 0.0 & 0.0 \\
\hline $\mathrm{CA} 3 \mathrm{PO} 42$ & 0.0 & $2.53192 \mathrm{E}-04$ & 0.0 & 0.0 \\
\hline CHAMOSITE7A & 0.0 & $5.92592 \mathrm{E}-05$ & 0.0 & 0.0 \\
\hline MG $3 \mathrm{PO} 42$ & 0.0 & $1.56351 \mathrm{E}-04$ & 0.0 & 0.0 \\
\hline MGOH2 & 0.0 & $3.88163 E-05$ & 0.0 & 0.0 \\
\hline PB3PO42 & 0.0 & 1. $41812 \mathrm{E}-06$ & 0.0 & 0.0 \\
\hline uIVO2 & 0.0 & $7.98317 E-06$ & 0.0 & 0.0 \\
\hline $\mathrm{ZN} 3 \mathrm{PO} 42.2 \mathrm{H} 2 \mathrm{O}$ & 0.0 & $1.00814 \mathrm{E}-05$ & 0.0 & 0.0 \\
\hline & $===========$ & $============$ & $============$ & $===========$ \\
\hline Total g/hr & 2.10458 & 0.232308 & 0.0 & 0.0 \\
\hline Volume, L/hr & 0.00191896 & $5.45597 \mathrm{E}-05$ & 0.0 & 0.0 \\
\hline Enthalpy, cal/hr & -6853.07 & -670.273 & 0.0 & 0.0 \\
\hline Density, g/L & 1096.73 & 4257.87 & & \\
\hline Vapor fraction & 0.0 & 0.0 & 0.0 & 0.0 \\
\hline Solid fraction & 0.0 & 1. & 0.0 & 0.0 \\
\hline Organic fraction & 0.0 & 0.0 & 0.0 & 0.0 \\
\hline Osmotic Pres, atm & 212.438 & & & \\
\hline Redox Pot, volts & 0.0 & & & \\
\hline E-Con, 1/ohm-cm & 0.517329 & & & \\
\hline E-Con, cm2/ohm-mol & 62.3255 & & & \\
\hline Abs Visc, cP & 0.453761 & & & \\
\hline Rel Visc & 1.67516 & & & \\
\hline Ionic Strength & 4.18054 & & & \\
\hline
\end{tabular}


WSRC-TR-2002-00567

Revision 0

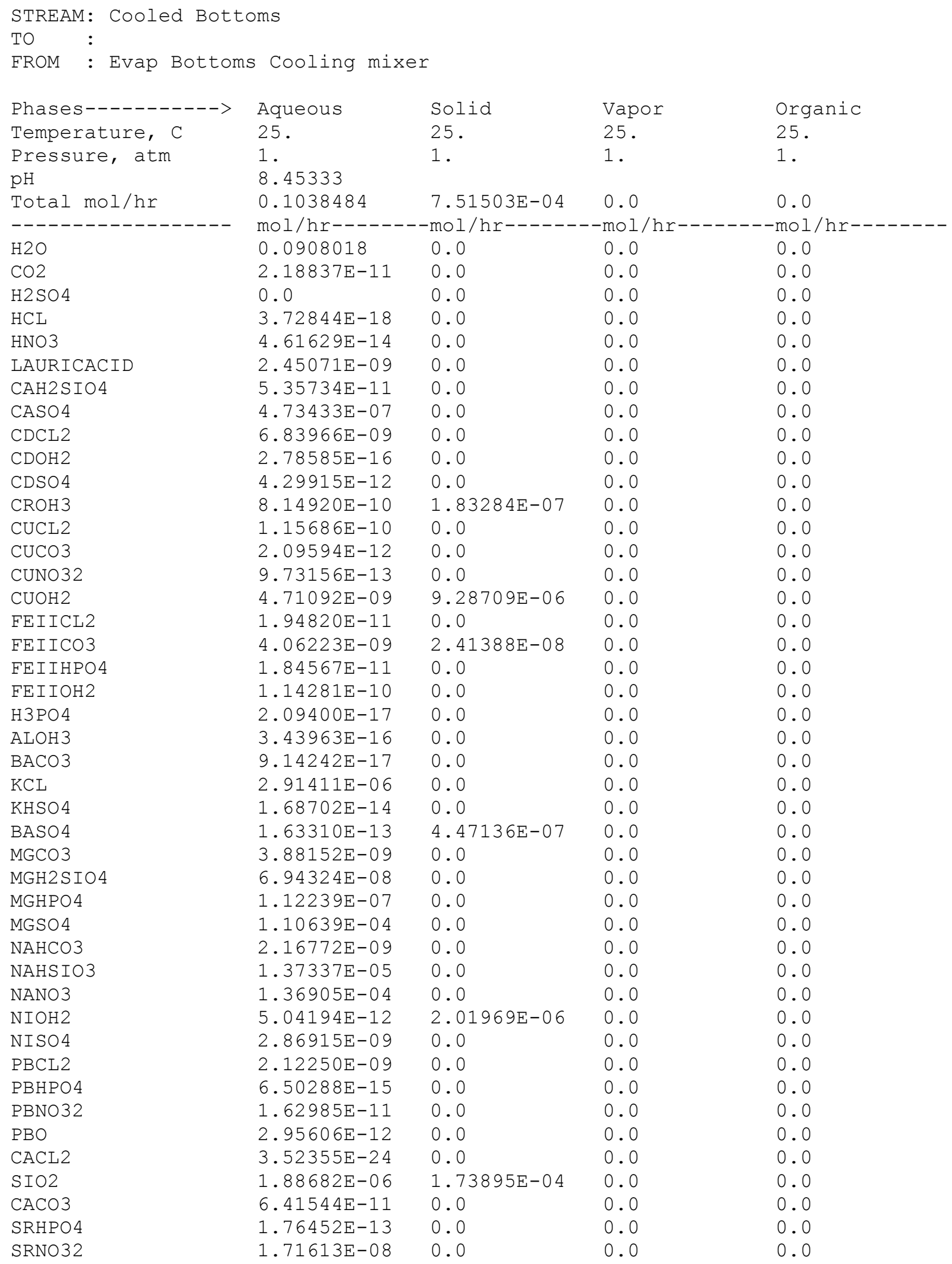




\begin{tabular}{|c|c|c|c|c|}
\hline $\mathrm{SRSO} 4$ & $3.42181 E-08$ & $2.91313 E-05$ & 0.0 & 0.0 \\
\hline UIVOH 4 & $1.77184 \mathrm{E}-13$ & 0.0 & 0.0 & 0.0 \\
\hline ZNCL2 & $1.95975 \mathrm{E}-07$ & 0.0 & 0.0 & 0.0 \\
\hline ZNHPO 4 & $3.84068 \mathrm{E}-11$ & 0.0 & 0.0 & 0.0 \\
\hline ZNNO32 & $2.90055 E-09$ & 0.0 & 0.0 & 0.0 \\
\hline $\mathrm{ZNOH} 2$ & $6.45414 \mathrm{E}-09$ & $4.84409 E-05$ & 0.0 & 0.0 \\
\hline OHION & $3.75646 \mathrm{E}-09$ & 0.0 & 0.0 & 0.0 \\
\hline ALION & $1.68417 \mathrm{E}-24$ & 0.0 & 0.0 & 0.0 \\
\hline ALOH2 ION & $7.72335 \mathrm{E}-19$ & 0.0 & 0.0 & 0.0 \\
\hline ALOH 4 ION & $7.44227 \mathrm{E}-14$ & 0.0 & 0.0 & 0.0 \\
\hline ALOHCLION & $7.90217 \mathrm{E}-21$ & 0.0 & 0.0 & 0.0 \\
\hline ALOHION & $1.19488 \mathrm{E}-21$ & 0.0 & 0.0 & 0.0 \\
\hline ALSO 42 ION & $9.33888 \mathrm{E}-24$ & 0.0 & 0.0 & 0.0 \\
\hline ALSO 4 ION & $7.51685 \mathrm{E}-24$ & 0.0 & 0.0 & 0.0 \\
\hline BAHCO3ION & $7.52584 \mathrm{E}-16$ & 0.0 & 0.0 & 0.0 \\
\hline BAION & $2.82791 \mathrm{E}-07$ & 0.0 & 0.0 & 0.0 \\
\hline BAOHION & $2.16291 \mathrm{E}-17$ & 0.0 & 0.0 & 0.0 \\
\hline CACLION & $4.23683 \mathrm{E}-11$ & 0.0 & 0.0 & 0.0 \\
\hline CAH2 PO 4 ION & $6.76513 \mathrm{E}-12$ & 0.0 & 0.0 & 0.0 \\
\hline CAHCO3ION & $1.18315 \mathrm{E}-10$ & 0.0 & 0.0 & 0.0 \\
\hline CAHSIO3ION & $4.19423 \mathrm{E}-09$ & 0.0 & 0.0 & 0.0 \\
\hline CAION & $8.57375 E-06$ & 0.0 & 0.0 & 0.0 \\
\hline CANO3ION & $3.70959 \mathrm{E}-06$ & 0.0 & 0.0 & 0.0 \\
\hline CAOHION & $9.45090 \mathrm{E}-11$ & 0.0 & 0.0 & 0.0 \\
\hline CAPO 4 ION & $1.66588 \mathrm{E}-09$ & 0.0 & 0.0 & 0.0 \\
\hline CDCL3ION & $3.42584 \mathrm{E}-09$ & 0.0 & 0.0 & 0.0 \\
\hline CDCL 4 ION & $4.81624 \mathrm{E}-08$ & 0.0 & 0.0 & 0.0 \\
\hline CDCLION & $4.71748 \mathrm{E}-10$ & 0.0 & 0.0 & 0.0 \\
\hline CDION & $2.10649 \mathrm{E}-11$ & 0.0 & 0.0 & 0.0 \\
\hline CDNO3ION & $3.52498 \mathrm{E}-12$ & 0.0 & 0.0 & 0.0 \\
\hline CDOH3ION & $4.40744 E-20$ & 0.0 & 0.0 & 0.0 \\
\hline CDOH 4 ION & $5.95045 \mathrm{E}-25$ & 0.0 & 0.0 & 0.0 \\
\hline CDOHION & $4.26988 \mathrm{E}-14$ & 0.0 & 0.0 & 0.0 \\
\hline CLION & 0.00393781 & 0.0 & 0.0 & 0.0 \\
\hline CO3ION & $1.53943 \mathrm{E}-09$ & 0.0 & 0.0 & 0.0 \\
\hline CRIIICL2ION & $1.01875 E-15$ & 0.0 & 0.0 & 0.0 \\
\hline CRIIICLION & $5.17648 \mathrm{E}-17$ & 0.0 & 0.0 & 0.0 \\
\hline CRIIIH2PO4 ION & $6.67944 \mathrm{E}-18$ & 0.0 & 0.0 & 0.0 \\
\hline CRIIIHPO4ION & $7.42140 \mathrm{E}-11$ & 0.0 & 0.0 & 0.0 \\
\hline CRIIIION & $9.58176 \mathrm{E}-21$ & 0.0 & 0.0 & 0.0 \\
\hline CRIIINO3ION & $2.10488 \mathrm{E}-13$ & 0.0 & 0.0 & 0.0 \\
\hline CROH2 ION & $2.95535 \mathrm{E}-12$ & 0.0 & 0.0 & 0.0 \\
\hline CROH 4 ION & $1.06600 \mathrm{E}-11$ & 0.0 & 0.0 & 0.0 \\
\hline CROHION & $4.13843 \mathrm{E}-10$ & 0.0 & 0.0 & 0.0 \\
\hline CRSO 4 ION & $1.44227 \mathrm{E}-11$ & 0.0 & 0.0 & 0.0 \\
\hline CUCL3ION & $2.51098 \mathrm{E}-12$ & 0.0 & 0.0 & 0.0 \\
\hline CUCLION & $3.73244 \mathrm{E}-10$ & 0.0 & 0.0 & 0.0 \\
\hline CUCO32ION & $6.82880 \mathrm{E}-15$ & 0.0 & 0.0 & 0.0 \\
\hline CUION & $3.51924 \mathrm{E}-10$ & 0.0 & 0.0 & 0.0 \\
\hline CUNO3ION & $7.17203 \mathrm{E}-11$ & 0.0 & 0.0 & 0.0 \\
\hline CUOH3ION & $2.70013 \mathrm{E}-12$ & 0.0 & 0.0 & 0.0 \\
\hline CUOH 4 ION & $6.00867 \mathrm{E}-16$ & 0.0 & 0.0 & 0.0 \\
\hline CUOHION & $3.18656 \mathrm{E}-10$ & 0.0 & 0.0 & 0.0 \\
\hline DODECION & $3.94431 E-05$ & 0.0 & 0.0 & 0.0 \\
\hline FEIICLION & $7.77142 \mathrm{E}-09$ & 0.0 & 0.0 & 0.0 \\
\hline FEIICO32ION & $2.94832 E-13$ & 0.0 & 0.0 & 0.0 \\
\hline FEIIH2PO4ION & $6.11747 \mathrm{E}-13$ & 0.0 & 0.0 & 0.0 \\
\hline FEIIHCO3ION & $1.12598 \mathrm{E}-12$ & 0.0 & 0.0 & 0 . \\
\hline
\end{tabular}




\begin{tabular}{|c|c|c|c|c|}
\hline FEIIION & $6.71690 \mathrm{E}-06$ & 0.0 & 0.0 & 0.0 \\
\hline FEIIOH 3 ION & $4.30902 \mathrm{E}-12$ & 0.0 & 0.0 & 0.0 \\
\hline FEIIOH 4 ION & $1.46323 \mathrm{E}-17$ & 0.0 & 0.0 & 0.0 \\
\hline FEIIOHION & $1.76917 \mathrm{E}-07$ & 0.0 & 0.0 & 0.0 \\
\hline H2 P2O 7 ION & $5.94515 E-19$ & 0.0 & 0.0 & 0.0 \\
\hline $\mathrm{H} 2 \mathrm{PO} 4 \mathrm{ION}$ & $2.37478 \mathrm{E}-10$ & 0.0 & 0.0 & 0.0 \\
\hline H2SIO4 ION & $5.04286 \mathrm{E}-11$ & 0.0 & 0.0 & 0.0 \\
\hline H3P2O 7 ION & $2.12731 \mathrm{E}-26$ & 0.0 & 0.0 & 0.0 \\
\hline H3SIO4 ION & $1.12575 \mathrm{E}-07$ & 0.0 & 0.0 & 0.0 \\
\hline HCO3ION & $1.05655 \mathrm{E}-08$ & 0.0 & 0.0 & 0.0 \\
\hline HION & $5.67212 \mathrm{E}-12$ & 0.0 & 0.0 & 0.0 \\
\hline HP2O7ION & $2.82958 \mathrm{E}-16$ & 0.0 & 0.0 & 0.0 \\
\hline HPBO2 ION & $2.36820 \mathrm{E}-14$ & 0.0 & 0.0 & 0.0 \\
\hline HPO4 ION & $5.52793 \mathrm{E}-08$ & 0.0 & 0.0 & 0.0 \\
\hline $\mathrm{HSO} 4 \mathrm{ION}$ & $1.02937 \mathrm{E}-11$ & 0.0 & 0.0 & 0.0 \\
\hline KION & $7.61191 \mathrm{E}-04$ & 0.0 & 0.0 & 0.0 \\
\hline KSO4 ION & $8.62274 \mathrm{E}-05$ & 0.0 & 0.0 & 0.0 \\
\hline MGH2 PO 4 I ON & $1.16567 \mathrm{E}-09$ & 0.0 & 0.0 & 0.0 \\
\hline MGHCO3ION & $3.83726 \mathrm{E}-08$ & 0.0 & 0.0 & 0.0 \\
\hline MGHSIO3ION & $1.03905 \mathrm{E}-06$ & 0.0 & 0.0 & 0.0 \\
\hline MGION & $4.05930 \mathrm{E}-04$ & 0.0 & 0.0 & 0.0 \\
\hline MGOHION & $1.21421 \mathrm{E}-07$ & 0.0 & 0.0 & 0.0 \\
\hline MGP2O7ION & $1.60805 \mathrm{E}-11$ & 0.0 & 0.0 & 0.0 \\
\hline MGPO 4 ION & $2.69500 \mathrm{E}-07$ & 0.0 & 0.0 & 0.0 \\
\hline NACO3ION & $7.15055 \mathrm{E}-10$ & 0.0 & 0.0 & 0.0 \\
\hline NAION & 0.00536897 & 0.0 & 0.0 & 0.0 \\
\hline NASO 4 ION & $2.62761 \mathrm{E}-04$ & 0.0 & 0.0 & 0.0 \\
\hline NICLION & $6.16331 \mathrm{E}-10$ & 0.0 & 0.0 & 0.0 \\
\hline NIION & $1.65129 \mathrm{E}-08$ & 0.0 & 0.0 & 0.0 \\
\hline NINO3ION & $4.51036 \mathrm{E}-09$ & 0.0 & 0.0 & 0.0 \\
\hline NIOH3ION & $5.85351 \mathrm{E}-14$ & 0.0 & 0.0 & 0.0 \\
\hline NIOHION & $8.46959 \mathrm{E}-11$ & 0.0 & 0.0 & 0.0 \\
\hline NO3ION & 0.0011333 & 0.0 & 0.0 & 0.0 \\
\hline P207ION & $7.39577 \mathrm{E}-15$ & 0.0 & 0.0 & 0.0 \\
\hline PBCL3ION & $6.84063 \mathrm{E}-09$ & 0.0 & 0.0 & 0.0 \\
\hline PBCL 4 ION & $1.79393 \mathrm{E}-07$ & 0.0 & 0.0 & 0.0 \\
\hline PBCLION & $8.61106 \mathrm{E}-10$ & 0.0 & 0.0 & 0.0 \\
\hline PBH2 PO 4 ION & $4.29542 \mathrm{E}-17$ & 0.0 & 0.0 & 0.0 \\
\hline PBION & $1.43765 \mathrm{E}-10$ & 0.0 & 0.0 & 0.0 \\
\hline PBNO33ION & $5.80028 \mathrm{E}-12$ & 0.0 & 0.0 & 0.0 \\
\hline PBNO3ION & $1.57552 \mathrm{E}-10$ & 0.0 & 0.0 & 0.0 \\
\hline PBOHION & $7.89056 \mathrm{E}-11$ & 0.0 & 0.0 & 0.0 \\
\hline PO4ION & $9.31693 \mathrm{E}-11$ & 0.0 & 0.0 & 0.0 \\
\hline SO4ION & $7.60284 \mathrm{E}-04$ & 0.0 & 0.0 & 0.0 \\
\hline SRION & $3.77430 \mathrm{E}-07$ & 0.0 & 0.0 & 0.0 \\
\hline SRNO3ION & $1.20222 \mathrm{E}-07$ & 0.0 & 0.0 & 0.0 \\
\hline SROHION & $7.43923 \mathrm{E}-13$ & 0.0 & 0.0 & 0.0 \\
\hline SRPO 4 ION & $3.18865 \mathrm{E}-13$ & 0.0 & 0.0 & 0.0 \\
\hline UIVOH2ION & $3.10919 \mathrm{E}-27$ & 0.0 & 0.0 & 0.0 \\
\hline UIVOH3ION & $2.71601 \mathrm{E}-20$ & 0.0 & 0.0 & 0.0 \\
\hline UIVOH5ION & $1.04767 \mathrm{E}-14$ & 0.0 & 0.0 & 0.0 \\
\hline UIVOHION & 0.0 & 0.0 & 0.0 & 0.0 \\
\hline ZNCL3ION & $4.38919 \mathrm{E}-07$ & 0.0 & 0.0 & 0.0 \\
\hline ZNCLION & $2.57605 \mathrm{E}-07$ & 0.0 & 0.0 & 0.0 \\
\hline ZNH2PO 4 ION & $2.07236 \mathrm{E}-13$ & 0.0 & 0.0 & 0.0 \\
\hline ZNHCO3ION & $6.75183 \mathrm{E}-12$ & 0.0 & 0.0 & 0.0 \\
\hline ZNION & $9.69682 \mathrm{E}-07$ & 0.0 & 0.0 & 0.0 \\
\hline ZNNO3ION & $1.18865 \mathrm{E}-07$ & 0.0 & 0.0 & 0.0 \\
\hline
\end{tabular}


WSRC-TR-2002-00567

Revision 0

\begin{tabular}{|c|c|c|c|c|}
\hline ZNOH3ION & $4.26534 \mathrm{E}-11$ & 0.0 & 0.0 & 0.0 \\
\hline ZNOH $4 \mathrm{ION}$ & $2.67470 \mathrm{E}-14$ & 0.0 & 0.0 & 0.0 \\
\hline ZNOHION & $2.73122 \mathrm{E}-08$ & 0.0 & 0.0 & 0.0 \\
\hline $\mathrm{CA} 3 \mathrm{PO} 42$ & 0.0 & $4.11373 E-04$ & 0.0 & 0.0 \\
\hline CHAMOSITE7A & 0.0 & $5.92593 E-05$ & 0.0 & 0.0 \\
\hline $\mathrm{CU} 3 \mathrm{PO} 42.2 \mathrm{H} 2 \mathrm{O}$ & 0.0 & $5.19274 \mathrm{E}-08$ & 0.0 & 0.0 \\
\hline FEII3PO $42.8 \mathrm{H} 2 \mathrm{O}$ & 0.0 & $1.79198 E-06$ & 0.0 & 0.0 \\
\hline MG3PO4 2 & 0.0 & $5.58503 E-06$ & 0.0 & 0.0 \\
\hline $\mathrm{PB} 3 \mathrm{PO} 42$ & 0.0 & $2.03019 E-06$ & 0.0 & 0.0 \\
\hline UIVO2 & 0.0 & $7.98319 \mathrm{E}-06$ & 0.0 & 0.0 \\
\hline & $===========$ & $===========$ & $============$ & $===========$ \\
\hline Total g/hr & 2.16101 & 0.175877 & 0.0 & 0.0 \\
\hline Volume, L/hr & 0.00181226 & $4.73683 E-05$ & 0.0 & 0.0 \\
\hline Enthalpy, cal/hr & -7145.9 & -519.676 & 0.0 & 0.0 \\
\hline Density, g/L & 1192.44 & 3712.97 & & \\
\hline Vapor fraction & 0.0 & 0.0 & 0.0 & 0.0 \\
\hline Solid fraction & 0.0 & 1 . & 0.0 & 0.0 \\
\hline Organic fraction & 0.0 & 0.0 & 0.0 & 0.0 \\
\hline Osmotic Pres, atm & 212.328 & & & \\
\hline Redox Pot, volts & 0.0 & & & \\
\hline E-Con, $1 / \mathrm{ohm}-\mathrm{cm}$ & 0.191752 & & & \\
\hline E-Con, cm2/ohm-mol & 28.9825 & & & \\
\hline Abs Visc, cP & 1.64804 & & & \\
\hline Rel Visc & 1.85024 & & & \\
\hline Ionic Strength & 4.99138 & & & \\
\hline
\end{tabular}


WSRC-TR-2002-00567

Revision 0

\begin{tabular}{|c|c|c|c|c|}
\hline \multicolumn{5}{|l|}{ STREAM: Condensate } \\
\hline TO $\quad:$ & & & & \\
\hline FROM : Condensate & mixer & & & \\
\hline Phases-----------> & Aqueous & Solid & Vapor & Organic \\
\hline Temperature, C & 25 . & 25 . & 25 . & 25 . \\
\hline $\begin{array}{l}\text { Pressure, atm } \\
\mathrm{pH}\end{array}$ & $\begin{array}{l}1 . \\
4.16393\end{array}$ & 1. & 1. & 1. \\
\hline Total mol/hr & 13.77792 & 0.0 & 0.0 & 0.0 \\
\hline------------------ & $\mathrm{mol} / \mathrm{hr}------$ & -mol/hr------- & -mol/hr-------- & $-\operatorname{mol} / \mathrm{hr}------1$ \\
\hline $\mathrm{H} 2 \mathrm{O}$ & 13.7762 & 0.0 & 0.0 & 0.0 \\
\hline $\mathrm{CO} 2$ & 0.00165552 & 0.0 & 0.0 & 0.0 \\
\hline $\mathrm{HCL}$ & $1.30038 E-20$ & 0.0 & 0.0 & 0.0 \\
\hline HNO3 & $2.79394 \mathrm{E}-16$ & 0.0 & 0.0 & 0.0 \\
\hline LAURICACID & $3.41000 E-05$ & 0.0 & 0.0 & 0.0 \\
\hline OHION & $3.70698 \mathrm{E}-11$ & 0.0 & 0.0 & 0.0 \\
\hline CLION & $3.23314 E-10$ & 0.0 & 0.0 & 0.0 \\
\hline CO3ION & $7.80462 \mathrm{E}-12$ & 0.0 & 0.0 & 0.0 \\
\hline DODECION & $6.11429 E-06$ & 0.0 & 0.0 & 0.0 \\
\hline $\mathrm{HCO} 3 \mathrm{ION}$ & $1.10661 E-05$ & 0.0 & 0.0 & 0.0 \\
\hline $\mathrm{HION}$ & $1.71809 \mathrm{E}-05$ & 0.0 & 0.0 & 0.0 \\
\hline NO3ION & $\begin{array}{l}8.25564 \mathrm{E}-11 \\
============\end{array}$ & $\begin{array}{l}0.0 \\
============\end{array}$ & $\begin{array}{l}0.0 \\
=============\end{array}$ & $\begin{array}{l}0.0 \\
=============\end{array}$ \\
\hline Total g/hr & 248.264 & 0.0 & 0.0 & 0.0 \\
\hline Volume, L/hr & 0.249024 & 0.0 & 0.0 & 0.0 \\
\hline $\begin{array}{l}\text { Enthalpy, cal/hr } \\
\text { Density, g/L }\end{array}$ & $\begin{array}{l}-9.41267 E+05 \\
996.949\end{array}$ & 0.0 & 0.0 & 0.0 \\
\hline Vapor fraction & 0.0 & 0.0 & 0.0 & 0.0 \\
\hline Solid fraction & 0.0 & 0.0 & 0.0 & 0.0 \\
\hline Organic fraction & 0.0 & 0.0 & 0.0 & 0.0 \\
\hline Osmotic Pres, atm & 0.16944 & & & \\
\hline Redox Pot, volts & 0.0 & & & \\
\hline E-Con, 1/ohm-cm & $2.75028 \mathrm{E}-05$ & & & \\
\hline E-Con, cm2/ohm-mol & 4.01269 & & & \\
\hline Abs Visc, cP & 0.890758 & & & \\
\hline Rel Visc & 1.00004 & & & \\
\hline Ionic Strength & $6.92274 E-05$ & & & \\
\hline
\end{tabular}


WSRC-TR-2002-00567

Revision 0

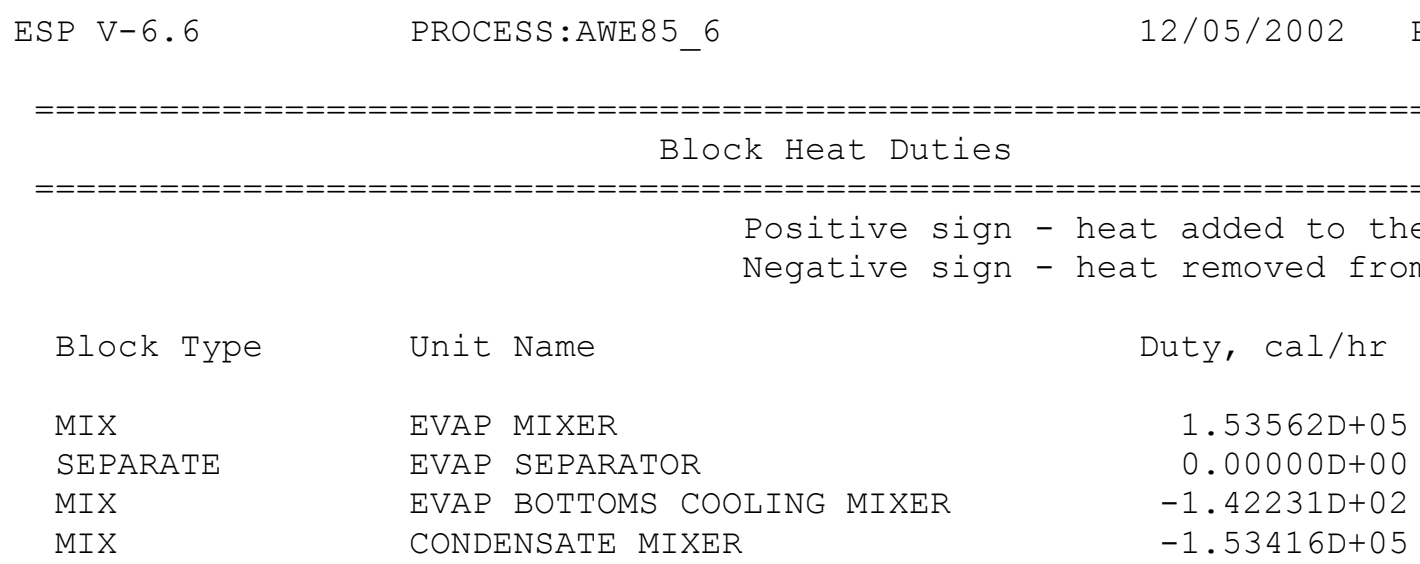


WSRC-TR-2002-00567

Revision 0

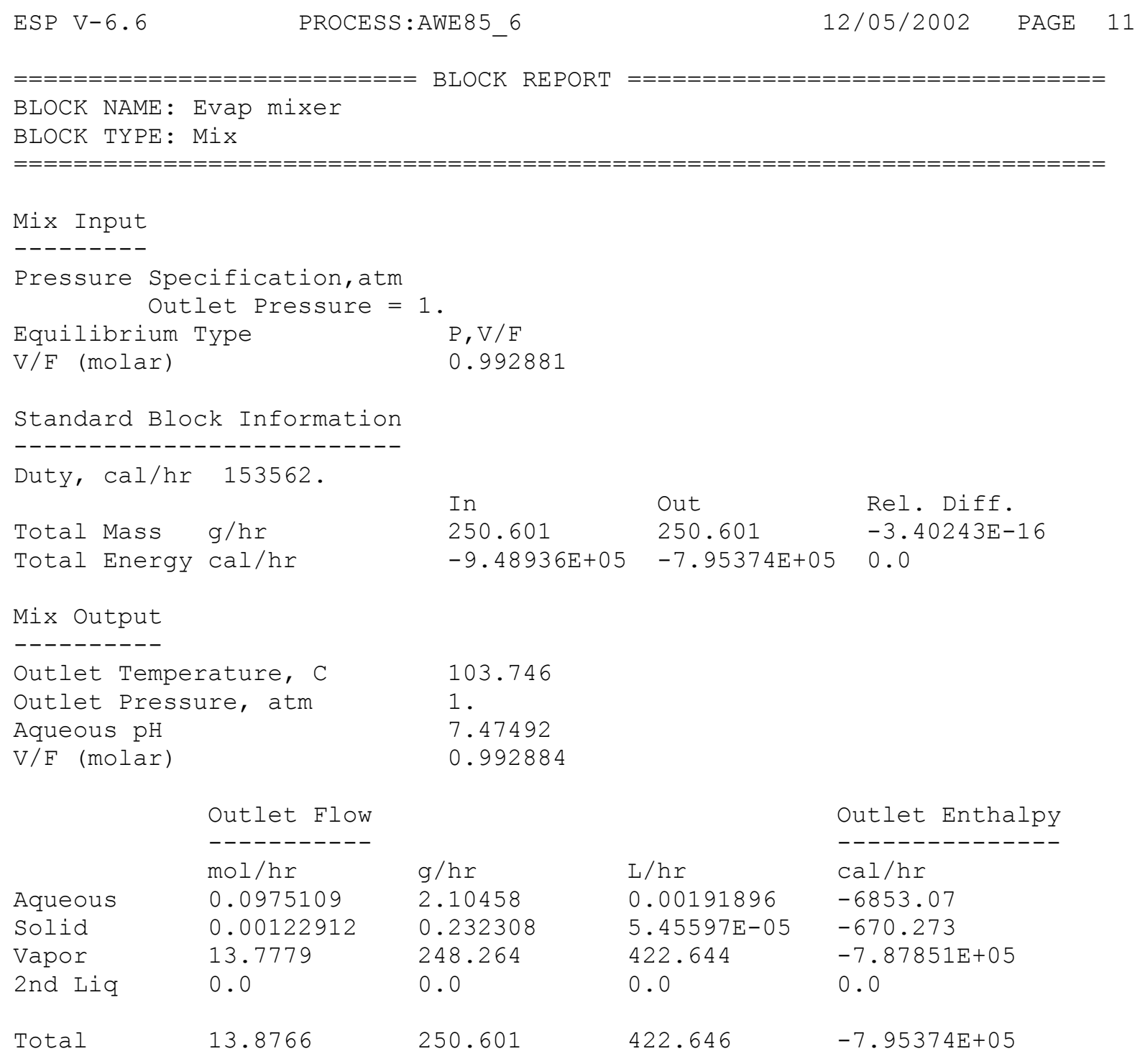


WSRC-TR-2002-00567

Revision 0

$\begin{array}{lllll}\text { ESP V-6.6 PROCESS :AWE85_6 } & \text { PAGE } & 12 / 05 / 2002\end{array}$

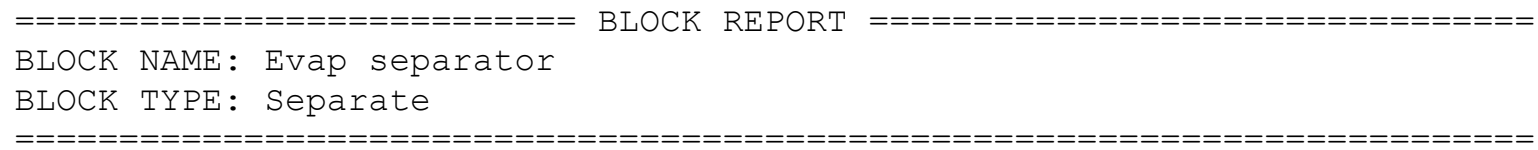

$0.0987401 \quad 2.33689$

$\mathrm{L} / \mathrm{hr} \quad \mathrm{cal} / \mathrm{hr}$

$5.45597 \mathrm{E}-05-670.273$

$0.0 \quad 0.0$

Total

$0.00197352 \quad-7523.34$

Outlet Enthalpy 
WSRC-TR-2002-00567

Revision 0

ESP V-6.6

PROCESS:AWE85 6

Vapor stream

\begin{tabular}{ll}
\multicolumn{2}{c}{ Overhead } \\
Outlet Flow \\
-------- \\
mol/hr & $\mathrm{g} / \mathrm{hr}$ \\
0.0 & 0.0 \\
0.0 & 0.0 \\
13.7779 & 248.264 \\
0.0 & 0.0
\end{tabular}

Total

248.264
$12 / 05 / 2002$

PAGE

13

$\mathrm{L} / \mathrm{hr}$

0.0

0.0

422.644

0.0

Outlet Enthalpy
-------------
cal/hr
0.0
0.0
$-7.87851 \mathrm{E}+05$
0.0

422.644 
WSRC-TR-2002-00567

Revision 0

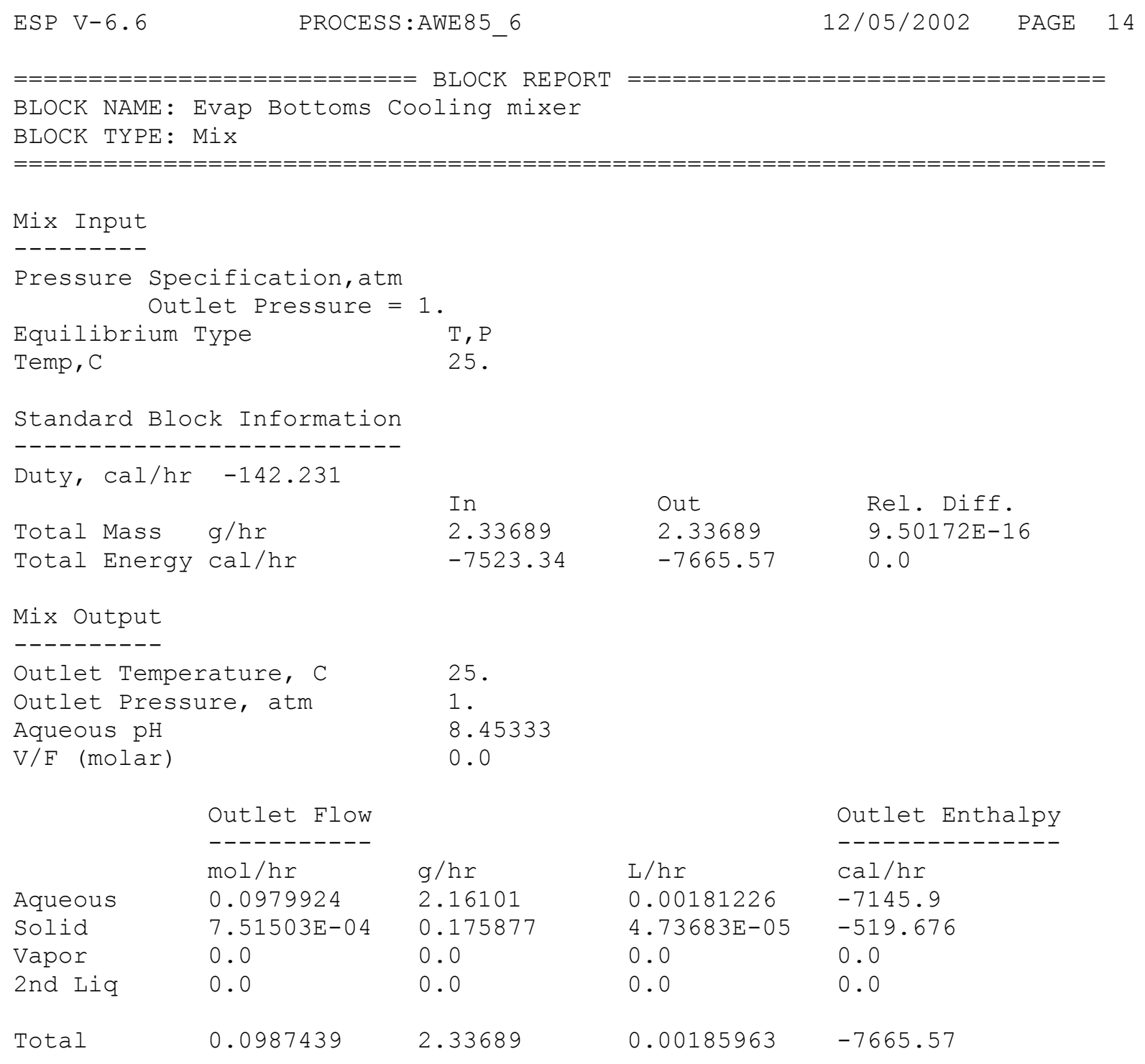


WSRC-TR-2002-00567

Revision 0

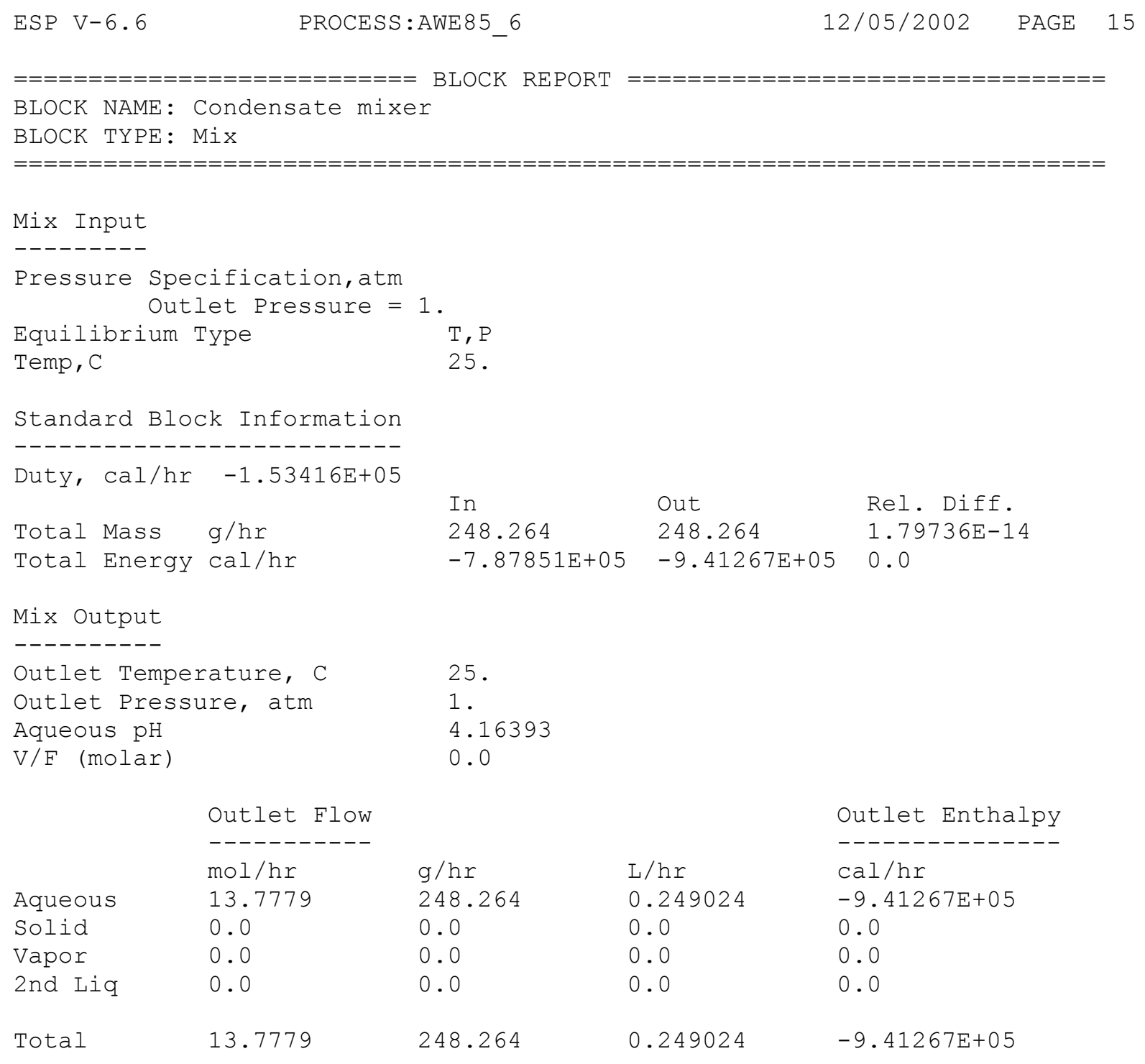


WSRC-TR-2002-00567

Revision 0

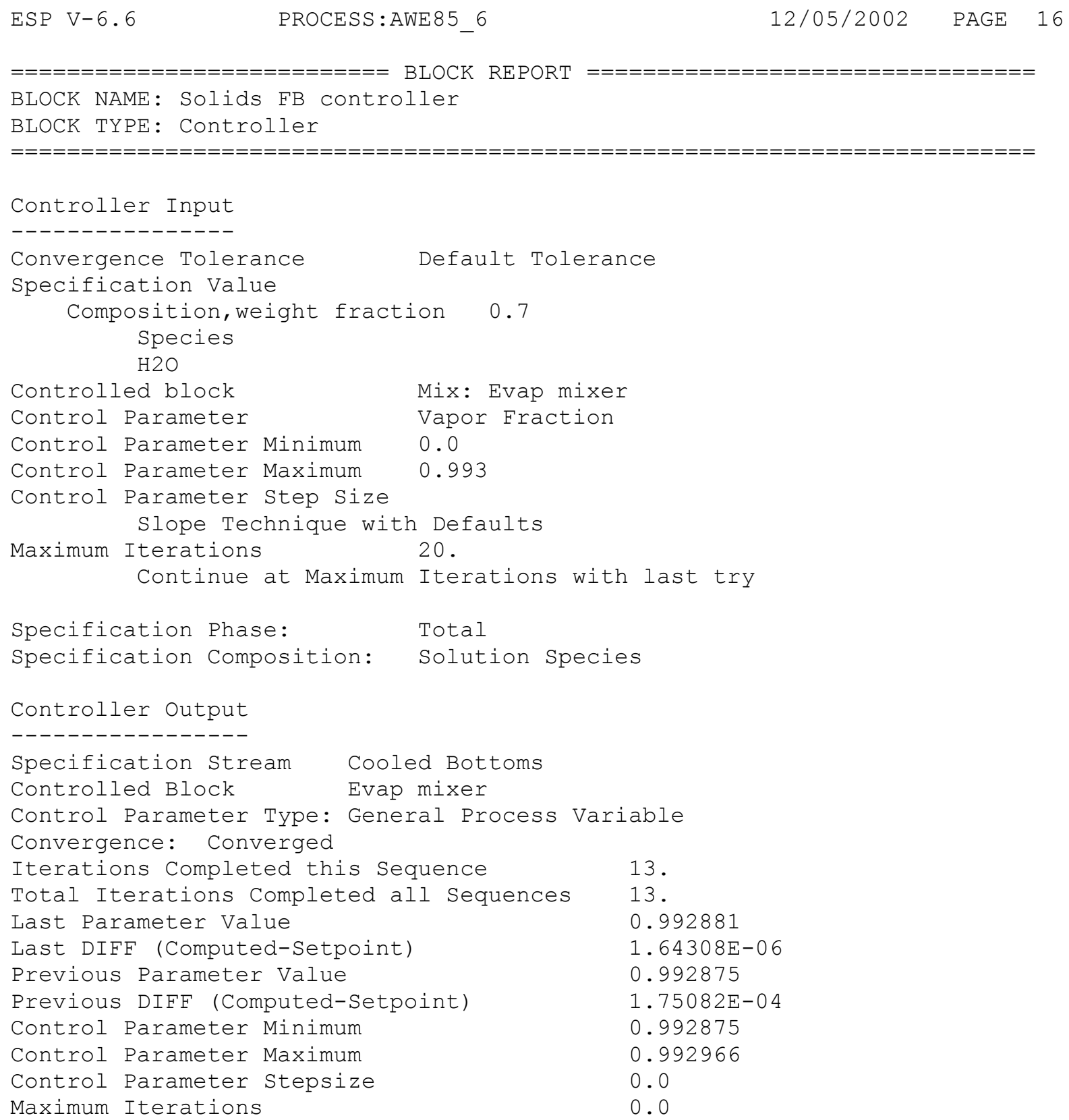


WSRC-TR-2002-00567

Revision 0

Influent Limit Composition 95\% Target $\mathrm{pH}=8.5$

8.5-95

\begin{tabular}{|c|c|c|c|c|}
\hline 0 & 0 & 0 & $\mathrm{~L}$ & $I I I I$ \\
\hline 0 & & 0 & $\mathrm{~L}$ & I \\
\hline 0 & & 0 & $\mathrm{~L}$ & I \\
\hline D & & 0 & $\mathrm{~L}$ & I \\
\hline D & & 0 & $\mathrm{~L}$ & I \\
\hline D & & 0 & $\mathrm{~L}$ & I \\
\hline 0 & & 0 & $\mathrm{~L}$ & I \\
\hline 0 & & 0 & $\mathrm{~L}$ & I \\
\hline 0 & 0 & 0 & L L L L L L L L L L & I I I \\
\hline
\end{tabular}

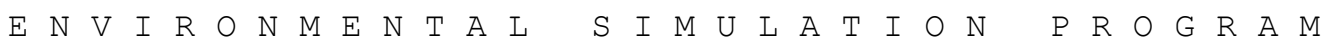

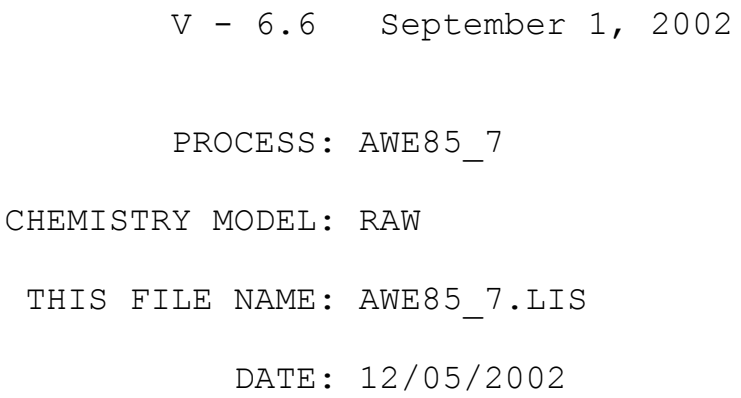


WSRC-TR-2002-00567

Revision 0

TABLE OF CONTENTS

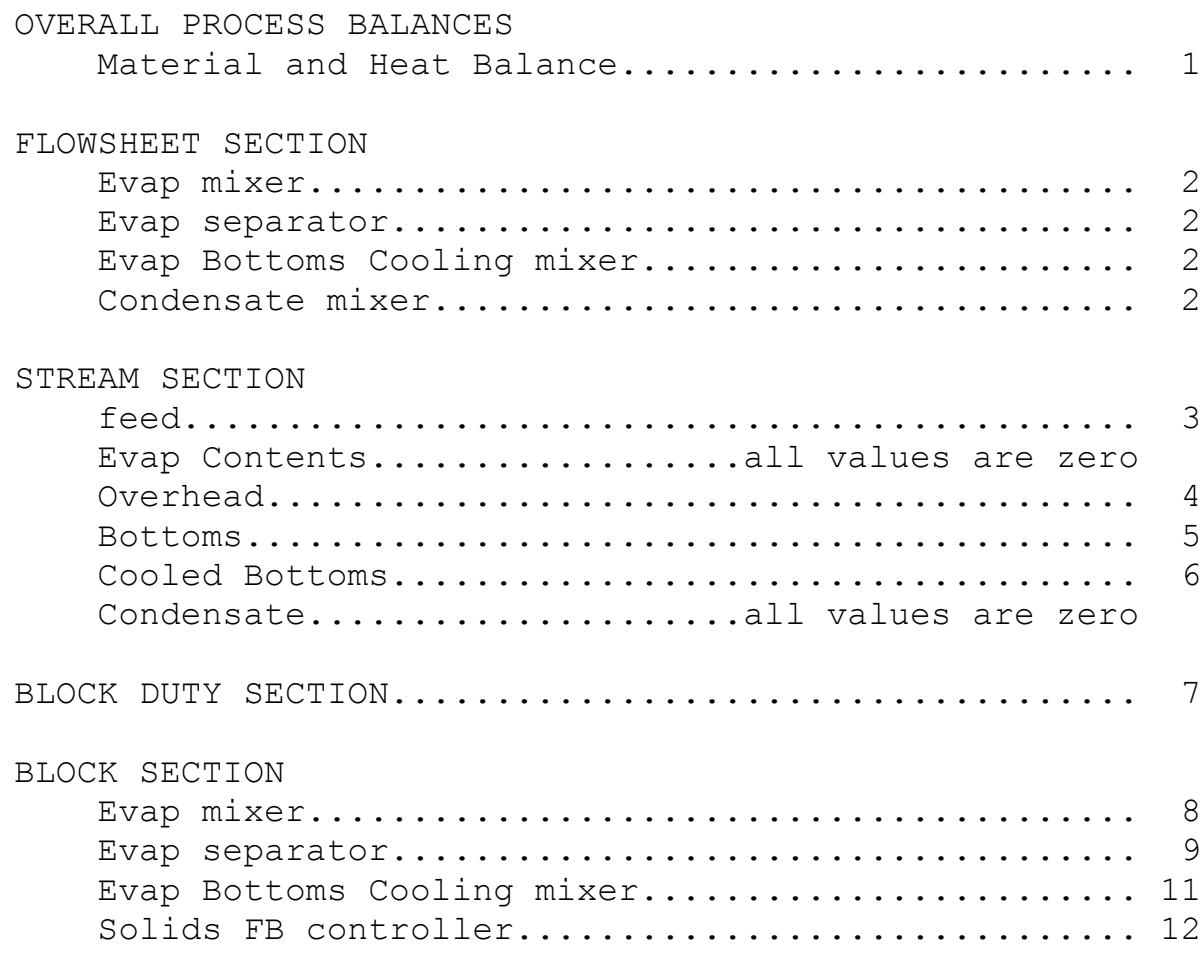


WSRC-TR-2002-00567

Revision 0

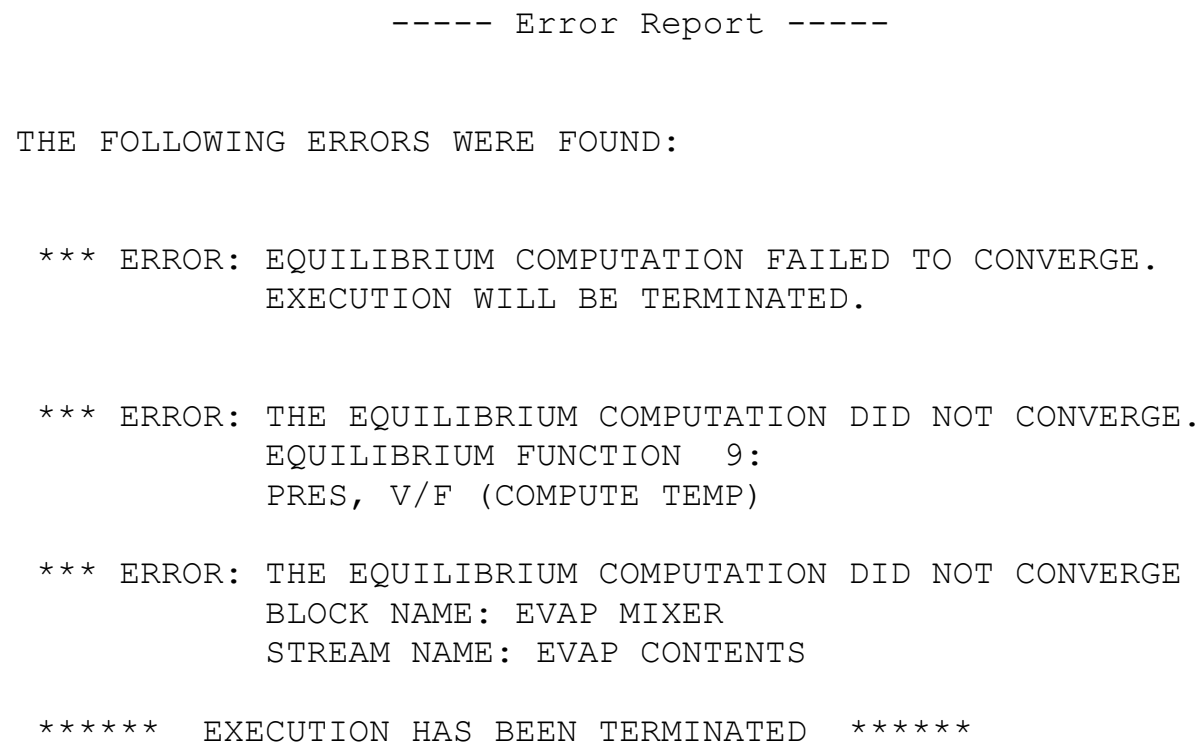


WSRC-TR-2002-00567

Revision 0

ESP V-6.6 PROCESS:AWE85_7
$\begin{array}{lcc}\text { Overall Process } & \text { Balances } \\ \text { Inlet } & \text { g/hr } & \mathrm{Cal} / \mathrm{hr} \\ ---------- & ---------- & --------- \\ \text { FEED } & 1.00000 \mathrm{D}+03 & -3.79027 \mathrm{D}+06 \\ \text { =========== } & ========== & ========= \\ \text { Total in } & 1.00000 \mathrm{D}+03 & -3.79027 \mathrm{D}+06\end{array}$

\begin{tabular}{lrr} 
Outlet & \multicolumn{1}{c}{$g / \mathrm{hr}$} & \multicolumn{1}{c}{$\mathrm{cal} / \mathrm{hr}$} \\
---------- & ---------- & ---------- \\
COOLED BOTTOMS & $3.63365 \mathrm{D}+00$ & $-1.23015 \mathrm{D}+04$ \\
CONDENSATE & $0.00000 \mathrm{D}+00$ & $0.00000 \mathrm{D}+00$ \\
$==========$ & $==========$ & $==========$ \\
Total out & $3.63365 \mathrm{D}+00$ & $-1.23015 \mathrm{D}+04$
\end{tabular}

Block Heat Duties

$\mathrm{cal} / \mathrm{hr}$

------------

EVAP BOTTOMS COOLING MIXER

-----------

$========$
Total Duty

$-2.31165 \mathrm{D}+02$

$=========$

$-2.31165 D+02$

DIFFERENCE $\quad-9.96366 \mathrm{D}+02 \quad 3.77820 \mathrm{D}+06$

REL DIFFERENCE $\quad-9.96366 \mathrm{D}-01-9.96815 \mathrm{D}-01$

Material Code Balances

\begin{tabular}{|c|c|c|c|c|}
\hline Code & $\begin{array}{l}\text { Input } \\
\text { mol/hr }\end{array}$ & $\begin{array}{l}\text { Outlet } \\
\text { mol/hr }\end{array}$ & $\begin{array}{l}\text { Difference } \\
\text { mol/hr }\end{array}$ & Rel Diff \\
\hline------------ & ------------ & ------------ & ------------ & ------------1 \\
\hline $\mathrm{H}(+1)$ & $1.10908 \mathrm{D}+02$ & $3.06712 \mathrm{D}-01$ & $-1.10601 D+02$ & $-9.97235 D-01$ \\
\hline$K(+1)$ & $1.08048 D-03$ & $1.08048 \mathrm{D}-03$ & $-2.60209 D-18$ & $-2.40828 D-15$ \\
\hline $\mathrm{NA}(+1)$ & $7.34738 D-03$ & $7.34738 D-03$ & $-5.20417 D-18$ & $-7.08303 D-16$ \\
\hline $\mathrm{BA}(+2)$ & $8.02920 \mathrm{D}-07$ & $8.02920 \mathrm{D}-07$ & $1.35525 \mathrm{D}-20$ & $1.68791 \mathrm{D}-14$ \\
\hline $\mathrm{CA}(+2)$ & $1.44638 D-03$ & $1.44638 \mathrm{D}-03$ & $2.16840 \mathrm{D}-19$ & $1.49919 \mathrm{D}-16$ \\
\hline $\mathrm{ZN}(+2)$ & $5.65749 \mathrm{D}-05$ & $5.65749 \mathrm{D}-05$ & $-6.77626 \mathrm{D}-21$ & $-1.19775 \mathrm{D}-16$ \\
\hline $\mathrm{CU}(+2)$ & $1.16535 \mathrm{D}-05$ & $1.16535 \mathrm{D}-05$ & $-1.69407 D-21$ & $-1.45369 D-16$ \\
\hline $\mathrm{FE}(+2)$ & $1.46953 \mathrm{D}-04$ & $1.46953 \mathrm{D}-04$ & $-2.71051 D-20$ & $-1.84447 D-16$ \\
\hline $\mathrm{MG}(+2)$ & $5.76132 \mathrm{D}-04$ & $5.76132 \mathrm{D}-04$ & $0.00000 \mathrm{D}+00$ & $0.00000 \mathrm{D}+00$ \\
\hline $\mathrm{PB}(+2)$ & $1.01449 \mathrm{D}-05$ & $1.01449 D-05$ & $-1.69407 \mathrm{D}-21$ & $-1.66986 \mathrm{D}-16$ \\
\hline $\mathrm{AL}(+3)$ & $2.25926 \mathrm{D}-04$ & $2.25926 \mathrm{D}-04$ & $0.00000 \mathrm{D}+00$ & $0.00000 D+00$ \\
\hline $\mathrm{NI}(+2)$ & $2.55537 \mathrm{D}-06$ & $2.55537 \mathrm{D}-06$ & $-8.47033 D-22$ & $-3.31472 \mathrm{D}-16$ \\
\hline$O(-2)$ & $5.54751 \mathrm{D}+01$ & $1.70828 \mathrm{D}-01$ & $-5.53042 \mathrm{D}+01$ & $-9.96921 D-01$ \\
\hline $\mathrm{CL}(-1)$ & $4.50704 \mathrm{D}-03$ & $4.50685 \mathrm{D}-03$ & $-1.93919 D-07$ & $-4.30258 D-05$ \\
\hline$C(+4)$ & $1.83333 \mathrm{D}-03$ & $8.23570 \mathrm{D}-10$ & $-1.83333 \mathrm{D}-03$ & $-1.00000 D+00$ \\
\hline$P(+5)$ & $1.15789 \mathrm{D}-03$ & $1.15789 \mathrm{D}-03$ & $0.00000 \mathrm{D}+00$ & $0.00000 D+00$ \\
\hline$S(+6)$ & $1.66667 \mathrm{D}-03$ & $1.66667 \mathrm{D}-03$ & $8.67362 \mathrm{D}-19$ & $5.20417 \mathrm{D}-16$ \\
\hline $\mathrm{N}(+5)$ & $1.77419 \mathrm{D}-03$ & $1.77413 \mathrm{D}-03$ & $-6.74142 \mathrm{D}-08$ & $-3.79971 D-05$ \\
\hline $\operatorname{SI}(+4)$ & $2.66667 \mathrm{D}-04$ & $2.66667 \mathrm{D}-04$ & $0.00000 \mathrm{D}+00$ & $0.00000 \mathrm{D}+00$ \\
\hline $\operatorname{SR}(+2)$ & $3.08219 \mathrm{D}-05$ & $3.08219 \mathrm{D}-05$ & $-6.77626 \mathrm{D}-21$ & $-2.19852 \mathrm{D}-16$ \\
\hline $\mathrm{CD}(+2)$ & $6.96429 \mathrm{D}-08$ & $6.96429 D-08$ & $-2.06464 \mathrm{D}-21$ & $-2.96461 D-14$ \\
\hline $\mathrm{CR}(+3)$ & $2.50000 \mathrm{D}-06$ & $2.50000 \mathrm{D}-06$ & $7.62330 \mathrm{D}-21$ & $3.04932 \mathrm{D}-15$ \\
\hline
\end{tabular}


WSRC-TR-2002-00567

Revision 0

$\mathrm{U}(+4)$

$\operatorname{DODEC}(-1)$
$1.17647 \mathrm{D}-05$

1.34809D-04
$1.17647 \mathrm{D}-05-1.69407 \mathrm{D}-21-1.43996 \mathrm{D}-16$

$2.31748 \mathrm{D}-07-1.34577 \mathrm{D}-04-9.98281 \mathrm{D}-01$


WSRC-TR-2002-00567

Revision 0

\begin{tabular}{|c|c|c|c|}
\hline \multicolumn{4}{|l|}{$\begin{array}{l}\text { PROCESS BLOCKS } \\
===========\end{array}$} \\
\hline $\begin{array}{l}\mathrm{BLOCK} \mathrm{NAME} \\
=========================\end{array}$ & $\begin{array}{l}\text { BLOCK TYPE } \\
===========\end{array}$ & $\begin{array}{l}\text { INLET STREAM }(\mathrm{S}) \\
==============\end{array}$ & $\begin{array}{l}\text { OUTLET STREAM }(\mathrm{S}) \\
==============\end{array}$ \\
\hline Evap mixer & $\operatorname{Mix}$ & feed & Evap Contents \\
\hline Evap separator & Separate & Evap Contents & $\begin{array}{l}\text { Overhead } \\
\text { Bottoms }\end{array}$ \\
\hline Evap Bottoms Cooling mixer & Mix & Bottoms & Cooled Bottoms \\
\hline Condensate mixer & Mix & Overhead & Condensate \\
\hline
\end{tabular}


WSRC-TR-2002-00567

Revision 0

ESP V-6. 6

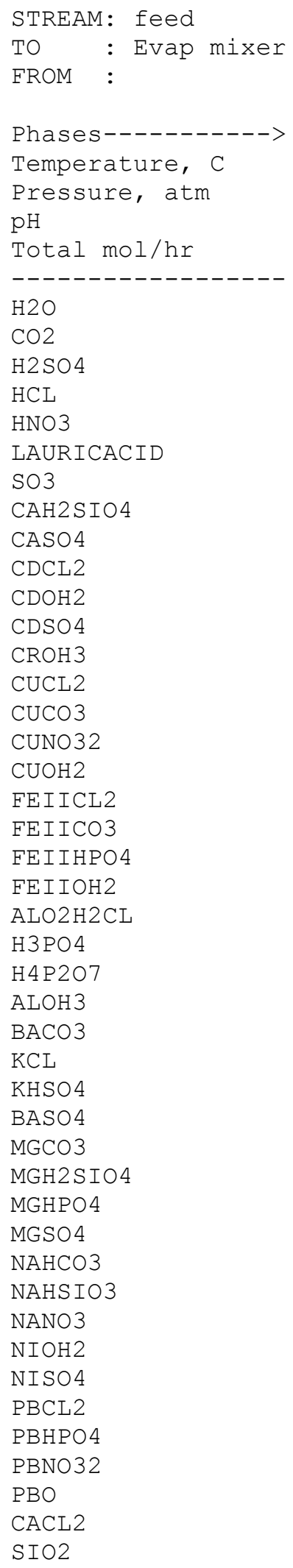

\begin{tabular}{|c|c|c|c|}
\hline Aqueous & Solid & Vapor & Organic \\
\hline 25 . & 25 . & 25. & 25 . \\
\hline 1. & 1. & 1 . & 1. \\
\hline \multicolumn{4}{|l|}{6.60357} \\
\hline 55.47341 & $3.82531 E-04$ & 0.0 & 0.0 \\
\hline \multicolumn{4}{|c|}{ mol/hr--------mol/hr--------mol/hr--------mol/hr-------- } \\
\hline 55.4523 & 0.0 & 0.0 & 0.0 \\
\hline $5.88079 E-04$ & 0.0 & 0.0 & 0.0 \\
\hline $2.14444 E-25$ & 0.0 & 0.0 & 0.0 \\
\hline $5.87155 E-16$ & 0.0 & 0.0 & 0.0 \\
\hline $1.93306 \mathrm{E}-11$ & 0.0 & 0.0 & 0.0 \\
\hline $2.37575 E-06$ & 0.0 & 0.0 & 0.0 \\
\hline $2.77646 E-29$ & 0.0 & 0.0 & 0.0 \\
\hline $1.65311 \mathrm{E}-12$ & 0.0 & 0.0 & 0.0 \\
\hline $2.15063 E-05$ & 0.0 & 0.0 & 0.0 \\
\hline $8.73138 E-10$ & 0.0 & 0.0 & 0.0 \\
\hline $3.06941 E-15$ & 0.0 & 0.0 & 0.0 \\
\hline $9.76996 E-09$ & 0.0 & 0.0 & 0.0 \\
\hline $3.66859 \mathrm{E}-12$ & 0.0 & 0.0 & 0.0 \\
\hline $2.66926 \mathrm{E}-13$ & 0.0 & 0.0 & 0.0 \\
\hline $7.66956 \mathrm{E}-09$ & 0.0 & 0.0 & 0.0 \\
\hline $8.70434 \mathrm{E}-15$ & 0.0 & 0.0 & 0.0 \\
\hline $9.38132 \mathrm{E}-10$ & 0.0 & 0.0 & 0.0 \\
\hline $6.99770 \mathrm{E}-15$ & 0.0 & 0.0 & 0.0 \\
\hline $2.31403 E-06$ & 0.0 & 0.0 & 0.0 \\
\hline $2.87252 \mathrm{E}-07$ & 0.0 & 0.0 & 0.0 \\
\hline $3.54278 E-12$ & 0.0 & 0.0 & 0.0 \\
\hline $1.13614 \mathrm{E}-28$ & 0.0 & 0.0 & 0.0 \\
\hline $1.78881 \mathrm{E}-08$ & 0.0 & 0.0 & 0.0 \\
\hline $5.69496 \mathrm{E}-19$ & 0.0 & 0.0 & 0.0 \\
\hline $2.39696 E-09$ & $2.25921 E-04$ & 0.0 & 0.0 \\
\hline $1.02454 \mathrm{E}-11$ & 0.0 & 0.0 & 0.0 \\
\hline $3.12107 \mathrm{E}-08$ & 0.0 & 0.0 & 0.0 \\
\hline $4.02679 \mathrm{E}-13$ & 0.0 & 0.0 & 0.0 \\
\hline $2.05430 \mathrm{E}-10$ & $5.96795 E-07$ & 0.0 & 0.0 \\
\hline $5.76744 \mathrm{E}-08$ & 0.0 & 0.0 & 0.0 \\
\hline $7.86632 \mathrm{E}-12$ & 0.0 & 0.0 & 0.0 \\
\hline $4.55648 E-05$ & 0.0 & 0.0 & 0.0 \\
\hline $1.84531 E-05$ & 0.0 & 0.0 & 0.0 \\
\hline $2.88488 E-06$ & 0.0 & 0.0 & 0.0 \\
\hline $1.19823 E-07$ & 0.0 & 0.0 & 0.0 \\
\hline $5.77473 E-07$ & 0.0 & 0.0 & 0.0 \\
\hline $2.26487 E-12$ & 0.0 & 0.0 & 0.0 \\
\hline $2.65836 \mathrm{E}-07$ & 0.0 & 0.0 & 0.0 \\
\hline $5.41616 \mathrm{E}-12$ & 0.0 & 0.0 & 0.0 \\
\hline $7.19010 \mathrm{E}-10$ & 0.0 & 0.0 & 0.0 \\
\hline $1.61226 \mathrm{E}-13$ & 0.0 & 0.0 & 0.0 \\
\hline $5.59764 \mathrm{E}-13$ & 0.0 & 0.0 & 0.0 \\
\hline $8.99132 E-27$ & 0.0 & 0.0 & 0.0 \\
\hline $2.66369 \mathrm{E}-04$ & 0.0 & 0.0 & 0.0 \\
\hline
\end{tabular}




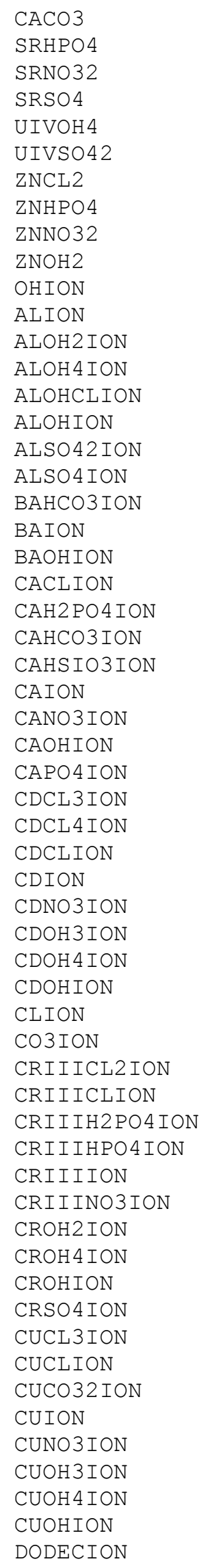

\begin{tabular}{|c|c|}
\hline $2.59629 E-07$ & 0.0 \\
\hline $3.89643 E-08$ & 0.0 \\
\hline $7 E-10$ & 0.0 \\
\hline $10437 E-06$ & 0.0 \\
\hline $92 \mathrm{E}-10$ & 0.0 \\
\hline $58 E-28$ & 0.0 \\
\hline $87 E-10$ & 0.0 \\
\hline $14 E-06$ & 0.0 \\
\hline $300 E-11$ & 0.0 \\
\hline $71 E-09$ & 0.0 \\
\hline $240 E-08$ & 0.0 \\
\hline $.52770 \mathrm{E}-13$ & 0.0 \\
\hline $46 E-10$ & 0.0 \\
\hline $.74052 E-09$ & 0.0 \\
\hline $84 \mathrm{E}-14$ & 0.0 \\
\hline $9.77565 \mathrm{E}-12$ & 0.0 \\
\hline $20 E-14$ & 0.0 \\
\hline $31 E-13$ & 0.0 \\
\hline$E-09$ & 0.0 \\
\hline $8 E-07$ & 0.0 \\
\hline $3 E-15$ & 0.0 \\
\hline $4 E-11$ & 0.0 \\
\hline $6 E-05$ & 0.0 \\
\hline $46 E-06$ & 0.0 \\
\hline $6 E-09$ & 0.0 \\
\hline 0232 & 0.0 \\
\hline $3 E-06$ & 0.0 \\
\hline $2 E-10$ & 0.0 \\
\hline $8 E-07$ & 0.0 \\
\hline $1 E-13$ & 0.0 \\
\hline $1 E-16$ & 0.0 \\
\hline $8 E-08$ & 0.0 \\
\hline $9 E-08$ & 0.0 \\
\hline $8 E-10$ & 0.0 \\
\hline $5 E-21$ & 0.0 \\
\hline $2 E-29$ & 0.0 \\
\hline $7 E-11$ & 0.0 \\
\hline 0684 & 0.0 \\
\hline $6 \mathrm{E}-07$ & 0.0 \\
\hline $6 E-19$ & 0.0 \\
\hline $5 E-15$ & 0.0 \\
\hline $8 E-12$ & 0.0 \\
\hline $73 E-06$ & 0.0 \\
\hline $7 E-13$ & 0.0 \\
\hline $31 E-14$ & 0.0 \\
\hline$O E-13$ & 0.0 \\
\hline $2 E-16$ & 0.0 \\
\hline $9 E-11$ & 0.0 \\
\hline $03 E-10$ & 0.0 \\
\hline $6 E-18$ & 0.0 \\
\hline $9 E-10$ & 0.0 \\
\hline $77 E-12$ & 0.0 \\
\hline $96 E-08$ & 0.0 \\
\hline $96 \mathrm{E}-11$ & 0.0 \\
\hline $47 E-15$ & 0.0 \\
\hline $96 \mathrm{E}-21$ & 0.0 \\
\hline $01 E-09$ & 0.0 \\
\hline $33 E-04$ & 0.0 \\
\hline
\end{tabular}

0.0

0.0

0.0

0.0

0.0

0.0

0.0

0.0

0.0

0.0

0.0

0.0

0.0

0.0

0.0

0.0

0.0

0.0

0.0

0.0

0.0

0.0

0.0

0.0

0.0

0.0

0.0

0.0

0.0

0.0

0.0

0.0

0.0

0.0

0.0

0.0

0.0

0.0

0.0

0.0

0.0

0.0

0.0

0.0

0.0

0.0

0.0

0.0

0.0

0.0

0.0

0.0

0.0

0.0

0.0

0.0

0.0

0.0
0.0

0.0

0.0

0.0

0.0

0.0

0.0

0.0

0.0

0.0

0.0

0.0

0.0

0.0

0.0

0.0

0.0

0.0

0.0

0.0

0.0

0.0

0.0

0.0

0.0

0.0

0.0

0.0

0.0

0.0

0.0

0.0

0.0

0.0

0.0

0.0

0.0

0.0

0.0

0.0

0.0

0.0

0.0

0.0

0.0

0.0

0.0

0.0

0.0

0.0

0.0

0.0

0.0

0.0

0.0

0.0

0.0

0.0 


\begin{tabular}{|c|c|c|c|c|}
\hline FEIICLION & $5.41330 \mathrm{E}-10$ & 0.0 & 0.0 & 0.0 \\
\hline FEIICO32ION & $2.55857 \mathrm{E}-11$ & 0.0 & 0.0 & 0.0 \\
\hline FEIIH2PO4ION & $1.65032 \mathrm{E}-07$ & 0.0 & 0.0 & 0.0 \\
\hline FEIIHCO3ION & $1.55723 \mathrm{E}-08$ & 0.0 & 0.0 & 0.0 \\
\hline FEIIION & $1.44045 \mathrm{E}-04$ & 0.0 & 0.0 & 0.0 \\
\hline FEIIOH3ION & $6.38887 \mathrm{E}-16$ & 0.0 & 0.0 & 0.0 \\
\hline FEIIOH 4 ION & $3.86809 \mathrm{E}-24$ & 0.0 & 0.0 & 0.0 \\
\hline FEIIOHION & $1.25535 \mathrm{E}-07$ & 0.0 & 0.0 & 0.0 \\
\hline H2 P2O 7 ION & $1.97284 \mathrm{E}-09$ & 0.0 & 0.0 & 0.0 \\
\hline H2 PO 4 ION & $5.79503 \mathrm{E}-04$ & 0.0 & 0.0 & 0.0 \\
\hline H2SIO4ION & $8.11052 \mathrm{E}-14$ & 0.0 & 0.0 & 0.0 \\
\hline H3P2O 7 ION & $7.39390 \mathrm{E}-14$ & 0.0 & 0.0 & 0.0 \\
\hline H3SIO4 ION & $1.74147 \mathrm{E}-07$ & 0.0 & 0.0 & 0.0 \\
\hline HCO3ION & 0.00121675 & 0.0 & 0.0 & 0.0 \\
\hline HION & $2.81373 \mathrm{E}-07$ & 0.0 & 0.0 & 0.0 \\
\hline HP207ION & $3.71199 \mathrm{E}-09$ & 0.0 & 0.0 & 0.0 \\
\hline HPBO2ION & $2.85838 \mathrm{E}-17$ & 0.0 & 0.0 & 0.0 \\
\hline HPO4 ION & $2.13262 \mathrm{E}-04$ & 0.0 & 0.0 & 0.0 \\
\hline HSO 4 ION & $2.50925 \mathrm{E}-08$ & 0.0 & 0.0 & 0.0 \\
\hline KION & 0.00107284 & 0.0 & 0.0 & 0.0 \\
\hline KSO4 ION & $7.60891 \mathrm{E}-06$ & 0.0 & 0.0 & 0.0 \\
\hline MGH2 PO 4 ION & $8.20271 E-06$ & 0.0 & 0.0 & 0.0 \\
\hline MGHCO3ION & $1.37777 \mathrm{E}-05$ & 0.0 & 0.0 & 0.0 \\
\hline MGHSIO3ION & $1.75499 \mathrm{E}-09$ & 0.0 & 0.0 & 0.0 \\
\hline MGION & $4.89600 \mathrm{E}-04$ & 0.0 & 0.0 & 0.0 \\
\hline MGOHION & $2.24740 \mathrm{E}-09$ & 0.0 & 0.0 & 0.0 \\
\hline MGP2O 7 ION & $2.39980 \mathrm{E}-08$ & 0.0 & 0.0 & 0.0 \\
\hline MGPO 4 ION & $4.48086 \mathrm{E}-07$ & 0.0 & 0.0 & 0.0 \\
\hline NACO3ION & $5.21340 \mathrm{E}-09$ & 0.0 & 0.0 & 0.0 \\
\hline NAION & 0.00728684 & 0.0 & 0.0 & 0.0 \\
\hline NASO 4 ION & $5.69499 \mathrm{E}-05$ & 0.0 & 0.0 & 0.0 \\
\hline NICLION & $6.22085 \mathrm{E}-10$ & 0.0 & 0.0 & 0.0 \\
\hline NIION & $2.28098 \mathrm{E}-06$ & 0.0 & 0.0 & 0.0 \\
\hline NINO3ION & $7.05143 E-09$ & 0.0 & 0.0 & 0.0 \\
\hline NIOH3ION & $1.05921 \mathrm{E}-16$ & 0.0 & 0.0 & 0.0 \\
\hline NIOHION & $8.74541 \mathrm{E}-10$ & 0.0 & 0.0 & 0.0 \\
\hline NO3ION & 0.0017705 & 0.0 & 0.0 & 0.0 \\
\hline P207ION & $1.82366 \mathrm{E}-11$ & 0.0 & 0.0 & 0.0 \\
\hline PBCL3ION & $1.19764 \mathrm{E}-14$ & 0.0 & 0.0 & 0.0 \\
\hline PBCL 4 ION & $4.39223 \mathrm{E}-17$ & 0.0 & 0.0 & 0.0 \\
\hline PBCLION & $4.26175 \mathrm{E}-10$ & 0.0 & 0.0 & 0.0 \\
\hline PBH2 PO 4 ION & $8.23231 \mathrm{E}-11$ & 0.0 & 0.0 & 0.0 \\
\hline PBION & $5.71629 \mathrm{E}-09$ & 0.0 & 0.0 & 0.0 \\
\hline PBNO33ION & $4.89837 \mathrm{E}-17$ & 0.0 & 0.0 & 0.0 \\
\hline PBNO3ION & $1.20764 \mathrm{E}-10$ & 0.0 & 0.0 & 0.0 \\
\hline PBOHION & $3.99297 \mathrm{E}-10$ & 0.0 & 0.0 & 0.0 \\
\hline PO4ION & $7.67244 \mathrm{E}-10$ & 0.0 & 0.0 & 0.0 \\
\hline SO4ION & 0.00155815 & 0.0 & 0.0 & 0.0 \\
\hline SRION & $2.75109 \mathrm{E}-05$ & 0.0 & 0.0 & 0.0 \\
\hline SRNO3ION & $1.67075 \mathrm{E}-07$ & 0.0 & 0.0 & 0.0 \\
\hline SROHION & $4.91284 \mathrm{E}-12$ & 0.0 & 0.0 & 0.0 \\
\hline SRPO 4 ION & $2.43898 \mathrm{E}-10$ & 0.0 & 0.0 & 0.0 \\
\hline UIVCLION & 0.0 & 0.0 & 0.0 & 0.0 \\
\hline UIVION & 0.0 & 0.0 & 0.0 & 0.0 \\
\hline UIVOH2ION & $3.74688 \mathrm{E}-21$ & 0.0 & 0.0 & 0.0 \\
\hline UIVOH3ION & $9.59876 \mathrm{E}-16$ & 0.0 & 0.0 & 0.0 \\
\hline UIVOH5ION & $7.18116 \mathrm{E}-14$ & 0.0 & 0.0 & 0.0 \\
\hline UIVOHION & $2.62284 \mathrm{E}-25$ & 0.0 & 0.0 & 0.0 \\
\hline
\end{tabular}


WSRC-TR-2002-00567

Revision 0

\begin{tabular}{|c|c|c|c|c|}
\hline UIVSO4ION & $1.97515 E-28$ & 0.0 & 0.0 & 0.0 \\
\hline ZNCL3ION & $9.50209 \mathrm{E}-13$ & 0.0 & 0.0 & 0.0 \\
\hline ZNCLION & $1.57635 E-07$ & 0.0 & 0.0 & 0.0 \\
\hline ZNH2 PO4 ION & 4.91130E-07 & 0.0 & 0.0 & 0.0 \\
\hline ZNHCO3ION & $5.85854 \mathrm{E}-07$ & 0.0 & 0.0 & 0.0 \\
\hline ZNION & $3.64782 \mathrm{E}-05$ & 0.0 & 0.0 & 0.0 \\
\hline ZNNO3ION & $1.12664 \mathrm{E}-07$ & 0.0 & 0.0 & 0.0 \\
\hline ZNOH3ION & $7.90121 E-14$ & 0.0 & 0.0 & 0.0 \\
\hline ZNOH 4 ION & $6.00796 \mathrm{E}-20$ & 0.0 & 0.0 & 0.0 \\
\hline ZNOHION & $1.22109 \mathrm{E}-07$ & 0.0 & 0.0 & 0.0 \\
\hline $\mathrm{CA} 3 \mathrm{PO} 42$ & 0.0 & $1.32534 \mathrm{E}-04$ & 0.0 & 0.0 \\
\hline $\mathrm{CU} 3 \mathrm{PO} 42.2 \mathrm{H} 2 \mathrm{O}$ & 0.0 & $3.87732 E-06$ & 0.0 & 0.0 \\
\hline $\mathrm{PB} 3 \mathrm{PO} 42$ & 0.0 & $3.37915 E-06$ & 0.0 & 0.0 \\
\hline UIVO2 & 0.0 & $1.17644 \mathrm{E}-05$ & 0.0 & 0.0 \\
\hline \multirow[t]{2}{*}{ ZN3PO $42.2 \mathrm{H} 2 \mathrm{O}$} & 0.0 & $4.45792 \mathrm{E}-06$ & 0.0 & 0.0 \\
\hline & $==========$ & $==========$ & $==========$ & $==========$ \\
\hline Total g/hr & 999.932 & 0.0682873 & 0.0 & 0.0 \\
\hline Volume, L/hr & 1.00238 & $2.08656 \mathrm{E}-05$ & 0.0 & 0.0 \\
\hline Enthalpy, cal/hr & $-3.79006 E+06$ & -206.797 & 0.0 & 0.0 \\
\hline Density, g/L & 997.561 & 3272.72 & & \\
\hline Vapor fraction & 0.0 & 0.0 & 0.0 & 0.0 \\
\hline Solid fraction & 0.0 & 1. & 0.0 & 0.0 \\
\hline Organic fraction & 0.0 & 0.0 & 0.0 & 0.0 \\
\hline Osmotic Pres, atm & 0.536462 & & & \\
\hline Redox Pot, volts & 0.0 & & & \\
\hline E-Con, 1/ohm-cm & 0.00128076 & & & \\
\hline E-Con, cm2/ohm-mol & 121.201 & & & \\
\hline Abs Visc, cP & 0.893498 & & & \\
\hline Rel Visc & 1.00312 & & & \\
\hline Ionic Strength & 0.0153053 & & & \\
\hline
\end{tabular}


WSRC-TR-2002-00567

Revision 0

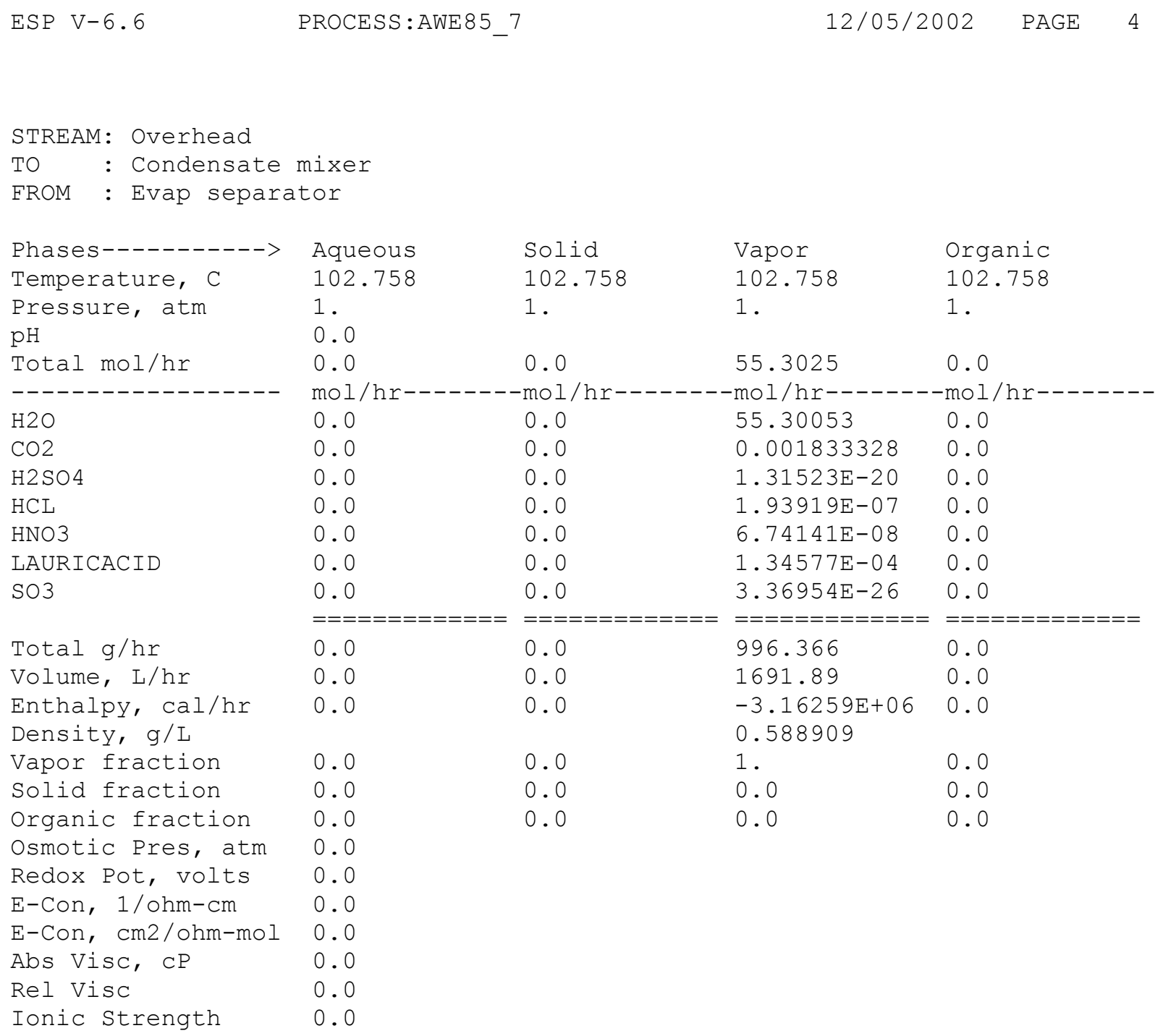


WSRC-TR-2002-00567

Revision 0

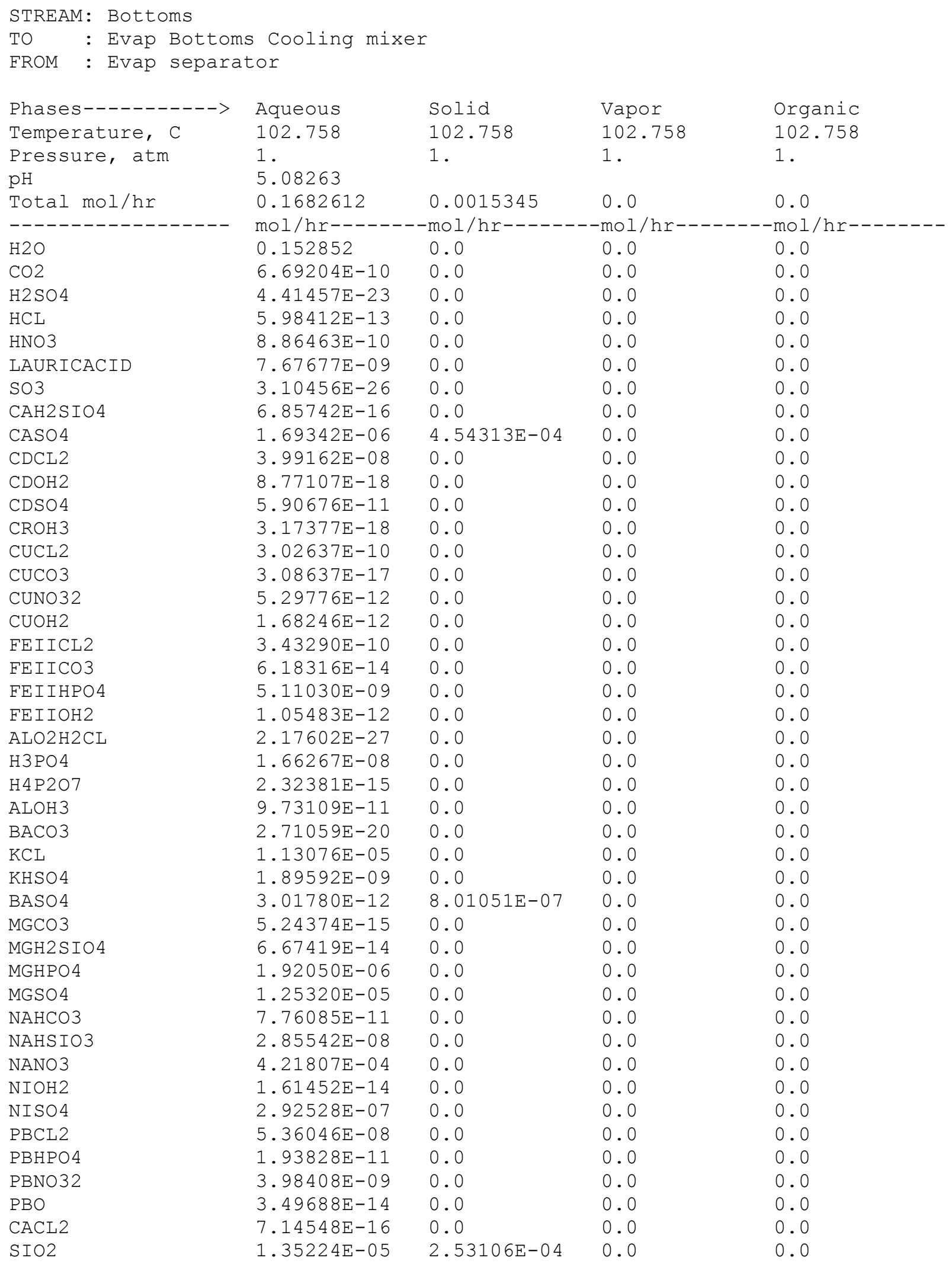




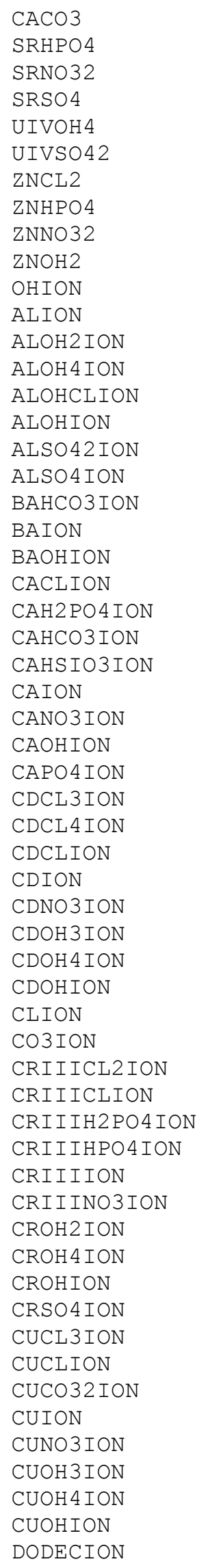

$\begin{array}{ll}1.61750 \mathrm{E}-15 & 0.0 \\ 3.38548 \mathrm{E}-11 & 0.0 \\ 4.67742 \mathrm{E}-08 & 0.0 \\ 2.88560 \mathrm{E}-08 & 3.05916 \mathrm{E}-05 \\ 3.69153 \mathrm{E}-12 & 0.0 \\ 2.07856 \mathrm{E}-23 & 0.0 \\ 1.82490 \mathrm{E}-06 & 0.0 \\ 2.09271 \mathrm{E}-09 & 0.0 \\ 1.98934 \mathrm{E}-09 & 0.0 \\ 1.45141 \mathrm{E}-12 & 0.0 \\ 3.07451 \mathrm{E}-10 & 0.0 \\ 4.05163 \mathrm{E}-14 & 0.0 \\ 1.09963 \mathrm{E}-11 & 0.0 \\ 1.09430 \mathrm{E}-10 & 0.0 \\ 2.49880 \mathrm{E}-12 & 0.0 \\ 1.30805 \mathrm{E}-12 & 0.0 \\ 3.94181 \mathrm{E}-13 & 0.0 \\ 2.98638 \mathrm{E}-13 & 0.0 \\ 3.70181 \mathrm{E}-16 & 0.0 \\ 1.86794 \mathrm{E}-09 & 0.0 \\ 5.75384 \mathrm{E}-17 & 0.0 \\ 3.18549 \mathrm{E}-08 & 0.0 \\ 1.33480 \mathrm{E}-06 & 0.0 \\ 7.19440 \mathrm{E}-13 & 0.0 \\ 5.56821 \mathrm{E}-11 & 0.0 \\ 1.76439 \mathrm{E}-05 & 0.0 \\ 4.62890 \mathrm{E}-06 & 0.0 \\ 1.18284 \mathrm{E}-11 & 0.0 \\ 1.73479 \mathrm{E}-10 & 0.0 \\ 2.20064 \mathrm{E}-08 & 0.0 \\ 3.80307 \mathrm{E}-09 & 0.0 \\ 3.72126 \mathrm{E}-09 & 0.0 \\ 1.08292 \mathrm{E}-10 & 0.0 \\ 2.85790 \mathrm{E}-11 & 0.0 \\ 8.54299 \mathrm{E}-24 & 0.0 \\ 5.09705 \mathrm{E}-30 & 0.0 \\ 1.59765 \mathrm{E}-14 & 0.0 \\ 0.00447894 & 0.0 \\ 8.66006 \mathrm{E}-15 & 0.0 \\ 1.21326 \mathrm{E}-16 & 0.0 \\ 6.56247 \mathrm{E}-16 & 0.0 \\ 9.98496 \mathrm{E}-16 & 0.0 \\ 2.49999 \mathrm{E}-06 & 0.0 \\ 1.40014 \mathrm{E}-18 & 0.0 \\ 1.16513 \mathrm{E}-13 & 0.0 \\ 9.30201 \mathrm{E}-18 & 0.0 \\ 4.83299 \mathrm{E}-23 & 0.0 \\ 1.54433 \mathrm{E}-13 & 0.0 \\ 8.17423 \mathrm{E}-12 & 0.0 \\ 2.87514 \mathrm{E}-12 & 0.0 \\ 3.09150 \mathrm{E}-09 & 0.0 \\ 4.71388 \mathrm{E}-25 & 0.0 \\ 1.15074 \mathrm{E}-09 & 0.0 \\ 2.18339 \mathrm{E}-10 & 0.0 \\ 1.02973 \mathrm{E}-17 & 0.0 \\ 1.45949 \mathrm{E}-22 & 0.0 \\ 2.30264 \mathrm{E}-11 & 0.0 \\ 2.24071 \mathrm{E}-07 & 0.0 \\ & \\ 1.0 & \end{array}$

0.0

0.0

0.0

0.0

0.0

0.0

0.0

0.0

0.0

0.0

0.0

0.0

0.0

0.0

0.0

0.0

0.0

0.0

0.0

0.0

0.0

0.0

0.0

0.0

0.0

0.0

0.0

0.0

0.0

0.0

0.0

0.0

0.0

0.0

0.0

0.0

0.0

0.0

0.0

0.0

0.0

0.0

0.0

0.0

0.0

0.0

0.0

0.0

0.0

0.0

0.0

0.0

0.0

0.0

0.0

0.0

0.0

0.0

0.0 


\begin{tabular}{|c|c|c|c|c|}
\hline FEIICLION & $1.17722 \mathrm{E}-07$ & 0.0 & 0.0 & 0.0 \\
\hline FEIICO32ION & $2.80230 \mathrm{E}-23$ & 0.0 & 0.0 & 0.0 \\
\hline FEIIH2PO4ION & $8.77411 \mathrm{E}-08$ & 0.0 & 0.0 & 0.0 \\
\hline FEIIHCO3ION & $2.99634 \mathrm{E}-14$ & 0.0 & 0.0 & 0.0 \\
\hline FEIIION & $2.32445 E-05$ & 0.0 & 0.0 & 0.0 \\
\hline FEIIOH3ION & $1.34161 \mathrm{E}-16$ & 0.0 & 0.0 & 0.0 \\
\hline FEIIOH 4 ION & $2.20440 \mathrm{E}-23$ & 0.0 & 0.0 & 0.0 \\
\hline FEIIOHION & $1.29778 \mathrm{E}-08$ & 0.0 & 0.0 & 0.0 \\
\hline H2 P2O 7 ION & $4.61453 \mathrm{E}-08$ & 0.0 & 0.0 & 0.0 \\
\hline H2 PO 4 ION & $2.09966 \mathrm{E}-05$ & 0.0 & 0.0 & 0.0 \\
\hline H2SIO4ION & $1.05073 \mathrm{E}-15$ & 0.0 & 0.0 & 0.0 \\
\hline H3P2O 7 ION & $8.42216 \mathrm{E}-12$ & 0.0 & 0.0 & 0.0 \\
\hline H3SIO4 ION & $9.82710 \mathrm{E}-09$ & 0.0 & 0.0 & 0.0 \\
\hline HCO3ION & $6.63714 \mathrm{E}-11$ & 0.0 & 0.0 & 0.0 \\
\hline HION & $3.53386 \mathrm{E}-08$ & 0.0 & 0.0 & 0.0 \\
\hline HP207ION & $6.45615 \mathrm{E}-09$ & 0.0 & 0.0 & 0.0 \\
\hline HPBO2ION & $2.12330 \mathrm{E}-18$ & 0.0 & 0.0 & 0.0 \\
\hline HPO4 ION & $1.41284 \mathrm{E}-06$ & 0.0 & 0.0 & 0.0 \\
\hline HSO 4 ION & $4.25872 \mathrm{E}-07$ & 0.0 & 0.0 & 0.0 \\
\hline KION & $8.96648 \mathrm{E}-04$ & 0.0 & 0.0 & 0.0 \\
\hline KSO4 ION & $1.72519 \mathrm{E}-04$ & 0.0 & 0.0 & 0.0 \\
\hline MGH2 PO 4 ION & $2.94029 \mathrm{E}-06$ & 0.0 & 0.0 & 0.0 \\
\hline MGHCO3ION & $9.53608 \mathrm{E}-12$ & 0.0 & 0.0 & 0.0 \\
\hline MGHSIO3ION & $7.11339 \mathrm{E}-10$ & 0.0 & 0.0 & 0.0 \\
\hline MGION & $4.40300 \mathrm{E}-05$ & 0.0 & 0.0 & 0.0 \\
\hline MGOHION & $6.38780 \mathrm{E}-10$ & 0.0 & 0.0 & 0.0 \\
\hline MGP2O 7 ION & $6.33897 \mathrm{E}-08$ & 0.0 & 0.0 & 0.0 \\
\hline MGPO 4 ION & $1.27839 \mathrm{E}-09$ & 0.0 & 0.0 & 0.0 \\
\hline NACO3ION & $1.16510 \mathrm{E}-15$ & 0.0 & 0.0 & 0.0 \\
\hline NAION & 0.00692554 & 0.0 & 0.0 & 0.0 \\
\hline NASO 4 ION & $1.34775 \mathrm{E}-09$ & 0.0 & 0.0 & 0.0 \\
\hline NICLION & $2.04376 \mathrm{E}-08$ & 0.0 & 0.0 & 0.0 \\
\hline NIION & $3.77255 \mathrm{E}-07$ & 0.0 & 0.0 & 0.0 \\
\hline NINO3ION & $5.05988 \mathrm{E}-08$ & 0.0 & 0.0 & 0.0 \\
\hline NIOH3ION & $2.47291 \mathrm{E}-19$ & 0.0 & 0.0 & 0.0 \\
\hline NIOHION & $6.65740 \mathrm{E}-11$ & 0.0 & 0.0 & 0.0 \\
\hline NO3ION & 0.00134731 & 0.0 & 0.0 & 0.0 \\
\hline P2O7ION & $9.93175 \mathrm{E}-12$ & 0.0 & 0.0 & 0.0 \\
\hline PBCL3ION & $7.15029 \mathrm{E}-08$ & 0.0 & 0.0 & 0.0 \\
\hline PBCL 4 ION & $3.48423 \mathrm{E}-07$ & 0.0 & 0.0 & 0.0 \\
\hline PBCLION & $2.47255 \mathrm{E}-08$ & 0.0 & 0.0 & 0.0 \\
\hline PBH2 PO 4 ION & $1.62179 \mathrm{E}-10$ & 0.0 & 0.0 & 0.0 \\
\hline PBION & $3.54662 \mathrm{E}-09$ & 0.0 & 0.0 & 0.0 \\
\hline PBNO33ION & $1.42914 \mathrm{E}-10$ & 0.0 & 0.0 & 0.0 \\
\hline PBNO3ION & $1.01521 \mathrm{E}-08$ & 0.0 & 0.0 & 0.0 \\
\hline PBOHION & $1.43266 \mathrm{E}-10$ & 0.0 & 0.0 & 0.0 \\
\hline PO4ION & $1.59596 \mathrm{E}-12$ & 0.0 & 0.0 & 0.0 \\
\hline SO4ION & $9.93468 \mathrm{E}-04$ & 0.0 & 0.0 & 0.0 \\
\hline SRION & $4.32871 \mathrm{E}-12$ & 0.0 & 0.0 & 0.0 \\
\hline SRNO3ION & $1.54787 \mathrm{E}-07$ & 0.0 & 0.0 & 0.0 \\
\hline SROHION & $8.22292 \mathrm{E}-14$ & 0.0 & 0.0 & 0.0 \\
\hline SRPO 4 ION & $3.91153 \mathrm{E}-14$ & 0.0 & 0.0 & 0.0 \\
\hline UIVCLION & $2.90894 \mathrm{E}-27$ & 0.0 & 0.0 & 0.0 \\
\hline UIVION & $1.74929 \mathrm{E}-29$ & 0.0 & 0.0 & 0.0 \\
\hline UIVOH2ION & $6.55200 \mathrm{E}-21$ & 0.0 & 0.0 & 0.0 \\
\hline UIVOH3ION & $9.58016 \mathrm{E}-18$ & 0.0 & 0.0 & 0.0 \\
\hline UIVOH5ION & $2.14115 \mathrm{E}-16$ & 0.0 & 0.0 & 0.0 \\
\hline UIVOHION & $4.98634 \mathrm{E}-23$ & 0.0 & 0.0 & 0.0 \\
\hline
\end{tabular}


WSRC-TR-2002-00567

Revision 0

\begin{tabular}{|c|c|c|c|c|}
\hline UIVSO 4 ION & $1.36358 \mathrm{E}-24$ & 0.0 & 0.0 & 0.0 \\
\hline ZNCL3ION & $1.40665 \mathrm{E}-06$ & 0.0 & 0.0 & 0.0 \\
\hline ZNCLION & $6.65518 E-06$ & 0.0 & 0.0 & 0.0 \\
\hline ZNH2 PO4 ION & $8.53166 \mathrm{E}-09$ & 0.0 & 0.0 & 0.0 \\
\hline ZNHCO3ION & $2.14254 \mathrm{E}-14$ & 0.0 & 0.0 & 0.0 \\
\hline ZNION & $5.57964 \mathrm{E}-07$ & 0.0 & 0.0 & 0.0 \\
\hline ZNNO3ION & $6.23505 E-08$ & 0.0 & 0.0 & 0.0 \\
\hline ZNOH3ION & $3.50168 \mathrm{E}-16$ & 0.0 & 0.0 & 0.0 \\
\hline ZNOH 4 ION & $1.76265 \mathrm{E}-21$ & 0.0 & 0.0 & 0.0 \\
\hline ZNOHION & $2.73823 E-08$ & 0.0 & 0.0 & 0.0 \\
\hline ALPO4 & 0.0 & $1.04450 \mathrm{E}-05$ & 0.0 & 0.0 \\
\hline $\mathrm{ALOOH}$ & 0.0 & $2.15482 \mathrm{E}-04$ & 0.0 & 0.0 \\
\hline $\mathrm{CA} 3 \mathrm{PO} 42$ & 0.0 & $3.22247 E-04$ & 0.0 & 0.0 \\
\hline $\mathrm{CU} 3 \mathrm{PO} 42.2 \mathrm{H} 2 \mathrm{O}$ & 0.0 & $3.88293 E-06$ & 0.0 & 0.0 \\
\hline FEII3PO $42.8 \mathrm{H} 2 \mathrm{O}$ & 0.0 & $4.11618 E-05$ & 0.0 & 0.0 \\
\hline MG3PO4 2 & 0.0 & $1.71548 E-04$ & 0.0 & 0.0 \\
\hline $\mathrm{NI} 3 \mathrm{PO} 42$ & 0.0 & $6.04829 \mathrm{E}-07$ & 0.0 & 0.0 \\
\hline $\mathrm{PB} 3 \mathrm{PO} 42$ & 0.0 & $3.20952 \mathrm{E}-06$ & 0.0 & 0.0 \\
\hline UIVO2 & 0.0 & $1.17647 \mathrm{E}-05$ & 0.0 & 0.0 \\
\hline $\mathrm{ZN} 3 \mathrm{PO} 42.2 \mathrm{H} 2 \mathrm{O}$ & 0.0 & $1.53426 E-05$ & 0.0 & 0.0 \\
\hline & $===========$ & $===========$ & $===========$ & $===========$ \\
\hline Total g/hr & 3.3568 & 0.276855 & 0.0 & 0.0 \\
\hline Volume, L/hr & 0.00313217 & $7.40418 E-05$ & 0.0 & 0.0 \\
\hline Enthalpy, cal/hr & -11260.2 & -810.201 & 0.0 & 0.0 \\
\hline Density, g/L & 1071.71 & 3739.17 & & \\
\hline Vapor fraction & 0.0 & 0.0 & 0.0 & 0.0 \\
\hline Solid fraction & 0.0 & 1 . & 0.0 & 0.0 \\
\hline Organic fraction & 0.0 & 0.0 & 0.0 & 0.0 \\
\hline Osmotic Pres, atm & 155.499 & & & \\
\hline Redox Pot, volts & 0.0 & & & \\
\hline E-Con, $1 / \mathrm{ohm}-\mathrm{cm}$ & 0.43356 & & & \\
\hline E-Con, cm2/ohm-mol & 68.1584 & & & \\
\hline Abs Visc, cP & 0.199162 & & & \\
\hline Rel Visc & 0.72786 & & & \\
\hline Ionic Strength & 3.30246 & & & \\
\hline
\end{tabular}


WSRC-TR-2002-00567

Revision 0

\begin{tabular}{|c|c|c|c|c|}
\hline Phases-----------> & Aqueous & SOLld & Vapor & Organic \\
\hline Temperature, C & 25 . & 25 . & 25 . & 25 . \\
\hline \multirow{2}{*}{$\begin{array}{l}\text { Pressure, atm } \\
\mathrm{pH}\end{array}$} & 1. & 1 . & 1 . & 1 . \\
\hline & 6.38737 & & & \\
\hline Total mol/hr & 0.1688008 & 0.00104875 & 0.0 & 0.0 \\
\hline------------------ & $\mathrm{mol} / \mathrm{hr}-----$ & -mol/hr----- & $-\operatorname{mol} / \mathrm{hl}$ & -mol/hr-------- \\
\hline $\mathrm{H} 2 \mathrm{O}$ & 0.15256 & 0.0 & 0.0 & 0.0 \\
\hline $\mathrm{CO} 2$ & $7.49846 \mathrm{E}-11$ & 0.0 & 0.0 & 0.0 \\
\hline $\mathrm{H} 2 \mathrm{SO} 4$ & $1.54162 E-26$ & 0.0 & 0.0 & 0.0 \\
\hline $\mathrm{HCL}$ & $5.41641 \mathrm{E}-16$ & 0.0 & 0.0 & 0.0 \\
\hline HNO3 & $9.53223 E-12$ & 0.0 & 0.0 & 0.0 \\
\hline LAURICACID & $2.10157 E-09$ & 0.0 & 0.0 & 0.0 \\
\hline $\mathrm{SO} 3$ & $2.21849 E-30$ & 0.0 & 0.0 & 0.0 \\
\hline $\mathrm{CAH} 2 \mathrm{SIO} 4$ & $6.34926 \mathrm{E}-15$ & 0.0 & 0.0 & 0.0 \\
\hline $\mathrm{CASO} 4$ & $6.31234 \mathrm{E}-07$ & 0.0 & 0.0 & 0.0 \\
\hline CDCL2 & $2.17858 \mathrm{E}-08$ & 0.0 & 0.0 & 0.0 \\
\hline $\mathrm{CDOH} 2$ & $1.93141 \mathrm{E}-19$ & 0.0 & 0.0 & 0.0 \\
\hline $\mathrm{CDSO} 4$ & $3.35318 \mathrm{E}-11$ & 0.0 & 0.0 & 0.0 \\
\hline $\mathrm{CROH} 3$ & $1.31259 \mathrm{E}-13$ & 0.0 & 0.0 & 0.0 \\
\hline CUCL2 & $6.11149 \mathrm{E}-11$ & 0.0 & 0.0 & 0.0 \\
\hline CUCO3 & $3.85348 E-16$ & 0.0 & 0.0 & 0.0 \\
\hline CUNO32 & $9.12732 \mathrm{E}-13$ & 0.0 & 0.0 & 0.0 \\
\hline CUOH2 & $5.41687 \mathrm{E}-13$ & 0.0 & 0.0 & 0.0 \\
\hline FEIICL2 & $9.39954 \mathrm{E}-12$ & 0.0 & 0.0 & 0.0 \\
\hline FEIICO3 & $6.82097 \mathrm{E}-13$ & 0.0 & 0.0 & 0.0 \\
\hline FEIIHPO4 & $4.60792 E-09$ & 0.0 & 0.0 & 0.0 \\
\hline FEIIOH2 & $1.20011 \mathrm{E}-14$ & 0.0 & 0.0 & 0.0 \\
\hline $\mathrm{ALO} 2 \mathrm{H} 2 \mathrm{CL}$ & $1.16492 \mathrm{E}-28$ & 0.0 & 0.0 & 0.0 \\
\hline H3 $\mathrm{PO} 4$ & $1.11647 \mathrm{E}-10$ & 0.0 & 0.0 & 0.0 \\
\hline H4 P 207 & $1.51436 \mathrm{E}-20$ & 0.0 & 0.0 & 0.0 \\
\hline ALOH3 & $3.90291 E-12$ & $2.25926 \mathrm{E}-04$ & 0.0 & 0.0 \\
\hline $\mathrm{BACO} 3$ & $2.66141 E-20$ & 0.0 & 0.0 & 0.0 \\
\hline $\mathrm{KCL}$ & $2.86969 \mathrm{E}-06$ & 0.0 & 0.0 & 0.0 \\
\hline $\mathrm{KHSO} 4$ & $2.88531 E-12$ & 0.0 & 0.0 & 0.0 \\
\hline $\mathrm{BASO} 4$ & $3.34496 \mathrm{E}-13$ & $6.21775 E-07$ & 0.0 & 0.0 \\
\hline $\mathrm{MGCO} 3$ & $4.39523 E-13$ & 0.0 & 0.0 & 0.0 \\
\hline MGH2SIO4 & 4. $91712 \mathrm{E}-12$ & 0.0 & 0.0 & 0.0 \\
\hline MGHPO 4 & $1.88970 \mathrm{E}-05$ & 0.0 & 0.0 & 0.0 \\
\hline MGSO 4 & $8.81484 E-05$ & 0.0 & 0.0 & 0.0 \\
\hline NAHCO3 & $4.75115 \mathrm{E}-11$ & 0.0 & 0.0 & 0.0 \\
\hline NAHSIO3 & $1.79910 \mathrm{E}-07$ & 0.0 & 0.0 & 0.0 \\
\hline NANO3 & $1.24533 E-04$ & 0.0 & 0.0 & 0.0 \\
\hline $\mathrm{NIOH} 2$ & $5.16589 \mathrm{E}-14$ & 0.0 & 0.0 & 0.0 \\
\hline $\mathrm{NISO} 4$ & $3.30719 E-07$ & 0.0 & 0.0 & 0.0 \\
\hline PBCL2 & $1.15570 \mathrm{E}-09$ & 0.0 & 0.0 & 0.0 \\
\hline PBHPO4 & $1.83223 E-12$ & 0.0 & 0.0 & 0.0 \\
\hline PBNO32 & $1.57557 \mathrm{E}-11$ & 0.0 & 0.0 & 0.0 \\
\hline $\mathrm{PBO}$ & $3.34805 E-16$ & 0.0 & 0.0 & 0.0 \\
\hline CACL2 & $1.91857 \mathrm{E}-24$ & 0.0 & 0.0 & 0.0 \\
\hline $\mathrm{SIO} 2$ & $3.64135 E-06$ & $2.62837 \mathrm{E}-04$ & 0.0 & 0.0 \\
\hline
\end{tabular}




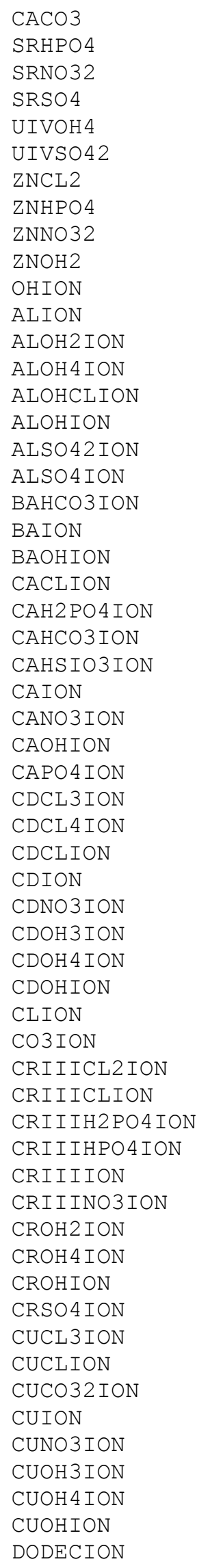

$\begin{array}{ll}1.21572 \mathrm{E}-14 & 0.0 \\ 5.95778 \mathrm{E}-11 & 0.0 \\ 1.98804 \mathrm{E}-08 & 0.0 \\ 5.46729 \mathrm{E}-08 & 0.0 \\ 3.97369 \mathrm{E}-13 & 0.0 \\ 3.18806 \mathrm{E}-27 & 0.0 \\ 1.41585 \mathrm{E}-07 & 0.0 \\ 1.43582 \mathrm{E}-08 & 0.0 \\ 3.72041 \mathrm{E}-09 & 0.0 \\ 1.01492 \mathrm{E}-12 & 0.0 \\ 6.65317 \mathrm{E}-11 & 0.0 \\ 3.93307 \mathrm{E}-14 & 0.0 \\ 8.58320 \mathrm{E}-13 & 0.0 \\ 6.41764 \mathrm{E}-12 & 0.0 \\ 5.34303 \mathrm{E}-13 & 0.0 \\ 1.94275 \mathrm{E}-13 & 0.0 \\ 9.66066 \mathrm{E}-14 & 0.0 \\ 8.41188 \mathrm{E}-14 & 0.0 \\ 2.01537 \mathrm{E}-17 & 0.0 \\ 1.81143 \mathrm{E}-07 & 0.0 \\ 3.85501 \mathrm{E}-19 & 0.0 \\ 1.68298 \mathrm{E}-11 & 0.0 \\ 1.75268 \mathrm{E}-07 & 0.0 \\ 2.08004 \mathrm{E}-12 & 0.0 \\ 4.37003 \mathrm{E}-11 & 0.0 \\ 1.37632 \mathrm{E}-05 & 0.0 \\ 3.51001 \mathrm{E}-06 & 0.0 \\ 1.09424 \mathrm{E}-12 & 0.0 \\ 3.18657 \mathrm{E}-09 & 0.0 \\ 5.25474 \mathrm{E}-09 & 0.0 \\ 4.02542 \mathrm{E}-08 & 0.0 \\ 2.15479 \mathrm{E}-09 & 0.0 \\ 1.40152 \mathrm{E}-10 & 0.0 \\ 1.96073 \mathrm{E}-11 & 0.0 \\ 2.17088 \mathrm{E}-25 & 0.0 \\ 0.0 & 0.0 \\ 2.89330 \mathrm{E}-15 & 0.0 \\ 0.00450254 & 0.0 \\ 2.81457 \mathrm{E}-13 & 0.0 \\ 6.62759 \mathrm{E}-14 & 0.0 \\ 3.57067 \mathrm{E}-13 & 0.0 \\ 2.32962 \mathrm{E}-11 & 0.0 \\ 2.49720 \mathrm{E}-06 & 0.0 \\ 6.64638 \mathrm{E}-15 & 0.0 \\ 2.78132 \mathrm{E}-11 & 0.0 \\ 4.67326 \mathrm{E}-14 & 0.0 \\ 1.21968 \mathrm{E}-17 & 0.0 \\ 4.14073 \mathrm{E}-10 & 0.0 \\ 2.33191 \mathrm{E}-09 & 0.0 \\ 6.38784 \mathrm{E}-13 & 0.0 \\ 2.82756 \mathrm{E}-10 & 0.0 \\ 1.14095 \mathrm{E}-22 & 0.0 \\ 3.62526 \mathrm{E}-10 & 0.0 \\ 6.53035 \mathrm{E}-11 & 0.0 \\ 2.23638 \mathrm{E}-18 & 0.0 \\ 3.92677 \mathrm{E}-24 & 0.0 \\ 3.59719 \mathrm{E}-12 & 0.0 \\ 2.29646 \mathrm{E}-07 & 0.0\end{array}$

0.0

0.0

0.0

0.0

0.0

0.0

0.0

0.0

0.0

0.0

0.0

0.0

0.0

0.0

0.0

0.0

0.0

0.0

0.0

0.0

0.0

0.0

0.0

0.0

0.0

0.0

0.0

0.0

0.0

0.0

0.0

0.0

0.0

0.0

0.0

0.0

0.0

0.0

0.0

0.0

0.0

0.0

0.0

0.0

0.0

0.0

0.0

0.0

0.0

0.0

0.0

0.0

0.0

0.0

0.0

0.0

0.0

0.0
0.0

0.0

0.0

0.0

0.0

0.0

0.0

0.0

0.0

0.0

0.0

0.0

0.0

0.0

0.0

0.0

0.0

0.0

0.0

0.0

0.0

0.0

0.0

0.0

0.0

0.0

0.0

0.0

0.0

0.0

0.0

0.0

0.0

0.0

0.0

0.0

0.0

0.0

0.0

0.0

0.0

0.0

0.0

0.0

0.0

0.0

0.0

0.0

0.0

0.0

0.0

0.0

0.0

0.0

0.0

0.0

0.0

0.0 


\begin{tabular}{|c|c|c|c|c|}
\hline FEIICLION & $5.37684 \mathrm{E}-09$ & 0.0 & 0.0 & 0.0 \\
\hline FEIICO32ION & $4.36860 \mathrm{E}-21$ & 0.0 & 0.0 & 0.0 \\
\hline FEIIH2PO4ION & $1.40434 \mathrm{E}-08$ & 0.0 & 0.0 & 0.0 \\
\hline FEIIHCO3ION & $1.92298 \mathrm{E}-14$ & 0.0 & 0.0 & 0.0 \\
\hline FEIIION & $8.69185 \mathrm{E}-06$ & 0.0 & 0.0 & 0.0 \\
\hline FEIIOH3ION & $3.38176 \mathrm{E}-18$ & 0.0 & 0.0 & 0.0 \\
\hline FEIIOH 4 ION & $8.77628 \mathrm{E}-26$ & 0.0 & 0.0 & 0.0 \\
\hline FEIIOHION & $1.81503 \mathrm{E}-09$ & 0.0 & 0.0 & 0.0 \\
\hline H2 P2O 7 ION & $3.98159 \mathrm{E}-10$ & 0.0 & 0.0 & 0.0 \\
\hline H2 PO 4 ION & $8.26242 \mathrm{E}-06$ & 0.0 & 0.0 & 0.0 \\
\hline H2SIO4ION & $5.78523 \mathrm{E}-15$ & 0.0 & 0.0 & 0.0 \\
\hline H3P2O 7 ION & $2.24978 \mathrm{E}-15$ & 0.0 & 0.0 & 0.0 \\
\hline H3SIO4 ION & $2.00177 \mathrm{E}-09$ & 0.0 & 0.0 & 0.0 \\
\hline HCO3ION & $2.57512 \mathrm{E}-10$ & 0.0 & 0.0 & 0.0 \\
\hline HION & $1.37164 \mathrm{E}-09$ & 0.0 & 0.0 & 0.0 \\
\hline HP207ION & $1.83562 \mathrm{E}-09$ & 0.0 & 0.0 & 0.0 \\
\hline HPBO2ION & $2.11654 \mathrm{E}-20$ & 0.0 & 0.0 & 0.0 \\
\hline HPO4 ION & $1.37372 \mathrm{E}-05$ & 0.0 & 0.0 & 0.0 \\
\hline HSO 4 ION & $2.07573 E-09$ & 0.0 & 0.0 & 0.0 \\
\hline KION & $9.76881 \mathrm{E}-04$ & 0.0 & 0.0 & 0.0 \\
\hline KSO4 ION & $1.00726 \mathrm{E}-04$ & 0.0 & 0.0 & 0.0 \\
\hline MGH2 PO 4 ION & $1.80459 \mathrm{E}-05$ & 0.0 & 0.0 & 0.0 \\
\hline MGHCO3ION & $4.39772 \mathrm{E}-10$ & 0.0 & 0.0 & 0.0 \\
\hline MGHSIO3ION & $6.46907 \mathrm{E}-09$ & 0.0 & 0.0 & 0.0 \\
\hline MGION & $4.50358 \mathrm{E}-04$ & 0.0 & 0.0 & 0.0 \\
\hline MGOHION & $8.40062 \mathrm{E}-10$ & 0.0 & 0.0 & 0.0 \\
\hline MGP2O 7 ION & $3.51310 \mathrm{E}-07$ & 0.0 & 0.0 & 0.0 \\
\hline MGPO 4 ION & $3.23603 E-07$ & 0.0 & 0.0 & 0.0 \\
\hline NACO3ION & $1.18179 \mathrm{E}-13$ & 0.0 & 0.0 & 0.0 \\
\hline NAION & 0.00674452 & 0.0 & 0.0 & 0.0 \\
\hline NASO 4 ION & $4.78149 \mathrm{E}-04$ & 0.0 & 0.0 & 0.0 \\
\hline NICLION & $4.16043 \mathrm{E}-08$ & 0.0 & 0.0 & 0.0 \\
\hline NIION & $1.81219 \mathrm{E}-06$ & 0.0 & 0.0 & 0.0 \\
\hline NINO3ION & $3.70768 \mathrm{E}-07$ & 0.0 & 0.0 & 0.0 \\
\hline NIOH3ION & $4.25884 \mathrm{E}-18$ & 0.0 & 0.0 & 0.0 \\
\hline NIOHION & $8.51938 \mathrm{E}-11$ & 0.0 & 0.0 & 0.0 \\
\hline NO3ION & 0.00164538 & 0.0 & 0.0 & 0.0 \\
\hline P2O7ION & $3.04621 \mathrm{E}-10$ & 0.0 & 0.0 & 0.0 \\
\hline PBCL3ION & $1.99221 \mathrm{E}-09$ & 0.0 & 0.0 & 0.0 \\
\hline PBCL 4 ION & $2.47665 \mathrm{E}-08$ & 0.0 & 0.0 & 0.0 \\
\hline PBCLION & $6.72434 \mathrm{E}-10$ & 0.0 & 0.0 & 0.0 \\
\hline PBH2 PO 4 ION & $1.11284 \mathrm{E}-12$ & 0.0 & 0.0 & 0.0 \\
\hline PBION & $1.94608 \mathrm{E}-10$ & 0.0 & 0.0 & 0.0 \\
\hline PBNO33ION & $3.59776 \mathrm{E}-12$ & 0.0 & 0.0 & 0.0 \\
\hline PBNO3ION & $1.49810 \mathrm{E}-10$ & 0.0 & 0.0 & 0.0 \\
\hline PBOHION & $9.18108 \mathrm{E}-13$ & 0.0 & 0.0 & 0.0 \\
\hline PO4ION & $1.99423 \mathrm{E}-10$ & 0.0 & 0.0 & 0.0 \\
\hline SO4ION & $9.98001 \mathrm{E}-04$ & 0.0 & 0.0 & 0.0 \\
\hline SRION & $3.06117 \mathrm{E}-05$ & 0.0 & 0.0 & 0.0 \\
\hline SRNO3ION & $1.35567 \mathrm{E}-07$ & 0.0 & 0.0 & 0.0 \\
\hline SROHION & $9.30352 \mathrm{E}-15$ & 0.0 & 0.0 & 0.0 \\
\hline SRPO 4 ION & $7.25223 \mathrm{E}-13$ & 0.0 & 0.0 & 0.0 \\
\hline UIVCLION & $6.32402 \mathrm{E}-30$ & 0.0 & 0.0 & 0.0 \\
\hline UIVION & 0.0 & 0.0 & 0.0 & 0.0 \\
\hline UIVOH2ION & $9.53054 \mathrm{E}-23$ & 0.0 & 0.0 & 0.0 \\
\hline UIVOH3ION & $5.88885 \mathrm{E}-18$ & 0.0 & 0.0 & 0.0 \\
\hline UIVOH5ION & $1.66927 \mathrm{E}-16$ & 0.0 & 0.0 & 0.0 \\
\hline UIVOHION & $9.45181 \mathrm{E}-26$ & 0.0 & 0.0 & 0.0 \\
\hline
\end{tabular}


WSRC-TR-2002-00567

Revision 0

\begin{tabular}{|c|c|c|c|c|}
\hline UIVSO4ION & $8.88058 E-28$ & 0.0 & 0.0 & 0.0 \\
\hline ZNCL3 ION & $1.69605 \mathrm{E}-07$ & 0.0 & 0.0 & 0.0 \\
\hline ZNCLION & $2.66885 E-07$ & 0.0 & 0.0 & 0.0 \\
\hline ZNH2 PO4 ION & $7.12375 E-09$ & 0.0 & 0.0 & 0.0 \\
\hline ZNHCO3ION & $1.56169 \mathrm{E}-13$ & 0.0 & 0.0 & 0.0 \\
\hline ZNION & $1.31954 \mathrm{E}-06$ & 0.0 & 0.0 & 0.0 \\
\hline ZNNO3ION & $1.49966 \mathrm{E}-07$ & 0.0 & 0.0 & 0.0 \\
\hline ZNOH3ION & $5.36613 E-17$ & 0.0 & 0.0 & 0.0 \\
\hline ZNOH 4 ION & $2.30984 E-22$ & 0.0 & 0.0 & 0.0 \\
\hline ZNOHION & $3.81361 E-10$ & 0.0 & 0.0 & 0.0 \\
\hline $\mathrm{CA} 3 \mathrm{PO} 42$ & 0.0 & $4.76099 E-04$ & 0.0 & 0.0 \\
\hline $\mathrm{CU} 3 \mathrm{PO} 42.2 \mathrm{H} 2 \mathrm{O}$ & 0.0 & $3.88424 E-06$ & 0.0 & 0.0 \\
\hline FEII3PO $42.8 \mathrm{H} 20$ & 0.0 & $4.60785 E-05$ & 0.0 & 0.0 \\
\hline $\mathrm{PB} 3 \mathrm{PO} 42$ & 0.0 & $3.37198 E-06$ & 0.0 & 0.0 \\
\hline UIVO2 & 0.0 & $1.17647 \mathrm{E}-05$ & 0.0 & 0.0 \\
\hline $\mathrm{ZN} 3 \mathrm{PO} 42.2 \mathrm{H} 2 \mathrm{O}$ & 0.0 & $1.81673 E-05$ & 0.0 & 0.0 \\
\hline & $===========$ & $===========$ & $===========$ & $===========$ \\
\hline Total g/hr & 3.4141 & 0.21955 & 0.0 & 0.0 \\
\hline Volume, L/hr & 0.00296175 & $7.05313 E-05$ & 0.0 & 0.0 \\
\hline Enthalpy, cal/hr & -11634.2 & -667.301 & 0.0 & 0.0 \\
\hline Density, g/L & 1152.73 & 3112.8 & & \\
\hline Vapor fraction & 0.0 & 0.0 & 0.0 & 0.0 \\
\hline Solid fraction & 0.0 & 1 . & 0.0 & 0.0 \\
\hline Organic fraction & 0.0 & 0.0 & 0.0 & 0.0 \\
\hline Osmotic Pres, atm & 147.94 & & & \\
\hline Redox Pot, volts & 0.0 & & & \\
\hline E-Con, $1 / \mathrm{ohm}-\mathrm{cm}$ & 0.163989 & & & \\
\hline E-Con, cm2/ohm-mol & 31.8205 & & & \\
\hline Abs Visc, $c P$ & 1.41151 & & & \\
\hline Rel Visc & 1.58469 & & & \\
\hline Ionic Strength & 3.74 & & & \\
\hline
\end{tabular}


WSRC-TR-2002-00567

Revision 0

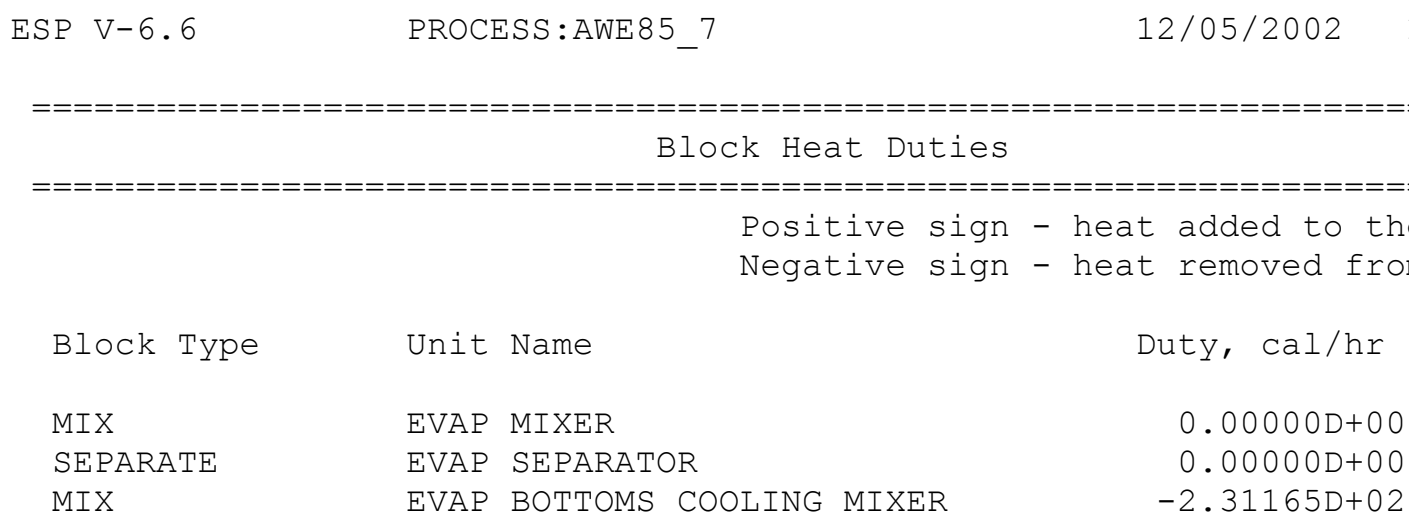


WSRC-TR-2002-00567

Revision 0

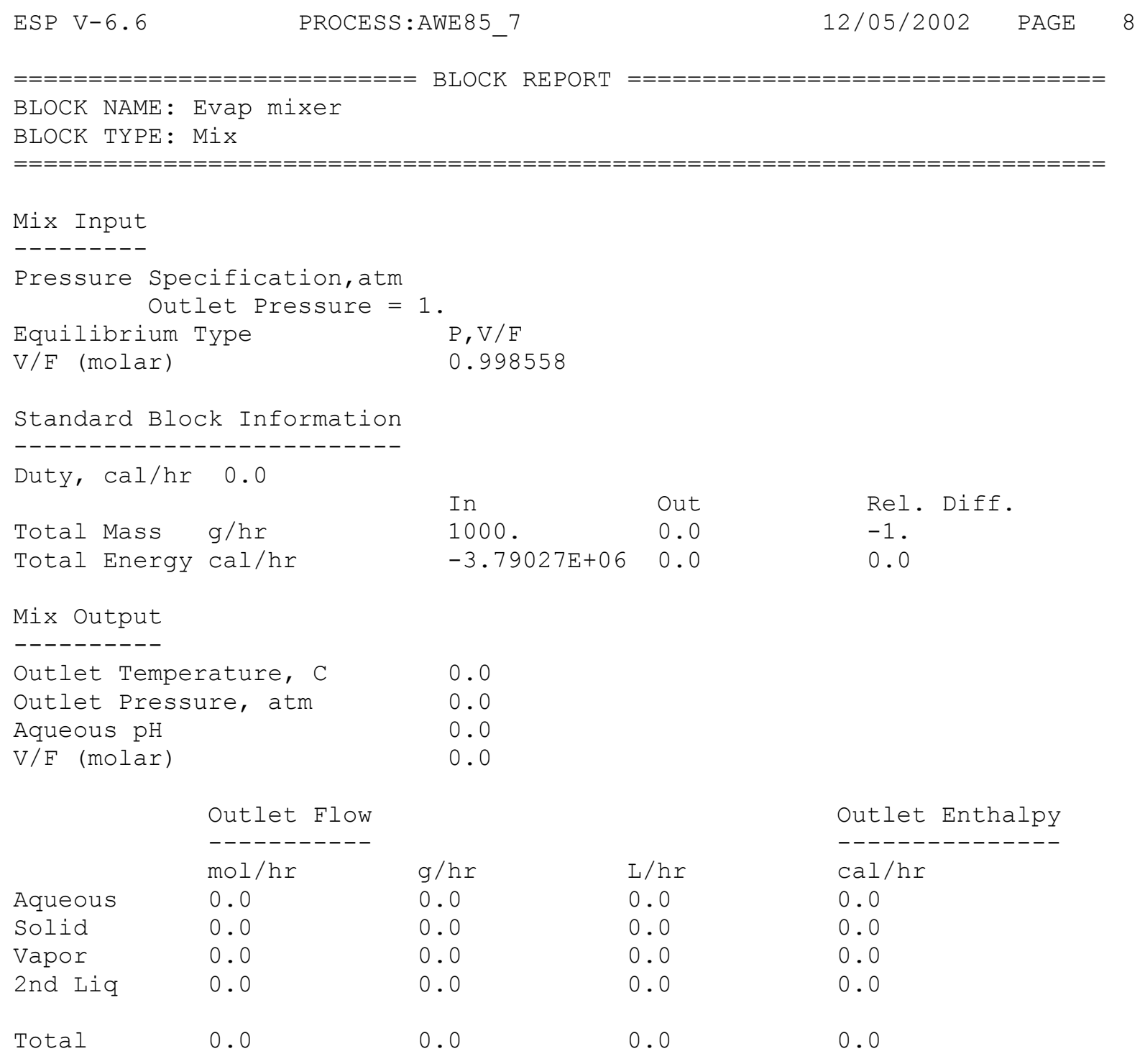


WSRC-TR-2002-00567

Revision 0

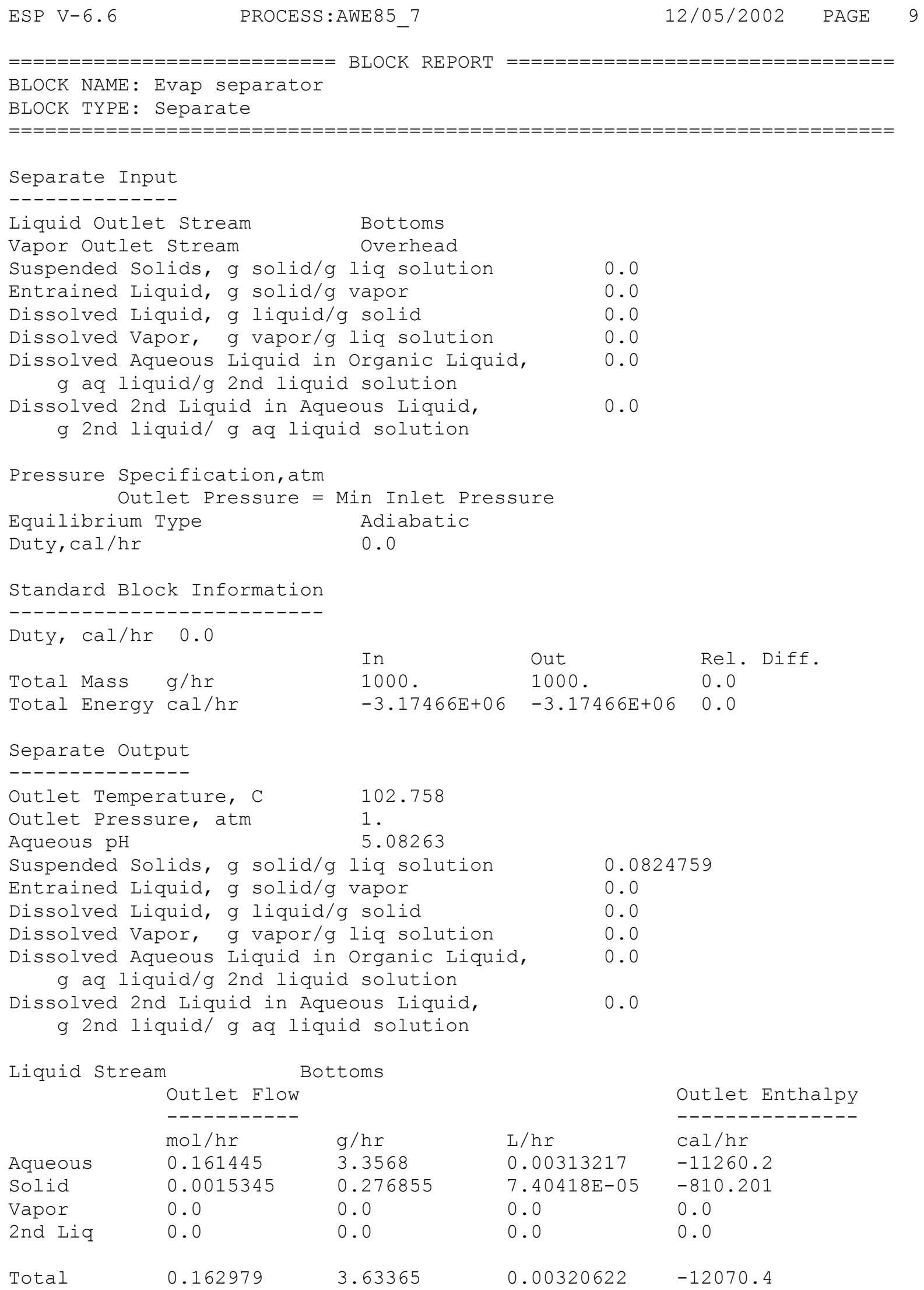


WSRC-TR-2002-00567

Revision 0

ESP V-6.6

PROCESS:AWE 85_7

Vapor Stream

Outlet Flow
--------
mol/hr
0.0
0.0
55.3025
0.0

55.3025
Overhead

g/hr

0.0

0.0

996.366

0.0

996.366
$12 / 05 / 2002$

PAGE

10

$\mathrm{L} / \mathrm{hr}$

0.0

0.0

1691.89

0.0

1691.89

\author{
Outlet Enthalpy \\ cal/hr \\ 0.0 \\ 0.0 \\ $-3.16259 E+06$ \\ 0.0
}

$-3.16259 E+06$ 
WSRC-TR-2002-00567

Revision 0

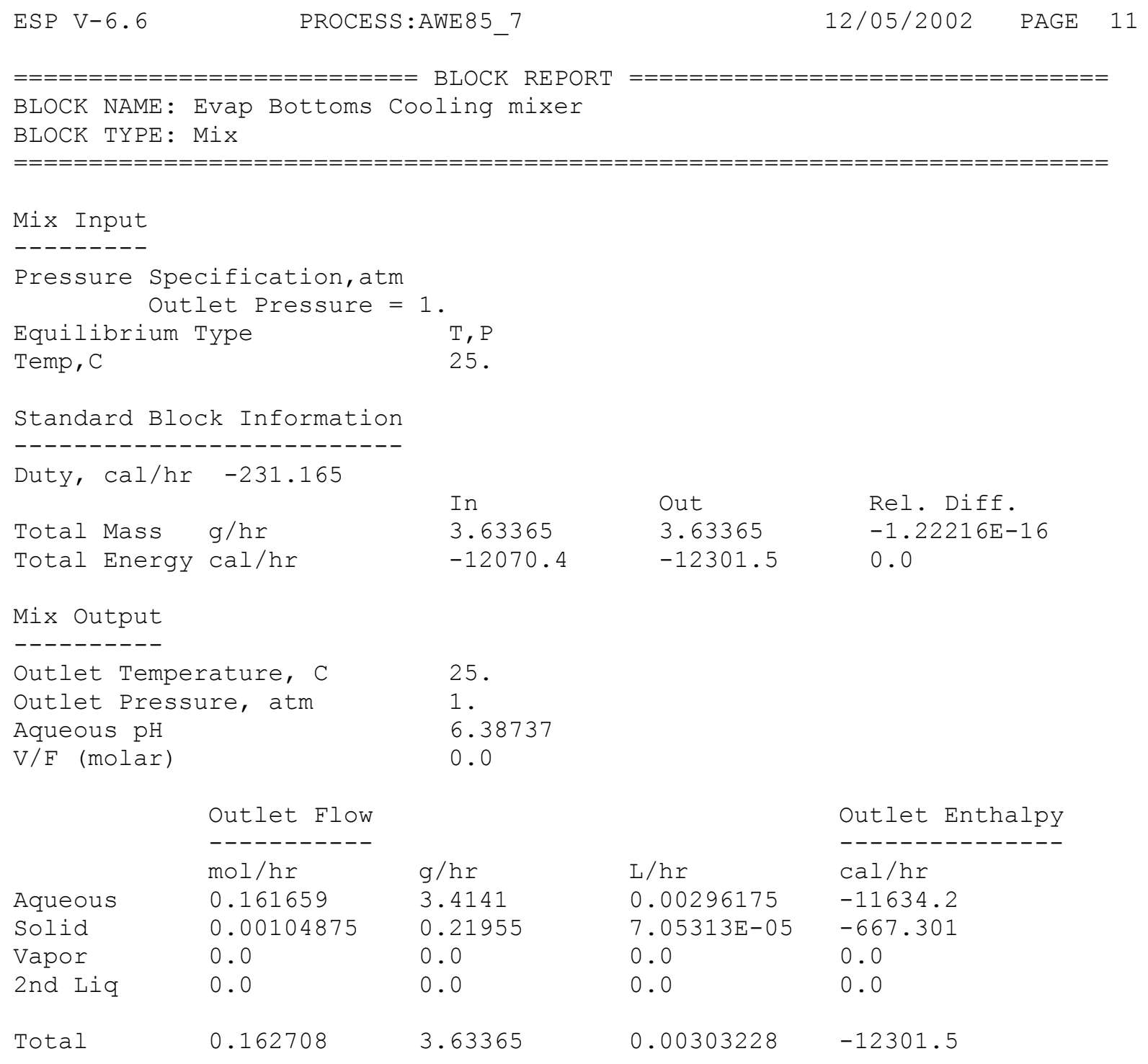


WSRC-TR-2002-00567

Revision 0

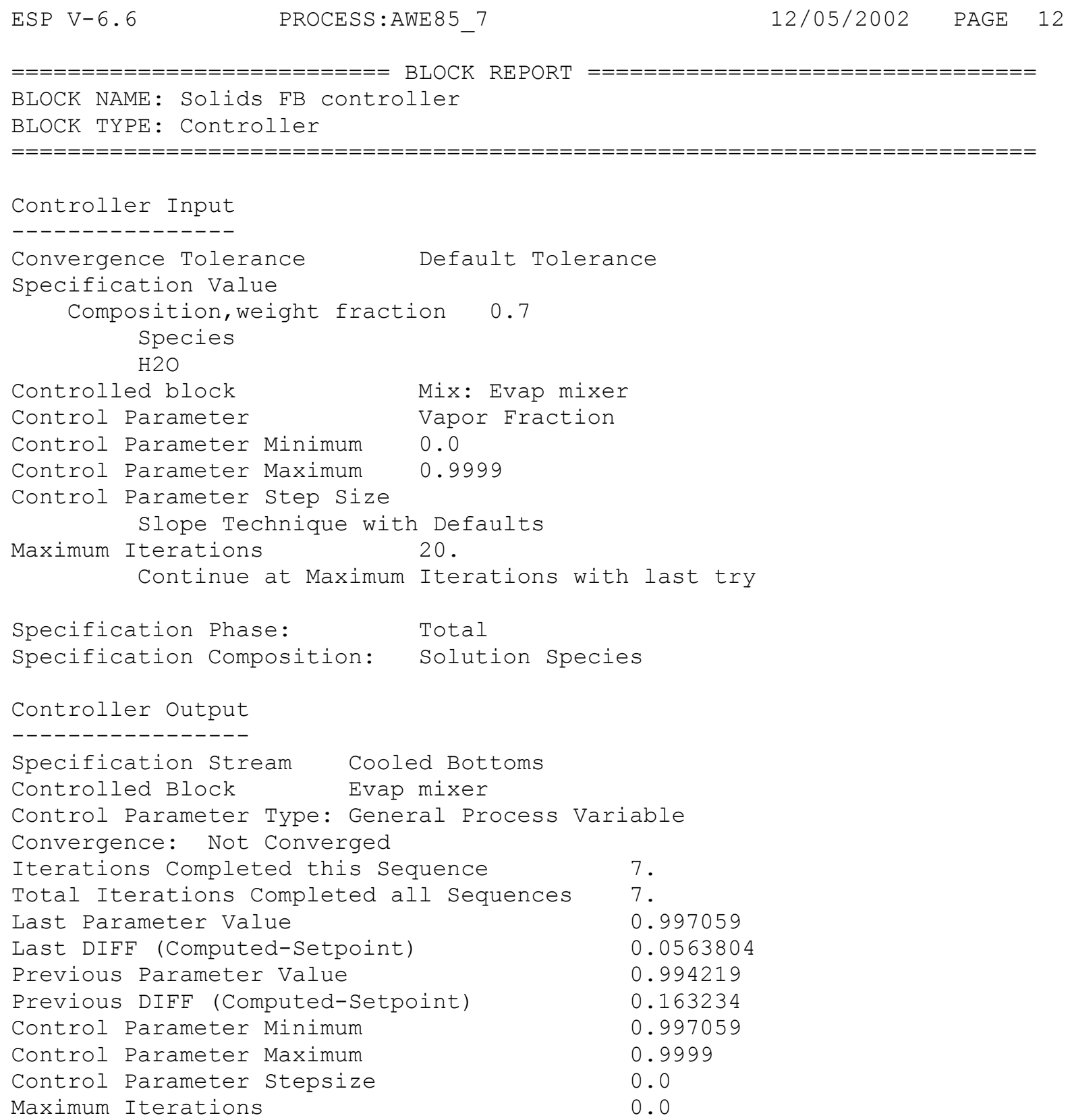

